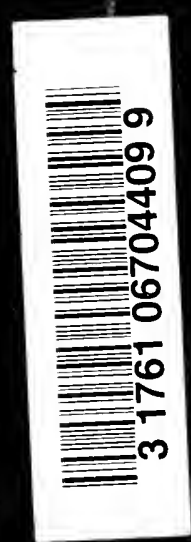




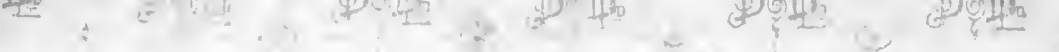

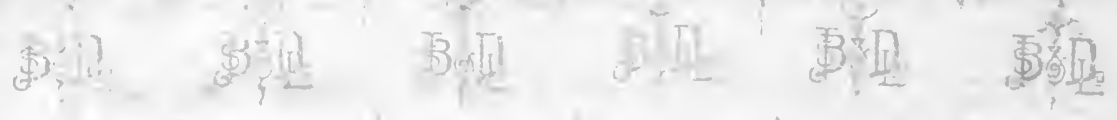

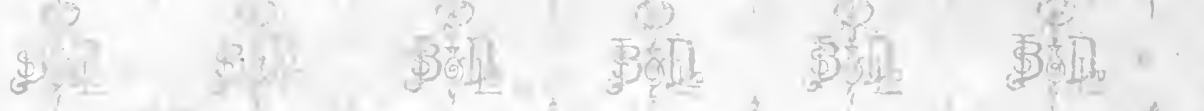

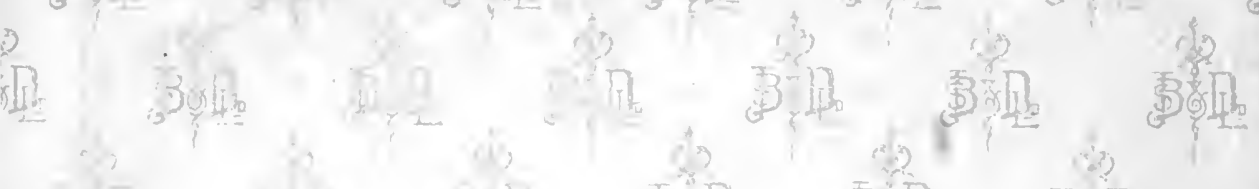

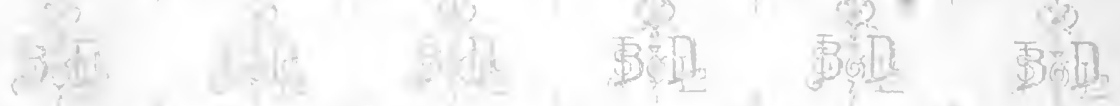
6
97
(2) $35+2$
(3)
$3+1=$
th: 7

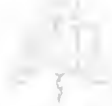
Thing
- AT)
政ID

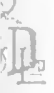

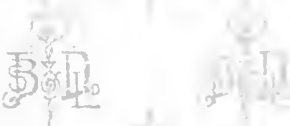

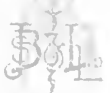
क्र
Sin
整㩆
(i) 512
正
承政
31.1
B. $P$.

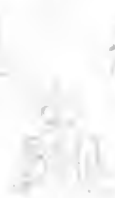
$\frac{1}{4}$
B.

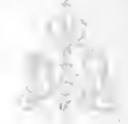
(3) 17

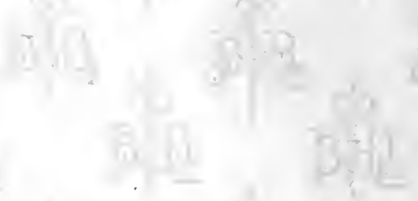
(5) 곤.

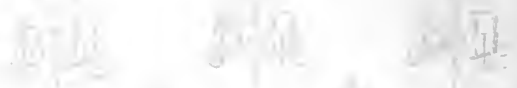
䐎

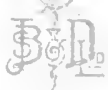
蛋
ब
5it.
bin
7y)
(2) 18
5 I. 38
$(1+5)$

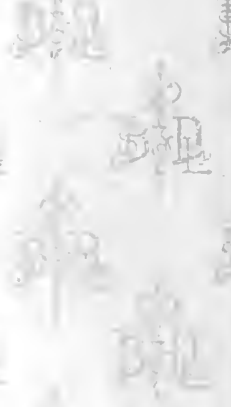
(1) 1
8) (10)
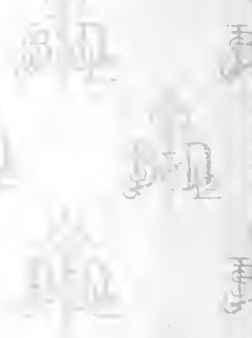

17

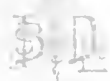
क्षेt
車国
6)
B) 슨
3e 
$(2,-6)+2$

)

\section{\$일}

多

क्षे?

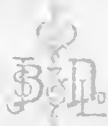

B.

$3=$

IIT

(\$)

(P)

(i)

Bing

車额

it is d

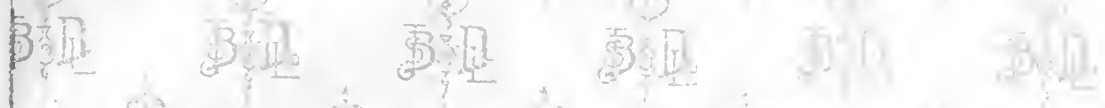

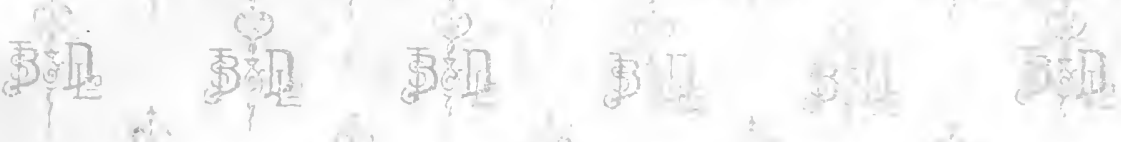

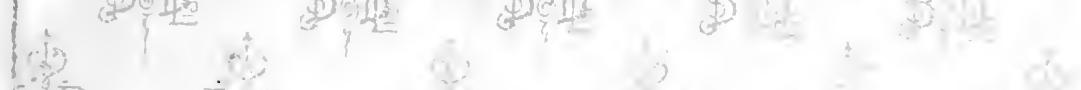

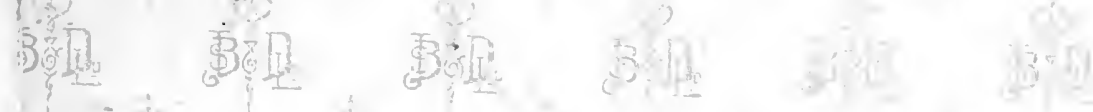

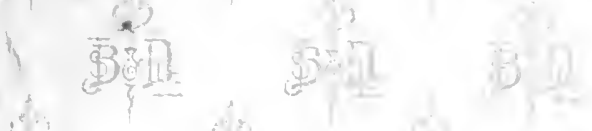

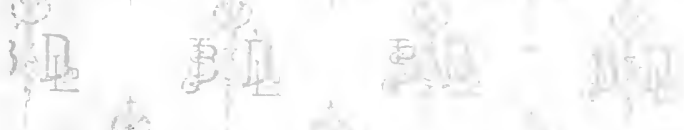

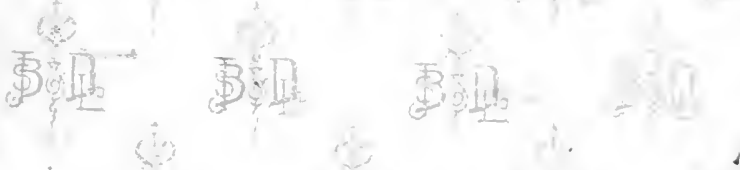

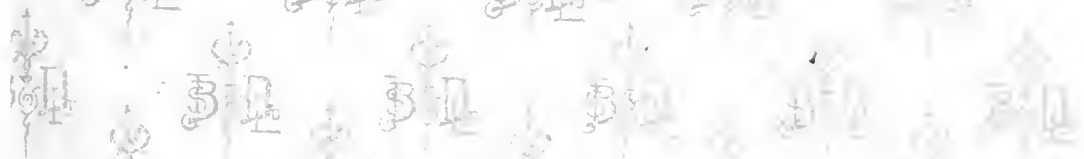

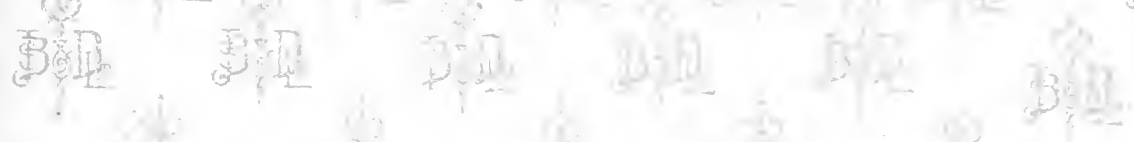

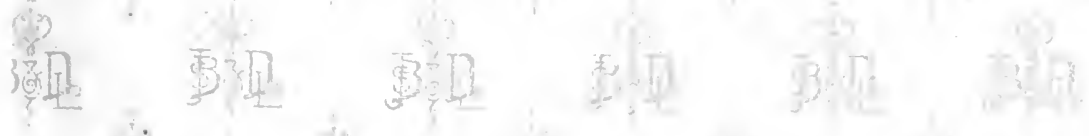

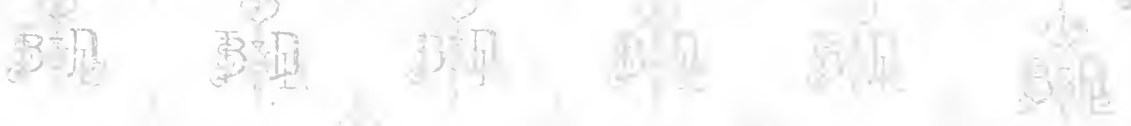

iste
雨是
94
is

के

得且

B. 12

$3+20$
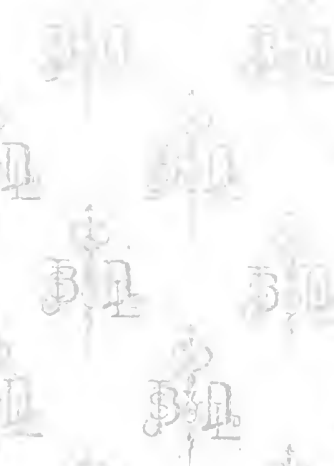

绕

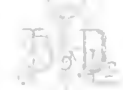

事情

335

If:

35.7
電的

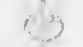

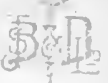

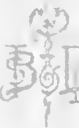



THE INTERNAL SECRETORY ORGANS: THEIR PHYSIOLOGY AND PATHOLOGY 



\section{The Internal Secretory Organs:}

THEIR PHYSIOLOGY AND PATHOLOGY

BY

Professor Dr. ARTUR BIEDL VIENNA

With an Introductory Preface

BY

LEONARD WILLIAMS, M.D., M.R.C.P.,

Physician to the French Hospital;

Assistant Physician to the Metropolitan Hospital

Translated by LindA Forster

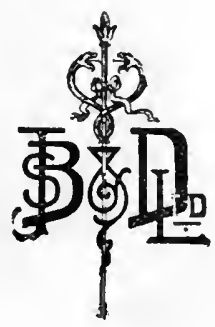

LONDON :

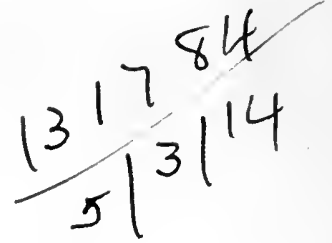

JOHN BALE, SONS \& DANIELSSON, LTD.

OXFORD. HOUSE

83-91, GREAT TITCHFIELD STREET, OXFORD STREET, IV.

I9I 3

All rights reserved 
Digitized by the Internet Archive in 2007 with funding from Microsoft Corporation 


\section{FOREWORD.}

THE subject of the Internal Secretions, or Glandular Physiology as it is sometimes called, is one which is destined to occupy the attention both of physiologists and clinicians for a long time to come. The admirable manner in which the microbic theory of disease has been developed and pressed into practical employment has served completely to revolutionize the surgery, and profoundly to modify the medicine, of thirty years ago. But the truth of the theory and the wonders which it has wrought leave unexplained certain problems in pathology which cry aloud for solution. Microbes do not explain gout, diabetes, Graves's disease or Hodgkin's disease, to mention but a few which inhabit the vast territory which remains to be conquered. Upon these and allied diseases the study of the internal secretions promises to throw some light. It has already illuminated myxœdema, acromegaly, Addison's disease, and some others, and the promise which it holds is as enticing and fascinating as its pursuit is baffling.

The condition precedent of any real progress on the clinical side is an exhaustive study of the physiological side. Professor Biedl's book supplies this in a painstaking and methodical manner. It comprises all the work of an experimental kind which has been done in any country up to within a very recent date. It is for the most part content to leave that work to speak for itself, and does not unduly burden the reader with explanations, or mislead him with premature theories.

To unravel the tangled skein of the inter-relation of the various internal secretory glands must be a task for many workers 
vi.

Forezord

over a long period. Professor Biedl has rendered this task very much more simple than it could have been in the absence of some co-ordinating and focussing text-books such as he has now given to labourers in this field.

The English translation has been done in a manner deserving of the highest praise. I have had many opportunities of comparing it with the original German text, and have in every case had cause to admire the faithful, accurate, and even graceful rendering of the meaning of obscure and highly technical passages.

LEONARD WILLIAMS. 


\section{O N T E N T S}

PART I.-GENERAL.

Historical and Introductory

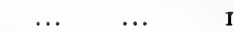

Definition and Limitation of the term "Internal Secretion " $\ldots \ldots$

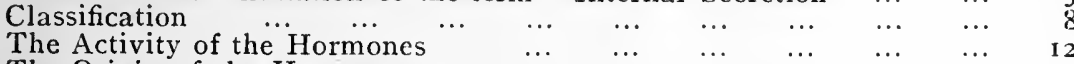

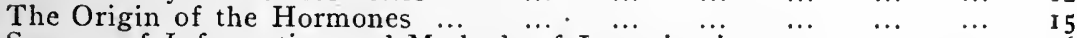

Sources of Information and Methods of Investigation $\quad \ldots \quad$...

PART II.-SPECIAL.

The Thiroid Apparatus

$\begin{array}{lllllll}\cdots & \cdots & \cdots & \cdots & \cdots & \cdots & \cdots\end{array}$

$\begin{array}{lllllllll}\text { Histology } & \cdots & \cdots & \ldots & \ldots & \ldots & \ldots & \ldots & \ldots \\ \text { Ch } & \ldots & \ldots & \ldots & \ldots & \ldots & \ldots & \ldots\end{array}$

26

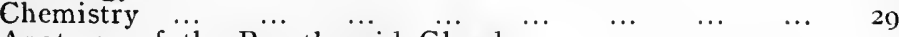

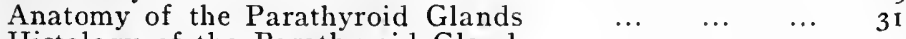

Histology of the Parathyroid Glands $\quad \ldots \quad \ldots, \ldots . \quad 32$

History of the Development of the Organs of the Neck ... 33

Comparative Anatomy of the Parathyroid Glands $\quad \ldots \quad 34$

$\begin{array}{llllll}\text { Physiology of the Thyroid Apparatus } & \ldots & \ldots & \ldots & \ldots & 36\end{array}$

$\begin{array}{llllllllll}\text { Historical } & \ldots & \ldots & \ldots & \ldots & \ldots & \ldots & \ldots & \ldots & 36\end{array}$

$\begin{array}{lllllll}\text { Tetania Parathyropriva } & \ldots & \ldots & \ldots & \ldots & \ldots & 36 \\ & \ldots & \ldots & \ldots & \ldots & \ldots & 39\end{array}$

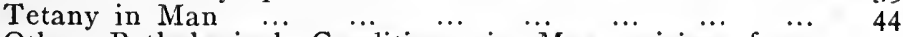

Other Pathological Conditions in Man arising from Derangement of the Function of the Parathyroid Glands

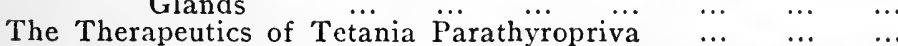

The Relationship between the Thyroid and Parathyroid Glands

The Function of the Parathyroid Glands ... ... ... ...

The Tetany Toxin $\ldots$. $\ldots$. $\ldots \ldots$ i $\ldots \ldots$

The Point of Attack of the Hypothetical Tetany Toxin ...

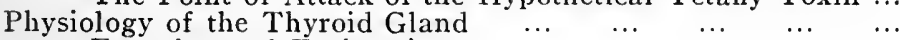

$\begin{array}{llllll}\text { Experimental Extirpation } & \ldots & \ldots & \ldots & \ldots & \ldots\end{array}$

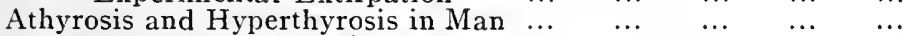

$\begin{array}{lllllll}\text { Operative Athyrosis } & \ldots & \ldots & \ldots & \ldots & \ldots & \ldots\end{array}$

$\begin{array}{lllll}\text { True.Athyrosis and Hyperthyrosis } & \ldots & \ldots & \ldots & \ldots\end{array}$

Substitution Therapy $\ldots$
The Metabolic Changes in Hypothyrosis and the manner in which they are influenced by the Exhibition of $\begin{array}{lllllll}\text { Thyroid Extract } & \ldots & \ldots & \ldots & \ldots & \ldots & \ldots\end{array}$

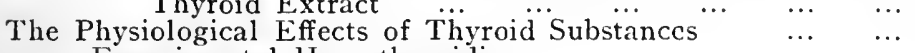

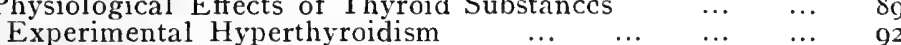

$\begin{array}{rlllll}\text { Hyperthyroidism in Man } & \ldots \\ \text { As Produced by the Exhibition of Thyroid } & \ldots & \ldots & \ldots & 92 \\ \text { Cland } & \ldots & 92\end{array}$

Conditions which may be classed as those of true Hyperthyroidism

$\begin{array}{llllllll}\text { Theory of the Thyroid Function } & \cdots & \cdots & \cdots & \cdots & \cdots & 93\end{array}$

$\begin{array}{llllllllll}\text { The. Thymus GLAND } & \ldots & \ldots & \ldots & \ldots & \ldots & \ldots & \ldots & \ldots & \text { I }\end{array}$

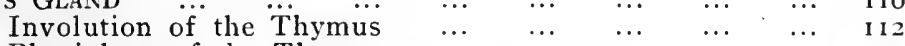

$\begin{array}{lllllll}\text { Physiology of the Thymus } & \ldots & \ldots & \ldots & \ldots & \ldots & 113\end{array}$

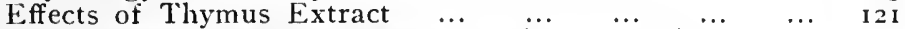

Mors Thymica and the Status Thymicolymphaticus $\quad \ldots .612$ 
viii.

The SUPRARENAL SySTEM

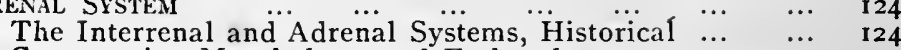

$\begin{array}{llll}\text { Comparative Morphology and Embryology } & \ldots & \ldots & \mathbf{1} 27\end{array}$

$\begin{array}{lllllllll}\text { Physiology of the Suprarenals } & \ldots & \ldots & \ldots & \ldots & \ldots & 134\end{array}$

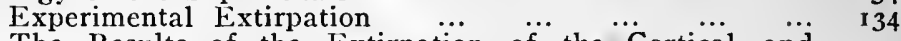

The Results of the Extirpation of the $\dddot{C}^{\circ}$ Cortical and

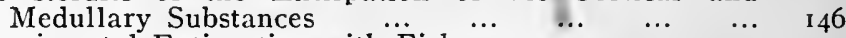

Experimental Extirpation with $\begin{array}{llllll}\text { Fish } & \ldots & \ldots & \ldots & \ldots & 146\end{array}$

Symptomatology of Suppression of the Suprarenal Function ... 153

Suppression of the Suprarenal Function in Man $\ldots \quad \ldots . \quad 158$

$\begin{array}{llllll}\text { The Symptomatology of Addison's Disease } & \ldots & \ldots & \ldots & \text { I63 }\end{array}$

$\begin{array}{llllllllll}\text { Suprarenal Substitution } & \ldots & \ldots & \ldots & \ldots & \ldots & \ldots & \text { I65 }\end{array}$

$\begin{array}{llllllllll}\text { Organotherapy } & \ldots & \ldots & \ldots & \ldots & \ldots & \ldots & \ldots & 165\end{array}$

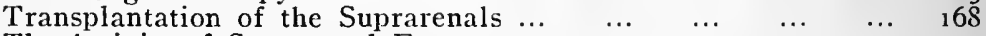

$\begin{array}{llllllll}\text { The Activity of Suprarenal Extract } & \ldots & \ldots & \ldots & \ldots & \ldots & 173\end{array}$

Chemistry of the Specific Active Substance present in the Supra-

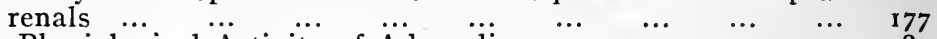

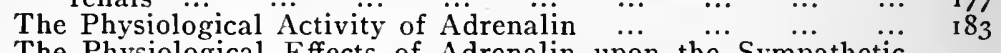

The Physiological Effects of Adrenalin upon the Sympathetic

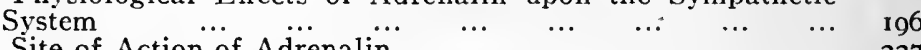

$\begin{array}{lllllll}\text { The Site of Action of Adrenalin } & \ldots & \ldots & \ldots & \ldots & \ldots & \\ \end{array}$

$\begin{array}{lllllllll}\text { The By-effects of Adrenalin } & \ldots & \ldots & \ldots & \ldots & \ldots & \ldots & 234\end{array}$

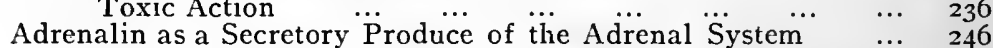

Experimental Suprarenal Stimulation, Experimental Hypersecretion

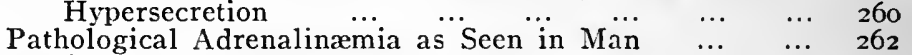

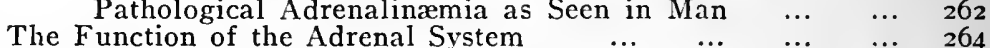

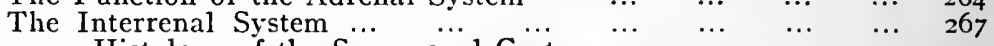

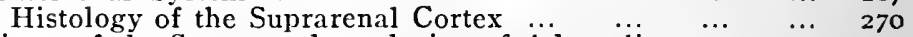

$\begin{array}{llllll}\text { Chemistry of the Suprarenal exclusive of Adrenalin } & \ldots & \ldots & 274\end{array}$

Theory of the Function of the Interrenal System $-\quad \ldots . \quad \ldots \quad 292$

$\begin{array}{lllllll}\text { The Suprarenal considered as an Entity } & \ldots & \ldots & \ldots & \ldots & 295\end{array}$

$\begin{array}{llllll}\text { Carotid Gland and Coccygeal Bodies } & \ldots & \ldots & \ldots & \ldots & \\ & \ldots & \ldots & \end{array}$

Hypophysis Cerebri (Cerebral Appendage, Pituitary Gland) $\ldots \quad \ldots \quad 305$

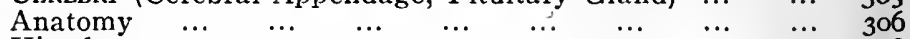

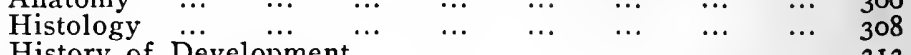

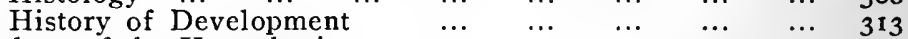

$\begin{array}{llllllll}\text { Physiology of the Hypophysis } & \ldots & \ldots & \ldots & \ldots & \ldots & \ldots & 315\end{array}$

$\begin{array}{llllllllll}\text { Pituitary Extract } & \ldots & \ldots & \ldots & \ldots & \ldots & \ldots & 321\end{array}$

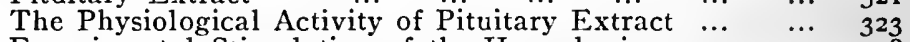

Experimental Stimulation of the Hypophysis $\quad \ldots . \quad \ldots .328$

$\begin{array}{lllllllll}\text { Diseases of the Hypophysis in Man } & \ldots & \ldots & \ldots & \ldots & \ldots & 330\end{array}$

$\begin{array}{lllllll}\text { Theory of the Function of the Hypophysis } & \ldots & \ldots & \ldots & \ldots & 348\end{array}$

The Pineal Body (Glandulæ pinealis, Epiphysis, Conarium) $\ldots \quad \ldots \quad 350$

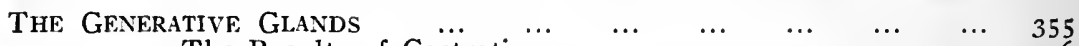

The Results of Castration $\ldots$. $\ldots$ Activity of $\begin{array}{lllll}\ldots & \ldots & \ldots & \ldots & 355\end{array}$

The Sexually Undifferentiated Activity of the Hormone
in the Generative Glands $\ldots$
...

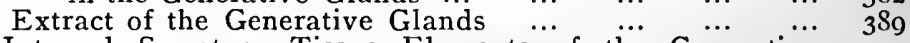

The Internal Secretory Tissue Elements of the Generative

$\begin{array}{llllllllll}\text { Glands } & \ldots & \ldots & \ldots & \ldots & \ldots & \ldots & \ldots & \ldots & 39 \mathrm{r}\end{array}$

Internal Secretion of other parts of the Genital Apparatus $\quad \ldots \quad 4$ ro

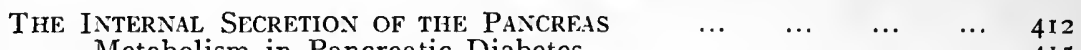

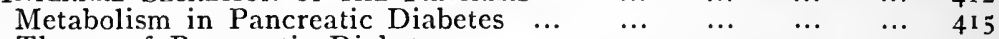

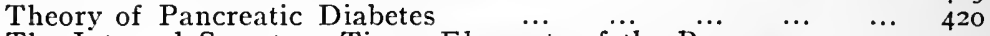

The Internal Secretory Tissue Elements of the Pancreas $\quad \ldots \quad 432$

The Internal Secretion of the Gastric and INtestinal Mecosa $\ldots \quad 438$

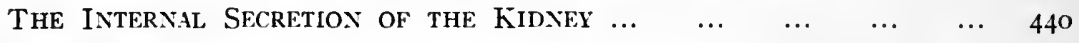

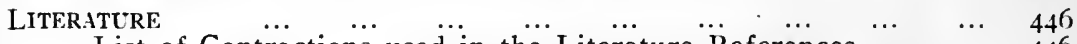

List of Contractions used in the Literature References $\ldots . . .64$ 


\title{
The Internal Secretory Organs: their Physiology and Pathology.
}

\author{
PART I.-GENERAL.
}

\section{HISTORICAL AND INTRODUCTORY.}

WE have long been aware of a reciprocity of activity between different parts of the body, and this inter-relation of function is called the consensus partium. But, until very recently, the haziest ideas prevailed as to the manner in which the individual organs react upon one another and the mechanism by which their interbalance is maintained.

We are now, however, in a position to advance the logical postulate, that the mediating agents can be those only which, while having access to each organ, yet come together at a common centre. The central nervous system supplies an excellent example of such a mechanism, affecting and controlling as it does the correlation of the various physiological activities. Messages are carried by the centripetal nerves from the extreme periphery to the centre, and this centre is enabled, by means of the centrifugal nerves, to convey ever-changing impressions to different parts of the body. When the so-called "reflexes" were discovered, partly by Descartes in the 17 th, and finally by Prochaska in the 19 th century, the prompt and purposeful intervention of the central nervous system in the regulation of organic function became firmly established.

An impression conveyed from the periphery to the nervous centre will excite activity in an entirely different part of the body. Though this is accomplished without the assistance either of the consciousness or of the will, it is neither casual nor purposeless in its happening. On the contrary, the degree of activity is calculated to the needs of the organism and is in accordance with changes in the conditions surrounding it. This reflex character is in part congenital and is an integral factor in the organization of the nervous system; in part it is acquired, and is the result of habits formed during life. By its means the co-ordinated activities of the different parts of the body are regulated and controlled and it was for this reason that scientists until recently 
believed that the nervous system was the sole mediating agent by which the consensus partium was maintained.

Such was, naturally enough, the view adopted by clinicians. to explain disturbances of functional equilibrium. Derangement of function in any direction was said to produce a change, both qualitative and quantitative, in the nature of the stimuli and, reacting through the nervous system upon other organs, was. believed to produce in them also a departure from the normal, thus giving rise to the most varied pathological symptoms. From this arose the doctrine of the reflex manifestation of diseases, a doctrine which was until quite recently universally accepted and is not, even now, entirely discredited. The changes, for example, which for centuries have been known to take place in the organism after suppression of the secretion of the sexual glands, whether by castration or by the physiological phenomena of the climacteric, were believed to result from a purely nervous reflex action.

But, logically faultless though this hypothesis be, and founded as it certainly is upon a sound physiological and anatomical basis, it has not always maintained an undivided supremacy. Looking back over the fields of biology and general pathology we find that the neural theory of the correlation of organs was. companioned by a belief in their humoral relationship, and that this latter theory is even the older of the two.

In the healing arts of primitive peoples, as well as in the earlier periods of scientific medicine, remedies were employed and measures resorted to, which are the direct forerunners of the organo-therapy of to-day. Hahnemann's homœeopathy threw out a branch-isopathy-which in certain directions proved itself stronger than the parent stem. In every organic disease it was the practice of isopathy to administer the healthy animal organ; fox-lung was given in pulmonary disease, wolf's liver in liver troubles, ox-eyes in ocular affections, and so on. In spite of the haziness of the ideas which prompted these measures, they were certainly carried out in the spirit which animates the humoral pathology of to-day, for they recognized the fact that each organ plays a part in maintaining the balance of secretion and has thus an influence upon the organism as a whole.

The discovery of the circulation of the blood supplied a second channel by means of which the different parts of the body may be brought into relationship. And yet in the early days of the new humoral pathology, at a time when changes in the constitution of the blood-plasma were universally recognized as causes of disease, the blood-stream was very rarely admitted as a factor in the consensus partium. This was due to the fact that at that time changes in the nature of the blood-plasma were believed to result, partly from external conditions and partly from retention in the blood of normal secretions. Thus, Bordeu (a predecessor of Brown-Séquard, who lived in the second half of 
the 18 th century and was discovered by Neuburger) ascribed the changes which occur after removal of the sexual organs and in certain other conditions, to a deficiency or superfluity of the glandular secretions. The existence of normal and pathological secretions was known, but no account was taken of substances which are formed by all the organs, including those which are not in the first instance secretary organs, and which contribute something to the composition of the plasma.

An important step forward was marked by Johannes Müller in his text-book of physiology. He drew a sharp distinction between secretion-the formation of a specific chemical substance by the organ-and excretion - the change of place of the already formed substance. He recognized secretory cells, secretory membranes, and secretory glands. Among the latter he included those organs which are known as the vascular ganglia or blood glands, namely: the spleen, the thyroid, the suprarenals, the thymus, and the placenta.

Müller believed that these ductless glands " exert a plastic influence upon the juices which are within them, which circulate through them, and which are carried from them by the general circulation. Unlike the other glands, however, they exercise no external influence."

It was Professor A. A. Berthold, of Göttingen, who, in 1849, first demonstrated by means of experiment the nature of the activity of a true ductless gland; that is to say, he showed the influence which an organ through which the blood-stream circulates, can exert upon the composition of the blood and thus upon the entire organism. He removed the testicle from cocks and grafted it upon another part of the body; and he found that " the animals remained male in regard to voice, reproductive instinct, fighting spirit, and growth of comb and wattles." He concluded that "the consensus is maintained by the productive influence of the testicle; that is to say, by its effect upon the blood and, through the blood, upon the organism." The honour of having first proved the existence of an "internal secretion" and of having recognized its significance, belongs undoubtedly to Berthold.

Berthold's discovery, however, received no recognition in his own day. The physiologist, Rudolf Wagner, was unable to confirm the experiments, and Berthold was probably unknown in general circles. Claude Bernard, in $\mathrm{r} 855$, is generally credited with having been the first to give expression to the doctrine of an internal secretion. He maintained that, not only the glands by their secretory processes-sécrétion externe-by means of which substances are withdrawn from the blood, but that every organ of the body, by a process of sécrétion interne whereby it yields its products up to the blood, determines the composition of the blood and can, under certain conditions, modify and even change it. 
Bernard showed that, in the case of the liver, in addition to the external secretion of gall there is an internal secretion or glycogenesis, that is to say, the aggregation of glycogen and the formation of sugar; the latter being afterwards conveyed into the blood-stream. He endeavoured to prove that the function of internal secretion is under the control of the nervous system.

That the composition of the blood is susceptible to the influence of the specific products of individual organs was, as we see, known as long ago as the middle of the last century. Even the designation "internal secretion" dates from that time. And yet the introduction of the theory of the internal secretions is universally ascribed to Brown-Séquard. This is because he was the first to express the idea* that all glands, with or without ducts, supply to the blood substances which are either useful or essential, ? and the lack of which may produce pathological signs. It was not until twenty years later that he was able to give experimental proof of his views.

The actual date upon which the doctrine of the internal secretions may be said to have been born is June I, I889. It was the occasion of that memorable meeting of the Société de Biologie, Paris, when Brown-Séquard, then 72 years of age, described the experiments which, in order to prove his hypothesis, he had carried out upon his own person, by the subcutaneous injection of testicular extract. He found that after the injections, and indisputably as a result of them, he experienced an access of physical strength and an invigoration of cerebral function which were quite extraordinary. Appetite improved, the bowels were regulated, and there was a marked increase in mental activity. This lecture and the detailed publication which followed it, a few weeks later, were not only the actual foundations of the theory of internal secretion, but they furnished the first practical application of a new method of treatment, namely, that now known as organo-therapy, or the Brown-Séquard method.

It is not possible to estimate Brown-Séquard's work at its proper value, unless it is kept distinct from the method which he inaugurated and more especially from the abuses which followed it, with which Brown-Séquard himself was unfortunately not entirely unconnected. What he really did do was to prove by experimental means the existence of a process of internal secretion, which is in the nature of a chemical interchange between the different organs and which is of the highest importance to the economy. His theories were at once accepted in France and to some extent in England. German physiologists and clinicians were extremely sceptical. Nevertheless it should not be forgotten that Hansemann, in Germany, soon afterwards adopted a similar standpoint. Like Brown-Séquard, he assumed a physiological

* In his address before the Medical Faculty of Paris in I869. 
interchange between the cells of different organs, and this functional interdependence he designated "cell-altruism." As the founder of the doctrine of internal secretion, Brown-Séquard has opened to physiology a new and fruitful field for experiment; he has pared the way for the right understanding of many pathological derangements; and he has pointed out a rational and, in many cases, a remarkably successful method of treatment.

\section{DEFINITION AND LIMITATION OF THE TERM "INTERNAL SECRETION."}

Two agents are concerned in ordering and maintaining the complex activity of the animal organism. In addition to the nervous intercommunication, which is admittedly the agent in effecting rapid adjustments, there is also a chemical correlation of the different organs. In accordance with the latter, each organ, each tissue, and even each cell, by means of its specific secretory products, acting through the agency of the blood-stream, is enabled to exert a definite influence upon other parts of the body. In this manner the equilibrium of the various parts is guaranteed.

It must not be imagined, however, that chemical communication is effected by the agency of the blood-stream only. We know, for instance, that the acid secretion which is elaborated upon the superficial membrane of the stomach, causes the pyloric sphincter to relax at regular intervals, and that movements of the intestinal canal are provoked by the bowel-contents. Granting that in these instances there is also a nervous reflex which is set in motion by the secretions of the gastric and intestinal mucosa, yet the formation of secretin is clearly an instance of chemical interaction taking place upon an internal surface.

As soon as it enters the duodenum, the acid gastric secretion (then called chyme) excites the cells of the intestinal mucosa to form secretin, which, in its turn, acting through the bloodstream, stimulates the liver to an increased production of bile, excites the formation of pancreatic secretion, and promotes secretion by the glands of the intestinal walls. Bayliss and Starling, who have made a special study of these processes, propose that the name "hormone" should be given to those physiological substances which act as chemical stimuli and serve as intermediaries between different parts of the body. "Hormone" is derived from the Greek op $\mu a ́ \omega$, meaning to awake or excite, and the term has been universally adopted.

All organs which supply substances which are capable of exciting or in any way of affecting the functions of other organs, may be described as "hormone-producing." Schiefferdecker proposes that they should be called "Beeinflussungsorgane" 
(organs of influence), which might be contracted into B-organs. It is, however, more expedient to call them internal secretory organs. It must be clearly understood, however, that not every change in the blood-plasma due to organic intervention, and not all metakerastic organic activity, is to be included under the heading "internal secretory." The term can apply to the formation of chemical substances only. Those organs, for instance, which are known to be the formative agents of the morphological elements of the blood (as the spleen, the lymphatics and the bone marrow)-in so far as the changes they effect in the character of the blood are due to the contribution of new form-elementsare not to be regarded as internal secretory.

Very little is known about the specific form in which the true internal secretions, or hormones, find their way into the blood. It may be that they enter the circulation as morphological constituents of the blood, or perhaps in combination with such. But, in the present state of our knowledge, we must bear in mind that, though the cellular elements of the blood may possess internal secretions, they are not internal secretions in themselves.

The internal secretions include the chemical products of organic metabolism only, though this limitation of the term is not accepted without question by morphologists.

Assuming always that the elaboration of substances from materials withdrawn from the blood and the returning of the changed materials back to the blood again, is a property common to all living tissue, yet, from the point of view of the morphologist, certain tissues only possess a true secretory value. According to this view, those tissues only are able to secrete which possess histologically differentiated epithelial cells, whether occurring singly or in groups. An excretory gland is no longer to be recognized in virtue of its possession of an excretory duct, but by its consti- $\Lambda$ tution in epithelial elements. Should the duct be absent but the epithelial structure present, then, and then only, should the term "internal secretory gland" be employed. Following out this idea, the internal secretory glands would include: The thyroid, the parathyroids, the epithelial layer of the suprarenals, the glandular portion of the hypophysis cerebri (pituitary gland), and the inter-tubular cell-agglomerations of the pancreas. Other tissues, as, for instance, the so-called carotid gland, the medullary substance of the suprarenals, and the chromaffine tissue in general, would be excluded from this group.

The last-named example clearly shows, however, that there is not sufficient grounds for adopting a morphological and genetic limitation of the term "internal secretion," and that to do so would not in any way conduce to a solution of the question. We have unequivocal proof of the internal secretory activity of the chromaffine tissue, for we are able to obtain its secreted substance in a state of chemical purity. Yet the chromaffine tissue cannot be 
described, either -genetically or morphologically, as epithelial or glandular tissue. If, in considering the activity of the true glands -whether ductless or otherwise-we are prepared to recognize one secretion only, and to describe it as internal or external according to the direction in which it is discharged, then we are compelled to regard all glands as having more than one secretory surface, and should need proof, moreover, that both internal and external secretion are elaborated by the same morphological elements. But it will be obvious, from what has gone before, that such a theory cannot be made to include all the facts. For instance, it is very possible that, in the case of the male sexual glands, the internal secretion is not elaborated from the spermatozoa-producing elements, but from the interstitial tissues.

Looking at the matter from the physiological point of view, we must assume that all organs, in addition to their special function which is determined by their structure, possess a specific productive activity, otherwise an internal secretion, which is largely independent of structure. The description " internal secretion " is hardly to be improved upon. It expresses the idea which has animated the doctrine from its earliest inception, namely, that the specific internal secretions are physiological hormones affecting the activity of other parts of the body and affecting the body as a whole, and that they are the means by which the chemical correlations of the different organs are brought about.

There is a large group of chemical adjustments which cannot be included among the internal secretions. It comprises all those readjustments which take place when drugs or poisons are introduced into the economy. During the last decades, investigation has revealed a not inconsiderable group of chemical interactivities which are aroused in the body for purposes of offence and defence, not only by the causes of disease, but by poisons of all sorts. There is no doubt whatever that the processes by which antitoxins and antibodies are formed, are the outcome of a modification of the chemical activity of the tissues, and that the most important part is played by the internal secretion of the cells. It is, however, expedient to the present purpose to confine the discussion solely to those chemical correlationships which are maintained between the different parts of the body without the intervention of external agents.

Nevertheless, it is not possible, in the nature of things, to keep entirely within these limits, seeing that no organism lives for itself alone, but is in constant relationship with its surroundings. Foreign materials are introduced into the economy by means of foodstuffs; in the intestinal canal they become so changed by the flora and the ferments that poisonous products of decomposition are formed, and these find their way into the blood-stream. The intermediary toxic substances formed from the splitting up of the foodstuffs, especially of albumen, are rendered innocuous by the 
agency of the internal secretions of various organs, more particularly the liver. It is a question of expediency and one which each physiologist will decide for himself, whether or not these antitoxic processes-the measures, that is, which are adopted on the part of the organism to guard against auto-intoxicationshould be included in the definition of the internal secretions, while the antitoxic activity of the liver, where exogenic poisons are concerned, is excluded from it.

\section{CLASSIFICATION.}

Having defined the field which the internal secretions cover, the next step is to classify them. Berzelius divided the true glandular secretions into recrementitial and excrementitial, and, up to now, a similar distinction has been maintained in regard to the internal secretions. As far as we can judge at present, the process of internal secretion has a twofold aspect. In the first place there is positive recrementitial secretion or production of hormones. In the second, there is that activity of the organs which is sometimes called negative secretion, which concerns itself with the neutralization of materials circulating in the blood which might otherwise impair the activity of other organs, or even endanger the well-being of the entire organism. The latter function is a parallel to the external secretion of the true excretory organs, as the skin, the intestine, the lungs and the kidneys, by whose agency the body is freed from useless and harmful substances.

But it is very obvious that there is an essential difference between the two processes. The excretory organs are able to expel undesirable material from the body, but in the case of the internal secretory organs this method is impossible. In the absence of any channel communicating with the exterior, the substances which have been withdrawn from the blood must, sooner or later, be returned to the blood. The organism as a whole, therefore, can be relieved in only one of two ways. Either the effete substances must be retained in the organ, or they must be rendered innocuous before they are again returned to the blood.

The former process comes into play chiefly in the management of those waste materials which circulate in the blood as formed elements. The adenoid or lymphatic tissue distributed over the body, the bone-marrow, the spleen, and the liver are all organs which, by a kind of filtering process, retain the broken-down remains of old red and white corpuscles, remnants of tissue, traces of pigment, \&c., and in this way they free the blood of useless morphological elements. The particles which have become fixed then undergo secondary changes, after which they supply the material for fresh organic activity; thus, iron goes to build up the red blood corpuscles, pigment to the preparation of bile. 
The second method of dealing with soluble toxic substances is by a process of chemical neutralization. Here also, to a large extent; the toxins are first fixed and then deposited in the individual organs. The juices of the body and the component parts of the cells and tissues play the part of chemical prophylactics. Thus, exogenous and endogenous toxins combine with the alkali and albumin of the blood; with the nuclein in the nuclei of the white corpuscles, and with glycogen and the biliary acids in the liver.

Neutralization in the narrower sense, that, namely, of the conversion of poisons into harmless substances, is carried on by chemical processes of various kinds; such are: oxidation and reduction, hydration and dehydration, building up and breaking down, \&c.

A special significance attaches to the chemical affinity which certain toxins display for substances normally occurring in the body. In I824, Woehter discovered the affinity of benzoic acid for glycocoll, a product of the decomposition of albumin. Baumann, in 1879 , was able to prove that the highly toxic bodies of a phenol-like nature which occur in the body and are the products of the decomposition of albumin, together with the sulphates derived from the same source, have an affinity for the non-toxic hydrogen ethyl sulphates which are discharged in the urine. Other chemical affinities were discovered later as, for instance, that of glycuronic acid, which is derived from the carbohydrates, for mercapturic acid, and carbamic acid for urea. The parts of the body where these chemical pairings take place are largely unknown. We only know that in the dog, and probably also in man, hippuric acid is formed in the kidney, while in the herbivora it is also formed in other organs, such as the liver and spleen.

The best known example of the antitoxic function of an organ is the formation of urea by the liver. Urea is the principal end-product of the metabolism of albumin, but the simple hydrolytic division of the albumin-molecule produces it in the most minute quantities only. It comes into existence by means of a synthetic process strictly in conformity with its constitution. There are various hypotheses regarding this synthesis; according to one, the base of urea is ammonium carbamate, according to another it is ammonium carbonate. It is certain, however, that ammonia is formed in considerable quantities by the body, and that the body is able to convert the ammoniacal salts which reach it from the exterior into urea. The organ which is most concerned in converting the toxic agents into the comparatively non-toxic urea is undoubtedly the liver. Blood containing ammonia is being constantly carried by the portal vein to the liver (Drechsel proved the presence of ammonium carbamate in the blood of dogs) and is there rendered innocuous. This can be shown 
experimentally by establishing a communication by means of Eck's fistula between the portal vein and the vena cava of dogs, thus enabling the blood from the portal vein to pass directly into the general circulation without traversing the liver. After a few days such animals show symptoms of acute poisoning; there are changes in the sensorium, intervals of excitement and depression, clonic and tonic spasms, and coma. These are sometimes spontaneous, but they are more likely to appear if the animals are fed on meat. A similar picture of intoxication can be produced in healthy dogs by the subcutaneous injection of carbamates. When given by the mouth, there is no reaction to these salts in normal animals; those with Eck's fistula present the same symptoms as after feeding with meat. The amount of ammonia in the urine is increased, while the amount of urea is proportionally diminished.

These experiments show that, by the formation of urea, the liver exercises a neutralizing activity, whereby the organism is safeguarded from poisoning by ammonia.

Up to the present, however, this is the only case in which we are in a position to point with certainty, not only to the exact nature of the poison but also to the organ which neutralizes it, and, though this is only partial, to the chemical process by which the transformation takes place.

In most cases suspension of function, in man or animals, gives rise to symptoms resembling those of auto-intoxication. It must not be forgotten, however, that an assumption of autointoxication founded upon the symptom-complex, has no real explanatory value as long as we are without definite information as to the nature of the toxic agent. Formerly, while the doctrine of internal secretion was still in its infancy, the antitoxic action of the organs was all too frequently invoked to explain the symptoms which appear upon the suppression of any organic function. But the fruitlessness of the search for the supposed poison has led to a considerable modification of this hypothesis. Indeed, the theory necessarily falls to the ground if we are able to find another satisfactory explanation of the symptoms which follow suppression.

As a matter of fact, we have ample foundation upon which to formulate another hypothesis. In every organ there is a store of potential energy, a reserve of labour laid by in a special manner, enabling it, upon adequate provocation, to fulfil its specific function. Under normal conditions the potential energy is kept in check and prevented from manifesting itself. The sight of an organism showing signs of extreme suffering, racked with pain, and with all the organs in a state of excessive activity, would suggest, even to the untutored intelligence, that the potential energy is relieved from its normal clieck, and that the bonds are burst which had hindered the individual parts from the full 
display of their powers. It has long been known that the nervous system has control not only of the stimuli which promote function, but also of those inhibitory activities which are essential to the regulation of the working economy.

That many chemical substances present in the body also exercise this inhibitory influence is a discovery of very recent date.

We know, from Loeb's interesting experiments, that certain activities of muscles, nerves, and glands, especially the muscular irritability which manifests itself as rhythmic contraction, depend upon the presence under certain conditions of $\mathrm{Na}_{-}, \mathrm{K}_{-}, \mathrm{Ca}$-, and probably also Mg-ions; and that every change in the relations of these ions changes the properties of the tissues. A sudden change in the relationship of the ions will, according to the nature of the change, bring about either stimulation or inhibition of function.

The Ca-ions, which are necessary to all life, act as inhibitors of muscular activity, and Loeb draws attention to the fact that, if it were not for the presence under normal conditions of $\mathrm{Ca}$-ions in the blood, the skeletal muscles of the body would be in a state of constant rhythmic contraction.

If, then, certain organs produce substances which are inhibitory to the activity of other organs, it is obvious that failure on the part of an organ to provide its inhibitory material may give rise to symptoms similar to those of poisoning.

Having obtained definite proof that in certain tissues substances are formed which, by acting as chemical stimuli, bring about a decreased activity on the part of other organs-in other words, they exercise an inhibitory influence upon functional activity -we are in a position to abandon the hypothesis of autointoxication and to adopt that of the presence of inhibitory hormones. This theory attains its highest measure of probability when we find it supported by the results of organo-therapy; when, in fact, the disturbances which regularly ensue upon the cessation of any organic function, may be prevented or even cured by the administration of the extract of that organ or of the chemical material obtained from it.

In those cases where organo-therapy has failed, while the symptoms of suppression have disappeared upon the direct transplantation of the organ, the results have usually been ascribed to an antitoxic activity. But this conclusion is by no means incontestable. The transplantation of an organ is a method which closely reproduces the natural conditions and, where successful, restores the organ to the body in its natural structure and function. All that has happened is that the organ in question has, to a certain extent, changed its locality. The medical method is, in reality, a substitute for the surgical and is a less perfect and less certain imitation of natural measures. Where the extract 
is employed, only that proportion of the active substance which is for the time being in the tissues, and this is doubtless very small, becomes absorbed; while under natural conditions or after transplantation, the increase is almost incalculable because there is constant production.

In face of the difficulties which beset the theory that the neutralization of toxins takes place within the organ itself, we are forced to assume that there is a relationship between the process of neutralization and that of secretion. The neutralization of the hypothetical toxin is assumed to take place, not within the organ itself, but in the blood-stream and by the agency of a specific antitoxic organic secretion. In that case the function exercised by the organ would not be merely excrementitial (using the word in its special sense), but truly secretory.

This hypothesis can only hold good where the presence of the supposed toxin on the one hand, and the process of neutralization on the other, can be demonstrated in vitro. As our knowledge of the internal secretions increases, the idea of a process of neutralization of toxins becomes narrowed in its application and, though to-day it may still be essential to the doctrine as a whole, in course of time it will probably disappear altogether.

\section{THE ACTIVITY OF THE HORMONES.}

We will now endeavour to classify the internal secretory organs according to the manner in which their secretions act. The secretory products fall readily into two groups. The first of these comprises those secretions which, either from their organic constitution or because they act as sources of energy, assist in the building up or the activity of other organs. These may be called the "nutritive secretions." The second group includes those substances whose sole influence is upon the manner in which the materials and energies, already collected in the organs, are employed. These are the true hormones.

In addition to the antitoxic and neutralizing substances already described, the first group includes the nutrient or productive internal secretions. These are the nutritive material of the organs, and they are derived by the chemical agency of the intestinal wall and glands of the appendix, from the foodstuffs. As products of assimilation, they pass into the blood- and lymphstreams and are carried to the different tissues. The albumin, which is absorbed by and circulates in the blood, and the sugar, formed from glycogen by the liver, are instances of such wastesecretions, for they supply the direct material for the renovation and activity of the organs.

A large number of these substances, however, act also as hormones and exert a modifying influence upon the very processes by which they are changed and consumed. We know, 
for instance, that an increased amount of albumin in the circulation, by its direct influence upon the organs, gives rise to an increased decomposition of albumin. Thus, albumin in the form in which it is produced by the digestive apparatus, is both a nutrient and a hormone. Another nutrient which acts likewise as a hormone, is the oxygen inhaled by the lungs, though its production is not the result of any organic activity.

In spite of this dual function it is, in many instances, not only possible but expedient to draw a distinguishing line between the nutrient secretions and the hormones. The factor of differentiation is to be found in the quantitative conditions. Substances which supply either material or energy, or both, must inevitably be present in considerable quantities. Hormones, on the other hand, which give rise to energetic processes, or influence them in a special direction, must be able to produce their effect even in minimal quantities. The practical outcome is this; that, where a nutrient secretion is lacking it is useless to attempt artificial substitution, because of the large amount of material which would be required; whereas we are frequently able artificially to replace a missing hormone.

The true hormones have been variously classified according to the manner in which they act. Thus we have formative and protective, growth-promoting and growth-retarding, stimulant and inhibitory.

If we adopt Hering's theories upon the chemical processes in living matter as a basis for classification, the hormones will fall into two groups; according to the nature of their activity they are either assimilatory or disassimilatory. Those in the first group promote the anabolic phase of metabolism-those processes, that is, which conduce to the building up of living matter. Those in the second promote the katabolic phase, for they favour and promote the decomposition of living matter. Each stimulus to assimilation necessitates an inhibition of disassimilation, and vice versa.

All the known hormones can be included in one of these two classes. The promotion of growth supplies an example of an assimilatory hormone which, at the same time, exerts an inhibitory influence upon disassimilation. In the case of retardation of growth the process is reversed. Every increase in normal activity, whether this be an improved secretion, a stronger muscular action, or any other augmented performance, is always associated with an increased disassimilation, which is the work of a disassimilatory hormone. But it must be remembered that, in the organism, as elsewhere, no work can be performed without expenditure. So long as the metabolic equilibrium is maintained, every decomposition must be inevitably succeeded by a regeneration. Even in the case of hyperactivity, so long as exhaustion does not supervene, there will be a continual regeneration of the 
living substance and therewith a restitution of provision for labour. With the cessation of the disassimilatory stimulus assimilation becomes excessive and, if the process is frequently repeated, the well-known phenomena of organic hypertrophy will make their appearance.

Some experiments by Kronecker and Cutter are interesting in this connection. These authors found that, after the exercise of the lower extremities by mountain climbing, the upper extremities, which did not participate in the exercise, showed a perceptible increase in the development of the biceps group. This increased capacity for labour without previous direct exercise of function, which, by repetition of the same process, would certainly lead to a "labour-hypertrophy without labour," can only be accounted for in one way. The assumption is that, by means of a chemical agent, a secondary increased assimilatory growth takes place in organs which are not affected by the disassimilatory decomposition.

That inhibition of function of certain organs may be due to the intervention of the nervous system is, of course, admitted, but we now know that such inhibition may also be induced by chemical substances. This is readily explained by a combination of increased assimilation with one of decreased disassimilation; while, by reversing the process, we account for the existence of organic activity. Some years ago the physiologist, W. Gaskell, pointed out that, if we assume an assimilatory activity on the part of the vagus nerve, its effects are no more mysterious and inexplicable than are those of the motor-nerves in their character of stimulants of disassimilation. As a matter of fact, there is considerable ground for the belief that the chemical changes brought about in the heart muscle by the agency of the vagus are assimilatory in their nature. Thus, after vagal irritation, there is increased muscular power and capacity for work and an improved conduction of stimulus, especially of the positive current oscillation. This positive current oscillation is the antithesis of the negative current oscillation, which is due to intervention of the motor-nerves and is to be regarded as a symptom of disassimilation.

By inhibitory hormone, then, we understand a chemical agent which excites an assimilatory process in another organ. The increased activity (muscular contraction, secretion, \&c.) which follows the suppression of a hormone, is to be regarded as a sign of the unchecked disassimilation consequent upon such suppression.

The method of classification, as defined above, does not pretend to be more than the outline of a plan into which the greater number of details have still to be fitted. The data which we possess are very scanty and, in many directions, are in need of revision. 


\section{THE ORIGIN OF THE HORMONES.}

The internal secretions may also be classified according to their origin. The distinction is between $(a)$ products of the chemistry of the organs which are simply the end-products of decomposition, i.e., the waste or by-products of the process of metabolism; and $(b)$ substances which are the outcome of a synthetic process. It would be wrong, however, to imagine that the true hormones are all included in the latter group. For, in many instances, it is just the products of decomposition formed during cell-activity which are the stimulating agents, by whose aid the function, not of the cells only, but of other tissues, is promoted and maintained.

That form of activity which is usually ascribed to automatic irritation of the nervous centre, is not really automatic; it is in reality brought about by the agency of the products of cellmetabolism and by the vital processes of the protoplasm. Thus, in suffocation, the changes in the respiratory and cardiac activity, the spasms of the voluntary muscles, and the tonic contractions of the muscular tissue of the vessels, are due solely to overirritation by the products of decomposition, an excessive quantity of which is circulating in the blood. The regulation of normal respiration, moreover, is due to the agency of chemical stimuli. Nervous irritation coming from the periphery is able to modify respiration, but is unable to regulate it. It is more probable that the so-called automatic irritation of the centre of respiration, is brought about by the effete products, formed during and by the activity of the tissues. According to Miescher, and there is good grounds for his view, the actual agent is carbonic acid $\left(\mathrm{CO}_{2}\right)$, one of the end-products of oxidation. The fluctuating quantity of $\mathrm{CO}_{2}$ in the blood brings about changes in the respiration and in the supply of oxygen, which are entirely suited to the momentary needs of the organism. If the amount of $\mathrm{CO}_{2}$ in the blood increases, the need of the tissues for oxygen is satisfied by means of deeper and accelerated respiration. It is possible that, where the activity of the tissues is excessive, especially in the case of muscular exertion, the breathing apparatus is influenced by other acid products of incomplete combustion, as lactic acid (Geppert and Zuntz). But in normal respiration, the disassimilatory hormone is carbonic acid, the common product of protoplasm. It must, however, be remembered that, in the opinion of many, the essential factor in the regulation of normal respiration is not the accumulation of carbonic acid, but the lack of oxygen. It may be a combination of both.

This example-it is not the only one but it is the best known -goes to show that the end-products of metabolism play an important part in the accomplishment of chemical correlations. 
Starling's idea, that many of the by-products of organic activity have become hormones only because certain apparatuses have a specific sensibility for them, seems very probable.

Our knowledge of the chemistry of hormone formation is very slight. All that can be affirmed with certainty is that substances of widely different origin and of the most varied chemical composition may operate as stimulating agents. Internal secretions may result from complicated synthetic processes, as iodothyrin in the suprarenals; they may be of very complex chemical structure, as adrenalin, which is a methylamino-alcohol of pyrocatechin; but they may just as easily be the simple products of decomposition, as carbonic acid and lactic acid.

As far as we know at present, the sole characteristic which all hormones possess in common is a negative one. They certainly do not belong to that group of substances which are known as antigens, which, according to Ehrlich, possess a haptophoric affinity with protoplasm and one or more by-affinities which are determined by specific activities. The hormones resemble the antigens in that, like the latter, they are active in minimal quantities, but they differ in certain. essential points. The hormones do not require a period of incubation and, what is still more important, they never provoke the formation of antibodies. These negative properties of hormones are readily understood when considered in the light of their physiological activity. The accomplishment of rapid adjustments and the frequent repetition of the same form of actirity, are not functions which can be performed by agents which are antigenic in character.

It is for the future to supply a minute characterization of the individual hormones and to decide whether their activity arises from their chemical constitution or is due to other chemicophysical causes.

\section{SOURCES OF INFORMATION AND METHODS OF INVESTIGATION.}

The oldest information about the internal secretions, and undoubtedly the most valuable, is that derived from what may be called clinical observation.

The castration of males, whether of man or of the lower animals, is a proceeding which illustrates in the clearest manner the results of the suppression of an organic function. It could. not fail to be remarked that castration is followed, not only by the cessation of sexual activity, but also by other phenomena pointing to a deep-seated change in the nature of the entire organism. These changes have long been very well known, and are brought about for definite ends. For instance, the knowledge that castration is followed by an increased deposit of fat is used 
in connection with the fattening of animals for slaughter. But the older and more obvious belief, that the somatic and psychic modifications resulting from castration were due to a change in the balance of the bodily secretions, was, as we have already seen, more and more undermined by advances in anatomy and physiology. With the knowledge of the intimate relationship to the nervous system in which the individual organs stand, the theory of nervous correlation became firmly established, and the results of castration were regarded simply as signs of the changed activity of the nervous system.

A closer study of the structure of the individual organs led to a better knowledge of their specific functions, but threw little light upon internal secretory activity, even in the case of what were known as the ductless glands.

The first fact in connection with these mysterious organs to receive experimental proof, was the discovery that the suprarenal capsules are indispensable to life, and this we owe to human pathology. It is to the undying credit of Thomas Addison (I 855 ) that he connected a pathological condition observed by him in man with a disturbance of function of the suprarenal capsules.

The results of human pathology, especially the comparison of the clinical signs with the post-mortem findings, have in other directions supplied the most valuable evidence in favour of the doctrine of internal secretion. Instances are afforded by myxœdema on the one hand and by exophthalmic goitre and acromegaly on the other. These pathological conditions have supplied facts concerning the physiology of the thyroid and pituitary glands, the value of which is incalculable. In Addison's disease and myxœedema, the normal processes become destructive or generally pathologically hypoplastic, whereby a decrease is brought about in the activity of certain organs, which manifests itself in pathological signs. In Graves's disease, on the other hand, and to a certain extent in acromegaly also, there is excess of activity on the part of the thyroid and pituitary glands, and these diseases may be taken as classic examples of hyperfunction by an internal secretory organ.

It is certain that in clinical medicine we have an almost inexhaustible source of knowledge, for we find that the careful observation of clinical signs when taken in conjunction with the anatomical findings, continually discloses fresh chemical relationships between the different organs. Thus, the form of pituitary disease described by A. Frohlich in Igor, under the name of dystrophia adiposogenitalis, revealed facts regarding the relationship of the pituitary gland to metabolism which were entirely new.

Operative surgery in man has not only contributed largely to our knowledge (cachexia strumipriva and tetany following the extirpation of the thyroid), but in doubtful cases it frequently 
plays the part of determining factor. Instances are afforded by the operative treatment of Graves's disease and the extirpation of pituitary tumours in acromegaly.

Experimental pathology has reached a high stage in its development and experiments upon animals have done much to clear up the mystery which surrounds the internal secretory processes.

Addison's discovery supplied the incentive to the first orderly experiments on living animals. It was thought that the extirpation or destruction of the suprarenals would not only throw light upon the pathogenesis of Addison's disease, but would also help to an understanding of the physiology of the organs themselves. The experimental extirpation of various organs has, in the first place, decided their indispensability to the life of the organism. In the second place, from symptoms arising after the suppression of any organic function, the most certain knowledge regarding the normal activity of that organ has been deduced. The internal secretory function of the pancreas, which plays a very important part in the economy, was discovered by means of experimental extirpation by v. Mehring and Minkowski. Quite recently, the removal of the pituitary body of animals has supplemented to a remarkable degree the observations of pathologists in regard to its function.

Extirpation experiments with animals have certain advantages over pathological destruction in man. In the first place it is possible to choose a subject suitable for experiment; and in the second, it is possible to localize exactly the destructive process, even to a single portion of a single tissue. It is to this that we owe the discoveries which have been made concerning the function of the parathyroid glands. The value of the method, when employed on different species of animals, will appear later when we are dealing with the suprarenal system.

Another method, that of the destruction of certain tissue elements by means of cytotoxic sera, has not fulfilled all that was expected of it. The method is based upon the experiments of Bordet, Landsteiner, Ehrlich and Morgenroth. These observers found that, if an animal is treated with the red blood corpuscles of another species, substances-the so-called hæmolysins-are formed in its serum which will dissolve, in vitro and in vivo, the blood corpuscles of the species from which the red blood corpuscles were taken. It was expected, therefore, that treatment with either cells or organic extracts would induce the formation of cytolysins (which correspond to the hæmolysins) and of cytotoxins, and that these could be employed to bring about by chemical means specific injury or complete destruction of the organs.

Acting upon this hypothesis, substances which were cytotoxic to different organic cells were prepared, and this method of 
functional and anatomical destruction was tried upon the internal secretory organs. But the results of a large number of experiments did not contribute very materially to the solution of the problems under discussion. In the most favourable instances, the destruction of tissue elements by specific cytotoxic sera merely confirmed the results which had been obtained in other ways. A closer study of their. action showed that, though they have an affinity for the organic cells, this affinity is by no means confined to specific organs. Moreover, the cytotoxic sera nearly always contain hæmolysins and other components, which exert a toxic influence upon other organs.

A more satisfactory method is the elective destruction of certain tissue formations by means of the Röntgen rays. Very interesting results have already been obtained and it is probable that this method will contribute largely to our knowledge of the internal secretory activity of certain tissue elements.

Extirpation is attended by certain by-effects by which the results of experiment may be prejudiced. These are: The degree of surgical shock; derangement of the circulation; and injury to the nerves. In human pathology, when any organic function is suddenly suppressed, an important factor is supplied by the vicarious intervention of another organ possessing the same or a similar function; but it must be borne in mind that such intervention is hardly to be expected in the case of the lower animals.

For this reason, partial destruction-especially the destruction of one of a pair of organs-will be found to yield very suggestive results. The compensatory hypertrophy of the other one of the pair is a proof of their functional relationship.

Compensatory or correlative hypertrophy (which is the anatomical expression of the functional over-activity of a tissue) appears primarily in the accessory organs, in tissues of a similar structure belonging to the same system, or, in the case of the paired organs, in the sound half of the pair. But where there is underactivity of any organ possessing chemical functions, tissues of entirely different structure may vicariously intervene and may even, as a result, become hypertrophied. An instance of this is the hypertrophy of the pituitary gland which follows extirpation of the thyroid. Finally, processes of growth may lead to hypertrophy in individual organs, if the function of another chemically correlated organ becomes deranged, thus increasing or diminishing the amount of hormone which it supplies. In such a case there is no compensatory activity on the part of the hypertrophied organ. The cardiac hypertrophy which accompanies hypertrophy of the thymus, as in the status thymicolymphaticus and the well-known " goitre heart," is an instance of such a condition. The cardiac enlargement in these cases may be due to the continued over-production of substances which stimulate cardiac activity. 
In the larger number of cases, experimental extirpation does not yield reliable results, unless these are supplemented by experimental substitution. This method offers the best means of combating the old argument, that the symptoms of suppression which follow the extirpation of an organ are really due to unavoidable secondary injuries. But if after transplantation-where the normal nerve connections are severed and the local conditions changed-there are no pathological symptoms, while these appear as soon as the transplanted organ is extirpated, then it is obvious that the correlative activity of the organ in question is not nervous in its origin, nor dependent upon an external secretory function, but is wholly chemical and internal secretory in its nature. Clinically successful transplantation, whereby a threatening pathological condition has been averted or a declared one cured, would complete the chain of evidence. Nor is such confirmation lacking. The testicle, ovary, thyroid, suprarenals, \&c., have all been transplanted with good result. It must be remembered, however, that, even where transplantation is apparently successful, the supply of blood and of nutrition may, under the new conditions, prove insufficient and the organ may eventually cease to function.

The discovery that the implantation of an organ which does not successfully heal in, may yet temporarily replace the suppressed function, pointed the way to a simpler method of substitution, namely, that known as organo-therapy, or the Brown-Séquard method. This method is founded upon the assumption that the internal secretion elaborated by an organ is contained in the tissue of that organ and, more particularly, in the juice expressed therefrom. It was thought that, even though the actual amount of active substance present in the organ must necessarily be small, yet, in view of the extreme activity of the extract, it must be possible to obtain all the effects of substitution, especially if the administration was continued over a long period of time. The brilliant results of organo-therapy in the pathological conditions due to suppression of the thyroid, amply prove the justice of this theory. Similar results, though by no means so striking, have been obtained in connection with other organs; while in the case of yet others, the extract of the organ in question, whether given by the mouth or by subcutaneous, intraperitoneal, or intravenous injection, produced but unimportant and, in some instances, barely perceptible, effects. The organic extract and expressed juices depend for their effects, not only upon the method of their preparation and employment, but also upon certain other conditions, of undoubted importance, but of which little is at present known. For reasons which have already been discussed, the failure of organo-therapy in any given instance is not to be regarded as evidence against the existence of an internal secretory function of the organ in question.

Quite apart, however, from their substitutive effects, the 
organic extracts have a distinct value in determining the nature of organic function.

The study of the chemistry of the organic extracts has brought many important facts to light and promises to yield still further results. The lipoid substances, which are of the highest biological importance but to which, as yet, little attention has been paid, deserve a closer investigation.

There is one direction in which the extracts of the various organs have been submitted to frequent tests. It has been found that, when injected under the skin or into the veins of normal animals, these substances exercise a more or less toxic action, leading, in some instances, to death. Where, however, the toxic action appears after intravenous injection only, the effect cannot be regarded as in any sense specific. Most tissues contain substances, in varying quantities, which promote the coagulation of blood-thrombokinesic substances-and these, when injected into the veins, may cause intravascular coagulation. It will depend, therefore, upon the localization and the extent of the thrombi whether there is more or less pronounced functional derangement of the circulation and respiration, or whether the condition becomes acute and terminates fatally. Tests of the coagulability of the blood together with the post-mortem findings may perhaps help the diagnosis. And it must be further borne in mind that, however slowly and carefully the injections are given, the introduction into the blood of substances having thrombokinesic properties, whether in large or small quantities, is very likely to provoke a negative action-that, namely, which prevents the coagulation of blood.

The toxic effects of the subcutaneous or intraperitoneal injection of organic extracts, on the other hand, may be regarded as specific-always supposing that the extracts have been prepared from fresh organs under sterile conditions. But though the method proves their toxicity, it does not permit of any definite conclusion as to their physiological activity, for we are dealing with substances, in exceptionally large quantities, which have been obtained from the tissues by the mechanical destruction, the literal breaking-up, of the cells.

Far more reliable data are supplied by those activities of the organic extracts which are purely physiological. Brown-Séquard's discovery that the watery extract of the testicle-his liquide testiculaire-possessed a marked tonic property, led to numerous experiments, the object of which was to discover the physiological activities of the extracts of various other organs. The most important results were those obtained by Oliver and Schäfer (I894), who discovered the peculiar influence which certain tissue extracts have upon the circulatory apparatus of normal animals. The intravenous injection of certain organic extracts, as suprarenal, pituitary, and renal, brings about a more or less charac- 
teristic increase in blood-pressure, while in the case of others the effect is to reduce blood-pressure. These changes of tension form the basis for a classification of the internal secretory organs into "hypertensive" and "hypotensive" (Livon).

But it must be remembered, and the warning will be repeated when we are dealing with the organs separately, the effect of an organic extract in changing blood-pressure is not a proof that the internal secretion of the organ is active in the same way. An organic extract is a mixture of substances, the chemical properties of which are by no means clearly defined. Moreover, the effects which we are able to observe are not necessarily due to the activity of a single substance specific to a single organ; but may result from the activity of cne or more substances, which may be common to many organs. Thus, the depressing action of many organic extracts may be due to the fact that they contain choline, which, it is known, reduces blood-pressure. In addition it may be remarked that changes in blood-pressure may also be caused by intra-vascular coagulation.

It is obvious, from what has been said, that conclusions as to the physiological and hæmodynamic activities of any organic extract, should be accepted only with the greatest caution. Welldefined results must be obtained and an exact knowledge of the chemical characterization of the active substance is essential. But even where these requirements are fulfilled, there must be definite proof that the active substance is formed intravitally, and that it does pass into the blood-stream. Then, and then only, are we in a position to state, that the substance in question is the product of the internal activity of a certain organ. This conclusion will be confirmed by the morphological proof of secretory cells in the organ in question, and by the histological evidence of the different stages of secretory activity.

The physiological activity of the organic extracts is not confined to the circulatory system, but may show itself in modification of the function of other organs. A closer investigation of their effects shows that many organic extracts contain substances which exercise a specific influence upon the nervous system, the muscular structure, the glandular cells, \&c., and which affect the general metabolic processes in a special manner. In cases where this influence is extended to functions which are modified inversely by suppression of the organ, there are eminently rational grounds for a substitution therapy.

Naturally, many of the physiological effects of the organic extracts upon certain tissues are to be observed only after prolonged use. They sometimes take the form of structural alteration.

The material which has been collected during the last two decades has materially altered the point of view with regard to the interrelation of the bodily parts. Twenty years ago, the 
only known organic relationships were those effected by means of the nervous system and, until very recently, these alone were considered worthy of attention. It was only after a long time and as the result of infinite labour, that the existence of the chemical correlationships was recognized, and the part which they play in the animal economy estimated at its proper value.

To-day, the doctrine of the internal secretions plays an important part in almost every department of physiology and pathology, and is employed in the solution of some of the greatest problems that biology affords. Nothing is more characteristic of this change of view than Schiefferdecker's hypothesis of the part performed by specific internal secretion in the functions of the nervous system. "Internal secretion," he says, " determines the effect which the products of metabolism, excreted by the nerve cells during the simple processes of nutrition, will exercise upon other nerve cells or upon the cells of the end organ, such activity being called 'trophic.' It also determines the effect which the products of metabolism excreted in the course of specific activity will produce, and this effect is known as 'irritation ' or 'stimulus.' "

This view of the origin of nervous activity can hardly be accepted as final, but the mere proposition of such a hypothesis illustrates the magnitude of the difference between "then" and " now." The older physiologists thought that each organic interactivity was due to nervous intervention; to-day we believe that even the nervous correlationships themselves are effected by means of chemical agents. 



\section{PART II.-SPECIAL.}

IN Part I, a brief account was given of the history of the doctrine of internal secretion, of its physiological basis, and of the general principles upon which the teaching is founded. In Part II we propose to take the internal secretory organs separately and to describe what is known of the nature and significance of their activities. In the present state of our knowledge anything in the nature of a systematic classification is impossible. The only rational basis would be a morphological one, but this is rendered impracticable by the insufficiency of the data at our disposal. There is a general tendency to ascribe a specific activity to every cell-form and to every kind of tissue, but though, in a sense, we are justified in so doing, there is as yet no certain proof of the internal secretory activity of the greater number of cells and tissues. From the structure of a tissue we are, no doubt, able to deduce its specific function, but we are a long way from being able to infer the nature of its chemical correlative function.

There are also drawbacks to a classification upon purely physiological grounds. In any such attempt organs and tissues of the most dissimilar type would, on account of the similarity of their function, be classed together; while organs possessing activities in more than one direction would be included in several different groups.

Such being the state of matters it is better to avoid all attempts at classification and simply to give a list of the internal secretory organs, taking them, as far as possible, in the order of their importance.

It is more in deference to custom than in support of any personal conviction that the so-called "vascular" "or "ductless" glands are first dealt with, leaving the second place to the other internal secretory organs. The first group includes:-

The organs of the thyroid apparatus, namely, the thyroid gland and the parathyroid glands;

The thymus gland;

The two suprarenal systems, namely, the interrenal and the adrenal, together with the carotid and coccygeal glands;

The hypophysis cerebri or pituitary gland, consisting of the anterior and posterior portions, together with the pineal gland.

The spleen, which was formerly considered a vascular gland, is now regarded as essentially hrematopoietic in its activity, its internal secretion having no great physiological significance.

The second group includes :- 
The sexual glands and other tissues concerned in the processest of reproduction;

The pancreas;

The intestinal and gastric mucosa;

The kidneys.

It is not proposed to enter into the questions of glycogenesis and the neutralizing activity of the liver. These secretory functions are the oldest known to us and are fully described in the current text-books of physiology.

The plan adopted in each instance is: First, to give the necessary information concerning the morphology and the development of the organ; second, to describe in detail its physiological and experimental aspects; and finally, to estimate its pathological significance by means of material gained from clinical observation.

\section{THE THYROID APPARATUS.}

The thyroid apparatus comprises two distinct organic systems which differ both in structure and in function. They are the thyroid gland, Glandula thyroidea, and the parathyroid glands, Glandula parathyroidea.

\section{Anatomy.}

The thyroid gland of man consists of two lateral lobes, situated upon the lateral surface of the larynx, united by a transverse portion, the isthmus. Accessory lobes (Glandula thyroidece aberrantes), distinct from the principal organ, may occur within a triangular area, the apex of which is at the root of the aorta, the base being formed by the edge of the.lower jaw. In animals these accessory glands may prejudice the results of experiment, while in man they may give rise to retrosternal goitres and so acquire a pathological significance.

The thyroid gland is remarkably vascular, being supplied with blood by no less than three to five large arteries. According to Tschuewsky, the whole of the blood in the body of the dog passes through the thyroid sixteen times a day.

The blood is carried away from the gland by a system of large veins. The lymph passages, which are present in large numbers, open into the deep cervical glands. The nerves, which are comparatively scanty, proceed in part from the ganglia of the cervical sympathetic nerve and in part from the superior laryngeal nerve.

\section{Histology.}

In an early stage of development the thyroid gland is a compound tubular gland with a duct (ductus thyroglossus), which 
opens at the foramen cæcum linguæ, but which becomes obliterated during fotal life. The developed thyroid contains numerous vesicles or follicles, formed by the subdivision of the embryonal tubuli.

The parenchyma of the thyroid, which is exceedingly vascular, consists of a stroma formed of connective tissue, in the meshes of 'which the follicles are situated. These follicles are surrounded by a network of capillaries and are bound together by means of connective tissue to form lobules. The follicles are hollow spaces of different sizes and shapes, lined with cubical or cylindrical epithelium. The epithelial cells, in both man and animals, contain a varying number of small and large granules. These are situated in the free end of cells, facing the lumen of the follicle. These granules are highly refractive and, from the reactions which they give, are believed to consist of fat in combination with oleic acid. Except during the first month of fotal life, these granules are always present; they continuously increase in number until old age is reached; and it is probable that they play a part in the formation of the secretion.

The follicle contents are composed of a peculiar substance, known as thyroid colloid. Morphologically it is a colloidal substance, though chemically it is not. It varies in consistency-and is insoluble in water, alcohol, ether, and diluted acids. It swells in acetic acid and strong alkalis, and is, in general, acidophile. It stains with acid aniline dyes and most readily with eosin. After staining by van Gieson's method it assumes an orange tone, the connective tissue and hyaline substance becoming a brilliant fuchsine red. As a general rule, it does not stain with the basic aniline dyes, hæmatoxylin, or the specific stains for mucus, though at times it appears to be readily basophile. Nearly all authors are agreed that the colloid contents of the follicles are a product of the secretion of the epithelial cells, though it is probable that a very small proportion is also evolved in the course of cell-decomposition. In sections of thyroid gland prepared by the Ehrlich-Biondi method, Langendorff was able to distinguish between principal cells and colloid cells, the former remaining uncoloured, the latter staining red and their nuclei green. The secretory processes of the thyroid have since bcen closely studied by many authors (Andersson, Bozzi, Galleotto, Hürthle, L. R. Müller, E. Schmidt), and it is now generally accepted as a fact that specific granules are formed in the protoplasm of the epithelial cells, which swell up and pass out of the cell body in the form of drops, and that these drops constitute the contents of the follicles. The various phases of secretory activity are accompanied by characteristic changes in the structure of the cells. The follicular contents, which at first are fluid, become viscid and undergo secondary changes, until finally they attain the characteristic colloid consistency. 
Microscopic examination of the thyroid gland shows that, in its finished state, it is what $\mathrm{F}$. Kraus very aptly terms a " storage " gland. In regard to the continuous formation of colloid, it is assumed that a proportion of the secretion passes into the bloodstream, though opinions differ as to the manner in which this takes place.

According to Biondi, the passage of the secretion into the lymph-ressels is effected by dehiscence of the epithelial cells and the breaking through of the colloid mass into the lymph-spaces; Hürthle believes that the secretion passes out by way of the intercellular passages; while Lewandowski is of the opinion that the process is osmotic. One thing, however, is certain, namely, that a substance is found in the lymphatics which appears to be identical with the colloid substance of the thyroid.

Although a certain amount of information is forthcoming concerning the effect which nervous excitement and other momenta exercise upon the secretory process, the data are insufficient to form the ground-work of a hypothesis. Huirthle found that electric stimulation of the thyroid nerves did not produce concomitant changes in the histological aspect of the secretion. Katzenstein found that, after resection of the superior and inferior laryngeal nerves of one side, there was histological degeneration of the thyroid upon both sides. On the other hand, Martini was unable to discover any departure from the normal tissue, and A. Exner found that the amount of iodine in the gland remained unchanged.

Wiener has recently discovered that pilocarpin is without effect upon the thyroid, while adrenalin brings about an increase in the amount of thyroglobulin. Marked effects were obtained with iodide of sodium, which also induced a hypersecretion of thyroglobulin. In cases where the gland had previously been removed, iodide of sodium not only induced a suppression of compensatory hypertrophy, but it is probable that it even gave rise to atrophy. The action of thyroidin, though much less intense, was identical with that of the inorganic iodine combinations.

According to Wyss and E. A. Schäfer, the cells of the thyroid gland react to pilocarpin in the same way as the cells of the true secretory glands. Hürthle described the histological signs of increased glandular activity in dogs after ligature of the gall-duct, and he believed that the bile, which thus passed into the blood, stimulated the thyroid gland to increased secretory activity. This, however, was not confirmed by Muiller's experiments with cats, which were highly jaundiced as a result of the ligature of the common bile duct. In dogs, however, this operation was followed by enlargement of the thyroid follicles and formation of tissue similar to that seen in colloid goitre. These changes are attributed, by both Müller and Lewandowski, to acute degeneration of the gland. 
H. Wiener found that, after removal of the inferior cervical ganglion, there was a decrease in the cell-mass, together with an increase in the thyroglobin contents, of the thyroid gland upon the same side. The resection of the fifth and sixth spinal nerves caused a reduction in the weight of the gland, unaccompanied, however, by a decrease in the amount of thyroglobin. Wiener concludes that the rami communicantes of these spinal nerves are the roots of the sympathetic nerves of the gland; though they convey only trophic impulses, the secretory fibres having another origin.

\section{Chemistry.}

The earlier investigators (Kühne, Oidtmann, Bubnow, Gurlay, Morkuton, Notkin, Drechsel, Hutchison) showed that, among the chemical constituents of the thyroid gland are the albuminoids-both albumins and nucleoproteids containing phosphorus-albumose, leucin, xanthin, hypoxanthin, paralactic acid, and succinic acid. Of the inorganic substances, it contains sodium chloride and oxalate of calcium.

E. Baumann showed, in 1895 , that the normal thyroid gland contains large quantities of iodine in organic combination. It was suggested by Kocher, even earlier, that the favourable effects of the iodine preparations in goitre might be due to the fact that iodine is a normal constituent of the thyroid gland.

The amount of iodine present in the normal gland of man and animals is $0.3-0.9 \mathrm{mg}$. to $\mathrm{I}$ grm. of the dried substance. The entire organ contains 2-9 mg., or, according to Aschbacher, an average of $6.48 \mathrm{mg}$. The thyroid gland of the foetus and of the new-born infant contains no iodine, and it appears from this that iodine is introduced into the organism with the food and is stored in the thyroid gland. In children the amount of iodine in the gland is less than in adults; according to Jolin, from I-Io years, it is o.I45 $\mathrm{mg}$. According to Aschbacher, the average up to 25 years is $2.43 \mathrm{mg}$. ; from $25-30$ years, it is $8.98 \mathrm{mg}$.; and over 50 years of age it is $4.6 \mathrm{mg}$. Among animals, the thyroid of herbivora contains a high percentage of iodine; that of omnivorz is low; while the lowest percentage of all is contained in the thyroid of carnivoræ, from which iodine may even be absent altogether.

The amount of iodine present in the thyroid may be taken as a measure of the amount of iodine taken into the economy, whether in food or as a medicament. According to Jolin, who gives the average amount of iodine in the normal human thyroid as $8.5 \mathrm{mg}$. the amount increases under iodine treatment to $15.6 \mathrm{mg}$. Baumann states that the proportion of iodine is lowest in localities where goitre is endemic-such as Freiburg-while the gland weighs very heavily. In localities which are free from goitre (Hamburg, Berlin) the opposite holds good, the weight of the 
gland being low and its iodine content high. The absolute iodine content of goitrous glands is, however, considerably higher, and may reach 50 , or even $100 \mathrm{mg}$. Oswald pointed out that the amount of iodine in the thyroid gland is a parallel to its colloid content. True parenchymatous goitres, the goitres of Graves's disease-in so far as these are parenchymatous - the purely fibrous goitres, portions of goitres which have undergone celldegeneration, and the hyperplastic goitre of calves, do not contain iodine, or, at least, contain very little; while, on the other hand, goitres containing much colloid substance are very rich in iodine. Oswald is of the opinion that, wherever there is pronounced colloid degeneration, the proportion of iodine decreases and may, indeed, disappear altogether.

Iodine is present in very nearly all the human organs, though this was not Baumann's original opinion. The thyroid gland is, however, 8-Io times richer in iodine than the other organs (hypophysis, liver, kidney, ovaries). According to Baldi, bromine is also present in the thyroid; and Gautier and Bertrand assert that arsenic is present in both the thyroid and the ectodermal organs. The presence of arsenic, however, is denied by Hödelmoser. Fürth and Schwarz, as previously stated, believe that choline is present in the thyroid.

Iodine is undoubtedly the most characteristic, as it is the most important, of the chemical constituents of the thyroid gland, and it occurs, according to Baumann, in combination with an albuminoid. Baumann gave the name of thyroidin to this substance, but the name was afterwards altered to iodothyrin. Iodothyrin is obtained by boiling the thyroid gland for several hours in Io per cent. sulphuric acid, and extracting the residuum with go per cent. alcohol.

Oswald believes that thyroid colloid is a mixture of two albuminoids, the thyroglobulins. Of these, one contains iodine and, after treatment with boiling mineral acids, yields iodothyrin as the product of hydrolytic division. Iodothyrin is a peculiar atom-complex contained in the larger molecule of thyroglobulin. Oswald has recently shown that, if it is treated with trypsin for several weeks, iodine-thyroglobulin also breaks up into the final products of hydrolytic division, for by this means the iodine is set free and passes into the ionised state. The second albuminoid is free from iodine, but contains phosphorus, and is a nucleoproteid.

Iodine-thyroglobulin forms by far the largest proportion of the weight of the dried thyroid gland, being one-third to one-half; while it forms three-quarters and more of the weight of colloid goitres. In hyperplastic goitre and the goitre of Graves's disease, the amount of iodine-thyroglobulin is reduced, while the amount of the albuminoids containing phosphorus is much increased. The amount of iodine in thyroglobulin obtained from the thyroid 
gland is not constant; this is ascribed to the fact that thyroglobulin is present in two forms, one of which contains iodine and one not. The relative quantity of iodine to the whole amount of thyroglobulin becomes smaller in proportion as the degenerative process is more advanced. There is no iodine in the thyroglobulin of goitres which are free from colloid substance.

Oswald believes that thyroglobulin is formed in the interior of the follicles, and that it is only after it emerges from them that it becomes iodized; or after iodization it may be excreted as a colloid into the follicular space. He thinks that the production of iodine-thyroglobulin is the physiological function of the thyroid gland; that iodine thyroglobulin is the physiologically active secretion; and that the degree of activity depends upon the amount of iodine present. Thyroglobulin obtained from goitres has a physiological activity precisely similar to that obtained from normal glands. Thyroglobulin which does not contain iodine does not produce physiological results.

It is Oswald's opinion that goitres, including the goitre of Graves's disease, are not to be regarded as a manifestation of increased thyroid activity; for, seeing how poor in iodine goitrous glands are, the value of their function is diminished rather than increased. They either do not form any secretion at all, or that which they do form has a smaller physiological activity than that of normal thyroid glands. F. Kraus, on the other hand, explains the lack of iodine in the goitre of Graves's disease by a decrease in the storage power of the gland, which thus parts with its secretion too readily. Oswald is unable to deny that goitrous glands are hyperactive, but he maintains that, though there is increased secretory activity, the substance secreted has less physiological value.

\section{ANATOMY OF THE PARATHYROID GLANDS.}

In development and function the parathyroid glands are entirely independent of the thyroid, but anatomically they are closely related to it. In man they are kidney-shaped bodies, 3-15 $\mathrm{mm}$. long and $2-4 \mathrm{~mm}$. broad and thick, and yellowish brown to a brown-red in colour. As a general rule, they are four in number and are situated in such a manner with regard to the lobes of the thyroid, that it is customary to distinguish between a posterior superior, and an inferior anterior parathyroid gland. The upper body usually lies upon the dorsal surface, and at the upper edge, of a nodulated process of the thyroid gland, known as the pyramidal process, and it is found by following up the inferior thyroid artery. The lower body is less constant in its localization ; it may be situated at the lower edge of the pyramid, or it may be found at the lower pole or on the medial surface of the thyroid gland. Accessory parathyroids are sometimes present, and these may extend downwards into the cavity of the thorax; 
or they may be simulated by lobules thrown out from the thyroid itself.

The parathyroids are extremely vascular and are supplied with blood from the inferior thyroid artery. The veins open into the lateral portion of the thyroid venous system. Up to now there is no anatomical proof that nerves are present.

\section{HISTOLOGY OF THE PARATHYROID GLANDS.}

The parathyroid gland usually has the appearance of a closed capsule composed of fibrous connective tissue. It is filled with parenchyma, which is divided into more or less regular columns of epithelial cells, by delicate septa which proceed from the envelope. These septa convey the capillary vessels, and they unite to form a kind of network, in the meshes of which the parenchyma is enclosed. Occasionally, the parenchyma appears to be a single coherent cell-mass traversed by a very close network of capillaries. In other cases, owing to pronounced development of the connective tissue, it is divided up into portions resembling lobules or follicles. Sometimes all three structural forms appear in one organ; in other cases the structure is uniform throughout.

The greater proportion of the parenchyma consists of rather large polygonal cells, called principal cells (Hauptzellen), the protoplasm of which does not stain readily. The nuclei, which stain well, are about the size of erythrocytes; they are furnished with a regular, net-like, chromatin framework and have several nucleoli. A second group of cells is oxyphile. These have a delicate granulated protoplasm, which colours strongly with eosin and other acid dyes, and a small central nucleus. The oxyphile cells are, as a rule, smaller than the principal cells, but there are numerous transition forms. Of these, one is believed by some authors to constitute a separate cell-form. It is intimately associated with the connective tissue of the capsule and the septa, and appears as a cubic or cylindrical cell with an elliptical nucleus. These cells unite to form palisade-like rows, which are arranged either radially or perpendicularly to the direction in which the septa run.

The numerous capillary vessels unite to form a close network; their endothelial cells rest partly on the epithelial cells and in part are covered with a delicate sheath of connective tissue. Occasionally, and more particularly in old age, the arteries are accompanied by large lymphatics. According to Sacerdotti and Anderson, the vessels in the interstitial tissue are accompanied by nerve-fibres by which they are enclosed as by a network, and which may also penetrate between the epithelial cells.

That the parathyroids possess a secretory function is shown by the presence of fat-granules in the epithelial cells of the parathyroids of both man and animals. These fat-bodies are 
found during the first month of life, and they increase in number with age, though they are not affected by the general nutritional conditions. Petersen found glycogen in the parathyroid glands of man at all ages; its distribution was irregular, the principal cells containing much, the oxyphile cells little. Glycogen is also present in the connective tissue and the blood-vessels. Petersen's view is endorsed by Guizetti, v. Verebély and Yanase. H. Koenigstein considers that upon histological grounds glycogen may be regarded as a secretory product of the parathyroid glands.

The parathyroid glands sometimes contain colloid, which occurs in agglomerations situated, as a general rule, near the edge, and which is occasionally contained in follicles similar to those of the thyroid. There is a follicle-like arrangement of the oxyphile cells, and at a later stage, the centre of this follicle contains a colloid deposit, generally circular in shape, the edge of which stains readily with eosin. Later on, the follicle cells group themselves round the colloid to form a gland-tubule. Colloid masses are also found within the larger cell-agglomerations (Petersen).

Chemical examination of the parathyroid glands is yet to come. Gley proved the presence of iodine, though Chenu and Morel found that both the external glands of the dog and the rabbit contained something less than $0.025 \mathrm{mg}$., a proportion far smaller than that in the same weight of thyroid tissue.

According to Berkeley and Beebe, the parathyroid glands contain a nucleo-proteid of specific activity.

\section{HISTORY OF THE DEVELOPMENT OF THE ORGANS OF THE NECK.}

To understand the anatomy of the parathyroid glands and their relation to the thyroid, it is necessary to have some insight into the history of the development of the organs of the neck. This may be briefly stated as follows :-

At a very early stage of development, epithelial thickenings form at the third and fourth branchial arches, one being situated on the dorsal or cranial side, and one upon the ventral or sacral side of the branchial clefts of both sides. The ventral thickening of the third branchial cleft is the definitive primordial beginning of the thymus gland, and is called thymus III. The thickening upon the ventral side of the fourth branchial cleft was formerly believed to be the primordial beginning of the thyroid gland, but it is now very generally regarded as one of the beginnings of the thymus, and is called thymus IV.

It was formerly believed that the thyroid gland was derived from the two ventral portions of the fourth branchial cleft, in combination with material derived from the median portion of the fauces. This view has become very much modified, and it is now 
held that, not the thyroid, but the thynus, is derived from the lateral branchial clefts and that, as a matter of fact, the lateral lobes of the thyroid gland have not a separate area of origin. This view is confirmed by discoveries made by Maresch and Peucker. They each describe an instance in man in which the thyroid was absent, while the parathyroids and the remains of the thymus were present in their full number. It is highly probable, therefore, that the lateral portions of the thyroid gland are derived from a diverticulum of the primitive median thyroid process.

The parathyroid glands are developed from the dorso-cranial thickenings of the branchial clefts, the parathyroid III from the third branchial cleft, and the parathyroid IV from the fourth branchial cleft. In the neighbourhood of the latter is a cyst-like hollow lined with pavement epithelium, which is called the postbranchial body. This is derived from a lateral portion of the fourth branchial cleft.

In a further stage of development, the entire complex evolved from the fourth branchial cleft becomes very intimately associated with the thyroid and is, eventually, entirely covered by it. In many species of animals, the parathyroid IV, together with the thymus IV and, in some cases, the post-branchial body, are found within the thyroid gland and, on account of their topographical position, are described as " internal."

It would be more accurate if we were to follow the example of French authors and call these branchial organs by a name expressing their origin, namely, glandulæ branchiales parathymica and parathyroidea. And it would serve still further to elucidate the matter if we were to add the numbers III and IV, in accordance with the branchial process from which the organ takes its origin.

The descriptions "internal" and " external" cannot be applied to the parathyroid glands of man, because these are all placed externally to the thyroid. The glandula branchialis parathyroidea III is situated at the aboral pole of the thyroid, and represents the inferior parathyroid body. The glandula parathyroidea IV, which represents the internal parathyroid gland of many animal species, lies, in man, on the dorsal surface and near the upper edge of the thyroid and is called the superior parathyroid gland.

\section{COMPARATIVE ANATOMY OF THE PARATHYROID GLANDS.}

The organs of the neck, even in the embryo, do not always occur in the classic number. The most constant is the thymus III, and the origins of the parathyroids are also of fairly regular occurrence. 'The primordial beginning of the parathyroid III 
has never been known to be absent. A. Pepere's investigations show that, in all mammals, including man, the development of the thymus is invariably accompanied by a persistent accessory parathyroid system, which is derived from the third branchial cleft. It is seen most clearly in the thymus or in the region of the thymus of live rabbits, where it appears in the form of single groups of epithelial cells, or of relatively large islands composed of parathyroideal tissue.

As a general rule, all the embryonic beginnings of parathyroid glands develop into definitive organs. Variations occur in their topographical relationship to the thyroid, and for this reason there is a wide diversity in the anatomical conditions of species and individuals.

The position of the parathyroids is most constant in cats. The glandula parathyroidea III, representing the external parathyroid gland, lies upon the dorsal surface of the thyroid gland and generally at its upper pole, though sometimes at the middle. The glandula parathyroidea IV, representing the internal parathyroid gland, is placed at about the middle of the thyroid, more or less deeply embedded in the tissue.

A similar arrangement is found in the dog. The externai parathyroid body lies in a depression situated at the upper pole of the thyroid, while the internal parathyroid body is found in the upper third or in the middle of the thyroid, and is likewise embedded in the tissue. Supernumerary parathyroid bodies are by no means infrequent in the dog, though in the cat they are scarcely ever met with.

In ruminants the topographical-anatomical conditions are aberrant. The glandula parathyroidea III of the sheep and the goat is much nearer to the cranium, and is found on the inner surface of the submaxillary gland or at the fork of the carotid. The glandula parathyroidea IV is embedded in the centre of the thyroid.

In the rabbit, the glandula thyroidea IV also remains in association with the thyroid, and is found in the interior of the gland. The glandula parathyroidea III, representing the external parathyroid body, lies from $\frac{1}{2}-1 \frac{1}{2} \mathrm{~cm}$. below the thyroid gland. Under typical conditions, it lies close to the carotid and depends from a delicate arterial peduncle. It is about $4-6 \mathrm{~mm}$. long, $\mathrm{I} \frac{1}{2} \mathrm{~mm}$. broad, and weighs $4-6 \mathrm{mg}$. Erdheim and Pepere found numerous accessory parathyroids in the thymus of rabbits. Cristiani's investigations show that, in the case of other rodents, one parathyroid body is either embedded in the thyroid tissue or projects from its surface. A glandula parathyroidea III is generally absent, but in the field-mouse there is a free parathyroid gland which lies at either the upper or the lower edge of the thyroid. Erdheim found that, in the rat, in addition to the one parathyroid body embedded in the thyroid tissue, there is fre- 
quently a second larger body. In every case, however, there is a larger or smaller number of small and minute accessory bodies, and these are found distributed over the space between the principal parathyroid body and the tip of the thymus gland, in and on the thyroid, below the lower pole of the thyroid, and about and within the tip of the thymus.

In apes, it frequently happens that both the parathyroid glands are situated outside the capsule upon the lateral surfaces of the thyroid gland. In Macacas rhesus an external parathyroid gland is frequently found at or about the middle of the lateral surface of the thyroid; or it may occur, though more rarely, at the upper or lower pole. The other gland is found embedded in the substance of the thyroid, near the internal surface, and at about the middle of the organ. This second gland is usually the larger.

In birds, the entire thyroid apparatus lies in the cavity of the thorax, on both sides of the trachea, and consists of the thyroid and I-2 parathyroids, which are placed at the upper or lower poles of the thyroid.

In the tortoise, Doyon and Kareff found at the base of the neck a parathyroid body of a yellow colour on each side of the thymus.

In fish, the only portion of the thyroid apparatus which is known is the thyroid gland, and this is situated at the fork of the branchial artery.

\section{PHYSIOLOGY OF THE THYROID APPARATUS.}

\section{HISTORICAL.}

From very early times the thyroid gland has been regarded as an organ possessing specific physiological functions. We gather from the older physiologists, Horsley (I89I), Hellin (I893), Langendorff (I 899), that before any definite information as to its structure was forthcoming, the most extraordinary notions regarding the nature of its activity prevailed. It was believed to enhance the beauty of the form and contour of the neck; to be a kind of cushion for the larynx; to act as a sounding-board for the voice, \&c.

An early theory, based on the anatomical aspects of the thyroid gland and, more particularly, upon its extreme vascularity, held that the function of this organ was the regulation of the cerebral circulation. As a matter of fact, the thyroid glands, with their exceptionally numerous blood-vessels-which are in direct communication with the great vessels of the brainand their enormously developed capillary system, do, to a certain extent, influence the blood which circulates through the brain. These conditions might seem to suggest that the thyroid apparatus 
serves as the blood reservoir for the brain, and that, when a change takes place in the circulatory conditions, it may either prevent a rush of blood to the arteries of the brain, or it may receive large quantities of blood from the cerebral veins.

This theory has recently been revived by E. von Cyon. He suggests that iodothyrin; the active substance formed by the thyroid gland, stimulates the nerves of the heart which regulate pressure, and that by this means the blood-pressure is lowered and the circulation retarded. At the same time, the central vagal irritation is conveyed by means of vasodilators to the vessels of the thyroid gland, causing them to dilate and thus still further reduce the flow of blood to the brain.

When we have discussed the activity of the thyroid extracts, we shall be in a position to judge whether or not there is justification for this hypothesis. Suffice it for the present that, though experimental proof of changes in the cerebral circulation due to chemical or nervous intervention of the thyroid, if they really exist, ought to be readily obtainable, and would be very convincing. Such proof is, up to the present, not forthcoming.

Another of the older theories has recently been advanced by J. F. Meckel. It postulates an intimate physiological association between the thyroid gland and the female organs of generation. This theory owes its origin to the fact that the thyroid gland of women is larger than that of men, and that it increases in size during menstruation, deflorescence, and pregnancy.

The swelling of the thyroid is a well-known phenomenon in menstruation and pregnancy. This increase in size may be partly due to general vascular turgescence, but, as we shall see later, there is an actual biological relationship between the thyroid and the sexual glands.

Tiedemann was the first to suggest that the thyroid is concerned in the formation of blood, and that it aids, or may even replace, the function of the spleen. He bases his view upon the histological construction of the thyroid and its structural analogy with the lymphatic glands and the spleen. The latest experiments show that there is some ground for the belief that the thyroid exerts an influence, though indirectly, upon the formation of the blood.

The functional significance of the thyroid gland and its importance to the life of the organism, were first demonstrated by the serious nature of the results which follow its extirpation.

Numerous instances in which goitre in man has been treated by total extirpation of the gland, confirm the results obtained by means of hundreds of experiments with animals, and there is no doubt that the removal of the thyroid gland gives rise to severe pathological conditions which often terminate fatally. The results of extirpation may show themselves in two conditions, 
which differ radically, both in their symptom-complex and in the course which they run. Of these conditions, one appears promptly and is attended by violent muscular spasms. It is obviously a disease affecting the nervous system and has been called "tetany," on account of its resemblance to a well-known idiopathic disease of man. The second condition is more chronic in its development and is characterized by nutritional disturbances of the most varied description. In man there is a peculiar œdematous swelling of the skin, a decrease in mental power, and, in young people, a remarkable arrest of development. This condition was named myxodème postopératoire by Reverdin, and by Kocher, general cachexia strumipriva or thyropriva. These two conditions were not regarded as separate pathological entities, but as etiologically and pathogenetically the same. For either might make its appearance in man after extirpation of the thyroid gland, and transition forms between the two conditions were known to exist.

It seemed a remarkable thing that the suppression of an organic function should be expressed by means of two totally different symptom-complexes. Experimënts with animals were undertaken to show that this was due to the operation of factors as widely divergent as species, age, external conditions such as temperature, and nutrition. The latter appeared to be the deciding factor; for it was invariably found that removal of the thyroid in the carnivora caused death, while in the herbivoræ it only produced nutritional derangements, these taking the form of chronic cachexia, or, in young animals, disturbance of growth.

The discovery of the parathyroid glands by Sandstroem in I 880 first provided a means of interpreting this difference in its relation to species. A long time, however, elapsed before anything was done in this direction. It was not until $r 890$ that the French physiologist, E. Gley, was able to show that herbivorous animals, such as rabbits, develop tetany if, in addition to the thyroid, the parathyroid glands, which lie at some distance away at the side of the carotid artery, are also removed.

The anatomy of the thyroid glands was finally cleared up by experiments undertaken by A. Kohn; and, in I896, Vassale and Generali showed that in the dog, extirpation of all the parathyroids was followed by tetany, while extirpation of the thyroid was unattended by pathological signs. These results were confirmed in many directions (Rouxeau, I896; Gley, I897; Moussu, 1897; Welsh, I898; Capobianco, 1899), but there was considerable divergence of opinion as to their interpretation. Surgeons were by no means unanimous in conceding to the parathyroid glands a pathogenetic significance in tetania strumipriva.

My own experience has shown that, not only does tetany follow extirpation of the parathyroid glands, but that extirpation 
of the thyroid, leaving the parathyroids intact, produces derangement of the processes of growth in young carnivoræ. I became convinced, from the results of my experiments, that cachexia and tetany cannot have a common origin; and I urged (1901) that, in the surgical treatment of goitre, the parathyroids should in all cases be left intact, and that, wherever possible, a portion of the thyroid should be spared.

My opinion was confirmed later by Jeandelize (1903), Walbaum (1903), Pineles (1904), Erdheim (1906), Pfeiffer and Meyer (1907), Hagenbach (1907), Haberfeld and Schilder (1909). There are observers who have adopted a different standpoint (Blum; Caro, 1903; Kishi, 1904), but they are very few.

We may, then, take it as established : that the acute nervous symptoms which appear after extirpation of the thyroid apparatus, are genetically independent of the thyroid gland and occur only where the parathyroids have also been removed; and that tetany arises from the suppression of the function of the parathyroids and should, rightly, be called tetania parathyropriva. Whether this form of tetany is identical with that which follows the extirpation of the entire thyroid apparatus is, as yet, undecided. It is, however, barely possible to distinguish between the clinical pictures presented by the two conditions in animals of similar species and of the same age. The only apparent difference lies in the intensity of the symptoms and the rapidity with which they make their appearance.

\section{TETANIA PARATHYROPRIVA.}

The results produced by extirpation of the parathyroid glands or of the entire thyroid apparatus of animals, vary somewhat with the species and age of the subject. In addition to the typical acute tetany, which shows itself in characteristic muscular convulsions, there are other acute forms which are associated with symptoms of paralysis. Abortive forms are occasionally seen, as well as a latent tetany, which is shown only by a hypersensibility of the nerves and occurs most frequently after partial parathyroidectomy. Finally, many animals develop a chronic tetany which is associated with certain trophic disturbances.

The typical picture of an acute tetany is most frequently seen in the dog and the cat, and may be described as follows: There is, first, a latent period lasting 24 to 72 hours, during which time appetite falls off and the animal may refuse food; thirst increases; the temperature may fall and the number of red blood corpuscles be diminished; and later on there may be mechanical hypersensibility of the peripheral nerves. This is followed by fibrillary contractions in certain muscles of the head, face, back and tail. In the course of the next day or two the temperature rises and 
the muscular spasms become more frequent and more intense; the animal rubs its snout, walks stiffly, and steps on the dorsum of the paws. Frequently, there is a peculiar position of the paws caused by tonic contraction, accompanied by diffuse clonic contractions in different groups of muscles and, more particularly, by the tremor of an extremity, due to lightning contractions.

As the condition progresses, the clonic contractions spread to nearly all the muscles of the body, when what is known as a tetanic seizure comes on. It begins with a marked increase in the respiratory and cardiac activity, and the temperature rises to $4 \mathrm{I}-42.5^{\circ} \mathrm{C}$. (about $106-108^{\circ} \mathrm{F}$.). The animal suddenly lies over on its side and the breathing becomes extremely rapid, interrupted by occasional deep convulsive inspirations; or there is opisthotonus and tetanic convulsion of the diaphragm and the animal dies from suffocation. Very frequently, however, there is spontaneous recovery even after severe attacks; the cardiac and respiratory activity become gradually slower; the temperature sinks and may become even subnormal; and a condition of extreme weakness supervenes. After an interval varying from one to forty-eight hours, the fibrillary contractions again become stronger and a fresh attack comes on. After several attacks the animals become very emaciated and, ten to fourteen days after operation, they die. Apart from pneumonia, which is frequent, the post-mortem finding is negative.

Gozzi has recently described severe and extensive lesions of the liver, which he found regularly in dogs after extirpation of the parathyroids, and which affected the vessels as well as the cells.

A slightly aberrant form of tetany is observed in young dogs. There may be clonic contraction of the muscles in certain regions, but the seizures are more frequently characterized by tonic contractions of the muscles of the glottis, respiratory apparatus and extremities. The body, especially in the hinder extremities, is stiffly extended and this seal-like attitude persists when the animal is held away from the ground. After frequent repetition of these seizures, a spastic paralysis develops in the hinder extremities and these are dragged along the ground when the animal tries to walk. The paralysis spreads and the animal dies.

Complefe thyroidectomy produces an extremely acute tetany in rabbits (to remove all parathyroids it is necessary to extirpate the whole or the major portion of the thyroid). For one to three days the condition of the animal remains unchanged; then there is quivering of the masseters, accompanied by single clonic contractions in various muscles; there is a sudden and violent tetanic seizure and death from suffocation results. Sometimes the extirpation of the external parathyroids only is sufficient to bring about a fatal tetany though it more frequently provokes the chronic form. Frequently, however, and especially in the 
case of old animals, the extirpation of all four parathyroids is not followed by tetany. Haberfeld and Schilder found that if, in such cases, the thymus and the accessory parathyroids embedded in it were also extirpated, fatal tetany followed. It is worthy of notice, however, that a proportion of animals continue to live, even after removal of all the parathyroid glands.

Christiani (1893) produced tetany in the rat by extirpation of the entire thyroid apparatus. The results of destroying the parathyroids by cauterization are minutely described by Erdheim, who distinguishes between four degrees of tetany in rats. The lightest form is shown by a rapid tremor of the extremities, accompanied by single coarser spasms when the animal is lifted from the ground. A more severe form consists in spontaneous clonic contractions, trembling of the extremities, twitching in the region supplied by the facial nerve, and quivering in nearly all the muscles of the body. The third degree manifests itself in tonic convulsions, which may be artificially provoked by ligaturing the extremities, but which also occur spontaneously; the animals walk with the feet doubled up and there are tetanic spasm in the muscles of the back and the extremities. The severest form is characterized by epileptiform seizures, or by an epileptic state lasting several hours. As a general rule the tetany of rats is chronic in character and is accompanied by trophic disturbances, to which we shall have occasion to refer later.

The parathyroid tetany of mice, as described by Pfeiffer and Meyer, is characterized by two stages. After a latent period of twelve to twenty-four hours, there are attacks of clonic convulsion alternating with tonic rigidity. The second stage is characterized by an extreme motor restlessness, ataxia, and tonic rigidity; somnolence follows, in which the animal slowly passes away.

Parathyroidectomy of goats, according to Christens, is followed by violent tetany. He finds, and his experiments are confirmed by my own, that similar effects are produced by extirpation of the thyroid and the superior parathyroids.

Horsley observed that extirpation of the thyroid apparatus of apes was in some cases followed by acute tetany. He believes that the acute nervous after-symptoms appear only in young animals when kept in a cold place. Horsley and others (Munk, Langhans, Murray, W. Edmunds, Kocher, v. Eisélsberg, Capobianco, Mazzioti) are of the opinion that a fatal termination is less frequent than in the case of the carnivora, and that the condition frequently passes into a stage of chronic cachexia with extreme apathy. After extirpation of the four parathyroids, I found that there was increased nervous irritability, fibrillary twitchings, and finally, there were tetanic seizures and a fatal termination. Pineles describes a case in which the symptoms were similar, but the condition ran a more chronic course.

Doyon and Jouty produced a typical tetany in birds by cauteri- 
zation of the parathyroid glands. The same method produced paralysis in tortoises, ending in death.

It would appear from the foregoing that the extirpation or destruction of the parathyroid glands in all those species of animals which have come under observation, gives rise to a pathological condition, characterized by peculiar symptoms of nervous derangement, and which, sooner or later, ends in death.

My attempts to destroy the parathyroid glands of cats by means of X-rays or radium emanations were, in all cases, unsuccessful.

According to Gontscharnkow, Mankowsky, Demoor, and van Lint, the injection of thyrotoxic sera is followed by nervous symptoms and by tetanic muscular convulsions. MacCallum tried to obtain a cytolytic serum by immunizing geese with parathyroid gland obtained from dogs. His careful experiments with dogs show that the repeated injection of this serum was not followed by clinical appearances, suppression of function, or by any pathologico-histological change whatsoever in the activity of the parathyroid glands.

Partial parathyroidectomy, two of the glands being left intact, is constantly performed in removing the thyroid from rabbits, goats and sheep; it is not usually followed by nervous symptoms. In these cases, the remaining glands are uninjured, but with the carnivora it is different. Here the partial removal of the parathyroids is attended either by unavoidable lesion of the remaining glands, or by a disturbance of their blood supply. These cases nearly always show more or less severe symptoms of tetany, which, however, disappear spontaneously. I observed this transient condition in dogs, in a fox, and in an ape where, in removing the thyroid, the enucleation of the parathyroids was attended with difficulty and these organs became unavoidably crushed. The animals recovered after a short time.

Tetany almost invariably follows the extirpation of more than two parathyroids, but here also, if one parathyroid is left, the effect is frequently only transitory. Should death, however, result, it will be found that the remaining gland is atrophied or, what is more frequent, that it is destroyed by secondary processes.

If, after partial parathyroidectomy (removal of two to three glands) no symptoms appear, the animal is then in the condition known as "latent" tetany. Vassale observed this condition for the first time in a bitch from which three parathyroid glands had been removed. She developed a passing tetany, but completely recovered and had two puppies, which were, however, not carried to term. She littered a second time, eighteen months after operation; she had four puppies and there was a profuse secretion of milk. She was attacked quite suddenly by violent tetany, but recovered after treatment with thyroid extract.

The occurrence of tetany after partial parathyroidectomy in 
pregnant animals has been frequently described. It has been proved, moreover (Adler and Thaler) that when animals, which appear perfectly sound after the removal of two or three parathyroids become pregnant, pregnancy is heralded by an outbreak of tetany. Fromme provoked tetany in such animals by injecting them with placental extract. Rudinger showed that a passing tetany may be produced in cats from which the external parathyroids have been removed, by the introduction of certain toxins (atropin, calomel, morphine, ergotin, tuberculin), and by profound ether anzsthesia. The assumption is that, in these cases, a relative parathyroid insufficiency is latent and that any metabolic derangement causes the symptoms of tetany to appear.

In addition to the nervous symptoms, every acute tetany is accompanied by secondary signs, such as shaggy appearance of the coat; falling of the hair; eczema; and extreme emaciation. These manifestations become more pronounced the longer the animal lives after operation. Similar conditions are seen in rabbits. If tetany does not develop after partial parathyroidectomy, and if the animals continue to live, the nutritional condition of all such animals is more or less seriously disturbed.

Tetany in rats is strikingly chronic in character. The animals in Erdheim's experiments survived from fifty-four to 162 days, and invariably showed changes in the teeth. Between the sixth and tenth weeks, opaque white spots appear upon the enamel of the anterior surfaces of the upper and under incisors and these move upwards from the base to the apex. These spots are circumscribed defects in the enamel, which may scale off and cause the tooth to break. The upper incisors grow again quickly after fracture, but fracture of the lower ones is frequently followed by necrosing ulcers. As the changes in the teeth prevent the animal taking a sufficiency of nourishment, extreme inanition and cachexia follow. Histological examination of the teeth shows that the trophic disturbances appear very soon after removal of the parathyroids, and that they result from imperfect calcification of the dentine together with hypoplasia of the enamel.

Erdheim's observations are confirmed by Pfeiffer and Meyer and by Iselin. Iselin also describes disturbances of the processes of growth in the bones of speckled mice after remoral of the parathyroids. He found that in young animals there was complete arrest of growth, unaccompanied, however, by secondary bony deformity.

Erdheim saw cataract in a rat with chronic tetany and he regards this as a symptom of trophic disturbance. Albuminuria, which was first observed in the tetany of animals by Pfeiffer and Meyer, is an almost constant finding. Massaglia considers the nephritis and albuminuria to be symptoms of an auto-intoxication consequent upon parathyroidectomy.

Iselin's account of the effects of the parathyroidectomy of 
the mother upon the offspring are very interesting. 'The young of rats from which the parathyroids had been removed were found to be highly sensitive to electric stimuli, and were so susceptible to parathyroidectomy that four to ten hours after operation they succumbed to tetany in its most violent form.

\section{TETANY IN MAN.}

Experiments with animals, such as those described above, paved the way for a closer investigation of the part played by the parathyroid glands in the tetany of man.

There is no doubt that the tetania strumipriva of man, like the tetania thyropriva of animals, is due to injury or removal of the parathyroids when extirpating a goitre. This view was originally propounded by Vassale and Generali and it has been confirmed by many other observers (Biedl, Jeandelize, Pineles). Benjamins showed that one to three parathyroids may be found attached to an extirpated goitre and this sufficiently explains the occurrence of post-operative tetany. Erdheim found that in three cases of tetany following partial excision of a goitre, all four parathyroids had been removed. In a fourth case, one parathyroid had been left, but it had become necrosed, as the artery below it had been ligatured.

Whether or not the extirpation of a goitre will be followed by tetany, depends entirely upon the amount of parathyroid tissue left intact and the functional capacity of the tissue thus remaining. The first care of the surgeon who extirpates a goitre should be to spare, wherever possible, all parathyroid glands. It is also essential, as we shall see later, that a sufficient amount of thyroid parenchyma should be left intact. For this reason, methods of intracapsular resection or enucleation are by far the best. Of such are Mikulicz's wedge-resection, and Kocher's resectionenucleation methods.

In man, as in animals, a post-operative transient tetany, due to mechanical lesions of the parathyroid glands or injury of their blood supply, is sometimes observed. Cases have been described where thyroidectomy has not been followed by any apparent symptom and yet a condition of latent tetany must have existed, for under the influence of certain momenta, such as pregnancy, tetany has appeared.

A second form of tetany, which we know from the results of both animal and human pathology to owe its origin to the parathyroid glands, is that known as the tetany of maternity, or puerperal tetany. That there is an intimate connection between tetany and the functions of the female organs of generation (menstruation, pregnancy, lactation) has been frequently pointed out by clinicians.

It has been shown that the partial parathyroidectomy of 
animals is not invariably followed by symptoms of tetany, but that these may be provoked by pregnancy or lactation. It appears highly probable that in man also, parathyroid insufficiency may be the originating cause of tetanic outbreaks, the determining causes of which are metabolic changes arising from alteration of function of the organs of generation. In the absence of any definite anatomical information regarding this condition in man it is impossible to be sure whether the assumed insufficiency of these organs was pre-existent, or whether it occurs as the result of changes in the female organism.

Another form of tetany is that seen in infants, and here we have definite anatomical grounds for associating it with the parathyroid glands. Erdheim found hæmorrhages, or traces of hæmorrhages, together with blood pigment, in the parathyroid tissues of children who had had tetany during life. Yanase, working in Escherich's clinic, was able to demonstrate the presense of hæmorrhages in the parathyroid glands of all children under one year who, during life, showed hypersensibility to electric stimulus. After one year, he found traces of old hæmorrhages or the finding was normal. Fleischmann found that the teeth of all the persons he examined who had a history of infantile tetany, showed hypoplasia of the enamel. Escherich concluded from these results that all forms of infantile tetany are due to parathyroid insufficiency.

There are certain authors, however, who adopt a different standpoint (Thiemich, I906; Forsyth, 1908). They point to the fact that the most diverse changes may be seen after death in the parathyroid glands of persons who, during life, never showed any symptom of tetany. But in these cases, it may very well be that there was sufficient healthy parathyroid tissue to carry on the function of the glands. Also, as Rudinger points out, the cardinal symptoms of tetany are those which are latent; they may be supplemented by secondary convulsive symptoms, but these are not an essential feature of the condition. And it is just the latent signs which most easily escape observation. The second point, namely, that cases occur where the clinical signs are unmistakably those of tetany, while the post-mortem findings are negative, loses its significance in face of the fact that a transient tetany may be provoked in man, as in animals, by a passing lesion of the parathyroid glands.

The lack of anatomical material makes it difficult to discuss the genesis of the other forms of tetany, namely, those of gastrointestinal origin, that which follows certain forms of poisoning, and the tetany which is associated with some nervous diseases; but from what we know of analogous conditions in animals, it seems highly probable that here also we have to do with insufficiency of the parathyroid glands.

Rudinger remarks the frequency with which the parathyroids 
become tuberculous and the striking frequency of the Chvostek phenomenon in tuberculosis, as noted by many observers. He mentions a very instructive case described by Carnot and Delion, that of a tuberculous woman who on the day before her death was seized by tetanic convulsions of the most violent description. The post-mortem examination showed that the internal parathyroids were atrophied, while the external parathyroids were entirely destroyed.

The generally accepted view to-day (Pineles, v. FranklHochwart, Chvostek) is that all forms of clinical tetany are of parathyroid origin.

\section{OTHER PATHOLOGICAL CONDITIONS IN MAN} ARISING FROM DERANGEMENT OF THE FUNCTION OF THE PARATHYROID GLANDS.

H. Lundborg (1904) was the first to associate not tetany only, but a number of pathological conditions included in the term " motor-neuroses," with the function of the parathyroid glands. He based his conclusions upon the results obtained by Vassale and Generali, Biedl, Jeandelize in the tetania parathyropriva of animals. According to his hypothesis, Myotonia congenita

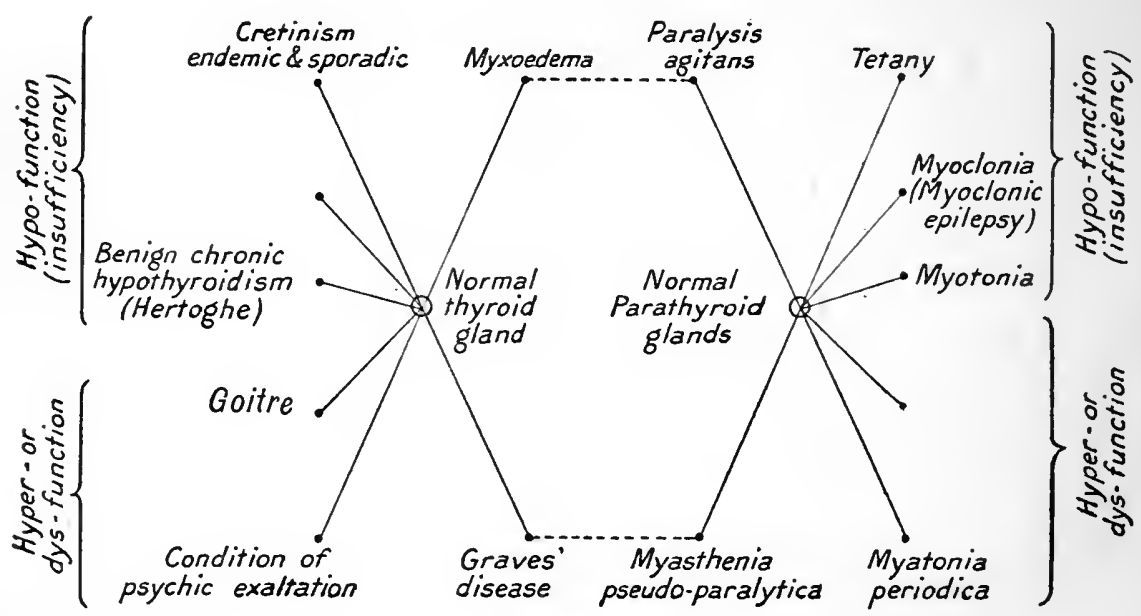

(Thomsen's disease), Paralysis agitans (Parkinson's disease), and myoclonia are to be regarded as chronic progressive conditions due to hypoparathyroidism.

On the other hand, Myotonia periodica and Myasthenia gravis pseudoparalytica (Jolly), or Paralysis myasthenica (Oppenheim) are diseases which, like Graves's disease, arise from an increased or disturbed function of the parathyroid glands (hyperparathyroidism or dysparathyroidism). Lundborg regards these 
glands as regulatory organs whose task it is to maintain the balance of neuro-muscular activity. 'This view is illustrated by the accompanying diagram, in which parallel conditions originating with the thyroid gland are also included.

Chrostek (I908) made a minute analysis of the clinical symptoms of Myasthenia gravis. - He rejected the hypothesis that this condition is primarily of nervous or muscular origin, and is of the opinion that, in all essential points, myasthenia and tetany are diametrically opposed. "They have the same relationship to one another that the negative has to the photograph," and yet they have certain points in common which make it impossible to deny them a common origin. Chvostek believes that tetany is produced by hypo-function of the parathyroids, while hyper- or dysfunction gives rise to myasthenia. The originating cause of this disturbance of function may lie in changes which take place either within the gland itself, or in the centres or channels by which its function is regulated. Thymus persistency, which so frequently accompanies myasthenia, has no pathogenic significance: it merely points to the existence of a constitutional anomaly, favourable to the manifestation of symptoms of functional parathyroid derangement.

\section{THE THERAPEUTICS OF TETANIA PARA- THYROPRIVA.}

Once we are assured that tetany results from the suspension of function of the parathyroid glands, the therapeutics of the condition become much simplified. The object of any therapy must be to restore to the organism the function of the gland which has become incapacitated. The simplest method is by transplantation, and before the parathyroid origin of tetany was recognized, the condition was frequently treated by thyroid transplantation. But the subjects of experiment were invariably animals in which, as we now know, the implantation of the thyroid necessarily included the implantation of the parathyroids.

In 1884 Schiff succeeded in keeping a thyroidless dog alive for some time by implanting fresh thyroid glands in the abdominal cavity. V. Eiselsberg (I892) showed more positively that the thyroid gland, when implanted in the abdominal fascia or in the peritoneum of cats, healed in and prevented the occurrence of tetany. When the healed-in tissue was removed, tetany followed and the animal died.

Enderlen (1898) recognized the significance of the parathyroids and in the experiments which he carried out with dogs and cats he found that these glands preserved their structure after implantation better than the thyroid.

Following E. Payr's method with the thyroid, I have grafted 
the parathyroids of dogs and cats in their spleen. I found that the subsequent removal of the thyroids and inner parathyroids was not attended by untoward results. Violent tetanic symptoms sometimes appeared, but these were usually transient. These animals may remain for months (a dog lived for eighteen months) without symptoms, but they eventually die of tetany. This applies also to animals in the spleen of which two to four parathyroids from other animals of the same species have been transplanted, their own thyroid being afterwards removed. Postmortem examination shows that only cicatricial tissue remains at the implantation site, the grafted parathyroids being completely absorbed.

In two experiments out of three carried out on young dogs by Pfeiffer and Meyer, the parathyroids were successfully implanted in the rectus muscle, and tetany did not follow the extirpation of the remaining portion of the thyroid apparatus.

Halstead, and later Hermann and Harvey, also described the successful transplantation in dogs of both their own parathyroid glands and those of other dogs. The latter authors are of opinion that the visceral peritoneum is a more suitable site for implantation than either the muscular structure or the spleen.

Walbaum (1903) found that after implantation of the external parathyroids in the serous membrane of the stomach of rabbits, the internal parathyroids being removed at the same time or later, the animals lived from five to nine months. There was no tetany, but there were trophic derangements, such as dermatitis, emaciation, and arrest of growth, which in the end caused death. The grafted organs generally retained their normal histological character for a certain length of time, showing few and inconsiderable changes. Christiani, who since the beginning of the nineties has carried out many hundreds of greffes thyroidiennes and parathyroidiennes with rats, finds that the structure of the implanted glands remains unchanged, though they at first show a slight central necrosis which is, however, soon replaced by normal tissue. After two years in rats, and after even five years in cats, the glands will be found to be practically unchanged.

Camus found that the parathyroid glands, when transplanted into the ear of rabbits, invariably atrophied if a sufficiency of active parathyroid tissue was allowed to remain in situ.

Leischner implanted the parathyroids of rats, with a portion of the thyroid tissue attached, in the abdominal wall. The simultaneous transplantation of both parathyroids provoked a passing tetany. The grafted organs healed in and, one to two months later, the removal of the abdominal rectus muscle in which they were implanted, produced tetany.

These experiments show that transplanted glands, whether of the subject of the experiment or of another animal of the same 
species, will not only heal into place, but will prevent the appearance of symptoms of suppression of the glands. The grafted organs undoubtedly retain their normal structure for some time, but after a few months retrogressive changes usually set in which lead, in time, to the disappearance of the organ.

The transplantation of a parathyroid gland has been successfully performed in man by v. Eiselsberg. The patient was a woman aged 42 who, twenty-seven years previously, had had the whole of her thyroid removed and was suffering from tetany. A parathyroid gland was implanted in the abdominal rectus muscle. This gland was taken from a woman who had had a cyst enucleated from one lobe of the thyroid gland, and in whom the other parathyroids were intact. The transplanted gland healed in, the tetany became reduced to isolated spasms of the glottis, and the sensibility to electric stimulus became normal.

The results of this experiment naturally suggested a substitution therapy by means of organic extracts.

Experiments by parathyroid extract with man as the subject have been repeatedly carried out. Some authors, especially those who employed Vassale's so-called parathyroidin, obtained good results; others (v. Frankl-Hochwart, Escherich, Reinsberg and Rey) were unsuccessful. Pineles treated three cases of tetany with 0.15 to $0.45 . \mathrm{grm}$. (about $2 \frac{1}{2}$ to $7 \mathrm{gr}$.) of dried parathyroid substance daily, and continued the treatment for months, but he was unable to report any change in either the frequency or the intensity of the seizures.

Quite recently Halstead (1907) and Putman (I909) described favourable results obtained in post-operative tetany with the parathyroid extract prepared by Berkeley of New York. When given by the mouth, or, better still, by subcutaneous injection, these nucleo-proteids are said immediately to subdue all symptoms of tetany.

J. Ott (Ig09) describes the physiological activity of this substance as well as that of the watery extracts of parathyroid gland. These substances increase intestinal peristalsis and the muscular contractions of the uterus. When injected in small doses into the veins of cats and rabbits, they produce a fall, which is followed by a rise, in the blood-pressure. In large doses they bring about a fall in blood-pressure unaccompanied by any change in the frequency of the pulse; there is a slight reduction in temperature, with slightly accelerated respiration. But the most marked symptom is the extraordinary increase in diuresis, which may rise to ten times the normal, and is due to stimulation of the epithelial cells of the kidneys.

It will be evident from what has gone before that the thyroid preparations play an important part in the management of postoperative tetany, whether therapeutically in man or experimentally in animals. At a first glance, it seems reasonable to suppose 
that the beneficial results of thyroid administration are due to the fact that parathyroid tissue is given at the same time; experiments by Pineles have, however, shown this view to be untenable. He found that the amount of parathyroid substance contained in the thyroid glands of sheep and calves is by weight $I$ in 309 and 379 respectively; while 200 times that amount of parathyroid substance, when added to thyroid tabloids, had no therapeutic effect whatsoever.

There are numerous accounts by reliable observers of the favourable effects of thyroid gland and thyroid extracts in the tetania parathyropriva of animals.

Kocher, the greatest authority upon the parallel condition in man, wrote in I906, at a time, therefore, when the significance of the parathyroids in post-operative tetany was well known to him, as follows :-

"We found that by energetically pushing the thyroid extract and iodotlyyrin in large doses, we were able to procure a rapid abatement of the symptoms of tetany and, by continuing the treatment over long periods, we obtained a cessation of the attacks."

The favourable influence of the thyroid extracts in postoperative tetany will not be questioned by those who have witnessed the remarkable improvement which takes place, almost immediately, after the subcutaneous injection of soluble thyroid preparation, in the condition of animals with severe tetanic symptoms; or that which, after one to two hours, follows the administration by the mouth of fresh or dried thyroid gland. Nor will the efficacy of these substances be held in doubt by anyone who has, by this means, kept animals alive for two to three weeks after parathyroidectomy, while the animals not so treated died of tetany in a very much shorter time.

But these results must not be accepted without criticism. It must always be taken into consideration that the clinical picture produced by experimental tetany is in the highest degree variable; that spontaneous remissions and far-reaching improvements are of as frequent occurrence as sudden relapses, and that in certain cases this renders the prognosis extremely difficult. The sudden termination of a tetanic seizure, the cessation of the muscular tremors, and even the temporary disappearance of the nervous hypersensibility, cannot always be taken as evidence in favour of the treatment, for all this may, in rare cases, occur spontaneously.

It is an important point in favour of thyroid extract that improvement follows rapidly upon treatment, and that the life of tetanic animals is very much prolonged by its use. Vassale tries to explain the results of thyroid treatment in tetany by the presence of supernumerary parathyroid glands, which are supposed to exercise the same effect as the implanted glands or those 
purposely left in situ. It seems, however, highly improbable that accessory glands should be present in just those cases which have been treated by thyroid extract, especially as such supernumerary bodies are extremly rare in certain species, such as the cat. But even allowing that there is some foundation for Vassale's assumption, a certain measure of efficacy must be granted to the thyroid extract, for it is undoubtedly the agent by which the animals are carried through a phase of disease in which they would otherwise certainly perish.

With so little knowledge at our disposal we are not in a position to give any definite explanation of the effects of the thyroid preparations in tetany, but I do not think this should prevent us from drawing from the analogy of experimental pathology a conclusion which is of the utmost importance to man. It is, that treatment with thyroid gland should always be tried where the extirpation of goitre is followed by symptoms of tetany. By this means time is gained, during which the parathyroids and any accessory parathyroids are enabled to attain the full measure of their functional activity; or a substitute for such activity may be provided by means of transplantation.

\section{THE RELATIONSHIP BETWEEN THE THYROID AND PARATHYROID GLANDS.}

Gley's view that the parathyroids are embryonal organs which, after the removal of the thyroid gland, assume the function of the principal organ, and thereupon acquire the structura! characteristics of thyroid tissue, has not been endorsed by later authors. His discovery that, after the removal of the thyroid and inner parathyroids, the external parathyroids became hypertrophied, is confirmed by many, but no proof is forthcoming of a histological structural transformation of parathyroid tissue into thyroid tissue. Gley himself, in combination with Nicolas, has recently repeated his experiments, and has been compelled to abandon his former view. Vincent and Jolly describe such structural transformation of the parathyroids in cats, but they are alone in their experience.

Quite recently-Vincent, Halpenny and Thompson found that the external parathyroids of a dog from which the thyroid and internal parathyroids had been removed eighty-three days previously, were hypertrophied and changed in such a manner that it was difficult to distinguish them from thyroid tissue. In addition to parathyroid tissue composed of compact rows of cells, the drawings of these organs show numerous irregular vesicles, lined with a single layer of epithelial cells, in part empty and in part filled with colloid. The histological picture of these glands has a strong resemblance to that of a thyroid which the 
authors found in a dog thirty days after total parathyroidectomy. This gland also contained irregular vesicles, together with an increase in the quantity of intravesicular tissue. The clinical course of this experiment is interesting. After removal of the last hypertrophied parathyroid gland, the animal lived for fortyfour days; it did not show any symptoms of tetany, but there were trophic disturbances, such as falling of the hair, eczema and idiocy. It is probable that this animal possessed accessory parathyroid glands.

The occasional occurrence of gland-like hollow spaces in the parathyroids has been frequently described. These hollows are lined with epithelium; they are sometimes empty and sometimes. filled with a homogeneous mass believed to be colloid.

I have found in numerous cases that removal of the thyroid, together with the internal parathyroids, is nearly always followed by hypertrophy of the external parathyroids. The hypertrophy is more or less clearly marked according to the length of time after operation. Many authors (Walbaum, Erdheim, Pepere) have failed to observe an appreciable increase in the volume of the parathyroids embedded in the thyroid, after extirpation of the two external glands. But Haberfeld and Schilder have recently shown that the internal parathyroid glands of rabbits show a very high degree of vicarious hypertrophy if, in addition to the external parathyroids, the accessory glands situated in the thymus are also removed.

I found that the parathyroids of a growing dog, from which the thyroid had been removed, were at the end of a year twice as large as those of control animals taken from the same litter. Hypertrophy of the parathyroids is by no means always associated with important structural changes, although gland-like hollows filled with colloid are of decidedly more frequent occurrence in hypertrophied than in normal parathyroid glands. Whether or not this colloid formation is a compensatory secretory process which is set up in the gland after the suppression of the thyroid, is at present unknown. It suggests, however, that there may be a functional relationship between the two organs.

That there is a relationship between the thyroid and the parathyroid glands is suggested by the fact that hypertrophy of the thyroid gland has been frequently (W. Edmunds, Lusena, Vassale and Generali, Halpenny, and Thompson) observed after removal of the parathyroids. The tissue changes in these hypertrophied organs consist in a new formation of intravesicular tissue, which either forms solid cell rows or causes irregularity im the shape of the follicles. There is also a wasting away of the colloid, together with vacuolization of the contents of the follicles.

These changes are difficult of interpretation, but the examination of a large number of thyroids shows that many apparently normal glands present a similar picture. Vassale thinks that 
the new formation of tissue points to a hyper-function of the gland, the wasting of the colloid to a hypo-function. If this view is correct the same operation, namely, the removal of the parathyroids, is able to produce conditions in the thyroid which are opposed to one another.

There are three directions, then, in which the evidence of a functional relationship between the thyroid and the parathyroid glands may be regarded as conclusive. In the first place, there is the favourable influence of thyroid treatment in the tetania parathyropriva of animals. In the second place we have certain proof of hypertrophy of the external parathyroids after extirpation of the thyroid gland. And in the third place, there is the hypertrophy of the thyroid gland, described by many authors, which follows the removal of the parathyroids. These facts do not, however, supply any definitive information concerning the manner in which this interrelationship is effected.

Vassale and Generali advanced the theory that toxic products of metabolism are formed by the thyroid, and that these become neutralized by the activity of the parathyroids. They based their theory upon the fact that after parathyroidectomy the symptoms are much more acute, the condition runs a more violent course, and that death follows far more rapidly than after extirpation of the entire thyroid apparatus. In the latter case, according to this theory, both the toxin-forming and toxin-neutralizing agents are removed. The explanation, however, can hardly be considered satisfactory. In the first place, it offers no solution of the problem as to why animals from which the whole of the thyroid apparatus has been removed, should yet develop fatal tetany, seeing that the toxic agent is supposed no longer to be present. In the second place, I was unable, in spite of repeated experiments, to obtain any confirmation of the results described by Vassale and Generali. I could not find any difference in the intensity of the tetanic seizures, nor could I discover that animals from which the thyroid apparatus had been removed in toto, lived longer than those from which the parathyroids only had been removed. Everyone who has had experience of postoperative tetany knows how the clinical picture varies in regard to intensity and duration, and will be careful how he allows these factors to influence his conclusions in regard to the functional relationship between the thyroid and parathyroid glands.

What then is the inference which we may safely draw from the results of these experiments? First, that the hypertrophy of the one group of organs after removal of the other is to be regarded, upon general pathological grounds, as compensatory; and second, that a vicarious activity of the thyroid and parathyroids is to be assumed.

It is remarkable that Rudinger employs these facts to support a theory of antagonism between the thyroid and parathyroid 
glands. He thinks that the hypertrophy of the parathyroids which follows thyroidectomy is due to the fact that an antagonistic organ has been removed. He remarks: "We have to suppose that, under normal conditions, the function of the one gland is controlled by the other, and that when this balance is disturbed by the extirpation of one, the remaining organ becomes functionally, and later organically, hypertrophied." Rudinger brings the following as evidence in support of this theory : Moussu and Charrin found that the exhibition of the parathyroid gland of horses was followed by unfavourable symptoms in myxœdema, but that in Graves's disease it had a very beneficial effect. Rudinger believes that the hyper-function of the thyroid in Graves's disease is restrained by the exhibition of parathyroid gland, while in myxœdema the hypo-function is intensified. He is also inclined, with Walbaum, to attribute the favourable action of Moebius's serum and of rhodagen - a preparation obtained from the milk of thyroidless goats-to the presence of a large proportion of parathyroid secretion. "The removal of the thyroid permits of an increased activity on the part of the parathyroids; thus the serum and the milk contain substances which, being stronger than those in normal serum and milk, are better able to counteract the activity of the thyroid gland." Rudinger also brings forward the results obtained by Eppinger, Falta and Rudinger in their investigation of the interrelationships which subsist between the different glands possessing internal secretory functions. These authors found that the effects of adrenalin upon dogs from which the entire thyroid apparatus had been removed, differed markedly from those which it exercised upon dogs in which the thyroid gland only was lacking. After thyroidectomy, the subcutaneous or intraperitoneal injection of adrenalin did not, as it invariably does in normal animals, produce glycosuria, and the intravenous injection of adrenalin was not followed by a rise of blood-pressure. But in animals with tetany after thyroparathyroidectomy, the same experiments provoked both the glycosuria and the rise in blood-pressure. From these results the observers infer that the thyroid secretion stimulates the sympathetic nerve, while that of the parathyroids has an inhibitory effect upon it. Stimulation and inhibition are so balanced by the activity of the two glands that, under normal conditions, oscillation is possible within a very small margin only. The suppression of the thyroid reduces the irritability of the sympathetic nerve, not only by robbing it of its stimulating agent, but by releasing the inhibitory activity of the parathyroid glands. With the extirpation of the parathyroids, the inhibitory agent is removed and this brings in its train a condition of hypersensibility of the sympathetic nerve:

The evidence which Rudinger advances cannot be regarded as a sufficient basis for his theory. The interrelationships between the thyroid and parathyroid glands, and between the pancreas 
and the chromaffine tissue will be discussed in a later chapter. The most important point in favour of the theory of antagonism, is that provided by the hypertrophy of the thyroid and parathyroid glands, which is provoked in each by the suppression of the other. But it must be borne in mind that anatomical hypertrophy is the result of functional hypertrophy; and that the hypertrophy of an organ can be ascribed to the suppression of its antagonist, only where evidence is forthcoming of the manner in which the one organ is excited to functional hyperactivity by the suppression of another, the normal function of which is antagonistic to it. A correlative hypertrophy of antagonistic organs is, so far, unknown to us, and such a state of matters does not seem very probable.

Rudinger himself points out that the well-known variation in intensity of the symptoms in Graves's disease makes it very difficult to estimate exactly the beneficial effects of any therapy. This does not enhance the value of Mossu and Charrin's experiments and of the experiments with Moebius's serum, as evidence in support of Rudinger's theory. And it has been shown, moreover, that there is no proof of the difference, described by Vassale and Generali, between the clinical picture presented by tetany in animals in which the thyroid is intact, and that presented by the condition in animals which have undergone thyroidectomy.

The inevitable conclusion is, then, that a hypothesis of functional antagonism between the thyroid and parathyroid glands, is not supported by the evidence at our disposal.

\section{THE FUNCTION OF THE PARATHYROID GLANDS.}

\section{The Tetany Toxin.}

The first attempt to account for the tetany which follows parathyroidectomy was the neutralization theory advanced by Vassale and Generali. We have seen that these authors regarded the thyroid as the organ in which the toxin was elaborated and that, in its original form, their doctrine was untenable. Later authors (Pineles, Pfeiffer and Meyer), however, are agreed that a poisonous substance, the tetany toxin, is elaborated within the organism, but that its exact source is unknown.

Pfeiffer and Meyer endeavoured to demonstrate the presence of this toxin in the blood of animals suffering from tetany. They found that blood taken from dogs dying of tetany had a more marked hæmolytic effect upon the blood corpuscles of guinea-pigs than the blood of normal dogs. The observers do not attach any particular significance to this result. On the other hand, they emphasize the fact that the serum of tetanic dogs (in six out of serenteen examined) was toxic to mice which had undergone partial parathyroidectomy, causing tetany which, in some cases, 
terminated fatally. They conclude from this that tetany causes a specific toxin of uniform activity to accumulate in the serum, and that this toxin does not pass into the urine. Seeing that latent tetany is produced by partial parathyroidectomy and that tetanic symptoms are occasioned by many poisons, this assumption cannot be regarded as having any very great value.

I have repeatedly found in the course of my own experiments that violent tetanic attacks are arrested by copious blood-letting and that the improvement may be maintained for as long as twenty-four hours by the transfusion of fresh blood into the veins. This seems to point-and far more conclusively than do the results obtained by Pfeiffer and Meyer-to the presence of a toxin, circulating in the blood, from which the organism may be temporarily freed by bleeding.

Ceni and Besta assumed that the toxin of tetany was of the nature of an antigen, and they endeavoured to obtain specific antibodies with which to treat the acute symptoms which follow thyroparathyroidectomy. They immunized rabbits and goats with serum obtained, at the height of an acute tetanic seizure, from dogs deprived of the entire thyroid apparatus. The immune serum they injected in doses of 9 to $15 \mathrm{c.cm}$. into dogs which had undergone thyroparathyroidectomy and which showed severe tetanic symptoms. They found that, although the course of the condition was as a whole very little affected, there was a rapid, and sometimes a complete, cessation of the acute phenomena in nearly all their cases. The authors lay stress upon the fact that the effects which they obtained with the immune serum are much more energetic, prompt and complete than those obtained by Gley with antipyrin, by Ughetti with chloral-hydrate, or by Canizzaro with bromide of potassium. But they were not able by means of the serum to produce a modification of the disease and a prolongation of life, such as that which follows the exhibition of thyroid gland.

The suggestion that parathyroid tetany is due to the agency of a toxic product of metabolism, was recently put forward by Berkeley and Beebe. They believe that the toxin is formed by the decomposition of albumin, for they found that with an exclusively flesh diet, the symptoms were more violent and there was a marked increase in the excretion of ammonia. They also found that the symptoms provoked by the injection of ammonia or xanthin, like those of tetania parathyropriva, are arrested by the exhibition of calcium or strontium salts.

Frouin found that the urine of animals from which the thyroid and parathyroids had been removed, contained an excess of ammonia and carbamic acid. He regards tetany as the result of carbamic-acid poisoning. He produced a typical tetany in from twenty-four to forty-eight hours after removal of the entire thyroid apparatus, by the exhibition of 3-4 grammes of carbamate of 
soda. When the same subjects were treated with this salt in combination with a soluble calcium salt, symptoms of tetany did not follow.

\section{THE PARATHYROID GLANDS AND THE METABOLISM OF CALCIUM.}

In the first part of this book reference was made to J. Loeb's discoveries in connection with the physiological significance of the kathions, especially of the effect which calcium salts have in reducing neuro-muscular irritability. These discoveries have some bearing upon the pathogenic aspects of tetany and they have led the way to other experiments, undertaken with the object of determining the connection between the parathyroid glands and the metabolism of calcium. Quest (1905) induced a condition of electric hypersensibility of the peripheral nerves in young dogs, by means of a diet from which lime was excluded. He attributed a genetic significance in infantile tetany to the scarcity of calcium in the organism, and his view is confirmed by the fact that the brains of children who die of tetany contain a smaller proportion of calcium than those of normal children. Oddo and Sarles found that the urine of tetanous children contained an increased amount of calcium salts. Quest's results, however, were not confirmed by those of either M. Cohn, or Leopold and v. Reuss.

Netter found that the convulsions of infantile tetany were favourably influenced by the exhibition of calcium chloride, but Stoeltzner obtained only an increased sensibility to electric stimulus by this means. Unlike Quest, Stoeltzner explains the spasmophilia as the result of calcium poisoning. He assumes that the galvanic irritability of the peripheral nerves depends on the calcium concentration in the tissues, and believes that an excess of calcium inhibits convulsions.

The relationship which the quantity of calcium in the urine and fæces bears to the general metabolism of calcium, has also formed the subject of investigation. Experiments conducted with dogs suffering from tetany, and similar clinical experiments, have not, however, yielded conclusive results. Czybulski found that, in a child of 7 months with tetanic symptoms, there was a far smaller retention of calcium at the time of the seizures than in the intervals between the attacks. In dogs from which the parathyroids had been removed and which were either starved or kept on a uniform diet, MacCallum and Vögtlin found a conspicuous increase in the amount of calcium in the urine and faces as compared with the control animal. V. Reuss and Welde found that, after partial parathyroidectomy, a dog showed increased retention of calcium.

The contradictory nature of these results induced v. Reuss and Leopold to try other methods to obtain more definite data 
concerning the metabolism of lime. They investigated the entire amount of lime, in both normal rats and those which had been deprived of the parathyroid glands. The latter showed no change which could be described as abnormal and there was certainly no reduction in the quantity of excreted lime. They found, however, that in young growing rats afflicted with tetany, the entire amount of lime was less than in normal rats; moreover, the proportion of lime in the bones to that in the soft parts, was invariably lower than in the control animal. The soft parts contained more lime, the bones less, and this finding is in accordance with the histological changes in the bones and the teeth.

As a matter of fact, a knowledge of the total amount of calcium does little to furnish a solution of the problem, for it supplies no information as to the amount of physiologically active free kathions. Leopold and $v$. Reuss think that the results of their experiments justify them in the belief that the amount of active calcium in the organism is reduced in tetany. This might occur as the result of the activity of the tetany toxin in precipitating calcium; while at the same time the passive calcium of the soft parts, which is ultimately excreted, is not only not reduced but, by means of food rich in lime, may even be increased.

The relationship between tetany and the metabolism of calcium, though not as yet fully understood, forms the basis of the therapeutic treatment of tetany by means of calcium salts. MacCallum and Vögtlin found that the administration of a 5 per cent. solution of calcium acetate and calcium lactate, whether by the mouth or by intravenous or subcutaneous injection, to dogs from which the parathyroids had been removed, was followed by disappearance of the tetanic symptoms. The improvement lasted twenty-four hours; the convulsions then recurred, but these were again subdued by a repetition of the treatment. Similar results were obtained with magnesium salts, but in this case the therapeutic effects were obscured by the anæsthetic toxic byeffects. Calcium salts increased the tetanic symptoms, but these were reduced by the exhibition of larger doses. MacCallum and Vögtlin are of the opinion that, taken in conjunction with their discoveries regarding the excretion of calcium, these therapeutic results point to an activity on the part of the parathyroid glands in controlling the metabolism of calcium. They believe that, after removal of the parathyroids, active calcium is withdrawn from the tissues and that there is, in all probability, an imperfect absorption and assimilation of calcium. Suppression of the calcium-ions causes muscular convulsion similar to that produced in normal animals by Loeb and MacCallum by the administration of oxalates which, in combination with calcium, form insoluble precipitates. In these cases also, the tetanic symptoms disappear after the administration of fresh soluble calcium salts. 
The results obtained by Parhon and Urechie, Berkeley and Beebe, and Bell and Martin, Ott, and by my own researches, furnish unquestionable proof of the susceptibility of tetany to the therapeutic influence of calcium salts. Slight, and even more severe, attacks are very much ameliorated by the exhibition of these salts, the symptoms sometimes disappearing altogether. After eighteen to twenty-four hours, the symptoms return in their former intensity. I did not find that the daily administration of calcium salts had any effect in prolonging the life of cats from which the thyroid and parathyroids had been removed. It must, however, be borne in mind that our knowledge of the therapeutic effects of calcium salts in tetania parathyropriva does not justify us in forming a definite conclusion with regard to the pathogenetic significance of these salts. It appears very probable that the calcium salts exercise an inhibitory effect upon the pathological muscular convulsions, similar to that which they exercise upon all forms of muscular contraction. This applies to the other kathions (magnesium, barium, strontium), which also exercise a favourable influence upon tetanic convulsions.

\section{THE POINT OF ATTACK OF THE HYPOTHETICAL TETANY TOXIN.}

The first experiments with the object of localizing the seat of tetany were carried out by O. Lanz in Horsley's laboratory. Similar investigations have recéntly been undertaken by MacCallum, by Falta and Rudinger, and by Biedl. The results are as follows :-

If, after parathyroidectomy, the nerve of one of the extremities is severed, no convulsion will take place in that limb. If the nerve is resected at a moment when the whole body is convulsed with tetany, the muscular contractions of that limb will cease, and the hypersensibility of the nerve will rapidly disappear. Pressure upon a nerve which has been severed some hours previously, will not produce a muscular contraction; also, the nerve is insensitive to galvanic stimulus. I have confirmed these results by repeated experiment, but the findings do not agree with those obtained by Falta and Rudinger. These observers severed the sciatic nerve after the appearance of tetany; they found that the electric hypersensibility persisted for several days and that the normal irritability of the nerve persisted until the death of the animal, which occurred nine days later. This discrepancy in the findings is probably explained by the fact that in each case I severed the whole of the nerve plexus of the front extremity, while Falta and Rudinger serered the sciatic nerve only. The resection of the sciatic nerve alone is not sufficient to arrest the muscular contractions in the limb, and MacCallum for this reason 
severed also the anterior crural nerve and the obturator nerve. Moreover, if the sciatic nerve is not divided high up, at the place where the plexus emerges from the pelvis, communication with the centre is not entirely cut off, and hence the persistence of the nervous irritability. In view of this fact, the explanation given by the authors appears superfluous. This explanation is that the increased excitement in tetany is due to the continued abnormal loading of the entire neuron from the trophic centre (the ganglion cell of the anterior cornu) with a substance, at present unknown and not definable; and that this substance remains for a time in the peripheral portion of the severed nerve.

The relationship of tetany to the spinal cord is best studied in animals in which the cord is severed at the sixth or eighth dorsal vertebra. The parathyroids may be removed at the same time, or subjects with tetany may be employed; the results in either case will be identical. After resection of the cord, there will be complete flaccid paralysis of the hinder part of the body with, at first, normal or exaggerated reflexes. If tetanic movements either remain or reappear, the whole of the paralysed portion of the body will show, in addition to slight fibrillary contraction, rapid, lightning-like spasms of large muscular areas, which resemble voluntary movements. It as an extraordinary thing to see the paralysed hinder extremities contract so rapidly and so violently that they appear to make running movements. But there is a marked difference between the behaviour of the fore and hinder parts of the body and this becomes particularly suggestive as the muscular spasms work up to the height of a tetanic seizure. The fore part of the body is affected with violent clonic muscular contractions which are interrupted by tonic contractions, the muscles being rigid in the intervals. The hinder part of the body is much less affected. The muscles are relaxed, there are fibrillary tremors, and single coarser lightning contractions; movements resembling running movements occur, but there is no trace of tonic rigidity. From these results it appears highly improbable that the seat of the tetanic changes is in the ganglion cells of the anterior cornua. MacCallum thinks that the site of hyper-irritation is in the higher motor neuron, probably just below the cortex.

Not enough attention has been given to the part played in tetany by the higher portions of the brain, the cerebrum and cerebellum. Horsley and Lanz are frequently cited as having proved that extirpation of the motor areas of the cortex of one side does not affect tetany of the other. But if the subjects are closely watched, it will be seen that the half of the body which has lost its motor centre is affected much more intensely than the other, and that during the tetanic seizures the tonic muscular rigidity of this side is very pronounced. During remission, on the other hand, whether spontaneous or, as in my experiments, 
effected by means of thyroid extract, the hemiparesis, both in dogs and cats, is far more pronounced than that usually seen after extirpation of the cortical areas, where this is unaccompanied by thyroidectomy. In one case extirpation of the cortical layer of one side of the cerebellum of a cat, was followed by a distinct increase in the tetanic symptoms of the same side of the body. Some years ago, Lanz described a similar but "not entirely conclusive result." He points out that the remarkable disturbance of equilibrium and the staggering walk of many thyroidless dogs, suggest that a portion of the cerebellum is concerned in the causation of tetany. Lanz believes that his experiments point to the medulla oblongata as the chief seat of tetany, but that the higher centres also exercise an influence upon the contractions.

All attempts to localize the site of tetany have not, as yet, led to any satisfactory solution of the problem. Rudinger's contention that the condition of hyperirritability arises in the ganglion cells of the anterior cornua, if not in the entire peripheral neuron, did not survive the test of experiment. In a later publication by Falta and Rudinger, the theory is modified to this extent, that the condition of hypersensibility is attributed to the sensory and motor ganglion cells, more particularly those of the peripheral nerves. The authors suggest that the hyperirritability after parathyroidectomy arises from the suppression of the inhibition which, under normal conditions, is conveyed from the parathyroids to the spinal cord. Hence, "the continued storing of fresh energy in the motor ganglion cells leads eventually to an overflow of irritability, and this constitutes a tetanic attack."

This complicated hypothesis derives its sole support from the fact that adrenalin glycosuria reappears in dogs from which both the thyroid and the parathyroids have been removed; the inference being that the parathyroids exercise an inhibitory effect upon the sympathetic nerve. The theory cannot be said to have stood the test of experiment, and it does not in any way add to our understanding of the symptoms which follow cerebral lesions and the resection of the spinal cord. These symptoms point to the involvement of other and higher nerve centres in the genesis of tetany, though they do not explain the manner in which the intervention of the higher portions of the brain takes place. The pathologico-histological findings, described by MacCallum, in the central nervous system of tetanous animals, which consisted in acute degenerative changes such as swelling of the nuclei of the cells of the anterior cornua and chromatolysis in the cortical cells, also point to the involvement of the central nervous system.

In the present imperfect state of our knowledge of tetany, all that we can do is to point to the increased irritability of the nervous centres and of the peripheral nerves connected with them, together with the spasmophilia to which this irritability gives 
rise as the characteristic features of the condition; but we are not in a position to define either the exact anatomical seat of tetany, nor indeed the nature of the tetanic changes. One thing only can be affirmed with certainty, and that is, that the affection of the muscles is not myopathic in origin.

\section{PHYSIOLOGY OF THE THYROID GLAND.}

\section{Experimental Extirpation.}

A certain amount of light is thrown upon the functional significance of the thyroid gland by the results which follow its extirpation. It will be clear from what has gone before that, before the function of the parathyroid glands was understood, experimental extirpation of the thyroid could be of value in the case of those animals only in which removal of the thyroid did not necessarily include removal of the parathyroids. Hence, the only results of any value were those obtained exclusively with the herbivoræ. As a matter of fact, it is only the more-recent experiments, those in which the parathyroids have been purposely spared, which can be regarded as a rational basis for the investigation of the phenomena associated with the thyroid gland. This applies more particularly to the investigation of the pathogenesis of those symptoms which characterize the suppression of the parathyroid, as well as of the thyroid, glands.

The results of thyrotoxic serum in the thyroid disturbance have been investigated by various authors (Gontscharnkoff, Mankowsky, Yates, MacCallum, Portis, Demoor and van Lint, Luithke, Slatineano), but with very partial success. Several of these describe a condition resembling cachexia thyropriva, but in the greater number of instances the symptoms are those of acute tetania parathyropriva. Slatineano found that, in addition to its hæmolytic action, thyrotoxic serum exercises a specific and very decided influence upon the thyroid. In small doses, it acts as a stimulant and promotes the secretion of colloid; after larger doses, the colloid diminishes and there is hypertrophy of the epithelial cells of the follicles, followed by acute degeneration of these cells and their rapid destruction. Portis found that the exhibition of thyrotoxic serum was followed by degeneration, not of the thyroid only, but of the liver, spleen and kidneys. Marchetti's finding, that the blood of patients with thyroid affections gives a precipitation with thyrotoxic serum from rabbits, was not confirmed by Schutze.

Both the recognition of the symptoms of suppression and the estimation of their pathological significance are influenced by one very important factor. It must always be remembered that accessory glands, distinct from the principal organ and situated 
at a greater or less distance from it, are of by no means rare occurrence. Many animals, especially dogs, are not necessarily thyroidless because the thyroid gland has been removed from the neck. After thyroidectomy, cases such as these frequently show only passing symptoms of suppression, or the signs may be absent altogether.

There is a certain amount of difference between the signs of thyraid suppression in young growing animals and in adults. This difference does not affect the essential character of the changes, the so-called athyrosis, which always involves the metabolic processes of the entire organism. In the case of young individuals, however, the derangement of metabolism is manifested with peculiar distinctness in the changes which take place in the processes of growth.

Hofmeister ( 1892 ) found that the extirpation of the thyroid of young rabbits, the external parathyroids being left in situ, was followed by a retardation of growth and the development of a condition of chronic cachexia.

Further experiment showed that the arrest of growth was due to a remarkable retardation of the process of ossification, both of the epiphyses and of the synchondroses. The long bones, the pelvis and the vertebral column, showed the greatest departure from the normal, the skull the least. The growth of the long bones was retarded to the extent of at least a third of their proper length, and microscopic examination of the epiphysial line showed specific degeneration, consisting in a reduction of normal cell proliferation, combined with vesicular swelling of the cartilage lacunæ and shrinkage, or even partial destruction, of the cells.

In the internal organs, Hofmeister found a condition similar to that described by Rogowitsch in the case of adult rabbits, namely, enlargement of the glandular portion of the hypophysis cerebri, and the appearance of large vacuoles in the protoplasm of the enlarged principal cells. Moreover, he observed a similar formation of vacuoles, together with degenerative changes, in the epithelial cells of the looped uriniferous tubules. The condition of the organs of generation was particularly striking. In addition to degenerative changes, the ovaries invariably showed premature maturity of many of the follicles. Jeandelize discovered later that, in male animals, there is imperfect development of the testicles.

v. Eiselsberg found that in sheep whose thyroids had been removed at the age of ten days, there was conspicuous retardation of growth and a change in the mental condition, which forcibly suggested the idiocy of cretinism. Post-mortem examination showed that, in addition to a high degree of derangement of the processes of skeletal growth, there was widespread atheromatous -degeneration in the aorta. The intima was covered with very 
hard, smooth, white plates which, under the microscope, showed either calcification or fatty degeneration. The testicles were very small.

In goats, operated on three weeks after birth, the same author was able to observe a difference in growth in comparison with the control animal as early as one month after operation. At the end of four months, the thyroidectomized goat was half the weight of the control animal. In addition to shortening of the bones of the extremities, there was marked shortening of the frontal part of the head, the occipital part appearing exaggerated by comparison. There were also remarkable changes in the growth of the hair. The thyroidectomized sheep had poor fleece and the fur of the young rabbits was scanty, but in the young goats there was a conspicuous increase in the growth of the hair, and the long hairs could be pulled out in handfuls. The horns, however, of both sheep and goats, were equally stunted in growth. There was also meteoristic swelling of the abdomen, reduction of temperature, and arrested development of the sexual glands. Both the general apathy and the atheromatous degeneration of the aorta were very marked in goats. Pick and Pineles found pronounced sclerotic changes in the aorta and enlargement of the suprarenals of two goats, from which the thyroid had been removed at the age of 6-10 weeks, one of which had been treated with iodothyrin. In a pig which had been thyroidectomized at the age of 4 weeks, v. Eiselsberg found that there was marked arrestation of growth unaccompanied, however, by apathetic idiocy, the animal being as lively as normal animals.

In pigs, which had been thyroidectomized at the age of a few weeks, Moussu saw a condition several weeks later, which closely resembled myxœdema. There was swelling of the abdomen and formation of infiltrated cutaneous folds; the skin became bald in places and, in others, was covered with remarkably long and thick bristles; there was also arrestation of growth.

The infantilism, the imperfect activity of the sexual glands and the general torpor, are very apparent after thyroidectomy in hens. Lanz found that such birds lay very few eggs and that these are abnormally small and covered with a very thin shell. The laying capacity may be increased by feeding with thyroid gland.

In those species where the parathyroids are in close anatomical relationship with the thyroid, and where extirpation of the latter necessarily included the removal of the former, the operation was invariably followed by acute symptoms of suppression. The violent and fatal tetany in carnivora was believed to result from suppression of the thyroid function. After the discovery of the parathyroid origin of tetany, I set myself to work to produce the same clinical picture in dogs as that presented by the herbivora after removal of the thyroid. I was especially anxious to reproduce the arrestation of growth in young animals. 
After the thyroid has been removed from dogs $3-5$ weeks old, the parathyroid bodies being left intact, the animals may be kept alive for months or even years, provided that post-operative tetany does not supervene. After about two months, the animals show a considerable difference in size, as compared with control animals of the same litter. After six months, this difference becomes so marked that the control animals will weigh two or three times as much as the thyroidectomized animals.

I found that the constitutional changes were also very noticeable.* The thyroidectomized animals are much smaller, the legs are short and fragile, and the skull is rounded, with a projecting infantile forehead. As a result, apparently, of disproportion between the size of the viscera and that of the skeleton, the abdomen appears to be very much distended. Occasionally there is a peculiar deformity of the breast-bone. It is remarkable that the mental apathy and impaired intelligence which, according to v. Eiselsberg, is invariably displayed by thyroidectomized sheep and goats, is absent in the case of young dogs. This is the more remarkable in view of the fact that spontaneous cretinism is known to occur in young dogs (v. Wagner). Examination of the skeleton shows that all the bones, and especially the hollow long bones, are remarkably fragile in thyroidectomized dogs, while in the herbivora, on the other hand, the bones are short, thick and heavy. The post-mortem examination of my thyroidless dwarfed dogs revealed the persistence of an exceptionally large thymus, where normally only slight traces of this organ are found; up to the second or third year there was distinct hypertrophy of the hypophysis cerebri with increase of colloid; a slight hypertrophy of the cortical part of the suprarenals; and a very noticeable hypoplasia of the sexual glands. In one instance I found a very much hypertrophied accessory thyroid gland embedded in the adipose tissue of the aorta. The skin showed neither the clinical nor the histological changes characteristic of myxœdema. Similar results have since been obtained with dogs by Massaglia, and by Parhon and Goldstein with cats. The arrest in the growth of the skeleton and in the development of the sexual organs is the typical and invariable result of the absence of the thyroid function in both carnivora and herbivora.

The changes seen in older animals after thyroidectomy are, as a general rule, much less clearly marked. They are principally confined to changes in the metabolic processes, the clinical manifestations of which are: Loss of appetite, sluggish digestion, increased emaciation, and, finally, cachexia thyropriva.

Loss of intelligence is rarely seen. The disinclination for

* Illustrations will be found in A. Biedl, "Innere Sekretion," Vorlesungen, 1902, Vienna, 1903. Wiener Klinik, xxix, 1903. 
active movement and the indolence of the movements are symptoms of apathy. Trophic disturbances of the cuticle are very frequent, and consist in falling of the hair, dryness of the skin, and more or less extensive eczema. A myxœedematous swelling of the subcutaneous cellular tissue, together with idiocy, has been described by Horsley in apes, by Moussu in pigs, goats and sheep, and by v. Wagner in cats. Personally I have never seen, in any animal species, changes in the skin resembling those of myxœdema.

The changes in the blood are also remarkable. There is a marked and continued decrease both in the number of red blood corpuscles and in the amount of hamoglobin, accompanied by an increasing leucocytosis, in which, more particularly, the number of the mononuclear and eosinophile cells is augmented (Falta and Berterelli).

The typical and, in my experience, the invariable resuit of suppression of the thyroid function in adult animals, is a progressive emaciation, which increases to pronounced cachexia and culminates in death. These results are absent only in those, not very rare, cases where accessory thyroids are present, the hyperfunction of which more or less completely replaces the suppressed activity of the principal organ. Such accessory glands are of somewhat frequent occurrence in dogs, and may be reckoned upon in one case out of five; in cats they are rarer.

In cats, the emaciation begins after two or three days, before there is any appreciable diminution of appetite. Later, the appetite disappears entirely, the animals refusing all food but milk, and after a few weeks become extremely emaciated in consequence. In dogs, the loss of weight begins a good deal later and progresses so slowly that the animals may live for months, even one to two years, after removal of the thyroid. They then present, however, a wretched picture of emaciation and trophic cuticular derangement. Death in such cases most frequently occurs from infective diseases accidentally acquired.

Of the post-mortem findings, the most characteristic is the enlargement of the hypophysis cerebri, which varies in degree according to the length of life after operation. In animals which have lived for some time after thyroidectomy, the hypophysis is frequently two or three times as large as in control animals.

The changes which take place in the pituitary after thyroidectomy were first described by Rogowitsch in 1888 . He found an increase in the size of this organ in rabbits and dogs as early as eight to fourteen days after operation. The histological changes consisted in an increase in the amount of cell protoplasm and an enlargement of the space between the nuclei; the number of vacuoles in the cell-body and the amount of colloid were both increased. In addition, there was a marked dilatation of the blood-vessels, and the organ contained a very large amount of 
blood. These findings were confirmed by those of later authors (Stieda, Tizzoni and Centanni, Hofmeister, Gley, Leonhardt, Pisenti and Viola, Alquier, Lusena, Thaon, Torri, Lucien and Parisot). Katzenstein and Traina were unable to find hypertrophy of the hypophysis after thyroidectomy, and this is obviously due to the fact that their subjects were examined too soon after the removal of the gland. Cimoroni showed that the hypertrophy of the pituitary gland is due to the removal, not of the parathyroids, but of the thyroid. According to this author, the hypertrophy of the hypophysis, consequent upon thyroidectomy, acquires a specific character from the presence of certain cells, which distinguish it, more particularly by their size, from the hypertrophy which follows castration.

Herring describes noticeable changes affecting the glia cells in the nervous parts of the hypophysis after thyroidectomy.

The enlargement of the spleen, which is sometimes seen after removal of the thyroid, cannot be regarded as typical of the condition.

The changes in the processes of metabolism which follow thyroidectomy are constant and, to a certain extent, characteristic. The older experiments, those in which thyroparathyroidectomy was carried out with dogs and cats, can scarcely be employed as evidence in this connection. The muscular contractions characteristic of the acute tetany which follows this operation bring about an augmented metabolism; moreover, as animals in this condition are either unable to take food or take it very spasmodically, it is obvious that accurate observation of metabolic changes is impossible. For this reason, a large number of experiments undertaken with the object of determining the metabolism of gases and of albumin (E. Maier, Smith, Michaelsen, Dutto and Monaco, E. Roos, Ver Ecke, Verstraeten, Gluzinski and Lemberger, Formanek, Ducceschi, \&c.) are highly contradictory in their results.

Extirpation of the thyroid alone brings about a diminution of metabolism, a fall in the physiological interchange of gases and a reduction in the amount of albumin required by the organism. Eppinger, Falta and Rudinger found that, in thyroidectomized dogs in which the parathyroids were left intact, the metabolism of albumin in the fasting state is lower than in normal dogs of the same weight. In larger animals, provided that hypertrophied accessory glands are not present, the reduction in the amount of albumin metabolized in the fasting state amounts to one-third. The exhibition of carbohydrates or fats either does not lower the metabolism of albumin, or does so in a lesser degree than under normal conditions.

The metabolism of the carbohydrates in thyroidless animals has been dealt with by various authors. According to Falkenberg and $\mathrm{R}$. Hirsch, the removal of the thyroid produces glycosuria and 
a diminished capacity for the assimilation of grape sugar. Eppinger, Falta and Rudinger found, on the contrary, that thyroidectomy never produced glycosuria, and that feeding with large quantities of sugar was not followed by sugar in the urine. They pointed to the remarkable fact that an adrenalin injection which, in normal animals, provokes extreme glycosuria together with an increased metabolism of albumin in the fasting state, does not produce glycosuria in thyroidectomized animals, even where sugar is given at the same time. Moreover, the decomposition of albumin in the fasting state is decreased in such animals after the injection of adrenalin. These authors account for the glycosuria and the diminution of the normal assimilation of sugar in Falkenberg and R. Hirsch's animals, by the fact that in all their experiments the entire thyroid apparatus was removed. After thyroparathyroidectomy, there is a disturbance of the metabolism of the carbohydrates; this, according to Underhill and Tadasu Saiki, is illustrated by the fact that, after the subcutaneous injection of 5-7 grm. sugar in 20 per cent. solution for every kilogramme of weight, there is a marked excretion of sugar, which is continued over a considerable period. Eppinger, Falta and Rudinger showed, further, that adrenalin produces in dogs from which the entire thyroid apparatus has been removed, a more marked glycosuria than in normal animals, and that, at the same time, the metabolism of albumin in the fasting state may be augmented. After the extirpation of several parathyroids, there was a more or less marked reduction in the assimilation of sugar, and this applies also to those cases where there were no apparent symptoms of tetany. The effect upon metabolism of the suppression of the parathyroid function is the reverse of that produced by suppression of the thyroid; for in true athyrosis the assimilation of sugar is increased and the exhibition of adrenalin is not followed by glycosuria. R. Hirsch, however, regards the reduced capacity for the metabolism of sugar and starch after parathyroidectomy as a typical symptom of tetany. The glycosuria makes its appearance in tetany after the nervous symptoms have become manifest, and this led Hirsch to believe that it is due to intervention of the nervous system.

The experiences of Eppinger, Falta and Rudinger in regard to the absence of glycosuria after the exhibition of adrenalin in thyroidectomized animals, was confirmed by Grey and de Santelle with dogs, and by Pick and Pineles with young goats but not with rabbits; while Underhill and Hilditch found that there was distinct glycosuria after the exhibition of adrenalin in dogs. Ritzmann found that, in the first two days after thyroparathyroidectomy, the intravenous infusion of diluted adrenalin solution did not produce glycosuria in cats, even where the quantity used would be considered large in the case of normal animals. The action of adrenalin becomes negative at the maximum development 
of the acute symptoms which follow extirpation, and the reduction of the adrenalin glycosuria keeps pace with the development of the symptoms characteristic of suppression. According to Ritzmann, this is not the result of suppression of the thyroid function, but is due to a change of conditions which arises while the organism is adapting itself to the absence of the thyroid function.

The relationship between the thyroid gland and the metabolism of the carbohydrates, suggests that this correlation is dependent upon the agency of an organ of the highest importance to the metabolism of the carbohydrates, that, namely, of the pancreas. Lorand was the first to suggest a relationship between the thyroid gland and the pancreas, and he endeavoured to support his theory by histological and experimental investigation. He pointed to the fact that after extirpation of the thyroid in dogs from which the pancreas has been removed, the sugar disappears from the urine; and that where the pancreas is extirpated and the thyroid spared, the colloid contents of the latter increase. Licini, more recently, describes the histological signs of increased thyroid activity, and Falta and Bertelli found that, after thyroidectomy, there was a distinct hypertrophy and increase in the number of the islands of Langerhans.

The results of the experiments undertaken by Eppinger, Falta and Rudinger with the object of determining the relationship between the thyroid and the pancreas may be summed up as follows :-

I.-If the thyroid is removed seven to thirteen days before extirpation of the pancreas, the resulting diabetes will be characterized as follows :-

(a) The metabolism of albumin in the fasting state is slightly more than that of the control animal; but if the pancreas only is removed, the metabolism of albumin will be increased three- or four-fold.

(b) The quotient $\mathrm{D}: \mathrm{N}$ (Dextrose : Nitrogen) is considerably higher than after extirpation of the pancreas alone. In the latter case it oscillates regularly between 2.8 and 3 , but after removal of the thyroid it rises to $3 \cdot 5$. These figures apply both to animals in the fasting state and those fed on flesh.

(c) The reduction in weight of dogs in the fasting state is comparatively slight.

II.-If the pancreas and thyroid are removed at the same time, the suppression of the thyroid is the first to make itself gradually felt. On the first day after operation, there is an increase in the decomposition of albumin in the fasting state, which exactly resembles that following removal of the pancreas alone. But in place of a further increase in the decomposition of albumin, the rise is followed by a fall which, in this case also, raises the quotient $\mathrm{D}: \mathrm{N}$ above the normal. 
These results led Eppinger, Falta and Rudinger to the conclusion that the thyroid and the chromaffine system, together with, as Falta and Berterelli discovered later, the infundibular portion of the hypophysis cerebri, constitute a group of vascular glands which augment and accelerate the processes of metabolism. The balance is maintained by the antagonistic activity of those other vascular glands which, like the pancreas and the parathyroids, exercise a retarding influence upon metabolism.

Now as these two groups of internal secretory glands possess physiological interrelationships with one another, the extirpation of a vascular gland is followed by two different sets of phenomena. In the first place, there are the direct results, due to suppression of the specific secretion; in the second, there are the indirect results, due to derangement of other glands, the functions of which, under normal conditions, were either stimulated or inhibited by the activity of the suppressed gland. The thyroid is believed to promote the activity of the chromaffine system and to inhibit that of the pancreas. The direct results of thyroidectomy consist in reduction in the metabolism of albumin, fat and salt. The indirect results are, on the one hand, the absence of stimulation of the chromaffine system, and on the other, a hyperactivity of the pancreas due to the removal of the inhibitory agent.

Eppinger, Falta and Rudinger believe that the nervous system is the agent by which the interactivities of the vascular glands are affected, and by means of which they are enabled to influence the processes of metabolism.

The relationship of these organs to the vegetative nervous system is two-fold. In the first piace, each gland is innerved by a definite portion of the regetative nervous system; and in the second, the internal secretion of each gland reacts upon the condition of irritability of the nerve by which it is supplied. That group of internal secretory organs which promotes metabolism has a sympathetic innervation and stimulates the sympathetic nerves, at the same time exercising an inhibitory effect upon the autonomous nerves. 'The group which retards metabolism, on the other hand, possesses an autonomous innervation and, while stimulating the autonomous nerres, inhibits the sympathetic. The thyroid gland possesses a double function; being furnished. with both an autonomous and a sympathetic innervation, it is able to affect both portions of the vegetative nervous system.

The suppression of the thyroid is followed by a decreased irritability of the sympathetic nerve, and this is manifested in a sluggishness of the circulation and in certain trophic disturbances. Adrenalin exercises a direct influence upon the sympathetic nerve, but, after thyroidectomy, its exhibition is not followed by glycosuria and it provokes only an inconsiderable rise in bloodpressure. At the same time, the athyrosis produces a decreased excitability of the autonomous nerves, as shown in the sluggish- 
ness of intestinal peristalsis, the insignificant effects produced by pilocarpin, and the intensified action of atropin upon the eyes (Asher).

That thyroidectomy has an effect in reducing the metabolism of fats has been proved by experiments.

With regard to the metabolism of salts, Falta, Tedesco, and Bolaffio found that dogs which had been thyroidectomized some time previously did not show a decreased assimilation during the first few days of the fasting state. Cases have been described, however, where the excretion of the chlorides and the amount of calcium, phosphorus and magnesia present in the urine were decreased after thyroidectomy, while the amount of calcium and phosphorus in the faces and of magnesia in the urine became increased after the exhibition of thyroid preparations (Falta).

These results would seem to show that the suppression of the thyroid function reduces the metabolism of salts in the same way as it does that of albumin, though the two processes do not proceed parallel to one another.

\section{ATHYROSIS AND HYPERTHYROSIS IN MAN.}

Sir William Gull (I873), under the title of "Cretinoid State in Adult Life in Women," described a symptom-complex which, later, was termed "myxœedema" by W. Ord (1877), and " cachexie pachydermique" by Charcot (I879), and which was regarded, by both these authors, as a separate clinical entity. Curling, at an earlier date, described the atrophy or absence of the thyroid gland in sporadic cretinism; and the pathogenetic significance of the thyroid gland in myxœedema was suspected even at that date. But until the publications of both Reverdin and Kocher in r882-1883, the clinical conditions which follow suppression of the thyroid were either unknown or were unappreciated. These authors, writing independently of one another, described the symptoms which are observed after the total extirpation of a degenerated thyroid gland. Reverdin named this condition Myxadème postopératoire; Kocher called it Cachexia strumipriva.

\section{OPERATIVE ATHYROSIS.}

The most constant symptom of thyroid insufficiency in the young growing individual, in man as in animals, is a remarkable disturbance of the processes of growth. This takes the form of a retardation of the growth in height, the growth in breadth continuing very much as in the normal (v. Bruns). The arrestation of development produces a dwarf-like appearance of the individual, due to imperfect endochondral ossification. The unclosed epiphysial lines may be seen in skiagrams, and post-mortem examination shows that the ossification of the ends of the 
epiphyses is extremely deficient, while the periosteal bone formation is undisturbed. But the changes are not in the skeleton only; the entire organism shows signs of arrested development. The most marked of these is the infantilism of the sexual organs, in consequence of which puberty is either very retarded, or is absent altogether. Of the other organic changes, the most important is the enlargement and degeneration of the hypophysis cerebri, first described by Schönemann. The enlargement is easily seen in life by means of skiagrams. In addition to the somatic changes, there is a very marked arrestation of mental development. As this shows itself in a decreased locomotion and an impaired intelligence, the clinical picture presented by such thyroidless children is very suggestive of that of cretinism.

In adults, where the signs of arrested growth are absent, the most conspicuous symptoms are those of nervous derangement. There are fatigue and pains in the limbs, followed by pronounced lethargy, and in spite of the fact that the muscular structure is well developed, there is a remarkable deficiency in muscular power. This is followed by a reduction in mental activity and power. Of the somatic signs, the most important are the changes in the skin, from which the disease myxodema derives its name. The skin is remarkably white and wax-like; it is thickened and puffy, yet at the same time dry. There is a peculiar oedematous swelling which appears first in the face and afterwards in the extremities; it yields very slightly to pressure and gives a peculiar awkwardness to the body and a fixed expression to the face. The circulation of the blood and the nourishment of the skin are both much impaired. The coldness to the touch, and pallor of the skin are objective signs, as the persistent sensation of chilliness is the subjective sign, of the reduced cuticular circulation. The dryness and scaling of the skin, the chronic eczema and secondary pigmentation, and the blanching and loss of hair, are all signs of trophic disturbance. The visible mucous membranes of the tongue, the uvula and the nose, all show signs of œdematous swelling. The changes in the function of the sexual organs are very remarkable. In women, menorrhagia is an unfailing accompaniment of athyrosis. Should pregnancy occur, as it sometimes does, the signs of athyrosis will be greatly intensified. Markedly cachectic men are sterile. The blood-changes consist in a somewhat high degree of anæmia, a very great reduction in the number of red corpuscles, accompanied by a proportionate increase in the number of the leucocytes and, more especially, of the mononuclear cells.

In the absence of the thyroid gland it is possible, according to Kocher, for man to live for seven years, though in a condition of pronounced cachexia. Apart from the supervention of complicating conditions, he may die as the result of the cachexia.

Symptoms of suppression occur in 70 per cent. of the cases 
of total extirpation. Where these do not appear, either the gland was not removed in its entirety, or accessory thyroids are present. Symptoms of cachexia may appear after partial extirpation of goitrous glands. In such cases, however, the symptoms are much milder and the condition is one of mitigated cachexia thyropriva, analogous to Hertoghe's spontaneous "chronic benign hypothyroidism."

The evil consequences of treating goitre by the surgical method may be largely avoided, if a proportion of the glandular tissue is allowed to remain in situ. It is hardly possible to define the exact quantity. Kocher thinks that, in the case of diffuse goitres, at least a quarter of the gland must be preserved if dangerous symptoms are to be avoided.

There is sometimes spontaneous recovery from the symptoms of suppression which follow total extirpation of the thyroid. These coincide with the growth of a so-called " goitre relapse," and the improvement is explained by the fact that the new formation is not of goitrous tissue, but is a hyperplastic development of remains of normal glandular tissue which were left in situ. These cases are instances of autotransplantation. On the other hand, cachexia may develop after partial extirpation of a goitre, the phenomena in this case making their appearance at the moment when goitrous degeneration appears in the remains of the lobe, or in the lobe of the other side. As Kocher points out, there are goitres and goitres. In one case, goitre may be the result of hyperplasia of functionally active thyroid tissue; in another, it may mean a degenerative process leading to suppression of the normal thyroid function.

\section{TRUE ATHYROSIS AND HYPERTHYROSIS.}

Having given an account of the results which follow the operative removal of the thyroid gland, it is fitting that those genuine clinical conditions which are brought about, to the best of our knowledge, by the suppression of its function should next be described. The first of these is myxœdema adultorum, generally known as myxœedema. It is not necessary to describe the symptoms of this condition, for to do so would be to repeat the clinical description of cachexia strumipriva. The identity of the symptoms justifies the name of "operative myxœedema," which is sometimes given to cachexia strumipriva. Moreover, though the post-mortem findings in true myxœema are somewhat scanty, they show that this condition, like cachexia strumipriva, owes its origin to the suppression, or to the conspicuous reduction, of the activity of the thyroid gland. The thyroid is invariably much reduced in size and occasionally completely atrophied; it is pale in colour, and, under the microscope, shows obliteration of the glandular parenchyma together with proliferation of the 
interstitial connective tissue. We are still, even at this date, entirely in the dark in regard to the etiology of this slow, progressive atrophy of the thyroid tissue. We only know that women are far more frequently affected than men (8o per cent.); that the disease is frequently associated with disturbance of sexual function, and occasionally with acute infective conditions; and that it occurs with much greater frequency in countries where goitre is not endemic (Great Britain, Belgium).

The abortive forms of true myxœedema, to which Hertoghe gave the name of Hypothyroidie benigne chronique, resemble the myxœedema postoperativum frustrum which appears after partial thyroidectomy.

These cases, which have latterly received a good deal of attention, generally show only isolated, though characteristic, secondary symptoms of myxœedema; such are trophic and vasomotor disturbance of the skin, absence of perspiration, falling of the hair, and, occasionally, œdema. Functional disturbances of the sexual organs, such as menorrhagia, also occur. The manner in which these cases respond to treatment with thyroid extract shows that the condition arises from a reduced thyroid activity, or hypothyreosis.

Another clinical condition which ought to be included in this group is Adiposis dolorosa, or Dercum's disease, but its pathogenetic relationship to the thyroid gland is by no means fully established. In I888, the American neurologist, Dercum, described a condition in many respects resembling myxœedema, but characterized by the appearance in the connective tissue at different parts of the body, of circumscribed and very painful fatty tumours. In addition to the localized fatty tumours, there are also diffuse fatty deposits and, at times, a remarkably obese bodily habit. Side by side with this, there is usually pronounced muscular weakness, the patients performing only the most necessary voluntary movements on account of the pain induced by muscular action. The will-power is generally unimpaired, though there is sometimes an apathy reminiscent of that of myxoedema, and more rarely a condition of psychic exaltation. No information concerning the metabolism of the condition is forthcoming; in some cases glycosuria has been observed. A remarkable feature of the condition is the absence of eosinophile leucocytes from the blood. Six cases have come under postmortem examination, and of these five showed more or less farreaching pathological changes of the thyroid gland. In one case, described by Dercum and MacCarthy, the thyroid was normal, but there was adeno-carcinoma of the hypophysis cerebri.

The pathological anatomical findings appear to point to the thyroid as the originating cause in adiposis dolorosa. But the hypophysis undoubtedly plays a part in the genesis of this condition, and it is probable that the sexual glands also are concerned, as in such cases they are frequently atrophied. 
Certain French authors (Féré, Kaplin, and Fédoroff) are of the opinion that this condition is an obesity due to hysteria, and that the thyroid is without pathogenetic significance.

The exhibition of thyroid gland in adiposis dolorosa has occasionally been attended with success, but, in many cases, it has been entirely without result.

A special pathogenetic significance is ascribed to thyroid insufficiency in the changes which occur in later life, and which are included in the term cachexia of old age, or senile degeneration. The foundation for the theory that old age results from changes in the thyroid gland lies in the fact, commented upon by Horsley, that in old age the thyroid becomes atrophied, its follicles shrink and retrogressive changes take place in the epithelial cells. This is reinforced by the fact that there is a profound analogy between the signs of advanced old age and those of myxœdema. The falling of the hair and the dropping out of the teeth, the dry and wrinkled skin, the lowered temperature of the body, the diminished perspiration, the indolent digestion and consequent emaciation, the reduced metabolism and consequent primary deposit of fat, followed by emaciation, the atrophy of the sexual organs, the decrease of mental power, and the diminution of the activity of the entire nervous system-these are all symptoms which characterize chronic myxœedema. Horsley holds the view, which is even more emphatically expressed by Vermehren, that senility is due, at any rate in part, to degeneration of the thyroid gland, while myxœedema may be described as a condition of premature senility.

Premature decay, called progeria by Gilford, was regarded by Rummo and his pupils as a separate clinical entity and was described by them under the name of senilismus or geroderma genitodistrofico.

This condition is characterized by the appearance, in youth, of senile changes in the skin and a defective development of the sexual apparatus; it is believed to depend upon changes in the thyroid gland and is regarded as a form of myxodema frustrum.

Lorand recently advanced the view that old age, or senile involution, has an etiological relationship, not with the thyroid only, but with all the organs which possess an internal secretory function. He points out the similarity between the changes of senile decay and the symptoms of myxœdema, and he lays particular stress upon the reduction of metabolism, the trophic changes in the skin, the general increase of connective tissue, and the cessation of sexual activity, all of which are symptoms characteristic of both conditions. He believes that the atrophy of the thyroid is the result of retrogressive changes in other internal secretory organs (hypophysis, suprarenals).

There is, however, one point in connection with this theory which must not be overlooked. We have no proof that there is 
an etiological relationship between the symptoms of senile cachexia and the retrograde changes which, in this condition, take place in the internal secretory organs. The possibility suggests itself that these changes are not the cause, but the consequence, of the general nutritional disturbance which occurs in old age. As Ewald rightly points out, the atrophy of the intestinal tract, which is an invariable and characteristic feature of senile cachexia, is absent in myxœedema.

We have not sufficient grounds for the assumption that senile decay results from the suppression of function of internal secretory organs other than the thyroid gland. The pathogenesis of this condition offers a wide field for investigation.

We are very much better informed in regard to the pathological consequences of thyroid insufficiency in youth.

In addition to myxœdema, conditions occur in young growing individuals as a result of suppression of the thyroid function, which, like those which follow operative athyrosis, are characterized by derangement of the processes of growth and serious mental deficiency. Owing to the similarity which these cases bear to those of endemic cretinism, they are generally included under the heading of sporadic cretinism. Out of the medley of these cases, Pineles, with considerable penetration, distinguished two distinct clinical entities, namely, congenital myxœedema, which he calls thyroplasia, and infantile myxoedema. In addition to myxœedematous signs, both conditions present symptoms of derangement of the processes of growth, though these are not as severe in the latter condition as in the former.

Infantile myxœedema appears at or after the fifth year; it resembles adult myxoedema in its distribution and in its clinical course, but more particularly in the fact that it is never congenital, but always acquired. In both cases the condition is due to pathological changes leading to atrophy of an already completely formed thyroid gland.

But in congenital myxœdema the case is otherwise, and we have to do with a congenital thyroid deformity, a thyroidaplasia, in fact. From evidence afforded by the literature of the subject, Pineles drew the conclusion that the complete absence of the thyroid, the parathyroids being present intact, is not the secondary result of a previous pathological condition, but is a congenital defect of development; it is, in fact, true spontaneous thyroid suppression. The clinical picture, as in operative athyrosis, is one of extreme severity; there are pronounced dwarfism, extreme idiocy, and marked symptoms of myxœedema, all of which are present from earliest childhood.

Congenital myxœdema, the symptoms of which closely resemble those of endemic cretinism, differs from the latter condition in the sporadic nature of its occurrence. Moreover, endemic cretinism is never, and goitre very rarely, found in the antecedents 
of congenital myxœdema, while goitre is almost invariable in the antecedents of endemic cretinism.

Hypothyrosis in children also includes the condition known as infantilism.

Infantilism, as defined by Laségue, is a disturbance of development, the salient feature of which is the persistence, in both the mental and physical state, of infantile characteristics. The chief clinical signs of this condition are the small size of the bones and internal organs, the defective development of the sexual glands and accessory sexual organs, and the childishness of the mental state.

The term infantilism was afterwards made to include those cases of arrested development which result from nutritional derangements and from diseases of the vessels, the brain, \&c. This form is. known as infantilismus dystrophicus or typus Lorrain, and its etiology is to be sought in a number of widely different originating causes. It may appear, as the result of hypoplasia of the vascular system (Virchow), in hereditary syphilis; after alcoholism and other forms of chronic poisoning in the parents; in primary cerebral disease; as the result of infective diseases contracted in early life (tuberculosis, pellagra); metabolic derangements (chlorosis); cardiac disease (pulmonary and mitral insufficiency); and finally, as the result of unhygienic surroundings and insufficient nourishment in early childhood.

A closer study of infantile myxoedema and cretinism, and the recognition of the pathogenetic significance of the thyroid gland in these conditions, led Brissaud, and after him a number of French scientists (Meige, Thibierge, Hertoghe, \&c.), to investigate the close relationship which exists between the abortive forms of myxoedema and certain forms of infantilism. Brissaud named these conditions infantilismus myxodematosus, or myxinfantilismus, and he believed that both the dystrophic and the myxœdematous types were thyrogenetic in origin.

Many authors, especially Hertoghe, ascribe all forms of infantilism to disturbance of the function of the thyroid. But there are others (De Sanctis, Ferranini, Anton) who lay stress upon the fact that, in many cases of infantilism both slight and severe, the thyroid is comparatively normal. Brissaud's type is, however, well known, and its pathogenetic relation to the thyroid is very generally conceded.

Since the discovery of the interactivity of the internal secretory organs and of the influence which certain of these organs (hypophysis, suprarenals, generative glands) exercise upon physical and mental development, we are accustomed to ascribe those forms of infantilism which are not dystrophic in their origin to an arrest of development consequent upon the abnormality of one or more internal secretory organs.

These developmental disturbances must not be confused with 
those of another group, which are also characterized by insufficient sexual development and sometimes by mental deficiency. The latter are recognized as more or less clearly defined clinical entities. Of these the most important is true dwarfism, the nanismus, microsomia, or nanosomia of Virchow. The pathological, as distinguished from the ethnological, aspect of true dwarfism has been minutely studied by A. Paltauf. D. v. Hansemann distinguished between two distinct forms of nanosomia. In the one, the individual at some stage of his development ceases to grow (nanosomia infantilis); in the other, the lack of size may be traced back to the earliest beginnings of fotal life (nanosomia primordialis). Individuals belonging to the latter group are very small when born and remain, through life, smaller than normal individuals of the same age, but they attain to a definite completion of their development. The epiphysial cartilages ossify, sexual maturity supervenes, and the characteristic signs of infantilism are absent. In infantile nanosomia, on the contrary, the individual is normal in size when born, but at some point in his history he ceases to grow. This cessation takes place gradually and in spite of the fact that the epiphysial synarthroses remain unclosed during life (Schaafhausen, Joachimsthal). But here also, the proportions of the body are maintained and the mental development is usually normal. Bayon describes these individuals as having the appearance of normal people when seen through the wrong end of an opera-glass. It is possible to mistake true drawfism for infantilism in those cases only where there is idiocy combined with anomalous formation of the bones, probably the result of rickets, though the latter condition is rare.

Achondroplasia (chondrodystrophia hyperplastica fotalis, rachitis fotalis, micromelia after Kassowitz) also presents certain clinical signs which resemble those of infantile myxœdema. Achondroplasia presents no mental symptoms, however, and is characterized by a lack of proportion between the shortness of the extremities and the extreme length of the head and trunk, which sufficiently distinguishes it as a distinct clinical type. The condition represents an arrest of the processes of longitudinal growth, caused by the absence, at the points of apposition, of the vertical cell groups of the cartilages of the long bones.

The differential diagnosis between sporadic myxœedema and so-called mongolism is a more difficult matter. Mongolism is an arrest of the natural processes of longitudinal growth, accompanied by abnormality of the structure of the skull; depression of the bridge of the nose; open fontanelles; thickened tongue; defective development of the sexual organs; and especially mental derangement, which at first takes the form of apathy and somnolence, and finally passes into insane desire for movement. According to Kassowitz, mongolism is distinguished from infantile myxœdema by the absence of myxoedematous cuticular changes, by 
the oblique angle of the eyelids, by the normal derelopment of the epiphyses and the centres of ossification of the short bones, and more especially by the fact that the exhibition of thyroid is attended by negative results. In mongolism the thyroid gland is usually normal, and suppression of its function cannot be regarded as pathogenetic in this condition. Alt and Schüller are of the opinion that mongolism results from an affection of the sexual glands and is, in fact, a primary dysgenitalism.

A condition resulting from thyroid insufficiency, which is of far greater clinical and social significance than those which have been described, is endemic cretinism. Its distribution alone makes it of importance. In $188_{3}$, the number of cretins in Italy was 12,882, and in Austria west of the Leitha, I2,8 I5. In France in 1873 , there were 120,000 cretins, distributed over Savoy, the Maritime Alps, and the Pyrenees.

Endemic cretinism is characterized by clinical signs similar to those which have been repeatedly described as resulting from athyrosis. Of these, the most noticeable is the disturbance of the processes of growth of the skeleton and soft parts, which is well seen in the more or less characteristic changes which take place in the face. Owing to the premature cessation of development of the base of the skull, the root of the nose is broad and sunken; the stature is dwarf-like; and the development of the genitals, remains at the infantile stage. The texture of the skin and the cuticular trophic changes are similar to those of myxœdema. But the most characteristic symptoms are arrest of mental development, idiocy, and disturbances of the sensorium. Of the latter, deaf-mutism is the commonest manifestation.

The thyroid gland plays an important part in endemic cretinism, though its responsibility in this direction has been variously defined. Several authors describe cases in which the thyroid was absent altogether and, according to $\mathrm{v}$. Wagner, this occurs in ro per cent. of the cases of typical cretinism. But this finding is purely clinical, and post-mortem evidence of a thyroidaplasia, or of the total atrophy of an already developed thyroid, is not as yet forthcoming. Congenital absence of the thyroid comes under Pineles' definition of congenital myxœdema. As a general rule, the post-mortem finding in cretinism is goitrous degeneration and, more rarely, atrophy of the thyroid (v. Eiselsberg). Histological investigation shows either goitrous hypertrophy, or simple or chronic inflammatory atrophy of the thyroid parenchyma, though both conditions are occasionally present in the same subject. W. Scholz lays special stress upon the fact, however, that in all his cases as well as in some of those of his predecessors, a proportion of normal, active thyroid tissue remained.

Both the origin and the nature of endemic cretinism have formed the subject of a multiplicity of hypotheses. As early as 
1867 , St. Leger mentioned forty-two different theories, propounded by 378 different authors, concerning the genesis of goitre and of cretinism. These possess at this date no interest other than a historic one.

The attitude which is now universally adopted towards the results of thyroid insufficiency, finds its expression in the theory advanced by Kocher and v. Wagner. They maintain that the cause of cretinous degeneration is to be sought in a disturbance of function of the thyroid gland, a chronic athyrosis; or, in other words, where the thyroid is normal cretinism is impossible (Ewald).

Whether, however, endemic cretinism is identical with athyrosis, and whether the pathological condition of the thyroid gland is the primary cause of all the symptoms, or is itself the outcome of other general degenerative processes, are questions upon which opinions differ.

W. Scholz adopts the view that cretinism and athyrosis are not identical conditions. He bases his conclusion upon his finding of active thyroid tissue in cretins, and upon the different effects upon metabolism and general condition produced by thyroid exhibition in cretins and in thyroidless subjects. In Scholz's opinion, endemic cretinism is " a goitrous degeneration, accompanied by cerebral disturbances of unknown origin, to which the inhabitants of certain localities are subject."

The demonstrable differences between the results of partial and total thyroid insufficiency and those of endemic cretinism, are commented upon with varying emphasis by different authors. Ewald lays great stress upon the fundamental difference in the growth of the bones. Athyrosis gives rise to a general and equal retardation of endochondral ossification, which results in dwarfism. In the cretin, however, skiagrams show that there is unequal retardation of ossification and deformity of the bony structure, which may be accompanied in isolated cases by premature synostosis. There is, moreover, an unmistakable difference in the clinical course, for cretins may live to old age, the condition after a certain time remaining stationary; but myxœdema, in all its forms, is progressive and leads ultimately to death.

An even more important factor is that furnished by the difference in the etiology of the two conditions. In endemic cretinism, as the name implies, certain territorial factors are operative and we have to assume the existence of an endemic noxa, the nature of which is at present unknown. It is possible that this harmful agent does not affect the thyroid gland only; it is even probable that it does not affect it primarily. Ewald concludes that endemic cretinism " is a condition of chronic physical and mental degeneration, which is dependent upon local causes, in combination with a more or less pronounced degeneration of the thyroid 
gland. As the latter factor develops under the influence of the former, a distinct clinical type is evolved, which is to be regarded as the resultant of both the operating causes."

There is undoubtedly a close etiological relationship between endemic cretinism and endemic goitre. Virchow pointed out that " true endemic cretinism always appears in goitre areas, and we do not know of a single spot on the inhabited earth where true endemic cretinism occurs which is not also the seat of endemic goitre." In addition to this territorial relationship, there is a large number of facts which point to a close etiological connection between the two conditions. The most suggestive of these is the fact, mentioned by Fodéré in I 800 and since repeatedly confirmed, that in the greater number of instances of cretinism the parents are either cretinous or goitrous, but that goitre is invariably present in the antecedents. From these observations Fodéré concluded that goitre was the first stage of a degenerative process, the last stage of which was cretinism. This view is supported by the large majority of observers, and is now very generally accepted.

The results of recent investigations supply certain factors of indisputable importance in the etiology of endemic goitre and endemic cretinism. The occurrence of both conditions is confined to specific localities. These are the higher mountainous districts in Europe, more particularly the region of the Central Alps; in Asia, the Himalayas; in Central America, the Cordilleras; in Africa, all the mountainous regions. A comparison of the conditions shows that the distribution of these endemics is dependent neither upon climatic nor meteorological factors, but upon the composition of the soil. Bircher's investigations led him to conclude that endemic goitre and cretinous degeneration occur only upon the marine deposits of the Palæozoic (Devonian, Silurian, coal, dyas), Triassic and 'Tertiary periods; while the crystalline formations of volcanic origin of the Archean period, the sedimentary deposits of the Triassic, Cretaceous and Quaternary periods, as well as all fresh-water deposits, are free from goitre. Kocher found that this classification required a certain amount of modification, but he agrees with Bircher that the causal factor lies, not in the mineralogical composition of the soil, nor in the chemical composition of the rocks, but in a certain definite contamination inherent in the soil, as the result of its geological formation. The direct causative agent of goitre and cretinism is water, derived from certain geological formations, and rendered impure by specific by-admixtures.

The etiological importance of drinking-water cannot be doubted when we remember that goitre may be developed in a short time by drinking from so-called "goitre springs"; while, on the other hand, even in districts where goitre is endemic, persons who refrain from drinking the "goitre water" are free 
from the disease. Conclusive experiments were conducted on a large scale at Bozel in the Tarantaise and at Rupperswil in Aargau, to which localities a fresh water supply was brought from goitre-free soil. In both districts goitre and cretinism were formerly rife, but since the institution of the new water supply these conditions have, in the space of twenty years, entirely disappeared. Further observation, and especially the discovery that goitre may be prevented by boiling and filtering the water, seems to point to the conclusion that the diseases are originated by one or more living agents. The chemical composition of the water and the presence or absence of certain substances, such as iodine, phosphates, lime or magnesium salts, can hardly be taken into account.

Though it seems highly probable that goitre and cretinism are chronic infective diseases, the originator of which is to be found in the drinking water, there is as yet no entirely satisfactory proof of this. Moreover, we have no conclusive proof that the diseases are due to a living agent. Kolle, who employed the most perfect of modern methods, was unable to prove the presence of a specific originator in either the intestinal flora, the goitrous thyroid gland, or in blood taken from goitrous subjects. He also endeavoured to prove the presence of specific substances by means of immunization and immunity reactions, as well as by anaphylactic reactions, but without success. Attempts to transmit goitre by surgical means were also negative in their result.

Animals in goitre districts frequently develop goitre, and though certain experiments have been undertaken with the object of producing the condition artificially, the material at our disposal is very scanty. Lustig and Carle found that they were able to produce a slow progressive enlargement of the thyroid in a horse and a young dog (the only one out of $r_{3}$ ) by means of goitre water from the Val d'Aosta. The enlargement disappeared when the animals were given filtered water.

Grassi and Munaron found that the results of their experiments varied considerably. In certain dogs brought from goitre-free localities to localities where goitre was endemic, goitre developed even after the food and drinking-water were boiled; while others, which were given goitre-water and the sediment obtained by filtering such water, remained free from goitre. But dogs which were kept in enclosures in which there was a quantity of damp earth and rotting material obtained from districts where goitre was endemic, developed unmistakable goitre. Grassi and Munaron conclude from this that goitre and cretinism result from toxins produced by a specific microbe, which does not inhabit the organism, but lives free in the outer world, always in damp material and preferably in the soil. These toxins enter the organism by way of the alimentary canal, to which they are conveyed in different media, of which the drinking-water is probably one. 
v. Wagner and Schlagenhaufer were unable to produce goitre in young dogs by means of. sediment obtained from the water of a goitre-spring in Zeltweg (Styria).

McCarrison affirms that goitre can be produced in man by the exhibition of the residuum left after filtering goitre-water.

Interesting experiments, intended to throw light on the etiology of goitre, were'latterly undertaken by Wilms and E. Bircher. They found that it was possible to produce both nodose and parenchymatous goitres in apes and dogs, and, more particularly in rats, by giving them water from certain springs. Moreover, when filtered by means of a Berkefeld filter, this water invariably produced goitrous degeneration of the thyroid gland, while the deposit was inactive. From these results the authors conclude that goitre is of toxic, not miasmatic, origin.

v. Wagner and Schlagenhaufer were unable to confirm the results described by Halsted, Lanz, Marine and Lenhart, namely, that the descendants of female animals with reduced thyroid activity frequently show goitre, thyroid insufficiency and stages of cretinism.

v. Wagner and Schlagenhaufer supply some interesting facts concerning endemic cretinism in animals. Goitres are of by no means rare occurrence in certain species (pig, calf, dog), but cretinism in animals appears to be extremely rare. v. Wagner and Schlagenhaufer give descriptions and very instructive drawings of five cretinous dogs which came under their notice. The conspicuous symptoms were clumsiness of shape, broad short skull, dry, scaly skin, hairless tail, defective sense of hearing, apathy, indolent and clumsy movements, lack of appetite, and retarded metabolism. Microscopic examination revealed a peculiar .change in the skin, namely, considerable deposits of a substance which, when treated with hæmotoxylin-eosin, coloured blue. The same substance was found in the skin of thyroidectomized goats. The thyroid gland of these cretinous animals varied in size but slightly from the normal, and showed no histological changes worth mentioning. The colloid contents were large, the follicular epithelium scanty, but there were no symptoms of degeneration. The thymus showed signs of premature involution. Both the clinical symptoms of cretinism and the cutaneous changes disappeared after the exhibition of thyroid gland, to reappear, however, as soon as the treatment was suspended.

\section{SUBSTITUTION THERAPY.}

The results which have been obtained from time to time by the substitution of the thyroid gland have a clinical, as well as a physiological, importance. Schiff was the first (1884) to whom the idea occurred of overcoming the symptoms of suppression by means of transplantation. He transplanted fresh thyroid glands into the peritoneal cavity of thyroidectomized dogs, and 
found that the animals remained alive for a considerable time. Later, by means of very exact experiments, v. Eiselsberg showed that the implanted glands will heal into place and will remain active. Prompted by the success which attended Schiff's experiments, Kocher attempted thyroid transplantation with the human subject, but with negative results; while Bircher (1890) obtained a transitory relief of the symptoms by the same means in the case of a woman with cachexia strumipriva. Horsley next proposed the implantation of thyroid glands from sheep, not only in post-operative myxœedema, but in true myxœedema and in cretinism. The attempt was made by several surgeons and was followed by favourable results, which lasted for a varying period of time. Operative transplantation is, however, a comparatively difficult and not altogether a harmless undertaking; hence the introduction of a method of substitution by means of thyroid extract marked an important advance in technique. Moreover, the results of this method have added considerably to our knowledge of the functions exercised by the thyroid apparatus.

In 1 S91 Vassale, who was soon followed by Gley, discovered that the symptoms which follow thyroidectomy may be aborted by the intravenous injection of the watery thyroid extracts. In those days, the tetanic symptoms were believed to be the most important, if not the only, signs of suppression, and it was just these symptoms which were relieved by the treatment. Tetany and cachexia were at that time still regarded as identical clinical conditions. But we now know that the acute nervous derangement which follows extirpation of the thyroid apparatus is due to the removal, not of the thyroid, but of the parathyroids; and it is obvious that the results of the older substitution experiments, whether by transplantation or by exhibition of thyroid extract, are much in need of revision.

The results obtained in tetany by transplantation of the parathyroids and by the exhibition of thyroid extract have already been fully discussed. It is proposed to quote here only those results which have been obtained by the transplantation of the thyroid gland itself.

The experiments of Schiff and v. Eiselsberg were followed by numerous others (Carle, Ribbert, Ferratti, Fano and Zanda, Zuccaro, Bourchard, Lamari and Sgobdo, Cannizzaro, Ughetti, Ferrari, Sultan, Pantaleone, Enderlen), and every method of auto- and hetero-transplantation, with subjects of a great variety of species, was attempted. The site of implantation was usually the preperitoneal or subcutaneous tissue. Payr preferred the spleen on account of the peculiarly favourable circulatory conditions. Kocher proposed the cancellous tissue of the hollow bones, bordering the epiphysial line-the metaphyses of the tibia -as sites of implantation, and the results of such implantation are described by Sermann. Salzer (I909) described the results of peritoneal transplantation in rabbits. 
Nearly all authors are agreed that the healing-in of the transplanted organ is preceded by a degenerative process, slight at the periphery, but becoming more intense towards the centre, where it culminates in complete necrosis. Soon blood-vessels find their way from the periphery into the interior of the organ, which shows a comparatively healthy peripheral zone passing into a granulation zone, and a necrosed centre. Regeneration starts in the peripheral follicles, and groups and rows of epithelial cells appear, which are transformed into follicles. The granulation tissue is replaced by glandular substance, while the necrosed centre becomes a connective tissue scar. Finally, as the organ becomes completely healed in and the vascularization improves, it shows a structure precisely similar to that of the normal thyroid.

Christiani showed, and his results were confirmed by those of Salzer, that the healing-in of the implanted gland does not take place in the same way in thyroidectomized as in normal animals. After extirpation, the process is more rapid and vascularization proceeds so quickly that about three weeks after transplantation the gland is large in size and proliferates actively; a condition which, in the normal animal, is not reached until seven to eight weeks after transplantation. Some degree of difference is apparent for five to six months. It is obvious that the rapid healing-in of the transplanted thyroid in the case of the thyroidless animal is due to the more rapid vascularization. Thus it appears that an early physiological demand upon the function of the organ promotes its rapid healing-in. Christiani also found that portions of thyroid implanted into the ear of normal rats, healed in well; while in the case of animals to which thyroid tabloids were given, only cicatricial tissue with no trace of glandular structure was found at the site of implantation. He concludes from this that the physiological demand of the organism upon the thyroid function facilitates and hastens the reorganization of the transplanted tissue, and he agrees with Salzer that the large healthy implanted gland of the thyroidectomized animal is an instance of vicarious hypertrophy.

The rapid healing-in of the implanted thyroid is accompanied by equally favourable results in regard to its function. Immediately after transplantation, the implanted organ is merely a chemical storehouse of active thyroid substances; with increasing regeneration, however, it is gradually enabled to fulfil the complete function of the normal thyroid gland. For this reason, animals having one implanted lobe, but which are otherwise thyroidless, may live for months without showing symptoms of suppression. Payr found that after extirpation of the spleen, into which the thyroid had been transplanted, several animals developed not only post-operative tetany, due to removal of the parathyroids, but also cachexia (emaciation, roughness of the coat, falling of the hair). 
Payr describes one case of remarkable interest, that of a child of 6 with extreme infantile myxœdema. The child was given thyroid gland for $3 \frac{1}{2}$ years without result, but the implantation in the spleen of a large portion of thyroid tissue taken from the mother was followed by a marked improvement in both the physical and mental conditions.

Under certain conditions, and more particularly where the exhibition of thyroid extract is negative, the transplantation of healthy thyroid tissue has a distinct therapeutic value. As Salzer conclusively shows, the site best suited for implantation is the subcutaneous or preperitoneal tissue, for here the colloid secretion is adequate, while the vascularization is certainly as profuse as when the thyroid occupies its normal site, if not more so. Moreover, as Christiani points out, in order to obtain a therapeutic result, it is frequently necessary to repeat the implantation, and for this reason alone the subcutaneous or preperitoneal tissue is preferable to the spleen or the bone.

There is, however, but a limited field for surgical intervention, the medical method supplying, in the great majority of cases, a thyroid substitution which is not only of equal reliability, but is far safer.

The results of the treatment, by means of extracts and other preparations of thyroid gland, of the pathological conditions which follow the suppression of the thyroid function are not only of profound interest, but they have a very important practical application. The experimental material, though considerable, is largely valueless, owing to the fact that substitution was almost invariably attempted with a view to its effects in post-operative tetany. It is certain that neither the exhibition of thyroid preparations nor of the fresh thyroid gland can permanently replace the complete thyroid apparatus; that is to say, thyroparathyroidectomized animals cannot be kept alive by this means.

There is a small amount of material forthcoming concerning the beneficial effects of thyroid extract upon the pathological changes, and especially the changes in growth in young animals, which follow the suppression of the thyroid. Pick and Pineles found that, in the case of thyroidectomized goats, the pathological signs disappeared after the animals were given thyroglobulin and the thyroid glands of swine.

According to Coronedi, the functions of the thyroid and parathyroids may be replaced by artificial organic halogen compounds, fats containing biniodide and dibromide of stearic acid, as well as by the iodine compounds contained in the thyroid itself. Animals which are treated with these fats before thyroparathyroidectomy are said to be immune for a certain time from its results. For instance, the hypertrophy of the hypophysis, otherwise a constant feature, is not observed under these conditions. 
According to Pick and Pineles, the secondary peptones obtained from pig's thyroid after a forty-eight hours' digestion by pepsin and hydrochloric acid, have very little influence upon the pathological symptoms of suppression. The primary albumoses, and the products of prolonged pepsin and trypsin digestion, are also inactive. They found, moreover, that iodothyrin was inactive, which appears to contradict the view that iodothyrin is the only active principle of the thyroid secretion.

There is a large amount of evidence in favour of the clinical value of substitution. Since the really surprising results obtained in myxœdema by Murray (I89I) with subcutaneous injections of sterile glycerine thyroid extract, and by Howitz, Mackenzie and Fox with thyroid gland given by the mouth, thyroid extract has become an indispensable factor in the management of certain conditions. The thyroid substances are specific remedies in all those pathological conditions which arise from absence or insufficiency of thyroid function.

Brilliant results were obtained by Kocher in cachexia strumipriva. The uninterrupted administration by the mouth of thyroid extract will prevent the appearance of cachexia, and may for years completely replace the function of the absent thyroid. Moreover, the treatment may be continued for any length of time without fear of ill-effects. Such subjects are as capable of work as those who are "the happy possessors of a thyroidin factory in their own bodies." But pathological symptoms make their appearance if the treatment is suspended, even for one day.

The results of thyroid extract are no less striking in myxœdema; sometimes the symptoms disappear almost immediately, sometimes not until after months of treatment. As a general rule, the exhibition of thyroid extract is followed by a reduction of the cuticular swelling and the patients lose their puffy appearance. The sweat-glands begin to secrete and the hair grows again upon the proper places. The temperature rises to the normal, sexual activity is renewed, and, in women, menstruation returns. The apathy and lack of interest disappear, and the whole personality changes to such an extent that the patient seems to be a different person. In infantile myxœedema and in cretinism, there is a remarkable promotion of the vertical processes of growth, and this may be seen in cretins even at a comparatively advanced age. Suspension of the treatment is followed, first, by a cessation of improvement, and, later, by a return of the pathological symptoms.

The systematic treatment of endemic cretinism by thyroid extract is warmly advocated by v. Wagner. His experiments extend over a period of at least five to six years, and the results show that every degree of cretinism is favourably influenced, even at an advanced age, by thyroid treatment. The slighter forms may completely recover and, provided that the treatment 
is continued for a sufficient length of time, the improvement will be maintained. In the severer forms, where the cases do not come under treatment until very late, the improvement is confined to certain symptoms. The administration of over-doses of thyroid may, however, be a cause of failure (W. Scholz). The state treatment of cretinism in Styria by means of thyroid extract has been followed in every case since 1907 by satisfactory results (v. Kutschera). In 85.7 per cent. of the cases, the growth was in excess of the normal growth proportionate to the age; in 42.8 per cent. there was a considerable improvement in all the cretinous symptoms; and in 48.6 per cent. there was a distinct improvement in certain symptoms.

Thyroid treatment has also been employed in the management of a large number of pathological conditions which are believed, frequently upon no very clear grounds, to have an etiological connection with insufficiency of the thyroid. Such are: Trophic oedema, scleroderma, several skin affections, osteomalacia, obesity, \&c.

\section{THE METABOLIC CHANGES IN HYPOTHYROSIS} AND THE MANNER IN WHICH THEY ARE INFLUENCED BY THE EXHIBITION OF THYROID EXTRACT.

The metabolic changes in hypothyrosis in man are minutely described by Magnus-Levy. In this condition, the entire energetic output (Kraftwechsel) of the organism is reduced, and patients increase in weight, even with a very small amount of nourishment. The diminution of the energetic output (Gesamtumsatz) is not entirely due to physical indolence, but is largely the result of the enormous reduction in the amount of the physiological turnover (Grundumsatz) - the physiological expenditure of the resting body. This falls to 50 to 60 per cent. of the normal, a figure unknown in any other chronic condition. The low metabolism of myxœdema is accompanied by a proportionate decrease in assimilation and in the metabolism of albumin. The daily excretion of nitrogen amounts to only 8 or 9 grammes. An increase in the amount of nourishment, and especially in the amount of albumin, may add to the amount of nitrogen.

This remarkable reduction of metabolism in myxœdema disappears as soon as the condition yields to treatment with thyroid gland. The gas metabolism reaches the normal and may, indeed, exceed it. Under the ordinary treatment, with one to three tabloids a day, this change is completed in three to four weeks. If the treatment is then suspended, the expenditure of oxygen will be again reduced, only to increase again as soon as the treatment is recommenced. In slight cases of myxœdema, 
where the gas metabolism approaches the normal, thyroid treatment increases the amount up to 15 per cent. The use of thyroid preparations produces a complete change in the conditions governing the metabolism of albumin. As a consequence of the improvement in appetite, the amount of albumin ingested is much larger, and the excretion of nitrogen becomes very much increased; consequently, there is an appreciable loss of nitrogen which is counteracted only by raising the entire caloric supply. The larger part of the N-excretion is derived from pathological deposits, which, at the same time, disappear. The increased supply of albumin may, at a later stage, occasion a deposit of albumin as tissue.

Scholx concludes that the metabolic conditions of cretinism are singularly like those of myxœdema. But the effect of thyroid extract upon the metabolism of nitrogen is not the same in cretinism as in myxœdema. In the former condition it closely resembles that seen in Graves's disease. Nevertheless, Scholz does not conclude from this that myxœedema and endemic cretinism are opposed conditions; he is of the opinion that, as far as metabolism is concerned, cretinism is a similar condition to myxœedema, but is more advanced.

\section{THE PHYSIOLOGICAL EFFECTS OF THYROID SUBSTANCES.}

Of the physiological effects of thyroid extract, the first to be considered is the effect which it produces upon the circulatory system. Oliver and Schäfer (I894) pointed out that the injection of watery or glycerine thyroid extracts into the veins was followed by a reduction in blood-pressure, the cardiac activity remaining unchanged. This is confirmed by the observations of many others (Haskovec, Georgiewsky, Guinard and Martin, Fenyvessy, Ocaña, Patta, v. Fürth and Schwarz, Farini and Vidoni). Certain authors (Heinatz, Livon, Patta) noticed that an increase in blood-pressure was brought about by thyroid extract. Farini and Vidoni found that, during artificial circulation through the hinder extremities of cats and rabbits, the effect was vaso-contractor, never vaso-dilator. Though Schäfer declares that the cardiac rhythm is unaffected by thyroid extract, yet Haskovec, Guinard and Martin, and Svehla all found that the decrease in bloodpressure was accompanied by a marked acceleration of the heart's action. Others, again, report a definite slowing of the pulse.

The effects of iodothyrin and of iodothyroglobulin upon the circulatory apparatus were tried by v. Cyon and Oswald. They found that, when injected into the veins of dogs and rabbits, these substances produced a fall in blood-pressure, together with a stronger and slower heart action, and that these phenomena persisted after resection of the vagi and after administration of 
atropine. v. Cyon believes that iodothyrin has a powerful effect upon the irritability of the terminals of the vagi and of the depressors, and that it also has an antagonism for atropine. v. Cyon's findings have been confirmed by those of others (Boruttau, Ocaña, Besmertny, Kraus and Friedenthal, Coronedi), but Harnack casts a doubt upon the antagonism of iodothyrin to atropine and ascribes the restoration of vagal irritability to the rapid passing of the effects of the atropine. Further investigation showed (Fenyvessy, Isaac and van der Velden, v. Fürth and Schwarz) that the irritability of the apparatus which inhibits cardiac activity is not affected by iodothyrin. v. Fürth and Schwarz also proved that the fall in blood-pressure which follows the exhibition of thyroid extract is not specific. As they point out, the thyroid gland, in common with many other organs (suprarenals [Lohmann]; brain [Vincent and Cramer]; spleen, thymus, lymph glands, intestinal glands, kidney, ovary, pancreas [Gautrelet]), contains choline, a substance which is concerned in the composition and decomposition of the lecithins and other lipoid substances; and it is probable that the reduction in bloodpressure which follows the exhibition of thyroid extract is due to the presence of this substance. They believe, moreover, that this effect of thyroid extract has no relationship to the physiological activity of the thyroid gland.

The direct and characteristic effect of iodothyrin upon the circulatory apparatus is absent in dogs and rabbits, but is seen to a very marked extent in cats. The intravenous injection of a quantity of iodothyrin corresponding to 0.2 to $0.3 \mathrm{mg}$. iodine is followed by a fall in pressure and by large slow vagus beats, lasting for several minutes. These "Aktionspulse," as they are called, result from stimulation of the vagus centre in the medulla, for they disappear after resection of the vagi. The reduction in pressure is due, not only to stimulation of the peripheral vessels but, as oncometric experiments show, to a direct action upon the heart itself. Further study of these phenomena shows, however, that they are not specific to the action of iodothyrin, but are also observed after the intravenous injection of iodized albuminoids. v. Fürth and Schwarz conclude that iodothyrin is a condensation product of melanoid nature obtained by the action of acids from the iodine albumin of the thyroid; and that its effects upon the circulatory apparatus do not in any way suggest a specific activity of the thyroid gland.

In addition to the immediate effects of thyroid extract, there are certain other phenomena which are produced by its continuous exhibition. Hyperthyroidism has been frequently produced experimentally, with a view to ascertaining its effects upon the circulatory system, and a more or less marked tachycardia appears to be the almost invariable result. v. Fürth and Schwarz produced considerable tachycardia in dogs and cats by the sub- 
cutaneous injection of large quantities of iodothyrin, but the result was by no means invariable. In many cases, in spite of flooding with iodothyrin, there was neither tachycardia nor any other symptom of thyroid poisoning. They believe that the production of tachycardia is not specific to the thyroid gland, but that it is common to iodothyrin and to certain other iodized derivatives of albumin. In the case of thyroid extract, they explain the effect by the large amount of iodized albumin which the thyroid gland contains.

Coronedi regards thyroid extract as a physiological diuretic. He bases his view upon results obtained with thyroparathyroidectomized animals having a reduced activity of the kidneys. A very marked diuresis was produced with thyroid extract in cases where the usual diuretics had failed.

The effects of thyroid extract upon metabolism are constant. It has already been shown that the exhibition of thyroid substances in myxœedema and other conditions arising from suppression of the thyroid gland, is followed by an enormously increased metabolism. Similar changes, though less marked in character, are produced in normal subjects, both man and animals, although the physiological turnover (Grundumsatz) is very little affected by thyroid extract. If, however, thyroid extract or iodothyrin are given continuously for two to three weeks, the amount of the $\mathrm{CO}_{2}$ excretion will be increased by $\mathrm{I}_{5}$ to 25 per cent. The nitrogenous interchanges, however, are invariably disturbed by the exhibition of thyroid extract. The increased decomposition of albumin is expressed by an increased excretion of nitrogen, hence the nitrogen balance is a negative one. By increasing the caloric food supply the nitrogen losses may be avoided. In obesity, owing to the large reserve, the loss of albumin is not as great as in the normal subject.

Thyroid preparations have been largely employed in the therapeutics of obesity and, in many instances, with success. Magnus-Levy thinks that the increase in oxidation during the resting state (Ruhegaswechsel) is not sufficient to account for the reduction of adipose tissue which follows the exhibition of thyroid. He believes that the result is largely due to an increased expenditure; for the effect of thyroid extract is to increase the capacity for nervous reaction and, as a consequence, these otherwise phlegmatic people display a greater energy in movement. There is a good deal of evidence to show that thyroid extract has an effect in reducing constitutional obesity, but that it has very little influence upon obesity due to overfeeding; and this seems to point unmistakably to the conclusion that, in this case also, we have to do with a "substitution," by means of thyroid extract, of the diminished function of the normal thyroid gland.

With regard to the metabolism of salts, Falta, with Bolaffio and Tedesco, showed that the administration of thyroidin produced 
in dogs in the fasting state a marked increase in the quotient $\mathrm{N}: \mathrm{P}_{2} \mathrm{O}_{5}$, which is expressed by a rise in the amount of nitrogen and a fall in the amount of phosphorus excreted in the urine. Thyroidin also brings about a considerable increase in the amount of calcium excreted in the faces, the calcium carrying off with it a large proportion of phosphorus. Scholz had previously shown that the exhibition of thyroid extract produces in man an increase in the amount of phosphorus excreted in the faces.

\section{EXPERIMENTAL HYPERTHYROIDISM.}

The continued exhibition of the expressed juice of the thyroid gland, or of thyroid extract, is followed in animals by a number of pathological symptoms, though the appearance of these is by no means constant or to be relied on. The tachycardia of hyperthyroidism has already been described. There are, however, other and various changes both in the body generally and in individual organs; such are: derangement of metabolism (emaciation, increased oxidation, increased requirement of albumin, polydypsia, polyphagia, polyuria, glycosuria); derangement of digestion (diarrhœe, intestinal hæmorrhage); trophic disturbances (œdematous swelling of the connective tissue); changes in the thyroid gland itself; and a large number of symptoms which point to a change in the activity of the nervous system. Kraus and Friedenthal found that the intravenous injection of thyroid juice almost invariably produced in rabbits enlargement of the palpebral fissures, projection of the eyeball, retraction of the membrana nictitans, and enlargement of the pupil. Hoennicke succeeded in producing exophthalmos in rabbits by the continued exhibition of thyroid juice. Eppinger, Falta and Rudinger then showed that it is possible to produce adrenalin mydriasis in both normal and thyroidless dogs by continued treatment with thyroid juice or thyroid preparations, exclusive, however, of iodothyrin. These authors regard the symptoms seen in hyperthyroidism as the expression of an increased hypersensibility of the sympathetic nerve. Certain symptoms point, however, to an influence affecting the autonomous nervous system, and indeed the symptomcomplex of hyperthyroidism is so extremely varied that it can only be explained by the assumption of a polyvalency of the thyroid secretion.

\section{HYPERTHYROIDISM IN MAN.}

\section{As Produced by the Exhibition of Thyroid Gland.}

Since the introduction of the thyroid preparations into clinical medicine, instances of artificially produced hyperthyroidism have frequently come under observation. The continued employment of thyroid extract, even in small medicinal doses, is frequently 
followed by various symptoms of derangement, such as a sensation of heat, increased perspiration, palpitation and, sometimes, by glycosuria. The most remarkable are those cases where toxic symptoms appear after a single large dose or the prolonged administration of moderate doses. In isolated instances a single large dose has failed to produce any evil effects; such is the case of a child of II, who swallowed roo tabloids of $0.3 \mathrm{grm}$. each. But Boinet and Ferranini found that the prolonged administration of six to eight tabloids a day provoked serious symptoms of intolerance. Conditions of psychic exaltation, sleeplessness, violent trembling, extreme emaciation and anæmia, digestive disturbances, extremely unpleasant subjective sensations, deranged cardiac activity, and tachycardia, are all described as symptoms of thyroid intolerance. v. Notthaft describes a case of artificially produced Graves's disease. A man, aged 43, suffering from obesity, who took nearly $\mathrm{I}, 000$ thyroid tabloids in five weeks, presented all the symptoms of true Graves's disease; these gradually disappeared, however, after the thyroid was suspended:

These cases suffice to show the enormous influence which the thyroid substances exercise upon the organism. It is obvious that their employment as therapeutic agents must be in rigid conformity to the clinical indications, the pathological conditions, and the idiosyncrasy of the subject.

\section{CONDITIONS WHICH MAY BE CLASSED AS THOSE OF TRUE HYPERTHYROIDISM.}

Of the pathological conditions arising from a disturbance of function of the thyroid gland, the most important is undoubtedly Graves's disease or exophthalmic goitre. Karl v. Basedow, in r840, first pointed out that the combination of exophthalmos, goitre and tachycardia constituted the syndrome of a definite clinical condition. To these three, other more or less characteristic secondary symptoms have from time to time been added, and the clinical definition of Graves's disease is now made to include the so-called forme fruste and the condition lately described by Stern as "Basedowoid."

The nature of Graves's disease has formed the subject of various conflicting hypotheses. The older neurogenic theory, which regarded the condition as an affection either of the sympathetic (more particularly the vago-sympathetic) nerve or of the medulla, was based upon inconclusive experiments and isolated and contradictory post-mortem findings. The thyrogenic theory, as first propounded by Gautier and ably confirmed by Möbius, produced a revolution in the point of view regarding the pathogenesis of this condition, though individual authors still continued to support the bulbar theory. Tedeski, for instance (I902), be. 
lieved that his experiments pointed to a lesion of the anterior portion of the restiform body as the originating factor of Graves's disease, but that the symptoms are manifested by the agency of the thyroid gland. The later attempts to prove the neurogenic origin of the disease were based upon a more complete knowledge of the innervation of the thyroid gland. Experiments to this end were undertaken by Briau at Morat's laboratory and they show that the sympathetic nerve of the neck contains fibres which act as vaso-dilators to the blood-vessels of the thyroid. Wiener has recently expressed the view, based upon his discoveries concerning the innervation of the thyroid, that the thyroid derangement is not the primary feature of Graves's disease, but is a secondary condition brought about by abnormal innervation of the gland.

Whether the thyroid affection is primary or is a secondary feature of nervous origin, one thing is universally conceded, and that is, that the thyroid gland is the pathogenetic centre from which the symptom-complex of Graves's disease proceeds. A rational explanation of the condition is offered by the assumption of a functional anomaly on the part of the thyroid gland. The grounds for this assumption are as follows: (I) The invariable occurrence of a pathological enlargement of the gland-the anatomically and histologically characteristic goitre; (2) the coincidence of the first clinical symptoms with the swelling of the gland, especially as seen in those instructive cases (acute inflammation, syphilitic changes, metastases of neoplasms) where the sudden affection of the gland is attended by the immediate appearance of the clinical signs; and (3) the remarkable therapeutic results by which partial extirpation is invariably attended.

The thyrogenic theory of the origin of Graves's disease receives remarkable support from the antagonism, described by Moebius, between the clinical symptoms of athyrosis as manifested in myxœedema and in Graves's disease. This is well seen in A. Kocher's comparison of the symptomology of Graves's disease with that of cachexia thyropriva.

\section{Cachexia Thyropriva.}

Absence or atrophy of the thyroid gland.

Slow, small, regular pulse.

Absence of vascular congestion and coldness of the skin.

Indifferent expression, lacking in movement and vitality.

Narrowed eyelids.

\section{Graves's Disease.}

Swelling, generally diffuse, of the thyroid gland, hypervascularization.

Rapid, frequently tense, jerky, occasionally irregular pulse.

Extreme excitability of the vascular nerves.

Anxious, roving glance which appears angry when fixed.

Wide open eyelids, exophthalmos. 
Cachexia Thyropriva.

Retarded digestive and excretory processes, small appetite, slight need for food.

Slow metabolism.

Thick, opaque skin, dry or scaly.

Short, thick fingers, frequently broadened at the tips.

Sleepiness and desire for sleep.

Retarded sensation, perception and action.

Mental vacancy and indifference, lack of emotion.

Awkwardness and slowness of movement.

Stiffness of the extremities.

Retarded skeletal growth, short, thick, frequently deformed bones.

Constant chilliness.

Difficult and slow respiration.

Increase in weight.

Senile appearance, even of Youthful, luxuriant developyoung people.
Graves's Disease.

Abundant excretion, generally abnormal appetite, increased need for food.

Increased metabolism.

Skin thin, transparent, finely injected, moist.

Long, slender fingers, with pointed tips.

Insomnia and restlessness during sleep.

Intensified sensation, perception and action.

Mental whirl, excitement to the point of hallucination, mania, melancholy.

Perpetual restlessness and haste.

Tremor of the extremities, increased mobility of the joints.

Slightly built skeleton, occasionally soft or thin bones.

Unbearable sensation of heat.

Superficial respiration, slight expansion of the thorax in inspiration.

Loss of weight. ment, at least in the earlier stages.

The antagonism between the principal symptoms of the two conditions is very marked, though occasionally in severe cases of Graves's disease symptoms appear which bear a close resemblance to those of cachexia strumipriva.

Other momenta, such as the deleterious effects of the exhibition of thyroid extract in Graves's disease and the artificial production of the symptoms of Graves's disease by hyperthyroidism, are to be regarded, not as direct proofs of the thyrogenic origin of this condition, but as powerful arguments in its favour.

We will now pass to a consideration of the symptoms and organic changes characteristic of Graves's disease.

The thyroid enlargement-the goitre-is one of the constant symptoms of the condition. Cases where the thyroid changes are absent are, in the opinion of all observers, extremely rare. The 
enlargement of the thyroid is not, as a rule, very considerable, and is generally readily distinguishable from other forms of goitre. Histologically, the goitre of Graves's disease is defined as a parenchymatose hypertrophy of the gland, with profuse vascularization. In addition to the active new formation of tissue there are retrogressive processes; and side by side with young follicles, formed by epithelial proliferation, old follicles are seen to be breaking up by means of cell desquamation: In older goitres there is also a marked increase of interstitial connective tissue. The goitre of Graves's disease is differentiated from all other goitres by the nature of its histological changes. But it must be borne in mind that the signs of an increased secretory activity are the same as in the colloid goitres.

Erdheim regards certain young cell formations with fat granules as characteristic of the goitre of Graves's disease; while according to Lobenhoffer, the acidophile granules in the protoplasm, which must be regarded as the secretory product of the epithelial cells of the thyroid, are increased in exactly the same way in Graves's disease as in colloid goitre.

Of the pathological anatomical findings in Graves's disease, hypertrophy of the thymus is among the most frequent (Thorbecke, v. Hansemann, Boit, Mönkeberg, Gierke, Capelle, Rössle). The enlargement of the thymus is a contra-indication for thyroidectomy, for after operation such cases frequently get worse, owing, it is believed, to thymogenic auto-intoxication (Gierke). The thymus hypertrophy is said to arise from a vicarious endeavour on the part of the thymus to make good the deficiency in the amount of secretion elaborated by the hypertrophied thyroid (Rössle). But the persistence or enlargement of the thymus may be explained by the fact that the victims of Graves's disease are frequently of lymphatic constitution; and the presence of the thymus as well as the hypoplastic arrest of development of the genitals, like the frequent swelling of the lymph glands and the formation of lymphatic deposits in the thyroid gland, may only be the signs of a status thymicolymphaticus.

The heart shows all the clinical signs of hypertrophy; more rarely, there is dilatation of the left ventricle with fatty degeneration or brown pigmentation of the muscle fibres. According to Askanazy, an interstitial lipomatosis of the voluntary muscles, which develops variously with the severity and age of the case, is a constant finding.

The other organic changes, and particularly the pathological changes in the central nervous system, are not constant.

The origin of the exophthalmos, which is almost pathognomonic of Graves's disease, has always been the most difficult problem in connection with the disease.

The exophthalmos consists in an enlargement of the palpebral 
fissure, which may be due to contraction of the levator palpebrum muscle, together with a protrusion of the eyeball, brought about by contraction of Müller's protrusor bulbi muscle, the existence of which in man has been proved by Landström. The latter muscle has a sympathetic innervation, while the levator palpebræ muscles are innerved by the autonomous oculomotor nerve. The irritation of the sympathetic at the neck causes a protrusion of the eyeball, in the course of which the space between the lids undergoes a certain amount of secondary mechanical enlargement. The exophthalmos is undoubtedly a symptom of the increased irritability of the sympathetic nerve, and this view is supported by the adrenalin mydriasis which $O$. Löwi saw in persons with Graves's disease. The enlargement of the space between the lids, which sometimes takes place independently of the protrusion of the eyeball, and Gräfe's symptom, which consists in the absence of synergy between the movements of the eyeball and of the upper lid, are both signs of irritation of the autonomous system (Eppinger and Hess).

Tachycardia is also a constant symptom in Graves's disease, though, as Chvostek points out, of very varying clinical significance. The results of experimental research point to the symptom as a sign of increased irritability of the sympathetic nerves. An irritation of the nervus accelerans, which hastens the heart's action and belongs to the sympathetic system, suggests itself in this connection. Experimental systematic hyperthyroidization almost invariably produces tachycardia, and there is ground for the belief that the acceleration of the pulse due to the increased activity of the sympathetic nerve, is an effect of the increased amount of thyroid substances circulating in the blood. The hypertrophy of the cardiac muscle is sufficiently explained by its continued hyperfunction.

Other signs of the increased activity of the sympathetic system are to be found in the greater vasomotor excitability, the increased secretion of the sweat glands, the tendency to a heightened temperature, and the increased production of heat. The increased amount of adrenalin in the blood points to the same conclusion. Kraus and Friedenthal discovered that the serum of persons with Graves's disease caused the pupil of an enucleated frog's eye to dilate, and later A. Fraenkel, by exact biological experiments with the uterus removed from the living body (" iiberlebend"), found that the blood of persons with Graves's disease contained a very much increased amount of adrenalin.

Eppinger and Hess brought together a variety of symptoms which they believe to be signs of irritation of the autonomous nervous system. In addition to the enlargement of the palpebral fissure and Gräfe's symptom, they include derangement of the respiratory rhythm and diarrhœa among the symptoms of an 
increased vagal activity; they also attribute (though somewhat arbitrarily) the increased perspiration, the congestions and the circumscribed œedema to irritation of the autonomous nerves. These authors divide their cases of Graves's disease into two. groups; one, in which the symptoms of sympathetic excitement predominate, they describe as sympathetico-tonic; while the other is described as vago-tonic, from the fact that here the most marked symptoms are those proceeding from the autonomous nervous system. Falta, Eppinger and Rïdinger assume a polyvalency of the thyroid secretion-and they regard the hyperthyroidism of Graves's disease as the outcome of a simultaneous, though probably independent, stimulation of both the sympathetic and autonomous nervous systems.

A special interest attaches to the metabolic changes of Graves's disease. In simple goitre, the metabolism is in no way different to the normal, and it is affected by thyroid extract in a manner precisely similar to that of other persons with normal thyroids. In Graves's disease, on the other hand, the metabolic changes are so characteristic of the condition that they constitute important diagnostic criteria. The metabolism of Graves's disease is characterized by an increased expenditure of energy. The respiratory interchange of gases shows an increase of 50 per cent., and even 70 to 80 per cent., in the amount of oxygen consumed (Magnus-Levy, Salomon), and these results are confirmed by Steyrer, who continued his experiments over a long period of time. The increased consumption and the increased and remarkably fluctuating caloric production, is partly the result of the augmented cardiac and respiratory activity; in part, and to an even greater degree, it is due to the nervous excitement and motor unrest, more particularly the tremor. But, after making due allowance for the operation of these factors, it will be found that in complete muscular repose there is still a large increase in the interchange of gases. When we remember the effect of thyroid extract upon metabolism, it seems obvious that this augmentation is only to be accounted for by a hypersecretion of the thyroid gland. That the caloric production may be still further increased by the exhibition of thyroid extract, has not as yet been proved (Magnus-Levy, Steyrer). It may be noted here that the administration both of rhodagen and of Moebius's anti-thyroidin serum is not followed by any demonstrable effect upon metabolism. This applies also to treatment with thymus extract, at one time much vaunted for its curative effect in Graves's disease. The enormously increased metabolism accounts for the emaciation, which is often very extreme. The increased caloric explains the subjective sensation of heat, the profuse outbreaks of perspiration, and the tendency, commented upon by Charcot, to a heightened temperature. That the increased caloric does not produce hyperpyrexia or even fever 
is probably due to an adequate degree of activity on the part of the apparatus which regulates heat production. 'The rise in temperature which occurs shortly before death (Kocher) is probably due to failure on the part of this apparatus.

The increased production of heat is frequently associated with an augmented demand for, and consumption of, albumin, together with a negative $\mathrm{N}$-balance, though the latter is not invariable. The metabolism of nitrogen varies with the fluctuating course of the disease and may, for longer or shorter periods, approximate to the normal. The albumin losses and the toxic decomposition of albumin may be counteracted, even when the disease is at its height, by a diet containing albumin, or by a diet containing carbohydrates but free from nitrogen (Rüdinger). The fats also undergo decomposition. In connection with the metabolism of the carbohydrates, it must be borne in mind that the assimilation of the carbohydrates is reduced in Graves's disease, and that for this reason an alimentary glycosuria is easily provoked (Ludwig and F. Kraus). The feature which characterizes the metabolism of Graves's disease is the increase in the total amount of oxidation.

An increased metabolism of mineral salts has not as yet been proved, though $\mathrm{A}$. Kocher describes an increase in the excretion of phosphorus. Investigation in this direction has recently been undertaken by Scordo and Franchini.

Certain features of Graves's disease are the clinical opposites of those seen in myxœdema. These are : the thin, soft, smootin, moist skin; the general nervous excitability; the restlessness and haste; the tremor; the exaggerated reflexes; and the mental exaltation and psychic changes, which may even amount to psychosis.

There are certain symptoms which are common to both Graves's disease and cachexia strumipriva as, for instance : trophic disturbance of the skin; œedema; dryness, falling and blanching of the hair; friability of the nails; abnormal pigmentation; and increased salivary and lachrymal secretions.

The changes in the blood are the same in both conditions. There is an increase of mononuclear leucocytes and a remarkable increase in the eosinophile cells. And in both conditions there are changes in the activity of the sexual glands, which are shown, in women, by a cessation of the menstrual processes.

It is interesting to note, in this connection, that symptoms resembling those of Graves's disease are associated with other pathological conditions. Such are the forme fruste seen in chlorosis (Chvostek); the pseudo-chlorosis, encountered in goitrous districts, and characterized by swelling of the thyroid. increased cardiac activity, mental excitement, fatigue, and pallor, without, however, chlorotic changes in the blood (F. v. Müller); and, finally, the symptoms resembling those of Graves's disease, which are seen at the climacteric (Gluzinski). On the one hand, 
therefore, hyperthyroidism leads, as in Graves's disease, to changes in the function of the ovaries; on the other, primary changes in the activity of the sexual glands may exercise a secondary influence upon the thyroid, and as a result, symptoms resembling those of Graves's disease are produced.

The brilliant results obtained by modern surgery in the treatment of Graves's disease illustrate very clearly the etiological connection between this condition and the thyroid gland. The operative method is based upon the view that a reduction in the volume of the gland, together with a decrease in, or prevention of, its abnormal vascularization, must reduce its secretory activity and so abolish the results of hypersecretion. The methods employed are : the ligature of one or more enlarged thyroid arteries; the extirpation of one-sided vascular goitres; and the resection or excision of more than one half. Kocher found that these measures vere followed, in 76 per cent. of his cases, by complete cure, and in another 14 per cent. by improvement. Similar results are described by others. But there is a strong feeling in many quarters that not all cases are suitable for operative treatment, and that success largely depends upon the constitution of the patient and, most important of all, upon conditions affecting the thymus. Surgical treatment is accompanied by certain dangers per se, and in a certain percentage of cases (3 to 4.7 per cent.) operation has resulted in death.

Another method of limiting the secretion of the goitrous gland and perhaps, also of destroying the hypertrophic parenchyma, is by means of the Röntgen rays (Görl, Stegmann, Holzknecht, \&c.). Up to the present, however, no very conclusive results as to the therapeutic value of this method have been forthcoming.

The results of organo-therapy in Graves's disease are interesting only in so far as they furnish direct evidence against the assumption of a hypothyrosis in this condition (v. Cyon, Oswald). The effect produced by thyroid extract is, invariably, the aggravation of the disease.

Attempts to treat Graves's disease with extracts of the thymus, ovary and suprarenal capsule, have a certain theoretic interest, but are of no practical significance.

The serum-therapy, first tried by Ballet and Enriquez, and afterwards developed by Moebius, is founded upon the belief that the surplus secretion formed by the hypertrophied thyroid of Graves's disease can be employed to neutralize the toxins contained in the serum of thyroidectomized animals. It is thought that in this way the surplus is removed from the tissues which have a specific affinity for the thyroid secretion, the reaction of which would otherwise be manifested by pathological disturbances. The serum of thyroidectomized animals, known as Moebius's antithyroidin serum, and rhodagen, which, upon the 
assumption that the toxins pass into the milk (Lanz), was prepared from the milk of thyroidectomized goats, were at one time very widely employed, and, it is said, with good result. But the earlier enthusiasm for this method appears to have somewhat abated; at least, very little has latterly been heard of it.

Isolated attempts have also been made to treat Graves's disease by means of thyrotoxic immune serum (Thompson, Stradiotti).

From the nature of the evidence before us, the etiological relationship between Graves's disease and the thyroid gland cannot any longer be doubted. The further question, as to whether the etiological factor is to be found in a quantitatively increased secretion, a hyperthyrosis, or in a qualitatively altered secretion, a dysthyrosis, is not so easily answered. Although the greater number of facts appear to be in favour of hyperfunction, yet the absence of any histological signs of an increased secretion, the failure of all attempts to produce a complete clinical picture of Graves's disease by means of experimental hyperthyroidization, and more particularly, the not infrequent occurrence of symptoms of thyroid insufficiency in Graves's disease, are factors not readily explained by a theory of hyperfunction. According to Moebius's theory, the thyroid secretion of Graves's disease is not only increased, but it is also perverted.

Nevertheless, an increased knowledge of the nature of thyroid secretion and, more particularly, of its chemical composition and physiological activity, together with a more accurate analysis of the clinical symptoms and of their significance, renders the probability of a dysthyroidism in Graves's disease more and more remote. As Kraus points out, this is a problem which, for the present at any rate, cannot be explained beyond a certain point.

The evidence in favour of a qualitative change in the nature of the thyroid secretion is becoming gradually less significant. The absence of any signs of hypersecretion, more particularly the small amount of iodine present in the goitres of Graves's disease, is sufficiently explained by the assumption of an abnormally small capacity on the part of the gland for the fixation of iodine, and by the over-rapidity with which the active substance is expended (Kocher, Kraus). The failure of hyperthyroidization to produce the entire symptom-complex of Graves's disease in animals, can scarcely be regarded as unequivocal evidence, when we remember that by the methods employed, only very imperfect imitations of the clinical conditions as seen in man were reproduced. The positive results of experiments with animals have a much greater value in their relation to individual cardinal symptoms. With regard to the symptoms of thyroid insufficiency sometimes encountered in Graves's disease, it must be remembered that similar phenomena are seen in pathological affections of other internal secretory organs as, for instance, the hypophysis and the 
sexual organs, signs of hyper- and hypo-function occurring sometimes side by side. There is a possibility that the continued increased activity gives rise to a temporary functional insufficiency, which may, later, find anatomical expression.

It is possible to account for all the symptoms of Graves's disease by the continuous circulation in the blood of thyroid substances in abnormally large quantities. For it must be borne in mind that, not only do the extent and intensity of the symptoms depend upon the degree of thyroid affection, but they also depend upon the modifying effect which the thyroid secretion itself exercises upon the different organic tissues and functions. As Eppinger and Hess with justice point out, the symptoms of derangement will vary according to whether the subject is sympathetico-tonic or vago-tonic.

Graves's disease is the direct outcome of the flooding of the organism with thyroid substances. These exercise an elective stimulating effect upon the sympathetic, and partly also upon the autonomous, portions of the vegetative nervous system; and they also influence the activity of those other internal secretory organs which have functional interrelationships with the thyroid gland (thymus, hypophysis, suprarenals, sexual glands). The question now arises as to whether the primum movens of Graves's disease is to be sought within the thyroid or without it. The etiology of the condition is still shrouded in darkness. The remarkable incidence of the disease in women, and the frequency with which its occurrence is associated with functional changes of the sexual glands, are undoubtedly factors of significance. Moreover, the comparative frequency with which Graves's disease occurs in persons with status thymicolymphaticus is probably something more than coincidental. A more complete knowledge of the innervation of the thyroid gland suggests the possibility that the cause of the anomalous secretion is to be sought in a primary affection of the sympathetic nerve, or of the nervous areas in which it takes its rise. Thus we arrive at a neurogeno-thyrogenic theory of the origin of Graves's disease.

Another pathological condition which is associated with clinical hyperthyrosis, is simple goitre. Goitre is a latent swelling of the thyroid gland, unaccompanied by inflammation. Anatomically, the degenerative process consists in hypertrophy and hyperplasia of the gland, by which different parts of the tissue may be affected in a varying degree. The functional disturbances arising from a derangement of the internal secretion are, however, comparatively unimportant. The profound metabolic changes produced by the hyperfunction of Graves's disease are absent in simple goitre, and up to now, an appreciable departure from normal metabolism has not been observed. Moreover, the etiological connection between the pathological signs of the disease and a change in the function of the thyroid, is very 
slight. At a first glance, it might appear that the cardiac and respiratory disturbances were referable to this cause. But more careful investigation shows that the respiratory disturbance results, partly from stenosis of the trachea due to pressure, and partly from mechanical injury of the nerves of the larynx. The cardiac derangement consists, anatomically and clinically, in hypertrophy and dilatation, more particularly of the right heart. It is brought about, partly by mechanical injury of the venous circulation (Rose's goitre heart), and partly by the difficulty of respiration (Kocher's goitre heart with dyspnoea) ; compression of the nerves of the heart may also be a contributing factor.

The cardio-vascular symptoms are the only ones which can be regarded as of hyperthyrotoxic origin. These are : palpitation, some tachycardia, slight cardiac enlargement, glittering of the eyes, slight tremor, liability to perspire, and dermographism (Kraus's goitre heart).*

There is undoubtedly an analogy between the symptoms of Graves's disease and those which are experimentally produced in animals by hyperthyroidization. Moebius describes a pathological sequence, commencing with simple goitre, the evidences of Graves's disease afterwards developing one by one, until the complete clinical picture of that condition is reproduced. Kraus, however, does not consider that there are sufficient clinical grounds to justify such a sequence. For, in the great majority of cases, the characteristic signs of Graves's disease are absent in simple goitre, and this applies very particularly to the typical metabolic changes, a fact of primary importance.

The functional aspect of simple goitre is not identical with that of hyperthyroidism, and the hyperfunction suggested by the anatomical conditions is most undoubtedly absent from the clinical signs. But it must be remembered that the goitrous degeneration of the thyroid gland represents the anatomical diagnosis only; that hypersecretion is not always demonstrable, even histologically; and that, though some portions of the gland are hyperplastically changed and are more actively secretory, others again are degenerated in such a way that their function is reduced or even abolished. That "goitre" and "goitre," as Kocher points out, "are not always identical," is proved by the fact that, after thyroidectomy, symptoms of suppression may be in some instances provoked, in others abolished, by the development of a goitre from a portion of the gland left in situ. That the anatomical hypertrophy of the thyroid may be associated either with slight symptoms of hyperfunction or

* E. Bircher (March, I9ro) has recently shown that in rats with parenchymatous goitres produced by means of goitre water, there is invariably an enlargement of the heart, shown by the-increased weight and the thickened walls. Bircher believes that the noxa of goitre has a direct influence upon the heart. 
severe symptoms of reduced function, is illustrated by endemic goitre and endemic cretinism, the etiological relationship between which is well known.

\section{THEORY OF THE THYROID FUNCTION.}

We now proceed to utilize the foregoing descriptions of the results of hypo- and athyrosis, of the activity of thyroid extract, and of the appearances which accompany hyperthyrosis in man and animals, as material for a more detailed account of the functional activity of the thyroid gland.

The thyroid gland was the first organ to be credited with an internal secretory function, and it was in connection with the thyroid that the two-fold aspect of this function was first suggested. On the one hand, we have to do with a positive productive activity on the part of the gland; and, on the other, with its neutralizing activity, or negative secretory function. Thus two theories regarding the function of the internal secretory organs arose, the one advocating a positive and metabolic activity, the other what may be called a negative or antitoxic activity. Both theories derived a measure of support from the neutralizing function of the thyroid gland. And, to-day, it is very generally believed that the secretion elaborated by the thyroid gland serves to neutralize the toxic products which are formed in the course of normal metabolism, or which are believed to be formed abnormally, either qualitatively or quantitatively, upon suppression of the function of the organ. The two theories are opposed only in regard to the actual place where the process of neutralization occurs. The secretion theory assumes that this process takes place in the blood-stream, while the antitoxin theory places the seat of operation within the thyroid gland itself.

With an improved knowledge of the results which follow suppression of the thyroid function and, more particularly, of the acute nervous symptoms induced by thyroidectomy, it was very natural that the idea of an auto-intoxication should present itself. It was not until the discovery of the presence in the thyroid gland of a body containing iodine, that its secretory activity assumed a physiological significance. The results of a substitution therapy were, however, invariably regarded as due to a neutralizing and antitoxic action on the part of the thyroid extract. This view is still so widely accepted that it is expedient to enter into a more detailed consideration of it.

The antitoxic theory was first propounded by Notkin, and was afterwards developed by F. Blum. It can have but few supporters at this date among scientists whose views are based upon their own clinical or experimental experiences. It is as well, however, that the theory should be reviewed, because it was advanced by Blum in opposition to the views which he formerly 
held, and which he himself declared to be erroneous; and it constitutes, in a sense, an attack upon those views.

Blum failed to recognize that the thyroid apparatus is not a single organ. According to his theory, the "thyroid gland is not a secretory gland, but is a neutralizing organ, which acts by withdrawing certain toxins from the blood-stream, which are neutralized within the gland, the process serving, more particularly, to protect the central nervous system." The toxins (entero-toxins) are supposed to be formed within the intestines, and to be largely derived from flesh foods; they become fixed in the thyroid gland, and are there gradually neutralized. In the course of this process, intermediate products (thyrotoxalbumins) are formed and these, when completely neutralized, are eventually discharged from the gland in the form of harmless excreta.

The serious pathological disturbances which follow the suppression of the thyroid function-the acute tetany, with degenerative changes of the central nervous system, the cachexia, idiocy and mental derangement, the interstitial nephritis and grave ocular troubles-these can all, according to Blum, be interpreted only as manifestations of a toxic condition. The influence which diet has upon post-operative symptoms, commented upon by Horsley, is regarded by Blum as proof of the activity of a toxic agent which, under normal conditions, would be retained and neutralized by the thyroid gland. Neutralization is supposed to take place by iodization of the toxic albuminoid, whereby an unsaturated thyrotoxalbumin, containing iodine, is formed as an intermediate product. This body has a toxic influence upon the heart and upon metabolism. In view of the fact that the amount of iodine contained in the iodized albuminoid of the thyroid gland varies very considerably, and that the amount of organically fixed iodine may be increased by iodide of sodium given by the mouth, it must be assumed that a continuous process of iodization goes on in the gland. The process of iodization is continued to the point of saturation, the saturated iodized albuminoid being nontoxic. Blum assumes a specific property of isolating iodine-an iodasis-on the part of the thyroid gland, and he believes, further, that the iodine stored up in the gland does not again leave it. He was unable to find the smallest trace of iodine in the lymph of the thoracic duct of animals which had been given iodide of potassium in large quantities. As Magnus-Levy points out, however, a careful examination of the quantitative condition robs this result of its significance, for the amount of lymph analysed by Blum was only $100 \mathrm{c.cm}$. , and it was certainly not all derived from the thyroid. Moreover, Gley and Bourcet found that iodine was a constant constituent of the blood, and their finding is confirmed by Bönninger.

Blum maintains that iodine is retained in the thyroid gland; 
for he found iodine in considerable quantities in the thyroid gland of dogs which had, for months, been given food which contained no halogens. If the thyroid furnished a continuous supply of iodine to the blood, it would in time, under these conditions, part with the whole of its supply. But, as Kraus points out, this argument is not convincing, for, judging by the analogy of other substances which are present in the organism in small quantities only, it is by no means improbable that the iodine which leaves the thyroid gland is again returned to it by the agency of the blood-stream. Further, Baumann, and also Miwa and Stoltzner, showed that, under conditions similar to those described by Blum, the amount of iodine present in the gland is actually reduced.

If Blum were right in his original assumption that the thyroid gland supplies a vital principle to the blood-stream, we should expect the ligature of the veins and lymphatics leading away from the gland to be followed by symptoms of suppression. Not only is this not the case, but Blum found that this operation was followed by an increased oxidation, a stimulated cardiac activity, destruction of the red blood-corpuscles, and serious lesion of the liver, the bile pigment passing into the urine. He deduces from this that the ligature of the vessels leading away from the gland causes obstruction, and consequent overflow, of its contents; that this produces a partial insufficiency of the organ, which gives rise to appearances similar to those which follow the exhibition of thyroid juice: But the results of these experiments contain no proof that an incomplete, and therefore toxic, toxalbumin proceeds from the gland. It is much more probable that, under the changed circulatory conditions, which do not, however, completely arrest the flow of blood from the gland, the amount of the normal active substance which passes into the blood-stream is very much reduced.

That Blum was unaware of much that has recently been discovered concerning the nature and function of the thyroid gland, is suggested by the fact that he speaks of the parathyroids as bodies composed of undeveloped thyroid tissue, which contribute nothing of importance to the general economy. He draws his conclusions from his own and Kishi's experiments with dogs, which lived for a considerable time after extirpation of the parathyroids, a small proportion of thyroid tissue having been left in situ. Blum describes the post-mortem findings as follows: "After a certain time, the remains of the thyroid showed a remarkable increase in the number of the interior cells, which produced a certain resemblance to the parathyroids. The follicles, with occasional isolated exceptions, had entirely disappeared, and the colloid substance was absent. Closer investigation showed that the cell-agglomerations formed a tubular structure, similar to that seen in true glands." After a comparison of this description with the plates reproducing the microscopic findings, it is not possible 
to repress a suspicion that the " remnants of the thyroid gland" left in situ were not thyroid tissue at all, but were the actual parathyroids themselves.

Blum also claims that he was able to cure, or at any rate very much ameliorate, acute tetany by means of a milk diet. In the chapter on tetania parathyropriva, instances were given of the effect upon this condition of certain forms of diet; a more careful investigation of this point is very desirable. As far as we can see, however, Blum's observations do not furnish grounds for the assumption of an auto-intoxication. They may add to our knowledge of the function of the parathyroids, but they cannot be said to supply any evidence as to an antitoxic activity on the part of the thyroid gland.

The pathological conditions which follow affections of the thyroid gland result, according to Blum's theory, from the interruption of the steady procedure of the process of neutralization. As soon as, owing to suppression of the thyroid gland, the fixing of the free poison ceases, tetany, cachexia, strumipriva, myxœdema, and other thyroid affections make their appearance. If the entero-toxins are fixed, but are not sufficiently quickly, or not completely, neutralized, the thyroid becomes overloaded. This gives rise to those forms of goitre which are favourably influenced by iodine-the neutralizing agent of the thyroid gland.

If, however, the fixation of the toxin continues uninterruptedly, but the toxin, as thyrotoxalbumin, passes into the blood before it is completely neutralized, a partial thyroid insufficiency results, similar to that produced experimentally by the exhibition of thyroid gland or by ligature of the vessels and lymphatics, and identical with the clinical picture presented by Grares's disease. Blum advances this as the clinical ground for the treatment of Graves's disease by means of an absolutely flesh-free diet, as opposed to operative treatment.

It must be evident from the preceding pages, that Blum's theories concerning the thyroid gland are not only insufficiently grounded, but that they are not adequate either to explain the physiological and pathological phenomena with which we are already acquainted, or to pave the way for further knowledge. Blum's theories offer no explanation of the undoubted effects of "substitution" in conditions arising from thyroid insufficiency, and he himself must admit the possibility of the neutralizing process being performed by the thyroid substances themselves. For the increased oxidation, by which Blum explains the beneficial effects obtained with thyroid extract in myxoedema, is the result of an activity on the part of the thyroid substance, which takes place externally to the gland, and is not, therefore, dependent upon the presence of the thyroid cells. Moreover, Blum takes no account of the significance of the suppression of the parathyroid 
function in the causation of tetany. For this reason, his doctrine represents a step backwards, and, though the risk of post-operative tetany is now, happily, much less than formerly, the acceptance of Blum's theory might very conceivably add to the seriousness of that risk.

The arguments which have been brought forward, and the observations which have been described, demonstrate very clearly the erroneous nature of Blum's theory of the antitoxic activity of the thyroid gland. At the same time, these results show that the function of the thyroid gland is, by means of its internal secretion, to supply a chemically active substance to the economy. What is the nature of the specific activity of the thyroid secretion? The theory advanced by Baumann and his pupils and, more particularly, by Oswald, is based upon the assumption that the specific activity of the colloid present in the thyroid secretion is directed to the neutralization of toxic substances, and that this neutralization takes place within the blood-stream. This is a combination of the theories of secretion and of neutralization. All attempts to obtain this toxin from the blood of thyroidless animals has, however, been attended by as little success as the search for the toxin of tetany.

The evidence in favour of a neutralizing function on the part of the thyroid gland is wholly indirect. It has been pointed out that thyroidectomized animals have a smaller resistance to inorganic poisons (calomel, according to Perrin and Jeandelize) than normal animals, and that experimentally induced tuberculous and post-tuberculous processes are favourably influenced by prolonged exhibition of thyroid extract (Frugoni and Grixoni).

The most important evidence in favour of an antitoxic activity on the part of the thyroid gland and the thyroid substances, is supplied by Reid Hunt's investigation of the neutralization of cyanmethyl (acetonitril, $\mathrm{CH}_{3} \mathrm{CN}$ ). He showed that, after the exhibition of a minimal quantity (I/ $10 \mathrm{mg}$. of the dry gland) of thyroid extract by the mouth, the resistance of white mice to the subcutaneous injection of acetonitril was increased from two- to ten-fold. Thyroid gland which is free from iodine exercises a certain amount of influence in this direction, but as a general rule the neutralizing activity of thyroid extract increases in direct proportion to its iodine contents. It is, however, hardly possible that the process of neutralization consists in a chemical combination of the cyanogen components with the sulphur present in the gland. For, in the first place, the thyroid substances do not neutralize the other cyanogen compounds, such as hydrocyanic acid and sodium nitrocyanide; and in the second place, the amount of acetonitril neutralized bears no relationship to the amount of thyroid substances.

Reid Hunt himself points out that the results of his experiments cannot be regarded in any sense as proofs of the 
neutralizing activity of the thyroid gland. He thinks that these results are sufficiently explained by the changes in metabolism produced by the thyroid substances. And, certainly, the evidence in favour of a neutralizing activity on the part of the thyroid gland cannot yet be regarded as sufficient, seeing that such activity is manifested only, in regard to acetonitril, and only in mice. Moreover, the resistance of animals, including mice, to other toxins, as, for instance, morphine, is not only not increased, but is appreciably lowered by thyroid extract. It is interesting to note in this connection that the blood of persons with Graves's disease produces the same increased resistance to acetonitril in mice as thyroid extract. This may be regarded as evidence in favour of a hyperthyrosis in Graves's disease.

It is evident, then, that direct proofs of the neutralizing function of the thyroid gland are not forthcoming, and that the indirect proofs advanced by Reid Hunt are capable of another interpretation. Such being the case, the hypothesis of an antitoxic thyroid function must be allowed to remain sub judice. At the time when the symptoms of thyroid suppression were regarded as manifestations of an acute auto-intoxication, this hypothesis was obvious and perhaps even necessary. To-day, it not only appears unproven, but, in the light of our present knowledge of the function of the thyroid gland, it is in reality superfluous.

All the known phenomena, both physiological and pathological, associated with the activity of the thyroid gland, are sufficiently accounted for by the following interpretation of the thyroid function.

The thyroid gland is a secretory organ, the end-product of the secretory activity of which is supplied to the blood in the form of an iodized albuminoid. Inasmuch as it modifies the activity of distant organs, this substance behaves as a hormone. As far as we know at present the thyroid secretion, by promoting katabolism and increasing normal function, plays the part of disassimilatory hormone to a large number of tissues and processes. This disassimilatory activity principally affects the metabolic processes, the cardiac activity, some portions of the sympathetic system, and certain of the internal secretory organs (hypophysis, suprarenals). Certain phenomena in other parts of the body suggest, however, that the thyroid secretion also possesses an inhibitory function and contains, therefore, an assimilatory hormone. The promotion of skeletal growth, the development of the sexual glands, and the limitation of the internal secretory function of the pancreas, are instances of such assimilatory activity.

The question arises as to whether this two-fold and antagonistic activity on the part of the thyroid secretion is due to the action of two different hormones; or whether the results 
are brought about by a single homogeneous hormone capable of producing two opposite sets of effects, according to the apparatus influenced. The nervous system supplies the only field for such activity, and so the further question suggests itself: Is the hormonic activity of the thyroid gland direct, or is it effected only by the agency of the nervous system? That it is possible for an organ to send out chemical stimuli which, in different parts of the body, produce entirely different results-in one part promoting assimilation and in another inhibiting it-cannot be doubted. It must further be taken into account that the endresults will also be different in cases where the influence upon the end-organ is not direct, but is effected by the agency of another internal secretory organ. Thus, for instance, the changes which take place in the sexual organs after suppression of the thyroid might easily result, not directly from the absence of an assimilatory thyroid hormone, but indirectly and as secondary results, due to a change in the function of the hypophysis. The solution of these problems demands a far more complete knowledge of the interactivities of the internal secretory organs than we at present possess.

The second theory, which explains the antagonistic activities of the thyroid gland by a single thyroid hormone, acting primarily upon the nervous system, demands serious consideration. Certain phenomena seem to point to the conclusion, that the changes in organic function brought about by the agency of the thyroid secretion, are, in reality, only the physiological expression of a changed condition of irritability of the vegetative nervous system. Although the thyroid secretion principally stimulates the sympathetic system, it does not affect it alone, for it also stimulates certain portions at least of the autonomous system; moreover, the sympathetic and autonomous innervation is, certainly in many parts, antagonistic. This antagonism is sufficiently explained by the assumption of a single homogeneous thyroid hormone, which exercises a disassimilatory effect upon the vegetative nervous system-in other words, increases its functional activity. But it is very important to remember that our knowledge of the manner in which the nervous system acts upon the processes of metabolism, and especially upon the new formation of tissue and the processes of growth, is extremely meagre. Investigation of recent date, especially that undertaken by the Vienna school of scientists, has been concerned solely with the chemistry of metabolism, while the morphological aspects of these, processes have up to now received but little attention.

\section{THE THYMUS GLAND.}

THE thymus (German : Briesel) develops in man as a paired sac-like diverticulum of the ventral portions of the third and fourth, and perhaps also partly from the second, pharyngeal 
clefts. It proliferates into the pleural space until it reaches the heart, at the same time becoming gradually separated from the branchial clefts. This sac-like primordial beginning of the thymus is entirely epithelial in structure; the lumen is very small and the cortex is composed of several layers of epithelial cells. Branch-like processes are early thrown out from the lower edge, the organ in man assuming a lobular structure at the second or third month of foetal life. Connective tissue and blood-vessels now begin to form and the communication between the central lumen and the pharyngeal cleft closes up. At birth, the two halves are grown together in the median line, and the thymus lies between the sternum, the pericardium and the great vessels, with the upper end extending upwards into the region of the neck.

The complete thymus is a greyish-red organ composed of lobes and lobules, the lobules being surrounded by a delicate sheath of connective tissue. The change which, in the course of development, takes place in the length and breadth of the organ, is accompanied by changes in its histological appearance, and the original epithelial organ develops into a structure resembling the lymph glands.

There are two theories as to the manner in which this histological change takes place. According to the one, the transformation theory, the epithelial cells divide and subdivide, becoming smaller and smaller so as to resemble leucocytes. According to the other, the theory of pseudor orphosis, the epithelial beginning of the primordial thymus is, with the exception of a small portion, entirely replaced by elements from the central germinal layer. From investigations carried out with a large number of animals of different species, Hammar concludes that the reticulum-the supporting and stellate cells, believed to be derived from the central germinal layer-is in reality also of epithelial origin. He regards the reticulum as the most important element of the thymus parenchyma.

Development is accompanied by changes in the epithelial thymus, both regenerative and degenerative in nature. In the human embryo, signs of involution appear in the course of the fourth month, the flat, horny epithelial cells becoming transformed into the concentrically disposed layers of Hassal's corpuscles. At the same time, leucocytes pass from the blood-vessels for the purpose of absorbing the broken-down medullary masses. According to Stoehr, the earlier view of the thymus as a lymphoid organ is no longer tenable. And he also points out that the so-called lymphocytes, upon the existence of which the analogy of the thymus with the lymph glands rests, are of epithelial origin and are epithelial in their permanent characteristics.

In many animals, the complete thymus is apparently entirely composed of lymphoid tissue, the epithelial cells having almost 
completely disappeared. In man, the medullary substance contains larger or smaller groups of epithelial cells, which are studded with leucocytes. The thymus also differs chemically from the lymph glands. Ivar Bang showed that the thymus cells have a different chemical reaction to that of the leucocytes, the bonemarrow cells, and the cells of the spleen. That the thymus cells are not identical with those of the lymph glands is proved by the fact that the amount of the nucleinates, the substances which are characteristic of the nuclear structure of the true glands, is at least five times as large in the thymus as in the lymph glands. The older idea, that the thymus was the seat of origin of the leucocytes (Beard), is disproved by the investigations of Stoehr.

\section{INVOLUTION OF THE THYMUS.}

The thymus is an organ which maintains its structure (as described above) for a certain period of life only, and its function is necessarily exercised only during that period. After a short period of progressive development and growth which, according to Friedleben, lasts in man until about the second year of life, retrogressive changes set in, which lead eventually to a complete disappearance of the parenchyma. The adenoid tissue is gradually replaced by adipose tissue, the original lobulated formation being retained until old age, and the fatty substance frequently enclosing small remnants of true thymus tissue. Involution of the thymus in man coincides normally with adolescence.

Hammar shows thii, in addition to the involution of adolescence, there is also an accidental involution, which may occur at any period of life and leads to a rapid reduction in the size of the gland. He found that the thymus gland of rabbits in the fasting state fell, during the first three days, to one-half its normal weight, and that toxic influences operated in the same direction. This accidental involution is characterized histologically by a diminution in the lymphocytes of the cortex, a reduction in the bulk of the lobes, a disappearance of the differentiation between cortex and medulla, and a remarkable richness. in blood. In the involution of adolescence, the microscopic changes are very similar, though the process sometimes affects certain portions only of the parenchyma and these may be of varying extent. The formation of adipose tissue takes place in the interlobular or vascular connective tissue.

The process of involution may, however, be arrested at any age, and a remarkably large and well-preserved thymus, the socalled persistent thymus, is sometimes seen in man. In these cases, according to Schaffer, the original organ is not preserved, but there is a new formation of thymus tissue, the original medullary substance assuming a fresh cortical layer. Friedleben and, latterly, Basch, found that after partial extirpation of the thymus, there was hypertrophy of the remaining portions. 
The normal weight of the thymus in man is shown by the following table (Hammar) :-

\begin{tabular}{|c|c|c|c|c|c|c|c|}
\hline At birtl & & & .. & $\ldots$ & $\ldots$ & I 3.26 & mmes. \\
\hline Between & the & ages & $I-5$ & $\ldots$ & $\ldots$ & 22.98 & ," \\
\hline , & , & , , & $6-10$ & ... & $\ldots$ & 26.I & ," \\
\hline ,' & ," & , , & I I - I 5 & $\ldots$ & $\ldots$ & $37 \cdot 5^{2}$ & , , \\
\hline , & ," & , & I6-20 & $\ldots$ & $\ldots$ & $25 \cdot 58$ & ,' \\
\hline , & ,' & , , & $5^{6-65}$ & $\ldots$ & $\ldots$ & I6.08 & ,' \\
\hline , , & , , & ,, & $66-75$ & $\ldots$ & $\ldots$ & 6.0 & , \\
\hline
\end{tabular}

At a more advanced age the organ is principally composed of adipose tissue.

The size of the thymus in the dog is estimated by Baum as follows: At birth, the weight of the thymus is to the body as I :250. In the first two weeks the proportion changes to I : I 70 . Involution now begins, and in the course of the first two to three months, the proportion falls to $\mathrm{I}: \mathrm{I} 200$, or $\mathrm{I}: \mathrm{I} 600$. Remnants of the thymus are found in dogs up to the second or third year. Basch found that the most frequent proportional figures were: in dogs in the third to fourth week, I : 300 ; and in rabbits in the third week, I :500 or 600 . According to Basch, the development of the thymus in dogs, during the same period, is at least twice as favourable as in rabbits and cats, and for this reason, dogs are more suitable subjects for experimental extirpation.

According to Noel Paton and A. Goodall, guinea-pigs are the best subjects for this purpose. In these animals the thymus appears in the forn of two flat lobes; it is situated in the neck upon both sides of the trachea and does not extend into the thoracic cavity. In new-born animals, its average weight is $0.19 \mathrm{grm}$. to about $80 \mathrm{grm}$. of body weight, and the weight of the organ continues to increase until the weight of the animal is about 300 grm., which occupies about two months. At this age the animal becomes sexually mature and retrogressive changes in the thymus begin to take place; in animals weighing $400 \mathrm{grm}$. and over, its average weight is only $0.03 \mathrm{grm}$.

According to the investigations of Soederlund and Backmann, the thymus of rabbits attains its maximum relative weight at the end of the third week after birth; and its greatest absolute weight during the fourth month, while the organism is preparing for spermatogenesis. The involution of the thymus seems to coincide, in rabbits as in guinea-pigs, with the completion of the development of the sexual organs.

\section{PHYSIOLOGY OF THE THYMUS.}

In 1858 , a monograph by Friedleben appeared, entitled "The Physiology of the Thymus Gland in Health and in Disease." 
This work was based upon the results of experimental investigation and clinical experience, and it must still be regarded as the text-book upon the subject. It not only brings together all that was previously known about the thymus, but it contains descriptions of the author's anatomical, physiological and chemical experiments, together with an account of his clinical experiences. He tested the physiological significance of the thymus by means of experimental extirpation, and he mentions that he was anticipated in this method by Restelli who, in 1845 , experimentally removed the thymus from sheep, dogs and calves. The greater number of Restelli's animals died after a short period of fever. Friedleben operated upon twenty dogs and three goats, his method of procedure being first to expose the jugular fossa, then to open the apex of the pleura, thus bringing the thymus into view. After this it was an easy matter to remove the organ, either whole or in portions, by inserting the forceps on the inner surface of the manubrium sterni. Friedleben found that none of his animals died, and he concludes from this that extirpation of the thymus does not affect the life of the organism any more than extirpation of the spleen. He describes metabolic changes, however, affecting the amount of albumin contained in the blood, the respiratory interchange of gases, and the products of decomposition found in the urine. But these results do not stand the test of the more perfect methods of our day, and the only findings of Friedleben's which can be regarded as really important are the changes which he noticed in the bones of thymectomized dogs (profusion of blood, pronounced tendency to bend, retarded growth).

It is not until thirty-five years later that we hear of the next attempt at experimental extirpation of the thymus. Langerhans and Sawaliew removed the thymus from rabbits three to six weeks old and found that this was not followed by symptoms of suppression. In 1894 , Gluck described the results of extirpation of the thymus after complete resection of the sternum, the animal remaining perfectly healthy. According to Ambrosini, Thiroloix and Bernard found that total extirpation of the thymus of young rabbits was followed by progressive emaciation, the animals dying three or four weeks later with symptoms of hyperthermia and convulsions.

Tarulli and Lo Monaco (I894-I897) describe the results of thymus extirpation in young dogs and young hens, and they conclude that, in the dog, the thymus is not an organ which is essential to life. They found that in very young dogs its removal was followed by nutritional disturbances; reduction in muscular power; reduction in the volume of the blood, in the number of red blood-corpuscles, and in the quantity of hamoglobin; derangement of the growth of the bones; and rickets-like changes in the skull and bones of the extremities. The changes were, however, quite transitory, and disappeared as the animals grew bigger. 
In young hens, operated upon when two to five days old, there was weakness of the legs, tremor of the entire body, and, finally, a somnolent condition ending in death. The removal of one lobe only of the thymus was followed by a transitory weakness, from which the birds quickly recovered. In birds of more than a week old, the removal of the whole of the gland, like that of one lobe, was not followed by pathological signs.

Abelous and Billard (1896) describe the remarkable results which follow extirpation of the thymus in the frog. According to these authors, frogs live from three to, at the outside, fourteen days after total extirpation; death is accompanied by intense muscular weakness, trophic disturbances of the skin (pallor, ulceration), changes in the nature of the blood (decrease in the number of red blood-corpuscles, leucocytosis) and general œedema. Removal of one lobe of the thymus is not dangerous to life, but extirpation of the portion left in situ is followed by the symptoms described. If the remaining portion is not removed until fifteen to twenty days after extirpation of the first, it will show a certain amount of hypertrophy.

Camia is the only author who is able to confirm these results. The careful investigations of $\mathrm{S}$. Vincent, ver Ecke and, more particularly, of Hammar," led them to different conclusions. Hammar found that, during an observation period of twelve to fifty-nine days after total thymectomy, his frogs showed no symptoms of pathological disturbance. It seems probable from Pari's experiments, that the symptom-complex seen by Abelous and Billard was due to the accidental infection of their subjects. Moreover, Hammar and ver Ecke found that removal of one side of the thymus was not followed by compensatory hypertrophy of the other side; on the contrary, owing to reduced nutrition, there was a more or less clearly defined accidental involution. Hammar concludes that the thymus is not more necessary to life in the frog than it is in mammals.

Experiments with mammals are described by Carbone, Ghika, Ventra and Angiolella. Carbone found that thymectomized rabbits and a thymectomized dog did not differ in their development from control animals of the same litter, and that they showed only a passing reduction in the number of their red bloodcorpuscles. Ghika removed the thymus from eight cats and thirteen rabbits; he found that in the first few days there was emaciation and arrest of development which lasted for weeks, though in some of his animals the losses were afterwards made good. In his opinion, the thymus is concerned in the formation of blood and the promotion of growth. Ventra and Angiolella saw cachexia, similar to that which follows extirpation of the thyroid, in young animals after thymus extirpation.

In $1903, K$. Basch first published the results which he obtained by experimental extirpation of the thymus; he added 
to these results later, and in 1906 published a detailed account of his investigations. Before discussing his discoveries in detail, however, it will be well briefly to describe the results obtained by means of extirpation by certain other authors.

Cozzolino removed the thymus from young rabbits weighing $300-400 \mathrm{grm}$. and, in the first two months after operation, was able to find no. change in their condition. In a second communication, however, he describes crookedness of the legs, arrest of growth, swelling of the tendons, and progressive cachexia in an animal upon which he had operated three months previously. The histological changes were: irregular proliferation of the cartilage in the region of the epiphyses, which were swollen, and considerable new formation of vessels in the bones.

S. Vincent did not observe any change in the condition of guinea-pigs after extirpation of the thymus.

Noel Paton and Goodall found that extirpation of the thymus produced no effect upon the growth and development of new-born guinea-pigs. The number of the red blood-corpuscles was unchanged, but up to the age of 2 months there was a reduction in the number of all kinds of leucocytes. Thymectomized animals showed the same leucocytosis, inflammatory conditions and artificial infections in pregnancy as formal animals; their resistance to the diphtheria toxin was normal, but to streptococci and staphylococci it was slightly reduced.

Noel Paton arrived at some remarkable results concerning the behaviour of the sexual glands after thymectomy. That the removal of the sexual glands of rabbits is followed by hypertrophy of the thymus, was discovered by Calzolari in r898. J. Henderson next showed that early castration is associated in cattle with a stimulation of the processes of growth and a retarded atrophy of the thymus. The normal atrophy of the thymus, which begins at the age of puberty, may be considerably hastened in cattle by using the young bulls for stud purposes and by -allowing the heifers to become pregnant. Paton found that the atrophy of the thymus was arrested in guinea-pigs after castration. He also made the interesting discovery that if thymectomy is performed on guinea-pigs before they reach the age of puberty, the operation is followed by a rapid development of the testicles.

$U$. Soli found that the weight of the thymus in capons was much larger than in cocks; in the latter it averages $0.6 \mathrm{grm}$. to each kilogramme of body weight; while in the capon its weight is I.I6 grm. to each kilogramme of body weight. In Soli's cocks, the removal of the thymus produced a reduction in the weight of the testicles.

It seems probable from these results that the thymus exercises an inhibitory influence upon the development of the testicles, and the appearances seem to show that the involution of the thymus is consequent upon the maturity of the sexual glands. 
The results obtained by $\mathrm{R}$. Fischl from his experiments with goats, rabbits and dogs were purely negative, and he concludes from this that the removal of the thymus has no effect upon the development of the subject. Basch, however, is of the opinion that Fischl's experiments were carried out under quite wrong conditions, and for this reason cannot be regarded as conclusive.

Basch points out that the method of extirpation practised by Friedleben, and by the greater number of investigators after him, that, namely, of removing the thymus by way of the suprasternal fossa, is not a good one, at any rate in the case of dogs. $\mathrm{He}$ preferred a median section of the sternum, and was careful to assure himself before finishing the operation that every particle of the thymus had been removed. Post-mortem examination some time after operation does not yield the same results as the exploration of the living animal. The almost inevitable pneumothorax does not, in his experience, produce unfavourable results. Of the animals upon which he operated (frogs, doves, rabbits, cats, guinea-pigs, dogs), dogs are most sensitive to the removal of the thymus. As in dogs the thymus continues to develop only during the first weeks after birth, and, at the second month undergoes rapid involution, Basch always employed suckling dogs for his experiments, from the same litter as control animals. In seventeen out of twenty litters, he succeeded in keeping at" least one subject and one control animal alive for longer than four weeks. He destroyed the greater number of the animals operated upon in the second month after extirpation; a few were kept for a longer period and, in one instance, the animal was kept for six months.

The most important changes which Basch observed in his thymectomized dogs were those affecting the skeleton. From the second to third week after operation the bones were generally distinctly softer and more pliable than in the control animals, and the thymectomized animals were more straddling and awkward in their gait. The hinder part of the body was weaker and more emaciated, and the upper and lower legs were turned outwards; there was less liveliness of movement than in the control animal, the thymectomized animal mostly sitting upon its haunches. A few weeks later, the front legs also became bandy; there was a broadening in the region of the lower radial epiphysis; and the animal walked on the flat of its feet. The animals were backward in development as compared with the control animals, and sometimes showed a change in the mental condition; there was less movement and less intelligence. There was, moreover, in the course of the first and second months, a distinct backwardness in weight.

In order to test the conditions of ossification, Basch fractured a leg-bone at the same time and under similar conditions in both 
the thymectomized and the control animals. He found that the amount of strength required to break the bone was much less in the case of the thymectomized than in that of the control animal. Five to seven days after the fracture the callus in the control animal was large, and felt more like a spindle-shaped swelling of the bone; in the thymectomized animal it was ring-shaped and considerably smaller. The difference in the development of the callus was perceptible for several weeks. The changes in the bones were well shown in skiagrams. In the thymusless animal, the periosteal apposition at the seat of the fracture was slight, the change of the soft parts into bone being only indicated. The difference in the formation of the callus was best seen in suckling puppies, more particularly in cases where the fracture of the bone took place three to four weeks after the extirpation of the thymus. This seems to show that the change in the regenerative processes of the bones does not make itself felt until after a certain time has elapsed. The changed character of the bones is most clearly seen in the hollow long bones, especially in the tibia and femur, and after them in the humerus and the forearm bones; the ribs and the bones of the hand and the foot are, in general, much less affected.

The examination of anatomical preparations also revealed a distinct backwardness in the development of the bones. In the animals from which the thymus had been removed, the bones were slighter and smaller than in control animals, while the epiphysal lines were frequently broadened and irregular. In two cases there were pronounced changes similar to those seen in the spontaneous rickets of dogs. Microscopic examination showed the changes typical of retarded ossification. In the thymectomized animal, the bone-compact is narrower and contains less lime than in the control animal; and the bony trabecula are more fragile and contain a larger proportion of the original cartilaginous formation. There is a larger proportion of cartilaginous tissue at the epiphysal diaphysis line than in the normal animal. The cartilaginous coating of the epiphyses is thicker and more voluminous, and the ossified bony portion is smaller. The long bones of the thymectomized animal talie longer to develop; calcification progresses slowly, and this causes the retardation of growth, the softness of the bones, and the imperfect apposition of the periosteal bony layers after fracture.

Basch does not regard the changes which follow extirpation of the thymus as being in any way related to rickets. He is much more inclined to look upon them as disturbances of the development of the skeleton, more especially affecting the calcification of the bones, and having an etiological connection with the suppression of the thymus gland. The thymus function being only temporary in its nature, it follows that the changes brought about by its suppression are temporary also. Basch believes 
that the changed conditions of ossification resulting from the suppression of the thymus may be the forerunners of rickets.

Basch endeavours to find an explanation of the changes in the bones, in the metabolic conditions which obtain in thymectomized animals. As C. Bracci pointed out (1905), the removal of the thymus of rabbits causes a reduction of $\mathrm{Ca}$ in the bones and other tissues. Basch investigated the amount of calcium salts excreted in the urine; he found that, during a period of eight days and under precisely similar conditions, the thymectomized animals excreted more than twice the amount of calcium excreted by control animals. This difference disappeared some considerable time after extirpation.

In contrast to this, however, Sinnhuber found that neither the extirpation of the thymus nor the exhibition of thymus gland was followed by an increased excretion of lime in the faces or the urine. Basch explains this discrepancy with his results by a difference in the conditions under which the experiments were performed, Sinnhuber's control animal being taken from a different litter to that from which the thymectomized animal was taken; and, moreover, the control animal had previously suffered from a serious nutritional disturbance characterized by exceptionally high excretion of lime.

Basch also describes a litter of puppies, a few weeks old, in which : the development of the bones was proportional to the growth of the thymus, while there was no apparent modification of the development of either the thyroid or the testicles.

He also found that, where a portion of the thymus was left in situ, there was less difference in the formation of the callus and that, in some instances, it was quite normal. In one such case he observed a hypertrophy of the remaining lobe of the thymus.

$\mathrm{U}$. Soli also found that thymectomy was followed in young. rabbits by changes in the development of the skeleton, these changes affecting the hollow long bones and the ribs.

Sommer and Floerken (I908) described the results of thymectomy and transplantation of the thymus in both dogs and cats. The thymus was removed from the animals at the age of two months; they lived for six to seven months, and differed from the control animals of the same litter in awkwardness of shape, clumsiness of movement, and roughness of coat. Skiagrams showed that the long bones were much shorter and clumsier than those of the control animals. In the case of animals in which a thymus had been implanted, the long bones showed, on the contrary, an increase in the processes of longitudinal growth.

Ranzi and Tandler (I909) showed some dogs from which the thymus had been removed by a median section of the sternum. To prevent pneumothorax, this was done in Sauerbruch's negative-pressure chamber. Three and a half months after operation the animals weighed less than control animals from 
the same litter; their bones were remarkably soft; they had a peculiar straddling walk; and they had not lost their milk-teeth. There was no eniargement of the testicles.

The implantation of the thymus in young dogs was followed by contradictory results. The continued subcutaneous injection of an extract of the thymus glands of sheep, produced in sheep a backwardness in development and in weight. Ranzi and Tandler assert that when applied to the enucleated eye of frogs, thymus extract produces enlargement of the pupil.

With regard to the influence of thymus extirpation upon the nervous system, Basch (I908) proved that, in young dogs, the well-known galvanic excitability of the peripheral nervous system is distinctly increased after extirpation of the thymus. In animals before thymectomy, or in control animals of the same age, the kathodic closure contraction takes place at I to 2 milliamperes, the kathodic opening contraction at over 5 milliamperes; after thymectomy, the kathodic opening contraction figures gradually fall, reaching their lowest, 3 to 4 or even $I .5$ to 2 milliamperes, in the third week, and at this they remain for several weeks. In two cases there was an increased irritability at the cortex of the brain of the motor-points affecting the anterior extremities.

The hypersensibility of the nerves to electric stimulus is less intense after thymectomy than after thyroparathyroidectomy, and for this reason only a small proportion-about one-tenth-of thymectomized dogs die of convulsions.

According to Sabatini, and his view is confirmed by Basch, in normal animals the direct application of soluble calcium salts reduces the irritability of the cortex of the brain ; in thymectomized animals, the subcutaneous injection of these salts produces a distinct reduction of peripheral hyperirritability, and their repeated injection may even restore the kathodic opening contraction to its normal figure.

In normal animals, the injection of thymus extract is believed to increase the sensibility of the peripheral nerves; in thymectomized animals, both thymus extract and suprarenal extract rather reduce this sensibility.

According to Basch, the thymus gland is physiologically associated with a definite reaction of the motor apparatus to the electric current. He is of the opinion that the causative factor of infantile tetany lies, not only with the parathyroid glands, but with the entire group of branchiogenic organs, of which the thymus is probably the most important; for not only is it very sensitive in its reaction to general disturbances, but it is functionally active in the first years of life only.

Now that we know that, in many species of mammals, the thymus gland contains accessory parathyroids (Pepere), it seems probable that the latent tetany associated with Basch's experiments was purely parathyroprive in origin, and not in any way due to suppression of the function of the thymus. 


\section{EFFECTS OF THYMUS EXTRACT.}

Svehla (x896) found that the watery extract of the thymus of man and of certain animals (pig, dog, ox), when injected into the veins of dogs, produces an acceleration of the pulse and a reduction of arterial tension. The fall in pressure is the expression of an enfeebling, or of a paralysis, of the vasoconstrictors, while the acceleration of the heart's action is the result of the direct influence of the extract upon the cardiac muscles themselves, and not upon the nervous apparatus which accelerates cardiac activity. The arrest of respiration and of cardiac activity which sometimes follow large doses of thymus extract, are secondary results of the peripheral vaso-motor paralysis.

Svelha showed further that this specific substance is not present in the thymus of the human embryo, but is formed after birth. Its activity apparently becomes stronger with time and, even at the age of 40 , the thymus in man still retains its specific activity. Both the toxicity of thymus extract and its influence in reducing pressure have been confirmed by other investigators (Basch, Livon, Vincent and Sheen). Vincent and Sheen, however, emphasize the fact that substances with similar depressant effects are obtained from many tissues besides the thymus. Farini and Vedoni also describe the depressant action of thymus extract; they found that blood containing thymus extract, when circulated by artificial means through the hinder extremities, did not produce a vaso-dilation, but a vaso-contraction, which lasted, however, only a short time. R. Popper (1905) discovered that the marked reduction of blood-pressure which, together with suffocation and heart failure, follows the intravenous injection of homologous thymus extract, is not the result of a specific toxic activity of the thymus extract upon the circulatory apparatus, but is due to a property common to all tissue extracts, that, namely of producing intravascular coagulation and so giving rise to circulatory disturbances. He found that, in rabbits which died after the injection of thymus extract, there were extensive areas of coagulation in the vascular system and in the heart ; dogs, however, usually recovered, the formation of thrombi being confined to single vessels. If the coagulation of the blood is prevented by means of extract of leeches, the action of the thymus extract ceases to be toxic, and the reduction in blood-pressure appears only after very large doses, and then in a very slight degree.

Thymus extract remains active after boiling, and this shows that its active principle is not, in itself, a ferment, but that it is a substance which causes the formation of a ferment having the property of coagulating blood. 


\section{MORS THYMIA AND THE STATUS THYMICO- LYMPHATICUS.}

The laryngeal spasm of infants was termed asthma thymicum by the older physicians, and its sudden fatal termination was supposed to result from changes in the thymus. After very careful investigation, Friedleben came to the conclusion that " neither the normal nor the hypertrophied thymus is able to provoke laryngeal spasm, and that there is no such thing as asthma thymicum." This view was, at first, very generally accepted. The fact, however, that in many cases of sudden death, post-mortem examination showed a distinct hyperplasia of the thymus, led Grawitz to conclude (I888) that, in such cases, death was due to suffocation from a mechanical compression of the trachea, and this explanation is still, in many instances, held to be rational and sufficient.

Another explanation was, however, offered by Arnold Paltauf (I889). He insisted upon the existence of a specific constitutional anomaly, suggesteâ in Rokitansky's text-book (186r), and which Paltauf describes as follows :

"Pallor of the skin; well-developed panniculus adiposus; hyperplasia of different parts of the lymphatic system, the lymphatic glands of the neck, axilla, mesentery; enlargement of the follicles of the naso-pharyngeal cavity, the intestinal walls, the base of the tongue and the spleen; the thymus larger than the average. This finding points to a general pathological condition of the body which is generally termed the lymphatic constitution. The hyperplasia of the thymus and its abnormally prolonged duration are not the causes of death, but are symptoms of the general nutritional derangement, which is further evidenced by the enlargement of the lymph-glands, tonsils, \&c."

Svehla next concluded, from the effects of thymus extract upon the heart and the vessels, that the mors thymica of children is due to hyperthymization; that is, to the action of a substance circulating in the blood, which is supplied in an increased quantity by the hyperplastic thymus.

The fact that the effects of thymus extract are not specific, withdraws from Svehla's theory its most important support. And yet Hart, writing quite recently, considers the theory of an accumulation in the organism of the toxic products of metabolism as the result of thymus hyperplasia, to be quite plausible. $\mathrm{He}$ tions, there was paresis of the hinder extremities, "the cardiac persistent thymus, rubbed down and prepared under sterile conditions, there was paresis of the hinder extremities, "the cardiac activity was much accelerated, the animals sat forward and stiffly upright, as if in cardiac distress, in which attitude, after a few moments, they died." Post-mortem examination showed that, apart from hyperæmia, all the organs were healthy; the suprarenals showed signs of extreme hyperæmia with some hæmor- 
rhages. Calf's thymus, rubbed down and prepared under sterile conditions, produced negative results when injected into the peritoneum of control animals.

Wiesel ascribes sudden death in the status thymicolymphaticus to a hypoplasia of the chromaffine system, as seen in the post-mortem finding of changes in the suprarenals. He thinks that the secretion of the chromaffine system, which raises bloodpressure and stimulates the heart and the vessels, is supplied in insufficient quantity, and that, in such individuals, slight noxæ may cause paralysis of the vessels and heart failure.

It is evident, then, that sudden death in children and adults as the result of laryngospastic convulsion, as well as those cases where death takes place at the commencement of chloroform anæsthesia, the post-mortem findings frequently being negative, are referable to a status thymicolymphaticus. The investigations of Richter, as well as those of v. Sury, show, however, that great caution should be exercised in identifying sudden death in childhood with mors thymica. Richter points out that an acute capillary bronchitis, unrecognized during life, may easily be the cause of sudden death.

We have, as yet, no sufficient explanation of the pathogenetic significance of the thymus in mors thymica, or of the manner in which this is brought about. And the part played by the thymus in the production of the so-called status thymicolymphaticus is equally unexplained.

Bartel recently interpreted the status thymicolymphaticus as one of the symptoms of a more or less general hypoplastic constitution. He describes the post-mortem findings in over Ioo cases. The superficial fascia was in all cases well developed; the height was frequently above the average for the sex and age; there was marked swelling and hyperplasia of the lymphatic glands, the lymphatic deposits of the mucous membranes (tonsils, lymph follicles of the tongue, stomach and intestines) and the follicles of the spleen; the bone marrow was frequently red, and there was, in the very large majority of instances, a much enlarged thymus. The average weight of the thymus considerably exceeded the average figures given by Paltauf and Mettenheimer, namely : from 15 to 25 years of age, 22.I grm.; from 25 to 35, 3.I grm. Bartel's figures do not differ greatly from those of Hammar, which are, however, remarkably high. Bartel also found congenital smallness in the lumen of the aorta and of the peripheral vessels, remarkable smallness in the size of the heart, comparatively large brain (hypertrophia cerebri), colloid degeneration of the thyroid gland and fragile structure of the bones. The only details needed to complete the clinical complex of the hypoplastic constitution are: hypoplasia of the genitals, defective growth of pubic hair ; in the female, narrow vagina, infantile uterus, usually abnormally large 
and smooth ovaries, menstrual anomalies and frequently anomalies of pregnancy; in the male, deficient growth of pubic hair and smallness of the external sexual organs.

Out of 15 cases of Addison's disease, Hedinger found 7 with marked status thymicolymphaticus, 5 in whom the condition was not so marked, and 3 in whom it was uncertain.

The pathological significance of the constitutional anomalies presented by the status thymicolymphaticus is shown, in the first place, by the greater sensitiveness of such individuals to harmful disturbances of all kinds (Laub). Their greater vulnerability is shown by the fact that more than half of these subjects die early in life ( 14 to 25 ) of infective diseases. Of these the most frequent is tuberculosis, but nephritis, eclampsia and diabetes are all to be met with; and suicide is a not infrequent termination to these lives (Bartel).

The older pathologists regarded this condition as a predisposition to tuberculosis and to certain other infective diseases; this view is confirmed by the results of modern investigators (Kundrat, Ort, Bartel).

\section{THE SUPRARENAL SYSTEM.}

\section{THE INTERRENAL AND ADRENAL SYSTEMS.} HistoricAL.

THE suprarenal capsules were first recognized as separate organs by Bartholomeus Eustachius Sanctoseverinatus, the great anatomist of the Renaissance, in the year ${ }^{5} 563$. It is commonly believed that these organs are mentioned in different passages in the Bible, but the notion is an erroneous one arising, as Blanchard points out, from incorrect translation of the Vulgate.

The manner in which our knowledge of the suprarenals has grown is interesting from many points of view. This is fully described in another place, the present intention being to give only a very brief summary of the more important historical data.

The work of Eustachius was followed up by a number of authors, who made exhaustive investigations into the anatomy and functions of the suprarenal capsules. It is nevertheless curious to find that many anatomical and medical works of the 17 th and I8th centuries contain no mention of these organs (Vesalius, Fallopius, Fabricius, Aquapendente and van Swieten).

In 1716, the Académie des Sciences of Bordeaux offered a prize for an answer to the question: “ Quel est l'usage des glands surrenales?" The judge was Montesquieu, then 29 years of age, who found himself unable to award the prize to any of the conflicting and, in some instances, very extraordinary theories offered in solution of the problem. He closed his searching criticism of these theories with the words: "Le hazard fera peut-être quelque jour ce que tous les soins n'ont pu faire." 
This fortunate chance was a long time in coming. In I8I9, Caillau made Montesquieu's judgment public, at the same time reviewing the work of the century following it, and his summary was this: "Les anatomistes n'ont pas pu découvrir l'usage des glandes surrénales . . . Nous ne sommes pas plus avancés aujourd'hui sur les glandes dont il est question que du temps du fameux Eustache qui en parle le premier."

Caillau's view was right in so far as it concerned the function of the suprarenals; but it must not be forgotten that the i 8 th century added largely to our knowledge of the anatomy of these organs. In $175^{2}, \mathrm{~J}$. B. Winslow published an exact description of the anatomy of the human suprarenals, and at the beginning of the igth century (1806) J. F. Meckel's comparative anatomy of the suprarenals appeared, a work the importance of which it is difficult to over-estimate.

In the course of the next fifty years, important additions were made to the morphology of the subject. In i $846 \mathrm{~A}$. Ecker gave an exact description of the minute structure of the suprarenals as revealed by the microscope. Leydig's work in comparative morphology (I85I-I853) and A. Kolliker's epoch-making description of the histological structure of the suprarenals (I854) were of the highest importance to the later development of our knowledge of the subject.

The year i 855 marks both a turning-point and the commencement of a new era in suprarenal research. All that had gone before served merely as the foundation for a profound and farreaching doctrine concerning the physiological and pathological significance of these organs, a doctrine which to-day is, in certain directions, still awaiting completion. "At that date, the work of nearly three centuries had supplied all that was to be known macroscopically of the anatomy of the suprarenals; there was a certain small amount of knowledge of the embryology, teratology and pathological anatomy of the subject; the keen research of the fifty previous years had supplied important data in the department of comparative anatomy; whilst as much was known of the minute structure as the means of that day permitted. But the physiology of the suprarenal capsules was a sealed book. The older authors, it is true, formulated vague and generally insufficiently grounded hypotheses concerning the functional significance of these organs; more modern observers, basing their views upon the results of investigation in comparative anatomy and histology, arrived at more or less well-founded suppositions; but in no case did either observation or experiment bring to light a single fact concerning the functional activity of these organs in any direction whatsoever.

It was in 1855 that Thomas Addison published his book " On the Constitutional and Local Effects of Disease of the Suprarenal Capsules" -a work in which a clinical condition observed 
in man was, upon pathologico-anatomical grounds brought into relationship with a disturbed condition of the suprarenals.

Addison described the condition, which was afterwards named after him, as an idiopathic anzemia, associated with extreme apathy and adynamia, disturbances of the digestive tract and of the nervous system, and a bronze-coloured pigmentation of the skin and mucous membranes; whose course was chronic; which presented all the features of a progressive cachexia, frequently accompanied by violent symptoms, such as uncontrollable diarrhœa, coma and convulsions; and with a termination which was always fatal. In every case examined, the post-mortem findings showed serious disease, generally tuberculous in character, of the suprarenals, and Addison concluded from this that both the pathological condition and its fatal termination were due to suppression of the suprarenal function.

Addison's observations supplied the first contribution, from clinical experience, to the physiology of the suprarenals; they showed that these organs are essential to the life of the individual; and they furnished certain inferences as to the nature and method of their functional activity.

Addison's discovery gave an immense impetus to research which, for the first time in the history of this subject, was conducted upon physiological lines; that is to say, by means of experiment with the living animal. It was believed that extirpation of the suprarenals of animals-planned earlier by various investigators (Ecker, Meckel) but never carried out-would supply the much desired information as to the function of these organs. Moreover, this method alone could decide whether the clinical condition observed by Addison in man, could be induced in animals by the experimental destruction of their suprarenals. These expectations were, however, not fulfilled. Although a large number of communications were forthcoming which confirmed the coincidence of the classical symptom-complex-pigmentation of the skin, muscular weakness, gastro-intestinal derangement, anamia, - with a disease of the suprarenals in man, yet in no case was it possible experimentally to reproduce a similar group of symptoms in animals. All that experimental investigation was able to achieve, was a definite answer to the question as to whether animals could survive after extirpation of the suprarenals -in other words, as to whether or not the suprarenals were essential to the life of the animal.

The first accounts of such experiments were published in the year 1856 . The results described were capable of various interpretations and led to a vast amount of discussion which, helped on

by numerous experiments, lasted until about the middle of the 'sixties. It seemed at that time that the part played by the suprarenals in the animal economy was not a vital one.

The interest of physiologists was, however, permanently 
aroused, and the suprarenals have since formed a constant subject of investigation. A strong impetus was furnished by the new doctrine of the internal secretions and, more particularly, by the discovery of the remarkable physiological activity of the watery extract of suprarenal.

Since I 896 a large number of publications have appeared (Brauer, Diamare, Fusari, Giacomini, Grynfellt, Kohn, Kose, Pettit, Poll, Soulié, Srdinko, Vincent, Wiesel) in which the suprarenal question is treated from the genetical and from the comparative morphological points of view.

\section{COMPARATIVE MORPHOLOGY AND EMBRYOLOGY.}

The study of the comparative morphology and of the embryology of the suprarenals led to a complete revision of the older theories, and created a new physiological foundation. The results of these investigations are, briefly, as follows:

Neither morphologically and genetically, nor physiologically and pathologically, are the suprarenals homogeneous organs, but they are the result of the combination of two different and independent organic systems. The suprarenal complex is derived, not from the whole of these systems, but from different larger portions of them.

The earlier view, which regarded the suprarenals as independent homogeneous organs, having a topographical relationship to the kidneys, was a perfectly justifiable one, for it was based upon purely anatomical data obtained from man and the higher mammals. That the physiology and pathology of the suprarenals are largely derived from this anatomical standpoint and continue to be affected by it, is readily comprehensible; but that this is a cause of much of the haziness by which the subject is surrounded cannot for a moment be doubted.

The conception of the suprarenals as homogeneous organs, early underwent a certain amount of modification; for in addition to the principal organs, other structures were frequently observed to be present, sometimes associated with the principal organs and sometimes isolated from them, which could only be regarded as accessory suprarenals. Although the presence, position and structure of these bodies is subject to considerable variation, yet they occur so frequently that they must be regarded as normal structures. The fact that their existence has so often been ignored and their physiological significance insufficiently understood, has been a very fruitful source of error.

If the conception of the suprarenals as homogeneous organs was shaken by the discovery of these accessory bodies, a consideration of their structure served to shatter the theory entirely. Section of the suprarenals shows, even to the naked eye, that they are composed of two sharply defined portions, differing from one 
another both in colour and consistency. This difference was recognized by the earlier investigators, by whom the two portions were distinguished as the "medulla" and the "cortex." These terms are not merely topographical, but possess a histological significance. The microscopic examination of the more minute structure shows that the medulla and the cortex of the suprarenals are composed of two different tissue formations.

The cortex is composed of rows and columns of cells containing a varying quantity of shining granules, which are partly double-refractive. The characteristic reactions of these granules show that they contain fat or are closely related to lecithin, and for this reason they are known as lipoid granules. They colour strongly with the specific analine dyes for fats (alkanet, sudan III, scarlet R.); they stain black with fluids containing osmium; and they are more readily soluble than fats in fat-dissolving agents (chloroform, xylol, ethereal oils).

The medulla consists of a tissue well furnished with nerves and vessels, and composed of spheroid or cord-like cell-agglomerations, the cells of which possess a specific affinity for chromium salts. The contents of these cells are very evanescent, but they may be preserved in chromic acid and solutions of the chromic acid salts, by which they are coloured a light yellow to dark brown (Henle, 1865). The finely granular cyptoplasm has a marked affinity for the specific stains for nuclei (carmine, hæmatoxylin, safranin) and colours a black-green with ferric chloride.

Thus, the anatomically homogeneous suprarenals consist histologically of a peculiar combination of two different tissues, the cells of one being characterized by their lipoid contents and those of the other by their chromaffinity.

This differentiation is not sufficient, however, when we come to the anatomical comparison of the homologous organs of the different classes of animals. It is only in the higher mammals that well-defined paired organs are met with which, from their position and partly also from their structure, can be identified as suprarenals. In birds these organs consist of the two characteristic kinds of tissue, but the topographical arrangement of medulla and cortex is absent. It is rather the intimate interweaving of two nets of cell-columns of which one-the system of principal columns-has all the histological characteristics of the suprarenal cortex, the other-the intermediary system-presents those of the chromaffine medulla. The conditions in reptiles are essentially the same, but it is worth noting that here the tissue with lipoid cell-contents is largely predominant; it is only in the hydrosauria and chelonia (crocodile and tortoise) that these cortical threads are interwoven with fine medullary threads. In the lepidosauria (lizards and snakes), isolated balls of medullary substance are scattered in a ventral direction in the cortex, and the greater part of the chromaffine tissue occupies a dorsal position. 
This, at least anatomically, isolated suprarenal of the amniota is absent in the anamniota, where it seems to be replaced by a number of small bodies, partly connected and partly entirely separate. The amphibia represent, to a certain extent, the transition stage between the higher and lower vertebra. In the anura (frogs, toads), the yellow bodies which represent the suprarenals are united to form a thin plate which is attached ventrally and medially to the kidneys; in the gymnophiona (blind worms), they are united at least at the head end to form a largish mass, and the cordal part only is broken up into separate portions; in the urodela (salamander, newts), they are divided into numerous small islands, situated close to the kidney and along its entire length. The histological relationship between these organs of the amphibia and the suprarenals of the amniota is shown by the fact that, in addition to the larger portions, composed of rows of cells with lipoid contents, chromaffine tissue is also present in isolated groups of cells and cell-masses which, in the anura, form an intercommunicating network of intermediary columns.

In all vertebrates lower than the batrachians, the suprarenals are replaced by two topographically separate systems of small bodies.* The only exception is the amphioxus, in which no cell-groups of a structure similar to suprarenal tissue have as yet been found.

The one system includes the entire body, described in the Selachii under the name of " interrenal body," lying between the caudal end of the kidneys; in the Teleostii, the so-called "corpuscle of Stannius "; and in the Ganoidei, the corpuscles distributed throughout the whole kidney, likewise described by Stannius; as well as the small structures which, in cyclostomata, are attached to the walls of the posterior cardinal veins and extend from the region of the pronephros (head kidney) to the tail. Histologically, all these bodies are exclusively composed of cells arranged in vesicles or tubules. The cells contain lipoid granules and their structure is therefore similar to that of the suprarenal cortex.

The second system is chromaffine in character and comprises those paired bodies, associated with the sympathetic ganglia and lying dorsally to the kidneys in the caudal rump-part of the body, which, in the Selachii, are called the suprarenal organs. But as this terminology is liable to lead to confusion (the German

* The question as to whether or not the invertebrates possess organs equivalent to the suprarenals was first propounded by Leydig. He observed peculiar cells with yellow granular contents against the nerve fibres and ganglia of Paludina, Pontobdella and Mermis, and regarded these as the counterparts of the suprarenals. Poll and Sommer found chrome-brown cells in the central nervous system of leeches, and Poll ' found similar cells in the abdominal ganglia of Hirudo, Aulastoma, Placobdella, and Nephelis. I myself found that the extract of the abdominal ganglia of Hirudo medicinalis and Lumbricus terrestris contained adrenalin, and this may be proved by biological methods. 
Nebennieren being called in English " suprarenal bodies "), it is expedient to call them, from their histological characteristics, chromaffine or phæochrome bodies; or, from the nature of their function, they may be termed adrenal organs.* In the Teleostei and Ganoidei, these adrenal bodies are situated in the walls of the cardinal veins. In the Cyclostomata, the adrenal system extends from the neighbourhood of the second pair of gills to the tail; it takes the form of bands, which are situated between the aorta and the cardinal veins, and are intimately associated with the blood-vessels.

Comparative anatomy shows that the two fundamental tissues, which in mammals are distinguished as cortex and medulla, are present in all vertebrates, and are therefore to be regarded as structures which are component parts of the vertebrate body. In fish, the two kinds of tissue appear as two different organic systems, anatomically separate from one another, and known as the interrenal and adrenal systems. The interrenal system includes all the structures which, like the suprarenal cortex, are composed of cells with lipoid contents; the adrenal system comprises all tissues the cells of which, like those of the medullary portion of the suprarenal, are characterized by their chromaffinity.

In the higher vertebrates, however, these systems have, to a differing extent, lost their independence, and have also become very much reduced in size. A partial amalgamation of both systems is seen in the amphibia, the independent portion of the adrenal system here forming isolated cell-agglomerations, which are intimately connected with the sympathetic system. In reptiles and birds, the association between the two systems is more intimate; though independent portions of the adrenal system are found, in the form of chromaffine bodies, throughout the sympathetic system in birds, while in reptiles they lie along the great abdominal vessels. In mammals, the amalgamation of the two systems is complete. This is the result of the topographical arrangement by which the adrenal system becomes the medullary portion, while the interrenal system becomes the cortical portion, of the suprarenal capsules. But even here, a considerable proportion of the adrenal system remains independent. It is distributed along the sympathetic system, partly in the form of isolated phæochrome cells and larger or smaller chromaffine bodies, and partly in the form of larger structures, invariably found at the bifurcation of the carotid artery and at the abdominal

* The terms "cliromophile" (Stilling), "chromaffine" (Kohn), "phæochrome" or "chrome-brown" (Poll) tissue, indicate a special histological characteristic; the term " paraganglia," suggested by Kohn, has an etiological significance; while the term "adrenal system," in so far as it is not a topographical description but is used as a synonym for "adrenalinproducing organs," expresses the functional significance of these glands. The chrome-brown cells are very aptly termed "cellules adrénalogènes" by Bonnamour. 
division of the aorta and named respectively the carotid gland and Zuckerkandl's accessory organs of the sympathetic system.

The greater number of what are usually called accessory suprarenal organs are rather, from their histological structure, free portions of the interrenal system, and should by rights be called accessory interrenal bediès. They are found in the neighbourhood of the suprarenals; in the substance of the kidneys; and distributed through the whole of the retroperitoneal space and extending downwards into the pelvis, in women chiefly in the ligamentum latum, in men in the vicinity of the spermatic cord and the testicles.

A homogeneous suprarenal is found only in the higher vertebrates, and even here it is only certain portions of the two systems, varying in different classes, which combine to form the apparently homogeneous organ. The suprarenal of mammals, with its cortical and medullary parts, represents a combination of portions of the adrenal and interrenal systems. The terms "cortex" and "medulla" merely represent the topographical conditions in mainmals; in reptiles and birds the systems are differentiated into "principal" and "intermediary columns"'; in the lepidosaurii and amphibia a portion of the "medullary substance" lies, not in the interior, but upon the surface of the "cortex." As histological definitions, "cortex" and "medulla" are synonymous with interrenal and adrenal tissue, but being only parts of these systems, they can never be employed to describe the systems as a whole.

It has been shown by means of comparative anatomy and histological investigation that the bodies of vertebrates contain two different and independent systems, certain portions of which combine to form the suprarenals of the higher orders. This is confirmed by the results of comparative embryology. In all orders of vetebrates during organogenesis, two independent and autonomous organic systems are evolved from two different primordial beginnings. These are the adrenal and interrenal organs which, according to the further development of their tissue, maintain their independence either wholly or in part (as in cyclostomata and fish). In the course of histogenesis, and after manifold changes, large portions of each system combine to form either a number of small bodies, as in the amphibia, or a single homogeneous organ, such as the suprarenals of the amniota.

The interrenal system is the direct descendant of the mesoderm. It is developed from the ventral portion, that part which forms an epithelial sheath, covering the lateral plates of the coelom in the so-called interrenal zone. At about the time when the sexual glands first begin to appear, thickenings of the peritoneal epithelium are formed by means of mitotic cell-proliferation at circumscribed areas. These thickenings take the form of buds, the cells of which early acquire a characteristic appearance. Later 
on, the buds become detached from the parent membrane and assume the status of interrenal organs which, in the form of isolated cell-agglomerations, lie free in the supporting tissue and, by their change of position, acquire fresh topographical relationships to the surrounding organs. At the same time, as growth continues, processes of degeneration and of coalescence take place, leading, in the former case, to a reduction in the length of the system, which originally extended over comparatively large portions of the body of the embryo; and in the latter, to the partial or complete negation of the primary discontinuity of the system. Thus, vigorous growth and the coalescence of separate buds is the primary origin of the principal organs, while accessory organs are primarily the result of imperfect involution. Accessory bodies may, however, be formed secondarily at a later date by fission of the principal organs.

The embryonal interrenal cells undergo further histological and cytological differentiation until the specific structure is assumed. In cyclostomata and in fish, the interrenal system remains in this condition of independence.

The adrenal system is. derived from the ectoderm; it shares its primordial beginning with the sympathetic system and develops as a part of it. In the course of the histogenesis of the sympathetic ganglia, two different cell-types are formed from the apparently homogeneous and undifferentiated seat of origin of the primitive ganglia. Of these, one is the prototype of the sympathetic nervecells, while the other includes certain specific elements of a peculiar character which are the first chromaffine cells (phæochromoblasts). The latter occur either singly or in larger or smaller groups in the midst of the nervous tissue and not divided from it. The apparently homogeneous primitive beginning may, however, divide into two in such a manner that the chromaffine tissue lies within or near a sympathetic ganglion, and may occasionally be separated from it by a sheath of connective tissue. In the Selachii, the elements of the adrenal system remain stationary at this stage of development, and are represented by the so-called suprarenal bodies of this order. Finally, there may be a complete topographical separation of the adrenal elements from the sympathetic system, the chromaffine bodies, as soon as they become separate structures, showing only very slight traces of their genetic association with the sympathetic. Separation of all three kinds is seen in amniota and in amphibia. In these orders the adrenal system consists of depositions of chromaffine tissue within the sympathetic ganglia and plexuses; of deposits of chromaffine bodies attached to the sympathetic; and of relatively autonomous structures like. the so-called accessory organs. The slight disposition to a separate existence by which the organs of the adrenal system in general are characterized, is also shown by the fact that portions of this tissue associate themselves at an early develop- 
mental stage with other heterogeneous tissues, such as the carotid gland and the interrenal tissue.

The development of the suprarenal represents a later chapter in the history of both systems; it is the last genetic event in which a combination of interrenal and adrenal tissue takes place. It is seen for the first time in the amphibia, where groups of characteristic cells, released from the sympathetic ganglia, wander along the wall of the aorta in a ventral and median direction, finally reaching the interrenal bodies, which are already fully formed and clearly defined. In the gymnophiona, these cell-groups place themselves by the side of the interrenal cell-bodies, but in the urodela and anura they become either partially or wholly intermingled with them. The result is the formation, not of a suprarenal, but of a number of small suprarenals.

In reptiles and birds the process is analogous. At the beginning, the interrenal system consists of isolated buds; at a later period, sympathetic elements associate themselves with the metamorphosed and well-defined interrenal body and, in the course of histogenetic development, this association becomes more intimate. Almost the entire interrenal system is used up in the formation of the suprarenal bodies, and the accessory interrenal bodies, which are rare in these orders, are probably formed by secondary division of the principal organ.

In mammals, at the time when the interrenal system has become clearly defined and histologically differentiated, which in the human embryo is at about the fifth week, undifferentiated sympathetic elements wander from the principal mass of the abdominal plexuses to the cell-columns of the interrenal bodies. These cells break through the capsule upon the median side and make their way between the cell agglomerations and columns of the interrenal tissue, at the same time keeping their structural relationship with the abdominal sympathetic. In the external mass, a differentiation between nerve-cells and chromaffine cells is soon perceptible; but the cells which made their way into the interior remain for a long time in their primordial state. This intrusion of sympathetic elements is continued during the whole of fotal life, the interrenal tissue becoming entirely interwoven with these elements, as seen in the completed suprarenal of birds. It is not until later, when the histological and cytological transformation of interrenal tissue into the three-layered suprarenal cortex is complete, that the interpolated sympathetic elements become, in a topographical sense, the suprarenal medulla. At about the beginning of the fourth month, they acquire the chrome-brown colour which is their chief histological characteristic. That in mammals, as in the other higher orders of vertebrates, the whole of the interrenal tissue is not used up in the formation of the suprarenals, is shown by the fact that accessory suprarenals frequently occur which contain no trace of chromaffine tissue. These structures, which should rightly be 
termed "accessory interrenal bodies" (Beizwischennieren), may be formed primarily by the persistence and after-growth of isolated interrenal buds placed in a caudal direction; they may also be formed secondarily by fission of the principal organ, due either to interproliferation of the sympathetic elements, or to circumscription of growth and consequent division as the result of connective tissue formations.

It is obvious that wherever, in the course of embryonal development, portions of the interrenal and adrenal systems come into close topographical relationship, true "accessory suprarenals" (Beinebennieren) may be formed; that is to say, organs composed of a cortex and medulla with a structure the exact counterpart of that of the suprarenals. The accounts of such bodies are, however, very scanty. In I884, d'Ajutolo found a true accessory suprarenal in the plexus pampiniformis of a newly-born boy; and Delamare found one in 1904, in the solar plexus. Aschoff (1903) describes chromaffine bodies and interrenal structures lying side by side in the paradidymis and parovarium of newly-born children.

Comparative embryology supplies etiological proof of the existence within the body of animals of two independent suprarenal systems; it also shows how, in the course of philogeny, these systems tend to an increasing and more perfect unification. The question arises as to whether this coalescence results in an organ the homogeneous character of which is purely morphological, or whether this combination of the two heterogeneous systems is the expression of an intimate functional relationship of the two, the suprarenal being perhaps functionally a higher organic entity.

But physiology, whose province it is to solve this problem, was from the beginning confronted with an anatomically homogeneous suprarenal and, up to the present, has occupied itself solely with the suprarenal function.

The following tables illustrate the suprarenal systems as they occur in all the animal classes. Table A shows all that is at present known concerning the invertebrate animals and the vertebrates up as far as the bactrians inclusive. In addition to the permanent conditions affecting the interrenal system, it shows those which obtain in the embryonal interrenal. Table B includes all vertebrates higher than the bactrians; it illustrates the conditions which govern the embryonal interrenal, those affecting the free portions of both the interrenal and adrenal systems, and the conditions governing those portions of both systems which unite to form true accessory suprarenals.

\section{PHYSIOLOGY OF THE SUPRARENALS.}

\section{EXPERIMENTAL ExtiRPation.}

It has already been pointed out that Addison's epoch-making discovery of the results which, in man, arise from the suppression 


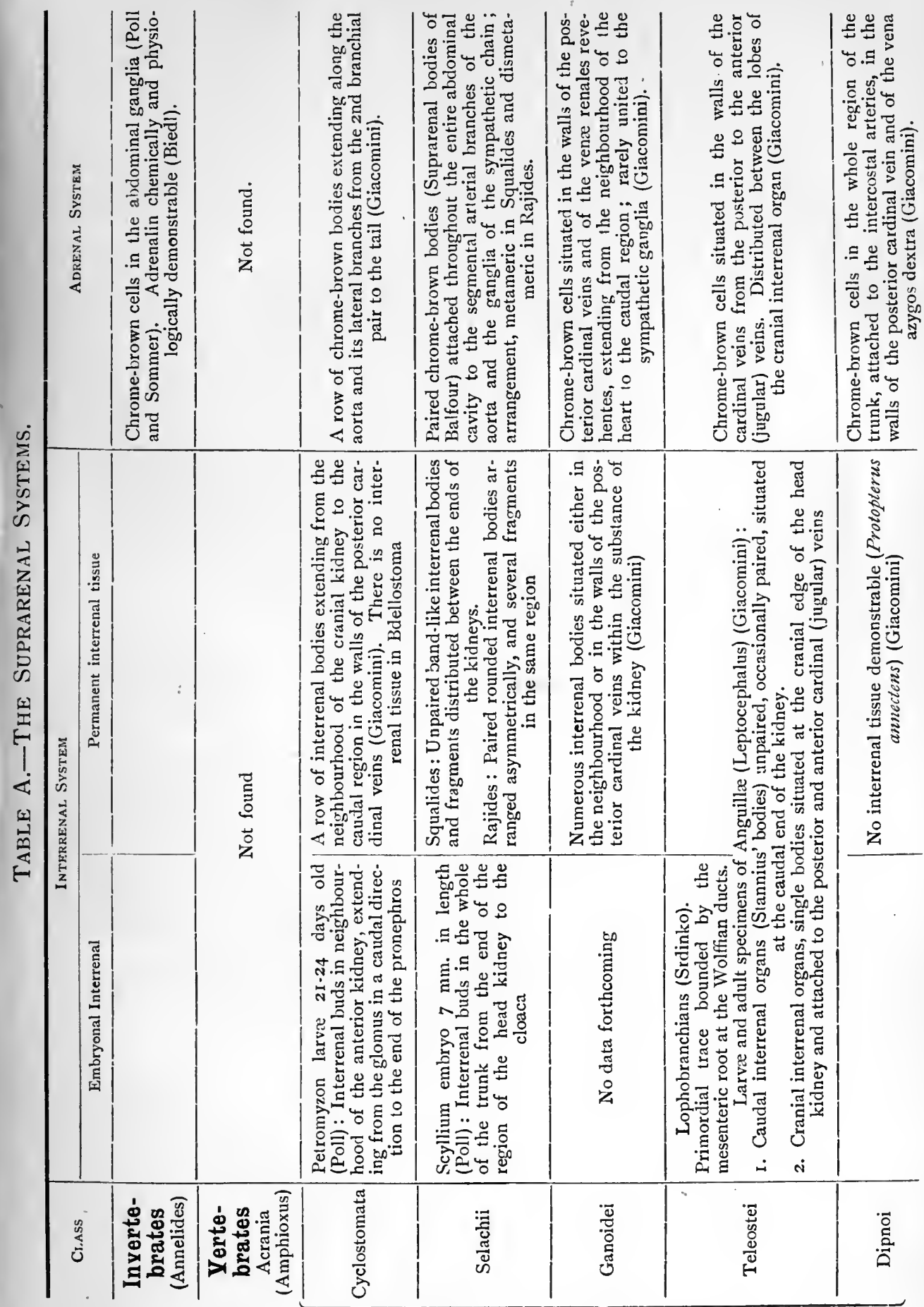

e)oluuru 


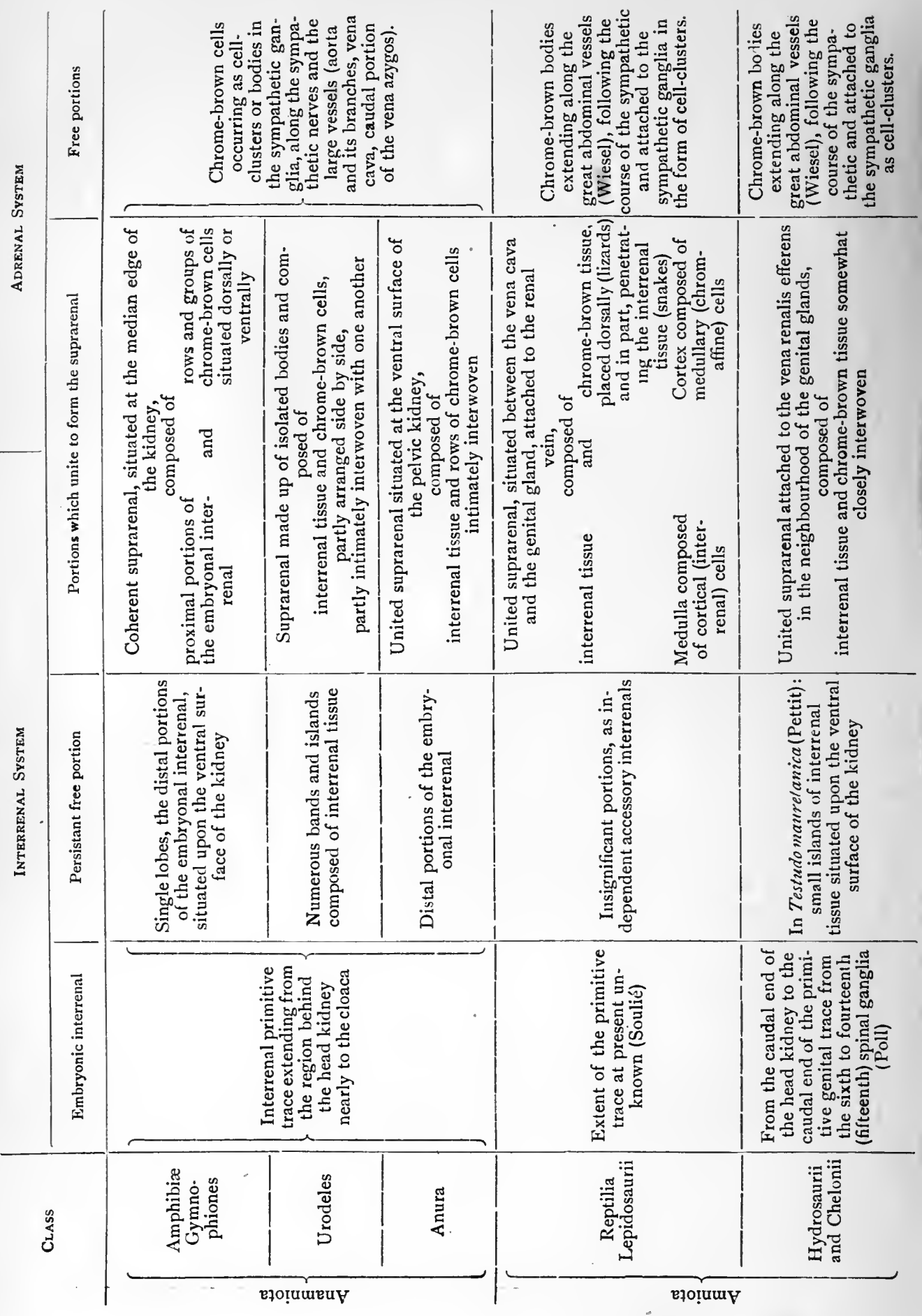




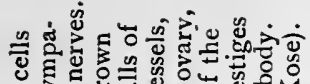

ह के व्रें

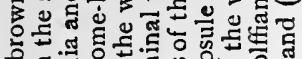
次.

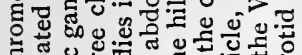

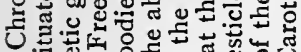

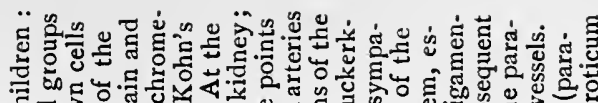

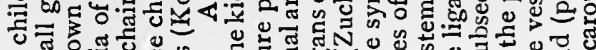

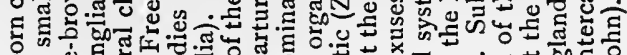

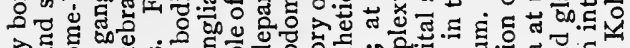
त和

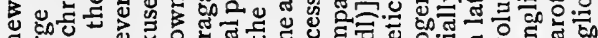

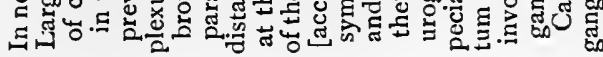
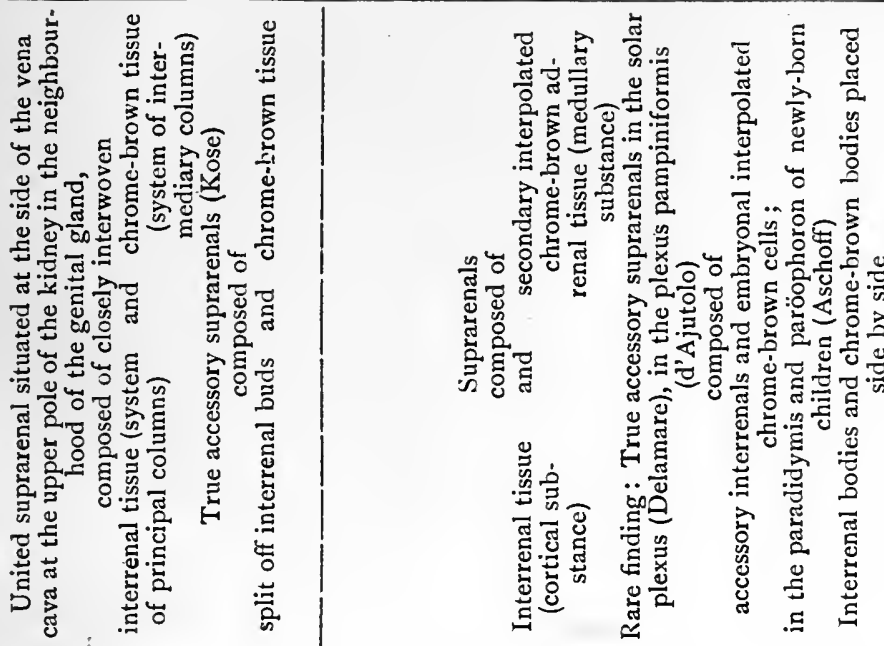

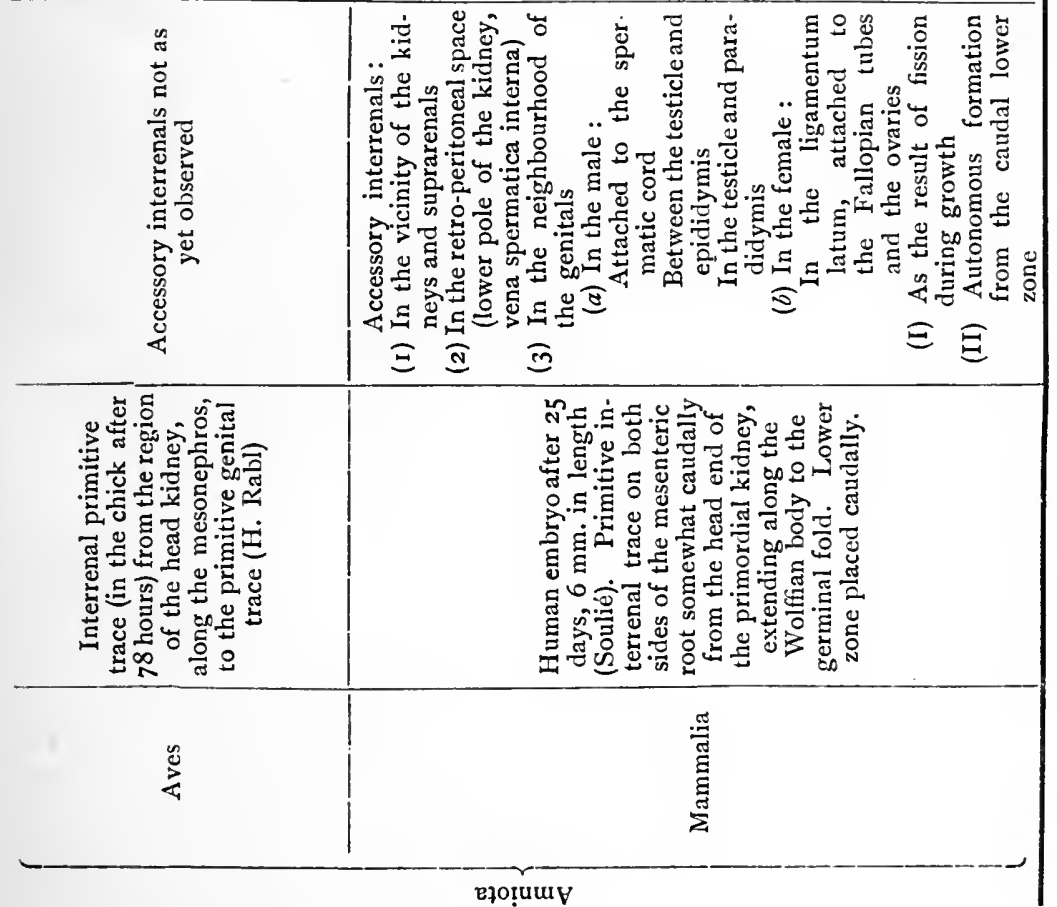


of the suprarenal function, was followed by a large number of experiments, undertaken with the object of discovering whether or not the suprarenals are essential to the life of the organism. The lack of a sound anatomical basis was, however, a great bar to physiological investigation. It was not until after nearly fifty years of experimental work of the most searching kind, that the conflicting evidence was reconciled and a satisfactory solution of the problem finally discovered.

A brief review of the evolution of the subject is not without interest.

As the result of his experiments with animals, Brown-Séquard came to the conclusion that the removal of both suprarenals, or even of one, as he originally believed, resulted in death within a very short time (average 34 hours), and that death in such cases was due to suppression of the suprarenal function. It was not long, however, before this view was disputed upon many sides.

It was pointed out by Gratiolet, Philipeaux, Berrutti and Perusino, and Harley that the death of the subjects might easily result from secondary affections, such as surgical shock; the lesion of numerous nerves and plexuses, the semilunar ganglion more particularly; hepatitis and peritonitis. But more important than this was the fact that numerous accounts were given by different authors (Martin-Magron, Berrutti and Perusino, Philipeaux, Harley, Chatelain, Schiff) of cases where animals, as mice, rabbits, cats, dogs, horses, and especially rats, lived without pathological signs for weeks and months after extirpation of both suprarenals.

With this negative solution of the problem of the vital importance of the suprarenals, general interest in the subject declined, and to such an extent that between 1863 and 1879 no publication dealing with the experimental side appeared.

Nothnagel's experiments in 1879 supplied a fresh stimulus to investigation. He, together with other scientists both of his own and of a somewhat later day (Foà, Burg, Russo-Giliberti and Di Mattei, Tizzoni, Alezais and Arnaud), endeavoured by means of lesions of the most varied description (crushing, cauterization, \&c.) to produce chronic inflammatory processes of the suprarenals, in the hope of obtaining experimentally the clinical complex of Addison's disease, as seen in man. These experiments were of little value in determining the vital importance of the suprarenals, for, in the large majority of cases, there is no proof that by the methods employed the whole of the suprarenal tissue was removed. It is much more probable, and the experimental evidence points directly to it, that where the animals lived for some time after operation, portions of suprarenal tissue had been left in situ, and that, as a result of regenerative processes, these remnants became enlarged later. This accounts also for the statement that animals will live for some time after sup- 
pression of both suprarenals, provided that they are destroyed separately, several weeks being allowed to elapse between the two operations.

From the results of his experiments with dogs and rabbits, 'Tizzoni came to the conclusion that the destruction of one or both suprarenals is followed by, death. He believed that death may occur soon after operation or that it may be postponed for weeks and even months, and that it occurs as the result of serious changes of the central and peripheral nervous systems. He describes farreaching disturbances of the nerve-fibres and ganglion-cells, accompanied by pronounced congestion, changes in the vessel walls, hæmorrhages, and leucocyte infiltration into all parts of the nervous system. Tizzoni's experiments do not prove that the suprarenals are essential to life, for his animals sometimes survived experiment for months and even years. Moreover, definite proof of a relationship between the operative procedure and the post-mortem findings would have to be forthcoming. Such proof is lacking; and, in view of the method of operation employed, by which total extirpation of the suprarenals has rarely been accomplished, Tizzoni's experiments seem rather to show that animals with even serious lesion of the suprarenals may live for a long time without pathological signs, provided that these organs are not entirely destroyed.

Tizzoni's statement that, whether one suprarenal or both were extirpated and, in the latter case, whether they were removed together or singly, the results were in all cases the same, is a proof of the unreliability of his conclusions. This statement was challenged by $\mathrm{H}$. Stilling in I89o. Stilling found that young rabbits, in which the left suprarenal had been destroyed, lived for several months to over a year without showing signs of disturbance.

Stilling was able to show that, after extirpation of the one suprarenal, the other became very much larger, and in fact showed compensatory hypertrophy. This is a discovery of the greatest importance in determining the functional significance of the suprarenals.

According to Stilling, the weight of both suprarenals in the rabbit is on an average o.I grm. to I,Ooo grm. of body weight, but after extirpation of the left gland he found that in six cases the right gland weighed 0.17 to $0.43 \mathrm{grm}$. per kilo of body weight. He also found that after the removal of both suprarenals, the remnants of tissue which it is almost impossible to avoid leaving upon the right side, may by proliferation of the undamaged cells, become as large as the normal suprarenal. Stilling, like Canalis, found accessory suprarenals in normal rabbits in two cases out of forty, and in every case where the principal organs had been removed; he makes the interesting statement that, after extirpation of one suprarenal, accessory suprarenals are 
very frequently found in the vicinity of the vena cava and vena suprarenalis. In his view, the suppression of the suprarenal function causes a strong proliferation of the isolated suprarenal bodies which, in the embryonal stage, split off from the main organs. This proliferation produces a compensatory hypertrophy which may end by completely replacing the suppressed suprarenal function.

Stilling's suggestive work contains the important statement that these newly-formed, accessory suprarenals possess a structure resembling that of the cortical substance, and that they contain no medullary tissue. Stilling, moreover, was the first to describe cells structurally similar to those of the suprarenal medulla. These cells stain brown with the chromium salts; and they are found, sometimes singly and sometimes formed into small bodies with a structure similar to that of the medulla, in the ganglia of the abdominal sympathetic and in the intercarotid ganglion. Stilling thinks that, owing to the presence of these cells, a proportion of medullary substance is present in animals from which both suprarenals have been removed. This, in combination with the fact that the cortical substance is always built up again by accessory suprarenals, explains the impossibility of completely destroying the suprarenals and of producing the clinical complex of Addison's disease experimentally in animals. The Addison's disease of human pathology is due to the destruction of the suparenals, and perhaps also of the structures by which the suprarenal function may be replaced. Enlargement of the accessory suprarenals and of the chromophile bodies would explain the occurrence of suprarenal derangement unaccompanied by Addisonian symptoms.

Stilling's work did not receive the recognition which it deserved. It appeared at a time when physiologists were first beginning to understand the importance of the blood glands. Brown-Séquard had introduced his theory of the internal secretions, and had expressed his conviction of the necessity of the suprarenals to the life of the organism. Stilling's discoveries seemed to contradict this view.

In Richet's physiological laboratory, a number of experiments were undertaken with the object of determining the essential character of the suprarenal function. Special attention was devoted to the technique of these experiments, and the removal of the suprarenals was effected according to the most approved rules of modern surgery.

Abelous and Langlois ( $189 \mathrm{I}$ ) next showed that, in frogs, the destruction of one suprarenal is not attended by results; but that the destruction of both glands invariably causes death, winter frogs living for twelve to thirteen days and summer frogs dying at the end of forty-eight hours. The animals also die after partial destruction of both suprarenals, unless at least a quarter of each 
organ is left intact. In such cases the animals live the same length of time as after one-sided extirpation.

Albanesi and Gourfein obtained similar results. The latter, however, was unable to confirm the difference in the duration of life in summer and winter frogs.

Experiments with tritons showed that one-sided suprarenal extirpation was borne without ill-effect, while the removal of both organs invariably caused death at the end of a few days. When, however, a portion of tissue the size of a pin's head was left in silu, the animals lived from eighteen days to nine weeks.

Abelous and Langlois obtained similar results from their experiments with guinea-pigs, and Langlois with experiments upon rabbits. Removal of one suprarenal was attended by transient emaciation only; removal of both was followed within quite a short time, nine to twelve hours, by death. The animals lived for a few hours longer if an interval of one to two weeks was allowed to elapse between the removal of the glands. Donetti found that guinea-pigs lived from fifteen to forty-eight hours.

Somewhat later (I897) Langlois published the results of a large number of experiments with dogs. He found that, after total extirpation, the maximal length of life was forty to fiftytwo hours; where an interval was allowed to elapse between the removal of the glands, the average length of life was twenty-eight hours, and still shorter where both glands were removed at once. There is some diversity of opinion as to the length of life in dogs after total suprarenal extirpation. De Domenicis (I893) gives it as two to four hours; Szymonowicz (1896) gives an average of fifteen hours; Thirololois (I893) gives twenty-five to forty hours; Kudinzew (1897) gives eighteen to twenty-four hours. Soddu (I899) and, more recently, Mariani (I906) describe experiments on dogs where the animals lived one to two days.

From the results of their experiments with dogs, Pal (I894) and Santi Rindome Lo Re (1895) disputed the essential character of the suprarenal function in the life of the organism. Pal found that of eight dogs from which the suprarenals had both been removed, three lived for two to three days; two lived for six days; and one lived for four months and eight days. He was unable to discover in this animal either remnants of suprarenal tissue or, at least within the area of operation, accessory suprarenal bodies.

One of Santi Rindone's dogs lived for thirty-six days after total extirpation.

Numerous reliable extirpation experiments upon dogs, cats, and rabbits are described by E. O. Hultgren and O. A. Andersson (I 898 ).

They found that extirpation of one suprarenal together with partial destruction of the other, was followed by a passing emaciation, but that the animals did not die. The removal of both suprarenals at the same time was followed, in cats, by death 1.: $1 \ldots$ 
after an average of sixty-eight hours; where the suprarenals were removed in two sittings, death followed after 134 hours; and where by three, in eighty-eight hours. In rabbits, after removal of both organs at the same time, death followed in five to six days; where, however, the organs were removed separately, with a long interval between the operations, the animals remained alive for months. These authors also state the remarkable fact that previous castration doubles the length of life of such animals. According to Krychtopenko (1906), rabbits frequently survive the total extirpation of their suprarenals.

From the results of their very careful experiments, H. Strehl and O. Weiss (Igor) concluded that all animals, from which both suprarenals have been removed, die. The following table shows the results of their experiments :

\begin{tabular}{|c|c|c|c|c|c|c|c|c|}
\hline \multicolumn{3}{|l|}{ Species } & \multicolumn{4}{|c|}{$\begin{array}{l}\text { Length of Life after } \\
\text { Operation ; in hours }\end{array}$} & \multicolumn{2}{|r|}{$\begin{array}{l}\text { Number } \\
\text { of Animals }\end{array}$} \\
\hline Dogs & $\ldots$ & $\ldots$ & $\ldots$ & $\ldots$ & $22-75$ & $\ldots$ & $\ldots$ & 7 \\
\hline Dogs & $\cdots$ & $\ldots$ & $\ldots$ & $\ldots$ & $75-138$ & $\ldots$ & $\ldots$ & 3 \\
\hline Cats & $\ldots$ & $\ldots$ & $\ldots$ & $\ldots$ & I $5-28$ & $\ldots$ & $\ldots$ & 15 \\
\hline Cats & $\cdots$ & $\ldots$ & $\cdots$ & $\ldots$ & $28-47$ & $\ldots$ & $\ldots$ & 2 \\
\hline Rabbits & $\ldots$ & $\cdots$ & $\cdots$ & $\ldots$ & S-I4 & $\ldots$ & $\cdots$ & 26 \\
\hline Guinea-pigs & & $\ldots$ & $\ldots$ & $\cdots$ & $4-9$ & $\cdots$ & $\ldots$ & 20 \\
\hline Rats & 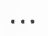 & $\ldots$ & $\ldots$ & $\ldots$ & $15-19$ & $\ldots$ & $\ldots$ & 4 \\
\hline Mice & $\cdots$ & $\cdots$ & ... & $\ldots$ & $8-13$ & $\ldots$ & $\cdots$ & 10 \\
\hline Hedgehog & & $\ldots$ & $\ldots$ & $\ldots$ & 14 & $\ldots$ & $\ldots$ & I \\
\hline Weasel & $\ldots$ & $\ldots$ & $\cdots$ & $\ldots$ & $2 \mathrm{I}$ & $\cdots$ & $\ldots$ & I \\
\hline Frogs & $\ldots$ & $\cdots$ & $\ldots$ & $\ldots$ & $22-45$ & $\ldots$ & $\ldots$ & 25 \\
\hline
\end{tabular}

One cat lived for a week after operation without showing signs of pathological disturbance. After death, a suprarenal of about the size of a pea was found attached to the spermatic vein. The other animals died in every case, and the postmortem finding was negative.

Where the suprarenals were removed separately, with an interval of a month between the operations, the animals lived longer. Thus, four dogs lived Iog to 2 I 4 hours; five cats 30 to I 70 hours; nine rabbits 2 I to 76 hours; twenty-one frogs from 30 to 66 hours. In every case the suprarenal which was removed last was enlarged.

My own method of extirpation, and one which I have practised on numerous dogs, cats, and rabbits, differs from those of the earlier investigators in certain important particulars. In each case, I first performed a lumbar section by which I was enabled to dislodge the suprarenals in a dorsal direction. Leaving the glands attached by means of the blood-vessels, I sewed them in between the cuticle and the muscular structure of the back. In this way they remained alive, and were readily accessible without the necessity of opening the peritoneum. Three or four days later, they were exposed by cutting through the cuticle; the vessels were ligatured, and the organs removed in the easiest 
manner possible. Many animals bore the extirpation of one gland without any sign of derangement, and lived for months afterwards. After the removal of both glands death invariably followed within two to four days. In two rabbits which lived respectively sixteen and twenty-eight days, there were accessory suprarenals of about the size of a pea, situated close to the vena cava, below the renal veins.

These experiments undoubtedly prove that the suprarenals are essential to the life of the organism. For not only was the extirpation of the dislodged organs followed shortly by death, but the operation was performed in such a manner that there were no secondary lesions, and there was no danger of infection.

The results of experimental extirpation of the suprarenals in rats and guinea-pigs require to be discussed separately. It must be remembered that, in the first accounts which were given of animals which survived the extirpation of both suprarenals, the subjects of the experiments were rats (Harley).

In a large number of his publications, Boinet lays particular stress upon the fact that grey rats will live for one to six months after extirpation of both suprarenals, whether these are removed separately or simultaneously, though many animals die a few days after operation. Abelous and Langlois explain this peculiar state of things by the fact that, in ten rats out of eleven, two to three accessory suprarenals are present in the neighbourhood of the principal organs and the hinder surface of the renal vein. Boinet next tried the effect of removing these accessory structures as well as the principal organs; he found that, out of twelve animals, seven survived the operation, and of these some lived for several months. He concluded from this that the vicarious function of these accessory organs is generally overestimated, and he emphasizes the fact that these accessory suprarenals contained no medullary substance.

Wiesel found that accessory suprarenals without medullary substance, or, as they would now be termed, accessory interrenals, were present in about 50 per cent. of the rats which he examined. They were situated between the testicles and the lower pole of the epididymis, and he found that, after removal of the suprarenals, these structures undergo compensatory hypertrophy. We shall again refer to the significance of this finding in the light of the remarkable frequency with which rats survive double extirpation of the suprarenals. It must, however, be pointed out that, contrary to the opinions of $\mathrm{H}$. and $\mathrm{A}$. Christiani-who found that, after total extirpation of both suprarenals, rats died in ten to twenty hours (out of twenty-nine animals only three survived)-white rats, especially male animals which have attained sexual maturity, in the greater number of cases, bear the removal of their suprarenals without evil results. It is evident from this that results obtained from experiments with rats 
do not furnish conclusive evidence as to whether or no the suprarenals are essential to the life of the animal organism.

A peculiar interest is attaching to the guinea-pig as the subject of experimental epinephrectomy, owing to the fact thdi there is no record of any instance where guinea-pigs survived the removal of both suprarenals; moreover, it is generally believed that accessory suprarenals are never present in these animals. A. Velich has devoted special attention to the study of the guinea-pig, and he found that, out of roo animals over the age of six months, only five possessed accessory suprarenals. In each case the structure was extremely small, about $\mathrm{I} .5 \mathrm{~mm}$. in diameter, and was placed, in four cases upon the right and in one case upon the left, of the vena cava, at the spot where the renal vein opens into it. He also found that, in young guinea-pigs, after extirpation of one suprarenal there was not only a compensatory hypertrophy of the other together with partial regeneration of the tissue left behind from the extirpated organ, but that accessory suprarenals, $2-3 \mathrm{~mm}$. in diameter, were invariably formed at the right of the vena cava, and that these new structures were entirely composed of cortical tissue. The further development of the minute accessory suprarenals - which frequently consist only of isolated cortical elements-after the partial extirpation of the principal organs, shows that even these structures are capable of compensatory activity. They are not able, however, entirely to replace the complete suppression of the principal organ, and for this reason total extirpation of both suprarenals of guinea-pigs invariably results in death.

Experiments have been undertaken quite recently which aim at the destruction of the suprarenals by means of specific cytotoxins. Several investigators (Bigart and Bernard, Abbot, Yates, Sartirana, Gildersleeve, Van Calcar) have endeavoured to produce an epinephrotoxin or suprarenolysin, but their attempts have been principally confined to a description of the pathological changes which take place in the suprarenals, and little has been added to our linowledge of the function of these organs. A. Bogomolez recently obtained a suprarenolytic serum from rabbits with the suprarenals of dogs, and from dogs with the suprarenals of cats; he found that the injection of this serum was followed by a marked increase in blood-pressure, a stronger cardiac impulse, an acceleration of the pulse and of the respiration, and later, by a period of prostration, which was, however, transient. He regards these symptoms as the result of the increased activity of the suprarenals and their consequent exhaustion, and of the medullary substance more particularly. Histological examination showed profound changes in the suprarenals of animals which had been treated with injections of suprarenolytic serum. The protoplasm of the cells of the medulla had become markedly spongy and vacuolized, and was, in parts, entirely dissolved. Amorphous deposits of basophile substance, which the author regards as 
the products of secretion, were not present in the medullary cells. With the exception of moderate enlargement of the vessels, the cortical substance presented no signs except those of an increased secretory activity.

L. della Vida employed immune serums obtained separately from the cortex and medulla of the suprarenals of guinea-pigs. $\mathrm{He}$ states that the action of the medulla-serum was toxic to his animals and produced hyperæmia of all the organs, together with degenerative changes in the suprarenals, kidneys, and liver. It is more than probable that the cytotoxic sera employed by della Vida were strictly specific in vitro, but that at the same time they were more or less hæmolytic in their activity; this renders it difficult to distinguish the toxic effects of the hæmolysis from any specific disturbances which may have taken place in the suprarenal tissue.

From the results which have been obtained by extirpation of the suprarenals the following conclusions may be drawn :-

(I) From the fact that it is followed by no pathological signs, the removal of one suprarenal must be regarded as negative in its effect upon the organism. The compensatory hypertrophy which invariably takes place in the remaining organ and in any accessory organs which may be present, shows that this negative effect is not due to an absence of functional significance, but to the fact that the suppressed function is replaced by the vicarious activity of the remaining organ and of other similar tissues. This hyperfunction is expressed anatomically by hypertrophy.

(2) Extirpation of both suprarenals is as a rule followed by the death of the animal, whatever its species, within a very short space of time (hours or a few days). That this is not due to the severity of the operation or to secondary causes, such as infection, \&c., but occurs solely as a result of the suppression of the function of the organs, is shown by the constancy of the result; for death follows even in those cases where, the organs having been previously dislodged, the operation is a slight one. It is evident then, that the suprarenals are not only important to life, but that they are essential to it.

(3) Whether the suprarenals are removed together or singly, with a longer or shorter interval between the operations, the final result of complete epinephrectomy will be the same. But where both the organs are removed at once, the duration of life is much shorter, and it would appear from this that the sudden suppression of the suprarenal function is not compatible with a continuation of life. That the duration of life is longer where the suprarenals are removed separately is explained by the fact that, in the interval, any accessory suprarenal tissue which may be present undergoes compensatory hypertrophy and, after removal of the second suprarenal, does what it can to replace the suppressed function, though necessarily to an insufficient extent. 
(4) In certain exceptional cases, where animals have survived the extirpation of both suprarenals and where the result is not due to imperfect extirpation, it arises from special anatomical conditions. In some animal species, as the dog and cat, such exceptional cases are extremely rare; in others, as in the rabbit, they are of more frequent occurrence; in rats, they are rather frequent, and extremely rare in the case of guinea-pigs. In these cases the survival of the animals is doubtless connected with the presence of accessory organs.

These structures are very rare in dogs and cats; they occur in about 15 to 20 per cent. of rabbits; in nearly 50 per cent. of rates; in guinea-pigs, at the most, 4 per cent.

(5) Partial destruction of the suprarenals, either by the removal of one and large portions of the other, or by partial destruction of equal portions of both, may be borne without results, or it may cause death. Survival depends, in the first instance, upon the amount of tissue destroyed.

Repeated experiments have been made with the object of discovering the amount of suprarenal substance necessary to life. Mathematically precise results were hardly to be expected. Langlois found that, in the case of rabbits and dogs, one-sixth to one-eleventh of the total weight was sufficient. I myself found that cats, rabbits, and dogs always survived if, after the removal of one suprarenal, one-quarter of the second was left in situthat is to say, one-eighth of the whole amount of suprarenal substance. In isolated cases even smaller quantities sufficed. $\mathrm{H}$. and A. Christiani found that, in the case of rats, the extirpation of one suprarenal together with half of the other, was borne without illeffects. If more than half of the second suprarenal was removed, they found that some animals survived, while others died. These authors are of the opinion that a very small quantity, less than one-quarter of the total weight, of suprarenal tissue may suffice to support life.

\section{THE RESULTS OF THE EXTIRPATION OF THE CORTICAL AND MEDULLARY SUBSTANCES.}

I found in the course of my experiments that partial suprarenal extirpation was followed by certain phenomena, which led me to the conclusion that the amount of tissue left in situ is not the only factor of importance, but that the part of the organ from which the remnant is derived is also of considerable significance. Experiments undertaken with the object of determining whether or not the suprarenals as homogeneous organs are necessary to animal life, served also to determine the significance of the different parts and the vital importance of the interrenal and adrenal systems.

The question as to whether it was the cortex or the medulla 
which rendered the suprarenals necessary to life, seemed at first to have already received an answer. The specific physiological effects of suprarenal extract, to which we shall refer later, were known. It was also known, or at any rate suspected, that the active principle of the extract was derived from the medullary substance, and that this essence passes intra vitam into the blood-stream by way of the suprarenal veins.

In view of the known physiological activity of the medullary substance, the conclusion seemed obvious that animals from which both suprarenals had been removed, died from the lack of an essence, elaborated by the suprarenal medulla, which is necessary to the performance of a large number of bodily functions. According to Cybulski, this essence maintains in a state of normal tonus, the activity of those nervous centres which control the cardiac and vascular muscles, the respiratory musculature, and perhaps the muscular tissue of the entire body. After removal of the suprarenals the animal dies, because, in the absence of perpetual stimulus, these nerve centres, the activity of which is essential to the life of the organism, cease to act. The evidence in support of this theory is that, in dying animals from which the suprarenals have been removed, the bloodpressure is extremely low, while interference with respiration, as by occlusion of the trachea, does not produce either a dyspnœic increase in blood-pressure nor dyspnœic cramps. The injection of suprarenal extract is followed by an increase in blood-pressure accompanied by a certain improvement in vitality, and the dyspnœic blood produces the usual symptoms of suffocation.

These experiments have no great value as evidence, especially when we consider that, in all moribund animals, there is a low arterial blood-pressure and a slight reaction to dyspnœic stimuli, and that the injection of suprarenal extract into moribund animals is always followed by a rise in blood-pressure and a certain return of vitality.

What seems to be more reliable proof of the part played by the medullary substance in death from suprarenal suppression, is the fact that extirpation of both suprarenals is immediately followed by a considerable reduction in arterial tension. Strehl and Weiss attribute this fall in blood-pressure to the cessation of the supply of the substance which stimulates blood-pressure. Their views are founded upon the results of the following experiment with rabbits.

If, after removal of one suprarenal, the vein leading from the other is severed or ligatured, in certain cases, "though in a small minority only," there will be a marked fall in blood-pressure. If the ligature is released, the blood-pressure will rise to the normal. Young and Lehmann performed a similar experiment upon dogs; they found that the ligature of both suprarenals gradually produced a slight reduction in arterial pressure, and 
that the loosening of the ligatures was followed, in from ten to thirty minutes, in three out of eight cases, by a distinct rise in blood-pressure.

The results of Strehl and Weiss's experiment, though interesting, have in my opinion been very much overrated. The experiment really only shows that the specific substance elaborated by the suprarenal is perpetually conveyed into the blood-stream by the blood from the suprarenals. If, after complete extirpation of the suprarenals, death is due to the absence of this substance, or rather to the reduction in blood-pressure caused by its absence, we should expect the operation to be immediately followed by a somatic sinking of the animal, accompanied by a continued fall in blood-pressure. The fall in blood-pressure described by Strehl and Weiss was, however, only transient, the authors describing it as lasting several seconds. Lewandowski found that thirty minutes after extirpation of both suprarenals - the blood-pressure was normal.

I myself found that, after removal of the suprarenals from the extraperitoneal situation in which I had placed them, there was a fall in blood-pressure lasting fifteen to thirty minutes, except in cases when the animal was much affected by the operation and the anzsthetic. At the end of that time the bloodpressure gradually rose and, by the next day, had reached the normal. Where the anæsthesia is more profound and the suprarenals are removed by laparotomy, the blood-pressure becomes very much reduced and in some instances there is no recovery. As a general rule, however, where the method of operation is technically above reproach, animals from which both suprarenals have been removed usually appear to be perfectly well, with normal blood-pressure for two or three days, after which they die (Hultgren and Andersson). This applies more particularly to cases where the suprarenals have previously been dislodged, the ultimate removal taking place extraperitoneally. A few hours before death there is a distinct gradually increasing fall in blood-pressure, though the same phenomenon is observed in all moribund animals. As neither ligature of the veins nor removal of the suprarenals is followed by an appreciable decrease in blood-pressure, there is no actual proof that death is due solely to the suppression of the agent which stimulates bloodpressure. The cause of death after complete extirpation of the suprarenals is, at present, unknown.

The assumption that death after total suprarenal extirpation may, in some measure, be due to intervention of the cortical portion-or, in other words, that the interrenal tissue is essential to life-is justified upon various grounds.

It has been repeatedly proved that the accessory organs which, after removal of the suprarenals, suffice to maintain life, are entirely composed of cortical tissue, or, in the terminology 
of the present day, belong exclusively to the interrenal system. There is also undoubted proof that, after extirpation of the suprarenals, this tissue undergoes compensatory hypertrophy. These are the two points upon which $\mathrm{l}$ base my assumption that the interrenal tissue has a vital significance in the economy of the organism.

In the course of my investigations into the question of the amount of suprarenal tissue necessary to the maintenance of existence, I found that mammals (rabbits and dogs) would live with one-eighth, or less, of their suprarenal substance, provided that the portion left behind was composed of cortical tissue. Further experiment showed that, after removal of one suprarenal in toto together with the medullary substance of the other, more or less of the cortex being left in situ, the animal not only continued to live, but showed no signs of pathological disturbance. I found in one of five surviving rabbits two small accessory interrenal structures situated to the right of the vena cava. In a number of other instances, the destruction of the medullary portion, in so far as this was possible by operative means, was entirely negative in its results; but I have never succeeded in keeping animals alive after removal of the cortex and preservation of the medulla, no matter how carefully the operation was carried out.

These results are not, however, confirmed by those of Vassale and Zanfrognini (1902). They found that the complete destruction of the medulla was followed, in cats and rabbits, by death in exactly the same way as total extirpation of both glands; while, after partial destruction of the medulla, the animals lived for three weeks and then died, showing signs of pronounced cachexia. This fatal termination was ascribed by these authors to the absence of the medullary tissue, while in those cases where the animals survived, they attribute the result to the presence of extracapsular chromaffine bodies.

H. and A. Christiani concluded from their experiments with rats, that in the case of these animals very minute portions of suprarenal tissue suffice to support life, always provided that the remnant contains a sufficiency of sound medullary tissue. Larger portions, if composed wholly of cortical substance, will not prevent a fatal termination.

Without a knowledge of the method of operation, it is very difficult to judge of the value of the results obtained by Vassale and Zanfrognini. The experiments of $\mathrm{H}$. and A. Christiani, though apparently free from objection, are of little value in the present connection, owing to the fact that accessory interrenal structures are frequently present, especially in the testicles, of the species which they employed as subjects. Rats always possess a large amount of interrenal tissue and in some recent experiments I found that these animals, especially adult males, 
very frequently survive the removal of both suprarenals. This shows that the remnants of tissue left in situ by Christiani have no appreciable functional significance. A. Kohn, who emphasizes the physiological importance of the chromaffine tissue and lays special stress upon its importance to life, is inclined to doubt the value of the last-mentioned experiments. He says: "The complete destruction of the medullary substance is supposed to cause death. But in many mammals, such as cats and rabbits, a considerable quantity of chromaffine tissue lies externally of the suprarenals upon the ventral surface of the abdominal aorta. Why is it that these structures do not prevent death, if a small portion of medullary tissue when left in situ is able to do so?" According to Kohn, it is even more difficult to reconcile this view with the results of experiments on amphibia. "In the amphibia, the amount of extracapsular chromaffine tissue in the sympathetic is considerably in excess of the medullary substance, and yet after complete destruction of the suprarenals, frogs and newts (tritons) invariably die. How is it that the large amount of extracapsular chromaffine tissue which remains after operation, does not prevent the death of these animals, if a minute proportion of suprarenal tissue, which may not even contain chromaffine cells, is able to maintain life for many weeks?" My answer to this question is: That the animals will survive without medullary tissue, for, in the free portions of the adrenal system they possess a sufficiency of extracapsular chromaffine tissue. If it were possible to extirpate all the free portions of the adrenal system, which is, of course, technically impracticable, then and then only should we be justified in referring the death of the animal to suppression of the function of this system.

The specific activity and physiological significance of the adrenal system, together with that of its subdivision, the suprarenal medulla, cannot, in face of the evidence, be doubted. That it is important and even essential to the life of the organism we know, but the proof of this is not supplied by the results of experimental extirpation. These experiments tend rather to show that the cortex, or more correctly the interrenal tissue, is necessary to the life of the animal.

\section{EXPERIMENTAL EXTIRPATION WITH FISH.}

During my stay at the zoological station at Naples, in I899, I undertook a series of experiments with the object of determining the vital importance of the interrenal tissue in fish. The most favourable conditions appeared to me to be provided by the topographically separate interrenal and adrenal organs of the Selachii. I was compelled at the outset, however, to abandon all attempts at extirpation of the adrenal system on account of its intimate association with the cardinal veins. Even the first pair of large 
adrenal bodies, formerly called the " axillary heart," cannot be removed. Conditions relatively more favourable for extirpation are offered by the interrenal system.

The interrenal body of sharks (Squalidæ) is a long narrow band, situated between the kidneys and partly covered by them; it is continued towards the cranium as a detached band, or in separated dot-like bodies. The interrenal body is exposed by raising the kidneys from the dorsal abdominal wall. In some species of Squalidæ, the interrenal body is embedded in the substance of the kidney and is only visible in preparations. I employed small specimens of Scyllium cattulus and Scyllium canicula as subjects for my investigations, and in twelve cases I succeeded in removing the interrenal body. Of these, one lived for eight and one for ten days, while the remaining animals lived for three weeks, after which they were destroyed. Anatomical examination of the first two animals showed an entire absence of interrenal tissue. In all the other cases, post-mortem examination showed remnants of interrenal tissue of a varying size, which upon histological examination were found to be hypertrophied.

Experiments with Raiidæ (skates and rays) were more instructive, and the anatomical conditions were decidedly more favourable. In most Raiidæ, round or oval paired bodies are found to right and left of the middle line between the kidneys; occasionally they are united by a bridge of varying width. In the species which I employed (Torpedo marmorata, T. occellata, Raia batis, $R$. punctata, $R$. clavata), there is generally a largish interrenal body, situated either to right or left, generally at the caudal point of union of the kidneys; while upon the other side, more in a cranial direction and at the medial border of the kidneys, a row of smaller bodies is sometimes found. For anyone who is acquainted with the anatomical conditions it is possible to remove the whole of the interrenal tissue; care must be taken, however, to avoid any isolated interrenal bodies which may be present.

In twenty-seven cases out of thirty-two, post-mortem examination revealed no trace of interrenal tissue; in one case there was a portion of about the size of a grain of millet seed, and in four cases portions as large as a pea. Animals which died within forty-eight hours were excluded, for these cases always showed changes which sufficiently explained the cause of death. Apart from violent hæmorrhage and destruction of the renal tissue, the severing of the abdominal sutures and consequent prolapse of the viscera was the most frequent cause of death. In successful cases, for the first three to four days after operation, there was no appreciable change in the condition of the animals, the first symptoms making their appearance at about the seventh or eighth day. The animals first showed a loss of liveliness and readiness to move about; spontaneous swimming about the aquarium became less frequent; and, compared with control animals in the same 
aquarium, they were much paler in colour; fourteen to eighteen days after operation they had become so weak muscularly that they could scarcely be induced to swim at all; their colour was very pale; and they lay all day in a corner of the aquarium, refusing all food. Their reaction to stimuli almost entirely disappeared, and at the latest, three weeks after operation they died with all the symptoms of general prostration. Post-mortem examination revealed no definite cause of death; the stomach was invariably empty and the blood pale in colour.

Of the five fish in which portions of interrenal tissue were accidentally left behind, one lived for over four weeks without symptoms of muscular weakness. It was finally destroyed, and was found to possess an interrenal body the size of a pea. Both in this case and the other four, all of which showed symptoms of suppression of the interrenal function, there was distinct histological proof of hypertrophy of the interrenal tissue left in situ.

In my opinion, these experiments incontestably prove that the complete destruction of the interrenal tissue is incompatible with the continuation of life; and that death occurs under conditions similar to those which follow extirpation of the suprarenals.

The results of my experiments with cartilaginous fish appear, however, remarkably antagonistic to those obtained by Pettit and by S. Vincent with osseous fish. Both these investigators found that eels lived for months after extirpation of the suprarenals and showed no change in their general condition. They considered that the suprarenals of osseous fish were represented by the corpuscles of Stannius, situated upon the ventral or dorsal surface of the kidneys and composed exclusively of interrenal tissue. These experiments with eels appeared to contradict the results which I obtained with Selachii, and the discrepancy seemed to me the more significant in view of the careful methods adopted by both Pettit and Vincent and the remarkable unanimity of their results. I was unable to detect the slightest inaccuracy either in their methods or observations, and for this reason it seemed that a repetition of their experiments was hardly likely to yield fresh results. For some time, I was unable to find an explanation of this difference in the behaviour of Teleostii and Selachii, but Giaconimi's recent anatomical work has explained the mystery in the most satisfactory manner. Giacomini shows that in the eel, in addition to Stannius's body and the interrenal tissue distributed in its vicinity, which he calls the posterior or caudal interrenal organ, an anterior cranial interrenal system is also present, composed of isolated bodies situated at the anterior edge of the head kidney and attached to both the anterior and posterior cardinal veins. The wide distribution of this anterior interrenal system, together with the large amount of interrenal tissue present, makes it very certain that the removal of Stannius's corpuscles 
alone supplies no evidence as to the essential character of the interrenal tissue in the economy of the organism. The large amount of tissue remaining in the system explains why eels invariably survive the removal of their suprarenals.*

\section{SYMPTOMATOLOGY OF SUPPRESSION OF THE SUPRARENAL FUNCTION.}

The symptoms which, in different animal species, follow the removal of the suprarenals, have been variously described. This is chiefly due to the fact that the duration of life after operation is so short, that it is difficult to determine which symptoms arise from suppression of the organ, and which are complications resulting from the operative intervention. The clinical complex presented by suprarenal suppression is by no means uniform, and is, moreover, so ill-defined in character that its classification as a clinical entity is impossible. Where operation is immediately followed by pronounced muscular weakness, extreme nervous depression which may amount to a paralytic condition, reduced cardiac and respiratory activity, and a lowered temperature, the idea which inevitably presents itself is that we have to do with the after-effects of serious surgical disturbance.

If, however, the suprarenals are removed by less drastic surgical means, the operation is not followed by any of the above symptoms, the animals appearing quite normal a few hours after their recovery from the anæsthetic. Where the suprarenals have been previously dislodged, they may be removed from their position under the skin of the back without an anæsthetic, and the animals will remain free from symptoms for one to two days. Upon the second day in some cases, though usually not until the third, there is loss of appetite. About twenty-four to forty-eight hours before death, which occurs four to six days after operation, the animal becomes spiritless and wears a pathetic look; it ceases to move about and refuses all food. The apathy and muscular weakness increase and there is considerable stiffness and uncertainty of movement of the hind legs. Extreme prostration follows, the animal becoming too weak to move; it has a flaccid paresis of the hind legs and lies in a peculiar position, flat on the abdomen with the extremities extended. At this stage the temperature is very low, respiration becomes increasingly difficult and laboured, the heart's action is irregular and weak, and the animal soon dies. Immediately before dissolution, single muscular contractions are sometimes observed and, though more rarely, convulsions.

* In a recent publication (Ascher-Spiro, Ergebnisse der Physiologie, gth year, 1910, p. 537) S. Vincent compares his experiments with eels with mine with Selachii, and he concludes that the cortex of the suprarenal is not essential to the life of the organism. It is evident from this that Giacomini's anatomical work ( 1908 ) has not come to Vincent's notice. 
Let us take these symptoms singly and examine them more closely.

The initial symptom, a reduction of $2-4^{\circ}$ in temperature immediately after operation, has very little clinical significance.

This fall in temperature is quite transient, the figure becoming again normal twenty-four hours after operation. Personally, I have never observed the initial fall in animals operated upon by my method. In cases, however, where the results of operation are complicated by hæmorrhage, prolonged narcosis, \&c., the initial fall in temperature may be continued and progressive. As a general rule the temperature begins to drop forty-eight hours before death, after the asthenic symptoms have appeared. From then onwards the reduction is progressive. The normal rectal temperature of $39^{\circ} \mathrm{C}$. drops to $34^{\circ} \mathrm{C}$., and immediately before death to $30^{\circ} \mathrm{C}$. and under. Hultgren and Andersson regarded this abrupt fall in the temperature curve before death as chraracteristic of animals from which the suprarenals had been removed.

The emaciation which follows removal of the suprarenals may, with greater justification, be regarded as characteristic of the condition. There is a considerable reduction in weight immediately after operation, and thus reduction is slowly progressive. The loss is undoubtedly partly due to the lack of appetite, the animal in the later stages refusing all food. That a relationship exists between the emaciation and the suppression of the suprarenal function is proved by the fact that the removal of the suprarenal from one side only is followed by a considerable loss of weight. This lasts from two to three weeks, when an increase in weight takes place. It is an interesting fact that intestinal derangements and diarrhoea are not usually associated with the condition.

Investigation of the metabolic conditions and systematic examination of the urine of animals from which the suprarenals have been removed, has rarely been undertaken. According to Alezais and Arnaud, the amount of phosphates in the urine of rabbits is increased after extirpation of the suprarenals. Nothnagel considers that the indican reaction, sometimes afterwards observed in the urine of rabbits, is due to secondary conditions. Many authors report polyuria.

Investigation of the metabolism of albumin was first carried out with rabbits and cats after both single and double suprarenal extirpation by Hultgren and Andersson. They found that there was no change in the metabolism of albumin. Where the animals were able to take nourishment, food containing a large proportion of carbohydrates possessed the same property of storing up albumin as in normal animals. Where the animals refused to take food, the decomposition of albumin was the same as in the normal fasting animal.

Interesting information concerning the metabolism of the carbohydrates in suprarenalless animals has recently been pub- 
lished. Bierry and Malloisel (1908) describe hypoglycæmia in dogs. O. Porges found that, a few hours after double suprarenal extirpation, the sugar contents of the blood are subnormal. He also found that, in three cases of Addison's disease in man, the sugar contents of the blood were below the average. Frank and Isaak, who experimented with rabbits, deny that there is a connection between hypoglycæmia and suprarenal suppression, but their experiments cannot be regarded as conclusive.

Eppinger, Falta and Rudinger showed the phlorizin produces only slight glycosuria in suprarenalless dogs. They also found that, in certain cases of Addison's disease, the toleration of sugar is remarkably high; while L. Pollak was unable to produce glycosuria with $2 \mathrm{mg}$. adrenalin in a case of Addison's disease. Similar doses when exhibited in normal people invariably produced glycosuria.

André Meyer describes his failure to obtain results by means of adrenalin injections; his results are confirmed by those of Kahn.

It appears from the foregoing that the regulation of the sugar contents of the blood is a function of the suprarenal, or rather of the adrenalin-secreting, tissue.

In a communication dated December 17, 1909, O. Schwarz describes certain appearances which follow extirpation of both suprarenals. He found that in sexually mature male rats, which it is well known live without any symptom of disease for long periods after removal of both suprarenals, phlorizin exercised its full glycosuric action; but that it produced toxic effects which, under certain conditions, were counteracted by the exhibition of adrenalin. Rats deprived of both suprarenals also showed a marked reduction in the amount of glycogen, an increased decomposition of albumin, and, in spite of these factors, an increase in weight.

These findings of Schwarz have a peculiar interest, but in my opinion they represent the results, not of suprarenal suppression, but of the suppression of a portion of the adrenal system. It is probable that many symptoms should properly be ascribed to a decreased function of the interrenal system; this applies particularly to the remarkable change in the behaviour of rats, wild and ferocious animals frequently becoming tame and quiet after operation. Schwarz thinks that, owing to the frequency with which accessory interrenals occur in white rats, these animals are not suitable subjects in which to study the functions of the suprarenal as a whole. Rats which have been deprived of their suprarenals are not in reality suprarenalless, they merely possess a more limited adrenal system than normal animals. Moreover, they cannot be regarded as suffering from the absence of adrenalin, but-and perhaps only temporarily-merely from scantiness of it. We know from the experiments of v. Haberer and Stoerk that adrenal tissue may undergo a very high degree of hypertrophy. 
In connection with these findings of Schwarz's, O. Porges reports that he found a diminution in the quantity of glycogen in dogs without suprarenals.

These results, when taken in conjunction with observations to be described later of the glycosuric activity of adrenalin, point to the fact that the internal secretion of the adrenal system affects the mobilization of the sugar in the blood as well as the new formation of glycogen in the economy.

The most conspicuous symptoms of suprarenal suppression are undoubtedly the muscular weakness, asthenia, and apathy, which increase to complete paralysis shortly before death. The extreme indolence of the muscular movements is very conspicuous in frogs; it makes its appearance upon the second day after operation and in the case of the hinder extremities passes ultimately into paralysis. Abelous and Langlois compare the condition with the effects of curare poisoning, for they found that the sensibility of the muscles to indirect electric stimulus by way of the nerves disappeared shortly before death, while the sensibility to direct stimulus remained. These results were not confirmed however by later investigators (Gourfein, Hultgren and Andersson, Biedl) with either frogs or warm-blooded animals. It is very important to remember that the clinical picture presented by suprarenal suppression bears not the slightest resemblance to that of curare poisoning, either at the beginning, when the condition is characterized by extreme muscular weakness and prostration, or later, when total paralysis has made its appearance. On the other hand, a liability to fatigue, first pointed out by Albanese, and an extreme sensitiveness to violent muscular exertion, are undoubted characteristics of all animals with suprarenal inadequacy. To compel such animals to extreme muscular effort is to greatly shorten their lease of life, and, in many cases, the animal falls dead while making the muscular effort.

The blood of animals which have lost their suprarenals is said to possess a peculiar toxicity. According to Brown-Séquard, the blood of such animals shortens the life of animals of the same species which have likewise lost their suprarenals, while the life of the latter is prolonged by the blood of normal animals. Abelous and Langlois found that symptoms of exhaustion were produced in frogs, guinea-pigs and dogs, and, in the case of epinephrectomized animals, death was hastened, not by the blood only of animals which had lost their suprarenals, but also by alcoholic extracts of their muscles, especially of tetanized muscles; similar effects were produced by extracts of tetanized muscles of normal animals. The intravenous injection of these extracts into healthy animals produced transient symptoms of poisoning. These findings are confirmed by Boinet and Mariani. Boinet also found that the viscera of epinephrectomized animals was markedly toxic, while Mariani found that there was an increased toxicity of the urine. 
Mariani affirms that the increase in blood-pressure, which is induced by the exhibition of suprarenal extract, is immediately abolished by the blood of epinephrectomized animals. According to Strehl and Weiss, there are certain data which point to the conclusion that the toxic action of the blood of suprarenalless animals may be counteracted by means of suprarenal extract. These findings, together with those of Soddu concerning the favourable effects of blood-letting and saline infusion upon suprarenalless animals, would seem to show that the peculiar prostration of such animals and. their eventual death is due to an accumulation, more particularly in the muscular structure, of the harmful products of metabolism. These results supply the foundation for the theory that it is the function of the suprarenals to render innocuous certain toxic products of metabolism, the chemical nature of which is as yet unknown. According to Charrin and Langlois, the suprarenals exercise this neutralizing activity upon other toxins also, such as nicotine and the bacteria toxins. This is a point, however, which we shall have occasion to discuss more fully later.

To return to the signs of suprarenal suppression; there still remain the conditions governing the morphological elements of the blood, and the question as to the deposition of pigment after suprarenal extirpation.

Brown-Séquard's original view was that death followed epinephrectomy as the result of an accumulation of pigment in the blood, whereby emboli and hæmorrhages were produced in the brain. This view did not find favour with later authors. Boinet alone held the opinion that, after removal of the suprarenals, a black pigment accumulated in many organs and tissues, and for this reason he employed the term "experimental" Addison's disease. Pigmentation of the skin and mucous membranes of rabbits was observed by Nothnagel in three cases, by Tizzoni in thirteen (twelve cases of single and one of double suprarenal extirpation). F. and S. Marino-Zucco found that fourteen to twenty-four days after the removal of one suprarenal from rabbits, slate-grey patches the size of a linseed appeared upon places where the skin had been shaved. These patches at first increased in size, but at the end of two months had entirely disappeared. Similar patches, were seen in rabbits which were not albinos after inoculation of the suprarenals with Pfeiffer's pseudotubercle bacilli and Eppinger's cladothrix, as well as after the subcutaneous injection of neurin. But it has been pointed out by many that these pigmented patches are quite accidental, and are frequently observed in rabbits which are not albinos. Of the later investigators, no one has been able to record anomalies of pigmentation in animals after removal of one or both suprarenals.

The blood of epinephrectomized animals presents no analogy with the anomalous conditions seen in Addison's disease in man. 
Szymonowicz found that removal of the suprarenals was followed by an increase in the number of the red blood corpuscles, while Bornet describes a decrease in the red and an increase in the white blood cells. But Hultgren and Andersson found that after very exact experiment they were unable to show any change in the hæmoglobin contents of the blood and in the number of its cellular elements.

\section{SUPPRESSION OF THE SUPRARENAL FUNCTION IN MAN.}

Complete suppression of the suprarenal function may occur in man as the result of the congenital absence of these organs. The accounts of the older authors of the absence, believed to be congenital, of the suprarenals from the cadavers of adult persons are entirely valueless, and are, in any case, very scanty. More recently such a finding has been described in the case of one person, aged 40, who had died of phthisis (Martini), and in one boy aged 6 (Averbeck); but in these instances no account was taken of the probable presence of accessory suprarenals. The absence of one suprarenal which, as experiment seems to show, is by no means dangerous to life, is of the rarest occurrence.

With regard to hypoplastic infantilism of the suprarenals, many instances of malformation have been described by J. F. Meckel. Weigert, Lomer, Zander, point to the hypoplasia of the suprarenals as a frequent occurrence in certain disturbances in the embryological development of the brain, more especially in anencephaly and hemicephaly. According to Zander, a reduction in the size of the suprarenals is to be expected in cases where, at a certain stage of embryonal development, the anterior portions of the cerebral hemispheres have perished. Czerny found that, in the five cases of congenital hydrocephalus which he examined, there was complete absence of the medullary substance of the suprarenals, the cortex remaining intact.

Total aplasia of the medulla in adults has been occasionally described as an accidental finding (Ulrich, Klebs). In these cases the suprarenals were atrophied, and consisted only of cortical substance. The condition of the extracapsular chromaffine tissue was not described.

Special interest is attaching to Wiesel's findings of hypoplasia of the chromaffine system. One case was that of a girl of eighteen with posteriolateral curvature of the spine, who died of cardiac insufficiency. The second was in a case of sudden death after heat stroke, and the third was in a youth of eighteen with status thymicolymphaticus. Hedinger describes hypoplasia of the chromaffine system in a number of cases of sudden death in the status thymicolyphaticus.

Acute suprarenal suppression is not infrequently brought 
about in man by hæmorrhage into the organ. Such suprarenal hæmorrhages are seen in still-born children, and these may perhaps occur intra partum. Sudden destruction of the suprarenals may, however, take place in later life as a result of hæmorrhage or suppuration of the organ.

The symptom-complex of this form of suprarenal disease was first described by Virchow. There are very severe nervous symptoms, together with peritonitis, and these end rapidly in death. Post-mortem examination shows that, in the greater number of these cases, the hæmorrhage is due to trauma, emboli, or thrombi; in rare cases, acute suppuration was found. Acute suprarenal suppression to a certain extent resembles the condition produced in animals by the extirpation of both suprarenals. In subacute cases pigmentation of the skin is rarely absent.

The cases of chronic suprarenal suppression which have been described have a greater significance. Bittorf has recently classified chronic suprarenal insufficiency under two headings. The first group includes those cases in which the disease is primary and is due to atrophy, hypoplasia, or cirrhosis of the organ. The second comprises those cases where the suprarenal insufficiency is secondary, and occurs as the result of tuberculosis, syphilis, or tumours. In the literature of the subject, Bittorf found accounts of forty-seven cases of primary atrophy, and to these he added five cases which came under his own observation, and of which two were clinical only. Anatomically, these were all cases either of simple atrophy or of chronic, inflammatory, connective tissue cirrhosis. The degenerative changes were not confined to any particular portion of the organ. In some instances the cortex only was affected; in others the medulla only; but in the majority of cases both portions were affected, though unequally. Clinically, all these cases were characterized by more or less clearly marked symptoms of Addison's disease; the pigmentation of the skin, in particular, was always present, though in a varying degree.

Primary disease of the suprarenals is of comparatively rare occurrence, "secondary conditions such as those which form the groundwork of Addison's disease being of far greater frequency. The typical anatomical finding in Addison's disease is tuberculosis of both suprarenals, which may be the sole finding, or it may be associated with a tuberculous condition of other organs. As a general rule, there is complete, or nearly complete, caseation and fibrous induration with chalky deposits or caseous softening. More rarely, syphilitic processes and tumours have been observed.

This extreme and very typical suprarenal degeneration is, however, not present in all cases. As early as 1885 , Lewin collected $38 \mathrm{I}$ cases of typical Addison's disease, and of these the suprarenals were affected in only $28 \mathrm{I}$ cases, in 37 they were intact. In a later communication he describes 316 fresh cases, 
and in 25 of these the suprarenals were sound. The occurrence of cases which present all the clinical signs of Addison's disease with anatomically sound suprarenals, when taken in conjunction with certain pathological changes which have been observed in the sympathetic system, suggest that the pathogenetic factor in this condition does not lie with the suprarenals, but is to be sought in the sympathetic system itself. Nevertheless, a critical consideration of the material at his disposal compelled v. Kahlden (I896) to adopt the view that the changes in the sympathetic system, and especially in the spinal cord, generally slight in character, though occasionally severe, are without any pathogenetic significance in this condition.

The nervous or sympathetic theory assumed that Addison's disease originated in a pathologically demonstrable affection of the nervous system, but in its original form this theory is no longer tenable. A certain measure of etiological responsibility is ascribed to the sympathetic system by Neusser, who regards Addison's disease as a systemic affection which may take its rise in any portion of the chain-in the spinal cord, the sympathetic nerves, or the suprarenals. Neusser's view is supported by a fact which I have proved, namely, that certain fibres of the splanchnic nerves act upon the suprarenals as vaso-dilators, while others may exercise an influence upon suprarenal secretion.

Owing to the advances which have been made in anatomy and embryology, the sympathetic theory has necessarily changed in form, but in certain directions it receives a measure of support from Wiesel's recent publications. Wiesel does not find, however, that the cause of Addison's disease lies with the nervous system itself, he believes that it is to be sought in the adrenal system, so intimately connected with the nervous system genetically, anatomically, and functionally. He describes six cases in which there was serious degeneration and even destruction of the chrome-brown cells, not in the medullary substance of the suprarenals only, but also in the free parts of the adrenal system in the neighbourhood of the sympathetic. In one case, that of severe double tuberculosis of the suprarenals unaccompanied by symptoms of Addison's disease, not only was there no absence of the chrome-brown tissue, but there was distinct hyperplasia of it. If these findings show that Addison's disease is due to a pathological condition of the chrome-brown tissue, then all the earlier observations of cases of "Addison's disease with sound suprarenals" lose their significance. For those observations were founded upon an imperfect knowledge of anatomy, the adrenal system being at that time little understood. Moreover, there was always the possibility that in such cases the intracapsular portion of the adrenal system, the suprarenal medulla, had at first remained sound, the free portions only being primarily or more severely affected. On the other hand, cases of "suprarenal affection un- 
accompanied by symptoms of Addison's disease " are to be explained, in the light of our present knowledge, by a pathological condition of the cortex only. Where, however, there were demonstrable changes in the medullary substance, the functional activity of the adrenal system might be carried on by its extracapsular portions.

Wiesel's findings are confirmed by those of Beitzke, but both v. Hansemann and Karakascheff are opposed to his views. The latter even goes so far as to ascribe Addison's disease to a pathological condition, not of the chromaffine tissue, but of the cortex of the suprarenals. He describes a case in which, notwithstanding complete caseation of both suprarenals, the symptoms of Addison's disease were absent. But in this case there was an accessory suprarenal consisting only of cortical substance-an accessory interrenal. Bittorf and Beitzke are of the opinion, however, that not only does Karakascheff's case prove nothing against Wiesel's theory, but that it does not establish his own,that, namely, of the paramount importance of the cortex in the etiology of Addison's disease. Bittorf believes that the pathogenesis of Addison's disease does not lie solely with the chromaffine system. He bases his view upon the localization in the suprarenals of the atrophic disturbances described above; and upon one case in which distinct symptoms of Addison's disease were present, in spite of the fact that the chromaffine tissue was intact, the suprarenals being only partially destroyed by hypernephrometastases. As Karakascheff and Bittorf pointed out, the chief evidence against Weisel's theory lies in certain findings by him and Hedinger of extreme hypoplasia of the chromaffine system, unaccompanied by any symptoms of Addison's disease.

It is still more difficult to account by Wiesel's theory for those typical cases of Addison's disease where there is either complete disorganization of the suprarenals or pronounced destruction of the cortex, the medulla being comparatively sound. In the latter class of case, considerable portions of the medullary substance and of the extracapsular chromaffine tissue may still be intact. Wiesel explains these cases by the assumption that, owing to the intimate topographical relationship between medulla and cortex and the fact of their common circulatory system, the affection of the cortex is a secondary condition resulting from the primary disease of the adrenal system. But until very definite grounds upon which to base such an assumption are forthcoming, the only tenable theory concerning the pathogenesis of Addison's disease is that which refers it to the suprarenals as a whole, and not to certain portions of them. From such a point of view, whether the medulla or the cortex is the part primarily affected is immaterial, for the functional disturbance of one part is invariably followed by that of the other; and it is the disease of the entire 
organ, whether the destructive process is partial or entire, which produces the typical symptom-complex of Addison's disease.

Bittorf, like Neusser, adopted the view that derangement of suprarenal function may be caused, not only by disease of the organ itself, but also by a pathological condition of the secretory nerves which regulate its function. Those cases of Addison's disease in which the suprarenals were apparently sound, are readily explicable by the assumption of a primary affection of the secretory nerves. Moreover, as Bittorf points out, and his view is confirmed by Beitzke, the recent literature of the subject contains no conclusive accounts of cases such as these, and he believes that the frequency of the findings is in an inverse ratio to the accuracy with which the cases were observed.

Cases of one-sided partial destruction of the suprarenals unaccompanied by Addisonian symptoms, are readily explained by the presence, either of functionally active suprarenal tissue in larger or smaller quantities, or of accessory suprarenals. It is a more difficult matter to account for those cases where one-sided suprarenal disease is accompanied by symptoms of Addison's disease. Neusser believes this to be due to the transmitted influence of the diseased organ upon the sound one, but Bittorf believes that the influence is rather chemical. He regards as analogous the "transmitted" anuria which, with other symptoms characteristic of total suprarenal suppression, is seen in one-sided suppression; and he points moreover to those cases of onesided suprarenal tuberculosis, where the removal of the diseased capsule is followed by complete cessation of all symptoms of Addison's disease.

This theory derives no support, however, from the results of experimental pathology. One-sided suprarenal suppression is not attended here by symptoms of a deranged organic function, and there is no insufficiency of the half of the pair which remains intact. On the contrary, there is rather a functional hyperactivity, which may ultimately be expressed by anatomical hypertrophy, of the sound capsule. It is evident from this that the pathogenesis of Addison's disease, where associated with the destruction of one suprarenal capsule, is in need of further investigation.

We are in a more favourable situation in regard to those cases, formerly so much discussed, in which, though the clinical signs of Addison's disease are absent, post-mortem examination shows a more or less complete destruction of both suprarenals. As Bittorf with justice points out, these are usually cases where the clinical observation has been faulty, the absence of the pigmentation of the skin in many instances being regarded as sufficient ground for the exclusion of Addison's disease, other clinical signs passing unnoticed. The clinical observation of these cases, if it is to be of value, requires to be scrupulously exact. 
Moreover, it must be borne in mind that, though the destruction of the suprarenals means the suppression of a considerable portion of an organic system, the suprarenals form a portion only of that system, the probability being that the adrenal system is not appreciably affected by such suppression. Moreover, considerable portions of interrenal tissue, which vary in size in individuals, may be present as accessory interrenals.

A critical survey of the facts which we possess concerning the pathogenesis of Addison's disease leads inevitably to the conclusion that Addison's theory, according to which the clinical complex presented by the condition is the result of an anatomical disease of the suprarenals, explains the large majority of cases, and that the instances in which the functional derangements are not associated with appropriate anatomical findings are exceptional. But the pathological anatomy of Addison's disease is as yet unable to explain the physiological and pathological significance of the different parts of the suprarenal system. We are not at present in a position confidently to assert that the suppression of a definite suprarenal system is the cause of the condition, and we are even less justified in forming conclusions upon the homogeneity of the suprarenal function from the pathological-anatomical conditions as seen in man.

\section{THE SYMPTOMATOLOGY OF ADDISON'S DISEASE.}

Addison's description of the clinical symptoms of chronic suprarenal derangement is so significant and comprehensive that, though the exhaustive clinical investigation of the last fifty years has strengthened and defined his view, it has added little to the symptomatology of the condition.*

Experimental pathology has not as yet provided a satisfactory explanation of the genesis of individual symptoms. As we have already pointed out, the destruction of the suprarenals is not followed in animals by a symptom-complex analogous to that seen in man; such an analogy, as we know, exists in the case of thyroid suppression. The well-defined and characteristic signs of Addison's disease as seen in man are represented in animals by vague and ill-defined symptoms of acute suprarenal suppression. This difference is most easily accounted for by the radical difference in the two subjects in the duration of functional suppression. It seems almost as if, in the case of the animal, there was not sufficient time after acute destruction of the suprarenals for the development of symptoms of chronic disease. Owing to the fact that partial destruction of the suprarenals gives rise to regenerative processes and to the vicarious activity of the sound portion,

* A comprehensive description of the clinical appearances in diseases of the suprarenals is given in a monograph by Bittorf, and also by v. Neusser and Wiesel. 
it is not possible to produce chronic progressive suppression of the suprarenal function experimentally and, by this means, acquire a more intimate knowledge of the consecutive symptoms.

Moreover, as the results of extirpation of the suprarenals do not in any way resemble the clinical symptoms of Addison's disease, our reference of the individual symptoms of the latter condition to a suppression of the suprarenal function is, necessarily, more or less hypothetical.

What is known of the physiological activity of the internal secretion of the adrenal system makes it easy to understand that the reduction or suppression of the function of that system should be followed by hypotonus, reduction in blood-pressure, and the consecutive symptoms of cerebral anæmia. There was also at one time a disposition to explain the adynamia-the extreme muscular weakness so characteristic of Addison's disease, which has been demonstrated objectively by Langlois by means of Mosso's ergograph-by a lack of the tonic suptarenal secretion. But a better acquaintance with the physiological effects of adrenalin shows that there is no justification for ascribing to this substance a controlling influence upon the tone of the striated muscles. As we shall see later, adrenalin affects the sympathetic nerve-endings only. The muscular asthenia of Addison's disease is to be explained only by the most complicated hypotheses. The most tenable theory is that, adrenalin being essential to the metabolism of the carbohydrates, its reduction is attended by a loss in the metabolism of glycogen, glycogen being essential to the performance of muscular function.

The asthenic symptoms and the gastro-intestinal disturbances are by many authors attributed to the antitoxic activity of the. suprarenal cortex. The sole symptom which invariably follows suppression of the suprarenal function in both man and animals, is emaciation. Up to the present, however, we have no knowledge of the metabolic changes in animals to which this emaciation is due. The information which we possess concerning the metabolic changes in Addison's disease in man is of too scanty a nature to supply an answer to the problem.

The origin of the pigmentation of the skin and mucous membranes, the typical bronze skin (melanoderma) of Addison's disease, is very difficult of explanation. Once again let it be said that, up to the present, no one has succeeded in producing, under experimental conditions free from objection, a pigmentation of the skin in animals. The pigmentation of the skin is a fairly constant, and frequently a very early, symptom in Addison's disease. It first shows itself in the face and hands, then it affects the neck and arms and, finally, spreads to the trunk and lower extremities. The parts of the skin which are normally pigmented are usually attacked first and acquire a deeper colour. The distribution is affected by external conditions, such as exposure to 
strong sunshine, pressure, and friction. Even more characteristic than the discoloration of the skin, is the affection of the mucous membranes of the cheeks, lips and gums, and of the conjunctiva. Anatomically, the pigment is principally deposited in the cells of the rete Malpighii, and, as far as we know (v. Kahlden, Riehl, Schmorl) is conveyed there from the blood-vessels of the cutis by means of wandering cells (chromatophores). It is almost exclusively deposited in the lower epithelial layers, the upper cells generally remaining free from pigment.

The pigment is free from iron, but nothing further is known regarding its chemical constitution, though, as far as we can judge, it is identical with that of other cuticular pigments. Its formation is generally believed to be associated with a colourless pre-stage in the blood, though certain observers consider that it may be formed autochthonously within the epithelium. It has also been referred to the increased destruction of blood corpuscles in Addison's disease, together with the retarded flow of the stream due to the decrease in blood-pressure, but there is no ground to warrant such an assumption.

The effects of adrenalin upon the pigment of Addison's disease have recently been investigated. v. Furth and $\mathrm{H}$. Schneider found that, when tyrosinase was added to tyrosin, the latter acquired a red, then a violet, and finally an ink-black coloration. Neuberg next discovered that the extract of a melanotic suprarenal tumour acted upon adrenalin in such a manner that pigment was formed; and he discovered a ferment in the ink-bag of Sepiidæe which converted adrenalin into a black product of oxidation. Adami supports the view advanced by Halle and Fränkel that tyrosin is the matrix of adrenalin, and he believes, further, that in suprarenal disease the matrix (tyrosin and allied bodies) is unable to form adrenalin, and therefore accumulates in the tissues. Where the skin is exposed, these substances become converted by the action of oxydases into a black-coloured pigment. The entire question of pigmentation in Addison's disease is urgently in need of further investigation.

\section{SUPRARENAL SUBSTITUTION.}

Numerous experiments have been undertaken with the object of counteracting the results of suprarenal suppression in animals by means of a substitution therapy. This has been attempted by two methods, the one by the exhibition of the organic extract (organo-therapy), the other by transplantation.

\section{ORGANO-THERAPY.}

Speaking generally, experimental organo-therapy has not been attended by satisfactory results. Abelous and Langlois attempted, but without success, to keep frogs, from which both 
suprarenals had been removed, alive by means of watery suprarenal extract obtained from animals of the same species. They obtained better results with guinea-pigs, and succeeded in prolonging the lives of these animals for a few hours by means of injections of suprarenal extract, given immediately after removal of the second capsule. In a later publication, Abelous asserts that he succeeded in keeping suprarenalless frogs alive for as long as twelve days, by the injection of alcoholic extract of the suprarenals of dogs. At almost the same moment, BrownSéquard communicated the fact that the injection of suprarenal extract will produce a marked improvement in the condition of moribund epinephrectomized guinea-pigs.

Numerous accounts are forthcoming of the favourable effects of organotherapy upon both the general condition and individual symptoms, in animals reduced to extreme prostration after extirpation of their suprarenals. Many authors lay special stress upon the fact that the intravenous injection of suprarenal extract raises the low blood-pressure and improves the much impaired respiratory activity. Hultgren and Andersson found, in addition, that the subcutaneous injection of suprarenal extract prevented the terminal fall in temperature, that it improved the general condition, and to a certain extent relieved the extreme muscular weakness. But in face of what is now known of the physiological activity of suprarenal extract, these results cannot be regarded as in any way establishing a specific substitution therapy. The results which follow the exhibition of suprarenal extract in suprarenalless animals are identical with those observed in normal animals and in dying animals, whatever the cause of death. To establish the value of a suprarenal substitution therapy, it is not sufficient to point to the results observed after a single exhibition (Einverleibung) of suprarenal extract. In my opinion such a conclusion would be justified only if it were possible to show, as in the case of substitution by the other organic extracts, that the exhibition of suprarenal substance is able to prolong the lives of suprarenalless animals. The material which is forthcoming does not, however, provide any such proof. Langlois's epinephrectomized rabbits and Hultgren and Andersson's epinephrectomized cats lived, after treatment with suprarenal extract, for at the most twenty-four hours. Strehl and Weiss were able to keep the animals which they treated with suprarenal extract alive for only nine hours longer than the control animals. I have treated a large number of animals, by feeding them with suprarenal tabloids previous to epinephrectomy, for periods varying from a few days to several weeks, the treatment being continued after operation by means of subcutaneous injections of the watery extract of suprarenal. But in spite of the flooding of the organism in this manner with suprarenal substance, there was little difference after operation in the condition of these 
animals from that of the control animals. Also, the average length of life of the animals under treatment was very little longer than that of the control animals. From the results which I obtained I was led to conclude that suprarenal substitution is not practicable by these methods.

Seeing that the exhibition of suprarenal extract is attended by doubtful results in experimental suprarenal suppression, it is not surprising that in Addison's disease the method has not accomplished all that was at first expected of it. Charrin and Langlois were probably the first to attempt the treatment of this condition by means of subcutaneous injections of the glycerine extract of suprarenal, obtained from dogs and horses. Since then the effects of organo-therapy in Addison's disease have been tried by many.

According to Gilbert and Carnot, the clinical material may be classified, according to the results obtained, in four groups.

(I) Those cases in which organo-therapy was attended by harmful results, the general condition becoming aggravated and the fatal termination hastened (Rendu, Foà and Pellacani, Zucco, Pitres, Posselt). Boinet, more particularly, has drawn attention to the symptoms of intolerance which follow treatment with organic extract in Addison's disease. He found that prolonged treatment produced nervous symptoms, the most frequent being a tremor similar to that of Graves's disease.

(2) The large group of cases in which the results of treatment were negative (Langlois, Chauffard, Grainger-Stewart, Murrel, Darier, P. Marie, Galliard, Allaria and Varanini, Bendix, Turner, Trevithick, Box, Huismans, Christomanos, Lange, Bockhaus, Osler, Ringer and Phear, Sibley, Bittorf).

(3) Those cases in which an improvement in certain symptoms was observed. The most frequent signs were a diminution of the muscular asthenia, improvement of the digestive derangements, and an increase in weight (Langlois, Mahé, Maragliano, Dieulafoy, Marie, Schäfer, Rolleston, Osler, Vidal, Hayem, Vollbracht, Dupaigne, Spillmann, Edel, Faisans, Anderodias, Althaus, Byrom-Bramwell, Gullau, Vernescu, Foster, Raven, Decks, Engelhardt, Ebstein, Sellier and Verger, Posselt).

(4) Those rare cases in which complete recovery is reported. Schilling found that, in the case of a patient 16 years old, a threemonths' treatment with suprarenal ( $\frac{1}{2}$ to I suprarenal capsule per day) was followed by the disappearance of all previous Addisonian symptoms, namely, the asthenia, anorexia, diarrhœa, and even the pigmentation of the skin and mucous membranes. The weight rose from 69 to $99 \mathrm{lbs}$. Some time after recovery the patient died suddenly from pneumonia, and the post-mortem examination showed that there was sclerosis and softening of both suprarenals.

Béclère, Hirtz, Lambotte, and Androdias also describe cases 
of recovery from Addison's disease. Kinnicut publishes the statistics of forty-eight cases of Addison's disease which were treated by organo-therapeutic measures. Of these, in two cases there was aggravation of the condition; in eighteen, the condition remained unchanged; in twenty-two, there was an improvement; and in six cases there was complete recovery.

It is evident from this that the treatment of Addison's disease by means of suprarenal extract is very variable in its results. The few cases of recovery are nullified by those where an aggravation of the condition ensued. In the large majority of instances the treatment produced negative results or the amelioration of single symptoms only. The muscular weakness and the digestive derangements appear to be the symptoms most favourably influenced.

That suprarenal extract, when given by the mouth, should affect the reduced cardiac activity, and the lowered blood-pressure is hardly to be expected. Nevertheless, Grünbaum asserts that, although suprarenal extract, when given by the mouth, does not raise the blood-pressure of normal subjects, it does have that effect when given to persons suffering from Addison's disease. Falta has recently shown that in man, the subcutaneous injection of adrenalin brings about a rise in blood-pressure.

The symptom which is least affected by this method of treatment is undoubtedly the pigmentation.

The foregoing accounts of the effects of suprarenal extract in Addison's disease suggest certain definite ideas. Of these, the most important concerns the influence of adrenalin upon the symptoms. But a certain measure of efficacy must be ascribed to other substances present in suprarenal extract, and notably those. which are derived from the suprarenal cortex. Experiments with animals seem to suggest that the exhibition of suprarenal substances in Addison's disease provokes hypertrophy of the portions of the organ which, in this condition, undoubtedly remain intact, and that by this means the functional inadequacy of the organ is reduced in a quantitative sense. The negative and harmful effects of suprarenal treatment are probably seen in those cases where the suprarenals have become completely destroyed.

\section{TRANSPLANTATION OF THE SUPRARENALS.}

The second method of suprarenal substitution is that of the surgical transplantation of the organ. In 1887 Canalis attempted the implantation in the kidney of small portions of suprarenal tissue, but the results which he obtained were purely negative, the implanted portions becoming necrosed and undergoing resorption. The experiments of Abelous, and later those of Abelous and Langlois, with frogs were equally unsuccessful. Courfein implanted the suprarenals of frogs and guinea-pigs in 
the lymph-sac of frogs, but in all his cases the implanted organ perished. Boinet implanted suprarenals intraperitoneally in rats and found that the implanted organ became atrophied and, in some instances, resorbed. De Dominicis removed the left suprarenal of dogs, leaving the vascular stem intact, and implanted the organ below the kidney; he found that, at the end of nine to ten days, there was no change. Jabulay implanted fresh suprarenals from dogs in two patients suffering from Addison's disease, and in each instance death followed after twenty-four hours. Hultgren and Andersson implanted suprarenals in three cats, in each case without result.

There is a special interest attaching to H. Poll's transplantation experiments. He removed the left suprarenal of rats and implanted it under the skin, or in the muscles of the back, of the same animal or another of the same species. By this means he was enabled minutely to follow the histological changes in the transplanted organ.

Poll found that, during about the first week, regressive changes preponderated. A largish necrosed focus appeared in the centre of the implanted suprarenal. In addition to the central medullary tissue, the zona reticularis of the cortex and the inner portions of the zona fasciculata underwent degenerative changes, and were destroyed.

During the second week, a large part of the necrosed tissue became gradually resorbed, giant cells being formed in the process. At the same time the cells of the zona glomerulosa and the external portion of the zona fasciculata became destroyed, while peculiar, light, transparent cells, filled with vacuoles, were formed in the cortical tissue, which afterwards disappeared, leaving a pigmented scar.

From the third week onwards, signs of progressive new formation appeared. Minute regenerative foci were formed which lay, partly internally and partly externally of the thickened connective-tissue capsule. They developed into largish bodies, shaped like the segment of a sphere, with cord-like arrangement of cells. Later on, these cell agglomerations assumed the exact appearance of cortical substance.

The regeneration of the cortical tissue and the degeneration of the medullary tissue were observed by Christiani in experimental transplantation performed on rats. And Stilling found that, when the suprarenals of rabbits were transplanted in their testicles, they showed typical cortical tissue for as long as one and a half to three years.

Further information concerning the results of transplantation is given by Imbert. He implanted the suprarenals of dogs in their kidneys and found, in one instance, a pseudolipoma, in another, a cyst, at the site of implantation. Strehl and Weiss sewed the suprarenals into pockets between the muscular structure and the 
abdominal cavity; they also placed them free in the abdominal cavity; and they implanted them in vascular organs, such as the kidney and liver. But in every instance the implanted organs perished. Small portions of implanted suprarenal tissue did not, in the vast majority of cases, heal in.

Better results were obtained by Schmieden, who implanted small portions of suprarenal tissue in the kidneys of rabbits and, in a large number of cases, these healed in. But he does not. believe that these implanted portions are likely to remain alive for any length of time, and his experiments seem to show that one year is the maximal period.

Payr used the spleen with considerable success as the site of implantation for the thyroid, but in the case of the suprarenals the choice of this locality was followed only by transient healing in, no permanent results being obtained (Coenen, Kreidl, Biedl). Shiota found that a suprarenal, when transplanted into the spleen or kidney of rabbits and cats, contained adrenalin during the first forty-eight hours only, the substance disappearing at the end of this time. Although the cortex will remain in a fairly healthy condition for from ten to seventeen weeks, the medullary substance ceases to stain with chromium twenty-four hours after implantation.

The admirable experiments of v. Haberer and Stoerk resulted in successful suprarenal transplantation with complete functional activity. Their method was to remove the suprarenal without severing its vascular peduncle and to implant it in the kidney. De Dominicis had already dislodged the left suprarenal without cutting through the peduncle (his experiment has been described in a previous chapter) and I had removed the suprarenals to an extra-peritoneal position, leaving their vascular stalk intact. These results undoubtedly showed that, if the normal nutritional conditions were maintained, the transplanted organ would preserve its functional activity. In 50 per cent. of their cases, v. Haberer and Stoerk succeeded in producing permanent results which were functionally, as well as anatomically, demonstrable. In the unsuccessful cases, the result was invariably attributable to insufficient nourishment of the implanted organ.

Microscopic examination of these successfully transplanted suprarenals showed that in no case did the organ retain its original structure, but that, in addition to sound and hypertrophied tissue, it invariably contained regressive and necrosed substance. In the first few days after transplantation with preservation of the peduncle, there is a regressive metamorphosis of the organ which is occasionally so widespread that the only part which remains alive is that in the immediate vicinity of the peduncle. Comparatively soon after operation, generally in the first week, active proliferation commences at this spot, the proliferating parenchyma spreading into the regressive or necrosed portions of the organ. 
From this a chaotic mixture of living and hypertrophied, and regressive and necrosed tissue results.

The hypertrophied tissue may in a later stage again become regressive and, in some cases, even necrosed. This interchange of proliferation and regression goes on for some time, and necrosed tissue may be observed for as long a period as three months. The newly-proliferated cell agglomerations may assume the structure characteristic of the normal suprarenal ; or there may be a re-arrangement, not only in the cells of the cortex, but also in the relative position of the cortex and medulla. The medullary substance frequently breaks through the cortex and forms enclosures between the cortical cell complexes; or these enclosures may be placed sub-capsularly, and even extra-capsularly. In many instances the regenerated cortex, when complete, assumes a form similar to that of suprarenal adenoma. It was formerly believed that, in successful transplantation, the cortex only remained intact, but it is now certain that the medullary substance also retains its vitality and its capacity for regeneration, and that it undergoes hypertrophy in the same manner as the cortex.

About five months after transplantation, the alternation of regenerative and degenerative processes ceases and the conditions become more stable. In the majority of cases an entirely new suprarenal has been formed; by proliferating into the old structure and becoming regeneratively hypertrophied, the new cortical and medullary tissue crowds out the regressive, necrosed tissue and replaces it.

The tests which have been made of the functional capacity of the transplanted suprarenal yield the following results : In experimental one-sided transplantation-and this applies to cases where the histological findings are unequivocal-it is not possible to pronounce upon the functional activity of the healed-in organ, for there is always the possibility of the function having been undertaken by the sound one of the pair. One-sided implantation is usually borne without symptoms by dogs, rabbits and cats. v. Haberer describes a control-experiment with a dog, in which one-sided suprarenal extirpation was accompanied by distinct symptoms of suppression, namely, pronounced spastic paresis of the hind extremities, failing appetite and general depression. The dog died at the end of eight days, the post-mortem examination showing that the second suprarenal was slightly hypertrophied.

In a second group of experiments, both suprarenals were transplanted into the kidney, each capsule being transplanted separately and a varying length of time allowed to elapse between the two operations. In many cases, where the first suprarenal had become necrosed or atrophied and had ceased to function while the preliminary regressive changes were still in progress in the second, the animals died as the result of suprarenal inadequacy. This group of experiments also brought the interesting 
fact to light that after suppression of one capsule, the condition of the second does not render it more favourable for transplantation; it does not, as a priori assumption would suggest, become hypertrophied but, on the contrary, it almost completely perishes.

The functional capacity of a transplanted suprarenal is tested by removing the second capsule some time after the first. In cases where there was pronounced necrosis of the implanted suprarenal, the animals lived for a short time only after the second operation. In nine cases, animals with only one transplanted suprarenal remained alive for many days and even for months. Subsequent microscopic examination revealed, in these cases, a splendid healing-in of the implanted capsule with new formation and reconstruction of the suprarenal tissue, together with complete degeneration of the original peduncle and a new vascular system derived from the kidney. As accessory suprarenals were in no case present, it is certain that these animals continued to live with their only suprarenal implanted in their kidney and that they showed no pathological signs. But these cases, as well as those of double transplantation, supply another remarkable fact, namely, that the animal has a better chance of life if an interval of about eleven to sixteen days is allowed to elapse between the transplantation of one suprarenal and the extirpation and implantation of the other. Where the interval is longer; the results are frequently unsuccessful. The impression conveyed is that the suprarenal function is so completely taken over by the sound capsule, that the regenerative processes in the second. damaged capsule are retarded. If the sound capsule is injured or removed at a moment when the regenerative process in the. second organ is at its height, the new formation of tissue will receive an additional stimulus.

Yet another group of experiments comprises those in which the transplantation of both suprarenals is undertaken separately, and one kidney, together with the suprarenal implanted in it, is removed by means of a third operation. Of eleven animals experimented upon, six died after the third operation as the result of suprarenal inadequacy. In each of these cases, however, it happened that the suprarenal removed was well healed in and hypertrophied, while the condition of the second, as seen post mortem, was regressive. Five dogs lived for years after the third operation and showed no pathological signs, and in these instances the extirpated transplanted suprarenal was also hypertrophied.

The degree of functional activity of the transplanted suprarenal has been further tested, by examination of its adrenalin contents by means of blood-pressure experiments and experiments with frog's eyes. The results showed a complete agreement between the microscopic structure and the adrenalin contents. 
The value of the experiments of $v$. Haberer and Stoerk, which will be described more fully later, lies in the proof which they supply that the suprarenal may be transplanted with success, both functional and anatomical, provided its vascular stem is left intact. Further, these experiments were the first to show that, after transplantation, the medullary substances lives and becomes hypertrophied in exactly the same manner as the cortical substance. The conclusion drawn by the authors from these findings, namely, that the part played by the medulla in suprarenal function is of equal importance with that of the cortex, is the more incontrovertible seeing that other observations have supplied proof, not only of the vital necessity of the medulla, but also of its functional significance. But, in my opinion, the successful transplantations of v. Haberer and Stoerk are decided evidence against the view, that the medulla is the only part of the suprarenal capsule which is essential to life. This theory was based upon experiments where transplantation, though technically successful, was followed by death, and it depended upon the fact that degeneration of the medullary substance, together with preservation of the cortex, were always found in such cases. All that these older experiments prove, however, is the greater sensibility and lability of the medulla. They show, and this is confirmed in other directions, that $a$ reduction in the vascular provision and a limitation of the nutritional conditions invariably kills the medulla, but that the cortex has greater powers of resistance.

Free grafting of chromaffine tissue is successful in those cases only where this tissue is normally situated upon a free surface as, for instance, the dorsal surface of the kidney in reptiles (Christiani's experiments with lizards). Parodi's experiments show that embryonal suprarenal tissue, in which the medulla lies upon the surface, possesses very little regenerative power; and, in an experiment recently described by Neuhäuser, the transplantation of embryonal tissue into the kidney of a rabbit was followed by a tumour-like growth.

The results of experimental transplantation, as far as they go to-day, not only prove the internal secretory function of the suprarenals and their vital importance to the organism, but they also justify the hope that the clinical symptoms produced by suprarenal suppression may be more successfully treated by surgical means than has been possible, up to now, by organo-therapeutic measures.

\section{THE ACTIVITY OF SUPRARENAL EXTRACT.}

Investigation of the clinical activity of extract of the suprarenals, has effected a clearer insight into the function of these organs.

As early as 1879 , Pellacani demonstrated the toxic action of 
subcutaneous and intravenous injections of the watery extract of suprarenal. He did not believe that this action was specific, for he found that similar results were produced by means of other organic extracts. But very soon after, Ziino and di Mattei denied the toxicity of suprarenal extract and they referred the results which followed its exhibition to infective processes, more particularly the influence of products of the decomposition of the albuminoids introduced into the system with it. On the other hand, Pellacani and Foà (I883) proved that the toxic substance in the suprarenal cannot be identical with the fibrin ferment, which is present even in the alcoholic extracts of these organs and is specific to the suprarenal. Guarnieri and Marino Zucco (I888) confirmed the extreme toxicity of the watery extracts and they considered that the active agents were neurin and the organic phosphorus compounds. The brothers Marino Zucco even went so far as to believe that the symptom-complex of Addison's disease could be produced by means of neurin injection. These authors, together with Dutto, proved that neurin was present in the urine of persons with Addison's disease, and, towards the end of the 'eighties, a hypothesis arose that the physiological function of the suprarenals consisted in the neutralization of neurin, which was a product of the decomposition of the nervous tissue. This doctrine seemed to find a support in Albanese's experiments with frogs. But both Boinet and Langlois were unable to confirm the experiments upon which the theory was founded; while Supino further showed that the toxic conditions which follow the injection of neurin are entirely different from the clinical complex produced by suprarenal extirpation. Alezais and Arnaud, and later Tizzoni, denied the existence of a toxic agent in the suprarenal, confirming the view of an earlier observer, namely, Alexander. They attributed the effects of suprarenal extract to post-mortem changes in the tissues.

The discovery by Oliver and Schäfer (I894) of the specific physiological action of suprarenal extract, drove all previous conclusions as to the general toxic activity of these organs into the background. The experiments which followed were, naturally enough, chiefly concerned with the more minute analysis of individual pharmacodynamic effects. But since the discovery that these effects are principally due to an active substance present in the medulla and, more particularly since the isolation of this active principle in the form known as adrenalin, inquiry into the general effects of suprarenal extract has somewhat languished. The lack of any method of exact dosage is a serious drawback to experiment.

A consideration of the material furnished by the subject shows that there is no sufficient justification for identifying the general effects of suprarenal extract with those of adrenalin. For, in addition to adrenalin, suprarenal extract contains other substances 
derived from the cortex, concerning the activity of which we as yet know nothing. Moreover, therapeutic measures, especially those aiming at substitution in the case of suprarenal suppression in man, have been attempted almost exclusively with preparations derived from the whole suprarenal. The fresh raw organ, the organ dried and powdered, suprarenal tabloids, and the watery extract of suprarenal, have all been employed in this connection. Until something is known of the physiological activity of the cortex, the empirical method of treatment with the whole organ must be regarded as superior to treatment by means of the active principle, adrenalin.

The following experiments serve to illustrate the present position. Oliver and Shäfer injected the watery extract of the suprarenal of calves into dogs, guinea-pigs and cats without producing toxic symptoms. In guinea-pigs and dogs, large quantities of the extract were followed by transient disturbances of the pulse and respiration, as well as by a fall in temperature. The subcutaneous injection of large doses into rabbits resulted, after a few hours and in some instances after a few days, in a fall in temperature followed by death. In frogs there were symptoms of central paralysis.

According to Cybulski the toxic action of suprarenal extract is seen in rabbits only after intravenous injection; it affects the central nervous system.

According to Gluzinski, watery glycerine extracts of the suprarenals of cattle, calves, pigs, dogs and rabbits produce a high degree of intoxication in normal animals. Intravenous injection is followed by paralysis and loss of sensibility in the hinder, and convulsions in the fore, extremities; there is acceleration of the respiration and enlargement of the pupils, dyspnœea and widespread paralysis, ending in death. Subcutaneous injection is less deadly; the animals sicken and the temperature becomes subnormal, but unless the doses are too large they recover.

Dubois found that suprarenal extract derived from rats was very deadly. When injected in even quite small doses, it produces lassitude, paresis, and in many cases paralytic symptoms ending in death. The degree of the toxicity of the extract yaries; it is increased if the animals are fed upon decomposing food, and after experimental infection. Suprarenal extract derived from rats kept in captivity is less toxic than that obtained from freeliving animals. The medullary substance contains a larger proportion of toxin than the cortex. The amount of the extract tolerated varies with individuals, and muscular fatigue previous to injection renders the animals more sensitive to its toxic action. According to Dubois, suprarenal extract contains at least two substances, one of which causes general vaso-dilation and is insoluble in 90 per cent. alcohol; while the other, which is soluble in alcohol, produces widespread paralysis, reduced cardiac activity, and asphyxia ending in death. 
By treating watery glycerine extracts of the suprarenals of cattle, sheep and calves with strong alcohol, Gourfein obtained a precipitate which was insoluble in alcohol and which, when dissolved in water, was less toxic in its action. The substance which remained dissolved in the alcohol and which resisted heat, produced progressive difficult respiration and weakness of the heart-beats, but it did not produce paralysis. The autopsy showed that the lungs were severely congested.

According to Wybauw, the extract of the suprarenal of guinea-pigs is less toxic than suprarenal extracts derived from other animals.

Very complete experiments are described by S. Vincent. The extract which he employed was mostly derived from sheep, though occasionally from oxen, dogs, cats and guinea-pigs, and was obtained by the agency of saline solution both with and without boiling. He found that the subcutaneous injection of this extract, if given in sufficiently large doses (equivalent to 0.5 to $0.6 \mathrm{grm}$. of the fresh organ) produced more or less pronounced muscular weakness, occasional paresis with ultimate paralysis of the extremities, accelerated and shallow, followed by deep and slow respiration, occasional dyspnœic convulsions, and a fall in temperature ending in death, in all the animals experimented upon (frogs, toads, rats, mice, rabbits, guinea-pigs). Hæmaturia and bleeding from the nose and mouth are not infrequently seen in rats and guinea-pigs. The injection of small doses of the extract is followed by a partial immunity lasting several weeks. Thirst is the first noticeable symptom in dogs, which drink water greedily and afterwards excrete a great profusion of urine. After a short stage of excitement accompanied by increased muscular activity and tremor, these dogs develop paresis and finally paralysis. The most noticeable symptom in cats is the quickened respiration, the paralysis being less marked. Late effects, those which only make their appearance after hours or even days, have never been observed. The administration of the extract in sufficiently large doses is invariably followed by symptoms within a few minutes. According to Vincent, the symptoms take their rise in the central nervous system, and are the outcome of the activity of the medulla, no toxic substance being present in the cortex.

Vincent also tested the physiological effects of separate extracts derived from the interrenal and the adrenal tissues of fish. He found that the Stannius's body of Gadus morrhua, composed exclusively of interrenal tissue, and the interrenal body of Selachii produced no effect when exhibited in mice, while the extract of the suprarenal body of Scyllium canicula and Raia clavata, when injected under the skin of mice, produced quickened respiration, reduction in temperature, and convulsions terminating fatally within, at most, five minutes. The toxicity of the extract of the suprarenal bodies of Selachii, when injected into the veins of dogs and rabbits, is confirmed by Biedl and Wiesel. 
The discovery that the intravenous injection of the watery extract of suprarenal is followed by specific and well-defined physiological results, started a new era in suprarenal investigation. This discovery was announced by Oliver and Schäfer before the Physiological Society of London on May Io, I894. A year later, Cybulski and Szymonowicz published their results which, though obtained independently, coincided in the main points with those of Oliver and Schäfer. An enormous number of experiments followed, which not only explored the physiological and pharmacodynamic activity of suprarenal extract down to the minutest detail, but utilized the facts so won to reconstruct in its entirety the chemistry of the suprarenals.

\section{CHEMISTRY OF THE SPECIFIC ACTIVE SUBSTANCE PRESENT IN THE SUPRARENALS.}

As early as ${ }_{1} 8_{5} 6$, Vulpian made the discovery that the suprarenal contains a substance which is distinguished by its remarkable colour reactions. It colours green with ferric chloride, and takes on a pink to carmine-red colour when exposed to the air and treated with alkalies or with oxydizing agents, such as iodine-water and chlorine-water. Virchow, who confirms Vulpian's discovery, found that the characteristic colour reaction is associated with the medullary substance and that it is demonstrable in microscopic sections. According to Virchow, the chromogenic colouring substance is identical with the substance which colours green with ferric chloride, and is not associated with the morphotic elements, but with the secretion, the intercellular substance. In I885, Kruckenberg drew attention to the important fact that this suprarenal chromogen possesses certain properties in common with pyrocatechin, and Brunner (1892) believed that the two substances were identical.

Oliver and Schäfer ascribed the peculiar hæmodynamic action of suprarenal extract to the medullary substance and, following them, Moore found that the substance which produces increased blood-pressure is identical with chromogen, but that its pharmacological properties are entirely different from those of pyrocatechin. S. Fränkel (1896) was the first to suggest that the substance which raises blood-pressure is a derivative, containing oxygen, of the pyrocatechin group. The experiments of $v$. Fürth and Abel were of great importance because they prepared the way for the isolation, in a state of chemical purity, of the active principle of the suprarenal. In Igo I Jokischi Takamine succeeded in obtaining this substance in a crystalline form, and he gave it the name of adrenalin. Almost at the same time and quite independently, Aldrich produced the same substance from the suprarenal, also in the form of well defined crystals.

The methods of isolating adrenalin are based upon the fact 
that, when alcohol or acetate of lead are added to suprarenal extract, the by-substances are released, and the addition of concentrated ammonia results in the precipitation of adrenalin in the form of microscopic crystals. These are further purified by repeated solution in acids and precipitation by means of ammonia, until eventually, clusters of crystals composed of prismatic needles and rhomboid scales are formed. These adrenalin crystals dissolve slowly in cold water and somewhat more readily in hot. They dissolve freely in diluted acids, and form salts; their reaction upon litmus paper is weakly alkaline. They are not readily soluble in alcohol, and they are insoluble in chloroform, amylic alcohol, carbon bisulphide, ether, acetone, and benzine. In accordance with its phenol nature, adrenalin is soluble in the caustic alkalis, but not in the alkali carbonates and ammonia. It cannot be precipitated by means of potassium mercuric iodide, picric acid, tannic acid, phosphoromolybdanic acid, phos̀phorowolframic acid, or mercuric chloride; but it is reducible by Fehling's solution and by ammoniated solution of silver. When exposed to the air, the watery solution colours red and afterwards turns brown; the addition of ferric chloride produces the characteristic green coloration.*

Numerous experiments were undertaken by Aldrich, Bertrand, Abderhalden and Berger, Jowett, Friedmann, v. Fürth, Pauly, Stolz, with the object of analysing the elements and ascertaining the weight of the molecules, and these revealed the more intimate characterization of adrenalin (epinephrin, according to Abel, suprarenin, according to v. Fürth). This substance is empirically formulated as $\mathrm{C}_{9} \mathrm{H}_{13} \mathrm{NO}_{3}$. The following formula expresses its conștitution :-<smiles>CNCC(O)C(C)CO</smiles>

Adrenalin contains a benzol nucleus which is substituted in the $2,5,6$ - position. Three hydroxyl groups $(\mathrm{OH})$ are present in the adrenalin molecule and, of these, two are placed in the vicinity of the benzol nucleus in the 5,6 - position. The molecule contains a methylimid group.

Stolz succeeded in obtaining a substance, by a synthetic process, which possesses pharmacological properties exactly similar to those of adrenalin.

If chloracetopyrocatechin-which is obtained from the action of chloracetylchloride upon pyrocatechin-is treated with ammonia

* The preparation known as "Adrenalin Takamine" (put upon the market by Parke, Davis and Co.) is adrenalin in combination with hydrochloric acid, in a solution $(\mathrm{r}: 1000)$ of sodium chloride and chloreton, the latter being a derivative of chloroform and aceton. 
or with primary aliphatic amine, amino-, methylamino-, and ethylaminopyrocatechin are obtained according to the following formula :

$$
\begin{aligned}
& \mathrm{C}_{6} \mathrm{H}_{3}(\mathrm{O} \cdot \mathrm{H})_{2} \cdot \mathrm{CO} \cdot \mathrm{CH}_{2}|\overline{\mathrm{Cl}+\mathrm{H}}| \cdot \mathrm{NH}_{2} \quad=\mathrm{C}_{6} \mathrm{H}_{3}(\mathrm{OH})_{2} \cdot \mathrm{CO} \cdot \mathrm{CH}_{2} \mathrm{NH}_{2} \\
& \text { Aminoacetopyrocatechin } \\
& \overline{+\mathrm{H}} \cdot \mathrm{NH} \cdot \mathrm{CH}_{3}=\mathrm{C}_{6} \cdot \mathrm{H}_{3} \cdot(\mathrm{OH})_{2} \cdot \mathrm{CO} \cdot \mathrm{CH}_{2} \mathrm{NH} \cdot \mathrm{CH}_{3} \\
& +\overline{+\mathrm{H}} \mid \cdot \mathrm{NH} \cdot \mathrm{C}_{2} \mathrm{H}_{5}=\underset{\text { Ethylaminoacetopyrocatechin }}{\stackrel{\text { Methylaminoacetopyrocatechin }}{\mathrm{C}_{6} \mathrm{H}_{3}(\mathrm{OH})_{2} \cdot \mathrm{CO} . \mathrm{CH}_{2} \cdot \mathrm{NH}} \cdot \mathrm{C}_{2} \mathrm{H}_{5}}
\end{aligned}
$$

According to Löwi and Meyer, the manner in which these bodies act is exactly similar to that of adrenalin, but the strength of their action is very much less. By reducing these ketone bases, Stolz obtained, by the addition of two hydrogen atoms, the complementary secondary alcohols, namely, amino-ethanol-, methylamino-ethanol-, and ethylamino-ethanol-pyrocatechin. In their action these alcohol bases are not only qualitatively identical with adrenalin, they also approach it quantitatively. Methylaminoethanolpyrocatechin, or orthodioxyphenylethanolmethylamin, is chemically identical with adrenalin obtained from the suprarenal.*

This synthetic adrenalin differs only from natural adrenalin in that it is optically inactive, while the natural substance diverts the polarized ray of direction towards the left. This synthetic racemic suprarenin is composed of equal parts of d- and l-suprarenin; it was split up into its component parts by Flächer.

Synthetic 1-suprarenin is, both physically and chemically, absolutely identical with the natural adrenalin obtained from suprarenal. The experiments of Cushney, Abderhalden and his co-workers show that the physiological action of the synthetic substance is both quantitatively and qualitatively the same as that of natural adrenalin.

The physiological action of d-suprarenin is in every respect similar to that of the natural substance, but is very much less intense; while the racemic body, in accordance with its composition out of d- and l-suprarenin, occupies a middle position.

The question which now arises is, of which substances is the organic adrenalin composed? The theory that adrenalin is formed from the waste products of muscle-metabolism (Boruttau, Battelli) is unsupported by any known fact. The experiments of Abelous, Soulié, and Toujans, which appear to confirm this assumption, and which also point to the cortex as the site of origin, will be discussed later.

The information which we possess in regard to the chemical

* The Höchster Farbwerke have recently put upon the market a I : 1000 solution of this combination, to which they give the name of "Suprarenium hydrochloricum syntheticum." At present they supply the 1-suprarenin only under this name. 
process by which adrenalin is formed, is very scanty. As far as its preceding stages are concerned, Friedmann supposed that adrenalin might be derived from an oxyphenylserin or an oxyphenylmethylserin, which substances might possibly have been previously formed in certain albuminoids. According to Halle and Fränkel, tyrosin and phenylalamin may possibly be waste products. The conversion of tyrosin into adrenalin is supposed to be a combination of four chemical processes (oxidation, evolution of carb̄onic acid, methylization of nitrogen, and a second process of oxidation of the lateral chain in the $\beta$ position), in the course of which para-oxiphenylethylamin is formed as an intermediary product. This supposition is supported by the results of experiments showing that the addition of tyrosin to an emulsion of ox's or pig's suprarenals, is followed after six days in an incubator by an increase in the adrenalin contents of from I 4 to 33 per cent.

Neuberg found that the extract from a melanotic tumour converted adrenalin in a short time into a dark brown product; and he also discovered that a melanin ferment obtained from the inkbags of cuttle fish (sepia) gives a similar melanin reaction with para-oxy-phenylethylamin and tryptophan as with adrenalin. By means of the tyrosinase from the fungus Prussula delica, Abderhalden and Guggenheim obtained the same colour-reactions with tryptophan and the derivatives of tyrosin (d-alanyl-1-tyrosin and 1-leucyl-1-tyrosin) as with 1- and d-suprarenin.

From these results it appears highly probable that the products of the decomposition of albumin, and especially tyrosin, are the matrix of adrenalin.

The most important chemical test of the presence of adrenalin in liquids and tissue secretions is the characteristic colour reaction, namely, green with ferric chloride and pink with iodine water. The same reactions have also been employed as quantitative tests in colorimetric experiments; ferric chloride by Battelli, and the iodine reaction by Abelous, Soulié and Toujan. But the results obtained by these methods cannot be regarded as by any means exact, for they are dependent upon the operation of certain extraneous conditions. The substance must be employed in a state of chemical purity and with an almost neutral reaction, for the presence of free acids and other bodies prevents the ferric chloride reaction, which, in any case, is common to all derivatives of orthodioxybenzol, and is not specific to adrenalin. Iodine staining is prejudiced by the fact that adrenalin solutions which are exposed to the air and light take on a red colouring.

In the last two years a number of chemical tests for adrenalin have been published.

The method described by $G$. Comessatti is based upon the fact, first observed by Muhlmann, that the addition of sublimate to suprarenal extract produces a red colour. A few drops of a 
1-2 : I 000 sublimate solution are added to the liquid containing adrenalin when, after one to three minutes, a diffuse red coloration is seen which lasts for several hours. Comessatti employed his method also as a quantitative test of the amount of adrenalin present in the suprarenal. In his view, the red coloration is due to the formation of oxyadrenalin (Battelli), and is identical with the red colour which adrenalin solutions acquire when exposed for some time to the air. Of allied substances, pyrocatechin gives a green reaction with sublimate, salicylic acid and resorcin produce shades of red. According to Comessatti, this reaction gives positive results with attenuated solutions, where Vulpian's ferric chloride reaction is doubtful (0.0025 grm. adrenalin : 1000 ; or, one drop adrenalin in twenty distilled water; or, one drop adrenalin in Ioo to $130 \mathrm{~cm} .{ }^{3}$ tap water). On the other hand, K. Boas found that, in the case of even concentrated extracts, the reaction did not take place at room-temperature, but that it made its appearance only after boiling.

Another chemical method of differentiating adrenalin is that of Cevidalli. If hydrochloric acid is added to suprarenal extract prepared with physiological saline solution, the albuminoids will be precipitated; the filtrate is then rendered alkaline with $\mathrm{NH}_{3}$, and a few drops of a concentrated solution of ferricyanide of potassium are added, when a pinkish colour makes its appearance which, after several hours, changes to a brownish black. By this method, Cevidalli and Leoncini carried out a series of investigations into the amount of adrenalin present in the suprarenals after death from various causes. They found that the "docimasia surrenale" is sufficient to decide the medico-legal question as to whether death has taken place suddenly, or is the outcome of prolonged illness. In the latter case, the reaction is weak, but it is very strong after sudden death.

The colorimetric method of A. Zanfrognini depends for its results upon the fact that, where adrenalin is present, the brown manganese dioxides are converted into the colourless lower oxides, in the course of which the solution acquires a red stain. The intensity of the colouring is proportional to the amount of adrenalin present in the solution. The reaction is extremely sensitive, and is clearly perceptible in a solution of $1: 1,000,000$; it remains unchanged for hours; and takes place independently both of the reaction of the medium and the presence of neutral salts. The reagent $(3 \mathrm{grm}$. permanganate of potassitim dissolved in $24 \mathrm{c.cm}$. distilled water, with the addition of $8 \mathrm{c.cm}$. lactic acid) is added drop by drop to the liquid containing adrenalin, and it immediately loses its characteristic colour and acquires a distinct pink coloration. An excess of the brown reagent does not affect the result, the superfluity being immediately carried off by the addition of a minimal quantity of peroxide of hydrogen. The colorimetric result is ascertained by 
progressive weakening of the liquid to be tested until a reaction takes place, which, in regard to the intensity of the stain, is equal to a standard adrenalin solution of $\mathrm{I}: \mathrm{I}, 000,000$.

The amount of adrenalin in the suprarenal is ascertained by cutting the organ into small pieces and reducing it to a paste by means of quartz sand; ten times the bulk of a 2 per cent. acetic acid solution is added, and the mixture is allowed to stand for about twenty-four hours; the filtrate is then twice treated, each time with five parts of the same acetic acid solution, in a water-bath at $70^{\circ} \mathrm{C}$., and is again filtered. The filtrate, which still retains its brown colour, is bleached with ammonium sulphate, and is then ready for use. According to Zanfrognini, the adrenalin present in the human suprarenal remains unchanged for several days after death.

S. Fränkel and R. Allers describe a new and extremely sensitive and characteristic adrenalin reaction. It depends upon the fact that hydroidic-acid, or potassium biniodide and dilute phosphoric acid, when added to adrenalin solution and heated, change in such a manner as to produce an eosin pink colour in weak solutions, and a splendid rose-red colour where stronger solutions are used. On the further addition of ammonia, the red colour changes to a rust-brown. The reaction is obtainable with an $\mathrm{n} / 5000^{-}$adrenalin solution, which is thus a .00365 per cent. solution, or, in other words, contains $I: 300,000$. The reaction is wholly specific to adrenalin, and none of the substances for which adrenalin may be mistaken give a similar red stain. The characteristic coloration is probably due to the formation of an iodo- or an iodoso-combination on the part of the adrenalin.

As G. Bayer has shown, the adrenalin colour reactions with ferric chloride and with chromates, as well as with the FränkelAllers test, may be rendered more sensitive by the addition of aromatic amidosulphonic acids ( $a$-naphthylaminosulphonic acid, p-amidobenzolsulphonic acid). The sulphanil acid modification of the ferric chloride and chrome reactions ought to have a distinct value in microscopic and histological work. The iodine reaction of adrenalin is intensified by alanin and by phenylalanin, and probably also by other products of the decomposition of albumin.

A method of testing the urine for adrenalin, recently described by Schur, makes use of the red iodine reaction in the following manner : an excess of tincture of iodine is added to the liquid to be examined; the surplus iodine is then removed by repeated shaking-up with ether, and this is continued until the ether ceases to acquire a yellow tinge. Where adrenalin is present the specimen stains pink, and though this colour is permanent in pure adrenalin solutions, in urine it disappears after some time. Adrenalin can be detected by this method in urine, in a proportion of $I: 200,000$; in normal saline solution, in a propor- 
tion of I : I, 500,000. Pure urine frequently gives a similar positive reaction, and Schur thinks it possible that adrenalin is one of the constituents of normal urine. The sensibility of this iodine reaction may also be made use of in quantitative adrenalin tests.

The biological methods employed for the detection of adrenalin will be discussed later.

\section{THE PHYSIOLOGICAL ACTIVITY OF ADRENALIN.}

Of the physiological effects of adrenalin, that which it exercises upon the vascular apparatus is the most important and the most characteristic. This is readily seen if a certain quantity (about one-twentieth $\mathrm{mg}$.) of adrenalin is injected into the veins of an animal, and its blood-pressure registered by means of a kymograph. The injection is followed almost immediately by a rapid increase in blood-pressure which rises to a considerable height, but this is partly concealed by the fact that, soon after injection, the pulse becomes extremely slow. The increase in blood-pressure lasts a short time only, from thirty seconds to two to three minutes according to the dose, and the maximum is obtained for a few seconds only. The retardation of the pulse soon disappears also, and is followed by a gradual fall in pressure, the starting point being reached in two, or at the most four, minutes.

The increase in pressure is very much more perceptible if the fall in the pulse-rate is avoided. This is done by eliminating, by means either of atropine or resection, the inhibitory influence of the vagi upon the cardiac contractions. If this is done, the blood-pressure rises to twice, or even three times, the normal figure. Blood-pressures reaching 250 to $300 \mathrm{~mm}$. $\mathrm{Hg}$. may by this means be observed in dogs and rabbits, figures which are not obtainable by the action of any other substance. Where breathing is spontaneous, the respiratory fluctuations in bloodpressure disappear during the rise and while the tension is at its height; they reappear as the tension gradually diminishes.

A rise in blood-pressure follows the exhibition, of even minimal doses; the amount of the increase and its duration are to a certain extent proportional to the amount of substance injected. A typical increase in pressure of about 15 to $25 \mathrm{~mm}$. $\mathrm{Hg}$. is obtainable with $.0005 \mathrm{mg}$., and is certain with .oor mg., to every kilo of body-weight, of both the natural adrenalin and the synthetic suprarenin. A dose of $.005 \mathrm{mg}$. will raise the bloodpressure $50 \mathrm{~mm} . \mathrm{Hg}$.; and after or $\mathrm{mg}$. it may be increased by as much as 80 to $100 \mathrm{~mm}$. $\mathrm{Hg}$. An increase in the dose gradually prolongs the period of hypertension. The maximal degree of pressure is usually obtainable with .I $\mathrm{mg}$. adrenalin, while a further increase of two to tenfold in the amount of the dose 
frequently produces only toxic effects, such as fluttering or sudden arrest of the heart, and occasionally œdema of the lungs.

These conditions militate to a certain extent against an accurate estimation by means of blood-pressure readings, of the quantitative value of adrenalin solutions. The presence of an amount less than .ooI $\mathrm{mg}$. in I c.cm. cannot be proved with certainty by means of animal experiments. The approximate values of solutions containing a larger proportion of adrenalin may be estimated by means of dilution.

The effect of adrenalin in raising blood-pressure is primarily the result of vaso-constriction, that is to say, of a contraction of the walls of the peripheral blood-vessels. This constriction of the blood-vessels is clearly shown by the pale colour of the mucous membranes. By means of oncometric experiments, Oliver and Schäfer ascertained the volume of the different organs. They found that the abdominal viscera supplied by the splanchnic nerve, such as the spleen, kidney, and intestine, become considerably reduced in volume; while the volume of the extremities becomes increased, owing to the rush of blood into them from the splanchnic region. A. Velich found by direct inspection that there was a distinct reduction in the volume of the intestine, kidney, and conjunctiva.

With regard to the origin of this peripheral vaso-constriction, Cybulski and Szymonowicz assumed that it resulted from stimulation of the central vaso-motor apparatus in the medulla oblongata. But other experiments have shown that suprarenal extract produces an enormous rise in blood-pressure after resection high up the spinal cord and destruction of the medulla (Oliver and Schäfer), after destruction of the entire central nervous system (Biedl, Velich), and after resection of both splanchnic nerves (Boruttau). I have kept the arterial pressure of animals in which the central nervous system was entirely destroyed, at 140 to $160 \mathrm{~mm}$. Hg. for hours at a time, by means of continuous transfusion of a diluted solution of suprarenal extract. The rise in blood-pressure also takes place where, as the result of profound chloroform or chloral anæsthesia, the intervention of the central nervous system is excluded (Gottlieb). That the vaso-motor nerve-centres play a part in the production of the vaso-constriction cannot be denied, if the quantitatively stronger action in the sound animal, and the increased pressure which immediately follows the direct introduction of adrenalin into the cerebral circulation (injection into the carotid towards the brain, Biedl and Reiner) are taken into account. It is probable that the stimulation of the vascular centre is not direct, but is a secondary effect brought about by the local anæmia.

The vaso-contraction does not affect all parts of the system equally. The introduction of adrenalin into the general circulation affects primarily those vessels whose innervation proceeds 
from the splanchnic nerve, and these undergo the maximum degree of contraction. As Jonescu has pointed out, the renal vessels are the first to contract, and this effect is produced by doses which are too small to raise the pressure of the circulation as a whole. If then, constriction within the splanchnic region gives rise to a marked increase in tension, the increase in pressure will produce a passive dilatation of the vessels in other parts of the system, and this dilatation will be most pronounced in those portions of the circulatory system which are not actively affected by the constricting agent. Portions of the circulatory system which are comparatively slightly affected by the vaso-constriction are, apparently, the vessels of the extremities (passive increase in the volume of the extremities has already been referred to), the cerebral vessels and vessels of the retina, the pulmonary vessels, and the coronary vessels of the heart.

Spina showed that the intravenous injection of suprarenal extract produces so marked a dilatation of the cerebral vesseis, that the opening of the dura mater may be followed by hyperæmia amounting to cerebral hernia. But Biedl and Reiner found that the cerebral vessels may be induced to contract by the direct application of the agent. As soon, however, as the increase in tension becomes general throughout the circulation, this cerebral anæmia disappears as a result of the general high pressure. That adrenalin causes the cerebral vessels to contract was proved by Wiggers, who allowed Locke's fluid, to which adrenalin had been added, to stream through the brain of a dog; he found that the liquid, after leaving the brain, was considerably reduced in quantity.

Velich was the first to discover the effects of suprarenal extract upon the pulmonary circulation. He found that there was a slight increase in pressure in the pulmonary artery, but believed that this might be due to back pressure from the left auricle. Direct inspection of the lungs showed that neither the intravenous injection nor the direct application of adrenalin was followed by the blanching observed in other organs under similar conditions. Gerhardt also observed a slight increase in tension in the pulmonary artery, and he ascribed it to the fact that, owing to the contraction of the svstemic vessels, a larger amount of blood is carried to the right heart. According to Gerhardt, the pulmonary vessels are unaffected by suprarenal extract, and Brodie and Dixon were unable to prove a vaso-constriction of these vessels after the employment of adrenalin. They ascribe this result to inadequacy on the part of the vaso-motor innervation of the pulmonary vessels. Plumier found that large doses were followed by distinct vaso-contraction of the pulmonary vessels, and Petitjean arrived at the same conclusion in regard to these vessels as Biedl and Reiner in regard to the vessels of the brain. Petitjean believes that adrenalin induces the pulmonary vessels to contract, but that 
the contraction is very quickly counteracted by the passive dilatation.

The effect of adrenalin upon the coronary vessels of the heart is, in one particular, quite distinctive-it does not induce contraction. It will be shown later that strips cut from the coronary vessels react to adrenalin differently from other vessels, and the cause of this startling phenomenon will be explained. It will, moreover, be shown upon which factor the localization of the vascular effects depends.

The vaso-contraction produced by adrenalin may be observed, in both warm-blooded animals (Pick, Biedl) and in frogs (Läwen), by allowing the substance to circulate through organs removed from the living body (iiberlebend); the effect is shown by the marked reduction in the venous outflow. Läwen transfused a liquid composed of two parts suprarenin (Hœehst) to ten million parts Ringer's fluid, through the hinder extremities of Rana esculenta, and he found a reduction of 87 to 96 per cent. in the amount of the outflow; while a solution of ten times less concentration produced a reduction of 50 to 81 per cent. in the outflow. If the vessels are washed out with an indifferent liquid, they resume their normal size. Owing to the sensitiveness of this reaction, it is possible to employ artificial circulation through the excised living organ both as a test for the presence of adrenalin and as a means of estimating its physiological value.

The effect of adrenalin in producing local vaso-constriction is well known and forms the basis of its therapeutic employment. Adrenalin produces a high degree of vaso-contraction in very vascular, and especially in hyperæmic, localities. It was introduced into the therapeutics of ophthalmics by Bates and Darier, who employed it as a means for the reduction of the hyperæmia of inflamed and reddened conjunctiva. It has also been employed to produce local anæmia in oto-rhinology and dermatology. The work of $\mathrm{H}$. Braun suggested this substance as an adjuvant to cocaine and its congeners, and it has been largely employed in minor surgery for the production of local anæsthesia. Adrenalin was early employed as a styptic, more especially in paren-, chymatous hamorrhage. As early as i895, I made use of the vaso-contractor properties of adrenalin by employing it in experimental surgery to reduce parenchymatous hæmorrhage. I also recommended, and with good result, tampons soaked with sterilized suprarenal extract as a remedy in profuse uterine hæmorrhage. To-day, adrenalin is largely employed for the reduction of hyperæmia; as an adjunct to other measures in the production of local anæsthesia ; for the prevention or reduction of hæmorrhage in operation; and as a styptic.

Adrenalin is unable to penetrate the epithelial cover, hence it produces no apparent effect upon the normal skin and mucosa as long as these are intact. For therapeutic purposes, adrenalin is 
usually introduced into the tissues by subcutaneous injection; where, however, the mucosa are acutely inflamed and hyperæmic, the local application, either by instillation or by painting the surface, has a similar effect.

J. Baum has pointed out that the effect of suprarenal substance in producing blanching of the skin will be the more energetic, prompt, and sustained, the more nearly the condition of the vessels approximates to the normal. In acute inflammatory conditions the action is marked; where, however, the condition of the vessels is chronically pathological, as well as in all forms of destructive disease, the anæmia produced by adrenalin is slight and very transient, and telangiectatic veins do not become emptied. As soon as the anæmia passes, normal tissue regains its normal appearance, and hyperæmia supervenes only after repeated employment of the drug; but where there are chronic pathological changes in the tissue, the first application is followed by pronounced venous hyperæmia.

Upon which part of the vessels does adrenalin act? There is no doubt that, in large and small arteries with muscular walls, adrenalin produces a contraction of the musculature. It is a remarkable fact that, where adrenalin is employed locally, those injections disappear first which are described as parenchymatous, and it is more than probable that this is due to an enlargement of the capillaries. Further, it has been observed that, where adrenalin is applied locally to reduce parenchymatous hæmorrhage, not only do the great vessels not contract, but, owing obviously to the increased peripheral resistance, the great arteries have a stronger beat. If the circulation of frogs is watched through the microscope it will be seen that, after the introduction of adrenalin, the circulation in the capillaries is suspended at a moment when the blood in the arteries supplying them is still flowing with undiminished rapidity (Bukofzer). When adrenalin is injected into the veins, the volume of the organ decreases and the parenchyma becomes pale, but the great arteries which supply the organ are rather dilated than otherwise, and their beat is stronger; this is well seen in the mesentery, after the exposure of a loop of the intestine.

Since the contractility of the capillaries and their motor innervation was proved by Steinach and Kahn, the part played by this portion of the vascular system in the vaso-contraction produced by adrenalin, can no longer be doubted. These authors showed that there is a similarity of process between the contraction of an artery and the contraction of a capillary; for, in response to certain stimuli, the branched cells in the capillary wall (discovered by Rouget and S. Mayer) contract, and may so reduce the vessel as to entirely obliterate the lumen. An object eminently suited to observation of capillary conditions is the nictitating membrane of the living frog; if this is watched under the microscope, it 
will be seen that stimulation of the isolated sympathetic is followed by a contraction of the capillaries.

The constricting effect of adrenalin upon the veins seems to be slight, and has, up to the present, received little attention.

The characteristic effect of adrenalin, namely, the increase in arterial tension, is chiefly the result of an increased peripheral resistance due to the contraction, and consequent reduction in the size of the lumen, of the smaller arteries and the capillaries.

The enormous power which suprarenal extract has of producing vaso-contraction, forms the rationale of its therapeutic employment in all those conditions, where the circulation threatens failure in consequence of the sluggishness of the stream. Of these, the most comprehensive are : vascular paralysis, such as may be produced experimentally by destruction or inhibition, by means of toxins (chloral hydrate, chloroform), of the vaso-motor centres, and which is frequently seen in the last stages of some infective diseases and in many toxic states; and cardiac asthenia, in which, owing to inadequacy of the heart muscle and the vascular paralysis, those symptoms of decreased circulation make their appearance which are known as "shock" or " collapse."

Paralysis of the vessels and cardiac weakness both produce a fall in arterial tension, and lead to a deficiency in the blood supply to the vital nervous centres, as well as to an insufficient filling of the chambers of the heart, cardiac anæmia, and ultimate arrest of the heart's action. By producing contraction of the engorged ressels in the splanchnic area and so raising the bloodpressure, adrenalin effects a more favourable distribution of blood; it promotes the filling of the chambers of the heart and increases the supply of blood to the nervous system. I found that, even in cases where the nervous system was entirely destroyed, suprarenal extract not only prevented the cessation of the circulation, but that, by its aid, a normal circulation with a respectable arterial tension was maintained for some considerable time. It was upon the basis of these findings that, in 1895 at Professor Albert's Clinic, I injected sterilized suprarenal extract into the veins in two cases of cardiac and vaso-motor paralysis. In one case, the results were negative, but here the autopsy showed that death was due to embolism of the pulmonary artery. The second case was one of extreme collapse with arrested respiration, the result of chloroform narcosis, and here the effect of adrenalin was really startling. The injection was immediately followed by deep, slow respirations which gradually became more frequent, and a full radial pulse was perceptible, which was, however, somewhat slow at first.

The resuscitating effect of adrenalin has been repeatedly shown in a large number of animal experiments where, owing to hæmorrhage, or the arrest of cardiac and respiratory activity in 
chloroform anæsthesia, a fatal termination was threatened. Similar results have been observed in cardiac paralysis induced by potassium salts (Mankowski, Winter, Thenen).

Kothe recommends the clinical employment of an intravenous injection of $\frac{1}{2}$ to I c.cm. of a I : IOOO adrenalin solution in extreme collapse; where hæmorrhage threatens a fatal termination, he advises the addition of a few (6 to 8 ) drops of adrenalin to the normal saline solution employed for transfusion. Heidenhain observed that adrenalin in saline transfusions was attended by favourable effects in septic peritonitis, this condition, according to Romberg and Pässler as well as Heinecke, arising from paralysis of the vaso-motor centres.

Diluted adrenalin solution in large quantities has been employed with favourable results in the cardiac inadequacy of diphtheritic children (Pospischil).

Quite recently, F. Meyer showed that the intravenous injection of adrenalin saline solution into rabbits poisoned with diphtheria toxin and already moribund, was followed by a rise in blood-pressure and in the pulse-rate, an appreciable improvement in the respiration, and a return of the corneal reflex; death did not supervene until seven hours later.

In addition to its effect upon the vessels, adrenalin, as we have already seen, exercises a tonic influence upon the heart muscle, and for this reason its exhibition is indicated in all forms of circulatory inadequacy. Kothe's dictum: "That adrenalin is the most powerful analeptic which we possess" is justified by clinical experience (Meissl, Rothschild, Calmann, John).

In considering the other physiological effects of adrenalin, it must be well borne in mind that this substance exercises an intensifying influence upon a number of muscular and glandular organs which derive their innervation from the sympathetic system. Lewandowsky (I899) was the first to show that the intravenous injection of suprarenal extract into cats produces a contraction of those muscles of the eye and orbit which have a sympathetic innervation, and this finding was confirmed by Boruttau. Langley, and after him his pupil Elliott, next showed by means of the most careful experiments, that the action of adrenalin is wholly confined to tissues with a sympathetic innervation; and, moreover, that the effect of adrenalin, in any part of the body, is identical with that produced by electric stimulus of the sympathetic nerve supplying that part. Where the influence of the nerve is stimulatory, exciting contraction or promoting secretion, the action of adrenalin will also be stimulatory. Where, however, irritation of the nerve is followed by inhibition, the injection of adrenalin will also produce inhibition.

Before passing to a consideration of the grounds upon which this hypothesis is founded, it is proposed to give a brief summary of the doctrine of the innervation of the vegetative organs, as formulated by the English school of physiologists. 


\section{THE VEGETATIVE NERVOUS SYSTEM.}

It has been the custom hitherto upon anatomical and functional grounds, to divide the entire nervous apparatus into two great systems, namely, the cerebro-spinal or animal system, and the sympathetic or vegetative system. The first, by means of efferent nerve fibres, supplies impulses to the voluntary, striated muscles, and, by means of afferent nerves, receives communications from the organs of sense. The second controls all the involuntary functions; it innervates the unstriated muscular tissue throughout the body, as well as the secretory glands and the striated muscle of the heart; and it also controls certain striated muscle cells at the beginning and the end of the intestinal tract and in the external genitals.

From the results of descriptive anatomy it has long been known that the two systems are not entirely distinct, and this is confirmed by experiment. The sympathetic system is not independent, but is connected by means of numerous rami communicantes with both the brain and the spinal cord. The essential difference between the two systems lies in the fact, that communication between a striated muscle and its nervous centre is effected by means of a single neuron, composed of a ganglion cell, a peripheral nerve-fibre, and the terminal expansion of the latter; while in the case of the vegetative system, ganglia are interspersed along the entire length of the nerve, from its source of origin in the brain or cord to the organ where it ultimately terminates. This arrangement is carried out, in the first place, by the ganglia of the prevertebral chains, and, later, by ganglia of the second and third order. These ganglia possess a special physiological significance, for they represent breaks in the continuity of the neuron, each ganglion cell with its nerve fibre marking the commencement of a second neuron. Thus the vegetative system is anatomically characterized by the presence of pre-ganglion and post-ganglion fibres. That this distinction has a functional significance, in other woras, that a change in the nature of the stimulus takes place in the ganglion cells, is shown by the fact that stimulation of the pre-ganglion nerve fibres before and after inhibition of the ganglia (as the result of anæmia or of the action of nicotine, which has a specific paralysing influence upon these ganglion cells) produces entirely different effects.

The English physiologist, Gaskell, has further drawn attention to the fact that, though all the anterior roots of the spinal nerves supply motor nerves to the voluntary muscles, the efferent fibres supplying the vegetative organs (which Gaskell calls the "visceral nervous system ") do not leave the cord in an unbroken series, but are divided into three groups, separated from one another by the nerve roots of the plexus of the anterior and pos- 
terior extremities. The system has been variously named "vegetative," "sympathetic," " organic," and " ganglionary," but Langley abandons these terms in favour of the more comprehensive word " autonomous." Following Gaskell's division of the system into three groups, he names them as follows: (I) the cranio-cervical; (2) the thoraci-columbal; (3) the sacral. The central portion of the thoraci-columbal system, together with the thoracic and ventral chains and their plexuses and branches, forms the "sympathetic" system in its more limited sense; and with these must be included the plexuses which Anerbach and Meissner regarded as forming a separate intestinal nervous system.

The term "autonomous nervous system," as employed by Langley and the English authors following him, includes, then, all those nerves by which the involuntary organs are supplied, and according to this nomenclature the "sympathetic system " is a subdivision only of the great autonomous system. Other later authors, and the Germans more particularly, regard the sympathetic as distinct from the autonomous system; they include under the former heading, the prevertebral chains, and those nerve fibres whose roots are centred in the cord in a series'extending from the first thoracic to the fourth lumbar nerves. Under this system of classification the autonomous system comprises: (I) the cranio-bulbar nerve fibres, which are derived from the mesocephalon and the medulla oblongata, and which include the oculomotor (III), the facial (VII), the glossopharyngeal (IX), and the vagus $(\mathrm{X})$ nerves; (2) the sacral nerve fibres, which proceed from the lower lumbar and sacral portions of the spinal cord, and which combine to form the pelvic nerve.

This system of classification is based upon the fact that nearly all vegetative organs possess a double innervation.

The sympathetic system supplies the involuntary muscles all over the body, including those of the organs and of the vessels, and it also innervates the glands. But an exclusively sympathetic innervation belongs only to the sweat glands, the hair muscles of the skin, and a portion of the muscular structure of the intestinal vessels. In all other parts of the body there is, in addition to the sympathetic innervation, a cranial-autonomous or sacral-autonomous innervation, and in certain instances, as in the vasodilators of the extremities, there is also a spinal nerve supply. In many cases, the autonomous nerves are actually the physiological antagonists of the sympathetic nerves, influencing the voluntary muscles, the glands, vessels and heart in a manner diametrically opposed to that of the sympathetic nerves. Where the sympathetic in such instances promotes activity, the autonomous nerves inhibit it.

It was at first believed that the stimulatory and inhibitory nerves must be derived from different portions of the nervous system, the one belonging to the sympathetic, the other to the 
Vegetative

Autonomous Nerves.

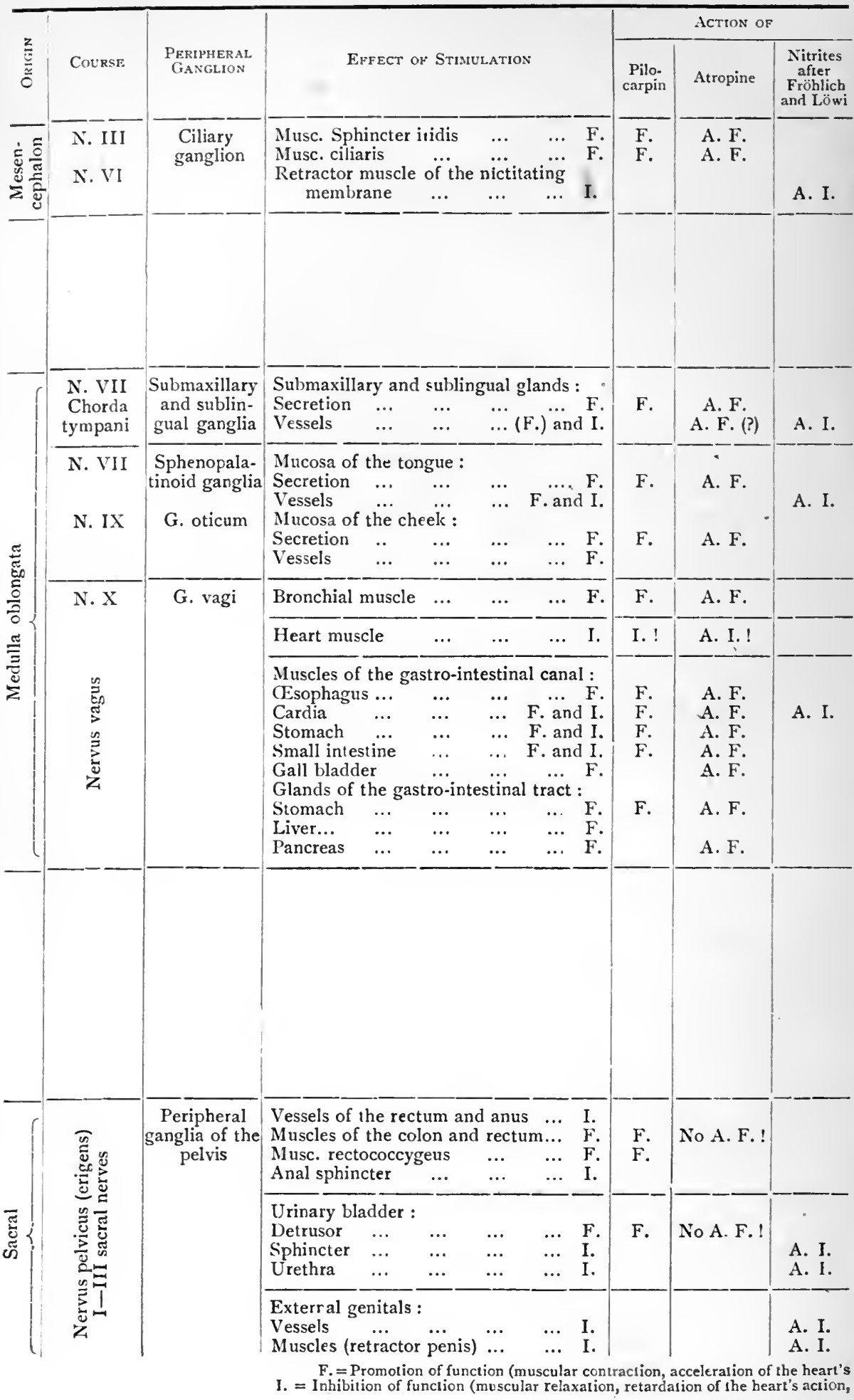


Sympathetic Nerves from the Thoraco-lumbar Roots.

\begin{tabular}{|c|c|c|c|c|c|}
\hline \multirow[b]{2}{*}{$\mathrm{O}_{\mathrm{RIG} 1 \mathrm{~N}}$} & \multirow{2}{*}{\multicolumn{2}{|c|}{ Effect of Stimulation }} & \multicolumn{3}{|c|}{ Action of } \\
\hline & & & $\underset{\text { alin }}{\text { Adren- }}$ & $\begin{array}{l}\text { Ergotoxin } \\
\text { after Dale }\end{array}$ & $\begin{array}{l}\text { Stimulation of the } \\
\text { sympathetic or by } \\
\text { adrenalin after } \\
\text { ergotoxin }\end{array}$ \\
\hline & $\begin{array}{c}\text { Retractor muscle of the } \\
\text { brane ... }\end{array}$ & $\begin{array}{c}\text { ctitating mem- } \\
\ldots \quad \ldots \quad \text { F. }\end{array}$ & F. & & \\
\hline $\begin{array}{l}\text { I-III Th. } \\
\text { I-II Th. }\end{array}$ & $\begin{array}{ll}\text { Muscle of the eyelid } & \ldots \\
\text { Orbital muscle } \ldots i l l & \ldots \\
\text { Musc. dilator pupillæ } & \ldots\end{array}$ & 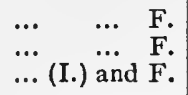 & $\begin{array}{l}\text { F. } \\
\text { F. }\end{array}$ & f $\begin{array}{l}\text { F. and } \\
\text { A. F. }\end{array}$ & Slight or no $F$. \\
\hline $\begin{array}{l}\text { II-IV Th. } \\
\text { IV-VII Th. }\end{array}$ & $\begin{array}{l}\text { Cerebral vessels ... } \\
\text { Vessels of the ear and hea } \\
\text { Muscles of the hair of the }\end{array}$ & $\begin{array}{lll}\ldots & \cdots & F . \\
\cdots & \cdots & F \\
\text { and neck } & \text { F. }\end{array}$ & $\begin{array}{l}\text { F. } \\
\text { F. }\end{array}$ & A. F. & No $F$. \\
\hline \multirow[t]{2}{*}{ II-IV Th. } & 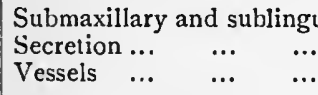 & $\begin{array}{ccc}\text { glands : } & & \\
\ldots & \ldots & \text { F. } \\
\ldots & \ldots & \text { F. }\end{array}$ & F. & A. F. & No $F$. \\
\hline & $\begin{array}{l}\text { Mucosa of the tongue : } \\
\begin{array}{l}\text { Secretion } \ldots \\
\text { Vessels } \ldots\end{array} \quad \ldots \\
\begin{array}{l}\text { Mucosa of the cheek : } \\
\text { Secretion } \\
\text { Vessels } \ldots\end{array} \quad \ldots\end{array}$ & $\begin{array}{lll}\ldots & \ldots & \text { F. } \\
\ldots & \ldots & \text { F. } \\
& & \\
\ldots & \ldots & \text { I. }\end{array}$ & $\begin{array}{l}\text { F. } \\
\text { F. } \\
\text { F. (?) }\end{array}$ & & \\
\hline I-V Th. & Heart muscle $\quad \ldots \quad \ldots$ & $\begin{array}{lll}\ldots & \ldots & F\end{array}$ & F. & A. $F$. & Slight or no F. \\
\hline \multirow{2}{*}{ 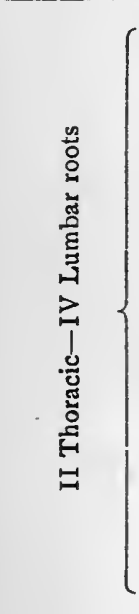 } & \begin{tabular}{llll}
\multicolumn{4}{l}{ Muscles of the gastro-inte } \\
CEsophagus & $\ldots$ & $\ldots$ \\
Cardia & $\ldots$ & $\ldots$ & $\ldots$ \\
Stomach & $\ldots$ & $\ldots$ & $\ldots$ \\
Small intestine & $\ldots$ & $\ldots$ \\
Gall-bladder & $\ldots$ & $\ldots$ \\
Gall-duct . & $\ldots$ & $\ldots$ \\
Ileocæecal sphincter (cat) \\
Secretion of the pancreas
\end{tabular} & 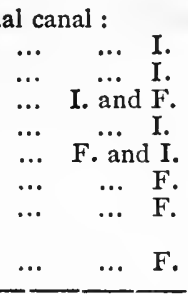 & $\begin{array}{l}\text { I. } \\
\text { I. } \\
\text { I. } \\
\text { I. } \\
\text { I. } \\
\text { F. } \\
\text { F. (?) } \\
\end{array}$ & $\begin{array}{l}\text { F. and A. F. } \\
\text { F. and A. F. } \\
\text { F. and A. F. }\end{array}$ & $\begin{array}{l}\text { I. } \\
\text { I. } \\
\text { I. } \\
\text { Negative action. }\end{array}$ \\
\hline & 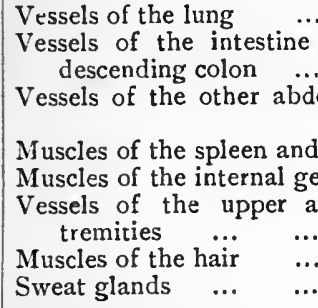 & 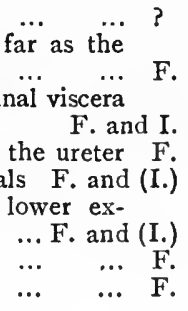 & $\begin{array}{l}? \\
\text { F. } \\
\text { F. } \\
\text { F. } \\
\text { F. } \\
\text { F. } \\
\text { F. }\end{array}$ & $\begin{array}{c}\text { F. and A. F. } \\
\text { F. and A. F. } \\
\text { F. and A. F. } \\
\text { F. and A. F. } \\
\text { F. and A. F. } \\
\text { F. and A. F. } \\
\text { A. F. }\end{array}$ & $\begin{array}{l}\text { I. } \\
\text { I. } \\
\text { I. } \\
\text { I. } \\
\text { I. } \\
\text { I. ? } \\
\text { Nessure } \\
\end{array}$ \\
\hline \multirow{3}{*}{ 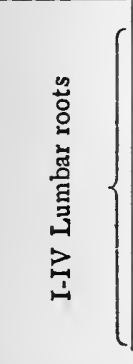 } & $\begin{array}{l}\text { Vessels of the rectum and } \\
\text { Muscles of the colon and } \\
\text { Anal sphincter (cat) }\end{array}$ & $\begin{array}{lll}\text { us } & \ldots & \text { F. } \\
& \ldots & \text { I. } \\
& & \\
\ldots & \ldots & \text { F. } \\
\end{array}$ & $\mathrm{F}$. & F. and A. F. & I. \\
\hline & 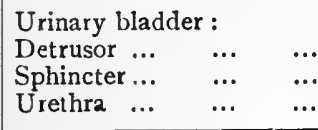 & $\begin{array}{ll}\ldots & \mathrm{I} . \\
\ldots & \mathrm{F} . \\
\ldots & \mathrm{F} .\end{array}$ & $\begin{array}{l}\text { I. } \\
\text { F. } \\
\text { F. }\end{array}$ & f. $\begin{array}{c}\text { F. and } \\
\text { A. F. }\end{array}$ & $\begin{array}{l}\text { I. } \\
\text { Negative action. }\end{array}$ \\
\hline & $\begin{array}{l}\text { External genitals : } \\
\text { Vessels \# \# \# } \\
\text { Muscles (retractor penis) }\end{array}$ & $\begin{array}{ll}\ldots & F \\
\ldots & F\end{array}$ & $\begin{array}{l}\mathrm{F} \text {. } \\
\mathrm{F} \text {. }\end{array}$ & F. and A. F. & Negative action. \\
\hline
\end{tabular}

activity, vasoconstriction, secretion). A. F. = Arrest of function.

vasodilation). A. I. = Arrest of inhibition. ! = Noteworthy exceptions. 
autonomous system.* Further investigation reveals the fact, however, that fibres which produce entirely opposite results are not necessarily derived from different sources. Indeed, it has been shown that both systems frequently send nerve fibres to the same organ and that the function of the fibres is apparently identical; on the other hand, nerve fibres producing completely opposite results appear to proceed from the same system.

We must assume that the complete nervous supply to a vegetative organ comprises a fourfold innervation, namely: (I) autonomous stimulatory; (2) autonomous inhibitory; (3) sympathetic stimulatory; and (4) sympathetic inhibitory. A number of instances of such fourfold innervation are already known, but investigation of the subject is of so recent a date that the information which is forthcoming is necessarily very incomplete. The conditions under which the innervation of the various vegetative organs takes place offer a wide field for inquiry in the future.

The table upon pp. 192 and 193 shows all that is at present known concerning the innervation of the vegetative organs. They also show the relationship between the individual organic functions and certain poisons.

The study of the action of certain toxins has brought important functional characteristics to light, and has revealed functional differences between the animal and vegetative systems on the one hand, and the autonomous and sympathetic portions of the vegetative system on the other. These differences are for the most part fundamental and in accordance with certain laws; and they depend upon the chemistry of the organs and, more particularly, upon the chemical differentiation of the nerve endings.

Langley showed that nicotine has an elective affinity for those ganglion cells, which, throughout the regetative system, produce an anatomical break in the continuity of the neuron, and in which a functional change in the nature of the nervous stimulus takes place. After the intravenous injection of nicotine-and the effect is even more marked if a diluted nicotine solution is applied directly to a ganglion-there is a transient stimulus, followed by paralysis, of those ganglion cells in which commutation of stimulus takes place. Electric stimulus produces certain effects upon nerve fibres of the vegetative system, and the results of this stimulus, whether applied before or behind a ganglion, are identical. But the effect of nicotine is to paralyse the obstructing ganglion cell-and its influence is entirely confined to this cellwith the result, that stimulus of the pre-ganglion fibre is negative, while the post-ganglion fibre responds in the ordinary way.

* Following a system of nomenclature adopted by English physiologists, it is proposed to describe any action, whether a muscular contraction, an increase in the cardiac function, a contraction of the vessels, or the promotion of the secretion of an organ as motor effect or function (F.). The prevention of function of whatever nature (muscular relaxation, cardiac insufficiency, vasodilation) is termed inhibition (I.). 
It is evident from this that the effects of nicotine are confined to the vegetative nervous system, for the efferent fibres of the cerebro-spinal system are nowhere interrupted by ganglia. Such being the case, nicotine may be used to determine whether certain results are due to stimulation of the vegetative or of the animal system; moreover, it will decide the question as to whether or not certain ganglia act as physiological commutators to certain nerve fibres. Anatomical investigation shows that each vegetative nerve contains several ganglia, but it is evident from the effects produced by nicotine that an actual interruption in transmission occurs at one point only in its course.

The elective action of other toxic substances upon the vegetative nervous system serves to differentiate between the activity of the sympathetic and the autonomous nerves. The elective, and at the same time antagonistic, effects produced upon certain organs and organic functions, by atropine on the one hand and by the so-called muscarine group of substances (muscarine, pilocarpine, physostigmine) on the other, have long been recognized and have, in a certain measure, been turned to account for therapeutic purposes. The substances of the muscarine group exercise a stimulatory effect upon those functions which are inhibited by atropine. Closer investigation shows that the organs influenced by these substances are exclusively those which possess an autonomous innervation, such as the eye, the salivary glands, and the organs supplied by the vagus nerve, namely, the heart, the bronchial musculature, and gastro-intestinal canal. The sweat-glands form a remarkable exception, for, although they are equally affected by the action of these toxins, their innervation, as far as we know at present, is entirely sympathetic. Moreover, as will readily be seen by referring to the table on pp. 192 and 193, the stimulatory action of pilocarpine, as representative of the muscarine group, and the inhibitory action of atropine, do not extend to all the nervous effects produced by the autonomous system, but are confined to those whose influence consists in the promotion of function. Pilocarpine is, then, a toxin which promotes autonomous activity; atropine is a toxin which inhibits autonomous activity. In the case of the heart muscle, on the contrary, the action of pilocarpine is inhibitory, while that of atropine is stimulating. Fröhlich and Löwi were able to prove that the nitrites are also inhibitory toxins whose action is confined to the autonomous nerve endings, but that their influence is extended solely to those whose function is inhibition.

Adrenalin is a substance possessing an elective affinity for the sympathetic system. The foundation for this theory will be fully discussed in the following chapter. It will be seen from the table given above (pp. 192 and 193) that adrenalin affects those tissues only which have a sympathetic innervation; and that its effects are identical with those which may be obtained by stimu- 
lating the sympathetic nerve fibres of certain organs. The invariable effect of adrenalin upon the sympathetic nerve fibres is to intensify their activity, whether the nature of that activity be stimulatory or inhibitory.

With regard to the two last columns of the table, the following facts must be borne in mind. The column before the last shows the effects of ergotoxin upon the sympathetic system, while the last column shows the effects of stimulation, by electricity and by adrenalin, upon a sympathetic system which has been poisoned by ergotoxin. Dale has shown that the toxic influence of preparations of ergot, and especially of ergotoxin, produces a stimulation, followed by inhibition, of the sympathetic nerve endings, this toxic influence being solely confined to their function-promoting activity, their function-retarding activity remaining unchanged. An injection of adrenalin given under these conditions produces, therefore, a paradoxical result. The conclusions to be drawn from these phenomena will be discusised later.

\section{THE PHYSIOLOGICAL EFFECTS OF ADRENALIN UPON THE SYMPATHETIC SYSTEM.}

The Vessels.--The striking effect which the-active principle of the suprarenal has in producing contraction of the vessels, naturally suggested the question: Is this result due to the direct action of the substance upon the contractile elements of the vessel walls; or is it a secondary result, the outcome of the stimulation of peripheral structures included in the sympathetic system?

Oliver and Schäfer at first assumed a direct action upon the muscular tissue, and among the proofs which they advanced in support of this theory was the effect produced by suprarenal extract upon the heart and upon the striated muscles.

Support is given to the theory of a direct action of the substance upon the vessel walls by the fact that, at a developmental stage when nervous elements are not demonstrable in the vessel walls, a distinct contraction of the vessels may be produced in the embryo chick by the direct application of a drop of suprarenal extract (Königstein).

By the artificial circulation of adrenalin through the hinder extremities of dogs, I discovered that the contraction of the vessels, which is shown by the decrease in the venous outflow, will also take place if the animal is first paralysed by means of large quantities of curarin, or if curarin has been previously added to the blood circulating through the extremities. Tillie proved that curarin produces paralysis of the vaso-constrictor nerve terminals, and these experiments of mine go to show that the effect of the substance in producing vaso-constriction is due, at least in part, to a direct action upon the muscular structure. I 
found that the effect of suprarenal extract is much stronger before than after the exhibition of curarin, and it seems certain from this that the peripheral nervous elements play the chief part in the production of vaso-constriction.

Langley found that, in the case of the submaxillary gland, even where the post-ganglionary fibres had undergone degeneration, the intravenous injection of suprarenal extract was followed by constriction only of the vessels, and he believed that the action of the substance was, in all probability, directly muscular. $\mathrm{He}$ emphasizes the faet, however, that the effects of suprarenal extract vary in different parts of the vascular system, and he draws attention to the parallel between the action of this substance and the effects of electric stimulus upon the sympathetic nerves.

According to Brodie and Dixon, the fact that the influence of adrenalin upon the pulmonary vessels is negative, is a sufficient proof that this substance affects the sympathetic nerve endings and these only, and has no influence whatever upon the unstriated muscles.

In order to test the effects of adrenalin upon the nerve terminals, these authors first induced, by means of cocaine, curarin, or apocodein, a paralysis of the systemic vaso-constrictor nerve fibres. They were unable to obtain satisfactory results with cocaine, but they found that, after curarin, the action of adrenalin, although not entirely neutralized, was very much slighter in effect. Läwen, on the other hand, found that the effects of suprarenin, when introduced into the circulation of frogs previously treated with curarin, were in no way diminished; he concludes from this that there is not a direct antagonism between curarin and suprarenin, and he believes that suprarenin exercises a direct action upon the muscular structure of the vessels.

Dixon's experiments with apocodein bring an even more certain proof of the effects of adrenalin upon the nerve terminals of the vessels of both the heart and the intestine. Dixon showed that apocodein, which is an alkaloid obtained by dehydration from codein, when given in moderate doses, affects the sympathetic nerve cells in exactly the same way as nicotine. A moderate dose of apocodein causes vaso-dilation with consequent hypotension; it accelerates the heart's action; and it intensifies the autonomous movements of the unstriated muscles of the intestine. Apocodein in moderate doses does not in any way affect the physiological action of adrenalin.

When given in large doses, apocodein produces a paralysis of the nerve terminals of the striated muscles, similar to that caused by curarin; a paralysis of the vagus terminals in the heart, similar to that produced by atropine; and a complete paralysis of the vaso-motor nerve endings. At this stage of apocodein poisoning, adrenalin does not provoke a rise in blood-pressure nor does it affect the portions of the intestine which have a sym- 
pathetic innervation. Substances such as barium, which exercise a direct influence upon the unstriated musculature of the vessels, produce an increase in blood-pressure even under these conditions. Dixon concludes from this that adrenalin acts on the sympathetic nerve endings.

That the effect of adrenalin upon the blood-vessels is identical with that produced by stimulation of the sympathetic nerves could not be doubted if proof were forthcoming that, where the sympathetic innervation is inhibitory and therefore vaso-dilator, the effect of adrenalin is also to produce vaso-dilation.

Dastre and Morat point out that the sympathetic system furnishes vaso-dilator fibres to the bucco-facial region, and $\mathrm{R}$. Bradford discovered that it also supplies vaso-dilators to the kidneys. Elliott was unable to obtain a primary vaso-dilation by means of adrenalin in any part of the body; upon the principle that the vaso-dilator nerves react to very slight stimuli, he reduced his doses to the minimum, but was still unsuccessful. Sollmann and Pari observed an occasional dilation of the renal vessels after adrenalin, while Jonescu has recently pointed out that minimal doses are followed by a constriction of the renal vessels.

Elliott and Dale were successful in proving the vaso-dilator properties of adrenalin by means of chrysotoxin. After the intravenous injection of $100 \mathrm{mg}$. of chrysotoxin, stimulation of the splanchnic nerve is followed, not by a rise, but by a fall in blood-pressure. Under similar conditions adrenalin also produces a fall in pressure, which lasts for about the same length of time as the rise which takes place under normal conditions. This fall in blood-pressure, whether brought about by the agency of adrenalin or by electric stimulus, is probably due to stimulation of the vaso-dilator nerve endings.

The vaso-dilator effect of adrenalin may also be seen in the coronary vessels of the heart. It has long been known that adrenalin rather increases than diminishes the circulation of blood through the heart, and this result has been attributed to the increased cardiac activity. Elliott showed that adrenalin will produce an increased circulation in a portion of ventricle flooded with Locke's fluid.

Owing to the fact that portions of arteries respond actively to the influence of adrenalin after their removal from the living body, a great deal of information has been obtained concerning the effect which adrenalin has upon the vessels and its dependence for this effect upon the nature of their innervation. The experiments of Bayliss, MacWilliam and v. Frey show that isolated blood-vessels, if suitably treated, will remain alive and sensitive to stimulus for days. Prompted by the results of these experiments, O. B. Meyer succeeded in working out a method by which the influence of different drugs upon the vessels might be studied. A circular strip is cut from an artery and suspended lengthwise in 
some Ringer's solution, which is kept at the temperature of the body and through which oxygen is allowed to circulate; the alterations in the length of the specimen are recorded graphically by means of a lever. The walls of the large arteries of cattle become appreciably shortened by the addition of adrenalin, and the degree of the contraction is, up to a certain point, proportionate to the amount of adrenalin added. The concentration necessary to produce an effect was found to be .oooor $5 \mathrm{mg}$. to $15 \mathrm{c.cm}$. Ringer's solution (I : 1000 million); by increasing the concentration, the amount of contraction was increased; and maximal results were obtained with a concentration of $\mathrm{I}: 50,000$.

Owing to its sensitiveness, this reaction is a reliable biological test both for the presence and the quantity of adrenalin.

Meyer proved the presence of adrenalin in defibrinated bullock's blood or serum by this method. We shall consider later the extent to which Meyer's method may be employed as a test for the presence of adrenalin in the heterogeneous blood serum. I have proved the sensitiveness of the reaction in numerous experiments. I was able, by this means, to detect adrenalin in the ganglia of the ventral nerve-cord of leeches; and I found that an extract, obtained from the fresh ganglion chains of ten leeches in $2 \mathrm{c.cm}$. of normal saline solution, produces a vascular contraction equal to that obtained with $10^{-8}$ to $10^{-7}$ adrenalin concentration. In this instance, the ferric chloride reaction was uncertain. Fraenkel's adrenalin test with the uterus taken from a living rabbit (to be described later) gave results almost identical with those which I obtained with portions of arteries.*

Meyer proved by his method that, not only does adrenalin act upon the customary preparations from the carotid and the subclavian arteries of bullocks, but that it also reduces the length of sections taken from the pulmonary artery and of longitudinal strips from the jugular vein. He also found by this method that there is an antagonism between the effects of adrenalin and those of atropin, curarin, and cocaine, and he assumes, therefore, that adrenalin affects the nerve terminals. As further proofs in support of his theory, he adduces the following : (I) That adrenalin is active in low concentration and within certain temperature limits only; (2) that it ceases to affect preparations which are a few days old, at a stage when they are still sensitive to muscular electric stimulus; and (3) that adrenalin stimulates the vascular muscles, while it inhibits the contraction of intestinal muscles which have been removed from the living body.

O. Langendorff obtained conclusive proof, by Meyer's method,

* The statement which appears in a monograph by v. Neusser and Wiesel, to the effect that "according to Wiesel " the physiological activity of the ganglia of the ventral cord of invertebrates exactly coincides with that of the chromaffine tissue of vertebrates, is the result, Wiesel informs. $\mathrm{me}$, of a mistake in the name of the author. 
that the effect of adrenalin upon the vascular muscles depends upon their vaso-motor innervation. The treatment, with adrenalin, of portions taken from the coronary vessels of the ox is followed, not by shrinking, but by a distinct lengthening of the specimens. In this instance, therefore, adrenalin does not provoke contraction but dilation. This result is explicable if we take into consideration the fact that the sympathetic innervation of all peripheral vessels is constrictor, while, according to $\mathrm{P}$. Maass, the sympathetic nerves supplying the coronary vessels of the heart (which include the first thoracic ganglion and the ansa Vieussenii) contain only dilator fibres, the contractors being conveyed by the pneumogastric nerve.

It may be noted in passing that the antagonism, which Langendorff discovered between the cardiac vessels and the other peripheral vessels, is demonstrable by means of substances other than adrenalin. Eppinger and Hess found that, unlike adrenalin, pilocarpin, physostigmin and cholin hydrochlorate (Merck) produce a shrinking-that is to say, a contraction-of portions taken from the coronary vessels, while in the case of the peripheral vessels, they induce dilation as shown by the increase in length. Certain substances influence both sets of vessels in the same manner, barium chloride, calcium salts and the digitalis bodies producing contraction, while atropine, ergotin and the nitrites produce dilation. According to Pal, pilocarpin contracts the coronary, mesenteric and femoral arteries and dilates the renal artery.

The observations described above justify the conclusion that the site of the influence of adrenalin is to be sought, not in the muscular structure of the vessels, but in those apparatuses which are in communication with the sympathetic nerve endings.

The Heart.-As we now know, the full effects of adrenalin upon the heart are to be seen only after the inhibitory vagal nerve endings have been paralysed by means of atropine. If the vagi are allowed to remain intact, the predominant effect will be the slowing of the rhythm, due to the increased arterial tension. Even the resection of the vagi at the necli does not entirely overcome the tendency to a retarded rhythm, interrupted by single arhythmic beats. The slowing of the pulse and the extra-systolic arhythmia are sufficiently explained by the increased venous tension and consequent impossibility of completely emptying the left ventricle.

The full action of adrenalin may, however, be observed in the mammalian heart in situ after the vagal terminals have been paralysed with atropine. There is, as a rule, a further acceleration of the contractions and there is, especially, a stronger ventricular systole which is well seen by registering the contractions or by means of a plethysmograph. This increased cardiac activity is the result, not of improved circulatory conditions arising out 
of the heightened blood-pressure, but of the direct action upon the heart of the substance itself. Thus, the intravenous injection of suprarenal extract will bring back life into an animal poisoned with chloral, in which the action of the heart is almost or entirely obliterated. The injection is followed by a progressive increase in the rapidity and power of the contractions; the blood-pressure, which had fallen almost to nothing, rises; and, after a second injection, the heart resumes its normal activity which is maintained. A similar result may be obtained after poisoning with potassium salts, which bring about a cessation of diastole and thus are typical cardiac poisons (Gottlieb).

The resuscitating effect of adrenalin upon the heart-the manner in which it hastens and strengthens cardiac contraction-is undoubtedly an important factor in the analeptic action, already described, which this substance has in conditions of collapse due to paralysis of the vessels or to temporary cardiac insufficiency.

The action of adrenalin may also be observed in hearts which have been removed from the living body. Oliver and Schäfer experimented with isolated frogs' hearts through which normal saline solution was allowed to circulate. They found that suprarenal extract produces spontaneous contraction in a motionless heart; in the case of a feebly beating heart, the beats become stronger and more rapid, the irregularity disappears, and rhythm is restored.

The tonic influence of adrenalin upon the isolated mammalian heart when flooded by Langendorff's method is very clearly shown by the acceleration and remarkable increase in power of the contractions (Gottlieb, Hedbom, Braun and Mager, Cleghorn). Weakness of the heart's action, arising from prolonged deprivation of the nutrient fluid or from the action of toxins, is invariably corrected by the application of adrenalin, and it is not until after the method has been continued for some time that the reduced cardiac activity again becomes apparent in the guise of arhythmia and reduction in frequency. Hering-Bock found that an increase in the pulse-rate and a stronger cardiac contraction also followed the employment of adrenalin in the isolated cardiopulmonary circulation.

Gottlieb endeavoured to discover with isolated frogs' hearts, whether suprarenal extract acts directly upon the cardiac muscles or upon the ganglia. The action of the ventricle was arrested by means of the first ligature of Stannius between the sinus venosus and the auricle; by the injection of a small quantity of suprarenal extract, it was next induced to contract rhythmically in the same manner as after the so-called second ligature of Stannius. After the application of the second ligature of Stannius, however, the ventricle, which was then entirely deprived of its ganglia, failed to contract even in response to injections of suprarenal extract, and it responded to mechanical stimulus with a single contraction 
only. These results led Gottlieb to the conclusion that suprarenal extract is a powerful stimulant of the vaso-motor ganglia of the ventricle.

The question as to whether the effect produced by adrenalin upon the heart represents a direct muscular action, or is due to an affection of the sympathetic nerve endings which reaches the heart by way of the nervi accelerantes, has formed the subject of much recent discussion. The following results are advanced as evidence in favour of the latter hypothesis.

According to Elliott, the cardiac action of adrenalin is not observed in all animal species. The heart of invertebrates, as the crab, does not appear to react to it in any way.

Gaskell's experiments go to show that, in the heart of turtles and birds, the auricle is provided with augmentary fibres, while the ventricle possesses neither stimulatory nor inhibitory fibres. Elliott found that, in the turtle, the effect of adrenalin is to strengthen and accelerate the contractions of the auricle only, the ventricle remaining entirely unaffected. When the heart is undamaged, the acceleration probably affects the ventricular rhythm only, though this has the effect of shortening the contractions. The isolated hearts of birds (pigeons) become very much stimulated if adrenalin is added to the fluid circulating through them. After separation of the auricles, the ventricular beat is suspended for a time and adrenalin produces no effect upon the activity of the ventricles, but portions of the auricle respond to the action of adrenalin by accelerated contractions. As a general rule, those parts of the heart react more readily to adrenalin which are innervated, either wholly or in part, by the sympathetic system; thus the auricles react better than the ventricles.

The effect of suprarenal extract upon the hearts of fish is to produce a much stronger contraction of the ventricles. This is clearly shown in the experiments which I carried out in I899 with the object of finding the curve of the cardiac activity of torpedoes. The rhythm of the heart when in situ is rather retarded than otherwise. The blood-pressure, which was taken at a branchial artery, rose to very nearly twice the normal figure. A similar effect was obtained with extract of the suprarenal bodies of animals of the same species.

By means of experiments with isolated hearts, GatinGruzewska and Maciag have recently discovered that the hearts of cold-blooded animals are much less sensitive to adrenalin than those of warm-blooded animals. The effect of adrenalin upon the former is to increase the duration of systole and this is followed by a slowing of the rhythm; while in the case of hearts taken from rabbits, the use of minimal doses is followed by stronger and more rapid contractions.

In the case of the embryonal heart, the effect of adrenalin seems to depend upon whether or not it possesses a separate 
sympathetic innervation. Scott Macfie found that the cardiac movements of an embryo chick, two to three days old, were entirely unaffected by adrenalin. According to Kuliabko, the heart of the human embryo is very little influenced by this substance. Quite recently, I found that the heart of the embryo chick is insensitive to adrenalin at a time when the vessels of the vascular area contract readily in response to this substance. Upon the fifth to seventh day, when the intrusion of sympathetic elements has already taken place and a substance which, upon the ground of its biological reactions, is assumed to be adrenalin, is present in embryonic extract, the response of the heart to adrenalin stimulus is clearly shown in the acceleration of the contractions and in single arhythmic beats.

Dixon's experiments with apocodein supply definitive information concerning the actual site which adrenalin affects in the mammalian heart.

The immediate effect of apocodein is to produce a paralysis of the inhibitory vagal nerve endings in the heart. At this stage, adrenalin acts in the same manner as upon a heart in which the vagal terminals have been paralysed by means of atropine; that is to say, it brings about an increase in the rate and the volume of the beats. Further doses of apocodein have the effect of entirely neutralizing the action of adrenalin. The increase in the pulserate (from 93 to 2 I I a minute) produced by adrenalin almost entirely disappears (93 to IOI), and a still further application of apocodein is followed by an actual fall (87). Even when used in large doses, adrenalin fails to either accelerate or strengthen the systole. The effect of apocodein is to paralyse the acceleratory nerve endings, upon which adrenalin exercises a stimulating influence.

These results seem to show that the action of adrenalin consists in a stimulation of that portion of the sympathetic apparatus whose function it is to promote cardiac activity.

This assumption is confirmed by certain phenomena, recently observed after intravenous adrenalin injection by means of the electrocardiograph, by R. H. Kahn, some of which phenomena are also obtainable by means of strong artificial stimulation of the vagus nerve. Such are the prolonging of the refractory period, the heart-block, and the automatic and weakened ventricular beat. The phenomenon which is specific to adrenalin is the disassociation of the auricular and ventricular beats, which is continued for some time, and in the course of which the ventricular beat gives normal electrograms. After resection of both vagi, all these phenomena disappear, and the rise in bloodpressure is recorded without important changes in the electrocardiogram.

The Digestive Tract.-Strips cut from the œsophagus or stomach of frogs, when fresh, possess considerable tone, but 
after they are wetted with suprarenal extract they become relaxed, cease to contract spontaneously, and are less sensitive to electric stimulus (Boruttau, Botazzi). The inhibitory action which adrenalin has upon the rhythmic contractions of the stomach-ring of frogs or eels, is observed with a concentration as low as $1: 10^{-6}$ (O. B. Meyer).

Langley found that the intravenous injection of adrenalin into rabbits was followed by an immediate relaxation of the cardia, in consequence of which a fluid present in the œesophagus was permitted to pass. In no case was there a contraction of the oesophagus muscles, and even the spontaneous contractions disappeared. The action of adrenalin is the reverse of that of the vagus, for peripheral irritation of the vagus is followed by a contraction of the gastric sphincter. If the stomach is exposed, it will be seen that the action of adrenalin is to produce inhibition of the spontaneous movements. As soon as the relaxation of the cardia ceases, rhythmic contractions of the stomach take place; thus the effect of suprarenal extract resembles that of irritation of the splanchnic nerve. Elliott was also able to show that the spontaneous movements of the stomach are inhibited in the same manner, by both the action of adrenalin and by stimulation of the splanchnic nerve, the entire muscular structure becoming relaxed; irritation of the vagus, on the other hand, produces contraction of the stomach and peristaltic movements. The pylorus, in rabbits, contracts in response to adrenalin in the same way as after stimulation of the splanchnic nerve.

The effect of suprarenal extract upon the small intestine is shown by inhibition of the peristaltic and oscillatory movements, though after a few minutes these reappear (Boruttau, Pal). According to Kuliabko and Alexandrowitsch, adrenalin improves the tone of the intestinal muscles, and gives rise to oscillatory movements.

The inhibitory action of suprarenal extract is even more marked in the case of the large intestine. In the rabbit there is relaxation of the internal anal sphincter and blanching of the mucosa. In the cat and the dog there is a contraction of the sphincter similar to that produced in these animals by irritation of the sympathetic nerve-fibres.

The similarity of the effects produced by adrenalin and by stimulation of the sympathetic are demonstrable along the entire length of the gastro-intestinal tract. With the exception of the three sphincters (pyloric, ileo-cacal, internal anal), which contract, there is a general muscular relaxation.

This similarity of result persists in the intestines of birds and amphibians, in spite of the fact that here the innervation conditions are not the same. The œsophagus of frogs and turtles relaxes, and the stomach contracts in response both to adrenalin and to stimulation of the sympathetic. In fowls, 
adrenalin produces a tonic contraction of the duodenum and, to a certain degree, of the rest of the small intestine; the large intestine relaxes, and the ileocæcal and internal anal sphincters contract. Irritation of the vagus produces the reverse effects; and irritation of the spinal roots of the sympathetic produces results identical with those of adrenalin.

The Urinary Bladder.-Lewandowsky found that the intravenous injection of suprarenal extract was followed by a relaxation of the detrusor fibres, which was shown by a reduction of the internal pressure. Langley found that the results which he obtained were similar to those produced by stimulation of the inferior mesenteric ganglion or of the hypogastric nerve. The local application of suprarenal extract produces blanching accompanied by local contraction, which is, however, followed by relaxation.

Elliott investigated the innervation conditions of the bladder and urethra in different varieties of mammals, and he found that the effect of adrenalin upon the muscular structure of the bladder was in all cases coincident with a sympathetic innervation.

In all mammals, sympathetic fibres proceed from the lumbar spinal roots to the urinary bladder by way of the hypogastric nerve, and autonomous fibres pass to it from the sacral roots by the agency of the pelvic nerve (errigens). Electric stimulation of the hypogastric nerve is followed, in cats, by a brief contraction of the bladder, which principally affects the fundus, and this is succeeded by considerable relaxation of the bladder accompanied by a violent contraction of the urethra. The intravenous injection of adrenalin immediately provokes a short contraction of the fundus and of the urethra, together with a complete relaxation of the body of the bladder. While the effect of adrenalin lasts, stimulation of the pelvic nerve fails to produce contraction.

In the ape (Macacus rhesus), the effect both of adrenalin and of hypogastric stimulation is to produce a slight relaxation of the bladder. In the rabbit, irritation of the hypogastric produces a slight increase in internal pressure and contraction of the urethra, similar effects being obtained with adrenalin. The urinary bladders of the guinea-pig, the hedgehog, and the rat do not react to adrenalin. In the dog, stimulation of the hypogastric produces only very slight contraction of the fundus, and the influence upon the muscular structure is equally insignificant. In the ferret (Mustelus putorius, var. domestica), irritation of the hypogastric is followed by complete contraction of the bladder. It is a remarkable fact that, in this animal, the motor innervation of the bladder is derived from the sympathetic system and the inhibitory innervation from the sacral autonomous system. A small quantity of adrenalin given intravenously is also followed, in this animal, by violent and prolonged contraction of the bladder. The remarkably strong 
peristaltic movements of the ureters are not influenced either by adrenalin or by stimulation of the sympathetic. In spite of the close relationship between the Mustelides and Herpestes mungo, the bladder of the latter does not react to adrenalin. The urinary bladder of goats and of the Indian civet-cat (Viverra zibetha) responds in the same manner as that of the ferret.

This difference in the innervation of the bladder in mammals serves to show very clearly that adrenalin may exercise an inhibitory action upon unstriated muscles, if these muscles receive an inhibitory innervation from the sympathetic. The muscular inhibition produced by adrenalin is entirely independent of the blanching provoked by this substance; for, in the cat, the latter effect is almost entirely absent, while the relaxation of the muscles is perceptible in every part of the bladder, and may be produced by direct local application.

That the specific action of adrenalin is, in the case of certain tissues, independent of the blanching process, is also seen in the heart, where the coronary vessels remain uninfluenced, while the rapidity and strength of the contractions is considerably increased. The direct effect of adrenalin independently of the blood supply, namely, relaxation and cessation of spontaneous moyements, may also be observed in a portion of living intestinal wall after its removal from the body.

The External Genital Organs.-After the intravenous injection of suprarenal extract, these organs become blanched and contract in a manner similar to that which follows stimulation of the lumbar sympathetic (Langley and Anderson). The tunica dartos of the testicle has a different reaction-it does not contract in response to suprarenal extract. According to $\mathrm{S}$. Lieben, the intravenous injection of adrenalin provokes relaxation of the tunica dartos.

The Internal Genital Organs.-Langley observed a high degree of contraction and marked blanching of the vagina and cornua in rabbits and cats, after the intravenous injection of adrenalin in small doses. In male animals, there was a contraction of the vas deferens.

The similarity between the effects of adrenalin upon the uterine muscles, and those of stimulation of the sympathetic, will be fully discussed later. For the present purpose, the following will suffice.

The effect which adrenalin has upon the uterus is among the most perfect and startling of the phenomena which this remarkable substance calls forth. The blanching and contraction are well seen in the virgin uterus, but these appearances are nothing short of astonishing in the uterus of the pregnant or puerperal animal observed in a normal saline bath. The intravenous injection of adrenalin produces a degree of uterine anamia and a violence of contraction, not obtainable by the 
agency of any other substance. I found that, in pregnant animals, the intravenous injection of adrenalin was frequently followed by abortion. If, in the later stages of pregnancy, a premature birth is obtained by artificial means, it is usually accompanied, at least as far as my experience goes, by serious, or even incurable, hamorrhage. The intravenous injection of . I mg. adrenalin will, however, immediately arrest such hamorrhage. It will be seen by direct inspection that adrenalin produces a high degree of contraction of the uterine muscles as well as of the vessels, and this is sufficient to account for the cessation of the hamorrhage. Adrenalin not only produces uterine contractions, but it increases the excitability of the organ to such a degree, that it reacts to direct or nervous stimuli to which it was previously insensitive.

The specific action of adrenalin upon the uterine muscles was turned to account by Neu in the therapeutics of obstetrics. He recommended the direct injection of adrenalin into the substance of the relaxed and enlarged uterus. By this means the desired contraction was obtained, while the possible ill-effects of intravenous injection were avoided. I have found from personal experience that the method is free from objection.

The action of adrenalin upon the living uterus is also seen after the removal of that organ from the body. Acconi, and after him Franz, proved the effects of certain pharmacological reagents upon both the automatic movements of the uterus, and upon portions of extirpated uterine tissue obtained from man and animals. Kurdinowsky next discovered that it was possible to prolong the life of the uterus of a rabbit for days at a time by the transfusion of Locke's fluid into the aorta. He found that adrenalin, even in very weak solutions, acts more powerfully upon the uterus than any known substance. By adopting Magnus's method with the intestine, Kehrer obtained graphic tracings of the spontaneous movements of the uteri of rabbits and cats. Still more recently, A. Fraenkel employed the uterus removed from the living body of rabbits as a quantitative test of adrenalin. He found that adrenalin solutions reacted upon portions of the uterus in such a manner as to produce a specific change in the curve of the spontaneous contraction-waves. The lever rises rapidly and very decisively to a point above the summit of the highest normal wave; it remains in this position for several minutes, oscillating slightly; and then falls slowly to the abscissal line, at the same time making wide oscillations. The curve produced by the action of adrenalin is the expression of the transitory maximal contraction effected by this substance. This contraction takes the form of a tonic stimulation, starting promptly and increasing slowly until, in some instances, a tetanic condition obtains. According to Fraenkel, and I am in a position to confirm his results, the uterus of rabbits responds to an adrenalin 
solution of $I: 20$ million. The amount of contraction is dependent, within certain limits only, upon the size of the dose. When employing this method as a quantitative test, the degree of contraction must not be taken as the standard; the latter is obtained by accurate estimation of the degree of concentration which will produce a typical tonic increase. Of the biological tests for adrenalin, registration of the uterine contraction is undoubtedly the most effective.

The Hair Muscles.-As Lewandowsky pointed out, suprarenal extract produces a contraction of the erector muscles of the hair in cats, and an erection of the quills in sea-urchins. Langley next discovered that, in the cat, the erection of the hair of the head takes place in the same way after extirpation of the superior cervical ganglion and degeneration of the post-ganglionary fibres. Taking this finding in conjunction with the fact that all the pilomotors are included in the sympathetic system, he concludes that suprarenal extract exercises a direct influence upon the unstriated muscle-fibres of the hair.

The unstriated muscles of the skin show a remarkable departure from the phenomena usually associated with the action of adrenalin and the results of sympathetic stimulation. The back and tail hairs of cats stand up immediately in response to stimulation of the corresponding sympathetic fibres, but they react only slightly and tardily to the injection of even large doses of adrenalin. In the ape, the hairs are observed to move upon the temples and forehead only. In the dog, the injection of adrenalin is followed by the erection of the hairs of the neck, and in Herpestes mungo by prompt erection of the tail hairs.

Elliott believes that the difference in the behaviour of the erector muscles of the hair is attributable to differences in the demand made upon them under normal conditions. The English domestic cat rarely puts up its hair, while the hair of the mongoose rises in response to every passing emotion. Thus, in the latter species, adrenalin acts very promptly upon the hair. The feathers of birds have a sympathetic innervation, and adrenalin causes erection in this case also; it is best seen in the cock, and is less clearly marked in hens and pigeons. Elliott found in the case of his own skin, the hair muscles of which normally possessed a very sensitive reaction, that subcutaneous injection of .03 mg. adrenalin produced a stronger effect upon the hair muscles than upon the vessels.

The Bronchial Muscles.-According to Einthoven, Beer, Brodie, and Dixon, the involuntary muscles of the bronchi receive their innervation from the vagus nerve. Stimulation of this nerve produces a contraction of the bronchi, and consequent contraction of the bronchial tubes. According to Roy and Brown, and also to Sandemann, the vagus contains fibres which cause the bronchial muscles to expand. As far as we know at 
present, then, the innervation of the bronchial muscles is autonomous in the sense of motor stimulation, while the presence of a sympathetic innervation has not, up to now, been demonstrated. From Dixon and Brodie's careful experiments, it seems as if suprarenal extract exercised very little effect upon the bronchial muscles. Small doses do not produce either contraction or expansion of the bronchioles, while large doses have been followed in isolated cases by transient contraction. These findings are confirmed by my own experiments with adrenalin. In anaphylactic shock (Auer and Lewis) in guinea-pigs, as well as after the intravenous injection of peptone (Biedl, Kraus), there is acute cramp of the bronchial muscles which is promptly relieved by atropine, the action of which is to paralyse the vagus. In cases such as these, the intravenous injection of adrenalin is negative in its result. According to Jagic, bronchial asthmatic spasm in man may be checked by the subcutaneous injection of I c.cm. adrenalin. Eppinger and Hess explain this result by the assumption, that the stimulation of the sympathetic by adrenalin overpowers the muscular cramp arising from the increased stimulation of the vagus. In view of these divergent findings, further investigation of the innervation conditions of the bronchial muscles and of the effect which adrenalin has on them, appears to be imperative.

The Eye.-The intravenous injection of adrenalin provokes retraction of the nictitating membrane, elevation of the eye-lid, exophthalmos, and dilation of the pupil. Thus, the musculus palpebræ tertius, the unstriated muscles of the eye-lid, the musculus protrusor bulbi, and the musculus dilatator pupilla, are all induced to contract in the same manner as after electric stimulation of the sympathetic nerve in the region of the neck. Adrenalin produces effects which are similar and even more powerful, after extirpation of the superior cervical ganglion and consequent degeneration of the post-ganglionary fibres (Lewandowsky, Langley). It seems certain from this that the site directly affected by adrenalin is peripheral.

The effect which adrenalin has in enlarging the pupil was next studied, and from two points of view. In the first place, it has been turned to account as a test for adrenalin and as a method of determining its physiological significance. Wesseley, and later Meltzer and Auer, found that, when applied to the eyes of frogs, adrenalin produced mydriasis, and the latter authors suggested (1904) the frog's eye as a suitable adrenalin reagent. They pointed out that the reaction takes place even after enucleation of the bulb. Ehrmann next showed that, when preserved in isotonic fluid, the enucleated frog's eye is considerably more sensitive than the bulb in situ, and upon these findings he based his quantitative adrenalin test. Ehrmann found that adrenalin reacts upon the dilator muscles of the pupil in a solution of $I: 20$ 
million; in other words, the dilation of the pupil demonstrates the presence of adrenalin'in a quantity as small as $.000025 \mathrm{mg}$. This mydriasis of frogs' eyes was subsequently employed as a test for adrenalin in liquids of the most varied description (blood, urine), but the results were not in all cases unequivocal; that is to say, they were not characteristic of adrenalin only.

It has since been discovered that, in addition to adrenalin, a large number of substances provoke dilation of the pupil of isolated frogs' eyes. Waterman and Boddaert produced this effect with weak watery solutions of pyrocatechin, with salicylic acid, hydrokinone, and resorcin; they think the reaction is dependent upon pyrocatechin. Ehrmann found that acetic acid and ammonia produce irregular dilation of the pupil, but that the pupil also contracts in response to these reagents. According to Pick and Pineles, tyrosin and phenylalanin produce mydriasis of the frog's eye; Cramer, Borchardt and Pal attribute the same effect to pituitary extract; Ranzi and Tandler to thymus extract. Pick and Glaessner obtained a mydriatic reaction with the pancreatic juice of man and of dogs, which was particularly marked where these subjects had been previously fed upon meat. According to Comessati and Diem, the mydriasis of frogs' eyes, when employed as a test for the presence of adrenalin in urine or in concentrated saline solutions, has very little value.

We shall have occasion to speak later of the mydriatic action of the blood serum, and of the theories which have arisen concerning the presence of adrenalin in the plasma under both normal and pathological conditions. For the present, it is sufficient for us to notice the fact that the mydriasis of frogs' eyes is, in itself, not a conclusive proof of the presence of adrenalin in the blood serum; this test should always be reinforced by other biological and chemical reagents.

The action of adrenalin has also been put to practical use in another direction. Lewandowsky was the first to discover that the instillation of suprarenal extract into the conjunctiva is, in the case of both man and animals, without effect upon the pupil. Melzer and Auer next showed that, after extirpation of the superior cervical ganglion of both rabbits and cats, the instillation of adrenalin produces a considerable enlargement of the pupil. We know that the sympathetic contains fibres which promote contraction of the dilator muscles of the pupils. If, therefore, stimuli which are inactive or only slightly active in the case of normal eyes, react strongly upon eyes which have lost their ganglion, we must assume the presence of sympathetic inhibitory fibres which take their rise in the ganglion and by which the peripheral irritability is reduced. O. Löwi assumed that the occurrence or non-occurrence of mydriasis after adrenalin instillation, was an expression of the functional activity of the sympathetic inhibitory nerves. He therefore endeavoured to find 
out whether mydriasis takes place under certain special conditions, in which a suppression of the sympathetic inhibition is assumed. He found that, after the instillation of adrenalin into the conjunctiva, enlargement of the pupil was invariable in dogs and cats from which the pancreas had been removed; that it was occasional in artificial pancreatic insufficiency; and that it was present in many clinical instances of diabetes and Graves's disease. Löwi assumes from this that the inhibition of certain organs with a sympathetic innervation is a normal function of the pancreas. He regards the mydriasis produced by adrenalin in Graves's disease as the expression of an increased irritability of the sympathetic, brought about by hyperthyroidism. This increased irritability is as readily attributable to a suppression of the inhibitory nervous function, as to an increase in the stimulatory nervous function. Eppinger, Falta and Rudinger succeeded later in producing adrenalin mydriasis in normal and thyroidectomized dogs by continuous treatment with thyroid extract.

The Glands.-Langley found that, when suprarenal extract is given in moderately large doses, it provokes a profuse secretion on the part of all the salivary glands, as well as of the mucous glands situated in the mouth, œsophagus, and trachea. This increased secretion becomes apparent a little later than the rise in blood-pressure, it quickly reaches the maximum, and then gradually subsides. Hence the irritability of the cervical sympathetic and of the chorda tympani is not by any means reduced, that of the latter being, in fact, rather augmented. That the secretory stimulus does not proceed from the central nervous system, is shown by the fact that it continues unchanged after resection of the superior cervical ganglion and of the chorda tympani. It is the outcome of peripheral stimulation, and it remains constant, though somewhat diminished in intensity, after comparatively large doses of nicotine. Moreover, atropine, in a quantity sufficient to paralyse the fibres of the chorda, does not inhibit the increased secretion, though this disappears in response to atropine in large doses. It is evident, from these results, that suprarenal extract stimulates those elements-whether nerve terminals or glandular cells-which are inhibited by the action of atropine. Adrenalin excites the secretion of saliva even after extirpation of the ganglion and degeneration of the postganglionary fibres, and this makes it certain that the effect cannot be due to stimulation of the sympathetic nerve terminals.

Under the influence of adrenalin, the vessels of the submaxillary gland contract and the gland blanches; after an interval of thirty seconds it becomes increasingly red until its colour is deeper than before the injection. The blanching is less than after stimulation of the sympathetic, the flushing less intense than after stimulation of the chorda. Here also, vaso-constriction 
takes place after degeneration of the post-ganglionary fibres and must, for this reason, be regarded as the outcome of the direct action of suprarenal extract.

The intravenous injection of suprarenal extract produces an increased secretion on the part of the lachrymal glands, and this persists after removal of the superior cervical ganglion.

Suprarenal extract increases the secretion of bile. This result is not apparent, however, unless the cystic duct is occluded; otherwise the relaxation of the gall bladder permits the gall to escape down it.

According to Langley, adrenalin does not apparently induce pancreatic secretion, but it does promote the activity of a secreting pancreas. In large doses, adrenalin inhibits pancreatic secretion (Bendicenti, Gläsener and Pick).

According to a communication by Yukawa, the gastric secretion increases under the influence of adrenalin.

As we know, the blood supply to the kidney is very much affected by the action of adrenalin. Minimal doses produce an appreciable reduction in the volume of the organ, consequent upon the contraction of the vessels; this is succeeded by pronounced vaso-dilation and increase in volume. The experiments of Bardier and Fraenkel show that the urinary secretion is influenced in such a manner that there is, first, a reduction in the amount of urine excreted by the ureters, or there may even be complete cessation of the flow; this is followed by marked polyuria which lasts for some considerable time. These authors found that, in those cases where vaso-constriction is absent, the primary reduction in secretion also fails to appear, and it seems evident from this that the changes in renal secretory activity which are brought about by the agency of adrenalin, are largely attributable to changes in the circulatory conditions.

According to Schlayer, adrenalin, when given intravenously, acts under certain conditions as a diuretic upon animals containing much fluid.

Biberfeld found that the subcutaneous injection of suprarenin, in doses of 1.5 to $2.5 \mathrm{mg}$. per kilo, produced a marked diuresis in rabbits, together with a considerable decrease in the sodium chloride contents of the urine.

The manner in which the suprarenals themselves react to the influence of adrenalin has, up to now, received but little attention. As far as the suprarenal circulation is concerned, I found that the intravenous injection of suprarenal extract produced an increase, followed by a decrease, in the venous outflow. These changes in the circulation are due only in part to the altered hremodynamic conditions. In part, they represent the results of the action of the extract upon the vessels themselves, and they are comparable to those effects, partly constrictor and partly dilator, which are obtained by stimulation of the sympathetic nerves supplying the organ. 
The increase in the amount of lymph excreted from the thoracic duct which, according to Camus, follows the intravenous injection of adrenalin, is in all probability largely due to changes in the circulatory conditions. It cannot be denied, however, that adrenalin may possibly exercise an effect upon the formation of the lymph itself.

After the subcutaneous injection of adrenalin, odema frequently appears at the site of injection, and the lymphatics leading away from it are sometimes found to be full. If the same quantity of adrenalin is employed, but in a very dilute solution, the œedematous swelling becomes much more marked and may last for some time. These phenomena must be largely due to a reduction in resorption, brought about by a local constriction of the vessels at the site of injection. This accounts for the fact that the subcutaneous injection of adrenalin produces so little effect upon the blood-pressure; to a certain extent, the substance blocks the way of its own resorption.

The gradual resorption of the adrenalin in minimal quantities explains why it is that the subcutaneous injection of even large doses does not produce a rise in blood-pressure, but is followed by considerable periodical changes in pressure (Biedl). Löwi and Meyer found that when methylaminoketone, which is closely related to adrenalin, is injected subcutaneously in large doses, the blood-pressure becomes elevated and will continue so for over an hour. This result is explained by the fact that methylaminoketone is considerably less active than adrenalin, hence the possibility of its better resorption. Meltzer and Auer found that the intramuscular injection of adrenalin produced an increase in blood-pressure ; but Patta pointed out that, whether given intramuscularly or subcutaneously, the injection of adrenalin is not followed by a rise in blood-pressure in animals, unless the substance is introduced by accident into a blood-vessel. In man, the subcutaneous injection of adrenalin produces an increased blood-pressure (Falta).

A. Exner showed that the intraperitoneal injection of adrenalin is followed by a reduced resorption on the part of the lymphatics of the peritoneum. For it is not only those toxins which enter the system by way of the blood-vessels, which undergo a tardy resorption after the introduction of adrenalin into the peritoneum or into the stomach; the same is seen in the case of substances which, owing to their physical properties, reach the blood-stream through the agency of the lymphatics.

Langley found that the sweat glands of the cat were unaffected both by the intravenous injection of adrenalin and by its subcutaneous introduction into the ball of the paw. Elliott found that a subcutaneous injection of $.025 \mathrm{mg}$. of adrenalin did not provoke secretion of the sweat glands in the palm of the hand in man; on the contrary, owing to the extreme local anæmia, 
secretion at, and surrounding, the site of injection was rather diminished than otherwise.

The failure on the part of adrenalin to stimulate the secretory activity of the sweat glands is undoubtedly a very remarkable phenomenon, for it is quite certain that these glands are furnished with a sympathetic innervation.

Adrenalin stimulates the sweat glands of the frog to active secretion. After the injection of small quantities of adrenalin intc the dorsal lymph space, the entire body of the animal becomes covered with secretion. If the secretory process in the glands of the nictitating membrane is observed under the microscope (Stricker and Spina), it will be seen that the introduction of adrenalin is followed by progressive enlargement of the glandular epithelium and by the extrusion of drops of secretion, in exactly the same manner as after stimulation of the sympathetic nerves (Drasch).

S. Lieben found that adrenalin produced marked contraction of the pigment cells of the skin of frogs, a darli-coloured frog becoming light in the space of ten minutes. This effect of adrenalin upon the pigment cells is not brought about by the local anamia, nor is it a reflex effect; it is the result of the direct action of the substance. According to Gaup, the pigment cells are controlled by the sympathetic.

Adrenalin exercises a similar agglomerating effect upon the pigment granules in the pigment epithelium of the retina of frogs, but this is seen only after local application. This agglomerating action may even, to a certain extent, inhibit the migration of the pigment in response to the stimulus of light, and where this forward movement has already taken place, a further agglomeration of the pigment granules will be produced in a varying degree by the action of adrenalin (Klett).

Among the physiological effects of adrenalin, those must be included which this substance produces in the composition of the blood. According to Foa, adrenalin reduces the alkalinity and produces acidity of the blood. Falta and Berterelli found that the injection of adrenalin is followed by an increase in the specific gravity; by hyperglobulia with isolated erythroblasts; and by marked leucocytosis affecting the neutrophile polynuclear cells, the eosinophile cells being very much reduced. In dogs with hypereosinophilia of about $\mathbf{2} 2$ per cent., the injection of adrenalin is followed by a reduction to .3 per cent. Eppinger and Hess discovered that the physiological eosinophilia of rabbits is reduced by the action of adrenalin. The morphological structure of the blood is influenced by adrenalin in a manner the reverse of that of pilocarpin which, it is well known, produces lymphocytosis and eosinophilia in both man and rabbits (Neusser).

Metabolism.-The influence which adrenalin exercises upon metabolism demands special consideration. The investigations of 
Kraus and R. Hirsch, as well as those of Quest, show that there is little alteration in the metabolism of nitrogen in healthy dogs after the intravenous or subcutaneous injection of adrenalin. The slight increase in $\mathrm{N}$-metabolism is explicable by the glocosuria which is coincident with it, as well as by the necrosis of the skin which follows subcutaneous injection. Eppinger, Falta and Rudinger found that, in starving animals, adrenalin caused a marked increase in the metabolism of albumin; feeding with fatty foods still further increased the effects of adrenalin. The increased excretion of nitrogen which, in starving animals, immediately followed the injection of adrenalin and which was also observed by Underhill and Closson, cannot in the nature of things be referred to necrosis of the skin. Eppinger, Falta and Rudinger regard the increase in the metabolism of albumin and of fats as the expression of an improved thyroid function.

According to the investigations of Falta, Bolaffio and Tedesko, adrenalin excites an increase in the metabolism of salt. The amount of phosphorus excreted in the urine may be increased to three times, that of potassium and sodium to four times, the normal. With regard to the metabolism of purin, Falta declares that the excretion of uric acid and of allantoin are both considerably increased.

This effect which adrenalin has in accelerating metabolism is regarded by authors of the Viennese school as an expression of the condition of increased excitability which this substance produces in the sympathetic system.

That aspect of metabolism which, it is conclusively proved, lies within the province of the sympathetic system, is the metabolism of the carbohydrates. The influence which adrenalin exercises upon these conditions has, therefore, a special interest.

When Blum, in Igor, made the important discovery that the experimental subcutaneous or intravenous injection of suprarenal extract produces glycosuria, which follows with equal certainty in both starving animals and those deprived of the carbohydrates, there was a general inclination to regard this result as the manifestation of a toxic activity on the part of suprarenal extract. It formed the subject of keen and very minute investigations, and these have revealed the fact that the glycosuria which follows the exhibition of adrenalin, is the expression of a physiological action on the part of this remarkable substance.

Blum's discovery was tested and confirmed in many directions. It soon became evident that the glycosuria makes its appearance after the exhibition both of the extract of the suprarenal and of adrenalin, its active principle. For this reason, it was very generally believed that glycosuria is one of the physiological effects of adrenalin. The sole author who opposed this view was Landau, who ascribed the glycosuria to a substance derived from the suprarenal cortex. 
The following facts concerning adrenalin glycosuria may be regarded as proved. The glycosuria makes its appearance after the exhibition of comparatively small doses (.o t to .I mg.) of adrenalin, and is, on the whole, more readily provoked by subcutaneous or intraperitoneal, than by intravenous, injection (Löwi). The cause of this remarkable phenomenon will appear later. The subcutaneous introduction of $\mathrm{I}$ to $2 \mathrm{mg}$. adrenalin is followed after half an hour, at the latest after two hours, by a glycosuria lasting about three hours. The repeated injection of similar doses into the same animal is not, however, followed by the regular appearance of the glycosuria. One day the urine will contain a large quantity of sugar, the next day perhaps none at all, and in the end the glycosuria may entirely disappear.

It has been observed by several authors that the glycosuria which follows the exhibition of adrenalin is accompanied by hyperglycæmia. The increase in the amount of sugar in the blood was proved by Bierry and Gatin-Gruzewska, and by Noel Paton, in the case of rabbits and dogs. These authors also showed that when given to starving animals, adrenalin diminished the amount of glycogen present in the liver and muscular structure, and may indeed be regarded as a certain method of eliminating glycogen from the system. Drumont and Noel Paton were unable to produce changes in the glycogen of the liver of well-fed rabbits by means of small doses of adrenalin, such changes being brought about by doses large enough to produce acute toxic symptoms. Later investigations have proved beyond any manner of doubt, that the glycogen largely disappears from the liver and muscles where the adrenalin action is very intense (Agadchanianz, Doyon, Morel, and Kareff). L. Pollak recently pointed out that, after previous feeding with glycose or levulose, the glycogen stored up in the liver may be induced completely to disappear by means of large doses of adrenalin. Small doses of adrenalin differ considerably in their effect, according to whether the glycogen accumulated in the liver is formed from glycose or levulose. Levulose-glycogen is markedly the more resistant.

Since Blum's first communication it has been definitely proved that, after prolonged fasting, glycosuria is present in all animals. Pollak's experiments testified to the remarkable fact, that the repeated exhibition of adrenalin in fasting rabbits and in rabbits which have been rendered absolutely free from glycogen by means of strychnine, is followed, not only by sugar in the urine, but by the accumulation of glycogen in the liver. The amount of glycogen which collects in the liver is as large as that which is usually seen only in animals fed with carbohydrates. It has been shown (Underhill and Closson, Eppinger, Falta and Rudinger) that adrenalin causes a very considerable increase in the metabolism of albumin in starving animals, and the remarkable phenomenon described above is probably attributable to the storing-up of 
glycogen as a result of this increase in albumin-metabolism. Pollak is not inclined to explain the large quantities of glycogen which he found, solely by the theory of an increased metabolism of albumin; he is disposed to leave the question open for the present.

In answer to the question as to why the intravenous injection of adrenalin is not invariably followed by glycosuria, and why the repeated subcutaneous injection provokes signs of tolerance, frequently expressed by the absence of glycosuria-Pollak discovered that, though the intravenous injection of adrenalin invariably causes hyperglycæmia, this is not sufficiently marked to produce glycosuria, seeing that there is, under these conditions, an invariable reduction in diuresis. Where, however, simultaneously with the adrenalin injection, measures are adopted with the object of promoting diuresis, sugar is invariably present in the urine. After subcutaneous injection of adrenalin, the hyperglycæmia is so pronounced that glycogen will be present in the urine apart from artificial promotion of the diuresis. The repeated subcutaneous injection of adrenalin produces a high degree of hyperglycæmia, such as is usually observed only in cases where there is no simultaneous diuresis. As, however, the glycosuria is not always present, it must be assumed that, either the kidneys acquire a tolerance of their large glycogen contents resulting from the continued employment of adrenalin, or that they are subjected to some specific influence.

W. Straub and his pupil, H. Ritzmann, have done much to further a more detailed knowledge of adrenalin glycosuria. Kretschmer discovered that the remarkable absence of constancy in the effect of adrenalin upon the vessels, depends upon the readiness with which adrenalin undergoes oxidation, and that a permanent adrenalin action upon the tone of the vessels is to be obtained only by a permanent employment of the drug. Ritzmann next showed that the glycosuria lasts for exactly the same length of time as adrenalin is present in the blood. If adrenalin in very weak solution is allowed to enter a vein and to flow continuously into the blood stream, it will be found that an adrenalin solution of a concentration of $\mathrm{I}: 2$ million, introduced at a velocity of about c.cm. to the minute, does not provoke glycosuria. Sugar does not appear in the urine until the velocity reaches 3 to $4 \mathrm{c.cm}$. per minute. Within certain limits, there is a direct proportion between the concentration of the adrenalin in the blood and the amount of sugar excreted in the urine; so that, other things being equal, each velocity of a solution of given concentration provokes a glycosuria which invariably yields the same amount of sugar within a given space of time. The glycosuria does not make its appearance immediately, but is preceded by a prolonged latent stage, which is probably accounted for by the fact, that a certain amount of time is required for the completion of the chemical process by which the sugar is set free. There is, moreover, an 
intimate connection between the glycosuria and the amount of glycogen present in the economy. In animals which have a large amount of glycogen in their economy, half the usual dose of adrenalin will produce an excretion of sugar quantitatively equal to that provoked in normal animals by the full dose. Where, however, the animal economy is poor in glycogen, similar results will be obtained only by the agency of much larger quantities of adrenalin.

According to Ritzmann, where experiments are undertaken with the object of studying the physiological processes by which adrenalin glycosuria takes place, it is essential that the adrenalin should be introduced directly into the blood stream; for the method of introducing it into the economy by a single subcutaneous injection does not reproduce the natural conditions. It has, moreover, been proved that, where adrenalin is injected subcutaneously, the quantity required to produce certain glycosuric effects is considerably larger than in cases where the substance is introduced by continuous intravenous transfusion. For example, in one case, a subcutaneous injection of $2 \dot{\mathrm{mg}}$. adrenalin was required to produce $0.64 \mathrm{grm}$. sugar in the urine, while the same result was obtained by means of intravenous transfusion with a diluted solution containing $0.4 \mathrm{mg}$. adrenalin. Thus of the $2 \mathrm{mg}$. which were injected subcutaneously, $1.6 \mathrm{mg}$. or 8 o per cent. were destroyed without producing glycosuria.

It is evident from Pollak's experiments described above, that the glycosuria produced by the intravenous exhibition of adrenalin is dependent upon the diuresis. And Ritzmann mentions that, in several of his experiments, the diuresis entirely ceased, and the animals died without excreting any part of the fluid which had been introduced into their economy. Even where adrenalin is introduced continuously into the blood stream, a velocity which is at first active becomes inactive after a time (this effect being the reverse of that demonstrated by Kretschmer in the case of the blood-vessels), and the glycosuria is restored only by increasing the concentration of the solution. This phenomenon is explained by a transitory exhaustion, either of the sugar supply or of the ultimate products of the sugar.

From the results of these investigations into the mechanism of adrenalin glycosuria, it seems highly improbable that adrenalin directly affects the formation of sugar in the urine. It is far more likely that it influences the process of the distribution of sugar in the blood. We know that, in its influence upon the blood-vessels, the effect of adrenalin is equal to that of stimulation of the sympathetic; and it is also well known that the diabetes produced by Claude Bernard's puncture is the outcome of hyperirritation of the sympathetic. The analogy between the mechanism of the glycosuric and the vaso-constrictor effects of this substance, suggests the hypothesis that the actual 
site of the direct action of adrenalin is to be found in those fibres of the sympathetic, the central irritation of which produces effects similar to those of puncture of the fourth ventricle. Through the agency of the sympathetic system, adrenalin increases the tone of the vessels, and in a similar manner it increases the sugar-tone (Zuckertonus) - that is to say, it increases the concentration of the sugar in the blood, so producing hyperglycæmia, which is followed by glycosuria.

Blum first pointed out the similarity which exists between the effects of Claude Bernard's puncture and the glycosuria produced by adrenalin, and he suggested that the former is expressed through the agency of the suprarenals. A. Meyer next showed that puncture of the floor of the fourth ventricle does not produce glycosuria in rabbits from which the suprarenals have been removed; and more recently, Waterman and Smit found that puncture of the floor of the fourth ventricle, similarly to stimulation of the sympathetic, increases the amount of adrenalin present in the blood. The far-reaching analogy between the effects of puncture of the fourth ventricle and experimental adrenalin glycosuria, is still further confirmed by the experiments of Eppinger, Falta, and Rudinger. These showed that, if the thyroid gland is extirpated, in neither condition does glycosuria result; but that a mobilization of carbohydrates takes place, as shown by the decreased excretion of nitrogen, and that this is consumed by the agency of the now hyper-active pancreas. A further similarity between the two conditions is shown by the behaviour of dogs from which the pancreas has been removed. These animals showed increased glycosuria and hyperglycæmia, both after puncture of the fourth ventricle (Hédon and Kaufmann), and after the exhibition of adrenalin. The authors conclude from these results, that the excretion of sugar after puncture of the fourth ventricle represents a discharge from the chromaffine system.

The difference in the processes by which glycosuria and hyperglycæmia result from puncture of the fourth ventricle and from the exhibition of adrenalin, lies in this: that, in the case of adrenalin, the results are brought about by peripheral stimulation of the sympathetic; after puncture of the fourth ventricle they are due to stimulation of the sympathetic centres.

L. Pollak found that, after resection of the splanchnic nerve, adrenalin invariably produces glycosuria; while the experiments of Claude Bernard and Eckhard show that, after such resection, puncture of the fourth ventricle is not followed by glycosuria. In the case of puncture of the fourth ventricle, irritation of the sugar centre in the medulla is conveyed by way of the sympathetic (splanchnic nerve) to the liver, where it excites that organ to part with its glycogen. Resection of the splanchnic nerve breaks the communication between the centre and the sugar-forming organ, 
thus hyperglycæmia becomes impossible. Adrenalin, on the other hand, affects the peripheral sympathetic nerve-terminals in the liver in exactly the same way as it does those of other organs, and for this reason, the glycosuria is uninfluenced by the resection of the communicating nerve.

Observation of experimental adrenalin-glycosuria has revealed the intimate relationship which subsists between the action of adrenalin and the internal secretory activity of the pancreas. That adrenalin-glycosuria is in some way related to pancreatic diabetes is evident from the results obtained by Herter and Wakeman. These authors discovered that, if the pancreas is painted with adrenalin, the resultant glycosuria is more intense than that which follows the application of adrenalin to other organs. Vosbourgh and Richards obtained marked hyperglycæmia by the same means. Herter and Wakeman assume that, whatever the site at which adrenalin is applied, its glycosuric action reaches the pancreas and reduces the capacity of that organ for the oxidation of sugar. If this view is correct, adrenalin should not produce an increase in the glycosuria present in animals which have lost their pancreas; and results obtained by Lépine, as well as by Bierry and Gatin-Gruzewska, seem to point in this direction. Velich, on the other hand, found that though extirpation of the pancreas is not followed by glycosuria in well-nourished frogs until after several days have elapsed, it may be brought on immediately after uperation by means of adrenalin. Noel Paton produced glycosuria by this means in geese and ducks after the removal of the pancreas ; while Doyon, Morel, and Koreff found that the injection of adrenalin was followed by an increase in the sugar contents of the blood of dogs which had lost their pancreas.

It is very evident from the results of these experiments that the theory that adrenalin glycosuria is a form of pancreatic diabetes, is insufficiently grounded.

Zuelzer adopted a different theory. He found that, in dogs without pancreas, if adrenalin was prevented from entering the blood stream by ligature of the suprarenal veins, the glycosuria was minimal. He succeeded in preventing glycosuria in rabbits by injecting them with pancreatic extract, and he concluded that Minkowski's "pancreatic diabetes" is in reality a positive " adrenalin diabetes."

In experiments which I undertook with Offer, we succeeded in producing both inhibition of the adrenalin glycosuria and inhibition of the adrenalin mydriasis of frogs' eyes, with chyle from the thoracic duct.

These experiments were based upon some observations (to be described later) which I carried out in 1898 , and which showed that chyle contains a substance which influences the amount of the sugar consumption of the organism. I found that, in the case of dogs which had become diabetic owing to the fact that the 
chyle was prevented from flowing into the blood stream, Löwi's characteristic mydriasis reaction after adrenalin instillation was not obtainable. This seems to suggest a parallel between the origin of pancreatic diabetes and that "which follows ligature of the lymphatic duct. Upon the assumption that chyle contains the internal secretion of the pancreas, which is necessary to the normal metabolism of sugar, we tested the action of chyle in adrenalin glycosuria; we found that the subcutaneous injection of 80 to $120 \mathrm{c.cm}$ of chyle from dogs, markedly reduced, and even completely neutralized, the glycosuric action of adrenalin when exhibited simultaneously. Adrenalin glycosuria in rabbits is promptly suppressed by the intravenous injection of hirudin, and also, as I discovered later, of the extract of the muscular structure of crabs; it is, therefore, amenable to substances which, according to Heidenhain, are included in the group Lymphagoga, Order I. By following up our experiments, Tomaczewski and Wilenko found that all the Lymphagoga possess the property of suppressing adrenalin glycosuria.*

In the course of their investigations into the interactivities of the internal secretory glands, Eppinger, Falta, and Rudinger discovered that, in dogs without pancreas, in which the metabolic derangement is at its height, the excretion of sugar and of nitrogen are enormously increased by the subcutaneous or intraperitoneal injection of adrenalin. The excretion of sugar becomes so much increased that the proportion of the quotient $\mathrm{D}: \mathrm{N}$ may rise to over seven. It is the view of these authors that, in addition to an excessive and perverted mobilization of the carbohydrates, adrenalin produces inhibition of the pancreatic function. The glycosuria which follows the exhibition of adrenalin results from the operation of both momenta. Zuelzer's experiment, in which he effected the inhibition of adrenalin glycosuria by means of pancreatic extract, is explained by the supposition that, in this instance, the excess of sugar set free by the adrenalin was metabolized by the action of the reinforced pancreatic hormone.

That adrenalin exercises an inhibitory influence upon the activity of the pancreas, may be assumed from Bendicenti's observation that pancreatic secretion is inhibited by large doses of adrenalin. - By experimenting on dogs with pancreatic fistula, Glässner and Pick proved that the pancreatic secretion completely ceases after large doses of adrenalin, while, at the same time, a marked glycosuria makes its appearance.

Acting upon the assumption that the internal secretion of the pancreas is governed by the autonomous vagal nerve, and that

* According to Aronsohn, adrenalin glycosuria is inhibited both by fever and by heat-puncture. According to Richter, Ellinger and Selig, the heat puncture does not affect the glycosuria, which seems to be reduced only where there is simultaneous bacterial infection. After renal injury there is no glycosuria. (Ellinger and Selig.) 


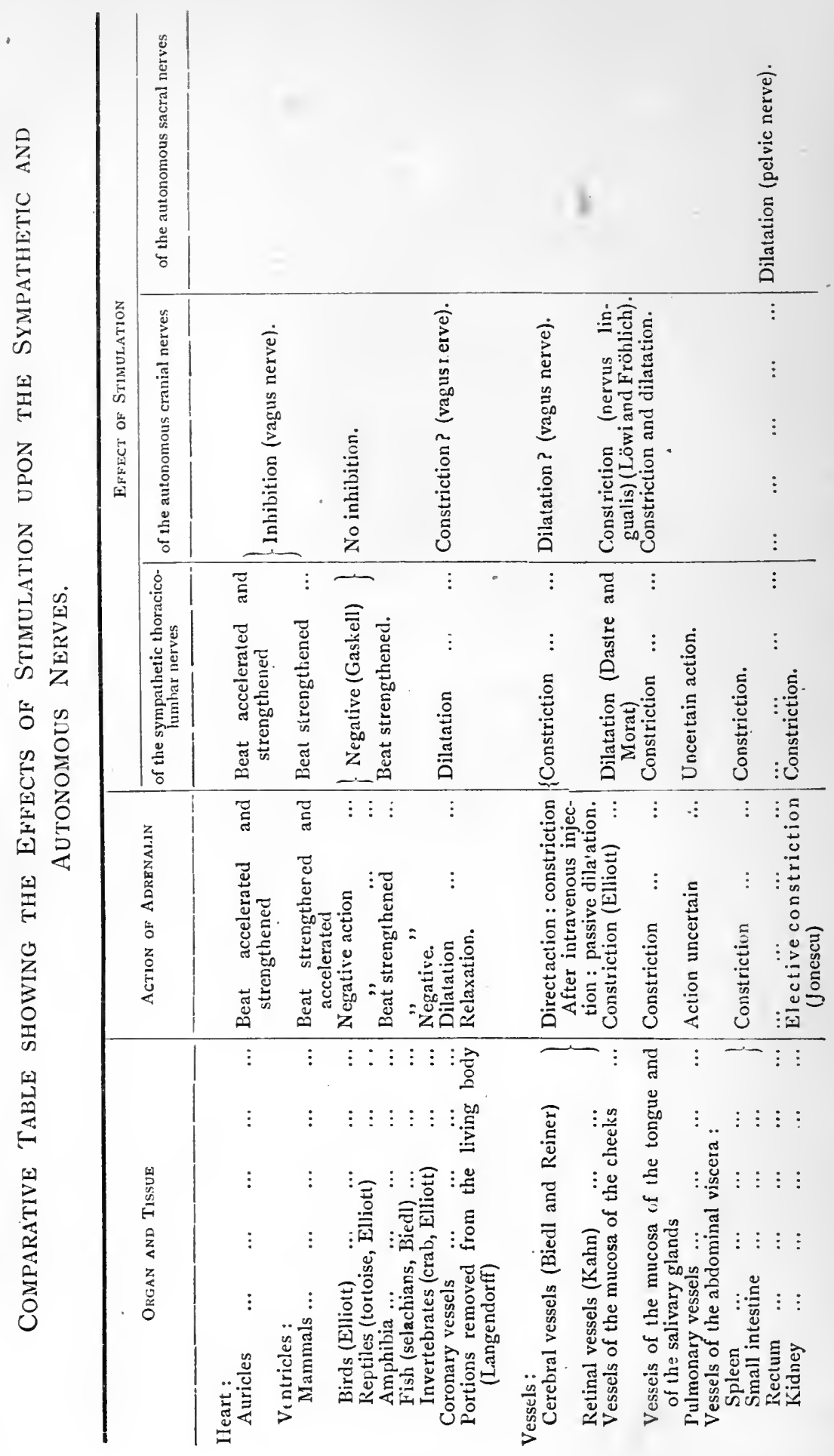




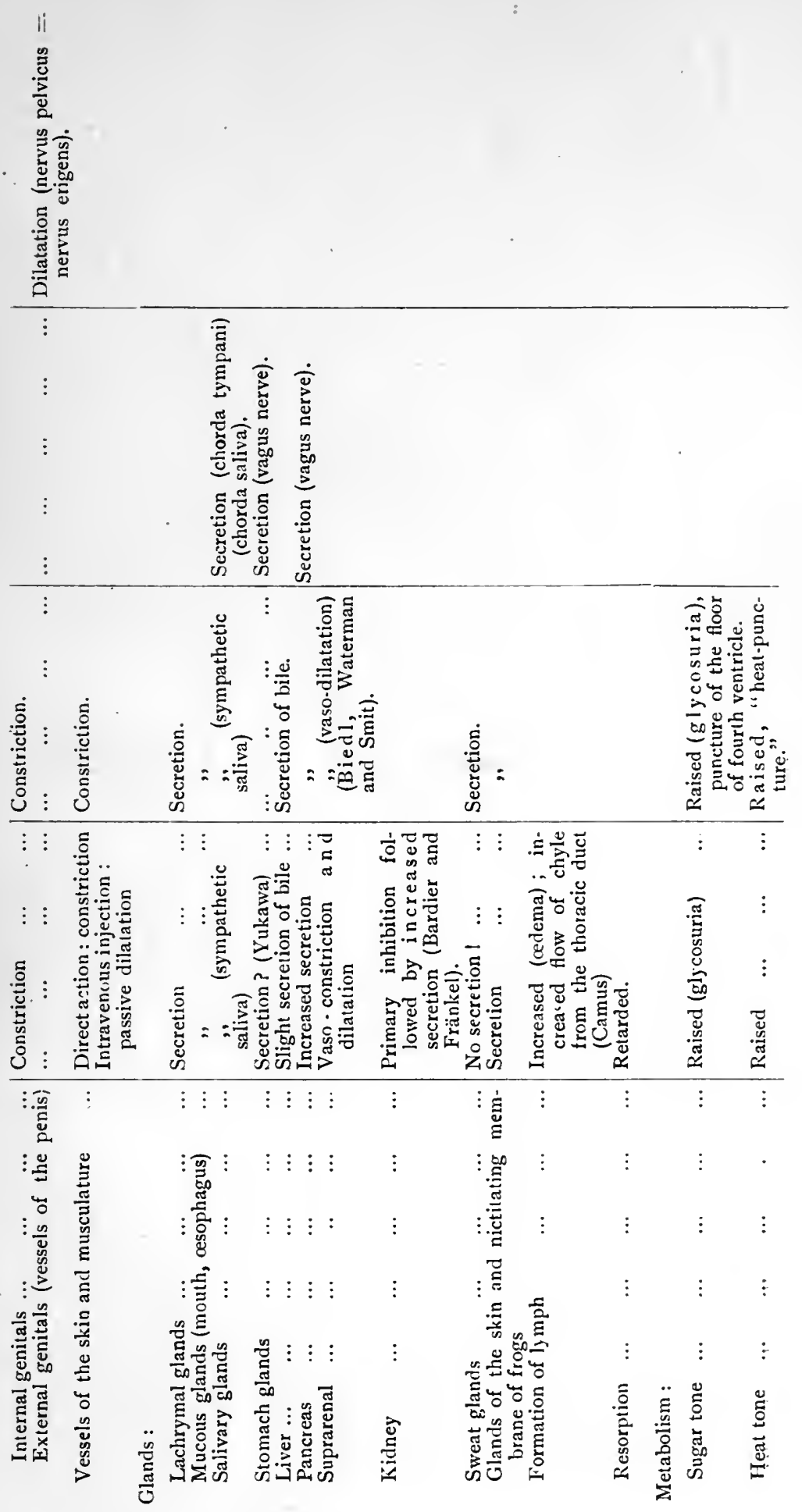




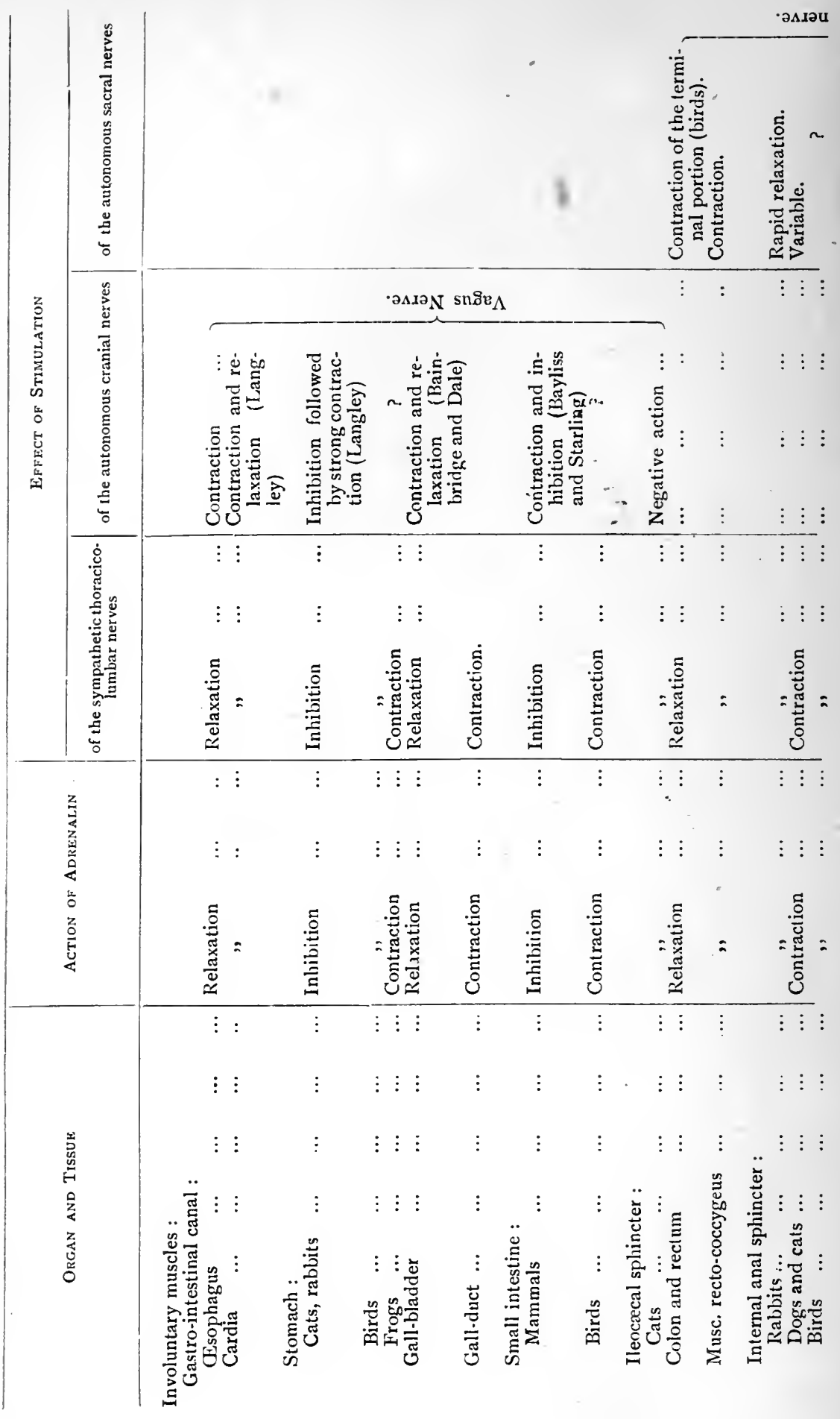




\section{$\min \left[\partial^{d}\right.$}

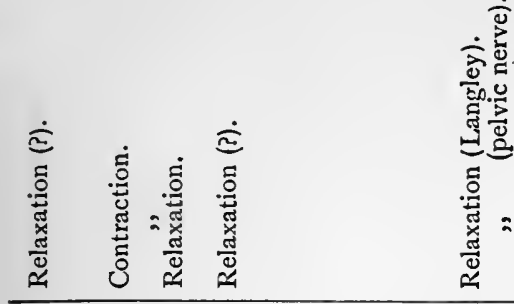

喜䇏

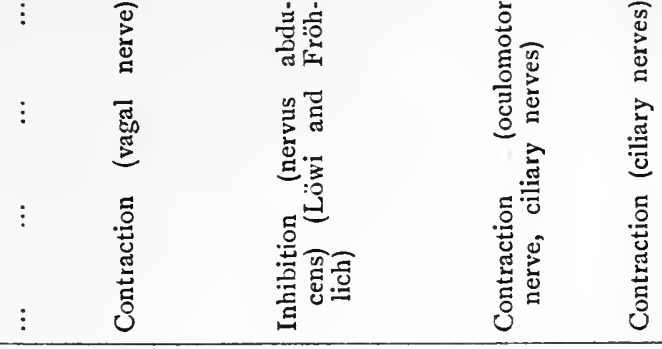

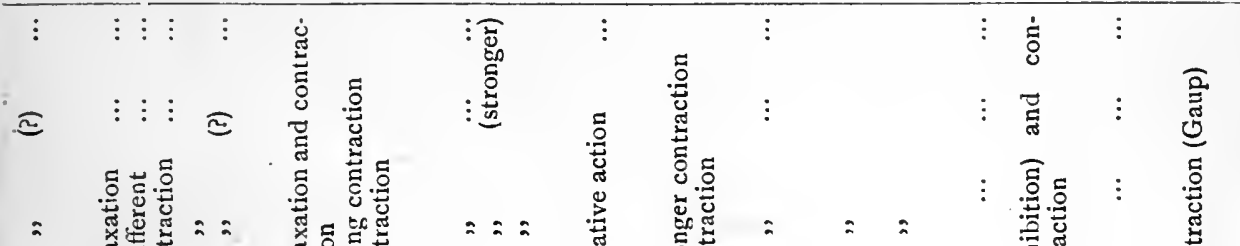

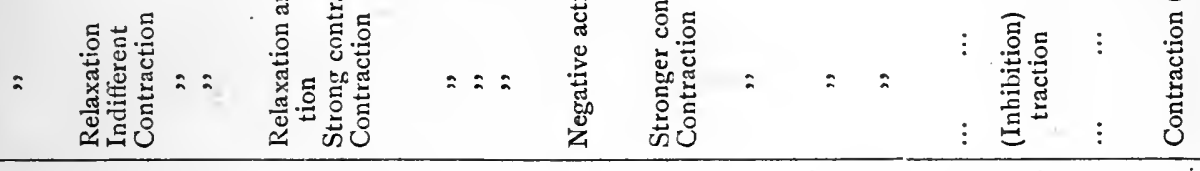

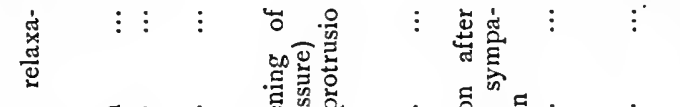

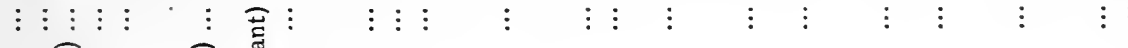

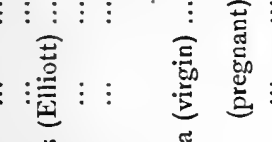

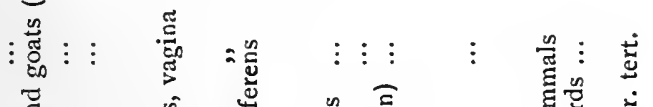

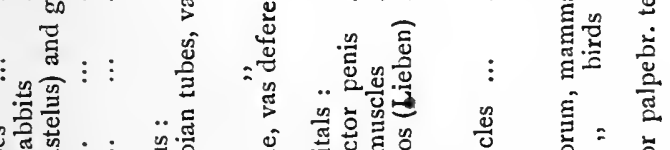

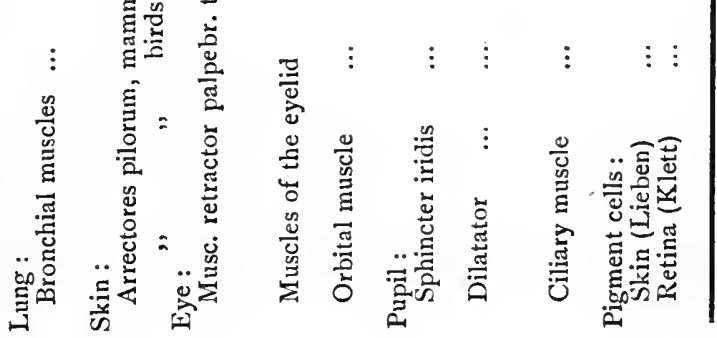


stimulation of the vagus produces hypersecretion of the pancreas, Eppinger, Falta, and Rudinger tested the effect of pilocarpinwhich is a specific toxin to the autonomous stimulatory systemin adrenalin glycosuria, and found that its action in this condition was completely inhibitory.

The conclusions to which the results described above seem to point are: that adrenalin exercises an inhibitory influence upon the internal secretory activity of the pancreas; and that the internal secretion of the pancreas limits the action of adrenalin in certain directions. The pancreas hormone regulates the metabolism of sugar in the organism, and its influence may serve to limit, or even to prevent, the hyperglycæmia and glycosuria which result from the superfluity of the carbohydrates, brought about by the agency of adrenalin. Löwi's reaction points further to the conclusion that the pancreas hormone exercises an influence upon the inhibition of certain organs possessing a sympathetic innervation. Where the activity of the pancreas is normal, the mydriatic action of adrenalin is not manifested, but appears only after suppression of the pancreatic internal secretion.

The manner in which the action of adrenalin is modified by the thyroid apparatus has already been discussed at length. It will be remembered that, according to Eppinger, Falta, and Rudinger, adrenalin fails not only to produce glycosuria, but also to raise the blood-pressure in animals which have been deprived of their thyroid. Ritzmann pointed out that, in animals with an excess of glycogen, the glycosuric action of adrenalin is less affected by thyroid suppression than the vaso-constrictor. Removal of the parathyroids increases adrenalin glycosuria. According to these authors, then, the thyroid promotes the action of adrenalin while the parathyroids inhibit it. They ascribe the increased metabolism of albumin and of fats, which follows the exhibition of adrenalin, to promotion of the thyroid function; so that it appears that adrenalin, in its turn, promotes the activity of the thyroid and inhibits that of the parathyroids.

There is yet another direction in which the animal organism is affected by the action of adrenalin, namely, the temperature of the body. The subcutaneous and intraperitoneal injection of adrenalin sometimes produces a considerable rise in temperature; the effect of intravenous injection is less marked. Whether this increase is due to an increased production of heat or to a diminished radiation, is at present unknown. It may be that both factors are in operation, the increase in combustion arising out of the excess of carbohydrates, the decrease in radiation from the extreme vaso-constriction. The analogy between the conditions under which the metabolism of the carbohydrates takes place, suggests a comparison between the effect. which adrenalin has upon temperature and the so-called " heat-puncture" ; for adrenalin raises the heat-tone (Wärmetonus) by acting upon the 
peripheral terminals of those nerves, the central stimulation of which produces the effect of "heat-puncture."

The tables on pp. 222 to 225 show the effects of adrenalin in so far as they are at present known. For purposes of comparison, they also include all that is at present known concerning the sympathetic and autonomous innervation of the vegetative organs.

\section{THE SITE OF AC'TION OF ADRENALIN.}

In his first work upon the effects of suprarenal extract (I90I), Langley emphasized the fact that the results produced by this substance are never identical with those obtained by stimulation of the cranial-autonomous or sacral-autonomous nerves. Phenomena exactly similar to those obtained in the eye, by stimulation of the oculomotor; in the heart and intestinal canal by stimulation of the vagus; in the rectum, urinary bladder, and internal genitals by stimulation of the pelvic nerve, are never produced by suprarenal extract. The increased secretion of saliva cannot be regarded as an exception to this rule, for it is not accompanied by vaso-dilation such as that which accompanies stimulation of the chorda tympani. On the other hand, it is noticeable that the effects which suprarenal extract produces are, in nearly all cases, similar to those obtained by stimulation of the sympathetic autonomous nerves. In many instances, the effect of the extract and that of electric stimulus of the sympathetic are identical. These results seem to point directly to the view that suprarenal extract exercises a specific stimulatory action upon the sympathetic nerveterminals. Yet, in view of the fact that, in the case of the eye, the vessels, the submaxillary gland, and the muscles of the hair, this action is constant after degeneration of the post-ganglionary fibres, Langley was compelled to admit that suprarenal extract may also have a direct influence upon the unstriated muscles.

Four years later, Elliott drew attention to the fact that, in spite of the difference in the method of the sympathetic innervation in different animal species, the effects of adrenalin upon the involuntary muscles were everywhere the same as those produced by stimulation of the nerves supplying them. The strength of the effect varies only with the frequency of the normal physiological impulse, which individual muscles receive during life by the agency of the sympathetic. The physiological stimulatory action of adrenalin is undoubtedly peripheral, but the question as to which portion of the periphery is the site of its activity demands further investigation. This site is most probably to be sought, either in the peripheral sympathetic nerve-terminals, or in the unstriated muscles themselves.

That adrenalin exercises a direct action upon the muscles, was suggested by various phenomena and was, until recently, assumed by the greater number of authors. Lewandowsky and even 
Langley were inclined to adopt this view, chiefly on account of the fact, so frequently illustrated, that the action of adrenalin is unaffected by so-called degenerative resection of the sympathetic nerves-that is to say, in cases where degeneration of the resected nerve has already taken place. On the other hand, the experiments of Brodie and Dixon show that, where the sympathetic nerve endings are paralysed by apocodein, adrenalin is inactive, in spite of the fact that the muscles have retained their contractility, as shown by the effect of agents, such as barium chloride, which have a direct action upon the musculature. It is hardly possible to imagine a chemical destruction of adrenalin by apocodein, hence the only assumption open to us is, that apocodein suppresses those portions of the tissue which, under normal conditions, react to the influence of adrenalin.

Dixon and Brodie localized the seat of action of adrenalin in the sympathetic nerve-terminals. These they defined as "connecting links between muscle and nerve, which do not, however, form a component part of either the muscular or the nervous fibres." They assume, moreover, that after resection of the nerves, this " neuro-muscular junctional tissue" does not undergo degeneration; and that this explains the constancy of the effects of adrenalin after degenerative resection of the nerves.

The histological evidence in favour of this theory is to be found in a discovery described by Fletcher (1897). By intra vitam staining with methylene blue, he discovered a nervous network in the retractor muscle of the penis of the rat, which does not undergo degeneration after resection of either the motor or the inhibitory nerve supplying it, but which does disappear after resection of both. If such a peripheral network were present in all involuntary muscles with a double innervation, the constancy of the action of adrenalin after degenerative resection of the sympathetic would be explained by the fact of this network remaining intact.

Elliott next investigated the manner in which different muscles react to adrenalin after: first, denervation (degenerative resection of the post-ganglionary sympathetic nerves); and second, decentralization (degenerative resection of the preganglionary sympathetic nerves). He found that he was unable to provoke contraction by electric stimulus of a denervated portion of the retractor of the penis of the dog, but that adrenalin was more active upon such muscle than upon that portion in which the innervation was normal. After decentralization and denervation, stimulus of the hypogastric and pelvic nerves produced no effect upon the urinary bladder of cats, though relaxation of the bladder and constriction of the urethra were obtainable by means of adrenalin. After removal of the superior cervical ganglion and the ciliary ganglion, together with the ciliary nerves, the instillation or intravenous injection of adrenalin provoked dilation of the 
pupil of maximal duration, while physostygmin produced myosis of the normal control eye only. All that these experiments prove is, that the tissue which reacts to the influence of adrenalin becomes increasedly sensitive to the action of this substance after decentralization, and even more so after denervation. But in the case of the retractor muscle of the penis, which undoubtedly possesses a dóuble innervation, this result can hardly be due to the persistence of Fletcher's nervous network, seeing that the integrity of the latter is dependent upon the nerve cells of the peripheral ganglion. It is evident from this that the site of the activity of adrenalin cannot be situated in the nerve endings in the general sense of the term; that is to say, in so far as they are demonstrable, anatomically by methylene blue, and physiologically by degeneration after nervous resection.

Upon the other hand, it is very difficult to account for the effects which adrenalin has upon the unstriated muscles; and especially for the different effects (contraction and relaxation) which it produces upon muscles of similar structure, by the theory of a direct action upon the muscles themselves. Hence, the seat of the elective influence of adrenalin can only lie in the junction of the sympathetic nerve-fibres with the muscles. We must, then, assume that the neuro-muscular system is composed of cell, fibre, and terminal derived from the nerve, and of muscle-cell and contractile fibrilla derived from the muscle, and that these two portions are connected by means of a third, the myoneural junction.

During the embryonal period, the muscle fibrilla possesses a direct contractility only, and in certain parts of the unstriated muscular structure, especially in the lower vertebrates, this developmental stage persists throughout life. But in the course of ontogenetic and philogenetic development, the greater number of the unstriated muscles acquire a relationship to the sympathetic nervous system. The myoneural junction, derived partly from the muscular substance, and partly from the sympathetic nerves, develops to form the mechanism which controls the manner in which the muscles react in response to nervous stimuli. Whether the muscle is influenced in a positive or a negative sense, whether in response to such influence it contracts or relaxes, depends upon the myoneural junction. Adrenalin exercises a specific stimulatory effect upon this portion of the neuromuscular system, and this is proved by the following facts : first, it does not influence muscles which have not a sympathetic innervation (as, for instance, the muscles of the bronchi); and second, it does not affect all muscles in the same way, but produces contraction or relaxation according to the nature of the myoneural junction through which it acts. This specific action of adrenalin has revealed the fact, that the neuro-muscular junction tissue is characteristic of the sympathetic system and distinguishes it from the cranial and sacral autonomous 
systems. In the case of the latter systems, the relationship between nerve and muscle is biochemical and similar to that between nerve and muscle in striated muscles.

The sensibility of a muscular tissue to adrenalin is proportional to the degree in which the myoneural junction is differentiated, and this, in its turn, seems to depend upon the frequency of the impulses which, under normal conditions, it receives through the agency of the sympathetic. In other respects, its irritability is independent of the nervous impulses.

Owing to the fact that its trophic centre is situated, not in the nerve cell but in the muscular nucleoplasm, the irritability of the myoneural junction is not destroyed by degenerative resection of the nerve. On the contrary, we sometimes encounter the startling phenomenon of a denerved and decentralized tissue having a hypersensibility to chemical stimulation by adrenalin. The "paradoxical dilation of the pupil" which, after denervation (extirpation of the superior cervical ganglion), follows the instillation of adrenalin into the eye of rabbits and of cats, while the normal eye of these animals does not react to adrenalin, has already been described. As we then pointed out, this phenomenon is explained by the suppression of the inhibitory sympathetic fibres. Elliott believes, however, that it is rather due to increased irritability of the denerved peripheral tissue.

After denervation, the blood-vessels, the retractor muscle of the penis, and the hair muscles all react to adrenalin. That, after denervation, adrenalin also increases inhibitory activity, such, for instance, as that of the urinary bladder, is highly probable, but as yet without definite proof.

This hypothesis explains a number of peculiarities in connection with the action of adrenalin, some of which have long been recognized. It is well known that, in cats, while adrenalin is active, stimulation of the vagi does not produce cardiac inhibition, stimulation of the depressors is not followed by a fall in blood-pressure, nor stimulation of the nervi erigentes by contraction of the urinary bladder. As soon as the effect of adrenalin has passed off, the muscles respond to stimulus in the usual manner. There was a general disposition to ascribe to adrenalin a transitory paralysing action upon the terminals of the cranial and sacral autonomous nerves. We now know that adrenalin effects an almost maximal stimulation of the myoneural sympathetic terminals, and that it produces results which are dependent upon these. It is obvious, therefore, that, in each organ, the response to electric stimulus can be changed by the action of adrenalin only in a manner and to a degree such as would result from a strong stimulation of the sympathetic nervefibres. While adrenalin is active, stimulation of the sympathetic does not produce more intense activity. When the adrenalin action has passed off, the results of such stimulation are neither 
increased nor diminished, but represent the normal response. In those organs, however, in which, in addition to a sympathetic, there is also an antagonistic autonomous innervation, the result of the specific action of adrenalin will be so marked an increase in the strength of the sympathetic effects, that the autonomous innervation will appear to be paralysed. Similar results are observed after the simultaneous stimulation of the sympathetic and autonomous nerves of any organ, the sympathetic effects, in this case also, far outweighing those of the autonomous innervation. In cases where, while the sympathetic is under the influence of adrenalin, stimulation of the antagonistic autonomous nerve (vagus, pelvic nerve) is without effect, the result is due, not to paralysis of the latter, but to a preponderance of the chemical over the electric influence upon the nerve-terminals. Hence, as soon as adrenalin ceases to act upon the sympathetic, electric stimulus of the autonomous nerve produces the customary result.

In the course of his investigations into the physiological action of the ergot preparations, namely, cornutin (Kobert), sphacelotoxin (Jacoby), chrysotoxin or ergotoxin (Dale), A. H. Dale discovered certain remarkable departures from the known method of action of adrenalin, to which a special interest attaches.

The first effect produced by small quantities of the ergot preparations is a stimulation of all the organs possessing unstriated muscle structure. Such doses bring about contraction of the blood-vessels and consequent increase in blood-pressure, together with contraction of the uterus and of the sphincter muscle of the iris. Wve have as yet no certain knowledge as to the site of origin of this stimulatory action. Larger doses produce progressive inhibition of the motor elements which were previously stimulated. At this stage, adrenalin fails to effect either a rise in blood-pressure or uterine contraction. A still further increase in the size of the doses of ergot produces the paradoxical result, that adrenalin in quantities which, in the first instance, effected an enormous rise in blood-pressure and were negatived where the ergot dosage was moderately increased, now bring about a fall in blood-pressure, the degree and duration of which is proportionate to the amount of adrenalin exhibited. In the same way, a quantity of nicotine which at first increased the tension of the vessels now diminishes it. Stimulation of the splanchnic nerve or of the spinal cord, instead of producing the customary rise in arterial tension, is followed by a fall. Moreover, substances such as barium chloride, which, under normal conditions, exercise a direct stimulatory action upon the involuntary muscles, are now followed by a rapid increase in arterial tension; while pituitary extract, the action of which is to raise blood-pressure, increases the tension of the vessels in animals at the third stage of ergot poisoning.

It is, moreover, easy to prove that this effect of adrenalin in 
lowering blood-pressure is not due to a decreased cardiac activity, but is the expression of a peripheral vasodilation.

A similar gradual inversion of the characteristic action of adrenalin is also seen in the case of other organs. The behaviour of the uterus is entirely analogous. After moderately large doses of ergot, the pregnant uterus will not contract in response either to adrenalin or to stimulation of the hypogastric nerve; on the contrary, both methods produce considerable uterine relaxation. This applies to the rabbit and the ape. In the case of the cat, stimulation of the sympathetic, whether direct or by means of adrenalin, inhibits the movements of the virgin uterus, while, in the pregnant uterus, it produces strong contraction. Toxic doses of ergot do not alter the inhibitory action of adrenalin upon the virgin uterus of the cat, but the pregnant uterus responds, no longer by contraction, but by relaxation.

The spleen which, under normal conditions, responds to adrenalin by contraction, after ergot poisoning responds by relaxation. The internal sphincter muscle of the cat, in which the action of adrenalin normally produces contraction, likewise relaxes under the toxic influence of ergot. The stimulatory effect observed in the urinary bladder of the ferret, as the result either of adrenalin or of irritation of the hypogastric nerve, becomes inhibitory under the toxic influence of ergot.

Acute ergot poisoning produces no change in the nature of the results which follow stimulation of the cranial and sacral autonomous nerves, or of the nerves supplying the skeletal muscles. Stimulation of the vagus produces the customary effects of inhibition of cardiac activity and contraction of the stomach, intestine, and gall-bladder. Stimulation of the chorda tympani provokes secretion of saliva; and stimulation of the pelvic nerve is followed by the customary contraction of the urinary bladder and large intestine, and by dilation of the ressels of the penis. After poisoning by ergot, the skeletal muscle contracts in response to stimulation of its motor nerves in the same manner as under normal conditions. It is evident, then, that the inhibitory action of ergot is confined to the myoneural junctions of the sympathetic system.

A closer investigation shows, however, that this conclusion is subject to certain modifications. The following table shows the effects which adrenal has in stimulating the sympathetic nerves, both under normal conditions and after large doses of ergot.

The results which this table includes may be classified in three groups :

(I) Where the sympathetic innervation is exclusively inhibitory, as in the stomach, intestines, urinary bladder, and virgin uterus, ergot poisoning produces no change in the effects brought about by adrenalin and by stimulation of the sympathetic.

(2) Where the sympathetic innervation is exclusively stimu- 
latory (motor), as in the arteries of rabbits, in the heart, the ileocæecal sphincter, the urethra, the dilator muscle of the iris, the pilomotor muscles and the retractor muscle of the penis, the stimulatory action of adrenalin upon the sympathetic system is more or less weakened by ergot poisoning, or may in some cases even be abolished.

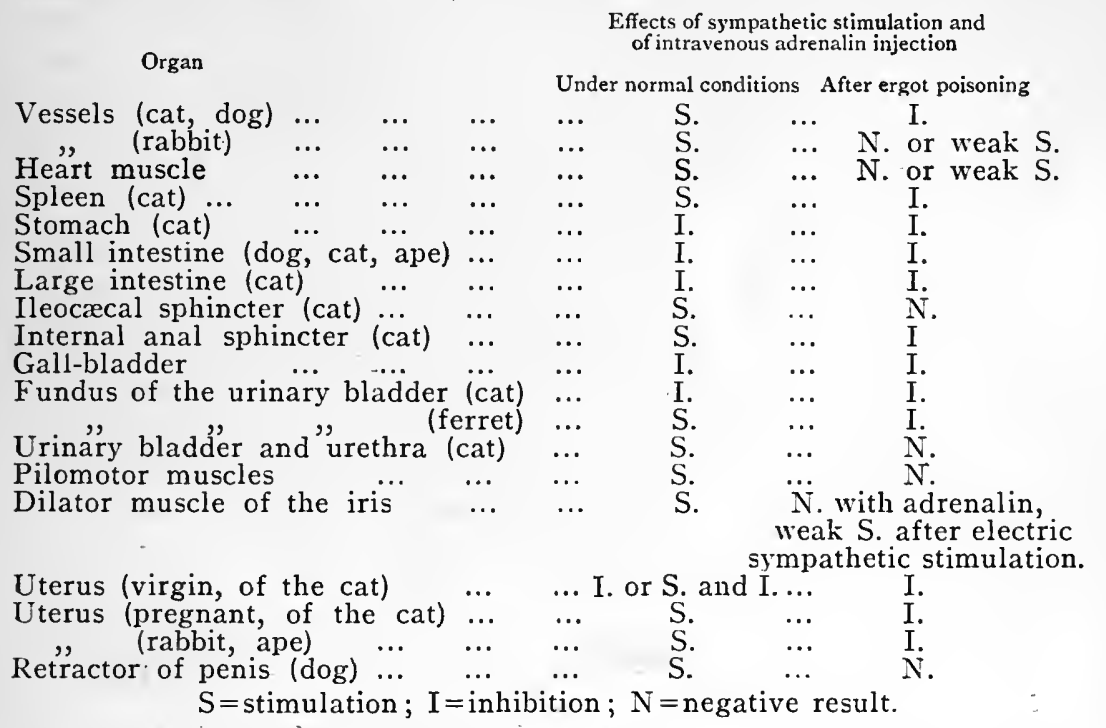

(3) Where the sympathetic innervation is twofold, both stimulatory and inhibitory in character, the stimulatory action preponderating under normal conditions, as in the vessels of the carnivore, in the spleen, the internal anal sphincter, the urinary bladder of ferrets, the uterus of rabbits and apes, and the pregnant uterus of cats, the action of adrenalin and the effect of electric stimulation of the sympathetic are exclusively inhibitory after ergot poisoning. It is evident from this that structures having a sympathetic innervation do not all respond to the action of ergot in the same manner. Those with inhibitory sympathetic innervation are entirely uninfluenced; those with stimulatory innervation become paralysed; where both stimulatory and inhibitory fibres are present, the inhibitory function, which was previously masked by the activity of the stimulatory fibres, becomes apparent as soon as these are put out of action.

Dale's experiments show that the same structural elements are influenced by the active principle of ergot as by adrenalin, and that the site of this influence lies in the sympathetic myoneural junction tissue. The inhibitory action of ergot is, however, confined to the motor myoneural junction tissue. Whether or no in organs with a double sympathetic innervation, the stimulatory and inhibitory functions are associated with the same substratum 
or are morphologically differentiated, is a question to which these observations of Dale's do not supply an answer. The results included in Group 3 render the former supposition the more probable; and these results are, moreover, evidence in favour of Langley's theory of the existence of a " receptive substance."

The results described above, together with extensive investigation into the action of nicotine and of curare, suggested to Langley that the cells possess two component parts: (I) a substance which performs the specific function of the cell, contraction and secretion; (2) receptive substances; which react to chemical influences and nerrous stimuli and which are able, in their turn, to affect the metabolism of the cells. According to this view, toxins such as nicotine, curare, atropine, pilocarpine, adrenalin, and strychnine, which were believed to exercise an influence upon the nerve terminals, do not act upon the terminals but upon a constituent of the reacting cell. This theory supplies a final answer to the question as to whether adrenalin acts directly upon the muscle itself, as Langley at first supposed, or whether it acts upon the sympathetic nerve endings. The myoneural junction forms a portion of the receptive substance, and is situated in the neighbourhood of the nerve terminal. The cells may contain either stimulatory or inhibitory receptive substances, or they may contain both; the effect of nervous stimulation will depend upon the extent to which both receptive substances are influenced by the nervous impulse.

According to Langley, not adrenalin only but other products of secretion, such as thyroidin and the internal secretion of the reproductive glands, react upon receptive substances in the cells: which are not necessarily connected with nerve fibres.

\section{THE BY-EFFECTS OF ADRENALIN.}

In addition to the physiological effects already described, adrenalin exercises certain other influences, partly physiological and partly toxic in character, which it is convenient to describe as "by-effects."

Such a physiological by-effect is the stimulation by adrenalin of the vasomotor and vagus centres in the medulla oblongata, to which reference has already been made. From what we now know of the action of adrenalin, those phenomena which appear after intravenous injection as the outcome of the stimulation of certain nervous centres, are due, not to the direct action of the substance itself, but to changes which it brings about in the circulatory conditions. In regard to its action upon the vagus and the consequent slowing of the pulse, there is no doubt that the result is due to stimulation of the vagal centre in consequence of the increased blood-pressure (Biedl and Reiner). The fact that the slowing of the pulse is immediately relieved by resection 
of the vagus or by atropine paralysis of the vagal terminals, is almost certain evidence in favour of this theory. It is, however, a more difficult matter to prove that the influence upon the vasomotor centres is secondary, though from what we know of the effects of high blood-pressure upon these centres, the assumption seems highly probable. Moreover, the exhibition of adrenalin is followed by other phenomena, due to the stimulation of certain other centres, which are certainly not provoked by the action of the substance itself, but are the result of the increased arterial tension.

To this group of phenomena belong the changes which are observed in the respiratory conditions after the injection of suprarenal extract or of adrenalin. After the injection of therapeutio doses, the respiration becomes shallow and diminishes in frequency. The shallowness is due to diminution of the inspiratory movements, the reduction in frequency to the prolonging of expiration. Sometimes there are isolated gasps and sighs, or there may be several prolonged expiratory pauses. If the injections are repeated, the shallowness remains, but the prolonged expirations disappear, and the breathing becomes slightly accelerated (Kahn). At the same time, the height of the expirations remains at the former level. The inspirations gradually become deeper, until, by the time the blood-pressure has fallen to the normal, the depth and frequency of respiration has also reached the normal.

Boruttau attributed this influence of adrenalin upon expiration to a direct inhibitory action, or at least to a reduction in the sensibility to stimuli of the respiratory centre in the medulla oblongata. It would be more accurate, perhaps, to speak of " an increased sensibility to stimulus on the part of the inhibitory centres," especially since Kahn has shown that the respiratory reflexes produced by centripetal stimulation of certain nerves, undergo a marked increase in intensity after the injection of suprarenal extract. Such are, the improved inspiration which follows weak stimulation of the central vagus stump; the improved expiration which follows stimulation of the superior laryngeal nerve; and the suspension of expiration (Kratschmer's reflex) which follows stimulation of the nasal branches of the trigeminus.

We have no proof that these changes in respiratory activity are the result of the direct action of adrenalin upon the respiratory centres; hence we are compelled to regard them, like the changes which take place in the bulbar and vagal vasomotor centres, as the result of changes in the circulatory conditions.

Among the effects of adrenalin of which we at present know very little, are those influences, perhaps physiological, but more probably toxic, which it exercises upon the striated muscles. According to Oliver and Schäfer, suprarenal extract, like veratrin, provokes an extension of the graphic curve of contraction of the 
gastrocnemius of frogs, as well as of the forearm muscles of dogs. According to Langlois, suprarenal extract produces a paralysis of the motor nerve endings, similar to that provoked by curare. Boruttau compared the action of adrenalin upon the striated muscles to the changes which take place in the first stage of fatigue. On the other hand, Dessy and Grandis believe that fatigued nerve muscle preparation of the frog is stimulated to renewed activity by the action of adrenalin. Panella, who confirmed this view, found that, in frogs, the action of the active principle of suprarenal was antagonistic to that of curare; it neutralizes the inhibitory effect which minimal doses of curare have upon the muscles, and in cases where curare paralysis has already made its appearance, it hastens the return to normal conditions. The results of Joteyko's investigations into the nature of adrenalin, seem to show that it produces neither stimulation nor inhibition of muscular action, but that it affects the muscular sarcoplasm in such a way as to render it more sensitive to nervous stimulus. These changes in irritability are, however, so slight as to be demonstrable only by special methods.

The experimental evidence which we at present possess is not sufficient to decide the question, as to whether the influence which adrenalin exercises upon the striated muscles is physiological or toxic in character. Observation of the heart muscle yields results which seem to point to the latter conclusion. For, in addition to the changes which have been described, certain phenomena are occasionally observed in connection with the heart muscle, which it is impossible to regard as other than toxic. In dogs, especially, both large doses of adrenalin and the injection into the veins of extract of the chromaffine bodies (Biedl and Wiesel), are frequently followed by arhythmia, and in some instances by a sudden fluttering which terminates fatally.

\section{TOXIC ACTION.}

When given in moderate doses, the influence of adrenalin is purely physiological, but when employed in large quantities its action is violently toxic. It exercises a destructive influence upon a number of tissues, and produces acute symptoms which may end in death.

The amount of the toxic dose of adrenalin is, to a certain extent, dependent upon the animal species and upon the method of exhibition. The intravenous injection of .I to .2 $\mathrm{mg}$. per kilo usually proves fatal to dogs, rabbits, and guinea-pigs. Amberg gives the toxic dose of epinephrin-though this is probably not identical with adrenalin-as I to $2 \mathrm{mg}$. per kilo in dogs. Cats, as a general rule, tolerate somewhat larger doses of adrenalin, the toxic dose being as high as .5 to $.8 \mathrm{mg}$. per kilo (Lesage). The size of the dose, the intravenous injection of which is immediately 
followed by death, is larger in proportion to the distance of the site of injection from the heart.

When injected subcutaneously, ten to fifty times the amount is borne. The lethal dose is about 8 to ro $\mathrm{mg}$. per kilo in guineapigs and rabbits, 5 to $6 \mathrm{mg}$. in dogs. Frogs tolerate a dose ten times larger in proportion to their weight than rabbits. About .I $\mathrm{mg}$. injected subcutaneously is fatal to mice weighing Io to 15 grms. Abderhalden and Slavu employed a similar dose of l-suprarenin. These authors found that, in the case of mice, the lethal dose was followed, either by convulsive symptoms lasting a few minutes, or by coma lasting several hours with a terminal fall in temperature. They found the action of $d$-suprarenin much less toxic, the fatal dose being .I to $.5 \mathrm{grm}$. The same authors also discovered that, if the animals are previously treated with increasingly large doses of d-suprarenin (.2 to $5 \mathrm{mg}$.), they acquire a definite tolerance of the l-component and will bear an amount two to ten times as large as under ordinary conditions. According to Watermann, the immunizing action of d-suprarenin is observed not only in regard to the fatal dose of 1-suprarenin, but also in the case of the dose which provokes glycosuria. $2 \mathrm{mg}$. 1-suprarenin produced little or no sugar in the urine of rabbits which had been previously treated with increasing doses (Io to 5o mg.) of d-suprarenin, though under ordinary conditions, such a dose of 1-suprarenin is invariably followed by glycosuria.

A. Fröhlich found that the intravenous injection of d-suprarenin ( 2 to $5 \mathrm{mg}$.) induced a condition in dogs and cats, in which the blood-pressure was entirely unaffected by the injection of natural adrenalin, by l-suprarenin in doses of I $\mathrm{mg}$., or by stimulation of the splanchnic nerve. In this state, death by asphyxia is not preceded by any rise in blood-pressure. According to Fröhlich, the non-toxic d-suprarenin, when given in large doses, combines with certain nervous or muscular portions of the blood-vessel cells and renders them completely inaccessible to the action of 1-suprarenin, to which these cells are, as a rule, very susceptible. Abderhalden, Kautzsch and Müller were unable to confirm Fröhlich's findings; they believe that the results of his experiments were largely influenced by the presence, in his animals, of relative cardiac insufficiency.

There is no doubt that the organism acquires a certain tolerance of the toxic action of adrenalin, for, by giving it at intervals of twenty-four hours or longer in increasingly large doses, rabbits, for instance, will take quantities up to $.4 \mathrm{mg}$. without signs of untoward effects. Moreover, it has been definitely proved by Elliott and Durham, that, where animals are under continuous adrenalin treatment, adrenalin antibodies are not formed in the organism.

When injected into the peritoneum, the lethal dose of adrenalin is about the same as when the injection is given subcutan- 
eously. Falta and Ivcovic recently discovered that, if exhibited by way of the stomach or intestines, or if given intravesicularly, enormous quantities of adrenalin are tolerated without toxic symptoms of any kind. No signs of poisoning followed the introduction with the probang of $20 \mathrm{mg}$. of adrenalin into guineapigs, 16 to $50 \mathrm{mg}$. into rabbits, and $150 \mathrm{mg}$. into dogs. The amount of adrenalin in the urine, after both subcutaneous and intraperitoneal injection, was insignificant. After exhibition by the mouth, however, considerable quantities of adrenalin, or of a substance possessing the chemical, physiological and toxic properties of adrenalin, were found in the urine. The authors believe that, when introduced into the stomach, adrenalin becomes fixed, either in the mucosa of the gastro-intestinal canal, or by the agency of the digestive secretions; that in this manner it loses its specific physiological and toxic properties; and that, when present in large quantities, it is again released in the kidneys.

Although adrenalin undoubtedly has a wide therapeutic application, it must be remembered that it is by no means a harmless substance. In its clinical employment, the subcutaneous injection of I $\mathrm{mg}$. is frequently sufficient to provoke toxic symptoms, such as palpitation and headache, while in older people whose vessels are sclerosed, there may be rigors (Falta). Some time ago v. Fürth pointed out the danger attaching to the indiscriminate employment of suprarenin.

The symptoms shown by animals dying of adrenalin poisoning, as well as the cause of death, vary to a certain extent with the method of employment of the drug. After the injection of large quantities into the jugular vein, the animals usually collapse at once and die after a few respiratory movements. In such cases, death is due to momentary arrest of the heart's action or to fluttering, consequent upon the cessation of circulation. The injection of the simple lethal dose, more particularly into the peripheral veins, frequently produces cedema of the lungs in rabbits, the animals dying within a few minutes. Many of the symptoms which appear in connection with the respiratory apparatus, such as the shallowness and infrequency of the breathing, the progressive dyspnœea, together with the fact that the animals may be kept alive for a long time by means of artificial respiration, point to inadequacy on the part of the respiratory nervous apparatus. This nervous inadequacy may be primary or, and this is more probable, it may be a secondary result of the changed circulatory conditions.

The subcutaneous or intraperitoneal injection of sufficiently large doses is, at first, followed by a stage of intense excitement, accompanied by repeated vomiting and sanguineous diarrhœea; later on, there is increasing weakness and, finally, extreme prostration with complete paralysis; death follows after a few hours-twenty-four at the most. The autopsy reveals a high 
degree of hyperæmia of all the internal organs; a serous, and occasionally sanguineous, fluid is present in the pleural, peritoneal, and pericardial cavities; there are sub-pleural, subpericardial, and epicardial ecchymoses, relaxed heart muscle, pulmonary hæmorrhages and, occasionally, pulmonary œdema. The abdominal viscera, especially the intestine, are not only markedly hyperæmic, but they are frequently covered with extensive hæmorrhagic patches.

There are, undoubtedly, good grounds for ascribing the fatal termination which follows the intravenous injection of large quaintities of adrenalin, to profound circulatory disturbances; but where the substance is introduced either subcutaneously or into the peritoneum, there is no evidence as to the cause of death. The destructive influence which adrenalin has upon the tissues, which will be fully discussed later, suggests, however, that the cause of death in these cases is to be sought in a destruction of the vital tissues.

Of the toxic effects which adrenalin exercises upon individual tissues, the most conspicuous is the necrosis which it sometimes produces at the site of either subcutaneous or intraperitoneal injection. As far as my observation goes, this process is of very frequent occurrence; it affects the subcutaneous cellular tissue and is more marked in proportion to the weakness of the adrenalin solution employed. The subcutaneous injection of .I mg. of the usual solution of $\mathrm{I}$ : $\mathbf{I} 000$ into rabbits, rarely produces necrosis; but necrosis almost invariably appears if the same dose is diluted with large quantities (50 to Ioo c.cm.) of sterilized normal saline solution. If this method is employed, marked œedema will appear at the site of injection, and this will be distinctly visible the next day, becoming hæmorrhagic later. In the course of the next few days, the skin becomes more or less extensively necrosed and finally heals, after forming a scab.

The subcutaneous or intravenous injection of adrenalin is followed by inflammatory and degenerative changes, not at the site of application only, but also in organs distant from it. These changes are observed after a single injection and are more marked where the injections are repeated. The kidneys show signs of inflammation and infiltration, together with hæmorrhages and necrosis; there are hæmorrhages and necrotic and cirrhotic changes in the liver; symptoms of inflammation appear in the lungs; and necrotic processes are seen in the mucosa and muscular structure of the stomach, intestines and urinary bladder. Schlayer found that the injection of adrenalin was followed by changes in the renal tissue resembling those of contracted kidney. Both Erb and Fischer found that the repeated injection of adrenalin into the veins of rabbits, was followed by multiple cerebral hæmorrhages, associated with pathological changes in the substance of the brain. The cause of these hremorrhages probably lies in a primary lesion of the cerebral vessels. 
The most conspicuous of the toxic effects of adrenalin is that which it exercises upon the circulatory organs. The repeated injection of adrenalin produces serious changes in the heart; hypertrophy of the left ventricle, myositis fibrosa, ischæmic infarction, foci which have undergone hyaline degeneration, and necrosed patches, have all been observed.

Of the changes effected by suprarenal extract, or by adrenalin, upon the circulatory apparatus, those which may be experimentally induced in the arteries have received the most attention. It is expedient to the present purpose to enter rather more fully into this subject.

Josué was the first to show, in 1902, that the repeated injection of adrenalin into the veins of rabbits produces atheroma of the ressels. Jores had previously tried to produce changes in the vessel walls by means of suprarenal tabloids given by the mouth, but his experiments were quite unsuccessful.

Later, experiments were carried out upon animals of varying species with the different suprarenal preparations; with adrenalin obtained from the suprarenal and with synthetic preparations, such as methylaminoacetopyrocatechin, by Sturli; and with synthetic suprarenin and its components, l- and d-suprarenin, by Biedl.

Experiments with dogs have produced somewhat variable results. Fischer and Mori, as well as Pearce and Stanton, obtained negative results; while Tarantini and Braun found that the intravenous injection of adrenalin was followed by vascular changes similar to those seen in rabbits immediately after such injection. According to Loeper, Lissauer, $v$. Hansemann and Boveri, the symptoms are intensified by feeding the animals with calcium; moreover, animals, the normal diet of which is rich in calcium, such as the hare, horse and cow, are more susceptible to vascular changes than animals whose normal food contains but little calcium (dog, cat).

The rabbit is a very reliable subject for such experiment,* and has been employed in the great majority of cases. It is evident that, in the first place, the appearance of changes in the ressel walls is dependent upon the manner in which the drug is exhibited. The results of subcutaneous injection have occasionally been positive, but far more often negative; in character. After intratracheal injection, Külbs observed typical changes in the aorta; Fischer found that intraperitoneal injection was not followed by such changes; Erb noticed slight changes in the aorta; d'Amato observed similar changes after the introduction of Vassale's preparation, "paraganglin," into the stomach; and Tarantini obtained only negative results by the latter method.

* Klotz and Bennecke think that the large calcium contents of rabbits' blood is significant in this connection. 
The most reliable results have been obtained by means of intravenous injection (Erb, B. Fischer, K. Ziegler, Lissauer, Braun, Minorescu, Scheidemandel, v. Koranyi, Pearce and Stanton, v. Rzentkowski, Külbs, Hedinger, Loeb, Loeper, v. Frey and Meyer, Pic and Bonnamour, \&c.). Kaiserling is the only author who is not convinced that the changes in the aorta are directly due to the action of adrenalin. The vast majority of authors hold the opinion that the results of adrenalin experiments are in no sense accidental, and that there is, as far as we know at present, no reason to doubt that the changes observed in the vessels of rabbits are the outcome of the action of this substance.

The vascular changes take place in response to quite small quantities of adrenalin. Braun found that, after a single injection of .I mg., several calcareous foci were present in the aorta; he found, also, that the repeated injection of .oor $\mathrm{mg}$. of Clin's adrenalin, or even of fractions of this quantity, offered the best means of studying the preliminary stages of " adrenalin sclerosis."

Speaking broadly, these changes take place more readily in old animals than in young ones; ${ }^{*}$ there are, moreover, certain idiosyncrasies peculiar to individuals and to varieties, $\uparrow$ but there is no sex incidence (Bennecke); and it is certain that diet exercises a modifying effect (v. Koranyi).$_{+}^{+}$

Where small doses are employed, the changes are distributed throughout the entire vascular system (Braun). Of the observers who employed larger doses, the greater number describe changes in the aorta only. The changes appear first and are most marked in the upper portion of the thoracic aorta, though they are occasionally met with in a slighter form in the abdominal aorta. According to v. Rzentkowski, the most frequent site is at the fork where the vessel branches. The macroscopic findings, as described by different authors, agree in their main points with those originally set forth by Josué.

At first, small, whitish, granulated patches, of about the size of a pin's head, make their appearance upon the inner surface of the aorta, and gradually combine to form round or band-like plaques. They early undergo calcification, patches no larger than a pin's head assuming an indented cup-like form. Later on, cup-like, round or oval aneurysmal pouchings are formed, often with rough chalky ridges between them. Diffuse calcification is also observed, though its occurrence is somewhat rare.

The histological changes (Braun) consist, in the first instance,

* Josué thinks that young rabbits are not susceptible to adrenalin. This view is confirmed by $\mathrm{O}$. Loeb and Githens, but is disputed by Bennecke.

+ Albinos and the more finely-organized breeds are less resistant to adrenalin.

$\ddagger$ According to $v$. Koranyi, rabbits fed on carrots have a greater power of resistance than those fed on oats.

I6 
of serous infiltration of the media; proliferation of and hypertrophic changes in the muscles of the vessels, and injury of the elastic elements in the vessel walls, especially of the lamina elastica interna, together with consecutive, and probably compensatory, proliferation of this tissue. In its earlier stages, it is difficult to define the exact nature of the injury to the muscular portion of the vessel walls. It is certain, however, that at a very early stage there are softening of the tissue, nuclear changes, and thickening of the fibres, the latter being probably compensatory. In the case of vessels of the muscular type, the changes in the intima-the "hyperplastic proliferation of the intima"-are, as a rule, apparent when the media are only slightly affected. At a later stage, the proliferation of the intima in vessels of both kinds becomes degenerative.

The final stage consists in necrosis of the muscle cells (Erb, Torri, Fischer, Hedinger, Lissauer, Külbs, Ziegler, Saltykow, \&c.), together with calcification of circumscribed areas, and various changes in the elastic fibres. The question as to whether the muscular changes are preceded by fatty degeneration has been differently solved by different authors. Klotz, Saltykow, Pearce and Stanton, Lissauer and Bennecke believe that this is the case; on the other hand, Fischer, d'Amato and Flagella, and Scheidemandel have never observed fat.

Where there are foci of calcification, the elastic lamellæ frequently appear to be stretched; their normal undulations disappear; and "granulated, elastic" fibres are observed. Later on, where the lesion is very pronounced, the elastic lamellæ may tear, or rather break, afterwards splitting and crumbling until they finally disappear. Fatty degeneration of the elastic fibres has also been described (Klotz).

Calcification is frequently present at a very early stage. According to Trachtenberg and Miesowicz, formation of cartilage also takes place. At the beginning, the calcified foci are, as a rule, sharply defined from the sound tissue. Where the media is completely destroyed, a deep and progressive pouching of the wall gradually takes place and an aneurysm is formed. The foci and the chalky scales upon the media become surrounded later by cellular proliferations, which consist of leucocytose and lymphocytose infiltration, granulation tissue and proliferation of muscle cells. It is my own experience (Biedl and Braun) that all these changes take place. The descriptions which have been given of giant cells are doubtless well founded. The hypertrophic muscle fibres, especially in section, closely resemble cartilage cells. In the later stages, there is connective tissue formation (Ziegler), diffuse sclerosis, fibrous degeneration of the media (Scheidemandel, Torri), reactive inflammation, mesarteritis, and endarteritis (B. Fischer).

The changes in the intima consist, at first, of endothelial pro- 
liferation (Erb, Külbs, Saltykow); fatty degeneration of the proliferated endothelium (Saltykow, Klotz); and connective tissue thickening of the intima (Papadia, Watermann). According to $\mathrm{K}$. Ziegler, the proliferation of the intima is wholly compensatory, its purpose being to fill up the inequalities of the inner surface of the vessel. This can apply, however, to a small proportion of cases only. F. Falk has frequently observed proliferation of the intima and of its component parts in portions of the small vessels which had become pouched as a result of the process. Fissure of the lamina elastica interna is frequently observed, as well as the occurrence of nuclei (unstriated muscles) in the intima of the small vessels. It is probable that changes (softening) do not take place in the media of the small vessels, or if they do, they can only be of the slightest description. Here and there, pronounced connective tissue traits appear in the portions of the media bordering upon the adventitia (Falk).

K. Ziegler explains the sporadic appearance of the diseased condition by the implication of the vasa vasorum. With regard to the primary localization of the disease, all authors, with the exception of Loeper, Papadia and Boveri, are agreed that the changes in the media are essential, the changes in the intima secondary; in character. Whether, however, this rule applies equally to the small vessels, is a point upon which no certain pronouncement can at present be given. The tissue which is first affected is the muscular structure of the vessels (B. Fischer, Falk, \&c.), although v. Rzentkowski, Marchand, Scheidemandel and Trachtenberg localize the site of earliest affection in the elastic portions of the vessel walls.

The pathogenetic factors in the changes brought about by adrenalin in the arteries are : its effect in raising blood-pressure; its toxic action upon the vessel walls; and perhaps, also, a direct influence upon the vasa vasorum.

Changes similar to those in the aorta are also observed in the pulmonary artery, in the veins (d'Amato), and in the lymphatics (Donzello and Vito Tardo), in all of which the conditions governing pressure could hardly be altered, to any appreciable extent, by the action of adrenalin. These facts militate against the view that the increased blood-pressure is the sole pathogenetic factor. Moreover, L. Braun, and later Waterman, both observed identical changes in the arteries in cases where, by injecting adrenalin in combination with amyl nitrite, they had excluded every possibility of increased blood-pressure. Similar results were obtained where adrenalin was administered simultaneously with other substances which lower blood-pressure; Boveri, for instance, employed adrenalin with opothyroidin; Mironescu adrenalin plus euphthalmin, which is a substance of the atropine group.

B. Fischer proved that changes in the vessels may be also 
brought about by the agency of many substances which do not raise pressure, if these are administered in a suitable manner. Such substances are: normal saline solution in large quantities; hydrochloric, phosphoric, and lactic acids; calcium phosphate, potassium bichromate, uranium nitrate, chloral amide, phlorizin, trypsin, pepsin, thyroidin, and dried mammary gland. It must be conceded, however, that these substances never produce changes as marked as those which result from the action of adrenalin.

The toxo-chemical action of adrenalin is still further proved by the results of Falk's experiments. While ignoring the rise in blood-pressure produced by adrenalin, he succeeded in intensifying or reducing the arterial changes which followed it by injecting, together with adrenalin, substances which have no influence upon blood-pressure, their action being purely chemical.* According to Falk, a repeated, transitory rise in pressure and functional hyper-stimulation of the muscles of the vessels, are essentially the predisposing causes of necrosis of the vessels. "High blood-pressure alone never gives rise to serious injury of the media, that is to say, to regressive changes; for hyperactivity is, in itself, far more likely to lead to a gradual hypertrophy of the various layers forming the walls of the vesseis. It is in this way that Falk explains the results of experiments in which changes in the vessels, in every way resembling those provoked by adrenalin, were brought about by repeated mechanical hypertension induced by the injection of gamboge or croton oil, with simultaneous compression of the abdominal aorta.

In company with L. Braun, I investigated the effect which purely mechanical hypertension exercises upon the vessels. We found that, in the rabbit, sheep, and ape, repeated compression of the aorta above the point of departure of the renal artery gives rise to lesions of the vessels, which upon the whole, are analogous to those produced by adrenalin. From the results of experiments carried out under special conditions, we concluded that the vascular lesions depend, not only upon the hypertension, but also upon the extreme pressure fluctuations which take place in the arterial system, and which make special demands upon the elasticity of the vessel walls. $\dagger$

* The arteriosclerosis was either prevented or arrested by the simultaneous injection of chemotaxic substances, such as: dead staphylococci, pyocyaneusprotein, oil of turpentine, aleuronate, and sesame oil ; it was enormously increased by the subcutaneous injection of croton oil, gamboge, and bacterial toxins. Falk thinks that the absence of arteriosclerosis is due to the increase in the amount of lymph. After Biedl and Offer had proved that the glocosuric action of adrenalin is inhibited by lymphagogue substances, Falk found that the injection of extract of leeches, which increases the circulation of lymph, was likewise unattended by changes in the vessels.

† A detailed account of "compression sclerosis" as observed by Braun and myself appeared in the Sitzungsberichten der $k$. Akad. d. Wissensch. in Wien. Vol. 119. 1911. 
Serious changes in the aorta of rabbits after compression of the abdominal aorta are described by W. Henwood Henry; and Klotz observed similar changes in rabbits after they had been repeatedly suspended with the head downwards.

These experiments illustrate the important part played by changed hæmodynamic conditions in the etiology of lesions of the vessel walls. They serve, moreover, to establish the probability of this agent as a factor in the causation of adrenalin sclerosis. The discovery that similar changes are produced in the vessel walls by other substances, whose property it is to increase blood-pressure, such as digalen, digitoxin, strophantin, barium chloride, ergotin, nicotine, and formaldehyde, may be regarded as confirmatory evidence, although it is not possible in these instances to exclude the operation of chemico-toxic factors.

In spite of much searching investigation, it has not been possible, up to the present, to arrive at any conclusive decision concerning the pathogenesis of adrenalin arteriosclerosis. But the increase and the fluctuation in pressure, on the one hand, and the toxic injury to the vessel walls, on the other, are undoubted factors in the causation of the condition.

These findings are confirmed by the results of certain experiments which I recently undertook, with the object of discovering the changes brought about in the vessel walls by means of $1-$ and d-suprarenin. I found that, when given in quantities too small to provoke a rise in blood-pressure, serious sclerotic changes followed the employment, not only of the 1-component whose property it is to raise blood-pressure, but also of the d-component which, owing to its slight toxicity, may be given in larger doses. In no case did I find that treatment by subcutaneous injection of d-suprarenin created a tolerance of subsequent intravenous injection of 1-suprarenin. The changes in the vessels to which d-suprarenin gives rise are undoubtedly in the nature of toxic injury, brought about by the agency of a substance, the affinity of which to certain components of the vessel walls is suggested by certain experiments of A. Fröhlich's, which have already been described.

The following facts are known concerning the part played by certain physical conditions in the causation of a sclerosed condition of the vessel walls.

Pregnant animals appear to be less susceptible than others to the influence of adrenalin (Loeb and Githens, Loeper, Boveri, v. Koranyi). It is certain that the conditions of pregnancy, castration (Thevenot), cachexia (L. Loeb and Githens, Pic and Bonnamour), and fatigue (Thevenot), are not favourable to the development of adrenalin lesions. During the period of lactation, the resistance to the action of adrenalin is lowered (Pic and Bonnamour, Thevenot). As far as the effect upon thyroidectomized animals is concerned, opinions are divided. It must 
be conceded that, upon the whole, the effect which different physical conditions have upon the enlargement of the vessels as induced by adrenalin, is at present insufficiently investigated.

The influence which certain drugs have upon the changes in the vessels produced by adrenalin, has formed the subject of extensive investigation. Of these, iodine has received the most attention.

v. Koranyi found that the simultaneous exhibition of adrenalin and iodine inhibits the changes which adrenalin provokes in the vessels. Boveri arrived at the same conclusion, but Biland obtained quite opposite results. He found that the simultaneous exhibition of iodide of potassium and suprarenin favoured the appearance of changes in the aorta. Hedinger, Loeb, and Fleisher, as well as Kalamkarow, bring forward a large amount of evidence in confirmation of Biland's findings; Klienenberger, on the other hand, is unable to show that the effects of adrenalin are inhibited by the action of iodine. The inhibitory influence of iodipin upon the development of adrenalin sclerosis, depends upon the amount of sesame oil which it contains (Schrank). According to Mansfield, the simultaneous employment of cholin with adrenalin does not arrest the changes in the ressel walls.

The vast majority of authors who have occupied themselves with the question of the changes which adrenalin produces in the vessels, have discussed this condition in its relation to the clinical form of arteriosclerosis. The views which different authors hold upon this subject are, however, very diverse. Josué, Loeper, Gouget, Gilbert and Lion, Boveri, Braun, etc., believe that the two conditions are more or less identical ; while Fischer, v. Rzentkowski, Scheidemandel, Hedinger, d'Amato, Lissauer, Loeb, Klotz, Saltykow, etc., lay stress upon the differences which subsist between the two conditions. Certain signs, such as the calcification of the media as seen in the clinical condition, are believed by many authors (v. Erb, Biland, Bennecke, Klotz, Marchand, Saltykow, etc.) to be common to both pathological forms.

\section{ADRENALIN AS A SECRETORY PRODUCE OF THE ADRENAL SYSTEM.}

It has been shown that adrenalin is a well defined chemical substance, minimal quantities of which produce definite physiological results. The next step is to discover whether this substance is formed and passes into the circulation during life, or whether it comes into existence post mortem.

The first question to be decided, however, is-in which of the two suprarenal systems is adrenalin formed? As stated in an earlier chapter, Vulpian and Virchow were able to obtain the colour-reactions characteristic of adrenalin with the medullary substance of the suprarenal only; with that tissue, in fact, which, 
as Henle proved, stains brown in watery solutions of chromic acid and the chromates:- Oliver and Schäfer next discovered that the specific action of suprarenal extract is obtained exclusively with the extract of the medulla, extract of the cortical substance being negative. It was shown by both Langlois and S. Vincent, that blood-pressure is raised by those extracts only which are obtained from animals, the suprarenals of which are composed of medullary and cortical portions. Vincent found that extracts of the socalled suprarenal bodies (Balfour) of Selachii, were physiologically active and, in combination with Moore, he succeeded in obtaining with these substances the chemical reactions characteristic of the chromogen of the suprarenal medulla. Extracts of the interrenal bodies were partially active owing, Vincent believes, to the inclusion of a proportion of medullary substance; extracts of the bodies of Stannius of Teleosteii were completely inactive. These tests were only employed to prove toxicity.

I next found that extracts obtained from the adrenal organs of Selachii possess all the physiological properties of adrenalin. It is a noteworthy fact that, if adrenal organic extract is injected into the branchial arteries of rays or torpedoes, a rise in bloodpressure takes place similar to that which follows the injection of adrenalin. Finally, it was shown by Biedl and Wiesel, that extracts of the extracapsular chromaffine tissue, more particularly extract of the accessory sympathetic organs in the neighbourhood of the abdominal aorta of children (Zuckerkandl), produce contraction of the peripheral vessels and increased cardiac activity, by which a rise in arterial tension is effected. These authors also discovered that the substances exercise a transitory injurious effect, first upon the right heart, and afterwards upon the left, which is manifested by the occurrence of arhythmic beats. They emphasize the fact, and in this they are supported by $A$. Kohn, that the physiological reaction which is regarded as the specific effect of suprarenal extract, is a property of the extract of the chromaffine cell-group. In view of these results, it is hardly possible to doubt that adrenalin is a chemical component of that tissue which is distinguished, histologically, by the chromaffinity of its cells, and which has received the name of adrenal tissue.

A certain amount of information as to the quantity of adrenalin present in the suprarenal and in the free portions of the adrenal system, is supplied by histological investigation. The chromaffinity of the adrenal cells, that is to say, the property which their cytoplasm has of staining brown or yellow-brown with chromium salts after suitable fixation, is the measure of their adrenalin contents.

The most suitable fixing fluids are mixtures of potassium bichromate and formalin (Kohn, Wiesel). The objects must be as fresh as possible when put into the fixing fluid, for the chrome reaction disappears with remarkable rapidity after death, the cells 
refusing to stain after twelve or, at the outside, thirty hours. It should be further borne in mind that all cells do not stain equally intensely, and that their chromaffinity is by no means always parallel to their adrenalin contents, as revealed by other methods. In the experiments of Schur and Wiesel, in which prolonged narcosis in rabbits was followed by reduction and total disappearance of the chromaffinity of the suprarenal medulla, I found a discrepancy between the chromaffinity of the suprarenal extract employed and its effect in raising blood-pressure. For instance, the hypertensive action of one extract was of about normal intensity, but the chromaffinity of the suprarenal from which it was derived was very much reduced. Whether, in these cases, the slight chrome reaction is due to insufficiency of the adrenalin contents, or to the presence of non-chromophile pre-stages of adrenalin, is at present undecided. Whatever the cause, however, findings such as these show very clearly that Henle's chrome reaction can be employed at best as a provisional quantitative test only for adrenalin.

Battelli was the first to attempt to record in actual figures the amount of adrenalin contained in the suprarenals. For this purpose, he employed a colorimetric method of his own with ferric chloride, but was able to obtain only approximate results. Battelli and his pupil, S. Ornstein, measured the amount of adrenalin in the suprarenals of man, rabbits, and guinea-pigs, by estimating the increase in blood-pressure produced by the extracts of those organs. The results of their experiments showed that the adrenalin contents of both suprarenals are approximately the same in the dog and the rabbit, but that in the guinea-pig the amounts differ, the excess being in the right or left organ indifferently. They also proved that, in the case of the dog and the rabbit, extirpation of one capsule did not lead, at any rate within the next few days, to a change in the adrenalin contents of the other. In the case of the dog, however, a reduction was occasionally observed in the adrenalin contents of the capsule left in situ.

Battelli's tables show that I grm. of suprarenal substance contains, upon an average, I mg., or slightly more, of adrenalin. It was estimated that is grm. of suprarenal substance of cattle contained a mean quantity of $4.3 \mathrm{grm}$. of medullary substance, and $.03 \mathrm{I} \mathrm{grm}$. of adrenalin; that is to say, .75 per cent. of adrenalin was present in the medullary substance.

According to Battelli, the adrenalin contents of the suprarenals of different animals, to every $\mathrm{I}, 000 \mathrm{~kg}$. of body weight, are as follows :

$\begin{array}{llll}\text { Man } & \ldots & \ldots & .0603 \\ \text { Ox } & \ldots & \ldots & .074-.077 \\ \text { Horse } & \ldots & \ldots & .0816--12 \\ \text { Sheep } & \ldots & \ldots & .115-.121 \\ \text { Pig } & \ldots & \ldots & .078-.084 \\ \text { Dog } & \ldots & \ldots & .0666-.116 \\ \text { Rabbit } & \ldots & \ldots & .083 \\ \text { Guinea-pig } & \ldots & .229\end{array}$

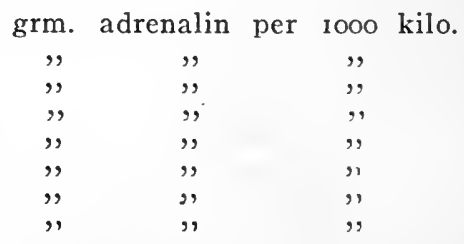


Although these figures possess an approximate value only, they show that the adrenalin contents in proportion to body weight are very much the same in different animals. The guinea-pig forms the only exception, the adrenalin contents in this instance being twice, or nearly three times, as large as in other animals.

Quantitative chemical tests for adrenalin have not as yet been carried out by means of the more improved modern methods. The following facts have been ascertained concerning the adrenalin contents of the suprarenals under both physiological and pathological conditions.

Langlois and Rehns found that the suprarenals of fœetuses yielded, at the end of the first half of pregnancy, extracts which produced a rise in blood-pressure, and also gave a positive reaction with ferric chloride. Judging by the chromium reaction, the portions of the adrenal system which are external of the suprarenals contain adrenalin earlier in the life of the embryo than the cells of the medulla (Wiesel).

That the adrenalin contents of the suprarenals are affected by changes in the physiological conditions, was first shown by Battelli and Roatta. These authors found that the amount of adrenalin in the suprarenals of dogs, which had been allowed to run in a treadmill until completely exhausted, was reduced to a third of the minimal normal amount. Where the muscular exertion was followed by muscular repose of several hours duration, the adrenalin contents became increased until far in excess of the normal.

Schur and Wiesel confirmed these results also with dogs. They found that, after running at the treadmill for six to seven hours, there was a distinct reduction in the cells of the medulla and total disappearance of their chromaffinity; there was no reaction to ferric chloride; and extract of these suprarenals failed to produce mydriasis. By means of the last-named test, they proved that, at the same time, the blood contained a large amount of adrenalin. Schur and Wiesel also discovered that prolonged anæsthesia, as the result of ether, chloroform, or A.C.E. mixture, produced in rabbits a reduction, or even complete disappearance, of the adrenalin in the medulla as well as of the chromaffinity of the latter, while at the same time adrennalin was found to be present in the blood. Kahn, however, was unable to confirm the results of Schur and Wiesel, either after muscular fatigue or after prolonged narcosis.

Considerable interest is attaching to the manner in which the adrenalin contents of the suprarenals are modified by pathological conditions. It has long been known that, in certain infective diseases, changes take place in the suprarenals, and these have been studied by means of experiment. Roux and Yersin described a high degree of hyperamia in the suprarenals of guinea-pigs, after experimental diphtheritic infection or poisoning 
by toxins. Charrin and Langlois found that the injection of pyocyaneus toxin was followed by pronounced hyperæmia and increase in the volume of the suprarenals, and Pettit observed histological degenerative changes. Bogomolez discovered serious changes in the suprarenals, namely, necrosed and hemorrhagic areas, after infection with diphtheria bacilli; and Tscherwenzow observed similar signs after infection with plague bacilli, Labzine after streptococcus infection.

According to Langlois, the amount of the active substance which raises blood-pressure is apparently unchanged in the hyperamic suprarenal; while, after chronic infective conditions, the capsule, though increased to three or even four times its original volume, yields extracts which are totally inactive. According to Luksch, the suprarenals of rabbits poisoned with diphtheria toxin or by the injection of other bacterial toxins (tuberculosis, typhus), do not contain a substance which either raises blood-pressure or produces enlargement of the pupil. This author also found that suprarenal extract obtained from uramic animals or those poisoned with phosphorus, is inactive, but that positive results were yielded by extracts obtained after hunger, fever, poisoning with hydrochloric acid, and resection of the spinal cord.

Schur and Wiesel found adrenalin in the blood after double nephrectomy, and after the excision of wedge-shaped pieces from the kidneys; there was, moreover, a change in the chromaffinity of the suprarenals, the medullary substance containing cellgroups which were strongly chromophile, together with other groups which were achromophile. After prolonged renal injury, both the suprarenal medulla and the free chromaffine bodies present appearances which suggest hypertrophy of the chromaffine tissue.

Molnár and Goldzieher found adrenalin in the blood of rabbits after the subcutaneous injection of pounded and rubbed-down kidney. They also found histological signs of hypertrophy in the suprarenals in thirty clinical cases of chronic nephritis.

There has been, up to now, no systematic investigation of the adrenalin contents of the suprarenals in different clinical conditions; and exact information as to the amount of adrenalin present in the suprarenals in Addison's disease is particularly scanty. In two cases of Addison's disease, I found that the extract obtained from the suprarenals, which had undergone serious pathological changes, when injected intravenously, produced no effect upon the blood-pressure. In a clinical case described by Neusser, which he believed to be one of suprarenal hypertrophy, I found that the extract obtained from the suprarenal, which had undergone pronounced carcinomatous degeneration, was exceptionally active. A portion of a suprarenal, obtained from a case of extreme cardiac hypertrophy, which was handed over to me by my colleague Störk, yielded an extract with strong hypertensive properties. On the 
other hand, I have always found that extracts of hypernephroma are negative in their results.

The above data concerning the variation in the adrenalin contents of the suprarenals, point with some degree of certainty to the suprarenal tissue as the site of origin of adrenalin.

The question as to whether adrenalin is formed during life, and is continuously supplied to the blood-stream by way of the veins of the suprarenal capsule, has already been answered in the affirmative in a communication of Vulpian's. Vulpian found that the blood in the suprarenal veins contains the substance which gives a green reaction with ferric chloride. By means of the vaso-contractor action of blood from the suprarenal veins, Cybulski showed that the active substance is formed continuously in the suprarenals and continuously discharged from them into the blood-stream. His results have been confirmed in several directions (Langlois, Bied1, Dreyer), and it has, moreover, been shown that the production of the active substance of the suprarenals is subject to the influence of the nervous system (Biedl). Ehrmann next proved, by means of his more sensitive test with enucleated frogs' eyes, that there is a continuous supply of adrenalin from the suprarenal to the blood-stream. He was able to show, moreover, that the amount of the substance supplied varies in different animal species, while in individuals of the same species it is substantially the same. The adrenalin contents in cats is low, in rabbits it is high. Ehrmann concludes that, upon the whole, those animals are most sensitive to adrenalin which have the larger amount of it circulating in their blood. The amount of adrenalin present in the blood in the suprarenal veins of rabbits, is equal to an adrenalin solution of between one in one million and one in ten million, that is to say, .0000005 $\mathrm{grm}$. in $\mathrm{I} \mathrm{c.cm}$. of blood serum.

Watermann and Smit found that blood serum from the vena cava produced mydriasis when undiluted only, and they estimated the adrenalin contents of the caval blood at .ooooor grm. per cubic centimetre. They found that electric stimulation of the suprarenal, by which all the sympathetic nerve fibres entering the organ are stimulated, produced an increase in the adrenalin contents of the blood in the vena cava, for the serum of such blood caused enlargement of the pupil, even in a 1 : 10 or 1 : 12 solution.

The suprarenal secretory process has long proved a subject of interest and investigation to the morphologist. The first point to be considered in this connection is the activity of the chromebrown adrenal tissue, which was investigated by Stoerk and v. Haberer under exceptionally favourable conditions. They were able to study the secretory process during excessive muscular exertion, and also to study the hypersecretion which accompanies compensatory suprarenal hypertrophy. As the result of their efforts, considerable light has been shed upon the nature of the secretory process of the suprarenal medulla. 
Seeing that the morphology of the adrenal system is everywhere the same, what is known of the secretory processes of the suprarenal medulla may be taken as typical of the entire suprarenal system. Whether forming the medullary portion of the suprarenal capsules, or present in the form of isolated bodies, either unattached or associated with the sympathetic nervous system, adrenal tissue is invariably composed of rows and spheroid agglomerations of cells, held together by a delicate structure of connective tissue, and forming a large-meshed net. The parenchymatous cells are always placed close against the bloodvessels, and are, as a rule, separated by the endothelium only, though occasionally. also by a delicate connective-tissue sheath, from the lumen of the capillaries and the enlarged sinus-like veins. The cytoplasm of the living chrome-brown cell contains extremeiy minute granules, which are, however, coarser in certain animal species. Some of these granules cluster elosely together, while others are arranged more loosely; they are not so highly refractive as fat-granules; they dissolve in water and in alcohol, but are not soluble in ether, acetic acid, the etherial oils, or in xylol. In fresh unhardened and unstained preparations, these granules are scarcely visible; they do not stain satisfactorily by any known method, the best, however, being iron-hamatoxylin with which they colour a pale grey. When unstained, they are most easily recognizable by means of a mixture consisting of Müller's fluid and formaline or by the various osmium solutions. By using Zenker's fixing fluid, it is sometimes possible to obtain a good eosin coloration. According to Poll, these granules have a certain relationship to the nuclear colouring substances. They colour pale pink with Ziehl's solution, lake by van Gieson's method, mauve with methylene-blue eosin, and greenish with UnnaPappenheim's methylene-green pyronin mixture.

It is principally these fine granules which respond to the ferric chloride and chromium tests, and give the reaction which is characteristic of adrenalin. It is true that microscopic preparations with ferric chloride are not entirely free from objection. But with fixing fluids containing chromium (chrome formol), these granules take on a characteristic coloration varying from yellow to brown, which does not, however, equally affect either all the cells or all the granules in the cells.

Ciaccio suggests that the granules are of two kinds; that they at first stain brown with chromium and afterwards pass into the iron-green stage, which represents the end product of secretion. The evidence in favour of this theory is, however, wholly insufficient, and particularly so in face of the fact that adrenalin itself reacts to both tests.

In addition to the fine granules, other.cell-contents, such as pigment granules, lipoid granules, etc., have been described. Stoerk and v. Haberer have described the occurrence of large rods 
and wedge-shaped granules which are arranged in a peculiar manner in the protoplasm, and which stain black with iron hæmatoxylin. But the biological significance of these bodies, that is to say, their relationship to the secretory process, is by no means clear.

According to Stoerk and v. Haberer, the process of the secretion of adrenalin takes place in the following manner : the chromebrown secretion is formed by the fine granules, which become gradually more heavily charged until a certain secretory climax is reached, when the chrome-brown secretion is discharged inta the intragranular cytoplasm. The cytoplasm, which is characterized by its pronounced affinity for basic aniline dyes, becomes saturated with the chrome-brown secretion and, at this stage, yields the diffuse ferric chloride reaction. The nuclei of the adrenalin-forming cells are distinguished by their small provision of chromatin, and by their peculiar situation at that cell-surface which is farthest removed from the vein against which the cell is situated. They sometimes react to chromium, which suggests that they also are saturated with the secretion.

When the cell contents have, up to a certain point, become saturated with the secretion, the latter gradually becomes diffused through the cell membrane and the capillary wall into the blood. At the same time, the cytoplasm and the granules gradually cease to react to chromium, and the process starts afresh. It appears from this that, if classified according to the nature of its secretory processes, the adrenal system belongs to the group of meroendokrinal glands (Poll).

After its transference from the cells to the blood-stream, the substance is easily recognizable in the capillaries by its yellowbrown coloration. Its tardy solubility in serum, and certain microscopic appearances, give it a certain resemblance to the mucous type of secretion. This adrenal substance is characteristic of the vessels of the suprarenal medulla, and is found neither in the vessels of the cortex nor in other vessels. It is distinguishable by its peculiar refractivity in native preparations, and was formerly observed in the venous blood of the suprarenals as a plastic mass (Gottschau, Biedl). It was at that time erroneously believed that the granules in this amorphous mass were the actual secretion. As Stoerk and v. Haberer showed, these shining granules are not identical with the chrome-brown granules, though their functional significance is, up to the present, not clear.

It may be regarded as proved that the product of the adrenalinproducing cells reaches the blood-stream in fluid form. Stilling supposes that the transference of the viscid secretion is effected by means of the numerous and important lymphatics; but in this he is wrong, for this function is performed by the veins. The suprarenal vein plays the part of excretory duct to the medullary substance of the suprarenal. 


\section{PHYSIOLOGICAL ADRENALINAMIIA.}

The secretion from the intracapsular portion of the adrenal system is continuously supplied to the general circulation by way of the suprarenal vein; thus the blood always contains a certain amount of adrenalin, and a physiological adrenalinamia is maintained.

Battelli was the first to prove the presence of adrenalin in the peripheral blood. After suitable concentration, he tested the vasoconstrictor action of normal serum from dogs, and found that its action was equal to that of an adrenalin solution of $I:$ IO to $I: 20$ millions.

Ehrmann was unable to obtain mydriasis of frogs' eyes with serum from the peripheral blood of rabbits. According to Schur and Wiesel, both Ehrmann's reaction and the ferric chloride test are invariably negative. O. B. Meyer, however, proved the presence of adrenalin in normal blood serum by means of portions of blood-ressels from the living body, this test being more sensitive than the above-named reactions. By the same method, Schlayer proved the presence in human blood-serum of a substance which produces vaso-contraction.

Fraenkel advanced conclusive proof of the presence of adrenalin in the serum of blood in the peripheral vessels. He found that serum from rabbits and cats, when diluted to twice its volume, was invariably active in the uterus of rabbits after removal from the living body. Human sera from healthy subjects, either undiluted or in solutions of $I: 5$ and $I: 10$, produce phenomena similar to those resulting from adrenalin. Similar results have been occasionally obtained with solutions of $1: 2$ and I : 40 and, in three instances, with $1: 50$. Judging by the results of Fraenkel's experiments, the minimal adrenalin contents of I c.cm. of peripheral blood may be estimated at .oooooo I mg., the maximal adrenalin contents at about .0000025 $\mathrm{mg}$.

Using these figures as a basis, the total adrenalin contents of a dog weighing $\mathrm{I}_{5} \mathrm{~kg}$. is as follows: The whole of the blood provision is $1200 \mathrm{c.cm}$. and contains a minimum of .ooor $2 \mathrm{mg}$., a maximum of $.003 \mathrm{mg}$. adrenalin. Now compare with these figures the amount which passes from the suprarenals into the rena cava. Before this can be done, however, we must know the velocity of the blood circulating in the suprarenals. There is no direct information as to the volume of the blood in the suprarenal veins. In certain older experiments of mine, I estimated the amount of venous outflow by counting the drops as they left the reins, and I reckoned that, in a dog weighing $15 \mathrm{kilo}$, the maximal velocity of the stream was roughly .I c.cm per second. These figures are the same, though this is by the way, as those more exact results which Burton-Opitz obtained with Hurthle's velocity register (Stromuhr) in the case of the blood flowing through the 
submaxillary gland. According to my figures, then, $6 \mathrm{c.cm}$. blood pass per minute from the suprarenals into the general circulation. If Ehrmann's calculation, that I c.cm. suprarenal venous blood contains $.0000005 \mathrm{mg}$. adrenalin, is correct and, judging from the comparatively slight sensitiveness of his method, this figure is probably too low-certainly not too high-.000003 $\mathrm{mg}$. adrenalin passes into the circulation per minute. The total production of adrenalin would be $.00432 \mathrm{mg}$. in twenty-four hours, but the quantity demonstrable in the blood is only .ooor $2 \mathrm{mg}$.about the fortieth part of the daily supply from the suprarenals. Even by Fraenkel's quantitative test for adrenalin, which is much more accurate, the maximal total of .003 $\mathrm{mg}$. in the blood is far less than the amount produced, as revealed by Ehrmann's not very sensitive reaction.

These figures represent approximate data only, but they show that a considerable portion of the adrenalin supplied to the blood very rapidly disappears from it.

The transience of the physiological effects of adrenalin injection points in the same direction. The increase in bloodpressure passes off at the end of a few minutes, and this happens equally after an amount which, when given refracta dosi, would serve to produce vaso-contraction 100 times or more.

What ultimately becomes of the adrenalin in the blood is a question which early came under discussion. Oliver and Schäfer held the view that, when injected intravenously, the active substance of the suprarenal accumulates and is, for a time, stored up in the tissues in which it eventually becomes active. They were, however, thinking chiefly of the muscles. They believe that the disappearance of the substance from the blood results, neither from its destruction in the blood, nor from its accumulation in the suprarenals, nor yet from its excretion in the urine, but from the fixation of the substance and its secondary oxidation within the reacting tissues. This theory is confirmed by Cybulski and Szymonowicz, with this reservation: that, seeing that after the exhibition of large quantities of suprarenal extract, the active substance is demonstrable in the urine, it is to be assumed that a certain proportion of the active substance is excreted by way of the kidneys.

It seems hardly probable, however, that an appreciable pro. portion of the adrenalin present in the blood is excreted in the urine. Urine drawn off from the bladder shortly after the intravenous injection of large quantities of adrenalin does not contain a substance which increases blood-pressure (Embden and v. Fuirth). That a minimal proportion of the adrenalin in the blood may pass into the urine is undoubtedly possible. Abelous and Bardier account for the vaso-contractor effect which normal human urine has, after suitable concentration, by the presence of a substance identical with or closely related to adrenalin, namely, 
urohypertensin. But it must be remembered that the accounts both of " urohypertensin " and of " urohypotensin," which is described by the same authors, are in need of further confirmation. As Comessatti showed, Ehrmann's reaction is useless as a test for adrenalin in the urine. Whether or not the substance, which Schur found by his chemical test to be present in normal human urine, is identical with adrenalin, is a question which must for the present remain unanswered.

As previously stated, according to Falta and Ivcovic, adrenalin, when introduced in large doses into the stomach, does not attain to specific activity within the organism, but is excreted in the urine.

More important than its excretion by way of the kidneys, is the manner in which adrenalin becomes destroyed within the organism itself. Such destruction takes place, in the first instance, in the blood and depends principally upon the alkalinity of the latter. According to Embden and v. Fürth, . I grm. of hydrochloric suprarenin, if added to $200 \mathrm{c} . \mathrm{cm}$. of defibrinated ox-blood and continuously aerated at incubation temperature, disappears almost entirely in two hours. In "laked " blood, the disappearance is even more rapid. A .I per cent. soda solution has a more energetic action than either blood or blood-serum. The relatively lower activity of the blood is probably due to the fact that a proportion of the alkaline contents of the blood is fixed.

That the degree of the alkalinity of the blood plays an important part in the disappearance of adrenalin, is shown by Kretschmer's experiments. By means of the intravenous injection of acids, he succeeded in prolonging to five or six times the normal, the hypertension produced by adrenalin in rabbits. The addition of acid-ions, inhibits the destructive action of the hydroxyl-ions in the blood and the tissues, upon the adrenalin.

Another factor in the destruction of adrenalin is the liability of the adrenalin molecule to oxidation, more especially in alkaline solutions.

Langlois believed that the destruction of adrenalin was principally oxidative. He and Athanasiu found that the vaso-contractor constituent of suprarenal extract may be rendered negative by means of ozonized air (aeration) and by the oxidase of crabs" blood. The fact that, in cold-blooded animals, the vaso-constrictor effect may be shortened by warming and lengthened by cooling, is accounted for, in Langlois's view, by the dependence of processes of oxidation upon temperature. He believes that all the tissues, and especially those of the liver, intestine and, to a certain small extent, the lungs, take part in the oxidative destruction of adrenalin. He bases his conclusion chiefly upon the results of experiments in which solutions of suprarenal extract were rapidly rendered inactive by the addition of the fresh pounded liver and intestine of rabbits; while, on the other hand, the artificial circu- 
lation through the lungs of saline solution containing suprarenal extract, produced only a slight lowering of the activity of the suprarenal extract.

Battelli found that the artificial circulation of blood containing adrenalin through the liver destroyed the activity of that substance, the alkali and the influence of oxygen converting it, as in vitro, into oxyadrenalin. Livon found that, when added to the pounded liver and striated muscles of guinea-pigs, adrenalin became rapidly reduced in quantity.

According to Embden and v. Fürth, however, the addition of pounded liver, lung or muscle to adrenalin solution and the artificial circulation of blood containing adrenalin through the liver or lungs, is followed by only slight reduction, if any, in the amount of the adrenalin. They suggest that this persistence of adrenalin has a connection with the formation of acids in the organs. They do not think that the transience of the effect upon the vessels is due to a rapid oxidation of the adrenalin, but explain this phenomenon by the fact that the contraction of the ressel muscles ceases as soon as, owing to diffusion or dilution with blood or tissue lymph, the concentration of the suprarenin falls below a certain point.

According to Elliott, however, the excessive quantities of adrenalin employed in these experiments render them unreliable as evidence regarding the disappearance of adrenalin from the tissues. He himself found that artificial circulation through the lungs did not reduce the adrenalin contents to any appreciable extent, but that artificial circulation through the intestine was followed by a marked diminution in the amount of the substance.

From the results of certain experiments, Langlois concluded that the liver played an important part in the destruction of adrenalin. These experiments showed that the amount of suprarenal extract which, when injected into the jugular vein, produced marked hypertension, produced no effect whatever when injected into a mesenteric vein.

Carnot and Josserand found, however, that the pharmacodynamic effect upon blood-pressure of a given adrenalin solution, was very much reduced where such a solution was injected into the carotid or femoral artery and allowed to flow with the stream in a peripheral direction, the adrenalin thus traversing the vessels supplied by these arteries before entering the general circulation. Passage through the liver also affected the action of adrenalin, but to a less degree. The loss was greatest after circulation through the intestine, the injection into a mesenteric artery of $.064 \mathrm{mg}$. adrenalin to every kilo of body weight producing no effect upon blood-pressure.

The absence of hypertensive effect on the part of adrenalin in localities where it normally produces intense vasoconstriction, may further be explained by the fact that the 
substance, to a certain extent, blocks its own passage into the general circulation. It is able to overcome, to a very small extent only, the resistance produced by the extreme contraction of the ressel walls; thus the major portion of the substance passes gradually, by a process of diffusion, into the surrounding tissue.

Elliott, whose experiments confirmed those of Carnot and Josserand, holds the view that the disappearance of adrenalin from the blood is in direct ratio to the development of its effects in the tissues. That a comparatively small amount of adrenalin disappears in the liver, in spite of the large amount of tissue which that organ contains, is probably explained by the fact that the vaso-constrictor effect produced by adrenalin in the liver is also comparatively small.

Although these observations seem to point to the fact that adrenalin is used up and destroyed by the tissue elements which it excites, they do not supply a sufficient explanation of the transience of the effect of large doses of adrenalin.

It has been shown by experiments of the most varied description that, after the specific action of adrenalin has passed off, the substance has not by any means disappeared from the blood. Weiss and Harris injected adrenalin into the general circulation of frogs after ligature of one iliac artery and observed the results in the webs of both feet. After the vaso-constriction had entirely passed off in the web of one foot, they removed the ligature from the artery and found that intense vaso-constriction appeared in the web of the other. These authors, similarly to Ehrmann and Miller, also found that blood taken from an animal after the effects of adrenalin had passed off, could be used to produce hypertension in a second animal.

These observations show beyond all doubt that, after its introduction into the blood-stream, adrenalin neither disappears nor does it undergo any depreciation of its pharmacodynamic value.

In face of this fact, the question as to the mechanism by which adrenalin achieves its effect requires further consideration. The experiments of Straub and Kretschmer, which supply evidence of the most valuable description concerning the part played by adrenalin in the physiology of the organism, also throw important light upon this matter.

These experiments are based upon facts supplied by Straub concerning the mechanism by which the effects of muscarin are brought about. These facts are as follows:-

(1) A toxin which has already penetrated the cell which has a specific sensibility to it, is inactive.

(2) A toxin which has not as yet penetrated such a specific sensitive cell, is also inactive.

(3) It is only in the course of, and by means of, the penetration of the toxin into the cell, that the specific toxic action takes place.

The observations, to which reference has been already made, 
of Weiss and Harris and others, seem to show that the action of adrenalin is similar in type to that of muscarin; that when, at the site of activity, a certain degree of saturation has been reached, no further effect is possible; and certainly that no permanent effec. is obtainable by repeated exhibition of the substance. Kretsch mer's experiments consisted in the repetition, at regular intervals, of the intravenous injection of a certain fixed quantity of adrenalin. They showed that, although the same hypertensive effects could be produced successively and as frequently as desired, a permanent rise in blood-pressure was not obtainable by the non-continuous exhibition of adrenalin, however large the doses. It is evident from this that, in the case of adrenalin, unlike that of muscarin, there is no cessation of absorption on the part of the specific cells, and that a condition of chemical balance does not obtain.

A permanent increase in blood-pressure, lasting for three hours, was, however, obtained by the continuous transfusion of adrenalin, the velocity being maintained at .0000I to $.00002 \mathrm{grm}$. per minute.* The hypertensive action increases the velocity of the transfusion up to a certain maximal point, beyond which the increase in velocity does not produce a further rise in bloodpressure. Interruption in the flow of the adrenalin is followed by a return of the blood-pressure to the normal, while the duration of the after-affects will be in proportion to the amount of adrenalin present in the blood at the moment of interruption. In view of the fact that the action of adrenalin lasts only as long as the substance is present in the blood, Kretschmer believes that adrenalin acts as an irritant poison, and that the method of action is, up to a certain point, similar to that of muscarin, the action in both cases depending upon the difference between the concentration in the blood and the concentration in the cytoplasm. Adrenalin differs from muscarin, however, in that the balance between the two concentrations, which takes place in the case of muscarin, is absent in the case of adrenalin. The disturbance of balance is probably due to the continued rapid destruction of adrenalin which goes on in the interior of the cell.

What is known concerning the action of adrenalin upon the vessels applies equally, as Ritzmann pointed out, to its glycosuric action. Observation of the method by which adrenalin produces glycosuria supplies certain data concerning the physiological significance of this substance. These suggest that the continuous flow of adrenalin, producing a condition of physiological adrenalinæmia, may exercise an immense influence upon the permanent tone of organs with a sympathetic innervation, more particularly upon the vascular tone and upon the sugar tone (Zuckertonus).

* By continuous transfusion of very dilute suprarenal extract I succeeded in obtaining and demonstrating ( 1895 ) a protracted vaso-contraction, so that the blood-pressure which, after destruction of the entire spinal cord, had fallen almost to zero, rose to $160 \mathrm{~mm}$. Hg. and remained at this level for several hours. 
The regulation and the maintenance of tone in every department of the sympathetic system, is a function of the internal secretion of the adrenal system.

\section{EXPERIMENTAL SUPRARENAL STIMULATION, EXPERIMENTAL HYPERSECRETION.}

Although the symptoms of suppression which follow extirpation of the suprarenals have formed the subject of exhaustive study, experiments in stimulation, by which the organs are impelled to hyperactivity, are becoming increasingly rare. In the course of his observations of the innervation of the intestinal movements, Jacobj (1892) found that extirpation of the suprarenals was followed, in fasting animals, by spontaneous intestinal movements; and that epinephrectomy, similarly to resection of the splanchnic nerve, negatives inhibition of intestinal movement without, however, producing paralysis of the vessels. These results induced him to attempt experimental stimulation of the suprarenals. He found that electric stimulation of these organs, as well as of the nerves which connect the suprarenals with the ganglion coeliacum, has the effect of arresting intestinal movements which are already in progress. The secretion of urine is at the same time very much diminished, and in two instances he found that suprarenal stimulation was followed by an increase in arterial pressure. According to Jacobj, the suprarenal nerves contain fibres which inhibit intestinal movement. These observations were not confirmed by later authors (Pal, Apolant), but in the light of our present knowledge concerning the action of adrenalin upon blood-pressure, peristalsis, and urinary secretion, they have a certain interest.

In 1897 , I investigated the physiology of the innervation of the suprarenais, a subject which, up to then, had received no attention. I found that the splanchnic nerve contains fibres which cause vaso-dilation within the suprarenal area, and that stimulation of these fibres produces suprarenal hyperamia, the direct result of the active vaso-dilation. That the suprarenals possess also a raso-constrictor innervation is to be inferred from the effect which suprarenal extract has upon these organs, although I was unable to trace the anatomical course of the vaso-constrictor nerves. I also investigated the secretory process of the suprarenals, together with the manner in which it might be modified by the presence of secretory nerves. I made use of the vasotensor action of the venous blood to determine the amount of active substance produced, but I was unable to obtain definite proof by this method of the presence of suprarenal secretory nerves. The results of my experiments, however, make it appear highly probable that both the production of the secretion by, and its transference from, the gland-or, more correctly, the medullary portion of the gland-. are subject to the control of the nervous system; and that, moreover, the splanchnic nerves contain suprarenal secretory, in addi- 
tion to suprarenal vaso-dilator, fibres. My results have since been confirmed by Dreyer, and completed by the successful demonstration of an increased vaso-constrictor action of the suprarenal blood during stimulation of the splanchnic nerve.

Erhmann was unable to discover, by means of the highily sensitive frog's-eye test, that the adrenalin contents of the blood in the suprarenal veins was changed by the addition of pilocarpin or atropine. As we have already pointed out, Waterman and Smit found an increase in the adrenalin contents of the blood in the vena cava after faradic stimulation. These authors showed at the same time that puncture of the floor of the fourth ventricle produces, in addition to glycosuria, an increase in the adrenalin production, the peripheral blood which is normally inactive acquiring a mydriatic action after this operation. This finding completes A. Mayer's discovery, already described, namely, that after total epinephrectomy puncture of the fourth ventricle ceases to produce glycosuria.

These experiments prove beyond a doubt that the secretory process of the adrenal system is under the control of the sympathetic nervous system; that the peripheral vasomotor and secretory fibres are contained in the splanchnic nerve; while their centre is probably situated in the medulla oblongata.

There have been, of course, numerous attempts to produce, by experimental measures, alteration in the function of the suprarenals and variation of their adrenalin contents. The results of such experiments have already been described and, as we have seen, a reduction in the adrenalin contents may be brought about by the most varied methods, more particularly by the experimental production of toxic and infective states. These findings are the expression of hypofunction on the part of the organ.

It is probable that hypersecretion on the part of the adrenal system is illustrated by the results of an experiment made by Battelli and Roatta. They found that, in the interval of repose which followed exhaustive muscular exertion, there was an increase in the adrenalin contents of the suprarenals of dogs. It is probable that the changes in the suprarenal medulla and in the extracapsular chromaffine substance observed by Schur and Wiesel after renal injury, and regarded by them as hypertrophic, also belong in this category.

An experimental hyperadrenalism is produced in animals after each exhibition of adrenalin; owing, however, to the transient action of the substance the effect is very fleeting. By means of Kretschmer's method of continuous transfusion of minute quantities of adrenalin, a condition of hyperadrenalinæmia may be maintained for several hours. The method seems likely to throw still further light upon the results of this condition.

The prolonged exhibition of adrenalin by subcutaneous, intraperitoneal, or intravenous injection does not, apparently, lead to hyperadrenalinism. These methods serve, however, to illus- 
trate the effects, either physiological or toxic, according to the size of the dose, which result from the action of adrenalin. In addition to the changes in the vessels, chronic glycosuria is observed, together with progressive emaciation. The autopsy shows that, in addition to the changes in the vessels and the organs, there is sometimes a marked increase in the volume of the suprarenals and hypertrophy of the suprarenal cortex.

\section{PATHOLOGICAL ADRENALINÆMIA AS SEEN IN MAN.}

Neusser was the first to assume a pathological hyperfunction on the part of the suprarenals. He based his conclusion upon a clinical case, in which the symptoms pointed to chronic Bright's disease, while the autopsy revealed carcinomatous degeneration of one suprarenal capsule, together with numerous cerebral hæmorrhages. Neusser was inclined to regard the arteriosclerotic changes and the thickening of the vessels, which Kolisko observed in young persons with suprarenal affections, as results of the continued high blood-pressure, brought about by suprarenal hypersecretion.

A large number of French authors (Darré, Pilliet, Vaquez, Aubertin and Ambart, Josué, Widal and Boidin, Ménétrier, Monod and Loumeau) emphasize the fact that the hypertension in chronic nephritis and in arteriosclerosis may be traced back to hypersecretion on the part of the suprarenals, the latter condition finding its anatomical expression in hyperplastic and adenomatous changes in those organs. The anatomical changes which they describe affect the suprarenal cortex only.

Wiesel, who was the first to inquire into the condition of the adrenal system in renal diseases, found that, in all the cases associated with cardiac hypertrophy of long standing, there was marked hyperplasia of the chromaffine tissue in the suprarenal medulla, in the solar plexus, and in the neighbourhood of the left coronary artery. He assumed from this that the cardiac hypertrophy present in renal affections, as well as certain cases of idiopathic cardiac hypertrophy, are of suprarenal origin. This discovery of Wiesel's paved the way for the experiments of Schur and Wiesel, undertaken with the object of demonstrating the presence of adrenalin in the blood-serum of persons with chronic renal disease; they found that such sera produced mydriasis of frogs' eyes and gave a positive ferric chloride reaction. The fact that the blood serum of persons with chronic nephrosis produces mydriasis is confirmed by a large number of authors (Kaufmann and Mannaberg, Goldzieher and Molnár, Eichler, Comessati, Reicher, Makaroff, Miesowicz and Macziag). Pal describes the frequent presence of mydriatic substances in the urine of pregnant women and persons with nephritis. According to Diem, however, the urine of persons, both with and with- 
out nephritis, gives a positive Ehrmann reaction in about the same percentage of cases; though Comessati believes that the Ehrmann test for adrenalin has a very limited scope when applied to urine.

As we have already pointed out, the mydriasis produced by blood serum is not a conclusive test, that is to say, the reaction is not confined to adrenalin. Bittorf, for instance, obtained mydriasis with the serum of healthy persons after the exhibition of salicylic preparations. That adrenalin is present in the serum in chronic nephritis, is not confirmed by other biological tests. Schlayer found that normal human serum produced marked contraction of portions of arteries removed from living oxen, while the contractions produced in such portions of arteries by the serum of nephritic persons were, in the greater number of cases, much veaker. The significance of these negative findings is, however, very much modified by a later discovery of Schlayer's; to the effect, namely, that $O$. B. Meyer's test for adrenalin by means of portions of arteries, is practicable with blood obtained from animals of the same species only. In the case of blood from animals of a different species, an increase in the adrenalin contents has the effect of reducing the contractions. That chronic nephritis is not associated with pathological adrenalinæmia, is well shown by the results which $\mathrm{A}$. Fraenkel obtained, by the most reliable of modern biological methods, with the uterus removed from living rabbits. He found that, in chronic interstitial nephritis with pathognomic hypertonicity, the variation in the adrenalin contents was the same as in the blood of subjects with sound kidneys.

It must be borne in mind that, although a pathologically increased adrenalinamia, producing abnormal tonicity of the vessels, would supply a satisfactory explanation of the hypertonicity and cardiac hypertrophy in chronic renal disease, certain proof of the existence of such a condition is, up to the present, by no means forthcoming. Schur and Wiesel, who based their experiments upon the assumption of a direct connection between hypertension and adrenalinæmia, were compelled to abandon this theory. They were unable invariably to produce mydriasis with serum from nephritic patients, and they were unable to establish a parallel between this reaction and a hypertonic condition.*

* The possibility was recently suggested by v. Neusser and Wiesel that a primary hyperplasia of the chromaffine system might lead to true renal processes. Conditions which demand an increased consumption of adrenalin, and of these the most important is forced muscular exertion, may produce hypertrophic processes of the adrenal system. In such a case the increased amount of adrenalin would cause a permanent vasocontraction, which might give rise to anatomical changes in the renal vessels, which would prepare the way for the development of a nephrosis. The authors lay stress upon the fact that, in cases of true contracted kidney, the etiology of which is still obscure, hyperplasia of the chromaffine tissue is invariably present. 
An abnormal activity on the part of the adrenal system would, in my opinion, not only produce a heightened vascular tone, but would also manifest itself in other signs of irritation on the part of the sympathetic nervous system. A manifestation of such a condition, in the form of a well-defined clinical entity, is, however, at present unknown. As far as we can judge, Graves's disease is invariably associated with an increase in the adrenalin contents of the blood, which is proved both by the mydriatic property of the serum and by the tonic action of preparations of the uterus. Fraenkel found that the adrenalin contents of the blood in Graves's disease were increased to from four to eight times the normal, the total amount in these patients being 50 to I oo $\mathrm{mg}$., as against the $\mathrm{I} 2 \frac{\mathrm{l}}{2} \mathrm{mg}$. present in the blood of normal persons. It is remarkable in this connection that an increased bloodpressure is not, as a rule, associated with Graves's disease.

\section{THE FUNCTION OF THE ADRENAL SYSTEM.}

From the facts which have been presented in the foregoing pages, the nature and functions of the adrenal system may be defined as follows :-

The adrenal system is an essential portion of the body of all vertebrate and some invertebrate animals. It has a specific structure, characterized morphologically and genetically by peculiar features; the nature of its function is distinctive and is probably dependent upon origin.

The structure of the adrenal system is characterized by the peculiar nature of the elements of which it is built up, namely, the chromaffine cells.

The adrenal system is distinguished anatomically by its wide distribution orer the whole of the body, as well as by the lack of independence of its parts. These conditions are explained by the history of the development of the system.

The chromaffine cells are derived from the same primordial beginning as the sympathetic ganglia and they develop, up to a certain point, as part of the sympathetic nervous system. A considerable portion of the adrenal system permanently retains its genetic relationship with the sympathetic, becoming associated only in part with the blood-ressels. The so-called free portions of the system are, however, found distributed all over the body in connection with the sympathetic nerves and ganglia. They occur partly in the form of more or less extensive agglomerations of chrome-brown cells, called paraganglia (Kohn); and partly as isolated structures attached to the great vessels-such as the suprarenal bodies of the Selachians, the carotid gland, and the accessory organs of the sympathetic (Zuckerkand1)-though the relationship of these bodies to the adrenal system has been recognized only in comparatively recent times. The lack of in- 
dependence is shown by the fact that, at a very early developmental stage, a portion of the adrenal system attaches itself to the embryonal interrenal and, in the higher vertebrates, combines with it to form an apparently homogeneous organ, the suprarenal. The medullary substance of the suprarenal is merely a somewhat considerable, intracapsular portion of the adrenal system and, as Kohn aptly remarks, is to be regarded as a large ganglion, the suprarenal paraganglion, formed by the fusion of several smaller ganglia.

In order to understand the functional significance of the adrenal system, an accurate knowledge of the quantitative value of the suprarenal medulla is essential. The fact that the medullary substance of the suprarenal was earlier recognized and has become altogether better known than the extracapsular portions of the adrenal system, produced this result, that morphological and physiological investigation has been almost exclusively confined to the intracapsular adrenal tissue. Investigation revealed the existence of an important internal secretory process, and this was at first regarded as a function of the suprarenal as a whole. But even later, after the peculiar position of the chromaffine tissue had been recognized, the physiological importance of the suprarenal medulla was very much over-estimated. There was an inclination to ascribe to the suprarenal medulla all the functions which are the property of the chromaffine system in general, for the sole reason that these functions had been proved to be attributes of the medullary portion of the system. For this reason it is very important to remember that the free portions of the adrenal system are not only genetically and structurally identical with the medullary substance of the suprarenal, but that they possess the same functional significance and that there is definite proof of this (the survival of animals without suprarenal medulla, compensatory hypertrophy of the extra-capsular chromaffine tissue, the similar effects of paraganglion extract). Not only is the suprarenal medulla not the sole active portion of the adrenal system, but it is not even the most important part of it. When we remember the wide distribution of the nervous system in the body, and consider that large and small groups of chrome-brown cells are attached to every one of its ganglia, that they lie in many plexuses and are found almost everywhere where sympathetic fibres and cells occur; and that, in addition to these, the adrenal system includes a number of independent structures-we can hardly doubt that the free portions collectively present a far larger mass of active, functional tissue than that section of the system which is enclosed in the suprarenals. These are facts which must not be lost sight of in any attempt to estimate the physiological significance of the activity of the adrenal system.

Functionally, the adrenal system belongs to the group of the 
internal secretory organs. The morphology of the secretory process is well known. The product of secretion is formed in the granules in the cells and is transferred to the blood-stream in fluid form. Not only have we morphological proof of the secretion, but its presence in the venous blood has been demonstrated chemically; in the face of these facts, it is not possible to doubt the secretory function of the adrenal tissue. The objections which Kohn advanced upon morphological and genetic grounds against the inclusion of the adrenal system among the internal secretory organs, is, however, justified to this extent; the chromaffine cells do not conform to the type of the epithelial cells; and agglomerations of chromaffine cells do not conform to the type of the true glands. But from the physiological standpoint, the specific productive activity of the adrenal tissue fully justified its inclusion among the internal secretory organs.

There is a continuous formation by the adrenal system of a substance of known chemical constitution, named adrenalin, which may also be obtained synthetically. This substance passes into the circulation and is carried by the blood-stream into all the tissues of the body. The passage of adrenalin by way of the nerves, which Lichtwitz assumed, has no physiological significance.

Adrenalin is a typical dissimilatory hormone, which acts upon certain tissue elements and reinforces their katabolic activity. The activity of this hormone is exclusively confined to organs with a sympathetic innervation, and it invariably coincides with the results produced by electric stimulation of the sympathetic nerves supplying those organs. Adrenalin produces its effect, not upon the specific functional portion of the reacting cell nor upon the nerve-ending itself, but upon a portion of the cell body situated in the neighbourhood of the nerve-ending, the so-called receptive substance which, in the involuntary muscles, is termed the myoneural junction. The activity of the hormone elaborated by the adrenal system, is the permanent expression, during the life of the individual, of the genetic relationship between the adrenal and the sympathetic nervous systems.

It must not be imagined that adrenalin is merely a sympathetic stimulant and therefore of use only in promoting the functions of the organism ; on the contrary, its activity is fundamentally and unceasingly necessary to the normal function of the entire sympathetic system. The parallel between the developmental processes and the dependence, shown in many directions, of the activity of the sympathetic upon the presence of adrenalin, as well as the continuous formation of this hormone-expressed by its presence as a physiological constituent of the blood-point to the fact that adrenalin is essential to the maintenance of sympathetic tone and to the normal function of the sympathetic system. These factors show, moreover, that changes in the condition of 
excitability of the sympathetic system, especially in so far as they make themselves felt in the periphery, are associated with variations in the activity of the adrenal system.

That the maintenance of tone by the sympathetic system in the cardiac and vascular muscles is an important factor in the normal circulation of the blood, has long been known and has been duly appreciated. A far-reaching significance is also attaching to the tonic innervation of all vegetative organs, this tone being maintained, partly by stimulation, and partly by inhibition, on the part of the sympathetic system. The sympathetic also regulates the sugar contents of the blood, and in this way exercises a determining influence upon the metabolism of the carbohydrates, which plays so important a part in normal muscular exertion. It seems probable from recent observations that the metabolism of albumin and of salt, as well as the composition of the blood, undergo a certain degree of modification by the agency of the sympathetic. Seeing that adrenalin is the hormone by which the irritability of the sympathetic system is regulated, it is evident from the foregoing, that the functional significance of the adrenal system is enormous.

It will be readily understood that the peculiar anatomical arrangement of the adrenal system renders its total suppression, whether experimentally or by pathological processes, impossible. Once again it must be pointed out that extirpation of the suprarenals is followed, not by suppression but, at the most, by inadequacy of the adrenal system, and that signs of a lowering of the sympathetic nervous tone would hardly be the outcome of such a condition. The most important of the symptoms of a lowered vascular tone, namely, the fall in blood-pressure, is, as a matter of fact, absent. It is true that accounts have been given of changes in the metabolism of the carbohydrates. Moreover, the symptomatology of Addison's disease does not permit the inference that the condition is exclusively the outcome of the cessation of activity on the part of the adrenal system. For it is highly probable that the symptom-complex of Addison's disease includes the signs of disturbance of the suprarenal cortex, a disturbance of which anatomical proof has frequently been forthcoming.

The effects which an abnormally increased adrenalinæmia has upon the organism may be exactly estimated by means of experiment. A condition of acute hyperadrenalinism is invariably accompanied by symptoms of increased irritability on the part of the sympathetic system.

\section{THE INTERRENAL SYSTEM.}

The interrenal system is present in all vertebrates; it takes the form of paired or unpaired bodies of various sizes. It is 
distinguished structurally by its lipoid cell contents and genetically by its mesodermic origin in what is known as the interrenal zone. During embryonal life, the interrenal system is extended over large portions of the body, but as development progresses the space which it occupies becomes more restricted. Principal bodies are formed by the amalgamation of several suprarenal buds, by regressive processes, and by the fission of single accessory bodies. In the cyclostoma and in fish, the interrenal system remains at this developmental stage. From the amphibia upwards, a large portion of the interrenal system combines, at the next developmental stage, with certain portions of the adrenal system to form either a row of small bodies, as in the amphibia, or an isolated organ such as the suprarenal of the amniota. In mammals and more particularly in man, the principal mass of the interrenal tissue is represented by the suprarenal cortex, while the structures which were formerly regarded as buds split.off from the principal organs, or as accessory suprarenals, are now known to be independent portions of the interrenal system, or accessory interrenals.

Such accessory interrenal structures (see table, p. I37) are frequently, though not constantly, found attached to the suprarenals and forming part of them; in the neighbourhood of the kidneys and suprarenals; in the genital region-the ligamentum latum in the female (Marchand, Rossa), and the spermatic cord, epididymis, and testicles of the male (Dagonet, Wiesel, Chiari); and occasionally in other parts of the abdominal cavity.

The accessory interrenals of the higher vertebrates have a considerable structural similarity with the cortical substance of the suprarenals. Occasionally these bodies possess all three layers, though, as a rule, the two outer layers only are present. In the earlier accounts of these organs, they are described as having a medullary portion; it is probable, however, that the error arose from mistakes as to the identity of the innermost zona reticularis.

That the free portions of the interrenal system have a functional significance is proved by the progressive development of the split-off portions; by the compensatory hypertrophy of the socalled accessory suprarenals in mammals, after removal of the suprarenals (Stilling, Velich, Wiesel); by the compensatory hypertrophy of portions of interrenal bodies left in situ in Selachians (Biedi); and by the survival of Teleostians after the removal of all except their anterior cranial interrenal system (Giacomini).

Experiments with the object of determining the function of the interrenal tissue are, at the present moment, chiefly concerned with that portion of the interrenal system which is represented by the cortical substance of the suprarenal. There is no lack of hypothesis, but the only fact supported by experiment which sheds light upon the functional significance of the suprarenal cortex is 
the following: The removal of the cortical substance-providing always that it is not replaced by the vicarious intervention of highly developed accessory interrenals, as in some animal species, notably rats-is not consistent with the continuance of life.

Some interesting anatomical data are to hand. Elliott and Tuckett compared the weight of the suprarenals in different animals and found that, as a general rule, the size of the suprarenal increases with the weight of the body and with the development of the muscles. They found, moreover, that the increase in the size of the organ is principally due to an increase in the cortical portion. The lower the status of the species in the order of vertebrates, the larger in proportion is the adrenal system and the part which it plays in the formation of the suprarenal; while, at the same time, the amount of interrenal tissue, and especially the suprarenal cortex, is proportionally reduced. There is, moreover, an increase in the volume of the suprarenal cortex in the course of the development of the individual. The medullary substance increases only slightly with the growth of the individual, but the volume of the cortical substance becomes very much augmented. In the guinea-pig, for instance, a five-fold increase in the weight of the body is accompanied by a twelve-fold increase in the weight of the suprarenal cortex, the medullary portion remaining practically unchanged. The exceptional size of the suprarenals is characteristic of guinea-pigs and is almost entirely the result of extreme development of the cortical part, this portion being in the guinea-pig nearly ten times as large as in the dog. The increase in the size of the cortex during extra-uterine growth is also observed in the cat, dog and rabbit.

A still further growth of this portion of the suprarenal takes place during pregnancy. Suprarenal hypertrophy in pregnancy was first observed by Guieysse in guinea-pigs and has since been observed in animals of different species (Stoerk and v. Haberer) and in man. This hypertrophy is not entirely the result of an increase in the cortical substance, although the cortex is the part chiefly affected. There is at the same time an increase in the chromaffine cells, in which numerous karyokineses are observed.

The similarity between the cells of the suprarenal cortex and those of the corpus luteum verum is specially commented upon by Mulon, Wallart and others. Mulon even regards the corpus luteum verum as a temporary suprarenal cortex.

That there is a connection between the suprarenals and the activity of the sexual glands, is shown by the observations of Marassini, Cecca, and Teodossieff. These authors found that, after castration, there was an increase in the weight of the suprarenal, principally due to hypertrophy of the cortical substance. Marchand describes suprarenal hypertrophy in pseudohermaphroditism. On the other hand, the observations of Bullock and Sequeira, Adams, Shattock, Linser, Thumin, Brotz, and others, 
have shown that tumours and hypertrophy of the suprarenals are associated with pubertas præcox; and it is believed by many that the suprarenals, and the cortex more especially, exercise an influence upon the genital glands and upon the somatic and psychic development of the individual during puberty. In Addison's disease (Kyrle) and in congenital hypoplasia of the suprarenals (Tandler), there are extensive changes in both the generative portion and the interstitial cells of the testicles. According to Elliott and Tuckett, there is no relationship between the suprarenals and the reproductive glands.

The occurrence of hypertrophy of the suprarenal cortex and of tumours of different kinds is by no means rare. Epithelial new formations may proceed from the cortical substance of the suprarenal and also from the accessory interrenal tissue. Of these, the most important are the renal hypernephroma, described by Grawitz under the name of struma suprarenalis; tumours of the female genitals, proceeding from Marchand's accessory suprarenals; and epithelial tumours of similar structure in other parts of the body. All these pathological findings have as yet, however, supplied no explanation of the function of the suprarenal cortex.

The physiology of the interrenal system is at present confined to the investigation of its chemical composition and the more minute study of its structure and of the structural changes which, under special conditions, are observed in the suprarenal cortex. The physiology of the interrenal system as it stands to-day is chiefly the histo-physiology of the suprarenal cortex.

\section{HISTOLOGY OF THE SUPRARENAL CORTEX.}

The connective tissue sheath of the suprarenal is connected with the centre of the organ by means, partly of thickish strands conveying the nerves and blood-ressels, and partly by delicate connective tissue lamellæ which radiate from the periphery to the centre. A short distance from the capsule, these strands and lamellæ become connected by cross and oblique anastomoses, and by this means the outer cortical zone is defined. Since Arnold's time this layer has been called the zona glomerulosa. These connective tissue processes, some of which are vertical, while others are joined together by means of a portion which curves outwards, enclose long fan-shaped portions, 20, 40 and 60 microns in breadth, which are again divided into smaller roundish spaces by means of transverse processes. It is in these rounded spaces that the parenchyma cells are situated. In the median portion of the cortex, the radial arrangement of the connective tissue strands become more open, enclosing parallel spaces in which isolated connective tissue septa conveying capillaries are arranged transversely and diagonally (zona fasciculata, Arnold). In the internal portion of the cortex, the connective tissue processes become still 
more delicate and, in the neighbourhood of the medulla, they break up into a fine net, composed of the finest connective tissue threads (zona reticularis, Arnold), in the meshes of which single parenchyma cells are situated. At the boundary between medulla and cortex there are again a certain number of thicker connective tissue threads running tangentially.

The fans formed by the lamellae of connective tissue are divided into meshes of different sizes by delicate septa running transversely and diagonally. These meshes contain the cortical cells, not as Ecker described them, in the form of bladders or tubules, but, both in man and in animals, in typical, solid cellcolumns without cavity-the cortical cylinders of Köllicker. These cell-columns are usually composed of a single row of cells, though occasionally of two or three; they traverse the external layer either directly or in a winding fashion, and end in the transverse connective tissue processes, or they may curve back like an arch and terminate in one another. In the median layer, these cell-cylinders are arranged in drawn-out radiating columns, in accordance with the shape of the meshes formed by the longitudinal connective tissue threads. In the interior, the columns break up into a network formed by single rows of cells, in the meshes of which the fine capillaries are situated. The meshes of the network, which at first are rounded, become flattened radially as they approach the medulla.

The form and size of the cells in the individual layers, varies with the changes in the arrangement of the connective tissue structure and of the cell columns. The cells in the external zone are, upon the whole, small in size and are of various shapes. In the human suprarenal these cells are mostly polygonal; in tangential sections they appear in the form of rounded balls and cell nests enclosed in connective tissue. The cells in the median layer are larger in size; in form, they are rounded polygons which are occasionally elongated in the direction of the column; and they are arranged several together, side by side, transversely of the column. As they approach the internal layer, the cells become smaller in size and either cubic or polyhedral in form; while the cells in the innermost cylinders are frequently oblong.

The connective tissue which runs between the cortical cell cylinders carries numerous capillaries and veins. These vessels pass between the cell columns and are somewhat dilated in the outer layer; they are especially numerous in the internal zone. Actual main arteries are not present in the cortex. The cortical cells are placed directly against the thin vessel walls, which are composed of endothelium and are sometimes enclosed in a delicate connective tissue membrane.

The arrangement of the cortical parenchyma is, in the main points, the same in all mammals, though the form, size and arrangement of the cells varies. In many species the zona 
glomerulosa is absent altogether. The form of the cortical cells is polygonal in man only; in the greater number of mammals the cells of the outer zone are long, narrow and cylindrical in shape. The most perfect cortical cylinders are observed in the horse and the dog. In microscopic sections taken from these animals the cell strands appear as perfect rings which closely resemble tubular glands. Whether or no the cortical cell groups enclose a lumen is a question of the utmost importance to both pathological and physiological histology, and one which has recently been subjected to searching investigation by Stoerk. He showed that, under normal conditions, there is no free space within the looplike arrangement of cell cylinders, although the appearance of these sometimes closely simulates a lumen formation. Where, for instance, owing to the over-filling of the suprarenal vessels, there is extravasation of blood into the external cortical layer, the cell columns become detached from the connective tissue by which the vessels are conveyed; thus, appearances are produced which, where the extravascular blood is not demonstrable as such, bear a misleading resemblance to transverse and oblique sections from glands. This effect is heightened by the fact that compression of the cell contents produces an apparently basal position of the nuclei. From the nature of his findings, Stoerk is convinced that the suprarenal cortex does not assume the formation of a true gland under either normal or pathological conditions (hypertrophy, adenoma).

The most important structural characteristic of the cortical cells is supplied by the lipoid granules which they contain; these resemble fat granules and are usually known as the cortical granules. The chemical nature of these granules will be described later; their histological characteristics are, briefly, as follows :-

If the cortex of a fresh mammalian suprarenal is teased out in physiological saline solution, the result will be a granular mass containing, in addition to single perfect polygonal cells filled with granules, an enormous number of free spheroids. These are pale yellowish in colour, very bright, double refractive, and $\mathrm{r}$ to 4 microns in size. Occasionally, these spheroids run together to form, in some cases, largish drops, in others, what are known as myelin forms. The large drop formation is very well seen after the addition of glacial acetic acid, concentrated mineral acids, or 33 per cent. liquor potassa solution; though, when diluted, the same acids and potash solution produce no change. The cortical granules are soluble in fat solvents (ether, chloroform, xylol, alcohol, though more slowly by the latter), and the addition of water to these solutions produces the same globular formations. The cortical granules do not stain with either fuchsin, methylene blue, or other similar colouring agents; but they stain vividly with the specific stains for fat (alkanna, sudan III, scarlet R); and in perosmic acid they colour a profound black. If, after colouring 
with osmium, the preparations are treated with alcohol, chloroform, or xylol, or cleared and mounted in Canada balsam or bergamot oil, it will be seen that the granules have become dissolved, leaving a diffuse brown coloration in the cells. Mounted in oil of cloves, however, they remain black for weeks, and in glycerine for even longer.

The cortical granules are best seen by means of the specific dyes for fat, provided that, after fixing the portions of suprarenal, no powerful fat solvents such as ether, chloroform, or alcohol, are employed in the further treatment. 'The best method of preparing the specimens is to fix in formalin, cut frozen, and stain with sudan III or scarlet $R$, when the granules will take on a red colour. If subsequently treated with osmium (twenty-four hours in Altmann's mixture), however, the granules in these frozen sections-unlike fat, which usually stains black immediately-take on a brown colour and become black only when further treated with alcohol. If, however, after treatment with osmium, the frozen sections are treated with bergamot oil, the brown granules will immediately dissolve; if ether or xylol are used, solution follows very rapidly; chloroform and benzine are slower in their effects, but solution follows within a quarter of an hour. If, after treatment with osmium, the granules are treated with alcohol until they become black (twenty-four hours), they will still dissolve rapidly and entirely in bergamot oil, though to a less extent in the other fat solvents; they will still dissolve, though very slowly, in ether and xylol, but they cease to be soluble in benzine and chloroform.

For the study of the cytological structure of the suprarenal cortex, the best results are obtained by fixing in formalin, formalinMïller, Zenker's fluid, and particularly in the different osmium solutions. By these methods the cortical granules are at first browned and become black only after treatment with alcohol.

On account of the solubility of the granules in ether and xylol, these reagents cannot be used to prepare sections for paraffin imbedding; the granules are, however, insoluble in chloroform or benzine, and these may be employed.

Sections should not be cleared in xylol or mounted in Canada balsam, as the cortical granules are to a large extent dissolved by these, so that, after a few days, only a few blackened or brown, osmium-stained cortical cells remain. When mounted in oil of cloves, the granules last for some weeks. The most satisfactory results are obtained by clearing and mounting sections which have been stained with osmium in glycerine or a saturated solution of potassium acetate.

In addition to the lipoid granules of the cortex, Altmann's fuchsinophile granules stain readily, even in embryonic suprarenals (Plecnik). These are round bodies of the same size as the lipoid granules; they are composed of several layers 
arranged in such a way that the smallest is nearest to the nucleus. At the cell periphery they are surrounded by the layer containing the lipoid granules. Where, however, as the result of the grouping of the cortical cells, the juxtaposing cell surfaces are free from lipoid granules, in fuchsin preparations these areas are seen to be crowded with fuchsinophile granules. There is no evidence to show that these granules are changed into lipoid granules. The effect of ether upon sections is to dissolve, not only all the lipoid granules stained with osmium, but also a large proportion of the fuchsinophile granules, the remaining portion of the latter remaining unchanged (Plecnik).

The cells of the suprarenal cortex also contain pigment. This is seen in the inner layer, the zona reticularis, where it takes the form of yellowish granules, even in sections coloured by the ordinary nucleus and cytoplasm stains. These granules colour black with Heidenhain's iron hæmatoxylin, and a deep grassgreen with Unna's polychrome methylene blue and differentiation with glycerine ether. In these preparations, even small quantities of pigment are clearly visible against the blue nuclei and the unstained cytoplasm. The sections should not have been previously fixed in osmium solutions. When fixed in Altmann's mixture the pigment loses its yellow colour, becomes a dirty green, and is readily distinguished from the intensely black lipoid granules. The embryonal suprarenal contains no pigment; Plecnik found that it was present in very small quantities in the suprarenal of an infant 6 days old.

The pigment is found both in free cells and in those which contain cortical granules. If sections which have been stained with osmium are treated with ether and then coloured with iron hæmatoxylin, large clear spaces will be observed in the zona reticularis in place of the black lipoid granules; while the pigment granules, also coloured an intense black, will still be visible in the alveolated body of the cell. According to Dostojewsky, this alveolate network represents, not the filamentous portion only, but the entire cell-plasm. Thus it appears that the lipoid granules occupy the plasmic spaces, while the pigment granules are situated in the cytoplasm itself (Plecnik).

\section{CHEMISTRY OF THE SUPRARENAL, EXCLUSIVE OF ADRENALIN.}

In spite of the immense interest which has been aroused in the physiologically active substance of the suprarenals, or perhaps because attention in general, including that of chemists, has been focused upon the physiological side, the advanced methods of modern chemistry have, up to now, supplied only the first beginnings of a knowledge of the chemical constitution of the suprarenal. 
Nabarro's experiments have acquainted us with the albuminoids of the suprarenal. These experiments show that the suprarenal contains an albumin which coagulates at $70^{\circ}$, certain globulines which coagulate at between $56^{\circ}$ and $75^{\circ}$; and one or more nucleoproteids. Thus far, then, the suprarenals do not differ from the other organs, seeing that the globulins and the nucleoproteids are present in all tissues. According to the older accounts, the suprarenal contains also the following: of the products of regressive metamorphosis it contains hippuric and taurocholic acid (Vulpian and Cloëz), benzoic acid and taurin (according to Seligsohn, but not confirmed by Stadelmann), leucin, tyrosin, xanthin bases (xanthin, methylxanthin, hypoxanthin, epiguanin, according to Okerbloom), inosit (Külz); of the carbohydrates it contains glycogen (Lubarsch), a substance resembling jekorin (Manasse), and salts, of which potassium phosphate is present in exceptionally large quantities (Halliburton).

A. C. Croftan discovered a diastatic ferment, and M. Jacoby an oxidative ferment, an aldehydase, in the suprarenal cortex. The suprarenal further contains a nuclease which splits up nucleoproteids, xanthin bases being formed (Jones).

The proof of the presence of cholin in the suprarenal was a discovery of the highest importance. As early as I888, it was stated by Guarnieri and F. Marino-Zucco that watery extracts of suprarenal contain neurin (in the terminology of to-day, cholin) and they assumed that the neutralization of the neurin in the body was a function of the suprarenals. Exact proof of the presence of cholin was, however, first offered by Lohmann (1907).

It was Lohmann's object to isolate from the suprarenal the substance present in suprarenal extract which produces an effect first observed by Gürber, namely, the lowering of blood-pressure. The suprarenal was first freed from fat and cut into small pieces, and, by means of hot water with the addition of a small amount of acetic acid, a triple extract was obtained. The concentrated extract was purified by Kutscher and Steudel's method with tannin, baryta and oxide of lead, and afterwards precipitated with phosphorotungstic acid. The precipitate was split up with baryta and the alloxur, or purin, bases were first extracted by means of nitric acid, and a further group of bases, "the arginin fraction," was afterwards extracted with baryta. "The filtrate was again precipitated with phosphorotungstic acid, the precipitate again split up with baryta, and, after removal of the superfluous baryta with carbonic acid, the bases were reduced to a thick syrup. A concentrated extract of this syrup was obtained by repeated treatment with boiling absolute alcohol. The bases obtained from the alcohol extract possessed marked hypotensive properties. The concentrated alcoholic extract was precipitated with alcoholic solution of platinic chloride. The platinates, which were insoluble in alcohol, were converted into chlorides with sulphuretted hydrogen, and then, by the addition of a watery solution of auric 
chloride, the aurate of cholin is obtained. By crystallization twice repeated, and by ascertaining the melting-point and the amount of gold present, the insoluble aurate was found to be homogeneous and was analysed. The result yielded by analysis was cholin auric chloride. The aurate was converted into the chloride with sulphuretted hydrogen, and this was employed in experiments upon animals. It appeared that, when injected into the veins of a cat in a quantity of $.036 \mathrm{grm}$. in $.5 \mathrm{c.cm}$. of Ringer's solution, this cholin chloride produced a sudden fall in blood-pressure and violent lachrymal and salivary secretion, followed by the death of the animal. That the influence of cholin was antagonistic to that of adrenalin was shown, not only by the effect upon the blood-pressure, but also by the effect upon portions of intestine removed from the living cat. The action of adrenalin in producing relaxation of the intestine was arrested by the addition of cholin, and violent muscular contractions followed. In further experiments, Lohmann tested the effects of adrenalin and cholin upon the heart when taken from the living rabbit; he found that the increased and accelerated cardiac activity produced by adrenalin was negatived by the weakening and slowing of the contractions which followed cholin. The antagonism between adrenalin and cholin was found to be mutual; it was especially noticeable that the profuse salivary flow produced by the subcutaneous injection of cholin, was absolutely controlled by the simultaneous injection of adrenalin. Lohmann was no more able than Frank and Isaac later, to prevent the occurrence of adrenalin glycosuria by means of cholin. According to Mansfeld, the subcutaneous injection of cholin will arrest the development of the changes in the vessels brought about by adrenalin.

Lohmann next investigated the distribution of cholin in the suprarenal and he discovered that it is possible to obtain nearly nine times as much from the cortex as from the medulla. He ascribes the cholin contents of the medulla to the presence of cortical substance and arrives at the conclusion that, in the same way that adrenalin is formed only in the medulla, so cholin is formed solely in the cortex.

Lohmann also described the manner in which he obtained a further auric salt, by the slow concentration of the mother-lye of the cholin auric chloride obtained from suprarenal extract. This auric salt was crystallized and found to be neurin gold.* This

* The constitutional formula of neurin (trimethylvinylammonium hydroxyd) is as follows :-

$$
\begin{aligned}
& \mathrm{CH}_{3}-\mathrm{CH}=\mathrm{CH}_{2} \\
& \mathrm{CH}_{3}-\mathrm{N}-\mathrm{OH} \\
& \mathrm{CH}_{3}-\mathrm{OH}
\end{aligned}
$$

This base is obtained from cholin by the splitting off of a molecule of water out of the ethylalcohol, by which the latter is converted into the vinyl residue. 
gold combination was converted, by means of sulphuretted hydrogen, into the chloride and was employed in experiments upon animals. The intravenous injection of .or grm. of neurin chloride into rabbits produced a fall in blood-pressure immediately followed by a considerable rise. There were at first several strong expiratory movements, followed by a reduction in the respirations.

That cholin was present in the animal economy had long been known, and the nature of its pharmacological activity had repeatedly been investigated (Gaehtgens, Böhm, Brieger, Cervello, Asher and Wood, Formánek).

Cholin (trimethyloxethylammonium hydroxyd) :-<smiles>C[N+](C)(C)O</smiles>

formerly called neurin, was first discovered in the bile by Strecker, and afterwards found, as a product of the decomposition of lecithin or of protagon, in watery extracts of the brain, and by Mott and Halliburton, in the cerebro-spinal fluid. The latter authors were inclined to regard the fall in blood-pressure observed by Schäfer and Moore after the injection of extract of the brain, and by Schäfer and Vincent after the injection of that constituent of pituitary extract which is soluble in alcohol, as an effect of cholin. Osborn and Vincent found that the injection of extracts of any portion of the nervous system produced a distinct temporary fall in blood-pressure. They concede that these extracts contain cholin in small quantities, but they do not ascribe the result to the action of this substance; for the lowered tension persisted after full doses of atropine, whereas, according to their experiments, the effect of atropine upon cholin is invariably to convert the vaso-dilator action into a vaso-contractor one. Vincent and Sheen next discovered that a substance which reduces blood-pressure is obtainable from muscular tissue of all kinds, as well as from the kidney, liver, spleen, testicles, pancreas, ovaries, lungs, thyroid, thymus, suprarenals and the hypophysis cerebri. Vincent and Cramer showed that watery extracts of nervous tissue contain two groups of substances, both of which, when injected intravenously, reduce bloodpressure. One group dissolves readily, the other is scarcely soluble, in absolute alcohol. The alcoholic solution contains two depressant substances. The action of the one is neutralized by atropine, that of the other is unaffected by it. The alcoholic solution yields a slight precipitate with platinic chloride, and this precipitate, when purified, yields prismatic crystals, the platinum contents of which are 32.8 per cent., which corresponds to the platinic double salt of dicholinanhydride. These observers found no cholin in extracts of the brain, and they are of the opinion 
that the hypotensive action of these extracts is independent of cholin.

The wide distribution of cholin in the organism was emphasized by Claude and Blanchetière (1907), who also pointed out that cholin is present in the greater number of tissues and secretions of the body, though not in a free state but in combination with lecithin. v. Fürth and Schwarz showed that cholin is present in intestinal extract and in secretin (so-called), and that it stimulates the secretory activity of the pancreas and of the salivary glands. The effect of cholin upon secretion is completely neutralized by atropine. They also found that the constituent of the thyroid which lowers blood-pressure, is identical with cholin. Schwarz and Lederer found depressant substances, which they identified with cholin, in extracts of thymus, spleen and lymph glands.

Finally, Gautrelet found cholin in the pancreas, spleen, ovary, thyroid, kidney, testicle, pituitary and salivary glands, bone marrow, and gastric and intestinal mucous membranes of animals of various species. He came to the conclusion that the common active principle of the extracts of those organs which were included by Livon in the description "hypotensive glands," is cholin, the activity of which is antagonistic to that of adrenalin. He divides the internal secretory organs into those which are cholinogenic and those which are adrenalinogenic (chromaffine), and he believes that the regulation of arterial pressure is brought about by the interactivity of these two antagonistic systems.

The evidence which these observations supply of the almost ubiquitous occurrence of cholin in the animal economy, has largely diminished the importance of Lohmann's discovery. The presence of comparatively large quantities of cholin in the suprarenal cortex can hardly be regarded as proof of the production of cholin as a specific function of that tissue. It is far more probable that cholin represents a product of the decomposition of the phosphatides which all organs contain, and that the comparatively large quantities of cholin which are present in certain tissues, merely show that such tissues are very rich in those lipoids. These phosphatides are very liable to hydrolytic decomposition, in the process of which they give off cholin. The presence of cholin is not a proof of its pre-existence under physiological conditions.

But the physiological activity of cholin, and especially its antagonism to adrenalin, requires more careful investigation. Modrakowski showed that the cholin (Merck) of commerce brings about a fall in blood-pressure and a slowing of the pulse, which may be arrested by means of atropine; that it also produces increased salivary and pancreatic secretion as well as increased secretion of urine; but that these results are wholly attributable to products, resembling muscarin, of the decomposition of cholin, and more particularly to trimethylamin. Pure cholin is a com- 
paratively non-toxic substance and, as Blum pointed out, in doses of .6 to $2 \mathrm{mg}$. per kilo of body weight, produces only a transitory increase in blood-pressure, and has no effect whatever upon the secretions. I was able to convince myself that Modrakowski was right in his findings, and they have since been confirmed by Busquet and Pachon. A pure cholin which was prepared by my colleague, S. Fraenkel, when injected into the veins of dogs in quantities of $4 \mathrm{mg}$. to every kilo, produced a distinct, though very transitory, increase in blood-pressure. After being kept for a few days, the preparation acquired a distinct odour of trimethylamin, and in doses of even $2 \mathrm{mg}$. produced marked hypotension and slowing of the pulse. After atropine, a fresh injection of the substance produced slight hypertension. This substance proved, after keeping, to be actively toxic, for animals into which it was injected in doses of $\mathrm{I} \mathrm{mg}$. per kilo died within a few hours.

According to these findings, then, cholin is neither specific to the suprarenal cortex, nor is its action antagonistic to that of adrenalin.

The suprarenal contains yet other substances, the importance of which to the vital processes has only recently been discovered. These are the lipoids. The presence of fat and substances similar to fat is mentioned in some of the older writings upon the suprarenals, but at that time our knowledge of the chemistry of these substances was very scanty. It is only recently, and indeed very recently, that we have arrived at any definite knowledge of the nature of the lipoids. The interest which has been aroused in both physiologists and pathologists by the lipoid substances in general, has drawn a certain amount of attention to those of the suprarenal in particular.

I have occupied myself with researches-in which I made use of the most recently discovered chemical methods-into the nature of the lipoid substances; and, in so far as my results concern the lipoids present in the suprarenals, they may be given here. In order fully to understand these results, it is necessary briefly to explain the older views, based almost entirely upon histological and biochemical material, and to show the manner in which recent chemical science has modified our views concerning the nature of the suprarenal lipoids.

It has long been known to morphologists that the suprarenal cortex, or rather the entire interrenal system, occurring in vertebrates of whatever species, possesses a remarkable cytological peculiarity, namely, the presence in the cells of fat-like lipoid granules, from which the name "lipoid tissue," used to describe the interrenal tissue, is derived. However much the structure and arrangement of the interrenal system may varyand according to H. Poll these variations are constant in mammals of the same species-the lipoid granules, or cortical granules as they are called, are a constant and characteristic feature both 
of the cortical cells and those of all interrenal tissue. What was known of the morphological relationships and the microchemical properties of these granules supplied the material for the investigation of their chemical composition.

This occurrence of peculiar granules in the suprarenal cortex was first described by Simon and Ecker (1846), and has been confirmed by all subsequent investigators. The older authors regarded them solely as fat-granules, and were content to describe their distribution in animals of different species and the quantities in which they were to be found in the different parts of the cortex. According to Kölliker, there are animals, such as carnivora and rodents, in which the amount of fat in the suprarenals is so large that it lends a yellow-whitish colour to the entire cortex. There is also a large amount of fat in the external portion of the cortex in the horse; while in the swine and in ruminants, on the other hand, the cortex is grey in colour, the cells being pale and having apparently little fat. The suprarenal of man occupies a middle place between the two, there being in youth but little fat, though this increases by degrees until finally the entire cortex, with the exception of the inner zone, acquires a yellow-white appearance. The greater number of authors find that the granules, which they believe to be fat globules, are most numerous in the median portion of the cortex; that they diminish considerably in number towards both the inner and outer cortical boundaries; and that the cells of the inner layer contain, in addition to the cortical granules, certain other cell-contents, more particularly pigment.

Moers and Arnold described the resistance which the more shining of these granules offer to alkalies and acetic acid; but A. von. Brunn was the first to inquire into the actual nature of these bodies. He found that the numerous shining granules in the cells of the external zone did not dissolve in acetic acid and ether, and that they did not stain black with osmic acid; he concluded from these findings that they could not be composed of fat. For the same reasons, Braun believes that the more strongly refractive bodies in the suprarenal cortex of reptiles are only partially composed of fat. Gottschau also describes the highly refractive granules, present in the inner layer of the cortex in mammals, as insoluble in acetic acid and liquor potassæ.

A detailed account of the microchemical relationships of the cortical granules was first given by $H$. Rabl. He found that cortical granules from the suprarenal of the hen were soluble in alcohol, ether and chloroform; that they stained black with osmic acid and red with alkanna. While, however, fat treated with osmium was insoluble in choloroform and bergamot oil, the blackened cortical granules proved to be soluble in these reagents and in xylol. For these reasons, Rabl held that the cortical granules, though not fat, were allied to it. These findings were confirmed by M. Pfaundler in the case of the dog and the 
horse. Alexander, however, lays special emphasis upon the fact that cortical granules did not stain black with osmium, but that they acquired a brownish tone, and for this reason must be composed of a chemical substance other than fat.

In 1895 , Kaiserling discovered that by far the greater number of the cortical granules are double refractive to polarized light. They had long been known to have a high refractivity, and Orgler, who carried Kaiserling's experiments still further, gives the following acćount of their microchemical nature. The granules measure .4 to 18 microns, and have an average dimension of 4 to 5 microns. They are soluble in water, readily soluble in ether and chloroform, and dissolve slowly in alcohol. They are unaffected by caustic soda solution, acetic acid, and concentrated sulphuric acid. They stain red with sudan III., at the same time losing their double refractivity. When treated with osmic acid they take on a grey-black tone, and if allowed to remain for a longer period in osmic acidfixing by Altmann's method, for instance-they stain black. Treatment with osmium, as well as fixing and hardening by other methods, destroys their anisotrophy, thus their optic differentiation from the fat-granules disappears. They are soluble in xylol, chloroform, and bergamot oil, and at this stage this is the only distinction between them and fat-granules treated with osmium, the latter being insoluble in these reagents. Orgler concludes that the cortical granules are not identical with fat; from their double refractivity, the manner in which they are influenced by osmium, and their resistance to acetic acid and caustic soda solution, he thinks it highly probable that they are related to the myelin of the medullary nerve-fibres.

The chemistry of the cortical granules was minutely investigated by Hultgren and Andersson. These authors found that the microchemical reactions of the cortical granules were in every case the same as those of fat. Their greater solubility after treatment with osmium is their only point of difference. Alexander showed that lecithin is present in the suprarenals in large quantities, and Hultgren and Andersson incline to a relationship between this substance and the cortical granules, taking further into consideration the large quantity of so-called myelin forms which appear in the etheric solution after the addition of water. They were unable to demonstrate phosphorus in the cortical granules, and were thus compelled to leave the question of their lecithin contents unsolved. All that they could say was that the cortical granules are composed of fat, but that they probably differ in certain respects from the other known fats. In 1902, P. Ewald discovered that the suprarenal cortex coloured a diffuse pale pink with scarlet R., and he believed that this was due, not to fatty degeneration, but to infiltration of fat.

Plecnik's observations (1902) are of exceptional interest. He 
found that, in the suprarenal cortex of a human embryo $5 \mathrm{~cm}$. long, all the cells contained granules, though these were smaller in size and in number than in the adult. In an embryo 2 to 15 $\mathrm{cm}$. long, the granules had increased considerably in size, and were much more numerous, especially in the peripheral cells. These conditions are maintained through the later developmental stages, and change very little during the post-embryonal period. The comparative maximal number of cortical granules is reached during the second six months after birth; from then onwards the number is subject to variation which is largely unexplained. But an increase in the number of granules in the cortex is by no means to be regarded as the result of fatty degeneration. Plecnik's experiments shed important light upon the nature of the cortical granules, because he applied to the suprarenals the test which Lewinson gives for fats, namely, a modification of Weigert's chrome-hrematoxylin stain for the medullary sheath of the nerves. He found that, after the suprarenals had been hardened for some time in Müller's mixture, the granules in the cortex were stained blue in exactly the same manner as the fat in the interstitial cells of the testicles, while subepicardial fat and the fat of phosphorus kidney did not stain by this method. The granules in suprarenals treated by this method frequently colour black after treatment with Altmann's mixture and rinsing in water. This is not the case, however, with subepicardial fat and phosphorus kidney fat.

By means of I per cent. osmic acid, Bernard and Bigart (I902) were able to differentiate between two cell types, or more properly, two cell conditions, in the external layer of the zona fasciculata, the so-called zona spongiosa. Of these two cell types, one contained larger or smaller globules of common fat which stained in the usual manner with osmium, while the other contained granules which, in response to osmium, took on a shade varying from light to dark grey. Mulon next showed that the grey granules are soluble in turpentine, xylol, bergamot oil, and cedar-wood oil. The cells known as spongiocytes are, according to Mulon, cells the protoplasm of which is filled with granules resembling fat. Their spongy appearance is merely the result of the secondary solution of the globules during mounting. Bernard and Bigart call this substance "labile fat," and consider that it is a product of normal cell-function, while ordinary fat may, under conditions, be a pathological product. Bernard, Bigart and Labbe believe that labile fat is lecithin or a mixture of lecithins. By extraction with ether and alcohol, they estimated both the total amount of fat and the total amount of phosphorus in the suprarenal, and found that the proportion of the phosphorus fat to the whole amount of fat was as follows :-45.3: Ioo in the horse, $48.8:$ I 00 in the sheep, 52.7: I00 in the rabbit. In the horse, the phosphorus fat forms 6.77 per cent. of the organ; in man the proportion of lecithin fat to the whole amount of fat is I3.I : IOO, the lecithin fat 
amounting to 2.08 per cent. of the total weight. According to these authors, lecithin is actively produced by the suprarenals. They found that, during muscular exertion, there was a considerable increase in the number and size of the labile fat granules in the spongiocytes, but that the ordinary fat remained unchanged.

Mulon (1903) points out the double refractivity of the cortical granules in the suprarenals of guinea-pigs, though this, as we know, had already been discovered by Kaiserling. Mulon also comments upon the fact that, as early as I866, Darest described double refractive granules in the ovum, in the suprarenals, and in other tissues; and that Dastre, in 1877 , showed that these granules are composed of lecithin. According to Mulon, oleate of soda and lecithin are the only double refractive granules which are soluble in ether and alcohol. He calls the zona spongiosa the zone lecithinogène. He found that the cortical granules, like those of the medullary sheaths, stain blue by Régaud's-modification of Weigert's hæmatoxylin copper method. Bonnamour and Policard proved that the cortical granules of the suprarenals of frogs possess the following characteristics: they stain grey-black with osmium; they are afterwards soluble in Canada balsam and in xylol; and they colour blue with Weigert's hamatoxylin. These authors consider that the cortical granules are composed of a phosphorus fat belonging to the lecithin group.

These double refractive granules, which were believed to be a specific product of the suprarenal cortex, were also found by Kaiserling in an amyloid kidney with pronounced fatty changes; and Kaiserling and Orgler discovered similar granules in organs which had undergone regressive and fatty degenerative changes, for instance, in regressive changes in the thymus, fatty degeneration of the intima cells of the vessels, in large white kidney, \&c. They believed these double refractive granules to be identical with the myelin globules found by Schmidt and F. Müller in the bronchial mucus, and they described the granules which they had discovered as "myelin" without, however, attaching a chemical significance to the term. Albrecht also described as myelin the globular formations and the figures which appear in the cell-body as the result of nuclear decomposition-as seen for instance post mortem in the kidney if kept at the temperature of the body-and which, unlike the nucleus, stain red with neutral red. Dietrich and Hegler also described the occurrence of myelin figures which were formed by autolysis in organs liept under aseptic conditions. By rapidly bringing crushed suprarenal cortex to a moderate degree of heat under the microscope, Albrecht produced every possible change in the appearance of the double refractive granules, from slight increase in size to enlargement equal to that of the globules seen in fatty infiltration of the liver, together with pronounced double refraction, and, in many instances, distinct formation of myelin forms. Certain other authors (Orgler, Loehlein, Rosenfeld) believed that the double-refractive substance 
seen in fatty degeneration and cloudy swelling is protagon, and this is in accordance with the theory of Kaiserling and Orgler.

O. Herrmann (1905) minutely investigated the double refractive substances resembling myelin present in the suprarenal cortex. The double refractive substances are present in varying quantities as physiological constituents of the human suprarenal from the embryonal state until old age. They are present in the form of round globules of varying size, and they are characterized by the following reactions : they resist alkalies and acetic acid; they stain slightly yellow with Lugol's solution; when warmed, pink with Millon's reagent; they are readily soluble in chloroform and ether; in alcohol they dissolve slowly; they stain brown with osmium, at the same time losing their double refractivity; when fixed in Flemming's solution they stain black.

The most important thing about these double refractive substances, however, is their attitude towards neutral red; they do not stain with this reagent. On the other hand, in addition to the globules, myelin masses are found, and these stain with neutral red. In badly fixed sections where the nuclei do not stain, the neutral red staining of myelin bodies is more easily recognized. It is evident then that the double refractive granules are not identical with the myelin substances, which colour with neutral red (Albrecht).

After hardening in formalin, needle-shaped crystals stained with fat-ponceaux are observed in addition to the globules; these crystals take up dyes, and are in many instances still refractive. Double refractive globules similar to those in man are found in large numbers in rats, but they are not present in guinea-pigs, nor, as a general rule, in rabbits. The cells of the suprarenal in rabbits are filled with small, polyhedral granules, which frequently combine to form largish scales, are not double refractive, and in fresh preparations do not stain with neutral red.

These granules stain with neutral red in preparations in which autolytic processes have occurred, either when kept in an incubator, or in the abdominal cavity, free or enclosed in indiarubber bags. Their double refractivity is not, however, increased, and there was no actual proof of the transformation of the polygonal granules into larger myelin forms or into globules. Colour reactions show that fatty acids are present in the external layer of disconnected suprarenal grafts. This is most readily explained by the assumption that the leucocytes in combination with the fluids of the body which penetrate into the external layer, bring about a process of resorption, which is attended with a splitting up of myelin.

Herrmann was unable to obtain from the results of his experiments any further information concerning the nature of the amorphous masses in the suprarenal of rabbits, as well as of the double-refractive globules which occur in profusion in the suprarenal of man, and in small quantities in the suprarenal of rabbits. 
Napp (1905) published the results of his investigations into the fat-contents of the suprarenals in different pathological conditions. He takes no account, however, of the manner in which the double refractivity is affected, and he describes as fat only that portion of the cell contents which stains with sudan red. Babes and Jonesco have also published communications respecting the distribution of fat in the suprarenals. These authors assert that the distribution of fat in the suprarenal cortex differs with the age and species of the individual, and that its amount is very much diminished by different pathological conditions, such as inanition and acute and chronic infective diseases. They found, further, that there is a reduction in the number of the fat-granules in the suprarenal cortex of rabbits, if hypertrophy of the organ is induced by the intravenous injection of adrenalin. Babes finds that, in suprarenals containing a large amount of fat and in suprarenal adenoma, the cortical cells are filled with crystalline double refractive granules, the reactions of which are similar to those of protagon. These double refractive granules may entirely crowd out the ordinary fat.

Elliott and Tuckett (1906) undertook minute investigations into the histology of the cortical granules. They found that the cortex contains four different substances, all of which may be regarded as the products of secretion; these are the fat granules, the double-refractive substance, the pigment granules of the cortex, and the chromaffine substance of the medulla. The fat in the suprarenal cortex differs from other fats in its ready solubility in etheric oils; it colours red with scarlet $\mathrm{R}$. The double refractive substance which is soluble in ether occurs in the suprarenal cortex in association with true fat, though the distribution of the two substances is not identical, and they may occur in different parts of the cortex. In the guinea-pig, true fat is found in the external third of the cortex only, while the double refractive substance occurs throughout the whole cortex. In the cat, the double refractive substance is generally situated in the external cortical layer, though it is sometimes met with in the internal layer, completely separated from the fat. In the rabbit, the distribution of fat is regular and, when viewed with a polarization microscope, glittering crystals appear to be also regularly distributed through the cortex. In the human suprarenal the conditions are the same. In certain species, though fat is present, the double refractive substance is absent, as for instance, in the case of the cortex of the sheep and the whale. In others, on the other hand, as for instance in the hen, the double refractive substance is present in such large quantities that the entire cortex appears to be filled with large needle-shaped crystals, irrespective of the cell boundaries. According to Elliott and Tuckett, the fat and the double refractive substance are two different secretory products of the suprarenal cortex, but are, however, intimately related to one another. When the body is in the resting state, the amount of the double refractive. 
substance increases, while the fat is reduced in proportion. Where muscular exertion is prolonged to fatigue, the double refractive substance disappears, and the fat spreads through the whole of the cortex. The authors point out, however, that these substances may neither of them represent the most important general product of the secretory activity of the cortex, for they may be absent in certain species, as in the case of the sheep. The brown pigmentgranules are specific to the suprarenal cortex of guinea-pigs. These accumulate during repose and disappear very rapidly during exhausting muscular exertion, fat being formed in the cytoplasm in their place.

These authors give no account of the chemical nature of the double-refractive substance; but they point out that it cannot be identical with fat, and they think that its refractive power probably points to myelin in combination with lecithin.

As we have seen, this double-refractive lipoid substance was almost invariably believed to be lecithin. The assumption was justified by Virchow's discovery, as early as 1857 , of the presence of a substance which he described as myelin (medullary) in the suprarenal, and which, from its chemical nature, he identified with the substance which Gobley described as lecithin. A little later, Bennecke pointed out that cholesterin, in combination with a peculiar substance of fatty nature, plays the chief part in the formation of myelin substances. But the peculiar richness in lecithin of the suprarenals was again emphasized by Alexander. He obtained his exceptionally high figures by multiplying by 8.8 , the phosphorus contents of the etheric extract of suprarenal. The brothers Marino-Zucco, as well as Guarneri and Marino-Zucco, obtained neurin and glycerite of phosphoric acid from etheric extract of suprarenal. Manasse obtained a body from the suprarenals, the phosphorus contents of which were 4.44 per cent., and which, after decomposition by means of acids, yielded a carbohydrate. Manasse concludes that this lecithin-carbohydrate combination is jecorin.

Orgler (1904) analysed the suprarenals with the object of ascertaining if they contained a body resembling protagon. Liebreich gave the name of protagon to a homogeneous substance which he regarded as the matrix of the lipoids found in the brain.* By means of alcoholic and etheric extraction, Orgler

* Later on, protagon was erroneously regarded as a body chiefly composed of cerebrin and lecithin, because it yielded, as the products of decomposition, cerebrosides, which are free from phosphorus, and phosphatides. Cerebrin is a body containing CON, as yet not exactly defined; while, from the results of Strecker and Diakonow's investigations, lecithin was commonly regarded as di-stearyl-glycerinite of phosphoric-acid-cholin. But Thudichum had already suggested, and it was afterwards proved by Fraenkel, that saturated lecithins of this description do not exist; that all lecithins are unsaturated phosphatides; and that Diakonow himself had isolated the unsaturated oleic acids from his own lecithin. Thus the finding of a saturated di-stearyl lecithin must be attributed to erroneous description of the experiments on the part of the text-books. 
obtained a yellow-white body from the suprarenals, which was found under the miscroscope to consist of fine, slightly bent needles arranged in rosette form. After repeated crystallization he obtained a snow-white body which, as it did not stain with osmium, he believed to be free from lecithin. The phosphorus contents were I.6 per cent.; higher, therefore, than the figure given for protagon by the majority of authors. Owing to the small amount of substance at his disposal, he was able to test for the carbohydrates by colour-reactions only, and the results of these were positive. From the results of his investigations, Orgler held the double refractive granules to be protagon.

After, however, Panzer had shown that the double refractive substances in the kidney are not protagons-that is to say, they are not combinations with phosphorus-but are cholesterinesters, Adami and Aschoff continued the investigations made by Lehmann and Schenk into the nature of the fluid crystals. They endeavoured to obtain by synthesis, combinations corresponding to those of the double refractive globules found in the body, corresponding therefore to the fluid crystals. They found that the most important ingredients are cholesterinesters, whether pure or in combination with oleic acids, fats, or cholesterin. These experiments pointed to the probability of the double refractive substance of the suprarenal being of a cholesterinester-like nature. On the other hand, Wright pointed out that mixtures of cholesterin with oleic acids may also yield double-refractive globules, and he lays stress upon the fact that the suprarenal cortex contains a mixture of cholesterin with lecithin and oleic acids, and that these are to be regarded as products of secretion.

Rosenheim and Tebb isolated a white crystalline substance from the suprarenal cortex of the ox, which contained 3.4 per cent. phosphorus, and the physical properties of which corresponded to Thudichum's diamidomonophosphatide, sphingomyelin, the most important constituent of the so-called cerebral protagon. This substance possessed a characteristic, which they describe, namely, that of sphero-rotation, and it crystallized into anisotropic spherocrystals.

In 1909 the same authors published their further investigations into the nature of the suprarenal lipoids.

In the first extract of dried suprarenal obtained with acetone, they found a large quantity of a crystalline substance which consisted principally of stearic acid, mixed with the cholesterinesters in small quantities. The latter were not identified, but their nature was assumed from analogy with the cholesterinesters which Panzer isolated from large white kidney. From the matrix of the cold acetone extract, they isolated free palmitic acid, an unsaturated oleic acid, and fat.

From the hot acetone extract they obtained a smaller quantity of a crystalline substance from which they isolated cholesterin 
stearate and cholesterin palmitate in a state of chemical purity. They obtained, further, the phosphatide, sphingomyelin, and the non-phosphoric galactocide, phrenosin. The phrenosin was identified by polarimetric estimation of the galactose obtained after hydrolysis. Free cholesterin was carefully watched for, but was not found to be present. Even with Windau's extremely sensitive digitonin reaction, free cholesterin could not be detected in either the hot or cold acetone extract of suprarenal. These authors lay stress upon the fact that their results do not support Powell White's assumption, that the double refractive substances of the suprarenal consist of a mixture of free cholesterin and fatty acids. On the contrary, the results of these experiments confirm another view held by Powell White, to the effect, namely, that the pure cholesterinesters do not yield double refractive granules, but that when in combination with fatty acids, the granules which they yield are double refractive. Seeing that the presence under physiological conditions of true cholesterinesters in the organism is denied by Bün, Tebb, and by Moore, this account of cholesterin palmitate and cholesterin stearate in the suprarenal is very remarkable.

According to Rosenheim and Tebb, the double refractive substances of the suprarenal consist of a mixture of free stearic and other fatty acids, together with a small proportion of sphingomyelin.

In his review of the morphology of the lipoid substances (1909), Aschoff affirms, upon the ground of results yielded by his own experiments, that the following bodies may show double refractive myelin forms, and, occasionally, globules :

(I) The phosphatides, including lecithin, though the latter is difficult to obtain in a state of chemical purity.

(2) The soaps of the oleic acids.

(3) The cholesterinesters.

(4) Solutions of cholesterin in the phosphatides, in the oleic acids, and the fats.

(5) Solutions of the cholesterinesters in the fats (perhaps also in the phosphatides, though, up to now, this has not been tested).

Of the double refractive substances included in these five classes, the cholesterinesters alone constantly and readily yield permanent emulsions of double refractive globules. The substances in classes $I$ and 2 frequently yield transient accidental globule formations with double refractivity, but the results cannot be relied upon. The substances in classes 4 and 5 , on the other hand, invariably yield emulsions containing globules, but these are either not double refractive, or there is coarse crystalline precipitation of the globules without typical crystalline formation. In all essentials, then, the double refractive substance is represented by the cholesterinesters.

In regard to the colour reactions of the cholesterinesters, 
Aschoff affirms that they colour a yellowish red with sudan, a weak reddish colour with Nile blue sulphate, and grey with osmic acid; that they do not stain with neutral red or by Golodetz's method; and that, after fixing with chrome, they are converted into insoluble lipoids with a positive colour reaction to sudan red.

The colour reactions of the double-refractive globules are identical with those of the cholesterinesters. They stain with sudan, Nile blue sulphate, and ponceau red in the same manner as the neutral fats, and they are unaffected by the Golodetz process which, by means of a mixture of formaldehyde and sulphuric acid, stains the smallest traces of cholesterin a brown to violet colour. Aschoff adopts the view that the presence of the double refractive globules may represent only a modification of the ordinary fatty degeneration, a special sort of storing-up of fat, and that the occurrence of the double refractivity does not point to a degenerative, endogenous formation of the globules. The processes are invariably pure infiltration processes. This is shown by the occurrence of isotropic and anisotropic globules side by side in the same cell, and especially by the physiological occurrence of such granules in the cells of the suprarenal cortex.

Nothing definite can be said concerning the physiological significance of anisotropic substances. The view which prevails at present is that, of the lipoids, lecithin plays the part of stimulant of the ferments which serve as protective agents to the cholesterins. It has been definitely proved by experiment that with substances of a saponin nature (saponin, digitonin), cholesterins give additive combinations which are insoluble and which do not decompose readily. Of the functions of the globular formations which are described as " myelin," we know practically nothing.

In most cases where the double refractive substance is of pathological origin, the process appears to be one of resorption or of accumulation, characterized by marked profusion of the cholesterin combinations.

Up to the present, no explanation has been found for the physiological occurrence of the double refractive substance in the suprarenal cortex. The assumption of Bernard and White, that the suprarenal cortex secretes lecithin and cholesterin in combination with fat, is pure hypothesis.

Aschoff also mentions Ciaccio's finding, that treatment with chromates renders the so-called myelin-forms of the suprarenal cortex difficult of solution, and that at this stage, in spite of treatment with alcohol and xylol and embedding in paraffin, they show a very distinct sudan coloration. Ciaccio carried out certain experiments with what he believed to be pure lecithin and protagon, and he considers, from the results which he obtained, that this sudan reaction is specific to lecithin, and he believes lecithin to be a product of cell-metabolism. 
On the other hand, as Aschoff justly points out, Ciaccio's chemical tests cannot be regarded as of any very great value, seeing that protagon does not exist as a chemical entity and that pure lecithin is scarcely obtainable. The term lecithin is not applicable to the lipoids of the suprarenal; all that can be positively stated is, that they are lipoids, that like the medullary substance of the nerves, treatment with chromates renders them insoluble, and that they give a sudan reaction. From what has been observed up to the present, it seems far more probable that they are cholesterinesters and sphingomyelin.

In my own researches into the nature of the so-called lipoid substances, the material was provided by the suprarenals of swine, while the method which I employed was an adaptation of S. Fraenkel's method for fractional distillation of the lipoids of the brain.* It must be explained, first of all, that the process consists in extracting the different groups of lipoid substances in such a manner as to separate the groups in the process of extraction. The tissues are first dehydrated with cold acetone, and are then successively subjected to the action of a number of suitable organic solvents. By this means, the cholesterin group is separated from that of the unsaturated phosphatides, and this again from that of the saturated phosphatides and the sphingogalactosides. By weighing the extracts it is possible to ascertain, not exactly, but approximately, the amount of the total lipoids as well as that of the single lipoid groups, together with the relationship which these bear to the total amount of lipoids.

It has been shown by Fraenkel and his co-workers that every organ contains lipoids which are, as a rule, characteristic of it; and that the same organ, in different classes of animals, contains phosphatides and lipoids which are also chemically differentiated. The differentiation which exists between the lipoids of different organs in different species on the other hand, is both qualitative and quantitative. Finally, it must be borne in mind that, in spite of their ready crystallinity, the lipoids which are normally present in the tissues may not be demonstrable by histological tests, for they may be present in a state of solution in one a nother. Under pathological conditions, the breaking-up of such a state of insolution may cause the one or the other substance to appear.

My experiments were conducted in the following manner: Fresh pig's suprarenals were freed from the surrounding fat, cut up in the mincing machine, dehydrated with plenty of cold acetone, and then exhaustively distilled by means of boiling acetone. The acetone extract contains the entire group of the cholesterins, free cholesterin, cholesterinester, possibly also the

* These experiments were carried out under S. Fraenkel's direction in the laboratories of the L. Spiegler Institute in Vienna. A detailed account, together with chemico-analytical results, will be published in the "Biochemischen Zeitschrift." 
neutral fats and fatty acids, together with single phosphatides soluble in acetone. Distillation with benzine follows, and this process carries off the whole of the unsaturated phosphatides, together with minute quantities of the sphingogalactocides. Subsequent distillation with benzol, absolute alcohol, diluted alcohol and ether yields the saturated substances.

The results of my analysis of the suprarenals of pigs were as follows: The organs contained 74.6I per cent. of water to 25.39 per cent. of dry substance. Of this dry substance, 6r.12 per cent. was albuminoids, \&c., and 38.88 per cent. was lipoid substances, together with extractive substances not as yet chemically defined. These figures show the richness of the suprarenals in lipoid material. But it must be remembered that, by employing the whole suprarenal for these experiments, no distinction between medulla and cortex is possible, and that the scantiness of the medullary lipoid contents considerably lowers the relative lipoid figure. By itself, the cortex would show a far higher lipoid proportion.*

In any case, however, the suprarenals are among the organs which are richest in lipoids, for more than a third of the total dried substance consists of lipoids.

\section{Table of Results obtained by Distillation of the Suprarenals OF PIGS.}

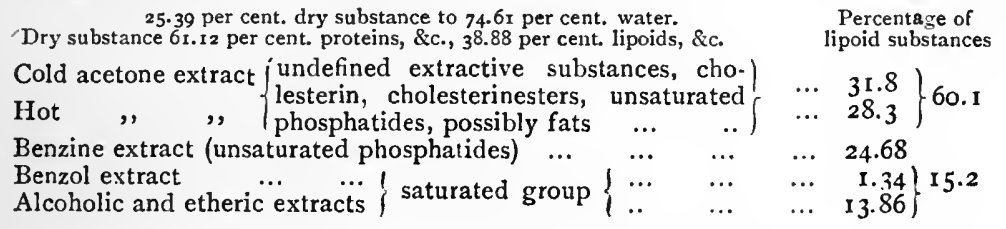

The figures which $\mathrm{K}$. Linnert obtained by the same process with the brain of pigs are given for the sake of comparison.

23.09 per cent. dry substance to $76.9 \mathrm{t}$ per cent. water.

Dry substance $37 \cdot 3^{8}$ per cent. proteins, \&c., and 62.62 per cent. lipoids

Percentage of

$\begin{array}{llllllll} & & & & & & & \\ \end{array}$

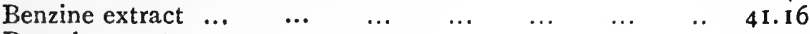

$\begin{array}{lllllllll}\text { Benzol extract } & \ldots & \ldots & \ldots & \ldots & \ldots & \ldots & \ldots & 25.02\end{array}$

$\begin{array}{llllllll}\text { Alcoholic extract } & \ldots & \ldots & \ldots & \ldots & \ldots & \ldots & \text { I I.35 }\end{array}$

From the hot acetone extract a cholesterinester was obtained in crystalline form which, from the shape of its crystals, its

* To obtain a clear idea of the lipoid contents of the interrenal tissue, experiments should be carried out with Selachians, and these I propose to undertake within the near future. Our knowledge in this direction is at present confined to Grynfellt's statement, that Ville found fat in the interrenal bodies of Selachians, and that, upon saponification, this fat yielded oleic acid, palmitinic acid, and stearic acid. Only the microscope test was employed. 
melting point, and its elementary analysis, was identified with cholesterinpalmitate $\mathrm{C}_{27} \mathrm{H}_{45} \mathrm{O}\left(\mathrm{C}_{16} \mathrm{H}_{31} \mathrm{O}\right)$. This substance was present in a comparatively large quantity. $.8 \mathrm{grm}$. was obtained in a state of chemical purity from $2,320 \mathrm{grm}$. of suprarenal.

Special interest is further attaching to the discovery of a cholesterinester which crystallized into very fine needles, which became illuminated under crossed Nicol's prism; molecular weight, 740. Elementary analysis showed figures corresponding to the formula $\mathrm{C}_{51} \mathrm{H}_{92} \mathrm{O}_{2}$; thus the substance may be described as carnauba-acid-cholesterinester.

These findings supplied material for a further investigation of the question as to whether or no the suprarenal is the source of the cholesterinester present in the blood. It is well known that Hürthle found cholesterinoleate, cholesterinpalmitate, and cholesterinstearate in blood serum. The examination of pigs' blood for cholesterinester shows that cholesterincarnaubate is not present in the blood in demonstrable quantities.

Of the further results of my experiments, I propose to mention here only that the benzine extract contains the monoamidomonophosphatide cephalin. The diamidomonophosphatide discovered by Rosenheim, namely, sphingomyelin, must be present in the alcoholic fraction, but, up to now, I have failed to isolate it.

My investigations into the nature of the suprarenal lipoids cannot be regarded as complete, and it is proposed to pursue them further at a future date. They show, however, that Aschoff's view, that the double-refractive substance present in the lipoid granules is represented by the cholesterinesters, receives considerable support from the results of chemical analysis. They suggest, moreover, that the cholesterinesters are substances specific to certain organs, and perhaps also to certain species, which are produced in the suprarenal, but which cannot be regarded as products of secretion, destined to be carried off by theblood-stream.

\section{THEORY OF THE FUNCTION OF THE INTERRENAL SYSTEM.}

At a first glance, the remarkable profusion of the lipoid substances in the suprarenal cortex suggests that this tissue is the locality in which the lipoid substances required by the entire organism are formed. Hence the hypothesis arose that the lipoid substances, the presence of which in each cell was believed to be essential to the acquirement by that cell of its nutritive material, were formed by a process of secretion in the suprarenal cortex, or rather in the suprarenal tissue. The evidence in favour of a secretory process on the part of the cortex was supplied by the different nature of the lipoid contents in different cells; by the difference between cells in different parts of the cortex, containing little 
fat, but much of the double refractive substance; by the finding of fatty spheroids furnished with a double refractive cap; and by other phenomena indicative of a developmental cycle on the part of the lipoid substances. Bardier and Bonne, who investigated the structure of the suprarenal cortex after tetanizing the muscles, found that there was an increase in the number of the cell vacuoles, together with an increase in the volume of the cortical granules; they interpreted these changes as the signs of an increased secretory activity. Not only the lipoid granules, but the pigment granules and other cell contents, were at that time regarded as products of cortical secretion.

A further consideration of the arguments in favour of secretion led, however, to the conclusion that they were insufficient to establish the existence of a secretory process occupied with the elaboration of certain definite substances. The evidence merely shows that the cortical cells are the locality in which certain substances, more particularly the lipoids, are formed, and that these lipoid granules form cell-contents of a kind subject to considerable variation in the matter of number and size; but it does not show that these cell contents are destined to pass into the blood-stream. Comparative chemical examination of the suprarenals and of the blood lends no support to this theory in so far as the lipoids are concerned. Bonnamour's own experiments, together with a critical survey of the literature of the subject, led him to the conclusion that the findings which, up to now, are forthcoming, do not justify the assumption of a secretory activity on the part of the interrenal tissue.

Bonnamour regarded the suprarenal cortex as an organ which, owing to its high lipoid contents, is peculiarly suited to the absorption and neutralization of the toxic products of metabolism.

The earlier investigators ascribed an antitoxic activity to the suprarenal as a whole. According to the brothers Marino-Zucco, the suprarenals neutralized neurin; Abelous and Langlois believed that they rendered harmless those products of muscular activity which produce the symptoms of fatigue. The antitoxic activity of the suprarenals was believed to extend also to exogenous toxins, and there are a number of communications (Oppenheim and Loeper, Bernard and Bigart) which describe the effects of suprarenal and of suprarenal extract in reducing the activity of various toxins. In so far as these results are concerned with toxinssuch as phosphorus, strychnine, cyanide of potassium, \&c.- the effect of.which is antagonistic to that of adrenalin, the apparently antitoxic action is sufficiently explained by the slowing of resorption which, as A. Exner showed, is a property of adrenalin. It may be mentioned here that the neutralization of strychnine by adrenalin which Falta and Ivcovic described, is, in Jaunschke's opinion, partly accounted for by this property of adrenalin and is in part the result of adrenalin stimulation. 
Matters are quite otherwise, however, in regard to the antitoxic activity of the suprarenal cortex. The anatomical and histological changes which take place in the suprarenal in infective diseases, and in experimental poisoning with bacterial toxins, have been described in an earlier chapter. Later experiments (Oppenheim and Loeper, Bernard and Bigart, Moschini, Nicolas and - Bonnamour) have shown beyond all doubt that poisoning with metals is accompanied by structural changes in the cortical cells, changes in the nature of the lipoid granules, as well as signs of hyperplastic processes in the suprarenal cortex; and that these changes are even more marked in experimental infective and toxic states induced by bacterial agents.

The suprarenal lipoids are able to fix and neutralize toxins in vitro. Myers showed that cobra toxin loses its toxicity if mixed with an emulsion of suprarenal cortex, but that its toxicity is unchanged by emulsion of the suprarenal medulla. According to Elliott, diphtheria toxin is not neutralized by suprarenal extract. Takati's experiments show that both phrenosin and the oleic acid obtainable from it (Thudichum's neurostearic acid), neutralize large quantities of tetanus toxin. Rosenheim and Tebb drew attention to the large amount of oleic acids which the suprarenal cortex contains.

Further experiment is required, however, before the antitoxic activity of the interrenal system and the part played therein by the lipoids can be established. It is the business of future investigators to decide whether the antitoxic process extends to the products of normal metabolism, and whether the neutralization of endogenous toxins is a physiological function of the interrenal tissue. An antitoxic activity on the part of the suprarenal cortex, and a relationship between this activity and the elaboration of adrenalin in the suprarenal medulla, have long been assumed, but the hypothesis is without sufficient foundation. A closer investigation of the arguments in its favour shows that there are no grounds for the assumption of a combined antitoxic and internal secretory activity on the part of the suprarenals.

If, however, we consider the possibility of the interrenal system being a true internal secretory organ, which supplies a specific hormone, different from adrenalin, to the blood-stream, the assumption is supported by observations which point to the suprarenal as an influential factor in the growth of the body, the development of the sexual glands, and the changes which take place during puberty in the normal human organism. There is sufficient evidence in this direction to justify the inclusion of the interrenal system among that group of organs which, like the thyroid, thrmus, hypophysis cerebri, and the sexual glands, exercise either a direct or indirect influence, by means of their assimilatory hormones, upon the somatic and psychic development of the individual. 


\section{THE SUPRARENAL CONSIDERED AS AN ENTITY.}

In the absence of any certain knowledge regarding the physiological activity of the interrenal system, it is difficult to arrive at a conclusion concerning the functional relationship between the portions of the interrenal and adrenal systems which are united to form the suprarenals. The two systems are morphologically so different and, in a large number of animal species, are topographically so entirely separate, that an independence and an autonomous functional activity on the part of both must be assumed. Even the anatomical union of portions of the two systems into a single organ cannot be regarded as proof of a functional interdependence. For the topographical association of morphologically and physiologically differentiated tissues is not without analogy - on the contrary, it is of frequent occurrence in the organism. But it cannot be denied that the inclination on the part of the interrenal and adrenal systems to unite to form a morphologically homogeneous organ - an inclination which increases and becomes more intimate with phylogenetic development-suggests that this specific association is not without significance. There may also be a functional connection, a coordinate or subordinate relationship between the two systems, which thus unite in the suprarenal for the performance of a higher organic function.

The absence of definite facts has not, however, militated against the formulation of hypotheses concerning the function of the homogeneous suprarenal. Even before it was known that the suprarenal system was biologically made up of two parts, Langlois attempted to combine into one the two theories at that time current concerning the function of the suprarenals. He believed that the internal secretory and the antitoxic activities were interdependent. This view, which was adopted by Neusser, Boruttau and partly also by Battelli, may be formulated as follows: That certain toxic products of metabolism, chiefly the result of muscular exertion, are neutralized by the suprarenal cortex, and that from the material thus supplied the adrenalin necessary to the working of the economy is elaborated by the medulla.

The experiments of Abelous and Langlois, already described, constitute the evidence in favour of the accumulation and neutralization, within the suprarenal, of the products of muscular fatigue. Boruttau then concluded that, after the transformation process, portions of the original products of fatigue remained unchanged in the suprarenal. His view is based upon experiments in which a change in the contractions, similar to the first stages of fatigue, was produced in frog's muscle after treatment with curarin by the application of suprarenal extract. Battelli and Roatta found a decrease in the adrenalin contents of the supra- 
renal of dogs exhausted by muscular exertion, and a progressive increase during recovery from such exhaustion. Battelli explains these findings by, in the first instance, an abnormal expenditure of the finished adrenalin; and in the second, by the deposition and accumulation of fresh precursors. He supposed that these precursors, which he terms proto-adrenalin, represented the material for the progressive increased production of adrenalin during repose. Schur and Wiesel demonstrated the increased adrenalin contents of the blood during muscular exertion by means of Ehrmann's pupil reaction, and the diminution in active substance present in the suprarenal by the weak chromium reaction. These findings of Schur and Wiesel's were, however, not confirmed by Kahn's very exact experiments.

Abelous, Soulié and Toujan investigated the formation of adrenalin in the suprarenal by means of a series of experiments, and the results at which they arrived are very remarkable. It must be assumed that these authors employed, as a quantitative adrenalin test, the colorimetric method with iodine which they themselves formulated. They first showed that there was an increase in the adrenalin contents of pounded suprarenal, if the latter was kept for twenty-four hours at a temperature of $40^{\circ}$. In their view, however, this post-mortem formation of adrenalin takes place principally in the cortical substance. For they found that, after twenty-four hours in an incubator, the adrenalin contents of the pounded cortex were 33 to $60^{\circ}$ per cent. higher than those of the control specimen kept at $0^{\circ}$. These results led them to the conclusion, that adrenalin is formed in the cortex and is only stored up in the medulla. In a further series of experiments, they next showed that the addition to pounded suprarenal of a small quantity of tryptophan, obtained by auto-digestion of the pancreas, was followed by a considerable increase in the adrenalin contents; and they think it probable that tryptophan is one of the matrices of adrenalin. They found, however, that the addition of pure tryptophan did not produce anything like the same effect as the fluid produc $\bar{t}$ of pancreatic auto-digestion. They tried, therefore, the effect of autolysates or extracts of putrefying organs, and found that here also there was an increase in the adrenalin contents of the pounded suprarenal; from this they conclude that adrenalin is formed post mortem in the cells of the suprarenal. The fact that putrid substances from the muscles produce a very large increase (the double and over) in the adrenalin contents, appears to them a proof of the relationship between the formation of adrenalin and the chemical processes of the muscles.

Toujan next carried out experiments supplementary to these; he summed up the results in a definite statement, that the suprarenal cortex contains a precursor of adrenalin, and that adrenalin is formed from it. 
Toujan found that cortical extract, which had been so completely freed from medullary substance that it ceased to give a chromium reaction, contained a vasotensor substance which did not react to ferric chloride. He regards this cortical product as a crude precursor of adrenalin and gives to it the name of proadrenalin; he identifies it with the granules which are histologically demonstrable in the cortical cells, and which are combined with a body of fatty nature. He found that, after twentyfour hours in a thermostat, the specific action of cortical extract is very much more intense than that of fresh extract, and he considers this sufficient proof of the formation of adrenalin from the crude substance which he discovered in the cortex.

The results communicated by Toujan are in need of further confirmation. H. Poll, who expected to be able to confirm " these remarkable findings," reports as follows: "Proadrenalin and adrenalin, cortical extract and medullary extract, all react to most tests; they respond in the same way to oxidizing substances (Battelli's iodine method), they produce glycosuria, contraction of the unstriated muscle fibres, and mydriasis of frogs' eyes." In regard to the latter, Poll mentions that the reaction was effected by Ehrmann; in the other instances he does not make it clear whether the experiments were his own or not.

In regard to the glycosuria test, it must be mentioned that Landau also believed that glycosuria originated with a substance present in the suprarenal cortex. In opposition to the wellfounded view that adrenalin is present in the medullary substance and in extracts of other portions of the adrenal system, Landau suggests the possibility that adrenalin obtained by Takamine's method is derived from the suprarenal cortex. He states that, after the intravenous injection of cortical extract, death followed rapidly, the same symptoms and post-mortem signs being observed as after the injection of medullary substance and of adrenalin. Landau's statements do not, however, bear analysis.

The only exact proof, up to the present, of the formation of adrenalin after death is supplied by G. Bayer's experiments, and these do not confirm the hypothesis advanced by Toujan.

Bayer found that, when pounded suprarenal was diluted with putrid material, a stronger and more rapid mydriasis was produced than when the suprarenal was diluted with water. But when employed alone, the putrid fluids also produced mydriasis, probably due to the presence of phenylalanin, and this was followed by myosis. An increase in the adrenalin contents was not demonstrable by Ehrmann's reaction. The iodine test produced a stronger reaction in suprarenal cells to which putrid fluids were added than in the control cells which were treated with water. Further experiment showed, however, that this result was not due to the post-mortem formation of adrenalin, but to an intensification of the chemical test. According to Bayer, the 
putrid liquids contain alanin, phenylalanin, and probably other products of the decomposition of albumin, and by these the colorimetric iodine test for adrenalin is intensified. Bayer believes that the increase in the amount of adrenalin which Abelous, Soulié and Toujan describe, is apparent only.

Thus the only experimental evidence which has been advanced up to the present, in favour of a functional interdependence between cortex and medulla; is proved to be unreliable.

Poll writes as follows concerning the hypothesis of a unity of function on the part of the suprarenals : "Whether right or wrong, it is undoubtedly true that this hypothesis brings into line in what is, at first sight, a most remarkable manner, a number of anatomical and physiological facts which are otherwise obscure." The morphology of the homogeneous suprarenal, and its vascularization-especially the intimate vascular association between cortex and medulla in mammals-undoubtedly suggest an important functional relationship between the two parts.

The remarkably rich vascularization of the suprarenal, and the peculiar relationship which subsists between the parenchyma cells and those of the surrounding capillaries-and there is no doubt that the capillaries act as ducts for the conveyance of the medullary secretion-have already been described. The circulation throughout the entire organ is remarkable and unique, and demands a short description.

The older scientists, of whom Ecker (I 844 ) was the first, noticed that, in certain classes of animals, the suprarenal possesses a portal system of its own.

Ecker's descriptions and drawings of the suprarenals and their vessels in the adder (Coluber natrix) show that these organs lie close to the venæ renales revehentes, on the inner surface of the ovary in the female, and on the inner surface of the testicle and vas deferens in the male; that they are furnished with venous vessels (venæ suprarenals advehentes) formed by the combination of two or three intercosto-spinal veins, which extend to the external surface of the organs where they form arcade-like anastomoses. From these proceed the minute branches which, together with the hair-like arterial vessels, form the capillary system of the suprarenals. Numerous fine venous branches are given off by the capillary network, and these combine to form larger veins leading away from the organ (venæ suprarenales revehentes) and opening either into the vena renalis revehens sinistra, or directly into the vena cava posterior. According to Ecker, then, Ophidians possess, in addition to hepatic and renal portal veins, a third or suprarenal portal system, the roots of which are situated in the wall of the body and the spinal cord, while its branches are distributed through the suprarenals. Ecker also states that the vascular conditions in Chelonians and tailed Batrachians to some extent resemble those in Ophidians. 
The small portal system of the suprarenals was minutely investigated in different animal classes by S. Jourdain (1859). $\mathrm{He}$ found that it was present, not only in Ophidians, but in Chelonians, crocodiles and Saurians (Corti, 1847), thus in all the reptilian orders.

In this "Beitrage zur Entwickelungsgeschichte des Venensystems der Amnioten" (I 892) F. Hochstetter gives a detailed description of the suprarenal portal system in reptiles, together with an excellent drawing showing the conditions in Lacerta viridis. In a later work he describes the suprarenal portal system in Crocodilus niloticus.

In Batrachians (anura), according to Jourdain, the branches of the vena renalis efferens, before uniting in the median line to form the vena cava posterior, form long anastomoses and a series of long arcades, which surround the yellowish bodies of the suprarenal, and to some extent pass into the suprarenal tissue. Jourdain does not, however, venture upon a decision as to whether this arrangement constitutes a suprarenal portal system. Jourdain found that, in Selachians, a small number of fine venous branches led into the external layer of the suprarenal cortex, while the median layer contained numerous veins leading into the venæ cardinales; he believes that this represents a small portal system. In Jourdain's drawings, the "suprarenals" are invariably associated with arterial branches, but it is not possible to say whether these suprarenals are interrenal bodies or whether they are adrenal organs. The intimate association, in fish, between the suprarenal system and the arterial and venous vessels, has been commented upon by all the more recent investigators, but a suprarenal system as such has not been described.

A special interest is attaching to the conditions which obtain in birds. The suprarenals are here furnished with two separate veins upon both sides. Upon the median side of the suprarenal - that which is nearest to the vena cava-is a comparatively short and broad vein (vena suprarenalis interna), which opens into the vena cava. Upon the lateral side of the organ is a second vein (vena suprarenalis externa), the course of which is complicated. In the majority of birds, the vena externa is formed by the union of one or two dorsal intervertebral and intercostal veins, together with a perforating dorsal cuticular vein. More rarely, a sacral intervertebral vein and a vein from the upper suprarenal surface empty themselves into the vena externa. As early as I844, Neugebauer foresaw the real significance of this vena externa. The comparatively large size of the lumen at the place where the vein enters the organ, together with the absence of a direct communication with the vena interna, suggested to him the idea that this vein might convey blood to the organ.

A few years later, the existence of a vena afferens upon the external surface of the suprarenal of birds was proved by Gratiolet. 
Jourdain found that it was present in more than thirty species, and he discovered that fluid injected into the vena externa passed readily into the vena interna, and vice versâ. He believes, however, that the communication between the two vessels is not direct but that it is effected by means of a network of capillaries, the vena externa representing the afferent, and the vena interna the efferent, portion of the portal system of the suprarenal. He was unable, however, to bring direct proof in support of his theory.

Since Jourdain, there has been, as far as my knowledge goes, no further investigation into the relationship between the two suprarenal veins in birds. It seemed to me advisable that the matter should be elucidated, and Professor F. Hochstetter was kind enough to lend me his help in the matter. Up to the present it has not been found possible to obtain the venous capillary network of hens by means of corrosion preparations. But I was able to follow the course of the vessels very exactly in a series of sections obtained from the embryo of a sparrow immediately before hatching. I found that the vein which approaches the suprarenal from the lateral side, enters the organ, forms large sinuous spaces within it, and probably afterwards breaks up into capillary vessels. In sections taken more towards the cranium, large veins filled with blood are seen at the median surface of the suprarenal; these are the vena suprarenales; they run in an oblique direction towards the vena cava and open into it. The arterial vessels also approach the organ upon the median side.

It is evident from this that a suprarenal portal system is present, not only in reptiles, but in birds, and that blood is conveyed to the suprarenals by veins as well as by arteries.

In mammals, however, blood is supplied to the suprarenals by way of the arteries only. According to Pettit, conditions resembling the portal system of birds are encountered in certain mammalian species. The last intercostal vein proceeds directly to the suprarenal, and opens into the vena cava at the place where the kidneys form an angle with it, in which angle the suprarenal lies, close against the walls of both vessels. If one or more branches from the suprarenal veins empty themselves into the intercostal vein, the appearance produced will suggest that the latter is not an efferent, but an afferent, vein. Pettit draws attention to a peculiar venous distribution which he has observed in monkeys and in certain rodents (kangaroo, sheep), and which has been observed in man by some French anatomists (Quénu and Léjars). Single branches proceed from the venous arches of the adipose capsule of the kidney to the suprarenal, which they cover, finally penetrating into the interior of the organ. Pettit is inclined to regard these venous branches as indications of a suprarenal portal system.

It is, however, very doubtful whether these rudimentary venæ afferentes suprarenales have a functional significance in mammals. 
However this may be, the vascular distribution in the suprarenal -that is to say, the arrangement of the arteries leading to it and the manner in which they communicate with the great central vein leading from it-is very remarkable. Flint's excellent work and the experiments of Srdinko have supplied very exact information concerning the suprarenal in mammals, and this is supplemented by Landau's corrosion preparations. Based upon the representations of these authors, the suprarenal circulation appears to be as follows.

A large number of the arteries which enter the suprarenal break up in the cortex into a network of capillaries, which traverse the connective tissue septa in a manner which accords with the structure of the latter, being straight in the zona fasciculata, and forming a network in the zona reticularis. Aftertraversing the entire cortex, the blood reaches the medulla in wide sinus-like vessels; in the medullary capillaries it flows round the chromaffine cells, and afterwards collects in small veins which increase in size, and all of which open into the great central vein. The arteries, and probably the afferent veins which supply the suprarenal capsule and the portions of the zona glomulerosa abutting on to it, also break up into capillaries, which again combine to form the venæe revehentes.

A certain number of the arterial vessels, the arteria perforantes (Srdinko) penetrate the cortex and reach the medulla directly; there they break up into capillaries, which also follow the course of the cell columns, and finally discharge their blood into the great efferent vein. This separate blood supply of the medulla is the outcome of the secondary invasion, during fotal life, of the interrenal organ by adrenal tissue.

This peculiar vascular distribution has been interpreted in the following manner (Poll). It is suggested that, in the first place, the blood from the body which has become deprived of its adrenalin contents, is carried by the vessels which exclusively serve the medulla directly to the adrenalin-forming cells, that these part with the secretion with which they are charged, and that the blood, with its reinforced adrenalin contents, is again returned to the general circulation. In the second place, the venous blood discharged from the cortex contains the products of the metabolism of this tissue, which constitute the raw material for the elaboration of adrenalin, and this raw material is immediately conveyed to the medulla. It would seem from this that, in certain species in which the adrenalin demand is very high, as in mammals and birds, there is a combination on the part of the two apparatuses which contribute to the elaboration of adrenalin, thus establishing a perfected method of production. In species in which the two suprarenal systems are independent, the semi-product supplied by the interrenal bodies is carried through the entire circulation before it reaches the site of its ultimate completion, namely, the adrenal tissue. 
This assumption of a function on the part of the interrenal tissue by which the raw material of adrenalin is provided, would explain the importance of the interrenal tissue to the life of the organism; and would account for those instances where animals survive double epinephrectomy, by the presence of accessory interrenals which thereafter become hypertrophied. In such cases as these, Poll believes that the organism returns, to a certain extent, to a previous developmental stage, that at which the two suprarenal systems are topographically independent, as in fish.

Fascinating though this hypothesis of an interdependent function on the part of the two suprarenal systems may be, it must be remembered that its sole foundation at present is upon morphological grounds; that it rests entirely upon the ontogenetic and phylogenetic association of portions of the interrenal and adrenal systems, together with the peculiar conditions which govern the vascular communication between the two systems. It must not be forgotten that, up to now, all attempts to prove a homogeneity of function on the part of the suprarenals, whether by experimental biology or from the results of human pathology. have signally failed.

It must be frankly confessed that, as things are at present, we have as little certain knowledge concerning the functions of the homogeneous suprarenal as we have of the function of the interrenal tissue; and it is only by means of investigation of the latter that we can hope to arrive at any definite information concerning the former.

\section{CAROTID GLAND AND COCCYGEAL BODIES.}

The carotid gland, which we have learnt to regard as a portion of the adrenal system, may be briefly described as follows. In man, this organ measures 5 to $7 \mathrm{~mm}$. in length, $2 \frac{1}{2}$ to $4 \mathrm{~mm}$. in breadth, and $1 \frac{1}{2} \mathrm{~mm}$. in thickness. It is situated at or within the bifurcation of the common carotid artery and, on account of its situation and its considerable nervous provision, was, by the older anatomists, called the intercarotid ganglion.

Luschka (I 862) was the first to undertake an exact microscopic examination of this body; his findings led him to the conclusion that it was a glandular organ auxiliary to the cervical sympathetic. He believed it to be a nerve gland, and named it glandula carotica. Arnold (I 865) next declared that the glandular structure described by Luschka was, in reality, an arrangement of blood-vessels, the walls of these vessels being formed of several layers of epithelium; he recommended that the organ should be called glomeruli arteriosi intercarotici. According to Eberth (r87o), however, the cell-agglomerations resembling epithelium by which the vessels are surrounded, do not form a true epithelium, but a vascular perithelium. Waldeyer (1872) compared the epithelial 
cells which he found in the vessels of the testicles with Eberth's perithelial cells from the glandula carotica, and with other perivascular formations also composed of large cells from the coccygeal bodies. Waldeyer classed these three structures together under the name of " perithelial organs."

The supposed glandular character of the carotid organ appeared at first to be borne out by the history of its development. Stieda (1881) came to the conclusion that the glandula carotica was a glandular organ developed from the epithelium of one of the branchial clefts. Jakoby (1895) then proved that the epithelial derivative of the third branchial cleft, which was supposed by many authors to be the primordial beginning of the carotid organ, is, in reality, the beginning not of that organ but of the external parathyroids (glandula parathyroidea III). This was confirmed by later authors (Prénant, Simon, Groschuf, Verdun, Fusari). At the present day, the branchial origin of the carotid organ is maintained by Maurer, but only in this sense, that he believes that it splits off from one of the parathyroids which take their rise in the second branchial cleft. These embryological discoveries have, to a large extent, undermined the theory of the carotid organ as an epithelial gland.

The examination of certain tumours of the glandula carotis led Marchand (1891) to the conclusion that this organ represents neither the development of a vascular network, nor is it a gland or a ganglion; he regards it as a rudimentary organ for which he proposes the name of nodulus caroticus.

R. Paltauf (1891) studied the development of the carotid organ and its tumours, and he classed this structure with the glandular organs, in the sense in which we are accustomed to describe the lymph-glands and the thymus as "glands." This definition involves neither a definite physiological function, nor a histological or histogenetic uniformity.

Schaper (1892) particularly emphasizes the fact that the typical cell agglomerations in the carotid organ are neither vesselwall cells nor gland cells; that, moreover, the organ is in no sense rudimentary, but, like the coccygeal gland and other large or small agglomerations of similar cells (perithelial cells and plasma cells), it may possess a definite physiological function.

Stilling (I892) was the first to describe cells in what, like the other authors, he terms the ganglion intercaroticum, which, like the medullary cells of the suprarenals, stain brown with potassium bichromate. He compared these cells with those which he found in the smaller bodies attached to the abdominal sympathetic. Stilling says: "The intercarotid ganglion is neither a simple vascular network, nor a rudimentary organ : it is a vascular gland, or blood-gland, with a structure analogous to that of the suprarenal capsules."

The absence of any certain knowledge concerning the 
character of this organ is expressed by the fact that, in the new anatomical terminology, its designation was glomus caroticum.

A. Kohn's (1910) minute histological investigations have completely elucidated the systematic position occupied by the carotid organ. It is essentially composed of a largish agglomeration of groups of chromaffine cells, and is traversed by a considerable number of nerve fibres, the majority of which are without medulla, and in which the cell balls and single ganglion cells are situated. The organ is extremely vascular and the structure and arrangement of the vessels are not in any way characteristic. Its primordial beginning is in embryonal ganglion cells of the intercarotid sympathetic nerve plexus. According to Kohn, the most fitting name for the organ would be "paraganglion intercaroticum," and it should be included in the group of the paraganglia, which he regards as members of the sympathetic nervous system. But, as we have previously shown, the paraganglia form part of the adrenal system, and the paraganglion intercaroticum cannot be classed separately from these.

The coccygeal body (glomus coccygeum) was termed by Luschka (1859) glandula coccygea, and was described by him as a reddish yellow body of about the size of a small pea, situated at the tip of the os coccyx and depending from the terminal branches of the medial sacral artery. Luschka himself held that this organ "might prove to be an integral portion of the sympathetic nervous system, but that in the meantime, owing to certain external characteristics, it was expedient to include it in the mixed company of the ductless glands." And there the socalled coccygeal gland has remained. The structural analogy between this organ and others of the same group (hypophysis cerebri, glandula carotis, suprarenal) has frequently been pointed out. Waldeyer compared the round cells of the interstitial connective tissue of the testis with the coccygeal and carotid glands, and classed them together under the name of perithelial organs.

Not only is there a similarity of structure between the carotid and the coccygeal glands, but, according to Jacobsson, they also resemble one another in their histogenetic relationship to the sympathetic. According to Kohn, the carotid and coccygeal glands are only the terminals of the continuous paraganglion chain by which the sympathetic is accompanied. Schapper expressed the same view.

And yet O. Stoerk (1907) showed that the cells of the coccygeal gland do not react to chromium during either fotal or post-foetal life; and further, that they probably have a genetic connection with the media of the vessels. According to Stoerk, moreover, the coccygeal gland has no developmental relationship to the sympathetic; the structures which Jacobsson identified with the primordial beginnings of this body have no connection with it whatever, but are in reality young chromaffine bodies. 
In his book on comparative anatomy and embryology (1907) S. v. Schuhmacher shows that the coccygeal gland of man corresponds to the glomuleri caudales of lower mammalian species, and that both are to be regarded as arterio-venous anastomoses. The primal origin of the coccygeal gland in man is to be found in a local thickening of the medial sacral artery and its branches. The muscular fibres of the vessels assume an epithelioid appearance even in the embryo. In adults the cells of the muscular structure of the artery are shortened and broadened, the nuclei are spheroid and react weakly to dyes. Thus it appears that the layers which, in the anastomotic vessels, are the continuation of the arterial muscles, have been taken for everything except what they are, namely, modified vessel muscles. According to v. Schuhmacher, this organ in man should rightly be termed glomus coccygeum; like the glomeruli caudales of animals, it consists of a network of branched and convoluted vessels, which cannot in any sense be credited with an internal secretory function.

\section{HYPOPHYSIS CEREBRI (CEREBRAL APPEND- AGE,* PITUITARY GLAND).}

From the earliest times the pituitary body has been regarded as an organ possessing a secretory function. Galen and Vesalius believed that the mucus (pituita) formed in the brain was excreted by the agency of the pituitary body. Later authors, however, (Willis, Vieussens, Sylvius, Boerhave, Monroe) inclined to the view that the cerebro-spinal fluid was secreted by that organ. Magendie (1847), who investigated the genesis of the cerebrospinal fluid, regarded the hypophysis as an organ resembling the lymph glands, and he believed that it collected the lymph from the brain and conveyed this lymph into the circulation.

Liégeois (1860) was the first to include the hypophysis among what were at that time known as the vascular or blood glands; he described histological findings which he believed pointed to the formation of blood in this organ. It is interesting to note that the brothers Wenzel (I8Io) believed that epilepsy was due to an accumulation of colloid in the hypophysis.

After the formulation of the doctrine of internal secretion by Brown-Séquard, a large number of observations were published which sufficiently justified the inclusion of the hypophysis among the internal secretory organs. The fundamental argument was supplied by Rogowitsch, who found that extirpation of the thyroid was followed by hypertrophy of the hypophysis. The

* Before the introduction by Soemmering of the term Hypophysis cerebri, the following names were also used: Glans pituitam excipiens Vesalius, caput rosæ, colatorium, labrum, lacuna, infusorium, concha pelvis, embotum, pelvis colatoria, sentina encephali. 
decisive evidence was, however, brought forward by Marie (1886) who, under the name of acromegaly (abnormal growth of the "acral" portions of the body), described a clinical condition, not sharply separated from gigantism, which had previously attracted the attention of a certain number of authors (Friedreich, hyperostosis of the entire skeleton; Lombroso, general hypertrophy or macrosomia; Fritsche and Klebs, gigantism). In combination with Marinesco (I89I), Marie showed that changes of the hypophysis, generally tumours, are invariable in this condition, and it seemed from this that the cause of the peculiar anomalies of growth must lie with the pathological condition of the hypophysis.

This momentous discovery of Marie's excited an immense amount of interest in the clinical and pathologico-anatomical aspects of anomalous development, while at the same time it pointed to the hypophysis as the centre of interest. These researches extended to the morphology of the organ and its pathological changes, with the result that a considerable number of facts have been brought to light which largely explain its functional significance.

Physiological research was first conducted by means of experimental extirpation. An important advance was made by Oliver and Schäfer (I894), who discovered that the watery extract of hypophysis possesses distinct physiological properties.

'The most instructive results have been obtained, however, by means of hypophysis surgery in man, and these results have been confirmed and completed by recent experiments with animals.

\section{ANATOMY.}

The hypophysis is situated within the cranium at the base of the brain; it is connected with the latter by the infundibulum; and it more or less completely fills the sella turcica. The sella turcica is lined with dura mater, which, in the form of a fibrous lamella (diaphragma sella turcica), covers the surface of the hypophysis, leaving a circular opening through which the infundibulum passes. The lateral boundaries of the hypophysis are formed by the walls of the cavernous sinus. The infundibulum is surrounded by the circular sinus of Ridley, formed from small anterior and posterior venous branches. The hypophysis is separated from the internal carotid artery by the sinuses, of the dura mater. It lies in the anterior angle of the optic commissure, the posterior portion of the organ being occasionally covered by the anterior edge of the commissure.

The hypophysis is flattened from back to front and upon the surface. Its average size in an adult man is given by Erdheim as follows: breadth, $14.4 \mathrm{~mm}$.; thickness, $21.5 \mathrm{~mm}$.; height, $5.5 \mathrm{~mm}$. The average weight at the average age of $33 \frac{1}{2}$ years is given by Comte as $59 \mathrm{cg}$. Caselli found that in fifty men 
the average weight was $66.7 \mathrm{cg}$., in fifty women, $73.1 \mathrm{cg}$. His figures are high owing to the fact that his subjects were mentally alienated; it is said that, in this class of case, the weight of the hypophysis is in inverse ratio to that of the brain. According to Erdheim and Stumme, the average weight of the hypophysis in the male is, in the second decade of life, $56.3 \mathrm{cg} . ;$ in the third decade, $59.3 \mathrm{cg} . ;$ in the fourth decade, $64.3 \mathrm{cg}$. From then onwards the weight gradually declines; in the fifth decade it is, on an average, $6 \mathrm{r} .4 \mathrm{cg}$.; in the sixth, $60 \mathrm{cg}$.; and in the seventh, just a shade higher, 6I.2 cg. The average weight of the hypophysis in women who have never borne a child is, decade for decade, almost identical with that of men; in women in whose pregnancy anamnesis is unknown, the average weight is generally higher- $71.6 \mathrm{cg}$. as against 60.1 in nullipara and $6 \mathrm{I}$ in men. During pregnancy the hypophysis undergoes a remarkable increase in weight. The minimum weight of $65 \mathrm{cg}$. in a primapara is larger than the minimum weight in a nullipara; even the maximum known weight of $75 \mathrm{cg}$. in a nullipara does not equal the average figure of $84.7 \mathrm{cg}$. in the primipara. The increase in the size of the hypophysis takes place almost exclusively in the length and breadth; in the anterio-posterior diameter it is barely noticeable. At the termination of pregnancy the weight is again reduced, but should pregnancy again occur it rises to a figure even higher than before. The average weight of the hypophysis in the multipara at the termination of normal pregnancy is $106 \mathrm{cg}$., a figure considerably in advance of the weights which have been ascertained in the case of the primipara. The difference in the maximal weights is even greater, that of the multipara being $165 \mathrm{cg}$. and that of the primipara $110 \mathrm{cg}$. The increase in weight is accompanied by structural changes which will be described later.

The hypophysis is supplied with blood by means of minute branches from the internal carotid artery; the blood is carried away into the circular sinus by small veins.

Section of the hypophysis shows, even to the naked eye, that the organ consists of two parts; the anterior portion is a hard, kidney-shaped lobe, concave posteriorly, pale yellow to grey-red in colour, and represents the epithelial or glandular portion of the hypophysis (the true pituitary gland). The posterior portion is a smaller, rounded, white, soft lobe, situated in the concavity of the anterior lobe; it represents the nervous or infundibular portion of the organ (the true hypophysis or neurohypophysis). The two lobes are joined together and enclosed in a common fibrous capsule. The hypophysis is attached to the brain, as previously mentioned, by means of the funnel-shaped infundibulum. The latter is a prolongation of the floor of the third ventricle, the tuber cinereum; it first forms a hollow sphere which represents a prolongation of the third ventricle, it then penetrates 
the diaphragma sellæe turcica and the dura mater, and finally enters the hypophysis, where it terminates in a slightly thickened end. The infundibulum usually enters the hypophysis at the centre of the hilus of the anterior lobe, though occasionally it enters the lobe itself. The tissue connection is always with the infundibular portion only.

\section{HISTOLOGY.}

The anterior lobe of the hypophysis consists of a connective tissue framework, with longish oval interstices at the periphery and larger rounded ones in the interior. These spaces contain groups of cell columns, which sometimes form glandular tubules provided with a lumen. Flesch divided the glandular cells of the anterior lobe into two classes in accordance with their size, form, and specific colour reactions; namely, the chromophile cells, and the chromophobe or adelomorphous cells.

Of the chromophile cells, some are spheroid or polyhedral; with a nearly homogeneous protoplasm, which is full of fine granules and stains strongly with eosin; while the nuclei are small and round, frequently in the centre of the cell, and stain well with hæmatoxylin. These are known as the eosinophile cells. A second group is composed of somewhat large cells, of regular shape, which stain a very good dark blue with hamatoxylin; they are filled with coarse granules, the nuclei are placed eccentrically, and the plasm contains rounded vacuoles. These are known as the cyanophile or basophile cells.

The chromophobe principal cells (Hauptzellen) are undefined in outline; the plasm is sometimes brittle and does not fix well by the ordinary methods; while the nucleus is large, either round or irregular in shape, and possesses a chromatin network which stains readily.

The topographical distribution of these three groups of cells is by no means constant. As a general rule, the principal mass of the eosinophile cells is found in the hinder portion of the anterior lobe, while the basophile cells usually lie towards the front. The principal cells are not peculiar to any precise locality, but are characterized by their topographical relationship to the chromophile cells; they frequently occupy the centre of alveoli or cell cylinders, thus becoming surrounded by chromophile cells.

The changes which Erdheim and Stumme observed in the enlarged hypophysis of pregnant women, chiefly take place in the tissue of the anterior lobe of the organ. The cut surface, which is normally grey-red in colour, becomes remarkably light and reddish-grey to grey-white; it becomes softer and yields a milky juice. These changes in colour and consistency are due to the appearance, in enormous numbers, of a new kind of cell, namely, the pregnancy cells; and these outnumber the eosinophile 
cells which, under normal conditions, largely predominate. The actual number of the eosinophile cells remains unchanged, but they are noticeably reduced in size. The basophile cells are entirely unaffected, the whole of the pregnancy changes taking place within the principal cells. These practically cease to exist as such, being almost entirely transformed into pregnancy cells. The pregnancy cells have large, light, irregular nuclei, with abundant plasm which is distinctly granular and stains red with eosin. They accumulate in large numbers in the two lateral portions of the hypophysis; in the posterior portion, where the eosinophile cells are ranged in compact masses, they are scanty but not entirely absent. The pregnancy cells are grouped in broad columns and in alveoli, generally occupying the centre of the alveolus. Occasionally, when pregnancy is at its height, these cells form agglomerations which suggest adenomatous hyperplasia. After parturition, involution of the pregnancy cells takes place and they largely reassume their former appearance of principal cells. There is, however, an increase in the number of the principal cells now present, and at the next pregnancy these undergo changes similar to the last, so that the result, though qualitatively the same as at the first pregnancy, is quantitatively very much more intense.

In the hinder boundary layer of the anterior lobe (Peremeschko's medullary layer, Thaon's région interglobulaire), a fissure is frequently, though not invariably, observed in the neighbourhood of the hilus; it is lined with a single layer of cubical cells or, more rarely, ciliated columnar cells, and its hollow is either empty, or contains a substance which stains pale blue with hæmatoxylin. This fissure represents the embryonal hypophysal cavity. Two kinds of vesicles or follicles are also observed in this region. By the side of the fissure, and sometimes in place of it, rounded vesicles are occasionally seen, which are lined with columnar epithelium and filled with an amorphous mass; they are probably formed from depressions in the posterior wall of the embryonal hypophysal cavity. A second variety of vesicles, found in the same neighbourhood, is considerably larger; both in appearance and in their colloid reactions, these vesicles are reminiscent of the follicles of the thyroid. The vesicle wall is composed of cubical cells, which do not stain readily and contain large nuclei; the lumen of the vesicle is sometimes filled with an amorphous homogeneous mass. In addition to a finely granular basophile substance, an eosinophile substance resembling the colloid of the thyroid is sometimes seen. Vesicles of this description are usually encountered in the posterior medullary layer, and occasionally also in the interior of the anterior lobe.

By means of Golgi's method, Gentès and Pirrone discovered nervous elements in the walls of these vesicles, similar to those in the sensory cells of the olfactory region of the nasal mucous membrane. 
Pisenti and Viola, as well as Caselli, found cells with large nuclei in this layer of the hypophysis, which might be regarded as residues of the endodermal portion of the embryonal hypophysis (v. Kupffer). According to Launois and Mulon and to Thaon, changes also take place during pregnancy in the posterior portion of the anterior lobe, which these authors interpret as signs of glanclular hyperplasia and hypersecretion.

The communication between the posterior lobe of the hypophysis and the brain, represented by the infundibulum, varies in man both in length and thickness; it is composed of glia fibres with few nuclei and the upper portion encloses a canal, the recessus infundibuli, which is lined with ependyma cells and represents the continuation of the third ventricle. This hollow is occasionally prolonged into the hypophysis and in some species, notably cats, it constantly extends as far as the posterior lobe.

The posterior lobe of the hypophysis, the pars nervosa, is considerably smaller than the anterior lobe; it lies posteriorly, in the hollow formed by the glandular portion, the opening of which is downwards. This lobe extends into the sella turcica, in the posterior wall of which a groove for its reception is sometimes observed. Histologically, the posterior portion consists of a loose stroma, which is a mixture of connective tissue elements and neuroglia, the latter predominating. In preparations coloured by Weigert's glia method, Thaon observed a small proportion of fine collagenic fibres, together with a manifold but not close network of glia fibres, in which isolated light, round nuclei were distributed. The tissue had few blood-vessels, and frequently appeared to be infiltrated with an amorphous substance, which resembled the substantia gelatinosa Rolandi of the spinal cord.

The question as to whether the nervous portion of the hypophysis contains nerve cells has formed the subject of repeated investigation. Both Krause and Berkley describe true nerve cells and nerve fibres; Ramon y Cajal found cells of an undefined kind in young rats, together with delicate fibres which were certainly nervous and which proceeded from nerve cells situated at the base of the brain behind the chiasma; these nerve fibres descended through the infundibulum, formed a plexus in the posterior lobe and terminated in free branches. Kölliker, as well as Caselli, did not believe that true nerve cells were present in the posterior lobe; they considered the cell elements present in it to be glia cells and ependyma cells. Gentès discovered a considerable network of nerve fibres by Golgi's method, and this finding was confirmed by Thaon.

According to A. Kohn, the foundation tissue of the neurohypophysis is to be regarded as primitive glia in this sense, that its elements are invariably associated with the ependyma, the matrix of all glia elements. He points to the occurrence of a peculiar pigment, which is found chiefly in the glia fibres and 
to a small extent only in the glia cells. This pigment is neither a fat nor a lipochrome, but is probably a product of decomposition quantitatively increasing with age. In consequence of the intimate relationship between the anterior and posterior lobes of the hypophysis, together with the penetration of epithelial cells into the neuro-hypophysis; the latter contains a considerable proportion of epithelial, glandular elements and products, all of which are derived from the anterior lobe and, in a histological sense, belong to it. Kohn is unable to confirm, from the morphological standpoint, Joris's view that the posterior lobe of the hypophysis is a secretory gland.

The structural indifference of the posterior lobe of the hypophysis forms a remarkable contrast to the proved physiological activity of the extract obtained from this portion of the organ.

Secretion.-Owing to its histological characteristics, and especially those presented by the anterior lobe, the hypophysis has been accredited a secretory function, though the views adopted concerning the nature of its secretory product are somewhat divergent. The fat granules which it contains are, however, unanimously regarded as a physiological product of cellular secretion. According to Erdheim, fine fat granules make their appearance at the beginning of post-fœtal life, and these become progressively larger until old age, when they are larger than nuclei. They are found in all the cells, in the shape of rings and spheres as well as in different vacuole forms. The granules in the chromophile cells are large in size, but few in number; those in the adelomorphous cells are small and very numerous. They colour with sudan, scarlet $R$. and osmium; they are soluble in ether and alcohol; and they are not double refractive. According to Thaon, the treatment of sections stained with osmium reveals different degrees of solubility, and he concludes from this that the granules are composed of fats of different kinds. He finds that the fat granules of the hypophysis contain little oleic acid, and believes that they are combined with an albuminoid molecule.

The cell granules represent another product of hypophysal secretion. Saint Remy and Benda, more particularly, do not regard the chromophile and chromophobe cells as different cell varieties, but they believe them to represent different stages of secretion on the part of a single cell variety. According to Benda, the adelomorphous cells which contain no granules and only isolated basophile fragments, are the young forms, the secretory starting point; the cells filled with acidophile granules represent the secretory climax; while the pale nuclear groups filled with dust-like amphophile granules are the product of the final secretory stage. Benda believes that the acidophile granules represent the measure of the secretory activity of the hypophysis cells; that they are produced during functional activity, and that at the resting stage their formation ceases. 
Quite recently, however, Gemelli expressed the view that the chromophile and chromophobe cells represent two different cell varieties. Pirrone, Guerini, Launois, Thaon and Joris adopt Benda's view, with this modification that they regard colloid as the end product of the secretory process.

The amorphous substance resembling the colloid of the thyroid is regarded by Benda, Gemelli and others as the product of degeneration. Erdheim regards it as rudimentary and reminiscent of a time when the organ possessed an external secretory function. By many authors, particularly those of the French school, the colloid substance is regarded as the product of normal secretion.

Of the chemical nature of this colloid, all that is known is that it is insoluble in water, alcohol and ether; that it does not yield gelatine after boiling; and that, unlike mucin, it swells in acetic acid and afterwards dissolves. It has been identified with the colloid substance of the thyroid and this is confirmed by the discovery of iodine in the hypophysis.

The colloid substance is present in the hypophysis of the human embryo at three and a half months; in adults, it is found in the cell columns and is particularly abundant in the vesicles in the neighbourhood of the hilus, and in the blood-vessels. In addition to a strongly coloured, eosinophile, homogeneous colloid, the hypophysis, like the thyroid, also contains a less homogeneous, granulated, basophile substance resembling coagulated albumin. The colour differentiation of the colloid substance depends upon the different granulation of the acido- and basophile cells.

The presence of the colloid substance between the cells, in the vesicles, and in the lumen of the capillaries is regarded as proof of its excretion by way of the blood-stream. Thaon showed the manner in which the secretion is excreted in series of sections from hypophyses which showed signs of hypertrophy. In addition to colloid, he found fat granules between the cells and in the capillaries; he was unable to demonstrate the presence of lymphatics in the hypophysis. According to Thaon, the secretion is eliminated from the hypophysis in two ways; one, through the cell base into the blood-vessel with which it is in contact; the other, through the opposite cell wall into the lumen of a vesicle, where present.

The presence of colloid in the vesicles may be considered to be evidence of the deposition of this substance; thus it is possible to regard the hypophysis as a storage gland, similar in character to the thyroid apparatus. Thaon believes that the physiologically active substance of the posterior lobe, is merely the secretory substance elaborated by the anterior lobe, which accumulates in the vesicles in the neighbourhood of the hilus. The method of preparing pituitary extract does not permit of any separation of 
this medullary layer from the posterior lobe. Silvestrini also locates the active substance in the medullary layer; Salvioli and Carraro, on the contrary, believe that it is contained in the pars nervosa.

It should be mentioned that Livon held the view that the internal secretion of the hypophysis passes into the brain by way of the nerve fibres in the peduncle.

\section{HISTORY OF DEVELOPMENT.}

Since the earliest investigations by Rathke in 1838 , the development of the hypophysis has formed one of the most widely discussed problems in the department of embryology. Even to-day, the views held concerning it are very divergent.

The subject of the earliest discussion, in which, in addition to Rathke, Reichert, Luschka, Kölliker, Fr. Müller, His, Maclay, and Duvry were concerned, was the supposed relationship between the hypophysis and the epithelium of the buccal cavity, Rathke's pouch more particularly. Mikalkovics's experiments with mammals, Goette's experiments with amphibia, and Dohrn's experiments with ammocœtes, appear to show that the hypophysis is exclusively ectodermal in origin. This view was shared by the vast majority of embryologists and finds expression in the textbooks of the subject.

The development of the hypophysis is described by Hertwig as follows: In the embryo of a rabbit, $6 \mathrm{~mm}$. in length, at a developmental stage when the cephalic flexure and the stomadæum have already made their appearance, the site of the future hypophysis is situated anteriorly and dorsally of the spot where the anterior end of the chorda dorsalis immediately approaches the insertion of the pharyngeal membrane. After the perforation of the pharyngeal membrane (in the chick on the fourth day, in the human embryo in the fourth month), a groove is formed, shallow at first, but gradually becoming deeper, which is known as Rathke's pouch or the hypophysis pouch. This groove, which develops in the direction of the funnel-shaped, depending base of the thalamencephalon, becomes clothed with several layers of cylindrical cells, and eventually forms a longish pouch (embryo rabbit, $12 \mathrm{~mm}$.).

At a later stage, this pouch is almost completely divided from the primitive mouth cavity by the development of the cartilaginous base of the skull, a narrow opening only being left. At the same time, the posterior wall of the pouch has reached the infundibulum and has been pushed backwards by it into the cavity of the pouch (embryo rabbit, $20 \mathrm{~mm}$.). A little later, the lumen becomes obliterated and the pouch forms a solid cell column. In Selachians, the pouch persists throughout life as a hollow canal, one end of which penetrates the cartilaginous base 
of the skull, while the other communicates with the epithelium of the oral mucosa. The hypophysis is now a closed structure, but in the human embryo, in the second half of the second month, hollow tubes are formed in the anterior and ventral wall of the cavity; these penetrate into the surrounding connective tissue, which is very vascular (embryo rabbit, $30 \mathrm{~mm}$.), and become cutoff from the cavity of the hypophysis to form isolated structures. The whole of the pouch now breaks up into tubes in the same way, thereby forming the anterior lobe, in the posterior portion of which traces of the original cavity still remain. The anterior lobe is now joined by means of connective tissue to the posterior lobe, which has in the meantime developed from the infundibulum.

Traces of embryonal development are observed in the human hypophysis during the entire course of post-fœtal life. The fissure lined with epithelium which is present in the anterior lobe, is the post-fotal representative of the original pouch; while the cavities filled with colloid, by which this fissure is frequently replaced, are the outcome of the division of the pouch. Erdheim discovered that, from uninvolved remnants of that portion of the hypophysis cavity which is inserted in the anterior lobe, agglomerations of pavement epithelium are formed; these are encountered within a circumscribed area of the anterior lobe, either in isolated groups or associated with adelomorphous cells or vesicles. According to Erdheim, these agglomerations of pavement epithelium may develop into pituitary tumours of the kind known as pavement epithelium sarcoma.

The road traversed by the hypophysis in the course of its development is represented, in all except a few isolated instances, by a bony channel which passes through the sphenoid bone and is called the canalis cranio-pharyngeus. In and under the mucosa of the pharyngeal roof of new-born infants, Erdheim discovered a cord composed of hypophysis tissue which he named the "pharyngeal roof hypophysis" (Rachendachhypophyse). Haberfeld, who carried Erdheim's investigations still further, found that this cord, which he termed "hypophysis pharyngea," was constant in man at all ages; he did not regard this structure as an embryonal remnant, but believed it to be a fully developed accessory hypophysis, with a function identical with that of the true hypophysis. Haberfeld showed that, in isolated cases, remnants of hypophysis tissue visible to the naked eye are situated along the entire length of this cord, and that they may form the site of origin of hypophysal tumours. As a matter of fact, Erdheim observed such a tumour in an unusual situation, the course of its development extending below the sella turcica, while the hypophysis, which was normal in size and shape, retained its position undisturbed.

These findings point to a purely ectodermal origin of the 
hypophysis, but the view has been contested upon many sides. First Hoffmann, and later Orr, assumed a relationship between the hypophysis and the ectoderm of reptiles. As the result of his later investigations (1894), v. Kupffer, who had previously accepted the ectodermal origin of the hypophysis in all vertebrates, arrived at the conclusion that, in addition to the ectoderm, the entoderm - the epithelium of the archenteron in particularis concerned in the formation of this organ. According to him, the hypophysis has three primitive sources of origin; the first is ectodermal and is a remnant of a phylogenetically old oral cavity (palæostoma), situated above the spot where the mouth afterwards develops, and which, later, becomes Rathke's pouch; the second, which is entodermal, is situated behind the pharyngeal membrane and is formed by an outgrowth, sometimes hollow and sometimes solid, of the cephalic intestine (preoral intestine), the blind end of which forms the pouch described by Seessel; the third or cerebral portion is provided by the infundibulum and infundibular gland. In the monorhines and myxinoids, the infundibular gland only is present, the palæostoma forming the permanent nasopharyngeal passage. In Petromyzons, Elasmobranchii, and Teleosteii, the infundibular gland and the dorsal gland-complex of the naso-pharyngeal passage are both present.

v. Kupffer's view is shared by Valenti and partly also by Nussbaum; but the inclusion of the entoderm in the formation of the hypophysis is denied by St. Rémy in the case of birds and mammals, by Hoffmann in the case of Selachians, and by $B$. Haller in the case of all vertebrates. Valenti, who has carried out a fresh series of investigations with embryo chicks and rabbits, maintains the view, however, that the hypophysis contains an ecto-entodermal portion, derived from the preoral intestine. Corming and Rossi, on the other hand, insist upon a purely ectodermal origin.

\section{PHYSIOLOGY OF THE HYPOPHYSIS.}

Experimental Extirpation.--It was Horsley (I885) who, in order to compare the results of the removal of the hypophysis with those which follow thyroidectomy, first undertook the extirpation of that organ. His experiments, like those of Dastre (1889) and Gley (189I) were completely negative, the animals dying immediately after operation. Following the theory propounded by Marie and Marinesco concerning the pathogenesis of acromegaly, Marinesco (I892) next carried out extirpation experiments with cats. Since that date, hypophysectomy has been performed on animals of different species, and according to various methods. But these attempts, if we except quite the most recent, supplied no satisfactory information concerning the importance of this organ to the life of the organism and the nature 
of its functional significance. The animals most frequently employed were the cat and the dog, though a certain amount of material has been provided by other animals (frog, tortoise, hen, rabbit).

Experimental extirpation from the oral cavity of the hypophysis of frogs, as carried out by Caselli (I900), did not yield results of any value. Gaglio (1902) operated upon frogs and tortoises by the same method, however, and found that, though some of his animals died very shortly after, apparently as the result of infective conditions, others lived one and a half to three months without pathological symptoms. Boteano, a pupil of Paulesco's, describes (I906) extirpation of the hypophysis of frogs by the supra- and latero-cranial methods; he found that, two to three days after total extirpation, his animals invariably died, with symptoms of neuro-muscular asthenia; while control animals operated upon by the same method, the hypophysis being only partially removed or left intact, remained perfectly normal, with the exception of slight symptoms of central nervous stimulation.

G. Fischera (1905) operated upon hens. He obtained access to the organ from the base of the skull, by means of an incision at the edge of the lower jaw ; with the aid of the thermocautery, he divided the basal sphenoid bone in the middle line, thus laying bare the hypophysis. Of forty hens, eleven died shortly after operation, twenty-nine survived and were destroyed after four months. The autopsy showed that, in eight cases, the hypophysis was undamaged; in seventeen, it was partially injured; and in four, completely destroyed. Operation was followed in the first few days by symptoms of fatigue and depression, which soon passed off. Two of the hens in which the hypophysis had been entirely destroyed showed signs of arrested development. The results of Fischera's experiments led him to conclude that, in young hens, the hypophysis is not essential to life, and that the symptoms which follow its removal are in no way characteristic. Even the arrestation of development cannot be regarded as a constant symptom.

The extirpation experiments which Gley carried out with rabbits are, on account of their faulty technique, of very little value. Experiments with cats and dogs are the only ones which have supplied reliable information concerning the function of the hypophysis; this is attributable, not only to the frequency with which these animals have been employed, but to the better operative technique by means of which such experiments have been accomplished. Cats and dogs have been employed as subjects for hypophysectomy by a large number of authors (Marinesco, Vassale and Sacchi, v. Cyon, Caselli, Gatta, Kreidl, Biedl, Friedmann and Maass, Della Vedova, Lo Monaco and van Rynberk, Pirrone, Gemelli). The method which was first employed and was later in most frequent use, was that of opening 
up the hypophysis from the, base of the cranium by way of the oral cavity. The organ was usually destroyed either with a thermocautery or by the injection of chromic acid; removal was effected with spoon-forceps, generally of the operator's own design.

Lo Monaco and van Rynberk followed up Gley's experiments with rabbits, the method they chose being from the vertex of the skull. After opening the dura mater, these authors penetrated between the hemispheres at a spot previously determined upon, and after passing through the corpus callosum they reached the infundibulum and from there they were able to reach the hypophysis. In twenty-eight cases out of forty-four they succeeded in removing the hypophysis by this method.

This method was very justly condemned by Paulesco; he pointed out that it is faulty in all essentials, for it inevitably provokes secondary lesions' of more or less grave a character, that it is accompanied by serious risk of infection, and that it does not properly accomplish what is, after all, the chief object of operation, namely, the exposure of the organ. Paulesco (Igo6) introduced a new method of hypophysectomy; he employed the lateral approach, beneath the temporal lobe.

I have employed this method myself in a large number of experiments. In my earlier experiments with cats, I used the buccal method of reaching the hypophysis, but in the later experiments with dogs which I carried out with Dr. M. Silbermark, we invariably employed the intercranial operation. This method, which was also adopted by Cushing, fulfils all requirements. After practising upon the cadaver, we were able to perform the operation with a considerable degree of certainty, and out of a series of fifty animals we did not lose one from post-operative hæmorrhage, meningitis or sepsis. It may be remarked in passing that this method may be employed with equal success for the purpose of exposing any structure at the base of the brain in the median cranial fossa, and is especially suited to the intercranial resection of the second to sixth cranial nerves. What is fundamentally new about the method is that it permits of a considerable dislocation of the brain without damage to it. An opening of suitable size is made in the squamous portion of both temporal bones, and the dura mater is opened upon both sides. With a flat bent spatula the temporal lobe may now be lifted and pressed to one side, without in any way crushing the substance of the brain. In a good light, after mopping out the cerebrospinal fluid, the hypophysis will be seen hanging like a berry on a stalk in front of the optic nerve, by the side of the internal carotid artery, and behind the oculomotor nerve. The organ is now clearly visible under conditions eminently suited to operative intervention. It may be severed from its peduncle and left free in the cavity of the brain; or it may be entirely removed; or 
single lobes or portions may be removed. This operation is very rarely followed by hamorrhage. After operation, the elevator is removed, the brain returns to its position, the dura mater is put into place and kept there by the temporal muscles, and the incision in the temporal cuticle is sewn up. We found, in our experiments, that Paulesco's resection of the zygomatic arch was unnecessary and, provided that the openings in the squamous portions of the temporal bones are sufficiently large, the structures in the sella turcica may also be easily reached without such resection.

What, then, are the results of experimental extirpation of the hypophysis? In order to arrive at a clear answer to this question, it is necessary to consider separately the results yielded by the older experiments, before the introduction of Paulesco's method. In a large number of instances the animals operated upon by the older investigators died either immediately or very shortly after operation. Under these circumstances it was not possible to decide whether death was due to the removal of the hypophysis, or whether it resulted from the serious surgical intervention with its attendant danger of infection. A small proportion of animals survived for a brief period, one for three weeks, and some lived even longer. It will be remembered that Lo Monaco and van Rynberk destroyed their animals after thirty-five to eighty-six days.

The relative short span of life after extirpation was regarded by many as a proof of the vital character of the hypophysis, though other investigators, whose animals lived for longer periods, ascribed the fatal termination to momenta other than suppression of the organ. Lo Monaco and van Rynberk, especially, laid great stress upon the fact that, after their transcerebral operation-which was undoubtedly very drastic, seeing that it necessitated a serious lesion of the infundibulum and the opening of the ventricle-their animals survived and showed no pathological symptoms. Single animals, with what the autopsy showed to be an uninjured hypophysis, yet showed all the symptoms of hypophysis suppression; while others again, in which the hypophysis was found to be absent, remained normal.

These contradictory views concerning the results of hypophysectomy are explained by the ill-defined and obscure nature of the symptoms by which the operation was accompanied. In a large number of cases, on the one hand, symptoms of depression are described, ranging from dulness and apathy to paralysis and even coma; while in others, symptoms of motor stimulation, fibrillary tremors and tonic cramps are reported; and in yet other instances, no symptoms, either of stimulation or of depression, were observed. The effects upon the vegetative nervous system were also very differently described. Many authors found polyuria, unaccompanied by abnormal constituents; others describe albuminuria; others again, glycosuria; while many were unable to report changes in either the circulation, secretion, or respiration. 
The only decisive observations are those obtained from later experiments carried out by Paulesco's method. Paulesco and Cushing have described the results of such hypophysectomies. I employed the method myself in combination with Silbermark, as well as with Leishner and Denk.

Paulesco performed total hypophysectomy with twenty-two dogs and two cats. His animals lived for ten to forty-eight hours at most. The autopsy revealed neither hæmorrhage nor suppuration at the site of operation, and the other findings were also negative. The autopsy showed that, in fourteen dogs, extirpation was not complete, portions of hypophysis tissue of varying size having been left in situ. These animals lived three to seven to ten days; where the amount of tissue left behind was large, they lived longer (seventeen, twenty-three, sixty-seven, $151,158,196$, and 395 days). The almost complete suppression of hypophysal activity was not revealed by any marked symptoms, and Paulesco found that even those animals which survived for a considerable time did not show any signs of trophic disturbance.

In three dogs, a portion of the anterior lobe only was removed. These animals lived without special symptoms for twenty-three, sixty-six, and 932 days respectively. The complete destruction of the cortical substance of the anterior lobe with the thermocautery proved fatal to seven dogs, death following as rapidly as after total hypophysectomy. Five animals survived the removal of the posterior lobe for thirteen and sixty-nine days, eight months, and two years respectively; there were no pathological signs, and the animals eventually died from accidental affections.

The loosening of the hypophysis from the sella turcica was found to be quite negative. According to Paulesco, death follows separation of the hypophysis from the base of the brain by resection of the peduncle, as rapidly as after complete, or almost complete, extirpation. That this result is not due to the opening of the third ventricle, is proved by the fact that the opening of the third ventricle alone is not followed by death. Neither can the fatal result be ascribed to the lesions at the base of the brain, for in the control animals such lesions produced only symptoms of motor stimulation, and these were invariably absent after removal of the hypophysis.

Paulesco concluded that the hypophysis is an organ which is essential to life, and that the suppression of its function is rapidly followed by death. In his opinion, the vital portion is the cortical substance of the epithelial anterior lobe.

Cushing confirms Paulesco's view of the vital character of the hypophysis. Nevertheless, his experiments seem to show that the results of total extirpation are less rapid in young dogs than in old. He was able to prolong the lives of his animals by the previous or simultaneous transplantation of the anterior lobe, as well as by the injection of extract of the anterior lobe. According to Cushing, total extirpation of the hypophysis is followed 
within a few days, or at latest a few weeks, by symptoms characteristic of cachexia hypophysopriva, terminating in death.

After extirpation of the posterior nervous lobe, the intermediate portion being generally removed with it, animals usually survive and show no pathological signs. Partial extirpation of the anterior lobe is consistent with the continuance of life. In isolated cases, however, it leads to remarkable symptoms, such as increased deposition of fat, polyuria, transitory glycosuria, and falling of the hair. The most striking result, however, was the reduction in sexual activity, shown anatomically by atrophy of the testicles and ovaries. Acute thyroid hypertrophy was also an occasional sequel to hypophysectomy.

Judging by Cushing's experiments, the anterior hypophysal lobe represents the vital portion of the organ, the complete extirpation of which is followed by death, its partial extirpation by disturbances of growth and of metabolism and by derangement of the activity of the sexual organs.

From the results of my own experiments I am able emphatically to confirm Cushing's views. We found that complete extirpation of the hypophysis was rapidly followed in both adult and young growing animals by death; the post-mortem findings were negative. Where the posterior lobe only was removed, the animals lived without symptoms for months, ultimately dying of intercurrent disease. Where extirpation was performed only on the anterior lobe, a portion of the tissue being left in situ, the animals lived for weeks and months, the autopsy showing, however, a remarkable deposition of fat in the omentum and retroperitoneal space, together with pronounced atrophy of the entire sexual apparatus. The ovaries and uterus of a 3 -year-old bitch presented appearances similar to those of an animal of a few weeks old.

Quite recently (December 3, I909), B. Aschner described extirpation experiments with dogs, in which he employed the older method of reaching the organ, namely, by way of the oral cavity. He succeeded in removing the complete hypophysis by this means. A proportion of his animals died within the first eight days, and he ascribes this to the removal, with the hypophysis, of too large a portion of the infundibulum. Other animals survived operation for several months. In full grown animals the results were practically nil; there was a certain change in the metabolism of the carbohydrates, similar to that seen in thyroidless dogs. The effects of adrenalin in producing glycosuria and symptoms of stimulation of the sympathetic were much less marked in these dogs than in normal dogs, and the different phases of alimentary glycosuria were much reduced.

The suppression of the hypophysis was followed, in young animals, by profound disturbances. These animals were very backward in growth and weight as compared with control 
animals; there was a shortening of the skull and especially of the muzzle, hypoplasia of the genitals, anomaly in the growth of the hair, considerable deposition of fat, even fatty degeneration of the internal organs (liver), and temperamental changes. The dogs ceased to bark, they breathed heavily, they became somewhat apathetic, the sensibility to electric stimulus and to pain became very much less, while their movements were indolent, and they seemed to have become less intelligent. In male animals, spermatogenesis ceased, in female animals there was involution of the ovarian follicles.

A glance at the material provided by experimental extirpation of the hypophysis shows that a good deal of light has been shed upon the functional and physiological significance of that organ. It is evident, in the first place, that the hypophysis is an organ of vital importance to the organism, the removal of which is followed by death. This is conclusively proved by the results of Paulesco's method of complete hypophysectomy, the contradictory results obtained by certain investigators being probably attributable to the faulty nature of their technique. "Total hypophysectomy" can only be described as such where, in addition to the removal of both lobes, the hypophysal peduncle is also severed. The peculiar significance of this peduncle is shown by the fact that, even where the hypophysis is allowed to remain in situ, death follows resection of the peduncle as promptly as after complete hypophysectomy. This remarkable phenomenon was first observed by Paulesco, and has since been confirmed by me; all that we can do is to record the facts, for no explanation of them is at present forthcoming.

The results of experimental extirpation also show the difference in the physiological value of the two lobes of the hypophysis. The removal of the posterior lobe is unattended, over a period of months, by pathological signs. Total extirpation of the anterior lobe, on the contrary, is immediately fatal. It appears, however, that this applies only to older animals, young dogs having a longer duration of life.

The results of total or partial destruction of the anterior lobe are also best seen in young animals. The suppression of function is manifested in a considerable increase in the fat body, which may amount to adiposis universalis, with pronounced hypoplasia of the sexual glands, together with arrested development and infantile habit. The influence which the hypophysis has upon the sexual glands is seen also in the case of older animals, partial destruction of the anterior lobe being followed by marked atrophy of the genitals.

\section{PITUITARY EXTRACT.}

Since the discovery by Oliver and Schäfer of the vaso-contractor action of pituitary extract, the symptoms which follow the 
exhibition of this substance have formed the subject of manifold investigation; further evidence, moreover, has been furnished of the internal secretory activity of the organ.

The chemistry of the hypophysis has, up to now, been hardly sufficiently investigated. We know that it contains a relatively large proportion of phosphorus and calcium; iodine (Ewald and Schwitzler), bromine (Paderi), and traces of arsenic (Gautier) have all been discovered. According to Gautrelet, the hypophysis, like many other organs, contains cholin.

Of the active pharmacodynamic substance of the hypophysis we know only that it is dialyzable; that it is not destroyed by reducing agents, such as boiling; and that it is insoluble in alcohol and ether. For experimental purposes, as well as in clinical medicine, the dried organ obtained from cattle, sheep, horses, cats, and dogs is employed, or extracts prepared from the dried or fresh organ in normal saline solution are used. As may be imagined, the dosage of such pituitary preparations is not very exact. An extract obtained with chloretone from the posterior lobe of the hypophysis has recently been put upon the market by Messrs. Parke, Davis and Co., under the name of hypophysin or pituitrin; the manufacturers state that I c.cm. of this extract is equal to o.I $\mathrm{grm}$. of the fresh, and .or $\mathrm{grm}$. of the dried, substance.

Pituitrin does not react either to ferric chloride or to the sublimate test given by Comessatti for adrenalin, or to the Fränkel-Allers test. It does, however, produce mydriasis of frogs' eyes. According to Allers, when boiled with caustic soda solution it gives off a distinct odour of alkylamin, while the liquid becomes brown; this author considers it probable that the hypophysis contains a substance which, similarly to adrenalin, possesses an alkaline amino-nitrogen constituent.

The general toxicity of pituitary extract is inconsiderable and not to be compared with that of adrenalin or suprarenal extract. Numerous accounts are given of extracts equal to $x_{2}^{\frac{1}{2}}$ to 3 fresh hypophyses (Mairet and Bosc, Conti and Curti, Garnier and Thaon, Salvioli and Carraro, Etienne and Parisot, Renon and Delille, Hallion and Carrion) being exhibited as a single dose, while Urechia reports a case in which the extract of ten ox-hypophyses was tolerated in a single dose. The direct effects of intravenous or subcutaneous injection of pituitary extract are transient apathy, somnolence, occasionally accelerated respiration and increased secretion of urine. After the intraperitoneal injection of large quantities of pituitrin (Io to 15 c.cm.), I observed transient excitement, followed by marked depression and muscular weakness, which afterwards developed into complete paralysis of the hinder extremities and terminated, at the end of twenty-four hours, in death. The intravenous injection of large quantities of pituitrin is sometimes followed by a sudden arrest of cardiac 
activity or by pulmonary œedema. After repeated exhibition of small quantities of the substance there was a marked loss in weight. The phenomena which Urechia observed after the reinjection of pituitary extract after an interval of ten days, were probably anaphylactic in character.

According to Delille, there is an essential difference between the toxic action of the anterior and the posterior lobes, the toxicity of pituitary extract being principally or even exclusively the property of the posterior lobe.

\section{THE PHYSIOLOGICAL ACTIVITY OF PITUITARY EXTRACT.}

It was first ascertained by Oliver and Schäfer that the injection of extract of the hypophysis into the veins produces a prompt and very marked increase in arterial pressure; this differs from that produced by suprarenal extract in its duration, which is longer by several minutes. This hypertension results, partly from contraction of the vessels, and partly from increased cardiac activity, and it is not disturbed by resection of the cord or of the medulla. The peripheral effect of this hypertension is illustrated by the fact that, if a fluid containing pituitary extract is circulated through the hinder extremities of frogs, there is a marked reduction in the venous outflow. The increased cardiac activity was shown in the myographic curve which Oliver and Schäfer obtained with the heart after its removal from the living body.

The effect of pituitary extract upon the circulatory apparatus is, in many respects, analogous to that of suprarenal extract, though it differs in certain important particulars. The vaso-constrictor action is much slighter, and the marked slowing of the pulse, together with inhibition of auricular contraction, produced by suprarenal extract where the vagi are intact, is absent in the case of pituitary extract. On the other hand, after resection of the vagi, pituitary extract produces, not an acceleration, but rather a retardation of the cardiac beats.

The findings of Oliver and Schäfer were confirmed in all important particulars by later investigators. Howell next discovered that the substance which produces vaso-constriction and slowing of the pulse is principally contained in the extract of the posterior lobe, the pars nervosa. He also discovered the remarkable fact that a second injection, given half to one hour after the first, has practically no effect upon the circulation. Howell's findings were confirmed by Schäfer and Vincent, who discovered, further, that the second injection is followed by a fall, instead of a rise, in blood-pressure. According to these authors, the fall in pressure is brought about by the action of a second substance with depressant properties, which is obtainable by extraction with 
absolute alcohol, treatment of the alcoholic residuum with ether, and solution in sodium chloride. If the substance obtained by this method is injected into the veins, a transient primary hypotension is observed, which is probably due to peripheral vasodilation and is not preceded by a rise in blood-pressure. This hypotension is similar to that obtained by the injection of cholin, but unlike the latter, it is unaffected by atropine.

Osborne and Vincent next showed that extracts of nervous tissue (brain, spinal cord, peripheral nerves) give rise to a considerable transient fall in blood-pressure, which is also unaffected by resection of the vagus and by atropine, and which is brought about by peripheral vasodilation. Contrary to the view expressed by Halliburton, it is certain that this result is in no way due to the action of cholin.

According to $\mathrm{v}$. Cyon, pituitary extract contains two substances which possess entirely different properties. The one, hypophysin, which is obtained by boiling pituitary extract, has an influence upon the heart; its intravenous injection is followed by groups of slow, strong beats, and this effect persists after resection of the vagi. Extracts obtained at a temperature of 38 to $40^{\circ} \mathrm{C}$. usually possess a vaso-constrictor effect which v. Cyon believes to be brought about by inhibition of the nervous depressor.

The effects of pituitary extract in raising pressure and slowing the pulse-the latter only where the vagi are intact, however-are confirmed by Livon, who inciudes the hypophysis in the group of the hypertensive glands; while Silvestri, on the contrary, obtained only a fall in blood-pressure with extract of the posterior lobe. After the injection of a quantity of infundibular extract equal to about one-fifth of the posterior lobe, Garnier and Thaon observed a rapid rise in pressure with slowing of the pulse, followed after ten to twelve seconds by a rapid transient fall in pressure, which was again followed by a marked rise. The slowing of the pulse was not observed after the resection of the vagi. Similar phenomena are described by Salvioli and Carraro, Pal, Lockhart-Nummery, and Legge.

By means of plethysmographs of different organs, Hallion and Carrion showed that the rise in pressure brought about by pituitary extract is associated with a vasoconstriction in different organs, in the nasal mucosa and thyroid gland more particularly. In the kidney there was, after an initial vaso-contraction, a continued vaso-dilation. These authors point out the antagonistic effects of pituitary to those of ovarian extract, the latter, as far as their experiments showed, producing a fall in blood-pressure together with a characteristic vaso-dilation in the thyroid gland.

Quite recently (December 17, Ig09), Falta and Ivcovic stated that they injected $2 \frac{1}{2} \mathrm{c} . \mathrm{cm}$. of an extract obtained from the glandu- 
lar portion of the hypophysis into dogs and rabbits, and produced a profound fall in blood-pressure which, in atropined animals, lasts for a comparatively long time. The lowered pressure, which, at the end of forty seconds, spontaneously returns to the normal figure, may be relieved by means of adrenalin. In two instances, after injection of the extract, the blood-pressure fell to zero, and respiratory and cardiac activity ceased. In one case the animal was saved by the injection of $2 \mathrm{c.cm}$. pituitrinum infundibulare; the heart-beats recommenced, the blood-pressure rose rapidly to normal, and the animal completely recovered.

Finally, L. Etienne and Parisot found that the repeated injection of pituitary extract into rabbits produced permanent hypertension and cardiac hypertrophy, which were apparent fifteen days after the last injection; there were, however, no sclerotic changes in the vessels.

The results which have been described comprise the whole of the material at present available concerning the influence of pituitary extract upon the circulation. It may then be taken as proven that pituitary extract, or rather the extract of the posterior hypophyseal lobe, produces a high degree of blood-pressure, which differs from that provoked by adrenalin in its slighter intensity and longer duration. The diminished, or negative, action of a second injection may be regarded as specific.

The hypotensive action of pituitary extract described by certain authors demands further investigation. It is very necessary that we should know whether the fall in pressure is a by-effect of pituitary extract, or whether it is due to a specific activity on the part of the glandular portion of the organ.

There is no doubt that the rise in arterial tension is mainly the result of peripheral vaso-contraction. But further investigation is necessary both to locate the seat of this vaso-contraction and to decide whether the vaso-contraction which takes place in the thyroid gland and the nasal mucous membranes, and the vaso-dilation which takes place in the kidney, are specific effects of pituitary extract.

Pituitary extract also influences the action of the heart, by slowing and strengthening its beats. This effect is in part attributable to stimulation of the cardiac inhibitory nervous centres. The experiments of Hedboom and Allen, Cleghorn, and, later, Herring, with the living heart from frogs and mammals, have proved, however, that the slowing of the pulse is unaffected by resection of the vagi, and it seems probable from this that pituitary extract also exercises a direct action upon the muscular substance of the heart. This conclusion is further justified by the fact that the constriction of the vessels is produced not, as in the case of adrenalin, by stimulation of the peripheral nerveterminals, but by a direct action upon the involuntary muscles; moreover, the effect which pituitary extract has upon the muscles is also demonstrable in the case of other organs. 
That pituitary extract exercises a direct contractor influence upon the vessel walls is proved by Salvioli and Carraro's experiments with artificial circulation. Dale's experiments, to which reference has been made in a previous chapter, show that pituitary extract resembles barium chloride, which is known to stimulate the involuntary muscles, in that it produces a rise in bloodpressure at that stage of ergotoxin intoxication at which a fall in blood-pressure is produced by adrenalin. Pal, de Bonis, and Susanna tested the action of pituitary extract by O. B. Meyer's method upon portions of arteries obtained from the living ox. According to Pal, pituitary extract produces a contraction of the carotid, mesenteric, and femoral arteries in the same manner as adrenalin, while its action upon the cardiac and renal vessels is the opposite to that of adrenalin. The coronary arteries, which enlarge in response to adrenalin, contract under the influence of pituitary extract. The peripheral portion of the renal artery which is situated in the pelvis dilates in response to pituitary extract, while the proximal portion contracts.

The direct muscular action of pituitary extract was proved by Cramer and Borchardt, as well as by $\mathrm{Pal}$, by the enlargement of the pupil of enucleated frogs' eyes.

Special interest is attaching to the discoveries of $\mathrm{v}$. FranklHochwart and Fröhlich concerning the effect of pituitrin upon the urinary bladder and the uterine muscles. In cats and dogs, pituitrin stimulates the muscles of the urinary bladder to a moderate degree, but it also increases the sensibility to electric stimulus of the autonomous motor nerve to the bladder, namely, the pelvic nerve. The sensibility of the inhibitory sympathetic, the hypogastric nerve, is unaltered. Maximal contraction of the uterus muscles of the rabbit is sometimes produced by pituitrin, and at the same time the uterus becomes considerably more sensitive to the influence of the uterine nerves which are supplied by the hypogastrics. Both these results occur independently of the blood-pressure and, like the effects upon the heart and vessels, they are observed after the first injection only. On account of its effect in increasing the sensibility of the muscles of the urinary bladder and the uterus, these authors suggest the employment of pituitrin as a therapeutic agent.

The effect which the extract of the posterior lobe has upon the muscles of the uterus and intestine is also described by Blair Bell.

According to v. Frankl-Hochwart and Fröhlich, hypophysin does not affect the sensibility of either the nerves of the salivary glands or the chorda tympani, nor does it influence erection, in so far as the latter is produced by peripheral stimulation of the autonomous erection nerve. In opposition to v. Cyon, these authors state that the sensibility of the autonomous vagus supplying the heart is unaffected by the influence of hypophysin. 
An ordered relationship, such as that which exists between the action of adrenalin and certain nerve terminals, has not been proved in the case of hypophysin.

Very characteristic of the action of pituitary extract is the increased diuresis which, according to Magnus and Schäfer, follows intravenous injection, and, if the injections are repeated, continues for some time. This is in accordance with the increase in the volume of the kidney, as shown by oncometric methods. Schäfer and Herring next proved that the substance which produces diuresis is contained in the posterior lobe of the hypophysis; that it is dialyzable, is insoluble in ether and alcohol, and is not destroyed by boiling; the extract of the anterior lobe they found to be non-diuretic. Moreover, they found that diuresis is also produced by pituitary extract when injected intravenously or into the peritoneum.

Borchardt found that the subcutaneous injection of pituitary extract into rabbits produced hyperglycæmia and glycosuria.

With regard to the manner in which metabolism is influenced by pituitary extract, Schiff found that, in one clinical case of acromegaly and one of paralysis agitans, there was an increase in the excretion of phosphorus, which he ascribed to the decomposition of organs containing much phosphorus, such as the nervous system and the bones. In one case of acromegaly Moraczewski found retention of $\mathrm{Ca}$ and $\mathrm{P}_{2} \mathrm{O}_{5}$; the administration of pituitary tabloids reduced the excretion of $\mathrm{Ca}, \mathrm{Mg}$, and $\mathrm{P}_{2} \mathrm{O}_{5}$.

The exhibition per os of pituitary tabloids in man is followed in the normal subject by increased metabolism of gases; in persons with acromegaly, metabolism is only very slightly increased (Magnus-Levy, Salomon).

Experiments intended to show the effect of pituitary extract upon metabolism in animals have produced conflicting results. Osward found that pituitary substance, when given by the mouth to dogs, produced no effect upon the excretion of phosphorus and nitrogen. Thompson and Johnson, on the other hand, found an increased excretion of nitrogen, urea, and phosphorus in the urine, together with a reduction in the weight of the body. Malcolm exhibited the glandular and nervous portions of the hypophysis separately; he found that, when given by the mouth, the anterior lobe produced retention of phosphorus, the posterior lobe excretion of phosphorus. The excretion of $\mathrm{Ca}, \mathrm{Mg}$, and $\mathrm{N}$ was increased by the exhibition of the fresh gland, but rather diminished than otherwise by the exhibition of the dried gland.

According to Falta, Bolaffio and Tedesko, the effect of pituitrin upon the metabolism of salt is similar to that of the thyroid substances. They found that, in fasting dogs, there was an increase of the quotient $\mathrm{N}: \mathrm{P}_{2} \mathrm{O}_{5}$ in the urine, together with an increased excretion of phosphorus in the fæces. The excess of the calcium excreted carries a proportion of phosphorus with 
it into the intestine. The proportion of the alkalies to nitrogen in the urine also increases after the exhibition of pituitrin in the same way as after thyroid. Thus both thyroid and pituitary increase the general metabolism of salt, the larger part of the excess being carried off by the intestines.

After the injection of pituitrinum infundibulare into dogs with normal nitrogen metabolism, Falta and his co-workers found that, whether the animals were fed on flesh or on milk and white bread, there was invariably an increase in the metabolism of albumin.

As far as the metabolism of purins was concerned, there was a rise in the excretion of uric acid with simultaneous reduction in the allantoin nitrogen. This is probably due to the increased diuresis, owing to which a larger proportion of uric acid is withdrawn from oxidation to form allantoin.

A definite influence of pituitary substance upon the metabolism of the carbohydrates has not been observed. The sugarcontents of the blood are not augmented; but the exhibition of adrenalin in dogs which have previously been treated with pituitrin is followed by a remarkable increase in the excretion of sugar.

It is interesting to note that four rabbits, which Delille treated with injections of pituitary extract over a period of fourteen months, became very fat.

What is at present known concerning the effect of injections of pituitary extract upon the growth of the bones, is insufficient to justify a definitive pronouncement. Caselli was unable to influence the processes of growth in young dogs and rabbits by means of glycerine extract of pituitary. Cerletti found that the subcutaneous or intraperitoneal injection of such extract produced backwardness in weight and in the development of the bones; the long bones, especially, were short in comparison with those of the control animal. Taking into consideration the fact that large quantities of the, by no means indifferent, extract were employed in these experiments, the results cannot be regarded as conclusive.

\section{EXPERIMENTAL STIMULATION OF THE HYPOPHYSIS.}

The changes produced in the general circulation by the mechanical and electric stimulation of the hypophysis were investigated by E. v. Cyon. Upon the results of these investigations, together with the known properties of pituitary extract, he based his theory concerning the function of the hypophysis. v. Cyon regarded the hypophysis and the thyroid as protective organs to the brain, whose function it is to prevent a dangerous flush of blood to that part. These organs, acting in concert, regulate the 
intercranial circulation and the conditions governing bloodpressure within the cranium. The regulation of pressure is to a certain extent automatic, for every rise in pressure within the cranium mechanically stimulates the hypophysis; this stimulation is conveyed reflexly to the vagus centre and a slowing and strengthening of the cardiac contractions results; while, at the same time, the vaso-dilator fibres contained in the vagus nerve produce an enlargement of the vessels in the thyroid, and by this means the superfluous blood is carried away from the brain. The intracranial rise in pressure also causes the hypophysis to part with a larger amount of its active substance, which produces a chemical stimulation of the vagus and simultaneous rise in blood-pressure. Thus, the auto-regulation by the hypophysis of intracranial blood-pressure is effected by stimulation of the vagi upon the one hand, and by increased arterial tension resulting from contraction of the peripheral vessels upon the other.

With regard to the latter point, v. Cyon believed that the contraction of the peripheral vessels resulted in a reduction in the amount of blood circulating within the cranium. But it must be remembered against this that, as all evidence up to the present goes to show, processes which give rise to a contraction of the vessels of the systemic circulation, thus producing an increase in arterial tension, also augment the blood contents of the cranial cavity. The higher the arterial blood-pressure, the larger the quantity of blood circulating through the brain, which, under these conditions, may amount to several times the normal. Thus it is evident that a temporary anæmia of the brain cannot be effected by this means, and that the effect produced upon the cranial circulation by the active vaso-tensor constituent of the hypophysis would be, not the result anticipated by v. Cyon, but one diametrically opposed to it (Biedl and Reiner).

As far as the stimulation of the vagus centre is concerned, the conditions are as follows: The dilation of the thyroid vessels as the result of stimulation of the vagus, which $\mathrm{v}$. Cyon described and which forms the groundwork of his theory, is not confirmed by Lusenna, who found that the vaso-dilators of the thyroid are contained, not in the vagus, but in the sympathetic. It is undeniable, however, that increased pressure within the cranial cavity produces stimulation of the vagus centres, and that by this means a slowing and strengthening of the cardiac beats is effected. But the question arises as to whether this so-called vagus phenomenon occurs as the result of high blood-pressure, brought about by the agency of the hypophysis. In support of this assumption v. Cyon states: (I) That mechanical or electric stimulation of the hypophysis produces changes in the bloodpressure curve, such as would follow a sudden compression of the aorta, namely, hypertension, slowing and strengthening of the heart-beats, and the so-called vagus pulse; (2) that the vagus 
phenomenon does not accompany compression of the aorta in cases where the hypophysis has first been destroyed. After the destruction of the hypophysis, it is not possible to produce the vagus pulse by either aortic compression or reflex stimulation of the aorta (by way of the nasal mucosa or trigeminus), the result in every instance being to accelerate the pulse. According to $v$. Cyon, there is no doubt that stimulation of the vagi, in consequence of hypertension within the cranial cavity, is effected by the agency of the hypophysis; in other words, that the increased pressure acts directly upon that organ and the stimulation of the vagi is reflexly produced by the stimulation of the hypophysis.

These statements of v. Cyon's do not, however, stand the test of minute investigation and they are entirely unconfirmed by later authors (Biedl and Reiner, Caselli, Pirrone, Lo Monaco, and van Rynberk, Livon, Gaglio). It is true that stimulation of the hypophysis, and electric stimulation more particularly, occasionally produces increased pressure and changes in the frequency of the pulse, together with respiratory changes, but similar phenomena may be produced in the same way by stimulation of the greater portion of the cerebral cortex and of the cerebral ganglia. Livon even states that the hypophysis itself is incapable of stimulation, and that the changes are due to the leakage of the faradic stream into the surrounding portions of the brain.

The destruction of the hypophysis does not bring about changes in either the pressure of the blood or the frequency of the pulse. Moreover, the vagus phenomenon in company with high blood-pressure is observed after, as before, this operation (Biedl and Reiner).

The theory of a mechanical hypophysal function must be regarded as demolished. The only author who confirmed the phenomena observed by v. Cyon after stimulation of the hypophysis is F. Masay, and he believes that stimulation of the hypophysis promotes the flow of secretion from the organ into the blood, and that by its action upon the muscles of the vessels a rise in blood-pressure is produced.

In the present state of our knowledge, the only assumption possible is that, if the hypophysis does influence the circulation, this result is effected by the agency of a chemical hormone, and is to be regarded as the outcome of the internal secretory function of the organ.

\section{DISEASES OF THE HYPOPHYSIS IN MAN.}

Of the clinical conditions which are associated with pathological changes in the hypophysis and the pathogenesis of which is undoubtedly in some way dependent upon that organ, the most important is the condition first described by P. Marie under the name of acromegaly. This disease usually attacks individuals 
who have reached the adult stage; the predisposing cause is unknown. The first symptoms are manifested through the nervous system; they are lassitude and muscular pain, progressive apathy and somnolence, and eventually headache. Derangement of sexual activity occurs very early in the course of the disease, shown in women by the cessation of menstruation and in men by impotence. The first pathognomic symptoms consist in changes in the external appearance of the patient; the face becomes distorted, due partly to unequal growth of the soft portions and partly to enlargement of the facial bones. In advanced cases, the entire face is enlarged, the eyebrows are prominent, the eyelids thickened, the nose enormously increased, the lips swollen and the zygomatic arches and lower jaw extremely salient. The peculiar appearance of persons with acromegaly is enhanced by the over-development of the soft parts of the mouth, the tongue in particular being so large as almost to prevent the closing of the mouth.

Simultaneously with these appearances or somewhat later, gradual progressive deformity of the extremities takes place. The hands and feet become clumsy-larger and broader than the normal-the long bones, however, remain almost unaltered. The enlargement of the ends of the extremities is due in part to a thickening of the skin, especially of the subcutaneous tissue, and in part to a characteristic growth of the bones of the terminal phalanges of the fingers and toes.

The clinical appearances of acromegaly are generally, though not invariably, accompanied by symptoms which point to the presence of a cerebral tumour. These are : headache, giddiness, vomiting, failing intelligence, and somnolence. Other symptoms permit of the localization of the tumour and point to the hypophysis as the site of the lesion. Of these, the most important are disturbances of vision, which consist, in the first instance, of bitemporal hemianopsia, followed by progressive amblyopia and amaurosis. At first the fundus is normal, later on there is primary atrophy of the optic nerve and, occasionally, choked disc. Exophthalmos is by no means rare, but it is noticeable that the projection of the eyeballs, like the derangement of vision, is periodic in character and is obviously dependent upon changes in the volume of the tumour. Paralytic symptoms are sometimes observed in other cerebral nerve areas. A valuable aid to the diagnosis of hypophysal tumours has recently been furnished by radiological methods. Skiagrams show a deepening of the sella turcica, the floor of which appears to be thinner; the dorsum sellæ shows usure and appears elongated. Where the tumour is outside the sella turcica and is developed from the cranio-pharyngeal canal, the sella is flattened and the entrance to it is enlarged; the further growth of the tumour may completely destroy the floor of the sella turcica (Schüller). 
Of the other clinical symptoms, glycosuria is the most important and is of comparatively frequent occurrence. The frequency of acromegalic diabetes, as reported up to now, is to to 35.5 per cent. and over. The intensity and the course of the glycosuria are subject to considerable variation.

The other metabolic changes observed in acromegaly are not typical. In some instances the total metabolism has been found to be increased (Magnus-Levy), in others it has been found to be unaltered (Salomon). Several authors found retention of nitrogen, and they associated this with the abnormal growth of the tissues. With regard to the metabolism of salt, several authors (Moraczewski, Edsal and Müller, Parhon) found retention of $\mathrm{P}, \mathrm{Ca}$, and $\mathrm{Cl}$. Franchini found retention of $\mathrm{N}, \mathrm{CaO}$ and $\mathrm{MgO}$, accompanied by excretion of $\mathrm{P}$ and $\mathrm{Cl}$. It is very difficult to estimate the metabolic changes in acromegaly, owing to the fact that the condition is frequently associated with myxœdematous symptoms (Pineles). On the other hand, MagnusLevy points out that the metabolic changes of acromegaly possess certain analogies with those of Graves's disease; such, for instance, are the excessive perspiration, so frequently observed; the increased combustion, proved in a certain number of cases; and slight or severe glycosuria.

The course of the disease is usually chronic and extends over a period of years. Cases are occasionally met with, however, where, owing to the rapid growth of the hypophysal tumour, the fatal termination takes place sooner and is accompanied by the general symptoms of intercranial pressure. In many instances the patients die as the result of intercurrent affections. In the typical course of the disease, progressive emaciation and cachexia make their appearance at the end of a term of years and lead, ultimately, to death.

Of the pathologico-anatomical findings in acromegaly, the most important are the changes in the hypophysis itself, first described by Marie and Marinesco. Before considering the pathogenetic significance of these changes, it is expedient first to describe the post-mortem findings in the other organs and tissues. The increased volume of the soft parts is brought about by thickening of the epidermis, hypertrophy and enlargement of the papilla of the skin, and pronounced infiltration of the subcutis, with proliferation of the subcutical connective tissue. The connective tissue proliferation extends also to the deep-lying soft parts and to the interstices of the muscle fibres; the latter show enlargement and proliferation of the nuclei and, later on, degenerative changes. The hypertrophy of the mucosa, especially noticeable in the case of the tongue, is caused by hyperplasia of the mucous membranes, proliferation of the papillæ, and multiplication of the connective tissue. Occasionally the visceral organs are also enlarged. A hyperplastic thymus is by no means 
a rare finding, and the thyroid may have undergone strumous degeneration or be markedly atrophied.

The changes in the bones are not as pronounced as the clinical symptoms would seem to show. The ends of the bones and the terminal phalanges are undoubtedly broadened and enlarged, but this is chiefly due to exostoses and irregular absorption of the cortical layers, and to bony outgrowths at the jointschanges which must be regarded as secondary results of the hyperplasia of the soft parts. The clavicle is usually very much enlarged. In addition to the changes in the sphenoid bone, the bony skull shows premature obliteration of the sutures; exostoses, especially at the muscle attachments; enlargement of the antrum and of the frontal sinus; increase in the volume of the superciliary arch, the zygomatic arch, and the inferior maxilla. Investigation of the histology of the bones shows that these results are due, not to specific pathological processes, but to the deposition and resorption of bony substance, in a manner identical with that of the normal growth of the bones. In addition to these changes, hypoplasia or degeneration of the sexual glands is a frequent accompaniment of the condition.

The most important point in regard to the pathogenesis of acromegaly is undoubtedly the behaviour of the hypophysis. Marie and Marinesco found that changes in the hypophysis were a constant feature of the condition; these consisted in increased volume, with hyperplastic proliferation and secondary sclerosis of the connective tissue, together with various neoplasms. The frequent, indeed almost constant, association of changes in the hypophysis with acromegaly is commented upon by the majority of the later authors. Enlargement of the hypophysis was found in forty-four out of forty-eight cases by Hutchinson, and in sixty-five out of seventy cases by Modena. In a large number of cases the hypophysal tumour is the expression of hypertrophic and hyperplastic processes within the organ. According to Benda, the process consists in an increase of the glandular epithelium of the anterior lobe, especially of the chromophile and eosinophile cells. The hyperplasia may even extend to the formation of adenomatous strumas. In the majority of cases, the tumours of acromegaly are true hypophysal tumours-adenoma, adenocarcinoma, sarcoma and, more rarely, teratoma and teratoid growths. Those cases of acromegaly in which no tumour was found (according to Cagnetto they are six in number) are probably explained by the fact that the new formation was situated externally of the sella turcica in the hypophysis pharyngea, and thus escaped observation. Pathological changes in the hypophysis in acromegaly may be regarded as constant post-mortem findings.

It is no longer possible to consider the hypophysal tumour in the light of an unimportant by-symptom, or as a co-ordinated 
splanchnomegalic symptom, of no etiological importance; on the contrary, it is essential that we should recognize its fundamental pathogenetic significance. Now that we know that pathological conditions of the hypophysis may produce widely different clinical conditions, the occurrence of hypophysal tumour without symptoms of acromegaly, can no longer be adduced as evidence against the hypophysal origin of acromegaly. So long as our knowledge of the nature of the changes in the hypophysis which lead to acromegaly is incomplete, these negative cases must be regarded with special interest; in so far as the pathogenesis of acromegaly is concerned, however, they are irrelevant.

The relationship between hypophysal tumour and acromegaly has been variously interpreted. The original view, of Pierre Marie, which was adopted by a large number of authors, was that acromegaly was the expression of insufficiency or suppression of the hypophysal function, in the same way that myxœdema expresses insufficiency on the part of the thyroid gland. This theory is supported by the frequent occurrence of destructive changes in the hypophysis. A further very strong piece of evidence in favour of this view is afforded by the acute form of acromegaly described by Sternberg, in which the condition, in its most clearly defined clinical form, develops practically under the eyes of the observer. In all these cases the post-mortem revealed an acute progressive tumour, frequently sarcoma, of the hypophysis. It was already known to Marie that the hypophysal changes frequently consisted only in hyperplastic processes, though this seems hardly consistent with the theory of a reduced activity on the part of the organ. It has been pointed out upon many sides, that simple glandular hyperplasia and adenoma are of far more frequent occurrence than destructive tumours associated with connective tissue proliferation. With regard to the malignant form, Hanau and Benda found that tumours, which at first sight appeared to be sarcomatous, contained cells derived from the hypophysal epithelium and they described these tumours as hyperplastic, malignant adenoma. Hanau suggests that the same adenomatous form may have been present in other cases which have been described as sarcoma.

Certain animal experiments which seem to show that the extirpation of the hypophysis does not produce pathological changes in animals, were at one time regarded as important evidence against the assumption of a suppression of hypophysal activity in acromegaly. But to-day this argument has little weight, for we know that the suppression of the hypophysis in animals, as in man, is not by any means an indifferent occurrence; on the contrary, it is followed by a number of pathological symptoms. 'The backwardness in development so clearly seen in young animals after hypophysectomy may be taken as proof of an influence exercised by the hypophysis upon the growth of 
the bones. Judging by this, the increased development of the "acral" portions is to be interpreted, not as a sign of reduced, but rather as the manifestation of an intensified, functional activity. But it is a remarkable fact, that the extirpation of the hypophysis of animals produces results in one direction, which are similar to those brought about by acromegaly in man; both lead to arrest of development, and particularly to defective function on the part of the sexual glands.

A second hypothesis, first propounded by Tamburini, regards acromegaly as the result of two pathological processes in the hypophysis. There is at first hypertrophy, leading to hyperfunction of the organ, and this explains the signs of increased growth in different parts of the body. The second phase consists in degeneration and reduction of functional activity, which produces the cachexic symptoms observed in the later stages of the condition. The assumption of a hyperpituitarism, of an increased internal secretory activity on the part of the hypophysis, was supported by a discovery of Benda's, since confirmed by others, that, in certain cases of acromegaly, the diffuse hyperplasia and hyperplastic adenoma, commonly known as struma, show not only regular increase in the number of the cells, but also a remarkable multiplication of those eosinophile cells, which Benda regards as the active functional elements of the hypophysis. A case of acromegaly described by Lewis is particularly interesting in this connection, for here, though the macroscopic appearance of the hypophysis was normal, the microscope showed that the number of the eosinophile cells was increased.

Benda, who regarded the hyperplastic proliferation of the chromophile cells as the cause of increased hypophysal secretion, was at first inclined to support Tamburini's theory. In a later publication, however, he modified his view. He pointed out that it is not possible to regard the clinical course of acromegaly as assuming two distinct phases, for in the classical form of the disease, malignant acromegaly, the development of the tumour in the hypophysis is intimately associated with the derangement of growth and the general deterioration of the organism. Benda regards the developmental disturbances as constant, but purely symptomatic, accompaniments, and defines the true primary disease as a tumour-like, connective tissue new formation, having an undeniable resemblance to myxœedema and elephantiasis; he ascribes the pathological symptoms to the excessive supply of a secretion of specific toxic action; the function of which, under normal conditions, is unknown, but the over-production of which produces serious lesions of the tissues of the body. Benda believes that the granules resembling zymogen in the chromophile cells represent the secretory product of the hypophysis.

The absence of any foundation for this last assumption, together with a critical appreciation of the known facts, led Cagnetto 
to deny the theory of hypersecretion in the causation of acromegaly. He pointed out that cases occur in which the hyperplasia and adenomatous struma of the hypophysis are associated with multiplication of the chromophile cells, but in which the symptoms of acromegaly are not present. Moreover, cases of acromegaly with adenoma of the hypophysis are observed, in which the adenoma do not contain chromophile cells. It must be borne in mind, however, that the chromophile cells are, almost certainly, not the only secreting element, and that, as Erdheim and Stumme showed, in the hypophysal hypertiophy of pregnancy, it is the chromophobe cells which play the leading part. That acromegalous thickening of the nose, lips and hands frequently takes place during pregnancy is another piece of evidence in favour of the hyperpituitary origin of acromegaly, although it contradicts Benda's assumption of the primal importance of the chromophile cells.

The whole of the anatomical material which we at present possess points to the hypophysal tumour, and consequent increased hypophysal internal secretion, as the causative factors in the production of the symptom-complex of acromegaly. The weightiest argument in support of this theory is the fact that, in all cases where hypophysal tumour is associated with acromegaly, the tumour is composed of true hypophysal tissue.

It remained for operative surgery to prove, in so far as experimental measures permit, the dependence of acromegaly upon hypophysal affection, and by this means to provide decisive evidence in favour of the theory of hypersecretion.

Sir Victor Horsley was the first to recommend the extirpation of the hypophysal tumour in acromegaly. The operation was first performed by Caton and Paul in 1893, but without success. In 1906, Horsley described the results of thirteen operations, without, however, supplying information as to the indications and symptomatic features of these cases. He obtained access to the hypophysis by opening up the dura mater from the central cranial fossa. Schloffer next considered the question as to whether or not the hypophysal tumour is operable, and by what methods; he concluded that the extracranial nasal method was the most practicable. In one instance, he operated for hypophysal tumour with what at first appeared to be favourable results, but after two and a half months the patient died. v. Eiselsberg next performed hypophysis operations upon three patients, one of whom presented the clinical signs of acromegaly and died as the result of acute sepsis. The other two cases will be described later. Borchardt also describes the result of extirpation of a hypophysal tumour in an anacromegalous case, the operation, in this instance, leading to an improvement of the headache.

Hochenegg ( 1908 ) was the first to succeed in removing, by way of the nose, a hypophysal tumour from a woman of 30 
with typical acromegaly; the promptness and the success of his results were literally startling. Five days after operation, the acute headache and periodic disturbances of vision had disappeared, and the teeth in the upper jaw had closed up; from the eighth day, the hands and feet became noticeably smaller; at the end of three months, with the exception of insignificant traces of thickening of the soft parts, the involution of the acra was complete. Menstruation reappeared and became regular. At the end of six months, the acromegalous symptoms had entirely disappeared, but the patient had an enlargement of the thyroid gland. Histological examination of the extirpated tumour showed it to be a malignant adenoma and quite free from typical chromophile cells.

Another case, that of partial recovery from acromegaly, is described by Cushing (I909). He performed partial hypophysectomy of the hypertrophied anterior lobe. The symptoms of intercranial pressure did not entirely disappear, but there was an appreciable reduction of the acral parts.

A second case of acromegaly was successfully treated in Hochenegg's clinic and is described by A. Exner. The patient was a woman, aged 37 ; seven years previously the menses ceased and the power of vision became progressively reduced; increased growth of the acral parts had been noticed for four years, and abnormal growth of hair for three. At the time of operation, the symptoms of typical acromegaly were well developed, and a tumour the size of a walnut was removed from the hypophysis. After operation, the headache, which had previously been very violent, ceased; the skin of the hands and feet became thinner; the circumference of the fingers became smaller; the bristly moustache hairs fell out; the teeth closed up; and the tongue became reduced in size. In this case also, the thyroid developed a middle lobe which could be distinctly felt upon palpation. In this case, as in the first, the tumour was a malignant adenoma (Wurmbrand).

The therapeutic results of hypophysis extirpation in acromegaly supply the last conclusive link in the chain of evidence in favour of the theory, that the causative factor in acromegaly is to be sought in hypersecretion of the hypophysis. Such being the state of things, it is not surprising that, in those cases where hyposecretion was assumed and the condition was treated by organo-therapeutic methods with pituitary substance, the specific acromegalic symptoms remained unchanged. In those rare instances where benefit has been derived from the use of pituitary substance, the subjective symptoms, especially the headache, were affected, while the developmental changes were entirely unaltered.

If we regard acromegaly as a manifestation of hyperpituitarism, the question arises as to why spontaneous recovery does not take place in cases where there is malignant degeneration of the hypophysal tumour and where, from the nature of the histological 
conditions, a reduction of the secretory elements is to be assumed. This, however, is readily understood, if we remember the enormous difficulty of estimating the functional activity of the different structural elements; moreover, judging by experience in other directions, it is by no means improbable that the specific function of the tissue of a malignant tumour is similar to, and may be even stronger than, the specific function of the normal tissue. It is a by no means rare thing in acromegaly for the anomalies of growth, after progressing to a certain point, to arrive at a standstill; there may even be regression, followed later by cachexia with regressive nutritional disturbance. It was upon these facts that Tamburini founded his hypothesis. Finally, it must not be forgotten that hyperfunction of the hypophysis is associated with changes in the activity of other internal secretory organs, the influence of which upon the general economy may continue, though in a perverted sense, after the primary factor, the hypophysal hyperplasia, has either ceased to exist or has undergone regressive changes.

This latter possibility leads us to the question: What is the primum movens in acromegaly? From the nature of the evidence, it appears unquestionable that the hypophysis is the site of origin of acromegaly, and that the nature of the illness is dictated by hypersecretion on the part of that organ. But the question as to whether we are to regard the pathological condition of the hypophysis as primary, or merely as the secondary result of organic affection in other parts of the body, remains unanswered. The latter assumption is permissible, for numerous experiments have shown that there is a physiological interrelationship between the hypophysis and other internal secretory organs, namely, the thyroid, thymus, suprarenals, and especially the sexual glands. Moreover, pathological conditions of these organs are associated with profound changes in the hypophysis.

The interrelationship between the thyroid gland and the hypophysis has already been described. Rogowitsch first showed that thyroidectomy is followed by hypertrophy of the hypophysis, and his finding is confirmed by all later observers. In the department of human pathology, it has also been shown that, in myxoedema and other related subthyroidic conditions, there is frequently enlargement and pathological change of the hypophysis. Of the manner in which hypophysectomy in animals affects the thyroid gland, we know only that Caselli found hypertrophy of the entire thyroid apparatus. In the two cases which recovered after hypophysectomy at Hochenegg's clinic, there was distinct increase in the volume of the thyroid. Pineles emphasized the clinical relationship between acromegaly and myxœdema, which he believes to be shown, not only in the comparatively frequent co-existence of the two conditions, but also in the similarity of many of the symptoms and in the occurrence in acromegaly of 
pathological changes in the thyroid gland. It is an undoubted fact that, in many of its aspects, acromegaly resembles Graves's disease. Enlargement of the thyroid apparatus is frequently observed in acromegaly, and Lancereaux believed that this condition results from a stimulation of the processes of growth, brought about by the enlarged thyroid gland, and modified and controlled by the hypophysis. As things stand at present, it is difficult to estimate the significance of the part played by the thyroid apparatus in acromegaly. That hypothyrosis produces an enlargement of the hypophysis is undoubtedly true, but it can hardly be regarded as the primary cause of such enlargement. There is an equal probability that the hyperpituitarism brings about a reduction of function, and consequent atrophy, on the part of the thyroid. The assumption of an interrelationship between the activities of the two organs is justified by clinical experience, and it remains for experimental pathology to crystallize this assumption into a certainty.

Persistence of the thymus in acromegaly is by no means rare. The inconstancy of the finding, however, deprives this phenomenon of the causative significance attributed to it by Klebs.

The relationship between the suprarenals and the hypophysis, especially in acromegaly, has not as yet been sufficiently investigated. After extirpation of the suprarenals, Alquier observed signs of moderate hyperplasia of the hypophysis in a dog; Boinet found that hypertrophy was exceptional in rats; while Marenghi found marked hypertrophy in guinea-pigs, rabbits and cats. Hyperplastic changes in the hypophysis have been described in suprarenal disease in man (Thaon, Delille); while, on the other hand, adenoma and cystic degeneration of the suprarenals have been observed in acromegaly. Delille points to the frequent occurrence of suprarenal hypertrophy; together with Rénon, he discovered that hyperplasia of the suprarenals may be produced by the repeated intraperitoneal injection of pituitary extract.

Best known is the interrelationship between the hypophysis and the sexual glands; the part played by the latter in the causation of acromegaly is as yet, however, undecided. The changes in the sexual organs observed in acromegaly, and especially the cessation of the menses in women, are among the earliest symptoms of the disease. Later on, there is cessation of sexual desire and sterility, accompanied by atrophy of the genital glands. The changes in the sexual glands in acromegaly have been minutely described by Tandler and Gross. Not infrequently, the secondary sexual characteristics disappear and there may be even a partial return to the heterosexual type. Many authors (Freund, Verstraeten, Campbell, Breton and Michaud) believe that the changes in the sexual glands are a causative factor in the production of acromegaly. There is a certain amount of foundation for this assumption, for it has long been known that the sexual glands 
exercise a considerable influence upon the general processes of growth.

In the meantime, a large number of physiological, pathological and experimental facts have come to light which show the influence of the sexual glands upon the hypophysis. Comte and a number of later authors (Launois and Mulon, Guerrini, Morandi Cagnetto, Thaon) found that the hypophysis regularly enlarges during pregnancy, and that during lactation it returns to the normal. These changes were from the first regarded as signs of hyperfunction, and the discoveries of Erdheim and Stumme afterwards supplied incontrovertible evidence of hypophysal hypertrophy during pregnancy and of the changes which take place in the structure of the organ. Fichera's valuable work next showed that the changes in the hypophysis during pregnancy are due to a reduction of activity on the part of the ovary. After the castration of male and female animals, he found hyperplasia and hypertrophy of the hypophysis which exactly resembled the hypertrophy of pregnancy. The weight of the hypophysis in capons and oxen, as well as in castrated female animals, was twice as large as in normal animals, and this hypertrophy was reduced by the exhibition of extract of the sexual glands. By examination of the skulls of skeletons, and by means of skiagrams of living castrated animals, Tandler and Gross next showed that there was anatomical enlargement of the sella turcica. In three animals from which the testicles or ovaries had been removed several years previous to death, Rössle found considerable enlargement of the hypophysis; as also in one animal, the uterus of which had been extirpated four days previously. The association of hypophysal hypertrophy with aplasia of the genitals and with the results of castration, was conclusively proved by Jutaka-Kon's investigations.

If the excessive growth of the hypophysis in acromegaly is considered in the light of these facts, it is readily conceivable that, as certain authors believe, the derangement of the internal secretory function of the sexual glands may be the primary cause of the condition; that it gives rise to secondary proliferation of the hypophysis; and that the latter, in its turn, brings about the typical changes of acromegaly. Stumme regards the anomaly of the internal secretory function of the sexual glands as a primary metabolic disturbance leading, where there is congenital predisposition, to tumour formation in the hypophysis. It cannot be denied that, in many of its aspects, the clinical complex of acromegaly suggests a primary affection of the sexual glands. It is suggestive in this connection, that the development of hypophysal tumour and of acromegaly are frequently preceded by symptoms of chlorosis, a condition which has been regarded as connected with the genital tract. On the other hand, there are reliable observers who affirm that, in many cases, the changes. 
in the genital organs in acromegaly do not appear until the condition is far advanced.

In the present incomplete state of our knowledge, it is not possible to define with certainty the primary origin of the hypophysal changes which give rise to the symptoms of acromegaly. It may be that the condition is not the outcome of a single, definitive cause. Hypertrophy of the hypophysis may take place primarily as the result of adenoma formation, which is extremely frequent in that organ; or it may be a secondary condition, the result of the primary affection of other internal secretory organs and of the sexual glands more particularly. From the known interrelationship between the hypophysis and the sexual glands, as well as from the fact that atrophy of the sexual glands is invariable at some stage of acromegaly, it seems highly probable that suppression of the function of the sexual glands plays a part, first in the causation of the clinical complex of acromegaly, and, afterwards, in the furtherance of the processes of growth by which the disease is characterized. Knowing, as we do, the manifold interactivities which subsist between the various internal secretory organs, it is not difficult to see that other internal secretory organs besides the hypophysis may be concerned in the production of acromegaly, in the same way that Graves's disease is attributable to the influence of organs other than the thyroid. Regarded from this point of view, acromegaly belongs to the group of the polyglandular diseases.

A second pathological condition which is associated with the hypophysis is gigantism (gigantismus, macrosomia). The classification of gigantism as a pathological condition is justified by the facts : first, that abnormal longitudinal development of the bones and increase in the volume of the organs must be regarded as a perversion of the processes of growth; and second, that abnormality of growth rarely occurs singly, but is almost invariably associated with derangements of the function, and of the anatomical constitution, of different organs. It is only the giants of fairy tales who, in addition to their size, are favoured with exceptional physical and mental advantages. A closer acquaintance with the giants of our day, as well as a careful analysis of the descriptions which have come down to us of the giants of old, shows that normal giants-that is to say, persons of exceptional size and free from deformity or other pathological signs, such as were assumed by Langer, and later by Sternberg - are very rarely encountered. The vast majority of giants are undoubtedly abnormal and belong to the class of pathological individuals.

Launois and Roy, who have made a special study of this phase of abnormality, define the condition as follows : Gigantism is an anomaly of skeletal growth which leads to a height of the body in excess of the average dimension of the race, and is associated with characteristic morphological and functional derangement. 
Gigantism attacks men more frequently than women, and usually takes its rise at the period of puberty. Thus at the age of I8 to 20 , the height of the subject may be 190 to $200 \mathrm{~cm}$., or more; and at 25 to 30 , when the normal individual has long ceased to grow, it may be still increasing.

The parts principally affected are the extremities, especially the lower ones, while the trunk is only slightly influenced, and the skull appears smail in comparison with the rest of the body. The excessive length of the lower part of the body, as compared with that of the upper part, is characteristic of typical gigantism. Separation of the epiphysal synarthroses, and hypertrophy of the osseous substance of the long bones, may be seen in vivo by means of skiagrams. Subsequent anatomical investigation confirms these findings, and shows the persistence of the cartilage at the junction between the epiphysis and diaphysis, as well as the imperfect ossification of the sutures and various signs of hyperostosis. The vertebral column frequently shows postero-lateral curvature (kyphoskoliosis). The most remarkable point about gigantism, however, is that, in the majority of cases, the skeleton of the face and of the acral portions shows changes which are distinctly characteristic of acromegaly. Exaggeration of the zygomatic arches, marked projection of the supraciliary arches, prognathism of the lower jaw, thickening of the bones of the skull, enlargement of the antrum and sinuses, and deepening of the sella turcica, are the usual facial characteristics of the gigantic subject. With these, changes of the hands and feet, entirely analogous to those of acromegaly, are frequently associated.

Of the internal findings, the most interesting is undoubtedly the condition of the hypophysis. The deepening of the sella turcica points to an enlargement of the organ, and this was established by Launois and Roy, who found that, in ten cases of gigantism which they examined post mortem, there was more or less marked increase in the size of the hypophysis. In the majority of cases there was hyperplasia of the glandular tissue and adenoma formation; more rarely there was vascular struma; and in a few cases there was sarcoma and epithelioma.

In the majority of cases of gigantism, the weight of the brain is remarkably small in proportion to that of the body. The reports of the condition of the thyroid glands are not unanimous. In the greater number of cases, the thyroid was much enlarged, the weight varying from two to six times the normal. In one case, Wells showed that the iodine contents, though relatively normal, were absolutely very much increased. On the other hand, there are descriptions of cases of gigantism in which the thyroid was normal or even comparatively small. Persistence of the thymus has not been described.

The most remarkable changes in gigantism are those presented by the sexual glands. Reduction of sexual activity, 
absence of menstruation in women, and sterility in both the male and the female, are among the clinical symptoms of gigantism. In rare cases, a preceding stage of sexual hyperactivity has been observed (Buday and Jancsó). In contrast to the other internal organs, which show hyperplasia, the sexual glands are usually atrophied with signs of degenerative lesion. The secondary genital organs and genital excretory ducts (prostate and penis, uterus and vagina) are also, as a rule, remarkably defective in development, and signs of infantilism are by no means infrequent. Lack of intelligence; mental apathy and indolence; lack of muscular tone and power; poorness of hair all over the body, especially that of the pubes, axillæ, and in the male, of the beard; and, in many cases, excessive development of the panniculus adiposus - these are all signs that the organism has remained at an infantile stage of its development. The slight powers of resistance of the subjects of gigantism is shown by the fact that they usually die at an early age as the result of accidental illness. Those cases of gigantism which pass on into definite acromegaly are the only ones which offer a better prognosis concerning the duration of life. The anomalies of metabolism are, as yet, uninvestigated. The comparatively frequent occurrence of glycosuria or even of serious diabetes, is worthy of remark.

The pathogenesis of gigantism was first discussed by Pierre Marie in the course of his investigations into the nature of acromegaly. Marie believes that true gigantism is characterized by an excessive increase of all the processes of normal bony growth; and that it is to be distinguished from those cases of symptomatic gigantism in which there is abnormal growth of certain portions only, as, for instance, in acromegaly, elephantiasis, and certain forms of congenital syphilis.

The similarity between certain of the symptoms in gigantism and in acromegaly-especially the enlargement of the sella turcica as seen in museum specimens-together with the fact that the subjects of gigantism frequently develop the typical signs of acromegaly, led many authors to dispute this theory of Marie's. Brissaud and Meige adopted the view, based upon the relationship between acromegaly and gigantism, that the conditions both originate in the same pathological processes, and that they differ from one another only in the moment at which the pathological process takes place. According to Brissaud, gigantism is the acromegaly of the period of growth, acromegaly the gigantism of the adult stage; and acromegalic gigantism is the result of a pathological process, which commences during the period of growth and continues on into the period in which growth has ceased.

This theory received valuable support from the exhaustive investigations of Launois and Roy. These authors also distinguished between two forms of gigantism, the acromegalic and 
the infantile; but they lay stress upon the fact that the number of cases included in the former class increases as the two forms are more carefully studied, and that it would be hardly possible to bring forward a case of infantile gigantism which did not present some of the signs of acromegaly. The subject of infantile gigantism, if he lives until his epiphyses are ossified, becomes acromegalic. After that, he ceases to increase in height and the endochondral is replaced by periosteal hyperossification. Launois and Roy showed that the skeleton of the giant Constantin, whose skeleton they very minutely investigated, presented the infantile type of gigantism in the extremities and the acromegalic type in the skull. Gigantism is the acromegaly of those individuals, irrespective of age, whose epiphysal cartilage has not undergone ossification. If all the subjects of gigantism are not acromegalic, they may at least become so. The fact that disturbances of growth are invariably associated with pathological changes in the hypophysis, led these authors to regard that organ as the source of origin of gigantism.

That the hypophysis plays a leading part in the pathogenesis of gigantism is undoubted. This applies to those cases particularly in which gigantism has, from the first, been associated with acromegaly. In infantile gigantism, on the other hand, primary disgenitalism must be included as a probable causative factor. This is suggested by the similarity of the symptoms in this condition with those observed after castration before the onset of puberty, as well as by the relatively slight changes in the hypophysis and the lateness of the stage at which these take place. According to Fichera, these changes are also only secondary results of the suppression of the activity of the sexual glands. The peculiar disposition of the hypophysis to pathological change, to which reference has already been made, may be the reason why, in these cases, the processes of growth are more prolonged, and the dimensions of the individual thus greater than is commonly the case after castration.

A further factor in favour of gigantism is supplied by the thyroid gland. After early castration the thyroid generally atrophies and the suppression of its function brings about an inhibition of the processes of growth. But in the majority of the subjects of gigantism the thyroid has been found to be normal or even hypertrophied; it seems possible that the condition may, in part, arise from the fact that the abnormal stimulation of the processes of growth, which results from the suppression of the sexual glands and the hypertrophy of the hypophysis, receives no inhibitory check from the thyroid gland.

A few attempts have been made to treat gigantism by organotherapeutic methods with ovarian and testicular extracts, but success has been very rare (Maisonave, Dor, Hudovernig). The results seem to show, however, that the primary genetic factor in 
infantile gigantism is supplied by the defective internal secretory activity of the sexual glands. Fischera succeeded in modifying the changes in the hypophysis after castration by the exhibition of extract of the sexual glands, and this seems to suggest the employment of the method in the treatment of secondary hypophysal disease.

All researches into the pathogenesis of gigantism suggest that the condition is the outcome of hyperpituitarism; that, in the acromegalic form, this hyperpituitarism is the primary cause of the abnormal development of the body; while, in the infantile form, it is probably the secondary result of primary disgenitalism, and acts in concert with the latter.

Another pathological condition associated with changes in the hypophysis is hypophysal obesity, or dystrophia adiposogenitalis (Bartels). This condition was first described by A. Fröhlich (Igor). He found that, in a case which showed symptoms of hypophysal tumour, there was a very rapid increase in corpulence, together with infantile character of the sexual glands, and he assumed that this peculiar clinical complex was produced by a specific action on the part of the hypophysis. Since that time Fröhlich's type has been described by a large number of observers.

The most striking symptom is the obesity, which may attain enormous dimensions. It consists, partly in the deposition of fat in certain localities-particularly the breast and the abdomen-and partly in a general increase of the entire fat-body. At the same time the skin is remarkably dry and low in temperature, the secretion of the sweat glands is reduced, and there are trophic disturbances of the hair and nails. The second important symptom is the hypoplasia of the genitals, which is associated with imperfect development of the secondary genital organs, together with infantile habit. Symptoms of cerebral tumour, with suggested localization in the hypophysis, complete the clinical picture.

The etiological significance of the hypophysis in this condition receives remarkable confirmation from a case described by Madelung, that of a 9-year-old girl, who developed signs of general obesity after a bullet wound in the head, the bullet remaining lodged in the sella turcica. But Erdheim disputed the hypophysal origin of this form of obesity, and showed that, in isolated cases, the hypophysis is found under the microscope to be entirely normal. He believes that the cause of the condition lies in an influence exerted by a tumour in some area at the base of the brain, and inclines to the infundibulum, in which a trophic centre may be assumed. According to Marburg, however, Erdheim's observations do not supply proof of his theory; but even if we allow it to stand and assume that the infundibulum only is affected by the lesion, the condition might yet be due to indirect disturbance of the hypophysis, owing to the destruction of the glandular nerves which enter the hypophysis by way of the infundibulum. 
Dystrophia adiposogenitalis is regarded by some authors (Schüller, Tandler, and Gross) as the result of primary genital atrophy. The influence of the internal secretory activity of the sexual glands upon metabolism, and especially the obesity which has long been known to follow the suppression of these organs, will be described in a later chapter. If we consider, however, that the hypoplastic constitution shows, in addition to the signs of infantile habit, hypoplasia of the genitals, together with a remarkable development of the fat-body, we cannot be far out in assuming adiposis cerebralis to be the result of defective genital function together with secondary changes in other internal secretory organs. It is, however, worth noticing that, out of thirty-two cases of hypophysal obesity, twelve only showed genital atrophy; and that genital involution was not always observed in the earlier stages of the disease.

It is hardly possible, in view of the results of experimental extirpation, to doubt the primary significance of the hypophysis in the causation of dystrophia adiposogenitalis. The extirpation of certain portions of the hypophysis, as well as of the entire organ, is followed by symptoms in animals which have a profound resemblance to the clinical type of hypophysal disease which Fröhlich described. Experimental investigation by Cushing and by Aschner, as well as the results of my own experiments, all show that partial hypophysectomy is invariably followed by a considerable increase in the fat body, and by hypoplasia of the sexual glands and of the entire genital tract. In the young animals which Aschmer operated upon there was also persistence of the infantile habit. These experimental results show, moreover, the nature of the derangement which takes place in the hypophysal function. If the symptoms described are induced in animals by experimental apituitarism, it is safe to conclude that the clinical type described by Fröhlich results from reduced hypophysal activity or hypopituitarism.

This assumption does not appear to be confirmed, however, by the results of the operative treatment of dystrophia adiposogenitalis. One of the first operations for hypophysal tumour successfully performed by $\dot{v}$. Eiselsberg was upon the patient described by Fröhlich. The operation was followed by the disappearance of the local symptoms due to the presence of the tumour, while at the same time there was an improvement in all the general symptoms which were attributable to the hypophysis. The weight fell, the obesity was reduced, hair grew at the pubes and in the axillæ, and sexual activity manifested itself by erections. The disappearance of the dystrophic symptoms after the removal of the hypophysal tumour appeared to be a weighty argument against the theory of hypopituitarism. There is, however, another side to the question. If we assume that the tumour only was remored, and that the hypophysal tissue was otherwise 
left intact, it is obvious that, with the removal of the tumour the obstruction of function was abolished, the latter being allowed to resume its normal course. This supposition is rendered more probable by the subsequent history of the case. The improvement lasted for nearly three years, the symptoms principally affected being those which had been caused by the tumour, while, after the first amelioration, the general symptoms ceased to make further progress. At the present time the patient is still of infantile habit, and there is no appreciable reduction of the corpulence. In three other cases of the same kind upon which v. Eiselsberg operated successfully, the tumour symptoms and the general condition were improved, but the state of the genital organs and the corpulence underwent no progressive improvement. The indication for surgical measures lies, not in the trophic disturbances, but almost exclusively in the progressive signs of cerebral tumour, such as violent headache and threatening blindness; hence, if these symptoms show improvement, it is perfectly justifiable to describe the result as "satisfactory." In certain cases, the operative removal of the tumour is undoubtedly the most rational method of procedure. The results which have just been described do not, however, disprove the significance of hypopituitarism as a factor in the etiology of dystrophia adiposogenitalis.

The results of organo-therapy in this condition are not unfavourable to the hypothesis of hyposecretion. Several accounts (Levy and Rothschild, Axenfeld, Delille) have been given of the beneficial effect of pituitary extract upon the obesity and the reduced sexual activity. On the other hand, there is a general consensus of opinion that the exhibition of pituitary extract in acromegaly is not only useless, but that in many instances it aggravates the symptoms.

In considering the diseases which originate in the hypophysis, the fact is brought home to us-perhaps more nearly than in the case of the other internal secretory organs-that disturbance of the function of one organ is so intimately associated with disturbance of the function of others, that it is sometimes difficult to decide with certainty in which organ the primary pathological affection is to be sought. As long as ten years ago, Pineles pointed out the fact that there appears to be an intimate anatomical and physiological interrelationship between the different blood glands, which is manifested clinically by the fact that the pathological disturbance of one gland is accompanied by symptoms pointing to the functional derangement of one or more of the others. Knowing, as we do, the many sided interactivity which subsists between the different internal secretory organs, it is readily conceivable that isolated diseases of single organs of this group are very much rarer than, at a first glance, they would appear to be. In the present state of our knowledge, the only course of 
investigation which is open to us, is to start with the known results of the functional derangement of any organ, and, by following these up, to seek the primary link in the pathological chain.

The scantiness of our knowledge concerning the function of the hypophysis and the experiments which have been undertaken, largely without success, with the object of determining the scope of the hypophysal function in animals, probably account for the fact that, in clinical conditions associated with changes in the hypophysis, other internal secretory organs (thyroid, thymus, sexual glands, pancreas, suprarenal) have always been accredited with an equal and simultaneous participation. This was the origin of the polyglandular syndrome of the French authors. Under the designation insuffisance pluriglandulaire, Claude and Gougerot (1907) described a symptom complex which they assumed to result from the general atrophy and functional insufficiency of a number of internal secretory organs. Since then, a number of similar cases have been described. That we have not to do here with a well defined clinical entity is shown by the fact that the functional derangement, in either the positive or negative sense, of one organ is not by any means always followed by corresponding changes in the activity of other organs of the group; for it more often happens that the hyperfunction of certain internal secretory organs is associated with hypofunction on the part of others, and vice versa. As a matter of fact, cases have been described in which polyglandular insufficiency was associated with either hyperpituitarism or with hypopituitarism (Delille).

The formulation of a separate clinical entity of polyglandular origin, appears upon the whole to be premature. There is no doubt that clinical observation and experimental research will shed a good deal of light upon the interrelationships between the different internal secretory organs; but until experimental investigation is put upon a sound physiological basis, we cannot hope for any certain illumination of the pathogenesis of the newer clinical types.

\section{THEORY OF THE FUNCTION OF THE HYPOPHYSIS.}

The study of the morphology, physiology and pathology of the hypophysis has supplied a good deal of information concerning the functional significance of that organ, but up to the present it has not sufficed to create a definite and finished picture of its physiological activity. The best that we can do is to draw from the facts at our disposal an approximate conclusion concerning the nature of the hypophysal function.

Morphologically, the hypophysis appears to be an organ composed, both genetically and structurally, of two entirely different portions. The anterior lobe may justly be regarded as a secretory 
gland; while the posterior lobe, which belongs genetically to the nervous system, is found to consist later of indifferent glia tissue. The intimate anatomical connection between these heterogeneous tissue formations is very remarkable.

The importance of the anterior glandular portion is shown by the results of physiological investigation and of clinical observation; for we know that the removal of this part of the organ is inconsistent with the continuance of life, and that its pathological disturbance is followed by marked clinical symptoms. On the other hand, it is not apparent from either post-mortem findings or the results of experimental extirpation, that the suppression of the posterior portion is attended by any consequences whatsoever.

If, however, the functional significance of the two portions is tested by the activity of the extracts which they supply, the results arrived at are very different. It must be remembered, however, that we possess no proof of any kind that the active substance present in pituitary extract, is formed in the organ during life and by it passed on into the blood-stream. The assumption of such a performance on the part of the hypophysis is founded upon analogy, and receives considerable support from the fact that the activity of pituitary extract is well defined and quite specific. It is, moreover, a very remarkable thing that the true glandula pituitaria yields an extract possessing no definite physiological properties; while the extract obtained from the neurohypophysis produces definite and characteristic effects upon the animal organism. Such are the vaso-constriction and immense rise in blood-pressure produced in a number of organs; the stimulatory action upon certain muscular structures, such as the heart, and especially the uterus; and finally the increased diuresis. But neither the operative removal of the neurohypophysis nor the clinical symptoms which accompany hypophysal disease, supply any proof that the suppression or perversion of the activity of this part is accompanied by appearances pointing to the absence or superfluity of the specific active substance. In face of these facts, it is only with the greatest reserve that the substances in the infundibular portion of the hypophysis can be described as physiologically active hormones. The internal secretory activity of the neurohypophysis and its functional significance are in need of further investigation.

We are more favourably placed in regard to the anterior lobe. In spite of the fact that the product of secretion has not as yet been obtained in the organic extract, there is no doubt that this portion of the hypophysis stands in intimate correlation with a number of other organs, and that their interactivity is effected by rhemical means. That the glandula pituitaria belongs to the group of the internal secretory organs is proved by two sets of facts.

In the first place, anatomical changes-that is to say, 
increased volume as the result of hyperplasia of the glandular cells-are produced in the anterior lobe of the hypophysis by alteration of function on the part of certain other internal secretory organs. Suppression or modification of the thyroid function and that of the sexual glands is followed' by such hypertrophy.

In the second place, functional and anatomical changes in the hypophysis are associated with changes in the thyroid gland, the genital glands, and perhaps also in the suprarenals. The exact manner in which these consecutive changes take place is not, as yet, exactly known. It is probable that hyperfunction of the hypophysis is associated with hypertrophy of the cortical portion of the suprarenals, together with a diminution both of function and of volume in the thyroid. The correlationship between the hypophysis and the genital glands appears to be even more complicated. Experiments with animals and observation of Frölich's dystrophia adiposogenitalis show that hypoplasia of the genitals is combined with reduced hypophysal function; while acromegaly and gigantism, which are regarded as manifestations of hyperpituitarism, are also characterized by defective sexual activity.

The internal secretory activity of the hypophysis is manifested, moreover, in the influence which this organ has upon the metabolic processes which take place in the body. The most important is undoubtedly the effect which it has upon development. Even the hyperactivity of the hypophysis which is observed during pregnancy as the result of inhibition of the ovarian function, is associated with developmental changes which affect not the soft parts only, but also the bones. A greater degree of hyperpituitarism leads to abnormal growth in height and to remarkable increase in the volume of the acral portions. On the other hand, the removal of the anterior lobe-of the hypophysis is followed in young individuals by a marked arrest of development. That form of hypopituitarism which leads, both in animals and man, to a conspicuous increase in the deposition of fat shows how important is the part which this organ plays in the metabolism of fat.

\section{THE PINEAL BODY.}

\section{(GLANDULA PINEALIS, EPIPHYSIS, CONARIUM).}

The structure which Descartes believed to be the seat of the soul must, upon clinical grounds, be included among those organs which, by the agency of their internal secretion, exercise a demonstrable influence upon the somatic and psychic development of the individual, as well as upon the nutritional condition of the body and of certain tissues. In a work published last year, O. Marburg gives a very complete account of the normal and 
pathological histology of the pineal gland, together with a description and critical survey of the available clinical and pathologico-anatomical material concerning the affections of that organ.

Anatomy.-The pineal gland of man is, in the adult, a small flattened body, nearly trilateral in shape, which is suspended free between the anterior pair of corpora quadrigemina, with its base directed forwards over the roof of the third ventricle; it is connected with the posterior commissure of the third ventricle by means of a medullary lamina issuing from its ventral portion. A second medullary lamina, called the commissura habenularum, issues from the dorsal part of the pineal body; it tails off into a thin band, the pineal peduncle or habenula, and this is attached to a thickening, the trigonum habenula, situated at the posterior end of the optic thalami. Between the two lamina is the narrow passage, blind at its caudal end, of the recessus pinealis. The tela choroidea lies against the posterior portion of the dorsal surface of the pineal gland; with the anterior portion it forms a pouch, the recessus suprapinealis, which opens into the third ventricle.

Embryology.-From the roof of the thalamencephalon (diencephalon) of the lower vertebrates, according to Edinger, two buds are developed, which, proceeding dorsally, represent: (I) the paraphysis; and (2) the pineal tubercle or dorsal pouch. The paraphysis is not present in mammals, but the dorsal pouch is represented by the recessus suprapinealis. At the boundary of the thalamencephalon and the mesencephalon, are two other buds : (3) the parietal vesicle; and (4) the epiphysis. From the parietal vesicle, in the lower vertebrates (fish, reptiles), the parietal organ is formed (though many authors believe it to be formed from a vesicular portion of the epiphysis), which breaks through the cranial capsule at the parietal foramen and persists as the socalled "frontal patch" ; or, in saurians, as the parietal eye, which has a formation similar to that of the eye of invertebrates. The changes which take place in the position of the organ during the embryonal stage are shown by the course of the parietal nerve, by which communication with the ganglion habenulæ is effected. In the loose connective tissue between the recessus suprapinealis and the pineal gland of new-born infants, Marburg found a structure resembling the parietal nerve and ganglion of reptiles. The pineal body is developed from the epiphysal protuberance, which throws out lateral buds, becomes vascular, and gradually changes into a glandular structure composed of solid cell masses. This gland is not furnished with ducts; it communicates with the brain by means of the pineal peduncle and the posterior commissure.

Histology.- In new-born infants the pineal gland consists of irregular lobes held together by a small quantity of connective tissue: the lobes are composed of cells of almost the same type, 
but arranged irregularly, being crowded together in the interior of the organ. The pineal cells have a pale tinted protoplasm and very characteristic nuclei, these being large and oval, and crowded, over their entire circumference, with granules. Dimitrowa distinguishes four different nucleus forms. Other important histological details are:(I) The peculiar nature of the ependyma of the recessus pinealis, in which cubical epithelium and epithelium composed of goblet-like cylindrical cells aiternate; and (2) the cysts which are observed at the base and in the interior of the pineal gland of new-born infants, and which probably represent obliterated blood-vessels. The pineal gland shows signs of involution before the age of puberty, the first symptoms being observed in the seventh year. The concretions known as pineal sand or acervulus, which consist of calcium phosphate and calcium carbonate, are found in the glia layer which covers the commissura habenularum. As age increases, a distinct increase of the connective substance at the expense of the glandular tissue takes place. The glandular lobes are replaced by plaques composed of connective tissue and a very fibrous glia tissue, and these plaques contain isolated glandular cells. The connective tissue septa further undergo hyaline degeneration, and may completely calcify. Occasionally, homogeneous masses are observed in the septa or in the interior of the glandular lobes and, after the deposition of calcium salts, corpora arenacea are evolved from them. The glandular cells also present signs of involution, but, even in extreme old age, glandular cells are encountered which are intact and, apparently, still functionally active. Cysts formed, in the majority of cases, from the glia plaques as the result of obliteration of the blood-vessels, are a very common finding in the pineal gland of adults.

Physiological experiments with the pineal gland are described by E. v. Cyon. This author investigated the activity of extract of the pineal gland of oxen and sheep. He found that, when injected intravenously, they produced no effect upon bloodpressure, but that small doses accelerated the heart's action, while large doses retarded and strengthened it. Irregularity of the cardiac beats, bigeminal and trigeminal pulses were also frequently observed. According to v. Cyon, extract of pineal gland, when given in small doses, excites the nervi accelerantes only; in large doses it simultaneously stimulates the inhibitory cardiac nerves, but in so irregular a fashion that the harmonious counteractivity of the two sets of nerves is unbalanced. The cardiac beats become irregular, but these irregularities disappear after resection of both vagi. Further experiments showed that glycerosodium phosphate acts in the same manner as weak doses of pineal extract, while glycero-calcium phosphate produces action beats, as they are called, in the same way as large doses of pineal extract; but unlike those produced by pineal extract, these action 
beats continue after resection of the vagi. The results of these experiments led v. Cyon to assume that it was the function of the pineal gland to store up certain salts in organic combination; and that the physiological action of pineal extract is explained by the presence of these salts, in comparatively large quantities, both in the concretions in the spaces and distributed throughout the tissue of the pineal gland. Calcium phosphate forms the larger proportion of these salts.

v. Cyon also describes the results of direct experimental stimulation of the exposed pineal gland of rabbits. He found that electric stimulation produced contraction, together with a change in the length and the shape of the organ. He supposes this contraction to be produced by the striated muscles, the presence of which in the pineal gland of oxen and calves has been proved histologically by Nicolas and Dimitrowa. According to v. Cyon, a contraction combined with a shifting of the pineal gland would regulate the in- and out-flow of the cerebro-spinal fluid in the third ventricle. Thus the pineal gland would fulfil the mechanical function of automatically controlling the flow of the fluid in the aqueduct of Silvius, enlarging or reducing the channel in accordance with the pressure in the third ventricle. v. Cyon thinks that, like the thyroid gland and the hypophysis, the pineal gland belongs to that group of organs which, acting partly alone and partly in physiological concert, control intercranial pressure.

v. Cyon's experiments have not, as yet, been confirmed. As far as the effect of pineal extract is concerned, Dixon and Halliburton (I OO9) state that its intravenous injection is without definite effect either upon blood-pressure or the cardiac contractions. The effect of pineal extract upon respiration, the intestines, and the urinary secretion, is negative.

Pathological Anatomy.-Virchow was the first to draw attention to the pathological formation of cysts in the pineal gland. Cysts have been recently described, the presence of which was attended by no clinical signs whatever. In a number of instances, serious pathological symptoms were present.

Teratoid formations are of frequent occurrence in the pineal gland. Since Weigert's classical description (I875), several accounts of pineal teratoma have been given. Askanazy (I906) regarded these formations as developments from a germ which gained access to the pineal gland at the earliest stage of embryonal life. Marburg believes the starting point of pineal teratoma to be the primordial beginning of the parietal eye. In so far as the clinical results are concerned, Askanazy supposes that these tumours act like a fœetus and produce certain anomalies of growth to be described later. According to Marburg, these anomalies of growth are the result of premature destruction of the active functional pineal tissue. 
Tumour forms of the most varied description-glioma, sarcoma, carcinoma, and tumours composed of different kinds of tissue-have all been observed in the pineal gland.

Clinical Signs of Pineal Affection.-Disease of the pineal gland is accompanied by certain very characteristic clinical signs, which are to an extent analogous with those which accompany pituitary disease.

Total destruction of the pineal gland, such as may be brought about by malignant tumours, was in six cases accompanied by profound cachexia with trophic derangement (decubitus). In a case of pineal carcinoma described by Hempel, there was obesity during the early stages which later developed into serious fatatrophy. In a case observed by Marburg, that of a 9-year-old girl with symptoms of cerebral tumour, there was extreme obesity. The autopsy showed a compound tumour of the pineal gland. The literature of the subject provides other instances in which pineal tumour is accompanied by remarkably good nutritional conditions and exceptional development of the fat-body. Genital atrophy, which invariably accompanies hypophysal obesity, is absent in pineal disease, and Marburg is inclined to regard pineal obesity as the result of increase in the amount of the active functional pineal tissue, in other words, as a manifestation of hyperpinealism. This deduction is by no means conclusive, for, in the first place, as Marburg himself points out, it is extremely difficult to form an opinion concerning the condition of the gland; and in the second, an exceptional development of the fat-body is also observed in forms of pineal disease, which both the clinical symptoms and the anatomical findings show to be the outcome, not of an increase, but of a reduction of the active functional pineal tissue.

Of paramount interest are the observations published by Ogle, Gutzeit, Oestreich-Slawyk, and more recently by v. FrankiHochwart. In boys of under 7 years, in addition to symptoms of cerebral tumour and disease of the corpora quadrigemina, these authors observed abnormal growth in height, abnormal growth of hair, premature development of the genital organs and of sexual instinct, and mental precocity. The autopsy revealed pineal tumour, generally teratoma. In these cases, the abnormal growth, the premature mental and physical development, and the remarkable genital hypertrophy were associated with a diminution of the pineal tissue, or with what Marburg defined as dispinealism.

These data point inevitably to the conclusion that the pineal gland is an internal secretory organ which influences metabolism, though the details of its functional activity are at present not ascertained. Still more important is the fact that, during the period of its complete development, that is to say, until the seventh year, this organ exercises a definite and apparently inhibitory influence upon the development of the sexual glands, and it is 
probable that it has a secondary effect upon mental development. The destruction of the pineal gland at this stage of existence leads to mental and physical precocity. That there is an antagonism between the activity of the pineal gland and that of the hypophysis is certain, for we know that pituitary insufficiency produces hypogenitalism.

I have occupied myself for some time with experiments, the main object of which has been to determine the clinical results of pineal suppression. I have succeeded, so far, in extirpating the pineal gland by a method similar to that which I employed in hypophysectomy. As far as my observations go, the pineal gland in the adult animal is a negligible quantity; my experiments with young animals are not as yet complete.

\section{THE GENERATIVE GLANDS.}

The generative glands possess chemical interrelationships with a large number of other organs and tissues. The importance of the part which these glands play in the general economy of the organism is shown by a wealth of evidence such as is forthcoming in the case of no other organ. It will be shown in the following pages that, not only are physiological and pathological processes modified by the function of the sexual glands, but that the activity of these organs is associated with the profoundest problems of "general biology and embryology.

The first problem is concerned with the sexual characteristics. In almost every class of animal, the type is represented and maintained by two different groups of individuals, which differ more or less widely from one another in habit, anatomical structure, functional activity, and certain other attributes. These groups are defined as the male and female sexes. The question arises as to whether this difference in the organization of the sexes, this dimorphism, as it is called, is the outcome of the difference in the nature of the organs of reproduction. That these organs are described as "sexual" organs is an illustration of the prevailing inclination to regard the stigmata of sex as dependent upon the organs of generation. Before the days of anatomy, and the view prevails to-day among primitive peoples (the Russian scopts, according to Pelikan, and the natives of India, according to Roberts), the external genital organs and, in women, the breasts, were regarded as the factors responsible for the characteristics of sex. The Russian scopts believe that women are rendered sterile by the removal of the breasts and the external genitals. Helmont said: "Propter solum uterum mulier est quod est," and this dictum was modified by the French physician Chérau into: "Propter ovarium solum mulier est quod est." Virchow expressed the same view in these words: "Woman is 
woman by reason of her generative glands. All the peculiarities of her body and mind . . . everything, in fact, which in the true woman we admire and revere as womanly, is dependent upon the ovary." There are others (Geoffroy St. Hilaire, Klebs, Puech, Hegar), however, who adopt a different view. Quite recently, weighty arguments have been advanced by several authors (Hegar, Halban, Pflüger) against the dependence of somatic and psychic sexual characteristics upon the genital glands.

In order to grasp the precise significance of the problem, we must first ask ourselves the question: What is the decisive factor in the determination of sex? What is it which decides whether from the fertilized egg, which contains both maternal and paternal chromosomes in equal number, a male or a female individual shall proceed?

It is possible that sexual distinction exists in the unfertilized egg; that, in fact, there are male and female ova which, after fertilization, develop in accordance with an already determined sex. In certain of the lower forms of life this is actually the case. Under certain favourable external conditions, the aphis produces only females; at a low temperature with slight atmospheric humidity, both males and females are produced, and these pair. The pregnant female does not produce young, but lays eggs, and from these, females are again hatched out. It is evident that the sex of the individual is here determined in the unfertilized egg.

Korschelt showed that Dinophilus apatris, a small worm, lays eggs of two different sizes and that, from the larger of these, females develop; while from the smaller, males proceed. Both Nussbaum and Maupas found that, in the case of wheelanimalculae (Hydatina senta), the larger eggs of which develop into females and the smaller into males, the results were affected by external factors acting on the mother, such as surrounding temperature and nutritional conditions.

A second method of sex differentiation, and one which recent discoveries have shown in process of action, is by two distinct varieties of spermatozoa. In certain insects, hemiptera and orthoptera, two kinds of sperma cells are present, which differ from one another in their chromosome contents. According to Wilson, females proceed from eggs fertilized by cells with accessory chromosome, males from eggs fertilized by the other cells.*

In bees, ants and wasps the conditions are different, there being only one kind of egg and one kind of spermatozoon. Males are produced by parthenogenesis, females proceed from fertilized ova. The queen bee is impregnated once only, the semen being

* According to Omelczenko, Selenew and others, two kinds of spermatozoon are also present in mammals and in man. Gram stains the body of the one violet, of the second red. The two kinds of spermatozoon-bodies are supposed to represent the two sexes. 
retained in the receptaculum seminis. From the eggs into which the sperm cells penetrate, females-that is queens and workersare hatched, while from the unfertilized eggs, males-that is drones-proceed. Lenhossék thinks it possible that the sperm cells find access only to those eggs which possess female characteristics and that they do not penetrate the male eggs.

These observations show that, in the lower forms of life, sex differentiation may already exist in the unfertilized egg, or it may be determined in the course of, or immediately after, fertilization. But it must not be imagined that these observations apply indiscriminately to animals of the higher orders. On the contrary, where these are concerned, we know neither the factor which determines sex nor the moment at which sex is decided. The fact that twins from one ovum-which are probably the outcome of division of the germ at a very early stage of segmentation-are almost invariably of the same sex, suggests that the determination of sex in man also takes place at a very early developmental stage.

This assumption is contradicted, however, by a large number of facts. In the first place, the primordial beginning of the body is, in all vertebrates, sexually indifferent. It is not until the embryo has made considerable advancement-at the fifth week in man-that a differentiation of the primitive genital trace into. a unisexual genital gland takes place. It may be that in vertebrates, as well as in invertebrates, both male and female glands develop simultaneously from the same primitive genital trace. As Pflüger showed in the case of Bufo cinereus, there is a developmental stage of true hermaphroditism, which later passes off. Cases of hermaphroditismus biglandularis are sometimes, though rarely, met with in man; these are individuals in which the generative glands of both sexes are present either on one or both sides, or a female gland on one side and a male gland on the other side. These findings show that the sex of the individual is not determined until a late stage of development, and that the differentiation of the unisexual glands does not take place until after the development of the undifferentiated sexual cells.

If the specific generative gland is regarded as the characteristic of sex, the body of the embryo must be regarded as sexless as long as the primitive genital trace remains undifferentiated. Such being the case we should be compelled to admit that the determining factor in the differentiation of sex is, as far as our present knowledge goes, unknown. We simply do not know why it is that, at a certain moment in the life of the embryo, the primitive genital trace develops, in one instance into a testis, in another into an ovary, and in certain very rare cases, into both.

But sexual dimorphism is not confined to dimorphism of the cells of generation; it extends to other bodily characteristics. 
There is a striking difference, in the two sexes, in the structure of the excretory ducts from the genital glands and in the external genitals, as well as in those organs which are called the secondary genital organs.

The study of embryology shows that the primitive beginning of these apparatuses is, in both sexes, common and indifferent. In addition to the primitive genital trace, it comprises the Wolffian body or primordial kidney, the Wolffian duct or primordial ureter, and Müller's duct. The latter runs along the lateral side of the Wolffian duct and has a common opening with the latter into the sinus urogenitalis. At the end of the third month of embryonal life, this primitive beginning becomes differentiated in accordance with the characteristics of one or the other sex, and develops to form either the male or the female sexual apparatus. But whichever the sex of the individual, portions of the characteristics of the other sex remain as rudimentary organs.

If a testis develops from the primitive genital trace, an epididymis is formed from the head portion of the Wolffian body ; the caudal portion forms blind ducts which eventually become the paradidymis and aberrant ducts; and the Wolffian duct becomes the vas deferens. Müller's duct disappears, but the caudal and cranial portions remain to form the hydatid of Morgagni and the sinus pocularis (uterus masculinus).

Where the primitive genital trace develops to form an ovary, Müller's ducts form the Fallopian tubes, uterus and vagina. That portion of the Wolffian body which is in communication with the ovarian tract shrivels up to form the parovarium (epoöphoron), which corresponds to the male epididymis. Vestiges of the caudal portion of the Wolffian body persist as the paroöphoron, situated in the ligamentum latum, and occasionally also as structures corresponding to the ductuli aberrantes. The cranial portion of the Wolffian duct forms a portion of the epoöphoron with the appendix vesicularis, while the remnants of the caudal portion form the so-called ducts of Gaertner.

This brief sketch of the developmental history of the sexual apparatuses shows that the primordial beginning of the secondary genital organs is independent of that of the generative glands. The secondary genital organs have a bisexual origin and, in the adult animal, these show a certain degree of hermaphroditism. This bisexual origin leads occasionally to the development of both sets of genital ducts and external genitals, thus producing instances of pseudo-hermaphroditism. Pseudo-hermaphrodites are individuals in whom the genital glands are unisexual, while the rest of the genital apparatus is, either wholly or in part, bisexual.

As a general rule, the development of the excretory ducts proceeds in accordance with the sexual character of the genital glands, though malformations have been observed which seem 
to disprove such dependence. Such are the cases of double congenital agenesis of the genital glands, with complete development of the external and internal sexual organs.

Interesting cases of hermaphroditic malformation have been observed in animals. Kölliker describes a pig i5 months old, which had well-developed testes, shrivelled seminal vesicles and Cowper's glands, and a large uterus with two cornua. v. la Valette St. George describes the findings in a striped water-newt (Triton teniatus) as follows: Testicles and male secondary sexual stigmata, large ovaries but no oviduct. Gerhartz observed a Rana esculenta with proponderating male characteristics, which also possessed a well-formed oviduct, but had no ovaries nor were eggs present in the testes. These observations show that the secondary genital organs are not only not derived from the same primitive beginning as the genital glands, but that their further development also is sometimes independent of the latter. There are certain exceptions to the rule that the sexual character of the secondary organs is in accordance with that of the genital glands.

In addition to the differentiated genital organs, which form the primary stigmata of sex, there are other phenomena by which the sexes are characterized. J. Hunter described these as " secondary sexual characteristics" and includes under this heading all the specific appearances which are not directly concerned with the processes of reproduction. It is true, as Darwin pointed out, that this definition is a loose one, for it is extremely difficult to decide in individual cases whether a detail of structure or of function is concerned with reproduction or not.

The term "secondary sexual characteristics" is inexact for another reason, namely, that it suggests a dependence of these characteristics upon the genital glands, and of this we have at present no proof. As we have shown in dealing with the secondary genital organs, such proof is difficult to obtain.

The only justifiable grounds for distinction is the differentiation between the cells of generation and the other somatic cells, which is manifested in their biological behaviour. The somatic cells are independent and capable of fission, but they are transitory structures. The cells of generation, on the other hand, are not autonomous, but they possess the capacity of forming another individual after their nuclear portion has combined with that of cells of the opposite sex.

Thus in all organisms with a sexual digenetic method of reproduction, there are two types of biologically incomplete cells of generation, the male and the female, and these only attain completion by means of the process of fertilization.

The somatic cells are completely autonomous, and a biological differentiation, such as that which characterizes the cells of generation, is superfluous to their development; sexual dimor- 
phism is absolutely excluded. But experience shows that there are recognizable differences between the greater number of the cells and tissues in the two sexes. Apart from the genital apparatus and the organs associated with its functions, such as the mammary glands, male and female individuals differ widely from one another physically, as in the details of the skeleton, the distribution and the amount both of the adipose tissue and the hair, the development of the larynx, \&c.; and these physical differences are accompanied by equally marked psychic divergence. These facts suggest the question: Are the somatic cells sexual in character? That is to say, are they formed from a male or female primitive origin? There is a second possibility, namely, that the sexual differentiation of the somatic cells is secondary and dependent upon the unisexual cells of generation, that is, upon the reproductive glands. Both these hypotheses have their supporters.

A large number of authors, Tandler and Gross more particularly, have laid stress upon the fact that what are called the secondary sexual characteristics are, in reality, only characteristics of the species, that is to say, properties peculiar to a species or an order of vertebrates and having no primary relationship with the organs of generation. Thus the mammary glands are the outcome of an agglomeration of sweat glands which, later, became the engine of a different function and was included within the sphere of influence of the organs of reproduction. The beard of the anthropoid apes and of man was also originally a characteristic of species, for the upper lip of the human fotus is clothed with 'a hirsute growth which attains equal development in both sexes. The antlers of the cervida, again, were originally specific characteristics of unknown function, which afterwards acquired a secondary relationship to sex. Tandler and Gross were able to prove that in the foetus of the roe-deer there is a primitive antler trace, the appearance of which is exactly similar in both sexes, the only difference being that in the case of the male development is progressive, while in the female further development takes place in very rare instances only. The formation of the antlers of reindeer is identical in both sexes, and the development of the antlers in the female is unaffected by castration.

But this translation of the characteristics of sex into characteristics of species merely postpones the solution of the problem; it does not supply any evidence in favour of either of the current hypotheses. The characteristic signs of species are undoubtedly foreshadowed in the embryo, but the direction of their subsequent development must depend on one of two determining causes : either ther already possess a tendency to develop in a certain manner, that is to say, they have sex; or their development is modified and differentiated by the internal secretory activity of a genital gland. Both are possible. If the secondary sex 
characteristics are regarded as characteristics of species, then the assumption of a primitive trace, which is equally developed in individuals of both sexes and which undergoes secondary differentiation through the agency of the genital gland, supplies a more probable hypothesis than that of Tandler, who held that the sexual nature of the secondary characteristics, like that of the genital glands, is determined in the ovum. But even if we accept the first hypothesis, we are compelled to concede that the normal development of the modified characteristics of species is dependent upon the existence of an active genital gland; and that the sexual differentiation of new-born infants is the outcome of the activity of the genital gland during fœtal life.

We will now proceed to a closer examination of the two conflicting hypotheses concerning the origin of the sexual dimorphism of the somatic cells.

The one theory assumes that all the primordial embryonal cells are sexually differentiated, and that the differentiation of the somatic cells takes place before that of the cells of the primitive genital trace. The assumption is that the unknown factor, which decides whether the indifferent genital beginning shall develop into a testis or an ovary, also acts upon the somatic cells, thus laying the foundation for those differences of body in the case of the two sexes, which make their appearance at a later developmental stage.

The evidence in support of this view is supplied by the following facts :-

In fœtuses and new-born infants, a large number of tissues are already sexually differentiated. That female babies have smaller hands and feet and a lower average weight when born than males, is well known. There is also a difference in the brain of the two sexes and there is a very marked differentiation of the bony framework of the pelvis. It is also known that certain anatomical variations occur only in the male, certain others only in the female. According to Rabl and Fischel, supernumerary ribs and vertebra are more frequently observed in males, while a deficiency in the number of lumbar vertebræ is most frequent in females.

These observations cannot, however, be regarded as conclusive; for differences between the tissues of male and female fotuses do not make their appearance until after differentiation of the genital glands, and the possible influence of the latter cannot be excluded. Fehling was unable to observe female characteristics in the pelvis before the fifth month of fotal life, while the primitive genital trace undergoes differentiation in the fifth week.

The condition of pseudo-hermaphroditism has supplied a large number of facts which point to the independence of the sexual differentiation of the somatic cells from that of the ceiis 
of generation. Not only does the genital apparatus in pseudohermaphrodites show abnormal development, though in a varying degree, but these individuals possess secondary somatic and psychic sex characteristics which are not in accordance with the type of their sexual glands.

There are, further, those numerous and undoubtedly bona fide instances in which individuals, with normal unisexual genital glands and normal internal and external genitals, possess more or less clearly marked heterologous sexual characteristics. Such are the large-boned muscular women with flat chests, narrow hips, deep voices, and more or less beard; and the beardless men with rounded outlines and soft flesh, with well-developed mammary glands and high-pitched feminine voices. Halban suggests the name hermaphroditismus secundarius for this class of abnormality, thus enlarging the definition of hermaphroditism to include the secondary stigmata of sex. Moreover, Halban considers that the psychic properties and peculiarities of character, which are described by the terms "masculinity" and "femininity," are congenital characteristics of sex, and the perversion of these he also includes under the heading of secondary pseudo-hermaphroditism.

To consider the more detailed aspect of hermaphroditism and to describe the abnormal combinations of sexual stigmata which have been observed would take us too far afield. The literature of the subject is considerable. Neugebauer, in his recent report, describes I,300 cases of pseudo-hermaphroditism; but these observations do not furnish certain evidence either for or against the dependence of somatic sexual distinctions upon the cells of generation. It is affirmed upon all sides that anomalies of the sexual system are very frequently accompanied by structural defects in other parts of the body, and that these defects are of a kind which arise, in part, at a very early stage of development (Hegar). So experienced a scientist as Neugebauer regards pseudo-hermaphroditism as actually one of the symptoms of general malformation. And Pick has recently shown that the genital glands of pseudo-hermaphrodites are primarily malformed from the time of the primitive genital trace; he points to the remarkable preponderance of teratoma and tumours developed from a teratomatoid primordial origin among the new formations of pseudo-hermaphrodites. Pick regards these tumours as tissue proliferations from a previous embryonal malformation, in a malformed embryonal organ. It is interesting in this connection that Bertkau, who has brought together all the known instances of hermaphroditism among arthropoda, arrived at the conclusion that all conditions leading to abnormality of any kind are favourable to the development of hermaphroditism.

These observations seem very clearly to suggest that the cause of the absence of the somatic stigmata of sex is to be found in the imperfect differentiation of the cells of generation. 
The presence of heterologous sex characteristics has been explained in various ways. Benda adopted the view that in the higher vertebrates the primitive trace of the entire sexual apparatus is female, and that the male type is a modified form of the female, the outcome of specific influences at present unknown. Herbst, on the contrary, assumes a primary male primitive trace, together with stimulatory and inhibitory activities on the part of the genital glands, by which the development of the sex characteristics is affected.

These hypotheses are superfluous, however, in view of the now certain fact, that the primitive genital trace is originally indifferent, therefore bi-sexual and, in the true sense of the term, hermaphroditic. Moreover, 'true hermaphrodites are not so rare as was formerly believed to be the case.

We now know that true hermaphroditism, in the morphological sense of the term, is observed in man, and that it occurs in the form which formerly appeared most doubtful, namely, hermaphroditism of the glands (ovotestis).

It has been asserted that physiological hermaphroditism, by which is meant the presence of both sets of glands in a state of physiological activity, is never encountered among the higher vertebrates; that certain species of fish (myxina, serranus, and some carp) are the only representatives of physiological hermaphroditic vertebrates.

If, by the function of the genital glands, we understand the production of semen and the release of ova, this pronouncement is undoubtedly right. But in view of the internal secretory function of these glands, we are justified in describing as physiological hermaphroditism those cases where tissue of the genital glands of both sexes is morphologically demonstrable; or rather, those tissue elements to which a specific internal secretory activity is ascribed. From the accounts of zoologists and veterinary surgeons, it appears that hermaphroditic glands, that is to say, genital glands composed of both testicular and ovarian parenchyma, are of very frequent occurrence. A communication made by Tourneux is particularly interesting; he states that in the female mole there is, in addition to a functionally active ovary, a rudimentary testicle, that is, rudimentary seminiferous tubules with interstitial cells.

It is highly probable that the cells of Leydig in the testicle and analogous cells in the stroma ovarii, represent the internal secretory tissue of the genital glands. If this is the case, the findings described in the previous paragraph must be regarded as proofs of the existence of physiological hermaphroditism in mammals.

Whether glandular hermaphroditism, either partial or complete, occurs in man is a question which can be settled only by exact anatomical and histological investigation. But, owing 
partly to the indefinite localization of hermaphroditic glands, and partly to the undefined nature of their histological characteristics, such observation is very difficult to carry out. If definite proof of glandular hermaphroditism were forthcoming, it would be easy to account for the presence of heterologous sex characteristics. The assumption would necessarily be that male and female formative influences are mutually exclusive, and that the sex characteristics of the body are in accordance with the nature of the genital gland which is best developed and functionally most active. But the important point in this connection would be, not the conditions governing the functions of external secretion (spermatogenesis, ovulation), but the degree of development and functional activity of that tissue of the genital gland by which the internal secretion is elaborated.

It is only by the assumption of a hermaphroditic primitive genital trace, together with the dependence of the somatic and psychic sex characteristics upon the internal secretory activity of the genital glands, that we can explain those cases in which complete alteration of single sex characteristics, or even of the entire sexual character, takes place during the life of the individual. These cases appear to be by no means exceptional, though they have not received the attention which they deserve.

A case of Friedrich-Grawitz's, recently described by Alberti, is very interesting in this connection. The subject was a girl of 20 , with clearly defined female habit, well-developed mammary glands, and regular menstruation, who gradually reverted to the masculine type, with cessation of the menses. The adipose tissue diminished and the body became covered with hairs; the breasts, which were previously very well developed, became soft; the hair of the head became short and thin; the voice deepened; and thick, dark whiskers and moustache made their appearance. Three years later she was operated upon for ovarian tumour; and she eventually died of peritonitis, the outcome of twisting of the pedicle by rotation of a multilocular cystoma.

A large number of analogous cases have been described, in which the female stigmata have, after puberty or even late in life, reverted to the male type, and vice versa (Zambini, Freund, Hegar, Wilson, Parhon and Goldstein, Hammond, DjemilPascha). In some instances, there was more or less complete disappearance of certain well-marked stigmata of the one sex, their place being taken by corresponding characteristics of the other. As a general rule, however, the alteration in the sex characters followed pathological processes, of traumatic or idiopathic origin, in the genital glands.

Important evidence in favour of the hermaphroditic nature of the primitive genital trace and of the dependence of the characteristics of sex upon the internal secretion of the preponderating specific genital tissue, is provided by those not very 
rare cases in which the physiological cessation of the function of the genital glands is followed by more or less alteration in the sex characters. Women at the menopause have been known to develop male characteristics, such as a deep masculine voice and hirsute growth upon the face and body.

Castration late in life is not followed by any demonstrable changes in the sex characteristics, and prepubertal castration, as we shall see later, produces not heterosexualism, but infantilism. But the perverted sex characteristics described above are the result of the suppression of the ovarian function. These facts suggest that in the case of castration, extirpation of the genital glands includes the removal of the rudimentary portions of heterosexual tissue; while in cases of ovarian inadequacy, these heterosexual rudiments have acquired a determining influence upon the development of primitive beginnings which are present in individuals of both sexes.

The same theory accounts for the perversion of sex characteristics in animals; such are, male sheep and goats which yield milk, and female goats and sheep with beard and horns; antlered females among the cervidæ; hens with cock's plumage or arrhenoidia, the reverse condition, thelyidia, in cocks, \&c.

The phenomenon of cock's plumage in hens has been extensively investigated by A. Brandt. It appears that the changes are not confined to the plumage, but that these birds acquire other male characteristics; they crow or sing and, in the absence of the cock, they lead the other females.

Brandt points out that arrhenoidia and thelyidia are normal conditions in certain species of birds, where they occur independently of changes in the genital glands," as the expression merely of variability of the external sex characteristics. They are congenital, however, in hermaphroditism and in other anomalies; they are acquired after loss or degeneration of the internal genitals; and they occur most frequently as accompaniments of senile degeneration of the ovaries with sterility, and of obliteration of the oviduct.

A. Rörig has made antler formation and the manner in which it is influenced by the reproductive organs the subject of careful investigation.

The antlers of the stag are acquired secondary characteristics, developed at a period when " the strong projecting canine teeth, the original weapons of his forefathers, had proved an inadequate means of defence, and the method of fighting forehead to forehead during the rutting season had been evolved."

The sexual character of the antlers is shown by the fact that " these organs of aggression invariably attain to full development at a period immediately preceding the rutting season, and that they are shed a short time after its close."

Rörig brings forward a large amount of material, chiefly 
anatomico-biological in character, to show that the absence of one or both antlers does not affect the virility of the animal. Absence of antlers may accompany abnormal formation of the male sexual organs.

Old females, which have become sterile, incline to antler formation, as do also females with diseased internal genitals. The antler development of hermaphrodites is said to be more influenced by the epididymis than the testes.

Rörig is unable to give a satisfactory explanation of abnormalities, and he closes his treatise with the remark that the originating cause of the malformation of antlers will probably remain a problem for some time to come.

Those cases in which the secondary sex characteristics are changed on one side only, usually upon the same side as the gland with which they correspond, may also be taken as evidence in favour of the theory that sex characteristics are dependent upon the genital glands.

The one-sided development of the mammary glands in men and the development of a single antler in female cervidæ are frequently advanced as instances of one-sided pervertion of the stigmata of sex. But that these deductions are inconclusive is shown by the fact that very different accounts are given of the manner in which the antlers of stags are affected by one-sided castration. Some authors state that one-sided castration is followed by loss of the antler on the same side; others again affirm that it is the antler of the opposite side which is lost.

A case described by M. Weber is frequently quoted as an instance of asymmetry in the somatic sex characteristics. Weber observed a finch (Fringilla coelebs) which possessed a right testis and had male colouring and plumage upon the right side; the left side was female in colouring and plumage, and was furnished with an ovary. This freak of nature is very difficult of explanation. Halban, and later Pflüger, regarded this bird as a one-sided hermaphrodite, not only in regard to the arrangement of its genital glands, but also in its somatic sex characters. Nussbaum, on the other hand, regards this case as proof of the great influence of the nerves upon the secondary sex characteristics. The stigmata of sex are usually arranged symmetrically upon both sides of the body; but it is important to remember, in this connection, that Virchow laid special stress upon the fact that, in the male pseudo-hermaphrodite Katharina Hohmann, the left side of the body, in which the genital anomaly was situated, was generally (extremities, rump, face) less well developed than the right.

The results of castration in both man and animals have frequently been adranced as evidence in favour of the congenital origin of the characteristics of sex. It is an undeniable fact that, if the genital glands are removed at the earliest possible date from two individuals of the same species, but of different sex, these 
individuals will not show complete identity of habit, either immediately after operation or later; certain characteristics, such as beard and the mammæ, will develop in accordance with their original sex. Castrated males grow a certain, though scanty, amount of hair on the face. These results are accounted for by the fact, that the sexual differentiation of the tissues probably takes place during foetal life through the agency of the already differentiated genital glands, and that after suppression of the genital glands the primitive beginnings of individual stigmata continue to grow and attain a certain imperfect degree of development.

The same applies to Ondermann's much-quoted experiments with Oeneria dispar, and those of Kellog with silk-worms. Castration of the caterpillar did not obliterate the signs of sexual dimorphism in the butterfly.

In all these cases, castration was performed at so late a period that the possible influence of the genital gland upon the development of the somatic stigmata was not entirely excluded.

In order to decide the question as to whether the sex of the soma is congenital or not, it would be necessary to extirpate the undifferentiated sexual glands of the fotus. The only evidence on this point is that supplied by Nature in the case of the socalled parasitic castration of crustaceans.

It has long been known that certain crabs are attacked by parasites which belong to the isopods of the family Bopyridæ. These parasites are never found in fully-developed male crabs and for this reason it has been asserted that female crabs are their only hosts. Giard, who investigated this question very thoroughly, found that the Bopyridæe enter their host only during the early youth of the latter; that they attack both male and female individuals; and that they effect the destruction of the genital glands of their host. The result of this early gonotomy is that the host does not develop sexual characteristics of any kind, but becomes neuter in every sense of the term. Even more interesting is the fact that, in certain orders (Stenorynchus), the males which have been castrated in this manner, after casting their shell several times, assume the habit of the female type so completely, that for a long time they were believed to be females or were regarded as of a different species. I believe myself that this remarkable occurrence is explained by the fact that the invading parasites are females only, which attain to sexual maturity within the body of their host, and that the female genital gland of the parasite has a determining influence upon the development of the sex characteristics of the host.

It appears then, that we have to do here with an instance of early castration accompanied by simultaneous transplantation of the heterosexual genital gland, and the results seem to show that the somatic sex characteristics are dependent upon the cells of generation. 
We are justified in assuming from the evidence which has been brought forward that the stigmata of sex are, from their earliest beginnings, dependent upon the genital gland, and that for this reason they are very rightly termed "secondary." That they attain to complete development by the agency of the cells of generation only, is a point which has never been questioned.

That the somatic differences between the sexes are dependent upon their genital glands is shown, in animals, by the signs which accompany the periodic recurrence of the rutting period, and in man by the phenomena of puberty. The maturity of the organs of generation at the rutting period is accompanied by remarkable changes in the structure and functional capacity of the body, which last only so long as the increased activity of the reproductive organs is maintained. Every one is familiar with the brilliant coloration of the plumage, the rich, full song, and the activity and love-play of birds in the mating season. In other classes, the amphibia, for instance, the males and females are distinguished by certain peculiarities during the mating season in the spring and early summer. The male watersalamander acquires a head-comb, the male batrachian, callosities upon the thumbs.

Nussbaum's careful experiments have proved beyond all doubt that, in the case of animals with recurrent mating season, the internal secretion of the genital gland of the male has an enormous influence, not only upon the genital apparatus, but upon the rutting organs which are closely related to the processes of reproduction. In the brown land-frog, Rana fusca, the seminal vesicles, the thumb callosities, and certain of the fore-arm muscles undergo annual cyclic changes which accord with the rutting season. These organs swell up, become hypertrophied, and at the expiration of the spawning period undergo involution.

The removal of the testicle from one side has no effect upon the rutting organs, but if castration is performed during the period of sexual quiescence, the rutting organs will not develop at the next rutting season. Nussbaum introduced portions of testicle into the dorsal lymph-space of such castrated male frogs, with the result that the rutting organs began to hypertrophy in exactly the same way as those of uncastrated animals. The portions of testicle did not heal in but gradually became resorbed, while at the same time the seminal vesicles, thumb-callosities and forearm muscles diminished in size. These experiments prove beyond any manner of doubt that the testicles supply a material to the economy which promotes development ; in other words, they elaborate an assimilatory hormone.

In the course of further experiments, Nussbaum discovered that after resection of the nerve of one fore-arm, neither the muscle of that fore-arm nor the papilla and glands of the thumbcallosity of that side underwent hypertrophy at the spawning- 
period, but that those of the sound side hypertrophied as usual. He concludes from this that the testicular secretion stimulates certain nervous centres and certain groups of ganglia only, and that these produce, by means of centrifugal nerves, changes in the form and the metabolism of the organs innervated by them. It is not possible to adopt this assumption without further proof, however. As Pflüger points out, the arrest of the motility and sensibility of the vaso-motor and secretory nerves produced by resection, is sufficient in itself to account for the absence of hypertrophy. It is superfluous, at the very least, to assume that the internal secretion of the genital gland produces its effect, not directly, but by the agency of the nervous system.

In man, the secondary sex characteristics attain their full development at puberty, that is to say, at a time when the genital glands are fully developed, and the individual has attained to sexual maturity. It is apparent, from what has gone before, that the beginnings of the somatic sex characteristics are largely present in the embryo, and that, like other tissues, they continue to develop from birth until puberty.

It is a remarkable fact, however, and one which points to an intimate association of the sex stigmata with the genital glands, that several of the attributes of sex do not make their appearance until the maturity of the genital glands is proclaimed by the development of their external secretory processes (spermatogenesis, menstruation, ovulation). The maturity of the secretory function of the genital glands is shown, in boys, by a slight enlargement of the testicle. In girls, it is expressed by the menstrual hæmorrhage which accompanies ovulation, that is, the expulsion of the ovum ready for fertilization. Many of the changes of puberty are common to both sexes; such are the rapid skeletal growth and the appearance of hair at the pubes and the axillæ; others, such as the growth of hair on the face, the alteration in the voice, and the appearance of hairs all over the body, are peculiar to the male; while others again, such as the development of the mammary glands, the peculiar pelvic formation, and the deposition of fat in certain localities are essentially feminine.

Among the secondary sex characteristics which make their appearance at puberty, are those reflex nervous mechanisms which are concerned in the performance of the sexual act. Through the agency of the internal secretion of the genital glands, the apparently low nerve centres which are here concerned acquire a specific sensibility. Goltz showed that, during the spawning season, the male frog reacts to every touch upon the skin of the chest with the embracing reflex, and that this reflex may still be obtained shortly after removal of the testicles and even after decapitation. That the increase in reflex sensibility is not general, is proved by the fact that the muscle-contractions are not obtained in response to every kind of touch, but are only provoked by the 
specific stimulation of the skin; the reflex movement is confined, moreover, to a certain group of muscles.

But the changes of puberty are not confined to the lower centres of the spinal cord. That the functions of the cerebral cortex are also affected is shown by the occurrence of sexual desire and by the psychic changes which accompany the "storm and stress period."

That all the somatic and psychic characteristics of puberty are dependent upon the maturity of the genital glands, is rendered probable by coincidence; the fact is made certain by the results of castration.

Before passing on to this subject, however, it is expedient to give a short description of the condition known as pubertas pracox.

Both the older literature and that of recent date contain numerous descriptions of premature puberty, especially in girls. Those cases, formerly regarded as freals, in which girls of very tender age showed menstruation, development of the mammæ, growth of hair at the pubes and axillae, and excessive growth, have been repeatedly brought together and criticized (Kussmaul, Ploss, Gebhard, Strassmann, Neurath). According to Neurath (I909) the known cases of precocious menstruation are eighty-three in number, but the descriptions of these are not in every instance complete. Precocity in boys has been observed in forty-three cases, many of which have been wrongly described as gigantismus infantilis. The similarity between these cases and gigantism is apparent only, the sole common symptom being the early tallness of the subjects. The abnormal skeletal growth is the outcome of very different causes. Skiagrams show that, in pubertas præcox, there is accelerated ossification, and a rapid approach of epiphysal ossification to the stage when the synarthroses close (Neurath). This finding is in accordance with that of physiological puberty, for in the latter condition also, the proliferation of the epiphyses soon ceases. Gigantism, on the contrary, is characterized in all its forms by persistence of the epiphysal synarthroses and the abnormal height to which this leads.

Pubertas præcox virilis is a condition affecting boys of 3 to 9 years of age. These children are remarkably large, heavy, and well developed, and they show all the signs of puberty-commencing growth of hair on the face, pubes and axilla, deep roice, and strikingly large external genitals, the testicle being the size of a plum when in accordance with the age it should not exceed the size of a bean. The psychic development sometimes corresponds to that of puberty, though the subjects are more frequently mentally defective, or even idiotic.

The most important point in regard to the etiology of pubertas pracox is whether or not the condition is primarily the outcome of precocious development of the genital glands. We know that 
the development of the genital glands is influenced by a large number of internal secretory organs, and we have to take into consideration the fact that hypergenitalism, and the pubertic precocity which accompanies it, may be the secondary results only of the primary affection of other organs. This applies to cases in which tumours were present either in the suprarenals or at the base of the brain, and especially where these affected either the pituitary or the pineal glands. Hypophysal affections are frequently associated with perversions of growth in the direction of gigantism, though as a general rule these are accompanied by genital atrophy. The cases of pineal tumour which have so far been described show all the signs of pubertas præcox; namely, abnormal growth in height and premature genital and sexual development, with corresponding mental precocity. The presence of suprarenal tumour in sexual precocity is remarkably frequent. Out of seventeen cases examined post mortem and four surgical cases, suprarenal tumours were found in eleven, nine being girls and two boys (Bulloch and Sequiera, Linser, Adams). All these cases were remarkable for the exceptional development of the body, considerable obesity, and physical precocity, together with the habit. of sexually mature persons, more especially extreme hypertrichosis; the functions of the sexual glands and certain secondary sex characteristics, such as the mamma in girls, were not, however, developed in proportion. In many instances, single heterosexual characters were peculiarly well developed.

If we exclude these observations, a large number of cases remain which cannot be explained otherwise than by primary hypergenitalism.

Such is the case of the boy R. K., described by $W$. Knöpfelmacher (I903 and 1906), who, at the age of 6, was $\mathbf{I}_{32} \mathrm{~cm}$. in height, and weighed $38.8 \mathrm{~kg}$. The testicles were the size of a plum, the secondary sex characters were almost fully developed, the amount of hair at the pubes being especially remarkable, while there was a distinct moustache, and the voice was deep. Skiagrams (Schüller) showed that the sella turcica was normal in size, while the inner surface of the skull manifested no signs of intercranial pressure.

The case described by Sacchi is even more instructive. The patient was a boy of $9 \frac{1}{2}$, who, until the age of $5 \frac{1}{2}$, was both physically and mentally normal. At that age, his entire being underwent a gradual and progressive alteration. His bones grew very rapidly, and his muscles became very much developed; the voice became deep; hair appeared at the pubes and upon the face; and his mental condition changed. At $9 \frac{1}{2}$ years of age he was $143 \mathrm{~cm}$. in height, and weighed $44 \mathrm{~kg}$. : he had a long black beard and long hair upon the pubes, chest and legs. The left testicle, owing to tumour formation, was enormously enlarged. After removal of the diseased testicle, which the microscope 
showed to be an alveolar carcinoma, the pubertic symptoms gradually disappeared, and the boy became mentally and physically the same as other boys of the same age. In this case the connection between the premature signs of puberty and the pathological hypergenitalism is abundantly clear.

Before passing to a consideration of the influence which castration has upon the secondary sex characteristics, it is expedient first to consider the significance of that organ which is regarded as the pronounced characteristic of the female sex, but which has no recognizable relationship to the function of reproduction, namely, the mammary gland. This gland is present in the embryonal beginnings of both sexes; in new-born infants it is confined to a few ducts in the neighbourhood of the nipple, which may produce a transient secretion known as "witches' milk" (Hexenmilch).

A remarliable development of the mammary gland takes place in female individuals at the period of puberty, though this increase in size is largely the result of the deposition of fat. The enlargement during puberty of the mammæ of boys is probably entirely due to fatty deposition; and gynæcomastia-that is, abnormal development of the mamma in males-is probably due, in many instances, to testicular hypoplasia, or to atrophy of the testicles as the result of pathological processes. Males which show abnormal development of the adipose tissue at certain points of predilection, are to be regarded as eunuchs or semi-eunuchs. An extremely instructive instance of such pseudo-gynacomastia is described by Foges.

As far as the female mammary gland is concerned, there is no doubt that its development at the period of puberty is influenced by the genital gland. It has been proved experimentally that the mammary gland does not develop in animals which have been castrated in early youth; while normal development takes place after ovarian transplantation. That the influential factor is supplied by the ovaries and not, as the simultaneous uterine atrophy suggests, by the uterus, has been proved by experiment (Foges).

The periodical swelling of the mamma observed in many women during menstruation, and the occurrence of similar phenomena in animals during the rutting period, are undoubtedly attributable to the internal secretory activity of the ovaries.

The most important changes in the mammæ are those which take place during pregnancy. At the commencement of this state, what must be regarded as a true hypertrophy takes place, which continues until parturition.

There was at one time an all-prevailing view that the mammary hypertrophy of pregnancy was the result of communication, effected by way of the nervous system, between the mammary gland and the pregnant uterus; this theory has, however, long 
been disproved. An experiment by Goltz and Ewald is interesting in this connection. These authors found that, after extirpation of the entire lumbo-sacral portion of the spinal cord of a bitch, the mammæ underwent normal enlargement during pregnancy, and that lactation after parturition was also normal. Ribbert transplanted the mammary gland of a guinea-pig into the neighbourhood of the ear, and found that it hypertrophied during pregnancy and secreted milk after parturition.

There is, then, no doubt that the growth of the mammary glands during pregnancy is the result of a chemically active stimulant. The next step is to discover the source of origin of this hormone. Pregnancy is associated with changes in the ovary (formation of the corpus luteum verum) and in the uterus (development of the muscles and mucosa); the fotus, moreover, develops; and from the combined maternal and fœtal tissue the placenta, an organ by which the fotus obtains nourishment, is formed. The active hormone may be the product of any one of these four factors. Consideration of the clinical material which the subject offers led Halban to the conclusion that neither the ovary nor the uterus is the source of the hormone. In the first place, castration early in pregnancy does not prevent the hypertrophy of the mammæ, and castrated women are able to suckle their children; in the second place, the development of the mammary glands is constant in extra-uterine pregnancy, in which the uterine hypertrophy is slight; and in the third place, after extirpation of the uterus, secretion of milk takes place in the same way as under normal conditions.

It would appear from this that the cause of the hyperplasia must lie with the ovum, that is to say, with either the foetus or the placenta. From the fact that pregnancy changes take place in the mamma where the embryonic body is absent, as, for instance, in molar pregnancy, Halban concludes that the stimulus cannot be supplied by the fœtus; by a process of exclusion he thus arrives at the result that the source of origin of the hormone is the placenta, especially the trophoblast and the chorion epithelium. Similarly, he regards the growth of the mamma in the fœtus at the eighth lunar month, as due to reaction on the part of the fœtal tissue to the stimulatory action of the placental substance.

According to Halban, then, the substances supplied by the ovary and the placenta produce similar results, those produced by the placental substance being, however, much more intense. During pregnancy, when the ovary ceases to be active, its internal secretory function is undertaken by the placenta, by which it is very much intensified.

Starling and Lane Claypon endeavoured to discover the origin of the hormone by experimental measures. They injected extract of fœtus, ovary, and uterine mucous membrane under the skin 
of virgin rabbits, with the object of obtaining hypertrophy of the mammary glands. In six cases they actually succeeded in producing a certain degree of hyperplasia with foetal extract, but the experiments with extracts of placenta, ovary, and uterine mucous membrane were negative in result. In one case, the injection of the watery extract of 160 fœtuses was followed by the formation of secreting acini at the periphery of the mammary gland.

The conclusiveness of Starling's experiments is disputed by Halban, who regards the number as insufficient to establish proof; he also points to the possibility that the changes in the mammary gland observed after injection of fotal extract were in reality symptoms of rut. 'This pronouncement of Halban's induced me to submit the question to further experimental test, the experiments being undertaken with the aid of Dr. R. Königstein.

While our experiments were still in progress a communication of Foà's appeared, in which he confirmed the findings of Starling and Claypon, and supplemented them with the statement that the action of the hormone is not confined to the species from which it is derived. Thirty-five injections of an extract obtained from fresh fotuses from cows produced swelling of the mamma in a virgin rabbit, and on isolated occasions a secretion resembling milk was observed.

Our experiments, which will be fully described in another place, showed that neither the repeated intraperitoneal injection of placental extract, nor the implantation of fresh placentas of rabbits in the abdominal cavity, produced histological changes of any kind in the mammary glands of virgin rabbits. There was, however, a more or less marked development of the mammary glands of all virgin animals treated by means of intraperitoneal injection of embryonal extract or by the intraperitoneal implantation of one or more embryo rabbits. Numerous glandular ducts appeared, frequently with well marked branches, or, at the least, formation of buds; the lumen of the ducts became enlarged, containing in some instances a cellular secretion; the number of epithelial layers usually increased; in nearly all cases there was hyperamia; acini formation was isolated only; the excretory ducts were enlarged and much more fully developed. The intensity of these changes was in direct ratio to the amount of extract injected or of material implanted. The glandular hypertrophy was more marked in animals treated by means of injection than in those in which embryos were implanted.

The results of these experiments seem to show that, contrary to the view expressed by Halban, the fœtus is the source of origin of the hormone which stimulates the growth of the mammary glands.

K. Basch has recently published a work which is chiefly concerned with the experimental investigation of those cases 
among animals, in which hyperplasia of the lactic glands persists for a short time after parturition. Basch states that he was unable to obtain hyperplasia of the mammary glands of virgin animals by the implantation of placental tissue. But the implantation of the ovaries from a pregnant bitch under the skin of the back of a I-year-old nulliparous bitch, was followed, fourteen days later, by proliferation of the mammary gland of the latter; at the end of six weeks the enlargement was considerable; and after eight weeks secretion of milk was induced by the injection of placental extract. Microscopic examination revealed the perfect picture of a gland in full secretory activity, while at the same time the uterus retained its virgin character and the ovaries were very small. Basch concluded that the hyperplasia of the mammary gland in pregnancy depends upon certain chemical substances, which are developed in the ovary, and which prepare the gland for prolonged functional activity.

It is difficult to reconcile this assumption of Basch's with the observations of other authors, which show that the internal secretory activity of the ovary is inhibited during pregnancy. It is, moreover, inconsistent with the fact that the hypertrophy of the mammary glands is sometimes unaffected by castration at an early stage of pregnancy. That the enlargement of the mammæe at puberty and during menstruation is the result of ovarian influence, is not to be denied; but the cause of the hypertrophy of pregnancy must be sought, not in the ovary, but in the foetus which develops from the fertilized ovum.

Before solving the further question, namely, as to why the secretion of the hyperplastic gland does not commence until after parturition, we must consider what happens upon suppression of the ovum, that is, of both fœtus and placenta. It has long been known that after intra-uterine death of the ovum milk forms in the mammary glands and the lacteal secretion begins. The same thing happens if the foetus is removed by surgical methods during the second half of pregnancy. Halban concluded from this that the important factor lay, not in the death or removal of the fotus, but in the removal of the placenta, and that it was the biological suppression of the placenta which supplied the impulse to the secretion of milk. He and others find confirmatory evidence of this theory in the "witches' milk" of new-born infants, which they regard as the expression of the puerperal involution of the fotal organs, which had previously reacted to the conditions of pregnancy.

Basch found that, by the subcutaneous injection of placental extract, he was able to produce secretion of milk, sometimes in considerable quantities, in animals in which the enlargement of the mammæ had persisted after the termination of pregnancy; he regards the results of these experiments as proof of the dependence of the lactic secretion upon a substance found in the 
placenta. In so far as they illustrate the therapeutic uses of placenta secretin, these experiments of Basch's are both important and interesting, but they do not afford a satisfactory explanation of the mechanism by which the impulse to secretion. of milk after parturition is supplied. If we assume that this impulse is supplied by the placenta, we are faced with the further problem, namely, that during the entire course of its development in pregnancy, this organ fails to excite the secretion of milk in the mammary glands; and that its activity in promoting secretion is developed at the moment when its connection with the uterine wall ceases. If the secretory hormone were elaborated in the placenta, it would be active during pregnancy. This physiological inconsistence is not explained by the assumption (Keiffer, Basch), that the amount of secretion supplied by the placenta to the blood stream is very much increased during labour.

The secretion of the hyperplastic mamma is not difficult of explanation, if viewed in the light of the theory laid down in Part I. of this book. The growth of the gland is the expression of an assimilatory increase of material with simultaneous inhibition of disassimilatory decomposition. The growing gland produces no secretion, or very little (colostrum). With the suppression of the assimilatory hormone supplied by the fotus, that is to say, at birth or by termination of the pregnancy during the second half, disassimilatory decomposition, as expressed by the secretion, is enabled to proceed unchecked.

The mammary gland is an organ which is present in the primitive genital trace of both sexes; during intra-uterine existence it undergoes a certain degree of development, and later assumes the part of a secondary characteristic of the female sex, attaining to further development under the influence of the ripening female genital gland. The function of the mammary gland, so intimately associated with the process of reproduction, does not become apparent until after the formation lof the fœetus from the fertilized ovum, for the developing fœetus supplies a hormone which promotes hyperplasia of the glandular elements, and it is the suppression of this hormone which permits the onset of the secretory function.

\section{THE RESULTS OF CASTRATION.}

The operative removal of the sexual glands is followed by a group of symptoms which, both in man and animals, has formed a subject of exhaustive investigation. The results of castration differ to a certain extent according to the age at which the operation is carried out.

The castration of young males is one of the oldest forms of operation, and one which is still in frequent practice in the East. 
The castration of domestic animals, such as stallions, bulls, rams, cocks, \&c., is also frequently performed, and at an early àge.

The first result of prepuberal castration is the defective development of the genital apparatus. Post-mortem examination shows that the seminal vesicles and the prostate of eunuchs are small and atrophied. The penis does not, as a rule, become atrophied, and for this reason it is customary to amputate the penis of eunuchs employed as harem guards. Atrophy of the seminal vesicles and prostate is observed in castrated animals, especially in rats which have been deprived of their genital glands at the age of 4 to 6 weeks (Steinach).

That the removal of the testes not only produces inhibition of prostatic development, but leads to involution of the fully-formed organ, is shown by a large number of animal experiments as well as by Athanasow's careful histological investigations. These observations suggested castration as a therapeutic measure in the treatment of hypertrophy of the prostate; the method was first carried out, with good result, by Ramm of Christiania in I 893 .

The results which have since been obtained justify us in regarding castration as a rational method of treatment in prostatic hypertrophy and one leading to complete or partial cure. It is somewhat difficult, however, to find a satisfactory explanation of these results.

The inhibition of development in the case of the growing organ is readily explicable. The hormone supplied by the testis is essential to the normal development of the seminal vesicle and the prostate. Walker showed that prostatic atrophy may be prevented in castrated dogs by the subcutaneous injection of testicular extract. In the case of hypertrophy of the prostate, which usually appears at a time when the external secretory activity of the testis is reduced, we have to assume an increase in the testicular internal secretion. Otherwise we are compelled to refer the results of castration in this condition to the operation of other momenta. The suppression of the external secretion of the testis might also lead to the atrophy of disuse in the secondary genital organs. It was this view which suggested the operative treatment of prostatic hypertrophy by the removal of one testis, and by the ligature or resection of the vas deferens. Both methods were found to be unsatisfactory and have been abandoned.

The etiology of hypertrophy of the prostate is to-day much less obscure than formerly, when the internal and external secretions of the testicle were regarded as functions of the same tissue, namely, the spermatogenetic elements. Now that we know that Leydig's cells in the interstitial tissue of the testicle are the probable seat of its internal secretory activity, it is a rational supposition that senile hypertrophy of the prostate results from the over-production of the hormone in combination with a reduced 
external secretion; and that for this reason the total suppression of the testicles may lead to the involution of the enlarged prostate, a result which is not obtainable by the suppression of the testicular external secretion only.

The secondary male characteristics do not as a rule attain complete development where castration is performed before the age of puberty. Observation of man and of animals of widely different species shows, beyond any manner of doubt, that castration does not impart characteristics peculiar to the opposite sex, and that transformation into the heterosexual type is never observed (Tandler and Gross).

All eunuchs show one male skeletal characteristic, namely, the development of the margo supraciliaris. The pelvis, moreover, is not female, but infantile in type. The hair on the body is scanty and is absent from the neighbourhood of the anus, but is present, though in small quantity only, at the pubes and the axillæ. The face is usually hairless, though well-developed beard and moustache may be present, especially in old age.

The larynx of eunuchs is not of the female type, but resembles the enlarged organ of a child; there is no prominentia laryngea, and ossification has not taken place. For this reason the voice retains its childish character and in no way resembles a woman's voice; it is a childish soprano which, late in life, undergoes the changes in timbre and tone characteristic of mutation. The general increase in the fat-body, which is sometimes very striking, is the only feature which may give to the eunuch a somewhat feminine appearance. Moreover, the mental and psychic development after castration in no way approaches that of the female, though the quiet, phlegmatic temperament of castrated men and animals is very characteristic.

The dependence of the secondary sex characteristics upon the genital glands is well seen in the domestic fowl. The cock, the male bird, is distinguished by well differentiated sexual signs, and it has been customary from the earliest times to castrate the cock in order, for domestic purposes, to produce the capon, in which the male characteristics are merely rudimentary. That there is a direct relationship between the genital gland and the sex characters of the cock, is shown by Berthold's experiments, but the incontestable proof of this was reserved for more recent researches. It has further been shown by various authors (Rieger, Sellheim, Foges) that the capons of commerce are not physiological capons; that is to say, they are not eunuchs, but animals produced by a combination of factors; namely, attempted, but rarely complete, castration; simultaneous removal of the primitive origin of the external sex characteristics; and forced feeding.

It may be remarked in passing that Sellheim showed that castration is never really carried out with hens, for the reason 
that complete extirpation of the ovary is impossible. Poulards are not castrated birds, but hens which have been crammed before arriving at sexual maturity, the oviduct being severed at the same time, so that the eggs are deposited in the abdominal cavity.

The true capon type is characterized by shrivelling of the comb, wattles and spurs; poor development of the neck and tail feathers; hoarse voice; and excessive deposit of fat. This type is obtainable only by the complete castration of young cocks. Whether castration is perfect or not is revealed only post mortem. Where the extirpation of the testicles is incomplete, the secondary characters of the cock are retained; in cases where these have already attained a certain degree of development they will continue to develop, provided that even a minimal amount of active testicular parenchyma is left in situ. Where, however, the amount of tissue is less than minimal, the secondary characters will not develop (Foges).

Transplantation of the entire testicle is invariably unsuccessful in castrated animals, and this explains the negative results bo R. Wagner's experiments. According to Foges, it is possible to get small portions of testicle to heal in so well, that months later they contain living spermatozoa. Castrated cocks with transplanted portions of active testicular tissue, do not acquire all the characteristics of normal cocks, but they cease to be true capons. The wattles and comb still retain their capon-like character, but the plumage and the entire external appearance of the bird, with the exception of the head, resembles that of the true cock. Whether the incomplete character of the male stigmata is due to the small size of the implanted portions of testicular tissue (Foges), or to absence of communication between them and the vas deferens (Nussbaum), is at present unknown. The first view finds support in the fact that A. Löwy observed a better growth of the comb and wattles in capons fed with testicular substance than in the control birds.

In the case of other animals (cattle, cervidæ), the invariable result of castration is insufficient development of the secondary sex characters, never transformation into the heterosexual type. A castrated cock never adopts the habit of a hen, a castrated bull never resembles a cow, and in the same way castrated men never resemble women.

When viewed in the light of the fact that cessation of the function of the genital glands, whether physiological or pathological, produces heterosexualism, the fact that castration never produces the positive characteristics of the opposite sex but results in a certain fixation of the infantile type, supplies a weighty argument in favour of the hermaphroditic origin of the sexual glands.

The information which we possess concerning the results of castration before the age of puberty in women, is extremely 
scanty. Roberts's observations in India have little value in this connection, for the operation in his cases consisted in mutilation of the external genitals only. It is known from the results of experiments with animals (Hegar, Kehrer), that if both ovaries are removed from female animals which have not reached sexual maturity, the development of the entire genital apparatus will be retarded, the uterus and Fallopian tubes remaining rudimentary. By transplanting the extirpated ovaries under the skin, Halban effected perfect development of the sexual apparatus, thus proving that the protective influence of the ovaries is effected, not by means of trophic nerves, but by the agency of an internal secretion.

Castration is frequently performed on women who have passed the age of puberty. It is common knowledge that this operation is followed by regressive changes in the secondary genitals, shrivelling and atrophy of the uterus and vagina being invariable findings. This atrophy was formerly explained by the suppression of a trophic nerve centre supposed to be present in the ovary. But Knauer's experiments have shown that, if the ovaries are removed from their place and implanted in another part of the body, atrophy of the uterus will not take place. Further experiment (Ribbert, Grigorieff, Rubinstein, Herlitzka, Marshall and Jolly, Carmichael and Marshall, Daels, \&c.) next showed that the presence of even a portion of one ovary in any part of the body is sufficient to maintain the complete anatomical integrity and functional activity of the genital organs. Pregnancy may even occur after intraperitoneal transplantation of the ovaries, and this result is a measure of the success which may be obtained by ovarian transplantation in women.

The implantation of ovaries from animals of another species has been repeatedly performed with successful results (Schultz, Katsch, Carmichael and Marshall, Bucura). These ovaries (for instance, those of the guinea-pig in a castrated rabbit, Bucura) not only heal in but remain functionally active, for their follicles become mature and atrophy of the uterus does not take place. The implantation of testes into a female animal succeeds to this extent, that the spermatogenetic tissue elements of the implanted organs remain intact and spermatozoa may even be produced, but the presence of the male genital gland does not prevent uterine atrophy. The implanted testicle does, however, exercise a certain influence upon the metabolism of the castrated female, the increase in the weight of the body being considerably less in these cases than after simple castration.

The subcutaneous injection of ovarian extract, whether from the same or another species, does not prevent uterine atrophy after castration (Jentzner and Beuthner). Bucura found that the injection of ovarin into castrated animals was followed, not only by atrophy of the muscles of the uterus, but by an increased dis- 
appearance of the interfascicular connective tissue. The exhibition of ovarin merely reduces, to a certain extent, the obesity which follows castration.

The influence which the ovary has upon the uterus is further shown by the fact that, after cessation of ovarian activity, menstruation ceases in women, and the analogous rutting symptoms in animals disappear.

That menstruation is dependent upon the ovary was first suggested by Sintema in the 18 th century, though upon no positive grounds. It was Bischoff (I844) who first suggested, upon histological grounds, that ovulation, that is the periodical maturity and release of the ovum, was coincident with menstruation, and that the latter was an expression of ovulation. Pflüger (1865) explained this relationship as follows : Stimuli accumulate in the maturing Graafian vesicle which, acting reflexly through the nerves, bring about a superfluity of blood in the genital organs; this causes the bursting of the follicle and release of the ovum on the one hand, and on the other, the discharge of blood from the uterus.

Strassmann succeeded in producing symptoms resembling rut by increasing the blood-pressure in the ovaries of bitches, and these results seemed to confirm Pflüger's theory.

But isolated findings in both man and animals suggested the possibility that the relationship between the ovary and the phenomena of menstruation and of rut, was effected by a chemical agent. Halban's experiments with apes proved that this assumption was well founded. Female baboons (Cynocephalus) have a menstruation similar to that of the human female. When the ovaries of these animals were extirpated and implanted under the cuticle of the abdomen, menstruation occurred in the same way as under normal conditions; it ceased, however, as soon as the implanted ovaries were removed. According to Marshall and Jolly, the implantation in castrated bitches of ovaries in a state either of, or preceding, rut, or the injection of the extract of such ovaries, is followed by swelling of the vulva and other phenomena of the rutting state.

It has frequently been found possible to maintain the process of menstruation in women by the re-implantation or transplantation of the ovaries (Cramer, Pankow).

van der Velde's statement that menstrual hæmorrhage may be procured at the physiological climacteric by means of ovarian tabloids, must be accepted with a certain amount of reservation.

It is evident then, from what has gone before, that the hormone which is formed in the genital glands and by them passed on into the blood-stream, supplies stimuli which are necessary to the normal development and subsequent anatomical integrity of the genital organs, as well as to their physiological activity.

It is certain, however, that the influence of the genital glands 
is not confined to the organs which are functionally related to them; that this influence extends to other physiological activities is shown by the following observations.

The menstrual flow is sometimes accompanied by certain nervous phenomena known as the molimen menstruale, and by regular fluctuations affecting different functions, such as temperature, pulse, blood-pressure, and muscular power. For this reason it is customary to speak of "undulations" in the female vital processes. These menstrual waves are not dependent upon the periodic loss of blood nor upon the activity of the uterus during menstruation; for the phenomena persist for a varying length of time after hysterectomy. In such cases, moreover, molimen menstruale without menstruation is also sometimes observed. But both menstruation and the menstrual wave disappear after suppression of the ovarian function, as at the menopause; after the operative removal of the ovaries; and as an afterconsequence of hysterectomy (Mandl and Bürger).

It is a common clinical experience (Chrobak, Landau) that the symptoms which follow cessation of the ovarian function, all the derangements, in fact, by which the climacteric is accompanied, are favourably influenced by the exhibition of ovarian extract. Bucura has recently recommended the milk of rutting cows in the management of the physiological or artificial menopause.

\section{THE SEXUALLY UNDIFFERENTIATED ACTIVITY OF THE HORMONE IN THE GENERATIVE GLANDS.}

The influence of the internal secretion of the generative glands is not confined to the development of the secondary somatic sex characteristics, but has a far-reaching effect upon a large number of organs and upon the general metabolism. The profound influence which this hormone has upon the organism is apparently independent of sex, and is thus not specific to the male or the female genital gland.

Of paramount importance is the effect of the genital glands upon the growth of the skeleton. That this is the case in man (eunuchs, and young males with atrophied testicles) was known to, and pointed out by, the older authors. Sellheim found that the castration of cocks has a modifying effect upon the growth of the bones. Changes take place in the skull, in the pelvis, and in the bones of the extremities; and these consist in increased longitudinal growth with retarded ossification of the epiphysal cartilages. Similar changes were afterwards observed by Sellheim in castrated dogs, horses, and cattle; and his results are confirmed by Poncet, Briau, Pirche, Möbius, and many others, whose observations extended to animals of other species. Poncet found that the entire skeleton was more powerfully formed; that the skull, 
as in eunuchs, was dolichocephalic; and that the epiphysal cartilages persisted longer than in normal animals.

The experiments of Launois and Roy and those of Tandler and Gross supply undoubted proof that in man, as in animals, castration is followed by excessive longitudinal growth, a lack of proportion between the length of the extremities and that of the trunk, and persistence of the epiphysal synarthroses (as seen in skiagrams) beyond the normal age.

Protracted epiphysal separation may result not only from the operative removal of the generative glands, but also from hypoplastic sub-development of them. The presence of this symptom in combination with the persistence of other juvenile traits, is described as immaturity of the organism or eunuchoidia (Tandler and Gross). These cases do not altogether fall into the group of pathological conditions classed as infantilism. For infantilism is characterized by the small size of the skeleton and its infantile proportions, namely, long trunk and short extremities. But hypoplasia of the genital glands is presumably accompanied by symptoms analogous to those of suppression; namely, abnormal longitudinal growth, especially in the legs, and considerable increase in the fat-body. Thus the only true cases of hypogenitalism would be those of infantile gigantism, already described, which are characterized by abnormal growth of the long bones, imperfect secondary sex characteristics, and deficient mental development; and in which the testicular atrophy and the absence of any signs of pituitary disease justify the assumption of primary hypogenitalism. Several authors (Alt, Schüller) ascribe mongolism to insufficiency of the genital glands. Moreover, genital insufficiency may well play a part in the pathogenesis of general hypoplasia (Bartel), for these cases are also characterized by abnormal height, well developed fat-body, and imperfect secondary sex stigmata.

That pregnancy is accompanied by reduced ovarian activity is shown by the changes which take place in the skeleton. Young women frequently grow in height during pregnancy (Halban), and changes are known to take place in the pelvis in this condition (Breuse and Kolisko).

According to Tandler, the age at which genital maturity takes place has a paramount influence upon the growth of the skeleton. The effect of late maturity, like that of genital hypoplasia, is to increase the height, and especially the length of the legs; while early maturity brings about premature closing of the epiphyses, and is consequently associated with shortness of the legs. In women, the upper part of the body is usually long in proportion to the legs, and inhabitants of warm countries are generally small of stature; these results are attributable to early sexual maturity. Among animals, those which develop early are characterized by the shortness of their extremities. 
In pubertas præcox, the acceleration of growth is accompanied by premature ossification and closing of the epiphysal synarthroses (Neurath). In chlorosis, precocity is said to be expressed by shortness of the legs and premature disappearance of the epiphysal synarthroses (Tandler).

A certain amount of evidence is also forthcoming concerning the effect upon growth of the adninistration of extracts of the genital glands.

Löwy showed that the skeletal pecularities described by Sellheim are not observed if young capons are given testicular substance, and that a premature arrest of skeletal growth may be obtained in young hens by feeding them with ovarian substance. Dor and Maisonave, Parhon and Antoniu, and Monziols describe inhibition of bony growth after the subcutaneous injection of testicular extract.

These observations all prove that there is an intimate relationship between the development of the skeleton and the internal secretory activity of the genital glands. In order to arrive at a fuller understanding of this relationship, it must be borne in mind that the increased skeletal growth is a secondary result, due to the imperfect ossification of the epiphysal synarthroses; this fact suggests that the genital glands elaborate a hormone which stimulates the processes of ossification.

The interrelationships which subsist between the genital glands and those other internal secretory organs which are known to influence the growth of the bones, are also very remarkable. This nexus finds expression in the fact that castration is followed by changes in the thyroid, thymus, and hypophysis; and that, further, the removal of the latter organs produces alteration in the structure of the genital glands.

In a eunuch dissected by Tandler and Gross, the thyroid apparatus was found to be remarkably small (weight $\mathrm{I} 3 \mathrm{grm}$. as against $45.8 \mathrm{grm}$. in normal persons) while thymus-persistency, so frequently observed in animals after castration (Calzolari, Henderson, Noel Paton), was observed in this instance also. By means of skiagrams of living scopts these authors further discovered hypophysal enlargement, their results being confirmed by the fact that in the eunuch which they dissected there was increase in the length, breadth, and depth of the sella turcica.

That the removal of the genital glands is associated with an increase in the size of the hypophysis was first shown in the case of animals by Fichera (1906), who succeeded in rapidly reducing this enlargement by the injection of testicular extract.

It is very difficult to estimate the extent to which the skeleton is directly influenced by the genital glands, owing to the fact that the thymus, thyroid, and hypophysis all affect the growth of the bones, their combined influence being extremely complicated, in part antagonistic, and in part co-operative.

The same applies to the influence of the genital glands upon 
certain other organic systems. It is only in isolated instances that we are acquainted with the mechanism by which the sexual glands produce changes in localities far removed from them. Thus, we are justified in ascribing certain developmental changes of pregnancy, which are reminiscent of acromegaly, to primary hypogenitalism and secondary hyperpituitarism; while other symptoms, we know, are due to secondary changes in the thyroid apparatus.

Whether directly, or indirectly through the agency of other hormone-producing organs, the genital glands exercise an influence upon the entire habit, as well as upon many of the functions, of the animal organism. With the maturity of the genital organs the juvenile habit makes its first appearance, and this is expressed by a youthful freshness and vigour of the entire being, affecting both the physical and mental capacities. The progressive physiological diminution of the function of the genital glands is accompanied by senile changes in the cuticle and cuticular glands, in the hair and teeth, and in the muscular and nervous systems. Castration is followed by changes, even in young persons, which largely resemble those of senile degeneration; and it is evident from this that senility, whether physiological or premature, bears a relationship to hypofunction of the genital glands. As we have shown in a previous chapter, senile decay is associated with regressive changes in other internal secretory organs, and especially in the thyroid. There is, however, no justification for ascribing a preponderating influence to one or more internal secretory organs in the causation of senile cachexia, seeing that we have as yet no definite information concerning the sequence of the different processes.

The effect which the genital glands have upon the general metabolism is very marked. That castration leads to increased deposition of fat is one of the oldest pieces of human knowledge, and has been utilized since very early times in the fattening of animals for market purposes. That the localization of the fatty deposit is dependent upon the sexual differentiation of the developing genital glands, and that it represents in part a sexual characteristic, is shown both at and after puberty.

The suppression of sexual activity, in women during pregnancy and at the menopause, and in men with advancing age, is accompanied by increased deposition of fat, the localization of which is peculiar and, to some extent, characteristic in both sexes. Certain forms of obesity, such as are observed in early childhood and in boys of feminine habit with shrivelled or cryptorchidic testes, and which are frequently associated with imbecility, should probably be regarded as the outcome of primary hypoplasia of the genital glands. It is frequently, however, a matter of extreme difficulty to distinguish between obesity of genital origin and that provoked by changes in the hypophysis. 
Exceptional obesity of castrated males is not invariable in man; and cases of abnormal leanness have been observed. Nevertheless, the obese type is very frequent among eunuchs and scopts, the entire fat-body being markedly over-developed and, at certain sites, such as the mammæ, the ventral region, the nates and the hips, attaining colossal proportions. Conspicuous obesity is observed in women both after castration and at the menopause, the percentage of cases in both classes being 42 to $5^{2}$ per cent.

With the object of determining the nature of this change in the nutritional conditions, as well as of discovering the cause of the abnormal deposition of fat-which was presumably to be sought in the changed manner of life, both physical and mental, the alteration, in fact, of the entire temperament-Löwy and Richter estimated the total metabolism (i.e., consumption of oxygen and excretion of carbonic acid) of castrated dogs of both sexes. They found that, after castration, metabolism was reduced in the proportion of 14 to 20 per cent. per kilo of body-weight, and that this reduction was maintained for months or even years. They found, moreover, that not only was there a fall in metabolism in proportion to body-weight, but that there was a considerable reduction in the total metabolism apart from body-weight, the latter frequently attaining a higher figure as the result of increased adiposity. The authors concluded from these results that the reduction of metabolism after castration is due to a diminution of the processes of oxidation; that the obesity is not necessarily degenerative, but may be constitutional.

Pächtner (1906) confirmed the results of Löwy and Richter. Lüthje obtained different results from his experiments with a dog and a bitch, but this is explained by the fact that obesity after castration is by no means invariable, being present in only $5^{\circ}$. per cent. of the cases under observation. L. Zuntz's statement that, of four castrated women, only one showed reduced oxidation during the resting stage, also offers no evidence against the theory of Löwy and Richter, for Zuntz's subjects did not become obese. This theory is very strongly supported, however, by the discovery that it is possible to raise the depressed metabolism to as much as 30 to 50 per cent. above the normal by the exhibition, either subcutaneously or by the mouth, of ovarian or testicular substance. In normal, sexually, mature animals, the exhibition of these substances is entirely negative. In the case of castrated animals, both male and female, the ovarian substance was found to be the more active, the effects of testicular substance being slight in males and negative in females.

As far as the influence of the sexual glands upon the metabolism of albumin is concerned, the experiments of Lüthje, Neumann, Vas, and others show that at no period of life does castration lead to changes in the metabolism of albumin; and that the increased excretion of nitrogen observed by certain 
authors after the exhibition of ovarian substance is to be ascribed to increased administration of food containing nitrogen.

These experiments prove, then, that the increase in the general metabolism which follows the exhibition of ovarian or testicular substances is entirely due to the decomposition of substances in the body which do not contain nitrogen.

In view of the relationship which has been assumed between osteomalacia and the genital glands, a certain interest is attaching to the influence which these organs exercise upon the metabolism of calcium and of phosphoric acid. The results which have been obtained up to the present are inconclusive. Curatolo and Tarulli, Neumann and Vas report reduction in the excretion of calcium and phosphorus after exhibition of ovarian substance; while Falk and Schulz, as well as Lüthje, were unable to demonstrate any change in the metabolism of calcium, phosphoric acid, and magnesia.

Heymann's finding, that in ovariectomized rats there is a marked progressive reduction in the total phosphoric acid contents, especially of the bones, seems to contradict the well-known fact that, in osteomalacia, castration inhibits the softening processes in the substance of the bones, and thus brings about a cure.

The pathogenesis of osteomalacia, which is still very obscure, requires a few words in passing. Fehling's theory that this disease originates in pathological hyperfunction of the ovaries, appeared to derive a certain support from the favourable effects which were observed after castration. It is, however, rendered practically untenable by the fact that the minutest histological investigation has repeatedly failed to prove that osteomalacia is accompanied by specific changes in the ovary, whether in the follicular apparatus, the germinal epithelium, or the corpus luteum. Wallart has recently observed peculiarly marked development of the interstitial stroma cells in cases. of osteomalacia in both pregnant and non-pregnant individuals.

The discovery of the interrelationships which subsist between the different internal secretory organs, especially between the ovaries and the thyroid and suprarenal glands, suggested the thyroid or suprarenal as the primary agent in the pathogenesis of osteomalacia. The thyrogenic theory was first propounded by Hoenicke (1904), and afterwards worked out by Parhon and Goldstein. That this hypothesis rests upon very insufficient foundation is shown by the fact that its authors have been unable to decide whether osteomalacia is the manifestation of hyper- or hypo-thyroidism.

Even more unsatisfactory is Bossi's theory, which explains the pathogenesis of osteomalacia by an affection of the suprarenals. That there is a relationship between the sexual glands and the suprarenals, or rather the suprarenal cortex, is suggested by the results of certain observations. There is no evidence to show 
that the suprarenal cortex has a direct influence upon the metabolism of the bones. The results of my own experience lead me very strongly to contest Bossi's statement that total or partial epinephrectomy is followed in animals by changes in the skeleton resembling osteomalacia in man. The sole evidence in favour of Bossi's view is the fact, which he observed and others have-since confirmed, that the subcutaneous injection of adrenalin produces favourable results in osteomalacia. It is a question, however, and one which must for the present remain unanswered, whether this result is due, less to the pathogenetic significance of the suprarenal, than to the pharmacodynamic action of adrenalin.

In the present obscure state of our knowledge, we are not justified in regarding osteomalacia as the expression of the pathological affection of a definite internal secretory organ or organs. We can only assume that several of these organs are concerned in the production of osteomalacia; that the principal rôle is played by the ovary (perhaps, also, as Bucura suggests, the parovarium) and, judging by the incidence among men, by the testis.

The supposed relationship between the ovarian function and chlorosis led to investigation of the effect of castration upon the composition of the blood.

Pinzani found that after castration there was increase of the hæmoglobin and red corpuscles in the blood of bitches, while Lithje was unable to observe any change in these elements. Breuer and Seiler carried out very exact experiments with bitches which had been castrated at the commencement of sexual maturity; they found that there was an invariable reduction in the hamoglobin contents and in the number of erythrocytes, and that this reduction disappeared again in the course of a few months. Unlike the conditions which obtain in human chlorosis, it was found that, in experiments with animals, the pigment contents always diminished in direct proportion to the number of red corpuscles.

From the earliest times, the ovaries have been credited with a pathogenetic significance in chlorosis, and the condition has been supposed to result from ovarian anomaly expressed by nervous or humoral agency throughout the entire organism. Similar views were held by the great physicians of antiquity, Hippocrates, Galen, and Avicenna. The theory which prevails to-day is that chlorosis is the manifestation of an alteration in the internal secretory activity of the genital glands. The nature of this alteration-whether hyper- or hypo-function-is, however, still unknown. The premature disappearance of the epiphysal synarthroses, as seen in skiagrams, is regarded as a sign of genital precocity (Tandler). Of the later theories the most interesting are those which attach a special significance to individiual tissue elements of the ovary. According to Wallart, there 
is a connection between the internal secretion of the so-called interstitial ovarian gland and the formation of the blood; while Villemin regards chlorosis as a toxic condition, due to the presence of the internal secretion of the corpus luteum, which has failed to discharge itself with the menstrual flow. This theory is suggested by the lipoid contents of the corpus luteum and the known hæmolytic effect of the lipoid substances. There is yet another hypothesis to which reference must be made in passing, that, namely, of the polyglandular origin of chlorosis.

\section{EXTRACT OF THE GENERATIVE GLANDS.}

It was by means of the peculiar and characteristic activity of testicular extract that Brown-Séquard obtained the first proof of internal secretion, and it is proposed, for this reason, to enter rather more minutely into the nature of the activity of the genital substances.

Since that date, liquide testiculaire has found therapeutic application in a large number of instances, but the scientific value of these experiments is very small. The same may be said of "spermin," first prepared by Poehl. The formula of this substance is $\mathrm{C}_{5} \mathrm{H}_{14} \mathrm{~N}_{2}$; it is not identical with the base, the phosphates of which form perfect crystals, which Schreiner originally discovered in seminal fluid, but which has since been demonstrated in other organic fluids. According to advertisements, this "Sperminum Poehl" acts as a "physiological catalytic" and ferment of intraorganic oxidation, and in this capacity is said to improve the general condition and increase muscular energy and cardiac activity, to promote appetite and sleep, and, above all, to stimulate sexual activity. Up to the present, exact and carefully controlled experiments with this substance have not been described. It has been largely employed by clinicians, but in estimating the results which in certain instances have been obtained, the suggestive influence of such measures is a factor to be reckoned with.

The influence of testicular extract upon muscular function has been demonstrated by Zoth and Pregl, by means of conclusive experiments (I896). These authors systematically excluded every possibility of error, including that of suggestion, and they found that the subcutaneous injection of orchitic extract, continued over a period of weeks, did not produce an increased capacity for muscular energy; but that when combined with muscular exercises, there was an increase of as much as 50 per cent. in the muscular performance, as shown by the ergograph and by dumb-bell experiments.

The other effects of the genital substances have been discussed in a previous chapter, where it was shown that the administration of these substances usually abolishes symptoms of suppression, 
and that both metabolism and the functions of certain individual organs may be intensified by this means.

According to the experiments of G. Loisel, extracts of the ovaries and testicles of fishes, frogs, birds, and mammals, all contain toxic substances and, when injected subcutaneously, these extracts may provoke serious pathological symptoms or even death (frogs, rabbits). Extracts of birds' eggs and, according to Lambert, extract of the corpus luteum may produce toxic conditions when injected under the skin of frogs.

The lesions produced in the Graafian follicles of normal animals by the repeated subcutaneous injection of ovarian extract obtained from animals of a different species, or even to a certain extent of the same species, are also very remarkable. The follicles may indeed be completely destroyed, while the rest of the ovarian tissue remains unchanged (Bucura).

The effect described by certain authors which extracts of the genital glands have upon the circulation of normal animals can hardly be regarded as physiological. According to Baum, spermin produces local vaso-dilation. According to Hallion, extract of dried ovaries, when injected intravenously in quantities of $5 \mathrm{mg}$. into dogs, produces a fall in arterial pressure with reduction in the volume of the kidney and of the nasal mucosa; at the same time the thyroid gland undergoes a preliminary diminution, followed by an enormous increase in volume, which is said to result from active vaso-dilation. Hallion regards the effect of ovarian extract in producing vaso-dilation within the thyroid as specific. I have carried out very careful hæmodynamic tests with ovarian extracts prepared in a variety of ways, but, personally, I have never been able to confirm this finding. The results which I obtained were never specific, but invariably pointed to the presence in the extracts of substances which promoted coagulation.

Serralach and Pares have made an interesting discovery, namely, that the internal secretion of the testis plays a part in the production of the genito-vesicular reflex. It is well known that the ejaculation of semen is accompanied by the muscular closing of the neck of the bladder, and by a relaxation of the bladder walls, the capacity of the bladder being by this means enlarged and the discharge of its contents at the same time prevented. Serralach and Pares found that the intravenous injection of a glycerine emulsion of testicular substance produces the same changes in the bladder as the genito-vesicular reflex.

The chemical constitution of the genital hormone has received as yet but little attention. A. Löwy's investigations are the first in this field of inquiry. They show that the active substance of the ovary must be an extremely labile body. The best effects are obtained with the dry substance, even after ethereal extraction; glycerine extracts are also very active; extracts obtained with 
physiological saline solution are very much weaker; and after precipitation with acids the effects are very uncertain. Alcohol seems to destroy the active principle.

According to Dixon (I9OI) the active constituents of testicular extract are nucleoprotein and certain toxic bases. Loisel arrived at a similar conclusion, as the result of his experiments with the ovaries and testes of animals of different species.

\section{THE INTERNAL SECRETORY TISSUE ELEMENTS OF THE GENERATIVE GLANDS.}

Although the correlative chemical activity of the generative glands has long been known, the question as to whether this internal secretion is the property of histologically differentiated portions of the organ and which tissue elements are the ones concerned, is of very recent date. The problem first arose about ten years ago, but has only latterly formed the subject of investigation. It was very generally assumed, though upon no very definite grounds, that the secretory elements of the sexual glands performed a double function; that they elaborated the specific external secretion, which was carried off by the excretory ducts; and that they also produced specific substances, which were conveyed by the lymphatics or the vessels into the general circulation, and which became active in distant parts of the organism. The genital glands were regarded as typical instances of glands with two surfaces, in which the epithelial cells elaborate both the internal and external secretions. In I906, Nussbaum adopted the theory that the generative portion of the testis is the site of production of the hormone. The coincidence of the development of the rutting organs with the most rapid development of the spermatic cells, suggested that it was the secretion of the spermatogonia, namely, the spermatides, which at the rutting season supplied the impulse to the development of the seminal vesicles and other rutting organs.

It had long been known that, in addition to the seminiferous tubules, the testis contained other morphological elements, but owing to the nature of their genesis and structure, these were not suspected of having an internal secretory function. It was not until after the most searching investigation in comparative morphology, chiefly by Bouin and his co-workers, that the biological differentiation of the elementary constituents of the male sexual gland was recognized.

The situation in regard to the internal secretory element of the ovary was more favourable. In this case also, it was at first assumed that a double secretory function was performed by the generative portion of the gland; but the persistence of the Graafian vesicle after the completion of ovulation suggested that the internal secretory function of this organ was not simultaneous 
with, but subsequent to, its external secretory function. Prénant (1898), and later Born and Fraenkel, recognized that both in genesis and structure the corpus luteum, which persists after the ejection of the ovum and is of epithelial origin, possesses the characteristics of an internal secretory gland. Later on, another ovarian tissue formation of connective tissue origin, which had long been known to histologists, but was first minutely investigated by Limon (1902), was recognized by Bouin as possessing internal secretory functions.

In describing the secretory elements in detail, it is expedient that the male and female glands should be treated separately.

Testis.-The germinative portions of the testicle are the seminiferous tubules which, in addition to the spermatic cells (spermatogonia, spermatocytes, spermatides and sperma), also contain the syncytium of Sertoli's cells. In addition to these, however, the testis of all mammals contains peculiar elementary constituents which are known as " interstitial" cells or "Leydig's betweencells." These cells have formed the subject of repeated investigation and it has been shown that they are characterized by special genetic and structural features. They are of mesodermal origin and appear in the primitive genital trace at a very early phase of embryonal development, before the seminal cells have assumed their cytological character. Enclosed in the cytoplasm of Leydig's cells are granules which stain with osmium and hæmatoxylin-copper-lake (Regaud), acidophile and basophile granules, pigment granules, and peculiar cell-crystals; and these enclosures give them a structural resemblance to secreting glandcells. Leydig's cells are situated in the interstitial tissue between the seminiferous tubules; they are sometimes few in number, but they may be present in extensive clusters, especially where, owing to lack of development or atrophy of the seminiferous tubules, there is room for fission to take place.

These between-cells were universally regarded as trophic tissue elements, whose function it was to take up nutrient material from the blood-vessels, and pass it on to the Sertoli's cells. This theory received fresh support from the discovery by Regaud (Igor) that a substance passes from Leydig's cells to Sertoli's cells. Reinke, however, who had proved that the cell crystalloid discovered by him in the human testis passes into the capillary lymphatics, suggested as early as I896 that Leydig's cells might elaborate an internal secretion which plays an important part in the production of sexual instinct. Regaud and Policard, Loisel, Mosselmann and Rubay also ascribed the internal secretory function of the testis to this tissue element, but Bouin and Ancel were the first to describe this tissue as the "glande interstitielle du testicule." In a large number of publications (1903-I904), these latter authors brought forward the proofs in support of their hypothesis, that this interstitial gland performs all the functions 
with which the internal secretion of the testicle has up to now been credited.

This ingenious theory was based, partly upon observations of the cryptorchidic testis, and partly on the results of experiment. In the greater number of instances (man, pig, horse, dog, ram) of cryptorchidic testicle, there is not only malplacement, but also, as histological investigation shows, complete absence of spermatogenesis; the cells of Sertoli in the seminiferous tubules are well preserved and the cells of Leydig are more or less developed. That animals with bilateral cryptorchidism are sterile has long been known to breeders, but it is equally well known that such animals possess normal, or even excessive, sexual instinct and that all the male sex characteristics are well marked. The external genitals and supplementary glands are fully developed. Where the cryptorchidism is unilateral, the removal of the normal testicle renders the animal sterile, but it retains its male appearance and habit. The completely castrated stallion is, in Germany, called "Walach," while the bilateral cryptorchis, or the unilateral cryptorchis from which the normal testicle has been removed, is called "Klopfhengst." Cryptorchidic individuals in man invariably show normal development of the external genitals and male secondary stigmata.

Later histological investigation (Felizet and Branca, Cunnéo and Lecène, Nielsen, Tandler, \&c.) confirms the finding that cryptorchidism is invariably associated in both animals and man with suppression of spermatogenesis, defective development of the seminiferous tubules, and apparently normal development of the cells of Leydig.

Similar changes are produced in the testicular tissue by experimental ligature of the vas deferens. Spermatogenesis ceases; the spermatocytes, and later the spermatogonia, degenerate and, at the end of a few months, they disappear. There is at first no change in Sertoli's cells of the seminiferous tubules, and the cells of Leydig retain their morphological integrity. No change is produced, however, in the manifestation of sexual instinct, nor in the development of the secondary signs of sex. Ligature of the vas deferens has frequently been performed by veterinary surgeons in place of castration, but as the animals retained the characteristics of the stallion, the results up to now can hardly be regarded as satisfactory. Similar results have occasionally been observed in cryptorchidic animals from which, in place of the testicle, the enormously developed epididymis was removed.

According to Shattock and Seligman, ligature of the vas deferens of sheep and poultry does not prevent the development of the secondary sex characters.

Further evidence in support of Bouin's theory is supplied by the results of clinical observation. Serious changes in the epidi- 
dymis (tumours, inflammation leading to atrophy of the vas deferens) do not inhibit sexual instinct nor do they affect the male secondary traits.

These observations prove that the internal secretory function of the testis does not lie in the generative elements. The question which now arises is, in which of the two remaining elements, Sertoli's cells or the cells of Leydig, is this function lodged. In the hope of solving this problem, Bouin and Ancel endeavoured by experimental measures to separate the functions of these two groups of cells. Their course of reasoning was as follows. The testicle resembles the other paired glands in that the removal of one gland provokes compensatory hypertrophy of the other unit of the pair. This hypertrophy presumably affects both the internal and external secretory tissue equally. If, however, the vas deferens of the testicle left in situ is ligatured, the semen-forming parts will be entirely destroyed while the hormone-forming tissue of the second gland will remain uninjured; such being the case, the compensatory hypertrophy will be expressed in those cells only the function of which is increased. Experiments with rabbits showed that, about six months after operation (unilateral castration, ligature of the vas deferens of the other side), the testicle left in situ appeared to be very much smaller, the seminiferous tubules contained a few spermatogoniæ, and Sertoli's cells were unchanged; while the interstitial gland was enormously developed and showed signs of secretory hyperactivity. At the end of ten to twelve months the seminiferous tubules had become very much shrivelled, Sertoli's cells were degenerated, and the entire testis was almost exclusively composed of extremely hyperplastic cells of Leydig.

Tournade showed further that, in the case of rats, longcontinued ligature of the vas deferens leads ultimately to degenerative destruction of Sertoli's cells.

Similar results were observed in the case of pigs with unilateral cryptorchidism, from which the second normal testicle had been removed before the age of sexual maturity. There was complete absence of spermatogenesis and, in many instances, the cryptorchidic testicle showed more or less marked hypertrophy, the weight sometimes reaching double the normal. This hypertrophy was due to development of Leydig's cells, while Sertoli's cells in the seminiferous tubules were unchanged. In three cryptorchidic pigs, Bouin and Ancel found that the testicles had remained at a primitive stage of development, Sertoli's cells being absent, but Leydig's cells well developed. These animals were typically male in appearance and the genital tract was fully developed.

It is evident, then, that the presence or absence of Sertoli's cells does not affect the secondary sex characters, and that the internal secretory function is the sole property of the interstitial 
gland. This gland not only exercises a protective influence, but it determines the appearance of the somatic male characteristics.

The behaviour of Leydig's cells during the embryonal period and at other stages of existence points to the same conclusions. The first appearance of Leydig's cells in the embryo precedes the sexual differentiation of the primordial genital cells. The structure of the former indicates an exceptional degree of activity during embryonal life. Bouin and Ancel thought it possible that it is these cells which, by means of their specific internal secretion, determine the sex of the generative cells-that it is their influence upon the indifferent primitive genital trace which causes the latter to take on the male aspect.

The sex of the somatic cells and, in consequence, the development of the secondary genital organs and the further development of all the male characteristics present at birth, are, then, dependent upon the interstitial cells of Leydig. The normal development of the secondary characteristics in the course of post-uterine existence can only be ascribed to the function of this tissue. From birth to about the beginning of puberty - at a time, that is to say, when somatic sexual divergence is least clearly marked-the interstitial gland shows the minimum relative development.

In quite young animals, ligature of the vas deferens does not at first inhibit spermatogenesis (Griffiths), and the results of the operation are first observed as secondary consequences at the period of puberty, when formation of semen normally takes place. Inhibition of development of the interstitial gland in early youth leads to arrest of the development of the secondary sex characteristics. In the course of their experiments with rabbits, Bouin and Ancel observed certain animals which, after ligature of the spermatic cord at the age of 7 to 8 weeks, developed the habit and behaviour of castrated animals. Microscopic examination of the testicle showed atrophy, not of the generative part only, but also of the cells of Leydig. These cells were few in number, they appeared reduced in size, and showed no cytological signs of secretory activity; they contained a large quantity of pigment granules. These investigators are of the opinion that the difference in the condition of animals after ligature of the vas deferens in early youth is dependent upon whether the excretory duct only of the testicle is ligatured or whether the nerves which supply the testicle are also included. Resection of the nerves contained in the spermatic cord, without ligature of the vas deferens, may lead to atrophy of the cells of Leydig. If the spermatic duct only is ligatured, or if the resected nerve-fibres grow together again, the lesion of the internal secretory tissue elements is either very slight or is quite transitory. S. Vincent and Copeman also found that complete suppression of both the internal and external secretion of the testicle is only to be effected by the ligature of the 
entire spermatic cord; and that ligature of the vas deferens destroys the generative portion of the gland only.

The changes of puberty are ushered in by an enormous development of the cells of Leydig. In species with well-marked rutting periods, this phenomenon invariably precedes spermatogenesis; it is very marked in the testicle of the mole (Tandler).

Histological investigation shows that, in the testes of old animals and of senile man, there is distinct diminution in the number and size, together with atrophy, of the cells of Leydig. The latter is expressed by the remarkable profusion of pigment in the cytoplasm and the absence of other enclosures.

The observations which have been made in connection with cryptorchidism are to be regarded as direct proof that the inception and development of the secondary male characteristics are exclusively controlled by the interstitial cells of Leydig.

In addition to these findings, which are common to the large majority of cryptorchids, further evidence is supplied by those rare instances in which cryptorchidism in man is associated with feminine secondary characters. Histologically, these cases show complete sclerosis of the testis, while at the same time the cells of Leydig are reduced to a few scanty groups (Felizet and Branca). According to Bouin and Ancel, cryptorchidism is peculiarly interesting in pigs, such animals showing considerable difference in the secondary sex signs, in sexual instinct, and especially in the development of the genital tract. These differences depend upon variation in the weight of the testicles, which in its turn results from the different degree of derelopment of the interstitial gland. The genital tract is well developed in proportion to the number of cells of Leydig which are present in the testes. In regard to genital organs, sexual instinct, and somatic characters, the cryptorchidic pig stands midway between the normal and the castrated animal. As a general rule there is insufficient development of the cells of Leydig in the testes, and the place which the animal occupies between the two extremes is determined by the degree of this insufficiency.

Tandler and Gross (I908) made use of the elective properties of the Röntgen rays to demonstrate the independence of the secondary characteristics of sex of the function of the generative portion of the testicle.

Since Albers-Schönberg, in 1903, reported that the Röntgen rays exercise a profoundly deleterious effect upon the male genital glands, numerous communications npon this subject have made their appearance; these were concerned, partly with the results of experiments and partly with clinical material, but they all confirmed Albers-Schönberg's findings. Experimental work is described by Selden, Buschke, Krause and Ziegler, Villemin, Bergonnié and Tribondeau, Regaud and Dubreuil, Herxheimer and Hoffmann. Clinical material is contributed by Philipp, and bv Brown and Osgood. 
Upon all essential points these investigators are unanimous. It is the specific cells of the testis, the spermatogoniæ, which are destroyed by the Röntgen rays; while Sertoli's cells, which take no part in spermatogenesis, remain intact and the entire interstitial tissue, with nerves and vessels, undergoes no change.

Tandler and Gross experimented with roebuck with perfectly developed secondary sex characters and found that, after exposure of the testicles to the Röntgen rays, the antlers remained perfect; while it is well known that castration results in the loss of these male characters.

This elective destructive action of the Röntgen rays upon the spermatic cells have recently been confirmed by Simmonds by means of very careful experiments. He points out, however, that even after prolonged exposure, isolated seminiferous tubules remain intact. Of his conclusions the following deserve mention :-

If the animal lives for a sufficient length of time after exposure, regeneration of the seminiferous tubules will take place and spermatogenesis may result. The destruction of the spermatic cells is almost invariably accompanied by proliferation of the cells of Leydig, and this proliferation disappears after regeneration of the spermatic cells.

Where the testicle had become withered by the action of the Röntgen rays, the animals frequently became very obese later. This obesity, like that which follows castration, must be regarded as the outcome of the destruction of the genital glands.

From the results of his experiments, Simmonds concludes that the maintenance of the sex characters, by means of an internal secretion, is a function of both the spermatic cells and the cells of Leydig; that under normal conditions the cells of Leydig are few in number, but, after the destruction of the spermatic cells, there is vicarious intervention on their part, in consequence of which they undergo proliferation.

If we summarize all that is known concerning the internal secretory tissue of the male genital gland, we are led to the in . evitable conclusion that the hormone which gives to the organism its male characteristics, is elaborated in the cells of Leydig in the interstitial tissue. In spite of their mesodermal origin, these cells are able actively to produce certain specific substances and to transfer these substances to the blood-stream; such being the case we are justified in describing them in their totality as an "interstitial gland."

It is highly probable that, by the agency of its secretory products, this gland is responsible for the development of the male sexual gland from the indifferent genital trace. That it has a determining influence upon the normal development and maturity of the generative portion of the sexual gland, upon the formation of the secondary genital organs, and upon the existence and 
persistence of those morphological and biological characters which are the property of the male sex, is undoubted.

The part played by Leydig's cells in the general effects produced by the genital hormone is still very obscure. Castration leads to changes in the growth of the bones; to modifications in the structure of certain internal secretory organs, as, for instance, the hypophysis; and to profound metabolic alteration.s which are, to a certain point, independent of sex and may be reduced by the exhibition of homosexual or even heterosexual genital gland extract. Up to the present, there is no evidence to show that the hormone which produces these results is elaborated in the cells of Leydig. In Simmond's experiments, the animals frequently put on fat after exposure of their testicles to the Röntgen rays, but there is no evidence to show that the changes in the metabolism of fat are not due solely to the destruction of the generative parts. Further investigation is required, especially of those effects produced by the genital gland which are independent of sexuality, before the significance of the individual tissues of the testicle can be understood. It appears by no means improbable that several hormones of different origin are supplied by the testicle to the blood-stream.

Very little is known concerning pathological changes in the cells of Leydig. According to Ancel and Bouin, this tissue hypertrophies in acute disease, in prolonged cachexia it atrophies. These investigators found that, in experimental intoxication or infection, the cells of Leydig were hypertrophied at the commencement of the condition, while in the final stages and in very severe illness they were atrophied. They regard the hypertrophy of the interstitial gland as a protective measure on the part of the organism. This finding is confirmed by Voinov, but he regards the increase in the number of Leydig's cells as a protective reaction on the part of the genital gland. It seems probable, from the observations which have been made up to the present, that the multiplication of Levdig's cells is in some manner dependent upon the behaviour of the semiferous tubules. Hyperplasia and hypertrophy of Leydig's cells are accompaniments of both imperfect development and secondary atrophy of the seminiferous tubules (Dürck). Hansemann believed that the cells of Leydig are concerned in the production of testicular tumour.

Ovary.-In discussing the internal secretion of the ovary it is necessary to consider this function in its relationship to three different tissues : (I) the Graafian follicles; (2) the corpus luteum; and (3) those peculiar stroma cells which Bouin described collectively as the glande interstitielle de l'ovaire.

Of these tissues, the two first have long been known, and investigation has revealed the nature of their interrelationship with one another. The third has recently become the subject of considerable attention. The epithelioid cells in the interstitial tissue 
of the ovary have, it is true, been minutely described and variously named (granule cells, migratory cells, plasma cells, medullary cells), but the functions accredited to them have been purely local and trophic in character. They were believed to be the renegerative matrix of the ovarian parenchyma, or of the nutritional cells of the membrana granulosa and of the ovum (Plato). Regaud and Policard were the first to suggest that these cells might be secretory glandular cells.

Bouin adopted the view that this cell-complex represented an internal secretory gland, and his foundation for this belief is given in a comprehensive work by Bouin's pupil, Limon (I9O2). According to Limon, the interstitial tissue of the ovary is composed of epithelioid cells, the protoplasm of which is filled with granules resembling fat, which stain black with osmium, but afterwards dissolve in Canada balsam. These cells are differently placed in different animals; they are sometimes arranged in compact masses and cords, sometimes they are scattered without any apparent arrangement. In the rabbit, the field-mouse, and the guinea-pig, they are clustered together to form lobes which occupy the major portion of the organ; in certain other species they are merely rudimentary. Concerning their genesis, Limon ascertained that these cells are derived from the cells of the theca interna folliculi, and are therefore of connective tissue origin. The metamorphosis of the theca interna into interstitial tissue accompanies the degeneration of the membrana granulosa and of the ovum. When the ovum becomes destroyed, the cavity in the vesicle becomes filled with young connective tissue and shrivels; scarcely anything remains of the epithelium of the membrana granulosa, and the ovum is recognizable only as a small, shrivelled, hyaline lump in a mesh of the network of connective tissue. At this stage, the metamorphosed vesicle resembles a small corpus luteum, the structure of the cells of the theca interna having a resemblance to those of the corpus luteum. These metamorphosed vesicles may be termed spurious corpora lutea, or, better, atretic vesicles, and, in their totality, they represent the interstitial ovarian gland.

The findings of Limon and Bouin, and the conclusions at which these authors arrived, are confirmed by Cohn (1903), who investigated the interstitial ovarian tissue of rabbits. From the results of researches which he carried out (1905), Fränkel came to the conclusion that in many species, especially rodents, the interstitial ovarian tissue is very perfectly developed, while in the adult human female there are no traces of it; for this reason he is unable to assign a physiological significance to this tissue. Wallart and Seitz, on the other hand, observed changes in the ovaries of pregnant women, such being enlargement, in many follicles, of the theca interna cells and the presence of fat and lutein in the protoplasm of these cells. These authors concluded 
that structures genetically and morphologically analogous to the interstitial gland of animals are present in man. Seitz does not, however, believe that these cells possess an internal secretion.

In later investigations, conducted with the utmost care, Wallart found that more or less well-developed interstitial tissue was invariably present in the human ovary when growing vesicles were also present. Similar cells are observed in new-born infants, but there are no fatty enclosures in the protoplasm of the elements of the theca interna. These fatty enclosures are invariably present in older individuals, except such as have died from serious exhausting diseases. The interstitial tissue continues to increase up to puberty and even until the end of the second decade, but its development is most marked and the cell agglomerations are thickest during the period between infancy and puberty. At a later stage, the development of this tissue is less marked than that of the other parts of the ovary. The interstitial gland attains its highest development during pregnancy. In many cases of pathological pregnancy (hydatid mole, chorionic epithelioma), the maximal development is attained. During menstruation an enlargement of the gland takes place which is reminiscent of the conditions during pregnancy. At the climacteric, the gland ceases to develop, and remnants only are found in the ovaries, though these persist for some time. According to Wallart, the situation which is most favourable to the conversion of the theca interna into the interstitial gland, is the portion of the ovary immediately below the cortex, and it is here that the atretic follicles chiefly undergo development.

Fränkel was the first to assume a functional interrelationship between the interstitial tissue and the corpus luteum; he suggested that the function of the corpus luteum might, under certain conditions, be replaced by that of the tissue which is formed from the atretic follicles. This assumption rests upon the presence of a cell form, the lutein cell, which is apparently identical in both tissues; the theory is negatived, however, by the histogenetic differentiation between the corpus luteum and the interstitial gland-a point upon which Seitz laid special emphasis.

Seitz gave expression to his view in a new and eminently appropriate form of nomenclature. He calls the lutein cells of the interstitial tissue, which are formed from the theca interna, theca lutein cells, in contradistinction to the granulosa lutein cells of the corpus luteum, which, according to Sobotta's exact investigations with mice, are formed from the hypertrophic follicular epithelium.

From the results of very careful observation of human ovaries, Cohn recently settled the vexed question of the origin of the lutein cells. According to Cohn, the development of the corpus luteum and that of the atretic follicles are two essentially different processes. Histogenetically, the lutein cell is not homogeneous. 
Lutein cells may be derived from epithelium and from connective tissue. The lutein cells of the corpus luteum are derived from the epithelium of the membrana granulosa. The connective tissue framework of the corpus luteum is formed, by means of invasion by connective tissue buds, from the theca interna. There is, however, no fundamental difference between the formation of the corpus luteum of pregnancy and that of the corpus luteum of menstruation. The compact corpus albicans is probably a product of the involution of the corpus luteum.

Atresia of the follicles is accompanied, both during and after degeneration of the ovum and the epithelium, by proliferation of the theca interna, which leads to the formation of a theca-lutein layer. This layer is observed during pregnancy and in pathological hyperæmia of the genitals; it is also observed under normal conditions. The atretic follicle is entirely a connectivetissue structure.

Involution of the atretic follicle is accompanied by the development of the theca-lutein cells into stroma cells, though in some species this transformation is accomplished very gradually. In such animals, the interstitial ovarian tissue is composed of theca-lutein cells which have not, as yet, undergone degeneration. The theca-lutein cell formation of the atretic follicles corresponds in man to the interstitial ovarian tissue of certain animals.

These investigations supply a complete morphological picture of the tissues of which the ovary is composed. We know that, in addition to the Graafian follicles, the ovary contains two other tissues, both of which, from the nature of their structure, might possess an internal secretory function, but which in their origin differ fundamentally from one another. These are : 'The corpus luteum, which is derived from the epithelium; and the interstitial stroma cells, which have a connective-tissue origin. That the interstitial stroma cells possess a secretory function is suggested by the presence of fat granules and other secretory enclosures, as well as by a certain resemblance, commented upon by many (Mulon, Wallart), to the cells of the suprarenal cortex.

Both tissues are present in a varying stage of development in all mammals. The knowledge of this fact is of vital importance to a right understanding of the ovarian secretory functions. And of equal significance is a second fact, namely, that there are no justifiable grounds for the distinction which has hitherto been drawn between the true corpus luteum of pregnancy and the so-called spurious corpus luteum of menstruation. As Ravano also points out, there is no difference whatever either in form and contents or in genesis, between the two structures; both are true corpora lutea, whether the ovum which proceeds from them is fertilized or not.

In the face of these facts, it is not possible to concur in the theory which Bouin and Ancel have recently advanced (1909). 
These authors assume that the mammalia are divisible into two groups, those in which ovulation is spontaneous, and those in which it is provoked by sexual advances. The first group includes man, the dog, horse, pig, cow and certain other species; females of this class possess, in addition to the corpus luteum graviditatis, a second corpus luteum, which develops periodically, is homologous to the interstitial gland, and fulfils the function of the latter. The second group, which is represented by the rabbit, guinea-pig, mouse and cat, is furnished with an enormously developed interstitial tissue and with a corpus luteum graviditatis, but possesses no periodic corpus luteum.

It is evident from the facts which have been advanced, that there is no justification, either for such a classification of mammals, or for any homology of the corpus luteum menstruationis with the interstitial tissue. The corpus luteum is a homogeneous structure, which proceeds from the epithelial portion of the Graafian vesicle; while the interstitial gland is another and different ovarian tissue element derived from the atretic follicles and of connective-tissue origin, the elements of which have a structural resemblance to true lutein cells and possess, moreover, a secretory function.

In what does the function of these two tissues consist? It is not possible, at present, to arrive at a satisfactory answer to this question. We shall have occasion later to describe the hypotheses which have been advanced concerning the functions of the corpus luteum. In so far as the interstitial ovarian gland is concerned, it is probable that this tissue, like Leydig's cells in the testis, is the site at which the specific hormone is produced, by which the correlative activity of the entire ovary is effected. The material at our disposal is, however, insufficient to support such a hypothesis, and the results of investigation are, moreover, largely contradictory.

The interstitial gland attains very marked development, not only before puberty and menstruation-that is to say, at the period of increased ovarian function-but also during pregnancy (under physiological and, to an even greater extent, under pathological conditions) when, for many reasons, we are led to assume a reduction of internal secretory function on the part of the ovaries. It is during pregnancy that extensive cystic degeneration of the follicles and formation of theca-lutein cells takes place (O. Fellner, Cohn). On the other hand, Wallart recently observed cases of osteomalacia unaccompanied by pregnancy, in which there was marked development of the interstitial gland.

If the functional activity of the interstitial gland is estimated in accordance with the degree of its anatomical development, it appears probable that this tissue is active at puberty, during menstruation, and in pregnancy; up to the present, however, no evidence is forthcoming to show the manner in which this internal secretory activity affects the organism. 
That the secondary female sex characters, especially those of the genital tract, are dependent upon the interstitial cells of the ovary in the same way as the secondary male stigmata are dependent upon those of the testis, is, at present, not proved. It has been shown that uterine atrophy after castration may be inhibited by the agency of the Graafian follicles alone, without the co-operation of the corpus luteum or the stroma cells (Bucura). But apart from this finding, Bouin, Ancel, and Villemin discovered that exposure to the $\mathrm{X}$-rays has an entirely different effect upon the ovary to that which it produces on the testicle. The $\mathrm{X}$-rays also exercise an elective destructive action upon the ovary, the specific tissue affected being the Graafian follicles. There is a reduction in the volume of the ovary, and a diminution of the follicles and corpus luteum; the interstitial tissue, on the other hand, not only remains intact, but it hypertrophies. The uterus becomes atrophied in the same way as after castration. In the case of the testicle, as we have already shown, the fact that the interstitial tissue remains intact guarantees the integrity of the genital tract and of the secondary characters.

From the knowledge which we at present possess, it appears probable that the interstitial ovarian gland exercises a local influence upon the physiological processes in the female genital apparatus itself, and especially upon menstruation. The older theory of an intimate connection between ovulation and menstruation is no longer tenable (Leopold and Ravano); moreover, the careful investigations of Hitschmann and Adler have shown that menstruation essentially consists, not in a hæmorrhage, but in a cyclic metamorphosis of the uterine mucosa. Hence we are forced to assume a continuous production of the hormone by which these changes are effected, the source of which can only lie either in the Graafian follicle itself or in the interstitial tissue. But the Graafian follicles are already present in the immature ovary, and for that reason they can hardly be credited with the same significance in this connection as the interstitial gland, which attains its full development at puberty, shows signs of a cyclic increase of function corresponding with menstruation, and which, at the climacteric, undergoes involution.

The morphological and functional resemblance between the premenstrual uterine mucosa and the decidua, suggests that the menstrual alteration of the mucosa is in the nature of a preparation, that it prepares the ground for the nidation of the fertilized ovum. It is interesting to note that, according to Keller's investigations, rut in bitches corresponds with human menstruation in that it is accompanied by changes in the uterine mucosa similar to those of the premenstrual period, though, in the case of the animal, hæmorrhage is absent or very slight. Thus, rut is also a preparation of the uterine mucosa for the reception of the fertilized ovum. 
If the interstitial tissue elaborates the hormone which brings about the uterine changes of menstruation and of rut, it is obvious that this tissue plays a part, first in the nidation, and later in the development, of the fertilized ovum. The assumption of a hyperfunction in this direction goes a long way towards explaining the remarkable hypertrophy of the interstitial gland during pregnancy.

'The further assumption of an antagonism between the interstitial cells and the other internal secretory tissues of the ovaryin other words, if we assume that the anatomical hypertrophy of the former takes place at the expense of the latter, the hypersecretion of the interstitial gland being associated with hyposecretion of the rest of the ovarian tissue-accounts for the signs of inhibition of the activity of the ovarian hormone which are observed in other organs during menstruation and pregnancy. The stroma cell-complex affects the cyclic processes in the genitals only, while hormones with other functions are elaborated in the other ovarian tissues. This theory is quite as plausible as the one which has gained credence up to now; namely, that the phenomena of menstruation, nidation, and development of the fertilized ovum are all promoted by the agency of one tissueformation, the corpus luteum, which at the same time exercises an inhibitory effect upon other correlative activities.

This brings us to a consideration of what is known as the corpus luteum hypothesis.

Prénant (i898) was the first to regard the corpus luteum, from the morphological peculiarities of its cellular elements, as a ductless gland, whose peculiar function it is to guard against the interruption of pregnancy, by preventing ovulation during this condition as well as during the intervals between the rutting seasons.

Gustav Born, the Breslau embryologist, suggested to L. Fränkel that, from the nature of its structure and developmental processes, the corpus luteum verum graviditatis is an internal secretory gland whose function it is to establish the ovum in the uterus and promote its development.

L. Fränkel undertook to supply the experimental evidence necessary to the support of this theory. His experiments were conducted with rabbits, the conditions governing their ovulation, impregnation, and nidation being very well known. Rabbits become pregnant every month, and immediately after littering they become pregnant again. If fertilization fails to take place, the rut occurs again at the end of about thirty-five days. The mature Graafian follicles burst soon after littering, when the animal immediately becomes ruttish and impregnation takes place. This act is fruitful; three hours later the spermatozoa have reached the ovary, seventy hours later the fertilized ova have passed down the Fallopian tubes into the uterus; upon the fourth 
to the seventh day, the blastula are formed in the uterus, and upon the seventh day they become intimately connected with the uterine mucosa.

Fränkel discovered that pregnancy is prevented by bilateral castration carried out between the first and sixth days, but that the presence of one ovary' is sufficient to ensure the development of the ova. Destruction by galvano-cautery of all the corpora lutea produced the same effect as bilateral castration, while cauterization of other parts of the ovary had no influence whatever upon pregnancy. It is evident then, that suppression of the corpora lutea prevents the occurrence of pregnancy. But the function of the corpora lutea is also to secure the development of the already established ovum, for both bilateral castration and the cauterization of all corpora lutea between the eighth and wwentieth days, are followed by involution of the embedded ovit. Further experiments showed that one corpus luteum is able to perform the function of at least two or three; and further that this trophic function is conveyed to the uterus by way of the blood stream, in other words, through the agency of the internal secretion of the corpus luteum. Fränkel finds support for his hypothesis in the hydatid moles and abortions which are by no means infrequent accompaniments of ovarian tumour, and in the corpus luteum cysts sometimes observed in tubular pregnancy.

In a further development of this hypothesis, Fränkel adopted the view that the corpus luteum menstruationis, which is formed in women in every fourth week and in animals at varying intervals, also possesses an internal secretion, and that it is the function of this secretion in man to supply a nutritional impulse of a cyclic nature to the uterus, and that this impulse prevents the uterus from reverting to the infantile, or anticipating the senile, stage. The mucous membranes are made ready for the reception of the fertilized ovum; if fertilization does not take place the hyperamia leads to the phenomena of menstruation and the corpus luteum undergoes involution. As evidence of the part played by the corpus luteum in the production of menstruation, Fränkel cites the fact that, in operative surgery, cauterization of the recent corpus luteum prevents the occurrence of the next menstrual period in six cases out of seven.

According to Fränkel, the corpus luteum is an ovarian gland which undergoes periodic regeneration, and which, from the time of puberty until the menopause, regulates the nutrition of the uterus.

The practical outcome of Fränkel's theory was the therapeutic employment of lutein tabloids, prepared by him from the corpus luteum of the cow, in the treatment of the clinical symptoms resulting from ovarian insufficiency. Fränkel obtained better results by this method than by the exhibition of ovarian tabloids.

The corpus luteum hypothesis, as propounded by Born and 
Fränkel, supplied the incentive to extensive experimental and morphological investigation, which produced no actual confirmation of the theory, but which supplied valuable information concerning the sites of origin of the ovarian hormones.

The point in regard to which the least confirmatory evidence is forthcoming, is Fränkel's theory that the originating cause of menstruation is to be sought in the corpus luteum. A small number of findings have been described (Magnus, Lindenthal) which seem to point to such origin; but the value of Fränkel's cautery experiments is disputed by the majority of authors, who bring forward the well-known fact that, for no assignable reason, menstruation frequently fails to appear at an expected date. The hypothesis assumes that the bursting of the Graafian follicle takes place ten to fourteen days before the commencement of menstruation, that corpus luteum being at the same time formed, the secretory activity of which produces the menstrual period following. Confirmation of this assumption was obtained in several instances by Ancel and Villemin, who found that, in ovaries removed from women ten to twelve days before the date of the next menstrual period, the Graafian vesicles were burst and quite recent corpora lutea were present. This finding is less significant than it appears, however, when we consider that the menstrual swelling of the uterine mucous membrane takes place concurrently with the ripening of the ovum in the Graafian follicle; that it certainly begins before the bursting of the follicle (Ahlfeld), and that it cannot, for this reason, be due to the agency of the corpus luteum. In one operation Mandl actually observed an ovary containing a mature, but intact, Graafian follicle, together with a uterus in which the mucosa had undergone premenstrual changes. The experiments of Hitschmann and Adler, to which reference has already been made, next proved conclusively that the hæmorrhage is the least important of the menstrual phenomena; that it is, as a matter of fact, merely an accompanying symptom of the cyclic changes in the mucosa; and that it is these which constitute the essential feature of the menstrual process. It is hardly possible that a stimulus of short duration, such as that provided by the bursting of the Graafian vesicle, can be the fons et origo of the entire cyclic process, nor can the hæmorrhage itself be directly due to that cause; but the two processes, ovulation as well as hæmorrhage, must be regarded as the co-ordinated results of an increased determination of blood to the genital organs.

The prevailing views upon the relationship between menstruation and ovulation are in need of considerable revision in the light of Leopold and Ravano's investigations. The material at the disposal of these authors was very large, and the conclusions at which they arrived are as follows:-

Menstruation, that is, the periodic emission of blood by the uterine mucous membranes, depends upon the presence of the 
ovaries and the development of the uterine mucosa, and not solely upon the bursting of a Graafian vesicle. In the greater number of instances there is, both before and during the bursting of the vesicle, a determination of blood to the ovary. This is, in all probability, the reason why ovulation and menstruation are frequently coincident. Ovulation may take place in accordance with a specific periodic cyclic process, or its occurrence may be irregular; as a general rule, however, its periodicity coincides with that of menstruation.

In more than a third of instances, ovulation and menstruation are not simultaneous. Ovulation may take place at any time, and is not necessarily accompanied by uterine bleeding. This fact makes it appear extremely probable that conception also can occur at any time.

Menstruation may take place without ovulation. At the period when the ovaries undergo senile involution, they sometimes contain normal Graafian follicles and corpora lutea, which seems to show that the process of ovulation may outlast that of menstruation.

It is evident from the above statements that Fränkel's theory of the dependence of menstruation upon ovulation and upon the internal secretory function of the corpus luteum, is untenable. As Regaud and Dubreuil have shown, rut in rabbits has no connection with the corpus luteum.

Fränkel's further assumption that the corpus luteum regulates the nutrition of the uterus from puberty to the menopause, is also unfounded. This theory is based upon experiments in which the destruction of the corpora lutea of rabbits was followed by permanent nutritional disturbances of the uterus, similar to those which follow bilateral castration. 'The results of exposure of the ovaries to the action of the X-rays-atrophy of the genitals, accompanied by diminution in the size of the ovaries and disappearance of the corpora lutea or Graafian follicles, with persistence of the interstitial cells-have alreadv been described, and these are advanced by Bouin, Ancel, and Villemin as further evidence in favour of the internal secretory function of the corpora lutea. Yet the results of both sets of experiments really only prove the functional importance of the corpora lutea and the Graafian follicles in the maintenance of the anatomical integrity of the genital tract. Both tissues are destroved by the action of the X-rays, and the possibility of damage to the Graafian follicles by cauterization of the corpora lutea cannot be excluded. Bucura's experiments, however, resulted in negative findings with regard to the corpora lutea. These experiments proved that atrophy after castration may be prevented by the transplantation of the ovary from an animal of another species, provided that the ovary contains developing Graafian follicles, the presence of an intact corpus luteum being alone insufficient to affect the result. That 
the Graafian follicles are the sole agents in the prevention of the uterine atrophy, is proved in the most conclusive manner by a case of Bucura's, in which a remnant of the ovary was left in situ after castration, and in which atrophy of the uterus did not take place. Examination of sections in unbroken series showed that this remnant consisted solely of maturing Graafian follicles, neither stroma cells nor any traces of corpus luteum being present.

Born's original hypothesis, that the function of the corpus. luteum is to provide for the nidation in the uterus and subsequent development of the fertilized ovum, finds considerable support in the results of morphological investigation as well as in Fränkel's. experiments. If the ovum, after its emergence from the Graafian resicle, becomes fertilized, lutein cells are formed from the epithelial cells of the membrana granulosa, probably in the same way as at the menstrual period; but the corpus luteum of pregnancy persists and, in accordance with both its genesis and structure, may be regarded as a secreting gland. What is the nature of its function? The similarity of structure and development which subsists between the menstrual and the gestative corpora lutea, inevitably suggests that the latter carries out, only in a more intense degree, the function inaugurated by the former. If the corpus luteum menstruationis prepares the uterine mucosa for the reception of the ovum, the corpus luteum graviditatis is responsible for the nidation of the fertilized ovum and for its further development. But, seeing that we are unable to attribute the responsibility for the menstrual changes in the mucosa to the corpus luteum menstruationis, the premises for the conclusion that the corpus luteum graviditatis also possesses a preparatory function cease to exist. On the other hand, Fränkel's experiments undoubtedly appear to bear this interpretation. These experiments show that in the rabbit, suppression of the corpora lutea in the first week after fertilization prevents the occurrence of pregnancy, and that suppression between the eighth and twentieth dars terminates it. With regard to the latter point, however, Kleinhans and Schenk found by rery careful experiments that the removal of the corpora lutea after nidation does not necessarily terminate a pregnancy of more than nine days' duration, and it seems evident from this that the corpus luteum has no significance in the after-derelopment of the ovum.

The function of the corpus luteum could only be to safeguard the nidation of the ovum. But even this manifestation of activity seems improbable in the light of an experiment carried out by

- Mandl. The left ovary of a pregnant rabbit was removed and. implanted between the fascia and the abdominal wall. After littering, pregnancy again took place, and two days later the second ovary was removed. The pregnancy ran a normal course, in spite of the fact that a corpus luteum graviditatis could not possibly have been present in the transplanted ovary, and post- 
mortem examination subsequently showed that there was no "spurious" corpus luteum. It is very evident from this experiment that the corpus luteum is not indispensable to either the nidation of the ovum or to its after development. Fränkel explains the results of this experiment by assuming that, in this instance, the function of the corpus luteum was vicariously undertaken by an atretic follicle, but this assumption is a complete departure from his own theory. For, as we know, the atretic follicle is entirely a connective tissue structure and the theca-lutein cells, formed in the course of its involution, compose the interstitial ovarian tissue. This proves the importance of the interstitial gland in the nidation of the ovum, while at the same time it reduces the function of the corpus luteum to a probable participation in this process.

Having disposed of Born and Fränkel's hypothesis, we return to the view, first expressed by Prénant and since confirmed by others (Sandes, Srobansky), that the internal secretory function of the corpus luteum persistens consists in the inhibition of ovarian activity and, more especially, in the prevention of ovulation during pregnancy.

It is common knowledge that, as a general rule, neither ovulation nor menstruation take place in pregnant women. In the cow, persistence and hypertrophy of one corpus luteum are by no means infrequent findings, and in such cases there is no recurrence of rut at the usual intervals of twenty-one days; if the hypertrophied corpus luteum is removed by surgical means, however, the signs of rut will appear a few days later. In the case of the mouse, the corpus luteum persists after littering, sometimes until after the second or'even third pregnancy (Sandes, after Mandl).

Ravano shows thathe current notion that, in women, the corpus luteum graviditatis persists during the entire period of pregnancy, is mistaken; it may even completely disappear, though the occurrence is rare. In about 5 per cent. of cases, complete ovulation takes place during pregnancy, while a tendency to ovulation is shown by the large majority of pregnant women.

The material we at present possess supplies no decisive evidence as to the part played by the corpus luteum in the inhibition of ovulation and the other ovarian functions during pregnancy. As we have previously shown, these processes may result, with equal probability, from hyperfunction of the interstitial cells and reduction of activity on the part of the other tissues, particularly of the Graafian follicles. The atresia, which extends during pregnancy to numerous follicles, consumes the material for the maturing of the ovum.

It is evident from the above that the third and most considerable tissue constituent of the ovary, the Graafian follicles, 
may possess important secretory functions. With the exception of the cyclic symptoms in the genital tract, and certain processes which take place during pregnancy which are referable to the activity of the hormone of the interstitial gland, the Graafian follicles are probably responsible for all the chemical correlationships which are ascribed to the ovary as a whole. There is proof that the maintenance of one important sex characteristic, the female genital tract, is dependent upon these follicles. The question as to which tissue is the primary origin of this, as of the other, female secondary characters, has scarcely as yet come under discussion.

That the tissue which performs the function of external secretion is also the site of production of the ovarian non-sexual hormone, which exercises an alterative influence upon a large number of tissues and functio:s, is suggested by analogy with the male sexual gland.

In conclusion, reference must be made to Bucura's assumption of an internal secretory function on the part of the parovarium or epoöphoron (remains of the Wolffian body), and his experimental attempts to prove such secretion. Bucura's investigations confirm the reports of earlier authors, that the epoöphoron is an organ which continues to develop after birth, that it attains the summit of its development at puberty, that it apparently hypertrophies during pregnancy, atrophies with old age, and, from the nature of its structure, may possess an internal secretory function. He found that the uterus reacts differently to ovarian extirpation if the parovarium is removed with it, and that both these results differ from that which follows the customary method of extirpating the uterine appendages. The difference in the appearance of the uterus is due to the manner in which the connective tissue is affected. Owing, however, to the extreme scarcity of experimental material, it is impossible at present to arrive at any definite conclusion concerning the function of this appendage of the female genital gland.

\section{INTERNAL SECRETION OF OTHER PAR'TS OF THE GENITAL APPARATUS.}

Prostate.--It is well known that the prostate is a glandular organ possessing an external secretion which promotes the activity of the spermatozoa, partly by a scecific stimulatorv influence, and partly by diluting and increasing the fluidity of the testicular secretion (Fürbringer, Exner, Steinach). From the results of experiments and from clinical observation, Serralach and Pares (I907) assumed an internal secretion on the part of the prostate. They found that after removal of the prostate of dogs, there was cessation of ejaculation of semen and suppression of the secretion of the preputial glands, temporary cessation of spermatogenesis 
and atrophy of the testes. The administration of glycerine extract of prostate subdued these symptoms or prevented their occurrence.

The conclusiveness of Serralach and Pares' experiments is questioned by I. P. Haberern on the justifiable grounds that prostatectomy, as carried out by them, was not complete. As I know from my own experiments, total extirpation of the prostate of dogs is attended by considerable technical difficulties, and I am prepared to support Haberern in his view, that the internal secretion of the prostate can be proved only by extirpation experiments which are free from objection, reinforced by the results of the transplantation of the organ. The implantation site most suited to the prostate is the bone tissue. Primary atrophy of the prostate in man may cause aspermatism.

Glycerine or watery extracts of the prostate of bulls (Thaon, Posner, and Kohn) and of dogs (Biedl) are extremely toxic. The intravenous injection of a few cubic centimetres produces a marked rise in arterial tension, followed by an equally marked fall; there is arrest of the heart's action and asphyxia. 'These symptoms are most probably due to intravascular coagulation.

Uterus.-The difference in the symptoms of suppression which follow extirpation of the uterus, extirpation of the ovaries, and extirpation of both uterus and ovaries, led O. O. Fiellner (Igo8) to assume that the uterus possesses an internal secretion. the action of which is both toxic and vaso-contrictor, and which starts the menstrual wave. Fellner supposes that there is an antagonism between the internal secretory activity of the uterus and that of the ovary, the function of the ovarian secretion being to neutralize the toxic product of the uterus. There is no experimental foundation for this hypothesis. As far as is at present known, extract of uterine tissue is physiologically inactive. As Fellner himself pointed out, the toxic effect which is obviously produced by the intravenous injection of homologous uterine extract, is probably the result of intravascular coagulation, brought about by the comparatively large amount of thrombokinase present in the uterine mucosa. In the course of my own experiments, I have been unable to confirm an effect of ovarian extract which is described by certain French authors (Hallion, Delille), namely, that it produces hypotension and has an elective vaso-dilator effect upon the vessels in the thyroid gland. The production of such a result by the intravenous injection of ovarian extract could hardly be regarded, moreover, as proof of an internal secretory function on the part of the ovaries, for, as we have frequently pointed out, depressants are present in many tissue extracts.

Placenta.-The internal secretion of this organ plays an important part in the modern theory of eclampsia. We will confine ourselves to a few brief comments upon the subject. 
Schmorl's anatomical finding of emboli composed of placenta cells in the lungs and other organs in eclampsia, paved the way for Veit's syncytiolysin theory (1905). Syncytiolysin is supposed to be an antibody, formed in the maternal organism in consequence of the passage of albumin from the syncytial villous coating. If the formation of this antibody does not take place, or is insufficient to combat the flooding of the organism with placental albumin, the latter acts as a toxin and produces the toxic condition known as eclampsia. Ascoli, on the contrary, regards the syncytiolysin as the toxic substance, while, according to Weichardt, the eclampsia toxin is a form of endotoxin which is liberated from the placenta cells by syncytiolysin.

The premises for these different and entirely contradictory hypotheses are provided by experiments which show that extracts obtained by various means from placental tissue, have a toxic effect; that after intraperitoneal injection they produce albuminuria and other derangements; and that after intravenous injection they may cause death. A more careful investigation showed, however, that the results are due in part to the action of albumin obtained from animals of a different species (Lichtenstein), and in part to intravascular coagulation (Dryfuss, Martin, Freund, Mathes) caused by the presence of thrombokinase in the placenta.

Liepmann found that the normal human placenta is practically non-toxic to rabbits, while the eclampsic placenta is extremely toxic, but Dryfuss was unable to confirm these results. He, like Hofbauer, Dienst, and others, holds the view that ferments, in certain cases in increased quantities, pass from the placenta into the maternal blood-stream; that these break up the leucocytes and bring about increased formation of fibrin-ferments and fibrinogen; and in consequence of this thromboses and degenerative processes occur in the liver and kidneys.

According to Massini, under normal conditions the placental toxin is neutralized in the thyroid gland, and eclampsia takes place only where there is thyroid insufficiency. According to Vassale, eclampsia is a motor neurosis due to decreased function of the parathyroid glands; he recommends the administration of parathyroidin, and describes favourable results.

\section{THE INTERNAL SECRETION OF THE PANCREAS.}

In the year 1859 , v. Mering and Minkowski discovered that extirpation of the pancreas of dogs is followed, not only by digestive disturbances due to the absence from the intestine of the pancreatic secretion, but by the continued presence of sugar 
in the urine and by all those further symptoms (polyuria, polyphagia, emaciation ending in death) which we are accustomed to associate with pronounced diabetes mellitus. An intimate relationship between diabetes and pancreatic disease had already been suspected by several clinicians, who, upon anatomical grounds, ascribed a pathogenetic significance to the pancreas in that condition. 'itheir hypothesis was now supplied with an experimental foundation, while at the same time a new and previously unknown function of the pancreas was brought to light.

A similar discovery to that of $\mathrm{v}$. Mering and Minkowski was made, at about the same time and quite independently, by de Domenicis, though he failed to recognize the significance of his findings. The results have since been confirmed by means of many hundreds of experiments, undertaken by numerous authors (Lépine, Hédon, Gley, Thiroloix, Caparelli, Harley, Schabad, Cavazzani, Sandmeyer, Selig, Rumboldt among others).

That glycosuria is a constant finding after extirpation of the pancreas was disputed by isolated authors, but these exceptional cases are fully explained by the, at first sight, remarkable fact, that characteristic sequele are observed only after complete, or almost complete, extirpation of the organ. If one-third to onefifth of any part of the gland is left in situ, glycosuria will not appear. If the remnant becomes destroyed by secondary processes, or is removed by surgical means, serious diabetic symptoms will make their appearance. These observations pointed to the fact that the cause of the metabolic derangement could not lie in the digestive disturbances arising from the absence of the pancreatic juice; they showed, moreover, the relative unimportance of the changes brought about by operative intervention, in the conditions governing the blood supply and the innervation of the organ. Minkowski, followed by E. Hédon, whose work, however, was quite independent, next showed by a well planned series of experiments, that neither lesion of the vessels nor of the nerves, nor suppression of the external secretion of the organ, is the causative factor in the production of pancreatic diabetes. The method employed was to remove that portion of the pancreas of the dog which is situated in the mesentery of the duodenum, and which Pflüger named processus uncinatus, and to implant it, together with its vascular peduncle, under the skin of the abdomen. If this transplanted portion is sufficiently vascularized and nourished, the rest of the pancreas may be removed by a second operation without producing diabetes. But if, by a third, very slight extraperitoneal operation, the transplanted portion of the pancreas is also removed, serious diabetes will follow, and death will ensue.

As the result of these experiments, pancreatic diabetes became the subject of eager experimental investigation. Total extirpation of the pancreas was next carried out with animals of different 
species. Minkowski observed diabetes not only in the dog, but in the cat and the pig, and Hédon observed the condition in apes. Destruction of the pancreas is followed in rabbits by slight diabetes expressed by alimentary glycosuria. In carnivorous birds, removal of the pancreas is followed by slight diabetes (Weintraud); while the herbivorous varieties show marked hyperglycæmia and reduction in the glycogen contents of the liver, but no glycosuria (Kausch). Aldehoff found that, of cold-blooded animals, the frog and the tortoise show glycosuria after pancreasectomy. Markuse and Pflüger also investigated the conditions in frogs, and found that, if the liver is removed with the pancreas, glycosuria does not appear. With regard to fish, in two cases out of eleven, Caparelli observed slight glycosuria in eels after pancreatic extirpation, while Diamare observed constant hyperglycæmia and glycosuria in Torpedo marmorata.

The dog has formed the subject of the vast majority of pancreatic experiments, and it was with this animal that an exact experimental analysis in diabetes was first obtained.

Total extirpation of the pancreas of dogs is followed by sugar in the urine in the course of a few hours or, at the most, a few days. If, during the following days, the animals are fed, the amount of sugar increases progressively, and, at the end of three to five days, reaches the maximum of 8 to 12 per cent. If the animals are kept fasting, the urine will contain sugar, but in smaller quantities. Taking into consideration the fact that, in addition to glycosuria, there is a very high degree of polyuria, the absolute amount of sugar excreted is considerable. Dogs weighing Io to 15 kilos. and fed on a mixed diet containing a large proportion of carbohydrates, excrete $\mathrm{I}$ to $\mathbf{I} \frac{1}{2}$ litres of urine containing 8 to ro per cent. sugar in twenty-four hours-that is, about I2O to I 50 grms. in the twenty-four hours. The other diabetic symptoms evidenced by these animals are polyphagia and polydypsia; in face of the large amount of food taken, the progressive emaciation is very remarkable. This is first shown by a rapid decrease in the adipose tissue, but the fleshy parts also soon show a marked falling away; and three to four weeks after operation the animals are reduced to skeletons and perish of extreme inanition. The emaciation, however, is attributable only in part to the digestive derangements which accompany the suppression of the external pancreatic secretion. The glycosuria persists until the end, the sugar disappearing from the urine on the day preceding death. In addition to sugar, the urine contains acetone, aceto-acetic, diacetic, and $\beta$-oxybutyric acid.

The duration of life depends, in the first place, upon the operative technique; in the second, upon whether the removal of the organ is effected without infective complications, especially, as so frequently happens, duodenal necrosis with subsequent peritonitis and perforation. It must also be borne in mind that, 
owing to the long time which it takes to heal, the surgical wound in experimental diabetes constitutes a certain danger to life. In the absence of all by-factors, however, the metabolic disturbances lead to death in three, or at most four, weeks after operation. Should the animal live for a longer period, it is safe to assume that extirpation was not complete, and that minute portions of the pancreas were left in situ.

The autopsy shows that, with the exception of emaciation, there are no noticeable changes in the organs. Fatty degeneration is sometimes present in the liver and kidneys, dilatation and hypertrophic changes are observed in the gastro-intestinal canal, and, according to Boccardi, there are changes in the central nervous system.

Partial Extirpation of the Pancreas.-If a portion only of the pancreas is removed, a quarter or one-fifth of any part of the organ being left in situ, glycosuria will not take place. An even larger proportion of the pancreas may be removed and, provided that the remaining portion is sufficiently well nourished and vascularized, the glycosuria will be transient only. Whether the remaining portion is left in situ, or is transplanted externally of the abdominal cavity, is a matter of indifference. The transplantation of the processus uncinatus with its vascular peduncle to a position under the abdominal cuticle, prevents glycosuria. Resection of the peduncle did not, in Hédon's experiments, provoke glycosuria; but Lombroso has recently shown that the subsequent ligature of the peduncle produced slight transient glycosuria. In one instance, where the implanted portion of pancreas was efficiently vascularized by a large cuticular artery, the removal of this portion eight days after implantation led to serious diabetes, which terminated fatally.

Extirpation is sometimes partial as the result of accident, small portions of pancreatic tissue being unintentionally left in situ. Such conditions produce "slight" diabetes, sugar appearing in the urine only after administration of the carbohydrates, and disappearing again with a flesh diet. In many instances, this remaining portion undergoes progressive degenerative atrophy, in consequence of which the diabetes passes from the slight into the severe form (Sandmeyer's diabetes).

\section{METABOLISM IN PANCREATIC DIABETES.}

The derangements of metabolism which follow extirpation of the pancreas are expressed by the glycosuria and the hyperglycæmia of which it is the immediate outcome; by the disappearance of the glycogen reserve; by the peculiar behaviour of the organism with regard to the different carbohydrates; by the increased formation of acetone bodies, due to the absence of the carbohydrates; by the over-charging of the blood with acids 
(acidosis), with its attendant danger, coma diabeticum; and by changes in oxidation and general metabolism, as well as by the secondary effects which these produce upon the nutritional conditions (polyphagia and emaciation).

Glycosuria.-The characteristic symptom, which appears earliest after pancreasectomy and is most readily demonstrable, is the presence of grape-sugar (dextrose) in the urine. The amount and the intensity of the glycosuria is subject to immense variation, partly the result of external factors, such as muscular exertion, surrounding temperature, \&c., chief among which is the amount and nature of the diet. Assuming these factors to be equal, however, the condition falls into two distinct types connected by transition 'stages, and these types correspond in all essential particulars to the "slight" and "severe" clinical forms of diabetes. The essential differences which Seegen pointed out between the two clinical conditions are not observed in experimental pancreatic diabetes. The differentiation is merely quantitative and depends upon the degree of pancreatic inadequacy.

The extirpation of relatively large portions of the pancreas produces slight glycosuria; extirpation of the entire organ is followed by severe glycosuria. If the portions left in situ undergo secondary atrophy, the slight condition may pass into the serious stage (Sandmeyer). Slight diabetes is characterized clinically by the fact that sugar appears in the urine only after a diet containing carbohydrates, and disappears rapidly if the carbohydrates are excluded from the diet. In the severe form, sugar is always present in the urine and, though the quantity is diminished by fasting or by an exclusively meat diet, the sugar never entirely disappears. As Minkowski showed, when the metabolic derangement, after total extirpation of the pancreas, has reached its height, the glycosuria has a certain definite intensity, which may be estimated by the proportion between the sugar and the nitrogen in the urine, and which, after fasting or a diet free from carbohydrates, is expressed by the quotient $\mathrm{D}: \mathrm{N}=2.8: \mathrm{I}$. It has been shown by numerous subsequent experiments that Minkowski's quotient of 2.8 varies but slightly in diabetes produced by total extirpation of the pancreas of dogs; and that within the limits of this quotient, sugar is formed from albumen. As we showed in a previous chapter, the previous or simultaneous extirpation of the thyroid of dogs without pancreas raises the quotient $\mathrm{D}: \mathrm{N}$ to $3 \cdot 5$, or even higher (Eppinger, Falta and Rudinger).

Alimentation increases the absolute amount of sugar excreted. Feeding with albumen is followed by a fluctuation of the sugar contents in proportion to the amount of nitrogen present in the urine (Minkowski, Berger, Lehmann, Bendix). The administration of fats does not, as a rule, increase the glycosuria, though Falta, Eppinger and Rudinger observed a transitory, but very 
marked, rise in the quotient $\mathrm{D}: \mathrm{N}$ in dogs without pancreas after feeding them with fats, and this result was intensified by the injection of adrenalin.

With regard to the different carbohydrates contained in the nutriment, Minkowski discovered that by far the greater number (starch, grape-sugar, milk-sugar, cane-sugar) are excreted, almost without residue, in the form of glucose. Levulose is the only carbohydrate of which a large proportion is used up in the organism. After the administration of levulose, a considerable deposition of glycogen takes place in the liver and in the muscles.

These findings of Minkowski's were confirmed by Sandmeyer and, more recently, by Pflüger. According to Eppinger and Falta, the pathological decomposition of albumin observed in dogs without pancreas is reduced practically to the normal by the administration of levulose; and it seems evident from this that a portion of the levulose is really consumed.

Of the other factors which contribute to the intensity of the glycosuria, the most important is the surrounding temperature. According to Lüthje, the quotient $D: N$ is very high when the surrounding temperature is low, and very low when the surrounding temperature is high. Minkowski and Allard, Falta, Mohr were unable to confirm this relationship between the amount of the glycosuria and the surrounding temperature.

The intensity of the glycosuria is influenced, moreover, by the temperature of the body. The increased combustion and the increased decomposition of albumin by which a heightened temperature, especially fever temperature, is accompanied, may produce an intensified glycosuria. But, as a general rule, the beginning of infective peritonitis and general sepsis in animals after operation, is accompanied by a marked diminution or even complete disappearance of the glycosuria. If experimental toxic nephritis is induced (with cantharidin or chromic salts) in dogs without pancreas, the condition will be accompanied by a considerable decrease in the amount of sugar excreted in the urine (Biedl). It is known that in spite of the fact that considerable hyperglycæmia is present, dogs with pancreatic diabetes cease at the preterminal stage to excrete sugar (Falta, Grote and Stahelin). Similar findings in the clinical condition (reduction in the sugar excretion with hyperglycæmia, in nephritis with fever) are explained by v. Noorden as the result of the increased impermeability of the renal filter to sugar; and this assumption is accepted by L. Pollak in explanation of the reduction in the glycosuria after repeated injection of adrenalin.

The effect which muscular exertion has upon the sugar excretion in animals with pancreatic diabetes is at present insufficiently investigated. Heinsheimer observed that glycosuria-submaximal, it is true-was favourably influenced by extreme muscular exertion; while Falta, Eppinger and Rudinger's dogs, 
which were totally devoid of pancreas, showed rapid decline both of the temperature and of the $\mathrm{N}$. and $\mathrm{D}$. excretion after an hour in the treadmill, and a few hours later they died.

Hyperglyccemia.-The immediate cause of glycosuria in pancreatic diabetes, as in all the experimental forms, except socalled renal diabetes (phlorizin, renal toxins), is an abnormal increase in the sugar contents of the blood. The renal filter is impermeable to the normal sugar contents of the blood, which are about .I per cent.; but if the sugar contents of the arterial blood exceed this figure, and especially if the hyperglycæmia is maintained, the kidney is unable to withstand the sugar in the blood and it passes, in consequence, into the urine. A high degree of hyperglycamia is invariably the result of extirpation of the pancreas; it is present also in those cases, such as herbivorous birds, in which glycosuria is absent (Kausch). The cause of hyperglycamia lies in a derangement of the regulatory mechanism by which the normal sugar contents of the blood are guaranteed. It is not proposed to enter here into a discussion of the nature of this derangement; it will be sufficient for the present purpose if we describe the phenomena by which hyperglycæmia is accompanied.

A factor of the first importance is supplied by the conditions covering the glycogen contents of the body. Very early after pancreas extirpation the glycogen in the liver is reduced until only traces of it remain. In all cases of severe pancreatic diabetes, the glycogen in the liver is reduced in the first few days to a minimal amount and this does not subsequently disappear. Even after generous feeding with carbohydrates only traces of glycogen are to be found. In the slight form of diabetes, the liver may contain considerable quantities of glycogen. Suppression of the pancreas is also attended by a marked diminution in the amount of glycogen in the muscles, though the muscles do not part with their glycogen as readily as the liver. In face of the disappearance of glycogen from the liver, the remarkably high glycogen contents of the leucocytes is very striking (Ehrlich); the probability is that these become crammed with superfluous glycogen from the blood. Not all the carbohydrates, however, escape conversion into glycogen. As Minkowski proved, the administration of levulose leads to a storing-up of glycogen in the liver and in the muscles. It has been already pointed out that the administration of levulose does not increase glycosuria; certain later observers (L. Pollak, Neubauer) regard this as the expression of a greater resistency on the part of levulose-glycogen than upon that of glucose-glycogen. As S. Fraenkel justly points out, the administration of any one of the carbohydrates is invariably followed by the formation of the same glycogen substance, that, namely, which directs the polarized ray to the right, and which, after hydrolysis, yields dextrose. No one has, up to the present, 
observed glycogen in another form, and for this reason the explanation of the levulose experiments must lie in some cause other than the formation of a different glycogen substance. Fraenkel draws attention to Henri's experiments, which have attracted little notice, and which show that levulose has an inhibitory influence upon the action of invertin. This result suggests that levulose may have an inhibitory effect upon the fermentative processes in the liver.

Liberation of the glycogen reserves cannot be the only source of the increased sugar contents of the blood, for these are independent of the conditions governing glycogen in the organism. After the exhaustion of the glycogen reserves and the rapid excretion of any carbohydrates which may have been present in the food, the source of the surplus sugar in the blood can only lie with the albuminoids and fats. Given a diet free from carbohydrates and rich in albumin, it is possible that glycogen would be formed as an intermediate stage in the conversion of albumin into sugar. This line of argument is excluded where the diet consists of fats, yet in this case also the glycosuria and hyperglycæmia are considerable.

The defective metabolism of the carbohydrates is the principal cause of another important symptom of diabetes, namely, the increase in the amount of the acetone bodies (oxybutyric acid, aceto-acetic acid, acetone) present in the urine, or, as it is called, the acidosis. Under normal conditions, the acetone bodies are formed, in part from the amino acids of the albumin (Embden), but principally free from oleic acids, as an intermediary product in the conversion of fat into carbohydrates. Although the cause is at present unknown, it is an undoubted fact that ketonuria, that is, excretion of the acetone bodies in the urine, takes place only when decomposition of the carbohydrates in the economy has ceased. This explains the occurrence of acetonuria in inanition and in those pathological conditions in which the nutritional conditions in general, and the assimilation of the carbohydrates in particular, are very much reduced. In severe diabetes, in addition to the carbohydrates contained in the diet, the major portion of the sugar obtained from other sources is also excreted; thus there is an enormous increase in the formation of acetone substances and, consequently, ketonuria of a very pronounced character.

The overloading of the blood with oxybutyric acid and acetoacetic acid gives rise to acid intoxication, which Naunyn and his school regard as the cause of diabetic coma. The organism of carnivora possesses a chemical protective substance in ammonia, which is formed by the decomposition of the albumin molecule, and this substance is able to neutralize the superfluous acids. In consequence of this, the increased production of acids increases the ammonia in the urine at the expense of the urea. But the amount of the ammonia is insufficient to combat the continued formation of acids, and a demand is made upon the fixed alkalis 
of the tissues; the amount of the fixed alkalis, calcium, and magnesia, present in the urine becomes increased (D. Gerhardt and $W$. Schlesinger); while the alkalescence. of the blood and tissues in very much diminished; and, as a result of the alkaline reduction, coma supervenes.

This theory of the origin of diabetic coma rests upon a sound clinical and experimental basis, and to-day is very generally accepted. The failure of the alkaline therapy, which was founded on this throry, led v. Noorden to conclude that diabetic auto-intoxication is attributable, not only to the over-production of acids in general, but also to the specific toxic action of isolated intermediary products (oxybutyric acid and aceto-acetic acid).

Acetonuria is a constant symptom in dogs without pancreas; its presence is the more remarkable from the fact that acetone bodies are not normally present in the urine of these animals, while it is extremely difficult to obtain ketonuria experimentally. The clinical picture presented by diabetic coma is by no means rare in dogs which have lost their pancreas and, in many instances, it is a terminal feature.

The general metabolism undergces a fundamental change after the extirpation of the pancreas. The accumulation of sugar in the blood was formerly regarded as due to imperfect oxidation of the carbohydrates and a lowering of the processes of oxidation in general. Falta, Grote, and Staehelin (190\%) showed, however, that in dogs with pancreatic diabetes, the processes of oxidation are enormously increased, for the metabolism of albumin of such animals when in the fasting stage may attain to three times the normal. The decomposition of fat is also very much increased. These findings suggest a toxogenic decomposition of albumin.

According to Eppinger, Falta, and Rudinger, the increased oxidation which follows removal of the pancreas is due to the fact that, owing to suppression of the inhibitory influence normally exercised by that organ, the activity of the thyroid in promoting metabolism is allowed to proceed unchecked.

It is certain that, owing to the increased metabolism, there is a reduction in the amount of the albumin, and that this gives rise to emaciation. The emaciation is further accounted for by the permanent caloric deficiency, due to the defective metabolisin of the carbohydrates. The increased demand for, and consumption of, food (polyphagia) does not entirely counteract the deficiency in the caloric, and does not prevent the rapid emaciation of the body. Dogs which have lost their pancreas become extremely emaciated within quite a few weeks, and usually perish of inanition.

\section{THEORY OF PANCREATIC DIABETES.}

Since it was first discovered that diabetes follows extirpation of the pancreas, this condition has formed the subject of searching 
and laborious investigation, as well as of lively discussion ; nevertheless, no final theory embodying a satisfactory explanation of its causes has up to the present been forthcoming. We are compelled, therefore, to confine ourselves to a short account of the theories concerning the condition which have been advanced by different authors.

v. Mering and Minkowski, the discoverers of pancreatic diabetes, believed the condition to be the outcome of the cessation of a specific function of the pancreas, a function which is essential to the normal employment of sugar by the organism. After consideration of the possible causes, Minkowski concluded that the pancreas supplies some substance which aids in the decomposition of sugar within the organism. He believed the cause of diabetes to lie in a cessation of the internal secretory, or positive, pancreatic function, using the term in the sense in which $v$. Hansemann employed it.

This conclusion was, however, opposed by de Domenicis, whose discovery that glycosuria followed extirpation of the pancreas was almost simultaneous with that of $v$. Mering and Minkowski, though he worked independently of them. In his opinion, glycosuria is not a constant result of pancreasectomy, but is merely an expression of the serious nutritional disturbances which are brought about by suppression of the external pancreatic secretion. Owing to the absence of this external secretion, the digestion of the nutrient material by the intestine is defective; toxins are formed and resorbed, and these disturb the chemistry of the tissues, and thus give rise to the formation of sugar. In the course of these earlier researches, de Domenicis discovered that the intestinal extract of dogs without pancreas produces glycosuria, though in a slight degree, in normal dogs. In a recent publication he has asserted that severe, permanent glycosuria may be produced by the injection of the duodenal secretion of dogs without pancreas. It is not to be denied that the external pancreatic secretion has a certain influence upon the resorption of nutrient substances in the intestine. Sandmeyer showed that the administration of pancreatic tissue promotes digestion, and resorption of nutriment. Similar results were observed by Lombroso after the introduction of pancreatic juice into the duodenum. In animals which have lost their pancreas, however, this method merely increases the sugar excretion, and where diabetes is present in the slighter form it provokes true glycosuria. The transplantation experiments carried out by Minkowski, Hédon, and Thiroloix prove beyond any manner of doubt that diabetes is not dependent upon the external pancreatic secretion.

The possession by the pancreas of an internal secretorv function was, however, denied for other reasons. In the case of the pancreas, as in that of all organs with internal secretion, the removal of which provokes pathological symptoms, the results of extirpation were explained by the nervous injuries inseparable 
from such operation. The brothers Cavazzani, as well as Thiroloix, endeavoured to explain the results of pancreasectomy by the injury to the nerves of the pancreas and liver; their view was based upon experiments in which extirpation of the solar plexus, or of the nerves in the neighbourhood of the pancreas, was followed by glycosuria. The theory of the nervous origin of pancreatic diabetes has recently been warmly advocated by Pflüger. He points out that, in his experiments with frogs, glycosuria followed, not extirpation of the pancreas only, but also resection of the duodenum and resection of the mesentery between the intestine and the pancreas; and that, in spite of the avoidance of any injury to the pancreas or disturbance of the pancreatic circulation, this result was constant. Herlitzka obtained similar results. The experiments in which dogs showed glycosuria after extirpation of the duodenum are not regarded as conclusive by Pfluger, for the reason that the duration of life in such animals is very short; but he draws attention to the earlier experiments by de Renzi and Reale, in which permanent glycosuria lasting until death was observed in dogs after resection of the duodenum.

That true diabetes follows duodenal extirpation was not confirmed, however, by Lauwens, Ehrmann, Rosenberg, and Minkowski, who ascribe the occasional slight glycosuria which they observed to nutritional derangement of the pancreas. Minkowski's experiment with a dog-in.which, after transplantation of the pancreas, the entire duodenum was excised, the animal remaining free from glycosuria for four weeks, at the end of which time the pancreas was removed, when severe diabetes made its appearance--is decisive evidence against the occurrence of duodenal diabetes.

The sole evidence in favour of the duodenum as a causative factor in diabetes is supplied by two clinical cases of Zak's, in which glycosuria followed cauterization of the duodenum; as well as by the results of certain experiments carried out by the same author, in which, after cauterization of the duodenum, sugar was found to be present in the urine and adrenalin present in the blood (frog's eye test).

The results of experimental transplantation of the pancreas, and of the extirpation of the organ when performed in several sittings, have completely destroyed the theory that pancreatic diabetes is the result of nervous lesions. Pfluger suggested that, in cases where the pancreas is implanted under the skin, a minute nerve branch may remain intact, and that by this means the resected nerve fibres may resume their connection with the nerve centres; this assumption is, however, almost too chimerical to need refutation. Pfluger himself has modified his views to this extent, that in his latest publication he concedes a certain degree of internal secretory activity to the pancreas. That this internal secretion may be influenced by the nerves is readily conceivable. 
That the pancreas possesses an internal secretory function must be conceded upon general grounds. But the two-fold significance attaching to the idea, and especially the earlier idea, of internal secretion, namely, that of positive production together with negative neutralization, suggests the possibility that the regular pancreatic function may consist in the destruction of socalled diabetogenic substances; that, after the extirpation of the organ, these substances accumulate in the body; and that they have a deleterious influence upon the normal metabolism of the carbohydrates. This is a theory which has been advanced by several authors (Hédon, in his first publication, Tuckett), and which seemed to derive support from Gaglio's statement, that ligature of the thoracic duct prevents glycosuria after pancreasectomy. This latter finding received no confirmation, however, from later investigators (Lépine, Hédon, Biedl). The views of Harley and of Vani are based upon de Domenicis's theory; both authors believe that the suppression of the pancreatic function gives rise to an auto-intoxication, the essential features of which are severe nutritional disturbances, glycosuria, and nervous symptoms being among the manifestations of the latter. According to Vani, this auto-intoxication is due to suppression of the endocrinal function of the pancreas.

The assumption that pancreatic diabetes is the outcome of an auto-intoxication never obtained much support. It has since been entirely abandoned; for the hypothesis that auto-intoxication occurs as the result of organic suppression becomes more and more limited in its application, owing to the fact that proof of the existence of toxic substances has never, as yet, been obtained. The possibility was recently suggested by Zuelzer that, after extirpation of the pancreas, harmful substances are retained in the economy which, under normal circumstances, would be excreted or destroyed by the agency of that organ. Zuelzer's findings permit, however, of a different interpretation.

All the known facts concerning pancreatic diabetes, and especially the profound changes in the metabolism of the carbohydrates to which it gives rise, point unequivocally to suppression of an internal pancreatic secretion-which, under normal conditions, plays a decisive rôle in the metabolism of the carbohydrates as the originating cause of the condition.

As evidence against a positive secretory function and formation of hormones by the pancreas, it is pointed out that the brilliant results expected of organo-therapy have been conspicuously absent in pancreatic diabetes. From all accounts, the results of the administration of fresh or dried pancreas and the subcutaneous injection of pancreatic extract, seem to be uncertain both in clinical pancreatic diabetes and in the parallel condition in animals deprived of their pancreas. The reports are, however, exceedingly contradictory. Positive results were observed by 
Caparelli, Zuelzer, Dohrn and Mayer; negative results by Hédon, Gley, Lépine, Forschbach, and others. For the reasons given in the first part of this book, the failure of organo-therapy does not necessarily disprove the internal secretory activity of the organ under consideration. It is no more reasonable to expect a restoration, by means of the administration of pancreatic extract, of the injured metabolic processes of pancreasectomised animals, than it is to expect the prolongation or the saving of life by means of suprarenal extract in the case of suprarenalless animals.

In face of the results obtained by a far more practical method, namely, that of pancreatic transplantation, the uncertain and contradictory results of organo-therapy lose their value as evidence against the formation of a pancreatic hormone. Instances are supplied by Minkowski's experiments, in which portions of the organ were transplanted together with their vascular stem; at a later stage, the vascular stem was severed and the healed in portions of pancreas sufficed to avert diabetes. Pflüger's unsuccessful implantation of the pancreas under the skin of the back in frogs has no bearing upon the question.

That the pancreas does possess an internal secretion has been absolutely proved by Forschbach's parabiotic experiments. The extirpation of the pancreas of one parabiotic dog was followed by slight glycosuria only. The manner in which the wound healed and the absence of cachexia further showed that the metabolic derangements characteristic of diabetes were absent. The internal secretion of the one pancreas sufficed for the needs of both animals. After separation, however, the animal which had been robbed of its pancreas developed diabetes.

Lépine was the first to formulate a theory concerning the nature and method of action of the internal secretion of the pancreas, and this supplied a new outlook in many directions and offered a stimulus to fresh investigation. But a glance at the results of nearly twenty years shows no discovery which in any way elucidates the causes in which diabetes takes its rise. According to Lépine's theory, a substance is formed in the pancreas which is necessary to the normal metabolism of sugar. The supposition is that the normal sugar in the blood is broken up and conveyed to the tissues by a glycolytic ferment, which is formed in the pancreas, reaches the blood by way of the thoracic duct, and attaches itself to the white corpuscles. By extirpating the pancreas, the source of origin of this ferment becomes destroyed. In both experimental pancreatic diabetes and in the spontaneous clinical condition, the amount of the glycolytic ferment present in the blood becomes very much diminished and, as a consequence, the decomposition of the sugar molecule is less than in normal blood. Thus an accumulation of sugar takes place in the blood and the excess is excreted by way of the kidneys.

If we inquire more closely into the fact upon which Lépine 
based his theory, namely, the diminution in clinical diabetes of the glycolytic ferment in the blood, we are led to the conclusion that the theory is untenable. Moreover, the disappearance at blood-temperature of sugar from the blood is no proof of the presence of a glycolytic ferment; for, on the one hand, it is difficult to ascertain the exact amount of sugar present in a fluid containing albumin, while, on the other, the alkaline contents are sufficient to account for an apparent decomposition of sugar (Bendix and Bickel). It is evident, then, that Lépine's theory is lacking in foundation.

Lépine himself, however, abandoned his original theory and assumed the existence in diabetes of a substance inhibitory to glycolysis. He and Boulud succeeded in isolating crystalline bodies from the urine of diabetic and other patients, the exhibition of which produced glycosuria in healthy animals. This substance is said to become decomposed by passage through the vessels of the pancreas. More recently still, Lépine again asserted his belief in a pancreatic internal secretion with the function of promoting glycolysis.

Although it was advanced against Lépine's theory that the normal decomposition of sugar takes place, not in the blood, but in the tissues, it seemed probable that the site of this decomposition lay in the pancreas. In such a case, absence of the pancreatic parenchyma would give rise to insufficiency of the glycolytic ferment and to consequent hyperglycæmia and glycosuria. This view was adopted by Baldi and was supported by the results of experiment. Pal, however, was unable to find a difference between the sugar contents of the arterial blood and those of the venous blood in the pancreas. Baldi, on the other hand, found a smaller amount of sugar in the pancreatic vein than in the carotid artery; he also found a diminution in the sugar contents of blood mixed with dextrose, after it had been allowed to circulate through the pancreas. Lépine discovered further that watery pancreatic extracts have a glycolytic action, and that this action is intensified by the addition of acids. From these findings Lépine assumed that a proferment was present in the pancreas, and that this proferment was converted, by the action of the acids, into a glycolytic ferment.

The presence of a destructive ferment in the pancreas has been confirmed by a large number of authors, but it has since been recognized that, not the pancreas only, but a number of other organs, such as the spleen, liver, lungs, kidneys, muscles, $\& c$. , exercise a glycolytic influence. The decomposition of sugar by the tissues even, was regarded as due to the action of oxidative ferments distributed through them. According to later experiments by Jacoby, Blumenthal, and N. Sieder, it seems probable. however, that glycolysis is a process sui generis, the details of which are still somewhat obscure. Stoklasa and his pupils re- 
garded glycolysis, in both the animal and vegetable economies, as typical alcoholic fermentation. Stoklasa found that, in addition to alcohol and carbonic dioxide, lactic acid, and later acetic and formic acids, were formed by organic enzymes in the process of the decomposition of sugar; according to him, the enzymic processes in the protoplasm are as follows: (I) Primary, the formation of lactic acid by lactolase; and (2) the formation by means of alcoholase, of alcohol and carbonic dioxide. The process of decomposition is completed by the enzymes acetolase and formylase, while combustion into carbonic dioxide and water is effected by the addition of oxygen.

It is true that the processes by which the oxidation of sugar is effected within the organism are in need of further investigation, yet this much may be taken as certain-that glycolysis is a process carried out by ferments which are present in all the tissues, and that in all probability the pancreas possesses no specific function in this respect. As Ehrmann and Wohlgemuth's very careful experiments have recently shown, the blood in the pancreatic vein does not contain a larger proportion of diastatic ferment than that in other vessels.

A specific function in the decomposition of sugar was ascribed to the pancreas by $\mathrm{O}$. Cohnheim, and simultaneously with him but quite independently, by R. Hirsch. According to Cohnheim's experiments, neither the expressed juice of the muscles nor that of the pancreas possesses any marked glycolytic action when employed alone; a complete destruction of glycogen is effected only by the combined activity of the two. Cohnheim assumes that the glycolytic ferment contained in the muscles is rendered active by an agent present in the pancreas. This "activator" is not destroyed by boiling, it is soluble in water and alcohol and insoluble in ether; hence it does not partake of the nature of a ferment, but is an internal secretion. Cohnheim investigated the activity of this agent in increasing quantities, and found that its action was inhibited at excess; he regards this as analogous to the complement deviation reaction.

Rahel Hirsch found that the glycolytic action of pounded liver was considerably strengthened by the addition of pancreatic extract. She assumes that either a proferment or a kinase is supplied by the pancreas, and that this lends to the liver a glycolytic activity.

Cohnheim's results at first received a measure of confirmation (by Arnheim and Rosenbaum and by Sehrt, whose experiments, interesting to relate, were conducted with muscles from mummies of a period previous to 300 B.C.); but Claus and Embden, who carried out a series of very careful experiments in v. Noorden's laboratory with the object of verifying Cohnheim's results, were unable to find any proof of the existence of a pancreatogenic activator, and they ascribe the disappearance of the sugar to 
bacterial impurity. Also J. de Meyer and Nanking were both unable to confirm Cohnheim's results.

Like Lépine, J. de Meyer (1904) believed that the decomposition of sugar took place in the blood. According to his experiments, the glycolytic ferment is situated, not in the muscles, but in the white blood corpuscles, and is rendered active by the agency of the pancreas. He believes that the pancreas contains a " sensibilizing substance," which changes a proferment -which is present in the leucocytes and is secreted by them and which possesses properties analogous to those of the fibrin ferment -into the active glycolytic ferment. De Meyer also believes that the cause of diabetes after extirpation of the pancreas lies in the suppression of the internal secretion, and that the latter is the agent which activates the glycolytic ferment. The evidence against this hypothesis is supplied by the arguments which were advanced in disproof of Lépine's theory.

Vahlen (1908) was also unable to demonstrate the presence in the pancreas of a substance which splits up sugar and thus promotes oxidation. He believes it possible, however, that the pancreas may contain a substance, at present unknown, which, without giving rise to direct fermentative decomposition, may yet bring about an increase in the decomposition of sugar in the organism. He succeeded in isolating a constituent from the pancreas which appreciably accelerated the alcoholic fermentation of sugar, and, in the case of phlorizin animals, effected a considerable reduction in the excretion of sugar. Vahlen did not, however, carry out conclusive experiments with pancreasectomized animals.

If we review the publications which, beginning with Lépine's theory, have attempted to explain the nature of pancreatic diabetes, it is evident that conclusive proof of the assumption that the pancreas supplies a substance which promotes glycolysis, or which is essential to the general metabolism of sugar, is not forthcoming. The generally accepted view, that pancreatic diabetes is a derangement of metabolism which consists in the arrest of the normal conversion of sugar, has not, as yet, been sufficiently confirmed, and for this reason, has little positive value.

The older hypothesis, that diabetes is the expression of an increased formation of sugar, has recently been revived; it is reinforced by certain new arguments and has received a large measure of support. This tendency marks a return to the teaching of Chauveau and Kaufmann (I893) which, at the time of its inception, found few supporters.

The central point round which the process of the metabolism of the carbohydrates revolves, is what Claude Bernard described as the "glycogénie animale"; the process, that is, by which glycogen accumulates and sugar is formed in the liver. From the carbohydrates derived from the nutriment, glycogen is formed 
in the liver and accumulates there as reserve material. It is probable that, when the supply of carbohydrates is deficient, the formation of glycogen from albumin also takes place in the liver; and here, also, the conversion of glycogen into the form in which it is used by the tissues, namely, grape-sugar, takes place. The sugar is withdrawn by the organs from the blood and is in part employed directly in the performance of function; in part, and in the muscles more particularly, it is converted into glycogen. The process of converting dextrose into a colloid polysaccharide, of condensing the glycogen, and of again converting it into sugar, is the common reversible function of the cells.

The sugar contents of the blood are dependent upon the amount of sugar formed in the liver. According to Chauveau and Kaufmann, this process is regulated and controlled by the nervous system. These authors believe that two nervous centres exist, one in the medulla oblongata and one in the cervical spinal cord, and that by means of the centrifugal nerves contained in the sympathetic, these regulate the formation of sugar, one centre exercising an inhibitory, the other a direct stimulatory influence upon the sugar production. According to the original theory of these authors, the nervous impulses are first conveyed to the pancreas, whence they pass by way of the nerves to the liver, and there bring about changes in the sugar production. Taking into account the many facts which point to a chemical correlation, these authors modified their view to this extent, that they ascribed to the pancreas an internal secretion influencing the liver, which was conveyed to the liver by the agency of the blood-stream. The production of the secretion they believed to be under the control of the nervous system.

Regarded in the light of our present knowledge, the theory of Chauveau, and Kaufmann signifies that the influence of the internal secretion of the pancreas is exercised directly upon the liver in part only; its main influence is directed to the nerve centres which control the sugar production, the effect upon the stimulatory centre being inhibitory and the effect upon the inhibitory centre stimulatory. In this way, a two-fold hindrance to the formation of sugar in the liver is effected by the pancreas with the aid of the nervous system. The suppression of the internal secretion of the pancreas not only abolishes inhibition, but it also relieves the check upon stimulation, and, in consequence, a pronounced glycosuria make its appearance. The suppression of inhibition appears, however, to be the decisive factor; for Hédon and Kaufmann discovered that the hyperglycamia, or rather the glycosuria, of pancreatic diabetes is by no means maximal and may be increased by puncture of the floor of the fourth ventricle, in other words, by irritation of the stimulatory centre. According to this theory, then, pancreatic diabetes consists in an increase in the sugar formation brought about by a 
disturbance somewhere in the functional chain, that is to say, in either the central nervous system, the pancreas, or the liver.

A similar view was expressed by Thiroloix and, more recently, by Pfluger. These authors assume that the sugar contents of the blood are dependent upon two opposing factors, both of which are under the control of the central nervous system. Of these two factors, one is the mechanism by which sugar is formed in the liver, which is governed by the centre in the medulla oblongata. The other factor, antagonistic to the first, is supplied by the so-called anti-diabetic activity, which limits the production of sugar; it is situated in the pancreas and is also under the control of the nervous system. Thiroloix assumes that the inhibitory action of the pancreas is performed by the agency of an internal secretion which is regulated by the nervous system; Pflüger, on the other hand, believes that its anti-diabetic property is supplied to the pancreas by nerves which originate in the duodenum. This view is supported by Herlitzka. But Pfluger concedes that the glandular substance of the pancreas may play a part in Sandmayer's diabetes; he suggests that the epithelial cells may possibly supply an anti-diabetic ferment to the blood-stream.

It is evident from these theories concerning the nature of pancreatic diabetes, that the conception of the pancreatic function as an inhibition of the formation of sugar, lies at the root of all the later teaching. Further information concerning the field of activity of the pancreatic hormone, hence a new point of view in regard to pancreatic diabetes, has recently been supplied by the discovery of the relationship between the metabolism of the carbohydrates and the activity of the other internal secretory organs.

Particularly important was the discovery and analysis of adrenalin glycosuria, for it showed the enormous influence of the suprarenal, or rather of the adrenal system, upon the sugar economy of the organism. Further, we know that adrenalin, by mobilizing the reserve glycogen and thus promoting the formation of sugar from other material, raises the sugar contents of the blood. The antagonism between the activity of adrenalin and that of the pancreatic hormone is chiefly symptomatic in its expression. The action of adrenalin is modified in certain directions by the internal secretion of the pancreas. In Zuelzer's experiments, the effect of pancreatic extract in inhibiting adrenalin glycosuria might be explained by the assumption that the excess of sugar in the blood undergoes combustion by the agency of the pancreatic hormone introduced into the economy. Zuelzer's results permit, however, of another explanation. It may be that the antagonistic effect of the two substances is the outcome of their antagonistic influence upon the same physiological site; in other words, the activity of an apparatus may be inhibited by 
the pancreas and stimulated by the adrenal system. What we know concerning the site of the activity of adrenalin suggests that the pancreatic hormone has an inhibitory action upon the nervous apparatus which regulates the formation of sugar. Löwi's reaction also points to an inhibitory action, on the part of the pancreas, upon organs with sympathetic innervation.

The investigations of Eppinger, Falta and Rudinger, to which frequent reference has been made, show that a certain influence is exercised by the thyroid apparatus upon the metabolism of the carbohydrates, and that this influence is apparently brought into play by the agency of the pancreas. The results of their experiments seem to show that, while the activity of the thyroid is opposed to the internal secretory activity of the pancreas, that of the parathyroids is favourable to it. The assumption is that the thyroid hormone stimulates the function of the vegetative nervous system; and that the pancreatic hormone affects that function in the opposite sense.

From the data which have here been set forth, we are justified in concluding that the pancreas, by means of its internal secretion, inhibits the formation of sugar in the liver, and that this inhibition is effected through the agency of certain nervous apparatuses. Suppression of pancreatic activity abolishes the normal check upon the formation of sugar, in consequence of which the glycogen present in the organism is released; that is to say, the glycogen derived from the carbohydrates in the food or, in default of these, from other material, is converted into glucose; and as a consequence hyperglycæmia and glycosuria follow. The pancreatic hormone appears to affect the same peripheral nervous apparatus as adrenalin; for, as Kaufmann showed, resection of the splanchnic nerve is followed by pancreatic diabetes in exactly the same manner as adrenalin glycosuria.

Attempts to isolate the pancreatic hormone have, up to now, been fruitless. Nothing definite is known concerning its chemical nature and we are forced to fall back upon hypothesis. The most probable assumption is, that the substance is of the nature of a ferment, and that it influences that process by which the decomposition and new formation of glycogen take place in the liver. The fact that, after extirpation of the pancreas, deposition of glycogen in the liver may be effected by the administration of levulose, suggests that suppression of the pancreatic function does not prevent the formation of glycogen, but that it permits the unchecked splitting up of this substance. The normal pancreatic hormone is a substance which inhibits the diastatic conversion of glycogen into sugar.

Many experiments have been undertaken with the object of discovering the path along which the pancreatic hormone travels, and the results were described by me in 1898 . I discovered that in the large majority of instances (66 to 86 per cent.), ligature

$$
\text { ₹ } ¥ .
$$


of the thoracic duct at the neck, or the diversion of the chyle by means of a fistula, was followed by permanent glycosuria, which was unaffected by either the fasting state or by feeding with carbohydrates. The fact that the thoracic duct is not the only channel by which the chyle is carried off, explains the negative cases.

The results of these experiments led me to conclude that a substance is contained in the chyle which influences the metabolism of sugar in the organism. I resumed this line of investigation in association with Offer in 1907 , and we discovered that the instillation of adrenalin into the conjunctiva produces a mydriasis, which, according to Löwi, is characteristic of pancreatic diabetes. We discovered, further, that the chyle in the thoracic duct possesses an inhibitory action in regard to adrenalin, and that adrenalin glycosuria may be reduced, or even abolished, by the subcutaneous injection of chyle, as well as by intensifying the normal lymph secretion by means of lymphagogue substances. It would appear from this that the substance contained in the chyle which influences the metabolism of the carbohydrates, is identical with the pancreatic hormone. The pancreatic substance which possesses the property of inhibiting the formation of sugar, reaches the circulation by way of the lymphatics.

Such being the case, then, it is to be expected that the injection of chyle from the thoracic duct of normal dogs into the veins of dogs without pancreas, will be followed by a diminution of the glycosuria. As a matter of fact, positive results were obtained in one case by Lépine, though Falta's results were negative.

In my later experiments, the action of the chyle in reducing glycosuria after pancreasectomy, was very clearly shown, the best results being obtained in cases where, owing to the presence of small remnants of pancreatic tissue in situ, the derangement of metabolism had not attained the maximal. On the days upon which large quantities (200 to $500 \mathrm{c.cm}$.) of chyle from healthy dogs were injected subcutaneously, the animals showed a diminution of the quotient $\mathrm{D}: \mathrm{N}$ to $\mathrm{I} .5$ or 1.2 ; while upon days when no lymph was given the quotient reached I.8, and later even 2.I. It should be noted in passing that these results suggest the therapeutic employment of chyle, or of lymphagogue substances, in those cases of clinical diabetes which suggest pancreatic inadequacy, but not pancreatic suppression.

The analogy between the symptomatology of true clinical diabetes and the experimental pancreatic form is so far-reaching, that it is not surprising that we frequently encounter the opinion that the metabolic disturbances of clinical diabetes are also pancreatogenic in their origin and are the expression of diminished or internal secretory activity on the part of the pancreas or its suppression. It should be borne in mind, however, that a 
pathological alteration may take place at any point in the complicated mechanism by which the sugar economy is regulated; and that a pathological condition of any one of the internal secretory organs associated with the metabolism of the carbohydrates, and consequent disturbance of their interrelationships, may give rise to slight glycosuria or even to permanent diabetes. But in these cases a relative insufficiency of the pancreas must also be assumed.

Such being the state of affairs, the question as to whether anatomically demonstrable changes take place in the pancreas in clinical diabetes, acquires an increased significance. Lesions of the pancreas in clinical diabetes were described before experimental pancreatic diabetes had been discovered, and the pathogenetic significance of these lesions was suggested by several authors. The comparative scarcity of instances in which diabetes is accompanied by serious anatomical lesions of the pancreas, and the enormous preponderance of cases in which no anatomical pancreatic lesion is demonstrable, together with the doctrine that serious disease of the pancreas may be unaccompanied by diabetes, are weighty arguments against the pancreas as a constant factor in the pathogenesis of clinical diabetes.

The changes which are observed in the pancreas in diabetes have recently become a subject of increased interest to anatomicopathologists. The morphological findings throw some light upon the question as to which tissue element it is which elaborates the pancreatic internal secretion. These, however, will be discussed later. All we need say here is, that recent investigations show that pancreatic lesions of the most varied description, chronic inflammatory and degenerative changes, atrophy and neoplasms, are observed in a comparatively large proportion (according to v. Hansemann about 70 per cent.) of diabetic patients. It is true that these are always cases in which the disease is present in its severest form; information concerning the slighter aspect of the disease, though its pathogenesis is far more interesting, is very scanty. Negative anatomical findings in the pancreas cannot be regarded as evidence against the pathogenetic significance of this organ: the disturbance of the metabolism of the carbohydrates may originate primarily in other links of the functional chain, or the chemistry of the pancreas may be altered without demonstrable morphological changes.

\section{THE INTERNAL SECRETORY TISSUE ELEMENTS OF THE PANCREAS.}

As in the case of all glands possessing an external secretion, it was at first assumed that the glandular cells of the pancreas were bipolar, that is to say, that both the internal and external secretory functions were performed by the same epithelial cells. 
But in addition to the acini and their excretory ducts, it was known from Langerhans's description (I869), that the pancreas contained irregularly distributed cell groups, which were later named, after their discoverer, the islands of Langerhans. The structure of these elements suggested that they might possess an internal secretory function.

The islands are composed of polygonal cells with ill-defined boundaries, containing large round nuclei and comparatively small and sparsely distributed granules. They are remarkably well furnished with capillary blood-vessels which are intimately associated with the cells, and they are surrounded with a delicate connective tissue sheath (Flint, Laguesse).

A question of paramount importance in the determination of function, namely, whether these islands. contain lumina communicating with the excretory ducts of the pancreas, is not as yet definitely decided. Lumina within the insular area have been described by Gianelli and Giacomini in reptiles, by Dale in toads, and by Vincent and Thompson in Kinosternon and in Amia calva. According to both Lewaschew and Mankowski, the islands in mammals may be injected from the excretory ducts, though this is denied by other authors (v. Ebner, Dogiel, Rossi). Laguesse found that, in the snake, the islands contained lumina which had an unbroken continuity with those of the acini. Welldeveloped excretory ducts have also been occasionally observed in the interior of the islands of the human pancreas. In his investigation of the regenerative processes which take place in portions of pancreatic tissue implanted in the spleen, Kyrle observed proliferation of the epithelial cells of the excretory ducts, in consequence of which the acini and the islands were formed separately and excretory ducts were present in the islands when fully developed. The embryological investigations of Weichselbaum and Kyrle led them to conclude that the islands of Langerhans are derived from the epithelium of the excretory ducts, and that their formation takes place, not during fœtal, but during the early part of post-fœtal life.

The islands of Langerhans were regarded by the older scientists, as well as many later authors, as lymph follicles to which no particular functional significance was attaching. A more exact knowledge of their genesis showed, however, that the cells of the islands are of epithelial origin. By some authors (Giannelli, Oppel) they are even regarded as the rudimentary remains of an atavistic gland. Many scientists held the view that, though the acini and the islands differ structurally from one another, they are not functionally differentiated. Harris and Gow, Gibbes, Gianelli and Giacomini assume that the islands play a part in the production of the external pancreatic secretion, especially of the diastatic ferment. Jarotsky observed signs of increased secretory activity on the part of the cells of the islands after the ad- 
ministration of carbohydrates in large quantities. According to Lewaschew and Pischinger, the islands are really acini in a state of functional exhaustion which, after a period of rest, resume their secretory activity.

Laguesse (1893) was the first to adopt the view that the internal secretion is elaborated by the cells of the islands of Langerhans, the islands thus possessing an endocrinal function which is of importance in the metabolism of the carbohydrates, while the external digestive secretion is supplied by the acini. The supposition is that a continuous transformation of acini into islands, and vice versa, takes place, by which the two secretory processes are carried out. Laguesse's theory principally depends upon the presence of transition forms; these he described with extreme minuteness and his findings are confirmed by a large number of observers (Mankowski, Gentès, Perdrigeat and Triboudeau, Dale, Vincent and Thompson, Marrassini). Changes in the number and size of the islands, and transition forms between acini and islands and vice versa, have been obtained by certain experimental measures, such as the injection of secretine, pilocarpine and phlorizin; fasting; and over-feeding. But these transformatory changes of acini into islands and vice versa have been questioned by several authors, first by Vassale, then by Diamare, Stangl, Rennie, and others, and more recently by Weichselbaum and Kyrle.

In his critical survey, Lombroso arrived at the conclusion that the morphological findings, obtained from experimental and comparative anatomical investigation, were insufficient to justify the theory of mutual transformation. According to Lombroso, mutual transformation does not prove a sharp differentiation of the internal and external secretions as functions of the two pancreatic tissues, acini and islands; but it confirms his view that both functions of the pancreas are undertaken by both tissues.

There are numerous authors, however (Kohn, Massari, Diamare, Rennie, Opie, \&c.) who deny Laguesse's theory of the intimate morphological relationship between the acini and the islands of Langerhans; who regard the latter as independent tissue formations, but believe them to be ductless glands-epithelial organs analogous to the parathyroids, the suprarenal cortex, and the anterior lobe of the hypophysis-and whose property it is to elaborate the pancreatic hormone.

Within the last fifteen years, clinical diabetes has provided a certain amount of anatomico-pathological material, which has a considerable bearing upon the internal secretory elements in the pancreas.

The numerous investigations which have been made have led, however, to very divergent results. The findings which they observed led a number of investigators to adopt the insular theory (Opie, Ssobolew, Herzog, Weichselbaum and Stangl, Sauerbeck, 
Diamare, Visentini, \&c.); while others (Hansemann, Gutmann, Karakascheff, Herxheimer, Schmidt) ascribed the internal secretory function to the acini. Others, again (Reitmann), adopted the view that both pancreatic tissues are concerned in the etiology of diabetes. According to Lombroso, the anatomico-pathological findings are distinct evidence against the assumption that the internal secretion of the pancreas is the function of one tissue formation only, whether of the acini or of the islands of Langerhans.

Experiments undertaken with the object of ascertaining the structural changes in the pancreas, which follow the exhibition of substances believed to have an action in increasing or diminish ing the hormone production, have also yielded contradictory results. Ssobolew and Lépine found that, in the dog and the guinea-pig, the intravenous injection of grape-sugar was followed by a reduction in the size of the islands and by a diminution in the number of granules in the island cells; while Diamare, on the contrary, observed a remarkable profusion of granules in the island cells of a Teleostean (Motella tricirrata), of frogs, and of guinea-pigs. Marrassini describes hypertrophy of the islands of Langerhans in rabbits, where prolonged administration of grapesugar was accompanied by continued glycosuria, and this hypertrophy was of so intense a nature that the neighbouring acini were flattened, deformed and atrophied. The island cells contained an enormous number of fine fuchsinophile granules. Reports of the effects produced by the injection of phlorizin and adrenalin upon the structure of the pancreas are equally contradictory. After the repeated injection of phlorizin into dogs, Ghedini observed a marked reduction in the volume of the pancreas, due to degenerative atrophy and necrosis of the acinous tissue, no cell changes taking place in the islands. Lazarus described marked hyperplasia and hypertrophy of the islands of Langerhans in guinea-pigs after prolonged treatment with phlorizin and adrenalin, and here also the acinous tissue was unchanged. After the administration of adrenalin, Herter and Wakeman found necrosed foci in the pancreas and changes in the cells of the islands of Langerhans; in chronic poisoning with arsenic, morphia, and phosphorus, Carnot and Amet found hyperplasia, together with frequent fatty degeneration, of the islands. Lépine and Vigliani observed absolutely normal histological conditions after the exhibition of phlorizin; and Tiberti reports similar findings after the injection of phlorizin and adrenalin, as the result of his very careful experiments with guinea-pigs and rabbits. The latter author, like Heiberg, believes that the hypertrophied islands which Lazarus described are normal formations in the pancreas of guinea-pigs.

A second experimental method of physiological differentiation between the two tissues of the pancreas seemed to promise better 
results. The method was based upon the known fact that ligature of the excretory ducts of the pancreas, and the injection of foreign substances into them, suppresses the external secretion and may lead to more or less pronounced atrophy of those portions of the gland by which the external secretion is elaborated. It was soon found that glycosuria did not, as a rule, make its appearance in experiments of this sort, and that the tissue of the islands of Langerhans remained in comparatively good condition. After ligature of the excretory ducts of the pancreas of guinea-pigs, Schultze observed disappearance of the acini, with persistence of the islands and absence of glycosuria; similar findings were described by Ssobolew in rabbits, dogs and cats; and by Laguesse in rabbits. Sauerbeck observed transient glycosuria in rabbits after ligature of the pancreatic duct; but the incidence, course and intensity of the glycosuria was said to run parallel with changes in the islands of Langerhans. Tiberti describes similar findings, while different results were obtained by other observers (Hansemann, Mankowski) who employed the same method.

From the results of his own experiments and those of other scientists, Lombroso concluded that, after ligature of the pancreatic duct of rabbits, changes take place in the acini, but that the acini do not entirely disappear, and that the islands also undergo modification, though more slowly. Glycosuria is either absent, or occurs in isolated instances only. He ascribes a particular significance to the fact that the resorption of nutriment remains unchanged.

In pigeons, ligature of the excretory ducts produced changes in the pancreas similar to those observed in rabbits; but though glycosuria was not present, the birds died after two to three weeks, in consequence of the derangement of the absorption of food. It must be remembered, however, that the absence of glycosuria in birds is no proof of the continuance of the hormone production; the diabetic disturbance of metabolism which follows extirpation of the pancreas is expressed only by hyperglycæmia, though Lombroso failed to take this fact into consideration.

The numerous and very careful experiments which have been carried out with the pancreas of dogs all show that, not only do the acini not disappear after ligature or resection of the excretory ducts, but that they do not undergo appreciable change. Lombroso found perfectly preserved normal acini fourteen days after operation. This finding has been explained by the presence of supernumerary excretory ducts, by which the passage of secretion from the gland was facilitated (Hess and Sinn). Lombroso rejects this explanation, however, and he emphasizes the fact that well-developed acini were present in implanted portions of pancreas which sufficed to prevent the occurrence of diabetes.

A consideration of all the available experimental material led Lombroso to conclude that both the epithelial tissues of the 
pancreas, namely, the acini and the islands of Langerhans, are concerned in the internal secretory function of that gland.

According to this author, the internal secretion of the pancreas not only influences the sugar economy, but also plays a part in the absorption of nutrient material. Previous to Lombroso, de Domenicis, as has been shown, believed the origin of pancreatic diabetes to lie in the disturbance of absorption which he observed after pancreasectomy. Abelmann's experiments ( 1890 ) showed that the decomposition of fat is, in a quantitative sense, only slightly affected by total extirpation of the pancreas, but that the absorption of fat entirely ceases after this operation. Fat absorption persists in a greater or less degree after ligf ure of the excretory ducts, as well as after partial extirpation and transplantation of a portion of pancreatic tissue to a position where it is in communication with the intestine. The absorption of fat in the latter instance has been explained by the assumption that the external secretion of the pancreas becomes re-absorbed and reaches the blood-stream, whence it is conveyed to the intestinal mucosa.

Lombroso found that the capacity for the absorption of fat was diminished by extirpation of the pancreas. The fæces of animals without pancreas contain precisely the same amount of fat as their food. If oleic acid is given, a portion becomes absorbed and this is replaced by fat having a higher melting point. After ligature of the excretory ducts, or where the pancreas has ceased to discharge secretion into the intestine, the absorption of fat amounts to about 80 per cent. of the amount of fat given. This absorption is not due to the agency of an external secretion, conveyed by some means into the intestine, but must be ascribed to an internal pancreatic secretion, the activity of which is necessary to the metabolism both of the fat in the tissues and that introduced from the exterior.

It has been advanced against this conclusion that, in Lombroso's experiments, the measures taken to prevent the external pancreatic secretion from reaching the intestine were insufficient. Hess and Sinn suggest supernumerary excretory ducts; Visenti points to the possibilities of the ligatured ducts again becoming passable, and of the formation of new ducts. Burckhardt, in one instance, obtained contradictory results and he believes that, in the case of animals with pancreatic fistula, the pancreatic secretion may be returned to the intestine by the animals licking themselves.

Lombroso's results have, however, been confirmed by Zunz and Mayer, and more recently, by R. Fleckseder in a series of extremely careful experiments carried out by methods designed to obviate all sources of error. Fleckseder's conclusion is that the absorptive power of the intestinal mucosa is chiefly dependent upon the internal pancreatic secretion. There is, however, com- 
pensation for the suppression of this secretion. Where the function of the pancreas has gradually become completely suppressed, the consequent severe diabetes may be unaccompanied by derangement of absorption, and the assimilation of nutriment may at this stage be better than where an active pancreatic fistula is present.

Lombroso, also, believes the internal secretory function to be the property of both pancreatic tissue formations.

\section{THE INTERNAL SECRETION OF THE GASTRIC AND INTESTINAL MUCOSA.}

Claude Bernard's observation, which later formed the subject of considerable investigation, first showed that the secretion of pancreatic fluid is dependent upon the filling of the duodenum and that it reaches its maximum when the ingesta have passed from the stomach into the duodenum. From the results of numerous experiments, admirable both in conception and technique, Pawlow came to the conclusion that the mechanism by which the pancreatic secretion is elaborated is by a reflex process on the part of the nervous system; the impulse proceeding from the gastric mucosa is carried by centripetal vagal fibres to a centre in the medulla oblongata, and from this centre stimuli are sent out which, travelling by way of the centrifugal vagus or splanchnic nerve, cause the pancreatic gland to secrete.

Under Pawlow's direction, Popielski next showed that, if acids are introduced into the duodenum, the pancreatic secretion appears after resection of both the vagus and the splanchnic: nerves, after extirpation of the solar plexus, and even after destruction of the spinal cord. Similar findings were observed by Wertheim and Lépage and they, as well as Popielski, concluded that the results were the outcome of a local peripheral reflex.

Bayliss and Starling, however, discovered that the pancreatic secretion is elaborated if acids are introduced into the lumen of a coil of the duodenum which has been freed from all nervous connections. This result showed very clearly that the release of the pancreatic secretion depends upon a chemical substance, and that chemical stimuli are conveyed to the pancreas by way of the blood-stream. According to Wertheim and Lépage, the direct introduction of acids into the blood-stream has no effect upon the pancreatic secretion; hence the only possible solution was that, by the aid of acids, a substance was formed in the mucous membrane of the intestine.

Bayliss and Starling obtained an extract by treating mucosa scraped from the upper part of the small intestine with hydrochloric acid, and this extract, when injected into the veins, provoked pro- 
fuse pancreatic secretion. To this active substance, which is not a ferment because it withstands boiling and is soluble in alcohol, they gave the name of "secretin." Bayliss and Starling assumed that, by the hydrolytic action of the acids, secretin is formed from "prosecretin," a precursor present in the cells of the duodenal mucous membrane. The secretin formed in the mucosa reaches the pancreas by way of the blood-stream; for Enriquez and Hallion succeeded in proving that, if the bloodstream is conveyed from the vessels of a $\operatorname{dog} \mathrm{A}$ into those of a $\operatorname{dog} B$, pancreatic secretion will make its appearance in B after the injection of acid into the small intestine of $\mathrm{A}$. These experiments proved the existence of an internal secretion of the intestinal mucosa, and they showed at the same time that there is a correlation of function between the intestinal mucosa and the pancreas, which is effected by the agency of hormones.

Bayliss and Starling's discovery has been subjected to repeated tests, and the actual findings have received almost general confirmation. The specific activity and the intimate chemical nature of secretin have formed the subjects of a controversy; into the details of which we cannot enter here. Suffice it to say that Popielski believes secretin to be identical with peptone, a product of the decomposition of albumin; while according to v. Fürth and Schwarz, secretin is not a homogeneous substance, but is amixture of several substances possessing the property of exciting secretion, of which the most important is cholin.

A far greater interest is attaching to the manner in which this discovery affects our conception of the mechanism by which the external secretion of the pancreas is effected. Is the secretory process entirely due to the agency of a hormone, as Bayliss and Starling are inclined to believe; or are the nervous reflexes, which Pawlow's experiments revealed, also brought into play? This question remains for the present unanswered. Several authors. (Fleig) assume, and apparently with justice, that two simultaneous processes are concerned in the passage of the chyme into the duodenum. Of these, one is the formation of secretin, which induces, by chemical means, secretion of the pancreas; the other is provided by the acid contents of the duodenum, which stimulate the nerve-terminals, and the reflex action of these also leads to pancreatic secretion.

According to Bayliss and Starling, the secretin formed in the duodenal mucosa stimulates the secretion of bile and increases its quantity.

With regard to the secretion of the intestinal juice, the succus entericus, opinions are divided. The intestinal juice serves to activate the proteolytic property of the pancreatic secretion, for: the trypsinogen contained in the latter is converted into the active trypsin ferment by the agency of the endokinase in the intestinal juice. The elaboration of the intestinal secretion is effected, 
according to Pawlow, first, by the mechanical stretching of the intestinal canal, and second, by the presence of the pancreatic juice. Bayliss and Starling believe it probable that the flow of intestinal juice from the glands in the intestinal wall is provoked by the chemical stimulus supplied by the pancreatic secretion; while Delezenne and Frouin hold the view that the discharge of all the three secretions which are active in the upper part of the small intestine, namely, the bile, the pancreatic fluid, and the intestinal fluid, is effected by the same mechanism, namely, the elaboration of secretin in the intestinal mucosa after the passage into the intestine of the acid contents of the stomach.

According to Frouin, the elaboration of secretion by the intestine may be increased by the intravenous injection of intestinal fluid.

Pawlow's careful experiments have shown that the secretion of the gastric fluid is largely the outcome of nervous impulses. But two to three hours after food the gastric secretion becomes increased; according to Pawlow, this increase is maintained after resection of the vagi, and it appears to be dependent upon the nature of the food.

Edkins next discovered that an extract is obtainable from the pyloric mucous membrane by extraction with hydrochloric acid, as well as by rubbing down with dextrose, maltose, or peptone. When injected into the veins in repeated small doses, this extract causes secretion of the gastric juice. According to Edkins, the active substance, gastrin, is not destroyed by boiling and appears to belong to the same class of bodies as pancreatic secretin. This "gastric secretin," as Starling calls it, is present only in the pyloric mucosa, extracts of the mucous membrane of the fundus being inactive.

\section{THE INTERNAL SECRETION OF THE KIDNEY.}

The assumption of an internal secretory function on the part of the kidney is among the earliest dicta of the Brown-Séquard doctrine. As early as i 869 , Brown-Séquard suggested that uræmia either was not, or was not exclusively, the result of the accumulation of urinary constituents in the blood, but was due to suppression of the internal secretion of the kidney. Following his first experiments with testicular extract, Brown-Séquard with d'Arsonval endeavoured to obtain practical proof of his assumption. A large number of clinical observations of anuria of long duration, in which uræmic symptoms were almost entirely absent. led him to conclude that the occurrence of uræmia does not depend solely upon the presence of urinary constituents in the blood, and that it is not the result of stimuli supplied by the diseased kidney; but that it is principally due to changes in the chemical composition of the blood, brought about by suppression of the 
renal internal secretion. Experiment showed that animals die much more rapidly after the removal of both kidneys than after ligature of both ureters, in spite of the fact that the retention of urinary constituents is the same in both cases, and this fact seems to bear a similar interpretation. Brown-Séquard and d'Arsonval further showed that if renal extract obtained from guinea-pigs and rabbits is injected into the veins of nephrectomized animals of the same species, symptoms of uræmia will appear later, and will be less intense in these animals than in control animals which have not received similar treatment. In animals treated with renal expressed juice, the duration of life' was as long, or longer, than in animals with ligature of both ureters.

Lépine's observations have an important bearing upon the passage of substances from the kidneys into the blood. He found that the ligature of both ureters of rabbits produced a fall in temperature, vomiting, and diarrhœea, terminating in death. An entirely different symptom-complex resulted, however, when the ureters were connected by means of cannulæ with a vessel containing normal saline solution, the pressure in the vessel being higher than that in the ureter. This procedure produced, not a fall, but a rise in temperature, dyspnoea and convulsions. According to Lépine, the filtrate of a kidney rubbed down in water, when introduced into the blood, also produces a rise in temperature, dyspnœea, and stronger movement; and Lépine believes that thermogenic and dyspnogenic constituents are present in renal substance.

E. Meyer, a pupil of Brown-Séquard's, next discovered that the blood of uræmic animals is inactive in normal animals, but that, in nephrectomized animals, it produces a remarkable dyspnœic slowing of respiration. In a second series of investigations, Neyer studied the periodic respiration, resembling the Cheyne-Stockes type, of uræmic dogs; he found that this remarkable uræmic symptom is reduced by the injection of renal expressed juice, as well as by the injection of normal defibrinated blood, the effect being so marked that for a short time the respiration becomes normal. Blood from the renal veins is said to produce particularly good and well-marked results.

These reports of prolonged life and improved uræmic symptoms in nephrectomized animals after the administration of renal extract or blood from the renal veins, have been tested by many investigators (E. Vanni, Manzini, Vitzou, Ajello, and Parascandalo, Mori, Bozzolo, Gilbert and Carnot, Spineanu, Maragliano, Chatin and Guinard, Fiori), but with very conflicting results. A definite pronouncement is hardly possible, however, seeing that the duration of life in nephrectomized animals is, in any case, very variable. As I know from my own experience, dogs and rabbits sometimes live five to six days after the removal of their kidneys, while other animals of the same species, operated upon 
in a manner precisely similar, perish at the end of thirty-six hours. In the second place, the appearance and course of the clinical symptoms of uræmia is not, in such animals as these, a reliable criterion of the condition. Many animals from which the kidneys have been removed show no symptoms for two, three, or four days, and then die, either suddenly or within a few hours, without having shown any characteristic signs. In the case of other animals, there is repeated vomiting and dyspnœa as early as the first day after operation; recovery is frequent, or there may be a condition of chronic uramia lasting for several days. Given such conditions, I hardly think it possible to estimate the results of any therapeutic measures at their proper value. Moreover, to base a so-called physiological treatment of uræmia upon the results of such experiments, and to apply this treatment, by the exhibition of renal extract, to clinical nephritis, in my opinion, shows a grave lack of judgment.

Attempts have been made to prove the elaboration of an internal secretion by the kidneys by means of renal extract. Oliver and Schäfer proved that the kidney belongs to that class of organs the extract of which, when injected into the veins, invariably produces an increase in arterial tension. Tigerstedt and Bergmann next showed (I898) that a substance is obtainable with cold water from the fresh kidney which, when injected into the veins of rabbits, produces within a comparatively short time amore or less marked rise in blood-pressure. This active substance; to which the name of "renin" was given by the discoverers, is derived from the cortex, and, in very small quantities, from the medulla; it is not dialyzable, it is soluble in water, in dilute saline solution, and in glycerine; it is insoluble in absolute and in 50 per cent. alcohol; it withstands heat up to 54 to $56^{\circ}$, but is destroyed by heating in a water bath and by boiling. A rise in blood-pressure is produced by this substance in very small quantities, and is due to the effect which it has upon the peripheral vessels, upon the nerve terminals, and perhaps also upon the muscles. From the results of two experiments, Tigerstedt and Bergmann assume that, under normal conditions, renin is conveyed into the blood which circulates through the kidneys. In these experiments, blood from the renal veins was injected in quantities of $2 \mathrm{c.cm}$. into the veins of rabbits which had been nephrectomized one to two days previously, and a rise in bloodpressure of 13 to 25 per cent. followed. These experiments were afterwards tested by Lewandowsky, who found that the results produced with 5 to $6 \mathrm{c.cm}$. of renal venous blood were in no way different from those produced by the same amount of venous blood from other parts of the body; for this reason he is not prepared to ascribe a specific vaso-contractor action to the blood in the renal veins.

The effect which renal extract has in raising blood-pressure 
had been confirmed by various authors (Riva-Rocci, Vincent and Sheen, Batty Shaw), who believe that the tonic internal secretion of the kidney forms the connecting link between renal disease on the one hand, and arterial hypertension and cardiac hypertrophy on the other. Seeing, however, that the hypertensive effect of renal extract is not specific but is the property of extracts obtained from a large number of other organs, it can hardly be regarded as proof of an internal secretion on the part of the kidney, and still less as a satisfactory explanation of the cardiac dhypertrophy of renal disease.

Rautenberg recently described marked cardiac hypertrophy and sclerotic changes in the vesst ls of rabbits after temporary ligature of the ureter.

The formation of what are known as "autonephrotoxins," has been regarded as a further argument in favour of the passage of the renal substance into the blood-stieam. Lindemann was the first to show that, after the repeated injection of the expressed juice of the kidney, an active poison, heteronephrotoxin, is formed in the serum of immunized animals, which has a specific and very injurious effect upon the kidney in animals of the same species as that from which the expressed juice was obtained. Nefediew next showed that blood taken from animals after ligature of the ureters or of the renal artery, produced diffuse nephritis when injected into animals of the same species. Lindemann's experiments showing that the blood of rabbits with chromiannephritis produced a parenchymatous nephritis in other rabbits, provided further evidence in favour of the existence of auto- or iso-nephrolysins.

As in the case of the other cytotoxic sera, however, these autonephrolysins do not represent physiological processes; they represent the toxic activities of substances formed under conditions of immunization, and are probably not even organ-specific.

Timofeew, a pupil of Lindemann's, has recently endeavoured to prove that nephritic œdema is chiefly the outcome of the presence in the blood-stream of decomposed constituents of the renal cells. These substances, which he calls " nephroblaptins," act in a manner similar to that of Heidenhain's lymphagogues of the first class. According to Timofeew, the suppression of renal activity which accompanies lesions of the renal parenchyma, produces disturbance of the osmotic interbalance between the organic fluids, and this leads to the retention in the tissues of an excess of water. The substances which pass from the diseased kidney into the blood-stream bring about changes in the blood similar to those produced by lymphagogues of the first class; and the consequent venous and capillary engorgement increases the permeability of the vessel walls. The excess of fluid in the organism, in combination with the increasing permeability of the vessel walls, produces œdema. 
'Timofeew showed that the watery extract of healthy kidneys (emulsions and filtrates), when introduced into the blood-stream of healthy animals, increases the flow of chyle from the thoracic duct to as much as eight to twenty-two times the normal. Normal blood serum, whether obtained from the systemic arteries and veins or from the renal vein, and blood from animals which have undergone double nephrectomy and are dying of uræmia, have no lymphagogue action. In animals in which only one renal artery or orre ureter is ligatured, the flow of chyle from the thoracic duct is increased to twice or even eight times the normal; and the serum from animals such as these has a marked lymphagogue action upon normal animals.

Kast (1902) has already pointed out that the blood of individuals with nephritic œedema contains a lymphagogue substance. Starling recognized that the œedematous fluid of persons with kidney disease was a strong lymphagogue. Blanck discovered (1906) that, if the serum or œdematous fluid from rabbits with uranian nephritis is injected into rabbits which develop nephritis without œdema after the exhibition of chromium or aloin, the latter animals become œdematous. According to Timofeew, the serum of nephrectomized animals is markedly toxic to healthy animals, but it does not increase the flow of chyle. It is said that sera from individuals with parenchymatous nephritis manifest both properties when exhibited in man; while, according to Strauss, sera from individuals with interstitial nephritis have a toxic property, but Kast does not believe it to be lymphagogue. Timofeew assumes that the lymphagogue action of the serum depends upon the presence of nephroblaptins, its toxic properties upon the presence of other substances. Non-albuminous odema may occur without the participation of the kidneys, brought about by the products of the decomposition of other organs which also possess a lymphagogue action.

These results require further confirmation, but they suggest that, where pathological changes take place in the kidneys, substances are supplied to the blood which may have a special significance to the organism. One thing is clear, however, and that is that these experiments supply no proof of a physiological internal secretion on the part of the kidneys.

The following observations have an interest in this connection. The experiments of J. R. Bradford (1899) showed that striking changes in metabolism may be produced by the reduction of the renal parenchyma. My own experiments, which have extended over a period of years, but have not as yet been published, have yielded interesting results. In dogs in which the nitrogenbalance was maintained for weeks, and whose daily excretion of urine was ascertained, the excision of a wedge-shaped portion of the kidney, equal to about a quarter of the organ, led to the following remarkable change, which was more pronounced after 
removal of the other sound kidney. The daily excretion of urine was considerably increased, and amounted to two, three, or even five times the normal. v. Haberer also observed polyuria after reduction of the renal parenchyma. The daily excretion of nitrogen also increased, though the diet was unaltered. This negative nitrogen-balance disappeared when the amount of food given was very much augmented. The animals showed conspicuous thirst and considerable appetite, and by increasing the amount of the food until the nitrogen was two to two-and-a-half times the former quantity, it was found possible to maintain the nitrogen balance. Reduction in the amount of the diet was followed by a progressive decrease in the excretion of nitrogen until a nitrogen deficit was again observed. The condition of animals in which the renal parenchyma had been reduced in this manner underwent no other change.

According to Bradford, the nitrogenous extractive substances of the blood and tissues, and especially of the muscles, are considerably increased in such animals; he believes that there is rapid decomposition of the tissues, particularly of the muscles. It is expedient that mention of these metabolic changes should be made here, though it is not possible at present to determine whether they arise from suppression of the internal secretion of the kidney or not. 


\section{LITERATURE.}

The reference table, although very extensive, makes no pretention to include the entire literature of the subjects dealt with. The older literature, that anterior to about the middle of the last century, has been omitted altogether. Detailed accounts of the manner in which our knowledge concerning the individual internal secretory organs has grown are given by various authors, and, as a general rule, these accounts include summaries of the older literature. To them the student is referred (Thyroid: Horsley, Hellin, Langendorff, W. Scholz; suprarenal : Pellegrino, Biedl ; hypophysis cerebri : Caselli, Paulesco ; generative glands : Hegar, Moebius).

The following list includes all the publications of which mention is made in the text, together with all works of a physiological or morphological nature. The clinical references include only those works which may be regarded as fundamental; while very few of the numerous publications of a casuistic or therapeutic nature have found mention.

In order to avoid repetition, the works are arranged, not according to subject, but under the authors' names, which are arranged in alphabetical order. The list of references is complete up to the end of the year I909; a few publications of the year igio also find mention.

\section{List of Contractions used in the Literature References.}

A. A. = Archiv f. Anatomie und Physiologie. Anatomische Abteilung.

A. P. = Archiv f. Anatomie und Physiologie. Physiologische Abteilung.

A. A. P: = Archiv f. Anatomie, Physiologie und wissenschaftl. Medizin von Joh. Müller, Reichert und du Bois-Reymond.

A. B. = Archives de Biologie.

A. d. F. = Archivio di Fisiologia.

A. d. P. = Archives de Physiologie normale et pathologique.

A. E. M. = Archiv f. Entwicklungsmechanik.

‥ G. = Archiv f. Gynäkologie.

A. H. = Archiv für Hygiene.

A. i. B. = Archives italiennés de Binlogie.
A. i. Ph. = Archives internationales de Physiologie.

A. J. A. = American Journal of Anatomy.

A. J. P. $=$ American Journal of Physiology.

A. J. M. S = American Journal of Medical Science.

A. k. Ch. = Archiv f. klinische Chirurgie.

A. m. A. $=$ Archiv für mikroskopische Anatomie.

A. $\mathrm{m}$. e. = Archives de médecine expérimentale et d'anatomie pathologique.

An. An. = Anatomischer Anzeiger.

A. P. P. = Archiv für experimentelle Pathologie und Pharmakologie. 
A. V.=Archiv für Verdauungskrankheiten.

B. C. = Biochemisches Centralblatt.

Bph. C. = Biophysikalisches Centralblatt.

B. d. ch. G. = Berichte der deutschen chemischen Gesellschaft.

B. G. G. = Hegars Beiträge zur Geburtshilfe und Gynäkologiè.

B. k. W. = Berliner klinische Wochenschrift.

B. Z. = Biochemische Zeitschrift.

C. a. P. = Centralblatt für allgemeine Pathologie und pathologische Anatomie.

C. f. B. = Centralblatt für Bakteriologie.

C. Ch. = Centralblatt für Chirurgie

C. $\mathrm{G}=$ Centralblatt für Gynäkologie.

C. G. M. C. = Centralblatt für die Grenzgebiete der Medizin und Chirurgie.

C. i. M. = Centralblatt für innere Medizin.

C. k. M. = Centralblatt für klinische Medizin.

C. m. W. = Centralblatt für die medizinischen Wissenschaften.

C. $P$. = Centralblatt für Physiologie.

C. r. A. = Comptes rendus de l'académie des sciences.

C. r. S. B. = Comptes rendus de la société de biologie.

D. A. k. M. = Deutsches Archiv für klinische Medizin.

D. Ch. = Deutsche Chirurgie.

D. K. = Deutsche Klinik.

Diss. = Inaugural-Dissertation.

D. m. $W .=$ Deutsche medizinische Wochenschrift

D. Z. N. = Deutsche Zeitschrift für Nervenheilkunde.

E. B. = Sitzungsberichte der physikal. med. Societät in Erlangen.

E. P. = Ergebnisse der Physiologie (Asher-Spiro).

F. M. = Fortschritte der Medizin.

G. $\mathrm{m}$. = Gazette médicale de Paris.

H. B. = Hofmeisters Beiträge zur chemischen Physiologie und Pathologie.

I. M. = Internationale Monatsschrift für Anatomie und Physiologie.

J. A. M. A. = Journal of American Medical Association.

J. o. A. a. P. = Journal of Anatomy and Physiology.

J. A. P. $=$ Journal de l'Anatomie et de la Pliysiologie.

J. B. Ch. = Journal of Biological Chemistry.

J. K. = Jahrbücher für Kinderheilkunde.
J. d. P. = Journal de la Physiologie de l'homme et des animaux.

J. d. P. P. = Journal de Physiologie et de Pathologie générale.

J. E. M. = Journal of Experimental Medicine.

J. o. P. = Journal of Physiology.

J. o. P. B. = Journal of Pathology and Bacteriology.

L. O. = Lubarsch-Ostertags Ergebnisse der allg. Pathologie und patholog. Anatomie.

M. G. G..= Monatschrift für Geburtshilfe und Gynäkologie.

M. G. M. C. = Mitteilungen aus den Grenzgebieten der Medizin und Chirurgie.

M. K. = Medizinische Klinik.

M. m. W. = Münchener medizinische Wochenschrift.

M. P. = Monatschrift für Psychiatrie.

M. U. = Moleschotts Untersuchungen zur Naturlehre.

N. C. = Neurologisches Centralblatt.

P. A. = Pflügers Archiv für die gesamte Physiologie.

P. m. = Presse médicale.

P. m. W. = Prager mediz. Wochenschrift.

P. R. S. = Proceedings of the Royal Society of London.

P. V. = Prager Vierteljahrsschrift

S. A. = Skandinavisches Archiv f. Physiologie.

$\mathrm{S} . \mathrm{m} .=$ Semaine médicale.

S. W. A. = Sitzungsberichte der $\mathrm{k}$. Akademie zu Wien. Mathematischnaturwissenschaftliche Klasse.

Th. G. = Therapie der Gegenwart.

Th. M. = Therapeutische Monatshefte.

V. A. = Virchows Archiv für pathologische Anatomie und Physiologie und klinische Medizin.

V. a. G. = Verhandlungen der anatomischèn Gesellschaft.

V. d. N. A. = Verbandlungen der deutschen Naturforscher und Arzte.

V. d. p. G. = Verhandlungen der deutschen pathologischen Gesellschaft.

V. ı. C. M. = Verliandlungen des Io. Congresses für innere Medizin.

V. g. M. $=$ Vierteljahrsschrift für gerichtliche Medizin und öffentl. Sanitätswesen.

V. H. = Virchow-Hirsch' Jahresberichte.

IV. B. = Sitzungsberichte der physikalisch-medizinischen Gesellschaft, Würzburg.

W. K. = Wiener Klinik.

W. k. W. = Wiener klinische Wochenschrift.

IV. k. R. = Wiener klinische Rundschau. 
W. m. P. = Wiener medizinische Presse.

IV. m. IV. = Wiener mediz. Wochenschrift.

W. V. = Verhandlungen der physikalisch-medizinischen Gesellschaft, Würzburg.

Z. A. = Zoologischer Anzeiger.

$Z$. a. P. = Zeitschrift f. allgemeine Physiologie.

Z. B. = Zeitschrift für Biologie.

Z. Ch. = Zeitschrift für Chirurgie.

Z. e. $P .=Z$ eitschrift für experimentelle Pathologie und Therapie.
Z. G. G. = Zeitschrift für Geburt shilfe und Gynäkologie.

Z. H. = Zeitschrift für Heilkunde.

Z. k. M. = Zeitschrift für klinische Medizin.

Z. ph. Ch. = Zeitschrift für physiologische Chemie.

Z. w. Z. = Zeitschrift for wissenscliaftliche Zoologie.

Ziegl. B. = Zieglers Beiträge zur allg. Pathologie und pathologischen Anatomie.

Z. r. M. = Zeitschrift für rationelle Medizin.

ABadie. Traitement chirurgical du goître exophtalmique. La France Méd., No. 2, 1898 .

AbDerhaldEN, E. Lehrb. d. physiolog. Chemie. 2 Aufl., Wien, 1909.

AbDerhalden, E., and Bergell, P. Über Epinephrin. M. m. W., I904.

- Zur Kenntnis des Epinephrins (Adrenalins). B. d. ch. G., 37, p. 2022, I 904 .

AbDerhalden, E., and Guggenheim, M. Weitere Vers. über d. Wirkung der Tyrosinase aus Russula delica auf tyrosinhaltige Polypeptide u. auf Suprarenin. Z. ph. Ch., 57, 1908.

ABDERHALDEN, E., and MÜLLER, F. Über das Verhalten des Blutdruckes nach intravenöser Einführung von l-, d- und dl-Suprarenin. $Z$. ph. Ch., 58, 1908.

ABderhalden, E., and Thies, F. Weitere Studien über das physiologische Verhalten von 1-, d- und dl-Suprarenin. II. Mitt. Z. ph. Ch., 59, pp. 22-29, 1909 .

ABDERHALDEN, E., and Slavu. Weitere Studien über das physiologische Verhalten von 1-. d- und dl-Suprarenin. Z. ph. Ch., 59, pp. 129I 37 , I909.

ABDERHalden, E., and Kautzsch. Weitere Studien über das physiologische Verhalten von 1- und d-Suprarenin. Z. ph. Ch., 61, p. IIg, I909.

AbDerhalDen, E., KaUtzSch, and MÜllLer, F. Weitere Studien über das. physiologische Verhalten von 1- und d-Suprarenin. V. Mitt. Z. ph. Ch., 62, 1909 .

ABEGG. Zur Kenntnis der Addisonschen Krankheit. Diss., Tübingen, I889.

ABEL, J. J. Further observations on the chemical nature of the active principle of the suprarenal capsule. Bull. of Johns Hopkins Hospital, No. 9o, September-October, 1898 .

- Über den blutdruckerregenden Bestandteil der Nebenniere, das Epinephrin. Z. ph. Ch., 28, I899.

- On epinephrin, the active constituent of the suprarenal capsule and its compounds.

- On the formation and composition of highly active salts of epinephrin.

- On the presence of cholin and neurin in the intestinal canal during its complete obstruction. The Chemistry of the Melanins. A. J. P., 2, March I, 1899 .

- Further observations on epinephrin. Johns Hopkins Hospital Bull., No. 120, March, 1901.

- On the behaviour of epinephrin to Fehling's solution and other characteristics of this substance. Ibid., No. I28, November, I901.

- On a simple method of preparing epinephrin and its compounds. Ibid., No. 130, 1902.

- On the elementary composition of adrenalin. On the behaviour of the suprarenal gland towards Fehling solution. On the oxidation of epinephrin and adrenalin with nitric acid. A. J. P., February 8, I903.

- Weitere Mitteilungen über das Epinephrin. B. d. ch. G., 36, p. 1839, 1903 ; and 37, p. 368,1904 . 
ABEL, J. J., and CRAWford, A. On the blood-pressure raising constituent of the suprarenal capsule. Johns Hopkins Hospital Bull., No. 76, July, 1897 .

ABEL, J. J., and TAVEAU, R. On the decomposition products of epinephrin hydrate. J. B. Ch., I, p. I, 1905 .

Abelmans. Über die Ausnutzung der Nahrungsstoffe nach Pankreasexstirpation. Diss. Dorpat, 1890 .

Abelous, J. E. Greffe de capsules surrénales sur la grenouille. C. r. S. B., 44, p. 864,1892 .

- Contribution à l'étude de la fatigue. A. d. P., pp. 437-446, 1893.

- La physiologie des glandes à sécrétion interne. Rev. gén Scienc., p. 273,1893 .

- Des rapports de la fatigue avec les fonctions des caps. surrén. A. d. P., 1893 .

- Toxicité du sang et des muscles des animaux fatigués. A. d. P., 26, 1894 .

- Sur l'action antitoxique de corps surrénales. C. r. S. B., 47, p. $45^{8}$, 15 juin, 1895 .

- Des rapports de la fatigue avec les fonctions des capsules surrénales. XI. Congrès intern. à Rome; and A. i. B., 22, I 895 .

- Sur l'origine musculaire des trouble consécutifs à la destruction des glandes surrénales. C. r. S. B., 56, p. 95 I, 1904.

- Les troubles de pigmentation de la grenouille à la suite de la destruction des glandes surrénales. Ibid., p. 952, 1904.

Abelous, J. E., and Bardier, E. Action hypertensine de l'urine de l'homme normal. Premières recherches sur l'urohypertensine. J. d. P. P., vol. $x$, p. 627,1908 .

- L'urohypotensine. J. d. P., vol. xi, p. 34, 1909.

- Über Urohypotensine und Urohypertensine mehrere Mitteilungen in C. r. S. B., vol. 64-66, 1908-1909.

Abelous and Billard. Recherches sur les fonctions du thymus chez la grenouille. A. d. P., p. 898,1896 .

Abelous, J. E., and Langlois, P. Note sur les fonctions des capsules surrénales chez la grenouille. C. r. S. B., 43, p. 292, 1891.

- La mort des grenouilles après la destruction des caps. surrén. Ibid., p. 855 .

- Note sur l'action toxique du sang des mammifères après la destruction des capsules surrénales. Ibid., 44, p. 165, 20 février, 1892.

- Destruction des capsules surrénales chez le cobaye. Íbid., 44, p. 388, 7 mai, 1892.

- Capsules surrénales. Toxicité de l'extrait alcoolique des muscles de grenouilles privées de capsules surrénales. Ibid., 44, p. 490, 1892 .

- Rech. expér. sur les fonctions des caps. surrén. de la grenouille. A. d. P., 1892 .

- Sur les fonctions des capsules surrénales. A. d. P., pp. 465-476, 1892.

- Sur les fonctions des capsules surrénales. C. r. S. B., 47, pp. 334-335, I895.

Abelous, Langlois, Charrin. Maladie d'Addison, Tracés ergographiquas. Diurèse par injections de capsules surrénales. C. r. S. B., 44, p. 623 , juillet, i 892 .

- La fatigue chez les addisoniens. Ibid., p. 721; A. d. P., 24.1892.

Abelous, J. E., and RIBAUT, H. Sur la substance hypertensive qu'on peut extraire par l'alcool des extraits de muscle putréfié. C. r. S. B., 64 , p. 907,23 mai, 1908 .

Abelous, Ribaut, Soulí and Toujan. Sur la présence dans des macérations des muscles putréfiés de subst. élév. la pression artérielle. C. r. S. B., 58 , i, 1906 .

- Sur la présence dans les macérations des muscles putréfiés d'une ptomaine élévant la pression artérielle. Ibid., p. 530, 1906.

Abelous, Soulié and Toujan. Dosage colorimetrique par le jode de l'adrénaline. C. r. S. B., 57, i, p. 301, 1905.

- Sur la formation de l'adrénaline par les glandes surrénales. Ibid., p. 533,1905 .

- Sur l'origine de l'adrénaline. Ibid., p. 57.4, 1905.

- Influence des extraits et des produits de l'autolyse des organs et tissus sur la formation de l'adrénaline par les glandes surrénales. Ibid., 57 , ii, p. 589,1905 . 
ABELOUS, SOuliê and ToujAN. Sur l'identité d'action des extraits des substances corticale et médullaire des capsules surrénales. Ibid., p. 530, 1905 .

- Influence des extraits et des organes et des tissus animaux soumis à l'autolyse sur la production de l'adrénaline (2nd note). C. r. S. B., 58 , ii, p. 16, 1906 .

- Sur un procédé de contrôle de dosages chimique et physiologique de l'adrénaline. Ibid., p. I74, 1906.

Авотт, A. C. The adrenal gland and its active principle in their relation to cytolysins and antitoxin production. C. f. B., 34, p. 696, 1903.

ABT. Le corps thyroïde et la grossesse. Thèse de Paris, rgo4.

ACHARD, M. Recherches cliniques sur le cœur, le pouls et la tension artérielle dans quelque convalescences. Thèse de Montpellier, 1906.

ACHARD and LOEPER. Gigantisme, acromégalie et diabète. Nouv. Icon. Salpêtr., I3, 1900.

ADAmI, J. G. The internal secretory activity of glands, \&c. New York Med. News, No. 19; and Montreal Med. Journ., May, 1897.

- The Myelins and potential fluid crystalline bodies of the organism. J. A. M. A., 48, 1907 .

- Principles of pathology. Phil. and New York, 1908.

ADAMI and ASCHOFF. On the myelins, myelin bodies and potential fluid crystals of the organism. Proc. Roy. Soc., 78, p. 359, I906.

Adams, E. IV. A case of previous development. Trans. Path. Soc., London, 1906.

ADDison, THOMAS. On the constitutional and local effects of disease of the suprarenal bodies. London, 1855 .

ADelheim, R. Über die Langerhansschen Inseln. Petersb. m. W., 30,

ADLER, J., and HENSEL, O. Über intravenöse Nikotineinspritzungen und deren Wirkung auf die Kaninchenaorta. D. m. W., 1906.

- Intravenous injections of nicotine and their effects upon the aorta of rabbits. Journ. of Med. Researrch, 15, p. 229, 1906.

AdLer, M., and MILCHNER, R. Untersuchungen des Kotfettes in einem Falle von Pankreasdiabetes und dessen Beeinflussung durch Pankreon. B. k. W., 1908.

ADLER, S., and THALER, H. Experimentelle und klinische Studien zur Graviditätstetanie. Z. G. G., 62, p. 194. Diskussion zum Vortrag Erdheim. W. k. W., 1906.

AfFanASSIEW, B. Über den Bau und Entwicklung der Thymus. A. m. A., $14,1877$.

AgaDSCHANIANZ, R. Über den Einfluss des Adrehalins auf das in der Leber enthaltene Glykogen. B. Z., 2, p. 148, 1907.

Agostini. Infantilismo distrofico e infantilismo mixedematoso. Riv. pat. nerv., 1902.

AHLBECK, VICTOR, and LOHSE, J. E. Ein Versuch, das Eklampsiegift auf experimentellem Wege nachzuweisen. Z. G. G., 62, p. II5.

AICHEL, O. Über die Entwicklung der Nebennieren bei Selachiern und über eine neue Homologie der Nebennieren in der Wirbeltierreihe. E. B., H. 31, p. 86, i 899 .

- Zur Kenntnis der Nebennieren. M. m. W., Igoo.

- Vergleichende Entwicklungsgeschichte und Stammesgeschichte der Nebennieren. A. m. A., 56, 1900 .

- Vorläufige Mitteilung über die Nebennierenentwicklung der Säuger und die Entstehung akzessorischer Nebennieren des Menschen. An. An., 17, p. 30 , 1900 .

- Eine Antwort auf die Angriffe v. S. Vincent in London. Ibid., 18, 1900.

AIMÉ, P. Les cellules interstitielles de l'ovaire chez le cheval. C. r. S. B., 58 , ii, p. 250 , 1906.

Ajello, G., and Parascandalo, C. Contr. sperim. alla patogenesi dell' uremia. Speriment, 1895.

D'AJuToLo, G. Intorno ad un caso di capsula soprarenale accessoria nel corpo pampiniforme di un feto. Arch. per le scienze, med., 8, pp. 203-306, 1884 .

- Su di una struma soprarenale accessoria in un rene. Bollet. delle sc. med. di Bologna, Ser. 6, vol. xvii, p. 9 , 1886.

AKopENKo. Über die Wirkung der Thyreoidektomie auf das Wachstum und die Entwicklung des Knochen- und Nervensystems bei jungen Tieren. L. O., 5, 1898 . 
AlaIkE, P. Le rôle de la fonction interne de l'ovaire et les essais de l'opothérapie ovarienne en pathologie nerveuse et mentale. Thèse de Montpellier, 1906.

Alamartine, M. H. La sécretion interne du testicule et la glande interstitielle. Gaz. des hop., 79, 1906.

ALbanese. Recherches sur la fonction des capsules surrénales. A. i. B., 18, 1893 .

- La fatigue chez les animaux privés des capsules surrénales. A. i. B., I7, 1892 .

ALBARRAN, J., and BERNARD, L. Etude sur les cytotoxines rénales. A. m. e., p. I3, 1903.

ALBERS-SCHÖNBERG. Uber eine bisher unbekannte Wirkung der Röntgenstrahlen auf den Organismus der Tiere. M. m. W., 1903.

ALBERTI. Kasuistik z. Hypertrichosis universalis. B. G. G., 9, Ig05.

ALBERTONI and TIZzONI. Sugli effetti dell' estirpazione della tiroide. Arch. p. le Scienze Med., x, p. I. I886.

Albrand, M. Die Anlage der Zwischenniere bei d. Urodelen. A. m. A., 72, 1908.

ALbRECHT. Ein Fall von Pankreasbildung in einem Meckelschen Divertikel. Ges. f. Morph. u. Phys. in München, 14, p. 52, 1901.

- Uber trübe Schwellung und Fettdegeneration. V. d. p. G., 6, I903.

- Die Bedeutung myelinogener Stoffe im Zellleben. Ibid., Igo3.

Albrecht, P. Ein Fall von Hypernephrom mit Metastasen. W. k. W., 1904.

- Beiträge zur Klinik und pathologischen Anatomie der malignen Hypernephrome. A. k. Ch., 77, 1905 .

ALDEHOFF. Tritt auch bei Kaltblütern nach Exstirpation des Pankreas Diabetes auf? Z. B., 28, 293, 1894.

ALDRICH, J. B. A preliminary report on the active principle of the suprarenal gland. A. J. P., 5, p. 457, August I, I901.

- Is adrenalin the active principle of the suprarenal gland? A. J. P., 7, p. 359,1902 .

ALESSANDRO. Influenza della macerazione acida di mucosa duodeno digiuuale (contenente secretina) sulla secrezione lacrimale, sulla produzione dell' umore aqueo e sulla circolazione del fondo dell' occhio. Arch. di ott., 1907; Arch. f. Augenhk., 61, 1908.

Alessandro, G., and Bonaventura, G. 'Le pouvoir sécréteur du pancréas. J. d. P. P.. x, 219, 1008.

Alexander. Behandlung $\bar{d}$. Morbus Basedowii mit Antithyreodin Moebius, M. m. W., I 905 .

Alexander, A., and EHRMANN, R. Untersuchungen über Pankreasdiabetes, besonders über das Blut der Vena pancreatico-duodenalis. Z. e. P., 5, p. 367 .

ALEXANDER, C. Untersuchungen über die Nebennieren und ihre Beziehungen zum Nervensystem. Zeigl. B., 1 1, 1892 .

Alezais, H. Contribution à l'étude de la capsule surrénale du cobaye. A. d. P., 1898 .

AlEZAIS and ARNAUD. Recherches expérimentales sur la toxicité des capsules surrénales. Marseille médical, p. 637,1889 ; pp. 81, 225, 1890.

- Sur le caractère du sang efférent des capsulés surrénales. Ibid., 393, I89I.

-- Recherches expérimentales sur les capsules surrénales. Ibid., pp. I I, 94, 13 I, 195, 1891.

- Etude sur la tuberculose des capsules surrénales et ses rapports avec la maladie d'Addison. Revue de méd., i 1, p. 283, I891.

Alezais and PeYron. L'organe parasympathique de Zuckerkandl chez le jeune chien. C. r. S. B., p. 1161, 1906.

- Particularités de dévoloppement des paraganglions lombaires. Ibid., I907.

- Sur les tumeurs dites gliomateuses des capsules surrénales. Ibid., p. 55 I.

- Un groupe nouveau de tumeurs épithéliales: les paragangliomes. Ibid., 65,1908 .

Allara, V. Der Cretinismus, seine Ursache und seine Heilung. Übersetzt von H. Marian. Leipzig, 1894.

ALLARD, E. Über die Beziehungen der Umgebungstemperatur zur Zuckerausscheidung beim Pankreasdiabetes. A. d. P., 59, p. I I I, 1908 . 
Allard, E. Die Acidose beim Pankreasdiabetes. Ibid., 59, p. 388, 1908.

ALLARIA, G. B., and VARANINI, M. Ricerche sul ricambio in un caso di morbo di Addison curato con l'opoterapia surrenale. Clinica med. ital., p. 40, 1902 .

Allen. Zur Frage der Tetanie bei Magendilatation usw. A. V., 5, 1898; B. k. W., I 899 .

ALLERS, R. Zur Kenntnis der wirksamen Substanz in der Hypophyse: M. m. W., I 1909 .

Almagia, M. Allattamento e funzione tiroidea. A. d. F., 6, p. 462.

ALMAGIA and EMBDEN. Über die Zuckerausscheidung pankreasloser Hunde nach Alanindarreichung. H. B., 7, p. 298.

ALonzo. Sulla funzione della glandola tiroide. Rif. med., vi, p. 208, 1891.

ALQUIER, L. Recherches sur le nombre et sur la situation des parathyreoides chez le chien. C. r. S. B., 58 , ii, p. 302 , 1906.

- Sur les modifications de l'hypophyse après l'exstirpation de la thyreoide ou des surrénales chez le chien. J. d. P. P., 9, p. 492, 1907.

Alquier, L. and SchmierGold. Deux tumeurs de l'hypophyse. L'encéphale, ii, No. 3.

Alquier, I., and Theuvery, L. Etat du testicule de chiens ayant subi diverses exstirpations partielles de l'appareil thyro-parathyroidien. C. r. S. B., 64 , p. 663 .

ALquIER, L., and' TOUCHARD, P. Lésions des glandes vasculaires sanguines dans deux cas de sclérodermie généralisée. A. m. e., p. 687, 1907.

Alterthum, E. Folgezustände nach Kastration und die sekundären Geschlechtscharaktere. B. G. G., ii, p. 13, I899.

Amabilino. Caso di morbo di Adddison con lesioni dei centri nervosi. Rif. med., 1899.

D'Amato, L. - Sulle alterazioni prodotte dagli estratti di capsule surrenali nei vasi sanguigni ed in altri organi. Giorn. internaz. d. scienze mediche, 27 Ref. Bph. C., i.

- Weitere Untersuchungen über die von den Nebennierenextrakten bewirkten Veränderungen der Blutgefäsze und anderer Organe. B. k. W., pp. 1100 and I131, 1906.

- Neue Unters. iuber d. exper. Pathologie d. Blutgefäsze. V. A., 192, 1908.

D'Amato, L., and Fagella, V. Della natura e del significato delle lesioni aortiche prodotte dagli estratti di capsule surrenali. Ref. Bph. C., I.

AMBerg. Über die Toxizität des wirksamen Prinzips der Nebennieren. Arch. intern. Pharmacod., II, p. 57, 1902.

Ambrosius, WW. Beiträge zur Lehre von den Nierengeschwülsten. Diss., Marburg, 1891 .

Amouroux. Etude expérimentale de l'athérome tabagique. Thèse de Toulouse, igo6.

AMPT. Über das Parovarium (Epoophoron). Diss., Berlin, 1895.

ANCEL, P. Histogenèse et structure de la glande hermaphrodite d'Helix pomatia. A. B., t. xix, I902.

- Les follicules pluriovulaires et le déterminisme du sexe. C. r. S. B., 55, p. 1049, 1903.

- Sur le déterminisme cytosexuel des gamètes. Période de différenciation sexuelle dans la glande hermaphrodite de Limax maximus. Arch. de zool. exp., i, 1903.

ANCEL, P., and Bouin, P. Recherches sur le rôle de la glande interstitielle du testicule. Hypertrophie compensatrice expérimentale. C. r. A., decembre, 1903 .

- L'apparition des caractères sexuel secondaires est sous la dépendence de la glande interstitielle du testicule. Ibid., janvier, I904.

- Histogenèse de la glande interstitielle du testicule chez le porc. C. r. S. B., 1903 .

- Sur l'existence de deux sortes de cellules interstitielles dans le testicule de cheval. C. r. S. B., 56, i, p. 8I, 1904.

- La glande interstitielle du testicule. Examen critique des essais de vérification expérimentale de son rôle sur l'organisme. Ibid., p. 83.

- Sur la glande interstitielle du testicule des mammifères. 'Rép. à M. G. Loisel.) Ibid., p. 95.

- Tractus génital et testicule chez le porc cryptorrhide. Ibid., 56, i, p. 281,3 février, 1904 . 
Ancel, P., and Bouin, P. L'infantilisme de la glande interstitielle du testicule. C. r. A., I février, 1904.

- Sur les relations qui existent entre le développement du tractus genital et celui de la glande interstitielle chez le porc. C. R. Assoc. des anat. Toulouse, 1904 .

- Sur la fonction du corps jaune. I. Méthodes de recherches. C. r. S. B., 66 , p. 454. - - II. Action du corps jaune vrai sur l'utérus, p. 505. III. Action du corps jaune vrai sur la glande mammaire, p. 605. - IV. Démonstration expérimentale de l'action du corps jaune, sur l'utérus et la glande mammaire, p. 689, I908.

- Le développement de la glande mammaire pendant la gestation est déterminé par le corps jaune. Ibid., 67, p. 466, 1909.

ANCEL and VILLEMIN. Sur la dégénérescence de la glande séminale déterminée par l'ablation du feuillet pariétal de la vaginale. C. r. S. B., 66, p. 6, 1907.

- Sur la cause de la menstruation chez la femme. Ibid., p. 200, 1907.

- Période cataméniale coincidant avec l'époque où le corps jaune a son tnaximum de développ. il est vraisemblable que la menstr. est sous la dépendance du corps jaune. Sur l'ectopie expérimentale de l'ovaire et son retentissement sur le tractus génital. Ibid., p. 227.

ANDERodias. Sécrétion interne. Journ. de Méd. Bordeaux, pp. 450, 467, $497,513,1900$.

ANDERSON. Reflex pupil-dilatations by cervical sympathetic nerve. J. o. P., p. 15,1904 .

- The paralysis of involuntary muscles with special reference to the occurrence of paradoxical contraction. I. Paradoxical pupil-dilatation. Ibid., p. 290.

ANDERSON and BERGMANN. Einflusz der Schilddrüsenbehandlung beim gesunden Menschen. S. A., viii, p. 326, 1908.

Andersson, O. Zur Kenntnis der Morphologie der Schilddrüse. A. A., p. 177,1894 .

ANDREZIEN, L. Origin and evolution of the thyroid function. Brit. Med. Journ., 1893 .

- The morphology of the pituitary body. Ibid., 1894 .

ANGIOLELLA, G. Sull' avelenamente sperimentale da tiroidina etc. Annali di Neurolgia, 15. Zit. N. C., 16, p. 644, 1897.

Anton, G. Über Formen und Ursachen des Infantilismus. M. m. W., Igo6.

- Nerven- und Geisteserkrankungen in der Zeit der Geschlechtsreife. W. k. W., 1904 .

- Wahre Hypertrophie des Gehirns mit Befunden an Thymusdrüse und Nebennieren. Ibid., 1902.

- Vier Vorträge über Entwicklungsstörungen beim Kinde. Berlin, 1908.

ANzILOTTI, G. Sulle proprietà tossiche dei nucleoprot. renali. La Clinica mod., No. 12, 1903.

- 'Sulle modificazioni indotte nel rene opposte della legatura dell' uretera. Ibid., 1203.

APERT. Les enfants retardaires. Actualités médicales, 1902.

- Hypertrophie parotidienne et insuffisance thyroidienne. S. m., 28, p. 7 r.

- Infantilisme et hypertrophie des glandes salivaires. Soc. méd. Hôp., 1908.

Apolant, H. Über Reizung der Nebennieren. C. P., 12, p. 721, 21 Januar, 1899.

ARGUSTINSKY. Über einen Fall von Thyreoplasie. B. k. W., I905.

ARNAUD, $F$. Les hémorrhagies des capsules surrénales. Arch. gén. Méd., 1900.

ARNDT. Untersuchungen über die Ganglienkörper des N. sympathicus. A. m. A., I0, 1874 .

ARNold, J. CUber die Struktur des Ganglion_intercaroticum. V. A., 33, 1865 .

- Ein Beitrag zu der Struktur der sogenannten Steiszdrüse. Ibid., 32, 1865.

- Über die feinere Strukter und den Chemismus der Nebennieren. Ibid., 35 ; 1866.

$\therefore$ Akromegalie, Pachyakrie oder Ostitis? Ziegl. B., 10, 1891. 
ARNold, J. Weitere Beiträge zur Akromegaliefrage. V. A., 135, p. 1, 1894. ARNOZAN and VAILLARD. Contribution à l'étude du pancréas du lapin. A. d. P., 1884 .

ARON, H., and SEBAUER, R. Untersuchungen über die Bedeutung der Kalksalze für den waclısenden Organismus. B. Z., 8, 1908.

ARONHEIM. Ein Fall von ausgedehnter Phlegmone, verursacht durch subkutane Injekt. einer Cocain-Adrenalinlösung bei einem 70 jährigen Manne. M. m. W., 1904 .

ARONsoHs, E. Die Zuckerausscheidung nach Adrenalininjektionen und ihre Beeinflussung durch künstlich erzeugtes Fieber. V. A., 174, 1903.

- Das Adrenalin zur Kupierung von Asthmafällen. D. m. W., 1903.

ARren. Essai sur les capsules surrénales. Thèse de Paris, 1894.

D'ARSONval. Note sur la préparation de l'extrait testiculaire. A. d. P., 1893.

ARTHUS. Glykolyse dans le sang et ferment glycolytique. A. d. P., 1891 and 1894

Aschner, B. Demonstr. hypophysektomierter Hunde. W. k. W., Dezember, 1909 .

AsChOFF, L. Cystisches Adenofibrom der Leistengegend. M. G. G., 9, 1899.

- Über einen Fall von angeborenem Schilddrüsenmangel. D.' M. IV., 1899 .

- Uber die Lage des Parophoron. V. d. p. G., vol. ii, pp. 433-439, I900.

- Über das Vorkommen chromaffiner Körperchen in der Paradidymus und in dem Parophoron Neugeborener und ihre Beziehungen zu den Marchandschen Nebennieren. Orth.-Festschrift, September-Abdr., pp. $1-9,1903$.

- Über Atherosklerose und andere Sklerosen des Gefässsystems. Beitr. z. M. K., 1908 .

- Zur Morphologie der lipoiden Substanzen. Ziegl. B., 47, 1909.

AsCHOFF and COHN. Bemerkungen zu der Schur-Wieselschen Lehre von der Hypertrophie des Nebennierenmarkes bei chronischen Erkrankungen des Nieren- und Gefässapparates. V. d. p. G., 1908.

AsCOLI and FIGARI. Über Nephrolysine. B. k. W., pp. 56I and 634, 1902.

AsCOLI, G. Vorlesungen über Urämie. Jena, 1903.

ASHER and WOOD. Einfluss des Cholins auf die Zirkulation. Z. B., 37, pp. 307-320, 1898 .

AsKaNazy, M. Die bösartigen Geschwülste der in der Niere eingeschlossenen Nebennierenkeime. Ziegl. B., 14, 1893.

- Path.-anat. Beitr. z. K. d. M. Basedowii, insb. üb. d. dabei auftret. Muskelerkrankung. D. A. k. M., $65,1898$.

- Teratom und Chorionepitheliom der Zirbel. V. d. p. G. in Jena, p. 58, 1906.

- Kommen in den Zellkomplexen der Nebennierenwände drüsenartige Lumina vor? B. k. W., 1908 (Gegen Stoerk bejahend).

ASKaNazY, M., and HǘBSchmañ, P. Über Glykogenschwellung der Leberzellkerne besonders bei Diabetes. C. a. P., I8, 1907.

Atkinson, R. T. The early development of the circulation in the suprarenal of the rabbit. An. An., 19, pp. 610-612, 1901.

Atrina. Porpora emorragica curato con l'adrenalina. Gazz. degli osped., 1904 .

Athanasiu, C., and Gradinesco, A. La circulation artificielle dans les muscles. Action de l'adrénaline sur l'endothélium vasculaire. C. r. S. B., 64, p. 613 , 1908 .

- Les capsules surrénales et les échanges entre le sang et les tissus. C. r. A., I49, I909.

Athasasiu and LaNGlois. Du rôle du foie dans la destruction de la substance active des capsules surrénales. C. r. S. B., 49, p. 575, 1894 .

Athanasow. Rech. histol. sur l'atrophie de la prostrate consécutive à la castration, à la vasectomie et à l'injection sclérogène épididymaire. Thèse de Nancy, 1898 .

AUberTin. L'hypertrophie cardiaque dans les infections et intoxications chroniques expérimentales. C. r. S. B., 63, 1907.

- Hyperplasie surrénale dans l'alcoolisme chronique expér. Ibid., 63, p. $270,1907$.

- Hypertrophie cardiaque dans l'alcoolisme expér. Ibid., p. 206, 1907.

AUBERTIN and AMAND. A propos de l'hypertension artérielle. S. m., p. 63, 1904 . 
AUbertin and CuNet. Hypertrophie cardiaque et hyperplasie médullaire des surrénales. S. m., I907; C. r. S. B., 63, p. 595, 1907.

AULD. Preliminary report on the suprarenal gland and the causation of Addison's disease. Brit. Med. Journ., May 12, 1894.

- A further report. Ibid., p. 745, October 6, 1894.

- A third report. Ibid., ii, p. 10, July, 1896 .

AUstoni, A., and TEDEschi, H. Sugli effetti degli estratti di ghiandolle surrenali e di ipofisi sul sangue. R. Accad. v. Padova Sed., January 29 , 1909 ; Bph. C., iv.

AverbeCK, H. Die Addisonsche Krankheit. Erlangen, I869.

AXENFELD. Sehnervenatrophie und Menstruationsstörung. D. m. W., 1903.

AzAM, J. Syndrome d'insuffisance hypophysaire aux cours des maladies toxi-infectieuses. Thèse de Paris, 1907.

BABER. Researches as to the minute structure of the thyroid gland. Philosoph. Trans. of the R. Soc., 172, $188 \mathrm{I}$.

BABES, V. Les rapports entre la graisse, le pigment et des formations cristallines dans les capsules surrénales. C. r. S. B., 64, p. 83 , I 908 .

- Lésions des capsules surrénales dans la tuberculose. Ibid., p. I94, 1908.

- Lésions inflammatoires et microbiennes des capsules surrénales. Ibid., 65, p. 235.

- Observat. asupra capsulelor suprarenale. Ann. Acad. Roman., Bucarest, 1908.

- La présence d'une hypertrophie et d'adénomes des capsules surrénales dans des cas d'adénomes ou du cancer primitif du foie. C. r. S. B., 66 , p. 479 , 1909 .

Babes, V., and JoNescu, V. Distribution de la graisse dans les caps. surrén. Ibid., 1908.

BABINSKI and ONANOFF. Tumeur du corps pituitaire. Rev. neur., 8, p. 531, 1900.

Baccarani, U., and Plessi, A. Ricerche cliniche sull' azione dell' estratto di sostanza modolare delle capsule surrenali (paraganglina Vassale) nell' atonia gastro-intestinale. Rif. med., I9, Nos. 13 and 14, pp. 358, 376,1903 .

BADUEL. Sopra alcune ricerche dirette a produrre un siero antiparagangliare. Boll. Soc. Eustach., vol. 3, Nos. 5-8, 1905; Ref. B. C. iv, p. 952 .

- Effetti delle iniezioni di adrehalina nell' arteria renale. Ibid.

- Le alterazioni delle capsule surrenali nei cardionefropatici. Riv. Clin. med., I 908.

- Lésions vasculaires produites par l'extrait d'hypophyse. Il Policlinico, 5 juillet, 1908 .

BAER and BLUM. Über den Abbau von Fettsäuren beim Diabetes mellitus. A. P. P., 59, p. 321 , 1908 .

- Zur Wirkung der Glutarsäure auf den Phloridzindiabetes. D. m. W., 1908.

- Über die Einwirkung chemischer Substanzen auf die Zuckerausscheidung und die Azidose, II. H. B., xi, p. 10I, 1907.

BAEZA. Über Adrenalinum-Chlorid u. Anwendung in d. rhinolog. Praxis. B. k. W., 1902 .

BaHrs, H. Beobacht. v. 38 Fällen v. Addisonscher Krankheit. Diss., Göttingen, 1905 .

BAINBRIDGE, F. A. Effects of ligature of one ureter. J. o. P. B., I1, 1907. - Prosecretin in relation to diabetes mellitus. Bioch. Journ., iii, 82, 1907.

BAINBRIDGE, F. A., and BEDDARD, A. P. Secretin in relation to diabetes. mellitus. Brit. Med. Journ., i, p. 429, 1906.

- The effects of partial nephrectomy in cats. J. o. P., 35, 1907.

- The relation of the kidneys to metabolism. Proc. Roy. Soc., Ser. B., $79,1907$.

B.LLI, D. Rapporto fra glicosuria ed acetonuria nel diabete sperimentale. Rif. med., 1892.

- Lo zucchero nell' organismo animale. Lo Sperimentale, 48, p. 1, 1894.

- La glicosuria in rapporte con la funzione del pancreas. Arch. di Farm., iii, 1895 .

- De la tiroide distingga un veleno nell organismo. Ibid., vi, 1898.

BALDONI. Der Lungengaswechsel des Hundes nach Exstirp. d. Schilddrüse. M. U., 17, 1900 
BaLfour, F. M. The development of Elasmobranch fishes. Journ. of Anat. ànd Phys., vol. x-xii, $1876-1878$.

- A monograph of the development of Elasmobranch fishes. London, 1878 and 1885 .

- Über die Entwickl. und die Morph. der Suprarenalkörper (Nebennieren). Biol. Cbl., No. 5, I881.

- Handbuch der vergleichenden Embryologie. Übers v. Vetter. Jena, I88I.

- On the nature of the organ in adult Teleosteans and Ganoids, which is usually regarded as the head-kidney or pronephros. Quart. Journ. micr. Sc., vol. xii, 1882 .

BALLET, G., and ENRIQUEZ, E. Des effets de l'hyperthryreoidization expérimentale. La méd. moderne, $1895 ; \mathrm{S}$. m., 1894.

BALLET, G., and LAIGNEL-LAVASTINE. Un cas d'acromégalie avec lésions hyperplasiques du corps pituitaire, du corps thyroide et des capsules surrénales. Nouv. Iconogr. de la Salpétr., xviii, 2, p. 176, 1905.

BALP, ST. Resultati di alcune esperienze di tiroidismo sperimentale. Giorn. di R. Acad. di Med. di Torino, Jhrg. 67, Nos. 9, 10.

BAMBERGER, H. Krankh. der Nebennieren u. d. chylopoetischen Systems. Erlangen, 1864 .

BANG, IVAR. Chemische Untersuchungen der lymph. Organe. H. B., 4 and $5,1904$.

- Biochemie der Zelllepoide. Asher. Spiro Ergeb., vi, 1907, and viii, 1909.

- Die biologische Bedeutung der Lepoidstoffe. Erg. d. inn. Med. u. Kind, iii, 1909.

BARBA. Sulla glycosuria surrenale. Rif. med, Ig02.

BARBERA, A. G. Über den Einfluss des J. Jodnatriums und Jodothyrins auf den Blutkreislauf. P. A., 73, 312, 1900.

BARBÉRA and BícCI. Strukturveränd d. Nebenniere. Boll. sc. med. Bologna, II, 1000 .

BARBIER. Des rapports entre les lésions des capsules surrénales, les lésions nerveuses et la maladie d'Addison. G. m., 1 Sgz.

Barbier, H., and Cruet. Opothérapie biliaire. Bull. gén. de Thérap., 1907.

BARDIER. Historique générale du rôle antitoxique des organes. P. m., I 896.

BARDIER and BAYLAC. De l'action de l'ad̂rénaline sur la pression sanguine des animaux atropinisés. C. r. S. B., 56, ii, p. 485, 1904.

BARDIER and BONNE. Modifications produites dans la structure des surrénales par la tétanisation des muscles. C. r. S. B., 55, ii, p. 355, I 903.

- Note sur les modifications produites dans la structure des surrénales par la tétanisation musculaire. J. A. P., 39, p. 296, 1903.

BARDIER and FRAENKEL. Action de l'extrait capsulaire sur la diurèse. C. $r$. S. B., 5I, p. 544, I $\$ 99$; J. d. P. P., i, p. 950, 1899 .

BARGIER, G., and JOWETT. Synthesis of substances allied to adrenalin. Proc. Chem. Soc., 21, p. 205.

BARLET, J. M. A case of Addison's disease with hyperpyrexia. Lancet, September 6, 1902 .

BARNabo, V. Sur les rapports entre la glande interstitielle du testicule et les glandes à sécrétion interne. Il. Policlinico, mars, 1908.

Baros, A. Diabetes suprarenalis. Utschennija Sapisski. Imp. Zit. n. Landau, 1906.

BARRAND. Etude de la vasoconstriction produite par l'application locale de l'extrait aqueux de capsules surrénales. Thèse de Lyon, is97.

Bartel, J. Der normale und abnormale Bau des lympliatischen Systems und seine Beziehungen zur Tuberkulose. W. k. W., 1907.

- Uber die hypoplastische Konstitution und ihre Bedeutung W. k. W., 1908.

BARTEL and STEIN. Über abnormale Lymphdrüsenbefunde und deren Beziehung zum Status thymicolymphaticus. A. A., p. 231, 1906.

Bartels, M. Über Plattengeschwülste der Hypophysengegend Zeitsch. Augenk., 16, 1906.

Bartsch, H. 'De morbo Addisonii. Diss., Königsberg, 1867. 
BarUCH, F., Ein Fall von Morbus Addisonii. Diss., Prag, 1895.

BASCH, K. 'Uber die Ausschaltung der Thymus. W. k. W., I903.

- Bemerkungen zu R. Fischls experimentellen Beiträgen zur Frage der Bedeutung der Thymusexstirpation bei jungen Tieren. Z. e. P., ii, p. 95,1905 .

- Beiträge zur Physiologie und Pathologie der Thymus. J. K., 64, I906.

- Beiträge zur Physiologie und Pathologie der Thymus, II. Über die Beziehung der Thymus zum Nervensystem. Ibid., 68, 1908.

- Uber experimentelle Auslösung der Milchabsonderung. Monatsschr. f. Kinderh., 8, No. 9, Dezember, r9o9.

B.sso. Über Ôvarientransplantation. A. G., 77, p. 51, 1906.

BATES, W. H. The use of extract of suprarenal capsule in the eye. New York Med. Journ., 63, p. 647, May 16, 1896.

- The use of the aqueous extract of the suprarenal capsule as a hæmostatic. New York Med. Record, p. 207, February 9, 1901; Arch. of Ophthalmol., 28, igoo.

Battell, F. Dosage colorimétrique de la substance active des capsules surrénales. C. r. S. B., 54, p. 57 I, 24 mai, 1902.

- Préparation de la substance active des capsules surrénales. Ibid., p. 608,1902 .

- Quantité de substance active contenue dans les capsules surrénales de différentes espèces animales. Ibid., p. 928, 12 juillet, 1902.

- Comparaison entre les propriétés colorantes, toxiques et les modifications de la pression artérielle produites par la substance active des caps. surrén. Ibid., p. 984 .

- Influence des injections intravéneuses continues d'adrénaline sur la survie des animaux décapsulés. Ibíd., p. I138, 18 octobre, I902.

- Présence d'adrénaline dans le sang d'animaux. Son dosage. Ibid., p. 1179 , 1902.

- L'adrénaline dans l'organisme des animaux decapsulés. P. I180, 25 octobre, 1902 .

- Toxicité de l'adrénaline en injections intraveineuses. 13 novembre, 1902.

- Transformation de l'adrénaline "in vitro." P. I435, I3 decembre, 1902.

- Transformation de l'adrénaline dans l'organisme. Ibid., p. 1518, 27 decembre, 1902 .

- Quantité d'adrénaline existant dans les caps. surrén. de l'homme. Ibid., p. 1205 .

- Influence du travail suivi de repos sur la quantité d'adrénaline existant dans les capsules surrénales. Ibid., p. I520, 27 decembre, I 902.

- Recherches sur les vasoconstrictiones du sérum sanguin. J. d. P. P., vii, p. 625 .

Battelli, F., and Ornstein, S. La suppléance des capsules surrénales au point de vue de leur richesse en adrénaline. C. r. S. B., 61, p. 677, 1906.

Battelli, F., and Roatta, G. B. Influence de la fatigue sur la quantité d'adrénaline existant dans les capsules surrénales. C. r. S. B., 54, p. I 203,8 novembre, I9O2.

Battelli, F., and Taramasio, P. Toxicité de la substance active des capsules surrénales. Ibid., 54, p. 815,28 juin, 1902.

BAtTEN, F. Hæmorrhage into the suprarenal capsule. Transact. of Path. Soc., London, i 898 .

BATtiER. L'anesthésie dentaire au moyen de l'extrait de caps. surr. S. m., I 902.

Baudouin, $\mathrm{H}$. Myatonie congénitale (maladie d'Oppenheim). S. m., I9o7. BAUER, K. Der chemische Nachweis d. degenerativen Nervenkrankh. H. B., I I, 1908 .

Baum, H. Die Thymusdruise des Hundes. D. Zeitsch. f. Tierh., 17.

BAUM, I. Die örtliche Einwirkung von Nebennierensubstanz, Brenzkatechin und Spermin auf die Zirkulation. B. k. W., 1905.

- Zur Wirkung und Verwendung der Nebennierenpräparate, insbesondere in der Dermatologie. Arch. Dermat. u. Syph., 74, p. 59, 1905.

BaUmanN, E. Über das normale Vorkommen von Jod im Tierkörper. Z. ph. Ch., 21, 319, 1896 .

.. Über den Jodgehalt der Schilddrüse von Mensch und Tieren. Ibid., 22, 1, I896. 


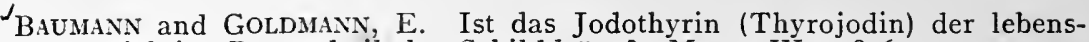
wichtige Bestandteil der Schilddruise? M. m. W., 1896.

BAUMANN and HERTER. Über die Synthese von Ätherschwefelsäuren und das Verhalten einiger aromatischer Substanzen im Tierkörper. Z. ph. Ch., p. 244, 1877-78.

Baumel. Pancréas et diabète. Montpellier med., sér. i, 47, 134, 1882.

- Capsules surrénales et mélanodermie. Paris, 1889.

- Nouvelle theorie pancréatique de diabète sucré. Montpellier med., 1889 .

BAYER, G Methoden zur Verschärfung von Adrenalin- und Brenzkatechinreaktionen. B. Z., 20, p. 178, I909.

- Über den Einfluss d. Drüsen mit innerer Sekretion auf die Autolyse. S. W. A., II 8 .

BAYLAC. Athérome expérim. de l'aorte consécutif à l'action du tabac. C. r. S. B., 1906.

BAYLAC and ALBARÈDE. Recherches expérimentales sur l'athérome de l'aorte consécutif à l'action l'adrénaline. C. r. S. B., 56, ii, p. 640, 1904.

BAYLISS, W. M. On the local reactions on the arterial wall to changes of internal pressure. J. o. P., 28, p. 220, 1902.

- Die Innervation der Gefässe. E. P., 5 , I906.

Bayliss, W. M., and StaRling, E. H. The mechanism of pancreatic secretion. J. o. P., 28, pp. 325-353, 1902 ; and ibid., 29, 1903.

- Die chemische Koordination der Funktionen des Körpers. E. P., 5, 1906.

Bayon, G. P. Erneute Versuche über den Einfluss des Schilddrüsenverlustes und der Schilddrüsenfütterung a. die Heilung der Knochenbrüche, W. V., 34,35 .

- Beitrag zur Diagnose und Lehre von Kretinismus, \&c. Ibid., 36, 1903.

- Über angeblich verfrühte Synostose bei Cretinen. Ziegl. B. 36 .

BEADLES, C. Tumour of the suprarenal body. Transact. of Path. Soc., London, 1898 .

BEARD, J. The development and probable function of the thymus. An. An., 9, 1894 .

- The sources of leucocytes and the true function of the thymus. Ibid., 18 , 1900 .

BEAUJARD, E. Les lésions surrénales dans les néphrites. S. m., 1907.

BECKER. Beitrag zur Thyroidinwirkung. D. m. W., 1895 .

BECKER, Ph. F. Der männliche Kastrat mit besonderer Berüksichtigung seines Knochensystem. Diss., Freiburg i, Br., I 898 .

- Über das Knochensystem eines Kastraten. A. A., p. 83, 1899 .

BÉCLÈRE. Opothérapie surrénale. Bull. Soc. méd., p. 171, 25 février, 1898.

- Le traitement méd. des tumeurs hypophysaires, du gigantisme et de l'acromégalie par la radiothérapie. Bull. de la Soc. méd. des Hôpitaux, pp. 274-293, I909.

BEEBE. Inhibition of tetany by extract of parathyroids. A. J. P., I9.

BEER, E. Die Nebennierenkeime in der Leber. Z. H., 25, I904.

BEIER. Untersuchungen iuber das Vorkommen von Gallensäuren und Hippursäure in den Nebennieren. Diss., Dorpat, I891.

Beissner. Die Zwischensubstanz des Hodens und ihre Bedeutung. A. m. A., $5 \mathrm{I}, 1898$.

Beitzke. Zur Biologie der Nebenniere. B. k. W., Igog.

BELAwENTz. Contribution à l'étude de l'action de l'adrénaline sur l'organisme animal. Ref. J. d. P. P., 5, p. 72I, I903.

BÉlenowSKY, G. Essai de préparation de sérum anti-intestinal. C. $r$. S. B., 63 , p. 9,1907 .

BeLL, W. B., and HICK, P. Observations on the physiology of the female genital organs. Brit. Med. Journ., p. 517, I909.

Bence, J. Exper. Beitr. zur Entstehung der nephritischen Ödeme. Z. k. M., 67 , 1909 .

BENDA. Uber den normalen Bau und einige pathol. Veränderungen der menschlichen Hypophysis cerebri. A. P., p. 373 , I900.

- Beiträge zur normalen und pathologischen Histologie der menschlichen Hypophysis cerebri. B. k. W., I 900 .

- Über vier Fälle von Akromegalie. D. m. W., I9or.

- Die Akromegalie. Deutsche Klinik, iii, 1903. 
BEndA. Patholog. Anatomie der Hypophyse in Flatau-Jacobsohn-Minor. Handb. d. path. Anat. d. Nervensystems. Berlin, Ig04.

- Die Arteriosklerose im Lichte exp. Forschung. Th. G., I909.

BENDER and LERI. Atrophie des caps. surrén. chez les foetus anencéphales. C. r. S. B., 1903 .

BENDIX. Intermittierende Polyurie bei Addisonscher Krankh. D. A. kl. M., 82, 1903 .

- Beiträge z. Kenntn. des glykolytischen Fermentes. Z. f. diät. u. phys. Th., I 899 .

BENDIX and BICKEL. Experimentell kritische Beiträge z. Lehre v. d. Glykolyse. D. m. W., 1902; and Z. k. M., 48, I903.

Benedicenti, A. L'azione 'dell' adrenalina sulla secrezione pancreatica. Giorn. della R. Accad. di. med. Torino, 1906; and A. i. B., 45, 1906.

Beneke, F. W. Studien über d. Vorkommen, d. Verbreitung u. Funktion von Gallenbestandteilen in d. tier. u. pflanz. Organismus. Giessen, 1862 .

BENEKE, R. Zur Lehre von der Versprengung von Nebennierengewebe in die Niere, nebst Bemerkungen zur allgemeinen Onkologie. Ziegl. B., 19.

Benjamins. Über die Glandulae parathyreoideae. Ibid., 31, 1902.

Benjamin, E., and A. v. ReUsz. Über den Stoffwechsel bei Myxödem. J. K., 67.

Bennet. Dangers in the use of adrenal preparations. J. A. M. A., I9o6.

BENSEN. Beitr. z. Kenntn. d. Organveränderungen nach Schilddrüsenexstirpation bei Kaninchen. V. A., I70, 1902.

BÉRARD and ALAMARTINE. Les glandules parathyroïdes et leur tumeurs. Lyon. chir., 1909. Ref. C. Ch., r909, p. 1019.

Berardi, S. Contributo alla patologia del morbo di Addison. Arch. ital. di. Clin. med., 35, p. I 7 , 1896.

BERDACH. Ein Fall von primärem Sarkom der Nebenniere. W. m. W., 1889 .

Berdach and Pal. Zur Pathologie der Nebennieren. B. k. W., 1894.

BERDÉs. Contrib. à l'étude des tumeurs des capsules surrénales. A. m. e., 4, 1802 .

Bergeat. Über 300 Kropfexstirp. a. d. Brunschen Klinik. Bruns Beitr., I $5,1896$.

BERGER. Fall von Tumor der Hypophysengegend mit Sektionsbefund. Z. k. M., 54, 1904 .

BergmaNn, G. v. Der Stoff- und Energieumsatz beim infantilen Myxödem und bei Adipositas universalis. Z. P. P., V. 43.

Bergonié and Tribondeau. Action des rayons $\mathrm{X}$ sur le testicule du rat blanc. C. r. S. B., 56, ii. 12 novembre, 17 décembre, $1904 ; 57$, i, 17 janvier, pp. I 54 and 155,1905 .

- Aspermatogenèse expérimentale après une exposition aux rayons $\mathrm{X}$. Ibid., p. 282 , 1905 .

- Action des rayons X sur l'ovaire de la lapine. Ibid., p. 284, I005.

- L'aspermatogenèse expérimen. des rayons $\mathrm{X}$ est-elle définitive? Ibid., p. 678 .

- Lésions du testicule avec des doses de rayons $\mathrm{X}$ comment se produisentelles? Ibid., p. 1029, 1905.

- Processus involutif des follicules ovariens après rœntgenisation de la glande génitale femelle. C. r. S. B., p. 105, 1907.

- Altérations de la glande interstitielle après roentgenisation de l'ovaire. Ibid., p. 274, I007.

BERKELEY and BEEBE. Extract of parathyroids. Journ. Med. Res., February, 1909.

BERkLEy, H. J. The nerve elements of the pituitary gland. Johns Hopkins Hosp., i 894 , and Brain, I894.

Bernard, CL. Leçons de Physiologie expér. au Collège de France. Paris, 1855 .

- Mémoires sur l'extirpation du pancréas. C. r. A., 1856.

- Leçons de pathologie expérimentale (au Collège de France, 1859-6o), publié 187 I.

- Rapport sur les progrès de la physiologie générale en France, 1865 .

- Vorlesungen über Diabetes. Übers. Berlin, 1878 . 
BERNARD, Cl. La science expérimentale. Paris, 1890.

BERNARD, L. Les syndromes surrénaux. P. m., I905.

- Du rôle des glandes surrénales dans les états pathologiques. Rev. de méd., I907.

BERNARD, L., and BIGART. Etude anatomo-pathologique des capsules surrénales dans quelques intoxications experimentales. J. d. P. P., iv., pp. 1014-1029, 1902.

- Sur les réactions histologiques générales des surrénales à certaines influences pathogènes expériment. C. r. S. B., 54, p. 1219, 1902.

- Réactions histologiques des surrénales au surmenage musculaire. Ibid., p. 1400, 1903 .

- Sur activité fonctionnelle des glandes surrénales dans l'intoxication saturnine expérimentale. Ibid., 56, i, p. 59, 1904.

-. Les processus sécrétoires dans la substance corticale de la glande surrénale. Ibid., 57, ii, p. 504, 1905.

- Lésions des glandes surrén. au cours de l'intoxication biliaire expérim. Ibid., p. 410 , I906.

- Etude anatom. pathologique des glandes surrén. des tuberculeux. J. d. P. P., I906.

Bernard, L., Bigart, and LABBÉ. Sur la sécrétion de lécithine dans les capsules surrénales. C. r. S. B., 55, ii, p. 120 , 1903.

Bernard, L., and LAEderich. Néphrites expérimentales par action locale sur le reins. C. r. S. B., p. 768, 1907.

BERNARDINI. Ipertrofia cerebrale e Idiotismo. Rivista sper. fren., I3, I887.

BERNATZKIJ, S. S. Das Lecithin des Knochenmarks normaler und immunisierter Tiere und die Verteilung des Phosphats im Organismus. Diss., St. Petersb., I908.

Bernstein, Bolaffio, and Westenryk. Über die Gesetze d. Zuckerausscheidung beim Diabetes. Z. k. M., 66, 378, 1908.

Berrutti and Perosino. Note sulle capsule surrenali. Giorn. dell' Accad. med.-chirurg. di Torino, 1857 and 12 giugno $1863 ; \mathrm{A}$. per le scienz. med., 1879 .

BERRY, J. M. Influence of adrenaline chloride on toxic doses of cocaine. A. J. M. S., I 905 .

Bertel. Zur Toxizität des Adrenalins. Öst.-ung. Vierteljahrsschr. f. Zahnh., 1905.

Berthold. Transplantation der Hoden. A. P., p. 42, I 849.

- Geschlechtseigentümlichkeiten. Wagners Handwört. d. Physiol., I, Braunschweig, 1872 .

BertKan, PH. Beschreibung eines Zwitters von Gastropacha Quercus. A. f. Naturgesch., 55, 1. 75, I889.

- Beschreibung eines Arthropodenzwitters. Ibid., 57, p. 229, I891.

BERTRAM, R. Betrachtungen über Adenomknötchen an den Nebennieren Neugeborener und über Tumoren der Nebennieren. Orth. Festschr., 1903 .

BERTRAND, G. Sur la composition chimique et la formule de l'adrénaline. Ann. Inst. Pasteur, I8, p. 672; Bull. soc. Chim., 3, sér, t. 3I-32, pp. I 188, I 2S9, I 904 .

- Sur les relations du chromogène surrénal avec la tyrosine. C. r. A., 138, 649, 1903.

- Sur la composition chimique et la formule de l'adrénaline. Ibid., r39, 12 , p. 502, 1904.

Bertrand, M. L'infantilisme dysthyroidien. Thèse de Paris, Igo2.

BESNIER. Dégénerescence cancéreuse complète des deux capsules surrénales. Bull. soc. anat. de Paris, I 850 .

- Mélanodermie généralisée avec pigmentation des angles, de la muqueuse buccale et du prépuce, sans signes certains de cachexie surrénale. C. r. des réunions clin. de l'hôp. St. Louis, Paris, I888-89.

BessmertNy, CH. Studien über antagonistische Nerven. Z. B. 47, p. 400, 1906.

BEST. Über Glykogen, insbesondere seine Bedeutung bei Entzündung und Eiterung. Zieglers Beitr., 33, p. 5 $\delta_{5}$, 1903.

- Über lokale Anästhesie in der Augenheilkunde. Vossius' Samml., vi, H. 3, 1905 .

Bestion. Le suc ovarien. Effets physiol. et thèrap. Thèse, Bordeaux, 1898 . 
BIAGI. L' azione del principio attivo della medollare della capsule surrenale sulla fibra muscolare liscia dell' utero. Rassegna di Ostetr. e Ginec., 1905 ; Ref. B. C., iv, p. 762.

Brasottr, A. Dell' influenza dell' estratto di ghiandiole surrenali sull' acceleramento dell'ossificazion dello scheletro. Soc. ital. di ostetr. e ginec., 1908; Pathologica, I908.

Biberfeld, J. Beitrag zur Lehre von der Diurese. XIII. Über die Wirkung des Suprarenins auf die Harnsekretion. P. A., I I9, p. 34I, 1907.

- Pharmakologische Eigenschaften eines synthetisch dargestellten Suprarenins und einiger seiner Derivate. M. K., I906; Pharmac. Journ., 1908 .

- Über Dosierung des in den Wirbelkanal gespritzten Suprarenins. D. m. W., 1907 .

BIECK, P. Beiträge zur Kenntnis der Nierengeschwülste. Inaug. Diss., Marburg, 1886.

BIEDL, A. Über die Centra der Splanchnici. W. k. W., p. 915, 1895.

- Vorl. Mitt. uber die physiol. Wirkung des Nebennierenextraktes. W. k. W., p. 157,1896 .

- Action de l'extrait de capsules surrénales sur la pression sanguine. S. m., 1896 .

- Beiträge zur Physiologie der Nebenniere. Die Innervation der Nebennieren. P. A., 67, 1897 .

- Schilddrüse und Jodothyrin. Hypophysisexstirpation. W. k. W., p. $195,1897$.

- Über eine neue Form des experimentellen Diabetes. C. P., 12, p. 624, I 898 .

- Zur Schilddrüsenfrage. W. k. W., p. 1278, 1901.

- Theoretisches über das Wesen und die Behandlung des Fiebers. W. m. W., 1902 .

- Innere Sekretion. Vorlesungen, 1902. Wein, 1903. Wiener Klinik, 29, 1903 .

- Diskussion zum Vortrage Erdheims. W. k. W., 26, 1906.

- Diskussion zum Vortrage Foges. W. k. W., 615, 1907.

- Diśkussionsbemerkung (zu Moskowicz). WV. k. W., p. 304, 1908.

- Diskussionsbemerkung (zu v. Haberer und Stoerk). W. k. W., p. 306, 1908.

- Die geschichtliche Entwicklung der Kenntnisse über die Nebenniere bis Addison (1885). Janus. Arch. int. pour l'hist. et la Géogr. médic., $15 \mathrm{Jg} ., 1910$.

- Über die Lipoide der Nebennieren.

BIEDL and BRAUN. Zur Pathogenese der experim. Arteriosklerose. W. k. W., 1909 .

- Das Bild der Kompressionsarteriosklerose.

BIEDL and DECASTELLo, V. Über Änderungen des Blutbildes nach Unterbrechung des Lymphzuflusses. P. A., 86, 1901.

BIEDL and KöNIGSTEIN, R. Über das Mammahormon.

BIEDL and Kraus, R. Experimentelle Studien über Anaphylaxie. W. k. W., 1908 and 1909 .

- Wirkung intravenös injizierten Peptons beim Meerschweinchen. C. P., $24,1910$.

BIEDL and OFFer, Th. R. Über Beziehungen der Ductuslymphe zum Zuckerhaushalt. Hemmung von Adrenalinwirkung durch die Lymphe. W. k. W., 1907.

BIEDL and REINER, M. Studien über Hirnzirkulation und Ödem. I. Mitt. Über das Vagusphänomen bei hohem Blutdruck. P. A., 73, 1898, II. Mitt. Die Innervation der Hirngefäsze. P. A., 79, I899.

- Offener Brief an den Herausgeber (gegen v. Cyon). 'P. A., 83, 1900.

BIEDL and ROTHBERGer, J. Die Volhardsche Methode der künstl. Atmung. C. P., 23, I909.

BIEDL and WIESEL, J. Über die funktionelle Bedeutung der Nebenorgane des Sympathikus (Zuckerkandl) und der chromaffinen Zellgruppen. P. A., 9I, 1902. 
Biedl and Winterberg, $\mathrm{H}$. Die Ammoniak entgiftende Funktion der Leber. P. A., 88, 1901 .

BIEglio, A. Il ricambio organico nella sindrome miotonica. Il Policl. 14, No. 8.

BieHLER, R. Ein eigentümlicher Fall von Morbus Addisonii. Diss., Rudolfstadt, 1892 .

BIERRING and ALBERT. Secondary manifestations of hypernephromata. A. M. A., 1904 .

BIERRY. Sérum nephrotoxique. C. r. A., mai, 190 I.

- Recherches sur les nephrotoxines. C. r. S. B., 4 avril, 1903.

BIERRY, H., and GatiN-GruzewSKa. L'Adrénaline produit-elle la glycosurie par son action sur le pancréas? C. $x$. S. B., 58, p. 904.

- Action physiologique de l'adrénaline pure. Ibid., p. 902.

- Effets de l'injection de l'adrénaline sur les animaux décapsulés. Ibid., p. 203, 1904 .

BIERRY and LALON. Variations du sucre du sang et du liquide céphalorachidien. Ibid., p. 253, 1904 .

BierRy, H., and Malloizel, J. Hyperglycémie après décapsulation, effets de l'injection d'adrénaline sur les animaux décapsulés. Ibid., 65, p. 232, 1908.

BIERry and Terraine. Sur l'amylase et la maltan de sur pancréatique de sécrétine. Ibid., 57, ii, p. 257, 1905.

BIEsing, K. Über d. Nebennieren u. d. Sympathicus bei Anencephalen. Diss., Bonn, 1886.

Bigart and Bernard. Sérum surrénotoxique. C. r. S. B., Igor.

BILAND. Über die durch Nebennierenpräparate gesetzten Gefäsz- und Organveränderungen. D. A. k. M., 87, p. 413, 1906.

BILharz, A. Die Genitalorgane schwarzer Eunuchen. Z. w. Z., x, p. 28I, 1860 .

BING, R. Nebenschilddrüsen und spontane Tetanieformen. M. K., p. 677, 1908.

Bingel, A., and Strauss, E. Über die blutdrucksteigernde Substanz der Niere. D. A. k. M., 96, p. 476,1909 .

BIONDI. Beitr. zur Struktur und Funktion der Schilddrüse. B. k. WV., I 888 .

- Contribution à l'étude de la glande thyréoïde. p. 475 . A. i. B., 17, 1892.

BIRCher, H. Das Myxödem und die kretinische Degeneration. Volkmanns Sammlung klinischer Vorträge, No. 357, 1890.

- Der endemische Kropf und seine Beziehungen zur Taubstummheit und zum Kretinismus. Basel, i 883 .

- Fortfall und Änderung der Schilddrüsenfunktion als Krankheitsursache. L. O., I, I 896 .

- Zur Implantation von Schilddrüsengewebe bei Kretinen. Z. Ch., 98, H., I.

BIRcher, E. Zur Pathogenese der kretinischen Degeneration. Berlin, 1908. - Experimenteller Beitrag zum Kropfherz. M. K., No. 10, 1910.

BIRnBaUm. Ovarium und innere Sekretion, Sammelreferat. Z. a. P., 8, 1908.

BIRnBaUm and Osten, A. Untersuchungen über die Gerinnung des Blutes während der Menstruation. A. G., 81, 1906.

BISCHOFF, TH. Beweis der von der Begattung unabhängigen periodischen Reifung und Loslösung der Eier, \&c. Gieszen, i844.

Bittorf, A. Die Pathologie der Nebennieren und der Morbus Addisonii. Jena, 1908.

- Adrenalinämie bei Nephritis? Die Loewische Reaktion. C. i. M., p. 33, 1909 .

Bizzozero and Vassale. Sur le tissu des glandes excrétantes. A. i. B., 9, 1887.

BlackBuRN, W. Primary sarcoma of the adrenal gland. A. J. M. S., August, 1906.

BLACKBURN, J. Hypernephroma. A histological study of three cases of hypernephroma of the kidney and one of the papilliferous adenoma. New York Med. Journ., No. 7, 1907.

BLAIR, BELL. The pituitary body and the therapeutic value of the infundibular extract in shock, uterine atony and intestinal paresis. Ibid., p. I609, I909. 
Blanchard. Note sur l'histoire de la découverte de la capsule surrénale. C. r. S. B., I882.

BLANCHETière and CHevalier. Sur la recherche de la choline dans la pancréas et la thyroïde. C. r. S. B., 67, p. 277, 1909.

Blanck. Exp. Beitr. z. Pathogenese d. Nierenwassersucht. Z. k. M., 6o, p. $472,1906$.

BletBTREU. Fall v. Akromegalie (Zerstörung der Hypoph. durch Blutung). M. m. W., I 1905 .

BLeibTREU, L. "Uber Beziehungen von Fettgewebsnekrosen und Arteriosklerose zum Diabetes mellitus. P. A., I 24, I908; and B. k. W., I908.

BLoch, I. Einflusz von Jod, Thyrojodin und Thyroiden auf den Stoffwechsel. Würzburg, 1806 .

Blum, F. Die Schilddrüse als entgiftendes Organ. B. k. W., I898.

- Die Schilddrüse als entgiftendes Organ. V. A., I58, I899.

- Zur Chemie u. Phys. d. Jodsubst. in d. Schilddrüse. P. A., 77, 1899.

- Neue experimentell gefundene Wege zur Erkenntnis und Behandlung von Krankheiten, die durch Autointoxikationen bedingt sind. V. A., 162 , p. 375 , 1900 .

- Über Nierenveränderungen bei Ausfall der Schilddrüsentätigkeit. V. A., 166, 1901.

- Über Nebennierendiabetes. D. A. k. M., 7I, Ig0I.

- Weitere Mitteilungen zur Lehre von dem Nebennierendiabetes. P. A., 00, p. 6I 7, I 902 .

- Gefassdrüsen und Gesamtorganismus. P. A., 105, p. 625.

- Neues u. Altes z. Physiologie und Pathologie der Schilddrüse. V., 23, C. M., 1906.

BLUMENTHAL, F. Über zuckerabspaltende Körper im Organismus. B. k. W., 1897.

- Über das glykolytische Ferment. D. m. W., I903.

- Über Organsafttherapie bei Diabetes mellitus. $Z$. f. diät. u. phys. Ther., 1,1808 .

- Über die nichtdiabetischen Glykosurien. Halle, I9o9.

- Glykolyse. Eulenburgs Realenzykl., 5, I909.

BlumReich, L., and JACOBY, M., Exp. Unters. über die Bedeutung der Schilddrüse und ihrer Nebendrüsen fur den Organismus. B. k. W., I 896 ; P. A., 64, I896.

Boss, K. Zur Methodik des Adrenalinnachweises. C. P., 22, p. 825, 20 März, Igog.

- Uber den Wert der Sublimatreaktion des Adrenalins. Ibid., 23, p. 252, I 909 .

Bock and Hoffmans. Über eine neue Entstehungsweise der Melliturie, Durchspülungsglykosurie. A. P., p. $550,187 \mathrm{I}$.

Bӧнм, B. Fortgesetze Unters. uiber die Permeabilität der Gesfaszwande. B. Z., I6, 1909 .

военм, R. Über d. Wirkung d. Barytsalze. A. P. P., 3, p. 216, I875.

- Über d. Vorkommen u. d. Wirkung d. Cholins. A. P. P., 19, p. 87, 1885 .

BoeniNGER, M. Beitrag zur Frage des Nierendiabetes. D. m. W., 1908.

BOERMA. Zur therapeut. Verwendung des Antithyreoidin. Ärztl. Rundsch., I905.

BoEse and LoRenz. Kropf, Kropfoperationen und Tetanie. W. k. W., 1909.

BöTTCHER, B. Eine neue Synthese des Suprarenins und verwandten Verbindungen. Chem. Ber., 42, p. 253, Januar, 1904.

Bogomolez, A. Zur Frage uiber die Veränderungen der Nebennieren bei experimenteller Diphtherie. Zieglers Beitr., 38, p. 5 10, 1905.

- Zur Physiologie der Nebennieren. Folia Serol., iii, Igog.

BOGULJUBOFF, W. L. Zur Chirurgie der Nebennierengeschwülste. A. k. Ch., 80, Igo6.

BorNet. Résultats éloign. de soixante-quinze ablations de deux capsules surrénales. C. r. S. B., 47, p. I62, 1895.

- Résistance à la fatigue chez les rats décapsulés. Ibid., p. 273, I895.

- Nouvelles recherches sur la résistance à la fatigue de rats décapsulés. P. 325 .

- Ablations des capsules vraies et accessoires chez le rat d'égout. Ibid., p. 498 . 
BorNet. Action comparée de la fatigue et de la décapsulation sur la toxicité des extraits musculaires du rat. Ibid., p. 646, 1895.

-- Maladie d'Addison expérimentale chez le rat d'égout. Ibid., 48, p. 164, 1 So6.

- Action antitoxique des capsules surrénales sur la neurine. Ibid., p. 364, 1896 .

- Nouveaux cas de la maladie d'Addison expérimentale. Ibid., 49, pp. 439 and $473,1897$.

- Diminution de résistance des rats décapsulés à l'action toxique de divers. subst. p. 466.

- Rech. expérim. sur les fonctions des caps. surrén. Ibid., 51, p. 671, 1899.

- Troubles nerveux et tremblements observés, chez un Addisonien, à la suite de trop fréquentes injections de capsules surrénales de veau. Ibid., 5 I, p. 891 , I 899 .

- Recherches expérimentales sur la pathogénie de la maladie d'Addison. Revue de méd., pp. 136-143, 17 février, 1897.

- Dangers de l'adrénaline dans certains cas de maladie bronzé d'Addison. C. r. S. B., 55, pp. 1471 and 1474,1903 .

- La mort dans mala die bronzée d'Addison. Arch. gén. de méd. I., 1903. - De l'Addisonisme. Ibid., ii, I904.

BoLdU, H. Glandula thyrcoudea u. Gl. thymus bei d. Amphibien. Zool. Jahrb., I2, I 899 .

BoLDT. Glokosurie nach Kropfschwund. D. m. W., I904.

DE BoNis. Action des extraits d'Hypophyse sur la pression artérielle et sur le cœur normal ou en état de dégénérescence graisseuse et de la nature du principe actif de l'hypophyse. A. i. Ph., p. 211, 1908.

DE BoNIS, W., and SusanNa, V. Über die Wirkung des Hypophysenextraktes auf isolierte Blutgefäsze. C. P., 23, p. 169, 1909.

Bonvamour, S. Recherches histologiques sur la sécrétion des capsules surrénales. C. r. assoc. anat. Montpellier, 1902.

- Etude histologique des phénomènes de sécrétion de la capsule surrénale chez les mammifères. Thèse de Lyon, igo5.

Bonnamour, S. and Pinatelle. Note sur l'organe parasympathique de Zuckerkandl. Bibliogr. anat. T. xi, pp. 127-136, 1902.

Bonnamour, S. and Policirin. Sur la graisse de la capsule surrénale de la grenouille. C. r. S. B., 55, p. 471, 1903 .

BonNamour and THEvenot. Variations de résjistance des lapins à l'adrénaline. C. r. S. B., 66, p. 509, 1909.

- Toxine diphtérique et adrénaline dans la production de l'athérome expérimental. Ibid., pp. 66 and 387 , 1909.

BONNET, R. Lehrbuch der Entwicklungsgeschichte. Berlin, 1907.

BORCHARDT, L. Experimentelles über den Diabetes bei der Akromegalie. Ref. D. m. W., 34, p. 946, Igo8.

- Die Hypophysenglykosurie und ihre Beziehung zum Diabetes bei der Akromegalie. Z. k. M., 66, p. 332, 1908.

- Diskussion zum Vortrage Hochenegg. Congr. d. G. Chirurg., 1908.

- Funktion und funktionelle Erkrankungen der Hypophyse. Erg. d. inn. Med. u. Kinderh., iii, p. 288, 1909 .

BORN, G. Über die Derivate der embryonalen Schlundbögen und Schlundspalten bei Säugetieren. A. m. A., 22, I882.

Borrmans, B. Ein Fall von Morbus Addisonii zufolge entzündlich-hyperplastischer Wucherung der Nebennieren auf traumatischer Grundlage. D. A. k. M., 86, 1906 .

BorutTAU, H. Erfahrungen über die Nebennieren. P. A., 78, 1899.

- Über den jetzigen Stand unserer Kenntnisse von den Funktionen der Blutgefäszdrüsen. D. m. W., 1899 .

- Zur Entstehung des Adrenalins im Tierkörper. C. P., 21, 1907.

- Innere Sekretion. Nagels Handb. der Physiologie, ii, I907.

Bossi, L. M. Die Nebennieren und die Osteomalacie. A. G., 83, p. 505, 1907.

- Nebennieren und Osteomalacie. C. G., pp. 69, 172, 1907.

- L'influenza delle capsule surren. sull' ossificazione d. scheletro. Gin. mod., 1908.

- The influence of the suprarenal glands on the bone skeleton in relation to osteomalacia and rickets. Brit. Med. Journ., 1908. 
Boteano, E. R. Contr. la physiol. glandei pituitare la brosca. Thèse. Bucarest, Igo6. Zit. n. Paulesco.

Botrazzi, F. Azione dell'adrenalina sul tessuto muscolare liscio esofageo di Bufo vulgaris. Atti della R. Accad. med. di. Genova, XVIII; C. P., 19, p. 100, 1904 .

- Sur quelques altérations de globules rouges du sang à la suite de la thyroïdectomie. A. i. B., 23,1895 .

Bottazzi and Costanzi. Nuove recerche sull' azione dell' Adrenalina (Clin.) e della Paraganglina (Vassale) sui muscoli lisci. Il tom., 1905; Ref. C. P., 20, p. 279, 1905.

BOTTAZZI, D'ERRICo and JapPELLI. Wirkung des Adrenalins auf die Speichelund Harnabsonderung. B. Z., vii, p. 431, I 908.

Botrazzi, F., and TORRETTA, A. Azione dell'adrenalina sulla muscolatura longitudinale dell' esofago di Bufo vulg. A. d. F., i, pp. 325-33 I, 1904 .

BouchaRD, CH. Involution sénile. Traité de path. gén., Igoo.

BOUCHARD and ClaUDE. Recherches expérimentales sur l'adrénaline. C. r. A., p. 928 , 1902 .

Bovchardat. Monographie sur le diabète. Paris, IS75.

Bouchart. Infiltration de la cornée et mydriase persistante attribuables à l'adrénaline. La clin. opht., 1904. - Accidents attribuables à l'adrénaline. Rev. d'opht., 1905 .

BouchÉ, F. Gegenseitige Beeinflussung von Adrenalin und Verdauungsstörungen. Diss., Freiburg, I909.

Bours. Les deux glandes à sécrétion interne de l'ovaire, la glande interstitielle et le corps jaune. Rev. méd. de l'Est., 1902.

- L'infantilisme et la glande interstitielle du testicule. C. r. A., janvier, 1904 .

Bouin, P., and ANCEL, P. Sur les cellules interstitielles du testicule des mammifères et leur signification. C. r. S. B., I4 novembre, I903.

- Sur la signification de la glande interstitielle du testicule embryonnaire. C. r. S. B., 19 décembre, 1903.

- La glande interstitielle chez les vieillards, les animaux âgés et des infantiles expérimentaux. Ibid., I3 février, I904.

- Recherches sur les cellules interstitielles du testicule chez les mammifères. Arch. de Zool. exp. et gén., vol. i, 1903.

- Sur les variations dans le développement du tractus génital chez les animaux cryptorchides et leur cause. Bibl. anat., tom. xiii, fasc. 2, 1904 .

- Sur l'hypertrophie compensatrice de la glande interstitielle du testicule. Réponse à M. G. Loisel. C. r. S. B., 56, ii, p. 97, 1904.

- Sur le déterminisme des caractères sexuels sccondaires et de l'instinct sexuel. Ibid., 56, ii, p. 335, I904.

- La glande interstitielle a seul, dans le testicule, une action générale sur l'organisme. Démonstration expérimentale. C. r. A., janvier, 1904 .

- Recherches sur la signification physiologique de la glande interstitielle du testicule chez les mammifères. J. d. P. P., vi, 1904 .

- La glande interstitielle du testicule et la défense de l'organisme. Hypertrophie ou Atrophie partielle de la glande interst. au cours de certaines maladies chez l'homme. C. r. S. B., 57, i, p. 553, 1905.

- Hypertrophie ou atrophie partielle de la glande interst. dans certains conditions expérimentales. Ibid., p. 554.

- A propos du trophospongium et des canules du suc. Ibid., 57, ii, p. 22 I, 1905 .

- Différenciation d'une membrane propre d'origine épithéliale. C. r. S. B., 65 , 1908.

- Sur le follicule de de Graaf mur et la formation du corps jaune chez la chienne. Ibid., p. 314.

- Sur les homologies et la signification des glandes à sécrétion interne de l'ovaire. Ibid., 67, pp. 464 and 497,1909 .

Bouin, P., ANCEL, P., and Villemin. Sur la physiologie du corps jaune de l'ovaire. Recherches faites à l'aide des rayons X. C. r. S. B., 58, ii, p. $417,1906$.

- Glande interstitielle de l'ovaire et rayons X. Ibid., p. 337, 1907. 
Boullenger. De l'action de la glande thyroïde sur la croissance. Thèse de Paris, I896.

Boulud and FAyol. Sur le dosage colorimétrique d'adrénaline. C. r. S. B.; 55 , p. 358,1903 .

BOURNEvilLe. Comparaisons entre les enfants norm. et anormaux au p. d. vue d. l. persistance ou l'absence du thymus. Progr. méd., 1900.

- Idiotie myxcedémateuse ou myxoedème infantile. Traité de méd. de Brouarde et Gilbert, ix, 1902.

BOverI; P. Über die Wirkung des Jods auf das durch Adrenalin erzeugte Atherom der Aorta. D. m. W., I 906 .

- Artériosclérose expérimentale chez le singe. C. r. S. B., 65, p. 597, 12 décembre, 1908 .

BOYCE and BEADLES. Enlargement of the hypophysis cerebri in myxœedema, \&c. J. o. P. B., October, I $S_{92}$, and February, I 893 .

BOY-TESSIER. L'adrénaline dans l'hypotension cardio-vasculaire. C. r. S. B., $57, \mathrm{i}$, p. 880,1905 .

- Durée de l'action de l'adrénaline. Ibid., p. I097.

Bozzi. Unters. über die Schilddrüse, \&c. Zieglers Beitr., I8, 1895.

Bоzzoto. Rapport sur l'opothérapie. Congr. méd. de Turin, 1898 .

BRA, M. La méthode Brown-Séquard, Traité d'histothérapie. Paris, 1895.

BRACCI, C. Timo e ricambio del calcio. Rivist. di Clinica pediatrica, iii, p. 572,1905 .

- L'échange du calcium et le calcium des os dans la Thyroïdectomie expérimentale. Gaz. des malad. infant. et d'obstétrique, septembre, 1905.

BRADFORD, R. J. The results following partial nephrectomy and the influence of the kidney on metabolism. J. o. P., 23, p. 414, 1899.

v. BRAMANN. Ubber Schilddrüsenimplantation bei Myxödem u. Kretinismus. D. m. W., 1909 .

BRAMWELl, BYROM. Acromegaly in a giantess. Brit. Med. Journ., p. 21, 1894 .

- A case of tetania treated by thyroid extract. Ibid., p. 1196, 1895.

- Two clinical lectures on Addison's disease. Ibid., January, 1897.

- Anæmia and some diseases of blood-forming organs and ductless glands. Edinburgh, 1899.

- A case of Addison's disease in which great improvement took place under open-air treatment and the administration of suprarenal extract. Brit. Med. Journ., October 28, 1905.

BRANCA, A. Le testicule chez l'axolotl, en captivité. C. r. S. B., 56, p. 243.

- Cellules interstitielles et spermatogenèse. Ibid., 350 .

BRANDT, ALEx. Anatomisches und Allgemeines über die sogenannte Hahnenfedrigkeit und über anderweitige Geschlechtsanomalien bei Vögeln. Z. w. Z., 48, p. 101, 1889.

- Uber den Zusammenhang der Glandula suprarenalis mit dem Parovarium resp. der Epididymis bei Hühnern. Biolog. Centbl., 9, r890.

BRANHAM. Tetany following thyroidectomy, \&c. Ann. of Surg., August, 1908.

BRat, H. Zur Wirkung des Chlorbaryums und Barutins. B. k. W., 1905.

BRAUER, A. Entwickelung der Excretionsorgane der Gymnophoinen. Z. A., 23, 1900.

- Beiträge zur Kenntnis der Entwickelung und Anatomie der Gymnophionen. III. Die Entwickelung der Excretionsorgane. Zool. Jahrb., xvi, pp. 1-176, 1902.

BRAUER. Beitrag zur Lehre von den anatomischen Veränderungen des Nervensystems bei Morbus Addisonii. D. Z. N., 7, pp. 54 and 415, 1895 .

Brault, A. Maladie du rein et des capsules surrénales. Traité de méd., v, Paris, 1893 .

Brault, A., and PERuchet, E. Maladie d'Addison. S. m., i 892.

BRAUN, H. Über den Einflusz der Vitalität der Gewebe auf die örtlichen und allgemeinen Giftwirkungen lokalanästhesierender Mittel und über die Bedeutung des Adrenalins für die Lokalanästhesie. A. k. Ch., 69, p. 541, 1903; and C. Ch., 1903 .

- Cocain und Adrenalin. Berl. Klinik. Heft 187, Januar, 1904

- Über einige neue örtliche Anästhetica (Stovain, Alypin, Novocain). D. m. W., 1905 .

- Die Lokalanästhesie. Leipzig, 1905 and 2. Aufl., 1907. 
BRaun, H. Zur Freilegung der zentralen Teile usw. Z. Ch., 87.

BRAUN, L. Zur Frage der Arteriosklerose nach Adrenalinzufuhr. W. k. W., 1905.

- Uber Adrenalinarteriosklerose. S. W. A., I16, 1907.

- Zur Pathogenese und Behandlung der Arteriosklerose. M. K., 1908.

Braun, M. Das Urogenitalsystem der einheimischen Reptilien, entwicklungsgeschichtlich und anatomisch bearbeitet. Arb. a. d. zool.-zoot. Inst., Würzburg, iv, p. I 13,1878 .

- Bau und Entwicklung der Nebennieren bei Reptilien. Ibid., v, p. I, 1882 .

- Über Bau und Entwicklung der Nebennieren bei Reptilien. Z. A., 1879 .

BRÉARD. Contr. à l'étude de myxœedème spontané et son traitement. Thèse, Paris, 1899.

BREGMANN. Zur Klinik der Akromegalie. D. Z. N., I7.

BREgManN and SteInhaus. Zur Kenntnis der Geschwülste der Hypophyse und der Hypophysengegend. V. A., 188, p. 360, 1907.

- Deux cas de tumeurs de l'hypophyse. J. de Neur. de Bruxelles, 1907.

BREISACHER, L. Unters. über die gland. thyr. A. A. Suppl., p. 509, I890.

- The clinical application of some thyroid gland experiments. J. A. M. A., 1903 .

BREMER. An improved method of diagnosing diabetes from a drop of blood. New York Med. Journ., March 7, 1896.

BRÉTON. La syndrome infantilisme, sa nature dysthyroïdienne. Thèse de Lille, 1901-02.

Breton, A. Note sur l'adrénaline. Gâz. des hôp., p. 761, 1903.

BRETON and MichaUt. Deux cas d'acromégalie. Ibid., p. 142, Igoo.

BRever, R. Beitr. zur Ätiologie der Basedowschen Krankheit und des Thyreoidismus. W. k. W., 1900.

Brever and v. SeIller. Über den Einflusz der Kastration auf den Blutbefund weiblicher Tiere. A. P. P., 5o.

BREUSS and KOLISKO. Die pathologischen Beckenformen. Wien, Igoo.

BRIAN, O. Über eine aus Knochenmark bestehende Geschwulst zwischen Niere und Nebenniere. V. A., 186, p. 258, 1906.

BRIAU. L'innervation du corps thyroïde. Thèse de Lyon, 1897.

- De l'influence d. 1. castration test. et ovar. sur le développ. du squelette. Gaz. hebd., I 90 I.

BRIGIDI, V. Delle capsule suprarenali accessorie. Lo Sperimentale, p. 581, 1882 .

BRIN. De l'évolution des tumeurs propres à la capsule surrénale. Thèse de Paris, 1892.

BrinckManN. Über Therapie bei M. Basedowii. Inaug.-Diss., München, 1905.

BRISSaUd. De l'infantilisme myxœdémateux. Nouv. Icon. de la Salp., 1807.

- Myxœdème thyroïdien et myxœdème parathyroidien. P. m., I898.

- Leçons clin. sur les maladies nerveuses. 2e série, p. 440, I899.

- L'Infantilisme vrai. Nouv. Icon. de la Salp., I907.

BRISSAUD and MEIGE. Gigantisme. Rev. neurol., p. $110 \mathrm{I}, 1904$.

- Type infantil du gigantisme. Nouv. Icon. de la Salp., I904.

BRODIE. An experiment upon the glomerula function of the kidney. C. P., p. 492,1901 .

BRODIE and DixON. Contributions of the physiology of the lungs and some observations on the action of suprarenal extract. J. o. P., 30, p. 476, I904.

BROOKS, H. and KAPLAN, D. M. The effect of prolonged adrenalin medication on the human circulatory organs with report of case. Med. Rec., New York, p. 708, 1908.

Brotz. Nebennieren und Geschlechtscharakter. A. G., 88, H. 3.

BROWN-SÉQUARD. Recherches expérimentales sur la physiologie et la pathologie des capsules surrénales. C. r. A., T. xliii, p. 422,25 août, 1856 .

- Recherches expérimentales sur la physiologie des capsules surrénales. Ibid., t. xliii, p. 542, 8 septembre, I ${ }_{5} 56$; Moniteur des hôpitaux, Paris, i8 56 .

- Recherches expérimentales sur la physiologie et pathologie des capsules surrénales. A. gén. de méd., pp. 385-401, 572-598, 1856 . 
BROWN-SÉQUARD. Nouvelles recherches sur les capsules surrénales. C. r. A., xlv, 9 fevrier, 1857 .

- Nouvelles recherches sur l'importance, des fonctions des capsules surrénales. Ibid., t. xlv, p. 1036, 21 décembre, 1857; Journ. de phys., i, 1858 .

- Des effets produits chez l'homme par des injections souscoutanées d'un liquide retiré des testicules frais de cobaye et de chien. C. r. S. B., pp. 415, 420, 430 and $45 \mathrm{I}$, I 889 .

- Expérience démontrant la puissance dynamogenique chez l'homme d'un liquide extrait de testicules d'animaux. A. d. P., p. 651, 1889.

- Exposé de faits nouveaux à l'égard de l'influence sur les contres nerveux d'un liquide extrait de testicules animaux. A. d. P., pp. 201 and 443, 1890 .

- Remarques sur les effets produits sur la femme par des injections souscoutanées d'un liquide retiré d'ovaire d'animaux. Ibid., pp. 456 and $65 \mathrm{I}, \mathrm{i} 890$.

- Exposé de faites nouveaux montrant la puissance du liquide testiculaire contre l'affaiblissement du certaines maladies et en particulier la tuberculose pulmonaire. Ibid., p. 224, $189 \mathrm{r}$.

- Remarques sur la spermine et liquide testiculaire. Ibid., p. 40I, I89r.

- Faits montrant l'influence du systeme nerveux sur la nutrition et secretions. Ibid., p. 747, I $\$ 91$.

- Injections de liquide extrait de la gl. thyroïde dans des cas de myxœdeme. Ibid., pp. 178 and 752 , I 892 .

- Liquide testiculaire. Ibid., pp. 151, 406 and 754, 1892.

- Progrès de nos connaiss. à l'égard de liquide testiculaire. Ibid., pp. 205 and 796,1893 .

- Importance de la secretion interne des reins. Ibid., p. 778, I893.

- Influence de l'extrait aqueux des capsules surrénales sur des cobayes. presque mourants à la suite de l'ablation de ces organs. C. r. S. B., p. 4 Io, mai, 1892.

- Influence heureuse de la transfusion du sang normal aprés l'exstirpation des capsules surrénales chez le cobaye. Ibid., p. 467, 14 mai, 1893.

BROWN-SÉQUARD and D'ARSONVAL. Recherches sur les extraits liquides retirés des glandes et d'autres parties de l'organisme. A. d. P., p. 49I, I 89 I.

- Injection dans le sang des extraits du pancréas du foie, du cerveau. Ibid., 1892.

- Nouvelles remarqucs sur les injections souscutanées et intraveneuses d'extraits organiques. Ibid., p. 200, r 893 .

- Influence physiolog. et therap. du liquide orchitique sur l'organisme. Ibid., p. 539 , i 893 .

Brown, O. H., and GUTHRIE, C. C. The effects of intravenous injections of bone marrow extract upon blood-pressure. A. J. P., 14, p. 328, I905.

BROWN and JOSEPH. The effects of intravenous injection of extract of the bone marrow of swine on the blood-pressure in dogs. Ibid., 16, p. I 10, 1906.

BRÜCHaxow, N. Zur Kenntnis der primären Nebennierengeschwülste. Z. H., 20,1898 .

BRUCKNer, J. Sur l'absence de l'adrénaline dans le sang des chiens thyroïdectomisés. C. r. S. B., 64, pp. $1123-24$, 1908.

- Sur la sécrétion thyroïdienne. Ibid., 66, p. $4 \delta$ г, 1909.

BRUCKNER, J., and JoNNESCO, V. Sur la résistance globulaire après thyréoïdectomie. Ibid., 64, 1908.

BruckNer, J., and Giascu, A. A. Disparition de la graisse des capsules. surrénales après fistule pancréatique chez le chien. C. r. S. B., 65, p. 697 , I909.

BRugsch, Th. Der Einflusz des Pankreassaftes und der Galle auf die Darmverdauung. Z. k. M., 58, p. 518 .

BRUgSCH, Th. and BAMBERG, $K$.' Zur Frage der Azidosis beim Pankreasdiabetes des Hundes. Centb. f. Phys. u. Path. d. Stoffw., 3, 1908.

Bruhs, J. Ein Fall von Addisonscher Krankheit. Diss., Kiel, 1869 .

BRUN, VItTorio. Azione dell' estratto di ghiandole' paratiroidi contro gli effeti di veleni convulsivante. Lo Pediastrio, No. 6, I908.

Brunet, L. Etat mental des acromégaliques. Thèse de Paris, i\$99. 
BRUnN, A. v. Ein Beitrag zur Kenntnis des feineren Baues und der Entwicklungsgeschichte der Nebennieren. A. m. A., 8, pp. 618-638, 1872 .

- Über das Vorkommen organischer Muskelfasern in den Nebennieren. Nachr. d. K. Ges. d. Wiss. Göttingen, pp. 421-422, 1873.

- Verdauungsorgane. Ergebnisse der Anat. u. Entw., 4, I894-5.

BRUNNER, $H$. Zur Chemie der Lecithine und des Brenzkatechins, Bestandteile der Nebennieren. Schweiz. Woch. f. Chem. u. Pharm., I 892.

BRunver, K. Experimenta nova circa Pancreas accedit diatriba di lymphae et pancreatis usu. 1863 .

Bruno, J. Über Morbus Addisonii. M. m. W., 1902.

BRUNs, P. Zur Frage der Entkropfungs-Kachexie. Bruns Beitr., 3, I 888

- Über den gegenwärtigen Stand der Kropfbehandlung. Volkmanns S., No. 76,1884 .

- Weitere Erfahrungen und die Kropfbehandlung mit Schilddrüsenfütterung. Beitr. K. Chir., 13, 1895 and 16, 1896.

BubNow. Beitr. z. Unters d. chemischen Bestandteile d. Schilddruise. Z. ph. Ch., 8.

BuchSTAB. Arbeit der Bauchspeicheldrüse nach Durchschneidung der Splanchnici und Vagi. Diss. St. Petersburg, Ref. B. C., I.

Bucura, K. J. Nachweis von chromaffinem Gewebe und wirklichen Ganglienzellen im Ovar. W. k. W., 1907.

- Über die Nerven in der Nabelschnur und in der Placenta. Z. H., 1.907 .

- Beiträge zur inneren Funktion des weiblichen Genitales. Z. H., 28, 1907.

- Zur Therapie der klimakterischen Störungen und der Dyspareunie. M. m. W., 1909 .

Buday, K. Beitr. z. Cystenbildung in suprarenalen Nierengeschwülsten. Zeigl. B., 24, 1898 .

BUDAY and JANCSó. Ein Fall von pathologischem Riesenwuchs. D. A. k. M., 60,1808 .

BÜNZ, R. Über das Vorkommen von Cholesterinestern im Gehirn. Z. ph. Ch., 46, 1905 .

BÜrger. Stoffwechsel d. gesunden Menschen b. Schilddrüsenfütterung. Diss., Halle, 1895 .

BÜRger and CHURChMAN. Der Plexus coeliacus und mesentericus und ihre Rolle beim Abdominalshock. M. G. M. C., 16, pp. 507-549, Igo6.

Bukofzer, M. Über Adrenalin und seine Wirkung auf die Kapillaren und deren aktive Kontraktilität. Allg. med. Cent. Zeit., 1902.

- Die Reaktion der Nasen- und Kehlkopfschleimhaut auf Nebennierenextrakte (Adrenalin). D. m. W., I903.

Bulius. Osteomalazie und Eierstock. B. G. G., I, 1898.

BULKELEY, F. S. The serum treatment of exophthalmic goitre. Boston Med. and Surg. Journ., clviiii, p. 626, 1907.

Bulloch and SEqueIRA. On the relations of the suprarenal capsules to the sexual organs. Trans. of the Path. Soc., lvi, London, I905.

Bultschenka and DRINKMANN. Blutunters. nach Exstirp. d. Schilddruise. Allg. med. Cent. Z., I897.

Buquet, H. and PACHON, V. Sur l'action vasoconstrictive de la choline. C. r. S. B., 67, p. 218 , 1909.

- Additions d'effets hypotenseurs de choline et d'adrenaline. Ibid., 67, p. 274,1909 .

BURANi. Caso classico osteomalacia mascuile. Rass. d. scienc. med. Modena, ii, I 887 .

Burchard, O. Acromegalie und Myxœdem. St. Petersb. med. Woch., p. $48 \mathrm{I}, 190 \mathrm{I}$.

BURCKHARDT, G. Über die Leistungen verlagerter Pankreasstücke für die Ausnuitzung der Nahrung im Darm. A. P. P., 58, p. 251, 1908.

- Ein Beitrag zur Ovarientransplantation (Transplantation von Ovarien in den Hoden bei Kaninchen). Zeiglers Beitr., 43, H. 3.

BURCKHARDT, L. Die klinische und pathologisch-anatomische Stellung der malignen Nebennieren-Adenome der Niere. Z. Ch., 55, p. 91, 1900.

Burg. Die Nebenniere und der Morbus Addisonii. Berlin, I863.

BURGHARD and BLrMeNTAL. Über die spezifische Behandlung des Morbus Basedowii. Th. G., I903. 
BURNETT, T. C. On the production of glycosuria by the intravenous injection of seawater made isotonic with the blood. J. B. Ch., iv, p. 57, 1908 .

- The inhibiting effect of potassium in sodium chloride glycosuria. J. B. Ch., v, p. $351,1908$.

BURR. A Case of adiposis dolorosa with necropsy. Journ. of Nerv. and Ment. Dis., 1900.

BURR, Ch. and ReIzManN, D. Un cas de tumeur pituitaire sans acromégalie. Ibid., xxvi, 1893 .

BURRESI. Morbo dell Addison. Lo Sperimentale, 1880.

BURTON-OpITZ, R. Stromuhr für d. Messung der Blutvolumina der Venen. P. A., 121 .

- Über die Strömung des Blutes in dem Gebiete der Pfortader. I. Das Stromvolum der Vena mesenterica. P. A., 124, p. 460.

- A method to demonstrate the changes in the vascularity of the submaxillary gland on stimulation of the secretory nerves. J. o. P., 30, p. 133,1904 .

BURY, S. and JUDSON. A case of Addison's disease in a child treated with suprarenal extract without benefit; characteristic lesions found post mortem. Lancet, I 897 .

BUSCH and v. BERGEN. Suprarenal transplantation with preservation of function. A. J. P., 16, p. 144, 1906 .

Buschan. Kritik der modernen Theorien über die Pathogenese der Basedowschen Krankheit. W. m. W., Nos. 51, 52, 1894 and No. I, 1895.

- Die Basedow'sche Krankheit. Wien, 1894.

- Die Brown-Séquard'sche Methode (Organsaft-Therapie). Heuser, 1895.

- Myxœdem und verwandte Zustände. Leipzig, 1896.

- Schilddrüsentherapie. Eulenburg Realenc., 1896.

BUSE, $O$. Über Bau, Entwicklung und Einteilung der Nierengeschwülste. V. A., 157, i 999 .

Bussano, G. Contributo alla fisiopatologia della ipofisi. Il Tommasi, No. 23, 1908 .

Busscher. A propos d'opothérapie rénale. La belg. Méd., No. 4, 1904.

BUTLER, H. O. A practical experience with adrenalin as a cardial and vasomotor stimulant. Lancet, March 3, 1906.

CACASE. Giorn. dell' ass. Napoli. dei medici e naturalisti, 1903 zit. nach Morat u. Doyon.

CAcciola, S. Un caso di capsula surrenale accessoria aderente al rene. In : Alcune osservazioni anatomiche. Padova, 1885.

CADÉAC and GUINARD. Quelques faits relatifs aux accidents de la thyréoïdectomie. C. r. S. B., p. 468 , 1894.

- Remarques sur le rôle du thymus chez les sujets atteints d'une altération du corps thyroïde ou éthyroïdés. Ibid., p. 508, I894.

- Modific. fonctionelles relevées chez les anim. éthyroïdés. Ibid., p. 509, 1894.

CAFIERo. Sulle alteraz. istolog. ind. nei tessuti dai succhi di organi e dai sieri citotoss. Rif. med., Nos. 30 and 31 , 1903.

CAGIATI, L. Sul rapporto fra le alterazioni delle capsule surrenali ed it morbo d'Addison. Ibid., vi, 92, 1890 .

Cagnetro, G. Hypophyse et acromégalie. Arch. p. Scienze méd., 31, p. 8o, 1907.

- Anatomische Beziehungen zwischen Akromegalie und Hypophysentumoren. V. A., 176, 1904 .

- Neuer Beitrag zum Studium der Akromegalie. Ibid., 187, 1907.

CaILlaU. Notice sur les glandes surrénales suivie d'un discours prononcé sur le même sujet de Montesquieu en 1718. Ann. clin. d. 1. S. d. méd. de Montpellier, i $\$ 19$.

CALCAR, R. P. vaN. Immunitätsreaktionen und einige ihrer praktischen Verwendungen für Klinik und Laboratorium. Leipzig, IgoS.

Calderara, A. Mixedema di atrofia della tiroide con ipertrofia della ipofisi. Giorn. R. Acc. med. Torino, vol. 13, fasc. 7-8, 1907.

Callari. Gérodermie, infantilisme, feminisme. Gazz. d. osped., 1901.

CALOGERo. Nebennierenexstirp. und access. Nebennieren bei der Ratte. Thèse de Paris, I901; C. r. S. B., 1903. 
Calzolari, A. Recherches expérim. sur un rapport probable entre la fonction du thymus et celle de testicule. A. i. B., xxx, I, S. 7 r.

CAMIA. Ricerche sulla funzione del timo nella Rana. Riv. di pat. nerv. e ment. I, 3, I900.

Camrs. Sul consumo di idrati carbonio nel cuore isolato funzion. $Z$. a. P., 8, 1908.

CAMpbell, A. W. Notes of two cases of dilatation of the central cavity or ventricle of the pineal gland. Transact. of the Path. Soc. of London, 50, p. 14, 1898 .

Camus, L. Action de l'adrénaline sur l'écoulment de la lymphe. C. r. S. B. 56,1905

- Greffes parathyroïdiennes chez l'animal normal et chez l'animal partiellement éthyroïdé. Ibid., p. 439, 1905.

- La sécrétine de l'intestin du foetus. Ibid., 61, p. 59, 1906.

CAMUS and LANGlois. Sécrétion surrénale et pression sanguine. Ibid., 52, p. $210,1900$.

Canalis, P. Contribution à l'étude du développement et de la pathologie des capsules surrénales. I. M., 4, pp. 312-334, 1887.

- Contributo allo studio dello sviluppo e della patologia delle capsule soprarenali. Atti della R. Accad. delle Scienze di Torino, xxii, 8 maggio, 1887.

CanNizaro. Über die Funktion der Schilddruise. D. m. IV., I892.

Cantani. Trattato delle malattie del ricambio. Il Diabete, Napoli, i875. Deutsch von S. Hahn, Berlin, isso.

Canter, Ch. Contr. à l'étude des fonctions de la gl. thyroïde. Merc. méd., I 895 .

Canturi, A. Sul gozzo esoftalmico. Gazz. Osped. e Clin., No. 62, 1908.

CAPELle. Ein neuer Beitrag zur Basedowthymus. M. w. M., I9o8.

- Die Beziehungen der Thymus zum Morbus Basedowii. Beitr. kl. Chir., 58.

Capobianco, F. La pneumonite da tiroidectomia e quella da recisione del vago nei conigli. Rif. med., p. 166, 1892 .

- Sulle fine alterazioni dei centri nervosi e delle radici spinali dopo la tiroidectomia. Rif. med., 1892; A. i. B., 18, p. 306, 1893 .

- La tiroidectomia nei mammiferi. Congr. med. int. Roma, 1894; Rif. med., 1895 .

- Ricerche microsc. e sperim. su gli effetti della tiroidectoma. I. M., II, 1894.

- Sur les effets de la thyroïdectomie chez les animaux. A. i. B., 22, I895.

- Reperto rarissimo e presenza di fibre muscolare striate nelle glandola tiroide. Rif. med., Ann. IX, vol. i, No. 73; Boll. di soc. di naturalisti in Napoli.

- Dell' azione di alcuni estratti organici sul lavoro muscolare. Atti della R. Acc. d. Sc. Fis. e Matem. Napoli, 11 giugno, rgo4.

Capobianco, F., and Mazziotti, L. Su gli effetti della Paratiroidectomia. Giorn. intern. delle sc. med., 1897 and A. i. B., 31, IS99.

CaPolonigo. Sulla penetrazione per diffusione dell' adrenalina nella camera anteriore. XVII. Ophthalmologenkongresz. Annalen der Ophthalmologie, 1905-6: Bph. C., ii, p. I19.

Capparell. Zur Frage des experimentellen Pankreasdiabetes. Biol. Cbl., $495,1893$.

Carbone, T. Das Neurin und die Nebennieren. Congr. int. med., Rom, 1894 ; C. a. P., 5,1894 .

- Esperience sull' estirpazione della ghiandola timo. Giorn. della real. Accad. di Torino, ix, 7, 1897.

CARle, A. Über die Exstirpation der Schilddrüse. C. P., p. 213, 1888.

CARLES, J., and Michel. Du pouvoir néphrotoxique de la macération rénale administré par ingestion. C. r. S. B., 5S, p. $276,1905$.

CARlier. Note on the structure of the suprarenal body. An. An., 8, 1893.

CARMICHAEL and MARSHALL. The correlation of the ovarian and uterine functions. Brit. Med. Journ., 1907.

- Compensatory hypertrophy in the ovary. J. o. P., 36, 1908.

CARNor. Diabète après injection de culture bactérique dans le conduit pancréatique. C. r. S. B., I 894.

Carnot and AMET. De la dégénérescence des îlots de Langerhans en dehors du diabète. C. r. S. B., 57, ii, p. 359, 1905 . 
CarNot and Delion. Parathyroidie tuberculeuse. Ibid., 57, ii, p. 321, 1905 .

CARNot and GILBERT. Action d'extrait hepatique sur la glycosurie exp. Ibid., 1806.

CARNot and Josserand. Sur la valeur hémostatique de l'adrénaline. Ibid., 54, p. 1346 .

- Des différences d'action de l'adrénaline sur la pression sanguine suivant les vois de pénétration. Ibid., 54, p. 1472, 1903.

- Influence du travail musculaire sur l'activité de l'adrénaline. Ibid., 55, p. 51,1903

CARO. Schilddrüsensekretionen und Schwangerschaft in ihren Beziehungen zu Tetanie und Nephritis. M. G. M. C., I7, I903.

- Wechselwirkung der Organe mit innerer Sekretion. M. K., 19ı.

CARPENTER, MACCARTY. Fall v. malignem Hypernephrom bei einem Kinde. B. k. WV., 1905 .

CARRACIDO, R. J. Un dato quimico para la explicación de la glucosuría pancreática. Revista ibero-americ. de ciencias médicas. T., xi, p. 294, Madr., 1904.

Carraro, A. Studio comparativo sugli effetti delle inizioni di estratto d'ipofisi e di ghiandola surrenale. Arch. sc. med. Torino, 32, pp. $42-80$, I908; and A. i. B., 32 .

CARRARO and KUSCHNITZKY. Regeneration der Nebenniere. B. k. W., Igog.

CARRel, A., and Guthrie, C. C. Exstirpation et Replantation de la glande thyroïde avec reversion de la circulation. C. r. S. B., 57, ii, p. 413, 1905 .

CARRIÉRE, G. Structure et fonctions du corps pituitaire. Arch. clin. d. Bordeaux, I 893 .

Carriére, N. Des dangers de la médication thyrö̈dienne. Nord. med., 1901 .

Carrière, G., and Deléarde, A. Sur un cas d'épithélioma atypique symétrique des capsules surrénales. A. m. e., 12, 1900.

CARRIERe and VANVERTS. Etude expérim. sur l'action de la thyroïdine dans la consolidation des fractures. C. r. S. B., p. 535 , 1900.

CASELli, A. Studii anatomici e sperimentali sulla fisiopatologia della glandola pituitaria. Reggio Emilia, igoo.

- Influence de la fonction de l'bypophyse sur le développement de l'organisme. Riv. sper. di fren., 37, I900.

- Hypophyse et glycosurie. Ibid., 38, 1900.

- Sui rapporti funzionali della glandola pituitaria coll'appareggio tiroparatiroideo. Ibid., p. 468, 1900.

CASPER, M. Stoffwechselregulierungsorgane bei Tieren. L. O., i, p. 522, 1896.

Castaigne, J. De l'élimination du pigment noir dans la maladie bronzée. Bull. de la Soc. anat., xi, 13, p. 510 , juin-juillet, 1897 .

CASTEIGNE, J., and RATHERY, F. Ligature unilatériale de l'artère rénale. C. r. S. B., 1901 .

- Sur les néphrotoxines. Ibid., 17 mai, 1902.

- Toxicité de la subst. rénale et néphrotoxines. P. m., I3 août, I903.

- Action exercée in vitro sur l'épithel. renal par les sérums. A. m. e., I 903.

- Néphrites primit. unlat. et lésions conséc. de l'autre rein. Ibid., 14, I 902.

DU Castel, J. Thyroïde et formule leucocytaire. C. r. S. B., 6j, pp. 443444,1908 .

CASTIGLIONi, G. Un nouveau cas d'acromégalie emélioré par l'opothérapie hypophysaire. Gaz. med Italiana, March 23, 1905.

Caton and Paul. Notes of a case of acromegaly, \&c. Brit. Med. Journ., 1893.

Caussade, G. Consid. sur la pathogénie de la maladie d'Addison. Union méd., I 895 .

- Sur les effets de l'injection souscoutanée d'extracts de capsules surrénales chez les animaux. C. r. S. B., is janvier, 1896 .

CAUSSADE and LANLery, C. Sarcome de la glande pituitaire sans acromégalie. A. m. e., I gog.

CAVAZZANi, E. Le funzioni del pancreas ed i loro rapporti colla patogenesi del diabete. I 892 , Venezia. 
Cavazzani, E. Zur Physiologie des Duodenums. C. P., p. 370, 1908.

- I nuovi dibattiti sul diabete pancreatico. Arch. di Farmacol., vii, 1908.

- Il latte atiroideo nella cura del gozzo esoftalmico. Riv. crit. di clin. med., ix, 1908 .

Cecca, R. Ovar und Nebenniere. Soc. méd. chirurg. de Boulogne, mars,

CECCA, R., and ZAPPI, F. Le ghiandole a secrezione interna dal punto di vista chirurgico. Boll. d. scienze med. di Bologna, Serie VIII, vol. iv, H. 3 .

CECIL, R. L. A study of the pathological anatomy of the pancreas in ninety cases of diabetes mellitus. J. e. M., xi, p. 200, March, 1909.

CECONI, A., and MICHELI. Intorno alla questione delle nefrolisine. Il. Morgagni, No. 4, 1904.

Ceconi, A., and ROBECHI, P. Cytotoxina ovarica. Rif. med., iii, Nos. 65, $66,1902$.

CENI and BESTA. Proprietà terapeutiche specifiche del siero di sangue di animali immunizzati con siero di animali stiroparatiroidiati. Riv. Sper. di Freniatria, 1903.

Centanni, E. La citoprecipitina e il suo valore diagnostica. Rif. med., $1901,1902$.

- Neurotoxin. Rif. med., I900; C. f. B., p. 988, 1901.

- Über die Autozytopräzipitine. Ibid., pp. 91, 239, 362, I903.

- Über Autozytopräzipitine. 2 Abteilung. Ibid., H. 5 and 6, 1907.

- Sugli edemi nefritici. Pathologica, No. I, 1908.

CERLETTI. Effets des injections de suc d'hypophyse sur l'accroissement somatique. A. i. B., 47, p. 123, 1907.

Cerletti and Perusini. Journ. of Mental Pathology, vii, No. 5 , 1906.

CERTENET DE DEMAY. Traitement des hémorrhoides par l'adrénaline. Journ. de Méd de. Bordeaux, No. 20, 1904.

Cervello, $\dot{V}$. Notices préliminaires sur l'action de la neurine. A. i. B., 5, p. 199,1884 .

CESA, D. and BIANCHI. Osservazioni sulla struttura e sulla funzione della cosidetta ghiandola interstiziale dell' ovaia. A. d. F., iv, H. 6.

CESARI, L. La choline dans le liquide céphalo-spinal du chien soumis à l'épilepsie expérimentale. C. r. S. B., p. 66, 1907.

Cesaris, DEMEL. Adenocarcinoma del pancreas. Arch. per le Scienze mediche, 1895 .

Cestan and Halberstadt. Epithelioma kystique de l'hypophyse. Rev. neurol., p. $1180,1903$.

Cevidalli, A. Di alcune reazioni dell' adrenalina. Lo Sperimentale, 62, 1908.

Cevidali, A. and Leoncini, F. Ricerche sul comportamento post-mortale del principio attivo delle capsule surrenali. Lo Sperimentale, p. 175, 1909 .

- La docimasia surrenale nella diagnosi medico-forense della morte in compendio. Ibid., p. 733, settémbre-ottóbre, I909.

ChampNeys, F. H. A note on the history of the toxæmia of pregnancy. Journ. of Obstet. and Gynæe., August, 19oS.

Champy, СH. Note sur les cellules interstitielles du lesticule chez les batraciens anoures. C. r. S. B., 64, p. $893,1908$.

CHANDLER, G. Hypernephroma as a result of traumatism. N. Y. Med. Journ., 1904.

ChaNTEMESSE and MARIE. Les glandes parathyroïdiennes de l'homme. Soc. méd. Hôp., 10, p. 202, 1893 .

Charpentier, A.' Ecrans testiculaires ayant pour base l'extrait de glande interstitielle. C. r. S. B., 56, p. 828 .

Charrin, A. Sur les élévations thermiques d'origine cellulaire. A. d. P. 21, p. 683,1889 .

- Les toxines; mécanisme de leur action. Rev. gén. des scienc., pp. 24-32, 15 janvier, 1895 .

- Les fonctions des capsules surrénales en physiologie pathologique. S. m., 1896 .

- Les défenses naturelles de l'organisme. Paris, 1898 .

-- Les poisons de l'organisme. 2 Bände, Encycl. scient., Paris. 
Charrin and Langlois. Lésions des caps. surrén. dans l'infection, C. r. S. B., p. 812,1893 .

- Action antitoxique du tissu des capsules surrénales. C. r. S. B., p. 4 10, 1894 .

- Hypertrophie expérimentales des capsules surrénales. C. r. S. B., p. 13I, I 896.

- Du rôle ces caps. surrén. dans la resistance à certaines infections. Ibid., 1896 .

Charrin and Levaditi. Action d. pancréas sur la toxine diphthérique. Ibid., 51,1899 .

Chassevant and Langlois. Des gaz du sang efférent des capsules surrénales. C. r. S. B., p. 700 , juillet, 1893 .

Chatelain. De la peau bronzée ou maladie d'Addison. Thèse Strassbourg, 1859; Ref. Canst. Jahresb., iv, p. 28r, 186r.

Chatin and GuiNard. De la sécrétion interne du rein. A. m. e., p. 137, 1900 .

Chauffard. Maladies du foie, etc. Traité de Méd. Paris, i, iii. I892.

- L'intoxication addisonienne. Sem. Méd., p. 74, 1894 .

Chauveau and Kaufmann. Le pancréas et les centres nerveux. C. r. A., p. 463, i 893 .

- Pathogénie du diabète. Ibid., 226, 1893.

CHÉNU and MOREL. Localisation de l'iode dans les glandules parathyroïdes externes. C. r. S. B., p. 680 , 1904 .

Chesseau. Tuberculose des capsules surrénales et insuffisance capsulaire. Paris, 1900.

Chevalier, J. L'adrénaline. Bull. de thérap., p. 856, I5 juin, I903.

- Sur la préparation et le tirage des produits opothérapiques. Ibid., 1908 .

Chevassu. Tumeurs de testicule. Paris, rgo6.

Chevrel, R. Sur l'anatomie du système nerveux grand sympathique des élasmobranches et des poisson osseux. Thèse Paris, Arch. zool. exp. et gén., v, Suppl. p. I, 1889.

- Recherches anatomiques sur le système nerveux grand sympathique de l'esturgeon. C. r. A., II7, p. 441, 1893; Arch. de zool. exp. et gén. sér., 3, i, ii, 1894.

CHIAJE, S. DELLE. Uber ein Zytotoxin, welches die Fettentartung des Eierstocks hervorruft. C. G., p. 705, 1908.

ChIARI, H. Zur Kenntnis der akzessorischen Nebennieren d. Menschen. Z. H., 5. 1884 .

Chiarcgi. Di un organo epiteliale, \&c. Mon. zool. Ital., 9, 1898.

Chidichimo, F. Azione dell' adrenalina sui muscoli lisci (utero, stomaco ed intestino). La Ginecol., ibid., I906; Ref. B. C., v, p. 297.

Chiene, G. L. Observations on the use of Eucain-B and Adrenalin as a means of inducing local anæsthesia. Scott. Med. Journ., September, 1904.

CHIRIÉ, J. L. Les capsules surrénales dans l'éclampsia puerpérale et la néphrite gravidique. C. r. S. B., 64, p. 799, 9 Mai, 1908; S. m., 1908.

Снолу, E. Influence du mode de préparation sur l'activité des extraits opothérapeutiques. Bull. gén. de Thérap., August, Igo8.

Chrétien, H. De la thyroïdectomie. Thèse, Paris, 1887.

CHRISTENS. Insuffisance parathyroïdienne chez la chèvre. C. r. S. B., 57, p. 337,1905 .

Christomanos. Über die Zahl der roten Blutkörperchen in zwei Fällen von Nebennierenerkrankung. B. k. WV., I 899 .

СнRовaK, R. Über Einverleibung von Eierstockgeweben. C. G., 20, 1896.

CHROBAK, R., and RosthoRN, A. v. Die Erkrankungen der weibl. Geschlechtsorgane. Nothnagels spec. Path. u. Ther., Bd. xx, II. Miszbildungen. Wien, 1908 .

Churtos. On the effects of total and of partial destruction of the suprarenal bodies. Lancet, i, p. 245, I886.

Chyostek, F., SEN. Suppurative Entzündung der linken Nebenniere. W. m. P., 1880 .

Chvostek, F., Jun. Störungen der Nebennierenfunktion. L. O., 3, 1896.

- Pathologische Anatomie der Nebennieren. Ibid., 9, 2. Abt. 1903.

- Bemerkungen zur Ätiologie der Tetanie. W. k. W., I905. 
Cirvostek, F., Jun. Beiträge zur Lehre von der Tetanie. Ibid., I907.

- My̆asthenia gravis und Epithelkörperchen. Ibid., I908.

- Die menstruelle Leberhyperämie. Ibid., Igog.

- Diagnose und Therapie der Tetanie. D. m. IV., Igog.

Criccio, C. Sopra i canaliculi di secrezione nelle capsule sopraren. An. An., 22, 1903 .

- Sopra una nova specie di cellule nelle capsule surrenali degli anuri. Ibid., 23, 1903.

- Ric. sui processi di secrezione cellul. nelle capsule surrenali. Ibid., 23, 1903 .

- Sui caratteri citologici e microchimici delle cellule cromaffini. Ibid., 24, 1903 .

- Sui processi secretorii della corteccia surrenale. Ibid., 28, 1906.

- Sur la fine structure et sur les fonctions des capsules surrénales des vertébrés. A. i. B., 43, p. 17, 1905.

- Rapporti istogenetici tra il simpatico e le cellule cromaffini. Arch. ital. di anat., vol. v, F. 2, pp. 256-267, 1906.

- Sur la sécrétion de la couche médullaire de la surrénale. C. r. S. B., I906.

- Sur la topographie de l'adrénaline. Ibid., pp. 333, 334, 1906.

- Sur l'entérokinase. Ibid., p. 676, 1906.

- Über das Vorkommen von Lezithin, \&c. C. a. P., 20, 1909.

- Contributo alla conoscenza dei lipoidi cellulari. Án. An., 35, rgog.

Cianni, A. Azione dell' adrenalina nel morbo maculoso di Werlhofi. Rif. med., 1906.

CIMORONI, A. Sull' ipertrofia dell' ipofisi cerebrale negli animali stiroidati. Lo Sperim., 61, H. I, 1907 ; and A. i. B., 48, H. I3, I908.

Cioffi, E. Contributo alla patologia e terapia dell' uremia. XII. Kongr. int. Med. in Rom, novémbre, 1902. Ref. B. C. i, p. 94, 1903.

- Contributo sperimentale alla patogenesi e cura dell' uremia. G. i. d. s. med., roo3.

- Sulla pretesa specificite delle nefrolisine. Clin. med. ital., 43, No. 5, I904.

- Smidollamente delle capsule surrenali in due tempi. Clin. med. ital., H. 6, 1905; Bph. C., i, p. 270 ; Gazz. d. Osp., anno 26, No. 4.

Crtron, J. Über die durch Suprarenin erzeugten Veränderungen. ' Z. e. P., I 905 .

Ciuffo, G. Azione antitossica sull' adrenalina del siero di animali scapsulati. Soc. fra i cultori delle sc. med. e. nat. Cagliari, June i8, I905; Ref. B. C., iv, p. 752.

Civalleri, S. Glandula paratirodea dell' uomo. Policlinico, No. 3, 1902.

- L'hypophyse pharyngienne de l'homme. Anat. Kongr., Marseille, I9o8.

Clairmont, P. Über ein Hyperneplirom-Impfrezidiv in den Broncliallymphdrüsen. A. k. Ch., 73, I904.

Claude, H. Acromégalie sans gigantisme. L’Encéphale, ann. ii, No. 3, pp. $295-298$, mars, 1907.

- Syndromes d'hyperfonctionnement des glandes vasculaires sanguines chez les acromégalique. C. r. S. B., 57, ii, p. 362 , I905.

Claude and Blanchetiere. La choline dans le sang. J. d. P. P., pp. 86IOI, 1907.

Claude, H., and Gougerot, H. Sur l'insuffisance simultanée de plusieurs glands à sécrétion interne (insuffisance pluriglandulaire). C. r. S. B., p. 785, I907.

- Les syndromes d'insuffisance pluriglandulaire, leur place en nosographie. Rev. de méd., No. 10, p. 86r ff.; No. I I, p. 950 ff., rgo8.

- Insuffisance pluriglandulaire endocrinienne (I. mémoire). J. d. P. P., p. 468 ; ii, mémoire, ibid., p. 505, I908.

Claude, H., and Schmiergeld, A. De l'état des glandes à sécrétion interne dans l'epilepsie. C. r. S. B., 65, S. 82,1908 .

- L'appareil parathyroïden dans l'épilepsie (II. note). Ibid., p. I39, I908.

- Etude de 17 cas d'épilepsie au point de veu de l'état des glandes à sécrétion interne. Ibid., juillet, rgo8; Congrès de Dijon, août, I908.

- Les glandes à sécrétion interne dans l'épilepsie (III note). L'hypophyse, les surrénales, les ovaires. C. r. S. B., 65, p. 196, igo8.

- Les glandes à sécrétion interne chez les épileptiques. 'L'Encéphale, I9o9. 
Claude, H., and Vincent, Cl. Un cas de myasthénie bulbo-spinale se rattachant peut-être à une insuffisance surrénale. Soc. d. Neurologie, 2 juillet, 1908 .

Claus, R., and Embden, G. Pankreas und Glykolyse. H. B., vi, pp. 214 and 393 .

ClaUS and v. d. STRIChT. Contribution à l'étude anatomique et clinique de l'acromégalie. Ann. et Bull. de la soc. de méd. de Gand, 1893.

Cleghorn. The action of animal extracts, \&c., on the mammalian heart muscle. Amer. Journ. of Physiol., 2, p. 273, 1899.

Clenens, P. Zum Stoff wechsel bei Morbus Basedowii. Z. k. M., 59, 1906.

CLEMENT. Les capsules surrénales sécrètent-elles de l'acide formique? Lyon méd. Ann. 37, pp. 1342-1 343, 1905. Ref. V.-H., i, p. 16, 1905.

ClopatT, V.-H. Jahresbericht, ii, p. 508, 1900 ; Finiska Läkaresell Handlungen, 1899 .

Clunet, J. Accidents cardiaques au cours d'un causes thyroïden basedowisié. (Réaction parathyroïdienne hypophys. et surrénale.) Arch. des malad. du cœur, p. 232, 1908.

COATS, J. An adenoid sarcoma with cartilage originating in the pineal gland. Transact. of the Pathol. Society of London, xxviii, p. 44, 1887.

COE, W. S., and Kunkel, B. W. The female urogenital organs of the limbless lizard Anniella. An. An., 26, pp. 219-222, 1905.

CoEnen. Über Nebennierenverpflanzung. A. k. Ch., 81, p. 288, 1906.

Cons, F. Zur Histologie u. Histogenese d. Corp. luteum u. d. interst. Ovarialgewebes. A. m. A., 62, 1903.

- Über das Corpus luteum und den atretischen Follikel des Menschen und deren cystische Derivate. A. G., 87, p. 367, 1909.

CohnheIM, O. Die Bedeutung des Dünndarms für die Verdauung. B. C., i, p. 169, 1903.

- Physiologie der Verdauung und Aufsaugung. Nagels Handb. d. Phys., ii, p. 516 .

- Die Verbrennung d. Kohlehydrate im Organismus und ihre Beeinflussung durch d. Pankreas. Z. ph. Ch., 39, p. 336, 1903.

- Über Kohlehydratverbrennung. II. Mitt. Die aktivierende Subst. d. Pankreas. Ibid., 42, p. 40I:

- Über Kohlehydratverbrennung. III. Mitt. Ibid., 43, p. 547.

- Über Glykolyse. IV. Mitt. Ibid., 47, p. 253.

Colasanti, G., and Bellati, L. Über die Toxizität des Harns bei der Addisonschen Krankheit. M. U., 15, 1895.

- Toxicité urinaire dans la maladie d'Addison. A. i. B., xxii, p. 283, 1895.

Coleman, Warren. Tuberculosis of the adrenal bodies unaccompanied by bronzing. New York Med. Record, 46, 18, 3 November, i894.

- Adrenal substance in the intestinal hæmorrhage of typhoid fever. Med. News, 1902.

Collis. Nervous impulses controlling menstruation and uterine hæmorrhage. Am. Gyn. and Obst., 6, 1895 .

Collin and Lucies. Nouveaux documents relatifs à l'évolution pondérale du thymus chez le foetus et chez l'enfant. C. r. S. B., 57, ii, p. 716, 1905 .

Collins. Sulla origine della gl. pituitaria. Riv. sper. d. fren., isgs.

- Sulla struttura d. gl. pituitaria. Riv. pat. nerv., 7, 1903.

Collinge, W. E., and VINCENT, S. On the so-called suprarenal bodies in cyclostoma. An. An., 12, pp. 232-241, 1896.

- The suprarenal bodies of the fishes. Nat. Sc., vol. x, No. 63 , pp. 318$322,1897$.

Collini, M. Siero citotossico dell' ipofisi. Nuovo Raccloglitore medico, No. 11 , 1902.

CollmanN. Beitr. z. Kenntn. d. Chondrodystrophia fœtalis. V. A., 166, I90I.

Colman, H. A case of Addison's disease with associated Leucoderma. Lancet, 1900 .

ColzI. Sulla estirpazione della tiroide. Lo Sperimentale, I884.

Comessatti, G. Contributo allo studio chimico clinico delle sostanze ipertensive. Gazz. Osped., No. 146, 1908.

- Beitrag zur Kenntnis der drucksteigernden Substanzen. M. m. W., $190 S$. 
Comessatti, G. Un metodo semplice per il dosamento dell' adrenalina contenuto nelle capsule surrenali del cadavere. Riv. de chim. et Microscop. Clin., ix, May, 1909.

- Beitr. z. chemischen Nachweis d. Adrenalins im Blutserum. B. k. W., 1909.

- Methode zur Bestimmung des Adrenalins im Nebennierengewebe. D. m. IV., I 909 .

- Über den Wert der Froschbulbusreaktion und einige Eigenschaften des Adrenalins. A. P. P., 60, p. 233.

- Pankreasextrakt und Adrenalin. Ibid., 6o, p. 243, 1900.

Comolli, A. Intorno al tessuto di sostegno del corpo surrenale. Monitore zool. Ital., an. xviii, Nos. 5 and 6, pp. $158-16$ r, 1907.

Compe. Contribution à l'étude de l'hypophyse humaine et de ses relations avec le corps thyroïde. Thèse de Lausanne, 1898 . Ziegi. B., 23, 1898 .

CoNDER, A. F. R. On Addison's disease, with two cases, and also a case which presented the symptoms of disease of the suprarenal capsules. Edinburgh Journ., p. 275, 1905.

CoNtr, A., and CURTI, O. 'Potere ipertensivo degli estratti surrenali dell' uomo in alcune forme morbose. Gazz. degli osped. I906; Bph. C., ii, p. 113.

- Effetti fisiologici degli estratti tiroidei ed ipofisari sul circolo. Boll. scienc. med., 1906.

Cooper, A. Anatomy of the thymus gland. London, 1832.

Coppez, H. Sur l'emploi d'adrénaline en thérapeutique oculaire. Journ. méd. Bruxelles, No. 51, p. Sog, 1902.

CORIAT. The production of cholin from lecithin and brain tissues. A. J. P., December 1, 1904 .

Corning. Über Entwicklungsvorgänge am Kopfe d. Anuren. Morph. Jahrb., 27, 1897.

Corona and Moroni. Contributo allo studio dell' estratto di capsule surrenali. Rif. med., Nos. 37-39, p. 433, May 16 to 18,1898 .

Coronedr, G. Innere Sekretion. Archivio di Fisiologia. Novémbre, I904.

- Rapporto fra tiroide e reni. Ricerche comparative interno all' azione dei dieuretici e dell' estratto tiroideo sul rene del cane etiroidato. Boll. scienz. med., 8o, p. $12 \mathrm{I}$.

- Importanza biologica degli alogeni nella funzione tiroparatiroidea; contributo sperimentale e teorico. VI. Intern. Kongr. f. Physiol. Bruxelles, September, B. C., iii, I 38 I.

- Studio intorno alla fisiologia della glandula tireoidea e delle glandole paratiroidi. Estratto degli Studi Sassaresi. Anno v, ser. ii, I-2. Sassari, 1906-1907.

- L'ablazione completa dell' apparecchio tiro-partiroideo nei conigli nutriti con grassi alogenati. Atti dell' Accad. med. fis. Fiorent., 1903. Ref. B. C., i, p. 789 , I903.

CoroNedi and LUzzatTo. L'ammoniaque dans l'urine du chien thyroïdectomisé. A. i. B., 47, 1907.

Coronedi and MarchetTI. Mixœedema sperimentale. Riv. ven. di scienze med., 21 , 10.

Cosma. Einige Worte über das Adrenalin. Ref. Schmidts Jahrb., 282, p. 250,1904 .

Cosmovici. Sécrétion et excrétion. C. r. S. B., p. 607, 1907

Costa. Goitre épidémique. Thèse, Lyon, 1897 .

DA Costa, C. Cellulas chromaffinas e adrenalina. Polytechnia Lisboa, i, pp. 16 and 137,1905 .

- Notes cytologiques sur les cellules corticales des glandes surrénales. C. R. du XV. Congr. intern. de méd. à Lisbonne, 1906.

- Extr. du Bull. de la Soc. Portug. de la Sc. nat. Séance du 29 octobre, 1907. Zit. n. Haberer.

D. Costa and PEREs, C. Glandulas suprarenales e suas homologas. Lisboa, I 29 pp., 1905 .

DE CouloN. Über Thyreoidea und Hypophysis der Kretinen sowie uber Thyreoideareste bei Struma nodosa. V. A., 147, 1896-1897.

Counsell, H. E. Case of Addison's disease without pigmentation. Lancet, 3 May, 1 Sgo. 
COUvée, H. Die Ursachen des Todes nach doppelseitiger Nephrektomie. Z. k. M., 54 .

CouzIN, P. Accidents aigus de la tuberculose des capsules surrénales. Paris, I899.

CozzI, C. Le alterazioni del fegato nei cani operati di paratiroidectomia totale. Med. Ital., lviii, No. 47.

Cozzolivo, O. Intorno agli effetti dell' estirpazione del timo. La Pediatria, 1903 .

- Deformazioni dello scheletro in seguito all' ablazione del timo. Ibid., p. 620 .

- La tetania infantile secondo le recenti vedute etio-patogenetiche. La Liguria Medica, i, H. 15, 1907.

Cramer, H. Uber die Verwendung des Adrenalins in der Gynäkologie. D. m. IV., 1903 .

- Transplantation menschlicher Ovarien. M. m. W., 1906.

- Zur Physiologie der Milchsekretion. Ibid., 1909.

- Ovarium und Osteomalazie. M. m. W., 1909.

CRAMER, W. On protagon, cholin and neurin. J. o. P., 31, No. I. p. 30.

- Note on the action of pituitary extracts upon the enucleated frog's eye. Quarterly Journ. of Exp. Physiol., I, p. 189, 1908.

CREIGHTON, C. A. Points of resemblance between the suprarenal bodies of the horse and $\operatorname{dog}$ and certain occasional structures in the ovary. P. R. S., 26,1877 .

- A theory of the homology of the suprarenals. J. o. A. a. P., 13, 1878 .

Crile, G., and Dolley, D. H. An experimental research into the resuscitation of dogs killed by anæsthetics and asphyxia. J. e. M., viii, pp. 713-725, 1906.

CRILE. Experimental researches into the means of controlling the bloodpressure. J. A. M. A., I903.

Cristiani, H. Sur les glandules thyroïdiennes chez le rat. C. r. S. B., p. 798,1892 .

- Nouvelles recherches sur les organes thyroïdiennes de rongeurs. Ibid., p. 44I, I893.

- Remarques sur l'anatomie et la physiologie des glandes et glandules thyroïdiennes chez le rat. A. d. P., 1893 .

- De la thyroïdectomie chez le rat. Ibid., p. 39, 1893 .

- Des glandules thyroïdiennes accessoires chez la souris et la campagnol. Ibid., p. 279.

- Effets de la thyroïdectomie chez les lézards. C. r. S. B., 1894.

- Etude histologique de la greffe thyroïdienne. Ibid., p. 716 , 10 novembre, 1894.

- De la greffe thyroïdienne et son evolution histologique. A. d. P., I895.

- Vascularisation comparée des greffes thyroïdiennes. Rev. méd. de la Suisse romande, 1901 .

- De l'accroissement des greffes thyroïdiennes. J. d. P. P., iii, rgor.

- Transplantation de thyroïdien dans des régions transparentes. C. r. S. B., p. 679 , 1903 .

- Infection streptococcique expérimentale de greffes thyroïdiennes. Ibid., 713.

- Lésions inflammatoires microbiennes de greffes thyr., p. 725 .

- Injection de subst. bactér. necrosante et d'essence de térébenthe dans des greffes thyr. Ibid., p. 726 .

- Hypertrophie compensatrice de greffe thyr. Ibid., p. 782.

- Vitalité des tissus séparés de l'organisme. Ibid., p. 828.

- Reimplantation de greffe thyr. reussies. Ibid., p. I457.

- De la greffe thyroïdiennes chez les oiseaux. Ibid., 56, i, p. 192, 1904.

- Conservation de tissu thyroïdien vivant dans l'eau salée physiologique. Ibid., p. I91.

- De la greffe thyroïdienne chez les poissons et les amphibies. Ibid., p. 227.

- La greffe thyroïdienne chez l'homme. Sem. méd., p. 8I, igo4.

- La guérison du myxodème par la greffe thyroïdienne. Ibid., 1005.

- Dégénerescence et atrophie experimentale des greffes thyroïdiennes C. r. S. B., 57, i, p. $68,1905$.

- Evolution des greffes thyroïdiennes superflues. Ibid., 36 r. 
CRISTIANI, H. De la persistance des greffes des glandes parathyroïdes. Ibid., 57, ii, p. 754 , 1905 .

- Propriétés différentes des tissus thyroïden et parathyroïdien. Ibid., 57, ii, p. 756, 1905 .

- Evolution histologique de greffes faites avec du tissu thyroïdien conservé. J. d. P. P., T. 7, p. 26I, 1905.

Cristiani, H., and A." Recherches sur les capsules surrénales. Ibid., 4. pp. $838-847,1902$.

- De la greffe des capsules surrénales. Ibid., 4, pp. 922, 979-982, 1902.

- Rôle preponderant de la substance médullaire des capsules surrénales dans la fonction de ces glandes. C. r. S. B., 54, 1902.

- De l'insuffisance fonctionelle des greffes des capsules surrénales. Ibid., 1902.

- Dégénérescence et atrophie expérimentale des greffes thyréoïdiennes par ingestion à dose toxique des pastilles de glande thyroide. C. r. S. B., 57 , ii, 1905 .

- Evolution comparée des greffes de jeune tissu thyroïden transplantation sur des animaux d'âge différ. Ibid., 57, i, 530, 1905.

Cristiani, H., and FERRARI, E. De la nature des glandules parathyroiddiennes. C. r. S. B., 49, 1897 .

Cristiani, and MLle. Frigoff. Altération des greffes thyroïdiennes par l'emploi de la "Subcutine" comme anesthésique local. C. r. S. B., 57, p. 689 , 1905 .

CRISTIANI and KÜMNER. Über funktionelle Hypertrophie der überpflanzten Schilddrüsenstückchen beim Menschen. M. m. W., 1906.

Cristiani, H., and OUSPENSKY. Effets de la cocainisation locale sur les greffes thyroiddiennes. C. r. S. B., 56 , p. $40,1904$.

- Action des solutions de cocaine sur le tissu thyroidien vivant. Ibid., p. 42 .

Croftan. Uber das diastatische Ferment der Nebennieren. P.A., 90, 1902.

- Notiz uber eine chemische Methode, Hypernephrome (NebennierenTumoren) der Niere von anderen Nierengeschwisten zu unterscheiden. V. A., 169, 1902.

- Concerning sugar forming ferment in suprarenal extract, a preliminary report on suprarenal glycosuria. American Medicine, 1902.

Croom. Überpflanzung des Ovariums mit nachfolgender Schwangerschaft und Geburt. Wer ist die Mutter des Kindes? Edinburgh. Obst. Soc. Ref. C. G., 1907.

CRovelLI. Frühzeitige Entwicklung der Geschlechtsorgane bei einem 18 Monate alten Mädchen. Journ. méd. de Bruxelles, 1 \$ 90

Crowe, S. J., Cushing, H., and Homans, J. Effects of hypophysal transplantation following total hypophysectomy in the canine. Quart. Journ. Exp. Physiol., ii, p. 389 , r 1909 .

Cú́not, L. Les moyens de défense dans la série animale. Encycl. scient., Paris.

Culles, G. M. The Carter literature of the thyroid gland. Edinb. Med. Journ., is9I-2, p. 531 .

Cummins, W. T., and Stout, P. S. Experimental arteriosclerosis by adrenalin inoculations and the effect of potassium iodide. Univ. of Pennsylvania. Med. Bull., xix, pp. 100-102, July, 1902; Ref. B. C., $\mathrm{v}, \mathrm{p}$. 702 .

CUNEo and LECÈne. Notes sur les cellules interstitielles dans le testicule ectopique de l'adulte. Rev. de chirurg., t. xxii, 1900.

Curatulo and Tarulli. Einflusz d. Abtragung d. Eierstöcke a. d. Stoff wechsel. C. G., 1895 .

- Sulla secrezione interna delle ovarie. Ann. di ostetr. e ginec, 1896.

CuRTIS, F., and GELLÉ. De la sclérose amorphe dissociante et de la fréquence des formes de transition des îlots de Langerhans dans certaines lésions du pancréas diabétique. C. r. S. B., 57, p. 942, 1905.

- Histogenèse de la sclérose amorphe dissociante du pancréas. Ibid.,

- De l'importance des formes de transitions acino-insulaires ou insuloaciniques dans l'interprétation des lésions du pancréas diabetique. Ibid., p. 966 .

CushiNG, H. The hypophysis cerebri. Clinical aspects of hyperpituitarism and of hypopituitarism. J. A. M. A., 53, pp. 249-255, July 24, 1909. 
Cushixg, H. Sexual infantilism with optic atrophy in cases of tumours affecting the hypophysis cerebri. Journ. of Nervous and Mental Diseases, xi, 1906.

Cushing, H., and Lewis, L. ReFord. Is the pituitary gland essential to the maintenance of life? Johns Hopkins Hosp. Bull., 20, p. 105, 1909

Cushry, A. R. The action of optical isomers. III. Adrenalin. J. o. P., 37, p. 130, 1908 .

- Further note on adrenalin isomers. Ibid., 38, p. 259, March 22, 1909.

Crbulski, N. Über die Funktion der Nebenniere. IV. m. IV., 1806.

- Sur les fonctions des capsules surrénales. Gaz. lekarska, March 23, 1 S95.

- Über den Kalkstoffwechsel des tetaniekranken Säuglings. M. f. Kinderh., I 906.

CYBulski and Szymoxovicz. Anz. d. Krakauer Akad. d. IV., 4 Februar und 4 März, 1805.

CYON, E. V. Beiträge zur Physiologie der Schilddrüse und des Herzens. P. A., 7o, I 898 ; and Bonn, i 898 .

- Jodothyrin und Atropin. Ibid., 70, p. 511 .

- Jodnatrium und Muscarin. Ibid., 7o, p. 643.

- Die Verrichtungen der Hypophyse. I. Mitt. Ibid., 7 1, 1898.

- Les glandes thyroïdes, l'hypophyse et le cœur. A. d. P., 1898 .

- Über die physiologische Bestimmung der wirksamen Substanz der Nebennieren. P. A., 72, p. 370.

- Die Verrichtungen der Hypophyse. II. Mitt. Ibid., 72, 635. I898.

- Die physiologischen Herzgifte. I. Teil. Ibid., 73, p. 42.

- Die physiologischen Herzgifte. II. Teil. Ibid., 73, p. 339.

- Die Verrichtungen der Hypophyse. III. Mitt. Ibid., 73, p. 483, 1898.

- Die physiologischen Herzgifte. III. Teil. Ibid., 74, p. 97, 1898.

- Die physiologischen Herzgifte. IV. Teil. Ibid., 77, p. 215, 1899.

-- L'innervation du cœur. Richet, Dict. d. Phys., 4, I 899 .

- Die physiologischen Verrichtungen der Hypophyse. P. A., 81, 1900.

- Die Beziehungen des N. Depressor zum vasomot. Centrum. Ibid., 84, I 90 I.

- Zur Physiologie der Hypophyse. Ibid., 87, 1901.

- Zur Physiologie der Zirbeldrüse. Ibid., 98, 1903.

- Einige Worte zur Untersuchung von J. Masay uber die physiologische Rolle der Hypophyse. Ibid., IOI, 1904.

CYON and OSWALD. Uber die physiologischen Wirkungen einiger aus der Schilddrüse gewonnenen Produkte. Ibid., 83, 1901.

CzyhlaRz v. Tetanie nach partieller Kropfexstirpation. W. k. IV., 1902.

CZECZOWICZKA. Zur Kenntnis der durch Zytotoxine im Tierkörper erzeugten Veränderungen. Z. H., 24, 1903.

Czerny, A. Hydrocephalus und Hypoplasie der Nebennieren. C. a. P., 10, p. $2 \$ 1,1899$.

Czirfusz, D. Tonogen gegen Hämorrhoiden. Gyóg yászat, 1904.

Dagonet, J. Beitr. z. patholog. Anatomie d. Nebennieren d. Menschen. Z. H., 6, 1885 .

DAнL, F. Nierengeschwülste, entstanden durch losgerissene (versprengte) Nierenkeime. Hosp. Tidende, 6 and 7 ; Ref. Monastb. d. Harn-u. Sexualapp., p. 125, 1900.

D.AKIN, H. D. The synthesis of a substance allied to adrenalin. P. R. S., B., 76, 1905.

- On the physiological activity of substances related to adrenalin. Ibid., p. 498 .

- Physiological action of synthetical substance allied to adrenalin. J. o. P., 32 .

- Fractionary hydrolysis of optically inactive esters by lipases. J. o. P., 32, p. 199, 1905.

- A synthesis of certain naturally occurring aliphatic ketones, with a suggestion of a possible mode of formation of this substance in the organism (Methyl-n-nonyl-ketone, Methyl-n-heptyl-ketone, Methyl-namyl-ketone). J. Biol. Ch., iv, p. 22 I, I9o8.

- A comparative study of the oxidation of the ammonium salts of saturated fatty acids with hydrogen peroxide. Ibid., iv, p. 227, 1908 .

- Note on the use of paranitrophenylhydrazine for the identification of some aliphatic aldehydes and ketones. Ibid., iv, p. 235, 1908. 
Dakin, H. D., and Kossel, A. Weitere Beiträge zum System der einfachsten Eiweiszkörper. Z. ph. Ch., 44, p. 342 .

DAKIN, H. D., and RANSOM. Note on the treatment of a case of diabetes mellitus with secretin. J. Biol. Ch., ii, p. 305, 1907.

Dalchế. Dystrophie ovarienne. Le Bull. méd., Igoi.

- La puberté chez la femme. Paris, 1906.

- Hyper- et hypovarie. Gaz. d. hop., 1906.

DALCHÉ and LÉPINE. Opothérapie ovarienne. Progr. méd., 1902.

DALE, H. H. The islets of Langerhans of the pancreas. P. R. S., 73, p. 84, London, 1904.

- Physiological action chrysotoxin. P. Pharmac. S., May 20; and J. o. P., 32,1905 .

Dallemagne. Trois cas d'acromégalie. A. m. e., 7, p. 589, 1895.

DALTon. A case of acromegaly with diabetes. 'Transact. Path. Soc., 48, 1897 .

DANA. On acromegaly and gigantism. Journ. of Nerv. and Ment. Dis., 1893 and 1804 .

DANIELSEN. Erfolgreiche Epithelkörper-Transplantation bei Tetania parathyreopriva. Bruns Beitr., 66, Igı.

DANIELSEN and LANDOIS. Transplantation und Epithelkörper. M. K., roro.

DANIS. De l'influence de la glande thyroïde sur le développement du squelette. Thèse Lyon, 1896 .

DANLOS, APERT and LÉVY-FRANKEL. Cyphose hérédofamiliale à début prècoce. Anomalies multiples (mamelons surnuméraires, incisives de troisième dentition, acromégalo-gigantisme sur plusieurs membres de la famille). Soc. méd. Hôp., 1909.

Dantec le, F. La sexualité. Scientia, No. 2, Paris.

DARBY. Anatomy, physiology and pathology of the suprarenal capsule. Charleston Soc. Rev., xiv, pp. 318-334, 1859.

Darcan-Mouroux. Contr. à l'étude de la ménopause précoce. Thèse Paris, 1904

DARIER. Nebennierenextrakt. Ophthalm. Ges., Heidelberg, 1896; S. m., I 805 .

DARRÉ. De l'influence des altérations du rein sur les glandes surrénales. Thèse de Paris, 1907; J. d. P. P., p. 714, 1907.

Dastre. . Sur le sucre et le glycogène de la lymphe. A. d. P., p. 532, 1895 .

Davidsohn. Das Melanom der Nebenniere. V. d. p. G., xiii, p. 287, 1909.

Davis, Th. G. N. Y. Med. News, p. 263, August i I, 1906.

DAVIS, A. N. A case of carcinoma of the suprarenal capsule, necropsy. Lancet, October 3, 1896 .

Debove. Adipose douloureuse. Arch. gén. méd., 1903.

- Du diabète hypophysaire. J. d. Prat., No. 50, 12 décembre, 1908.

DECKERS and MEESCEN. Suprarenin in der Chirurgie. Gaz. méd. Belg., $15,1,1903$.

DEHLER, A. Beitrag zur Kenntnis vom feineren Bau der sympathischen Ganglienzellen des Frosches. A. m. A., 46, 1895.

Delamare, G. Glandes surrénales. Traité d'anat. p. Poirier et Charpy, iv, 2, 1904 .

- Coloration de l'hypophyse par le triacide d'Ehrlich. C. r. S. B., 56, p. 743,1904 .

- Recherches sur la sénescence des capsules surrénales. Ibid., pp. I4331483,1904 .

Delaunay. Etude sur le rôle fonctionnel de la glande thyroïde. Thèse Paris, 1896 .

Delbet, P. Sémiologie des organes génitaux. Bouchard, Traité de path. gén., $v$, I901.

DeLezFNNE, C. Activation du suc pancréatique par les sels de calciums. C. r. S. B., 57 , ii, p. 476,1905 .

- Action des sels de calcium sur le suc pancréatique, préalablement dialysé. Ibid., p. 523.

- Sur l'activation du suc pancréatique par les sels de calcium. Action antagoniste des sels de potassium. Ibid., p. 614.

- Sur les rôles de sels dans l'activation du suc pancréatique. Spécifité du Calcium. C. r. S. B., 57, ii, pp. 478 and $481,1905$.

Delitala, F. Il fegato del cane dopo l'ablazione completa dell' apparato tiro-paratiroideo. Lo Sperimentale, anno xli, fasc. 4. 
Delille, A. L'hypophyse et la modification hypophysaire. Paris, I9og. Daselbst zitiert mehrere Arbeiten desselben Autors mit Rénon,. Monier-Vinard, Vincent. I907-Igog.

Demiéville. Deux cas de maladie d'Addison. Rev. méd. de la Suisse: rom., iv, No. 9, 1884 .

DEMoor, J., and A. VAN LINT. La sérum antithyroïdien et son mode: d'action. Mémoires couronnés de l'acad. de Belgique. Bruxelles, 1903 .

DERCUM. Adiposis dolorosa. Univers. med. Magaz., 1888.

- A subcutaneous connective tissue distrophy, \&c. A. J. M. S., 1892 .

DERCUM and CARTHEY. Autopsy in a case of adiposis dolorosa. Ibid., 190z.

DERRIEU, E. Troubles de l'utilisation des noyaux aromatiques dans. l'insuffisance surrénale? Montpellier méd., 2, t. 25, p. 589, 1908.

Descroizilles. D'un cas de maladie bronzée. Union méd., No. 53, 1893.

- Un cas de maladie d'Addison à évolution lente. G. m., 1893 .

DESGREZ, A. Influence de la choline sur les sécrétions glandulaires. C. r. S. B., p. 52,1902 .

Desgrez, A., and ChEvalier, J. Action de la choline sur la pression artérielle. C. r. A., 146, pp. 89-90, 1908.

Dessy, S., and GRANDis, V. Contribution à l'étude de la fatigue. Action de l'adrénaline sur la fonction du muscle. A. i. B., 4I, pp. 225-233. 1904 ; Rev. Sud-Amer. de scienc. méd., an. i, No. 2, April, 1903.

Dexler. Über endemischen Kretinismus bei Tieren. Berl. tierärztl. Arch.,. 1909.

Dezirot, H. Etude sur la maladie d'Addison chez l'enfant. Paris, 1898.

DHÉRÉ. Sur l'absorption des rayons ultraviolett par l'adrenaline. Bull.. de la soc. chimique de France, 4, série i, p. 834, 1907.

DIAMARE, V. I corpuscoli surrenali di Stannius ed i corpi del cavo. addominale dei Teleostei. Notizie anatomiche e morfologiche. Bollet. soc. natur. Napoli, ix, 1895.

- Ricerche intorno all' organo interrenale dégli Elasmobranchi ed ai corpuscoli di Stannius dei Teleostei. Mem. soc. ital. sc., x. Roma, 1896.

- Sulla morfologia delle capsule surrenale. An. An., 15, p. 357, 1899.

- Del valore anatomico e morfologico delle isole di Langerhans. An. An., 16, 481, 1899 .

- Sulla constituzione dei gangli simpatici negli Elasmobranchi e sulla. morfologia dei nidi cellulari del simpatico in generale. An. An., 20, $418,1902$.

- Metaplasma ed immagini di secrezione nelle capsule suprarenale. Archivio zoolog., vol. i, pp. 12 I-178, 1903.

- Sullo sviluppo e morfologia delle capsule suprarenali. Bollet. soc. natur. Napoli, vol. xvii, pp. 55-62, 1903.

- Varietà anatomiche dell' interrenale. Arch. ital. d'anat., vol. iv, H. 2, pp. 366-369, 1905 .

- Studii comparativi sulle isole di Langerhans. I. M., 16, 1899.

- Studii comparativi sulle di Langerhans. 2. Mem., I. M., 22, I905.

- Zur vergleichenden Physiologie des Pankreas. Versuche über die. Total-exstirpation des Pankreas und weiters über die Glykolyse bei Selachiern. C. P., I9, I905.

- Zwiveite Mitt. über die physiologische Bedeutung der Langerhansschen Inseln im Pankreas. Ibid., I9, No. 4.

- Sul nuovo indirizzo della questione del rapporto fra pancreas ed economia del glucosio nell'organismo. Tommasi, 1907.

- Vergleichend-anatomische-physiologische Studien über den Pankreasdiabetes. 3. Mitt. C. P., 21, No. 26, 1908.

- Sullo funzione endocrino del pancreas e sugli elementi che la disimpegnano. Arch. di fisiol., v, p. 253, 1908.

- La secrezione interna del pankreas. Tommasi, No. 9, Igog.

Dickins, S. J. O. Notes on a case of acute goitre. Lancet, October 24, 1908.

Dide, D'Auxerre. Les glandes vasculaires sanguines chez aliénés. Congrès de Dijon, août, 1908.

DieckHOFF. Beiträge zur pathologischen Anatomie des Pankreas. Diss., Rostock, I 894. - Festschr. Thierfelder. Leipzig, 1895.

DIEM, M. Über das Vorkommen mydriatisch wirkender Substanzen im Harn. D. A. k. M., 94, 1908. 
Dienst, A. Die Pathogenese der Eklampsie und ihre Beziehungen zur. normalen Schwangerschaft, zum Hydrops und zur Schwangerschafts niere. A. G., 86.

Diesing, E. Die Regulierung des Mineralstoffwechsels. V. 26 C. M., p. 127,1909 .

- Beiträge zur Kenntnis der Funktion der Stoff wechseldrüsen. C. f. d. g. Phys. u. Path. d. Stoff w., iv, p. 209, I 909 .

DiETERLE. Die Athyreosis und die Skelettveränderungen. V. A., 184.

DIETRICH, A. Morphologische. Veränderungen in der Autolyse. V. d. p. G. iv, 1903, p. 81 .

- Fettbildung. Ibid., ix, p. 212, 1905.

- Fettgehalt pathologischer Nieren. Ibid., xi, p. 10, 1907.

- Naphtholblausynthese und Lipoidfärbung. C. a. P., I9, 1908.

- Wandlungen der Lehre von der fettigen Degeneration. Arb. path. Inst., Tübingen, 1904.

- Die Fettbildungen im implantierten Gewebe. Ibid., 1906.

DIETRICH and HEYLER. Morphologische Veränderungen bei Autolyse. Ibid., 1904 .

DiEvlafoy. Accidents urémique avec anurie, \&c. Soc méd. des. hôp., 1892.

Dimitrowa, $Z$. Recherches sur la structure de la glande pinéale chez quelques Mammifères. Le Névraxe, ii, $H .3$, r 1901 .

Disse, J. Die Nebennieren. In K. v. Bardelebens Handbuch der Anatomie des Menschen, vii, 1, pp. I62-179. Jena, 1902.

Dixon, W. E. The composition and action of orchitic extracts. J. o. P., 26, 1900-0r.

- The innervation of the frog's stomach. Ibid., 28, 1902.

- The paralysis of nerve cells and endings with special reference to the alkaloid apocodeine. Ibid., 30, 1903 .

Dixon, W. E., and HalliburToN, W. Der Pinealkörper. Quart. J. e. P., ii, p. 283 , r909; Ref. B.C. IX.

Dixon, W. E., and Hamill, $P$. The mode of action of specific substance with special references to secretin. J. o. P., 38, p. 314, 1909 .

Dobrowolskaja, N. A. Zum Chemismus der Verdauung im tierischen Körper XXII. Mitt. Die Bedeutung des Blutes für die Magenverdauung. Z. ph. Ch., 56, p. 408.

- Contribution à l'étude des hypernéphromes. A. Sc. biol., xii, p. 359, 1907.

DoBRowOLSKy. Über Cytotoxine der Ovarien. Gyn. Rundsch., 1, 3.

DoEbBELIN. Pseudoacromegalie und Acromegalie. Diss., Königsberg, 1895.

DöNITZ. Cocainisierung des Rückenmarkes unter Verwend. v. Adrenalin. M. m. W., I903.

DöRNER. Über Nebennierenblutungen bei Neugeborenen. V. g. M., 3, F., xxvi, p. 27,1903 .

Doeschate, A. ten. Ửber das Vorkommen von Milchsäure bei Eklampsie. Diss., Utrecht, 1907; Z. ph. Ch., 54, 1907.

Dogiel, A. S. Die Ausführungsgänge des Pankreas des Menschen. A. A., p. 117,1893 .

- Die Nervenendigungen in den Nebennieren der Säugetiere. Ibid., pp. 90-104, 1894 .

Dohrs, A. L. Urgeschichte des Wirbeltierkörpers. V. Mitt. d. zool. Station in Neapel, 1884 .

Dolega. Ein Fall von Kretinismus. Zieglers Beitr., 9, 1891 .

DOLLARD. L'Adrénaline et ses applications thérapeutiques. Thèse de Toulouse, décembre, 1902.

Domenicis, DE. Studii sperim. intorno agli effetti delle estirpazione del pancreas. Giorn. int. dell. scienz. med., 801, 1889; Atti d. congr. intern., 3, 391. Roma, I 894 .

- Noch einmal über den Diabètes pancréaticus. M. m. W., 1891 .

- Esperimenti intorno alla glicosuria ed al diabete. Acc. Med. Ch., Napoli, 1898.

- Patogenesi della glicosuria che segue all' esportazione del pancreas. Ibid., I908.

- Ricerche sperimentali per la fisiologia della capsule surrenali. Gazz. surrenali. Atti d. R. Accad. medic. chirug. di Napoli, p. 268 ff., 1892.

- Cause de la mort après l'exstirpation des capsules surrénales. A. d. P., p. 810,1894 . 
Domenicis, DE. Richerche sperimentali per la fisiologia della capsule surrenali. Gazz. degli osp. Milano, xvii, No. 140, p. 1474, 1896; Ref. C. i. M., p. 1017, 1897.

- Experimentelle Untersuchungen zur Physiologie der Nebenniere. Wirkung der Transplantation derselben. W. m. W., 1897.

DoNath. Beiträge zur Path. und Ther. der Basedowschen Krankheit. Z. k. M., 1899 .

DoNati. Ipernefroma maligno del fegato. Arch. per le sc. med., 29, No. 8, 1905.

DonetTI. Les lésions des cellules du système nerveux central après l'ablation des capsules surrénales. C. r. S. B., 29 mai, I 897.

DonkIN. Addison's disease of very rapid course; remarks. Lancet, February 2, 1895 .

Donzello, G., and Vito TARDo, G. Linfoangiosclerosi da adrenalina. Sperimentale, 68, 1909 .

DOPTER and GOUROD. Les capsules surrén. dans l'urémie expérim. C. r. S. B., 1904 .

Dor. De l'action vasoconstrictive exercée par l'extrait de capsules surrénales sur le conjonctive oculaire. Sem. méd., No. 36, 1896; Province méd., I2 juillet, 1896.

DOR, MAISONNEUve and MEURIDS. Ralentissement expérimental de la croissance par l'opothérapie orchitique. C. r. S. B., 57, p. 673, 1905.

DORNHÖFER. Über Addisonsche Krankheit. Diss., Würzburg, 1879.

DostoIEWSky, A. Zur mikroskopischen Anatomie der Nebennieren. Diss (russisch), Petersburg, I884 and A.m. A., 27, 1886.

- Über den Bau des Vorderlappens des Hirnanhangs. A. m. A., 26, I 886.

DOUGLASS. The effect of suprarenal preparation on living protoplasm. Amer. Journ. Med. Science, 129, p. 98, January, 1905.

Doyon, M. Action comparé de la choline et de la pilocarpine sur la teneur en glycogène du foie. C. r. S. B., 64, 1908.

Doyon, M., and GaUTIER, Cl. Action de l'adrénaline sur la glycogène du foie. Influence de l'atropine. Ibid., 64, pp. 866-868, 16 mai, 1908.

Doron, M., and JouTy, A. Ablation des parathyroïdes chez l'oiseau. Ibid., p. I I, I904.

DOYON, MOREL and KAREFF. Action de l'adrénaline sur le glycogène. Ibid., I904.

- Action de l'adrénaline sur le glycogène hépatique et le sucre du sang. Ibid., 1905 .

- Les parathyroïdes chez la tortue (tortue d'Afrique). Ibid., p. 719, I904.

- Action de l'adrénaline sur le glycogène du foie. J. d. P. P., vii, p. 998, 1906.

DRECHSEL. Die wirksame Substanz der Schilddrüse. C. P., 9, 704, I896.

DREveT, LouIs. Effets thérapeutiques du corps jaune de l'ovaire en particulier dans l'hypofonction de la glande ovarienne, la ménopause naturelle, la ménopause post-opératoire. Thèse de Paris, 1907.

DReyer, G. P. On secretory nerves to the suprarenal capsules. A. J. P., 2, 1899 .

DREYFUS. Die Therapie des morb. Basedowii in den letzten Jahren. C. ges. Th., xxi, 1905 .

DROBNIK. Experimentelle Untersuchungen über die Folgen der Exstirpation der Schildrüse. A. P. P., 25, 1888 .

DRUMMOND. The histological changes produced by the injection of adrenalin-chloride. J. o. P., 3I, p. 8I, 1904.

DRUMMOND and Paton, D. $N$. Observations on the influence of adrenalin poisoning on the liver, \&c. J. o. P., 31, 92, 1904 .

DRyfuss, J. Chemische Unters. uib. die Ätiologie der Eklampsie. B. C., vii, $492,1908$.

DuboIs, L. A. L'action des extraits de capsules surrén. C. r. S. B., I 896 .

- Des variations de toxicité des extraits de capsules surrénales. A. d. P., 28,1896 .

- De la pathogénie et du traitement de la maladie d'Addison. Nancy, 1896 .

- Action de l'adrénaline et de l'anagyrine sur la circulation des muqueuses linguales et bucco-labiale. C. r. S. B., 56, p. 355, 1904.

- Les changements de la coloration de la muqueuse linguale comme indicateur du mécanisme d'action des agents vasoconstricteur. Ibid., p. 562 . 
Dubois, R. The ductless glands. Brit. Ass. Adv. of Sc., 1906; V. H. i, 1907.

Dubreuille. Un cas de mélanodermie généralisée. Ann. de dermat., p. 76, I 891 .

DuBs. Kech. histol. s. le pancréas diabét. et non diabétique. Thèse Paris, 1907.

Du CASTEL. Mélanodermie. Maladie d'Addison on acanthisis nigricans. Ibid., p. 1282, 1896.

DucCESCHI, V. Sur les albuminoïdes du sang chez le chien thyroïdectomisé. A. i. B., 24, p. 23,1896 .

- Les processus d'oxydation de reduction et de synthèse chez les animaux thyroïdectomisés. Ibid., p. 209, 1896.

Ducceschi, V., and TALlarico, G. Sulla determinazione sperimentale del sesso. A. d. $F_{i}$, i, 1904.

DUChESNEAU. Contribution à l'étude anatomique et clinique de l'acromégalie. Thèse de Lyon, 1891 .

Duclos. Contribution à l'étude des capsules surrénales dans la race nègre. Rev. gén. de clinique et de thérapeut. Paris, 1890.

DUCROT, R. L. Etude sur le rôle sécrétaire du liquide cephalorachidien par les plexus choroïdes. Thèse de Bordeaux, 1905 .

Dudgeon, S. L. The etiology, pathology and diagnosis of adrenal hæmorrhage. A. J. M. S., 1904 .

Dufour, G. La pathogénie capsulaire de la maladie bronzée. Thèse de Paris, I894.

Duncanson, J. Gray. A note on the therapeutic value of adrenalin. Brit. Med. Journ., Igo3.

- The danger of suprarenal treatment in hæmophysis. Ibid., p. 603, March 12, 1904.

Dungern, v. Histologie d. Nebennieren bei Morbus Addisonii. Diss., Freiburg i. B., I 892 .

Dupargne. Opotherapie surrénale chez les Addisoniens. Thèse de Paris, 1896.

DUPRAS, A. Hypernephrome de la capsule surrénale droite. Exstirpation Guérison. Etude chimique et anatomo-pathologique. Rev méd. de la Suisse rom., No. 3 , 1906.

DUPRÉ and PAGNIEz. Infant. dégénératif (type Lorain) compliqué de dysthyroïdie pubérale (type Brissaud). Nouv. Iconographie de la Saltpêtrière, No. 2, 1902.

Dürck. Atrophie und Hypoplasie der Hoden. Münch. Ärztever., Ref. in M. m. W., März, 1907 .

DÚRIG. Ein Beitrag zur Serumbehandlung des Morbus Basedowii. M. m. W., 1905 .

Durst, H. Über einen Fall v. kompensat. Hypertrophie d. Nebenniere. Diss., Zürich, 1899 .

Dutro. Diabete pancreatico. Bull. della R. Accad. Med. di Roma, 283, 1893.

Dutro and Lo Monaco. Sur le metabolisme chez les chiens privés du thyroide. A. i. B., 24,1895 .

DwORNITSCHENKo. Über die Thymus der Erwachsenen in gerichtlich medizinischer Beziehung. V. g. M., 3, F., Bd. 14.

EASTERBROOK. Action of thyroid and parathyroid extract upon metabolism. Lancet, ii, I 898 and Brit. Med. Journ., September, 1900.

EASTwOOD, A. Malignant tumours from adrenal remnants. Transact. Path. Soc., 53, 1902.

Eberth, J. C. Die Nebennieren. Strickers Handb. d. Lehre v. d. Geweben. i, Leipzig, 1871 .

- Intrakranielles Teratom mesodermalen Ursprungs. V. A., 153, 1898.

- Die männlichen Geschlechtsorgane. Bardelebens Handb. d. Anat., vii, 1904 .

EBNer, V. v. Köllikers Handbuch der Gewebelehre. 6 Aufl., Bd., 3, Leipzig, I899.

Ebstein, W. Peritonitisartiger Symptomenkomplex im Endstadium der Addisonschen Krankheit. D. m. W., 1897.

ECKer, A. Der feinere Sau der Nebennieren beim Menschen und den vier Wirbeltierklassen. Braunschweig, 1846. 
Ecker, A. Blutdrüsen in R. Wagner's Handwörterb. d. Phys., iv, p. 128, 1853.

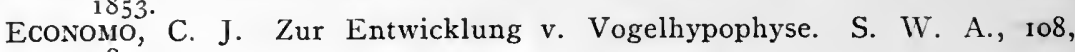
I 899 .

EDEL. Über einen günstigen Ërfolg durch Behandlung mit Nebennierentabletten in einem Falle von Morbus Addisonii. M. m. W., I90o.

EdelmanN, J. A. Die Bewegungen des Magens und der Übergang des Mageninhaltes aus dem Magen in den Darm. Diss., rgo6.

EDINGER. Bau der nervösen Zentralorgane. 7 Aufl., igo8.

EDkins, E. S. The clemical mechanism of gastric secretion. J. o. P., 34 , p. 133, 1906.

EDkins, E. S., and TweEDY, M. The chemical mechanism of gastric secretion. Ibid., 38, p. 263, 1909 .

EDMUNDS, C. W. Experiments of, on the thyroid and parathyroid glands. J. o. P., 1895 .

- Further observations and experiments on the thyroid and parathyroid. J. of Path. and Bact., i, p. 64, 1899; ii, p. 71, 1901 ; iii, p. 288, 1903.

- Thyroid of puppy of thyroidless bitch. Transact. Path. Soc. London, 51, p. 221,1900 .

- The pathology of exophthalmic goitre. Brit. Med. Journ., rgor.

- The parathyroid glands. Lancet, vol. I74, 1908.

- The treatment of Graves's disease with the milk of thyroidless goats. Lancet, April 1o, 1909.

EDMUNDS, IT. The influence of digitalis, strophanthus and adrenalin upon the velocity of the blood current. A. J. P., 18, pp. 129-148, 1907.

EDSALL and Miller. Chemical pathology of acromegaly. Med. Bull. Univ. Pennsylvania.

Eecke, A. ver. Corps thyroïde et échanges organiques. A. int. de pharm., 4,1892 .

- Modifications de la cellule pancréatique pendant la sécrétion. A. B., xiii, 1895 .

- Nouvelle contribution à l'anat. et phys. du thymus chez la grenouille. Bull. d. l'acad. R. d. med. de Gand, Belgique, t. 78, p. 103, 1899 .

EGDAHL, A. A study on the effect of intravenous injections of solutions of pancreatic tissue and the cause of collapse in acute pancreatitis. J. E. M., 9, 1907 .

Eggeling, H. Eine Nebenniere im Lig. hepatoduodenale. An. An., 21, 1902.

EhrenberG, R. Über alimentäre Glykosurie bei Psychosen. M. Psych., 25, pp. 1-13, I909.

EHRHARDT, O. Uber epileptisches Auftreten der Tetania thyreopriva. M. G. M. C., 10, 1902.

- Morbus Basedowii. D. Ch., 38, rgor.

EHrICH, E. Klin. u. anat. Beitr. z. Morbus Basedowii. Beitr. kl. Chir., 28, 1900 .

EHRMANN, R. Über eine physiologische Wertbeştimmung des Adrenalins und seinen Nachweis im Blut. A. P. P., 53, p. 96, r905.

- Über die Wirkung d. Adrenalins auf. d. Hautdrüsensekr. d. Frosches. Ibid., p. 137.

- Zur Physiologie und experimentellen Pathologie der Adrenalinsekretion. A. P. P., 55, p. 39, 1906.

- Über d. Einfl. d. Ausschaltung des Zwölffingerdarms auf die Zuckerausscheidung. P. A., $119,1907$.

- Über Substanzen, die die Zuckerausscheidung nach Injektion von Adrenalin zu hemmen imstande sind. B. k. W., I9o8.

--- Beitrag zur Physiologie der Nebennieren und über im Blut vorhandene und andere pupillenerweiternde Substanzen. D. m. W., igos.

- Zur Methode des qualitativen und quantitativen Nachweises kleinster Adrenalinmengen in Blut und Körperflüssigkeiten. D. m. IV., 1909 .

- Bemerkungen zu der Arbeit von R. H. Kahn zur Frage der inneren Sekretion des chromaffinen Gewebes. P. A., 129, I909.

EhrMaN,, R., and LEDERER, R. Über das Verbalten des Pankreas bei Achylie und Anazidität des Magens. D. m. WV., 1009.

EICHLER, F. Über die adrenalinähnliche Wirkung des Serums Nephrektomisierter und Nierenkranker. B. k. WW., 1907.

- Experimentelle Beiträge zur Diagnose der Pankreaserkrankungen. Ibid, 1907. 
EIChler, F., and Silbergleit. Über Glykosurie, hervorgerufen durch Verätzung und Verschorfung der Innenfläche des Darmes. B. k. W., I 908 .

EijSselsteyn, G. VAN. Een geval van Morbus Addisonii. Neederl. Weekblad, I4 Mai, I 898 .

EIselsberG, A. v. Über Tetanie im Anschlusz an Kropfoperation. Wien, 1890.

- Weitere Beitr. z. Lehre von d. Folgezuständen der Kropfoperation. Festsch. f. Billroth. Stuttgart, 1892 .

- Uber Vorkommen und Behandlung der Tetania parathyreopriva beim Menschen. Beitr. Phys. Path. Festschr. Hermann, pp. I-21, igo8.

- Uber erfolgreiche Einheilung der Katzenschilddrüse in die Bauchhöhle und Auftreten von Tetanie nach deren Exstirpation. W. k. W. I I 92 .

- Wachstumsstörungen bei Tieren nach frühzeitiger Schilddrüsenexstirpation. A. k. Ch., 49, 1895.

- Schluszbemerk. zu H. Munks Aufsatz. V. A., I 54, 1898.

- Diskussionsbemerkungen zum Vortrag von Exner. Ref. M. m. W., I 909 .

- Die Krankheiten der Schilddrüsen. D. Ch., Lief, 38, I901.

- Diskussion zum Vortrag Erdheim. W. k. IW., 78, ", 906.

- Über operative Behandlung der Hypophysentumoren. W. k. W., I907.

- Diskussion zum Vortrage Hochenegg. Kongr. d. d. Ges. f. Chir. Berlin, 1908.

EiselsberG, A. VON., and Frankl-Hochwart, L. v. Operative Behandlung der Tumoren der Hypophysisgegend. N. C., No. $21,1907$.

- Neuer Fall von Hypophysisoperation bei D̈egen. adiposo-genitalis. W. k. W., igo8.

EISELT, R. Vymena látek pri onoemocneui Addisonoen (Über den Stoffwechsel bei der Addisonschen Krankheit). Sbornik lekarsky, X. H. 5 , I 909.

EISENLOHR. Zur Kasuistik der Tumoren der Hypophysis. V. A. 68, i876. Ellenberger, W. Vergleichende Histologie der Haussäugetiere. Berlin, 1887.

EliLINGer and Seelig. Der Einflusz von Fieber, Infektion und Nierenschädigung aut die Suprarenin-Glykosurie. M. m. W., 1905.

- Festschrift zum 6o. Geburtstag von Max Jaffé, p. 347, Braunschweig, I 901 .

ELLIOTT. On the innervation of the ileocolic sphincter. J. o. P., 31, p. 57, I 904 .

- Antiperistaltic and other muscular activities of the colon. Ibid., 3I, p. 272.

- The action of adrenalin. Ibid., 32, p. 401, I905; and Brit. Med. Journ., I 905 .

ElliotT and DurhaM. On subcutaneous injections of adrenalin. Ibid., 34, p. 490, 1906.

Elliot and TuCKetT. Cortex and medullar in the suprarenal glands. Ibid., 34, p. 332 , I906.

Eloy, CH. La méthode de Brown-Séquard. Paris, 1893.

ELSÄSSER, O. Über die Häufigkeit und die Bedeutung der isolierten primären Nebennierentuberkulose. Arb. a. d. Geb. d. path. Anat. u. Bakt. v. p. 45 , Tübingen, 1904 .

EMBDEN and AlmaGiA. Über Zuckerausscheidung pankreasloser Hunde nach Alanindarreichung. H. B., 7, p. 298, I905.

EMBDEN, G., and FÜRTH, O. v. Über die Zerstörung des Suprarenins (Adrenalins im Organismus). H. B., 4, pp. 421-429, 1904

EMBDEN and SALOMON. Über Alaninfütterungsversuche am pankreaslosen Hunde. H. B., 5, $507 ; 6$, p. 63, 1904 .

EMBDEN, SALOMON, and SCHMIDT. Über Azetonbilding in der Leber. H. B., 8, 1906.

EmbDen, LÜTHJe and LiefMANN. Über den Einflusz der Auszentemperatur auf den Blutzuckergehalt. H. B., Io, p. 265, 1907.

EMERSON, H. Cardiac insufficiency due to high arterial pressure. Proc. Soc. Exper. Biol. Med., vol. v, pp. I-3.

Emery, C. Zur Morphologie der Kopfniere der Teleostier. Biol. C., i, pp. $527-529,1881$.

- Studi interno allo sviluppo ed alla morfologia del rene dei Teleostei. Mem. R. Accad. Lincei Roma, vol. xiii, pp. 43-50 (see also A. i. B., T. ii, I 882$)$. 
EMERy, C. Zur Morphologie der Kopfniere der Teleostier. Erwiderung an Herrn G. Grosglick. Z. A 2 , No. 212, Jahrg. 8, pp. 742-744, 1885 .

- La determinazione del sesso dal punto di vista biologico. Bologna, 1904.

EMMERT, J. Die Wirkung subkutan einverleibten Adrenalins. V. A., 194, p. $114,1908$.

ENDERLEN. Untersuchungen über die Transplantation der Schilddrüse in die Bauchhöhle von Katzen und Hunden. M. G. M. C., 3, p. 474, 1898 .

ENGEL, C. Über Diabetes insipidus. Z. k. M., 67, 1909 .

ENGELBERTZ. Morbus Addisonii. Diss., Bonn, 1892.

EngelmanN, F. Die Placentartheorie der Eklampsieätiologie. C. G., 33, 1909.

- Adrenalinbehandlung bei Osteomalacie. Ibid., 32, 1908.

ENGELManN, K. Über die Beziehungen von Erkrankungen der Nebennieren zu Morbus Addisonii. Diss., VWürzburg, 1906.

ENGLÄNDER, B. Adrenalin bei Knochenerweichung (Osteomalacie). C. G.,

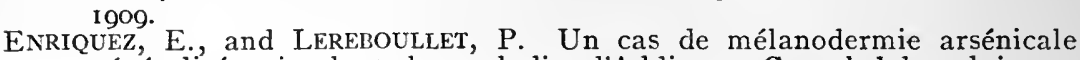
généralisée simulant la maladie d'Addison. Gaz. hebd., xlvi, 54, juillet, 1899 .

Epelnaum. Contribution à l'étude de l'organothérapie. Corps thyroïde. Capsules surrénales. Thèse Paris, 1895 .

EPPINGER, H., FALTA, W., and RUDINGER, C. Über die Wechselwirkungen der Drüsen mit innerer Sekretion. Z. k. M., 66, 1908; vorl. Mitt., W. k. W., I 908 .

- II. Mitteilung. Z. k. M., 67, 1909 .

- Über den Einflusz der Schilddrüse auf Stoffwechşel und Nervensystem. V. 25 , C. M., 1908 .

- Bemerkungen z. Arbeit Tomaszewski u. Wilenko. B. k. W., 1908.

- Über den Antagonismus sympathischer und autonomer Nerven in der inneren Sekretion. W. k. W., Igo8.

EPPINGER and HESS. Zur Pathologie des viszeralen Nervensystems. I. Mitt., Z. k. M., 67, p. 345, 1909; II. Mitt., Ibid., 68, p. 205, 1909; III. Mitt., Ibid., 69, p. 23I, I 909 .

- Zur Pathologie der Basedowschen Krankheit. 26 C. i. M., Ref. C. i. M., p. 539 , 1909 .

- Versuche über die Einwirkung von Arzneimitteln auf überlebende Coronargefäsze. Z. e. P., V. p. 622, 1909.

ERB, W. Acromegalie. D. A. k. M., 42, 1888 .

ERB, W.; JUN. Über experimentell erzeugte Arterienerkrankung beim Kaninchen. V. 21, C. M. April, 1004.

- Experimentelle und histologische Studien über Arterienerkrankung nach Adrenalininjektionen. A. P. P., 53, 1905.

- Uber Gehirnblutungen bei Kaninchen nach Adrenalininjektionen. Zieglers Beitr., Festschrift für J. Arnold, S. 500, 1905 .

ERBEN, F. Notiz zur Therapie des Diabetes mellitus. P. m. W., 1908.

ERDHEIM, J. Beitr. z. Kenntnis der branchiogenen Organe des Menschen. W. k. W., 1901 .

- Zur normalen und pathogischen Histologie der Glandula thyreoidea, parathyreoidea und Hypophysis. Zieglers. Beitr., 33, p. 158, 1903.

- Über Schilddrüsenaplaisie bei Cretinismus. Geschwülste des Ductus thyreoglossus. Über einige menschliche Kiemenderivate. Zieglers Beitr. 35 , p. 366 , 1904 .

- Beiträge zur pathologischen Anatomie der menschlichen Epithelkörperchen. Z. H., 25, Abt. f. pathol. Anat., I904.

- Uber Hypophysenganggeschwiulste und Hirncholesteatome. W. S., II3, 3,1904 .

- Zur Kenntnis einiger Kiemenderivate. "An. An., 29, Igo6.

- Tetania parathyreopriva. W. k. W., ı006; M. G. M. C., I6. 1006.

- Über Epithelkörperbefunde bei Osteomalacie. S. W. A., i i6, 1907. - Über einen Hypophysentumor von ungewöhnlichem Sitz. Zieglers

ERDHEIM and STUMME. Schwangerschaftsveränderung der Hypophyse. 37. Chirurgen-Kongresz, Berlin, 1908. B. k. W., 25 Mai, 1908. - Über die Schwangerschaftsveränderung der Hypophyse. Ziegl. Beitr.,
46, 1909. 
Erlandsen, A. Exper. Unters. über den Phloridzindiabetes. I. B. Z., 23, IgIO.

ErLanger. Les capsules surrénales. Thèse de Paris, No. 162, 1902.

ESAU, P. Exper. Unters. über den Phloridzindiabetes. M. G. M. C., 19, 1908.

EsCHERICH, TH. Bemerkungen über den Status lymphaticus der Kinder. B. k. W., 1896 .

- Zur Kenntnis der tetanischen Zustände des Kindesalters. M. m. W., 1907.

- Die Tetanie der Kinder. Wien, 1909.

Essen-Möller. Doppelseitige Ovariotomie im Anfgang der Schwangerschaft. C. G., 28, 1904 .

ESSER. Ein typischer Fall von Morbus Addisonii. D. m. W., I 899 .

ETIENNE and FRITSCH. La rôle athéromatisant du chlorure de calcium dans l'athérome expérimental n'apartient pas à sa chaux. C. r. S. B.,

p. 937, 1909.
ETIENNE, G., and PARISOT, I. Action sur les vaissaux de l'adrénaline employée simultanement avec les vaso-dilatateurs codes. Congr. franç. Méd., Paris, 1907.

- Athérome aortique et extract d'hypophyse. C. r. S. B., 64, p. 751,7 avril, 1908.

- Les composés iodés jouissent-ils de propriétés anti-athéromatisantes. Soc. de Méd. de Nancy, 26 février; Revue Méd. de l’Est, I juin, 1908.

- Pression artérielle et artério-sclérose. Congr. de Méd. Geneve, septembre, igo8.

- Action sur l'appareil cardio-vasculaire des injections répétées d'extrait d'hypophyse. Comparaison avec l'action de l'adrénaline. A. m. e., $20,1908$.

- La rôle de l'élévation de la pression artérielle dans l'étiologie de l'athérome. J. d. P. P., x, pp. 1055-1065, is novembre, I908.

ETTLINGER and NAGEOTTE. Lésions de cellules du système nerveux certral dans l'intoxication addisonienne expérimentale (décapsulation). C. r. S. B., 48,1896 .

EULENBURG. Antithyreoidinbehandlung der Basedowschen Krankheit. B. k. W., 1904 .

Eulenburg and Guttmans. Die Pathologie des Sympathicus. Berlin,

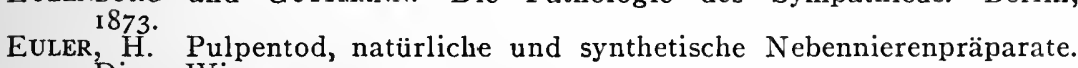
Diss., Wien, 1907.

EustachI, BARTH. Opuscula anatomica. De renum structura officiis, ed administratione. Cap. vi, Venezia, ${ }_{15} \sigma_{3}$.

EWALD, C. A. Die Erkrank. der Schilddrüse. Nothnagels. Hdb., 1896; 2 Aufl. Wien, Igog.

- Klinische Vorstellung von Hypophysistumoren. M. m. W., p. 1853, 1908.

- Dercumsche Krankheit. Encyclop. Jahrb., 16, 1909.

EW,ALD, J. R., and ROCKWELL. Exstirpation der Thyreoidea an Tauben. P. A. 47,1890 .

EwaLd, P. Fettgehalt und multiple Adenombildung in der Nebenniere. Diss., München, 1902 .

Ewans, H. W. Addison's disease following enteric fever. Lancet, i9 June, 1900.

ExNer, A. Kehlkopfnerven und die Funktionen der Thyreoidea. P. A., $68,1897$.

- Über die durch intraperitoneale Adrenalin-Injektion veränderte Resorptionsfähigkeit des tierischen Peritoneums. Z. H. 24, 1903.

- Über durch intraperitoneale Adrenalininjektion verursachte Verzögerung der Resorption von den in den Magen eingebrachten Giften. A. e. P., 50, p. 313,1904 .

- Exstirpation der Hypophyse bei malignem Adenom, gefolgt von Besserung. W. k. W., I5 Januar, 1909 ; and V. d. N. A. Ref. in M. m. W., No. 39 , 1909.

- Beitr. z. Pathol. u. Pathogenese der Akromegalie. M. G. M. C., 20, I909.

ExNer, S. Physiol. d männl. Geschlechtsorgane. In Fritsch-Zuckerkandls Hdb. d. Urologie. 
ExNcr, S. Männlich und weiblich. Beitr. z. Geb. u. Gyn., Festschr. Chrobak. Wien, 1903.

FABOzZI, S. Sugli effetti del succo di capsule surrenali negli animali scapsulati. Il Morgagni, 42, p. 177, 1900; Gazz. d. Osp., xxi, p. I16, Milano, Iooo.

Fabritius, J. Über Cysten an der Tube, \&c. A. G., 50, 1896.

FAIRBAIRN. A renal tumour arising from adrenal remnants. Transact. Path. Soc. London, vol. 53, p. 184, 1902:

FALK, F. Über die durch Adrenalininjektionen an Kaninchen hervorgerufenen Gefäszveränderungen und deren experimentelle Beeinflussung. Z. e. P., 4, p. 360, 1907.

- Über Adrenalinveränderungen an den Gefäszen und deren experimentelle Beeinflussung. M. m. W., 1907.

- Über die Adrenalin-Arterionekrose. iW. k. W., 1909.

FALKENBERG. Zur Exstirpation der Schilddrüse. 'V. 10, C. M., p. 502, 1891 .

FALTA, W. Über den zeitlichen Ablauf der Eiweiszzersetzung im tierischen Organismus. D. A. k. M., 86, p. 517.

- Über die Gesetze der Zuckerausscheidung beim Diabetes mellitus. IV. Mitt., Z. k. M., 65, p. 300; VI. Mitt., ibid., 65, p. 463; VIII. Mitt., ibid., 65 , p. 489 and ibid., 66 , p. 401,1908 .

- Über physiologische Bedeutung des Pankreas. W. k. W., p. 1559, 1907.

- Diskussion zum Vortrage Biedl-Offer. W. k. W., 1907.

- Beziehungen zwischen der inneren Sekretion der Schilddrüse, des pankreas und des chromaffinen Systems. V. 25, C. M., 1908.

- Über den Eiweiszumsatz beim Diabetes mellitus. B. k. W., 1908.

- Weitere Mitteilung über d. Wechselwirkung d. Drüsen m. innerer Sekretion. Ibid., I909.

- Concerning diseases that depend on disturbances of internal secretion. A. J. M. S., 1909 .

-. Uber die Bedeutung der Blutdrüsen in der Pathologie des Diabetes mellitus. P. m. W., rgio.

- Die Therapie des Diabetes mellitus. Ergebn. f. i. M. u. Kinderh., ii, 1908 .

FALTA, W. Diabetes mellitus. V. Mitt., Z. k. M., 65, p. 313, 1908.

- Über den Einfluss stickstofffreier Energieträger auf den zeitlichen Ablauf der Eiweiszzersetzung. B. Z., 13, p. 267, 1908.

Falta, GROTE and STAEHELIN. Versuche über den Kraft- und Stoffwechsel und den zeitlichen Ablauf der Zersetzungen unter dem Einfluss verschiedener Ernährung beim Hund. H. B., 9, p. 333, 1907.

- Über den Stoffwechsel u. Energieverbrauch bei pankreaslosen Hunden. Ibid., 10, 1907.

Falta, RUdinger, Bertelli, Bolaffio and Tedesko. Über Beziehungen der inneren Medizin zum Salzstoffwechsel. V. 26, C. M., 138-149, I 909 .

FALTA and WhitNey. Zur Kenntnis des Eiweisz- und Mineralstoff wechsels pankreasdiabetischer Hunde. H. B., I I, p. 224, 1908.

FALTA and Ivcovic. Adrenalin als Antidot. B. k. W., I909.

- Über die Wirkungsweise des Adrenalins bei verschiedener Applikation. W. k. W., igo9.

FAxo, G., and R̉ossi, G. Sulla viscosità del siero sanguigno nelle lesioni sperimentali dell' apparecchio tiroparatirioideo. A. di Fisiol., ii, 1005 .

FARRINI, A., and VIDONI, G. Azione degli estratti di tiroide delle soluzione di tiriodina, degli estratti di timo sistemo circolatorio. Lo Speriment., 62 ; and $\bar{A}$. i. B., 52, 1909 .

Fassin, L. Rôle de l'iode dans l'augmentation des propriétés du sérum sous l'influence des produits thyroïdiens. C. r. S. B., ii, p. 457, 20 mars, 1904 .

FASsint, R. Effetti terapeutici della paraganglino Vassale in alcune forme dispeptiche della tuberculosi pulmonare. Gaz. Osp. e Cliniche, I9o8.

FAURe, L. Contr. à l'étude de la maladie d'Addison. Thèse Paris, isgs.

FAURET-FrEMIET, E. Sur une sécrétion interne chez le cochliopodium pellucidium. C. r. S. B., 58, p. 905 , 1906. 
FAvorSKI, A. V. Traitement de l'acromégalie. Revue Neurcl, 1900.

FEDERICI, F. Della struttura delle capsule surrenali e delle alterazioni consecutive alle infezioni sperimentali. Lo Sperim., 58, p. 419, 1904.

FEDOROFF, S. P. Über Befund von Adrenalin in einem Nierentumor. Folia Urologica, H. 5, 1908 ; Bph. C., iii, No. 1626.

Fehling. Die Form des Beckens beim Foetus und Neugeborenen. A. G.,

- Über Wesen und Behandlung der Osteomalacie. A. G., 28, 1890 and 29, 1891 .

FEILCHENFELD. Fall von Tumor cerebri (Gliosarkom) der Zirbeldrüse. N. C., 1885 .

FELDKIRChNER, J. Zwei Fälle von Morbus Addisonii. Diss., Lindau, 1871 .

Felicine, L. Beitrag zur Anatomie der Nebenniere. An. An., 22, p. 152, 1903.

- UUber die Beziehungen zwischen dem Blutgefäszsystem und den Zellen der Nebenniere. A. m. A., 63, pp. 283-312, 1903; and Diss., Bern, 1905.

FÉlizet and Branca. Histologie du testicule ectopique. J. A. P. I 898 .

- Recherches sur le testicule en ectopie. Ibid., $38,1902$.

- Sur les cellules interstitielles du testicule ectopique. C. r. S. B., 53, 1901 .

FELLNER, O. Über die Tätigkeit des Ovariums in der Schwangerschaft. A. G., 87, 1909 .

- Die wechselseitigen Beziehungen der innersekretorischen Organe, insbesondere zum Ovarium. Volkmanns Sammlung, No. 508, 1908.

- Zur Histologie des Ovariums in der Schwangerschaft. A. m. A., 73, p. 288 , 1909 .

- Uber physiologische Graviditätserscheinungen bei Mutter und Kind. Z. G., 1909 .

- Úber intravasale Gerinnungen nach Injektion von Uterusextrakten. C. P., 23, 1909 .

Fellner and Neumans. Über Röntgenbestrahlung der Ovarien in der Schwangerschaft. C. G., 30, 1906 .

Fenyvessy, B. v. Über die Wirkung Schilddrüsenstoffe auf die Zirkulation, \&c. W. k. W., 1900.

Feodossiew, N. E. Hypertrophie der Nebennieren nach Entfernung der Eierstöcke. Russkij Wratsch.; No. 5, p. 135, 1906; Ref. Bph. C., ii, p. 160 .

FÉRÉ, C. L'instinct sexuel évolution et dissolution. Paris, 1899.

- L'adipose douloureuse, syndrome de Dercum. Rev. d. méd., août, 1901.

- Rapports des testicules avec le corps thyroïde. C. r. S. B., 57. p. 436, 1905.

FÉRÉ and BEChasi. Etude de l'action du suc ovarien sur le cobaye. Gaz. hebd., $44,1897$.

FERGusson, J. S. The veins of the adrenal. A. J. A., 5, p. 63, 1906.

FERRARI. Contr. à l'étude des glandes parathyroïdiennes. Thèse Genève, 1897.

FERRANINI, L. Über von der Schilddrüse unabhängigen Infantilismus und Stoffwechselbilanz in einem Fall von Mitralinfantilismus. Arch. f. Psych., 38, p. 296.

- Il geroderma genito distrofico. Policlin., Suppl. 1898 u. Congr. ital. med., Roma, 1899.

- Gifte und Toxine auf das überlebende Säugetierherz. C. R. du XXI. Congr. de la Soc. ital. de chirurg., Rome, October 27-29, 1908; J. d. P. P., xi, p. 341, March 15, 1909 .

Feuillé. Contribution à l'étude de l'athérome expérimental. Thèse de Lyon, 1905-1906.

FICHERA. Hypertrophie du corps pituitaire consécutive à la castration. Il policlinico, sez. chir., juin-juillet, 1905 .

- Sur l'hypertrophie de la glande pituitaire consécutive à la castration. A. i. B., 43, p. 405,$1905 ;$ and Boll. Acc. med., Roma, 1905.

- Sulla distruzzione dell' ipofisi. Lo Sperim., 59, 1905.

Filatow. Über den Einfluss der Blutsera auf das Auge. Diss., Odessa, 1908 .

FilehNe. Zur pathogenese der Basedowschen Krankheit. E. B., 14 Juli, 1878. 
Filipello, G. R. Sopra un caso di acromegalia. Annali di frenatria, 12, p. $57,1902$.

FilipPI. Azione della adrenalina sulla musculatura della siomaco di rana. Sperimentale, p. $764,1904$.

FINKELNBURG, R. Klinische und experimentelle Untersuchungen über Diabetes insipidus. D. A. k. M.: 91, 1907.

Finotrr. Zur Pathologie und Therapie der Leistenhoden nebst einigen Bemerkungen über die groszen $Z$ wischenzellen des Hodens. A. Ch., $57,1897$.

Finotri, R., and TEDESCHI, S. Alterazioni delle capsule surren. e pellagra. Rif. med., 1902.

FIORI, C. Legatura dell' uretere e suoi esiti. Clinic. Chirurg., Modena.

- L'azione dell' estratto renale e del siero di sangue sottoposti alla nefrectomia bilaterale. Gazz. degli osp., 1903.

- Sull' azione delle iniezioni di sangue venosa emulgente e di emulsioni di parenchyma renale negli animali della stessa specie. Pisa, 1903.

- Patologia sperimentale del rene. Il policlinico, 1903.

FischEL, A. Untersuchungen über die Wirbelsäule und den Brustkorb des Menschen. Anat. Hefte, 31, p. 463, igo6.

FISCHER, B. Über Arterienerkrankung nach Adrenalininjektionen. V., 22, C. M., 1905 .

- Experimentelle Erzeugung von Aneurysmen. D. m. W., I905.

- Experim. Arterienerkrankungen durch Adrenalininjektionen. M. m. W., 1906.

- Zur Frage der experim. Arterionekrose durch Adrenalininjektion. B. k. W., 1907 .

FISchER. Üb. Veränderungen d. Pankreas u. d. Leber bei Diab. mellitus. V. A., 172,1903 .

FISCher, J. Die Schilddrüse und der weibl. Genitalapparat. W. m. P., 1895 .

Fischer, $M$. H. Über die Hervorrufung und Hemmung von Glykosurie durch Salze. P. A., 109, 1905.

Fischer, PH., and HOPPE, J. Über Pankreon. M. m. W., I907.

FISCHL, R. Experimentelle Beiträge zur Frage der Bedeutung der Thymusexstirpation bei jungen Tieren. Z. e. P., i, 1905.

FLÄCHER, F. Über die Spaltung des synthetischen dl-Suprarenins in seine optisch aktiven Komponenten. Z. ph. Ch., 58, 3, pp. 189-194, 1908.

- The resolution of synthetic suprarenin into its optically active components. Pharm. Journ., January 9, 1909.

FlataU. Die Ovariotomie während der Schwangerschaft. A. G., 82, 1907.

FLECK. Zur Frage der inneren Sekretion von Ovarium und Plazenta. C. G., 29, 1905 .

Fleckseder, K. Uber die Rolle des Pankreas bei der Resorption der Nahrungsstoffe aus dem Darm. A. P. P., 59, 1908 .

Fleig, C. Essai de préparation d'une antisécrétine. C. r. S. B.. 57, ii, $795,1905$.

- Les sucs digestifs normaux et les sucs d'hypersécrétions provoqués artificiellement. C. r. S. B., 64, 1908 .

Fleiner, W. Addisonsche Krankheit mit Nebennierenkrebs. B. k. W., 1889 .

- Uber die Veränderungen des sympathischen und cerebrospinalen Nervensystems bei zwei Fällen von Addisonscher Krankheit. V. Io, C. M., 1891 ; D. Z. N., 2, 1892.

- Uber den heutigen Stand der Lehre von der Addisonschen Krankheit. Volkmanns Sammlung, No. 38, 1892 .

- Neuer Beitrag zur Lehre von der Tetanie gastrischen Ursprungs. D. Z. N., 5, 1900 .

- Zur Frage der Tetanie bei Magendilatation. A. V., 5, rgor.

- Über die Tetanie gastrischen und intestinalen Ursprungs. M. m. W., 1903.

Fleischman, L. Die Ursache der Schmelzhypoplasien. W. k. W., 1907, und Österr.-ungar. Viertelj. f. Zahnk., Igog.

FleischmanN, P., and DavidsoN, $H$. Über Zytotoxine. Folia serol., i, p. $173,1908$.

FLEISCHER, M. S. Über die Einwirkung von Jodopin und einigen anderen Substanzen auf die durch Adrenalin hervorgerufenen Arterienveränderungen, sowie über die WVirkung sehr groszer Adrenalindosen. Ca. P., 20, 1909 . 
Fleming, R. A., and Miller, J. A family with Addison's disease. Brit. Med. Journ., April 28, 1900.

Fleming. A case of acromegaly. Clin. Soc. Transact., 23, p. 253, 1890.

FLESCH. Über die Hypophysis einiger Säuger. V. d. N. Å., Magdeburg, I 884

FLint, J. M. Reticulum of the adrenal. An. An., 16, 1899.

- The blood-vessels, angiogenesis, organogenesis, recticulum and histology of the adrenal. Johns Hopkins Hospital Reports, ix, pp. 153-229. I900.

- The framework of the glandula parathyreoidea. A. J. A., 4, 1905.

- Das Bindegewebe der Speicheldrüsen und des Pankreas und seine Entwicklung in der Glandula submaxillaris. A. A., p. 6i, 1903.

Flockemans. Beeinflussung der Ausfallsersch. kastr. Frauen durch Ovarialpräparate. M. m. W., 1901 .

FloersheIM, S. The use of suprarenal capsule in diseases of the lower air passages. Med. Record, November 17, 1900; N. Y. Med. J., May 14 and 18 , igor.

- A report of forty-five unpublished cases of hæmorrhage treated by the internal administration of the suprarenal capsule. Am. Med. News, p. 17, January 4, 1902.

- The status of suprarenal therapy. Med. News, p. 587, April 1, 1905.

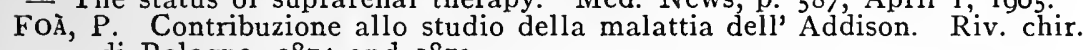
di Bologna, 1874 and 1879 .

FOÀ, C. Contr. anatomica e sperimentale alla patologia delle capsule surrenali. Arch. p. 1. scienze med., 24 , No. 22, 1900, and 25, p. 437, 1901.

- Sull' innesto delle ovaie e dei testicoli. Riv. gen. d. biol., 19or; and A. i. B., 35 .

- Contribution anatomique et expérimentale à la pathologie des capsules surrénales. A. i. B., 35, p. 250, 1901 ; ibid., 36, p. 237, 1902.

- Sui fattori che determinato la funzione della ghiandola mammaria. A. d. F., 5, 1909 .

Fò̀ and GATIN-GRUZEWSKA. Action de l'adrénaline sur la réaction du sang. C. r. S. B., 57, ii, p. 145,1905 .

- Influence de la piqûre diabétique sur la réaction du sang. Ibid., p. 144.

FOA and PELlaCani. Sur le ferment fibrinogène et sur les actions toxiques exercées par quelques organes frais. Arch. p. l. scienz. med., vii, p. 9,1883 ; A. i. B., iv, 1883 .

- Interno agli effetti tossici delle diluzione aquose degli organi freschi. A. per le scienz. med., iii, p. 24, 1879 .

FODERÃ and PITTAU. Studi sull' Hypophysis cerebri. Pathologica I, Igog.

FoDERÉ, F. M. Über den Kropf und Cretinismus. Aus d. Franz. v. Lindmann. Berlin, 1796 .

FölgER, A. Untersuchungen über die pathologische Anatomie der Nebennieren bei einigen Haustieren. Biolog. Ges. in Kopenhagen, 1907; S. A., 21, p. 277 , 1909.

FÖRSTER. Fall von Markschwamm mit ungewöhnlich vielfacher metastatischer Verbreitung. V. A., 13, p. 271, 1858 .

Försterling, W. Kasuistische Beitr. z. Kenntnis d. Morbus Addisonii. Diss., Berlin, I 898 .

Foges, A.' Zur Hodentransplantation bei Hähnen. C. P., 1898.

- Schwangerschaftshypertrophie der Mammæ und Nebenmammæ. W. k. W., I901.

- Zur Lehre von den sekund. Geschlechtscharakteren. P. A., 93, p. 39, 1902.

- Ein Fall von Hermaphroditismus masculinus internus. Beitr. z. G. u. G., Festschr. f. R. Chrobak. Wien, 1903.

- Ovarientransplant. in d. Milz. W. k. W., p. 615, 1907 and p. 271, 1908.

- Zur physiolog. Beziehung zwischen Mamma u. Genitale. C. P., 19, $1905 ;$ W. k. W., 1908.

Forsy. Nouveau procédé d'anesthésie des tissus enflammés. C. r. S. B., $55,1903$.

FoNTANEL, P. L'Asthénie motrice bulbo-spinale. Thèse de Lyon, 1905.

FORMÁNEK, E. Action de la choline sur la circulation. Arch. intern. d. Pharm., 10, 1903.

FORMÁNEK, E., and EISELT, R. Über die therapeutische Wirkung des Nierenextraktes bei chronischer Nephritis. Ibid., xvii, p. 231, 1907. 
FORMÁNeK and HaSkovec. Über die Funktion der Schilddrüse. Klin. Z. u. Streitfr., 9, Wien, I 895 .

ForschBaCH, J. 'Parabiose und Pankreasdiabetes. Vorl. Mitt. D. m. W., 1908.

- Zur Pathogenese des Pankreasdiabetes. A. P. P., 60, I909.

- Behandlung d. Diabetes mellitus mit Zuelzers Pankreashormon. D. m. W., 1909 .

FORSYTH, D. The Parathyroids. Quart. Journ. of Med., vol. i, p. 2, 1908.

- The comparative anatomy, gross and minute, of the thyroid and parathyroid glands in mammals and birds. J. A. P., 42, 1908.

FossatTI, G. Di alcuna proprietà dell' estratto etereo dell' urine specie in rapporte al puerperio ed all' allattamento. Ann. Ostetr. Ginec., 28, p. 88.

Foster, A. Case of Addison's disease treated with suprarenal extract. Lancet, June 10, 1899 .

Foster, N. S. Cases of diabetes treated with secretin. J. B. Ch., 2, p. 297, I907.

- Beobachtungen über die Wirkung des Sekretins bei Diabetes und Betrachtungen über seine Anwendung. M. K., 1907 .

Fraenkel, A. Über den Gehalt des Blutes an Adrenalin bei chroniscner Nephritis und Morbus Basedowii. A. P. P., 6o, p. 395, Juni, 1909.

Fraenkel, F. Ein Beitrag zur Therapie des Morb. Add. mit Nebennierenpräparaten. Diss., Berlin, I900.

FRAENKEL, L. Versuche über den Einfluss der Ovarien auf die Insertion des Eies. V. d. d. Ges. f. Gyn., rgor.

- Zur F unktion des Corpus luteum. A. G., 68, 1902.

- Weitere Mitteilungen über die Funktion des Corpus luteum. C. G., 28, 1904.

- Vergleichende histolog. Unt. über das Vorkommen drüsiger Formationen im interstitiellen Eierstocksgewebe. A. G., 75, p. 433, 190.

- Ovarialankörper und Osteomalakie. M. m. W., Nos. 25 and 26, 1908.

FraENkEL and COHN, F. Experim. Unters. über den Einfluss des Corpus luteum auf die Insertion des Eies. An. An., 20, 1901.

Fraenkel, S. Präparate aus der Nebenniere und Schilddrüse. W. k. W., 1896.

- Beitrag zur phys. Chemie der Thyreoidea. W. m. Bl., Nos. 13-15, 1896.

- Beiträge zur Physiol. und physiol. Chemie der Nebennieren. Ibid., Nos. $14-16$, 1896.

- Über Gehirnchemie und die Phosphatide der Gewebe. W. m. W., Igog.

- Gehirnchemie. E. P., 8, 1909.

- Über Lipoide. VI. Mitteilung. Über ein neues Verfahren der fraktionierten Extraktion der Gehirnlipoide. B. Z., 19, 1909.

Fraenkel, S., and ALLERS, R. Über eine neue charakteristisclie Adrenalinreaktion. B. Z., 18 , p. 39 , I909.

FRÄNKEL, STADELMANN and BENDA. Klinische und anatomische Beiträge zur Lehre von der Akromegalie. D. m. W., rgor, and Leipzig, I901.

FRANCHINI, GUISEPPE. Beiträge zum chemischen und histologischen Studium des Blutes bei Acromegalie. B. k. W., 1908 .

- Contrib. à l'étude de l'acromégalie. Riv. sper. di fren., 33, p. 888, 1907.

Franchini, G., and GIGlioli, G. J. Encore sur l'acromégalie. Nouv. icon. de la Salpêtrière, No. 5, p. 325, 1908.

Francillon, M. Essai sur la puberté chez la femme. Thèse de Paris, 1905.

FRANCIS, E. Suprarenal extract in Addison's disease. Brit. Med. Journ., May 2, 1896.

- A case of hæmophilia treated with adrenalin chloride. Ibid. May 28, 1904.

Franck, Fr., and HaLlion. Recherches sur l'innervation vasomotrice du corps thyroïde. J. d. P. P., $\mathrm{x}, \mathrm{pp} .442-454,15 \mathrm{mai}, 1908$.

Franke. Akromegalie. Th. M., p. 35, 1896.

FRANK and ISAAC, C. Die Bedeutung des Cholins und des Adrenalins fürdie Erforschung des Zuckerstoffwechsels. Z. e. P., vii, p. 326, Oktober, I 909 .

FrankL, O. Zur Frage der Plazentartoxine. Gynaek. Rundsch., Bd. 3, H. 3 .

FRANKL, TH. Über den Antagonismus der Chloride der Erdalkaliẹn und des Kaliums gegenüber dem Adrenalin. P. A., 130, 1909. 
Frankl-Hochwart, v. Die Tetanie der Erwachsenen. Wien, 189r. 2. Aufl., 1907.

- Die Schicksale der Tetaniekranken. W. m. W., 1906; and N. C., Nos. 14 and $15,1906$.

- Diskussion über Bergers Fall. Verein f. Psych. u. Neurol., Io Mai, 1904 .

- Die Diagnostik der Hypophysentumoren ohne Akromegalie. 16. Int. med. Kongr., Budapest, 1900; W. m. W., Igog.

- Úber die Diagnose der Zirbeldrüsentumoren. D. Z. N., 37, p. 455, 1909.

FRANKL-HOCHWART, v., and FRÖLICH. Über die Wirkung des Pituitrins. W. k. W., 1909 .

FraNz. Aus experimentellen Arbeiten über Adrenalin, seine physiologische Wertbestimmung und Wirkung. M. K., 1907 (Sammelreferat).

- Exstirpation eines groszen Nebennierentumors. W. m. W., I904.

FREDELI. Ricerche sull'azione terap. dell'ovarina in rapp. con una nuova teoria della clorosi. Rif. med., I 896.

FrerichS. Über den Diabetes. Berlin, 1884.

FREUND, H. W. Beziehungen der weiblichen Geschlechtsorgane zu anderen Organen. Z. Ch., 18, 1883; L. O., 3, 1898 .

- Über Akromegalie. Volkmanns klin. Vortr., Nos. 329-330, 1889.

Freund, H. Lokalanästhesie mittelst Eukain-Adrenalin. C. G., 1904.

FREUND, R. Zur plazentaren Eklampsie-Ätiologie. B. k. W. No. 15, p. 682,1909 .

FREY, $H$. On the suprarenal capsules. Todd, Cyclopædia of Anat. and Physiol., London, 1849 .

FREY, v. Beiträge zur Kenntnis der Adrenalinwirkung. W. V., 1905.

FREYER, O. Zur Kenntnis der von versprengten Keimen der Nebenniere. ausgehenden Abdominalgeschwülste, \&c." Diss., Kiel, 1901.

FRIEDENTHaL. Zur Wirkung der Schilddrüsenstoffe. M. m. IV., 1908.

FRIEDJUNG, J. K. Erkrankungen der Thymus, Status lymphaticus und plötzliche Todesfälle. Handbuch der Kinderheilk. von. Pfaundler und Schloszmann, Bd. iii, 2. Auff., 1910.

FRIEDLAND, F. Über einen Fall von accessorischen Nebennieren in den beiden Samensträngen. P. m. W., 1895.

Friedleben, A. Die Physiologie der Thymus in Gesundheit und Krankheit. Frankfurt, 1858 .

FRIEDMANN. Rhachitol bei Rachitis. Der Kinderarzt, Igoo.

- Über Nebennierensubstanz in der Therapie der Rhachitis. Th. G., p. 283,1901 .

FriediANN, E. Die Konstitution des Adrenalins. H. B., 6, 1904; ibid., 8, p. $95,1906$.

FRIEDMANN, F. Beiträge zur Kenntnis der Anatomie und Physiologie der männlichen Geschlechtsorgane. A. m. A., 52, 1898 .

FRIEDMANN and MAas. Über Exstirpation der Hypophysis cerebri. B. k.W., 1900.

- Noch einige Erfahrungen über Exstirpation der Hypophysis cerebri und über Transplantation von Carcinom u. Thyreoidea auf die Hypophysis. B. k. W., I902.

FRIEDREICH. Psammoma kystomatosum haemorrhagicum der Glandula pinealis in Kombination mit Medullarsarkom. - V. A., 33, p. 165, 1865.

FRISCH, A. v. Adrenalin in der urologischen Praxis. W. k. W., Igoz.

FrIsco. Giorn. internaz. d. sc. med., Napoli, 1897.

FrITSCHE and KLEBS. Beitrag zur Pathologie des Riesenwuchses. Leipzig, 1884 .

FRÖHLICH, A. Fall von Tumor der Hypophysis cerebri olne Akromegalie. W. kl. Rundschau, Igor.

- Eine neue physiologische Eigenschaft des d.-Suprarenins. C. P., 22, 1909.

Fröhlich and Loewi, O. Unters. z. Physiologie und Pharmakologie des autonomen Nervensystems. A. P. P., 59, 1908.

Fromaget, C. De l'extrait aqueux de capsules surrénales en ophtalmologie. Ann. de la Policl. de Bordeaux, V. 8, p. 613, mars, 1898.

Fromue, V. Experimentelle Untersuchungen zur parathyreoidalen Insuffizienz in bezug auf Eklampsie und Tetanie mit besonderer Berücksichtigung der antitoxischen Funktionen der Parathyreoideae. M. G. G., 24, 1906 . 
Frouln, A. Sur les variations de la sécrétion du suc intestinal. C. r. S. B., 58, p. 653 .

- Action du suc intestinal sur la sécrétion entérique. Ibid., p. 702; C. r., I40, p. I I20.

- Action sécrétoire du suc gastrique sur la sécrétion stomacale. Ibid., p. 887.

- La sécrétion et l'activité kinasique du suc intestinal ne sont modifiés par le régime. Ibid., p. 1025, 1905 .

- Sur la présence et l'origine d'acides organiques dans le suc gastrique pur. Ibid., 59, p. 392, I905.

- Influence des produits de la digestion des albuminoïdes et des sucres Sur l'action secretoire de l'HCl sur la sécrétion pancreatique. C. r. S. B., 63, p. 519 , 1907 .

- Ablation des capsules surrénales et diabète pancréatique. Ibid., 64, p. 216 , I 908 .

- Sur la possibilité de conserver les animaux après l'ablation complète de l'appareil thyroïdien, en ajoutant des sels de calcium eu de magnésium à leur nourriture. C. r. A., Bd. 148, p. 1622, 1908.

- Sur l'asepsie du suc pancréatique. Arch. int. de Phys., vi, p. 253, I908.

FroIs, G., and RIVET, L. Adénome des capsules surrénales et l'hypertension dans l'hémorrhagie cérébrale. Gaz. des hôp., No. 67, 1906.

Frugoni, C. Adrenalin-Glykosurie und ihre Beeinflussung durch das Extrakt und den Saft des Pankreas. B. k. W., Igo8.

-. La glycosurie adrénalinique et l'influence qu'exercent sur elle l'extrait et le suc pancréatique. A. i. B., 50, p. 209, 1908.

Frugoni, C., and Grixoni, G. Tubercolosi e tiroide. Riv. crit. clin. med., I0.

- Einflusz der wirksamen Elemente der Schilddrüse auf die experimentellen, tuberkulösen und pseudotuberkulösen Infektionen. B. k. W., 1909.

FRUGONI and StRadiotTI. Interno alla funzione dell' isole del Langerhans. Lo Sperimentale, 1909: and A. i. B.. 51, p. 186, 1909.

Fuchs. Zur Frühdiagnose der Hypophysistumoren. W. k. W., I903.

- Die Diagnose des Hypophysentumors. Jahrb. f. Psych., 26, 1005.

FürRRINGER. Die Störungen der Geschlerhtsfunktionen des Mannes. Nothnagels Handbuch, io, 3. Teil.

FÜRTH, O. V. Zur Kenntnis der brenzkatechinähnlichen Substanz in den Nebennieren. Z. ph. Ch., 24, p. 142, 1897; II. Mitt., ibid., 26, 1898; III. Mitt., p. 105, 1050.

- Mahnung zur Vorsicht beim Gebrauche von Nebennierenpräparaten. D. m. W., 1002 .

- Zur Kenntnis des Suprarenins. H. B., I, p. 243, I901.

- Zur Kenntnis des Suprarenins (Adrenalins). S. W. A., i12, 1903.

- Neuere Untersuchungen über die chemische Zusammensetzung der gefäszverengernden Substanz in den Nebennieren. B. C., ii. I903.

- Die Beziehungen der Schilddrüse z. Zirkulationsapparate. E. P., 8, I 909 .

FÜRTH, O. v. and SCHWARZ, K. Über physiol. Wirkungen d. Jodothyrins. W. k. W., r 908 .

- Uber die physiologischen Wirkungen des Jodothyrins und der Schild. drüsenextrakte. V. 25 , C. M. Wien, p. 400, April, I908.

- Über die Einwirkung d. Jodothyrins auf d. Zirkulationsapparat. P. A., I24, 1908 .

- Über die Natur der blutdruckerniedrigenden Substanzen in den Schilddrüsen. P. A., I24, p. $36 \mathrm{r}, \mathrm{r} 908$.

- Zur Kenntnis der Sekretine. P. A., 124, 427, 1908.

- Bemerkungen zur Jodothyrinfrage. C. P., 22, 1909.

- Zusatz zu der Abhandlung: Úber die Natur der blutdruckerniedrigenden Substanzen in der Schilddruise. P. A., 125, 1909.

Fuhr. Die Exstirpation der Schilddruise. A. P. P., 2I, 1886.

- Der Kropf im Altertum. V. A., I1 $2,1887$.

FunRmann, F. Der feinere Bau der Nebenniere des Meerschweinchens. An. An., 24, p. 606, 1904, and Z. w. Z., 78, p. 522, 1905.

Furnivall. On a case of acromegaly, \&c. Transact. Path. Soc., 49, London, 1908.

FUSARI, R. Osservazioni sulle terminazioni nervose e sullo sviluppo delle capsule surrenali. Rendic. d. Accad. dei Lincei, 6, p. 250, i89o; and A. i. B., I6, p. I9I, I891. 
FUsari, R. Contribuzioni alla studio dello sviluppo delle capsule surrenali e del simpatico nelle pollo e nei mammiferi. Arch. p. le scienze med., 16, p. 14, 1892 ; and A. i. B., 18, pp. 161-182, 1893.

- Sullo sviluppo delle capsule surrenali. Accad. di sc. med. e nat. di Ferrara, I 893 .

- Sullo sviluppo degli organi paratiroide del uomo. Ac. med., Torino, febbrájo, I 899 .

GabBi. Casi clinici di Morbo dell' Addison Considerazioni sulla Genesi del Pigmento. Rivista clin. di Bologna, p. 569, r 886.

- Forme fruste od incomplete del morbo di Addison. Clin. med. ital., 1899 .

Gaglio, G. Sul diabete, che segue all' estirpazione del pancreas. Rif. med., I89ז.

- Ric. sper. sulle rane int. alle funzione della ipofisi. Acad. Pelorit. Messin., 1900.

- Recherches sur la fonction de l'hypophyse de cerveau chez les grenouilles. A. i. B., 38, p. 117, 1902.

- Influenza della temperatura sulle glucosurie tossiche. A. P. P., p. 224, Suppl., 1908.

Gahmig, Max. Über einen Fall von Osteomalakie bei Mäusen. Diss., Leipzig, 1907.

GaILlaRD. L'hyperplasie surrénale dans ses rapports avec l'hypertension artérielle permanente la néphrite chronique et l'athérome. C. r. S. B., 63 , p. $569,1907$.

- De l'hyperplasie surrénale, \&c. Thèse de Paris, Ioo8.

Gamgee, A., and Jones, IV. Über die Nukleoproteide des Pankreas, der Thymus und der. Nebenniere, \&c. H. B., iv, p. 10, 1903; P. R. S., 71, p. 385,1903 .

Galdi. La glycosuria surrenale. Il Tommasi, I907.

GaLEOTtr. Z. Kennt. d. Sekretionsersch. in den Epithelzellen d. Schilddrüse. A. m. e., 1896 .

GANGHOFNER, F. Über plötzliche Todesfälle im Kindesalter. V. d. N. Ä., 1902.

GANFINI, C. Alcune particolarità morfologiche e topografiche delle glandulae suprarenales dell' uomo. Arch. ital. d'anat., iv, F. I, pp. 63-8o, 1905 .

GarbiNI, G. La structure de la fonction de l'hypophyse dans qq. formes graves, congénitales ou consécutives, de psychopathie. Riv. di pat. nerv., x, p. 440, 1905 .

- Contr. clinique et anatomo-pathologique à la connaissance du myxœedème postopérat., avec considérations sur la fonction de l'hypophyse. Ibid., xi, 1906.

Gardner, iV., and Allen. Azoospermia. Boston Med. and Surg. Journ., I34, I 896.

GARNIER, CH. Les filaments basaux des cellules glandulaires. Bibliogr. anat., 1897 .

- De la structure et du fonctionnement des cellules glandulaires. J. A. P., 1900.

GARNIER. Influence de l'adrénaline sur le développement des gangrènes microbiennes. C. r. S. B., 54, p. 1440, 1903.

GARNIER, M., and THAON, C. Action de l'hypophyse sur la pression artérielle et le rythme cardiaque. Ibid., 6o, p. $285,1906$.

- Recherches sur l'ablation de l'hypophyse. Ibid., 62, p. 659, 1907.

- De l'hypophyse sur la pression artérielle et le rythme cardiaque. J. d. P. P., 1906.

GaRRÉ, Transplantationen in der Chirurgie. V. d. N. Ä., Stuttgart, 1906.

GarroD, A. E. Pineal cyst. Transact. of the Path. Soc. of London, B. 50, p. 12,1898 .

GARROD, A. E., and DRYSDALE, J. Hæmorrhage into both suprarenal capsules. Ibid., 1898 .

GASKELL, W. E. On the origin of vertebrates deduced from the study of ammocœtes. Part II. The origin of the vertebrate body cavity and excretory organs; the meaning of the somites of the trunk and of the ductless glands. J. o. A. a. P., 27, 1903. 
GaSkelL, W. E. The contraction of cardiac muscle. Schaefer Text-book of Physiology, ii, London, 1900.

Gaspero, DI. Der psychische Infantilismus. A. f. Psych., I907.

GatiN-GRuzewska, J. Composition du foie des chiens nourris en vue de la production de la quantité maximale de glycogène. C. r. S. B., 58, p. 423,1905 .

- Influence de la dilution des solutions d'adrénaline sur la disparition du glycogène dans le foie et dans les muscles. Ibid., 60 , p. 940, I906.

Gatin-GruzEWSKA, J., and MACiAG, M. L'action de l'adrénaline pure sur le cœur isolé. Ibid., 63, p. 28, I5 janvier, 1907, and J. d. P. P., xi, pp. 28-31, 1908 .

GitTA. Sulla distruzione della ghiandola pituitariae tiroide. Gazz. degli osped., I 896.

GatTI, G. Über die von abgesprengten Nebennierenkeimen ausgehenden Nierengeschwülste. V. A., 144, 1896.

- Der Lecithingehalt der Grawitzscben Nierenstrumen. Ibid., I50, p. 4I7, I 896.

GaUdERER, L. Zur Kasuistik der Zirbeldrüsentumoren. Diss., Gieszen, I 889 .

GaUChLER and Roussy. Sur un cas d'acromégalie avec lésions associées de toutes les glandes vasculaires sanguines. Rev. Neur., p. 356, 1905.

Gaultier, R. Glycosurie expérimentale par destruction étendue de la muceuse duodénale à l'aide d'un caustique. C. r. S. B., 64, 1908.

- Recherches sur la rôle de la tension artérielle dans la production de l'athérome expérimental par l'étude de l'action simultané de l'adrénaline substance hypertensive et de "l'extrait aqueux de gui" subst. hypotensive. Ibid., 64, p. I1 50 , 1908.

Gaupp, E. Anatomie des Frosches. Braunschweig, 1901.

GaUssel. Un cas d'acromégalie. Nouv. Icon. de la Salpêtrière, 19, p. 39I, 1906.

Gauthier, G. Un cas d'acromégalie. Le Progr. méd., p. I 109, 1890; p. 4, 1892 .

- Les toxines microbiennes et animales. Paris, $\mathbf{1} \$ 96$.

- Localisation, élimination et origine de l'arsénic chez les animaux. Bull. acad. de méd., I90o.

- Fonctions du corps thyr., pathogénie du goitre endémique, du goitre sporadique, du goitre exophtalmique, \&c. Revue de Méd., 20, 1900.

- La fonction menstruelle et le rut animaux. Bull. ac. méd., 1900.

- Les médications thyroïdiennes. Paris, 1902.

- Réactions comparées de l'adrénaline et de la pyrocatéchine avec le permanganate de potasse. C. r. S. B., 66. p. 426, 1909 .

- Remarques sur la réaction d'Ehrmann. Ibid., 67, p. 426, I909.

-- Application de la réaction d'Ehrmann à la mise en évidence de l'adrénaline dans les surrénales de la grenouilles. C. r. S. B., 67, p. 490, I909.

Gautrelet, J. Présence de la choline dans certains glandes. Action de leur extrait sur la glycosurie adrénalinique. Ibid., 64, p. 174, 1908.

- Mécanisme de l'action hypotensive de certains glandes. Ibid., p. 176, 1908.

- Choline et glycosurie adrénalique. C. r. S. B., 65, pp. 173-174, 1908.

- La choline dans l'organisme antagonisme des appareils chromaffine et cholinogène. Ibid., 65, p. 448,1908 .

- Du rôle hypotenseur de la choline d. l'organisme. C. r. A., 148, 1909.

- La choline, son rôle hypotenseur dans l'organisme. J. d. P. P., xi, p. 227,1909 .

Gautrelet, J., and Thomas, L. Le sérum normal neutralise la glycosurie adrénalique. C. r. S. B., 66, p. 438.

- L'ablation des surrénales supprime la glycosurie adrénalique, et glycosurée phloridizine. Ibid., 66, p. 798, 1909.

- La respirations après ablation des surrénales. C. r. S. B., 66, p. 1042.

- La choline dans le sérum de chien de capsulé. Ibid., 66, p. 1o9o, 1909.

- Contribution à l'étude du cœur est de la pression artérielle chez le chien décapsulé. Ibid., 67, p. 231, 1909.

- Chez le chien décapsulé, l'excitation du splanchnique ne produit pas de glycosurie. Ibid., p. 233.

- Action hypotensive du sérum de chien privé de surrénales. C. r. A., 149,12 juillet, 1909 . 
Gautrelet, J., and Thuan. Influence de la polypnée sur la glycosurie adrénalique. C. r. S. B., 64, 1908 .

Gayme. Essai de pathogénie basedowienne. Dauphiné médical, Nos. 8 and 9,1899 .

GEBHARD. Menstruatio praecox in Veits Handb. d. Gynäkologie, 3, I, 1808.

GEDDES, A. G. Examination of the acromegalic subject. Edinb. Med. Journ., 1909.

GEIS. The parathyroid glands.' Annals of Surg., 47, p. 532, I908.

GELLÉ. Du retentissement des lésions canaliculaires sur le parenchyme pancréatique et leur importance dans la genèsè du diabète. J. d. P. P., iv, p. 645 .

Gemelli, A. Contr. alla conosc. d. struttura d, ghiand. pituitaria. Bull. Soc. med., Pavia, Igoo.

- Nuove ric. sull' anatomia e embriologla dell' ipofisi. Ibid., 1903.

- Structure du feuillet juxta-nerveuse d. la portion glandulaire de l'hypophyse. Ibid., 55, 1903.

- Contribution à la physiologie de l'hypophyse. A. d. F., iii, novembre, 1905 .

- Les processus de la sécrétions de l'hypophyse des marnmifères. A. i. B., $47,1907$.

- Nouvelle contribution à la connaissance de la fonction de l'hypophyse. Societa milanese di medicina e biologica, December 13, 1907.

- Ulteriori contrib. alla conosc. della funz. d. ipofisi cerebrale. Rif. med., 1908 .

GENTĖs, L.' Morphologie et structure des îlots de Langerhans chez quelques mammifères; évolutions et signification des îlots en général. Thèse de Bordeaux.

- Ilots de Langerhans du Pancreas du chien. C. r. S. B., 54, 535; and ibid., 55,334 .

- Note sur la structure du lobe nerveux de l'hypophyse. C. r. S. B., $55,1903$.

- Lobe nerveux de l'hypophyse et sac vasculaire. Ibid., 5 mars, I907.

- L'hypophyse de vertébrés. C. r. S. B., 63, 190\%.

- La glande infundibulaire des vertébrés. Ibid., 3 juillet, I907.

GEORGIEWSKY, K. Wirkung der Schilddrüsenpräparate auf den tierischen Organismus. Z. k. M., 33, $153,1897$.

Georgopulos. Exp. Beitr. z. Frage d. Nierenwassersucht. Z. k. M., 6o, I 906.

Gerber, H. Nebennierencarcinom mit in den rechten Vorhof reichender Geschwulst. Thrombose der Vena cava. W. m. IV., I 904 .

GerhardT, D. Über die Wirkungsweise d. blutdrucksteigernden Substanzen der Nebennieren. A. P. P., 44, r9oo.

- Diskussionsbemerkung zum Vortrage von Kocher. V. C. M., 1906.

GERHARDT and SCHLESINGER, W. Über Kalk- und Magnesiumausscheidung beim Diabetes. A. P. P., 42, I 890 .

Gerhartz, H. Geschlechtsorgane und Hunger. B. Z., 2, p. 154, I906.

- Rudimentärer Hermaphroditismus bei Rana esculenta. A. m. A., 65, 1005 .

- Zur P̈hysiologie des Wachstums. B. Z., 12, p. 9\%, 1008.

- Männliche Geschlechtsorgane in Oppenheimers Handb. d. Bioch., iii, I, 1909 .

GESSARD. Sur le pigment des capsules surrénales. C. r. A., I38, I904.

Getrowa, S. Über die Thyreoidea von Cretinen und Idioten. 'Diss., Bern, 1905.

- Uber die Glandula parathyreoidea. V. A., I88, 1907.

GHEDINI, G. Untersuchungen über die Wirkung einiger Organextrakte. C. f. B., 34, p. $72 \mathrm{I}$, I903.

- Contrib. alla anat. patol. del pancreas. Rif. med., 933, 1904.

- Adipose non-douloureuse. Gaz. degli osp., p. 1639, I907.

GHEDINI and MASCHERPA. Sulla glicosuria adrenalinica. Acc. med., Genova, aprile, Igog; Pathologica, i, No. I2, I mai, 1909.

GHika, C. Etude sur le thymus. Thèse de Paris, 1901.

GIACOMINi, E. Sulle terminazioni nervose nelle capsuli surrenali degli uccelli. R. Accad. dei Fisiocritici di Siena. November, I897; A. i. B., 29, pp. $482-483,1898$.

- Sopra la fine struttura delle capsule surrenali degli Anfibii. Ibid., I 898 . 
Giacomisi, E. Brevi osservazioni intorno alla minuta struttura del corpo interrenale e dei corpi soprarenali dei selaci. R. Ac. dei Fisiocritici di Siena. Io, p. 835 , I $89 \$$.

- Sopra la fina struttura delle capsule surrenali degli Anfibii e sopra i nidi cellulari del simpatico di questi vertibrati. Contributo alla morfologia del systema delle capsule surrenali, Igo2.

- Sull' esistenza delle sostanza midollare nelle capsule surrenali dei Teleostei. Monit. zool. ital., anno 13, pp. 182-189, I902; Ibid., I3, 7, 1903.

- Contributo alla conoscenza del sistema delle capsule surrenali dei capsule surrenali dei Petromizonti. Ibid., pp. I-20, 1902.

- Contributo alla conoscenza delle capsule surrenali dei Ganoidi e particolarmente sulle esistenza della loro sostanze midollare. Ibid., anno 15, pp. 19-32, 1904 .

- Contributo alla conoscenza delle capsule surrenali dei Ciclostomi. Sulle capsule surrenali dei Missinoidi. R. Accad. delle sc. dell' Istituto di Bologna, 29 maggio, 1904.

- Contributo allo conoscenza del sistema delle capsule surrenali dei Telostei. Sulla sostanza midollare (organi soprarenali o tessuto cromaffine) di Aiurus catus. R. Accad. delle Sc. dell' Istituto di Bologna, pp. 183-189, 1905 .

- Sulle capsule surrenali e sul simpatica dei Dipnoi. Ricerche in Protopterus tanectens. Rendiconti della R. Acc. dei Lincei, vol. xv, $I^{\circ}$ sem., sér. 5a, fasc. $7^{\circ}$, I 906 .

- Il sistema interrenaTe e il sistema cromaffine (sistema feocromo) nelle anguilla adulte, nelle cieche e nei leptocefali. R. Accad. d. Science di Bologna. 24 maggio, 1908.

- A propos de l'action physiologiques des capsules surrénales. Biol. méd. Ann., 6, pp. 133-1 58, 1908.

GiANNELLI, L. and Giacomini, E. Ricerche istologiche sul tubo digerente dei rettili 3 nota. Intestina media e terminale, fegatopancreas. Acc. d. Fisiocritici di Siena, i 896 .

Grannell, L. Sullo sviluppo del pancreas. R. Acc. dei Fisiocritici Siena, ser. 4, 10, 1898 .

- Sul valore morfologico degli accumuli di Langerhans. Ibid., I2, I900.

- Sulla disposizione degli accumuli di Langerhans nel Pancreas degli anfibi urodeli. R. Acc. dei fisiocritici Siena, IS99; and Monit. Zool. Ital., 12, p. 207, 1900.

- Pancreas interepatico negli anfibi urodeli. Monit. Zool. Ital., 10, Suppl., I 890 .

- Ricerche istologiche sur pancreas degli uccelli. Monit. Zool. Ital., I3, 1902.

GIARD, A. De l'influence de certains parasites rhizocéphales sur les caractères sexuels extérieurs de leur hôte. C. r. S. B., 103, p. 84, I 886 .

- Sur la castration parasitaire chez l'Eupagurus Bernhardus Linné et chez la Gebià stellata Montagu. Ibid., I04, p. III3.

- Sur un Copépode (Cancerilla tubulata Dalyell) parasite de l'Amphiura squamata, Delle Chiaje. Ibid., ro4, p. I189.

- Sur la castratión parasitaire de l'Hypericum perforatum par la Cecidomya hyperici Bremi et par l'Erysiphe Martii Leo. Ibid., Iog, p. 324 .

- Sur la castration parasitaire de Typhlocyba par une larve d'Hyménoptère et par une larve de Diptère. Ibid., 109, p. 708.

- Comment la castration agit-elle sur les caractères sexuels secondaires? C. r. S. B., 56, p. 4,1904 .

Giard and Boxnier, J. 'Sur la philogénie des Bopyriens. C. r. A., Io4, p. I309.

Giard, A., and Julie, A. La castration parasitaire et ses conséqu. biologiques. Rev. gẹ́n. de sciences, ann. v, No. I5, 26 août, I904.

GibBes. On some points in the minute structure of the pancreas. Quat. Journ. of Microsc. Science, 24, 183, 1884 .

Gibelli, C. La funzione delle capsule surrenali in rapporto col processo di riparazione delle fratture e coll' etiologia dell' ulcera gåstrica. Pathologica, i, p. 131, 1909.

GIERKE, E. Das Glykogen in d. Morphologie des Zellstoffwechsels. Zieglers Beitr., 37, p. 516, 1905 . 
Gierke, E. Das chromaffine System und seine Pathologie. L. O., 10, p. $503,1906$.

- Über Knochenmarksgewebe in der Nebenniere. Ziegl. Beitr., Festsch. Arnold, 1905.

-- Die Persistenz und Hypertrophie der Thymusdrüse bei Basedowscher Krankheit. M. m. W., I907.

- Drüsen mit innerer Sekretión. Handb. d. path. Anat. von Aschoff, 2, p. 751,1909 .

GIGoN, A. Die Menge des aus Eiweisz entstehenden Zuckers beim Diabetes. D. A. k. M., 97, p. 376 , 1904 .

- Über die Gesetze der Zuckerausscheidung beim Diabetes mellitus. III. Mitteilung. Z. k. M., 63, 1907.

GILBERT and CARNOT. L'opothérapie, Paris, 1898.

GILBERT and CASTIGUE. Infection thyroïdienne et goitre exophtalmique. C. r. S. B., 5 I, p. 463,1899 .

GILDERSLEEVE, N. A study of the properties of the serum of rabbits treated with the adrenal glands and erythrocytes of guinea-pigs. Univ. of Pennsylvania, Med. Bull., 17, p. 183; Ref. B. C., iii, p. 169.

Ginsburg. Contr. to the anat. of parathyroid bodies. Un. Pennsylv. med. Bull., January, 1908.

GIOFFrEDI, C. La distruzioni dell' adrenalina nel' organismo. Arch. Farmac., vi, p. 145, 1907.

GIOFFrEDI, C., and ZiINO, A. Contr. alla pathologia del Morbo di Addison. Rif. med., 1895 .

GIORDONI. Diagnostic des tumeurs de l'hypophyse par la radiographie. Thèse Paris, Igo6.

GIUFFrIDA-RUGGERI. Considerazioni antropologiche sull' infantilismo e conclușioni relative all' origine delle varietà umane. Monit. zool. Ital., apríle and maggio, 1903 .

Gizelt, A. Einflusz des Darmextraktes und des Pepton Witte auf die Harnsekretion. P. A., 123 , 1908 .

GLÄSER. Untersuchungen über Cholesteatome und ihre Ergebnisşe für die Lehre von der Entstehung der Geschwülste. V. A., 122, 1899.

GLASERFELD, BRUNO. Die Epithelkörperchen und ihre Beziehungen zur Pathogenese der Tetanie. B. k. W., 1909.

Glassner, K., and PICK, E. P. Über d. 'Ausscheidung d. Arenalins. V. 25 , C. M., 1908.

- Unters. üb. d. gegenseitige Beeinflussung v. Pankreas und Nebennieren. Z. e. P., 1909.

GLEY, E. Détermination de la toxicité du sérum -sanguin chez les chiens thyroïdectomisés. A. d. P., p. 283, 1894; 771, 1895 .

- Sur les effets de l'exstirpation du corps thyroïde. C. r. S. B., 1891 .

- Note sur les fonctions de la glandule thyroïde chez le lapin, \&c. C. r. S. B., p. 843,1891 .

- Sur le diabète alimentaire chez les animaux privé du pancréas. Ibid., 752 , 1891 .

- Des effets de la thyroïdectomie. Remarque à Moussu. Ibid., $1 \$_{92}$.

- Des troubles tardifs consécutifs à la thyroïdectomie chez le lapin. Ibid., p. 666, 1892 .

- Action d'un liquide extrait du pancréas sur les chiens diabétiques. A. d. P., 753, 1892 .

- Effets de la thyroïdectomie chez le lapin. Ibid., 1892.

- Nouvelles recherches sur les effets de la thyroïdectomie chez le lapin. Ibid., 1892.

- Contr. è l'étude des effets de la thyroïdectomie chez le chien. Ibid., 1892.

- Remarques sur quelques travaux de la phys. de la glande thyroïde. Ibid., 1892 .

- Glandes et glandules thyroïdes du chien. Ibid., p. $217,1893$.

- Nouvelle preuve de l'importance fonctionelle des glandules thyroïdes. Ibid., p. 396.

- Accidents consécutifs à la thyroïdectomie chez deux chèvres. Ibid., p. 453,1894 .

- Les résultats de la thyroïdectomie chez le lapin. Ibid., 1893.

- La question des rapports entre la rate et la glande thyroïde. Ibid., 1894 and 1895 .

- Des effets de l'exstirpation des glandules parathyroïdes chez le chien et le lapin. Ibid., p. 18, 1897. 
GLEY, E. Bemerkungen über die Funktion d. Schilddrüse. P. A., 66, 1897. - Glande thyroïde et thymus. C. r. S. B., p. 1017, 1909.

- Resumé des preuves des relations qui exist entre la glande thyroïde et les glandules parathyrö̈de. V. Physiol. Kongr. Turin. Progr. méd., p. 251 , 1901 .

_. Essais de philosophie et d'histoire de la biologie. Paris, 1900.

-Données exp. sur les corrélations fonctionelles chez les animaux. Ann. biol., i, p. 313,

GLEY and CHARRIN. 'Diabète expérimental. C. r. S. B., 836, 1893.

GLEY and LANGLOIS. Sur l'antagonisme réciproque de sécrétion déversés dans le sang par divers glandes. C. r. S. B., p. 109,1898 .

GLEY and Nicolas. Recherches sur les modif. histologiques, \&c. Ibid., p. 216,1895 .

GLEY and PHISALIX, C. La nature des glandules thyroïdiennes des chiens. ' C. r. S. B., 1893 .

- Sur les effets de la thyroïdectomie chez la salamandre. Ibid., I894.

- Sur la fonction des glandules thyroïdes. Congr. med. int. Rome, 1894.

GLIKIN, W. Zur biologischen Bedeutung des Lecithins. B. Z., vii, 286, 1907.

GLuck, TH. Thymuspersistenz bei Struma hyperplastica. B. k. W., I 894 .

GLUZIŃSKI. Physiologische Wirkung der Nebennierenextrakte. W. k. W., I 895 .

- Sur la toxicité de l'extrait des capsules surrénales. Przelgrad lek., March 2, 1895.

- Einige Bemerk. z. klin. Bilde des Klimakteriums. IV. k. W.. I9a9.

GLUZIŃSKI and LEMBERGER. Einflusz der Schilddrüsenbehandlung beim gesunden Menschen. C. i. M., I 897.

Godall, A. The postnatal change in the thymus of guinea-pigs. J. o. P., 32, p. I91, 1905 .

GODARD. Recherches sur les monorchides et les cryptorchides chez l'homme. C. r. S. B., mars, 1856 ; and Ibid., 1857 .

GODART. Recherches sur la transplantation de la glande thyroïde. Journ. d. méd. Bruxelles, 49, I 894 .

GöBELT. Versuche über Transplantation des Hodens in d. Bauchhöhle. C. a. P., 9, 1898 .

GörL. Ein neues Feld für Radiotherapie (Strumenbehandlung). Mr. m. W., 1905 .

Goetze, J. Physiologie und Pathologie der Nebennieren. Diss., Berlin, 1897.

GötZL and ERdheim. Zur Kasuistik der trophischen Störungen bei Hirntumoren. Z. H., 1905.

GolDBERG. Der Einflusz der Schilddrüsenexstirpation auf die Entwicklung junger Tiere. Podwyssozki' Arch. f. Path., 8, p. 912, 1897.

GoldBERG, B. Das Prostatasekret bei d. Prostatahypertrophie. Folia urolog., 1907.

GoldschmidT, A. Anwendung des Nebennierenextraktes in der Therapie der Nasen- und Halskrankheiten. Monatsch. Ohrenhk., p. 358, 1902.

- Beit z. Kenntnis der Pathologie d. menschl. Nebenniere. D. A. k. M., 98,1909 .

GOLDSCHMIDT, B. Unters üb. d. Vorkommen v. alim. Glykosurie bei M. Basedowii. Diss., Berlin, 1896 .

GoldsCHMIDT, S. Materialien zu einer Monographie über Nebennieren und Nebennierentherapie. Diss., Halle, 74 pp., 1904.

- Adrenalin in der Urologie. D. m. W., 1902 .

Goldstern, L. Die Krankheiten der Nebennieren. Zuelzers Handbuch, I 894 .

GOLDzIEHER and MolNar. Beiträge zur Frage der Adrenalinämie. W. k. IV., I 1908 .

-- Pathologie der Nebennieren. Orvosi Hetilap, No. 16, 1908.

Golodetz, L. Neue Reaktionen für Cholesterin und Oxycholesterin. Chem.-Ztg., I 1908 .

GolTz. Einflusz des Nervensystems auf die Vorgänge während der Schwangerschaft. P. A., 9, 1874 .

GOMES. De l'opothérapie ovarienne. Thèse de Paris, i 808 .

GoMez, OCAÑa. Nuevos hechos y viejas kysotesis sobre et aparato tireideo. Riv. ibero-amer. d. sc. med., 1899 ; Ref. J. d. P. P., i, 1899. 
Gomez, Ocaña. Sur les secrétions internes des glandes. Phys. Congr., Brussels, 1904.

- Assoc. font. entre thyroïde et les testic. on les ovaires. Madrid, I904 (Spanish).

Gonis. Du tractement de l'urémie par les inj. souscout. de néphrine. Lyon. Med., 1894.

GONTIER DE LA ROCHE. Modification histol. du pancréas après exclusion partielle chez le cobaye. Biol. anat., II, 232, 1902; and Thèse de Lyon, 1903.

GONTSCHARNKow. Über die Herstellung eines für die Schilddrüse spezifischen Serums. C. a. P., xiii, p. 121, Igoz.

GoodsIR, J. On the suprarenal, thymus and thyroid bodies. Philos. Trans. R. Soc., pp. 633-641. London, 1846.

GoRdEEFF, J.'M. Die sekretorische Arbeit der Magendrüsen bei verschiedenen Speisesorten. Arb. d. Ges. russ. Ärzte, November, 1905.

Gottlieb, R. Beitr. z. Physiologie und Pharmakologie der Pankreasfunktion. A. P. P., 33 .

- Über die Wirkung von Schilddrüsenpräparaten an thyreoidektomierten Hunden. D. m. W., 1896 .

- Über die Wirkung der Nebennierenextrakte auf Herz und Blutdruck. A. P. P., 38 , pp. 99-112, 1896 ; and Ibid., 43, p. 286, 1899 .

- Über Herzmittel und Vasomotorenmittel. V. 19, C. M., Igor.

GotTSCHALK, S. Fall von akzessorischer Nebenniere im Ligamentum suspensorum ovarii bei einer Erwachsenen. Z. G. G., 38, p. 332, 1898 .

- Über die Kastrationsatrophie der Gebärmutter. C. G., I 186.

GotTsChaU, M. Über die Nebennieren der Säugetiere und des Menschen. IV. B., pp. $454-462,1882$.

- Struktur und embryonale Entwicklung der Nebennieren bei Säugetieren. A. A.; pp. 412-488, Jg. 1883 .

- Über die Nebennieren der Säugetiere. Biolog. C., iii, pp. 565-576, 1883.

GotTsteIN. Versuche zur Heilung der Tetanie m. Implant. v. Schilddr. D. Z. N., 6, 1895 .

Gouget, A. Maladie bronzée. Bull. de la Soc. anat., 5, p. 77, 11 juillet, 1897 .

- Saturnisme expérimental. Hypertrophie considérable des capsules surrénales Sclérose aortique. C. r. S. B., 55, pp. 1659 and.1953.

- Injections d'adrénaline et sérum athéromatogène. C. r. S. B., 66, p. 375,1909 .

Gouinet and ANDExaRd, P. Fonction du thymus chez les bovidés. Ibid., 6o, pp. 342-344.

GoURFEIN. Recheches physiologiques et chemiques sur une substance toxique, extraite des capsules surrénales. Rev. med. de la Suisse rom., xv, 10, p. 513,1895 .

- Contribution à l'étude pathologique des capsules surrénales. Ibid., janvier, 1895 .

- Recherches physiologiques sur la fonction des glandes surrénales. Ibid., $\mathrm{xvi}, 3$, p. 113,1896 .

- Sur une substance toxique extraite des capsules surrénales. A gén. de méd., p. $500, .1895$; C. r. A., 121, p. 311,1895 .

- La rôle de l'autointoxication dans le mécanisme de la mort des animaux décapsulés. Ibid., 125, p. 188, 1897 ; C. P., p. 423, 1897.

- Trav. du Labor. de Phys. de l'Univers. de Genève, 1900.

GourLaY. The proteids of the thyroid and the spleen. J. o. P., I6, I894.

GRÄFENBERG, E. Eine Nebennierengeschwulst der Vulva als eine Metastase eines malignen Nebennierentumors der linken Seite. V. A., 194, pp. 13-32, 1908.

GraEsER. Adrenalin gegen Darmblutung bei Typhus. M. m. IV., 1903.

GRAF, R., and LANDSTEINER, $K$. Versuche uber die Giftigkeit des Blutserums bei Eklampsie. C. G., 1909.

Grafts, L. M. Glandes à sécrétion interne et le métabolisme. J. A. M. A., 1908.

GramegNa, A. Un cas d'acromégalie traité par Radiothérapie. Rev. Neurol., 1909.

GrandCLÉMENT. Comment et dans quel cas faut-il employer d'adrénaline pour guérir le glaucome sans opération. Lyon. méd., 37, 2, 1905.

GrandRY, M. Sur la structure de la capsule surrénale. J. A. P., 4, 225 and 389,1867 . 
Grant-LaEhLan. L'opothérapie contre l'hémophilie. Lancet, 5 novembre, 1904 .

Grassi and Munaron. Rendic. d. R. accad. dei Lincei, vols. xii and xiii, 1903 and 1904.

GRATIOLET, P. Système veineux des reptiles. L'Institut, i6 février, 1853.

- Veine porte du rein dans les oiseaux. Ibid., p. 386, 16 novembre, 1853

- Note sur les effets qui suivent l'ablation des capsules surrénales. C. r. A., 43 , p. 468 , septembre, 1856 ; A. de Méd., 1856 .

GraupNer, R. Malignes Hypernephrom mit hyaliner Degeneration. Zeigl. B. 24,1898 .

GRAVES, W. Über Lückenbilding zwischen den einzelnen Zähnen, ein frühdiagnostisches und bisher wenig bekanntes Zeichen der Akromegalie. M. f. Psych., p. is, 1904.

Grawitz, P. Die sogen. Lipome der Niere. V. A., 93, I883.

- Die Entstehung von Nierentumoren aus Nebennierengewebe. A. k. Ch., 30, 1884 .

- Über Wachstumsstörungen bei Kretinismus. D. m. W., 1885 .

Gray, A. M. H. On the development of the ductless glands in the chick. Philos. Trans. R. Soc., pp. 205-309, London, 1902; Sem. méd., 1902.

- A case of myxœdema with unusual features. P. R. S., December, 1907.

GraZIA, F. DE. Ricerche di ricambio nel diabete insipido. Rif. med., Bd. 23 , No. 29.

GRECo. Nuova entità clin. geroderma genito distrofico. Riv. pat. nerv., 1898 .

Green, E., and Moore. Report of a case of Addison's disease. New Y. Med. Rec., igoz.

GREENHORD, E. H. On Addison's disease. London, 1866.

GREER, J., and WELLS, G. H. The absence of adrenalin in malignant renal hypernephromas. A. int. Med., iv, p. 291, 1909.

GREGOR. Über die Unschädlichkeit der Verfütterung groszer Mengen von Thyreoidea an Kinder. Monatsch. f. Kinderh., i, p. 318, 1902.

Gregoire. Physiologie du corps thyroïde. Press. méd., p. 162, 1901.

GRENET and TANON. Acromégalie et diabète. Soc. de Neurol., 1907; Rev. neurol., p. 84 .

Grey, E. G., and DE SANTELLE. The relation of the thyroid glands to glycosuria. J. E. M., p. 659, 1909 .

GRIFFITHS. The results upon the testicle of ligature or division of the vas deferens. Lancet, April, I 895 .

GrigoriEFF. Die Schwangerschaft bei Transplantation d. Eierstöcke. C. G., 1897 .

Grimani, E. Sugli effetti del trapianto del timo e sulle correl. funtim. fra testicolo e timo. Arch. d. anat. patol., i, 1906; Ref. Bph. C.. i, 1560 .

GROBER, J. Über den Einflusz von Muskelarbeit und Auszentempatur auf das Masz der alimentären Glykosurie. D. A. k. M., 95, p. 137, 1908.

Grober. Massenverhältnisse d. Herzens bei künstlich. Arterienstarre. W. k. W., 1907 .

- Zum erblichen Auftreten der Basedowschen Krankheit. M. K., I9o8.

- Adrenalinerkrankung der Kaninchenleber. C. i. M., Igo8.

GroschUFF, K. Bemerk. zu der Mitteilung von Jacoby usw. An. An., 12, p. 20,1896 .

- Über das Vorkommen von Thymussegm. der vierten Kiementasche beim Menschen. Ibid., p. 161, I900.

Grosglick, S. Zur Frage über die Persistenz der Kopfniere der Teleostier. Z. A., Jahrg. 9, No. 219, pp. 196-198, 1886.

- Zur Morphologie der Kopfniere der Fische. Ibid., 8, pp. 605-611, 1885.

Gross, E. Beziehungen der Tetanie z. weiblichen Sexualapparat. M. m. W., 1906.

- Zur Funktionsprüfung des Pankreas. D. m. W., I909."

GRUBE, K. Über chemische Korrelationen im Organismus. M. K., 1909.

GRUBER, W. Untersuchungen einiger Organe eines Kastraten. A. A. P., p. 463 , Jahrg., 1847 .

GRÜNBAUM, O. F. F. A note on the effect of the administration of suprarenal gland by the mouth in health and disease. J. 0. P., 24, 2, p. 24 , 1899 .

- Suprarenal gland extract as a hæmostatic. Brit. Med. Journ., November, I900. 
Grynfeltt, E. Sur le corps interrénal des Plagiostomes. C. r. A., 135, pp. 349-441, 1902.

- Structure des corps suprarenaux des Plagiostomes. Ibid., 135, p. 373, 1902.

- Vascularisation des corps surrénaux chez les scillium. C. r. S. B., 1902.

- Recherches anatomiques et histologiques sur les organes surrénaux des Plagiostomes. Bulletin scientifique de la France et de la Belgique, 38 , pp. 1-137; et Thèse de Paris, 1903.

- Notes histol. sur la capsule surrénale des amphibiens. J. A. P., 40, p. 180,1904 .

- Sur la capsule surrénale des amphibiens. C. r. A., 137, pp. 77 and 78, 1903.

- Sur la capsule surrénale des amphibiens. Paris, 1904.

- Les organes chromaffines. Montpellier méd., 1903.

GUALDRINI. Dell' emostasi nella chirurgia epatica e nuovo contributo col cloruro di Adrenalina. Gazz. d. osped., p. 259, 1904.

GUARNIERI and MARINO-ZUCO. Recherches expérimentales sur l'action toxique de l'extrait aqueux des capsules surrénales. A. i. B., 10, 1888 ; and M. U., 14, pp. 59 and $617,1892$.

GUARNIERI and Magini. Etude sur la fine structure des capsules surrénales. A. i. B., I0, 1888 .

Guay. Essai sur la pathogénie de la maladie d'Addison. Thèse de Paris, 1893.

GUbler. Fall von akuter maligner Akromegalie. Korrespbl. f. Schweiz.

Güntz, L. ${ }^{30}$, 1900 . Z. Ch., 44,1908 .

Gürber. Üb. d. Einfl. des Thyreoidins auf den Stoffwechsel. W. B., 1806.

- Über die wirksame Substanz der Nebenniere. M. m. W., 1897; W. B., 1897.

- Zur Kenntnis der wirksamen Substanz der Nebenniere. C. P., I898.

GuERMONPREZ. Contribution à l'étude de la maladie bronzée d'Addison. Thèse Paris, 1875 .

Guerrint, G. Sur les fines modifications de structure de quelques organes dans le cours de la fatigue (fou, rein, hypophyse, capsules surrénales). A. i. B., 49, pp. 16I-170, I 908 .

- Sulla funzione della ipofisi. Speriment., 58, p. $837,1904$.

- Sur une hypertrophie secondaire expérimentale de l'hypophyse. A. i. B., 43,1905 .

- Uber die Funktion der Hypophyse. C. a. P., I6, 1905.

GuiaRT. Etude sur la glande thyroïde, dans la série des vertébrés. Steinheil, Paris, 1896.

GuiEysSE, A. La capsule surrénale chez la femelle du cobaye en gestation. C. r. S. B., 51, p. 898,18 novembre, I 899 .

- La capsule surrénale du cobaye. J. A. P., 37 ; and Thèse de Paris, 1901.

Gurhal, E. Le syndrome addisonienses. Gaz. des Hôp., ıоor.

GUILlaIN and AlQuiEr. Etude anatomopathologique d'un cas de maladie de Dercum. A. m. e., p. 680, septembre, 1906.

Guinard, L., and Martin, E. Action de l'extrait capsulaire de l'homme sain sur le rythme du cœur et sur la respiration. C. r. S. B., pp. 96 and 98,4 février, 1899 .

- Action cardio-vasculaires du suc thyroïdien. Ibid., p. 16I, 4 mars, 1899.

- Contrib. à l'étude des effets du suc surrénal. J. d. P., i, 744, 1899 .

GUINDER. Über Nierendekapsulation bei Eklampsie. M. m. W., 1908.

Guinon. Un cas d'acromégalie à début récent. Nouv. Icon. de la Salp., 3 , p. $16 \mathrm{I}, \mathrm{I} 890$.

- Infantilisme. Soc. de Pédiatrie, ro mai, Igor.

GuizetTI, P. Modificazioni del glicogene delle paratiroidi umane nelle malattie. Atti Soc. Ital. Progress. Sc. I Riun., Parma, pp. 283 and 284 .

Gulbenk. Deux cas de maladie d'Addison traités avec succés par l'adrénaline. Bull. gén. thérap., 150, p. 88, 1905.

Gulcke, N. Verhalten der Nebennieren bei kongenitaler Syphilis. V. A., 173 , 1903 .

Gull. A cretinoid state supervening in adult life in women. Transact. Clin. Soc., London, 1874; and Brit. Med. Journ., November 1, 1873 . 
Gullax, A. G. Exophthalmic goitre. Lancet, September 5, 1908.

- A contribution to the discussion on Exophthalmic goitre, with special reference to the antithyroid treatment. Liverpool Med.-Chirurg. Journ., July, igo8.

GullaN, A., and GorDoN. Two cases of Addison's disease and the effect of the administration of suprarenal extract. Lancet, August 19, 1905.

Gulli, V. Il diabete surrenale. Gl' Incurabili, 1905.

GuREwitsch, R. Über die Wirkung des Jodkaliums auf die Pulszahl. Diss., Basel, 1907.

Gutmans. Beitr. z. Pathologie d. Pankreas bei Diabetes. V. A., 172, p. 493,1903 ; and 177, Suppl., p. 128, 1904.

GuttmanN, P. Addisonsche Krankheit. Eulenburgs Real-Encyclopädie, 3 Aufl.

- Über die Addisonsche Krankheit. Diss., Berlin, 1868; D. m. W., 1885.

Gutzeit, R. Ein Teratom der Zirbeldrüse. Diss., Königsberg, 1896.

HaAS, L. Über die Addisonsche Krankheit. Diss., Würzburg, 1862.

Haberer, $v$. H. Experiment. Verlagerung der Nebenniere in die Niere. A. k. Ch., 86, 1008 .

- Exp. Unters. üb. Nierenreduktion u. Funktion d. restierenden Parenchyms. M. G. M. C., $17,1907$.

HABERER, v., and STÖRK. Beitrag zur Marksekretion der Nebenniere. W. k. W., No. 9, pp. 305 and 338,1908 .

Haberern, J. P. Physiol. norm. et path. de la prostate. Congr. intern. d'urolog., ii, 1908.

HABERFELD, IV. Die Rachendachhypophyse. Zieglers, B., 46, p. 133, 1909.

Haberfeld, W., and Schilder, C. Die Tetanie der Kaninchen. M. G. M. C., 20, I909.

HACKerT, H. Morbus Addisonii. Diss., Jena, I 884.

HageNbach, E. Exp. Studien üb. d. Funktion d. Schilddrüse und der Epithelkörperchen. M. G. M. C., $18,1907$.

- Schilddrüsen und Epithelkörperchen. M. K., 1908.

HaHN, G. Über die Wirkung der Nebennierenpräparate speziell des Adrenalins mit besonderer Berücksichtigung der Oto-, Rhino-. Laryngologie. Diss., Leipzig, 1903.

Halász. Beitr. z. Kennt. d. hist. Veränderungen des Pankreas bei Pankreasdiabet. C. f. norm. u. path. An., i, I904.

Halban. Über den Einflusz der Ovarien auf die Entwicklung des Genitales. M. G. G., xii, p. 496 , I901.

- Ovarium und Menstruation. S. IT. A., I Io, p. 7I, 1901 .

- Die Entstehung der sekundären Geschlechtscharaktere. A. G., 70, I903; W. k. IV., No. 2S, igo3.

- Schwangerschaftsreaktionen der fötalen Organe und ihre puerperale Involution. Z. G. G., 53, 1904 .

- Die innere Sekretion von Ovarium und Placenta und ihre Bedeutung für die Funktion der Milchdruise. A. G., 75, 1905 .

HALBERSTäDTER. Die Einwirkung der Röntgenstrahlen auf Ovarien. B. k. W., 1905.

Halle, iV. Über die Bildung des Adrenalins im Organismus. H. B., viii, 1906.

Haller, Albert, v. Elementa physiologiae corp. humani. Bernae. 1765.

Haller, B. Über die Urniere von Acanthias. Morph. Jahrb., 29, I90I-2.

- Unters. über Hypophyse und Infundibularorgane. Ibid., 25, 1896.

- Über die Hypophyse niederer Placentalier. A. m. A., 74, I909.

HALLIBURTON. Report of chemical investigation of the tissues and organs from cases of myxœdema in man and animals. Report on Myxœdema, London, i888.

- The physiological effect of extracts of nervous tissues. J. o. P., 26, 1901 .

- Die Biochemie der peripheren Nerven. E. P., 4, 1905.

- Handbook of Physiology. London, 1907.

HALLIBURTON and OSBORNE. The physiological action of cholin and neurine. Proc. Phys. Soc., i 7 I, I899.

Halliburtox, CANDlER, P. J., and Sikes, A. W. The human pituitary body. J. o. P., 38, 1909.

Hallion, L. Corp thyroïde et capsules surrénales. Arch. gén méd., nouv. sér. ii, p. 488 , 1899 . 
Hallion, L. Physiologie normale et pathologique du corps thyroïde. Ibid., iv, p. $490,1900$.

- Corps thyroïde et capsules surrénales. Ibid., vi, p. 604, 1901.

- Effet vasodilatateur de l'extrait ovarien sur le corps thyroïde. C. r. S. B., I 907.

- La fonction glycogénique du foie et sa régulation. Gaz. des hôpitaux, No. II, 1909.

Hallion, L., and Alquier, L. Modifications histologiques des glandes à sécrétion interne par ingestion prolongée d'extrait d'hypophyse.

C. r. S. B., 64, p. 5, 1908 . Sur l'essai expérimental de l'extrait opothérapeutique
HALlion and CARRION. Sur d'hypophyse. Soc. de thérap., 13 mars, 1907 ; Bullet. gén. de thérap., p. 450 , mars, 1907.

- Sur le traitement sérothérapeutique du goitre exopht. d'après la méth. de Ballet et Enriquez. Presse méd., ig05.

Hallion and LAIGNEL-LAvastine. Recherches sur l'innervation vaso-motrice dès glandes surrénales. C. r. S. B., 55, p. 187,1903 .

Hallion and NeEPER. L'influence excito-motrice de la bile sur l'intestin. I. Action sur le rectum. Ibid., 63, p. I82, 1907. - II. Action sur l'intestin grêle. Ibid., p. 254.

Halcot, G. L'extrait de capsule surrénale et son emploi dans la thérapeutique oculaire. Thèse de Paris, 1897.

Halmagrand. Etat actuel de l'infantilisme. Thèse de Paris, Ioo7.

HalpenNy, J., and Thompson, F. D. On the relationship between the thyroid and parathyroids. An. An., 34, I909.

HaIsted, W. S. An experimental study of the thyroid gland of dogs, \&c. Jolins Hopkins Hospital Reports, vol. i, 1896 .

- An experimental study of the thyroid glands of dogs, \&c. Baltimore, I 806 .

- The transplantation of parathyroid glands in dogs. Proc. Path. Soc., Philad.; and J. E. M., 5, pp. 74-77, 1908.

- Auto- and isotransplantation in dogs of the parathyroid glandules. J. E. M., xl, p. 175 , 1909 .

HAMbURGer, W. The action of intravenous injections of glandular extracts and other substances upon the blood-pressure. A. J. P., I I, p. 282,

HAMEL. Klinische Beobachtungen über zwei Fälle von Morbus Addisonii mit besonderer Berücksichtigung des Blutbefundes. D. A. k. M., 7I, p. 240,1901 .

Намм. Suprarenin (Höclist). D. m. W., I904.

HAMMAR, A. Über die normalen Durchschnittsgewichte der menschlichen Thymusdrüse. V. g. M., 3, Folge, 37, p. I.

- Über Thymusgewicht und Thymuspersistenz beim Menschen. V. a. G., Genf, 1905 .

- Zur Histogenese und Involution der Thymus. An. An., p. 23. 1905.

- Über Gewicht, Involution und Persistenz der Thymus im Postfötalleben des Menschen. A. A., 1906, Suppl.

- Ist die Thymusdrüse beim Frosch ein lebenswichtiges Organ? P. A., 110,337 .

-. Zur Kenntnis der Tellostierthymus. A. m. A., 73, p. 1, 1909.

HAMON DU FOUGERAY. De l'origine rhino-pharyngienne de goitres. Progr. méd., No. 21, 1901 .

HaNaU, A. Versuche über den Einflusz der Geschlechtsdrüsen auf die sekundären Geschlechtscharaktere. P. A., 65, p. 5 I6, 1806.

HANDELSMANN. Experimentelle und chemische Úntersuchungen über Cholin und dessen Bedeutung für die Entstehung von epileptischen Krämpfen. Gazetta lekarska, Nos. 32-35; Ref. Virch-Hirsch' Jahresb. über, 1908 , i, p. I31: D. Z. N., 35, 1008.

HansemanN, D. v. Über Anaplasie, Spezifizität und Altruismus der Zellen. Berlin, $189 \mathrm{~s}$.

- Die Beziehungen d. Pankreas zum Diabetes. Z. k. M., 26, I 894 .

- Über die sogenannten Zwischenzellen des Hodens und deren Bedeutung bei pathologischen Veränderungen. V. A., 142, ISo5.

- Ein seltener Fall von Morbus Addisonii. B. k. IV., I896.

- Über Akromegalie. Ibid., 1897.

- Über die Struktur und das Wesen der Gefäszinseln des Pankreas. V. d. p. G., 4, p. 187 , I 90 I. 
HaNSEmanN, D. v. Echte Nanosomie. B. k. W., I902.

- Demonstration eines kretinistischen Schakals. B. m. W., I908.

HAPPEL. Üb. d. Folgen der Unterbindung d. Ausführungsgänge des Pankreas beim Hunde. Diss., Marburg, 1906.

Hardman, W. A case of hypothyroidism. Brit. Med. Journ., August 29. 1908.

HARLEY. An experimental inquiry into the function of the suprarenal capsules and their connection with bronzed skin. Briefl. Mitteil. an Virchow, November, 12, 1857; The British and Foreign Med. chir. Review, No. 41, pp. 204-221 and No. 42, p. 498, 1858; Med. Times and Gaz.,p. 564, November 28, 1857.

- Living specimen of a rat from which both the suprarenal capsules and the spleen had been removed. Trans. Path. Soc. London, vol. 9, p. 401, February 9, 1858 .

- Discussion in Roy. Med. and Chir. Soc., February 9, 1858.

- The histology of the suprarenal capsules. Lancet, 1858 .

Harley, W. Pathogenics of pancreatic diabetes. Brit. Med. Journ., I892.

- Absorption and metabolism in obstruction of ductus pancreaticus. J. o. P. B., 1895 .

HARMER. Über die Wirkung des Nebennierenextraktes auf die Schleimhaut der Nase und des Kehlkopfes. W. k. W., rgor.

Harnack, E. Zur Pathogenese des Diabetes mellitus. Diss., Dorpat, 1873.

- Über von Cyons antagonistische Versuche mit Jodothyrin-Atropin, \&c. C. P., 1898 .

HARRIS, I. Die Zerstörung des Adrenalins im lebenden Tier. Diss., Königsberg, I904.

HARRIS, V., and Gow. Comparative histology of the pancreas. J. o. P., 15, 349, 1894 .

HART, K. Thymushyperplasie bei Morbus Addisonii. W. k. WV., 1908.

- Thymuspersistenz und Thymushypertrophie. M. m. W., I908; C. G. M. C., 1909 .

HARVEY, B. C. The chromaffine characters of certain parietal cells of the stomach. Brit. Med. Journ., p. 1703, 1906.

HASKOVEC, L. Einwirkung des Schilddrüsensaftes auf den Kreislauf. W. m. Bl., 18g6.

- Nouv. contrib. à la question de l'action du liquide thyroïdien sur le système nerveux central. A. m. e., 13, 1903; and Arch. intern. d. Pharmac., 8, 1901 .

HaUshalter and LuCIEN, M. Polyurie simple et tubercule du l'hypophyse. Rev. Neur., 1908.

HAYEM and LESAGE. Pseudotuberculose bacillaire chez l'homme. Maladie d'Addison. Bull. et mém. de la soc. méd. des hôp., No. 21. Paris, I 89 r.

HECKER. Zur Pathologie der Schilddrüse und Nebenschilddrüsen. Jahresber. d. Ges. f. Naturheilk., p. 62, Dresden, 1906-1907; M. m. W., 1907.

НЕDвом, K. Über die Einwirkung verschiedener Stoffe auf des isolierte Säugetierherz. S. A., viii, pp. 147 and 169,1898 .

HEDINGER, E. Über Beziehungen zwischen Status lymphaticus und Morbus Addisonii. V. d. p. G., xi, 1907; Frankf. Zeitsch. Path., i, p. 527.

- Über familiäres Vorkommen plötzlicher Todesfälle, bedingt durch Status lymphaticus. D. A. k. M., 86, p. 248, 1905.

- Über experimentell durch Adrenalin und Hämostasin erzeugte Arterienerkrankungen bei Kaninchen. Korr.-Bl. f. Schweiz. Ärzt., 35, 20, 1905.

HEDINGER and LOEB. Über Aortenveränderungen bei Kaninchen nach subkutaner Jodkaliverabreichung. A. P. P., 56, p. 314, 1907.

HÉDon, E. Sur la production du diabète sucré après l'exstirpation du pancréas. C. r. S. B., I89o.

- Exstirpation du pancréas; diabète sucré. A. m. e., I and 3-4 H., 1891 .

- Pathogénie du diabète maigre. Ibid., I892.

- Exstirpation du pancréas. Les effets sur la nutrition générale. A. d. P., I891.

- Greffe sous-cutanée du pancréas. Ibid,, p. 617, 1892 .

- Les travaux récents sur la physiologie des glandes vasculaires sanguines. Nouveau Montpellier médic., Supp., pp. 467-494, 1893. 
HÉdos, E. Physiologie norm. et path. du pancréas. Encyc. scient., Paris.

- Diabète pancréatique. Paris, 1898; and in Richet, Dict. de physiol., iv, 1900.

- Expériences des transfusion réciproque par circulation carotidienne croissée entre chiens diabétiques et chiens normaux. C. r. S. B., 66, p. 699 , 1909 .

HÉdoN, E., and FleIG, C. Chloralose et inhibition. Arch. internat. de Phamac., 13.

Hegar, A. Die Castration der Frauen. Volkmanns Samml., Nos. 136-138, 1878 .

- Der Geschlechtstrieb. Stuttgart, 1894.

- Zur abnormen Beliaarung. B. G. G., 4, I901.

- Korrelationen der Keimdrüsen und Geschlechtsbestimmung. Ibid., 7, p. $201,1903$.

HeiberG, K. A. Ein Verfahren zur Untersuchung der Bedeutung der Langerhansschen Inseln im Pankreas. Z. ph. Ch., 49, p. 243.

- Hypertrophie der Langerhansschen Pankreasinseln. M. m. W.' I907. 1907.

Heidenhain, R. Die Bauchspeicheldrüse. Hermanns Handb. d. Physiol., Bd. 5 .

HeIdenhain, L. Über die Behandlung der peritonitischen Blutdrucksenkung mit intravenösen Suprareninkocksalzinfusionen. M. G. M. C., 18,1908 .

HEIM. Dissertatio de renibus succenturiis. Berlin, 1824.

Heinitz. Altes u. Neues uber die Schilddrüse. (Diss.) Russisch. Zit. nach Georgiew'sky, Z. k. M., 33, I64.

HeInsheIMER, F. Uber die Ursache der Zuckerausscheidung im Pankreasdiabetes der Hunde. Z. e. P., 2, 1905.

Heinz, R. Handb. d. exper. Path. u. Pharmakologie. Jena, 1904-1906.

HELLIN. D. Struma und Schilddrüse. München, 1893.

- Über den wirksamen Bestandteil der Schilddrüse. 1898 .

HELLY, K. Studien über Langerhanssche Inseln. A. m. A., 68, 124, 1905.

HÉMET. L'opothérapie surrénale chez les Addisoniens. Thèse de Paris, 1898 .

Hempez. Ein Beitrag zur Pathologie der Glandula pinealis. Diss., Leipzig, I90I.

- Behandlung des Morbus Basedowi mit Anthyreoidinserum (Moebius). M. m. W., 1905 .

Henderson, L. J., and SpIRo, K. Zur Kenntnis des Ionengleichgewichtes im Organismus. I. Teil. Über Basen- und Säuregleichgewicht im Harn. B. Z., 15, pp. 105-113.

HeNDERSON, J. On the relationship of the thymus to the sexual organs. J. o. P., p. 38,1904 .

HengGe, A. Pseudohermaphroditismus u. sekundäre Geschlechtscharaktere. M. G. G., 1903 .

Hente, J. Aligemeine Anatomie, pp. 996, 1004-1006. Leipzig, 184r.

- Über das Gewebe der Nebenniere und der Hypophysis. Z. r. M., 24, 1865 .

- Handb. d. system. Anatomie. II. Blutgefäszdrüsen. Braunschweig, 1866.

HENRI, V. Note sur l'action du suc pancréatique. C. r. S. B., 57 ii, 1905.

HENRot. Des lésions anatomique et de la nature du myxodème. C. r. Ass. franç., 82 .

HENSCHEN, K. Uber Struma suprarenalis cystica hæmorrhagica. Beitr. z. klin. Chir., 49, S. 217, 1906.

Herbst, K. Formative Reize in der tierischen Ontogenese. Leipzig, Igor.

HERLITZKa, A. Sul trapiamento dei testicoli. A. E. M., 9.

- Contributo allo studio del diabete duodenale di Pflüger. Giorn. R. Accad. di Med. di Torino, 71 , p. 57,1908 ; and A. i. B., 50.

- Zur Kenntnis des Pflügerschen Duodenaldiabetes. P. A., I23, p. 331, 1908 .

HERRING, P. J. The effect of thyroidectomy upon the mammalian pituitary. Quart. Journ. of Exp. Physiol. London, vol. I, pp. 281-285; Brit. Med Journ., igo8. 
HERRING, P. J. Action of pituitary extracts on the frog's circulatory system. J. o. P., 3I, p. 429 .

- The physiological action of extracts of the pituitary body and saccus vasculosus of certain fishes. Quart. Journ. of Exp. Physiol., i, 187, 1908 .

- A contribution to the comparative physiology of the pituitary body. Ibid., p. $26 \mathrm{I}$.

Hermann, G., and Verdun, P. Persistance des corps postbranchi aux chez l'homme. C. r. S. B., 1899 .

- Remarques sur l'anatomie comparée des corps postbranchiaux. Ibid.

HERMANN. O. Über das Vorkommen und Veränderungen von Myelinsubstanzen in der Nebenniere. Arb. a. d. path.-anat. Inst. zu Tübingen. V, 1906; Diss., Tübingen, 1905.

HerTer, C. A. On adrenalin glycosuria and allied forms of glycosuria due to the action of reducing substances and other poisons on the cells of the pancreas. Med. News, 80, 19, p. 865, May, 1902; A. J. M. S., 1903 .

- Note on the newly recognized sugar-controlling function of the suprarenal glands. Amer. Med. News, October 25, 1902.

Herter, C. A., and Richards, A. N. Note on the glycosuria following experimental injections of adrenalin. Med. News, T. 8o, p. 201, February, 1902 ; Ref. Sclimidt, 278, p. 118.

Herter, C. A., and WAKEMANN, A. J. Über Adrenalin-Glycosurie und verwandte durch die Wirkung reduzierender Substanzen und anderer Gifte auf die Pankreaszellen hervorgerufene experimentelle Glycosurien. V. A., 169, p. 479, 1902.

HERTOGHE. De l'hypothyroidie bénigne chronique ou Myxœdème fruste. Nouv. iconog. de Salpêt., juillet-août, 1899 .

- Eine Reihe von Arbeiten in Bulletin de l'Acad. royale de médecine de Belgique, 10-13, 1895-1899.

- Die Rolle der Schilddrüse bei Stillstand und Hemmung des Wachstums und die Entwicklung und der chronisch gutartige Hypothyreoidismus. Übers. von Spiegelberg. München, rgoo.

Herzog, M. Liefert das Pankreas ein dextrosespaltendes, Alkohol, und $\mathrm{CO}_{2}$ lieferndes Enzym? H. B., 2, 1902.

- Zur Histologie und Pathologie des Pankreas beim Diabetes. V. A., I68, 1902.

Hervieux, C. Les ferments solubles de la glande interstitielle du testicule. C. r. S. B., Bd. 60, pp. 653 and 683 .

Herxheimfr, G. Über Pankreaszirrhose bei Diabetes. V. A., 183.

- Pankreas und Diabetes. D. m. W., 1906.

- Zur Frage des Verhaltens der Langerhansschen Zellinseln im Pankreas bei Diabetes. Festschr. Orth., 1903.

Herxheimer, G., and HOFFMANN, K. F. Über die anatomischen Wirkungen der Röntgenstrahle auf den Hoden. D. m. W., I908.

Hess, O. Beiträge zur Anatomie und Pathologie des Pankreas. Med. naturw. Arch., Berlin, i, 1907.

- Die Ausführungsgänge des Hundepankreas. P. A., 118, 1907.

Hesse, A., and MOHR. Über Glykosurie und Glykämie des pankreaslosen Hundes. Z. e. P., vi, p. 300 , April, 1909.

Heyn. Ein Beitrag zur Lehre vom Myxödem. Arch. f. Psychiatrie, 41, 1906.

HildEBRAND, O. Über den Bau gewisser Nierentumoren, ihre Beziehungen zu den Nierenadenomen und zur Nebenniere nebst Mitteilungen über den Glykogenbefund in diesen sowie in anderen Geschwülsten. A. k. Ch., 47, pp. 225-273, 1894 .

- Adrenalin in der chirurgischen Praxis. B. k. W., 1905.

HILDEBRANDT. Zur Lehre von der Milchbildung. H. B., 5, 1904.

Hillemand, C. Organothérapie ou Opothérapie. Paris, 1 \&g9.

HinselmanN, $H$. Glykogenabbau und Zuckerbilding in der Leber normaler und pankreasdiabetischer Hunde. Z. ph. Ch., 61, p. 265, I909.

- Über das Wesen des Pankreasdiabetes. B. k. W., I909.

Hippel. Ein Beitrag zur Kasuistik der Hypophysentumoren. V. A., 126, p. 124,1891 .

Hirsch, A. Die Geschwülste der Nebennieren und Nebennierengeschwülste der Niere. Diss., Würzburg, 1902. 
Hirsch, R. Über das glykolytische Ferment der Leber. H. B., 4, 1903.

- Glykosurie nach Schilddrüsenexstirpation bei Hunden. Z. e. P., iii, 1906.

- Schilddrüse und Glykosurie. Z. P., v, p. 233, 1908.

- Innere Sekretion. Oppenheimers Handb. d. Bioch., Bd. 3, 1909.

Hirschel, J. Beiträge zur Kenntnis des Morb. Basedowii. Jahrb. Psych., 1002.

HirschberG, A. Beitrag zur Behandlung mit Oophorin. M. m. W., igo8.

HiRTz. Traitement de la maladie d'Addison par les injections de capsules surrénales. Bull. de Thérap., 30 juillet, 1902.

HiRzel. Beitrag zur Kasuistik der Addisonschen Krankheit. Diss., Zürich, 1860 .

His, IV. Beobachtungen über den Bau der Säugetiereierstöcke. A m. A., I, 1865 .

- Der Tractus thyreoglossus und seine Beziehungen zum Zungenbein. A. f. Anat. u. Entw. v. His und Braune, 1801 .

- Beitrag zur Kenntnis der zum Lymphsystem gehörigen Drüsen. $Z$. W. Z., 10 and 11 .

HitschilanN, E. Ein Fall von "Gefäsztod" durch hämorrhagische Zerstörung beider Nebennieren. W. k. W., igoz; Jahrb. f. Psych., $21,1002$.

HITSCHMANN and ADLER. Bau der Lterusschleimhaut des geschlechtsreifen Weibes mit besonderer Berücksichtigung der Menstruation. Berlin, 1908.

Hoche, A. Kretinismus und Myxödem. Im Lehrbuch der Psychiatrie von Binswanger und Siemerling, 1904 .

HocheneGG, J. Geheilter Fall von Hypophysentumor. 37, Kongr. d. G. Ch., 1908 .

- Ablation. von Hypophysentumoren. WV. k. WV., 1909.

- Zur Therapie der Hypophysentumoren. Z. Ch., 100, $100 \mathrm{I}$.

HOCHSTETTER, F. Beiträge zur Entwicklungsgeschichte des Venensystems der: Amnioten. II. Reptilien (Lacerta, Tropidonotus). Morph. Jahrb., xix, 3, H., pp. 428-501

- Beiträge zur Anatomie und Entwicklungsgeschichte des Blutgefäszsystemes der Krokodile. Sonderabdruck aus Voelzkow, Reise in Ostafrika in den Jahren, 1903-1905, Bd. iv, pp. 105-106. Stuttgart, 1906.

HODDIK. Über die Behandlung der peritonitischen Blutdrucksenkung mit intravenösen Adrenalin-Kochsalzinfusionen. C. Ch., p. I193, 1907.

HödLMOSER. Tod durch Embolie der Pulmonalarterie, ausgehend von einen in die Hohlvene gewucherten primären Angiosarkom der Nebenniere. Z. H., 25, 1904.

HönigsBerger. Behandlung der Rhachitis mit Nebennierensubstanz. M. m. IV., 1900 .

HoExicke. Zur Theorie der Osteomalacie. B. k. IV., 1004.

- Über das Wesen der Osteomalacie. Halle, I905.

- Über experimentell erzeugte Strumen. Chirurgenkongresz, 1908.

HössliN. Tumor der Hypophysis cerebri. M. m. IV., 1894 .

HofBAUER, S. Beiträge zur Ätiologie und zur Klinik der Graviditätstoxikosen. Z. G. G., 61, H. 2, 1907.

- Über experimentelle Beeinflussung von Gravidität. B. k. IV., ı go8.

HofFmanN, C. K. Zur Entwickiungsgeschichte der Urogenitalorgane bei den Anamnia. Z. w. Z., 46, pp. 570-643, i 886.

- Zur Entwicklungsgeschichte der Urogenitalorgane bei ben Reptilien. Ibid., 48,1880 .

- Etude sur le développement de l'appareil uro-génital des oiseaux. Verh. d. Ak. d. IViss. Amsterdam, 1892 .

- Zur Entwicklungsgeschichte des Sympathikus bei den Selachiern (1900), bei den Urodelen. Ibid., 1902.

HOFFMANN, E. Über Hypernephrometastasen. D. m. IV., 1907.

HofFMAN, H. Verwendung synthet. Suprarenins in der Lokalanästhesie. M. m. W., 1007 .

HofFMAN, J. WVeiterer Beitrag zur Lehre von der Tetanie. D. Z. N., 9, p. 278,1897 .

HoffMAN , K. F. Über den Einflusz der Röntgenstrahlen auf den Kaninchenhoden. Diss., Bonn, 1 oos. 
HOFFMaNN, R. Versuche mit Cholin. W. k. W., I905.

- Serumuntersuchungen bei Thyreoidose. M. m. W., 1908.

- Beiträge zur Lehre vom Morbus Basedowii. Z. k. M., 69, 1909.

- Antithyreoidin Moebius bei Osteomalakie. Z. G., 1908.

- Die Affinität des Adrenalins zu Jod. M. m. W., Igog.

HOFMEISTER, F. Untersuchungen über die Zwischensubstanz in den Hoden der Säugetiere. S. W. A., 65, Abt. 3, 1872 .

HofMeIster. Zur Physiologie der Schilddrise. F. M., 10, 1892.

- Exper. Unters. über die Folgen des Schilddrüsenverlustes. Bruns. Beitr., II, 1894 .

- Zur Frage nach der Folgezuständen bei Schilddrüsenexstirpation. D. m. W., 1896 .

- Über Störungen d. Knochenwachstums bei Kretinismus. Fortsch. d. Röntgenstr., 1 .

Holland, C. J. The X-ray treatment of exophthalmic goitre. Liverpool M. Ch. J., July, 1908 .

HOLLINGer. Über die Verteilung des Zuckers im Blut. B. Z., 17. 1904.

HoLM, F. Über die nervösen Elemente in den Nebennieren. S. W. A., 53, 1866.

- Uber die chemischen Bestandteile der Nebennieren. J. prakt. Ch., i, p. $150,1867$.

HolmgReN, E. Über die Saftkanälchen der Leberzellen und der Epithelzellen der Nebenniere. An. An., 22, p. 9, 1903.

- Weitere Mitteilungen über die Trophospongienkanälchen der Nebennieren vom Igel. Ibid., pp. 470-481, I903.

HOLSCHEWNIKOFF. Ein Fall von Syringomyelie, verbunden mit trophischen Störungen (Akromegalie). V. A., II9, p. Io.

Holsti. Ein Fall von Akromegalie. Z. k. M., 20, 298, 1892.

HOMBERGER, E. Über Addisonsche Krankheit. Zeitschr. f. prakt. Ärzte, No. $4,1897$.

HoNigMaNN. Adrenalin und Lokalanästhesie. C. Cl1., H. 25, 1903.

HONORÉ. Recherches sur l'ovaire du lapin. A. B., I6.

HOPPE-SEYLER, G. Über chronische Veränderungen des Pankreas bei Arteriosklerose und ihre Beziehung zum Diabetes mellitus. A. k. M., 8 I, p. II 9 .

HORN, G. Beiträge zur Histogenese der aus aberrierten Nebennierenkeimen entstandenen Nebennierengeschwülste. V. A., 126, pp. 191-217, 1891.

HornowskI, J. Recherches sur la pathologie du système chromaffine. A. m. e., 21,1909 .

- Veränderungen im Chromaffinsystem bei unaufgeklärten postoperativen Todesfällen. V. A., 198, p. 93, 1909.

HORNOWSKI, J., and NEWICKI, WW. Histologische Untersuchungen über die Nebennieren bei Arteriosklerose sowie uber den Befund in Nebennieren und Aorta von Kaninchen bei intravenösen Adrenalininjektionen. V. A., 192, pp. 338-356, 1908.

HORSLEY. Die Funktion der Schiliddrüse. Festschr. Virchow, 1891; Brit. Med. Journ., i, 287, 1890 .

- Remarks on the function of the thyroid gland. Repr. of the Dep. of Path., Univ. Coll., London, 1894.

- Functional nervous disorders due to loss of thyroid gland and pituitary body. Lancet, I, p. 5, 1886; Brit. Med. Journ., vol. i, 323; vol. ii, p. 4 II, 1906.

HoweLL, W. H. The physiological effects of extracts of pituitary body. J. E. M., 3, pp. 215 and 245,1898 .

Houghton, E. M., and MerRILL, C. H. The diuretic action of adrenalin and the active principle of the pituitary gland. J. A. M. A., 5 I, p. $1849,1908$.

HUChard and LANNoIs. Gigantisme acromégalique. Soc. med. d. hôp., p. 1444,1903 .

HudoverNig and Popovicz. Gigantisme précoce avec développement précoce des organes génitaux. Nouv. Icon. Salpètr., No. 3, 1903.

- Un cas de gigantismo précoce, étude complémentaire. Ibid., No. 4, 1006.

HÜRTHLE. Beitrag zur Kenntnis der Sekretionsvorgänge in der Schilddrüse. P. A., 56, I894.

- Über den Sekretionsvorgang in der Schilddrüse. D. m. W., 1894. 
HUISMANs, L. Über Morbus Addisonii. M. m. W., 1900 and $190 \mathbf{1}$.

- Über zwei Fälle von mechanischem Thymustod. B. k. WV., Igo8.

Hutst. Ein Tumor der Glandula parathyreoidea. C. a. P., i6, p. io3.

Hultgren, E. O. Bemerkungen zu der Arbeit Olaf Scheel : Über Nebennieren. V. A., I43, pp. 551-553, 1908.

Hultgren, E. O., and Andersson, O. A. Studien zur Physiologie und Anatomie der Nebenniere. Leipzig, i899. S. A., ix, pp. 73-312.

Hunt, R. The comparative physiological activity of some commercial suprarenal preparations. J. A. M. A., September 8, igo6.

- Influence of thyroid feeding and of various foods and of small amounts of food upon poisoning by acetonitril. Proc. Soc. Exp. Biol., New York, October 18, 1905 ; J. B. Ch., I, p. 33, October, 1905.

Hunt, R., and SEIDELL, A. Studies on thyroid and the relation of iodine to the physiological activity of thyroid preparations. Hygien. Lab. Bull., No. 47, I909.

Hunt, R., and TAVEAU, R. On the physiological action of certain cholin derivatives and new methods for detecting cholin. Brit. Med. Journ., p. I 788, December 22, I906.

Huot, M. E. Sur les capsules surrénales, les reins et le tissu lymphatique des poissons lophobranches. C. r. A., 124, pp. 1462-1464, 1897 and 126, p. 49, 1898 .

-- Recherches sur les poissons lophobranches. Ann. des sc. nat., t. xiv, pp. 197-288, I902.

HuschKe. Lehre von den Eingeweiden und Sinnesorganen des menschlichen Körpers von v. Soemmering. Leipzig, I 844 .

Hetchinson, R. The chemistry of thyroid gland and the nature of its active constituent. J. o. P., 20, 1896; and Ibid., 23, 1898.

HUTCHINSON-WOODS. The pituitary gland as a factor in acromegaly and giantism. Ref. C. a. P., ix, 1898.

IKEDA. Zum Einflusz des Ganglion hypogastricum auf die Geschlechtsfunktionen. C. P., 1907.

ILBERG. Das Zentr.-Nervensyst. eines Hemicephalus m. Aplasie d. Nebennieren. Arch. Psych. 36, 1902.

IMBERT. 'Recherches expérimentales sur les greffes des capsules surrénales dans la rein. IV. Sess. de la soc. franç. d'urol., 1899; Proc. verb., p. 527, 1900 .

ImMoDA. Su 5 casi d'infantilismo maschile. Arch. d. Psychiatria, I7, p. 526,1896 .

INABA-MASAMARO. Notes on the development of the suprarenal bodies in the mouse. J. of the Coll. of Sc., Imp. Univ., Japan, vol. vi, Part I, pp. $215-235,1891$.

Indemanns. Hel Antithyreoidin Moebus by de Behandlung van Morbus Basedowii. Tijdschrift voor Geneseskunde, No. I7, 1904.

INGERMANN. Zur Kasuistik der Hypophysistumoren. Diss., Bern, i88g.

IRSAI, VAS and GARA. Thyreoideapräparate bei Strumakranken. D. m. W., 1896.

Irvance, A., and PaCE, C. Nebennierensubstanz und Rachitis. A. f. Kindheilk., 49, r 909 .

ISAAC, S., and VELDEN, R. v. D. Kreislaufwirkung jodierter Eiweiszkörper. Verh. d. Kongr. f. inn. Med., p. 307, 1907; und Med.: naturwiss. Arch., I, I05, 1907.

Iscovesco, H. Pancréas et Catalase hépatique. C. r. S. B., 57, ii, p. 44, 1905 .

- Les lipoïdes du corps thyroïdea. Ibid., 65, pp. 84, 106, 218, 1908.

ISELIN. Tetanie jugendlicher Ratten nach Parathyreoidektomie. Steigerung der tetanischen Reaktionsfähigkeit jugendlicher Ratten bei Nachkommen parathyreoidektomierter Ratten. Z. Ch., 43, pp. 397-401.

- Wachstumshemmung infolge von Parathyreoidektomie bei Ratten. Ibid., p. 494, 1908.

ISRAEL, O. Experimentelle Untersuchung über den Zusammenhang zwischen Nierenkrankheiten und sekundären Veränderungen des Zirkulationssystems. V. A., 86, 188 I.

- Zur Diagnose der Nebennierengeschwülste. D. m. IV., I905.

- Der akromegale Kauerauf. V. A., 164, p. 344, 1901.

IssaKówiTsCH, A. Geschlechtsbestimmende Ursachen bei den Daphniden. A. m. A., 69, p. 223, 1907 . 
JABoulay, M. Capsules surrénales accessoires dans un ganglion sémilunaire et au milieu du plexus solaire. Lyon méd., pp. 300-302, 1890.

- La greffe de corps thyroide et de capsules surrénales dans la maladie de ces glandes. Ibid., 29, p. 399, 1897.

JACKSON. The prolonged existence of adrenaline in the blood. J. P., 23, p. $226,1909$.

ЈасовJ. Beiträge zur physiologischen u. pharmakologischen Kenntnis der Darmbewegungen mit besonderer Berücksichtigung d. Nebennieren. A. P. P., 29, 1892 .

JACOBS. Thérapeutique ovarienne. La Policlinique, 1896.

JACOBY, M. Studien zur Entwicklungsgeschichte der Halsorgane der Säugetiere und Menschen. Über die Entwicklung der Kiemenderivate. Diss., Berlin, I895.

- Uber die Entwicklung der Nebendrüsen der Schilddrüse und der Carotisdrüse. An. An., 12, 1896; and 13, 1897 .

- Uber das Aldehyde oxydierende Ferment der Leber und Nebenniere. Z. ph. Ch., 30, p. 135 .

JACoprni, G. La secrezione siderofila delle capsule surrenali. Clin. mod., p. $25 \mathrm{I}, 1906$.

JACQUES. Deux cas d'ectopie thyroïdienne. C. r. S. B., 57, ii, p. 714, 1905.

JAGIC. Zur Behandlung des Asthma bronchiale. B. k. WW., Igog.

JAKOBSON, J. H. Beitr. z. Kenntnis d. fötalen Entwickelung d. Steiszdrüse. A. m. A., 53, 1899 .

JAKSCH and ROTKY. Über eigenartige Knochenveränderungen im Verlaufe des Morbus Basedow. Fortschritte auf dem Gebiete der Röntgenstrahlen. Bd. xiii, 1908.

JANEwAY and OERTEL. Beitr. 2. Pathologie der Zuckerharnruhr. V. A., $171,1903$.

JANKowSKI. Beitr. z. Entstehung des Corpus luteum d. Säugetiere. A. m. A., 64, 1904 .

JANNIN. Jodisme constitutionel, thyroïdisme et maladie de Basedow. Revue méd. de la Suisse romande, 19, 1899.

JANosıK, J. Bemerk. über d. Entwickelung der Nebenniere. A. m. A., 22,1883 .

- Histologisch-embryolog. Unters. über das Urogenitalsystem. S. W. A., 9 I, 1885 .

- Bemerkungen über die Entwickelung des Genitalsystems. Ibid., 99,1890 .

- Uber die Entwickelung des Nachniere (Metanephros) bei den Amnioten. A. A., pp. 23-82, 1906.

JANowSKI, W. Primary suppuration of both suprarenal glands. Lancet, 1898.

Jaxowsky, Тн. G. Nebennierenpräparate bei Erkrank. d. Osophagus. A. V., 10, 1904.

Januschke, H. Adrenalin ein Antidot gegen Strychnin? W. k. W., 1910.

JAPPELLI, A. Untersuchungen über die Speichelabsonderung. Z. B., 5I, p. 405 .

JappelLi, G., and D'Errico. Contributo alla linfogenesi. Arch. fisiol., iv, $\mathrm{H}$. 4 .

JAQuet. Note pour servir à l'étude de la maladie d'Addison. A. P., ro, pp. $679-601,1878$.

- Des effets de l'extrait de rein dans le traitement, \&c. Thèse de Lyon, 1897.

- Über Schilddrüse und Schilddrüsenpräparate. Korr. Schweiz., Ae., 1899.

JAROTSKY, A. J. Über die Veränderungen in der Grösze und im Bau der Pankreaszellen bei einigen Arten der Inanition. Diss., St. Petersburg, 1898.

JaYLE. Die Opotherapie mit Ovarium. Revue de gynéc. vii, 1903.

JEANDELIZE. Insuffisance thyroidienne et parathyroidienne. Thèse de Nancy, 1902 .

JEANDELIZE and PARISOT. De la pression artérielle dans le goitre et l'insuffisance thyroïdienne. IX. Congr. franç. de médec. Paris, 14-16 octobre, 1907.

Jeandelize, P., and Perrin, M. Moindre résistance des lapins thyroïdectomisés à l'intoxication par l'arseniate de soude. C. r. S. B., 64, p. 233,1908 . 
JeNNER, W. Wirkung des Adrenalins bei Hämaturie. D. m. IV.. 1903.

JeNTZNER and BeUTTNER. Exper. Unters. z. Frage d. Kastrationsatrophie. Z. G. G., 42, 1900 .

JOACHIMSTHAL. Über Z Zwergwuchs u. verwandte Wachstumsstörungen. D. m. W., 1899 .

Joannovics, G. Über das Hepatotoxin. W. k. W., igog.

- Der Cytotoxine. Zeitschr. f. Immun.-Forsçh. Ref. i, I909.

JOESTEN. De glandularum suprarenalinum structura. Bonn, i863.

- Der feinere Bau der Nebennieren. A. phys. Heilk., 5, I864.

JoHN. Klinische Erfahrungen über intravenöse Suprarenininjektion bei schweren Herz- und Gefäszkollapsen. M. m. WV., 1909.

Johnston. Suprarenal extract in Addison's disease. 'Brit. Med. Journ., p. 419 , 1900 .

Johnston and Manko. Case of acromegaly. Glasg. Med. Journ., 50, p. 112,1898 .

Jolin. Über den Jodgehalt der menschlichen Schilddrüsen in Schweden. Upsala Läkaref. Fört, xi, Suppl.; Hammarsten Festschrift, No. 8, I 906, Ref. B. C., v.

Jones, PH. Mills. Epinephrin, the active principle of the suprarenal gland. California. Journ. of Med:, p. 302, October, I902.

Jones, W. On the enzyme of the thymus. On the enzyme of suprarenal gland. A. J. P., x, No. 6, p. 24 ; and Z. ph. C., 4I, p. Ior.

JoNes and WhIPpLE. The nucleo-proteid of the suprarenal gland. A. J. P., 7, p. 423 .

Jonescu, D. Notiz über eine besondere Affinität der Nierengefäsze zu Adrenalin. W. k. W., 1908 .

Jonson, A. Studien über Thymusinvolution. Die akzidentelle Involution bei Hunger. A. m. A., 73, p. 390, r909.

JORES. Uber Nebennierensarkome. D. m. W., I8c4.

- Wesen und Entwicklung der Arteriosklerose. Wiesbaden, 1903.

Joris, H. Contribution à l'étude de l'hypophyse. Mém. Acad. méd. d. Bélgique, 9, 1907.

- Le lobe postérieur de la glande pituitaire. Ibid., ıgo8.

- L'hypophyse au cours de la gestation. Bull. Acad. méd. d. Bélg., rgo8.

JosepH, J. R. Further investigation upon the influence of organ extracts of cold-blooded animals on the blood-pressure. J. E. M., 19, p. 606, 1907.

Joseph, J. R.; and Meltzer. Eine Vergleichung der Giftigkeit der Chloride des Magnesiums, Calciums, Kaliums und Natriums. C. P., 22, p. 244, 1908 .

Josine, R. Über das Antithyroidin Mœbius bei Morb. Basedowii. M. m. W.., I 904 .

Josserand, P. Contr. à l'étude phys. de l'adrénaline. Paris, 1905.

Josué, O. La vasoconstriction déterminée par l'adrénaline n'est pas due aux centres sympathiques. C. r. S. B., 55, p. 30, 1903.

- Athérome expérimentale par injections répétées d'adrénaline dans les veines. C. r. S. B., 55, p. 1374 , 14 novembre, 1903.

- La pression artérielle chez le lapin à la suite d'injections répétées d'adrénaline dans les veines. C. r. S. B., 56, ii, 14 and 21 octobre, 1o décembre, $1904 ; 57, \mathrm{i}, \mathrm{p} .319$, 1905 .

-- Les capsules dans trois cas d'athérome artes. Soc. hôp., 19 février, 1904.

- Athérome artériel et artériosclerose. Press. méd., 4 mai, Igo4.

- Pathogénie de l'athérome artériel. Congr. franç de médicine, 24 octobre, I 904 .

- Contribution à l'étude histologique de l'athérome artériel. J. d. P. P., p. 690,1905 .

- Traité de l'artériosclérose. Bruxelles, igo8.

Josú́ and BLOCH. Action hypertensive de la couche corticale des capsules surrénales. C. r. A., 144, p. 1295, 1907.

Joteyko, J. Action toxique curarisante de la neurine. S. m., p. I22, I897.

- Influence de l'adrénaline et de quelques autres produits glandulaire sur la contraction musculaire. Journ. méd. de Bruxelles, Nos. 27-29, pp. $417,433,449,1903$.

JouchtENKo, A. J. Influence de la thyröidine, de la spermine et de l'adrénaline, de l'ablation du corps thyroïde et des testicules sur les processus d'oxydation, la toxicité urinaire et les échanges gazeux chez les animaux. Russki Wratsch., 1907-1908; Ref. J. d. P. P., x, p. I IO3, I5 novembre, Igo8. 
Joukovsky. Hydrocephalie et tumeur congenitale de la glande pinéale chez un nouveau-né. Rev. mens. des maladies de l'enfance, xix, p. I97, I901.

Joung, J. A., and LeHMAN, J. E. Internal secretion of the suprarenals. J. o. P., 37, I 908 .

Jourdain, S. Sur le système V. porte rénale. Ann. des sc. nat. ser., iv, t. I2, 1859 .

Jovane, A., and PACE, C. Capsule surrenali e rachitismo. La pediatria, B. xvii, p. 195 .

Jowert. Journ. of the Chem. Soc., p. 193, 1904; Jahrb. f. Tierchem., 34, p. $58 \mathrm{I}, 1904$.

Julien. Rech. sur les suites éloignées de la castration chez la femme. Thèse de Lille, igoo.

Julin, CH. Le système nerveux grand'sympathique de l'Ammocoetes (Petromyzon Planeri). An. An., pp. 192-201, 1887.

- Recherches sur l'appareil vasculaire et le système nerveux périphérique de l'Ammocoetes (Petromyzon Planeri). A. B., vii, pp. 759-902, 1887.

Kaessmann, F. Adrenalinbehandlung der Osteomalakie. C. G., 3I, p. 1376 , 1907 .

KAHLDEN, v. Beiträge zur pathologischen Anatomie der Addisonschen Krankheit. V. A., I 14, p. 65, 1888 .

- Über Addisonsche Krankheit. Zieglers Beiträge, Io; D. m. W., I89ı.

- Über Addisonsche Krankheit und über die Funktion der Nebennieren. Zusammenfassendes Ref. C. a. P., 7, pp. 464-509, 1896.

KahN, R. H. Beobachtungen über d. Wirkung d. Nebennierenextr. A: P., pp. 522-537, 1903.

- Über Beeinflussung der Gefäszweite in der Netzhaut. C. P., I8, 1904.

- Über die Beeinflussung des Augendruckes durch Extrakte chromaffinen Gewebes. C. P., 20, No. 2, p. 33, 1906.

- Zur Frage nach der inneren Sekretion chromaffinen Gewebes. P. A., I28, p. 519,1909 .

- Die Störungen der Herztätigkeit durch Adrenalin im Elektrokardiogramm. P. A., I29, 1909 .

KAHN and LiEnEN. UUber d. scheinbaren Gestaltsänderungen d. Pigmentzellen. A. P., 1907.

KAISERLING, D. Beitrag zur Wirkung intravenöser Suprarenininjektionen auf die Kaninchenaorta. B. k. W., I907.

Kaiserling, G., and Orgler, G. Über das Auftreten von Myelin in Zellen und seine Beziehung zur Fettmetamorphose. V. A., 168, pp. 296-310, 1902 .

KajIURA, G. La choline se trouve-t-elle dans le liquide cephalo-rachidien des épileptiques? Quart. Journ. of Exper. Physiology, i, pp. 291-296, 1908 .

KalamkarofF, J. G. Zur Frage über experimentelle Atheromatose der Aorta beim Kaninchen und über die Wirkung der Jodverbindungen auf diesen Prozesz. Diss., 1907; Ref. Bph. C., iii, No. 674.

Kantorowicz, L. Zur Histologie des Pankreas. Diss., Gieszen.

KAPLAN, D. On the hypodermic use of adrenalin chloride in the treatment of asthmatic attacks. Med. News, 86, 19, p. 871, May, 1905.

KaPLAN and FFDOROFF. Adipose douloureuse. Rev. suisse d. méd., 1902.

KARAKASCHEFF, v. Beiträge zur pathologischen Anatomie der Nebennieren (Atrophie, vikariierende Hypertrophie, Tuberkulose). Ziegl. Beitr., 36, p. 401,1904 .

- Weitere Beitr. z. pathol. Anatomie der Nebennieren. Ibid., 39, p. 373, 1906.

- Über das Verhalten der Langerhansschen Inseln des Pankreas bei Diabetes mellitus. D. A. k. M., 82, 1904 ; and ibid., 87, 1906.

KAREWSKI. Zwei exstirpierte Nierentumoren. B. k. IV., IgO4.

KARG. Über Hautpigment und Ernährung der Epidermis. An. An., p. 377, 1887.

Kasahara. Über das Bindegewebe des Pankreas bei Krankheiten. V. A., I I I, 1896 .

KASCHKE. Cachexia thyreopriva und Myxödem. Diss., Berlin, 1892.

KASSEL, K. Epinephrinum solutum. Th. M., p. 422, August, I903.

KAST. Über lymphagoge Stoffe im Serum Nierenkranker. D. A. k. M., 73, 1902. 
Kastschenko. Das Schicksal der embryonalen Schlundspalten bei Säugetieren. A. m. A., 30, I 887 .

KASSOWITZ. Allgemeine Biologie. 4 Bände, ı̊99-19o6.

- Infantiles Myxödem, Mongolismus und Mikromelie. Wien, Ig02.

KASTEN, F. Üb. d. therapeutischen Wert d. Nebennierensubstanz. Diss., Rostock, 1902 .

KaTz, A. Harnuntersuchungen in einem Falle von Morbus Addisonii. W. m. Bl., is9o.

KATZ and WiNkLER. Experimentelle Studien über Fettgewebsnekrose des Pankreas. A. V., 4, 289, 1898 .

KATZENSTEIN. Über Veränderungen in der Schilddrüse nach Exstirpation der zuführenden Nerven. A. P., I897 and 1899.

- Über exp. Beobachtungen an der Schilddrüse. D. m. W., 1899.

Kaufmann. Sur le diabète pancréatique et le mécanisme de régulation de la glycémie normale. A. d. P., p. 209, 1895.

- Mode d'action du système nerveux dans la production de l'hyperglycémie. Ibid., p. 266.

KaUfmanN, E. Über Zwischenzellengeschwülste des Hodens. V. d. p. G., II, 1907 .

KAUFMANN, M. Stoffwechselbeobachtung bei einem mit Nebennierensubstanz behandelten Falle von Morbus Addisonii. C. Stoffw. u. Verdauungskr., ii, 7, 1901.

- Über Diabetes und Psychose. M. m. W., 1908.

- Organotherapie der Nephritis. F. M., pp. 633, 665, 1905.

KaUfmann and ManNaberg. Diskussionsbemerk. zu Schur u. Wiesel. W. k. W., I 1907 .

KaUsCH. Über den Diabetes der Vögel nach Pankreasexstirpation. A. P. P., 37, p. 274,1896 .

KEDZIOR and ZANIETOWSKY. Zur pathologischen Anatomie der Basedowschen Krankheit. N. Z., No. 10, p. 438, 1901.

KeHRER. Zur Menstruationslehre. B. G. G., 2, I 884

- Versuche über Kastration und Erzeugung von Hydrosalpinx. Ibid., I887.

KEHRER, E. Physiologische und pharmakologische Untersuchungen an den überlebenden und lebenden inneren Genitalien. A. G., 8I, p. 129, 1907 .

KeIBEL, F. Uber die Entwickelung des Urogenitalapparates von Echidna. Ergänzungsheft. An. An., 22, pp. 14-19, 1903.

KeISAKU-KobUkO. Über die kongenitale Syphilis der Nebennieren. C. a. P., I4, 1903 .

KEITLER. Über das anat. und funkt. Verhalten der belassenen Ovarien und Exstirpation des Uterus. M. G. G., 20, I904.

Keller, K. Über den Bau des Endometriums beim Hunde. Anat. Hefte, I I 8, I 909 .

KEILY, A. O. J. Über Hypernephrome der Niere. Ziegl. Beitr., 23, 1898.

KeNwORTHEY, IVM. B. The use of suprarenal capsule in hæmoptysis. New York Med. Record, p. 415, I9oI.

KERKIS, C. Les vasoconstrictions des sérums normaux. Thèse de Genève,

KING, J. H. The influence of the thyroid on carbohydrate metabolism. J. E. M., Igo9.

KINNER. Über die Behandlung der Rachitis mit Nebennierensubstanz. Diss., Breslau, ז9or.

KINNICUT, F. P., A clinical study of the therapeutic value of the calcium salts in gastric tetany. A. J. M. S., 138, pp. 1-10, 1909.

KINZLER. Über den Kausalzusammenhang zwischen Zerstörung der Nebennieren und Bronzed skin. Diss., Tübingen, 1895.

Kirkatdy, J. W. On the head-kidney of Myxine. Quart. Jour. of Micr. Scien., 35, 1894 .

KIRCH, R. Über Adrenalinanwendung bei schweren Blutungen. D. m. W., I 903 .

KISCH, H. Die Schilddrüsentherapie bei Fettleibigkeit. W. m. P., I899.

KISHI. Beiträge zur Physiologie der Schilddrüse. V. A., 176, I904.

KLAPP, R. Experimentelle Beiträge zur Kenntnis der Wirkung der Nebennierenpräparate. Z. Ch., 71, p. I87, 1904.

KLeBs. Handb. z. path. Anat., I, Abt. ii, 1876; and Allg. Path., ii, 1897. 
KLEestadT, W. Über cystische Tumoren im Bereiche des Infundibulum. Diss., München, 1906.

KLEIN. Ü̉ber die Bedeutung der bei Morbus Basedowii im Zentralnervensystem nachgewiesenen pathol.-anatomischen Befunde. D. Z., p. 431, 1904 .

- Neue Arbeiten über sog. fötale Rachitis. C. a. P., 12, I00I.

KLeINHANs, F., and ScHENK, F. Experimentelles zur Frage nach der Funktion des Corpus luteum. Z. G. G., 66, p. 283.

KLEMPERER, G. Addisonsche Krankheit. In Eulenburg, Real-Enc., 4. Aufl, i, 1907.

KLEMPERER, G., and UMBER, H. Z. Kenntnis d. diabetischen Lipämie. Z. k. M., 65, 1908 .

KLETT. Zur Beeinflussung der phototropen Epithelreaktion in der Froschretina durch Adrenalin. A. A. Supplementbd., p. 213, 1908.

KLIENEBERGER, C. Üb. d. Wirkung v. Nebennierenpräparaten auf die Kaninchenaorta bei gleichzeitiger Anwendung von Jodipin und Jodkali. C. i. M., 28, 1907.

KLotz. Experimental production of arterio-sclerosis. Brit. Med. Journ., 1906; J. E. M., viii.

KNAFFL-LENz, v. Beziehungen $z w$. Lipoidverflüssigung und Zytolyse. P. A., 123,1908 .

KNAUER. Einige Versuche von Ovarientransplantation am Kaninchen. C. G., 20, p. 524,1896 .

- Uber Ovarientransplantation. W. k. W., 1899.

- Die Ovarientransplantation. A. G.. 6o, p. 322, 1900.

KxöPfELMACHER, W. Alimentäre Glykosurie und Myxödem. W. k. W., 1904.

- Demonstrationen. Ibid., 1903 and 1906.

KNY, E. Fall von isoliertem Tumor der Zirbeldrüse. N. Z., p. 281, 1889 .

KOCHER, TH. Über Kropfexstirpation und ihre Folgen. A. k. Ch., 29, 1883 .

- Vorkommen und Verteilung des Kropfes im Kanton Bern. Bern, 1889.

- Zur Verhütung des Kretinismus. Z. Ch., 34.

- Die Schilddrüsenfunktion. Korr. Schweiz. A., 1895.

- Zweitausend Kropfexstirpationen. A. k. Ch., 64, 1901.

- Die Pathologie der Schilddrüse. C. i. M., Müunchen, 1906.

- Chirurgische Operationslehre, 1907.

KocHER, A. Über Morbus Basedowii. M. G. M. C., 9, 1902.

- Über die Ausscheidung des Jods im menschlichen Harn und ihre Beziehung zum Jodgehalt und zur Verkleinerung der Strumen. M. G. M. C., 14, 359, 1905 .

KocurEK, R. Über Zwergwuchs. V. d. naturf. V. Brünn, 45.

KöHLER. Einflusz der Auszenteinperatur auf experimentelle Glykosurien. Z. k. M., 1908 .

KöLLIKER, v. Mikroskopische Anatomie oder Gewebelehre des Menschen. Leipzig, 1854 .

- Handbuch der Gewebelehre des Menschen. 2. Aufl., 1855; 3. Aufl., I859; 4. Aufl., I863; 5. Aufl., 1867.

- Entwicklungsgeschichte des Menschen und der höheren Tiere. 2. Aufl., 1879.

- Über Zwitterbildungen bei Säugetieren. W. B., I884.

- Über die Nerven der Nebennieren. V. d. N. Ä., 66, p. 363. I894.

- Über die feinere Anatomie und die physiologische Bedeutung des sympathischen Nervensystems. W. k. WT., 1894 .

KöNIG. Über ein Psammosarkom der Zirbeldrüse. Diss., München, 1894.

KöNIGSTEIN. Anwendung des Extractum suprarenale haemostaticum. W. m. P., 1897 and 1898 .

KöNIGSTEIN, JUN. Demonstration von Sekretbildern im Epithelkörper. W. k. W., p. 778, 1906 .

KöRber, E. Kann Nebennierengewebe durch biochemische Reaktionen nachgewiesen werden? Diss., Greifswald, 1903.

- Uber die Croftansche Methode zur Erkennung von Nebennierengewebe auf biochemischem Wege. V. A., 142, p. 356, 1908 .

KoHN, A. Studien über die Schilddrüse. I, A. m. A., 44, 1895 : II, Ibid., 48,1896 .

-- Die Nebennieren der Selachier nebst Beiträgen zur Kenntnis der Morphologie der Wirbeltiernebenniere im allgemeinen. A. m. A., 53 , pp. $281-312,1898$. 
Кон., A. Über die Nebenniere. P. m. W., pp. 194-195, 1898 .

- Die chromaffinen Zellen des Sympathicus. An. An., I5, pp. 293-400, I 899 .

- Die Epithelkörperchen. Erg. d. Anat. u. Entw., ix, pp. 194-252, 1899 and 1900 .

- Uber innere Sekretion. P. m. W., I900.

- Über Bau und Entwickelung der sogenannten Carotisdrüse. A. m. A., 56, 1900.

- Chromaffine Zellen, chromaffine Organe, Paraganglien. P. m. W., I 902.

-- Die Paraganglien. A. m. A., 62, pp. 263-365, 1903.

- Das chromaffine Gewebe. Erg. d. Anat. u. Entw., xii, pp. 253-348, 1902 and 1903 .

- Die Blutgefäszdrüsen. P. m. W., 1903.

- Üb. d. Entwicklung d. sympath. Nervensystems d. Säugetiere. A. m. A., 70, 1907.

-- Uber das Pigment der Neurohypophyse des Menschen. Ibid., 75, p. 337, 1910 .

KOLISCH. Über sklerotische Veränderungen durch Phloridzinzufuhr. G. d. A., I 1905 .

Kolisch and PICHLEr. Ein Fall von Morb. Addisonii mit Stoffwechseluntersuchung. C. k. M., 1893 .

KOLLARITS. Hypophysentumor ohne Akromegalie. D. Z. N., 28, p. 88, r905.

Kolle, W. Über Ziele, Wege und Probleme der Erforschung des endemischen Kropfes. Korr.-B1. Schweiz. Ärzte, rgog.

KON JUTAKA. Hypophysenstudien. Zieglers Beiträge, 44, 2, 1909.

Koplik. Sporadic cretinism and its distinction from idiocy. N. Y. Med. Journal, I 897.

KorANyI, A: v. Über die Wirkung des Jods auf die durch Adrenalin erzeugte Arteriosklerose. D. m. W., 1906.

- Berichtigung zu meiner Mitteilung uber die Wirkung des Jods auf die durch Adrenalin erzeugte Arteriosklerose. Ibid., 1907.

Kose, WV. Über das Vorkommen chromaffiner Zellen im Sympathicus des Menschen und der Säugetiere. Lotos, No. 6, I898.

- Über das Vorkommen einer Carotisdrüse und der chromaffinen Zellen bei Vögeln. An. An., 22, pp. 162-170, 1902.

- Über die Carotisdruise und das chromaffine Gewebe der Vögel. Ibid., 25, 1904.

- Die Paraganglien bei den Vügeln. A. m. A., 69, 1907.

Kostlivy, S. Leber- und Pankreasläsionen in ihren Beziehungen zum chromaffinen System. M. G. M. C., 19, pp. 616-636, 1909.

KRAUPA, E. Untersuchungen über das synthetische Suprarenin. M. K., I 908 .

KraUs, F. Über die Zuckerumsetzung im Blute auszerhalb des Gefäszsystems. Z. k. M., 21, p. 315, 1892 .

- Über das Kropfherz. W. m. W., 1899 .

- Myxödem, Cachexie pachydermique. v. Mehrings Jahrb. d. inn. Med., I 901 .

- Über Fettdegeneration und Fettinfiltration. V. d. p. G., 6, 1903.

- Pathologie der Schilddrüse. V. C. M., München, rgo6.

KRAUS, F., and FRIEDENTAL, H. Über die WVirkung der Schilddrüsenstoffe. B. k. W., p. $1709,1908$.

KRAWKOFF. De l'action des prép. jodées sur l'artérionecrose expérim. Wratsch., 1008; Ref. J. d. P. P., Io, p. 549, 1908.

KRECKE. Chirurgische Behandlung des Morbus Basedowii. M. m. W., 1909 .

KREHL, L. Über die Störung chemischer Korrelationen im Organismus. D. A. k. M., 88, pp. $351-384,1907$; and V. d. N. Ä, Stuttgart.

KRESS, K. Wirkungsweise einiger Gifte auf den isolierten Dündarm. P. A., 109, 1905.

KRETSCHMER, WV. Über den Mechanismus der Adrenalinwirkung und dauernde Blutdrucksteigerung durch Adrenalin. A. P. P., 57, p. 348, 1907.

- Über die Beeinflussung der Adrenalinwirkung durch Säure. Ibid., 57, p. 438,1907 .

KREUZFUCHS. Erfahrungen uber innere Adrenalindarreichung. W. m. P., 1906. 
KRÜCKmann. Adipositas universalis bei 2 Geschwistern. D. m. W., Igo8. KRUkenberG, C. FR. W. Die farbigen Derivate der Nebennierenchromogene. V. A., 101, pp. 542-571, 1885 .

Krychtopenko, A. Caps. surrénales. Thèse de St. Pétersb., 1904; A. d. sc. biol., 12, 1906.

KUDINZEW. Zur lehre von den Glandulis suprarenalibus. Wratsch., I897; Ref. St. Petersburger m. W., 22.

KÜHN, A. Über das Vorkommen von akzessorischen Nebennieren. Z. r. M., $28,1866$.

KüLBS. Exper. Studien über die Wirkung des Nebennierenextraktes. A. P. P., 53, p. 140 ; M. m. W., 1905.

KÜlz, E. Beitr. z. Pathologie u. Therapie d. Diabetes. Marburg, I874 and 1875 .

KÜLIABKO and ALESSANDROWITSCH. Wirkung verschiedener Substanzen auf die automatischen Bewegungen des isolierten Darmes. C. P., IS, p. 280,1904 .

KÜRSTEINER. Die Epithelkörperchen in ihrer Beziehung zur Thyreoidea und Thymus. Anat. Hefte, I 808 .

KÜSTER. Zur Entwicklung der Langerhansschen Inseln im Pankreas beim menschlichen Embryo. A. m. A., 64, 158, 1904.

- Über Gliome der Nebennieren. V. A., I80, pp. 117-130, 1905.

- Chirurgie der Nieren der Harnleiter und der Nebennieren. D. Ch., 52, 1902 .

KuH, S. Treatment of acromegaly with pituitary bodies. J. A. M. A., p. 295, 1902 .

Kumita. Lymphgefäsze der Nierenkapsel und der Nebenniere. A. A., p. 49, I 1909 .

Kummer. ${ }^{2}$ Zur Kenntnis des Morbus Addisonii. Korresp.-B1. Schw. A., No. $15,1886$.

KUNDRAT, H. Über Wachstumsstörungen des menschlichen Organismus. Wien, I S S9o.

KupfFer, v. Die Deutung des Hirnanhangs. Sitzungsber. d. Gesellsch. f. Morph. u. Phys. München, 1894 .

KURDINowSKI. Physiologische und pharmakologische Versuche an der isolierten Gebärmutter. C. P., p. 323, 1904; A. P., Suppl. 3, 1904.

Kusmine, K. Untersuchungen über die Lymphe. 6. Mitt. Über den Einflusz der Lymphagoga (Lebergifte) auf die Lymphe. Z. B., 46, p. 554 .

Kussmaul, A. Über geschlechtliche Frühreife. Würzb. med. Zeitschr., 3, I 862 .

Kutschera, A. v. Zur Epidemiologie des Kretinismus. Off. Ber. über den Amtsärzte-Kongresz. Deuticke, Wien, Ioog.

- Das Groszenwachstum bei Schilddrüsenbehandlung des endemischen Kretinismus. W. k. W., 1909 .

KYRLE. Über die Regenerationsvorgänge im tierischen Pankreas. A. m. A., $72,1908$.

Labadie-Lagrave. Maladie bronzée ou maladie d'Addison. Traité des mal. du sang, Paris, 1893.

- Toxicité urinaire chez la cobaye en gestation. C. r. S. B., 4 juillet, 1897.

Labadie-Lagrave and Degny. Associations morbides de l'acromégalie. Arch. gén. de méd., N. S., i, p. 129, 1899.

LABBE, H. V. Physiologie pathologique des diabètes sucrés. Rev. de méd., 1907.

LABBÉ and GiRAUd, G. Dosage de l'iode contenu dans les corps thyroïdes des tuberculeux. C. r. S. B., 65, pp. 371-372, 1908.

LABzine, M. M. De la régénération des glandes surrénales. Arch. scienc. biol., II, p. 319, 1905; Thèse de St. Pétersbourg, 1904.

LäwEN, A. Quantitätive Untersuchungen über die Gefäszwirkung von Suprarenin. A. P. P., 5 I, p. 415, 1904 :

- Experimentelle Untersuchungen über die Gefäszwirkung von Suprarenin in Verbindung mit örtlich anästhesierenden Mitteln. Z. Ch., 74, p. 163,1904 .

- Die örtliche Anästhesie bei Zahnextraktionen, mit besonderer Berücksichtigung der Cocain-Adrenalingemische. A. k. Ch., 72, p. 231, 1904. 
LAFOND, M. Sclérodermie et Corps pituitaire. Thèse de Lyon, rgor-1902.

LAGUESSE, E. G. Sur la formation des îlots du Langerhans. I893.

- Structure et développement du pancréas. J. A. P., I894.

- Sur la variabilité du tissu endocrine dans le pancréas. C. r. S. B., p. 900, r 899 .

- Structure d'une greffe chez le chien. Ibid., p. 853, I902.

- Sur la structure du Pancréas chez quelques Ophidien et particulièrement sur les îlots endocrinés. Arch. d'anat. microsc., 4, 190r.

- Ilots de Langerhans et sécrétion interne. C. r. S. B., 57, ii, p. 368, i 905.

- Numération des îlots endocrinés dans le pancréas humain. Ibid., p. 504, 1905 .

- Lobule et tissu conjonctif dans le pancréas de l'homme. Ibid., p. 539.

- Ilots endocrinés et formes de transitions dans le lobule pancréatique, p. 542 .

- Etude d'un pancréas de lapin, \&c. Arch. d'anat. microsc., 9, p. 90, r9o6.

- Le pancréas. Lyon, 1906-1907.

- Sur les rapports des îlots endocrinés avec l'arbre excréteur dans le pancréas de l'homme adulte. Ibid., 65, p. 139, roo8.

- Preuve expér. du balancement dans les îlots endocrinés du pigeon. Ibid., 67, p. 94, 1909 .

- Sur l'évolution des îlots endocrinés dans le pancréas. Arch. d'anat. microsc., I r, 1909 .

LAGuesse and GONTIER DE LA ROCHE. Les îlots de Langerhans après ligature. C. r. S. B., p. 584, 1902.

LAIGNEL-LAVASTINE. Application de l'imprégnation argentique de Cajal à l'étude histo-chimique de la cellule médullo-surrénale. C. r. S. B., 58, p. $66 \mathrm{r}, \mathrm{r} 905$.

- Structure des cellules nerveuses de la substance médullaire de la surrénale humaine. Ibid., pp. 697-700, I906; pp. 331-332, r905.

- Inclusion surrénale d'un ganglion solaire. Bull. de la soc. anat., Paris, No. 5, r905.

- Les troubles glandulaires dans les syndromes neuropsychiques. Trib. méd., r 908 .

- Des troubles psychiques par perturbation des glandes à sécrétion interne. XVIIIe Congr. des médecins aliénistes et neurologistes. Dijon, 1908; Sem. méd., igo8.

- La corrélation des glandes à sécrétion interne et leurs syndromes pluriglandulaires. Gaz. des Hôp., p. 1563, rgo8.

LAIGNEL-LAVASTINE and AUBERTIN. Adénome médullaire de la capsule surrénale. A. m. e., 20, rgoo.

Laignel-L.Avastine and Bloch. Syndrome de Basedow. Arch. gén. méd., 1904.

LAIGNEL-LAVASTINE and HALBRon, $P$. Trois cas le tuberculose surrénale sans mélanodermie. Bull. de la soc. anat. Paris, No: 4, r907.

LAmbert. Sur l'action des extraits du corps jaune de l'ovaire. C. r. S. B., 62 , p. 18,1907 .

LANCERAUX. Notes et réflexions à propos de deux cas du diabète sucré avec altération du pancréas. Bull. acad. de méd., III. sér., 7, 1215, 1877.

- Nouveaux faits de diabète sucré avec altération du pancréas. Ibid., r 888 .

LANCERAUX and THrrolorx. Le diabète pancréatique. C. r. A., II 5 .

- Les rapports des lésions des capsules surrénales et de la maladie d'Addison. Arch. gén. méd., p. 5, janvier, I \$9o.

- Les glandes vasculaires. Sem. méd., I893; Cinquant. de la Soc. de Biol., r 899 .

- La trophoneurose acromégaligue. Sem. méd., 1 \$95.

LANDAU, E. Zur Morphologie der Nebenniere. I. Mitt. Protokolle d. Naturf.-Ges. b. d. Univ. Dorpat, rgor; II. Mitt. Allruss. Versamml. d. Naturf. u. Ärzte, St. Petersburg, Igor; III. Mitt. Sitzungsber. d. Naturf.-Ges. b. d. Univ. Jurjew [Dorpat], 1905; IV. Mitt. I.M., 24, pp. $43 x-446$, r 907 .

- Über die anatomischen Veränderungen in den Nebennieren bei Arteriosklerose. Z. k. M., 64, p. 237 , 1907.

- Altersveränderungen des Venensystems der Nebennieren. Petersb. m. W., 1908 .

- Experimentelle Nebennieren-Studien. Dorpat, rgo8.

LANDAU, M. Zur Behandlung der Beschwerden der natürl. u. anticept. Klimax mit Eierstocksubstanz. B. k. W., I 896 . 
LANDERER, P. Zur Kasuistik der Addisonschen Krankheit. Diss., Tübingen, 1878 .

LANDO. Veränderungen der Pankreas bei Lebercirrhose. Z. H., 17.

LaNDOIS, F. Die Epithelkörperchen. Erg. d. Chir. u. Orthop. I. 1910.

LANDOLT. Über die Verwendung des Nebennierenextraktes in der Augenheilkunde. C. Augenh., xxiii, p. 321, November, 1899.

LANDSTRÖM, J. Neuere Anschauungen über die Basedowsche Krankheit. M. K., 1909.

LANE; M. A. The cytological characters of the areas of Langerhans. J. A. Z., 1907.

LANGE, O. Über die Anwendung des Adrenalin bei Blutungen. M. m. W., 1903.

LANGENDORFF, O. Beitrag zur Kenntnis der Schilddrüse. A. A., Suppl. 218,1889

- Ältere und neuere Ansichten über die Schilddrüse. Biol. C., 9, 188 g.

- Über Veränderungen in den periph. Nerven bei Cachexia thyreopriva. V. A., 128,1892 .

- Über die Innervation der Koronargefäsze. C. P., 21, 1907.

LANGER, C. Wachstum des menschlichen Skeletts mit Bezug auf den Riesen. Denkschrift d. Akad. d. Wiss. Wien, 31, 1872 .

LANGERHANS. Beiträge zur mikr. Anatomie der Bauchspeicheldrüse. Diss., Berlin, 1869 .

- Nebennierenveränderungen beim Morbus Addisonii. V. d. p. G., 7, p. 254, 1903.

Langerhans and Saweliew. Beitr. zur Physiologie der Brustdrüse. V. A., 134,1893 .

Langians. Über die epithelialen Formen der malignen Struma. V. A., $189,1907$.

LANGLEX, J. N. Observations on the physiological action of extracts of the suprarenal bodies. J. o. P., 27, p. 237, 1901.

- On the reaction of cells and nerve-endings to certain poisons. Ibid., 33, p. 375 , I905; and 39, p. 235 , 1909 .

- The sympathetic and other related systems of nerves. Schäfer Textbook of Physiol. ii, 1900 .

- Das sympathische u. verwandte nervöse System der Wirbelthiere. E. E., 2 , ii. p. 818.

Langlois, P. Destruction des capsules surrénales chez le chien. C. r. S. B., p. 444,1893 ; A. d. P., pp. $488-498,1893$.

- Maladie d'Addison. Richet Diction. de Phys., i, 1895.

- De l'opothérapie dans la maladie d'Addison. 'P. m., 19 septembre, 1896.

- Action différente de l'extrait des capsules surrénales sur la pression sanguine suivant l'état d'altération morbide de ces organes. C. r. S. B., p. 942,1896 .

- Sur l'homologie fonctionnelle des capsules surrénales des grenouilles et des mammifères. C. r. S. B., pp. 184-186, Paris, 1897.

- Action des agents oxydants sur l'extrait de capsules surrénales. Ibid., p. 524,1897 .

- Du foie comme organe destructeur de la substance active des capsules surrénales. Ibid., p. 571, 1897.

- Physiopathologie des capsules surrénales. A. d. P., 30, p. 125, 1897.

- Les capsules surrénales. Paris, 1897 . T. iv des travaux du labor. de Phys. de $\mathrm{Ch}$. Richet.

- Le mécanisme de destruction du principe actif des capsules surrénales dans l'organisme. A. d. P., 30 janvier, i $\$ 98$.

- Sur l'identité physiolog. des corps surrénaux chez les batraciens et les mammifères. A. d. P., 10, 1898 .

- A propos de la destruction de l'adrénaline dans l'organisme. C. r. S. B., p. 93,1904 .

Langlois and Charrin. Du rôle des capsules surrénales dans la résistance à certaines infections. C. r. S. B., p. $708,1896$.

L.ANGLOIS and REHNS, J. Les capsules surrénales pendant la période fœale. Ibid., 1899 .

Langstein, L. Behandlung d. Rhachitis mit Nebennierensubstanz. J. K.. 53,1901 .

LaNz, O. Zur Schilddrüsenfrage. Volkmanns Sammlung. I eipzig, İ̃ọ

- Über Thyreoidismus. D. m. W., I895. 
LANZ, O. Zur Schilddrüsentherapie des Kropfes. Korr.-Bl. f. Schweizer Ärzte. No. 2, 1895 .

- Ein Vorschlag zur diätetischen Behandlung von Basedowkranken. Ibid., No. 23,1899 .

- Onderzoekingen over de afstammelingen van thyreopriven. Nederl. Tijdschr. voor Geneeskunde, No. 20. 1904.

- Progenitur thyreopriver Tiere. A. k. Ch., 74, 1904.

- Adrenaline en heelkunde. Weekblad, p. 1335, II Juni; Virch.-Hirsch, i, p. 736. 1904.

- Cachexia und Tetania thyreopriva. C. Ch., p. 339, 1905.

LAPIENTE and LEZENE. Gliome primitif de la capsule surrénale. A. m. e., 19, p. 59,1907 .

L.ARGUIER DES BAUCELS. Activation du suc pancréatique pur sous l'influence combinée des colloïdes et des électrolytes. C. r. S. B., 57, ii, p. 130, 1905.

LATTES, L. Über die Zuckerbildung in der künstlich durchbluteten Leber diabetischer Tiere. B. Z., 20, 1904.

Latzko, W. Zur Diagnose und Frequenz der Osteomalazie. M. G. G., I, 1895 ; and W. k. W., 1893 and 1894 .

- Uber Nebennieren und Osteomalazie. W. k. W., p. 239, 1907.

Latzko, W., and Schnitzler, J. Beitr. z. Organotherapie bei Osteomalacie. D. m. W., 1897 .

LaUB, M. Klinischer Beitrag zur Lehre vom Status thymicus. W. k. W., I899.

- Über die Wirkung einiger dem Adrenalin verwandter Ketonbasen in der rhinologischen Praxis. W. m. W., 1906 .

LaUnoIs, L. Do l'atrophie de la prostate. De la castration dans l'hypertrophie de la prostate. Ann. d. mal. d. org. gén. ur., 1894.

- Les cellules sidérophiles de l'hypophyse chez la femme enceinte. C. r. S. B., 55, 1903 .

- Glycosurie et hypophyse. Arch. gén. d. méd., 1903.

- Recherches sur la glande hypophysaire de l'homme. Thèse de Paris, I904.

- La cellule pancréatique dans l'intoxication par la pilocarpine. C. r. S. B., p. 245 , 1904 .

- Diapédèse et sécrétion pancréatique active. Ibid., 56, p. $2117,1904$.

- Action de la pilocarpine sur la sécrétion pancréatique. Ibid., p. 579 .

- Contr. à l'étude histophysiol. de la sécrétion pancréatique. A. i. Ph., $3,1905$.

- Traveaux récents sur l'anatomie fine des capsules surrénales, particulièrement en ce qui concerne la cellule cliromaffine. Biol. méd., No. 7, pp. $265-283$, 1905 .

- Contribution à l'étude du sérum des animaux éthyroïdés. C. r. A., 147, p. 263,1908 .

- Nouvelle contrib. à l'étude du sérum des animaux éthyroïdés. Ibid., 147,1908 .

LAUNoIS and Esmen. Essai d'interprétation du syndrome de Basedow. IXe Congr. français des Médicins. 14-16 octobre, 1907.

LAUNoIS, LOEPER and ESMONET. La sécrétion graisseux de l'hypophyse. C. r. S. B., 56, 575,1904 .

LAUnoIs and MǘLON. Etudes sur l'hypophyse humaine à la fin de la gestation. Arch. de gyn. et d'obstétr., I904.

LaUnoIs and Roy. Etude biologique sur les géants. Paris, 1904.

- Gigantisme et infantisme. Nouv. Icon. de 1. Salp., I9o2.

- Gigantisme et castration. Rev. intern. méd., 1903.

LAURECK. Zur Kasuistik des Morbus Addisonii. Diss., Bonn, 1880 .

LAUWENS, R. Exstirpation des Duodenum. Brief an den Herausgeber. P. A., $120,1907$.

LavroNA. L'action de l'iode sur les processus pathologiques expérimentaux provoqués par l'adrénaline dans les vaisseaux sanguins. Arch. scienc. biol., 13, 1909 .

Lawrence. 'Tumour of pineal body. Transact. Pathol. Soc. London, 50, 1898 .

LAZARÚS, P. Experimentelle Hypertrophie der Langerhansschen Pankreasinseln bei der Phloridzinglykosurie. M. m. IV., No. 45 and Nos. $5 \mathrm{I}$ and 52,1907 . 
LECKY, H. C. Notes on a case of Addison's disease, rapidly fatal, with symptoms of acute toxæmia. Lancet, February 17, 1906.

LECONTE. Etude sur les hémorrhagies des capsules surrénales. Thèse de Paris, 1899.

LECorché. Traité du diabète. Paris, 1877.

LE DENTU. Affection chirurgicale des reins et des capsules surrénales. Paris, 1889 .

LefÈvre. Contribution à l'étude de la maladie d'Addison. Thèse de Paris, 1890.

LEHMAN. Über Adrenalin (Tierversuche). M. m. W., Igoz.

LEHMANN, A. Beiträge zur Kenntnis der Zuckerbildung aus Eiweisz. Diss., Halle, 1902.

Lehmans, O. Flüssige Kristalle. Leipzig, 1904.

LEHNERDT, Fr. Zur Frage der Substitution des Calziums im Knochensystem durch Strontium. Ziegl. Beitr., 47, roog.

LEICHTENSTERN. Über Morbus Addisonii. D. m. W., I891.

LEISCHNER, H. Über Epithelkörperchen-Transplantationen und deren praktische Bedeutung in der Chirurgie. A. k. Ch., 84, 1907.

Lemoine and LaUNoIS. Lésions du pancréas dans le diabète. A. m. e., 3, 1891 .

- Beiträge z. Pathologie u. Therapie d. Pankreaserkrankungen. Z. k. M., 51 and 52, 1904 .

Lenhossék, M. Das Problem der geschlechtsbestimmenden Ursachen. Jena, 1003 .

LENNÉ. Die Zuckerkrankheit. Berlin, 1898.

LEONHARDT. Experimentelle Untersuchung über die Bedeutung der Schilddrüse für das Wachstum im Organismus. V. A., 149, I897.

LEOPOLD, J. S., and REUSS, A. v. Üb. d. Beziehungen d. Epithelkörper z. Kalkbestand d. Organismus. W. k. W., 1908.

LEOPOLD and RAVANo. Neuer Beitrag zur Lehre von der Menstruation und Ovulation. A. G., $83,1907$.

LÉPINE, R. Sur la présence normale dans le chyle d'un ferment destructeur du sucre. C. r. A., 1 10, 1890 .

- Etiologie et pathogénie du diabète sucré. A. m. e., I89I.

- Die Beziehungen des Diabetes zu Pankreaserkrankungen. W. m. P., I 892.

- Sur une autointoxication d'origine rénale. C. r. A., p. 99r, r889; and S. m., 1893 .

- Sur la participation du pancréas à la thermogenèse consécutive aux lěsions cérébrales. C. r. S. B., 5 I, p. 835, 1899.

- Présence du glucose dans le liquide céphalo-rachidien. Sem. méd., 18 février, 1903.

- De la glycolyse dans ses rapports avec le diabète sucré. Sem. méd., I903.

- Sérum antithyroïden. Lyon méd., 29 novembre, 1903.

- Etat du pancréas dans certaines glycosuries toxiques. pp. 161 and 1288 , 1903 .

- Excitation fonctionnelle du corps thyroïde au moyen de rayons $\mathrm{X}$. Ibid., 56,1904 .

- Sur la question du diabète rénale. B. k. W., 1905.

- Le goitre exophtalmique devant la sérothérapie. Revue du méd., p. 984, 1906.

- Du rôle des sécrétions dans la pathogénie du diabète sucré. D. A. k. M., $89,1907$.

- L'adrenaline agit-elle directement s. 1. fibres sympathiques? C. r. S. B., 65,1908 .

- Le diabète sucré. Paris, rgog.

LÉPINE, R., and BoUlud. Sur la glycolyse dans le sang in vitro. C. r. S. B., p. 73,1903 .

- Influence de la macération du pancréas sur la glycémie et sur le pouvoir glycolytique du sang. Ibid., 57, ii, p. 160, 1905 .

- Influence des globules blancs sur la glycolyse. Ibid., 58, i, p. $901,1906$.

- Sur le pouvoir glycolytique du sang des animaux phloridzinés. Ibid., 58 , ii, p. 93.

- Effets sur la glycémie de la compression de l'aorte près de sa bifurcation. Ibid., 62, p. $1108,1907$. 
LÉPINE, R., and Boulud. Sur la glycosurie sans hyperglycémie. C. r. A. B., 143, p. 949 , 1907 .

- Sur le sucre total du sang. Ibid., 147, 1908.

LÉPINOIs, E. Etude sur le chromogène des capsules surrénales et sur l'origine de la coloration rouge, que ces glandes prennent au contact de l'air. C. r. S. B., 5I, pp. 310, 315, 1899.

LERMITTE, A. Suprarenal gland extract as a hæmostatic. Brit. Med. Journ., 1899 .

LERmoYez, M. Un grand médicament de l'avenir: l'adrénaline. Presse méd., No. $37,1902$.

LESAGE, J. Recherches expérimentales sur l'adrénaline. Arch. int. de Pharmac., 13, 1904.

- Toxicité de l'adrénaline en injection intraveneuse pour le chien. C. $r$. S. B., 56, pp. 632 and 709,1904 .

- Toxicité de l'adrénaline en injection intraveneuse pour le chat. Ibid., pp. 665 and 754 .

- Phénomène d'accoutumance du cœur du chat à l'adrénaline. Ibid., 56, p. 800,1904 .

- Effets physiologiques du suc pancréatique naturel en injection intraveneuse. Action sur la circulation et respiration. Ibid., p. 938, 1904.

LESNÉ and DREYFus. Contr. à l'étude du pouvoir glycolytique du sang. Ibid., p. I 140 , 1904 .

- A propos de la pancréatectomie expérimentale chez le chien. Ibid., p. 528,1906 .

LÉtienne. De la senilité. P. m., 1906.

LETULLE, $M$. Note sur la dégénérescence graisseuse de la capsule surrénale. Bull. soc. anat., pp. 263-264, 1889.

- Surrénalité nodulaire hyperplastique et adénomes, de la capsule surrénale. Ibid., pp. 314-337, 1892.

- Mort subite dans la tuberculose des capsules surrénales. Press méd., 1894 .

- Adénomes de la glande surrénale. Arch. . de scienc. méd., i, I, p. 8o, 1896.

LEva. Zur Lehre des Morbus Addisonii. V. A., 125, 1891.

- Über den Einflusz gewisser Gifte (Alkohol, Adrenalin, Nikotin) auf die Produktion spezifischer Immunsubstanzen. M. K., 1907.

Levi, Della Vida. Sieri tossi specifici per le capsule surrenali. Lo Sperim., 58 , 1904 .

- Sulla particolare struttura del pancreas in un Lemuride. Ibid., 58, 1904.

LÉVI, L., and DE ROTHSCHILD. Oedèmes thyroidienne transitoires. C. r. S. B., 29 décembre, 1906.

- Hypothyroïdie et autoinfections à répétition. Ibid., 58, i, p. 797.

- Autothérapie thyroïdienne de la grossesse. Ibid., p. $1018,1906$.

- Corps thyroïde et intestin. Ibid., 62, p. 68 I, April, 1907.

- Intestin, thyroïdin et ioncalcium. Ibid., 62, p. 703 .

- Traitement thyroïdien. Ibid., 64, p. 932 .

- Hyperthyroïde basedowienne. Ibid., 19 décembre, 1908.

- Contrib. à l'opothérapie hypophysaire. Soc. de Neurologie, 7 février, I907.

- Sur un cas de myopathie atrophique progressive ou de myatonie améliore par l'opothérapie hypophysaire. Ibid., 6 juin, 1907.

- Etude sur la physiopathologie du corps thyroïde et de l'hypophyse. Paris, rgo8.

- Le syndrome oculaire de l'instabilité thyroïdienne. C. r. S. B., 66, 1909.

LEVy. Ein Beitrag zur Kasuistik der Hypophysentumoren. Diss, Berlin, 1800 .

LEvy, A. G. The blood-changes after experimental thyroidectomy. Brit. Med. Journ., I 898 .

LE WALD. A case of Addison's disease. New York Path. Soc., p. 237, I 90 I.

LEWaNDowsky, M. Über die Wirkung des Nebennierenextraktes auf das Auge. C. P., 12, p. 599, 1898 .

- Über die Wirkung des Nebennierenextraktes auf die glatten Muskeln, im besonderen des Auges. A. A., p. 360, I 899 .

- Zur Frage der inneren Sekretion von Nebenniere und Niere. Z. k. M., 37,1899 .

- Wirkungen des Nierenextraktes auf die glatten Muskeln der Haut. C. P., 14, 1900 . 
LEwandowsky, M. Das histologische Bild der Schilddrüse in Beziehung zu ihrer Funktion. Festschr., Leyden, ii, 1902.

LEwaschew, S. IV. Über eine eigentümliche Veränderung der Pankreaszellen warmblütiger Tiere bei starker Absonderungstätigkeit der Driise. A. m. A., 26, 953, is $\$ 86$.

LEwIN. Studies on the blood of animals deprived of the adrenalin. A. J. P., p. $358,190 \mathrm{r}$.

_. Physiologie und Pathologie der Hypophysis. Diss., Berlin, 1906.

LEwIN, C. Uber Epinephrin (Epirenan). Fortschr. d. Med., 23, p. 6, I905.

LEwIN, G. Studien über die bei halbseitigen Atrophien und Hypertrophien namentlich des Gesichtes vorkommenden Erscheinungen, mit besonderer Berücksichtigung der Pigmentation. Charité-Annal.. pp. 6ro704,1884 .

- Über Morbus Addisonii mit besonderer Berücksichtigung der eigentümlichen abnormen Pigmentation der Haut. İbid., pp. 630-726, 1885.

- Über Morbus Addisonii. II. Teil. Ibid., I892.

LEwix, M. Das Suprarenin, ein ideales Extraktions- und Dentinanästheticum. D. zalın-ärztl. IV., No. 16, 1904.

LEwIN and BOER. Quetschung und Ausrottung des Ganglion coeliacum. D. m. W., I 894 .

LEwIS. Hyperplasia of the chromophile cells of the hypophysis as the cause of acromegaly. Bull. of the Johns Hopkins Hosp., 16, p. 157, 1905; and J. M., 20, 102 .

LEIDIG, F. Lehrbuch der Histologie der Menschen und der Tiere. Frankfurt, 1857 .

LiBorio, P. Govez. The anatomy and pathology of the carotid gland. Amer. Journ. Med. Scien., 136, pp. 98-111, 1908.

Lichtexstein. Kritische und experimentelle Studien zur Toxikologie der Plazenta. A. G., 86, H. 2, 1908; C. G., p. 1639, 1908.

- Gegen die plazentare Theorie der Eklampsie-Ätiologie. C. G., No. S, p. 265,1909 .

LICHTwITZ, L. Über einen Fall von Skelodermie und Morbus Addisonii nebst Bemerkungen über die Physiologie und Pathologie des Sympathikus und der Nebennieren. D. A. k. M., 94, 1908.

- Über Wanderung des Adrenalins im Nerven. A. P. P., 58, p. 22 I, 1908.

Licini. Der Einflusz der Exstirpation des Pankreas auf die Schilddrüse. Z. f. Ch., p. 107, 1909 .

LiebeN, S. Wirkung v. Extrakten chromaffinen Gewebes a. d. Pigmentzellen. C. P., 20, 1906.

- Zur Physiologié der Tunica dartos. P. A., 124, 1908.

LIEBMANN, A. Über die Nebennieren und den Sympathicus bei Hemicephalen und Hydrocephalen. Diss., Bonn, i 886.

LIEFMAN, E., and STERx, R. Über Glykämie und Glykosurie. B. Z., i, p. $209,1006$.

LréGeOIS. Des glandes vasculaires sanguines. Thèse Paris, i 86o.

LiePMANN, IV. Zur Technik und Kritik der Plazentarforschung. C. G., 1909 .

Lier, E. H. vas. Über Adrenalin. Ned. Tijdschr. v. Geneesk., i907. Ref. B. C., VI.

LILIENFELD and MoNTr. Sur la localisation microchimique du phosphore dans les tissus. A. i. B., 19, I 893

Lmon. Etude histologique et histogénique de la glande interstitielle de l'ovaire. Arch. d'anat. micr., v, fasc. ii, septembre, 1902.

LindemanN. Über das Verhalten der Schilddrüse beim Ikterus. V. A., 1897 .

- Sur la mode d'action de certains poisons rénaux. Ann. Inst. Pasteur, p. 49,1900 .

LINDSTÄDT, C. Neuere Forschungen über die Verrichtung der Schilddrüsc. Berlin, Io04.

LiNNeRT, K. Vergleichend chemische Gehirnuntersuchungen. W. k. IV., 1910 ; and B. Z., 26.

Linossier, G., and Lemoine, G. H. Note sur l'action néphrotoxique des inj. de sérum normaux. C. r. S. B.,25 avril, 1903.

LINSER. Über die Beziehungen zwischen Nebennieren und Körperwachstum, besonders Riesenwuchs. B. z. kl. Ch., 37, p. 282, 1903.

Lipschitz, B. Über Myxoedem. Diss., Berlin, I 897 . 
IIsIs, F. Rech. expér. sur les médicaments cardiovasculaires. Arch. intern. Pharmacod., 17, p. $465,1908$.

LISSAUER, M. Experimentelle Arterienerkrankung beim Kaninchen. B. k. W., 1905 .

- Zur Kenntnis der Nebennierenblutungen. V. A., 193, pp. 137-148, 1908.

LISSER. Traitement du diabète par les lavements de pancréas. P. m., i 896.

Livini. Parathyroïdes et lobules thymiques. A. i. B., 34, 1900.

Livon, CH. Sécrétions internes, glandes hypertensives. C. r. S. B., p. 98, 25 janvier, 1898.

- Corps pituitaire et tension sanguine. C. r. S. B., 51, p. 170, 1899; and C. r. A., 1899 .

- Danger du principe actif des capsules surrénales dialysé. Ibid., 54 p. 1501,1903 .

- Action de l'adrénaline sur les vaissaux. Ibid., 55, p. 271 , 1903.

- Action des vieilles solutions d'adrénaline. C. r. S. B., 56, p. $125,1904$.

- Que devient l'adrénaline dans l'organisme? Ibid., 56, p. 539, I904.

- Destruction de l'adrénaline dans l'organisme. Ibid., 56, 26, p. 1118 I 904 .

- A propos de la destruction de l'adrénaline dans l'organisme. Ibid., 56, ii, p. 118 , 1904 .

- Sur le rôle de l'hypophyse. C. r. S. B., 17 juin, 1907.

- Action différente des lobes hypophysaire sur le sang du chien. Ibid., p. 618 .

- Présentation d'un chien hypophysectomé. Ibid., 18 février, 1908.

- Inexcitabilité de l'hypophyse. Ibid., 64, p. 177, 1908.

- Pénétration par la voie nerveuse de la sécrétion interne de l'hypophyse. Ibid., 65, p. 744,1908 .

- Sur l'action des extraits.du corps jaune de l'ovaire. Ibid., 66, p. 549, 1909 .

- Contribution à la physiologie de l'hypophyse. J. d. P. P., xi, pp. 16-27, 15 janvier, rgog.

Lloyd, J. Notes on a case of Addison's disease; recovering during the administration of extract of suprarenal body. Brit. Med. Journ., August 24, 1895 .

LOBENHOFFER. Beiträge zúr Lehre der Sekretion in der Struma. M. G. M. C., 20, 1909.

LOchte, G. Zur Kenntnis der epithelialen Umwandlung der Thymus. C. a. P., 10, I899.

Lockhart Mummeri, J. P., and Symes, IV. Legge. Some points on the experimental production of the vascular atony of surgical shock. Brit. Med. Journ., 1908.

LOCKWOOD, C. B. Upon the presence of adrenal structures in the inguinal canal. J. o. A. a. P., pp. $79-83,1899$.

Lode, A. Zur Transplantation des Hodens bei Hähnen. W. k. W., I 895

- Úber Zahlen- und Regenerationsverh. d. Spermatozoiden. P. A., 50, I891.

- Exp. Beitr. z. Physiologie d. Samenblasen. S. W. A., 104.

LODI, M. Sur un cas des germes aberrants des capsules surrénales dans les ovaires. A. i. B., 27, pp. $486-487,1902$.

Loeb. Hypophysis cerebri und Diabetes mellitus. C. i. M., 1898.

LOEB, J. Vorlesungen über die Dynamik der Lebenserscheinungen. Leipzig, I906.

LOEB, L. Influence of iodine preparations on the vascular lesions produced by adrenalin. Ibid., 133 , p. 903 , June 6 , 1907 .

LOEB, L., and FLEISCHER. Vergleichende Úntersuchung über die Ausscheidung von Jod bei Verabreichung von Jodkali und Sajodin. Z. e. P., iv, p. 716, 1907.

- Über den Einflusz von Jodpräparaten auf die durch Adrenalininjektionen hervorgerufenen Gefäszveränderungen. D. m. W., I907; A. J. M. S., 133, p. 903 , 1907 .

LOEB, L., and GITHENS, TH. C. The effect of experimental conditions on the vascular lesions produced by adrenalin. Ibid., 130, p. 658, October, 1905.

Loeb, M. Beitrag zur Lehre vom Diabetes mellitus. C. i. M., No., 35, 1898 .

LoeniNG, K., and FUss, S. Schilddrüsenveränderungen bei Adipositas dolorosa (Dercumsche Krankheit). V. 23. C. M., pp. 224-232, 1906. 
LOEPER. Action de l'adrénaline sur les organs hématopoétiques. C. r. S. B., 55 , p. 1452 .

-- L'action de l'adrénaline sur l'appareil cardiovasculaire et sur la capsule surrénale. Ibid., p. 1453, I903.

Loeper, M., and Crouzon, O. L'action de l'adrénaline et des extraits surrénaux sur le sang. Ibid., 55, p. 1376, 1903.

- L'action de l'adrénaline sur le sang. A. m. e., 16, p. 83, 1904.

- Purpura hémorrhagique traité par l'adrénaline, guérison. Bull. méd., 1903.

LOEPER and ESMONET. La sécrétion graisseuse de l'hypophyse. Thèse de Paris, 1904; C. r. S. B., 56, p. 575, 1904.

Loeper and OPPENHEIM. Maladies des capsules surrénales. Paris, 1906.

LOEPER and ROY. Glycosurie et hypophyse. C. r. S. B., 55, pp. 382-384, 1903.

-Gigantisme et acromégalie. Nouv. Icon. de la Salpêtrière, 16. p. 163, 1903.

Loewenstein. Die Entwicklung der Hypophysisadenome. V. A., 188, p. $44,1907$.

Loewenthal, N. Lésions cérébrales des chiens éthyroïdés. Rev. méd. Suisse rom., 1887 .

- Über eigentümliche Zellengebilde im Sympathicus des Frosches. I. M., I I, I 894 .

Löwenthal and Wielrecht. Die Behandlung der Tetanie mit Nebenschilddrüsenpräparaten. M. K., I907.

LoewI, $O$. Eine neue Funktion des Pankreas und ihre Beziehung zum Diabetes mellitus. W. k. W., p. 747, 1907; and A. P. P., 59, 1908.

- Arzneimittel und Gifte in ihrem Einflusz auf d. Stoffwechsel. v. Noordens Handb. d. Pathologie d. Stoffwechsels, ii, 1907.

Loewi, O., and MeYer, H. Über die Wirkung synthetischer dem Adrenalin verwandter Stoffe. A. P. P., 53, p. 213, I905.

LoEwrT, M. Diabetesstudien I. Kältediabetes und Organfunktion. II. Ein Beitrag zur Lehre von der Adrenalinämie beim Frosche. A. e. P., 6o, p. I, I 909 ; ibid., 6o, p. 420 .

-- Diabetesstudien. III. Mitt. D. Pankreasdiabetes b. Frosche. A. P. Th., 62 , 1909 .

- Vorlesungen über allg. Pathologie. Fieber, Jena, I 898 .

Loewx, A. Neuere Unters. z. Physiologie der Geschlechtsorgane. E. P., 2, 1903.

Loewy and Richter. Sexualfunktion und Stoffwechsel. A. P., Suppl., i 899.

- Zur Frage nach dem Einflusz der Kastration auf den Stoffwechsel. C. P., 1902 .

Lohmans, A. Cholin, die den Blutdruck erniedrigende Substanz der Nebenniere. P. A., I 8 , p. 215, 1907.

- Über die Verteilung des blutdruckherabsetzenden Cholins in der Nebenniere. C. P., 21, p. 139, 1907.

- Zur Physiol. d. Schilddrüse. Sitzungsber. d. Gesellsch. Naturw. Marburg, 25 Mai, Igo\$.

- Über die antagonistische Wirkung der in den Nebennieren enthaltenen Substanzen Suprarenin und Cholin. P. A., 122, p. 203, 1908.

- Neurin, ein Bestandteil der Nebennieren. P. A., I28, p. I42, 1909.

LOISEL, G. Grenouille femelle présentant les caractẹ̀res sexuels secondaires du mâle. C. r. S. B., 53, p. 204, 190 I.

- Terminaisons nerveuses et éléments glandulaires de l'épithélium séminifère. Ibid., 54, p. 346 , 1902 .

- Sur l'origine embryonnaire et l'évolution de la sécrétion interne du testicule. Ibid., p. 952, and 1037, 1902.

- Etude sur la spermatogenèse chez le monceau domestique. J. A. P., pp. $112-177,1902$.

- La sexualite. Revue scient. série 4, t. xix, No. 22, mai, 1903.

- Croissance comparée en poids et en longueur des fotus mâle et femelle dans l'espèce humaine. C. r. S. B., 55, pp. I235 and 1237, 1903.

- Les poisons des glandes génitales. Première note. Recherches et expérimentation chez l'oursin. Ibid., 55, p. I329, I903.

- Sur les sécrétions chimiques de la glande génitale mâle (à propos d'un prétendue glande interstitielle du testicule). Ibid., 56, p. 27, 1904 .

-- Contributions à l'étude des sécrétions chimiques des glandes génitales. Les pigments élaborés par le testicule du poulet. Ibid., 56, p. 104, 1904 . 
LoISEL, G. Les caractères sexuels secondaires et le fonctionnement des testicules chez la grenouille. Ibid., 56, p. 446, I904.

- Sur l'origine et la double signification des cellules interstitielles du testicule. Ibid., p. 448 .

- Recherches sur les ovaires de grenouilles vertes. Ibid., p. 504.

- Recherches comparatives sur les toxalbumines contenues dans divers tissus de grenouille. Ibid., 56, pp. 883 and 889,1904 .

- Les poisons des glandes génitales (suite.) IV. Recherches sur les mammifères. Conclusions genérales. Ibid., 57, i, p. 77, 1905.

- Conservation des poisons génitaux. Ibid., p. So.

- Substances toxiques des œufs extraites de tortue et de poule. Ibid., p. 133 .

- Les phénomènes de sécrétion dans les glandes génitales. Revue générale et faits nouveaux. J. A. P., ann. 15, pp. 536-562, 1904; and 41, p. 58, 1905.

- Stérilité et alopecie chez les cobayes soumis antérieurement à l'influence des poisons ovariens de grenouille. C. r. S. B., 57, ii, p. 463, I905.

- Recherches des graisses et des lecithines dans les testicules de cobayes en évolution. Ibid., 57, ii, p. $584,1905$.

- Les substances graisses dans les glandes génitales d'oursin en activité sexuelle. Ibid., p. 586 .

- Considérations générales sur la toxicité des produits génitaux. Ibid., 57 , ii, p. 5 I I, 1905.

- Expériences sur la toxicité des œufs de canards. Ibid., 57, ii, p. 460, 1905.

- Toxicité des œufs de poule et de tortue. Ibid., p. 403.

- Croissance des cobayes normaux ou soumis à l'action du sel marin ou du sperme de cobaye. Ibid., p. 506.

- Toxicité du liquide séminale de cobaye, de chien et de tortue. Ibid., p. 500 .

- Contribution à l'étude de l'hybridité. (Eufs de canards domestiques et de canards hybrides. Ibid., p. 587.

- Expérience sur la toxicité des œufs de canards. Toxicité des œufs de poule et de tortue. Ibid., p. 400.

Lombroso, $U$. Sul diabete sperimentale e la secrezione pancreatica esterna. Giorn. R. Accad. Medic. di Torino, rooz.

- De l'influence des phénomènes lipolytiques dans l'absorption des graisses chez les chiens dépancréatisés. C. r. S. B., 55, p. 400, I904.

- Observations histologiques sur la structure du pancréas du chien, après ligature et résection des conduits pancréatiques. Ibid., 57, p. 610, I 904 .

- Observations histologiques sur la structure du pancréas du pigeon, après ligature et résection des conduits. Ibid., p. 6 i i.

- Über die Rolle des Pankreas bei der Verdauung und Resorption der Kohlehydrate. H. B., 8, p. 5 I.

-- Sui fenomeni consecutivi all' estirpazione del pancreas non più funzionante regolarmente. Giorn. della $R$. Accad. di Med. di Torino, I I, anno 68, pp. $5-6,1905$.

- Sur la structure histologique du pancréas après ligature et section des conduits pancréatiques. J. d. P. P., No. 1, I905.

- Sugli elementi che partecipano alla funzione interna del pancreas. Arch. di fisiol., 3, F. 2, 2 1 5, 1906.

- Sulla funzione del pancreas nel ricambio materiale. Torino, 1906.

- Über die Beziehungen zwischen der Nährstoffresorption und den enzymatischen Verhältnissen im Verdauungskanal. P. A., I 1 2, 1906.

- Über die enzymatische Wirksamkeit des nicht mehr in den Darm sezernierenden Pankreas. H. B., I I, 8I, 1907.

- A proposito della nota del dottore Visentini: Sulla Funzione del pancreas. Gazz. med. Ital., No. 48, Pavia, 1907.

- Zur Beantwortung. von O. Hess: Die Ausführungsgänge des Hundepankreas mit Bezug auf Fettresorption. Med. Naturwiss. Arch., I, Heft, 3, Berlin.

- Zur Frage über die innere Funktion des Pankreas mit besonderer Rücksicht auf den Fettstoffwechsel. A. P. P., 56, 1907.

- Su gli elementi che compiono la funzione interna del pancreas. Arch. Farmac., 5, 20I, 1908 . 
Lombroso, U. Sulla lipasi del secreto intestinale. Rend. R. Accad. dei Lincei, 17, ser. 3 , 136. Roma, 1908 .

- Kann das nicht in den Darm sezernierende Pankreas auf die Nährstoffe einwirken? A. P. P., 6o, p. 99, 1908.

- Influenza di un segmento di pancreas completamente separato dai suoi normali rapporti nervosi. Boll. Associazione cultori Scienze mediche e naturali. Roma, 1908.

- Sulla teoria umorale degli "Ormoni." Il meccanismo della secrezione pancreatica ed intestinale. Lo Sperimentale, 1908.

- Zur Frage der Beziehung zwischen innerer Pankreasfunktion und Nervenwirkung. Folia Neurobiologica, iii, No. 2, 1909.

- La secrezione interna del pancreas. Il Tommasi, I9og.

- Die Gewebselemente, welche die innere Funktion des Pankreas besorgen. E. P., IX, 1909 .

Lombroso, N., and SACERDote, A. Sulle modificazioni istologiche del pancreas di coniglio dopo la ligatura del dutto di Wirsung. Rend. R. Accad. di Lincei. Roma, 1908.

Lomer, R. Eigentümi. Verhalten der Nebennieren bei Hemicephalen. V. A., 98 , p. 366 , I 884 .

- Antithyreoidin Moebius bei Basedowkrankheit mit Psychose. M. m. W., 1905 .

Lo Montco and v. RYNBerk. Sulla funzione dell' ipofisi cerebrale. Atti Accad. dei Lincei, 10, pp. 117, 212 and 265, 1901.

- Ric. sulla funzione della ipofisi cerebrale. Riv. di Neuropath. Roma, Nos. 9 and ro, I901.

Longworth, S. G. The action of some suprarenal preparations. Brit. Med. Journ., 1902.

Lorand, A. Les rapports du pancréas avec la thyroïde. Ibid., 56, p. 488, 25 mars, 1904.

- Pathogénie du diabète dans l'acromégalie. Ibid., 56, p. 554, I avril.

- On the blood glands as pathogenic factors in the production of diabetes and obesity. Transact. of the Path. Soc. of London, 57, i, 13, 1906.

- Klinische Beiträge zur Frage über die Beziehungen der Schillddrüse zum Diabetes. 79, V. d. N. A., pp. 40-4I; Klin. therap. W., 1907.

- Sur les rapports de la thyroïde avec les reins, avec considération sur la pathogénie de la goutte. C. r. S. B., 62, p. 129 , 1907.

- Note sur la pathogénie de la narcose. Ibid., 6o, p. go8.

- Die rationelle Behandlung der Zuckerkrankheit. Berlin, 1909.

- Das Altern. 2 Aufl. Leipzig, igoo.

LORD. The pineal gland, its normal structure, some general remarks on its pathology. Transact. of the Path. Soc. of London, 50, p. 18, 1898 .

LORTAT-JACOB, L. Influence de la thyroïdectomie partielle sur la gestation et la lactation chez la lapine. C. r. S. B., 56, p. 61, 1904.

Lortat-Jacob, L., and SabarÉand, G. Pathogénie de l'athérome artériel et thyroidectomie. Ibid., 19 novembre, 1904.

- Du rôle de la castration dans la production de l'athérome expérimental. Ibid., 57, p. 583,17 avril, 1905 .

LOTHRINGER. Untersuchung an der Hypophysis einiger Säugetiere und des Menschen. A. m. A., 28 , p. 257,1886 .

LUBARSCH, O. Über die Abstammung gewisser Nierengeschwülste von embryonal versprengten Nebennierenkeimen. V. A., 137, 1894.

- Beitr. z. Histologie d. von Nebennierenkeimen ausgehenden Nierengeschwülste. Ibid., 135, 1894 .

- Allg. Pathol. u. path. Anatomie der Nebennieren. L. O., i, p. $4 \$ 8,{ }_{1} 896$.

- Über d. Vorkommen kristall. Bildungen in d. Zellen d. Hodens. V. A., 145,1896 .

- Über die Kedeutung der pathologischen Glykogenablagerungen. V. A., $183,1906$.

Lublisski. Jodismus acutus und Thyreoiditis acuta. D. m. W., 1906.

- Über die Anwendung des synthetischen Suprarenins. B. k. W., 1907.

LuCIANi, L. Fisiologia dell' uome. II. Le secrezioni interne protettive. Milano, 1904 .

LUCIBELLI. Contributo all fisiopatologia della capsule surrenali. Gazz. d. osp., p. 1221, 1901.

Lucien, M. Thymus et athrepsie. C. r. S. B., 64, p. 559.

- Etude anatomo-pathologique sur l'hypertrophie du thymus. Ibid., 64, p. 921 . 
LUCIEN, M. Considération anatomo-pathologiques sur l'athrepsie. Réun. biol. de Nancy, ro juillet, 1908 .

- Capsules surrénales et athrepsie. C. r. S. B., März, 1908.

LUCIEN and PARISOT. Etude physiologique et anatomique du thymus dans l'athrepsie. Ibid., 64, p. 747.

- Variations pondérales de l'hypophyse consécutivement à la thyroïdectomie. İbid., 65, pp. 261 and $77 \mathrm{I}, 1908$.

- Influence de la thymectome sur la croissance. Soc. med. Nancy, 22 Juli, 1908 .

LÜDKE, $\mathrm{H}$. Über Zytotoxine mit besonderer Berücksichtigung der Ovariotoxine und Thyreotoxine. M. m. W., 1905 .

- Die Antikörperproduktion als zellulärer Sekretionsprozesz. B. k. W., 1905.

LÜтнJE, H. Zur Frage der Zuckerbild. im tier. Organismus. M. m. W., 1902 .

- Ist die Zerstörung des Zuckers nach Pankreasexstirpation vollständig aufgehoben? Ibid., 1903.

- Über die Kastration und ihre Folgen. A. P. P., 48 and 50, 1903.

LÜrzone, E. Über den Einflusz von diuretisch wirkenden Mitteln auf das Zustandekommen der alimentären Glykosurie. Diss., Göttingen, I907.

LUFF, A. P. On the uses of the calcium salts in morbid conditions. Brit. Med. Journ., 1909.

Luksch, F. Funktionsstörungen der Nebenniere bei Allgemeinerkrankungen, Intoxikationen und Infektionen. W. k. W., 1905.

- Über die Störung der Nebennierenfunktion bei Infektionskrankheiten. B. k. W., 1909 .

LUNA, E. La morfologia delle ghiandole soprarenali dell' uomo nelle varie fasi del loro sviluppo. An. An., 33, p. 383, 1908.

LunDBORG. Die Entwicklung der Hypophysis bei Knochenfischen und Amphibien. Z. A., 7, 1894 .

- Spielen die Glandulea parathyroidex in der menschlichen Pathologie eine Rolle? D. Z. N., 27, p. $217,1904$.

- Hypothese betr. d. Natur d. kataton. Symptomenkomplexe. Centbl. f. Nervenh., 1905 .

LUPó. Tiroidectomie. Alterazioni nei centri nervosi. Progresso med., Napoli, I 888.

LuschKa, H. Die Steiszdrüse des Menschen. V. A., 18, 1859.

- Der Hirnanhang und die Steiszdrüse. Berlin, 1860.

- Über die drüsenartige Natur des sog. Ganglion intercaroticum. V. A., pp. 405-411, 1862 .

Lusena, G. Sulla patogen. del morbo di Basedow. Cron. d. Clin. med. di Genova, 1897 .

- Sull' organoterapia paratiroidea. Ibid., 1898 .

- Fisiopatologia dell' apparecchio tireoparatiroidea: Diss., Florenz, 1899.

- Sui rapporti funzionali fra la tiroide e la glandule paratiroide. Ibid., 1 goo.

- La resistenza alle intossicazioni bacteriche dopo l'estirpazione delle capsule surrenali. Boll. della R. Acad. med. di Genova, anno xviii, No. I, 1903 ; Ref. B. C., ii, p. 246.

- Nuove ricerche sull' apparecchio tireoparatiroidea. Rif. med., 1906.

LUSK, G. The influence of cold and mechanical exercise on the sugar excretion in phlorizin glycosuria. A. J. P., vol. 22, p. I63, 1908 .

- The production of sugar from glutamic acid ingested in phlorizin glycosuria. Ibid., p. 144.

Lustig. Sugli effetti dell' estirpazione del plesso celiaco. Lo Sperim., I890-1891 ; Arch. per le scienz. med., 13, p. 129, 1889; Zieglers Beitr., 7 , p. 431,1889 .

- Über die Ätiologie des endemischen Kropfes. X. Kongr. intern. Berlin, I 890 .

LyMAN GREEN, CH. Akromegaly associated with symptoms of myxœdema. New York Med. Journ., October, 1905.

MAAS, O. Über Veränderungen im Zentralnervensystem nach Unterbindung der Schilddrüsengefäsze. B. k. W, 1902.

MaAs, P. Exper. Untersuchung über die Innervation der Kranzgefäsze. P. A., 74, 1899 . 
MacARoff, G. Action mydriatique du sérum et de l'urine dans les néphrites et chez les animaux néphrectomisés. Presse méd., 13 janvier, rgog.

MacCallex, F. Adrenalin und Drucksteigerung. Engl. ophth. Ges., 6, 5, 1903 .

MacCallum, W. G. On the production of specific cytolytic sera for thyroid and parathyroid, with observations on the physiology and pathology of the parathyroid gland, especially in its relation to exophthalmic goitre. Med. News, October 31, I903.

- Tumour of the parathyroid gland. Johns Hopk. Hosp. Bull., i6, No. 168, 1905 .

- Die Beziehung der Parathyroïddrüsen zu Tetanie. C. a. P., 76, I905.

- On the relation of the islands of Langerhans to glycosuria. Bull. Johns Hopk. Hosp., 20, p. 265, 1909.

MacCallum, W. G., and VoEGTLIN, C. On the relation of the parathyroid to calcium metabolism and the nature of tetany. Bull. of the Johns Hopk. Hosp., I9, p. 91, March, I908; and C. G. M. C., 1 I, 1908.

- On the relation of tetany to the parathyroid glands and to calcium metabolism. J. E. M., I I, p. i 8 , 1909.

- On the relation of calcium metabolism to tetany and the cure of tetany by administration of calcium. Proc. Soc. Exper. Biol. Med., vol. v, p. 84 .

MACCARRISOx, R. Observations on endemic cretinism in the Chitral and Gilgit valleys. Lancet, October 3 I, 1908 .

- A summary of further researches on the etiology of endemic goitre. P. R. S., vol ii, p. I, 1909 .

- Further researches on the etiology of endemic goitre. Quart. Journ. of Med., vol. ii, 7, p. 279 , I909.

MACCURDY, J. The influence of thyroidectomy on alimentary glycosuria. J. E. M., xi, p. 798 .

MACCRCDDEx. The effect of castration on the metabolism in osteomalacia. A. J. P., xvii, p. 2 II.

- The composition of bone in osteomalacia. Ibid., xvii, p. 32.

MACKExzIE, DAN. Suprarenal gland extract in the epistaxis of hremophilia. Brit. Med. Journ., pp. 1009 and I226, April 27, I901.

MACLANTHLIN. Report of a case of Addison's disease. Med. News, i 895.

MacLexwis, A. An experimental investigation into the function of the thymus gland. Glasg. Med. Journ., August, igos.

MACLEOD, J. J. R., and Dollex. Studies in experimental glycosuria. I. On the existence of afferent and efferent nerve fibres, controlling the amount of sugar in the blood. A. J. P., 19, p. 338, 1907.

- The effect of expressed tissue juices of muscle on the mammalian heart beat. Ibid., p. 426 .

- Studies in experimental glycosuria. II. Some experiments on the nature of the glycogenolytic fibres in the great splanchnic nerve. Ibid., 22, p. 373,1908 .

- Studies in experimental glycosuria. IV. The cause of the hyperglycæmia produced by aspliyxia. A. J. P., 23, p. 278, 1909.

- Experimental glycosuria. J. o. P., 32, July, 1905.

MACLEOD and RUH. Studies in experimental glycosuria. III. The influence of stimulation of the great splanchnic nerve on the rate of disappearance of glycogen from the liver, deprived of its portal blood supply or of both its portal and systemic blood supplies. A. J. P., 22, p. 397, 1908 .

MACMUNx. Sulle funzione della capsule surrenali. Il Morgagni, ii, No. 9, p. 106, I 889 .

MACWILliam, MaCkie, and MURRAY. Intravascular injection of salts and of nucleo-proteid. J. o. P., 30, p. 381 , 1904 .

Madelung. Über Verletzungen der Hypophysis. A. k. Ch., 73, p. ıо66, I 904.

MAGENDIE: Rech. physiol. et cliniques sur les liquides céphalo-rachid. Paris, 1842 .

MAG.NUS. Über das anatomische Verhalten der Nebennieren, der Thyreoidea, Thymus und des Sympathicus bei Hemicephalen. Diss., Königsberg, 1889 .

- Ovariets betydning for svangerskabet med saerligt hensyntil Corpus luteum. Norsk. Mag. f. L., Ref. in Frommels Jahresber., I902. 
Magnus. Serumbehandlung von M. Basedowii. Norsk Magazin für Lägevidenskob., No. 7 , 1905 .

- Über die Entstehung der Hautoedeme. A. P. P., 42, 1899.

- Suprarenin bei Sipunculus, WVirkung auf glatte Muskeln. A. P. P., 50, I903.

- Die Bewegungen des Darmkanales. Zusammenfassend. E. P., 7, 1908.

MAGNuS-Levy, A. Über den respiratorischen Gaswechsel und den Einfluss der Thyreoidea, \&c. B. k. W., I 895 .

- Untersuchung zur Schilddrüsenfrage, Gas- und Stoffwechseluntersuchung bei Schilddrüsenfütterung, Myxödem, Morbus Basedowii und Fettleibigkeit. Z. k. M., 33, 1897 .

- Über Myxoedem. Ibid., 52. Die Therapie des Myxoedem. Th. G., 1907.

- Untersuchungen über Azidosis bei Diabetes mellitus. A. P. P., 45, 1901.

- Die Azetonkörper. Erg. d. inn. Med. u. Kind., I, I908.

- Organtherapie und innere Sekretion. Mod. ärztl. Bibl., Berlin, 1906.

- Der Stoffwechsel bei Erkrankungen einiger Drüsen ohne Ausführungsgang. Noordens Handb. d. Path. d. Stoff wechsels, ii,. pp. 352-354, 1907 .

- Der respiratorische Gaswechsel in Krankheiten. C. k. M., 1906.

- Chylurie und Diabetes. Z. k. M., 67, 1909 .

MAGNUS and SCHAEFER. The action of pituitary extracts upon the kidney. J. o. P., 27, p. 9, 1901-02.

MAHE. Essai sur le traitement de la maladie d'Addison. Thèse de Paris, 1894 .

MAHU and ICHARD. Adrénaline. Journ. de méd., Io avril, Ig04.

MaIGNON, F. Mode de répartition du glycogène musculaire chez les sujets alimentés et inanitiés. C. r. A., 145, p. 334, 1907.

- Du rôle des graisses dans la glycogénie chez les sujets sains et les diabétiques. C. r. S. B., 64, pp. 11 and 14, 1908.

MAINZER. Vorschlag z. Behandlung d. Ausfallserschein. nach Kastration. D. in. W., 1896 .

MAIRET and BOSC. Recherches sur les effets de la glande pituitaire administrée aux animaux, à l'homme sain et à l'épileptique. C. r. S. B., p. 348 , 1896; A. d. P., p. 600, 1896.

MaISCH, CH. O. Three cases presenting indications of Addison's disease during infancy and childhood, with post-mortem and histological reports on two. Postgrad. 14, 1899 .

MaIsonave. Contr. à l'étude de l'opothérapie orchitique. Thèse, Lyon, Igo3.

MaJ, A. Su di un caso di Morbo d'Addison venuto a guarigione. Clin. med. Ital., igor.

MaLCOLM, JOHN. On the influence of pituitary gland substance on metabolism. J. o. P., 30, p. 270, December, 1904.

Malsen, H. v. Geschlechtsbestimmende Einflüsse und Eibildung des Dinophilus apatris. A. m. A., 60, p. 63, 1907 .

Malvoz, E. Corps thyroïde et immunité. C. r. S. B., 65, pp. 69-7o, 1908.

MAMLOCK. Zusammenfass. über Adrenalin. Zietschr. f. diät. u. phys. Ther., 7, 1904 .

MaNasse, P. 'Über die hyperplastischen Tumoren der Nebenniere. V. A., I33, 1893 .

- Über die Beziehungen der Nebennieren zu den Venen und dem venösen Kreislauf. Ibid., 135, 1894.

- Zur Histologie und Histogenese der primären Nierengeschwülste. V. A., 143, p. 278 ; ibid., 145, pp. 113-157, 1896.

- Über zuckerabspaltende phosphorhaltige Körper in Leber und Nebenniere. Z. ph. Ch., 20, p. 478, 1895 .

Manca, P. Il rene del cane dope l'ablazione completo dell' apparecchio tiroparatiroidea. Arch. di biol. norm. e pathol., 59, 1906.

Mand, L. Beitr. z. Kenntn. d. Funktion d. weibl. Keimdruise. Festschr. f. Chrobak, 1903.

-- Diskussionsbemerkungen zu Fraenkels Vortrag. C. G., 28, 1904.

MANDL, L., and BÜRGER, $O$. Die biolog. Bedeutung der Eierstöcke nach Entfernung der Gebärmutter. Leipzig. u. Wien, 1907.

Mandlebaum, F. S., and Celler, H. L. Contr. à l'étude de l'anatomie pathologique de la myasthenie grave. J. F.. M., x, 3, 1908.

MANICARDI G. La paraganglina Vassale nelle atonie gastro-enteriche. Rif. med., 1903 . 
MaNkowski, A. Nouvelles données sur la microphysiologie du pancréas, le rôle des îlots de Langerhans. Séance du XIII. Congr. int. de méd., à Paris, I900, 3, p. 302 .

- Über die mikroskopischen Veränderungen des Pankreas nach Unterbindung einzelner Teile. A. m. A., 59, 286, 1901.

- Uber die belebende Wirkung des Nebennierenextraktes bei drohendem Chloroformtode. Wratsch., 1897, Ref. St. Petersb. med. W., I897.

- Les moyens les plus simples pour préparer l'extrait des capsules surrénales et son action sur l'organisme animale. Arch. rus. de Path., iii, 3 , p. 387 , mars, 1898 .

MANkoWsKY. Zur Frage nach den Zellgiften (Zytotoxinen). Russki Wratsch, I902; Russ. A. f. Path., xiv, I; Ref. in C. f. B., 32, No. Ig.

MankiEwICZ, O. Über die bösartigen Tumoren der Nebenniere. Diss., Strassburg, i 888 .

Mann, J. Dixon. On Addison's disease. Lancet, p. 655, March 21, p. 7I1, March 28, p. 764, April 4. London, I891.

ManN, Fr. Über Bronzehaut. Diss., Greifswald, I893.

Manouchine. Blutgerinnung, Leukocyten und Pepton. Wratsch, J. d. P. P., $\mathbf{x i}, \mathbf{I} 909$.

Mansfeld, G. Die Verliütung der Adrenalinarteriosklerose. W. k. W., p. 1650,1908 .

MANSFELd and RABDELEO. Die Verhinderung der Adrenalinarteriosklerose. Orvosi Hetilap, 1908 .

Maragliano. Maladie d'Addison. Rif. med., I894.

- La cosa della malattia di Basedow. Gazz. Osped., I9oo.

- Note clinic. chim. e sperim. sulle nefriti. La Clinic. Med. Ital., p. 437, 1902.

Marassini. Dei fenomeni consecutivi all' estirpazione del plesso celiaco. A. Sc. Med., Payia 97, 1903 .

- Ricerche sopra l'ázione tossica dei nucleo-proteidi del fegato. Contributo allo studio del meccanismo d'azione delle cosidette citotossine specifiche. Ref. B. C., i, p. 804 , 1903.

- Contribution à l'étude de la structure et de la fonction des capsules surrénales. A. i. B., 46, pp. 73-82.

- Sopra le modificazioni che si hanno nelle capsule surrenali in rapporto con alcune variazioni della funzione genitale e della funzione renale. Lo Sperim., 40, p. 197, 1906.

- Sopra la minuta struttura dei vari elementi delle capsule soprarenali e sul loro probabile valore funzionale. Monit. zool. Ital., xvii, Nos. 2 and 3 , pp. $42-60$, I 906 .

- Contr. sper. allo studio della funzione dell' isole di Langerhans. Riv. d. Sc. Nat., Pavia, 1907.

- Sopra una particolare modific. delle glandule duoden. dopo l'allaciatura d. cond. d. Nirsung. Ibid., I9o8.

Marbé, S. Les opsonines dans les états thyroïdiens. I. Les opsonines des animaux hyperthyroïdes. C. r. S. B., 64, p. 1058, 13 juin, 1908.

- II. Les opsonines des animaux éthyroïdés. Ibid., p. 1113 , 20 juin, I908.

- III. Les opsonines et la phagosytose chez les myxœdémateux. Ibid., 12 décembre, 1908.

- IV. Actions directe, in vitro, du corps thyroïde. Ibid. 13 mars, 1909.

- La phagocytose chez l'animaux hyperthyr. et ethyr. Ibid., 26 Juni, Igog.

MarburG, O. Die Adipositas cerebralis. Beitr. z. Pathol. der Zirbeldrüse. W. m. WV., 1908 .

- Zur Kenntnis der normalen und pathologischen Histologie der Zirbeldrüse. Die Adipositas cerebralis. Arb. a. d. neurol. Institute a. d. Wien Univ., xvii, Igog.

Marchand, F. Über eine eigentümliche Erkrankung des Sympathicus der péripherischen Nerven (ohne Bronzehaut). V. A., 8I, i880.

- Über akzessoriche Nebennieren im Ligamentum latum. V. A., 92, 1883.

-- Beiträge zur Kenntnis der normalen und pathologischen Anatomie der Glandula carotica und der Nebennieren. Festschr. f. R. Virchow, i, p. 537 , I891.

- Über den Prozesz der Wundheilung. D. Ch., Lief. I6. Stuttgart, I90 I.

MARCHESI. La meccanica respiratoria dei cani tiroïdectomizzati. Arch. p. le Scienc. Med., I 7.

Marchett, S. Über eine Degenerationszyste der Nebenniere mit kompensatorischer Hypertrophie. V. A., I 72, pp. 472-479, 1903. 
MarchetTI, S. Beitrag zur Kenntnis der pathologischen Anatomie der Nebennieren. Ibid., 177, pp. 227-247, 1904.

- Schilddrüsenpräzipitine. Rif. med., t907.

MaRCUSE, IV. Über die Bedeutung der Leber für das Zustandekommen des Pankreasdiabetes. Z. k. M., 26, p. 225, 1894.

MARENGHI, G. Sull' esportazione delle capsule surrenali in alcuni mammiferi. Rendiconti del' Inst. Lombard., 2, 36, p. 543, I903; Autoref. in B. C., ii, p. 152 ; and Lo Sperim., 57 , 1903 .

Maresch. Kongenitaler Defekt der Schilddrüse, \&c. Z. H., 19, 1898.

MARIANI, F. Ricerche sperimentali sulla funzione delle capsule surrenali. Clin. medic. Ital., 1906.

MARIE. Sur deux cas d'acromégalie, hypertrophie singulière non congénitale des extrémités supérieures, inférieures et céplialiques. Rev. de méd., p. $298,1886$.

- L'acromegalie. Nouv. Icon. de la Salpêtrière, I, p. 173, 1888; 2, p. 45, I889; Progrès méd., 1889 .

- Acromégalie. Brain, I2, pp. 59-8I, July, I890; and Soc. m. Hôpit., 1896 .

Marie, P., and Marinesco, G. Sur l'anat. patholog. de l'acromégalie. A. m. e., $189 \mathrm{I}$.

MARINE, D., and LENHIRDT, C. H. Relation of iodine to the structure of human thyroids. Relation of iodine and histologic structure to ciseases in general, to exophthalmic goitre, to cretinism and myxcedema. Arch. Int. Med., iv, p. 440, 1909.

- Effects of the administration or the withholding of iodine-containing compounds in normal, colloid or actively hyperplastic (parenchymatous) thyroids of dogs. Ibid., iv, p. 253, 1909.

Marinesco, G. De la destruction de la glande pituitaire chez le chat. C. r. S. B., 44, p. 509, 1892 .

- Trois cas diacromégalie traités par des tablettes de corps pituitaire. Semaire méd., p. $484,1895$.

- Tetanie d'origine parathyroïdienne. S. m., I905.

- Contr. à l'étude de la myasthénie grave pseudo-paralytique. Ibid., p. $24 \mathrm{I}, 1908$.

MARINESCO and MINEA, J. Note sur les changements morphologiques des cellules des ganglions greffés sur des animaux privés de leur appareil thyro-parathyroïden. C. r. S. B., pp. 239-241, 1908.

MARINESCO and PARHON, C. Sur l'influence de la thyroïdectomie sur la survie des animaux en état d'inanition. C. r. S. B., 67, p. 306, r909.

- L'influence de l'ablation de l'appareil thyro-parathyroïdien sur la graisse surrénale. C. r. S. B., 64, p. 768 , 1908.

MARINI, N. Considerazioni sulla patogenesi dell' ateromasia adrenalinica e dell' ateroma in generale. Gaz. degli Osped., 26, 22, 1905.

MaRINo-ZvCCO. Recherches chimiques sur les capsules surrénales. A. i. B., Io, 325 , I 888 .

- Présence de la Neurine dans le sang. A. i. B., 21, p. 437, 1894.

MARINo-ZucCo and DutTo. Chemische Unters. üb. die Addisonsche Krankheit. M. U., 14, p. 617, 1892 .

MaRiNo-Zucco, F., and S. Untersuchungen über die Addisonsche Krankheit. Ibid., xiv, pp. 59-63, I892. Ital. : Rif. med., viii, 64. I892.

MARISCHLER, J. Ein Fall von lympliatischer Leukämie und einem Grawitzschen Tumor der rechten Niere. W. k. W., 1896 .

Marro. La puberté. Paris, I9oo.

MARShall, C. F. A case of Addison's disease; necropsy; remarks. Lancet, October 3, 1891.

Marshall, F. H. A., and Jolly, W. A. Physiology of reproduction. Lancet, 1905 . On the results of heteroplastic ovarian transplantation as compared with those produced by transplantation in the same individual. Quart. Journ. of Exp. Phys., I and 2, I 908.

Martin, A. L'adrénaline dans le traitement des hémopthysies. Progr. méd., p. 732,1907 .

MartiN, CH. The nerve theory of menstruation. Brit. Gyn. Journ., 1893.

Martineav. De la maladie d'Addison. Paris, 1864.

MARTINI, DE CH. Sur un cas d'absence congénitale des capsules surrénales. C. r. A., 43, pp. 1052-1053, 1856 .

MartiN-Magros and ORDONNEZ. Anat. et physiol. des glandes vasc. sang. Thèse, is6o, Canstatts Jahrb., vi, p. 272, i 863 . 
Martinotti, C. Sull' estirpazione del pancreas. Giorn. R. Accad. Med. di Torino, 348,1888 .

- Sui fenomeni consecutivi all' estirpazione del pancreas. Ibid., 338.

- Contribution à l'étude des capsules surrénales. Giorn. della R. Acc. di med. di Torino, lv, pp. 299-301, I\$92; A. i. B., 27, pp. 284-286.

- Sur les altérations des capsules surrénales consécutives à l'occlusion des veines centrales respectives. A. i. B., 49, pp. 236-240, 1908 .

MaRum, A. Über die Beziehungen über den Glykogengehalt der Organe und der Azidose beim Phloridzindiabetes. H. B., x, p. 105, 1907.

Masay, F. L'Hypophyse. Etude de Physiologie pathologique. Thèse de Bruxelles, I9o8.

- Recherches sur les effets d'un sérum hypophyse toxique. Ann. de la Soc. méd. et nat., p. 91, 1908 .

Masoin, P. Influence de l'exstirpation du corps thyroïde sur la toxicité urinaire. A. d. P., I894.

- Influence de l'exstirp. du corps thyroïde sur la quantité d'oxyhémoglobine. Acad. de med. Belg., 1895 .

Massaglio, A. Contributo alla patogenesi del mixedema. Rivista di patol. nervosa e mentale, 7 ; and ibid., 13; A. i. B., p. 343, 1908.

- L'influenza della fatica nei cani parzialmente sparatiroidati. Gazz. degl. Osp., 1907.

- Il trattamento del mal di mare con la paraganglina Vassale. Gazz. Osped., 1907.

Massaglia, A., and Sparapani, G. Eclampsie expérimentale et éclampsie spontanée des animaux. A. i. B., 48, pp. 109-114.

Massalongo. Sull' Acromegalia. Rif. med., I 892 .

Massari. Sul pancreas dei pesci. R. c. della R. Accad. dei Lincei, 7, 139. Roma, 1808 .

Massini. Contr. al estudio del patogen. de la ecclampsie. Ref. C. G., 1008 .

MASSONE, M. Il sistema cromaffine addominale in casi di "encephaloschisis." Pathologica, i, October 1, 1000.

Mastricht INDEMANNS. Die Störungen der inneren Sekretion der Gefäszdrüsen und ihrer Beziehungen zueinander. Centrbl. f. Therap., Mai, 1909 .

Mathes, P. Beobacht. an mit Plazentarsaft durchströmten Hundenieren. Z. G. G., 62 .

- Über die Einwirkung des Oophorin auf den Stoffwechsel. M. G. G., 1903.

MatTEI DI. Sull' iperplasia compensatoria delle capsule surrenali. Giorn. Accad. d. med. di Torino, 3, xxxiv, 127, 1886.

- Sulle fibre musculari liscie delle capsule surrenali allo stato normale e pathologic e sulle adenoma di questi organi. Ibid., 322-331.

- Sulla pretesa azione tossica delle diluzione acquose degli organi animali freschi. Arch. per le sc. med., vi, pp. 245-288, 1883 .

MatTEI DI, and RUsso. GILIBERTI. 'Sull' influenza dell' estirpazione delle capsule surrenali sull' organismo. Ann. della soc. de scienz. natur di Palermo, 1886 .

Mathied. Action de l'adrenaline sur le cœur. J. d. P. P., 6, p. 435, 1904.

MAURER, F. Die Schilddrüse, Thymus und andere Schlundspaltenderivate der Eidechse. Morph. Jahrb., 27, p. 119, 1899.

- Die Schlundspaltenderivate von Echidna. An. An., 16, Ergänzungsb., I 899 .

- Die Entwicklung des Darmsystems. Hertwigs Handb. d. vergl. Entwickl., 2, 1, 1906.

Maus, J. Glandula thyreoidea und Hyphophysis cerebri. Diss., Greifswald, 1895 .

Mawis, S. Über die Glykosurie erzeugende Wirkung der Thyreoidea. B. k. IV., 1897 .

MAY. Beiträge zur pathologischen Anatomie der Nebenniere. V. A., 108.

Mayer, A. Sur le mode d'action de la piqûre diabétique, rôle des capsules surrénales. C. r. S. B., p. $1124,1006$.

- Ablation des surrénales et diabète pancréatique. Ibid., 64, pp. 219-221, I 908 .

MAYER, K. Zum thyreogenen Eiweiszzerfall. B. k. W., 1908.

MAYER, L. Umgekehrte Ecksche Fistel. M. m. W., I907. 
MAYER, S. Beobachtungen und Reflexionen über den Bau und die Verrichtungen des sympathischen Nervensystems. S. W. A., 66, Abt. 3, pp. $1-52,1872$.

- Das sympathische Nervensystem. Strickers Handb. d. Lehre v. d. Geweb. ii, 1871.

- Die peripherische Nervenzelle und das sympathische Nervensystem. Arch. Psych., vi, 1876 .

- Zur Lehre von der Schilddrüse und Thymus bei den Amphibien. An An., 3, 1888.

Mayo, CH. 'The Parathyroid Question. Ann. of Surg., July, Igog.

MAYOR, A. Über intraarterielle und intravenöse Injektionen. Th. M., 1908.

MazurkIEWICz, W. Über den Einflusz des Pankreassaftes auf den Blutdruck und auf die Funktionen des Pankreas und der Submaxillardrüse. C. P., 20.

- Die festen Bestandteile des Bauchspeichels und die Theorie der Sekretionstätigkeit des Pankreas. P. A., I2 I, 1907.

Meckel, J. F. Abhandlungen aus der menschlichen und vergleichenden Anatomie und Physiologie. I. Über die Schilddrüse, Nebennieren und einige ihnen verwandte Organe, pp. I-276; II. Fragmente aus der Entwicklungsgeschichte des menschlichen Foetus, pp. 277-381. Halle, r8o6.

MehriNG, v. Über experimentellen Diabetes. V. 5, C. M., I 886.

- Über Diabetes mellitus. Z. k.'M., I4 and r6, i889.

MehriNG, v. and MINKowSKI. Diabetes mellitus nach Pankreasexstirpation. C. k. M., p. 393,$1889 ;$ A. P. P., 26, I 890.

Meige, H. L'infantilisme. Gaz. d. Hôp., p. 207, février, 1902.

- L'infantilisme chez la femme. Nouvelle Iconographie de la Salpêtrière, p. 21.9, 1902 .

- Le gigantisme. Arch. gen. méd., 1902.

MIEGE and FEINDEL. Infantilisme myxoedémateux et maladie de Recklinghausen. Revue Neurologique, p. 837,1903 ; Nouv. Icon. de la Salp., juillet-août, I903.

MEINERT. Tetanie in der Schwangerschaft. A. G., $55,1898$.

MeISENHEIMER, J. Ergebnisse über einige Versuchsreihen von Exstirpation u. Transplantation der Geschlechtsdrüsen bei Schmetterlingen. Zool. Anz., 32, 1908.

MEIXNER. 'Zur Frage des Hermaphroditismus verus. Z. H., 26, p. 318, I905.

MelchIORI. Nebennierentherapie bei Aorteninsuffizienz. Gazz. degli Osped.,

- No. 77 , 1908 .

Mellin. Über die Einwirkung einiger Gifte auf den kleinen Kreislauf. S. A., 1904 .

Meltzer, S. J. Geschichte der Schilddrüsentherapie. New York med. Monatsschr., 1895 .

- Wandert Adrenalin in den Nerven? A. P. P., 59, p. 458, 1908.

- Ideas and ideals in medicine. J. A. M. J., 50, p. I577, I908.

- Bemerkungen zur Wirkung von Adrenalin auf die Froschpupille. D. m. W., Igog.

MeLtzer and MÉtZer-AUER. Üb. d. Einwirkung v. subkut. Einspritz. und Einträuf. in d. Bindehautsack von Adrenalin auf d. Pupille d. Kaninchen. C. P., I7, 65I.

- Üb. d. Einfluss des Nebennierenextraktes a. d. Pupille d. Frosches. C. P., 18, p. 316,1904 .

- Studies on the "paradoxical pupil-dilatation caused by adrenalin." A. J. P., xi, 1904 .

- The effect of subcutaneous injections and instillations of adrenalin upon the pupils of rabbits. Ibid., p. 28 .

- On the influence of subcutaneous injections of adrenalin upon the eyes of cats after removal of the superior cervical ganglion. Ibid., p. 37.

- A discussion on the nature of the paradoxical pupil dilatation caused by adrenalin. Ibid., p. 40.

- The effect of suprarenal extract upon the pupils of frogs. Ibid., p. 449 .

- Influence of suprarenal extract upon absorption and transudation. Trans. of the Assoc. of Amer. Phys., 19, p. 208, 1904; Amer. Journ. Med. Sc., p. I14, I 905 . 
Meltzer and Meltzer-Acer. The action of ergot upon the stomach and intestines. A. J. P., xvii, p. 143, 1905.

Meltzer, S. J., and SALANT, W. Study on the toxicity of bile. The effect of intravenous injections of bile upon blood-pressure. J. E. M., vii, p. 280, 1905 .

MÉNarD. De l'origine thyrö̈dienne du rheumatisme chronique. Thèse Paris, 1908 .

MENDE, R. v. Ein Beitrag zur Anatomie der menschlichen Nebenniere. Diss., Königsberg, i, P. 1902.

Mendel, E. Ein Fall von Akromegalie. B. k. IV., 1805.

- Obduktionsbefund eines Falles von Akromegalie. Ibid., 1900.

- Diskussionsbemerkung betr. operatives Eingreifen bei Ákromegalie. Ibid., p. 67, 1903.

Mendel, K. Myxödem und Kretinismus. Diss., Berlin, 1896

MÉNÉTRIER, P. Note sur les rapports des adénomes des caps. surr. avec la néphrite interst. atroph. Soc. hôp., io février, 1904.

- A propos de la hypertension artérielle. Sem. méd., 1904 and 1005.

MENGeLberG. Adrenalin und Atropin. Wochenschr. f. Ther. u. Hyg. d. Auges. VI, 1902 .

MerckeL. Krankheiten der Nebennieren. Ziemsens Handb., viii, 2, pp. $281-314, \quad 1875$.

MERRIL. Adrenalindiurese und Hypophysisextraktwirkung. J. A. M. A., No. 22, 1908 .

Messed AGli..' Acromégalie et gigantisme viscéral. Morgagni, mai, 1908.

MetTENheimer, H. v. Zum Verhalten der Thymusdrüse in Gesundheit und Krankheit. Jahrb. f. Kinderheilk., 46, 1897 (1898).

MetzGer, L. Zur Kenntn. d. wirks. Substanzen in den Nebennieren. Diss., IV ürzburg, I 897 .

- Zur Frage von Nebennierendiabetes. M. w. M., 1902.

METZNER, H. Beiträge zur Kenntnis der primären Nierengeschwülste. Diss., Halle, 1888.

Meurox, PH. DE. Recherches sur le développement du thymus et de la thyroide. Recueil zoolog. Suisse, Bd. 4, 1886.

MeYer, A. Klinisches Bild des Infantismus und der Hypoplasie. M. w. M., 1910.

- Thymustumor und Myasthenie. M. m. W., 1908.

Mexer, E. Contrib. à l'étude de la pathogénie de l'urémie. A. d. P., p. $760,1803$.

- Faits relatifs à la sécrétion interne des reins. Ibid., p. 179, 1894

Meyer, F. Beiträge zur Kenntnis der Diphtherievergiftung. A. P. P., 6o; igog.

- Beitrag zur Anatomie des Urogenitalsystems der Selachier und Amphibien. Sitzungsber. d. Naturf.-Ges. Leipzig, Nos 2-4, pp. 38-44, 1875.

Meyer, H. Über den Antagonismus der Gifte. IV. k. WV., No. 17, 1908 .

Meyer, H., and GotTlieb. Experimentelle Pharmakologie. Wien, 19 ro.

Meyer, J. DE. Note à propos des expériences de M. Cohnheim. A. i. Ph., 2,131 .

- Note prél. sur la signific. physiol. de la sécr. interne du pancréas. Journ. méd. de Bruxelles, 1904.

- Contr. à l'étude de la sécr. int. du pancréas et de l'utilisation du glucose dans l'organism. Ann. Soc. roy. de Bruxelles, 15, p. 155.

- Hyperglycémie et glycosurie provoqué par injection d'un sérum antiglycolytique. C. r. S. B., 63, p. $385,1907$.

- Glycolyse, hyperglycosurie, glycosurie et diabète. Ann. d. l'Inst. Pasteur, No. 10, 1908.

- Recherches sur le diabète pancréatique. Inhibition de la sécrétion interne du pancréas par un sérum (3e mém). A. i. Ph., vii, p. 317, 1909.

- Contribution à l'étude de la pathogénie du diabète pancréatique. Variations de la perméabilité pour le glycose. Relations entre le pancréas et le rein. 4e mém. A. i. Ph., viii, p. 121, 1909.

- Nouvelle méthode de circulation artificielle à travers le foie, appliquée à l'étude de la glycogénie hépatique. Ibid., p. 204, 1000 .

Merer, O. B. Über einige Eigenschaften der Gefäszmuskulatur mit besonderer Berücksichtigung der Adrenalinwirkung. Z. B., 48, pp. $352-397,1006$.

- Versuche mit Kokainadrenalin und Andolin an überlebenden Blutgefäszen. Z. B., Bd. 50, p. 93, 1907. 
MEYer, R. Akzessorische Nebennieren im Ligamentum latum. Verh. d. Gesellsch. f. Gynäkol. u. Geburtsh. zu Berlin, 38, pp. 3 I5 and 542, 1898 .

- Die subserösen Epithelknötchen an Tuben, Ligamentum latum, Hoden und Nebenhoden (sogenannte Keimepithel- oder Nebennierenknötchen). V. A., 17I, 1903.

MeYer-ThraP. Ein Fall von M. Basedowii mit Blut und Milch von thyreoidektomierten Ziegen behandelt. Norsk Magazin f. Lägernd., No. 7, 1905 .

MEYers, M. K. Die Wirkung von intravenösen Injektionen von Hypophysenextrakt und Brenzkatecliin, sowie von einmaligen Adrenalininjektionen auf die Aorta von Kaninchen. C. a. P., 20, Igog.

MEZNICESCU. Les modific. du sang après l'estirpation du corps thyroïde. A. m. e., IgO2.

Michael, J. Zum Vorkommen der akzessorischen Nebennieren. A. k. Ch., $43,1888$.

MICHAELIS and RONA. Untersuchungen über den Blutzucker. B. Z., I4, 1908 .

MICHaLSKI. Die Therapie des Morbus Basedowii. Bruns Beitr., 49, Igo6.

MICHelsoN. Über die Wertlosigkeit des Zusatzes von Nebennierenpräparaten bei der Lumbalanästhesie. M. m. IV., I907.

Miescher, F. Histochem. u. physiolog. Arbeiten. Leipzig, I897.

Miesowicz, E. Untersuchungen über die Veränderungen in den inneren Organen des Kaninchens nach intravenöser Injektion von Adrenalin. Bull. intern. Acad. d. Sc. Cracovie, pp. 257-265, I906.

- Uber experimentelle Herzhypertrophie. iV. k. W., Igog.

Miesowicz, E., and MaciaG, A. Beobachtungen iber das Verhalten der mydriatischen Substanzen im menschlichen Serum. Gazeta lekarska, No. 49, 1008 ; Fol. serol., ii, Januar, 1909.

MignoN. Neue Verwendungsart des Adrenalins in der Rhinologie. A. int. Laryng., 1903.

Mihalcovics, V. v. Untersuchungen über die Entwicklung des Harn- und Geschlechtsapparates der Amnioten. I. M., 2, I $88_{5}$.

- Beiträge zur Anatomie und Histologie des Hodens. Ber. d. sächs. Ges. d. WViss., I 873 .

-. Entwicklung des Gehirnanhangs. C. m. IV., I874.

- Wirbelsäule und Hirnanhang. A. m. A., I I, 1874 .

MIKA. Infantilisme (Type Lorain). Revue Neurolog., p. 250, I902.

Miklassewski, W. Neuere Anschauungen über das Wesen der Addisonschen Krankheit nebst einem selbst beobachteten Falle. Kronika Lekarska Warschau, Nos. I7-I9, I899; V.-H., ii, p. 57, 1899.

Mikulicz. Über die Resektion des Kropfes. C. Ch., i 885.

-- Über Thymusfütterung bei Kropf und Basedow. B. k. W., i 895 .

Milian, M. G. Le prurigo dans la maladie d'Addison. Gaz. hebdom., No. 45,1897 .

Mrller, J. L. The action of the extract of the suprarenal gland and the method and indications for its use. J. A. M. A., 48, pp. I66I-I664, May 18 , 1907.

- Experimental arterial degeneration. Ibid., p. 593, April, 1907.

- Über Lebererkrankungen nach intravenösen Adrenalininjektionen beim Kanninchen. M. K., I 908 .

Miller, J. L., and BENNET. Dangers in the use of adrenal preparations. Brit. Med. Journ., 1906.

MILLs. Adrenalin in hæmatemesis. Brit. Med. Journ., March 28, Ig03.

MINelli, SPARTAEo. Sopra un caso di angiosarcoma primitivo di una capsula surrenale con metastasi. Gaz. med. ital., 38-39, p. 372, I904.

Minervini, R. Des capsules surrénales. J. A. P., 40, pp. 449-492. 634-667, I904.

Minerbi and Alessandri. Akromegalie und Adams-Stokes' Symptom. M. m. W., I 908 .

Minkel. Beitrag zur Kenntnis der Addisonschen Krankheit. Diss., Bonn, 1803 .

Minkiewitsch. Tetania parathyreopriva und Hyperparatlyyreosis. Diss., Basel, 1908 .

Minkowski, O. Untersuchungen über den Diabetes mellitis nach Pankreasexstirpation. A. P. P., 31, 1893. 
Minkowski, O. Störungen des Pankreas als Krankheitsursache. L. O., i, 1896 .

- Diabetes nach Pankreasaffektion. B. k. W., I899.

- Bemerkungen über den Pankreasdiabetes zur Abwehr gegen. Eduard Pflüger. A. P. P., 53, p. 331, September, 1905.

- Die Totalexstirpation des Duodenums. A. P. P., 58, p. 271, 1908.

- Zur Kenntnis der Funktion d. Pankreas beim Zuckerverbrauch. Ibid., Suppl., I 908 .

MiNich, W. Das Kropfherz. Leipzig and Wien, Igo4.

Minot, CH. S. Morphology of the suprarenal capsules. Proc. of the Amer. Assoc. for the Advance. of Science, xxxiv, 1885.

- Lehrbuch der Entwicklungsgeschichte des Menschen. Deutsch von S. Kästner, I904.

Mironescu, TH. Beiträge zur Wirkung des Adrenalins und Euphthalmins auf den Blutdruck beim Kaninchen. Romania med., I905 and I907; Th. M., 20, p. 39, I906.

- Sur qq. lésions des glandes parathyroïdes chez les pellagreux. C. r. S. B., 64, p. 515 .

MitsaKURI, R. B. On the development of the suprarenal bodies in mammalia. Quart. J. Micr. Sc., 22, pp. I7-29, I $\delta 82$.

Mittenzweig. Traumatische Verblutung aus den Gefäszen der rechten Nebenniere. Zeitschr. Medicinalb., p. 616, I893.

Miwa, SA., and STÖLTZNER, IV. Ist das Jod ein notwendiger Bestandteil der normalen Schilddrüse? Jahrb. Kinderh., 45, I 897 .

MLodzejewskI, W. K. Zwei Fälle von Morbus Addisonii (russisch.) M. Rundsch., 1895 .

ModenA. L'Acromegalia. Riv, sperim. di Fren., xxix, I 895 .

- Un cas d'acromégalie avec Myxœdème suivi d'autopsie. Annuario del Manicomio provinciale di Ancona, Igo3.

Modrakowsky, G. Zur Innervation des Pankreas. Wirkung des Atropins auf die Bauchspeicheldrüse. P. A., I 14, I906.

- Über das gegenseitige Verhältnis der Wirkung von Atropin und Physostigmin auf das Pankreas. P. A., 118 , p. 52, 1907.

- Uber die physiologische Wirkung des Cholins. Ibid., 124, pp. 601-632, Igo8.

Modugno, G. Sui nidi cellulari del simpatico della Rana. Contributo alla conoscenza dei caratteri citologigi delle celluli cromaffini. Boll. Soc. Natur. Napoli, Bd. 20, p. 42.

Möbius, P. J. Schilddrüsentheorie. Schmidts Jahrbücher, 210, p. 237, 1886.

- Uber das Wesen der Basedowschen Krankheit. N. Z., p. 8, 1887 .

- Über Morbus Basedowii. D. Z. N., I, 400, I891.

- Die Basedowsche Kranklieit. Nothnagels Handb., 22, I896.

- Über das Antithyreoidin. M. m. W., I903.

- Über die Wirkungen der Kastration. Leipzig, I903; and Halle, 1906.

Möller, J. Om Adrenalinet Nordisk Tijdskrift f. Terapi Copenh. Ref. V.-H., ii, 1903.

MölLER, S. Kritisch-experimentelle Beiträge zur Wirkung des Nebennierenextraktes (Adrenalin). Th. M., 19, pp. 547, 622, 1905; 20, pp. 25, 85; Diss., Würzburg, 1906.

MönckeberG. Die Tumoren der Glandula carotica. Zieglers Beitr., 38, 1905 .

- Über die reine Mediaverkalkung der Extremitätenarterien und ihr Verhalten zur Arteriosklerose. V. A., I I I, p. I41, Jg., I903.

MOERS. Über den feineren Bau der Nebenniere. V. A., 29, pp. 336-358, I 864 .

MofFit, H. C. Kidney tumours of adrenalin origin. Ref. V.-H., i, p. 73I,

МонR. ${ }^{1904 .}$ Bemerkungen zur Behandlung der Hydrocele. Adrenalineinspritzungen in den Sack. M. m. W., August, 1908.

Moнr, L. Uber die Herkunft des Zuckers im Pankreasdiabetes von Hunden. Z. e. P., ii, I9o6.

- Über die Zuckerbildung aus Eiweisz. Ibid.

- Über die Beziehungen der Fette und Fettsäuren zur Zuckerbildung. Ibid.

- Ubber die Ausscheidung von Aminosäuren im diabetischen Harn. Ibid.

- Untersuchungen iber den Diabetes mellitus. Z. e. P., iv, p. 910, 1907. 
MOHR, L., and FREund, R. Exper. Beitr. zur Pathogenese der Eklampsie. B. k. W., 1908 .

MoLlow, $\mathrm{W}$. Über Vorkommen von Mundschleimhautpigmentierung. M. K., I 1009 .

MOLNAR, V. Über die interne Anwendung des Adrenalin mit besonderer Rücksicht auf die scarlatinöse hämorrhagische Nephritis. W. k. R., I904; Orv. Hetilap., I904.

Molos, C. Sulla glicosuria adrenalinica. Gazz. d. Ósped., I 908.

MoNÉry, A. Sur un nouveau cas de concrétion osseuses dans la glande thyroïde. J. d. pharm. et d. Ch. (6), I9, p. 203.

- Recherches sur la fonction jodée de la glande thyroïde. Ibid. (6), 19, p. 288.

- Etudes nouvelles sur le métabolisme de l'iode dans l'économie et sur la fonction thyroïdienne. J. d. P. P., Bd. vii, p. 61I.

Montuori, A. Sulla termo secretina. Gazz. med. int. di med., No. 13, 1905 .

- Sull' importanza del fègato nel diabete pancreatico. Gazz. d. Osped., 1895 .

Montuoro. Sulle cellule midollari dell' ovaje del coniglio. Arch. d. Anat. e. Embr., I903.

Moov, R. O. Acromegaly with illustrative cases. Lancet, p. 27, January 2, I909.

MOORE, B. On the chemical nature of a physiologically active substance occurring in the suprarenal gland. J. o. P., I7, i895.

- On the chromogen and on the active physiological substance of the suprarenal gland. Ibid., 2 I, pp. 382-389, 1897.

MOORE, EDIE, and ABRAM. On the treatment of diabetes mellitus by acid extract of duodenal mucous membrane. J. B. Ch., i, p. 28, 1906 .

- Further observations on the treatment of diabetes mellitus by acid extract of duodenal mucous membrane. Ibid., p. 446, 1906.

Mooke, B., and Purintox, C. On cardiac thrombosis following complete removal of the suprarenal glands. A. J. P., 4, pp. $5 \mathrm{I}$ and $57,1900$.

- On the effects of complete removal of the suprarenal glands. A. J. P., 5 , p. 182 , I90I.

- On the absence of the active principle and chromogen of the suprarenal gland in the human embryo and in the child at birth. Ibid., Igoo.

- Uber den Einfluss minimaler Mengen Nebennierenextrakts auf den Blutdruck. P. A., 81, 1900.

MOORE, B., and VINCENT, S. The comparative chemistry of the suprarenal gland. P. R. S., 6I, pp. $280-283$, I897.

- Further observations upon the suprarenal capsules with remarks upon the non-existence of suprarenal medulla in Teleostean fishes. Ibid., 62 , pp. $252-256,1898$.

Moore, F. C. Cholesterin. Medical Chronicle, December, I907.

MORACZEWSKI. Stoffwechsel bei Akromegalie, \&c. Z. k. M., 43, p. 336, 1901 .

Moraxo. Studio sulle capsule surrenali. Napoli, i87o.

MORANDI. Ricer. sull' istologia norm. e patol. della ipofisi. Arch. sc. med., 28, 1904 .

Morat, J. P., and Dorox, M. ,Traité de physiologie. I. Sécrétion interne. Paris, 1904 .

Morat and DUFourt. Les nerfs glyco-sécréteurs. A. d. P., 1894.

MOREAU, C. Contr. à l'étude de la thyroïdectomie. Acad. med. Belg., I89o.

- De l'opothérapie ovarienne dans la maladie de Basedow. Thèse Paris,

Morel, L. E. L'adrénaline. Progrès méd., Nos. 31 and 32 , pp. 65 and 81 , I903.

- Les parathyroïdes dans l'ostéogenèse. C. r. S. B., 67, p. 780, 1909.

MOREsco. L'opoterapia soprarenale. Gazz. degli osped., No. 98, I903.

MORI, A. Sull' uso terapeutico del succo renale per iniezioni. Clin. Med. Ital., 1898 .

MORISON, B. G. Suprarenal hæmorrhage in an infant. Lancet,.19o8.

MORKOTUN. Über einen phosphorhältigen Eiweiszkörper in der Schilddrüse im Zusammenhange mit der Frage über die Funktion derselben. Petersb. m. W., I 895 .

Morlat, A. Infantilisme et insuffisance surrénale. Thèse de Paris, 74 pp., 1903; Ref. J. d. P. P., 6, p. I66, I904. 
MORRIS, H. Primary tumours of the suprarenal gland and their removal by operation. Brit. Med. Journ., November, 1899 .

Moschini, A. Sulle alterazioni delle capsule surrenali nella morte da scottatura. Gaz. med. Lombard., Ig05.

- Studio sulla capsula soprarenale. Atti Fondaz. Cagnola, Milano, vol. 21, 176 pp., 7 tav.

Moskowicz. Zur Technik der Operationen an der Hypophyse. W. k. W., I907.

Mosse, M. Beitrag zur Lehre von der Autointoxikation beim Morbus Addisonii. F. M., 15, p. 21, 1897.

-- Über Leberzellenveränderungen nephrektomierter und hungernder Tiere, ein Beitrag zur Lehre von der Azidose. Z. k. M., 60, p. 373, I908.

- Zwei Fälle von Morbus Basedowii. Reichs-Medizinal-Anz., No. 18, 1905.

Mossé. Etat actuel de l'opothérapie. VI. C. m. française, Montpellier, 1898 .

- Note sur deux cas d'acromégalie. C. r. S. B., p. 686, 1895.

MosSÉ, P., and OULIÉ. Influence de l'ovariotomie double et de l'ingestion d'ovaires sur quelques éléments de la sécrétion urinaire chez la chienne. C. r. S. B., 5 I, p. 447, r 899 .

Mosselman and RUBAY. Cryptorchidie et Spermatogenèse chez le cheval. Ann. de méd. vétér. Bruxelles, Igoz.

Mosso. Le leggi della fatica. Atti della R. Acad. dei Lincei, 1888.

MOTT and HALLIBURTON. Physiological action of choline. J. o. P., 24, ix, I 899 .

- The suprarenal capsules in cases of nervous and other diseases. J. o. P., 34, iii, 1906.

Mouret, J. Contribution à l'étude des cellules glandulaires (Pancréas). J. A. P., 1895 .

- Lésion du pancréas produites par l'injection d'huile dans le canal de Wirsung. C. r. S. B., pp. 132 and 23, 1895 .

- Sclérose de greffes du pancréas chez le chien. Ibid., p. 22 I.

Mousset. Note sur l'adrénaline. C. r. S. B., 54, 36, p. 1471, 1903.

- Les principes actifs des capsules surrénales, leur emploi en thérapeutique. Paris, 1903.

Moussu. Recherches sur les fonctions thyroïdienne et parathyroidienne. Paris, 1887.

- Effets de la thyroïdectomie chez nos animaux domestiques. C. r. S. B., p. 271,1892 .

- Sur la fonction thyroidienne. Ibid., p. 972, 1892; pp. 280 and 394, 1893.

- Fonction parathyroïdienne. Ibid., $1897-98$.

- De la médication parathyr. C. r. S. B., 51 , p. 242, 1899.

- Influence de l'alimentation thyroïdienne sur la croissance régulière. Ibid., p. $24 \mathrm{I}$.

MOUSSU and LE PLAY. Recherches expérimentales relatives à l'exstirpation et à la destruction des capsules surrénales. Ibid., 66, p. 36, 1909 .

- Essais de greffes de capsules surrénales sur la rate. Ibid., p. 83, 16 janvier, 1909 .

Moxter. Beitr. zur pathol. Anat. der Akromegalie. Char. Ann., 24, I894.

MOYER, FleISHER, S., and LOEB, L. The influence of the addition of adrenalin to solutions of sodium chloride upon the production of urine ascites and intestinal fluid. J. E. M., I I, pp. $470-479$ and $480-488$.

MUdGE, G. P. On intravascular coagulation in albinos and pigmental animals and on the behaviour of nucleo-proteids of testes in-the production of intravascular coagulation. P. R. S., Ser. B., 79, p. 103, 1907.

MÜhlMANN, M. Zur Histologie der Nebenniere. V. A., 146, 1896.

- Zur Physiologie der Nebenniere. D. m. W., 1896 .

MÜLLER, B. Über die Verwendung der Nebennierenpräparate. W. klin.ther. W., I 1904 .

- Über eine künstliche Blutleere. M. m. W., 1904.

- Zur Anwendung des Suprarenins und Adrenalins bei Blutungen. W. k. R., I904.

MÜller, JohanNes. Lehrbuch der Physiologie. I, Koblenz, 1844.

MÜLLER, F. Ein Beitrag zur Kenntnis der Gefäszmuskulatur. A. A., Suppl., p. 4II, 1906. 
MÜller, H. F. Über einen bisher nicht beachteten Formbestandteil dẹs Blutes. C. a. P., 7, p. $929,1896$.

MÜller, L. R. Beiträge zur Histologie der normalen und der erkrankten Schilddrüse. Zieglers Beitr., 19, 1896.

Müller, WV. Beitr. z. Lehre von der Addisonschen Krankheit. ' Diss., Göttingen, 1900 .

- Das Verhalten der Langerhansschen Inseln beim Diabetes mellitus. Diss., Berlin, 1905.

- Uber die Beeinflussung der Menstruation durch zerebrale Herderkrankungen. N. C., p. 790 , 1905.

MÜlLeR. Über Entwicklung und Bau der Hypophysis cerebri und des Proc. infundibuli. Jena. Zeitschr. f. Nat., 6,1871 .

MÜNzer, A. Die Hypophyse. Sammelref. B. k. WV., 1910.

Mulon, P. Note sur la constitution du corps cellulaire des cellules dites spongieuses des capsules surrénales chez le cobaye et le chien. C. r. S. B., $1310,1902$.

- Excrétion des capsules surrénales du cobaye dans les vaissaux sanguins. Ibid., 54 , p. 1540 , 1902.

- Réaction de Vulpian au niveau des corps surrénaux des plagiostomes. Ibid., 55, p. 1156, 1903 .

- Sur une réaction de l'adrénaline in vitro son application à l'étude des surrénales. Ibid., 56, p. I15, 1904.

- Spécificité de la réaction chromaffine glandes adrénalogènes. Ibid., 56, p. 113,1904 .

- Graisse internucléaire dans les surrénales des mammifères. C. r. A., 129,1904 .

- Les glandes hypertensives ou organes chromaffines. A. gen. de méd., No. 52,1904 .

- Sur la couche germinative de la corticale des surrénales chez le cobaye. C. r. S. B., 57, p. 592 , 1905 .

- Evolution de la corticale surrénale du cobaye avec l'âge de l'animal. Ibid., 57 , p. 337,1905 .

- Sur la réaction osmique de la médullaire des surrénales. Ibid., p. 757.

- Sur le pigment des capsules surrénales (cobaye). Bibliogr. Anat., xiv, pp. 177-182, 1905 ; ibid., pp. 223-234, 1905.

- Parallèle entre le corps jaune et la cortico-surrénale chez le cobaye. C. r. S. B., 6r, p. 292, 1906.

- Sur certaines cellules des corps jaunes chez le cobaye. Ibid., p. 6r5, I 906.

- Importance fonctionelle du pigment dans la surrénale. C. r. S. B., 6z, p. $905,1907$.

- A propos de la fonction des corps jaunes chez le cobaye. Ibid., 64, p. $265,1908$.

- Cristaux de pigment dans les surrénales. Bibliogr. Anat., xvi, F. 4, 1907.

Munk, F. Kommen doppeltbrechende Substanzen (Myelin) bei der fettigen Degeneration vor? Diss., Berlin, 1908.

Munk, H. Untersuchungen über die Schilddrüse. Ber. d. preusz. Akad., $40,1887$.

- Weitere Untersuchungen über die Schilddrüse. Ibid., r 888.

- Zur Lehre von der Schilddrüse. V. A., 150, 1897; and Ibid., 154, I898.

MUNSCH. L'injection intraveineuse d'adrénaline comme moyen de traitement de la mort apparente. Semaine méd., 18 mars, 1903.

MUREL. Case of Addison's disease treated unsuccessfully with extract of suprarenal capsules. Lancet, 1896 .

Muret. De l'organothérapie. Rev. méd. Suisse Rom., 12, 1896.

MURRAY, C. R. Myxœdema, its treatment. Brit. Med. Journ., 1883.

- Remarks on the treatment of myxœdema with thyroid juice.' Ibid., 1892.

- The signs of early disease of the thyroid gland. Ibid., November 25, 1900 .

MYERS, W. A preliminary note on the antidotal properties of normal tissue emulsions with respect to cobra poison. Path. Trans., 49, p. 368,1898 .

MYRTLE, A. S. Adrenalin in neurotic heart. Brit. Med. Journ., April, rgo4.

NABARRo, D. N. The proteids of suprarenal capsules. J. o. P., 17, 1895.

NAGEL. Úber die Struktur der Nebennieren. A. A. P., 366 ff., 1836. 
NAgel, W. Die Weiblichen Geschlechtsorgane. Hdb. d. Anatomie, vii, 1896.

NAGY, L. v. Über die chromaffinen Organe. Orvosi Hetilap, p. 452, I905. NAKAHARA. Über Veränderungen des Nebennierenmarks nach Nephro- und Nephrektomien. V. A., 196, pp. 68-73, 1909.

NAPIER. Note on administration of animal extracts during the menopause. Brit. Gyn. Journ., 46.

NAPP. Über den Fettgehalt der Nebenniere. V. A., 182, p. 314, 1895.

NARBOLTE. L'hypophyse cérébrale et sa signification pour l'organisme. St. Pétersbourg, 1903.

NAUnyx. Diabetes mellitus. Nothnagels Hdb., 7, 1898, 2 Aufl., 1906.

NAZZARI. Contributo allo studio anatomo-patologico delle cisti dell' ipofisi cerebrale e dell' infantilismo. Il Policlinico, I9o6.

Neboux. Contr. à l'étude des hémorrhagies surrén. d. les infect. et intox. Thèse Paris, I905.

NÉFÉDIEFF. Sérum néphrotoxique. Ann. de l'inst. Pasteur, p. 17, rgor.

Nelson, L. Über das Thymamin: ein Protamin aus der Thymusdrüse. A. P. P., 50 .

Nerkivg, J. Narkose und Lezithin. M. m. W., rgo8.

- Beiträge zur Kenntnis des Knochenmarkes. B. Z., Iо, p. 167, 1908.

- Zur Verteilung des Lezithins im tierischen Organismus. Ibid., p. I93, roo8.

Ness, R., and BarcLar: Case of probable Addison's disease associated with leucoderma and tuberculosis. Glasgow Journ., October, igoo.

NetTer, A. Le chlorure de calcium dans la tétanie, les spasmes de la glotte, la laryngite striduleuse, les convulsions. Revue neur. d. mal. d'enfants, p. I87, ig07.

Netter, E. Die Behandlung der Rhachitis mit Nebennierensubstanz. J. K., p. 600,1900 ; ibid., 52, p. 601 .

NEU, M. Experimentelles zur Anwendung des Suprarenins in der Geburtshilfe. Gynäk. Rundsch., p. 507, 1907.

- Verwendbarkeit des Suprarenins in der geburtshilflichen Therapic. Th. G., 1907.

- Adrenalin bei Osteomalakie. C. G., 31, 38, 1907.

- Untersuchungen über die Bedeutung des Suprarenins für die Geburtshilfe. A. G., 85, pp. 617-7II, igo8.

Necbauer, E. Ist der Unterschied im Verhalten der Glykogenbildung aus Lävulose bzw. Dextrose beim Diabetes für diesen charakteristisch? A. P. P., 6i, 1909 .

Neubauer, O., and Langsters, L. Über die chemische und biologische Bedeutung der Osmiumschwärzung. V. d. N. Ä., Karlsbad, In̨oz.

Neviberg. Zur chemischen Kenntnis der Melanome. V. A., 192, p. 514 , IgOS.

- Enzymatische Umwandlung von Adrenalin. B. Z., 8, p. 383 , rgo8.

NEUBERT, IV. Über Glykogenbefunde in der Hypophyse und im Zentralnervensystem. Zieglers Beitr., 45, Igoo.

Necgebauer, F. Eine Gefahr des Adrenalins. C. Ch., p. 1417, 1903.

- Hermaphrodismus beim Menschen. Leipzig, igo8.

NEUHÄUSER. Über die Fortentwicklung jugendlicher in die Niere implantierer Nebennieren. D. m. IV., I9o9.

Neujean, V. Contr. à l'étude exp. de l'adrenaline. Arch. int. de pharmacodyn., 13, p. 45 , 1904 .

Neuman, H. Ửber den mongoloiden Typus der Idiotie. B. k. IV., I899.

- Heilung eines Falles von Addisonscher Krankheit. D. m. W., I894.

- Über das Zusammentreffen von Gravidität u. Diabetes mellitus. Z. k M., 69, I909.

Neumans, M. Zur Kenntnis der Zirbeldrüsengeschwülste. Monatschr. f. Psych., 9, 1901.

Neumans, P. "Ein neuer Fall von Teratom der Zirbeldrüse. Dišs., Königsb., 1900.

NEUMANN and VASS. Über den Einfluss der Ovariumpräparate auf den Stoff wechsel. M. G. G., I 5 .

Nevrath, R. Die vorzeitige Geschlechtsentwicklung. Erg. d. inn. Med. u. Kinderheilk., 4, ii, 46, 1909.

- Die vorzeitige Geschlechtsentwicklung (Menstruatio praecox). W. m. W., 1909 . 
Neusser. Die Pellagra in Österreich und Rumänien. Wien, 1887.

Die Erkrankungen der Nebennieren. Nothnagels Handb., 18 , 1899.

Neusser, v., and WIESEL. Die Erkrankungen der Nebennieren. Wien, I0IO.

Niccollini, C. Sulla citotossica renali. Boll. della R. Accad. di Genova, xvii, No. 6.

Nicholsov, B. St. Abnormal position of suprarenal gland. Brit. Med. Journ., is 94 .

NICOLAIDE and MELINISSOS. Untersuchungen über einige intra- und extranucleare Gebilde im Pankreas der Säugetiere auf ihre Beziehung zu der Sekretion. A. A. P., 1890 .

Nicolas, A. Contribution à l'étude des cellules glandulaires. Les éléments des canicules du rein primitif chez les mammiferes. I. M., viii, I 891 .

- Le protoplasma des éléments des glandes albumineuses. A. d. P., 1892.

- Glande et glandules thyroïdes (parathyroïdes) chez les cheiroptères. Bull. d. séances de la Soc. d. Scienc. de Nancy, Mai, 1893 .

- Rechierches sur vésicules à épithélium cilié annexées aux dérivés branchiaux. Bibl. anat. Nancy, 1896.

- Nouvelles rect. sur les glandules parathyroides. Ibid., 1897.

Nicolas, J., and BonNaMOUR, S. Karyokinèse dans la surrénale du lapin rabique. C. r. S. B., pp. 213-214, 1905 .

Nicolas and CADE. Un cas de Maladie d'Addison traité par extrait surrénale. Province méd., i 899 .

Nicolas, J., and Dumoulis, F. Influence de la Splénectomie sur la sécrétion urinaire chez le chien. G. d. P. P., 5, p. 589 , 1903.

Niedes. Fall von Tumor (Hydrops cisticus) glandulæ pinealis. C. f. Nervenh., ii, 1899.

Niemans, A. Die Beeinflussung der Darmresorption durch den Abflusz des Pankreassaftes nebst anatomischen Untersuchungen über die Histologie des Pankreas nach Unterbindung seiner Gänge beim Hunde. Z. e. P., v, p. 466, 1909 .

Nikolajew, î. Über den Einflusz der Jodeiweiszverbindungen auf die Pulsfrequenz. A. P. P., 53, p. 447, 1905.

Niosi, F. Die Mesenterialzysten embryonalen Ursprungs nebst einigen Bemerkungen zur Entwicklungsgeschichte der Nebennieren-Rindensubstanz, sowie zur Frage des Chorion-epitheFioms. V. A., 190, p. 217,

Nishi, N. Über Glykogenbildung in der Leberpankreas diabetischer Schildkröten. A. P. P., 62, p. 170, 1910.

- Uber den Mechanismus der Blutzuckerregulation. Ibid., 61, p. ı 86, 1909.

Niskoubina, N. Sur la structure du corps jaune pendant et après la gestation. C. r. S. B., 65, p. 767 , 1908 .

- Recherches expérimentales sur la fonction du corps jaune pendant la gestation. Ibid., p. 760 .

NobÉCOURT, P., and TIXIER, LÉon. La pression artérielle dans la scarlatine de l'enfant. J. d. P. P., p. 484, 1908 .

Noellner, F. Die Anatomie des Splanchnicus und der Nierennerven beim Hunde. Eckhards Beitr. z. A. u. P., iv, 1869 .

NoIr, J. L'opothérapie capsulaire, nouvel lémostatique; l'adrénaline. Progr. méd., igoz.

Noordes, C. v. Handb. d. Pathologie d. Stoffwechsels. 2 Aufl., Berlin, 1907.

- Die Fettsucht, II. Aufl., Wien, igro.

- Die Zuckerkrankheit, 4. Aufl., 1907; and 5 Aufl., 1910.

- Die Bleichsucht. Nothnagels Handbuch. VIII', Igor.

NoTHAFFT. Ein Fall von artefiziellem akuten thyreogenen Morbus Basedowii, zugleich ein Beitrag zur Frage der Schilddrüsenfunktion und zur Frage der Ätiologie des Morbus Basedowii. C. i. M., No. ${ }^{5}$, 1898 .

NothNagel. Experim. Unters. über die Addisonsche Krankheit. Z. k. M., I, p. 77,1879 .

- Zur Pathologie des Morbus Addison. Ibid., 9, p. 195, 1885.

- Geschwulst der Vierhügel. W. m. Blätt., 1888 .

$\rightarrow$ Morbus Addisonii. Allg. W. med. Zeit., I 890.

NotKIn. Beitrag zur Schilddruisenphysiologie. WV. m. W., 1896 ; and V. A., 144, Suppl. 
Novi, I. Amygdale pharingée et hypophyse. Soc. med. chir. di Bologna, I5 février, 19o8.

NovikofF, M. Über die Wirkung des Schilddrüsenextraktes und einiger anderer Organstoffe auf Ciliaten. Arch. f. Protistenkunde, I I, p. 309.

NÜRNBERG, A. Zur Kenntnis des Jodothyrins. H. B., 10, p. 125, 1907.

- Zur Kenntnis des Jodthyreoglobulins. Ibid., 16, p. 87, 1909.

Nussbaum, M. Zur Differenzierung des Geschlechts im Tierreich. (Von der Bedeutung der Hodenzwischensubstanz, p. 85.) A. m. A., 18, 1880.

- Untersuchung des Hodensekretes auf die Entwicklung der Brunstorgane der Landfrösche. Sitzber. d. niederrh. Ges. f. Nat. u. Heilk., 23 Oktober, I904; and $22 \mathrm{Mai}$, 1906.

- Über den Einflusz der Jahreszeit, des Alters und der Ernährung auf die Form der Hoden und Hodenzellen der Batrachier. A. m. A., 68, p. I, I906.

- Über die Beziehungen der Keimdrüsen zu den sekundären Geschlechtscharakteren. P. A., 129, p. 110, 1909.

- Innere Sekretion und Nerveneinflusz. Merkel-Bonnet, Ergebn., 15, 1905 .

OBERndORFER, P. Keimversprengung von Nebennieren in die Leber. C. a. P., II, 1900 .

OBERNDORFER,'S. Lymphcysten der Nebenniere. Zieglers Beitr., 29, p. 516, IgOI.

OBERNDORFER. Über Untersuchungen an Nebennieren. V. I3. d. p. G., p. 273,1908 .

OBERNDÖRFFER, E. Über den Stoffwechsel bei Akromegalie. Z. k. M., 65, 1908 .

Orregia, Parhon and Florian. Note sur un cas de myxœdème infantile avec qq. considérations sur la pathogénie de myxœème. J. de Neurol., 1905 .

OCAÑA, GOMEZ. Siehe Gomez Ocaña.

OCEANU, P., and BABES, A. Les effets physiologiques l'ovariotomie chez la chèvre. C. r. A., 140, p. 173, 16 janvier.

Oestreich, R. Operative Heilung eines Falles v. Morbus Addisonii. Z. k. M., 31, 1896 .

OESTREich and SLAvyk. Riesenwuchs u. Zirbeldrüsengeschwulst. V. A.,

OGLe, I57, p. 475 , I 899 . Lond., 50, I 899 .

OHLEMANN, M. Zur Basedowschen Krankheit. M. m. W., 1908.

OHLMACHER, J. C. The relation of the islands of Langerhans to disease of the liver. Transact. Chicago Path. Soc., 83 $_{3}$ I904. Ref. B. C., ii.

OHNACKER, H. Uber Glykosurie bei Erkrankungen des Zentralnervensystems. Diss., Kiel, 1905.

Ordmans, D. Die anorganischen Bestandteile der Leber, Milz und der meisten anderen tierischen Drüsen. Preisschr. Würzburg, 1858 .

OKerbloM, J. Zur Frage uber das krystallinische Fibrin. Z. p. Ch., 28, p. 60, 1899. Diss., Petersburg, 1901.

OKouNEFF, B. Quelques remarques concernant certaines actions indirectes de l'adrénaline et sur l'idiosyncrasie pour l'adrénaline. Arch. int. Lar., xxii, I, p. So, 1906.

Oliver, G. The action of animal extracts on the peripheral vessels. J. o. P., $21, \quad$ i 897 .

OLIver, G., and SchaEFER. The plrysiological effects of extracts of the suprarenal capsules. J. o. P, 18, p. 231, I895; ibid., 16 and 17; Proc. Phys. Soc., I 894 .

-- On the physiological action of extracts of pituitary body and certain other glandular organs. J. 0. P., 18, p. 277, 1895; Proc. Phys. Soc., March Io, I 894 .

Omelczesko, TH. İber zweierlei Spermatozoen bei Menschen und Tieren. Russky Wratsch, No. 48 , 1908.

Oxody, A. D. Entwicklung des sympathischen Nervensystems. A. m. A., $26,1886$.

OPIE, E. L. Histology of the islands of Langerhans of the pancreas. Johns Hopkins Hosp. Bull., 2, No. 119, 1900 .

- On the relation of chronic interstitial pancreatitis to the islands of Langerhans and to diabetes mellitus. J. E. M., 5, p. 397, Igor. 
OPIE, E. L. The relation of diabetes mellitus to lesions of the pancreas. Hyaline degenerations of the islands of Langerhans. Ibid., p. 527..

OPPEL, A. Lehrbuch der vergleich. mikr. Anat. der Wirbeltiere.' Jena.

- Verdauungsapparat. Merkel-Bonnets Ergeb., 9, 1899.

OPPENHEIM. Lehrbuch der Nervenkrankheiten. Berlin, 5 Aufl., 1908.

OPPENHEIM, R. Rôle des capsules surrénales dans la résistance à quelques infections expérimentales. C. r. S. B., 53, p. 314, 1901 .

- Rôle des capsules surrénales dans la résistance à la toxinfection diphtérique. Ibid., 53, p. 316.

- Les capsules surrénales, leurs fonctions antitoxique. Thèse de Paris, 1902.

OPPENHEIMER, R., and LOEPER. Lésions des capsules surrénales dans quelques infections expérimentales. C. r. S. B., 53, p. 318, I901.

- Lésions des capsules surrénales dans quelques maladies infectieuses aiguës. Ibid., 53, p. 765,1901 .

- Lésions des capsules surrénales dans quelques infections expérimentales aiguës. A. m. e., 13, pp. 332, 683, 1901.

- Lésions des glandes surrénales dans quelques intoxications expérim. Ibid., 1902.

- Insuffisance surrénale chronique expérimentale par injections intracapsulaires des poisons du bacille tuberculeux humain. Ibid., 55, ii, p. 330, 1903 .

- L'insuffisance surrénale expérimentale par lésions directes des capsules. Ibid., p. 332.

- Syndrome surrénal chronique expérimental. A. gén. de méd., i, p. 128I, 1903.

- La médication surrénale. Actual. méd. Paris, I903.

OPPENHEIMER, C. Die Fermente. Leipzig, I900; and 2 Aufl., 1903.

OPPENHEIMER, E. H. Beitr. zur Verwendung d. Nebennierenpräparate. D. m. IV., 1904 .

ORD, WV. WV. On myxœedema, a term proposed, \&c. Medico-Chirurg. Trans. 61,1878 .

- Some cases of sporadic cretinism. Lancet, 1893.

Orgler, A. Zur Physiologie der Nebennieren. Diss., Berlin, 1898 .

- Über den Fettgenalt normaler u. in regressiver Metamorphose befindlicher Thymusdrüsen. V. A., 167, 1902.

- Über Beziehungen zwischen chemischem und morphologischem Verhalten pathologischer Nieren. V. d. p. G., 6, 1903.

- Über das Vorkommen eines protagonartigen Körpers in den Nebennieren. Salkowskis Festschr., p. 285. Berlin, I904.

- Über den Einfluss von Schilddrüsendarreichung auf den Stickstoff wechel von Kindern. Z. e. P., 5, p. 1, 1008.

ORLOwSKI. Atheromatose expér. par'l'injection d'adrénaline, \&c. Przeglad lek., No. 15, 1906 ; Ref. J. d. P. P., p. 558, 1005.

ORNSTEIN, SARAH. La suppléance des capsules surrénales au point de vue de leur richesse en adrénaline. Thèse de Gerève, 1906.

ORRU, E. Sviluppo d'isolotti del Langerhans nel Gongylus ocellatus. Monit. zool. italiano, anno 2, I19, I 900 .

- Sulle sviluppo dell' ipofisi. 'I. M., 17, 1900.

ORTNER. Zur angeborenen regelwirdrigen Enge des Aortensystems. W. k. W., r89ı.

Osawa, G. Beiträge zur Lehre von den Eingeweiden von Hatteria punctata. A. m. A., 49, pp. 1 13-226, I\$97; and ibid., 5 I, p. 98ı, 1900.

Osborne, O. T. Graves's thyroid disease. Med. News., 77, 1900.

OSBORNE, W. A., and VINCENT, S. The physiolog. effects of extracts of nervous tissues. J. o. P., 25, 1899-1900; and Brit. Med. Journ., 190o.

OSER. Erkrankungen des Pankreas. Nothnagels Handb., 18, I 898.

OSLER, W. Addison's disease treated with extract of suprarenal capsules. Dublin J. M. Sc., p. 102 , I 896.

- Case of Addison's disease. Johns Hopkins Hosp. Bull., NovemberDecember, 1897 .

- The principles and practice of med. London, $190 \mathrm{I}$.

OswaLD, A. Über den Jodgchalt der Schilddrüsen. Z. ph. Ch., 23, 265,

- Die Ë Eiweisskörper der Schilddrüse. Z. ph. Ch., 27, 1899.

- Über die chemisclie Beschaffenheit und die Funktion der Schilddrüse. Habil.-Schrift, Strassburg, 1900.

- Der Morbus Basedowii im Lichte neuerer experimenteller und klinischer Forschung. W. k. WV., 1900. 
Oswald, A. Was wissen wir über die Chemie und Plyssiologie der Schilddrüsc? P. A., 97, 1900.

- Zur Kenntnis des Thyroglobulins. Z. ph. Ch., 32, 190 r.

- Die Chemie und Physiologie des Kropfes. V.A., 169, pp. 444-479, 1902.

- Weiteres über Thyreoglobulin. H. B., 2, 545.

- Die Schilddrüse und ihr wirksames Prinzip. B. C., i, p. 249, 1903.

- Neue Beiträge zur Kenntnis der Bindung des Jods im Jodthyreoglobulin nebst einigen Bemerkungen über Thyreoglobulin. A. P. P., 60, p. II 5 , igo9.

- Zur Klärung der Jodothyrin-Frage. P. A., 129, p. 103, I909.

OTT, J. The parathyroid glandules from a physiolog. and path. standpoint. Philad., 1909.

OTT, J., and ScOTT, J. C. The action of glandular extracts upon the contractions of the uterus. J. E. M., xi, 2, p. 326.

OYA. Vortr. auf dem jap. gynäk. Kongr. in Osaka, April, rgo3. Zit nach Sata.

Packard, Fr. A., and Steel, J. DutTox. A case of sarcoma of the lung, with symptoms of Addison's disease from involvement of the suprarenal capsules. Amer. Med. News, September 1I, 1897.

Padot, G. Le capsule surrenali in alcune infezioni e intossicazioni batteriche sperimentali. Kiv. crit. di Clin. Med., vii, 1907; Bph. C., ii, p. 618.

Paechtser. Kastration u. Stoffwechsel. Verh. d. Berl. phys. Ges., 1906.

PAL, J. Beitr. z. Kenntn. der Pankreasfunktion. W. k. WV., I89I.

- Über die Hemmungsnerven des Darmes. Ibid., I $\$ 93$.

- Nebennierenexstirpation bei Hunden. W. k. IV., I894.

- Physostigmin, ein Gegengift des Curare. C. P., 1900.

- C̈ber den motor. Einfluss d. Splanchnicus auf den Dünndarm. A. V., 5 , 1900 .

- Z̈ber d. Beziehungen zwisch. Zirkulation, Mobilität u. Tonus d. Darmes. II. m. P.. 1901 .

- Über das Vorkommen mydriatisch wirkender Substanzen im Harne. D. m. W., 1907 .

- Ǔber die Gefässwirkung des Hypophysenextraktes. W. m. W., I909; and C. P., 23, 1909 .

Paladiro. Gli effetti della tiroidectomia. Acc. Medico chirurg. di Napoli, I 894 .

- Della caducità del parenchimo ovarico. Giorn. Int. scienz. med. Napoli, I $88 \mathrm{I}$.

PALTALF, A. Über intralaryngeale Schilddrüsentumoren. W. k. W.. ISgI.

- Der Zwergwuchs in gerichtlicher und anatomischer Beziehung. WVien, 189 I.

PaltaUf, R. Über Geschwülste der Glandula carotica nebst einem Beitrage zur Histologie und Entwicklungsgeschichte derselben. Zieglers Beitr., I1, 1892 .

- Diskussion zum Vortrag Erdheim. IV. k. W., 1906.

Paxella, A. Azione anticurarica del principio attivo delle capsule surrenali. Atti della Soc. Toscano di Sc. Nat., Bd. 22, 1906.

- Action anticurarique du princip actif de la capsule surrénale. A. i. B., $47,17.17,1907$.

- Azione del principio attivo surrenale sul cuore isolato di mammiferi. Soc. Toscana di Sc. Nat. Pisa, 1907.

- Action du principe actif surrénal sur le cœur isolé. A. i. B., 49, p. 321 , 1908 .

- Action du principe actif surrénal sur la fatigue musculaire. A. i. B., 48,1908 .

PAxkow. Was lehren uns die Nachbeobachtungen von Reimplantation der Ovarien beim Menschen. C. G., S. 1040, 1908.

- C̈ber die Reimplantation der Ovarien beim Menschen. B. G. G., 12, 1908 .

Pantaleone. Contributo sperimentale all' inesto tiroideo. Ref. in C. Ch., p. $101,1907$.

Pantanetri, O. Saggio di ricerche sull' affatticamento muscolare. Il Policlinico, 1893 .

- Sur la fatigue musculaire dans certains états pathologiques. A. i. B., 22,1895 . 
Panzer. Über das sogennante Protagon der Niere. Z. ph. Ch., 48, p. 5 I9, 1906.

- Doppelbrechende Substanzen aus pathologischen Organen. Ibid., 54, p. 239, 1907 .

Parhon, C. Pathogénie et traitement de l'acromégalie. Revista médicale, No. 2, 1005 .

- Considérations sur le rôle des altérations endocrines dans la pathogénie de la généréscence. Congr. de Dijon, août, 1908 .

PARHON and CAZACOU. Sur un nouveau cas de throphœedéme chronique. Nouv. Iconogr. de la Salpêtrière. décembre, 1907.

PARHON, DUTRIMESCO and NISSIPESCO. Recherches sur la teneur en calcium des centres nerveux des animaux thyréoparthyréodisectomisés. C. r. S. B., 66, p. 792, I909.

PARHON and GolDSTEIN. Sur l'existence d'un antagonisme entre le fontoinncment de l'ovaire et celui du corps thyroïde. C. r. S. B., 28 février, 1903; Arch. gén. de méd., 1905.

- Les sécrétions internes. Paris, 1909. Zusammenfass. d. rumän. Literatur.

PARHON and MiHAÉlesco. Sur un cas d'infantilisme dysthyroïden et dysorbitique. Journ. de Neurol., r 1908 .

PARHON, C., and PAPINIAN, J. Nota relativa la actinuea corpului tiroid si ovarului in asimilarea si desasimilarea calcelui. Romania medicala, 1904 ; Nos. I1, 12, 1905.

Parhon, C., and URECHIE, C. Die Rolle der Schilddrüse in der Behandlung des Ekzems. Spitalul, No. 7, 1908.

- Note sur les effets de l'opothérapie hypophysaire dans un cas de syndrome de Parkinson. Soc. de Neurol., 7 novembre, 1907.

PARHon, C., and Zalplasta, J. Sur un cas de gigantisme précoce avec polysarcie excessive. Nouv. Icon. de la Salp., pp. 9I, 97, janvier, février, 1907.

PARI, G. A. Azione locale dell' adrenalina sulle pareti dei vasi ed azione delle minime dosi di adrenalina sulla pressione del sangue. Arch. di Farm. sper., iv, april, I905.

- Action locale de l'adrénaline sur les parois des vaisseaux et action des doses minimes d'adrénaline sur la pression du sang. A. i. B., 46, p. $209,1006$.

- Uber den Einfluss der Schilddrüse auf den zeitlichen Ablauf der Zersetzungen. B. Z., xiii, 1908 .

PARISOT, M. Influence de l'injection du suc pancréatique dans la veine porte sur la disparition du glycogène du foie. C. r. S. B., 56, p. 720, 6 mai, 1904 .

- Hyperglycémie et glycosurie par injection de suc pancréatique dans le système veineux. Ibid., $58, \mathrm{i}, \mathrm{p} .64,1906$.

- L'injection du sécrétine dans la veine porte ne produit pas du sucre dans le sang de la veine sushépatique. İbid., p. 66.

- Athérome expérimental par injections répétées de chlorure de baryum. Soc. de méd. de Nancy, i I mars, 1908; Rev. méd de l'Est, I5 juillet, 1908.

- Action de l'extrait de thymus sur la pression artérielle. C. r. S. B., 64, p. 749,1908 .

- Action de l'extrait d'hypophyse dans la maladie de Basedow. IXe Congr. franç. de Médecin, i 4-16 octobre, 1907.

- Pression artérielle et glande à sécrétion interne. Paris, Igo8.

- Hypertension artérielle, hypertrophie cardiaque hyperplasies hypophysaire et surrénale. Arch. des malad. du cœur, p. 426, juillet, I908.

- Le rôle de la choline dans les effets cardiovasculaires produits par les sécrétions internes. C. r. S. B., 67, p. 749, 1909.

PaRISOT and HARTER. Lésions des capsules surrénales consécutives à des altérations expérimentales du rein et du foie. C. r. S. B., 63, p. 821 , 1907.

Parisot and Lucien, M. Etude physiologique et anatomique des capsules surrénales chez les tuberculeux. Ibid., 63, p. $525,1907$.

- Etude physiologique du thymus dans l'athrepsie. C. r. S. B., 64, p. 747, 1908.

PAROdI, U. Dell' innesto della capsula surrenale fetale. Sperimentale, 58, p. 47,1904 .

Parona. Acromegalie. Riv. crit. di clin. med., igor. 
Passler, H. Beitr. z. Pathologie der Basedowschen Krankheit. M. G. M. C., 1903.

PATOx, NOEL, $L$. 'The influence of adrenalin and thyroid extract on the metabolism in diabetes mellitus. Scot. Med. and Surg. Journ., II, p. 6, 1905 .

- On the nature of adrenalin-glycosuria. J. o. P., 29, p. 286, 1903.

- The relationship of the thymus to the sexual organs. Ibid., 32, p. 59, 1904 .

Patox, N., and Goodall, A. Contribution to the physiology of the thymus. Ibid., p. 49, 1904.

PatTA, A. Contributo critico sperimentale alla studio dell' azione degli estratti di organi sulla funzione circolatoria. Arch. di Farm., vi, 1907 ; and A. i. B., 48, 1908 .

- Osservazioni intorno alle iniezioni ipodermiche ed intramuscolari di adrenalina. Arch. di Farm., iv, Nos. 7-8, 1905; and A. i. B., 46, p. 463 , 1906.

Paulesco, N. C. Recherches sur la physiologie de l'hypophyse du cerveau. L'hypophysectomie et ses effets. J. d. P. P., 9, p. 44I, 1907.

- L'hypophyse du cerveau. Paris, I9o8.

PaUlY, H. Zur Kenntnis des Adrenalins. B. d. ch. G., 36, 1903; and 37, p. 1388,1904 .

- Bemerkungen zu der Abhandlung des Herrn Böttcher: Eine neue Synthese des Suprarenins usw. Ibid., 42, p. 484, Februar, I909.

Pavy. Carbohydrate Metabolisme und Diabetes. London, 1905.

PAWLIK, K. Kasuistischer Beitrag zur Diagnose und Therapie der Geschwülste der Nierengegend. A. k. Cl.. 53, p. 530, 1896 .

Pawlow. Folgen der Unterbindung des Pankreasganges beim Kaninchen. P. A., I6, I878.

- Die Arbeit der Verdauungsdrüsen. Wiesbaden, I 898 .

PAYR, E. Transplantation von Schilddrüsengewebe in die Milz. A. Ch., 8o, I 906.

Pearce, R. M. An experim. study of nephrotoxines. Univ. Penns. Med. Bull., ז6, p. 217, 1903.

- The development of the islands of Langerhans in the human embryo. A. J. A., 2, 445 .

- The relation of lesions of the adrenal gland to chronic nephritis and to arteriosclerosis: an anatomical study. J. E. M., 10, 6, p. 735, 1909.

PEARCE, R. M., and BaLdaUf, L. K. A note on the production of experimental vascular lesions in the rabbit by single injections of adrenalin. Albany Med. An., xxviii, p. 5I, I January, 1907; Amer. Journ. of Med, Sci. 132, p. 737, November, 1906.

- Experimental myocarditis; a study of histological changes following intravenous injections of adrenalin. Ref, Schmidts Jahrb., Ibid., pp. 42,62 and 67

PEARCE and JACKSON, H. C. Experimental liver necrosis. J. E. M., 9, p. 534,1907 .

Pearce and Stanton, E. M. Experimental arteriosclerosis. Ibid. 8, p. 74, I 906.

Pechkrasz. Zur Kasuistik der Hypophysentumoren. N. Z., I8, pp. 203 and 254,1899 .

Peham, H. Aus akzessorischen Nebennierenanlagen entstandene Ovarialtumoren. M. G. G., Io, pp. 685-694, i 899 .

- Über Fütterungen mit Ovarialsubst. z. Beeinflussung d. Geschlechtsbildung. M. G. G., 25, 1907.

PetPer. Experimentelle Studien über die Folgen der Ausrottung des Plexus coeliacus. Z. k. M., I7, p. 498 , I890.

PeIser, J. Über die Beeinflussung der Schilddrüse durch Zufuhr von Schildrüsensubstanz. Z. e. P., p. 515, 1906.

PEL. Acromégalie partielle avec infantilisme. Nouv. Icon. de la Salp., I906.

PELIKAN. Gerichtlich-medizinische Untersuchungen über das Skopzentum in Russland. Übersetzt von N. Ivanoff. Giessen, 1876 .

Pellacani, P. Intorno agli effeti tossici delle diluzione acquose degli organi freschi introdotte nell' organismo di alcuni animali. Arch. per 1. scienze med., 2, p. I, I879.

- Sull' azione tossica delle diluzione acquose degli organi freschi. Rivista sperim. di Frenetira e di Med. leg., vi, fasc. 1 e, 2, p. 169, 1880. 
Pelligrino, M. Studio sugli estratti surrenali. Congr. di med. intern., 1903 .

- Sopra una particolare disposizione della sostanza midollare nella capsula surrenale. Bol. d. Soc. di Natural. in Napoli. Ser. 1, vol. 18, 1904.

- Contributo allo studio della morfologia dell' organo parasimpatico dello Zuckerkandl. Monit. zool. Ital., xvii, No. 8, pp. 254-264, 1906.

- La storia e la bibliografia delle capsule surrenali. Il Tommasi, 1906.

- La Patagenesi delle Surrenopatie. Ibid., No. 22, 1906.

- A proposito di capsule surrenali. Ibid., No. 26, 1906.

- Le surrenopatie spurie. Contributo critico e clinico. Ibid., Igo6.

- La capsule surrenale del bue. Ibid., i, No. 35, No. 36, 1906.

- La glicosuria adrenalinica, contributo sperimentale alla sua patogenesi. Ibid., iii, Nos. 14-15, 1908.

Pemberton, R., and Seveet, J. E. The inhibition of pancreatic activity by extracts of suprarenal and pituitary bodies. Arch. of Intern. Med., i, p. 628, July, 1908 .

PENDE. L'alterazioni delle capsule surrenali in seguito alla resezione del plessi celiaco e dello splancnico. Policlinico, 1903.

- Contr. alla fisiopatol. d. pancreas con spec. riguardo agli isolotti di Langerhans. Policlinico, 12, 1905.

- Le modificazione del pancreas dopo l'occlusione dei canali pancreatici. Ric. Lab. Anat. Roma, 1907.

- Uteriore contr. alla questione dei rapporti fra pancreas e diabete. II Tommasi, 1907.

Pexza, A. Osservazioni sulla distribuzione dei vasi sanguigni e nervi del pancreas. Boll. della Soc. medico-chirurg. di Pavia. 24 giügno, 1904.

PEPERE, A. Sulla penetrazione delle capsule surrenali accessorie dei parenchimi degli organi addominali. Monit. zool. Ital., 24, pp. 261-273, I 903.

- Schwangerschaftseklampsie und Parathyroidkörpeninsufficienz. V. d. Ital. path. G. in Rom, 1905: Ref. C. a. P., 17, p. 313, 1906.

- Della cosidetta sostanza colloide paratiroidea. Clinica Moderna, 13, fasc. 16 .

- Le ghiandole paratiroidea. Ricerche anatomico e sperimentale. Torino, 1906.

- Di un sistema paratiroides assessoria (timica) costante in alcuni mammifere. Giorn.

- Sur les modifications de structure du tissu parathyroidien. A. m. e., 20, 1908 .

- Real. Accad. medica di Torino, 1907, Nos. 7-8, 1909.

PEPPER, W. A study of congenital sarcoma of the liver and suprarenal, with report of a case. A. J. M. Sc., March, Igor.

PeremeschKo. Über den Bau des Hirnanhangs. V. A., 38.

PERRIN, M., and JEANDELIZE, P. Sur la posologie des produits opothérapeutiques. IXe Cong. franç. de Méd., Paris, I907.

- Moindre résistance des lapins thyroïdectomisés à l'intoxication par le chlorure mercurique. C. r. S. B., 67, pp. 849 and $85 \mathrm{I}, 1909$.

PERShING. A case of acromegaly, \&c. 'Journ. of Nerv. and Ment. Dis., 21,

1894. Zur Anwendung des Adrenalins und ähnlicher Nebennierenpräparate in der Gynäkologie. Der Frauenarzt, No. 12, 1904.

PETERSEN. Anatomische Studie über die Glandulae parathyroideae des Menschen. V. A., 174, p. 413, 1903.

Petitjean. Action de quelques médicaments vasomoteurs sur la circulation pulmonaire. J. d. P. P., to, p. 412, 1908 .

Petrén. Über das gleichzeitige Vorkommen von Akromegalie. V. A., 190, p. $1, " 1907$.

Petrone and Bagalá. Le modificazioni della milza e del timo in alcune anemie sperimentali. La Pediatria, p. 505, 1903.

PEtTrt, A. Sur les capsules surrénales de l'ornithorynchus paradoxus. Bull. Soc. Zool., 25 décembre, is94.

- Sur les capsules surrénales et la circulation porte surrénale des Reptiles. Ibid., Io décembre, 1895 .

- Remarques anatomiques et physiologiques sur les capsules surrénales des Teleostéens et des Dipnoïques. Bull. d. Mus., No. I, I896.

- Sur les capsules surrénales et la circulation porte surrénales des Oiseaux. Ibid., No. 3, 1896 . 
PETtit, A. De l'action de quelques substances toxiques sur la glande surrénale. Ibid., No. 4, 1896.

- Sur le mode de fonctionnement de la glande surrénale. C. r. S. B., 1896.

- Recherches sur les capsules surrénales. Journ. de l'anat. et de la phys. Par. Année 32, pp. 301-362 and 369-419; and Thèse de Paris, 1806.

- Modifications structurales des glandes surrénales développées chez des nouveau-nés son l'influence des maladies de la mère. Cinquantenaire de la Soc. de Biol., p. 516, Paris, 1899.

Pecker. Über einen neuen Fall v. kongen. Defekt d. Schilddrüse. Z. H., 20, 1899 .

PexA, VÁclav. O purodo tetanie detské. (Über Ätiologie der Tetanie bei Kindern.) Sborník lékarsky, x, H. 4, roog.

Pfaundler, M. Zur Anatomie der Nebennieren. S. W. A., ioi, p. 518, 1892.

PeEIfFer, WV. M. Über Addisonsche Krankheit. Diss., Freiburg i. P., 1896. PfEIfFER, H., and MaYer, O. Über funktionstiichtige Einheilung v. transplantiert. Epithelkörperchen. IV. k. W., 1007.

- Exper. Beitr. z. Kenntnis d. Epithelkörperchenfunktion. M. G. M. C., 18, 1007.

PFEIfFER, TH., and SCHOLz, IV. Stoffwechsel bei Paralysis agitans und im Senium. Einfluss der Schilddrüsentabletten. D. A. k. M., 63, 369, I 899 .

PFörrixGer. Zur Entstehung des Hautpigments bei Morbus Addison. C. a. P., I I, 1, I 900.

Pflüger, E. Über die das Geschlecht bestimmenden Ursachen und die Geschlechtsverhältnisse des Frosches. P. A., 29, "1882.

- Die teleologische Mechanik der lebendigen Natur. Ibid., I5, Bonn, 1877.

- Über die Eierstöcke der Säugetiere und des Menschen. Leipzig, 1863.

- Über die Bedeutung u. Ursache d. Menstruation. Unters. a. d. phys. Labor., Bonn, 1865 .

- Le glycogène. Dictionn. de physiol. (Ch. Richet.), Paris, 1905.

- Das Glykogen und seine Beziehungen zur Zuckerkrankheit. 2 Aufl., Bonn, 1906.

- $\mathrm{Ob}$ die Totalexstirpation des Pankreas mit Notwendigkeit Diabetes beciingt. P. A., 106, 1905.

- Ein Beitrag zur Frage nach dem Ursprung des im Pankreasdiabetes ausgeschiedenen Zuckers. Ibid., 108, H. 3 5, Mai, 1905.

- Das Fett wird als Quelle des Zuckers sichergestellt. Ibid., 1o8, H. 8-9.

- Prof. Minkowskis Abwehr gegen meine ihn treffende Kritik. Eine Antwort. Ibid., I 10, H. I-2.

- Über die durch chirurgische Operationen angeblich erzeugten Glykosurien. Nachtrag zu meiner zweiten Antwort an O. Minkowski. Ibid., I 1 I, 1906.

- Ob der Zucker im Harn durch Gärung mit Sicherheit nachgewiesen werden kann. (Eine Antwort an E. Salkowski.) P. A., 111.

- Untersuchungen über den Pankreasdiabetes. Ibid., 118, Igo7.

- Über den Einfluss einseitiger Ernährung oder Nahrungsmangels auf den Glykogengehalt des tierischen Körpers. Ibid., 119, H. 3-4, 1907.

- Über die Natur der Kräfte, durch welche das Duodenum den Kohlehydratstoff wechsel beeinflusst. Ibid., 1 I9, H. 5, 1907.

- Bemerkungen zu Rud. Ehrmanns Exstirpationen des Duodenums. Ibid., I19, H. 5, 1907 .

- Unter gewissen Lebensbedingungen nimmt die im Tierkörper enthaltene Menge des Glykogens trotz vollkommener, über Monate sich ausdehnender Entziehung der Nahrung fortwährend erheblich zu. Ibid., I 20, H. 6-9, 1907.

- Über den Duodenaldiabetes der Warmblüter. Ibid., 122 and 267, 1908.

- Durch neue Experimente gestützte Bemerkungen zu den jüngsten Arbeiten über den Duodenaldiabetes des Hundes. P. A., 123, p. 323, 1908.

- Über die durch Resektion des Duodenums bedingten Glykosurien. Ibid., I 24, H. I-2, 1908 .

- Die Aufklärung, welche Errico de Renzi und Enrico Reale soeben (August, 1908)' uber ihre den Duodenaldiabetes betreffenden Versuche gegeben haben. Ibid., 124, H. 11-12, 1908. 
Pflüger, E. Über Parabiose und Pankreasdiabetes. Ibid., 124, H. I1-12, 1908.

- Experimentelle Untersuchung über den Darmdiabetes. Ibid., I28, H. 3, Igog.

- Ob d. Entwickl. d. sekund. Geschlechtscliarakt. v. Nervensystem abhängt. P. A., I 16, 1907 .

Philipeaux. Note sur l'exstirpation des capsules surrénales chez les rats albinos. (Mus rattus.) C. r. A., 43, pp. 904 ánd 1155 , 10 novembre and 22 décembre, 1856 .

- Ablation successive des capsules surrénales, de la rate et des corps thyroïdes sur des animaux qui survivent à l'opération. Ibid., 44, p. 396,23 février, I 857 .

PHILIPS. Addison's disease with simple atrophy of the adrenals. J. E. M., 4, p. 58I, 1899 .

Philoche. Action comparée de l'amylase et du suc pancréatique sur le glycogène et l'amidon. C. r. S. B., 57, i, p. 263 , 1905.

PIC and BONNamour. Contribution à l'étude du déterminisme de l'athérome aortique expérimental. Ibid., 57, ii, p. 219 , 1905; J. d. P. P., p. 460, Igo6.

Pick, E. P., and PiNeles, F. Über die Beziehungen der Schilddrüse zum Gefässsystem. V. 25 ; C. M., p. $360,1908$.

- Uber die Beziehungen der Schilddrüse zur physiologischen Wirkung des Adrenalins. B. Z., 12, pp. 473-484, 1908.

- Unters. uib. d. physiologisch wirksame Substanz d. Schilddrüse. Z. e. P.,

PICK, F, Igog. ändernde Mittel. A. e. P., 42, I899.

PICK, L. Die Marchandschen Nebennieren und ihre Neoplasmen nebst Untersuchungen über glykogenreiche Eierstockgeschwülste. A. G., 64, pp. 670-835, u. Festschr. f. L. Landau; B. G. G., pp. 434-603, I901.

- Tetanie und Gravidität. C. G., p. 1312, 1902 .

- Über Umbildungen am Genitale bei Zwittern. A. G., 76, p. I9 I, I905.

PickarT, M. Die Beeinflussung des Stoffwechsels bei Morbus Addisonii durch Nebennierensubstanz. B. k. W., i 898 .

PIERSOL. Über die Entwicklung der embryonalen Schlundspalten und ihrer Derivate bei Säugetieren. Z. w. Z., 47.

Pilliet, A. H. Tuberculose d'une capsule surrénale sans mélanodermie. Bull. de la Soc. anat., 65,4 , octobre-novembre, 1890.

- Débris Wolffien surrénal de l'epididyme chez le nouveau-né. Bull. Soc. anat., 65, p. 471,1890 .

- Recherches sur l'adénome des capsules surrénales. Ibid., 66, avril, 189 I.

- Capsule surrénale dans le plexus solaire. Ibid., 66, pp. 267-268, 1891.

- Capsule surrénale située sous la capsule fibreuse du rein droit. Ibid., 68, I 893.

- Etude histologique sur les altérations séniles de la rate, du corps thyroïde et des capsules surrénales. A. m. e., 5, I893.

- Pigmentation et hémorrhagies expérimentales des capsules surrénales. C. r. S. B., pp. 97-99, 1894 .

- Etude expérimentale sur les lésions des capsules surrénales dans quelques empoisonnements. A. d. P., 27, 1895 .

Pilliet, A. H., and VEAU, V. Capsule surrénale aberrante du ligament large. C. r. S. B., pp. 64-68, 1897 .

Pineles, F. Die Beziehungen der Akromegalie'zum Myxödem und zu anderen Blutdrüsenerkrankungen. Volkmanns Samml., N. F., 242, I 899.

- Über die Beziehungen der Akromegalie zum Diabetes mellitus. Jahrb. Wien. Krankenanst., 1899.

- Über Thyreoaplasie (kongenitales Myxödem) u. infantiles Myxödem. W. k. W., 1902.

- Zur Physiologie und Pathologie der Schilddrüse. Ibid., I904.

- Klinische und experimentelle Beiträge zur Physiologie der Schilddruise und der Epithelkörperchen. M. G. M. C., 14, 1904.

- Über die Funktion der Epithelkörperchen. S. A. W., I13, I904; and I 17,1908 .

- Zur Pathogenese der Tetanie. D. A. k. M., 85, 1906.

- Tetaniestar — Zuckerstar - Altersstar. W. k. W., I9o6.

- Zur Pathogenese der Kindertetanie. Jahrb. f. Kinderkh., 66, 1907. 
PINetes, F. Behandlung der Tetanie mit Epithelkörperpräparaten. Arb. neurol. Inst., I907.

- Über parathyreogenen Laryngospasmus. W. k. W., igo8.

PIRche. Influence de la castration sur le squelette. Thèse Lyon, 1902.

PIRONE, R. Contribution à l'étude des tumeurs rénales d'origine surrénale hypernéphromes): Arch. de scienc. biol., Io, No. I, 1903.

- Nouvelle contribution à l'étude des tumeurs des reins d'origine surrénale (hypernéphromes). A. m. e., 1903.

- Sulla fina struttura e sui fenomeni di secrezione dell' ipofisi. A. d. F., 2, 1905 .

-. L'hypophyse dans la rage. A. m. e., p. 688, septembre, I9o6.

PIRRONE, D. Contr. sper. allo studio della funzione dell' ipofisi. Rif. med., 19,1003 .

PISENTI. Sulla interpret. da darsi ad alcune particolarità istol. d. gland. pituitaria. Gazz. d. Osp., 16, I895.

PISENTI and VIOLA. Beitrag zur normalen und pathologischen Histologie der Hypophysis, etc. C. m. IV., 2S, 1 Soo.

Pischinger, D. Beitrag zur Kenntnis des Pankreas. Diss., München.

PI SUÑ̃er and TuRRó. Sur l'inconstance de la glycosurie après l'exstirpation totale de Pancréas. C. r. S. B., 66, 1909.

Pitini, A. Influenza dell' adrenalina sulla secrezione biliare. $A$. int. Pharmac., 16, 1906.

- Ricerche farmacologiche sugli amminochetoni. Nota II. Arch. di Farmac., 12, 1907 .

PITTARD. La castration chez lihomme. C. r. A., 1903.

PLITT, H. IV., and STEELE, P. Treatment of serous effusions by injection of adrenain chloride. Brit. Med. Journ., July I 5 , I905.

Plato, J. Die interstitiellen Zellen der Hoden und ihre physiologische Bedeutung. A. m. A., 48 , 1806 .

- Zur Kenntnis der Anatomie und Physiologie an Geschlechtsorganen. Ibid., 50, I 807 .

Play, L., and Corpechot. Action des Néphrolysines. C. r. S. B., p. 207, 1904 .

Ploss, H. Das Weib in Natur- und Völkerkunde. Herausg. von Bartels. 6 Aufl., i 890 .

Plumier, L. Action de l'adrénaline sur la circulation cardiopulmonaire. J. d. P. P., 6, pp. 655-670, 1904.

Pleckik, O. Zứr Histologie der Nebenniere des Menschen. A. m. A., 6o, p. 414, I002.

Pоснох. Beiträge zur Kenntıis der Langerhansschen Inseln des Pankreas. A. wiss. u. prakt. Tierrheilk., 34, p. 581 , Oktober, 1908; Bph. C., iii, No. 1710 .

PoEHL. Einwirkung des Spermins auf den Stoffumsatz bei. Autointoxikationen. Z. k. M., 26, I $\$ 94$.

- Verwendung physiologischer Katalysatoren als Heilmittel. D. m. W., 1903 .

POEHL, A., TARCHANOFF and WaCHS. Rationnelle Organotherapie. Übersetzt aus dem Russischen. Petersburg, 1905.

Poll, H. Über das Schicksal der verpflanzten Nebenniere. C. P., I\$98.

- Veränderungen der Nebenniere bei Transplantation. A. m. $\mathbf{A}$, 54, I $899 ;$ Diss., I 900 .

- Die Anlage der Zwischenniere bei den Haifischen. Ibid., 62, pp. 138174,1903

- Nebenniere, Enzyklopädie der mikroskopischèn Technik (herausgegeben von Ehrlich, Krause, Mosse, Rosin, VVeigert), 2, pp. 920-925, 1903.

- Die Anlage der Zwischenniere bei der europäischen Sumpfschildkröte (Emys europaea) nebst allgemeinen Bemerkungen über-die Stammesund Entwicklungsgeschichte des Interrenalsystems der Wirbeltiere. I. M., 21, pp. 195-29I, I 904 .

- Allgemeines zur Entwicklungsgeschichte der Zwischenniere. A. A., 24, 1904 .

- Die vergleichende Entwicklungsgeschichte der Nebennierensysteme der IVirbeltiere. O. Hertwigs Handb. d. vergl. u. exp. Entwicklungsgesch., iii, 1, pp. 442-6is, 1 go6.

-... Die Biologie der Nebennierensysteme. Zusammenfassender Bericht B. k. W., $648,1886,1973 ;$ 1909. 
Poll, H. Zur Lehre von der Nebennierentransplantation. A. A. P., 1006; M. K., 1905.

- Gibt es. Nebennieren bei Wirbellosen? G. d. naturf. Fr., Berlin, 1908; S. pr. A. W., 36 and 889,1900 .

Poll, H., and SoMMER, A. Über phäochrome Zellen in Zentralnervensystem. V. Physiol. Ges. Berlin, p. 77, Jg., I902-1903.

Pollak, L. Demonstration eines Falles von Morbus Addisonii. W. k. W., 1909.

- Experimentelle Studien über Adrenalindiabetes. A. P. P., 6I, p. 149, I909.

-- Kritisches und Experimentelles zur Klassifikation der Glykosurien. Ibid., 61, p. 157 .

- Untersuchungen bei Morbus Addisonii. W. m. W., 1910.

Pollak, O. Kritisch-experimentelle Studien zur Klinik der puerperalen Eklampsie. Wien, 1904.

Polte. Paranephrin, ein neues Nebennierenpräparat. Arch. Augenh., 51, p. 54,1904 .

Poxcet. Influence de la castration sur le dévelop. du squelette. Congr. Ass. franc., Havre, 1877.

PoNfick. Myxödem und Hypophysis. Z. k. M., 35 and 38,1899 .

- Über die Beziehungen zwischen Myxödem und Akromegalie. C. a. P., 1899 .

- Zur Lehre vom Myxödem. Verh. d. p. G., I899.

PoNTOPPIDAN. Ein Fall von Tumor der Zirbeldrüse. N. C., p. 553, I885.

Poot. Tetany parathyreopriva. Ann. of Surg., October, I907.

POPIELSKI, L. Über das peripherisch reflektorische Nervencentrum d. Pankreas. P. A., 86, 190r.

- Die Sekretionstätigkeit der Bauchspeicheldrüse unter dem Einfluss von Salzsäure und Darmextrakt (des sog. Sekretins). P. A., 120, p. 451, 1907.

- Uber den Charakter der Sekretionstätigkeit des Pankreas unter dem Einfluss von Salzsäure und Darmextrat. Ibid., I 2 I, H. 5-6, 1908.

- Uber die Wirkungsweise des Chlorbaryum, Adrenalin und Pepton Witte auf den peripherischen, vasomotorischen Apparat. A. P. P., Suppl., pp. $434-442,1908$.

- Uber eine neue blutdrucksteigernde Substanz des Organismus auf Grund von Untersuchungen von Extrakten von Glandula Thymus. Speicheldrüsen, Schilddrüsen, des Pankreas und Gehirns. C. P., 23, p. 137, 1909.

- Uber die physiologischen und chemischen Eigenschaften des Peptons Witte. P. A., I26, H. 9-10, 1909 .

- Über die physiologische Wirkung von Extrakten aus sämtlichen Teilen des Verdauungskanales (Magen, Dick- und Dünndarm) sowie des Gehirns, Pankreas und Blutes und über die chemischen Eigenschaften der darin wirkenden Körper. Ibid., 128, H. 4-5, 1909.

POPIELSKI, L., and PANEK, K. Chemische Untersuchung uiber das Vasodilatin, den wirksamen Körper der Extrakte aus sämtlichen Teilen des Verdauungskanales, dem Gehirn, Pankreas und Pepton Witte. P. A., 128,1900 .

POPper, $R$. Über die Wirkungen des Thymusextraktes. S. W. A., 114, 1905 ; ibid., $115,1906$.

POPPER. Das Verhältnis des Diabetes zu Pankreasleiden und Fettsucht. Öst. Z. f. prakt. Heilk., No. 11, 1868 .

Porcher. Sur la physiologie de la mamelle. Journ. de l'éc. vét. de Lyon, 15 septembre, 1905 .

PORGES, M. Exp. Beitr. z. Wirkung u. Nachwirkung v. Schilddrüsengift. B. k. IV., I 900 .

Porges, O. Wirkung der Nebenniere auf den Blutzucker. Ibid., p. 2817, 1908 .

- Diskussionsbemerk. zu. Pollak. "Ein Fall von Morbus Addisonii." W. k. W., igog.

- Über Hypoglycämie bei Morbus Addisonii sowie bei nebennierenlosen Hunden. Z. k. M., 69, p. 341 , 1909 .

Porosz, M. Das Tonogen suprarenale sec. Richter, Adstringens und Anästheticum. Monatsschr. f. Dermat., 39, I904.

Portis, M. Experimental study of the thyrotoxic serum. Journ. of Infect. Dis., 1, p. 127. 
Poschariski, J. F. Über Hypernephrome. Wratscheb. Gaz., N 4I, 1904. Posner, C. Die norm. u. path. Physiol. d. Prostata. Cong. int. d'urologie., i, I 908 .

Pospelow, J., and Gautier-Dufoyer, E. W. Zur Kasuistik und Pathogenese des Melasma suprarenale. (Morbus Addisonii.) Arch. f. Derm. u. Syph., 66, 3, p. 355 , 1903.

PospischIL. Über Diphtherietherapie. Versuch einer Behandlung der schwersten Fälle mit Adrenalin-Kochsalzinjektionen. W. k. W., 1908.

PosseK, R. Lassen sich Linsentrübungen organtherapeutisch beeinflussen? W. k. W., I909.

Posselt. Bericht uiber fünf zur Obduktion gelangte Fälle von Morbus Addisonii. Ibid., pp. 630, 652, 672, 714, 1894 .

- Morbus Addisonii. Ibid., 1?p. 17 and 24, 1897.

PotSEK. Schilddrüse und Auge. Klin. Monatsbl. f. Augenhk., Beilageheft, 1907.

PotTIEN. Beiträge zur Addisonschen Krankheit. Diss., Göttingen, I889.

POWER, D'ARCY. Symmetrical tubercular disease of the suprarenal capsules. Path. Trans., xi, p. 300,1890 .

- Case of hæmorrhages into the suprarenal capsule of both sides. Lancet, April 5, I89o.

Prandi, T. Syndrome myasthenique post-typhoidique. Gaz. degli osped., $28,1907$.

PREGL, F. Zwei weit. ergograph. Vers. uib. d. Wirk. orchitischen Extraktes. P. A., 62,1896 .

PRÉNANT, A. Etude sur la structure du tube séminifère des Mammifères. Thèse de Nancy, I887.

- Contribution à l'étude du développement organique et histologique du thymus, de la glande thyroide et de la glande carotidienne. La Cellule, 10, I, 1900 .

- Sur le développement des glandes accessories de la glande thyroïde, etc. An. An., xii, 1896 .

- Rectification au sujet de la commun. de M. Maurer : Die Schlundspaltenderivate von Echiona. Ibid., xvi, 1899.

- De la valeur morphologique du corps jaune son action physiologique et thérapeutique possible. R. gén. d. sc., I898.

Presbéanu, N. De l'hypophyse dans l'acromégalie. Thèse de Paris, 190 g.

Prevost, J. L., and Mioni, J. Convulsions de la thyroide par courants alternatif's après exstirpation. C. r. S. B., 57, p. 69, 1905 .

Pribram, H. Beitr. z. Kenntn. d. Schicksals d. Cholesterins u. d. Chlosterinester. B. Z., 1, 1906.

Pribram, E., and Porges. Über den Einfluss verschiedenartiger Diätformen auf den Grundumsatz bei Morbus Basedowii. W. k. W., 1908 .

Prieur, G. Le tabac et l'appareil vasculaire. Thèse de Paris, 1905.

PROCA, G. Insuffisance thyroidienen et bacillus mucinogènes de l'eau. C. r. S. B., 6o, p. 989 .

PROTOPOPOFF. Action de l'adrénaline sur la circulation intracranienne (russisch), Ref. J. d. P. P., 6, p. I1 22, 1904; and 7, p. 145, 1905.

Prouchinsky, $J$. Über den Einfluss von Adrenalin auf das Zirkulationssystem. (finn.). Ibid., 6, p. II21, 1904; and W. k. W., I904.

Proust, R. La chirurgie de l'hypophyse. Journ. de chir., Igo8.

Pruneau. Note sur la sécrétion interne du testicule. Rec. de Méd. vétérinaire, 1900 .

Prym. Milz und Pankreas. II. Teil. Versuche mit Infusen beider Organe. P. A., 107.

Puech. Des Ovaires et leur anomalies. Paris, Levy.

PUPPEL, E. Behandlung der Osteomalakie mit Nebennierenpräparaten. C. G., 1907.

PURTSCHER, D. Über Starbildung und Kropfoperation. Cbl. f. Augenh., 1909 .

Putnam. Case of myxœdema and acromegaly, treated by sheeps' thyroids. A. J. M. S., June, 1893 .

- The clinical aspects of the internal secretions. Ibid., 1898.

Pugliese. Zur Lehre v. d. Schilddrüse v. H. Munk. V. A., I 50 , 1897.

- Wirkung v. Thyreoideapräparaten nach Exstirpation d. Schilddrüse. P. A., 72, 1898 .

Pugnat, CH. A. Recherches sur l'histologie du pancréas des oiseaux. J. A. P., 33, p. 268. 
Pug.sat, Ch. A. Note sur la structure histologique du pancréas des oiseaux C. r. S. B., 48, p. 1071 .

QUADRI, G. Un caso di albuminuria delle gravido curato con la paratiroidina. Gaz. Osp. e cliniche, No. 116, 1908.

QUÉNU and LEJARS. Etude sur la système circulatoire. Paris, 1894.

QUERVAIN DE. Über Veränderungen des Zentralnervensystems bei exp. Cachexia thyreopriva der Tiere. V. A., 133, I 893 .

QUEST, ROBERT. Über den Einfluss der Ernährung auf die Erregbarkeit des Nervensystems im Säuglingsalter. IV. k. W., 1906.

- Uber die Bedeutung der Nebennieren in der Pathologie und Therapie der Rachitis. Z. e. P., 5, p. 43.

QurNCKE, H. Über Athyreosis im Kindesalter. D. m. W., Igoo.

RABAUD, E. Fotus humain paracéphalien hémiacéphale. J. A. P., 39, 1903.

RABL, C. Theorie des Mesoderms. Morph. Jahrb., 19, pp. 85-144, I893.

- Uber die Entwicklung des Urogenitalsystems der Selachier. Ibid., 24, p. 756,1896 .

- Zur Bildungsgeschichte des Halses. P. m. IV., I886.

RABL, H. Die Entwicklung und Struktur der Nebennieren bei den Vögeln. A. m. A., 38 , pp. $492-523,1891$.

RABL-RÜCKHARD. Des gegenseitige Verhältnis der Chorda, Hypophysis, etc. Morph. Jahrb., 6, I880; and A. A., I883.

RADASCH, H. E. Ectopia of the adrenal. A. J. M. S., 127, p. 286, 1902.

RADZIEJEWSKI, B. Über den augenblicklichen Stand unserer Kenntnis von den Nebennieren und ihren Funktionen. B. k. W., I 898 .

RäLBER, H. Zur feineren Struktur der Nebennieren. Diss., Berlin. 188 I.

RAINIERI. Le capsule surrenali in rapporto alla ovariectomia, all ovaroisterectomia e all' isterectomia. Folia gynecologica, I, fasc. 2, Igo8; B. k. IV., I 909 .

Ramond and Hulot. Dégénéresc. experim. du foie et des reins. C. r. S. B.,

RAc, IV. C̈ber die Abstammung von Nierensarkomen aus versprengten Nebennierenteilen. Diss., Bonn, i 806 .

RAxGE. Contr. à l'étude pathogénique du syndrome de Basedowii. Thèse de Paris, I 899 .

Ravkin, G. Myxodema. Practitioner, lxxxii, 2, p. 204, I909.

RANZI and TANDLER. Demonstration. W. k. W., 1909 .

RANZY. Intoxication thyreoïdienne. Thèse de Lyon, I898.

RAPAPORT. Exp. Unters. über Glykolyse. Z. k. M., 57, p. 208.

RATH. Ein Beitrag zur Kasuistik der Hypophysentumoren. Diss., Göttingen, I888.

- Zur Symptomenlehre der Geschwülste der Hypophysis cerebri. Gräfes Arch., 34.

RathKe. Über die Entstehung der Glandula pituitaria. Müllers Arch. f. A. u. P., 1838 .

RAUTENBERG: Experimentell erzeugte, mit Arteriosklerose verbundene chronische Albuminurien. V. d. N. u. Ä., Salzburg; and D. m. W., 1909 .

Ravano. Über die Frage nach der Tätigkeit des Eierstockes in der Schwangerschaft. A. G., 83, p. 587,1907 .

Raves, H. M. Adrenalin in Addison's disease. Brit. Med. Journ., January, 1904 .

RAYER. Die Nebennieren und der Morbus Addisonii. Berlin, I883.

RAYMOND. De la pigmentation dans la maladie d'Addison. A. d. P., 1892.

- Morbus Addisonii mit Integrität der Nebennieren. Soc. méd. des hôp., März, I $8 q^{2}$.

RAYMond and Lejonne, P. Deux cas de myasthène bulbospinale. Soc. de Neur., 5 avril, I 906.

REBENDI, ST. Eierstock, Corpus luteum und Langerhanssche Zellinseln. C. G., $4 \mathrm{I}, \mathrm{p} .1332$.

RECKE, F. M. Vergleichende experimentelle Untersuchungen Iokalanästhesierender Mittel. Diss., Leipzig, Ig03.

RECKLINGHAUSEN v. Über die Akromegalie. V. A., I 19, p. 36, I89o.

REGaUD, CL. Glandules à sécrétion interne juxta-épididymaires chez le lapin. C. r. S. B., I899. 
Regaud, Cl. Etude sur la structure des tubes séminifères et sur la spermatogenèse chez.les Mammifères. A. d'Anat. micr., iv, fasc. 2, 3, 1901 .

REGaUD and BLANC. Action des rayons $\mathrm{X}$ sur les diverses générations de la lignée spermatique. C. r. S. B., 58, ii, p. $163,1905$.

- Action teratogène des rayons $X$ sur les cellules séminales. Ibid., p. 390 .

REGAUD and Dubreull. Action des rayons de Roentgen sur le testicule du lapin. I. Conservation de la puissance virile et stérilisation. C. r. S. B., 63, p. 647,1907 .

- Variations macrosc. de la glande interstitielle de l'ovaire chez la lapine. Ibid., p. 780 .

- Glande interstitielle de l'ovaire et rut chez la lapine. Ibid., 64, p. 217, 1908.

- Gravidité et glande interstitielle de l'ovaire chez la lapine. Ibid., p." 396.

- A propos des corps jaunes de la lapine: ils n'ont avec le rut relation. Ibid., p. 442 .

- L'ovulation de lapine n'est pas spontanée. Ibid., p. 552.

- Observations nouvelles relatives à l'indépendance des corps jaunes et du rut chez lapine. Ibid., 64, p. 602, 1908.

- Karyokinéses des cellules luteinique dans les corps jaunes en regression chez la lapine. Ibid., p. 858 .

-. Parallelisme des variations macroscopiques et microscopiques de la glande interstitielle dans l'ovaire de la lapine. Ibid., p. 90I.

-- Perturbation dans le développement des œufs fécondés par des spermatozoïdes roentgenisés chez le lapin. Ibid., p. 1014.

- Sur les relations fonctionnelles des corps jaunes avec l'utérus nongravide. Ibid., 66, p. 257, 1909.

- Acceleration du rut par la cohabitation. Ibid., p. 130.

- Variations de volume de l'uterus par rapport a l'état des ovaires. Ibid., p. 299.

- Etats de l'utérus aux diverses phases de la période prégravidique. Ibid. p. 413 .

- Nouvelles recherches sur les modifications de la glande interstitielle de l'ovaire consécutives à l'isolement et la cohabitation avec le mâle. Ibid., 67, p. 348, 1909.

- Existe-t-il des relations entre les phénomènes du rut et la présence de corps jaunes ovariens chez la lapine. Ibid., p. I76.

- Effets de la rupture artificielle follicales de l'ovaire au point de vue de la formation des corps jaunes chez la lapine. Ibid., 67, p. 166, 1909.

Regaud, Cl. , and Policard. Etude comparative du testicule du porc normal impubère et ectopique au point de vue des cellules interstitielles. C. r. S. B., avril, 1901 .

Regerat. Thèse de Paris, r 903.

REHN. Die chirurg. Behandl. d. Morbus Basedow. M. G. M. C. 7, igoo.

ReICHE. Primäres Trachealkarzinom. Metastase in der linken Nebenniere. Melasma suprarenale. C. a. P., 4, p. 1, iso3.

ReICHER, K. Beziehungen zwischen Ảdrenalsystem und Niere. B. k. WV., I 908.

Reikhtmann. Thèse de St. Pétersbourg, 1902.

ReIL. Nebenniere. Berl. tierärztl. Woch, 28, I902.

Reinhold. Fall von Tumor der Zirbeldrüse. Diss., Leipzig, 1886.

REIMANN, G. Melanotisches Karzinom der Nebennieren bei einem Säugling. P. m. W., 1902 .

ReinbaCH. Zur Chemie des Kolloids der Kröpfe. C. Ch., No. 21, is98.

REINHARDT. Adrenalin und osteomalakie. C. G., p. 1613, 1907.

REINKE. Über Krystalloidbildungen in den interstitiellen Zellen des menschlichen Hodens. A. m. A., 42, i 896 .

REINL. Die Wellenbewegung der Lebensprozesse des Wiebes. Volkmanns Samml., 243, 1900.

ReItMANN. Beitr. z. Pathologie d. menschl. Bauchspeicheldrüse. Z. H., 26, 1905 .

RENAUT. Sur les organes lympho-glandulaires et le pancréas des vertébrés. C. r. A., 89 .

-_. Essai d'une nomenclature méthodique des glandes. A. d. P., I881.

- Observation pour servir à l'histoire de la maladie d'Addison et des tuberculoses locales. Ibid. 
RENAUT. Pouvoir sécrétoire et signification glandulaire des epithéliums des tubes contournés du rein et valeur thérapeutique de leur produits. Bull. gén. de Thérap., 147, p. 3.

- Opothérapie rénale dans' l'albuminurie. Sem. méd., p. 418, 1903.

RENDu. Note sur deux cas de maladie d'Addison avec autopsie. Bull. Soc. méd des hôpitaux, 24 février, 1899.

RENNER. Neue Symptome der Addisonschen Krankheit. Ver. Bl. f. Pfälzer Ärzte, xii, 3, p. 53, März, 1896.

Renon, L. Action de l'opothérapie associée sur le syndrome de Basedow. Acad. de méd., 5 mai, 1908.

- Les syndromes polyglandulaires et l'opothérapie associée. Journ. des prat., 1908.

Renon, L., and AzAM. Maladie de Basedow traitée par l'opothérapie hypophysaire. Soc. méd. d. hôpit., 24 mai, $190 \%$.

Revon, L., and Delille, A. Sur quelques effets opothérapeutiques de l'hypophyse. Soc. de thérapeutique, 22 janvier, 1907; Bull. gén. de Thérap., 1 53, p. 178.

- De l'utilité d'associer les médications opothérapeutiques. Ibid., 12 juin, I907.

- Sur les effets des extraits d'hypophyse, de thyroïde, de surrénale, d'ovaire, employés en injections intrapéritonéales. C. r. S. B., 64, p. 1037 , and 65, p. 499, 1908.

- L'insuffisance hypophysaire et la myocardite. IXe Congr. franç. de Méd., 1907.

- Insuffisance thyro-ovarienne et hyperactivité hypophysaire (troubles acromégaliques) médication par l'opothérapie thyro-ovarienne; augmentation de l'acromégalie par la médication hypophysaire. Soc. méd des hôp., 19 juin, Igo8.

- Syndrome polyglandulaire par hyperactivité hypophysaire (gigantisme avec tumeur de l'hypophyse et par insuffisance-thyro-ovarienne). Soc. méd. d. hôp., 4 décembre, rgo8.

- La médication hypophysaire dans les cardiopathies. Soc. d. Thérap., 9 décembre, I908.

- L'opothérapie indirecte. C. r. S. B., 16 janvier, 1909.

Renon, L., Delille, A., and Monier-Vinard, R. Syndrome polyglandulaire par dyshypophysie et par insuffisance thyro-testiculaire. Soc. méd. des hôpit., 5 février, Igog.

RExzi DE. Action néphrotoxique et hémolyt. du rein. Sem méd., p. 35i,

RENzI, E. DE, and BOERI, G. Ricerche sperimentali sull' asportazione di alcuni organi abdominali e sulla suppressione della loro circolazione. Gazz. d. Osped., 1903.

- Ancora sull' asportazione di alcuni organi abdominali e sulla soppressione completa della loro circolazione. Nuova riv. clin.-terap., vii, 2 , 1904 .

- Sull' azlone nefrotossica ed emolitica del reni. Ibid., 1905.

RENZI DE and REALE. Über den Diabetes mellitus nach Exstirpation des Pankreas. B. k. IV., I892; and Nuov. riv. clin.-terap. August, I908.

RENNIE, J. Über die physiologische Bedeutung der Langerhansschen Inseln im Pankreas. C. P., is: and Quart. J. Micr. Sc., 48, I904.

RENNIE, J., and FRASER. The islets of Langerhans in relation to diabetes. J. B. Ch., 2, 1907 .

Restelli. De thymo. Ticini Regis, 1845.

Revelli, C. Perchè si nasce maschi o femmine. Torino, 1899.

REverdin, J. and A. Note sur 22 opérations du goitre. Rev méd. Suisse rom., 1883 .

- Contribution à l'étude du myxoedema. II. Congr. Chir. Franç.. 1886.

REYNIER and PaUlesco. Glandes thyroïdes. Journ. de méd. int. is99.

RHEINBOLDT, M. Zur Fettsuchtsbehandlung mit Schilddrüse. B. k. IV., I 906.

Ribadeau, Dumas, and Pater. La syphilis congen. des caps. surrénales. A. m. e., 21,1909 .

RIBBERT. Über die Regeneration des Schilddrüsengewebes. V. A., Bd. II7, 1889.

- Über kompens. Hypertrophie der Geschlechtsdrüsen. V. A., I20, I89o.

- Über Veränderung transplantierten Gewebes. E. M., 6, p. i31, is97. 
RIBBERT. Über Transplantation von Ovarien, Hoden und Mamma. Ibid., 7, I 898 .

RichoN, L., and Jeandelize, P. Influence de la thyroïdectomie sur la castration chez la lapine. Effets de la thyroïdectomie sur la lapine adulte. C. r. S. B., 56, p. 19 , 1904 .

-- Tyroidectomie et accidents aîgus au cours du gestation. Ibid., p. 22

- Castration pratiquée chez le lapin jeune. Etat du squelette chez l'adulte. Examen radiographique. Ibid., 57, i, p. 555, 1005.

Insuffisance thyroïdienne expérimentale fruste. Ibid., p. 728, 1905.

Richter, M. Über plötizliche Todesfälle im Kindesalter. V. d. N. Ä., p. $290,1902$.

RICHTER, P. FR. Eiweisszerfall nach Schilddrüsenfütterung. C. i. M., 65, 1896.

-.. Fieber und Zuckerausscheidung. B. k. W., 1903.

- Experimentelles über Nieren wassersucht. İbid., 1905.

RICKER, G. Beiträge zur Lehre von den Geschwülsten in der Niere. C. a. P., S, 1897 .

-- Zur Histologie der in der Nebenniere gelegenen Nebennierenteile. Diss., Jena, 1806 .

- Thymus. In L. O., i, p. $462,1896$.

RIEDER, K. Undurchlässigkeit der Froschbaut für Adrenalin. A. P. P., 60,1909 . RIEDL, F. Errfolgreiche Anwendung gefässerweiternder Einspritzungen.

RiEger. Die Kastration in rechtl., sozial. und vitaler Hinsicht. Jena, Igoo. RIEHL, G. Zur Pathologie des Morbus Addisonii. Z. k. M., Io, p. 521, 1886. - Mycosis fungoides und Morbus Addisonii. Ibid., No. 5, 1895.

RIEläNDER. Das Paroophoron. Diss., Marburg, IgOt.

Riess, L. Die Addisonsche Krankheit. D. K., iii, pp. 227-260, 1903.

RINGER, A., and PHEAR, A. G. A case of Addison's disease treated with suprarenal extract. Transact. of the Clin. Soc. Lond., 29, 1806.

RISEr. Spasmophilie und Kalzium. Arch. f. Kinderheilkunde, xviii, Nos. 3 and 4.

RITCHIE. The specificity and potency of adrenalytic and thymolytic sera. Journ. of Pathol. and Bact., i2, p. 140, 1908.

Ritter, C. Die Einwirkung des Ädrenalins auf die Lymphgefässe. M. K., 1906 .

RitzmaNN, H. C̈ber den Mechanismus der Adrenalinglykosurie. M. m. W., 1909 ; A. P. P., 6r, p. 231, 1909.

RIVA-ROCCI. Le sostanze ipertens. del secrete renale. Gazz. med. Torino, Nos. $19-23,1898$.

Rivas Mateos, Calleji, M. and C., Folch, R. Contribución al estudio de la estructura de las cápsules suprarenals. Bolet. d. soc. españ. d. hist. nat., iv, pp. 262-264, 1904.

Riviere, C. Hæmorrhage of adrenals. Pathol. Transact., London, vol. 53, 1902.

ROAF and NiERENSTEIN. The physiol. action of the extract of the bypobronchial gland. of purpura labillus. J. o. P., 36, p. 5, 1907.

-. Adrénaline et purpurine. (Reply to M. R. Dubois.) C. r. S. B., 63, p. 773,1907 .

RoberTSON. The parathyroid glands. Scot. Med. and Surg. Journ., 1897.

RODE, F. Das Adrenalin in der Rhino-Laryngologie. W. k. R., rgoz.

RÖMER, P. Spezifische Organtherapie des beginnenden Altersstars. D. m. W., No. 7, I909.

RÖsIER, R. Adrenaline als hæmostaticum. Tijdschr. v. Geneesk., 44, V.-H. i, 1904 .

RÖRIG, A. Über die Wirkung der Kastration von Cervus (cariacus) mexicanus auf die Schädelbildung. A. E.-M., viii, 633 , IS99.

-Welche Beziehungen bestehen zwischen den Reproduktionsorganen der Cerviden und der Geweihbildung derselben. Ibid., viii, p. 382,1899 .

-_ Über Geweihbildung und Geweihentwicklung. Ibid., x, pp. 525, 6r8; xi, $65,225,1000-1901$.

RöSSLE. Über Hypertrophie und Organkorrelation. M. m. W., 1907.

RÖSSLE, R. Die Leber beim Diabetes. V. d. p. G., II, 1907.

- Beiträge zur Pathologie der Nebennieren. M. m. W., No. 26, г9ı.

ROGER, H. Lésions des caps. surrénale dans l'infection pneumo-bacillaire. C. r. S. B., p. 52, 1894 . 
ROger, H. Physiologie norm. et path. du foie. Encycl. scient. Paris.

ROGER and GARNIER. Des lésions d. 1. glande thyr. dans l'intox. phosphorée. C. r. S. B., 1900 .

Roger, H., and' JosuÉ, O. Action du foie sur les extraits intestinaux. Ibid., 60, p. 580 .

- Action de l'extrait d'intestin. sur la pression artérielle. Ibid., 6o, p. 37 I.

Rogowitsch. Zur Physiologie der Schilddrüse. C. m. W., No. 36, i886.

- Sur les effets de l'ablation du corps thyroïde chez les animaux. A. d. P., is88.

- Veränderungen der Hypophysis nach Entfernung der Schilddrüse. Ziegl. Beitr., 4, 1889 .

RokITANSKY. Handbuch der path. Anat., I861.

Rolleston, H. D. Note on the anatomy of the suprarenal bodies. J. o. A. a. P., 26, 1892 .

- The Goulstonian lectures on the suprarenal bodies. Brit. Med. Journ., pp. $629,745,687$.

-- Some problems in connection with the suprarenals. Lancet, 1907.

ROLLESTON and MARKS. Primary malignant disease of the suprarenals. A. J. M. S., 1898 .

Rolly. Über die Neubildung von Glykogen bei glykogenfreien und auf Karenz gesetzten Kaninchen. D. A. k. M., 83, 1-2.

Roloff. Ein Fall von Morbus Addisonii mit Atrophie der Nebennieren. Ziegl. Beitr., 9.

ROMBERG, v. Lehrbuch der Krankheiten des Herzens und der Blutgefässe. Stuttg., 1906.

Romiti, G. Atti della Soc. Tosc. di Sc. nat., vol. viii, I892.

RónA, D. Beiträge zur Frage der syphilitischen und tuberkulösen Degeneration der Nebennieren. Ung. med. Presse, 1899.

Roos, E. Über Schilddrüsentherapie und Jodothyrin. Habil.-Schrift, Leipzig, 1897 .

- Über die Wirkung des Jodothyrin. Z. ph. Ch., 22, 16, 1896.

- Unters. über d. Schilddrüse. Ibid., $28,1898$.

- Klinische Erfahrungen mit Jodothyrin. M. m. W., 1902.

Rose, E. Der Kropftod und die Radikalkur der Kröpfe. A. k. Ch., 22, 1878 .

RosenberG, A. Nebennierenextrakt in der Rhino-Laryngologie. B. k. W., 1902.

- Urticaria nach endonasaler Anwendung von Nebennierenextrakt. B. k. IV., 1903.

Rosenberg, S. 'Ub. d. Einfluss d. Pankreas auf d. Resorption d. Nahrung. P. A., 70 .

- Pankreas und Diabetes. B. C., i, p. 777, 1903.

- Zur Frage des Duodenaldiabetes. P. A., 121, p. 358, 1008.

-- Weitere Untersuchungen zur Frage des Duodenaldiabetes. Ibid., p. 209.

- Innere Sekretion. Pankreas und Glykolyse. Handb. d. Bioch., iii, I9o9.

Rosenfeld, G. Über Antithyreoidinserum. Allg. med. Zentralz., 1903.

- Über die Fettleibigkeit des Mannes und der Frau. M. K., 1907.

Rosenhaupt. Beitr. zur Klinik der Tumoren usw. B. k. W., Igo3.

RosenheIM, O. Choline in cerebro-spinal fluid. J. o. P., 35, p. 465, 1907.

Rosenheim, O., and TeBB, CHRISTINe. On a new physical phenomenon observed in connection with the optical activity of so-called "protagon." Ibid., 37, pp. 348-354.

- On the lipoids of the adrenals. Ibid., 38 , I900.

- The lipoids of the brain. Ibid., 38, I909.

— On the so-called "protagon." Quart. Journ. of Exp. Phys., i, I9o8.

Rosenstein, E. Beiträge zur Pathologie der Nebennieren. Arb. aus d. path.-anat. Inst. in Posen. Herausg. v. Lubarsch, 1901-1902.

Rosenstirn. Die Harnbestandteile bei Morbus Addisonii. V. A., 56, pp. $27-37,1872$

RosItsky, v. Über den Jodgehalt von Schilddrüsen in Steiermark. W. k. W., 1897.

Rossa, E. Über akzessorisches Nebennierengewebe im Ligamentum latum und seine Beziehungen zu den Cysten und Tumoren des Ligaments. A. G., 56, 1898 .

Rossi, G. 'Di alcune proprietà microchimiche delle isole di Langerhans. Il Monit. Zool. Italiana. Firenzi, 205. 
Rossi, G. Sullo sviluppo dell' ipofisi, etc. Accad. med. Perugia, I899; and Lo Sperim., Igoo.

- Sulla struttura della ipofisi e sulla esistenza di una ghiandola infundibulaire nei mammiferi. Monit. Zool., 15, 1904.

ROSSMEISL, JOSEF. Untersuchungen über die Milch kastrierter Kühe. B. Z., 21, p. 164.

ROUFFART and DELPORT. Etude d'une série de malformations congénitalés des organes génitaux de la femme. Ann. de la Soc. belge de chir., 2-3, 1910.

Rотн, P. Ein Fall von Morbus Addisonii. Diss., Würzburg, 1888.

ROTHBERGER, I., and WINTERBERG, H. Über Vergiftungserscheinungen bei Hunden mit Eckscher Fistel. Z. e. P., i, 1905.

Roud, A. Contribution à l'étude du développement de la capsule surrénale de la souris. Bull. de la soc. vaudoise des sciences naturelles, 38, Lausanne, 1903.

Rovqués. Substance thermogènes extraites des tissus animaux sains et fièvres par auto-intoxication. Thèse de Paris, 1893.

ROUTIER. Annales des maladies des organes génito-urinaires, xix, igor.

Roux, J. Sclérodermie et corps pituitaire. Rév. neurol., pp. 72 I-723, 1902.

Rouxeau. Note sur 65 opérations de thyroïdectomie chez le lapin. C. $r$. S. B., p. 638, 1895 .

- De l'influence de l'ablation du corps thyroïde sur le développement en poids des glandes parathyroïdiennes. İbid., 1896 .

- Résultats d. l'exstirpation isolées d. glandules parathyroïdes. Ibid., p. 16,1897 .

- La bronchopneumonie consécutive à la thyroïdectomie chez le lapin. Ibid., 27 juillet, 1897 .

- Relation des cent-trois opérations de thyroïdectomie. A. d. P., p. 136, 1897.

RUBERT. Wirkung des Adrenalins auf den intraocularen Druck. Z. f. Augenh., 21, 1909.

Rubisato. Osserv. sul rapporto fra diabete ed isole di Langerhans. Soc. Med. Chir. di Bologna, 1908.

RUBENSTEIN. Über die Verhältnisse des Uterus nach Exstirpation beider Ovarien und nach ihrer Transplantation an eine Stelle der Bauchhöhle. Petersb. m. W., ז 899 .

RUbNer, M. Die Gesetze des'Energieverbrauchs. Leipzig, I902.

- Das Wachstumsproblem und die Lebensdauer des Menschen rom energetischen Standpunkte aus betrachtet. A. H., 66, p. I27, 1908.

RUCKERT, A. Über die Einwirkung von Oidium lactis und Vibrio cholerae auf Cholin-chlorid. Arch. de Pharmac., p. 676, 1908.

Rudinger, Carl. Zur Ätiologie und Pathogenese der Tetanie. Z. e. P., 5,1908 .

- Über Eiweissumsatz bei Morbus Basedowii. IV. k. WV., 1908

- Physiologie und Pathologie der Epithelkörperchen. Erg. d. inn. Med. u. Kind., ii, rgog.

RUdiNGER, C., and Jonas. Über d. Verhältnis d. Tetanic z. Dilatatio ventriculi. 1904 .

RUHRAEH, J. The relation of the thymus gland to marasmus. Lancet, 1903.

RUMMO and FERRANINI. Geroderma genitodistrofico. Rif. med., 1897.

RUSSEL, A. E. Cysts of the pineal body. Trans. Path. Soc. Lond., 5o, 1888 .

Ruyter, DE. Kongenitale Geschwulst d. Leber und der Nebennieren. A. k. Ch., 40,1890 .

RYNBERK, G. v. Sulla funzione endocrina del pancreas nei vertebrati e sugli elementi morfologici che partecipano ad essa. Arcl., di Fisiol., $4,497,1907$.

- A proposito di una rivista sintetica sugli elementi istologici che componio la funzione interna del pancreas. Arch. di Farm., vii, H. 3 .

- Ancora la secrezione interna del pancreas. Il Tomassi, Igog.

RZENTKOWSKI, V. Atheromatosis aortae bei Kaninchen nach intravenösen Adrenalininjektion. B. k. W., 1904.

SabolotNow, P. Zur Lehre von den Nierengeschwülsten suprarenalen Ursprungs. Ziegl. B., 4I, 1907.

SABRAZÉS, J.' and BONNES, J' Examen du sang dans l'acromégalie. C. r. S. B., 57, 1905. 
SABRAzÉS, J., and HuSNOT, P. Hypertrophie avec adénomes enkystes multiples des surrénales chez les vieillards. C.r. S. B., 61, p. 445, 1906.

... Tissu interstitiel, macrophages et cellules à l'engrais des capsules surrénales chez l'homme et les animaux. Gaz. hebd. de Bordeaux, 1907.

- Tissu interstitiel des glandes surrénales. Folia hæmatol., iv, r907.

- Névromes et fibromes des surrénales. A. m. e., 20, 1908.

SACAzE. Syphilis avec syndrome Addisonien. Gaz. des hôp., p. 58, 1895.

SACCHI. Di un caso di gigantismo infantile con Tumore del testicolo. Riv. sper. di freniatria, 21 , p. I 49, 1895.

SACHS, H. Die Cytotoxine des Blutserums. B. C., i, 1903.

SACERDOTE. A proposite d. Marassini : "Sopra una particolarità, etc." Riv d. Fisic. Sc. Nat. Pavia, 9, 1908.

Sacerdotti, C. Sui nervi della tiroide. Acc. d. Torino, 29, 1893.

- Über die Nerven der Schilddrüse. I. M., I I, 1894 .

SaINTON, P. Les troubles psychiques dans les altérations des glandes à sécrétion interne. L'Encéphale, Nos. 3 and 4, 1906.

- Pathogénie et traitement du goitre exophtalmique. IXe Congr. franç m., 1907 .

Sainton, P., and Fernet, Jean. Corps thyroïde et glande mammaire. Progr. méd., igos.

Sainton, P., and FERkand, J. L'adipose douloureux ou maladie de Dercum. Gaz. des hôp., p. 957, 1903.

SaInton, P., and PISANTE. Goitre exopht. traité par le sérum de mouton ethyroïdé. Revue Neurol., p. I109, 1904.

Sainton, P., and Rathéry, F. Myxœdème et tumeur de l'hypophyse. Soc. méd. hôp., 1908.

ST. RÉMY. Contribution a l'histologie de l'hypophyse. C. r. A., 1892; and A. B., 12, 1802 .

- Sur la signification morph. de la poche pharyngienne de Seessel. C. r. S. B., I 895 .

SaIz, G. Beitrag zum Vorkommen u. zur Behandlung der Tetanie. W. k. W., 1908 .

SAJOUs, DE. The internal secretions and the principles of medicine. I, Philadelphia, 1903.

- Physiological action and uses of adrenal extractives. Med. Rec. New York, 70, p. 800, 1906.

- Les sécrétions internes, l'appareil hypophyséo-surrénal, son rôle à l'état normal et à l'état pathologique. IXe Congr. franç. de méd. Paris, 1907 ; Gaz. des. hôp., 1908.

Sakorraphos, M. Examen du sang dans l'acromégalie. C. r. S. B., 57, p. 831,1905 .

SALECKER. Verwendung v. Nebennierensubstanzen z. örtlichen Analgesierung. D. militärärztliche Z., p. 1904 .

Salmon. L'hypophyse et la pathogénie de la maladie de Basedow. Rev. de méd., 1905.

- Sur l'origine du sommeil. Ibid., 26, p. 368, 1906.

SALOMON. Gaswechsel bei Morbus Basedow u. Akromegalie. L. O., 5, I900; B. k. W., I904.

SALTYKow. Atherosklerose bei Kaninchen nach wiederholten Staphylokkeninjektionen. Zieglers Beiträge, 43, p. 147, 1908

- Über experimentelle Arteriosklerose. V. d. D. p. G., 1908; C. a. P., I9, 1908.

- Experimentelle Forschung in der Lehre der Arteriosklerose. C. f. Stoff w., iii, rgo8.

Salvioli. Du mode d'agir de l'extrait des capsules surrénales sur le tissu musculaire lisse. A. i. B., 37 .

Salviol. and Carraro. Sulla fisiologia dell' ipfisio. Arch. scienze med., 31, I907; and A. i. B., 49, 1908.

SAlvioli and Pezzolini. Sur les différents modes d'agir des extraits médullaire et cortical des capsules surrénales. A. i. B., 37, p. 380 .

- Contr. à l'étude de la fonction des capsules surrénales. Ibid., 37, p. 390.

SAlZER, H. Zur Entwicklung d. hypophyse bei Säugern. A. m. A., 5 r, I 898 .

- Zur Frage der Schilddruisentransplantation. A. k. Ch., $\delta_{9}$; W. k. W., 1909.

SAMBERGER, F. Über die Wirkung wiederholter Injectionen von Nebennierenextrakt. IV. k. Rundschau, p. 577, 1902. 
SAMUEL. Über Gewebssafttherapie und innere Sekretion. D. m. W., I9o9. Sanctis DE, S. Gli infantilisme. Riv. Sperim. di Fren., 31, 1905.

S.ANDES. The corpus luteum of Dasyurus viverrinus. Zit nach Sobotta.

Sandmeyer. Die Folgen der partiellen Pankreasexstirpation beim Hunde Z. B., 31, 1894 .

S.INDSTRÖM, J. Über eine neue Drüse beim Menschen und bei verschiedenen Säugetieren. Ref. in Hofmann-Schwalbes Jahresb., ix, I Abt.; Schmidts Jahrb., i 880 .

S.ANo, T. Über die Entgiftung v. Strychnin u. Kokain d. d. Rückenmark. P. A., $120,1907$.

S.ANQuirico. Sur l'exstirpation du corps thyroide. A. i. B., 9, 1888.

SANQUERICO and ORECCHIA. Accad. d. Fisiol. d. Siena, 6, 1887 and 1893.

SANSOM. Addison's disease treated by suprarenal extract. Brit. Med. Journ., November 16, 1895 .

SANTI RINDONE LO RE. Sulla estirpazione delle capsule surrenali. Rif. med., I 895 .

SANTIDE. Parathyroïdgeschwulst. Laryng. Ges., London, 1899; Int. Cbl. f. Laryng., 1900 .

Sartirana. Neuer Beitrag zur Kenntnis der zytotoxischen Sera. C. f. B., $36,37,1904$.

Sata. Über die Wirkung und die Spezifität der Zytotoxine im Organismus. Zieglers Beitr., 36 and 39, 1906.

SATRE, A. Insuffisance hypophysaire traité avec succès par l'opothérapie. Dauphiné médical, 1907.

Sauerbeck, E. Die Langerhansschen Inseln des Pankreas und ihre Beziehung zum Diabetes mellitus. V. A., 177, Suppl. u. L. O., 8, p. 473, 1902.

- Zur Frage des Pankreaszytolysins. Kritische Bemerkung. C. f. B., 34,1905 .

- Neue Exper. z. Frage n. d. Bedeutung d. Langerhansschen Inseln. V. d. p. G.; 1904 .

Sauré, L. Physiologie du Pancréas. Arch. gén. de méd., p. 25, 1908.

SAVAGNONE, E. Contr. alla conosc. della fine struttura dell' ipofisio. Riv. ital. di Neuropath., In Patalog., i, igog.

SAvarI, M. Üb. d. giftigen Bestandteil des Harns bei Eklampsie. H. B.,

II, 1907. Untersuchungen über den feineren Bau des Pankreas.
SAviotTI, G. A. m. A., 5, 1869 .

SAWELIEW, S. Uber die Wirkung der Adrenalininjektionen auf das Blut und über Adrenalinimmunität. Diss., St. Petersburg.

SCAFFIDI, $V$. Feinerer Bau und Funktion der Hypophysis des Menschen. A. m. A., 64,1904 .

SCIALLERo. L'organoterapia testiculare et l'orchipina. Rif. med., Jg. 21, No. 5 .

SCHABAD. Über Diabetes pancreaticus. Diss., Moskau, 1895. Ref. V. H., ii, 1895 .

SCHADE, $H$. Diabetes und Katalyse. M. m. W., 1907.

SCHAEFER, E. A. On internal secretion. Brit. Med. Asso. Lancet, ii, p. 320,1895 ; Brit. Med. Journ., ii, 1895 .

- On certain practical applications of extract of suprarenal medulla. Brit. Med. Journ., I901, April 27, I901.

- Schäfer's Text-book of Physiology, i, 1898; ii, 1900.

- Les vaisseaux coronaires ont-ils des nerfs vasomoteur? A. d. sc. biol., II, Suppl. Festschrift für Pawlow, p. 251, 1904.

- On the present condition of our knowledge regarding the functions of the suprarenal capsules. Brit. Med. Journ.; Lancet, i, 1908.

- Functions of the pituitary body. P. R. S., 81, p. 550, October, 1909.

SCHAEFER and HERRING. The action of pituitary extracts upon the kidney. P. R. S., 77, p. 571, 1906; Phil. Trans. of the Royal Soc. of London, ser. B, 199, $190 \%$.

SCHAEFER and MOORE. On the contractility and innervation of the spleen. J. o. P., 20, 1906.

Schaefer and Scharlieb. Chloroform poisoning. Trans. Roy. Soc. of Edinb., 4I, 1904.

SCHAEFER and VINCENT. On the action of the pituitary extract injected intravenously. J. o. P., 24, xix, 1899; and ibid., 25, p. 87, 1899 . 
SCHAEFFER, G. La physiologie de l'appareil thyro-parathyroidien d'après les travaux récents. Biol. méd., ann. 4, pp. I-34.

SCHÄFFLE, KARL. Notes on family goitre. J. A. M. A., lii, p. 212. January 16,1909 .

SCHAFFER, J. Über das Vorkommen eosophiner Zellen in der menschlichen Thymus. C. m. W., 1891 .

- Über den feineren Bau der.Thymus. S. W. A., 102, 1893.

SCHAFFER, J., and RABL, H. Das thyreothymische' System des Maulwurfs und der Spitzmaus. I. u. II. Th., S. W. A., 118, I909.

Schaper, A. Beiträge zur Histologie der Glandula carotica. A. m. A., 40, pp. $287-319,1892$.

- Über die sogenannten Epithelkörperchen. A. m. A., 46, I895.

- Einige Bemerkungen über das Wesen und die morphologische Stellung der Glandula coccygea (Glomus coccygeum.). An. An., 25, 1904.

SChatilofF, P. Die Nierensekretion im Lichte der Adrenalinwirkung. A. A. P., 1908.

SCHEEL, OLAF. Über Nebennieren. Sekretkörnchen-Ödem-Gewicht. V. A., 192, 1908.

- Erwiderung auf E. O. Hultgrens Bemerkungen. V. A., 194, p. 566.

SCHEIDEMANDEL, E. Über die durch Adrenalininjektionen zu erzeugenden Aortenverkalkungen der Kanninchen. V. A., 181, p. 363, 1905:

Scuenk, F. Giftwirkung des menschlichen Plazentasaftes bei Kaninchen. C. G., 1909 .

ScheUlt, R. A case of Addison's disease in a negress. Lancet, ii, 1907.

SCHIEFFERDECKER, P. Über das Verhalten des Bindegewebes bei der Hypertrophie und Atrophie der Muskeln und über die Schlüsse, welche man aus diesem Verhalten über die Symbiose verschiedener Körpergewebe machen kann. S. d. niederrhein. Ges. f. Natur- u. Heilk., pp. 75-9r, 26 Oktober, 1903.

- Nerven- und Muskelfibrillen, das Neuron und der Zusammenhang der Neuronen. Ibid., pp. 85-93, Bonn, rgo4.

- Über die Neuronen und die innere Sekretion. Ibid., pp. 46-54, 23 Oktober, 1905.

- Uber Symbiose. Ibid., pp. 44-54, 13 Juni, I904.

- Indikationen und Kontraindikationen des Radfahrens. Leipzig, 1901.

- Neuronen und Neuronenbahnen. Leipzig, Barth, 1906.

SCHIEFFERDECKER and SCHULTZE. Beitrag zur Kenntnis der Myotonia congenita, der Tetanie mit myotonischen Symptomen, der Paralyse agitans und einiger anderer Muskelkrankheiten, zur Kenntnis der aktiven Hypertrophie und des normalen Muskelbaues. D. Z. N., 25, $27,345,1903$.

SCHIF, A. Beeinflussung des Stoffwechsels durch Hypophysis- und Thyreoideapräparate. Z. k. M., 32, Suppl., pp. 284-290, 1897; W. k. W., 1897 .

SCHIF, M. Unters. iuber d. Zuckerbildung in d. Leber. Würzburg, 1859. Zur Physiologie des Pankreas. A. f. Heilk., 27I, 1862.

- Sopra l'estirpazione delle capsule soprarenali. L'imparziale, pp. 234237, 1863; Union médicale, p. 347, 1863.

- Bericht über eine Versuchsreihe betr. die Wirkungen d. Exstirpation der Schilddrüse. A. P. P., 18, 1884; and Rev. méd. de la Suisse Rom., Nos. 2 and 8,1884 .

- Gesam. Beitr. z. Physiologie. 3 Bände. Lausanne, 1894.

SCHILLING, F. Morbus Addisonii und Organotherapie. M. m. W., 1897.

SCHIPEROWITSCH, W. Zur Ätiologie und Therapie des Morbus Addisonii. (Russisch.) Wochenschr. prakt. Med., No. 13, 1895 .

SCHIROKOGOROFF. Die skleroitische Erkrankung der Arterien nach Adrenalininjektionen. V. A., I91, p. 482, 1908.

- Sur action phagocytaire des capsules surrénales. C. r. S. B., 65, p. 300. 17 octobre, 1908 ; Wratsch. Gaz., No. 44, 1908; Fol. haem, iii.

SCHLAGENHAUFER and v. WAGNER. Beiträge zur Ätiologie und zur Pathologie des endemischen Krentinismus. Leipzig-Wien, 1910.

SchlaYer. Zur Frage der drucksteigernden Substanzen im Blute bei chronischer Nephritis. D. m. W., 1907; and M. w. M., 1908.

Schlayer and Hedinger. Exper. Studien über toxische Nephritis. D. A. k. M., 90, 1907 .

Schlecht, H. Einfache Methode zur Prüfung der Pankreasfunktion. M. m. W., 1908. 
SCHLESINGER, H. Therapeutische Erfahrungen bei Behandlung schwerer innerer Blutungen mit Nebennierenextrakt. W. k. W., 1904 .

SCHLESINGER, IV. Über den Ursprung des diastatischen Fermentes im Blute und über Beziehungen zum Diabetes mellitus. D. m. W., 1908 .

SCHLOFFER. Erfolgreiche Operation eines Hypophysentumors auf nasalem Wege. W. k. IV., p. 621, 1907 .

- Berichtigung zu dem Artikel "Erfolgreiche Operation." W. k. W., No. 22, 1907.

- Weiterer Bericht über den Fall von operiertem Hypophysentumor. W. k. WV., 1907 .

- Zur Frage der Operation der Hypophysis. Bruns. Beitr. z. k. Ch., 50. Schmaltz. Zur Kasuistik des Morbus Addisonii. D. m. W., i89o.

Schmid, E. Der Sekretionsvorgang in der Schilddrüse. A. m. A., 47, I8g6.

SCHMID, J. Beeinflussung von Druck und Stromvolumen in der Pfortader durch die Atmung und durch experimentelle Eingriffe. P. A., 126, I909.

Schmid, E. Beziehung der Langerhansschen Insel des Pankreas zum Diabetes mellitus. M. m. IV., I 902 .

- Über Cholin, Neurin and verwandte Verbindungen. Lieb. Ann., 337.

Schmid, M. W. Über Pseudo-Hermaphroditismus bei Rana temp. A. m. A., 72,1908 .

Schmieden. Ërfolgreiche Einheilung exstirpierter Nebennieren b. Kaninchen. P. A., 90, 1902.

- Erfolgreiche experimentelle Verlagerung von Nebennierengewebe. Z. Ch., 70, 1903.

SCHMiERGELD, A. Lésions des glandes à sécrétion interne dans deux cas d'alcoolisme chronique. A. m. e., 21, 1909.

- Les glandes à sécrétion interne dans la paralyse générale. L'Encephale,

SCHMORL, G. Patholog.-anat. Untersuchungen über puerperale Eklampsie. Leipzig, 1893 .

-.- Zur Kenntnis der akzessorischen Nebennieren. Zieglers Beitr., 9, I89ı.

Schxaudigl, O. Das Suprareninum. Opht. Kl., No. 13, 1903.

SCHNEIDEMÜHL. Vergl. anat. Unters üb. d. Bau der Cowperschen Drüsen. D. Z. f. Tierm. u. vergl. Path., vi.

SchNiTZLER and EWALD. Uber das Vorkommen von Thyreoidin in der Hypophyse. IV. k. W., 1896.

Schön, N. Zwei Fälle von Ińfantilismus. Moskau, 1898; Ref. N. C., 1899.

SCHÖNBORN, S. Zur Wirkung der Thyreoideastoffe. A. P. P., 60, 1909.

SchÖNDORFF, B. Über den Einfluss der Schilddrüse auf den Stoffwechsel. P. A., 67, 395, 1897 .

- Ausscheidung von Zucker im Harn von gesunden Menschen. Ibid., I2I, 1908 .

SchöNE. Transplantation von Geschwïlsten und normalen Geweben. B. z. k. Ch., 6i.

Schönemain. Hypophysis und Thyreoidea. V. A., 129, 1892.

Scholz, W. Untersuchungen uber den Kretinismus. Zusammenf. Arbeit, Berlin, I 906.

- Uber den Stoffwechsel der Kretinen. Z. e. P., 2, 1905.

-- Schilddrüsenbehandlung und Stoffwechsel bei Morbus Basedowii. C. i. M., 1895 .

Scholz, W., and ZINGERLe, H. Beitr. z. path. Anatomie der Kretinengehirne. Z. H., 1906.

ScriraNk, F. Experimentelle Beiträge zur Wirliung der Jodpräparate auf die Adrenalin-Arterionekrose. Z. k. M., 64, p. 471, 1908.

- Uber die Wirkung des. Spermins bei Adrenalin-Arterionekrose. Ibid., 64,1907 .

-.- Zur antagon. Wirkung des Adrenalins und Chlorkalciums. Ibid., 67, I909.

SchraUbE, K. Die Beziehungen der Thymusdrüse zum Morbus Basedowii. Diss., München, I9o8.

Schreiber, L. Beiträge zur Kenntnis der Entwicklung und des Baues der Glandulae parathyreoideae (Epithelkörperchen des Menschen). A. m. A., 52, 1908 .

Schryver, S. D. Autolysis, the influence of the thyroid. J. o. P., 32, p. 159 .

Schubiger-Hartmann, F. Adrenalin. Schw. Korr.-Bl., 1902. 
ScHÜCKING. Hautverfärbung nach Injektion von Nebennierenextrakt. M. m. WV., 1903 .

Schüller, A. K̇eimdrüsen u. Nervensystem. Arb. a. d. Neurolog. Inst. WVien, 1907.

- Über Infantilismus. W. m. IV., 1907.

- Die Schädelbasis im Röntgenbild. Fortschr. a. d. Geb. d. Röntgenstr. Ergänzungsbd. II.

SCHÜLLER. Antithyreoidinserum Moebus bei 5 Fällen von Morbus Basedowii. D. m. Z., I 905 .

SchützE, A. Beiträge zur Kenntnis der zellenlösenden Sera. D. m. W., 1900.

- Antinebennierenserum. Z. k. M., 12, 1908.

Schultz, P., and Zuelzer, G. Totalexstirpation des Pankreas beim Hunde. C. P., I9.

Schultz, î. H. Der Einfluss der Instillation von Adrenalin in Augen von Säugetieren. C. f. P., 22, p. 713, 1908 .

Schultze, F. Über Melanoplakie der Mundschleimbaut und die Diagnose auf Morbus Addisonii. D. m. W., I 898 .

Schultze, O. Zur Frage von den geschlechsbildenden Ursachen. A. m. A., $63, p_{1} 97,1904$.

Schultze, WV. Die Bedeutung der Langerhansschen Inseln im Pankreas. Ibid., 56, $49 \mathrm{I}$.

- Fall von Ákromelagie. D. m. W., 1904.

Schulz, O. Über die Lebenswiclitigkeit der Schilddrüse und des Schilddrüsenapparates. D. m. W., 1900.

-- Neure und neueste Schilddrüsenforschung. Festschr. f. J. Rosental. Leipzig, I906.

Schulz, R. Tumor der Zirbeldrüse. N. C., iv, 1886.

Schumacher, S. v. Über das Glomus coccygeum und die Glomeruli caudales der Säugetiere. A. m. A., 71, pp. 58-115, 1907.

ScHur, H., and WIESEL, J. Über eine der Adrenalinwirkung analoge Wirkung des Blutserums von Nephritikern auf das Froschauge. W. k. W., Nos. 23 and 27, 1907.

- Beiträge zur Physiologie und Pathologie des chromaffinen Gewebes. V. d. p. G. Dresden, II ; and W. k. W., No. 40, 1907.

- Uber das Verhalten des chromaffinen Gewebes bei der Narkose. IV. k. W., I908.

- Zur Frage d. drucksteigernden Subst. im Blute bei chron. Nephritis. D. m. W., 1907 .

Schivarz. Alcune considerazioni a proposito delle consequenze della completa tireoidectomia. Lo Sperimentale, 1891 .

- Tumeur de la capsule surrénale droite; néprectomie. Bull. et Mém. de la Soc. Chir., p. $764,1893$.

SCHWARz, C. Beitrag zur Wirkung des Cholins auf die Pankreassekretion. C. P., 23 , No. 16,1909 .

Schwarz, C., and Lederer, R. Über das Vorkommen von Cholin in der Thymus, in der Milz und in den Lymphdrüsen. P. A., 124, 1908.

SCHWARz, O. Über einige Anfallserscheinungen nach Exstirpation beider Nebennieren. W. k. W., 1909.

SCHWARZWALD, R. T. Über das Verhalten des chromaffinen Gewebes beim Menschen unter dem Einfluss der Narkose. V. 13, p. G., p. 268, 1909.

Schioner. Über hereditäre Akromegalie. Z. k. M., 32, Suppl.-Heft.

SCHWyzer, F. Zur Ätiologie des Morbus Addisonii. New York m. M., $\mathbf{x}, 1898$.

Scramasna, C. Patologia et Terapia del Gran simpatico. Milano, 1894.

ScotT-MACFIE, J. W. Action of tissue extracts on protoplasm. J. o. P., 30,1905 .

SCOTT-WARTHIN, A. Accessory adrenal body in the broad ligament (adrenal of Marchand). Amer. Journ. of Obstet., vol. 42, 1900.

Sebsistiani, V. Contributo alla studio del potere tossico ed emolitico del sieri di sangue nei basedowiani e nuove vedute a proposito della sieroterapie di questi ultimi. Riv. crit. di clin. med., ix, p. 22.

SEDGWICK, A. Development of the kidney in relation with the Wolffian body in the chick. Quart. Journ. Micr. Soc., 22, pp. 146 and 372, 1880 .

SEEGEN. Der Diabetes mellitus. Berlin, I875; 3 Aufl., 1893.

- Die Zuckerbildung im Tierkörper. Berlin, 1900.

SeELIG. Beitrag zum Diabetes pancreaticus. B. k. W., 1892. 
SeEmanx, Chave. Über eine neue Methode der Transplantation des Schilddrüsengewebes. $Z$. Ch., 96,1908 .

SeEmanN, J. Die blutbildenden Organe. E. P., 3, I. Abt., p. 45.

SEESSEL. Zur Entwicklungsgeschichte des Vorderarms. A. A., 1877.

SEHRT, E. Zur Fermentwirkung des Mumienmuskels. B. k. W., 1904.

SEIDELL, A. The determination of iodine in thyroid. J. Am. Chem. Soc., 31 , p. 1326, 1900.

SEITZ. Die Follikelatresie während der Schwangerschaft, insbesondere die Hypertrophie und Hyperplasie der Theca-interna-Zellen (ThecaLuteinzellen) und ihre Beziehung zur Corpus luteum-Bildung. A. G., $77,203,1905$.

- Eklampsie und Parathyroidea. A. G., 89, 1909.

SELLEI, J. Tonogen in der Urologie. Orvos. Lapja, p. 895, 1904.

SELIGSOHN. De pigmentis pathologicis ac morbo Addisonnii, adjecta chemia glandularum suprarenalium. Diss., Berlin, 1858 ; and V. A., 18,

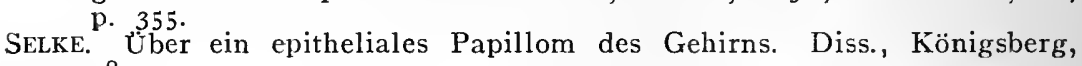
189 I.

SELherM, H. Zur Lehre von den sekundären Geschlechtscharakteren. B. G. G., i, 1898 .

- Kastration und Knochenwachstum. Ibid., ii, 1899.

- Kastration und sekundäre Geschlechtscharaktere. 'Tbid., v, p. 409, I90r.

SEMON, R. Die indifferente Anlage der Keimdruisen beim Hühnchen und ihre Differenzierung zum Hoden. Habilitationsschrift, Jena. I887.

- Uber die morphologische Bedeutung der Urniere in ihrem Verhältnisse zur Vorniere und Nebenniere und über ihre Verbindung mit dem Genitalsystem. A. A., I890.

-. Studien uiber den Bauplan des Urogenitalsystems; dargelegt an der Entwicklung dieses Organsystems bei Ichthyosis glutinosus. Jena, Zeitschr. f. Nat., 19, I891.

-- Das Exkretionssystem der Myxinoiden in seiner Bedeutung für die morphologische Auffassung des Urogenitalsystems der Wirbeltiere. Fests. Gegenbaur, 3, 1896 .

Semper, C. Das Urogenitalsystem der Plagiostomen und seine Bedeutung für das der übrigen Wirbeltiere. Arb. a. d. zool.-zoot. Inst. Würzburg, 2, pp. 195-509, 1875 .

- Stammesverwandtschaft d. Wirbeltiere u. d. Wirbellosen. Ibid., 2, 1875 .

SENATOR, H. Osteomalacie und Organtherapie. B. k. W., 1897.

- Untersuchung des Stoffwechsels bei einem mit. Nebennierensubstanz behandelten Falle von Addisonscher Krankheit. Charité-Ann., 22, p. 235,1897 .

SERGENT, E. L'insuffisance surrénale aigue. P. m., I902 and 1903.

SERGENT, E., and BERnard, L. Note pour servir à l'étude de la pathol. des caps. surrén. C. r. S. B., i 898 .

- Sur un syndrome clinique non Addisonien à évolution aigue, à l'insuffisance capsulaire. A gén. méd., nouv. sér., ii, I899.

- L'insuffisance surrénale. Encycl. scient. Paris, I902.

- Les Surrénalites chroniques d'origine infectieuse et l'insuffisance surrénale lente. A gén. de méd., i, No. 1, p. 14, 1904.

- Le Diagnostic du syndrome d'Insuffisance surrénale pure. Soc. d. hôpit., 1904.

SERralach and PAREs. Quelques données sur la physiologie de la prostate et du testicule. C. r. S. B., 63, p. 790, 1907.

SEzary. Les glandes surrénales des tuberculeux. C. r. S. B., 65, p. 603, 12 décembre, 1908.

- Structure métatypique de la corticale des surrénales. Ibid., 65, p. 430.

Shatтock, C. G. Ovarian Teratomata. Lancet, February 15, 1908.

- Cretinism in calves. J. o. P., 29, 1903.

Shattock, C. G., and SeligmanN, C. G. Some experiments made to test the action of extract of adrenal cortex. P. R. S., 8o, pp. 473-477, 1908 .

- Verschluss d. Vasa deferentia. Ibid., 72, Februar, I904.

- Example of incomplete glandular hermaphroditism in domestic fowl. Ibid., 1907.

SHAw, H. BATTY. Organotherapy. London, 1905.

- Autointoxication. Goulstonian Lect. Lancet, i, 1906. 
SHIMA, R. Über die Erweiterung der Pupille bei Adrenalineinträufelugen in ihrer Abhängigkeit vom Zentralnervensystem. P. A., 126 and 127, I909.

- Zur Frage der nach Adrenalinwirkung auftretenden Veränderungen des Zentralnervensystems. N. C., I908; and Arb. a. d. neurol. Inst. Wien, I908.

Shiota, H. Über das Schicksal und die Funktion der transplantierten Nebennieren. P. A., 128, 1909.

SHORT-RENDLE, A. Blood-pressure and pigmentation in Addison's disease. Lancet, 1006 .

SICARD. Surrénalite hémorrhagique. Sem. méd., 1904.

SICK, K. Flimmerepithelcysten in der Nebennierenkapsel. V. A., 172, 1903.

SIEGEL. Abkühlung als Krankheitsursache. D. m. W., I9o8; Z. e. P., 5, I908.

SIEGERT. Zur Pathologie der infantilen Myxidiotie, des sporadischen Kretinismus oder infantilen Myxödems der Autoren. Jahrb. f. Kinderh., I901.

Sikemeier, W. Adrenalin und Cocain-Adrenalinanästhesie. A. k. Ch., 78,

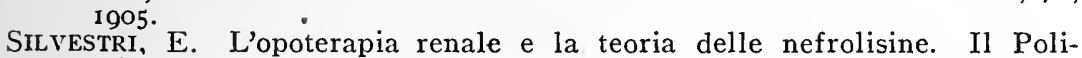
clinico, 9, 53, 1904 .

Silvestrini, E., and ToSatri, C. Degli effetti della capsulectomia in cavie e coniglie gravide. Soc. med. chir., Modena, 7 dicémbre, 1908; Pathologica, I909.

SilvestrinI, R. Sull' azione dell' estratto acquoso del lobo posteriori dell' ipofisi sulla pressione sanguinea e sul cuore. Riv. crit. di clin. med., No. 28, 1905.

Silvestrini, R., and BADUEL, A. Recherches pour préciser quelle est la partie. active du lobe postérieur de l'hypophyse. Nouv. Icon. de la Salp., 13, 1900.

SILVESTRINI, R., and DADDI, G. Ricerclie istologiche in un caso di morbo di Addison. Settimana med., lii, 1898 .

SImmoNDS, M. Über kompensatorische Hypertrophie der Nebenniere. V. A., I 53, 1898 ; and M. m. W., 1898 .

- Uber Nebennierenblutungen. V. A., I70, pp. 242-256, I902.

-.. Über Nebennierenschrumpfungen bei Morbus Addisonii. Ibid., 172, 1903.

-- Weitere Beobacht. üb. kompensator. Hypertrophie der Nebenniere. C. a. P., 13, 1902 .

- Über Bronzediabetes und Pigmentzirrhose. B. k. W., 1909.

- Über die Einwirkung von Röntgenstrahlen auf die Hoden. Fortschr. a. d. G. d. Röntgenstr., 14, 1909-10.

Simor, $\mathrm{CH}$. Thyroïde laterale et glandule thyroïde chez les mammifères. Thèse de Nancy, iso6.

Srmon, J. A physiological essay on the thymus gland. London, 1845.

SimoNowitsch. Contr. à l'étude de l'action et d'empoí d'adrénaline. Thèse de St. Pétersb., 1903: Ref. J. d. P. P., 6, p. 564, 1904 .

Singer, H. Die Nebennieren und ihr wirksames Prinzip. Th. M., 1902.

SINIBALDI. Arch. d'anat. patholog. e sc. affin., fasc. ii, 1906.

SINN. Der Einfluss exper. Pankreasgangunterbind. auf d. Nahrungsresorption. Diss., Marburg, I907.

Sinnhuber, F. Beziehung der Thymus zum Kalkstoffwechsel. Z. k. M., $54,1.904$.

SIPPEL. Ein typisches Krankheitsbild von protrahiertem Chloroformtod. A. G., 88,1908 .

SKROBANSKY, K. Über Immunisierung von Tieren mit Ovarien einer anderen Tierspezies. Russ. Zeitschr. f. Geb. u. Gyn., Januar, 1904.

- Diskuss. zu Fraenkels Vortrag. C. G., I904; and M. m. WV., I903.

SLADE, J. G. The physiological action of muscle extract. J. o. P., 35, 1907.

Slatineano. Recherches sur le sérum thyréotoxique. C. r. S. B., 57, ii, p. 76,1905 .

Smallwood, W. M. Adrenal tumours in the kidney of the frog. An. An., 26,1905 .

SmirnofF, A. Struktur d. Nervenzellen im Sympathicus d. Amphib. A. m. A., 35, I 890 .

Sмiтн, T. A case of "Thymic Death." Lancet, Igo8. 
SoBotTa. Über die Bildung des Corpus luteum bei der Maus. A. m. A., 47. - Über die Entstehung d. Corpus luteum d. Säugetiere. An. Hefte, II, Igor.

Soct. Sur un cas de sommeil prolongé pendant sept mois par tumeur de 1'hypophyse. Nouv. Icon. de la Salpêtrière, I3, p. IOI, Igoo.

SoDDu, L. Intorno agli effetti della estirpazione delle capsule surrenali nel cane. Sperimentale, 52; Ref. C. i. M., 20, 1899 .

Soederlund, G., and BackmanN, A. Studien über die Thymusinvolution. Die Altersveränderungen der Thymusdrüse beim Kaninchen. A. m. A., 73, 1909 .

SoKolOFF, B. Über die Bauchspeicheldrüse in verschiedenen Phasen ihrer Tätigkeit. Diss., St. Petersburg, I 883 .

- Einfluss der Ovarialexstirpation auf Strukturveränderungen d. Uterus. A. G., $5 \mathrm{I}$.

SOLDI, T. Casi di osteomalacia guariti colla terapia adrenalinica. Pathologica, 1908 .

SOLGER. Nebenniere. In Zuelzers Handb., I894.

SOLI, U. Les testicules chez les animaux ayant subi l'ablation de thymus. P. m., 1907 .

- Comment se comportent les testicules chez les animaux privés de thymus. A. i. B., 47, 1907 .

- Influenza del timo sullo sviluppo scelettico. Soc. ital. di Patol. Modena, 1909.

Sollier and Chartier. L'opothérapie ovarienne et hypophysaires dans certains troubles mentaux. Congr. de Dijon, août, Igo8.

Sollmans, T. The comparative physiologic activity of some commercial suprarenal preparations. A.J. M. S., 47, pp. 792-793, 1906.

Sommer, A., and FlörCKEN, H. Über die Funktion der Thymus. Sitzungsber. d. phys. med. Ges. z. Würzburg, H. 3-4, 1908.

Souza-Leite. De l'acromégalie. Thèse de Paris, i8go.

Soulié, A. Sur les premiers stades du développement de la capsule surrénale chez quelques mammifères. C. r. de l'Assoc. des Anatomistes, IVe session. Montpellier, I902.

- Recherches sur le développement des capsules surrénales chez les vertébrés supérieurs. Journ. de l'anat. et phys. Thèse de Paris, I903.

- Sur le développement de la substance médullaire de la capsule surrénale chez quelques mammifères. C. r. de l'Assoc. des Anat., 5 sess., p. 63 , Liège, 1903 .

Soulít and VERDÚN, P. Sur les premiers stades du développ. de la thyrö̈de. C. r. S. B., I897; and J. A. P., 33, I897.

Soup.uult. Maladie de Basedow. Bull. de la Soc. d'Anat., p. 592, 1897.

SOUQUES and MOREL. Traitement de l'hémoptysie par l'adrénaline. Soc. des hôp., 1902 .

Sptllman, L., and Hoche, L. Maladie d'Addison à dénouement rapide. A. gén. de méd., ii, p. 2369, I903.

- Sirint, A. Experimentelle Untersuchungen über den Einfluss von Rückenmarksdurchtrennungen auf den Kreislauf des Gehirns. W. k. W., 1897 .

- Beitr. zur Lehre von der Erektion und Ejakulation. W. m. B1., I897.

Exp. Beitr. z. Kenntn. der Hyperämie des Gehirns. Ibid., i $\$ 98$.

Exp. Unters. uber die Bildung d. Liquor cerebrospinalis. P. A., 76 and 8o, $1899-1900$.

Spriseanu. Thèse de Boucarest, I899; zit. nch Vitzou.

SPITTA, $W$ Über Morphiumdiabetes. Z. e. P., 5, p. $94,1908$.

SPITZER. Die zuckerzerstörende Kraft des Blutes und der Gewebe. P. A., 6o, 1896 .

SRdinko, O. Über Bau und Entwicklung der Nebennieren des Frosches (böhmisch). Sitzungsb. d. böhm. Kaiser-Franz-Joseph-Akad. in Prag, 2 Kl., No. I2, 1898 .

- Beiträge zur Kenntnis ubber die Fntwicklung der Nebennieren bei den Amphibien (böhmisch). Ibid., No. 32, 1900.

- Bau und Entwicklung der Nebennieren bei Anuren. An. An., 18, pp. $500-508,1900$.

- Beiträge zur Kenntnis der Nebenniere der Knochenfische. Über Bau und Entwicklung der Stanniusschen Körperchen der Lopobranchier. A. m. A., 66, pp. $773-802,1903$. 
SRDixko, O. Beitrag zur vergleichenden Anatomie und Embryologie der Nebenniere, 1903 .

- Über die Blutzirkulation in der Nebenniere der Wirbeltiere. I-IV. Sitzungsb. der böhmischen Kaiser - Franz-Joseph-Akad. Prag (böhmisch), Nos. 12, 16, 28, 1905.

- Uber die Kreislaufbahnen in der Nebenniere des Menschen und deren Beziehungen zu gewissen Affektionen dieser Drüse. Ibid., 1905; Ref. Schmidts Jahrb., 29I, p. 33.

- Beitrag zur Sinusoidenfrage (böhmisch), 1905.

- Eine sichere Methode zur Differenzierung der Rinden- und Markelemente in dẹr Nebenniere, besonders bei Säugetieren und Menschen. An. An., 26 , 1905 .

- Uber d. Entwicklung d. Nebenniere bei d. Lophobronchien (böhmisch). 1907.

SSAWELJEW. Über den Einfluss der subkutanen Adrenalininjektion auf das Blut und über Adrenalinimmunität. Ref. B. C., iii, No. 326, 1904.

Ssobolew, L. WV. Zur normalen und pathologischen Morphologie der inneren Sekretion der Bauchspeicheldrüse. V. A., 168; Diss, St. Petersburg, 1901.

- Über die Struktur der Bauchspeicheldrüse. C. a. P., 16, 1902.

- Über die Struma der Langerhansschen Inseln. V. A., 177, Suppl., I904.

- Zur Lehre über die Entwicklung von Paraphysis und Epiphysis bei den Schlangen. A. m. A., 70 , p. $318,1907$.

Stabel, $H$. Vers. $m$. Thryrojodin u. Thyraden an thyreoidektom. Hunden. B. k. W., 1897 .

StaChini. Rivista sperimentale di Freniatria, 29; and Rev. Neur., 1903.

STADELMANN. Über das Vorkommen von Gallensäuren, Hippursäure und Benzoesäure in den Nebennieren. Z. p. Ch., 18, p. 380 , I 893 .

- Zur Lehre von der Akromegalie. Z. k. M., 55 , 1904.

Stamatiade, A. Beiträge zum Studium der Läsionen der Nebennieren bei verschiedenen Krankheiten. Diss., Bukarest, 1907; Ref. C. i. M., p. $764,1907$.

Stangl, E. Zur Histologie des Pankreas. W. k. W., I90I; V. 6 d. p. G., 1902 .

- Tumor der chromaffinen Nebenorgane d. Symphaticus (Zuckerandl). W. k. W., 1902 .

- Zur Pathologie der Nebenorgane des Symphaticus. V. G. d. N. Ä. Karlsbad, I 1902.

StaRgart. Nekrosen nach Suprarenininjektionen. Klin. Monatsbl. f. Augenh., 1906.

STARLING, E. H. On the mode of action of lymphagogues. J. o. P., 17, 1894 .

- On the physiological factors involved in causation of dropsy. Lancet, I 894 .

- The chemical correlation of the functions of the body. Lancet, August, 1905.

- Die chemische Koordination der Körpertätigkeiten. V. d. N. Ä., Stuttgart, 1906.

- Physiology of digestion. London, 1006.

STARLING and LaNe ClayPON. Hormon der Brustdrüse. P. R. S., 77, 1905.

State. La forme douloureuse de l'acromégalie. Thèse de Paris, Igoo.

STATKEWITSCH. Über Veränderungen des Muskel- und Drüsengewebes sowie der Herzganglien beim Hungern. A. P. P., 33, 1894.

Stauber, A. Über das embryonale Auftreten diastatischer Fermente. P. A., I14, p. 619 .

STÄUbl. " Zur Pathologie und Therapie des Diabetes mellitus. D. A. k. M., 03,1908 .

STECKSÉN, A. Befund von Adenomknötchen an Nebennieren und von akzessorischen Nebennieren bei Erwachsenen. Arb. a. d. path. Inst., Tübingen, iii, I901.

Stefani, A. Azione del vago sugli scambi e sulla temperatura interna. A. d. F., 1908 .

STEFFECK. Menstruation und Ovulation. Jahresb. f. Geb. u. Gyn., 9, 1005.

StegmanN. Die Behandlung der Struma mit Röntgenstrahlen. M. m. WV., 1905.

Stein. Zur Kenntnis des Morbus Basedowii. Ibid., 1905. 
Steinach, E. Z. vergl. Physiol. d. männl. Geschlechtsorgane. P. A., 56, I 894.

Steinach, E., and Kann, R. Wr. Echte Kontraktilität und motorische Innervation der Blutkapillaren. P. A., 97, p. 105, I903.

Steinlein, M. Über den Einfluss d. Schilddrüsenverlustes auf die Heilung " von Knochenbrüchen. A. k. Ch., 6o, I896.

STEINSCHNEIDER. Adrenalin bei Gebärmutterblutungen. M. m. W.. 1905.

STÉPANOFF. Le corps thyroïde et les défenses naturelles. C. r. S. B., 66, I909.

STERN. Innere Sekretion der Niere. Trav. de labor. de phys. de Genève, I $901-1902$.

Sternberg. Akromegalie. Nothnagels Handb., 7, i 897 .

STERRI. Intorno alla struttura dell' ipofisi nei vertebrati. Padova, 1904.

STIEDA. Untersuchungen über die Entwicklung der Gl. thymus, Thyreoidea u. Carotica. Leipzig, I881.

- Über das Verhalten der Hypophyse des Kaninchens nach Entfernung der Schilddrüse. Zieglers Beitr., 7, 1890 .

Stilling, H. Zur Anatomie der Nébennieren. V. A., Iog, pp. 324-346, 1887.

- Über die kompensatorische Hypertrophie der Nebennieren V. A., I I8, 1889 .

- Note sur l'hypertrophie compensatrice des capsules surrénales. Rev. méd., T. 9, pp. 459-461, 1888 .

- Quelques expériences nouvelles sur la maladie d'Addison. Ibid., Io, I 890 .

- Du ganglion intercarotidien. Recueil inaugural. Lausanne, 1892.

- Zur Anatomie der Nebenniere. II. Mitt. A. m. A., 52, pp. I76-195, i8gs.

- Die cliromophilen Zellen und Körperchen des Symphaticus. An. An., 53,1899 .

- Einige Fragen als Antwort auf die Erwiderung von A. Kohn. A. m. A., 53, I 899 .

- Die Entwicklung transplantierter Gewebsteile. V. d. p. G., Igo3.

- Über das Ergebnis der Transplantation von Nebennierengeweben. Zieglers Beitr., 37, pp. 480-484, 1905 .

- Versuche über Transplantation. III. Mitt. Über den Bau und die Transplantation des Epoophoron. Zieglers Beitr., 43, p. 263, 1908.

Stina, NADÉJDA. Des fonctions de la thyreoide. Lausanne, 1900.

STörr, PH. Lehrbuch der Histologie. Jena, 9 Aufl., I90I.

- Über die Thymus. S. d. phil. Ges. Würzburg, \& Juni, 1905.

- Über die Natur der Thymuselemente. An. Hefte, 95, 1906.

STÖLTZNER. Behandlung der Rachitis mit Nebennierensubstanz. J. K., 51, I 900 .

- Histologische Untersuchung der Knochen von 9 mit Nebennierensubstanz behandelten rachitischen Kindern. Ibid., 53, iii, 5 and 6, 1901.

- Pathologie und Therapie der Rachitis. Berlin, 1904.

- Die Kindertetanie eine Calciumvergiftung. J. K., 63, p. 66r, 1906.

- Nebennieren und Rachitis. M. K., I 908 .

- Menstruatio praecox. M. K., I908.

STÖLTZNER and SALGE. Vorkommen von eigentiumlichen Krystallen in den Knochen von mit Nebennierensubstanz behandelten rachitischen Kindern. B. k. W., igoo.

Stoerk, O. Über Protagon. W. k. W., I906; and S. W. A., I15, 1906.

- Üb. Chromreaktion v. Gl. coccygea u. Bezieh. z. N. sympathicus. A. m. A., 69, 1907.

- Beiträge zur normalen Histologie der Nebennierenrinde. I. Gibt es eine Lumenbildung an den Rindenzellenveṛbänden. B. k. WV., No. I6, 1908 .

- II. Über adenomartige Bildungen in der Nebennierenrinde. Ibid., No. I9, I908.

- Zur Histogenese der Grawitzschen Nierengeschwülste. Zieglers Beitr.. 43,1908 .

Stoerk, O., and Haberer, H. v. Beitrag zur Morphologie des Nebennierenmarkes. A. m. A., 72 , pp. $481-496$, 1908 .

- Über das anatomische Verhalten intrarenál eingepflanzten Nebennierengewebes. A. k. Ch., 87,4 , 1008.

SToklisA, J. Alkoholgärung im Tierorganismus. P. A., IoI; dort zahlr. Arb. v. Stoklasa und seinen Schülern. 
Stolz. Über Adrenalin und Alkylaminoacetobrenzkatechin. B. d. ch. G., $37,1904$.

- Synthese der wirksamen Substanz der Nebennieren, synthetisches Suprarenin. V. d. N. A. Stuttgart, I906; Pharm. Zeitg., No. 80, 1906.

STOOKEY, L. B., and VERA-GARDNER. On the pharmacology of the iodides. Proc. Soc. exper. Biol. Med., 5, pp. 122-123.

- Relation of the thyroids to autolysis. Ibid., 5 , p. 120.

STOOKEY, L. B. On the physiology of the thyroids. Ibid., 5, p. I21.

Stradiotti, G. Di un tentativo di cura del Morbo Flajani-Basedow mediante un siero tireotossico. Riv. crit. clin. med., No. 7, 1907.

- Paraganglioprecipitine e siero ipotensivo. Acc. med. fis. fiorent., 1905.

Strassmann. Beitrag zur Lehre von der Ovulation, Menstruation und Kastration. A. G., 52,134 .

- Handb. d. Geburtsh. v. Winckel, I, 1903.

StraUb, H. Akuter Morbus Addisonii nach doppelseitiger Nebennierenthrombose. D. A. k. M., 97, H. I and 2.

Straub, W. Mechanismus der Adrenalinwirkung und die mögliche Bedeutung der Substanz für den Kreislauf. M. m. W., I 907 .

- Über den Mechanismus der Adrenalinglykosurie. M. m. W., 1909.

STRAUSS, H. Neurogene und thyreogene Glykosurie. D. m. W., 1897 .

- Die chronischen Nierenentzündungen. Berlin, 1902 .

- Untersuchungen über den Wassergehalt des Blutes bei Herz- and Nierenwassersucht. Z. k. M., 6o, r.9o6.

STRÄUSSLER. Zur Symptomatologie u. Anatomie d. Hypophysengangsgeschwülste. Arb. d. psych. Klinik, Prag, I907.

Strehl and WeISS. Beiträge zur Physiologie der Nebenniere. P. A., 86, p. 107 , I901.

STRICKER, S. Handbuch der Lehre von den Geweben. Leipzig, i 871.

- Vorlesungen über allgemeine und experimentelle Pathologic. Wien, 1883 .

STRICKER, S., and SPINA, A. Untersuchungen über die mechanischen Leistungen der acinösen Drüsen. S. W. A., 8o, 1879.

STRÜMPELL v. Akromegalie und Diabetes. D. Z. N., 1I 1897

STUDNicka, F. K. Die Parietalorgane. Lehrb. d. vergl. Anat., Jena, 1905.

STƯTZ, L. Über den Einfluss von Körperarbeit und Überwärmung auf die Zuckerassimilationsgrenze eines gesunden Menschen. Diss., Jena, 1908.

STÜve. Unters. über den respiratorischen Gaswechsel bei Sehilddrüsenfütterung. Frankfurt a. M., 1896.

Stumme, E. Ein Fall von Basedow mit Tuberkulose einer Glandula parathyreoidea. Z. Ch., 9o, p. 265 , 1907.

Akromegalie und Hypophyse. A. k. Ch., 87, 1908 .
STURLI. Gefässveränderungen n. Injektionen von Methylaminobrenzkatechin. M. m. W., 1905 .

STURSBERG. Ein Beitrag zur Addisonischen Krankheit. M. m. W., 1907.

STYBR, J. Deux cas de carcinome primitif des glandes surrénales non-suivis de la maladie d'Addison. Arch. Bohém. de Méd., iv, p. 100, 1890.

SudECK, P. Über die Strukter der Nierenadenome. V. A., 133, pp. 405$449,1893$.

- Zur Lehre von den aberrierten Nebennierengeschwïlsten. Ibid., 136, I 894 .

Sultan, G. Beiträge zur Involution der Thymus. V. A., 144, 1896.

- Zur Histologie der transplantierten Schilddrüse. C. a. P., 9, 1898.

- Beitrag zur Kenntnis der Schilddruisenfunktion. A. k. Ch., 63.

SunNer, A. Sur l'action inhibitoire du sang urémique sur la sécrétion urinaire. C. r. S. B., Bd., 58, p. 775 .

- Sur le pouvoir antitoxique des reins. J. d. P. P., vii, p. 935, 1905.

Supino. Sur la physio-pathologie des capsules surrénales. A. i. B., 18, 1893 .

- Sulla fisio-patologia delle capsule surrenali. Rif. Med., Settémbre, 1892.

SurY, K. v. Über die fragl. Bez. der sogenannten Mors thymica zu den plötzlichen Todesfällen im Kindesalter. Viertelj. f. ger. Med., 36,1908 .

SUTHERLAND. The differential diagnosis of Mongolism and Cretinism. Lancet, I 900 .

SuzukI. Chromaffiner Tumor des Nebennierenmarkes. B. k. W., 1908. 
Svenla, K. Exp. Unters. üb. d. Einwirk. des Thymussaftes a. d. Kreislauf. W. m. Bl., i 896 .

- Experimentelle Beiträge zur Kenntnis der inneren Sekretion der Thymus, der Schilddrüse und der Nebennieren von Embryonen und Kindern. A. e. P., 43, 1900 .

Symingtox, J. Über Thyreoidea, G1. parathyreoideae und Thymus beim Faultier. A. A. Suppl., 1897

Szurek, St. Die Lehre von Alexander Poehl und die mit seinen Präparaten erzielten Erfolge. W. k. W., Igo8.

SzymoxowICZ, L. Über die Erscheinungen nach der Nebennierenexstirpation bei Hunden und über die Wirkung der Nebennierenextrakte. Anz. d. Krak. Akad., i 895 .

- Die Nebennieren vom Standpunkte der Morphologie und Physiologie (polnisch). Krakau, 1895 .

- Die Funktion der Nebenniere. P. A., 64, pp. 97-164, 1896.

TADDEI, D. Contr. allo studio del cosidetto ipernefroma del, rene. Rif. med., 1909 .

TAKAMINE, J. The isolation of the active principle of the suprarenal gland. J. o. P., 27, p. xxix, 1901 ; A. J. P., 73, 1901.

TALBOT, E. Cases of hæmorrhages into the suprarenal capsules. St. Barth. Hosp. Rep., 1900.

Tambach. Zur Chemie des Jods in der Schilddrüse. Z. B., 36, 549, 1898.

TAMBRONi and LAMBRANZi. Una varietà di mixoedema frusto. Riv. di pat. nerv., 1899 .

Tamburini, C. Beitrag zur Pathogenese der Akromegalie. C. f. Nervenh. u. Psych., 1894.

- Contributo allo pathogenesi dell' acromegalia. Riv. sperim. di fren., vol. $\mathrm{xx}, \mathrm{i} 894$.

TANDLER, J. Untersuchungen an Skopzen. W. k. W., I908.

- Über den Einfluss der innersekretorischen Anteile der Geschlechtsdrüsen auf die aussere Erscheinung des Menschen. W. k. W., 19 Io.

- Einfluss der Geschlechtsdrüsen auf die Geweihbildung bei Renntieren. Anz. d Wien, Akad., igro.

TANDLER, J., and Grosz, S. Einfluss der Kastration auf den Orgnismus. W. k.'W., 1907.

- I. Mitteilung: Beschreibung eines Eunuchenskelett. A. E. M., 27, 1909.

- II. Mitteilung: Die Skopzen. Ibid., 30, 19 I0.

- III. Mitteilung: Die Eunuchoide. Ibid., 29, igro.

TANTURRI. Osteomalakie, geheilt mit den Adrenalineinspritzungen. C. G., 1907.

TARabiNi, L., and MAssagli, A. La paratiroidina Vassale contro il tremore senile. Gaz. Osp. e clin., No. 140, 1908.

Taramasio, P. Etude toxicologique de l'adrénalin. Rev. méd. Suisse rom., 22,1902 .

Tarantini. Contributo allo studio delle alterazioni aortiche prodotte della paraganglina. I1 policlinico, 13, p. 321, Lúglio, 1906 .

Tarchasow. Uber cinige physiologische Wirkungen des Adrenalins auf Tiere. Russki Wratsch, No. 47, 1902; Ref. B. C., i, p. 369, I903.

TARgetT, J. H. Accessory adrenal bodies in the broad ligaments. Trans. Obst. Soc., 39, 1898 .

Targhetta. Etude sur le thymus. A. i. B., 30, $1898-99$; Thèse de Paris, 1902.

TARUfFI, C. Sulla struttura delle capsule soprarenali. Boll. d. scienc. med. Bologna, 1866 .

- Della Microsomia. Riv. clin. di Bologna, 1878 .

- Hermaphroditismus und.Zeugungsfähigkeit. Übersetzt von Teuscher. Berlin, I903.

Tarulli. Sur les effets de l'exstirpation du Thymus. A. i. B., 37.

TARUlli, L., and Lomonaco, D. Ricerche sper. sul timo. Boll. della R. Accad. Med. di Roma, p. 311,1808 .

Tassini, R. Effetti terapeutici della paraganglina Vassale. Gazz. Osp. e Clin., 1908.

Tauret, C. Sur l'ergotinine. Journ. de Pharm. et de chim., Bd. 24, p. 397. TAUSzK and VAS. Beitr. z. Stoffwechsel bei Akromegalie. Pest. med.-chir. Presse, I 899 .

TEBB, CH. The cholesterins of the brain. J. o. P., 34, 1906. 
Teueschi, B. Contr. à la pathogénie du goitre exophtalmique. Rev. Neur., p. $683,1902$.

- Ancora sulla Patogenesi del gozzo esoftalmico. Autoref. B. C., i, p. 631, 1903.

TeIssier, J. A propos l'hypertension artérielle. S. m., I904.

- Arteriosclérose et atheromasie. Paris, igo8.

TEISSIER and FRAENKEL. Effets phys. des inj. souscont. d'extrait rénal. A. d. P., 1808 .

TeISSIER and THAON, L. La pression artérielle dans la scarlatine de l'adulte. J. d. P. P., I5 mai, 1908 , p. 481 .

Teissier and Thevecot. Antagonisme de la choline et de l'adrén. C. r. S. B., 64, p. 425 .

Tescione. Modific. istolog. della gland. tiroide in seguito all' ablazione dell' ovaie. Arch. ital. d. Ginec., ro07.

Thaos, P. Note sur la sécrétion de l'hypophyse et ses vaisseaux évacuateurs. C. r. S. B., 62, avril, 1907 .

- Toxicité des extraits de prostate; leur action sur la compression artérielle et de rythme cardiaque. C. r. S. B., 63, p. $111,1907$.

- Contribution à l'étude des glandes à sécrétion interne: L'hypophyse ̀̀ l'état normal et dans les maladies. Thèse de Paris, 1007.

TheoARI, A., and BABES, A. Über ein gastrotoxisches Serum. mit einem Studium des Chemismus des Magens und der von diesem Gastrotoxin veranlassten histologischen Veränderungen. C. f. B., 38, H. 6; 39, H. 1 and 2 .

Thevenot. Athérom aortique expérimentale. Paris, 1907.

ThibIERGE. Maladie d'Addison chez un nègre arabe. Gaz. des Hôpit., I899.

- Radiographie dans un cas d'infantilisme myxœdémateux. Ibid., Io février, I $\$ 99$.

Thiele-Nehring. Untersuch. des respir. Gaswechsels unter dem Einfluss von Thyreoideapräparaten. Z. k. M., $30,1806$.

THIEmich. Anatomische Untersuchungen der Glandulae parathyreoideae bei der 'Tetanie der Kinder. Monatsschr. f. Kinderhk., 5, p. 165, 1906.

- Entwicklung eklamptischer Säuglinge in der späteren Kindheit. J. B., 1906.

Thienger. Einige Beobachtugen über Moebius' Thyreoidin. M. m. IV., 1905.

Thies. Der Gebrauch des Adrenalins zur Unterstützung der Lokalanästhesie. Diss., Leipzig, I 903.

- Wird die Giftigkeit des Cocains durch Kombination mit Adrenalin herabgesetzt? Z. Ch., 74, p. 434, 1904 .

Tiriroroix. Diabète pancréatique. Bull. de la Soc. anat., 62, 1891.

- Fonction des capsules surrénales. Soc. anat., p. 207, 1892; C. a. P., 5, p. 87 .

- Procédé d'ablation sur lé chien des capsules surrénales, ectopie de ces organes. Mercredi médic., p. 557, 1892.

- Données exp. p. servir à éclairer la palhogénie du Diabète sucré. Gaz. Hôp., 1894 .

Thoinot and Diamare. Etude sur le pancréas diabétique. A. m. e., 19, 1907.

Тном. Unters. über d. norm. u. patholog. Hypophysis cerebri. A. m. A., 57,1901

Thompson. Die physiologische Wirkung der Protamine und ihrer Spaltungsprodukte. Z. ph. Ch., 20, 1900 .

Thompsos, G. Atrophy of the parathyroid glandules in primary infantile atrophy. A. J. M. S., October, 1907.

Thompson and HARRIS. Path. histology of the parathyroid glands, \&c. Journ. of Med. Res., Boston, igo8.

- A study of Addison's disease and of the adrenals. Ibid., October, I893.

THOMPSON and JOHNSON. Note on the effect of pituitary feeding. J. o. P., 33,1905 .

THOMPSON and LEIGHTON. The results of chronic parathyroiditis in the dog. Ibid., 1008 .

Thorel. Zur Frage der Erweichungescysten in den Geschwülsten der Nebenniere. Festschr. d. Nürnberger ärztl. Vereins, 1902.

Thumin, L. Geschlechtscharaktere und Nebenniere in Korrelation. B. k. W., Igog.

- Beziehungen zwisclien Hypophysis und Eierstöcken. Ibid., 1909. 
TIBERTI, N. Über die Sekretionserscheinungen in den Nebennieren der Amphibien. Zieglers Beitr., 36, p. 161, 1904.

- Intorno alla estirpazione totale del duodeno. Lo Sperimentale, 62, 479, 1908.

- Intorno-al modo di comportarsi dell' isola del Langerhans in seguito alla ligatura del condotto pancreatico. A. i. B., 5 I, 1908.

- Intorno alla rigenerazione del pancreas. Arch. d. Fisiol., 5, 1908.

- Ulteriori ricerche sperim. int. all' isole di Langerhans. 'Sperim., 62, 1908 .

Tiberti, N., and Franchetti, A. Sugli effetti della estirpazione parziale e totale del pancreas ne cani. Sperimentale, 62, 81-118, 1908.

Tigerstedt, R., and BERgmanN. Niere und Kreislauf. S. A., 8, 1808 .

TILLÉ. L'opothérapie ovarienne dans la maladie de Basedow. Thèse de Paris, 1899

TiLNeY, F. Un cas de myasthénie grave pseudoparalytique avec adénome du corps pituitaire. Neurograph. New York, vol. i, March 20, 1907.

TimofeEw, S. Zur Frage von der Pathogenese der nephritischen Odeme. A. P. P., 6o, 1909.

Tiтo. Morbo maculoso Werlhof curato con injez. ipoderm. d'adrenalina. Gazz. d. Osped., 1904 .

TizzonI. Physiologie et pathologie des capsules surrénales. A. i. B., 5, 1884 .

-. Ric. sper. int. alla fisiopat. di corpo tiroide. Gazz. d'Osped., I885.

- Sur physiologie pathologiques des corps surrénales. C. r. A., p. 832, 1886.

- Ablations des capsules surrénales chez le chien. A. i. B., 10, pp. 372378, I 888 .

- Über die Wirkungen der Exstirpation der Nebennieren auf Kaninchen. Zieglers Beitr., 5, pp. 3-100, 1889 .

Tizzoni and Centanni. Sugli effetti remoti della tiroidectomia nel cane. Arch. scienc. med., 14, 1890 .

TODARo. Sur l'épiphyse et l'hypophyse des Ascidiae. Arch. de Biol., I881.

TOGAMI. Experimentelle Untersuchung über den Einfluss einiger Nahrungsund Genussmittel auf die Pankreassekretion. Z. physik.-diät. Th., xii, p. 403,1908 .

TOMASZFivSKI and Wilexko. Beitrag zur Kenntnis der antagonistischen Wirkungen des Arenalins und der Lymphagoga. B. k. W., 1908.

TOPOLANSKI. Zur Frage des chromaffinen Systems. W. k. IV., 1907.

TORETTA. Les glandules parathyroidiennes chez les mammifères. Ann. malad. de l'oreille. Ref. C. Ch., I902.

TOROPOFF, D. J. Über die pathologisch-anatomischen Veränderungen des Gefässsystems bei intravenöser Injektion von Adrenalin. Diss., Petersburg, I907; Bph. C., iii.

TORRI. L'ipofisi nelle infezioni. Pisa, 1004.

TORRINI, U. L. Ricerche sperimentali sulla modificazioni istologiche delle ghiandole surrenali in seguito ad alcuni interventi operatorii sul rene. Lo Sperim., 63, pp. 79-98, 1909 .

Toujas, G. S. P. Recherches expérimentales sur l'adrénaline. Son dosage, sa formation, son origine, sa destruction. Thèse de Toulouse, 1905 .

TOURNaDE. Etudes sur les modifications du testicule consécutives à l'interruption du canal déférent. Thèse de Lyon, 1903-04.

Tourneux, F. L'organe de Rosenmueller (Epoophore) et le parovarium (Paroophore) chez les mammifères. J. A. P., 24, 1886.

- Hermaphroditisme de la glande génitale chez la taupe femelle adulte et localization des cellules interstitielles. Ass. des Anat., VI session. Toulouse, I904

Tourneux, F., and Herrmans. Sur l'évolut. histologique du thymus. C. r. S. B., I 887 .

TOURnEux and Soulié. Sur les premiers devel. d. 1. pituitaire ch. l'homme. Ibid., 1898 .

Tourneux, F., and Verdun, P. Sur le développ. de la thyr., du thym. et de gl. parathyr. J. A. P., p. 305, 1897.

TrachTENBERG. Über experimentelle heteroplastische Knorpelbildung in der Aorta bei Tieren. Charkower med. Journ., No. 5, 1906; Ref. Bph. C., ii.

TranA. Ric. sperim. sul sistema nervoso degli animali tireoprivi. Policlinico, 1898 . 
Traina. Sulle modificazioni delle paratiroidi del cane in diversi stati morbosi sperimentali. Lo Speriment., H. 1-2, 1908.

Tramonti, E. Contr. clinique à l'étude de l'acromégalie. Il Policlinico, I906.

Tranchini. Ricambio materiale in Acromegalia. Boll. scienc. med., Bologna, t905.

Tranjen. Das Altern als abwendbare Krankheit. Halle a. S., 1908.

Trautmann, A. Anatomie und Histologie der Hypophysis cerebri. A. m. A., 74,1900

TrebitzSCH, $H$. Über eine ungewöhnliche Form der Hautpigmentierung beim Morbus Addisonii. Z. k. M., 32, Suppl.-H., p. 163, 1897.

TREuPEL. Stoff wechselversuche bei Myxödem. M. m. IW., p. 885 .

Trerotoli, A. Sull' azione dell' estratto acquoso del lobo posteriore dell' ipofisi nei cardiaci e nei nefritici. Riv. crit. clin. med., 8, 1907.

TrewiThICK, E. G. Addison's disease; treatment with suprarenal extract. Lancet, 1900.

TrIEPCKE, O. Über Blutcysten in Nebennierenstrumen. Diss., Greifswald, 1901 .

Trinci, GIUlio. Cellule cromaffine e "Mastzellen" nella regione cardiaca dei mammiferi. R. Accad. delle Scienze dell' Istituto di Bologna, 26 maggio, 1907.

Trivas. L'adrénaline en otorhinolaryngologie. Thèse de Bordeaux, 1892; C. r. S. B., 1903.

Trousseau. Maladie d'Addison. Bull. de l'acad. de méd., séance du 26 août, 1855 .

TSCHASSOWNIKOFF, J. Ưb. d. histol. Veränderg. d. Bauchspeicheldrüse nach Unterbindung des Ausfuihrungsganges. A. m. A., 67, 1906.

TschEBOKSAROFF. Sur les nerfs sécréteurs des surrénales. Russki Wratsch., viii, 873. 27 juin, 1909; Ref. J. d. P. P., 11, p. 962.

TSCHERNIACHOvSKI, E. Gibt es einen Duodenaldiabetes? Z. B., 5.3, 1907.

TSCHERWENTzOFF. Des altérations des capsules surrénales dans la peste bubonique. Arch. de Scienc. biol., I2, St. Pétersbourg, 1906.

TsCHIRKOFF. Blutveränderungen bei der Addisonschen Krankheit. Z. k. M., $19, \cdot 1891$.

TschuewSKY, J. A. Über den Blutstrom in der Schilddrüse. P. A., 97, 1903.

Tsubor, H. Experimentelle Studie über die Urämie. M. med. Fak. Tokio, vii, 1907.

TUFFIER. Le capsule adipeux du rein. Revue de Chirurgie, I8go.

TUFFIER and Lejars. Les veines de la capsule adipeux du rein. A, d. P., 1892 .

TUNICLIFFE. Über die Wirkung des Piperidins auf den Kreislauf. C. P., 10, p. $777,1896$.

TURNER, F. CH. Spindle cell sarcoma of pineal body containing glandular and carcinomatous structures. Transact. of the Path. Society of London, 36 , p. 27 , I 885 .

- A case of Addison's disease in which the blood-pressure was taken two days before death. Lancet. June io, 1899 .

TURRó, R., and SUÑER, A. Inkonstantes Auftreten von Glykosurie nach Totalexstirpation des Pankreas. C. f. Phys. d. Stoffw., Igog.

- Der Mechanismus der natürlichen Immunität. C. f. B., 39.

UHтноFғ. Ein Beitrag zu den Sehstörungen bei Zwergwuchs und Riesenwuchs, respektive Akromegalie. B. k. W., 1897.

UlRICH, A. Anatomische Untersuchungen über ganz und partiell verlagerte und akzessorische Nebennieren und über die Frage der von den Nebennieren ausgehenden Geschwülste. Ziegl. Beitr., 18, Diss., Zürich,

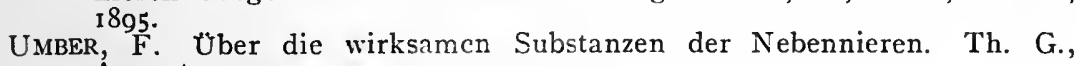
August, 1902.

UNDERHILL, F. P. Certain aspects of experimental diabetes. J. B. Ch., i, p. II3.

UNDERHILL and Closson, O. E. The mechanism of salt glycosuria. A. J. P., $15,1906$.

- Adrenalin glycosuria and the influence of adrenalin upon nitrogenous metabolism. Ibid., 17, 1907. 
UNDERHILl and Closson, O. E. The influence of subcutaneous injections of dextrose upon nitrogenous metabolism. J. B. Ch., ii, I906.

UNDERHILL and HILDITSCH, $\mathrm{W}$. W. Certain aspects of carbohydrate metabolism in relation of the complete removal of the thyroids and partial parathyroidectomy. A. J. P. October 25, 1909.

UNDERHILL and KLEINER. Further experiments on the mechanism of salt glycosuria. J. B. Ch., iv, p. 395 , 1908.

UNDERHILL and SAIKI, T. The influence of complete thyroidectomy and thyroid feeding upon certain phases of intermediary metabolism. Ibid., v, p. 225, October, 1908 .

URECHIA, C. J. Action de l'extrait hypophysaire en injections intrapéritonéales. C. r. S. B., 65, p. 278, 1908.

USPENSKI. Organotherapie. St. Petersburg, I896 (Russisch).

VAhlen, E. Pankreas und intermediärer Stoffwechsel. C. P., 22, No. 7, 1908.

- Über die Einwirkung bisher unbekannter Bestandteile des Pankreas auf den Zuckerabbau. I. Mitt. Z. ph. Ch., 50, p. 194, I909.

VALENTI, A. Del ricambio materiale nello gravidanza e nell' allattamento. Arch. Farmacol., vii, H. 8 and 9.

VALENTI, G. Sulla sviluppo delle capsule surrenale nel pollo ed in accuni mammiferi. Atti d. Soc. Tosc. $x$, Pisa, 1889; and Accad. med. chir. di Perugia, I894.

- Sulla origine e sul significato dell' ipofisi. Ibid., 1895 .

- Sopra la piega faringea. Monit. zool. Ital., 9, 1898 .

VALENTin, F. Der Einfluss letaler Verbrennungen auf däs histologische Bild der Schilddrüse. V. A., I91, p. 42.

la VAlETte, A. v. ST. GEORGE. Zwitterbildung beim Wassermolch. A. m. A., 45,1895 .

VALUDE and DÚCLOS. Effets de l'adrénaline en instillations longtemps prolongées. Ann. Ocul., Paris, 138, p. I I 5, 1907.

VAMOSSY, Z. V., and VAS, B. Experimentelle Untersuchungen über die Wirkung des Jodothyrins. M. m. W., I897.

VANNi, E., and Manzini. Sulla parte che spessa al rene nella patog. dell' uremia. Gazz. degli Osped., 16 dicémbre, 1893.

VANORI, L. Sugli effetti dell' estirpaz. del pancreas. A. ital. clin. med., 33,1894 .

VAQUEZ, H. Hypertension artérielle et ses conséquences au cours de l'intoxication saturnine aiguë de l'éclampsie et de l'urémie. Sem. méd., igo4.

VAQUEZ and AUBERTIN. Cour de Traube et hyperplasie médullaire des surrénales. C. r. S. B., 62, 967, 1907.

VARALDO, F. Capsule surrenali aberrenti nell' ovajo. Arch. d'ostetr. e ginec., xi, 1905 .

VARIOT and BEZANCON. Indépendance de la spermatogenèse et de la sécrétion testiculaire proprement dite. Bullet. de la Soc. d'Anthropologie de Paris, sér. 4, T. iii, 1892.

- Influence de la sécrétion testiculaire sur le développement organique. Indépendance de cette fonction et de la spermatogenèse dans certains cas. Gaz. méd., 1892 .

VAS. Über das Chromatin in den sympathischen Ganglienzellen. A. m. A., 40.

VASSALE, G. Ricerche microscopiche e sperimentali sull' alterazioni del pancreas consecutive alla legatura del dutto di Wirsung. Reggio Emilia, $1889-1891$.

- Ulteriori esper. int. alla gland. tiroide. Riv. sper. fren., 1892.

- Tetanie provoquée par l'allaitement chez une chienne partiellement parathyréoïdectomisée. A. i. B., 30, p. 49, 1897.

- Funzione paratiroidea e funzione tiroidea. Riv. sperim. di fren., 27, I9OI.

- L'hypophyse dans le myxœdème et dans l'acromégalie. Ibid., 38, I902.

- Physiopathologie de l'appareil des capsules surrénales. A. i. B., 43 , p. 256,1905 .

- Le traitement de l'éclampsie gravidique par la parathyroïdine et considérations sur la physiopathologie des glandes parathyroïdes. A. i. B., 43 , p. 256 , 1905 .

- Schwangerschaftseklampsie und Insuffizienz der Parathyfoiddrüse. M. m. W., 1906 . 
VASSale and Donaggie. Les altérations de la moëlle épin. chez les chiens opérés d'exstirp. des gl. parathyroïdiennes. A. i. B., 27, 1897.

Vassale, $G_{\text {., }}$ and General. Sur les effets de l'exstirpation des glandes parathyroïdes. A. i. B., 25; p. $459 ; 26$, p. 6r, 1896 ; and 33, 1906 .

- Sopra le ghiandoli paratiroidee dell' uomo. Rif. med., 2, p. 631, 1897.

- Ulteriori ricerche intorno alle ghiandole paratiroidee. Ibid., 3, p. 77, 1897.

- Intorno agli effetti dell' estirpazione delle ghiandole paratiroidee. Ibid., I, p. 800, 1897 .

- L'alimentazione tiroidea contro gli effetti dell' estirpazione delle ghiandole paratiroidee. Ibid., 2, p. 235, I897.

VASSALE and ROSSI. Sur la spleno-thyroïdectomie chez le chien. A. i. B., 21,1893 .

VASSALE and SACCHI. Sur la destruction de la glande pituitaire. Ibid., 1893.

- Sulla distruzione della glandola pituitaria. Riv. sperm. di fren., 18, 1892 ; and 20, 1894 .

VASSALE, G., and ZÁNFROGNINI. Sur l'exportation de la substance medullaire des capsules surrénales. A. i. B., 38, p. 175, 1902; and Rif. med., 1902.

VECCHI, DE. Über experimentelle Tuberkulose der Nebennieren. C. a. P., 12,1901 .

- Wirkung einiger Organextrakte bei den akuten Infektionsprozessen. C. f. B., 35, pp. 577 and 708 .

Vedova, Dalla. Per la funzione dell' ipofisi. Bull. Acc. med. Roma, 29, 1903 and 1904 .

- Ein Fall von Hypernephrom der Leber. V. A., 177, 1904.

VEIEL, TH. Pruritus cutaneus bei Erkrankung der Niere und Nebenniere. A. Derm. Syph., 8o, 1906.

VEIT, D. Verschleppung der Chorionzotten. Wiesbaden, I905.

VELDE, VAN DE. Über den Zusammenhang zwischen Ovarialfunktion, Wellenhewegung und Menstrualblutung. Jena, 1905.

VELICH, A. Über die Einwirkung des Nebennierensaftes auf den Blutkreislauf. W. m. Bl., Nos. 15-21, 1896; and Allg. W. m. Ztg., 1897 .

- Über.die Folgen der einseitigen Exstirpation der Nebenniere. W. k. R., 1897.

- Vergleichende Untersuchungen über die Einwirkung des Piperidins und des Nebennierenextraktes auf den Kreislauf. W. k. R., i898.

- Über die Einwirkung der örtlichen Applikation des Nebennierenextrakts auf die Blutgefässe der Haut des Menschen. W. m. Bl., 1897.

- Beitrag zum Experimentalstudium von Nebennieren. V. A., 184, 1906.

VELITS, D. v. Über Adrenalinwirkung bei Osteomalakie. C. G., 31, 29, p. 929,1907 .

Vellesen. Morbus Basedowii mit bes. Berücksichtigung der Pathogenese. Z. k. M., I899.

VERDUN. Sur les glandes satellites de la thyroïde dans les mammifères. Thèse, Toulouse, 1897 .

- Dérivés branchiaux cliez les vertebrés superieurs. Toulouse, i 898 .

VEREBÉLY, J. v. Beitr. zur Schilddrüsenzirkulation. M. G. M. C., I7, 1907.

- Beitr. zur Pathologie der branchialen Epithelkörperchen. V. A., 187, 192.

VERNON. Intercellular Enzymes. London, Igo8.

VERSTRAETEN. L'acromégalie. Rev. d. méd., pp. 377 and 493, 1889.

VERTRA and ANGIOlella. Manicomio moderno. Nos. 1 and 2,1899 .

VERWORN. Allgemeine Physiologie. 4 Aufl., Jena, 1903.

- Zur Analyse der dyspnoischen Vagusreizung. A. P., p. 65, 1903.

Vialleton. Anat. des caps. surrénales. Montpellier méd., is98.

VICOLINI. Cura del prolasso rettale con la paraganglina.' Gaz. d. Osped.,

VIDAL and BoIdin. Adénomes des capsules surrénales avec athérome généralisé et hypertension artérielle. Soc. méd. des hôpit., séance du 2 I juillet, 1905; Sem. méd., p. 356, 1905.

VIDAL, ROY and FROIN. Un cas d'acromégalie sans hypertrophie, \&c. Rev. de Méd., 1906.

VIDAL and SuTIER. Atrophie congénitale complète des testicules. Absence d'infantilisme et de féminisme. Bull. de la Soc. méd. des hôpitaux, 20 mai, 1902.

Vigliani, R. Contr. allo studio delle funzione del pancreas. Lo Sperim., 1905. 
Vigoureux and Delmas. Infantilisme. Bull. de la Soc. anat., p. 686, 1906.

VIGNES. L'Adrénaline en ophtalmologie. Presse méd., ro mai, 1902.

VilLEmin. Sur la régénération de la glande séminale après destruction par les rayons X. C. r. S. B., p. $1077,1905$.

- Sur le rôle du corps jaune ovarien chez la femme et la lapine. Ibid., 64, p. 363 , I908.

- Sur les rapports du corps jaune avec la menstruation et le rut. Ibid., p. 444 .

- L'ovulation est-elle spontanée chez la lapine? Ibid., p. 662.

- Le corps jaune considéré comme glande à sécrétion interne de l'ovaire. Thèse de Lyon, 28 janvier, 1908.

VINCENT, S. The suprarenal capsules in the lower vertebrates. Proc. of the Birmingham Nat. Hist. and Phil. Soc., vol. 1o, pp. I-26, 1896.

- The physiology of the suprarenal bodies. Birmingham Med. Rev., 1896.

- The suprarenal gland. Brit. Med. Journ., 1896.

- Contribution to the comparative anatomy and histology of the suprarenal capsules. The suprarenal bodies in the fishes and their relation to the so-called head-kidney. Trans. Zool. Soc., vol. 14, part 3, pp. 44-84, I 895 . Published 1897 .

- The comparative physiology of the suprarenal capsules. P. R. S., 61, pp. $64-77,1897$.

- On the general effects of the extracts of the suprarenal capsules. J. o. P., 22, pp. III-I 20,1897 .

- The effects of subcutaneous injections of extracts of suprarenal capsules. J. o. P., 2 I, 1897 .

- On the morphology and physiology of the suprarenal capsules in fishes. An. An., 13, pp. 39-48, 1897 .

- The effects of extirpation of the suprarenal bodies of the eel. P. R. S., $62,1898$.

- The nature of the suprarenal bodies of the eel and the effect of its removal. J. o. P., 22, p. 48,189 S.

- On the suprarenal and the lymphatic tissue of Teleostean fishes. An. An., 14, pp. I5I-152, 1898 .

- The comparative histology of the suprarenal gland. I. M., 15, I898.

- Addison's disease and the functions of the suprarenal capsules. Birmingham Med. Rev., 1898.

- Further observations upon the comparative physiology of the suprarenal capsules. P. R. S., 64 , 1899.

- The carotid gland of mammalia and its relation to the suprarenal capsule, with some remarks upon internal secretion and the phylogeny of the latter organ. An. An., I8, pp. 69-76, 1900 .

- A discussion of some points in connection with the suprarenal glands, cortical and medullary. 'J. A. P., 38, pp. 34-48, 1903.

-- On the results of extirpation of the thymus gland. J. o. P., 30, 1904.

- Internal secretion and the ductless gland. Lancet, I, 348, p. 1198, 1906.

- The ductless glands. Science Progress, No. II, January, Igog.

- Innere Sekretion u. Drüsen ohne Ausführungsgang. Zusammenfass. Ref., E. P., ix, 1910.

VINCENT, S., and CRAMER, W. The nature of the physiologically active substances in extracts of nervous tissues and blood, with some remarks on the methods of testing for choline. J. o. P., 30, pp. 143-154, 1904 .

VINCENT, S., and HARrison. Spleen and lymphatic glands. J. o. A. and P., 3I, 1887 .

Vincent, S., and Jolly, W. A. Function of thyroid and parathyroid glands. Ibid., 32.

VINCENT, S., and SHEEN. The effects of intravascular injections of extracts of a nimal tissues. Ibid., 29, 1903 .

Vincent, S., and THOMPSON, F. D. On the relation between the islets of Langerhans and the zymogenous tubules of the pancreas. I. M., 24, 1907 .

VIRCHOW, R. Die Bronzekrankheit in Canstatts Jahresber., 1856-1858.

- Zur Chemie der Nebennieren. V. A., I2, 1857.

- Gesammelte Abhandlungen zur wissenschaftlichen Medizin, p. 747, 1862.

- Die krankhaften Geschwülste, 3 Bde., Berlin, 1867.

- Fötale Rachitis, Kretinismus und Zwergwuchs. V. A., 94, 1883.

- Zur Frage der Kropfkachexie. Ibid., 144, 1896. 
VisentiNI, A. Zur Frage der Duodenalglykosurie. M. K., 1908.

Sulla questione dell glicosuria duodenale. Il Morgagni, No. 8, 1908.

- Über das Verhalten des Pankreas nach Unterbindung und Durchschneidung seiner Ausführungsgänge. A. A. P., 1908. Suppl.

- Über die anatomische und funktionelle Wiederherstellung der unterbundenen und, durchschnittenen Pankreasausführungsgänge. V. A., 195, 1909.

VitRY, G., and GIRAND, G. Lésions histologiques du corps thyroïde des tuberculeux, leur rapports avec la teneur en iode. C. r. S. B., 65, pp. $404-406,1908$.

VITzou. Recherches expér. sur la sécretion interne des reins. J. d. P. P., 1901.

VIvier. Sur l'infantilisme. Thèse Paris, 1897.

VOENA, G. Azione sulle capsule suprarenali di alcune particolari sostanze introdotte nell' organismo con injezioni ipodermiche. Bibliogr. Ital., ii, 92 ; Ref. V. H., ii, 1893.

VoGt. Über Mongoloidentypus der Idiotie. Jahresvers. d. d. Ver. f. Psych. in München, 1906.

VoIgt, B. Über Anwendung und Wirkung des Adrenalins. M. m. W., I904.

VoINov. Rôle probable de la glande interstitielle. C. r. S. B., 57, p. 414, 1905 .

Glande interstitielle et spermatoxines. Ibid., p. 688 .
VoIT, FR. Stoff wechseluntersuchungen am Hund mit frischer Schilddrüse und Jodothyrin. Z. B., 35, 1897.

Vollbracht, F. Ein Fall von Morbus Addisonii nach vorausgegangener Purpura haemorrhagica mit einer Stoffwechseluntersuchung. W. k. W., 1899 .

VollbRACHT and PANZER, Th. Aufklärung zu voriger Mitteilung. Ibid., No. 34 .

Vos, J. DE, and Kochmans. De la rapidité avec laquelle la principe actif des caps. surrén. disparait du sang. A. intern. Pharmacod., 14, p. 8I, 1905 .

VOSBURGH and RICHARD. An experimental study of the sugar content and extravascular coagulation of the blood after administration of adrenalin. A. J. P., 9, 1903.

VUlPIAN. Note sur quelques réactions propre à la substance des capsules surrénales. C. r. A., xliii, pp. 663-665, Paris, 29 septembre, 1856 .

- Note sur quelques réactions propres au tissu des capsules surrénales chez les reptiles. Ibid., p. $223,1856$.

- Lecons sur l'appareil vasomoteur. T. ii, p. 38, 1875 .

VulPIAN, A., and ClOEz, S. Note sur l'existence des acides hippuriques et cholériques dans les capsules surrénales des animaux herbivores. C. r. A., $x l v, 7$ septembre, 1857 .

Wagner v. JauregG, J. Über die Folgen des Exstirpation der Schilddrüse. W. m. Bl., No. 25, p. 771,1884 .

- Weitere Versuche über Exstirpation der Schilddrüse. Ibid., No. 30, p. 931 .

- Über endemischen und sporadischen Kretinismus und dessen Behandlung. W. k. W., 1900 .

- Behandlung des endemischen Kretinismus mit Schilddrüsensubstanz. Ibid., 1904.

- Über marinen Kretinismus. Ibid., 1906.

- Zweiter Bericht über die Behandlung des endemischen Kretinismus mit Schilddrüsensubstanz. Ibid., p. 33, 1907.

WAHNCAU. Zur Kasuistik des Morbus Addisonii und der akzessorischen Nebennieren. Jb. der Hamb. Staatskrank., Jg., i, p. 158, 1889.

Walbaum. Unters. über die Epithelkörperchen beim Kaninchen. M. G. M. C., 12, 1903 .

WaldEYER, W. Die Rückbildung der Thymus. Ber. d. preuss. Akad., I8go.

- Die Entwicklung der Carcinome. V. A., 55, 1872 .

- Über Bindegewebszellen. A. m. A., 11, 1875 .

WALLART, J. Chem. Unters. über den Luteingehalt des gelben Körpers während der Gravidität. B. G. G., xiv, No. I.

- Untersuchungen uiber die interstitielle Eierstockdrüse beim Menschen. A. G., 8I, 27 I. 
Wallart, J. Über das Verhalten der interstitiellen Eierstockdrüse bei Osteomalakie. Z. G. G., lxi, p. 581 .

WallmanN, $H$. Über das akzidentelle Vorkommen physiologischer Gewebe. Zeitschr. d. Ges. Ärzte, Wien; 1859 .

WALTER and Dixon. Pharmacology, Adrenalinwirkung, p. 425, 1906.

WARDA. Über Akromegalie. D. Z. N., 19, p. 358, rgor.

WARrington, W. B. Exophthalmic Goitre. Liverpool Med. Chir. Journ., 1908.

WASSERTRILling. Die funktionelle u. klinische Bedeutung der Epithelkörperchen. W. m. W., p. 1439, 1908.

WATERMAN, N. Einige Bemerkungen zur Frage: Arteriosklerose nach Adrenalininjektionen. V. A., 191, pp. 202-208, 1908.

- Über den Nachweis von Nebennierenprodukten im Blut und Harn. P. A., 128, 1909 .

- Über einige Versuche mit Rechtssuprarenin. Z. ph. Ch., 63, p. 290, Igog.

Waterman, N., and BoddaerT, R. J. Über den Nachweis von Nebennierenprodukten im Blut und Harn. D. m. W., is Juni, Igo8.

Waterman, N., and Smit, H. J. Nebenniere und Sympathicus. P. A., I24, p. I98, 1908 .

WATSON, $\mathrm{CH}$. Influence of a meat diet on the thyroid and parathyroids. J. o. P., 32, 1905 .

- The influence of meat diet on the thyroid gland in the second generation of meat-fed rats. J. o. P., 34, I906.

Wersiloff. Ein Fall von Akromegalie. Ges. d. Neurol. u. Irrenärzte zu Moskau., 6 Oktober, 1900; Ref. N. C., 21, p. 35, 1902.

Weber, F. P. Symmetrical adenomata or nodular hyperplasia of the suprarenal glands and extreme sclerosis of the aorta and coronary arteries. Transact. of Path. Soc., 57, p. 3, London, 1906.

Weber, H. Über Anästhesie durch Adrenalin. 'V. 2I, C. M., 1904.

Weber, L. Über die Behandlung des M. Addisonii mit Tuberkulin. B. k. W., I89I.

Weber, M. Über einen Fall von Hermaphroditismus bei Fringrilla coelebs. Zool. Anz., I3, p. 508, 1890.

Webster, W. Choline in animal tissues and fluids. Biochem. Journ., iv, Nos. 3 and 4 , rgog.

WeIchaRDT. Zur placentaren Theorie der Eklampsieätiologie. A. G., 87, I 909 .

WeichSElBaum. Diskussion z. Vortr. Erdheims. W. k. W., 1906.

WEICHSELBAUM and KYRLE. Über das Verhalten d. Langerhansschen Inseln d. menschlichen Pankreas im fötalen und postfötalen Leben. A. m. A., 74, I909.

WeichSELBAUM and Stangl. Z. Kennt. d. feineren Veränderungen des Pankreas bei Diabetes mellitus. W. k. W., rgor.

- Weitere histolog. Unters. . . Ibid., 1902.

WEIGANDT, W. Der heutige Stand der Lehre vom Kretinismus. Halle, 1903-4.

WeigerT, C. Zur Lehre von den Tumoren der Hirnanhänge. V. A., 65, p. 212,1875 .

- Hemicephalie und Aplasie der Nebennieren. Ibid., Ioo, pp. 176-179, 1885.

- Nachtrag zur Mitteilung. . . . Ibid., 103, p. 204, 1886.

WeIL, M. Über Adrenalin bei Asthma. D. m. W., Igo3.

WEIL, E., and BOYE. Action différente des lobes hypophysaire sur la coagulation du sang chez l'homme et le lapin. C. r. S. B., 67, pp. 192 and 428,1909 .

Weil, E., and Mouriquand, G. Ichthyose et corps thyroïde. Press. méd., Igog.

WeILAND. Über d. Einfl. ermüd. Muskelarb. auf d. Blutzuckergehalt. D. A. k. M., 92,1908 .

Weiler, J. Die Bildungsanomalien der Nebenniere und deren pathologische Bedeutung. Diss., Kiel, 1885 .

Weintraud. Pankreasdiabetes der Vögel. A. P. P., 34, I894.

WeISS, B. Zur Kenntnis der von versprengten Nebennierenkeimen ausgehenden Geschwülste. Zieglers Beiträge, 24, pp. 34-55, 1898 .

Weiss, F. Über den Jodgehalt von Schilddrüsen in Schlesien. M. m. W., 1897. 
WeISS, N. Zur Pathologie und pathologischen Anatomie der Tetanie. W. m. W., p. 683,1883 .

- Uber Tetanie. Volkmanns Samml. klin. Vortr., 7. Serie, No. I89, p. 1696,1880 .

WEISS, O., and HARris, J. Die Zerstörung des Adrenalins im lebenden Tier. P. A., 103, p. 5 I0.

WeissmanN and REISMANN. Die Veränderg. d. weibl. Sexualorg. n. Exstirpation d. Geschlechtsdruisèn. Jahresb. f.' Geb. u. Gyn., 6, 1890.

WELDON, W. F. R. On the head kidney of Bdellostoma with a suggestion as to the origin of the suprarenal bodies. Quart: Journ. Micr. Soc., 24 , pp. 171-183, I 884 .

- On the suprarenal bodies of vertebrates. Ibid., 25, pp. 137-I50, 1885 .

WELECKI. Bull. intern. Acad. Cracovie, p. 768, 1907.

WELLS, H. G., and BENSON, R. L. Studies on calcification and ossification. J. o. M., Res. xvii, p. $15,1907$.

WELSH, R. On the parathyroid glands of the cat. J. o. P. B., 5, p. 202, 1898 .

- Concerning the parathyroid glands. J. o. A. a. P., 32 (12), 1898.

- Influence de l'extrait de rate sur la digestion pancréatique. A. i. Ph., vii, p. $247,1908$.

WENDEL. Zur Chirurgie der Nebennierengeschwülste. A. k. Ch., 73, 1904.

WENCKEBACH, K. F. Zur Entwicklungsgeschichte der Knochenfische A. m. A., $28,1886$.

WERNECKE, TH. K. Zur Wirkung des Thyreotoxin auf das Auge. Klin. Monatstbi. f. Augenhk., I 908 .

WERSILOFF. Ein Fall von Akromegalie. Ref. N. C., I902.

WeRTHEIMER, E. De l'action sur le lait du suc pancréatique sécrété sans l'influence de la pilocarpine. C. r. S. B., 64, H. 10, I908.

Wertheimer and BAtTEz. Sur le mécanisme de la piqûre diabétique. Ibid., Bd. 66, p. 1059, 1909.

Wertheimer and DuboIs. Des effets antagonistes de l'atropin et de la physostigmine sur la sécrétion pancréatique. C. r. S. B., 56, p. 195, I904.

WERTHEIMER and LEPAGE. Sur l'association réflexe du pancréas avec l'intestion grêle et sur les propriétés réflexes des ganglions du sympathique. Ibid.. 51, p. I I 46 , I 899 .

- De l'action du chloral sur la sécrétion pancréatique. Ibid., 52, p. 668, I900.

WesseLY, K. Zur Wirkung des Adrenalins auf Pupille und Augendruck. Vortr. in der Berl. ophth. Ges., I903; and Zeitschr. f. Aug., I3, 1905.

- Zur Wirkung des Adrenalins auf das enukleierte Froschauge und die isolierte Warmbluteriris. D. m. W., 1909.

WESTENHOEFFER. Ein bemerkenswerter Fall von rapid verlaufener Addisonscher Krankheit. Militärärztl. Ztschr., No. 2, Igor.

WestphaL. Weitere Beiträge zur Lehre von der Tetanie. B. k. W., 190 I.

WEYMEERSCH, A. Lésions du thymus obtenues à la suite d'injections d'un sérum thymotoxique. Bull. d. 1. soc. de sc. méd. et nat., Bruxelles, p. 206,1908 .

Whijhe, J. W. van. Über die Mesodermsegmente des Rumpfes und die Entwicklung des Exkretionssystems bei den Selachiern. A. m. A., 33, pp. $46 \mathrm{I}-516$, I 880 .

White, C. P. On the so-called fatty degeneration of the adrenals. J. o. P. B., 1908 .

White, W. H. On Addison's disease. Practitioner, 82, I909.

WHITEHEAD, R. H. The histogenesis of the adrenal in the pig. A. J. A., 2, 1903.

WhItNEY, J. Über die Gesetze der Zuckerausscheidung beim Diabetes mellitus. Z. k. M., 65, p. 476,1908 .

WIEDERSHEIM, R. Lehrbucl der vergleichenden Anatomie der Wirbeltiere auf Grundlage der Entwicklungsgeschichte. 2. Aufl. Jena, 1886.

WIEDERSHEIM, B. Beiträge zur Entwicklungsgeschichte des Urogenitalapparates Krokodile und Schildkröten. A. m. A., 36, 1890; An. An., $5,1890$.

WIEchowsKI, W. Über exper. Beeinfl. d. Kontraktionszust. d. Gefässe im Schädelinnern. A. P. P., 52, 1905 .

Wiefel, A. Über Adenome der Nieren. Diss., Bonn, 1883. 
WIENeR, H. t'ber den Thyreoglobulingehalt der Schilddrüse nach experimentellen Eingriffen. A. P. P., 61, 1909.

WIESEL, J. Akzessorische Nebennieren im Bereiche des Nebenhodens. W. k. W., 1898 .

- Über akzessorische Nebennieren am Nebenhoden beim Menschen und über Kompensationshypertrophie dieser Organe bei der Ratte. S. W. A., 108, 1899 .

- Über Kompensationshypertrophie der akzessorischen Nebennieren bei der Ratte. C. P., 12, pp. 780-783, 1899.

- Über die Entwicklung der Nebenniere des Schweines, insbesondere der Marksubstanz. Anat. Hefte, xvi, H. 1, pp. I17-150, 1900.

- Zur Entwicklung der menschlichen Nebenniere. C. P., 15, pp. 614-615, 1902.

- Beiträge zur Anatomie und Entwicklung der menschlichen Nebenniere. Anat. Hefte, 63 , xix, 3, pp. 481-522, 1902 .

- Chromaffine Zellen in Gefässwänden. C. P., 16, 1903.

- Zur pathologischen Anatomie der Addisonschen Krankheit. Zeitschr. f. Heilk., 24, pp. 257-281, 1903.

- Zur Pathologie des chromaffinen Systems. V. A., 176, pp. 103-114, 1904.

-- Bemerkungen zu der Arbeit von Dr. Iv. Karakascheff. Beiträge zur pathologischen Anatomie der Nebennieren. Zieglers Beitr., 37, p. 169, I904.

- Bemerkungen zu der Arbeit H. Küsters "Über Gliome der Nebennieren." V. A., I80, p. 553,1905 .

- Uber Befunde am chromaffinen System bei Hitzschlag. Ibid., 183, p. 163 , 1906.

- Über Erkrankungen der Koronararterien im Verlaufe akuter Infektionskranheiten. W. k. W., I906.

- Chromaffines Gewebe im Herzen. W. k. W., igo6.

- Renale Herzhypertrophie und chromaffines System. Mitt. d. Ges. f. inn. Med., Wien, 1907.

- Der heutige Stand d. Lehre v. d. Arteriosklerose. Wien, I009.

WIGGERS, C. J. Of the action of adrenalin on the cerebral vessels. A. J. P., 14,1905 .

$\rightarrow$ The innervation of coronary vessels. Ibid., 24, 1909.

Wijhe, W. VAN. Ưber die Mesodermsegmente des Rumpfes und die Entwicklung des Exkretionssystems bei Selachiern. A. m. A., 33, 1889.

WILCox, R. W. Thymic opotherapy. Bost. Med. and Surg. Journ., August I3, 1908 .

Wildt, A. Beitrag zur mikroskopischen Anatomie der Speicheldrüsen. Diss., Bonn, 1894.

WilenKo, G. G. Zur Kenntnis der Glutarsäurewirkung auf den Phloridzindiabetes. D. m. W., I 908 .

Wilks, S. Addison's disease and leucoderma. Lancet, July 28, 1900.

WILlRICH, E. Sklerodermie in Verbindung mit Morbus Addisonii. Diss., Göttingen, I 892 .

Windaus. Die Entgiftung der Saponine durch Cholesterin. B. d. ch. G., 32, 1909 .

IVINTER. Über Wirkungen des Nebennierenextrakts auf das durch grosse Chloroformdosen vergiftete Säugetierherz. W. k. W., I 905.

IVINTERnitz, N. C. Tuberculosis of the parthyroid gland. Bull. Johns Hopkins Hosp., 20, I909.

Wirth, KarL. Tetanie bei Phosphorvergiftung. W. k. W., rgo8.

WithingtoN, Ch. F. Addison's disease with and without adrenal tuberculosis. Amer. Med. News, September 24, 1904.

Wrtzel, O. Die Technik der Pankreasexstirpation beim Hunde. P. A., 106.

WöLFLER, A. Über die Entwicklung und den Bau der Schilddrüse mit Rücksicht auf die Entwicklung der Kröpfe. Berlin, i880.

- Über die Entwicklung und den Bau des Kropfes. A. k. Ch., 29.

- Die chirurgische Behandlung des Kropfes. Berlin, i887; and III. Teil, I89i.

Wolf. Zur Histologie der Hypophysis des normalen und pathologischen Gehirns. V. p.-m. G. Würzburg, N. F., 31, p. 233, 1898.

WolownIK, B. Experimentelle Untersuchungen über das Adrenalin. V. A., 180, p. $225,1905$. 
Wooley. Adrenal tumours. Amer. Journ. Med. Scienc., 125, p. 33, 1903.

WORMSER, E. Experimentelle Beiträge zur Schilddrüsenfrage. P. A., 67, 505,1897 .

- Adrenalincocain in der Geburtshilfe. Schw. Korr.-Bl., No. 23, 1904.

WRAY. Treatment of post-operative shock by pituitary extract. Brit. Med. Journ., 1905.

WRIGHT and JOSLIN. Degeneration of the islands of Langerhans in diabetes mellitus. J. Med. Research 6, 1901.

WurmBraNd, GF. Histol. Unters. an drei oper. Fällen v. Akromegalie mit Hypophysentumor. Ziegl. B., 47, I909.

Wurtz. Maladie d'Addison. Manuel de méd. de Debove et Achard.

WYBAUW. Contribution à l'étude des capsules surrénales dans les maladies infectieuses expérimentales. Bruxelles, 1897 .

IVyss, R. v. Beitr. z. Entwickl? d. Skeletts von Kretinen. Diss., Bern 1899.

YANASE, J. Über Epithelkörperbefunde bei galvanischer Übererregbarkeit der Kinder. W. k. W., 39, 1907; J. K., 67, Ergänzungsh., pp. 57-122.

YATES, J. L. Notes on the experimental production of specific cytolysins for the adrenal, thyroid and parathyroid glands of dogs. Univ. Penns., Med. Bull., 16, 1903 .

YoNewaY and OERTEL. Bemerk. z. Pathologie der Zuckerharnruhr. V. A., $171, \mathrm{p} .547$.

Young, F. A., and LEHMANN, J. E. Internal secretion of the suprarenals. Experiments with the blood-stream from the suprarenal glands of the dog. J. o. P., 37, 1908 .

YUKaWA. Klinisch experimentelle Untersuchungen der Adrenalinwirkung auf die Magendrüsen. A. V., xiv, 166, 1908.

ZaHRADNicky, F. Medulläre Anästhesie kombiniert mit Adrenalin und Suprarenin. Lékarské rozhledy, xiii, 1, 1905; Ref. Schmidts Jahrb., 289, p. 163,1905 .

ZAK, E. Über Hypophysistumoren. W. k. R., p. 165, 1904.

- Glykosurie bei Verätzung des Duodenums. W. k. W., I908.

- Zur Kenntnis der Adrenalinmydriasis. V. 25, C. M., 1908.

- Exp. u. klin. Beobacht. über Störungen sympathischer Innervationen und.intestinale Glykosurie. P. A., 132, 1910.

Zamboni. Sugli effetti della resezione dei nervi del pancreas. Rif. med., 1905.

ZANDER, R. Über funktionelle und genetische Beziehungen der Nebennieren zu den anderen Organen, speziell zum Gehirn. Zieglers Beitr., 7, pp. $489-535,1890$.

- Über die Lage der Dimensionen des Chiasma opt. D. m. W., 1897.

ZANDY. Peritonitisartiger Symptomenkomplex im Endstadium der Addisonschen Krankheit. Z. k. M., 38, r899.

ZANETTI, G. Sur les paralysies des nouveaux-nés et sur la myotonie généralisée d'Oppenheim. Clinica Moderna, 1906.

ZaNfrogniNI, A. Insufficenza paratiroidea e gravidanza. Boll. Acc. Med. di Genova, 1905.

- Eclampsia e anomalia paratiroidea congenita. Istituto ostetr. Genova, 1905.

- La paratiroidina Vassale nel trattamento dell' eclampsia puerperale. Clinica Ostetrica, anno 7, fasc., 9, 1905.

- Eine neue kolorimetrische Methode zur Adrenalinbestimmung. D. m. W., 1909 .

Zappert. Histologische Nervenbefunde eines $1^{\frac{1}{4}}$ Jahre alten Kindes mit tetanischen Krämpfen. IV. k. W., 1898.

ZEIGAN. Untersuch. über subdurale Injektion von Adrenalin und Kokain. Th. M., 1904.

ZESAS, D. G.' Über d. physiol. Zusammenhang zw. Milz u. Schilddrüse. A. k. Ch., 31 .

ZIEGLER, K. Über die Wirkung intravenöser Adrenalininjektionen auf das Gefässsystem und ihre Beziehung zur Arteriosklerose. Zieglers Beitr., $38,1,1905$.

ZIenes. Fall von Gigantismus. Ges. d. Char.-Ärzte, 28 Juni, 1906.

ZiELINSKa, M. Beiträge zur Kenntnis der normalen und strumösen Schilddrüse des Menschen und des Hundes. V. A., 136, 1894 . 38 
ZietzschaAN, Otтo. Ein Beitrag zum Studium der Folgen der Schilddrüsenexstirpation. Thyreoidektomie bei Ziegen. M. G. M. C., I9, 1908 .

ZirNo. Giorn. intern. di scienz med., 3, 1880.

ZINNER. A case of tumour of the pineal gland. The Alienist and Neurologist, xiii, 4, p. 470, I 892 .

ZIMMERMANN, W. Über die Karotisdrüse von Rana esculenta. Diss., Berlin, 1887 .

ZIVIERI, A. Sulla presenza di colina e potassio nel liquido cefalorachideo e nel sangue in alcune malattie mentali. Riv. it. di Neurop., Psich. ed Elettroterap., i, rgo8.

Zöllser. Tumor der Schädelbasis, ausgehend v. d. Hypophyse. A. Psych.,

Zотн, ${ }^{44}$, ${ }^{1908}$ Zwei ergographische Versuchsreihen uber die Wirkung orchitischen Extraktes. P. A., 62, I 896.

- Neue Versuche (Hantelversuche) über d. Wirkung orchitisch. Extraktes. Ibid., 69, 1898 .

Zschech, B. Versuche über die Entstehung von Hautalterationen und Glykosurie bei der subkutanen Anwendung der Nebennierenpräparate. Diss, Mại, 1904.

ZuckerkaNDL, E. Über Nebenorgane des Sympathicus im Retroperitonealraum des Menschen. An. An., Ergäzungsh. zu Bd. 19, pp. 95-107, I9OI.

- Die Entwicklung der Schilddrüse u. Thymus bei der Ratte. Anat. Hefte, 66, 1902 .

-- Über akzessorische Nebennieren bei Torpedo marmorat. An. Hefte, 31, H. I, pp. $219-232,1906$.

Zuelzer, G. Zur Frage des Nebennierendiabetes. B. k. W., rgor.

- Untersuchungen über den experimentellen Diabetes. V. 24, C. i. M., 1907.

- Über Versuche einer spezifischen Fermenttherapie des Diabetes. Z. e. P., $\mathrm{V}, \mathrm{I} 908$.

Zuelzer, G., Dohrs, M., and Mayer, A. Neuere Untersuchungen über den experimentellen Diabetes. D. m. IV., 1908.

- Spezifische Anregung der Darmperistaltik durch intravenöse Injektion des Peristaltik-Hormons. B. K. WV., I 908 .

Zuntz, L. Über den Einfluss der Ovarien auf den Stoffwechsel. I. Menstruation und Stoffwechsel. A. G., 78, 1906.

- Einfluss der Kastration auf den respiratorischen Stoffwechsel. Z. Ch., 95, I 908 .

- Weibliche Geschlechtsorgane. Handb. d. Bioch., iii, Igog.

Zunz, E. A propos du mode d'action de la sécrétine pancréatique. A. i. P., viii, I 1000 .

Zunz and MEYER. Sur les effets de ligature des canaux pancréat. chez le chien. Bull. Ac. méd., Bruxelles, I905. 


\section{N D E X.}

ABORTION following injection of adrenalin, 207

Accessory hypophysis, 314

- organs, compensatory trophy, 19

Aceto-acetic acid, 419, 420

Acetone bodies, normal formation, 4 I9 - - in urine, increase of, see Ketonuria.

-, hot, used in distillation of pigs' suprarenals, 290, 29 I

Acetonitril, subcutaneous injection, resistance to, increased by blood from subjects with Graves's disease, 100

- - - increased by oral administration of thyroid extract, 108, 109

Achondroplasia, 78

Acidosis, 419

Acromegaly, clinical appearances of, 331

-, exophthalmos in, 33 I

-, glandular hyper-function in, 17

-, hypophysal tumour in, 332, 333

-, metabolic changes in, 332

-, pathologico - anatomical findings in, 332

-, sexual derangement in, 331

-, symptoms in, gigantism similar to, 343

$\rightarrow$, therapeutics of, 347

-, tumours of hypophysis associated with, 306

Addison's disease, 126

- - adrenalin contents of suprarenal capsules in, 250

- -, asthenia in, 164

- _, clinical signs absent, with destruction of suprarenal capsules, 162

- - -, with healthy suprarenals, I59, I60

- -, emaciation in, 164

- _, " experimental," 157

- - fatality of, I26

_- nervous or sympathetic theory of, 160

- - normal processes becoming hypoplastic in, 17

- - , pigmentation in, $164,165,168$

- -, symptoms, 126, 163-165

- -, symptom-complex of, 267

tract, I68
Addison's disease, typical findings in, 159

Adiposis dolorosa, 74

Adrenal cells, measure of adrenal contents, 247

- organs, Selachian, extracts obtained from, 247

- system, 129, 130, 131

- - , condition in renal diseases, 262

- - connected with nervous system, I60, 162

- -, development, 132

- - distribution over body wide, 264,265

- - , effect of extirpation of supra-

- renal capsules on, 267

- -, functions, defined, 264

- - nature, defined, 264

- - secretory process, how controlled, 26 I

_- _ - , morphology, 266

- - total suppression impossible, 267

- tissue, chrome-brown, activity of, $25 \mathrm{I}$

Adrenalin, 177 , action of, altered by increase of doses of ergot, 231

- - large doses on dogs, 236

- - limited by internal secretory activity of pancreas, 226

- - modification by thyroid apparatus, 226

- - - by body temperature, 226

- - peculiarities in, 230,231

- - , relation to internal secretory activity of pancreas, 220

- - site, 227,228

- - specific, different from blanching process, 206

- -, tables showing, 222-225

-, action on blood-pressure, 213

- - - , altered by administration of ergot, 231, 232

- - on blood-supply to kidney, 2 I 2

- - on bronchial muscles, 208

- - on composition of blood, 2 I4

- on digestive tract, 203, 204

- on embryonal heart, 202, 203

- on eye, 209

- on gastric secretion, 212

- on genital organs, 206

- - on hair muscles, 208

- on heart after removal from living body, 201 
Adrenalin action, experiments to determine nature, 202

- - neutralized by apocodein, 203

- on heart of cold and warm blooded animals compared, 202

- on lachrymal glands, 212.

- on lymphatics of peritoneum, 213

- - on metabolism, 214, 215

- on muscles, 228,229

- on organs innervated by sympathetic system, ISo

- on pancreas, 212

- on pigment cells and granules of frog, 214

- - on pregnant uterus, altered by ergot, 232

- on pupil, 230

- on respiration, 235

- on salivary glands, 211

- on submaxillary glands, 2 I I

- on sweat-glands, 214

- on sympathetic and autonomous nervous system compared, 230, 231

- - - _- contrasted, 231

- - on urinary bladder, 205

_. on uterus, 206, 207

- - as quantitative test, 207

- - - therapeutic, 207

-, amount in suprarenal capsules, $182,248,249$

-. - - modification by pathological conditions, 249

-, antagonism of cholin to, 276,278

-, by-effects of, $234-236$

- causing abortion, 207

- - mydriasis in Graves's disease, 211

-, cells producing, 253 .

-, chemical tests for, in liquids and secretions, ISo, IS I

- - - - colorimetric methods, I $S_{1}$, I $S_{2}$

- - contents in cats and rabbits compared, $25 \mathrm{I}$

- - of dog, 254

- - of suprarenals in Addison's disease, 250

-, conversion of tyrosin into, 1 So

-, destruction of, factors in, 256,257

- -, diabetes, 220

-, disassimilatory hormone, 206

-, disassociation of auricular and ventricular beats under, 203

--, drugs antagonistic to, in action, 200

-, effects after thyroidectomy and thyroparathyroidectomy, 54, 55

- - modified by previous administration of curarin, 196, 197

- - determined by Meyer's method, 199

- on arteries, 198

- on coronary vessels of heart, I 86
Adrenalin, effects on heart, how and when determined, 200

- on nerve-endings, 197, $19 \delta$

- on pigment in Addison's disease, 165

- on pulmonary circulation, $1 S_{5}$

- - - - vessels negative, 197

- on thyroid gland, 28

-, excretion of, 256

- - of lymph from thoracic duct promoted by, 213

-, formation of, relation to chemical processes of muscles, 206

-, function of, 266

-- -, connected with sympathetic system, 266, 267

Adrenalin-glycosuria, 215, 259, 429

- - accompanied by hyperglycæmia, 216

- - effect of subcutaneous injection of chyle on, 431

- - experimental, and puncture of fourth ventricle, 219

- - inhibition of, 220,221

- - - by fever, 221 (footnote)

-..., by hirudin, 221

_ - - by lymphagoga, 221

- - - by pancreatic extract, 429

- - by pilocarpin, 226

- - mechanism of, 217,218

Adrenalin in blood, 250,251

- - , disappearance, $256-258$

- - persisting after cessation of specific action, 258

- - , ultimate fatc of, 255

_., see also Adrenalxmia

- in collapse, $1 \$ 8,189$

- in heart failure, 188,189

- - - following diphtheria, 180

-, increases secretion of bile, 212

-, inhibitory influence on activity of pancreas, 221,226

-, injection of, contraction of bloodvessels following, 106

-... followed by glycosuria after parathyroidectomy, 68

- - not followed by glycosuria in. thyroidectomized animals, 68

-, intravenous injection, antidote topoisoning by chloral, 201

- - causing dilatation of cerebral vessels, $18 \overline{5}$

- in urine, merhod of testing for, 182 .

-, isolation methods, 177,178

-, matrix of, iso

.- mydriasis, inhibition of, 220

-., neutralization of strychnine by, 295

-, organic composition, 179, 180

-, physiological activity, $18_{3}$

- effects on sympathetic system, 106

- poisoning, symptoms of various animals dying from, $23 \mathrm{~S}$

-, portion of blood-vessels acted on. by, $1 \$ 7$

-, precursors of, 296, 297 
Adrenalin, production of anæmia by, 187

- - of hyperæmia by, 187

- raises heat-tone, 226

--, reaction of suprarenal capsules to, 212

--, resuscitating effect on heart, 201

-, rise in blood-pressure due to, 183 , 184

-, secretion of, process, 253

-, stimulation of myoneural sympathetic terminals. by, 230

- - sympathetic nerves by, under normal conditions and after large doses of ergot, 232, 233

-, subcutaneous injection followed by hyperglycæmia, 217

-, synthetic, 178,179

-, toxic action, 236-246, 259

- _ - on dogs, 240

_ _ _, inflammatory and degenerative, 239

- - intravenously, 236, 239

- _ - on rabbits, 239, 240

- - - subcutaneously, 237, 239

_ - _, on various animals, 236, 237

- - - on vascular system, 240-246

-, toxo-chemical action, 244

-, toxic dose, 236

-, transfusion, continuous, permanent increase of blood-pressure under, 259

-, tyrosin and, 165

-, tests for, 297

-, therapeutic applications, 188

_, time of formation, 251

-, vasoconstriction due to, $I_{4}$

_-, vasodilator properties of, 198

- and apocodein, action on muscles compared, 228

- and muscarin, action compared, 258,259

- and sympathetic system, 219

- as diurecic, 212

- as secretory process of adrenal system, 246

-, see also Phlorizin and adrenalin

Adrenalinæmia and Graves's disease, 264.

-, mydriasis as test for, 262,263

-, pathological human, 262

-, physiological, 254-26o

- - transient, 255

Alanin, 298

Albumin, decomposition of, I 3

-., metabolism of, 267,386

- - after extirpation of suprarenals, I 54

Albuminoids in suprarenal capsules, 275

Albuminuria in tetany of animals, 43 Ammonia poisoning, experimental production, by formation of Eck's fistula, 10

Amniota, suprarenals of, 129

Amphibia, organs representing suprarenal capsules in, 129
Amphibia, suprarenal bodies in, development, 133

Amphioxus, absence of structure similar to suprarenal tissue. in, 129

Anæmia following exhibition of adrenalin, 187

Animals, cold and warm-blooded, action of adrenalin on hearts of compared, 202

-, endemic cretinism in, 83

-, extirpation of hypophysis in, 334

-, goitre in, experimental production, $\mathrm{S}_{2}$

- - natural development, 82

-, suprarenal-less, metabolism of carbohydrates in, 154, 155.

-, thyroidectomized, changes in, 62$7 \mathrm{I}$

-, various, histology of suprarenal cortical granules in, 285

- Anisotropic substances, 289

Antibodies, formation not provoked by hormones, 16

Antigens, hormones and, 16

Antitoxic action of suprarenal capsules, 293, 294

- activity of thyroid glands, 108

Antlers as secondary sexual characters, 360,365

Aorta, atheromatous degeneration following thyroidectomy, $6_{3}$

-, toxic action of adrenalin on, 24

Apathy following suprarenal suppression, 156

Apes, acute tetany in, 41

-, parathyroid glands in, 36

Apocodein, 197

- and adrenalin, action on muscles compared, 228

- neutralizes action of adrenalin on heart, 203

- paralysing effect on nerve endings, 197

Arhythmia, production of by large doses of adrenalin and extract of chromaffine bodies, 236

Arteries, changes due to toxic action of adrenalin on, 24 I, 242, 243

-, effect of adrenalin on, 198 - - $-\frac{}{198}$, when isolated from body,

-, isolated, suspension in Ringer's solution, 198, 199

-, muscles of, effect of adrenalin on, 200

- - sympathetic innervation, 200

-, treatment with adrenalin, 199, 200 ; see also Meyer's method

Arteriosclerosis due to adrenalin, 245, 246

-, hypertension in, 262

Artery, pulmonary, toxic action of adrenalin on, 243

Athyrosis and dwarfism, 80

- and endemic cretinism, 8o

- in man, 7 
Athyrosis, operative, changes following, 71,72

Asthenia in Addison's disease, 164

- following suprarenal suppression I 56

Asthma thymicum, 122

Atropine, paralysis of vagal nerve endings by, 200

- and pilocarpine, action compared, 195

Auto-intoxication in pancreatic diabetes, theory of, 423

Auto-nephrotoxins, formation of, 443

BARIUM chloride, action of, altered by increase in doses of ergot, 231

Basophile cells, 308

Batrachians, suprarenal capsules in, 299

Benzoic acid, affinity for glycocoll, 9

Bile, secretion, increased by adrenalin, 212

Biology, problems of, solved by doctrine of internal secretions, 23

Birds, acute tetany in, 41, '42

-, castration in, results of, 378

-, heart of, action of adrenalin on, 202

-, perversion of sex characteristics in, 365,366

-, portal svstem of suprarenals present in, 300

-, suprarenal capsules in, 128, 130, 133, 292, 300

-, thyroid apparatus in, 36

Bladder, urinary effect of pituitary extract on, 326

- - action of adrenalin on, 205

- - experiments to determine, 205

-, innervation of, differences in mammals, 205, 206

- - - - experiments to determine, 205

Blood, action of thymus extract on, 121

--, adrenalin in, 250, $25 \mathrm{I}$

- - - disappearance, $256-258$

- - remaining after cessation of specific action, 258

_.., ultimate fate of, 255

-. , see also Adrenalæmia

-, alkalinity of, disappearance of adrenalin under, 256

-, changes in, following extirpation of suprarenals, 157,158

-. - following thyroidectomy, 66

-, cholesterinester in, source of, 292

-, circulation of, and humoral pathology, 2

- - in suprarenals of mammals, 300,301

- - , pulmonary, effects of adrenalin on, 185 .

-, composition of, action of adrenalin on, 214

_., effect of castration on, 388
Blood formation, influence of thyroid gland on, 37

-, sugar-contents of, on what dependent, 428

-- , toxicity after extirpation of suprarenals, 156

Blood-letting in tetany, 56

Blood-pressure, action of adrenalin on, 213

-._ _ _ , altered by previous administration of ergot, 231, 232

- - of methylaminoketone on, 213

-, condition of, after extirpation of suprarenal capsules, 147, 148

$\rightarrow$, effect of intravenous injection of organic extracts on, 21, 22

- - of pituitary extract on, 323

- - of renal extract on, 442,443

-, fall of, induced by cholin, 276,277

-, increase induced by suprarenal extract, how abolished, 157

-, intracranial conditions governing,

-, permanent increase following continuous transfusion of adrenalin, 259

$\overrightarrow{7}$, rise in, due to adrenalin, 183,184

Blood-serum, mydriatic, 262

Blood-supply to kidney, action of adrenalin on, 212

Blood-vessels, contraction, drugs inducing, 200

-, contraction following injection of adrenalin, 196

-, dilatation, drugs inducing, 200

-, effect of pituitary extract on, 326

-, portion of, acted on by adrenalin, I87

-, pulmonary, effect of adrenalin on, negative, 197

- , toxic action of adrenalin on, 240

Boiling, activity of thymus extract not affected by, 12 I

Bones, changes in, in acromegaly, 333

-, growth of, effect of pituitary extract on, 328

- - influence of generative glands on, 382

Brain, blood-vessels of, dilated by adrenalin, 185

-, fourth ventricle, puncture of, and experimental adrenalin-glycosuria, 219

- of pigs, lipoid substances in, $290,29 \mathrm{I}$

-, relationship of tetany to, 6o, 61

--, weight of, in gigantism, 342

Bronchi, muscles of, action of adrenalin on, 208

CACHEXIA, chronic, following thyroidectomy, 63

-, following total thyroidectomy, 71, 72,73

-, strumipriva and Graves's disease, symptoms common to, 99 
Cachexia strumipriva, treatment by thyroid extract, 87

- thyropriva, symptoms compared with those of Graves's disease, 94,

- $\stackrel{95}{-}$ see also Myxœdema, postoperative

Ca-ions, 11

Calcium metabolism in relation to parathyroid glands, 57

- salts, treatment by, of tetany, 58 , 50

Capons, characteristics of, 378

Carbohydrates, metabolism, action of adrenalin on, 215

- - after extirpation of suprarenal capsules, 154, I 55

- - central point respecting, 427

- - in Graves's disease, 99

- _, in suprarenal-less animals, I54, 155

-_ - influence of thyroid apparatus on, controlled by pancreas, 430

-..., in thyroidlcss animals, 67,68

Carbonic acid, affinity for urea, 9

- - effect on respiratory centre, 15

Carnauba-acid-cholesterinester, 292

Carotid organ (gland), 302

- - origin, theories respecting, 303

Castration, effects of, 17

- - , on composition of blood, 388

- - on thyroid gland, 344

-, hypertrophy of hypophysis after, 340

-, in relation to thymus gland, 116

-, obesity after, 386

-, results of, 366

- -, in animals, 379

-.. - in birds, 378

- -, on generative glands, 376-382

- . in man, 378,380

-, weight of suprarenals increased after, 260

Cat, acute tetany in, 39,40

-, adrenalin contents in, 25 I

-, effect of iodothyrin on circulatory apparatus in, 90

-, parathyroid glands in, 35

-, suprarenal capsules in, experimental extirpation, I 4 I

Cell-altruism, 5

Cell-complex of ovary, 399

Cell formation of hypophysis, 308

Cells of generation, 359,362

-, somatic, 359,362

Cerebellum, relationship of tetany to, 60,61

Cervidx, secondary sex characteristics in, 360,365

Children, hypothyrosis in, 76,77

Chloral, poisoning by, adrenalin antidote to, 201

Chlorinester, crystallizing, from hot acetone used to distil pigs' suprarenals, 292
Chloroform anæsthesia, sudden death at commencement of, in status lymphaticus, 123

Chlorosis, relationship to ovarian function, 388

Cholesterin palmitate and stearate in suprarenals, 288

Cholesterinesters, 287,288

-, colour reactions, 289

- in blood, source of, 292

-, permanent emulsions of double refractive globules yielded by, 288

Cholin, antagonism to adrenalin, 276 , 278

-, constitutional formula, 277

-, distribution throughout body, 278

- _ - in combination with lecithin, 278

-, effects of, 324

-, fall of blood-pressure induced by, 276,277

- in suprarenal capsules, 275,276

- - , method of extraction, 275 . 276

-, origin of, 278

, physiological activity, 278, 279

Chromaffine bodies, 304

- - extract of, action on dogs, 236

- cells, 132, 133

- -, derivation, 264

- - , staining of, 247,248

- system, effect on metabolism, 7o

- - , hypoplasia of, 158

- - effect, 123

_. - in sudden death in status thymicolymphaticus, ${ }_{5} 8$

- tissue, 6

- -, free grafting of, 173

- - extracapsular, extracts from, action, 247

- - hyperplasia, in true contracted kidney, 263 (footnote)

- - in suprarenal medulla, hyperplasia, 262

Chromates, treatment of myelin forms of suprarenal cortex with, 289

Chrome-brown cells, attached to ganglia of nervous system, 265

Chromophile cells of hypophysis, 308 , 3 I I

Chrysotoxin, action of, 198

Chyle, flow of, how increased, 444

- - subcutaneous injection, effect on adrenalin-glycosuria, 431

Circulatory system, effect of pituitary extract on, 323

- - physiological effects of thyroid extracts on, 89 , 90

Coccygeal gland, 304

-- , human, primal origin, 305

Collapse, adrenalin injection in, 188 , 189

Colloid in parathyroid glands, 33

- substance of the hypophysis, 312

Coma, diabetic, cause of, 419,420 
Conarium, see Pineal body

Corpus luteum, function of, 400, 402, 407

- -, hypothesis, 404

- -, menstruation originating in, 406

- - verum, cells of, similarity of cells of suprarenal cortex to, 260

Crabs, parasites causing neuter characteristics in, 367

Cretinism, endemic, 79

- - and athyrosis, 8o

- -, clinical signs, 79

- -, deaf-mutism in, 70

-..., geographical distribution, 79

_ _ - , same as that of endemic goitre, 81

-_ - in animals, $8_{3}$

_ _, metabolism in, 89

_ - treatment by thyroid extract,

Cryptorchidism, suppression of spermatogenesis, 393

-, unilateral, 393-394

Curarin, effects of adrenalin, modified by previous administration of, 196,197

Cyanophile cells, 308

Cytotoxins, specific, attempted destruction of suprarenal capsules by, 144

DEAF-MUTISM in endemic cretinism, 79 Decomposition, products of, effects of over-irritation by, 15.

- - , formation in intestinal canal, 7 Dercum's disease, see Adiposis dolorosa Diabetes, changes in pancreas in, 432 -, clinical, and experimental pancreatic, analogy between, 431, 432

-, duodenal, 422

-, pancreatic, 220

- - and auto-intoxication, 423

- -, experimental in animals of different species, 414

- - , in $\operatorname{dog}_{2}+14$

- -, failure of organo-therapy in, 423,424

- - glycosuria in, factors influencing, 416,417

- -, metabolism in, 415

- -, mydriasis characteristic of, production, 431

- - , nervous origin, theorv of, 422

- -, permanent factors originating, 432

_- -, theories respecting, 420-432

-, see also Adrenalin-diabetes

Diet, effect on glycosuria in pancreatic diabctes, 416

Digestive tract, action of adrenalin on, 203, 204

Dimorphism, sexual, 361

Diphtheria, heart failure following adrenalin administration in, 189

Diseases, reflex manifestation of, 2

Diuresis due to pituitary extract, 327
Diuretic action of thyroid extract, 91

-, adrenalin as, 212

Dog, acute tetany in, 39, 40

-.., adrenalin contents of, 254

-, effects of large doses of adrenalin and extract of chromaffine bodies on, 236

- effects of ligature of excretory ducts of pancreas on, 436

-, effect of thyroidin on, 92

-, emaciation in, following extirpation of pancreas, 420

-, extirpation of hypophysis in, 319 , 320

-, parathyroid glands in, 35

__ - , supernumerary, 35

-, production of pancreatic diabetes in, 414

-, suprarenal capsules in, experimental removal, 14 I

-, thymus gland in, 113

- _ - extirpation, results, 114,117

- - implantation into, I 19,120

-, thyroidectomized, changes in, 65

- - , staggering gait of, 61

-, toxic action of adrenalin on, 240

Ductless glands, functions of, early discoveries, 3

- - importance of, 17

Duodenum, see Diabetes, duodenal

Dwarfism, 78

- , athyrosis and, so

Dystrophia adiposogenitalis, 17, 345

Eck's fistula, formation of, leading to ammonia poisoning, Io

Eels surviving removal of suprarenal capsules, 153

Eggs, fertilization of, 356

Electric current, thymus gland and reaction of motor apparatus to, 120

Emaciation in Addison's disease, 164

- following extirpation of pancreas, 420

- following extirpation of suprarenals, 154

- , following thyroidectomy, 66

Embryo, development of hypophysis in, 313

Enzymes, organic substances formed by, in process of decomposition of sugar, 426

Eosinophile cells of hypophysis, 308

Epilepsy, colloid in hypophysis, as cause of, 305

Epinephrectomy, see Suprarenal capsules extirpation

Epinephrin, 178

-, toxic dose, 236

Epiphysis, see Pineal body

Epoöphoron, 410

Ergot, administration of, increase in, alters action of adrenalin, 231

-, alters action of adrenalin on pregnant uterus, 232 
Ergot, effect of adrenalin on bloodpressure, altered by, 231, 232

-, inhibitory action of, site of discussed, 233, 234

- , poisoning by, acute, 232

-, stimulation of sympathetic nerves by adrenalin after large doses of, 232, 233

Ergotoxin, effect on sympathetic system, I96

Excretion, distinction from secretion, 3

Excretory organs, expulsion of toxic substances by, 8

Exophthalmos in acromegaly, 331

- in Graves's disease, 96,97

Extremities, upper, effect of mountain climbing on, 14

Eunuchs, characteristics of, 378

Eye, action of adrenalin on, 209

-, diseases of, in acromegaly, 331

-, symptoms following intravenous injection of thyroid juice, 92

FASTING, prolonged, followed by glycosuria, 216

Fat, absorption after ligature of excretory ducts of pancreas, 437

- - $\rightarrow$ ceases after total extirpation of pancreas, 437

- distribution in suprarenal capsules, 285

- granules in epithelial cells of parathyroid glands, 32,33

--, labile, in spongiocytes, $2 \$ 2$

Fatigue, muscular, suprarenal capsules in relation to, 295

Fats, metabolism of, effect of thyroidectomy on, 7 I

Females, incidence of Graves's disease among, 102

Femininity, 362

Ferments in suprarenal capsules, 275

Ferret, motor and inhibitory innervation of bladder in, derivation, 205

Fever, inhibiting adrenalin glycosuria, 221 (footnote)

Fish, cortex and medulla of suprarenal capsules distinct bodies in, I 30

-, embryology of pineal body in, $35 \mathbf{I}$

-, heart of, action of adrenalin on, 202

-, interrenal body in experimental extirpation, I 50,152

-, suprarenal capsules in, extirpation not fatal, 152, 153

$\rightarrow$, thyroid apparatus in, 36

Fistula, pancreatic, 437,438

Flesh-free diet in Graves's disease, 107

Fotus, extract of, effect on mammary gland, 373

-, sexually differential tissues in, 361

Fowls, extirpation of thymus in, i14, I 16

Frog, extirpation of hypophysis in, 316
Frog, extirpation of thymus in, results, I 5

-, pigment cells and granules of, action of adrenalin on, 214

-, suprarenal capsule in, experimental extirpation, 140 , I 4 I

Frog's cye, isolated, action of adrenalin on, 209, 210

Function, suspension of, effects, to

Functional equilibrium, disturbances of, explanation, 2

GANGLION cells, particular, affected by nicotine, 194

Gastric and intestinal mucosa, internal secretion, 438

- secretion, action of adrenalin on, 212

Generation, cells of, 359,362

-, organs of, female, physiological association of thyroid gland with, 37

Generative glands, developmental history of, 358

- - , effect of Röntgen rays on, 396

_. - extract of, $389-391$

- - , hormone in, sexually undifferentiated activity of, $382-380$

- - influence upon growth of skeleton, $3 \delta_{2}$

- - internal 'secretory tissue, elements of, $391-410$

- - relationship to osteomalacia, 387

_...., results of castration, $376-382$

Genitals, defective, in gigantism, 342 , 344

- (external), action of adrenalin on, 206

-, female, tumours of, derived from Marchand's accessory suprarenals, 270

-, internal secretory tissue, elements of, 410-412

-, hypoplasia of, 340,349

- (internal), action of adrenalin on, 206

Genito-vesicular reflex, 390

Gigantism, infantile type, 344

-, pathogenesis of, 343

-, relationship to hypophysis, 341

-, sex incidence of, 342

-, symptoms in acromegaly similar to, 343

-, weight of brain in, 342

Glandulæ pinealis, see Pineal body

Glandular secretions, classification, $\delta$

- - , deficiency or superfluity effects,

Globules, double refractive bodies showing, 288

- - colour reactions, 280

Glycogen, accumulation in liver, 427, 428

-, content of leucocytes, increase in, cause, 418 
Glycogen, conversion into grapesugar, 428

-, deposition in liver after extirpation of pancreas, how effected, 430

-, formation in liver, 216

-. in liver, reduced after extirpation of pancreas, 4 I 8

- in muscles, diminishing after extirpation of pancreas, $4 \mathrm{I} S$

Glycogenesis, 4

Glycogoll, affinity of benzoic acid for, 9 Glycolytic ferment in muscles, 426

- - , theory of, 424,425

Glycosuria, absent after adrenalin injection in thyroidectomized animals, 68

_ _ - after partial extirpation of pancreas, 415

-, alimentary, in Graves's disease, 99

-, factors originating, 432

- following administration of adrenalin, sec Adrenalin-glycosuria

- following inhibition of internal secretion of pancreas, 428

- ligature of thoracic duct, 431

- prolonged fasting, 216

- - total extirpation of pancreas, 413

- in acromegaly, 332

- in pancreatic diabetes, factors influencing, 416, 417

- - immediate cause of, 418

-, marked after adrenalin injection following parathyroidectomy, 68

-, production following phlorizin in suprarenal-less animals, 155

-, site of origin, 297

-, slight, follows extirpation of larger portions of pancreas, 416

-, test for, 297

Glycuronic acid, affinity for mercapturic acid, 9

Goat, parathyroid glands in, 35

-, tetany in, $4 \mathrm{I}$

-, thyroidectomized, administration of thyroid extracts to, 86

- - changes in, 64,65

Goitre, cardiac symptoms, 103

-, causal agent unknown, 82

-, endemic, etiology, $S_{1}, 8_{2}$

-- - geographical distribution of endemic cretinism same as that of, 8 I

-, extirpation, preservation of parathyroid glands during, 44

-, geological distribution, $\delta_{\mathbf{I}}$

-, hyperplastic, reduction of amount of iodine-thyroglobulin in, 30

- in animals, $8_{2}$

- in Graves's disease, 95

-, relation of iodine in thyroid gland to, $29,30,31$

-, removal, thyroidectomy must be partial in, 73

-., simple, 102
Goitre, simple, functional aspect not identical with hyperthyroidism, 103

-, soil in relation to, $8 \mathbf{I}$

-, transmission by drinking water, $S_{I}$

Goitre-heart, 19

Graafian follicles, bursting of, 406

-- - function of, 401

Grape-sugar, conversion of glycogen into, 428

-, intravenous injection, effect on islands of Langerhans, 435

Graves's disease, 93

- - alimentary glycosuria in, 99

- - and cachexia strumipriva, symptoms common to, 99

-_ _ associated with adrenalæmia, 264

-, , blood from, increases resistance to acctonitril, subcutaneously injected, 100

- - , due to flooding of organism with thyroid substances, 102

- - etiological connection with thy roid gland, 100, 101

- - exophthalmos in, 96, 97 .

- - Hesh-free diet in, 107

- - glandular hyperfunction in, 17

- - goitre in, 95

- - , hypertroplyy of heart in, 96

_. - - of thymus in, 96

- - incidence among females, 102

- - metabolism in, greatly increased, 98

- - mydriasis produced by adrenalin in, 211

- - myxoedema and, difference between, 99

- - reduction of amount of iodinethyroglobulin in, 30

- - symptoms and organic changes in, $95-98$

- - compared with those of cachexia thyropriva, 94, 95

- - in relation to those of experimental hyperthyroidization, I03

- _ - resembling those of found

in other diseases, 99

- - treatment by adrenalin causing mydriasis, 211

- - by serum-therapy, 100

- - by thyrotoxic immune serum, 10

- - operative, 100

- - by organo-therapy, 100

- - by X-ray, 100

- - vasomotor excitability in, 97

Guinea-pig, suprarenal capsules in, exceptional weight of explained, 269

- - cxperimental removal, I4I, 144

thymus gland in, 113

- - extirpation, results, 116

Gynæcomastia, 372

H.EMOLYSINS, action of, is 
Hair-muscles of different animals, action of adrenalin on, compared, 208

Heart, action of adrenalin on, experiments to determine nature, 202

_- neutralized by apocodein, 203

- after removal from living body, action of adrenalin on, 201

-, coronary vessels of, effect of adrenalin on, 186

-, effect of adrenalin on, how and when determined, 200

- - of pituitary extract on, 323-325

-, embryonal, action of adrenalin on, 203

- failure, adrenalin in, $188,{ }_{1} \$ 9$

- - following diphtheria, adrenalin in, 189

-, hypertrophy accompanying hypertrophy of thymus, 19

- - in Graves's disease, 96

-, resuscitating effect of adrenalin on, 201

-, toxic action of adrenalin on, 240; see also Goitre-heart

Heart-beats, auricular and ventricular, disassociation under adrenalin, 203

Heart-muscle, chemical changes in, nature, 14

Heart-punctire, 221 (footnote), 226

Heart-symptoms of goitre, 103

Heat-tone raised by adrenalin, 226

Hens, extirpation of hypophysis in, 316

- , thyroidectomized, changes in, 64

Hermaphroditism, 357, 362

$\rightarrow$ glandular, 363

Hippuric acid, where formed in body,

Hirudin, suppression of adrenalinglycosuria by, 22 I

Homœopathy, isopathy, a branch of, 2

Hormone, 5, 6

-, definition of, 5

-, dissimilatory, action of thyroid gland secretion as, Iog, I 10

- -, adrenalin as, 266

-, genital, relationship of Leydig's cells to, 398

- in generative glands, activity of, $382-389$

-, mammary, origin of, $374-376$

-, pancreatic, 431

- -, action of, 429, 430

- - isolation, so far, impossible, 430

Hormones, activity of, 12

- and antigens, 16

-, chemistry of, 16

-, classification, 13

-, dissimilatory, 13, 109, 266

-, distinction from nutrient secretions, 13

-, formation of antibodies not provoked by, 16
Hormones, inhibitory, I4

-, negative properties, 16

-, origin of, 15

-, pancreatic, 423,424

- , production of, 8

Hydrogen ethyl sulphates, toxic bodies with affinity for, 9

Hyperadrenalinism, experimental, 261

Hyperemia, following exhibition of adrenalin, 187

Hyperglycæmia accompanying adrenalin-glycosuria, 216

- following subcutaneous injection of adrenalin, 217

-, immediate cause of glycosuria in pancreatic diabetes, 418

- in suprarenal-less dogs, 155

-, phenomena accompanying, 4 I 8

- , source of, 419

Hypernephroma, extracts of, 251

- , renal, 270

Hyperparathyroidism, diseases following, 46

Hyperpituitarism, 335, 337, 345

Hyperthyroidism, experimental, 92

-, following administration of thyroid extracts, 90

-, functional aspect of simple goitre not identical with, 103

-, human, following administration of thyroid extracts, 92, 93

- , true, conditions classed under, 93 Hyperthyroidization, experimental symptoms in relation to those of Graves's discase, 103

Hyperthyrosis in man, $7 \mathrm{I}$

Hypoparathyroidism, diseases following, 46

Hypophysectomy, experimental, 315319

Hypophysis, accessory, 3 I4

-, anatomy of, 306-30S

-, chemistry of, $\mathbf{3 2 2}$

-, change in function, 110

-, death following removal of, 3 s.$32 \mathrm{I}$

-, development of, history, 313

-, diseases of, $330-348$

-, effects of, 326

- - on blood-pressure after stimulation of, 329

-, embryonal, development of, 313

-, enlargement, following thyroidectomy, 63,66

-, experimental extirpation of, 315319,334

- - stimulation of, 328-330

-, function of, 306

- - determined by experiment, 18

- $\ldots$ theory of, 305, 309, 348-50

-, histology of, 308

-, hyperfunction of, 338

-, hypertrophy of, 335

- - following extirpation of thyroid, in

-, influence of pregnancy on, 307. 309,310 
Hypophysis, nervous portion of structure of, 310

-, physiology of, 315-32 I

-, relationship to gigantism, 34I

_.. to sexual glands, 339-34 I

- - to suprarenals, 339

- - to thymus, 339

- - to thyroid gland, 338

--, sarcoma of, 334

-, secretion of, histology of, 31 I

-., sources of origin, $3 \mathrm{I}_{\mathbf{5}}$

-, tumour of, extirpation of, in man, 336

- - , in acromegaly, 306, 332, 333

- - obesity associated with, 345-347

-, weight of, 307

_, see also Pituitary extract

Hypothyrosis, metabolic changes in 88

$\rightarrow$ in children, 76,77

Humoral pathology, 2

_- and circulation of blood, 2

INDICAN reaction in urine after removal of suprarenal capsules, I 54

Infantilism, 77

-, dystrophic, 77

-, following thyroidectomy, 64

-, myxodematous, 77

Infants, laryngeal spasm of, 122

- tetany in, 45

Infective diseases, mortality from, great among subjects of status thymicolymphaticus, 124

Internal secretion, definition and limitation of term, 5

- - doctrine of, date of origin, 4

- - - when first formulated, 3

Interrenal body in fish, experimental extirpation, 150

- bodies, accessory, 131, I34

- - compensatory hypertrophy of portions of, 268

- in fish, experimental extirpation, fatal, 152

- structures, accessory, 268

- - - connection of suprarenal capsules with, 268

-- systems, 129-131, 267-270

- -, development, 131, 132

- _, functional significance of free portions, 268

- - function of theory as to, 292294

- tissue, importance of to life, 302

Interrenals, accessory, in white rats, 155

Intestinal canal, formation of products of decomposition in, 7

- mucosa, secretion, mechanism of, 440

- tract, atrophy present in senile degeneration, absent in myxcedema, 76

Intestines, action of adrenalin on, determined by experiment, 204
Intracranial blood-pressure, conditions governing, 329

Invertebrates, adrenal system in, 135

-, suprarenal systems in, tables illustrating, $135-137$

Invertin, action of, inhibited by levulose, 419

Iodine, distribution in human organs, 30

- in thyroid gland, 29, 30, 106

- - , relation to goitre, 29, 30, 31

Iodine-thyroglobulin, 30

-, reduced in hyperplastic and exophthalmic goitre, 30

Iodization, process of, in thyroid gland, 105

Iodothyrin, 30

-, administration of, after thyroid suppression, 87

-, effects on circulatory apparatus, in animals, 90

-, function, 37

Iodothyro-globulin, effects on circulatory apparatus, $\delta_{0}$

Ions, function of, I I

Islands of Langerhans, see Langerhans, islands of

Isopathy, branch of homoopathy, 2

-, defined, 2

JECORIN, 286

Ketonuria, expcrimental, difficult to obtain, 420

-, symptoms of diabetes, 419

Kidney, blood-supply to, action of adrenalin on, 212

-, contracted, true hyperplasia of, chromaffine tissue in, 263

-, disease of blood from subjects of, containing lymphagogue substance, 444

- disease, condition of adrenal system in, 262

-, effects of pituitary extract on, 325, 327

-, internal secretion, 440

- - - effect of renal extract on, 442

--, parenchyma of, reduction of, effects, 444, 445

-, transplantation of suprarenal capsules in, $170,171,172$

LACHRIMAL glands, action of adrenalin on, 212

Lactic acid and respiratory centre, is Langerhans, islands of, concerned in internal secretory function of pancreas, 436,437

- - effect of injection of phlorizin and adrenalin on, 435

- - - of intravenous injection of grape-sugar on, 435

- - function, 433,434

Lecithin, cholin combined with, in body, 278 
Lecithin, function of, 289

-, pure, hardly obtainable, 290

- in suprarenal capsules, 286

Lecithin-carbohydrate, 286

Leucocytes, glycogen content, cause of increase, 418

Levulose, administration of, effect, 430

-, , experimental results, 417,418 , 419

- inhibits action of invertin, 419

"Leydig's between-cells," 392

Leydig cells, 394

- -, relationship to genital hormone, 398

Lipoid granules in cells of suprarenal cortex, 272

- substances, 2 I

- - in suprarenal capsules, 292, 293

Lipoids, suprarenal, 279, 287

Liver, accumulation of glycogen in, 427,428

-, deposition of glycogen in, after extirpation of pancreas, how effected, 430

- , effect on destruction of adrenalin, 257

-, formation of glycogen in, 216

- - of sugar in, inhibited by pancreas, 430

- - - , how controlled, 428,429 urea by, antitoxic function, 9

-, glycogen in, reduced after extirpation of pancreas, 418

Lutein cells, origin of, 401

- tabloids, therapeutic use of, 405

Lymph, excretion from thoracic duct, promoted by adrenalin, 213

- glands, cells of thymus gland not identical with those of, 112

Lymph-vessels, passage of thyroid secretion into, 28

Lymphagoga, suppression of adrenalinglycosuria by, 221

Lymphagogues, 443,444

-, blood from subject of kidney disease acting as, 444

Lymphatic ducts, ligature of, 22 I

Lymphatics, peritoneal, action of adrenalin on, 213

Mammals, blood-circulation in suprarenals of, 300,301

-, cortex and medulla of suprarenal capsules amalgamated in, 130

-, innervation of bladder, differences in, 205, 206

_-, suprarenal bodies in, development, 133

Mammary glands, 355,360

_ - development of, 372,375

Marchand's accessory suprarenals, tumours of female genitals derived from, 270
"Masculinity," 362

Menstruation, connection of ovulation with, 403, 406

-, disordered, in acromegaly, 331, 337

-, origin of, 406

-, relationship of ovary to, $3 \delta$ I

Mental changes following thyroidectomy, 65

Mercapturic acid, affinity of glycuronic acid for, 9

Metabolic changes in hypothyrosis, 88

- - - how influenced by thyroid extract, 88

Metabolism, action of adrenalin on, 214,215

-, effects of pituitary extract on, 327

- $\rightarrow$, thyroid extract on, 9 I

-, end-products of, function, $1 j$

- in endemic cretinism, 89

- in Graves's diseasc grcatly increased, 98

- in myxœdema, 88,89

- in pancreatic diabetes, 415

-, processes of, changes following thyroidectomy, 67

- -, glands accelerating, 70

- - - retarding, 70

Methylaminoketone, action on bloodpressure, 213

Meyer's method, effects of adrenalin determined by, 199

Mice, parathyroid tetany in, 41

Milk diet in acute tetany, 107

- , secretion of, 372,375

Mongolism, diagnosis from sporadic myxœdema, 78

Monoamidomonophosphatide cephalin, 292

Mors thymia, see Status thymicolymphaticus, sudden death in

Mountain climbing, effect on upper extremities, 14

Muscarin and adrenalin, action compared, 258, 259

Muscles, action of adrenalin on, 228 , 229 - - pared, $22 \mathrm{~S}$ apocodein on, com-

-, chemical processes, relation of formation of adrenalin to, 296

-, effect of pituitary extract on, 326

-, glycogen in, disappearing after extirpation of pancreas, 418

-, glycolytic ferment in, 426

-, involuntary nerve supply to, I9 1

-, toxicity of, following extirpation of suprarenals, 156

-, unstriated, action of adrenalin on, 229

- - and sympathetic nervous system, 220

$\rightarrow$, vascular, see Arteries, muscles of

Muscular exertion, effect on glycosuria in pancreatic diabetes, 417

- weakness following suprarenal suppression, 156 
Myasthenia gravis pseudoparalytica, cause of, 46,47

Mydriasis, characteristic of pancreatic diabetes, production of, 43 I

- due to adrenalin in Graves's disease, 211

- due to blood-serum from chronic nephrosis, 262

- due to pituitrin, 322

- following suppression of sympathetic function, 210, 211

- following treatment by thyroid extract, 211

- of frog's eye, due to adrenalin, 200, 210

- produced by adrenalin in Graves's disease, 211

-, production of, 297

-, test for adrenalamia by, 262, 263

-, sec also Adrenalin mydriasis; Pupil, dilatation of

Mrelin, 283

-, double refractive substances in suprarenal cortex resembling, $2 \mathrm{~S}_{4}$

- forms, double refractive bodies showing, 288

Myoneural junction, 229, 233, 234

Myotonia congenita, cause of, 46

- periodica, cause of, 46

Myxodema, abortive, 74

-, appearances in, 72

.., atrophy of intestinal tract present in, 76

-, congenital, 76

- following thyroidectomy, 66

-, infantile, 76

-, metabolism in, $s \delta, S o$

-, normal processes becoming hypoplastic in, 17

-, post-operative, $3 \delta, 7$ I

-, sporadic, diagnosis from mongolism, 78

-, treatment by thyroid extract, 8\%, IO7

- and Graves's disease, differences between, 99

Neck, organs of, development, 33

Nephrectomy, experimental, 44I, 442

Nephritis, chronic, hypertension in, 262

-, toxic, experimental results, 417

Nephroblaptins, 443,444

Nephrosis, chronic, blood serum from, causing mydriasis, 262

Nerve cells in hypophysis, 310

Nerve-endings, effect of apocodein on, 197

-, sympathetic, as site of action of adrenalin, 228

- - effect of adrenalin on, 197

- - myoneural stimulation adrenalin, 230

-, vagal, paralysis by atropine, 200

Terve-fibres, efferent, supplying vegetative organs, 190
Nerve-roots, spinal anterior, function, 190

Nerves, autonomous, table showing, 192

-, resection, experimental, to discover seat of tetany, 59, 60

-, sympathetic and autonomous, effects of stimulation on, comparative table showing, 222-225

- - stimulation by adrenalin, under normal conditions and after larger doses of ergot, 232, 233

- - table showing, 193

Nervous centre, impressions conveyed from peripluery to, effect of, I

- excitement, effect on thyroid gland, 28

- origin of pancreatic diabetes, 422

- system, autonomous, 19I

- - - definition, 19 I

- - - differentiation from sympathetic, 104, 105

- - adrenal system connected with, 16o, 162

- - central, regulation of organic function bv, I

- -, centres of, in relation to formation of sugar, 428,429

- - cerebrospinal, 190

- classification, 190, 191

- - effect of extirpation of thymus on, 120

- - , ganglia of, chrome-brown cells attached to, $26_{5}$

- - sympathetic and autonomous, action of adrenalin on, compared, 230, 231

- - adrenalin and, 219

- - and autonomous, action of adrenalin on, contrasted, 231

- - - effect of adrenalin on organs controlled by, i 89

- - - on dilatation of pupils, 210

- - - , function, 191

- - - of adrenalin connected with, 266, 267

- - hyperirritation of, 218

_- - physiological effects of adrenalin on, 196

- - - unstriated muscles and, 229

- - vasodilator function, 198

- -, vegetative, 100-196

- -..., effects of nicotine confined to, 195

- - - effect of internal secretory organs on, 70

- - - effect of toxic substances on, 195

$-\cdots-$, irritability of, 110

Neural theory of correlation of organs, 2

Neurin, constitutional formula of, 276 (footnote)

- gold, 276, 277

Neuro-hypophysis, histology of, 310 
New-born infants, pincal gland in, $35 \mathrm{I}$

in, 361

Nicotine, action of, altered by increase in doses of ergot, 231

- effects confined to vegetative nervous system, 195

-, particular ganglion cells affected by, 194

Nitrogen, excretion after reduction of renal parenchyma, 445

-, metabolism of, in Graves's disease, 99

Nutrient secretions and hormones, distinction between, 13

OBESITY associated with hypophysal changes, 345

-, origin of types of, 385

- reduction by thyroid extracts, $9 \mathrm{I}$

Organic extracts, intravenous injection, effect on blood-pressure, 21,22

- _, physiological action, 21,22

-. - toxic action, 2 I

Organotherapy, 20

- after experimental extirpation of suprarenal capsules, 165-168

-, failure in pancreatic diabctes, 423, 424

-, forerunners of, 2

- in Graves's disease, 100

-, uses of, II

Organs of body, chemical correlation,

- 5 , experimental extirpation, advantages, 18

- - - - by-effects, 19

- - internal secretory, 6

_ - partial destruction, i9

- - potential energy, Io

Ossification, effect of extirpation of thymus on, 117, I 18

-, influence of generative glands in, 382

-, retardation of, following thyroidectomy, 63

Osteomalacia, relationship to genital glands, 387

Ovarian extract, effects of, 380

Ovaries, relationship of, to chlorosis, 388

$\rightarrow$, transplantation of, 380

Ovary, influence of X-rays on, 403

-, internal secretion of, 398-406

-, interstitial tissue of, 399, 402

- relationship to menstruation, $38 \mathrm{I}$

Ovotestis, 363

Ovulation, connection of menstruation with, 403,406

Ovum, nidation of, 408

Oxidation, increased, following extirpation of pancreas, 420

Oxybutyric acid, 419,420

PANCREAS, action of adrenalin on, 212
Pancreas, agency in influence of thyroid apparatus on carbohydrate metabolism, 430

-, changes in, in diabetes, 432

-, effects on metabolism, 70

-, excretory ducts, experimental ligature, results, 436

- _ _ _ _ , absorption of fat after, 437

- external secretion, mechanism of, 439

-, extirpation, deposition of glycogen in liver after, how effected, 430

- -, experimental, change in metabolism following, 420

-. - - increased oxidation following, 420

- - - emaciation following, 420

- -, partial, not followed by glycosuria, 415

- - of large portions followed by slight glycosuria, 416

- - total, followed by cessation of fat absorption, 437

- _ - , followed by glycosuria, 413; see also Diabetes (pancreatic)

- _ - , reduction of glycogen in liver under, 418

-, function of, inhibitory, 2 I I

- - in relation to decomposition of sugar, 426

-., internal secretion of, 412

… inhibition followed by glycosuria, 428

- _ _ _ - of formation of sugar in liver by, 430

- - secretory activity of, action of adrenalin and, 220

- - - _ , inhibited by adrenalin, 221,226

- - - limiting action of adrenalin, 226

- - - elements of, $432-438$

- _, function, $18,421,423$

- _ - , acini and islands of Langerhans concerned in, 436, 437

-, transplantation experimental, 422

- and metabolism of sugar, 424,425

- and thyroid gland, relationship between, 69,70

-, see also Hormones, pancreatic; Langerhans, islands of

Pancreatic extract, inhibition of adrenalin glycosuria by, 429

Paralysis agitans, cause of, 46

Parathyroid extract, 49

- -, physiological activity, 49, 50

- -, treatment by, of post-operative tetany, 49

- glands, accessory, 31

- -, anatomy, 31

- -, colloid in, 33

- -, comparative anatomy, 34

- -, development, 34

- -, effect on metabolism, 70 
Parathyroid glands, extirpation, results, 38

__ , fat granules in epithelial cells, 32,33

- - function, 55

- - - determination of, 18

- - functional derangement, diseases, following, 46

- - glycogen in, 33

- -, histology, 32

- - preservation, at extirpation of goitre, 44.

- - in relation to calcium metabolism, 58

- - , secretory function, 32

-. - , supernumerary, 35

- -, transplantation, 48

__ and thyroid gland, relationship between, 51-55; see also under Thyroid gland

Parathyroidectomy, effects of, 53, 54, 55,84

--, experimental, results, $4 \mathrm{I}$

-, tetany following, 55

- with remnant of thyroid tissue left, result, 106

Parietal eye in saurians, 351

Parkinson's discase, see Paralysis agitans

Penis, retractor muscle in rat, nervous network, 228, 229

Periphery, impressions conveyed from, to nervous centre, effect of, I

Peritoneum, lymphatics of, action of adrenalin on, 213

Phenylalanin, 297

Phlorizin, action on suprarenal-less dogs, I 55

- and adrenalin, injection, effect on islands of Langerhans, 435

Phosphates in urine after removal of suprarenal capsules, 154

Phosphorus, excretion of, due to pituitary extract, 327

Phrenosin, 288

Pigeons, effects of ligature of excretory ducts of pancreas on, 436

Pigment cells and granules of frog, action of adrenalin on, 214

Pigmentation, anomalies of, following extirpation of suprarenals, 157

-, disordered, in Addison's disease, I64, 165,168

$-\frac{1}{165}-$, effect of adrenalin on,

Pigs, brain of, lipoid substances in, $29 \mathrm{I}$

-, suprarenals of, lipoid substances in, 290,291

- thyroidectomized, changes in, 64

Pilocarpin, adrenalin-glycosuria inhibited bv, 226

- effect on thyroid gland, 28

_- and atropine, action compared, 195

Pineal body, anatomy of, 35 I

- - clinical signs of diseases of, 354
Pineal body, embryology of, 351

- -, histology of, 351

- - in newborn infants, 351

-_ - pathological anatomy of, 353

Pituitary body, see Hypophysis

- extract, action of, altered by increase in doses of ergot; 231

$\ldots \ldots$, cardiac effects of, 323-325

- - characteristics of, 349

- - , effect on circulatory system, 323

- - - on metabolism, 327

- - , hypotensive action of, 324, 325

- - in acromegaly, 347

- - physiological activity of, 323

- - toxicity of, 322

- - vasoconstrictor action of, 321 , 324

- gland, see Hypophysis

Pituitrin, mydriasis due to, 322

Placenta, extract of, effect on mammary gland, 374

$\rightarrow$, internal secretion. of, 4 I I

Pneumothorax following experimental extirpation of thymus, 117, 119

Poisons, inorganic, resistance to, small after thyroidectomy, 108

Polyuria after extirpation of suprarenals, 154

- following reduction of renal parenchyma, 445

Portal system of suprarenals 298, 299 - - in birds, 300

$\rightarrow-$ in reptiles, 299

Potential energy of organs of body, I I

Precocity, sexual, 370

Pregnancy, enlargement of hypophysis during, 340

-, increase of weight of hypophysis in, 307, 309, 310

- - of suprarenal capsules during, 269

- interstitial ovarian tissue developed during, 400

-, mammary growth during, 372, 375

-, mydriatic blood serum in urine of, 262

Progeria, 75

Prostate, effect of castration upon, 377

-, hypertrophy of, cause of, 377

- , internal secretion of, 410

Protagon, 286, 287

- not a chemical entity, 290

Proto-adrenalin, see Adrenalin, precursors of

Pseudo-hermaphroditism, 358, 362

- , hypertrophy of suprarenals in, 269

Puberty, precocious, connection of hypertrophy of suprarenals with, 270

- , sexual development at, 369

Pulse, effect of pituitary extract on, 323-325

Pupil, action of adrenalin on, 230

-, dilatation of, effect of sympathetic system on, 210 
Pupil, dilatation under adrenalin, 209; see also Mydriasis

- of frog's eye (isolated), action of adrenalin on, 209, 210

$--\frac{-}{210}-$, oí various drugs on,

Pylorus, action of adrenalin on, 204

R.ABBIT, acute tetany in, 40

-, adrenalin contents in, 25 I

-, effects of ligature of excretory ducts of pancreas on, 436

-, extirpation of hypophysis in, 317

-, gestation in, 404

--, parathyroid glands in, 35

-, suprarenal capsules, amorphous masses in, 284

- - - in, experimental removal, 139, 141

-, thymus gland in, 113 $--\frac{1}{116}$, extirpation, results, 114,115 ,

-, toxic action of adrenalin on, 239, 240,245

Rats, effect of suppression of suprarenals on, 155

-, retractor muscle in penis of, nervous network, $22 S, 229$

-, suprarenal.extract from, toxicity, 175

-, survival after extirpation of both suprarenals, 143

-, tetany in, 41, 43

-, white, accessory intcrrenals in, 155

Reflex manifestation of diseases, 2

Renal extracts, administration, effects of, 441

- - and internal secretion of kidneys, 442

- - effect on blood-pressure, 442, 443

Renin, production of, 442

Reptiles, embryology of pineal body in, 351

-, suprarenal bodies in, 128, 130, 133

Respiration, action of adrenalin on, 235

Respiratory centre, effect of carbonic acid on, 15

- -, lactic acid and, 15

Ringer's solution, suspension of isolated arteries in, 19\$, 199

Röntgen rays, action of, on spermatic cells, 397

- - , elective destruction of tissue foundations by, is

- - influence on ovary, 403

- -, treatment by, of Graves's disease, 100

Rutting and menstruation, 403

SALIVARY glands, action of adrenalin on, 2 I I

Salt, metabolism of, 267

-- - increased by adrenalin, 215

- - effect of thyroidectomy on, 7 I

Secretin, discovery of, 439
Secretin, formation of, 5

-, function of, 439

- , nature of, 430

Secretion, distinction from excretion, 3

Secretory organs, internal, 6

- tissue elements of generative glands, $391-410$

Selachians, suprarenal capsules in, 299

Senile degeneration, 75

- and thyroid gland, 75

- - atrophy of intestinal tract present in, 76

Sera, cytotoxic action of, 19

Sertoli's cells, 394

Scrum (immune) in tetany, 56

- therapy in Graves's disease, 101

-, thyrotoxic immune, in Graves's disease, IOI

Sex, determination of, 356

Sexual characteristics, 355

- _ - , development of, 358

- - - secondary, 359

- dimorphism, $36 \mathrm{I}$

- derangement in acromegaly, 331

- glands, activity of, connection with suprarenals, 269

- -, changes in, in gigantism, 342

- - relationship to hypophysis, 33934 I

- - , secretion of, changes following suppression, how explained, 2

- organs, changes following thyroid suppression, how effected, I 10

-. - removal, changes following, how once explained, 3

- precocity, 370

- stigmata, 362, 364

Shark, interrenal body of, $15 \mathrm{I}$

Sheep, parathyroid glands in, 35

$\rightarrow$, thyroidectomized, changes in, 64

Skeleton, influence of generative glands on growth of, $3 \$ 2-384$

Soil in relation to goitre, $S_{I}$

Somatic cells, 359,362

Spasm, laryngeaî, of infants, 122

Species, characteristics of, 360

Spermatogenesis, suppression of in cryptorchidism, 393

Spermatozoa, varietics of, 356

"Spermin," 389

Sphingomyelin, 2SS, 292

Spinal cord, relationship of tetany to, 60

Splcen, action of adrenalin on, altered by increase in doses of ergot, 232

-, enlargement, following thyroidcctomy, 67

-, implantation with thyroid tissue, results, 86

- into which thyroid had been implanted, extirpation, cffects, 85

-, transplantation of suprarenal capsules into, 170

Spongiocytes, 282

- , labile fat in, $2 S_{2}$ 
Staining methods for granules of suprarenal cortex, 274

Stannius, corpuscle of, 129, 152

Status thymicolymphaticus, 122

- - enlargement of thymus in, 122, 123

- -, mortality from infective diseases great among subjects of, 124

- _, sudden death in, 122, 123

- _ - at commencement of chloroform anæesthesia in, 123

- - - in, with hypoplasia of chromaffine system, ${ }_{5} 8$

- - - symptoms, 122

Stigmata, sexual, 362, 364

Stomach, action of adrenalin on, 204

Struma suprarenalis, 270

Strychnine, neutralization by adrenalin, 293

Submaxillary glands, action of adrenalin on, 2 II

Succus entericus, 439

Sugar-contents of blood, on what dependent, $42 \mathrm{~S}$

Sugar, decomposition of, function of pancreas in relation to, 427

-., formation in liver, how controlled, 428

- - - inhibited by pancreas, 430

- - , nervous centres in relation to, 428,429

-, metabolism of, in relation to pancreas, 424,425

-, oxidation of, 426

Supernumerary ribs and vertebra, 361

Suprarenal capsules, accessory, I34, I39, 143

- - - , compensatory hypertrophy after removal of suprarenals, 268

- - - compensatory power of, 140, 146

- - - - , connection of interrenal structures with, 268

- - - tumours of female genitals proceeding from, 270

- - active substance in chemistry, 177,178 ; see also Adrenalin

- - adrenalin contents in Addison's disease, 250

- -, adrenalin in, how to ascertain amount, 182

- - , albuminoids of, 275

- - , amount of substance necessary for life, 146

- - and activity of sexual glands, connection between, 260

- -, antitoxic action, 293, 294

- - , attempted destruction by specific cytotoxins, 144

- .., blood circuiation of, in mammals, 300, 30I

- - , chemistry of (excluding adrenalin), 274-292

- - cholesterin, palmitate and stearate in, 288

- -, cholin in, 275
Suprarenal capsules, chrome-brown cells of, granules in, 252

- - colour of, 133

- -, comparative morphology and embryology, 127

- - connection with precocious puberty, 270

- - cortex of, 128,131

- - - , cells of lipoid granules, 272

- - - method of examining, 274

- - - pigment in, 274

- - - essential to life, 268,269

- - - experimental extirpation, $146-150$

- - - histology, 270-274

- - - , hypertrophy, connection with tumours, 270

- - - granular, comparative histology, 285

- - granules in, 280

- _ - - , double refractive, 283

- - - - - chemistry, 286,287

284

----7 , histology, staining of, $28_{4}$

- - - -, micro-chemical relationships, 28o

- - -, importance of, 148, I 50

__ _ and medulla, amalgamated in mammals, 130

$\rightarrow--$, methods of staining granules, 274

- - - myelin - forms, treatment with chromates, 2 So

- - - , similarity of cells to those of corpus luteum verum, 260

-. destruction without clinical signs of Addison's disease, 162

- - , development, I33

- -, disease of, see Addison's dis-. ease

- - effect of injected toxic substances on, 250

-. - extirpation, anomalies of pigmentation following, 157

......, changes in blood, 157,158 $--\frac{-}{267}$, effect on adrenal system,

- -, experimental extirpation, I 38

-. - - of one after another, finally fatal, 14 I, 142, I 45

- - - of both, survival after, 146

- $-\frac{14}{\text { - }}$, condition of blood-presure after, 147,148

- - - of one, not fatal, I4I, I 44, I 45

- - - of both at once, rapidly fatal, 138, 140, 14 I, I45

_ _ _ _ of one, followed by, compensatory hypertrophy in other, 139, 145 $--\frac{1}{\text { rat, } 143}$, not followed by death in 
Suprarenal capsules, experimental extirpation, death following, 142

$--1-$, followed by toxicity of blood, 156

- $\ldots \ldots$, followed by toxicity of muscles and viscera, 156

- - $-\frac{18}{-}$, organotherapy after, 165168

- - - - symptoms, $153-158$

- -, fat-contents, distribution, 285

- -, ferments in, 275

- -, functions of, historical account of discoveries, $124-127$

- - in relation to muscular fatigue, 295

- _ -, suppression chronic, 159

_. _ _ _, due to hæmorrhage into organ, 159 $--\frac{\mathrm{plex}, 159}{159}-$, symptom - com-

- - - in man, 158

- - - - symptoms, $153-158$

- - hypersecretion, experimental, 26o; see also Hyperadrenalism

- -, hypertrophy in pseudo-hermaphroditism, 269

- -, hypofunction, 26r

- -, hypoplastic infantilism of, 158

- - in adder, 298

- - in Batrachians, 299

- - in birds, 299, 300

- - indispensable to life, 17

- -- in fish, extirpation not fatal, 152,153

- - innervation, physiology of, 260

- in Selachians, 299

- -, lecithin in, 286

- - lipoids in, 279, 287, 292, 293

- 一, medulla of, 128,131

- - - experimental extirpation, 146-150

- - - hyperplasia of chromaffine tissue, 262

- - - quantitative value, 265

- - - - over-estimated, 265

- - - secretory processes, morphology, 252

- - - total aplasia of, 158

- - not homogeneous organs, 127

- - of pigs, distillation with hot acetone, 290, 29I

- - - lipoid substances in, 290, 291

- - of rabbits, amorphous masses in, 284

- -, physiology of, 134, 138

- -, portal system, 298, 299

- - - in birds, 300

- - - in reptiles, 299

- - primary disease, rare, 159

_- , reaction to adrenalin, 212

- - , reduction in size of, 158

- - , relationship to hypophysis, 339

- -, secretory process of, 251

-_ - sound, but with clinical signs of Addison's disease, 159, 160
Suprarenal capsules, stimulation, experimental, 260

- -, substitution, 165

- - transplantation, 168

- - - , functional capacity, 171

- - - , histological observations, 169

$--\frac{1}{-}$, regression and proliferation of tissue following, 170, 171

- - , site of, 170, I7 1, 172

- - , successful, 170

- - - - conditions for, 173

- - tuberculosis of both, typical feature of Addison's disease, 159

- - vascularization of, 298

- - , weight of, increased after castration, 269

$--\frac{-}{-}$ increase during pregnancy, 269

.. _ - in different animals, 269

-.. extract, blood-pressure increased by, how abolished, 157

- - in Addison's disease, I68

- - physiological action of, 173, 174

- -, specific action centred in adrenal body, 247

- -, toxic action, 175, I76

- -, watery, intravenous injection, I77; see also Adrenalin

- organs, accessory, 131, see also Interrenal bodies, accessory

- systems in invertebrates, tables illustrating, $135^{-1} 37$

Suprarenin, 178

-, d, toxic dose, 237

-, 1, toxic dose, 237

-, synthetic racemic, 179

Sugar in urine, see Glycosuria

Surgery, operative, knowledge as to internal secretions obtained through, i7

Sweat glands, action of adrenalin on, 214

- -, innervation of, 191, 195

TACHYCARDIA, following administration of thyroid extracts, 90

Temperature, bodily, modified by action of adrenalin, 226

Testicle, cryptorchidic, 393

-., development of , $366,369,371$

-, secretory elements of, 392

-, watery extract, 21

Testicular extract, effects of, 389

$-\ldots$, injection of, effect, 4

Tetania parathyropriva, $38,39,47$

Tetany, acute, experimental, 37, 39, 40 - -, milk diet in, 107

- _, secondary symptoms following, 43

- - , tuberculosis and, 46

-, chronic, experimental production, 43

--, experimental, albuminuria accompanying, 43 
Tetany, following extirpation of thyroid gland, 38

- parathyroidectomy, 55

-, human, 44

-, infantile, 45

- - causative factor, 120

-, localization of seat of, experiments for, 59

__ - - undetermined, 6r

-, post-operative, 44

- - treatment by parathyroid extract, 49,50

-, puerperal, 44,45

-, relationship to brain and cerebellum, 6o, 6 I

- - to spinal cord, 60

-, symptoms of, latent, 45

$\rightarrow$, toxin of, 55,56

_. - nature of, 56

- - point of attack, 59

-, treatment by blood-letting, 56

bv calcium salts, 58,59

- - by immune serum, 56

- - by thyroid extract, 50, 51

Thomsen's disease, see Myotonia congenita

Thoracic duct, excretion of lymph from, promoted by adrenalin, 213

- - ligature causing glycosuria, 431

Thymus extract, action of, I2 I

- - o on blood, 121

- - - depressant, 121

- - - toxic, 121

- - , activity not affected by boiling, 121

- gland, 110

- - , anatomy, I I I

- - cells of, not identical with those of lymph glands, II 2

- -, development, 34. II

-, enlargement in status thymicolymphaticus, 122, 123

- -, extirpation, effect on nervous system, 120

$\ldots+\ldots$, experimental, II5

$-\ldots-$, effect on ossification, I I 7 , $\therefore 18$

- - - - followed by pneumothorax, 117

- -, hypertrophy in Graves's disease, 96

- _ - accompanying cardiac hypertrophv, 19

- - implantation, experimental, 119,120

-. normal weight in man, I I 3

- -, involution, 112

- - persistent and enlarged, following thyroidectomy, 65

- -, physiology, I I3

- - reaction of motor apparatus to electric current, 120

- - relation of castration to, i 16

- - , relationship to hypophysis, 339
Thymus extract, secretion, action as disassimilatory hormone, I09, 110

- -, size in dog, guinea-pig, and rabbit, 113

Thyroglobin, 29

Thyroglobulin, 28, 3 I

Thyroid apparatus, 26

- - influence on metabolism of carbohydrates controlled by pancreas, 430

- - modification of action of adrenalin by, 226

- extract, administration after suppression of thyroid function, 86

- - - in cachexia strumipriva, 87

- - in endemic cretinism, 87

- - - followed by hyperthyroidism, $90,92,93$

- _ - - by tachycardia, 90

- - in myxœdema, $\$ 7$

- - oral, resistance to subcutaneous injection of acetonitril, increased by, 108, 109

- — - physiological effects, 89-92

- - antitoxic theory, 104, 105

- -, diuretic action, 9 I

- - , effect on metabolism, or

- - favourable influence on tuberculous processes experimentally produced, io 8

-. in myxœdema, 107

-- - neutralizing activity, I08, I09

- - , physiological effects on circulatory system, $S_{9}, 90$

- - , reduction of obesity by, 9 I

- - treatment by, mydriasis following, 2 I 1

- gland, administration, effect on metabolic changes in hypothyrosis, 88

- -, anatomy, 26

- - , antitoxic áctivity, 108

- autotransplantation, 73

- -, chemistry of, 29

- - colloid of, 27,28

- - comparative anatomy, 34-36

- - , development, 34

- - diseases of, cause of pathological conditions following, 107 effect of adrenalin on, 28

- - of castration on, 344

— - on metabólism, 70

- - of nervous excitement on, 28 .

- - - of pilocarpin on, 28

- - etiological connection with Graves's diśease, Ioo, 101

- - extirpation, followed by hypertrophy of pituitary gland, 19

- - function, secretory, negative, I04

- - - - positive, I04

- - - suppression, 105

- - histology, 26, 27

- - - influence on blood-formation, 37 
Thyroid gland, iodization process in, 105

$\rightarrow-$, iodine in, 20, 105

- ligature of vessels leading a'way from, result,' 106

- - parenchyma of, 27

- - physiological association with female organs of generation, 37

- - physiology, 62

- - - theories as to, 36,37 .

_ -, relationship to hypophysis, 338

- -, secretion, passage into lymph vessels, 28

- - , secretory processes, 27

- -, storage in, 28 substitution of, treatment by, 83

- - suppression, changes in sexual organs, how effected, 110

- -, transplantation, 84

二- -, experimental, $47,83,84$ processes following healing in, 85

- - and pancreas, relationship between, 69,70

- - and parathyroid glands, relationship between, 5 1-55

- -1 - experimental evidence, 52, 53

- - and senile degeneration, 75

- glands, accessory, 62, 63

- insufficiency in youth, 76

- -, partial, 107

- juice, intravenous injections, eyesymptoms following, 92

- preparations, treatment by, of tetany, 50, 51

- substances, Graves's disease due to flooding of organism with, 102

- tissue, implantation into spleen, results, 86

Thyroidectomy, effects of, 53, 54

- - adrenalin after, 54

-, experimental, 62,63

- - changes following, 62-71

- - resistance to inorganic poisons, small after, 108

_ - , results, 63

-, partial, 42

- necessity for, in removal of goitre, 73

-, results, $37-40$

-, symptoms following, how aborted, 84

-, total, cachexia following, 72, 73

Thyroidin, 30

-, effect on dogs, 92

Thyroparathyroidectomy, effects of adrenalin after, 54

-, results of, 68

Thyroplasia, 76

Thyrotoxalbumin, formation of, 105 , 107

Tissue-formation, elective destruction by Röntgen rays, 19

Tortoise, parathyroid bodies in, 36
Toxic substances, effect on vegetative nervous system, 195, , $\therefore, \cdots, \cdots, i$ - - chemical neutralization; 9 '

- - expulsion by excretory, organs, 8

- - injection, effect on suprarenal capsules, 250

Toxins, chemical affinity for: substances occurring in body, 9\%:

-, method of production, $105 . i$

$\rightarrow$ see also Tetany, toxin of , : : Transplantation of glands, results of, 379

- of organs, 11, 20

- - of body, see also Spleen, Thyroid, Parathyroid glands

Tritons, experimental removal of suprarenal capsules in, 141

Trypsin ferment, 439

Tryptophan, 296

Tuberculosis and acute tetany, 46

-, experimentally produced, effect of thyroid extracts on, 108

- of both suprarenal capsules, typical feature of Addison's disease, 159

Tumours, co-existence with hypertrophy of suprarenal cortex, 270

Turtle, heart of, action of adrenalin on, 202

Tyrosin and adrenalin, 165

-, conversion into adrenalin, 180

UREA, affinity of carbamic acid for, 9

- , formation by liver, 9

Ureters, ligature of, experimental, 441

Urine, acetone bodies in, increase of, see Ketonuria

-, adrenalin in, method of testing for, 182

-, examination after removal of suprarenal capsules, I 54

- of pregnancy, mydriatic substances in, 262

-, sugar in, see Glycosuria

Urohypertensin, 256

Uterus, action of adrenalin on, 206

- - - altered by increase of doses of ergot, 232

-, effect of pituitary extract on, 326

-, internal secretion of, 411

-, muscles of, action of adrenalin on, 206, 207

-, pregnant, action of adrenalin on, altered by-ergot, 232

VAGI, stimulation of, effect on bloodpressure, 329

Vagus nerve, assimilatory activitv, I4

Vas deferens, ligature of, effects of, 393,395

Vaso-constriction due to adrenalin, 184

- - to pituitary extract, 321,324

Vaso-dilator effect of adrenalin, 198

Vasomotor excitability in Graves's disease, 97 
Vegetative organs, innervation of, table showing, 190, 192, 193, 194 Vertebrates, embryology of pineal body in, 351

- lower than Batrachians, two systems of bodies replacing suprarenals in, 129

Viscera, toxicity of, produced by extirpation of suprarenals, 156

Vision, disturbances of, in acromegaly, 33 I, 337
WATER (drinking), transmission of goitre by, $8 \mathrm{I}$

Women, castration of, results of, 380 -, sexual characteristics of, 355

$\mathrm{X}$-RAYS, see Röntgen rays

YOUTH, thyroid insufficiency in, 76

ZONA glomerulosa, 270

- reticularis, 274

- spongiosa, 282, 283 


\title{
John Bale, Sons \& Danielsson, Ltd.,
}

\author{
MEDICAL PRINTERS AND PUBLISHERS.
}

\section{Books dealing with Tropical Medicine, \&c.}

The Journal of Tropical Medicine and Hygiene, issued fortnightly, price 1s. net. Annual Subscription 18s. net, post free to any part of the world. The oldest and best independent Journal of its kind.

\section{TROPICAL MEDICINE AND HYGIENE.}

Issued in Three Parts. Demy 8vo, limp cloth, gilt lettered. Price 7s, 6d. net each part; Inland, 8s.; Abroad, 8s. 3d.

Part I. (Second Edition.)

Diseases due to Protozoa. By C. W. Daniers, M.B.Cantab., M.R.C.P. Lond., and E. Wrukixson, F.R.C.S., D.P.H., D.T.M. and H.Camb., Major I.M.S.

PART II.

Diseases due to the Metazoa. By C. W. Danjels, M.B.Cantab., M.R.C.P.Lond. With an Article on Venomous Snakes by A. Alcock, C.I.E., M.B., F.R.S., Lt.-Col. I.M.S. (retd.)

\section{PART III.}

Diseases due to Bacteria and other Yegetable Parasites. Those probably associated with certain articles of diet as well as of unknown origin and others probably directly or indirectly due to meteorological and climatic conditions.

Studies in Laboratory Work. By C. W. DANIELs, M.B.Camb., M.R.C.S.Eng. Third Edition. Thoroughly revised, with many new and additional illustrations and 6 coloured plates. Demy 8 vo, cloth boards, gilt lettered. Price $16 \mathrm{~s}$. net, postage $6 \mathrm{~d}$.

The Diseases of China, including Formosa and Korea. By W. Haymtox JefFerys, A.M., M.D., and JAMES L. MAXwell, M.D.Lond. The work has been produced in the best manner and illustrated with 5 Coloured Plates, 11 Noso-Geographical Maps, and 352 other Illustrations. Price 25s. net, postage $7 \mathrm{~d}$.

The Principles of Hygiene, as applied to Tropical and Sub-Tropical Climates and the Principles of Personal Hygiene in them as applied to Europeans. By W. J. R. Srupson, M.D., F.R.C.P., D.P.H., Lecturer on Tropical Hygiene at the London School of Tropical Medicine. Demy 8vo, about 400 pp., with many illustrations and coloured plate, cloth limp, gilt lettered. Price 15s. net, postage $5 d$.

Maintenance of Health in the Tropics. By the same Author. Crown 8 vo, 119 pp., cloth. Price 2s. 6d. net, postage 3d.

A Handbook of the Gnats or Mosquitoes, including a Revision of the Anophelina. By Lievt.-CoL. GEorge M. Gires, I.M.S. (Retd.), M.B.Lond., F.R.C.S. Second Edition. Mainly intended for the use of Students of Tropical Medicine, giving the Anatomy and Life History of the Culicidæ. Cloth, gilt lettered. Price 23s. 6d. net; post free abroad, 25s.; postage $7 \mathrm{~d}$.

Climate and Health in Hot Countries, and the Outlines of Tropical Climatology. By the same Author. Medium 8vo, cloth, gilt lettered. Price $7 \mathrm{~s}$. 6d. net, postage $4 \mathrm{~d}$.

Sprue and its Treatment. By W. Garnegie Brown, M.D., M.R.C.P. Fcap. 4to, 270 pp., with two plates, cloth, gilt lettered. Price $6 \mathrm{~s}$, net, postage $4 \mathrm{~d}$.

Amcbic or Tropical Dysentery : its Complications and Treatment. By the same Author. Fcap. 4to, 271 + viii. pp., cloth, gilt lettered, price $7 \mathrm{~s} .6 \mathrm{~d}$. net, postage $4 \mathrm{~d}$.

Yenoms, Yenomous Animals and Antivenomous Serum Therapeutics. By A. Caluette, M.D., Corresponding Member of the French Institute and of the Academy of Medicine, Director of the Pasteur Institute, Lille, Translated by ERxest E. Austen. Size 9 in. by 7 in. Illustrated with all the original figures, and a Short Preface by the Author. Cloth boards, 15s. net, postage $5 d$.

83-91, GREAT TITCHFIELD STREET, LONDON, W. 


\section{JOHN BALE, SONS \& DANIELSSON, Ltd.}

Blackwater Fever (Bilious Malignant Tertian Ague). By A. G. Neweld, M.D.Glas., C.M., L.M., D.P.H.Cantab. Demy 8 ro, 127 + viii. pp. Illustrated with one Plate and Charts in the text. Cloth limp. Price 5s. net, postage 4d.

A Handbook of Practical Parasitology. By Dr. MAX Braun and Dr. M. LüHE. Translated from the German. Royal 8ro, $208+$ viii. pp. Cloth, lettered. Price 10s. 6d. postage $4 \mathrm{~d}$.

Progress Report on the Investigation of Pellagra. By Lours W. SAmpos, M.D. Crown 8vo, 126 pp., price 7s. 6d. net, postage 3d.

Some Factors Influencing Health in Tropical and Sub-Tropical Countries. By T. Gerald Garry, M.D., M.A.O., M.Ch., R.U.I. Crown 8vo, cloth, lettered. Price 2s. 6d. net, postage 3d.

Field Seryice Notes for R.A.M.C. By Major T. H. Goodwix, R.A.M.C. Crown 8vo, cloth, $94 \mathrm{pp}$. Price $2 \mathrm{~s}$. 6d. net, postage $3 \mathrm{~d}$.

The Etiology of Endemic Goitre. By Robert McCarrison, M.D., R.U.I., M.R.C.P.Lond., Major I.M.S. Being the Milroy Lectures for 1913. Price 10s. 6d. net, postage $4 \mathrm{~d}$.

\section{Books dealing with Tuberculosis, \&c.}

A Clinical System of Tuberculosis. By Dr. B. BANdelier, Medical Director to the Sanatorium Schwarzwaldheim at Schömberg, near Wildbad, and Dr. Roepke, Medical Director to the Sanatorium for Railway Workers at Stadtwald in Melsungen, near Cassel. Describing all forms of the Diseases. Translated from the Second German Edition by G. B. Huxt, M.D., B.S., late Physician to the Scarborough Hospital. Royal 8 vo, about $530 \mathrm{pp}$. and 6 coloured plates. Price $21 \mathrm{~s}$. net, postage $7 \mathrm{~d}$.

Tuberculin in Diagnosis and Treatment. By Drs. Bandelier and RoEPKE. Translated from the Seventh German Edition by W. B. Christopherson. Royal 8vo, 15s. net, postage $7 \mathrm{~d}$.

Annual Report of the Results of Tuberculosis Research, 1911. By Dr. F. KoHler, Head Physician to the Holsterhausen Sanatorium. From the Clinical Year Book, edited by Dr. Naumans, Wirkl. Geh. Ob.-Reg.-Rat und Ministerialdirektor, and Dr. M. Kinchner, Wirkl. Geh. Ob.-Med.-Rat und Ministerialdirektor and Professor. Translated by Ronald E. S. KRoHN, M.D. (Lond.). Price 7s. 6d. net, postage 4d.

Sahli's Tuberculin Treatment. By Dr. HeraranN SaHLI. Including a discussion on the Nature and Action of Tuberculin and of Immunity to Tuberculosis. Translated from the third German edition by WILFRED B. Christopherson, with an Introductory Note by Egbert MorLaxd, M.B., and B.Sc.Lond., M.D.Berne. Demy 8vo, cloth. Price 7s. 6d. net, postage $4 \mathrm{~d}$.

The Ophthalmic and Cutaneous Diagnosis of Tuberculosis (Cutaneous and Conjunctival Tuberculin Reactions according to v. PIRQUET AND Wolff-Eisner). By Dr. Wolff-Eisner, Berlin. With Introductory Note by C. Theodore Williavs, M.D. Royal 8vo, cloth, 7s. 6d. net, postage 4d.

Tuberculin Chart No. 21. Arranged for seven days, starting any day. and records fully Local, General and Focal Symptoms, also Dose and Time of Injection. Price, 60 copies, 3s.; 100, 5s.; 250, 10s. 6d.; 500, 20s.

Sanatorium Chart No. 19. This Chart has been designed by the Superintendent of a large Sanatorium in order that the particulars of each case may be kept uniformly from day to day for three months, and be available at any moment for reference. Perdozen, 1s. 3d.; per 100, $7 \mathrm{~s} .6 \mathrm{~d}$.; per $1,000,70 \mathrm{~s}$. Bound In books of 25 , with paper wrapper, $2 \mathrm{~s}$. $3 \mathrm{~d}$. each. These are bound at the head and open perfectly flat to facilitate ise.

Consumption : Its Preyention and Treatment. By E. W. Drver, M.D. Crown 8 vo., 140 pp., with 6 Illustrations, cloth, gilt lettered. Second Edition. Price 2s. 6d. net, postage 3d.

Surgery of the Lung. By C. Garrì, Professor of Surgery at the University of Bonn, and H. QU1NCKE, formerly Professor of Medicine at the University of Kiel. Second Edition, with 114 coloured and other Illustrations, and two coloured plates. Translated from the German by DAviD M. BARCroft, M.D. Royal 8vo, 258 pp. Price 12s. 6d., postage 6d.

83-91, GREAT TITCHFIELD STREET, LONDON, W. 


\section{JOHN BALE, SONS \& DANIELSSON, Ltd.}

Clinical Surgical Diagnosis. For Stúdents and Practitioners By F. DE Quervars, Professor of Surgery and Director of the Surgical Clinic at the University of Basle. Translated from the Fourth Edition by J. SNowmax, M.D. Royal 8vo, about 800 pp., with 510 Illustrations and 4 Plates. Price $25 \mathrm{~s}$. net, postage, Inland $7 \mathrm{~d}$.; Abroad $2 \mathrm{~s}$.

Epidemic Infantile Paralysis (Herne-Medin Disease). By Professor Paul H. Röner. Translated by H. Ridley Prentice, M.B., B.S.Lond., M.R.C.P. Royal 8vo, cloth, with 57 Illustrations. Price 7s. 6d. net, postage 5d.'

Orthopadics in Medical Practice. By AdoLF Lorenz and Dr. ALFRED SAXl. Authorised Translation from the German by J. C. PEEL Ritchie, Ch.M., M.D., F.R.C.S. Demy 8vo, cloth, lettered, about 300 pp., with 39 Illustrations. Price $7 \mathrm{~s} .6 \mathrm{~d}$. net, postage $5 \mathrm{~d}$.

Thompson's Compendium of the Pharmacopœias \& Formularies. A Handy Pocket Book of Reference for Medical Practitioners and Pharmacists. Fourth Edition. Revised, Enlarged and Partly Rewritten. Pp. $360+$ vi. Bound in blue leather. Price $5 \mathrm{~s}$. net, postage $3 d$.

Studies on the Influence of Thermal Enxironment on the Circulation and the Body-Heat. By EDGAR R. LYTH, M.B., M.R.C.S. Demy 8vo, cloth, lettered, with 15 Charts. Price 2s. 6d. net, postage $3 \mathrm{~d}$.

The Hunterian Oration. Delivered at THE ROYAL College of Surgeons, 1913. By Sir Rickman J. Godlee, Bart., P.R.C.S., B.A., M.S., M.D.Dub. Price 2s. 6d. net, postage 3d.

On Conjugal Happiness. Experiences, Reflections, and Adyice of a Medical Man. By Dr. L. Loewenfeld, Munich. Translated from the Third German Edition by Ronald E. S. Krohs, M.D.Lond. Demy 8vo, cloth, 7s. 6 d. net, postage 4 d.

How to Cut the Drug Bill. By A. H. Hart, M.D. Third Edition. Crown 8vo, xiv. +57 pp., cloth, lettered. Price 2s. 6d. net, postage 3d.

Some Successful Prescriptions. By A. H. HART, M.D. 1s. net, postage $1 \frac{1}{2} d$.

A Handbook on Surgery, intended for Dental and Junior Medical Students. By. ARthur S. Underwood, M.R.C.S., L.D.S., late Examiner Royal College of Surgeous of England, and BAYFoRD UNDERWood, I.R.C.P., M.R.C.S.Eng. Crown 8vo, cloth, lettered. Price 3s. 6d. net, postage $3 \mathrm{~d}$.

Studies in Clinical Medicine. By C. O. Hawthorne, M.D. Being a series of Essays and Lectures reprinted from various sources. Demy 8vo, cloth. lettered. Price $6 \mathrm{~s}$. net, postage $4 \mathrm{~d}$.

The Internal Secretory Organs: Their Physiology and Pathology. By Professor Dr. ArTur Bied, Vienna. With an Introductory Preface by Leonard Williams, M.D., M.R.C.P., Physician to the French Hospital; Assistant Physician to the Metropolitan Hospital. Translated from the German. Royal 8vo, cloth, lettered. Price 21s. net, postage 6d.

Theory and Practice of Thyroid Therapy. By Herbert Ewan WALLER, M.R.C.S., I.R.C.P. Being some experiences of the results of Thyroid medication, with deductions concerning the influence of Thyroid secretion in bealth and disease, and certain effects of drugs and various circumstances upon Thyroid secretion. A book for General Practitioners. Demy 8vo, cloth, gilt lettered. Price 5s. net, postage 3d.

Air, Light, and Sun Baths in the Treatment of Chronic Complaints. By Dr. A. Monteuvis. Translated from the French by Fred RothweLl. Crown 8vo, cloth, gilt lettered. Price 2s. net, postage 3d.

Lectures on Biology. By Dr. Curt Thesing. Translated from the Second Edition by W. R. BOELTER. With the original coloured and other illustrations. Medinm 8vo, 334 + viii. pp. 10s. 6d. postage 5d.

Modern Surgical Technique in its Relation to Operations and Wound Treatment. By C. Yelverton Pearson, M.D., M.Ch., F.R.C.S. Demy 8vo. Second Edition. Price 10s. 6d. net, postage 5d.

The Bacteriologists' Aid to Memory. A Handy Reference Sheet for the Laboratory. By J. W. S. SEccombe, M.R.C.S., L.R.C.P., D.P.H., Captain R.A.M.C. Price 3s.6d.; mounted on linen, 4s. 6d.; mounted on linen with rollers, 5s, $6 \mathrm{~d}$.

83-91, GREAT TITCHFIELD STREET, LONDON, W. 
The Campaign Against Microbes. By Étienne Burnet, M.D., of the Pasteur Institute, Paris. 'Translated from the French by E. E. Austex, F.Z.S. Demy 8ro, oloth, lettered, 5s. net, postage $4 d$.

A History of the Reading Pathological Society. By J J Mreson B. Hurry, M.A., M.D.Cantab., President of the Society. Demy 8vo, 185 pp. Cloth boards. Gilt lettering and edges, with illustrations. Price 7s. 6d. net, postage $5 d$.

The Anatomy and Topography of the Accessory Air Sinuses of the Nose in Childhood. By Dr. A. Ononr, Director of the University. Clinic for Diseases of the Throat and Nose in Budapest. An Atlas containing 102 specimens reproduced in natural size from photographs, with a preface by Dr. W. WALDEYER, Director of the Anatomical Institute of the University of Berlin. Imperial 8vo. Price 2ls. net, postage $8 \mathrm{~d}$.

Relations of the Lachrymal Organs to the Nose and Nasal Accessory Sinuses. By Prof. Dr. Oxodr. An English Translation by Dr. Dax McKenzte. With 45 plates. Price 10s. net, postage 5d.

Atlas of Killian's Tracheo-Bronchoscopy. By Dr. MANN, Translated by Thov.s Guthrie, M.B., F.R.C.S., Liverpool. 15 Coloured Plates representing Pathological Preparations from cases examined during life by means of Tracheo-Bronchoscopy. Demy folio. Price 22s. net, postage $8 \mathrm{~d}$.

A Lecture on Means for the Prolongation of Life. By Sir Hermann WEBER, M.D., F.R.C.P. Third Edition. Revised and enlarged. Demy 8vo, 222 pp., cloth, gilt lettered. Price 4s. 6d. net, postage 4 d.

The Rat Problem. By W. R. Boelter. 170 pp., fcap. 4to, price 2s. 6d. net, postage 4d. With numerous interesting illustrations.

Household Pests and Household Remedies. By the same Author. Fcap. 4to. Price 2s. 6d. net, postage 3d. With numerous illustrations.

Patent Foods and Patent Medicines. By Robert Hutchison, M.D., F.R.C.P. Two Lectures. Second Edition. Crown 8 vo, 48 pp., ls. net, postage 1 .

On Gastroscopy. By Willian Hill, B.Sc., M.D.Lond., Surgeon for Diseases of the Throat, Nose and Ear, St. Mary's Hospital, London. With a description of a New, Easy and Efficient Method of Esophago-Gastroscopy, combining Direct and Indirect Vision; and a Plea for its Employment by Gastric Experts. With 47 Illustrations. Demy 8vo, cloth. Price 3s. 6d. net, postage 3d.

Medical Gymnastics and Massage for the Treatment of Disease, Deformity and Injury. By Fredk. F. Middleweek, L.R.C.P., L.R.C.S.Ed., Director of Mechano-Therapeutics and Massage, West End Hospital for Nervons Diseases. With an Introduction by Dr. J. ARvedson, Stockholm. Crown 8vo, cloth, 2s. 6d., postage 3d.

Adenoid Growths of the Naso-Pharynx. By G. A. Garri Sinpson, M.R.C.S. Second Edition. Crown 8ro, 49 pp. Price 3s. 6d. net, postage 2d.

Notes and Thoughts from Practice. By W. J. Tyson, M.D., F.R.C.P., F.R.C.S., Hon. Physician, Victoria Hospital, Folkestone. Crown 8vo, paper cover, 2s. net; cloth, $2 \mathrm{~s}$. $6 \mathrm{~d}$. net, postage $2 \mathrm{~d}$.

Synoptic Chart of Cardiac Examination. Arranged by JoHs D. Coxrie, M.A., B.Sc., F.R.C.P.E., Assistant Pathologist, lately Clinical Tutor, Royal Infirmary of Edinburgh. Consists of Cardboard Sheath stamped with an outline of the Chest and Heart, inside of which there is a slide printed with the names of the various diseases which affect the heart, together with their physical signs. By the simple manipulation of two tapes the different diseases are made to show in a space at one side of the Chart and as each disease appears its various signs automatically come into place at the openings corresponding to the areas of auscultation. Price 2s. $6 \mathrm{~d}$. net, postage $4 \mathrm{~d}$.

Breathing Exercises. For use after Removal of Postnasal Growths (Adenoids). By Duncan Matheson Mackay, M.D., Senior Clinical Assistant, Hospital for Diseases of the Throat, Golden Square. Adapted from the Report of the Interdepartmental Committee on the Model Course of Physical Exercises, $190 \pm$ (by permission of the Controller of His Majesty's Stationery Office), and rearranged with modifications and additions. Demy 8vo, 4 pp.

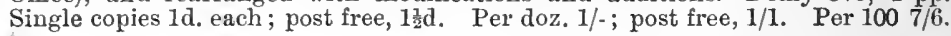

83-91, GREAT TITCHFIELD STREET, LONDON, $\mathbf{W}$. 

$\$$ \$s

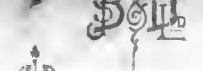<smiles>[GeH3]</smiles>

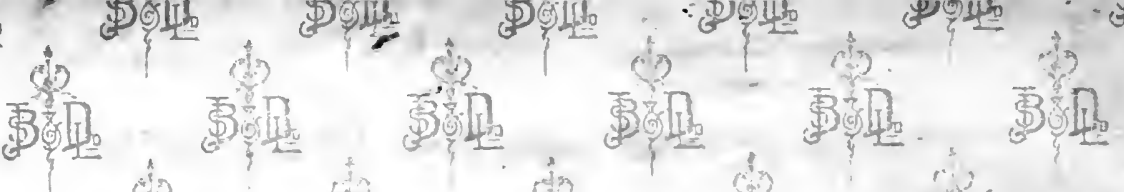

$\Phi$ DS

स्थ

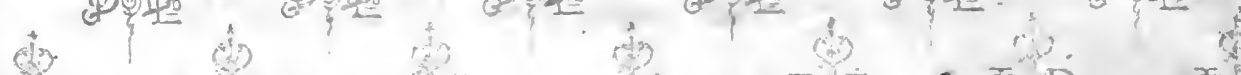

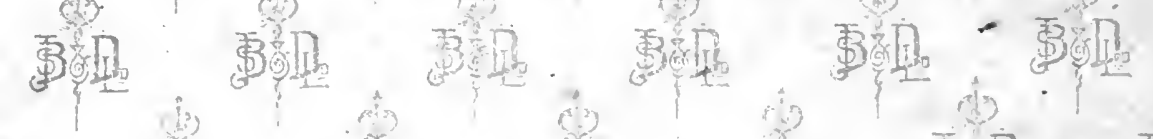

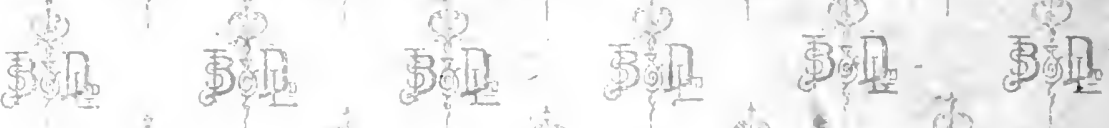

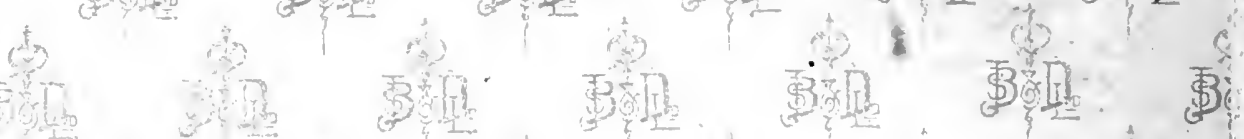
2.

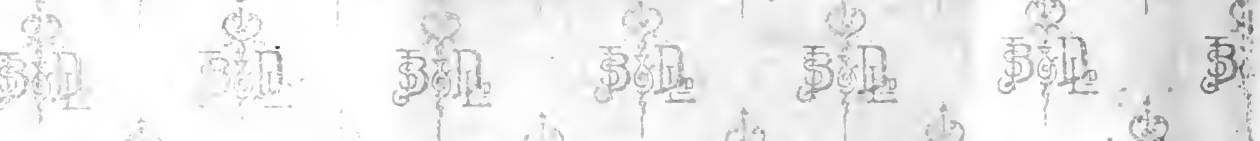
$\Phi=$ SOL

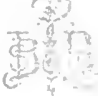

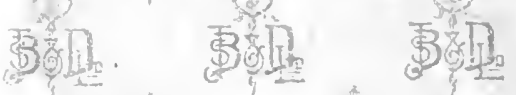

S.

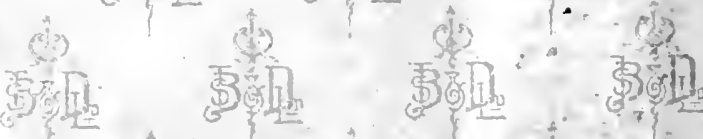
$\Leftrightarrow$ \& 1 (b)

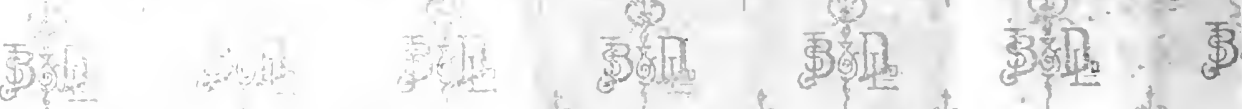
in

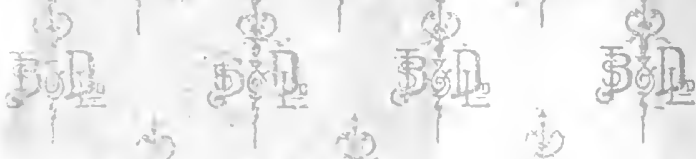

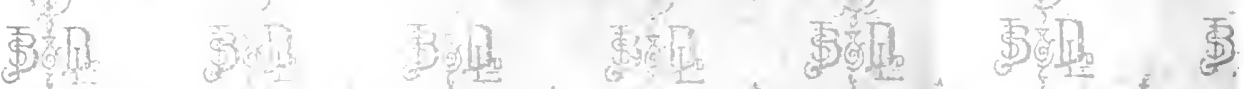

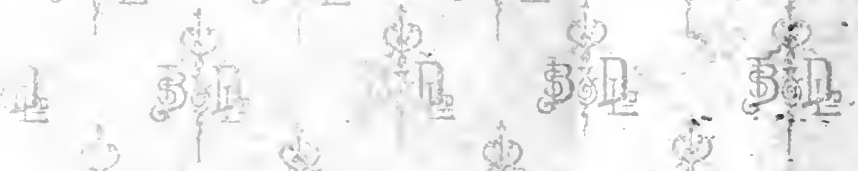
W W W

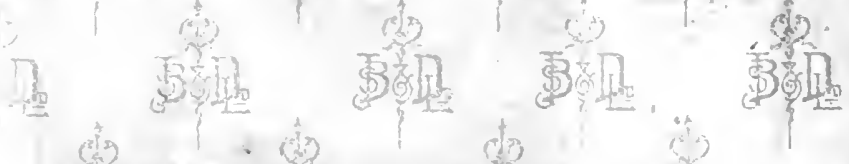

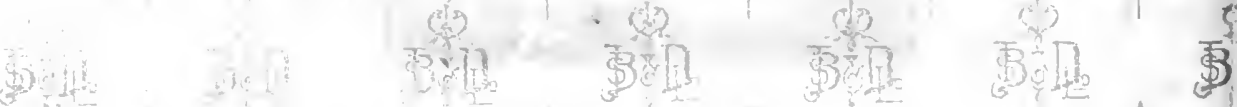
S ith. 
